

\section{DISCLAIMER}

This report was prepared as an account of work sponsored by an agency of the United States Government. Neither the United States Government nor any agency Thereof, nor any of their employees, makes any warranty, express or implied, or assumes any legal liability or responsibility for the accuracy, completeness, or usefulness of any information, apparatus, product, or process disclosed, or represents that its use would not infringe privately owned rights. Reference herein to any specific commercial product, process, or service by trade name, trademark, manufacturer, or otherwise does not necessarily constitute or imply its endorsement, recommendation, or favoring by the United States Government or any agency thereof. The views and opinions of authors expressed herein do not necessarily state or reflect those of the United States Government or any agency thereof. 


\section{DISCLAIMER}

Portions of this document may be illegible in electronic image products. Images are produced from the best available original document. 


\section{PREFACE}

Fast reactors are gradually attaining a very prominent place in the research and development programs of reactor research centers throughout the world. It is appropriate, therefore, that international conferences be held periodically to foster direct communication among fast reactor specialists. To achieve this purpose, the Argonne National Laboratory and the U. S. Atomic Energy Commission sponsored this conference, hopefully as the first in a series of annual or nearly annual conferences on various specific aspects of fast reactor science and technology. Subjectwise, this first conference was limited to the topics of breeding, economics and safety in large fast power reactors, with particular emphasis on the interrelation among them. Many other topics remain to be covered in future meetings.

In October, 1959, a related conference, "Conference on the Physics of Breeding," was held at Argonne. The proceedings of that conference were issued as report ANL-6122.

We at Argonne appreciate very much the assistance of all who participated in this conference and the cooperation given by their home organizations. We appreciate, too, the cooperation of the $\mathrm{AEC}$ in cosponsoring this program.

This proceedings has been edited by A. Glassner, Joyce Kopta and D. Okrent. Part of the transcript of the very considerable dis cussion period was found to be in incomplete form, or even garbled. In these cases the editors have tried to formulate sentences which conformed to the speakers' words and intent. In the interest of speedy publication, the speakers themselves have not been given an opportunity to edit the discussion. The editors hope that a reasonable portrayal of the actual discussion has been accomplished.

David Okrent

Program Chairman 


\section{SESSION I}

October 7, 1963

NUCLEAR DATA - REACTIVITY COEFFICIENTS

Chairman: R. Avery

Page

NEUTRON CROSS-SECTION MEASUREMENTS AT HARWELI IN SUPPORT OF

THE U. K. FAST REACTOR PROGRAMME

A. T. G. Ferguson and N. J. Pattenden, UKAEA Harwell. . . . . . . . . . 11 .

RECENT CHANGES IN HEAVY ELEMENT CROSS SECTIONS

A. B. Smith, ANL . . . . . . . . . . . . . . . . . . .

RECENT IMPROVEMENTS IN CALCULATIONS OF DOPPLER AND SODIUM REACTIVITY EFFECTS FOR LARGE FAST REACTORS

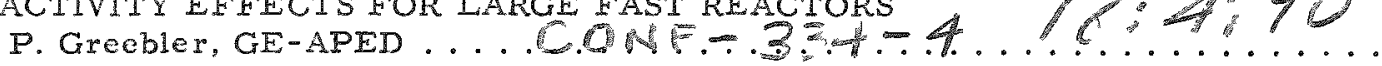

CALCULATIONS OF SODIUM VOID REACTIVITY EFFECT FOR LARGE FAST

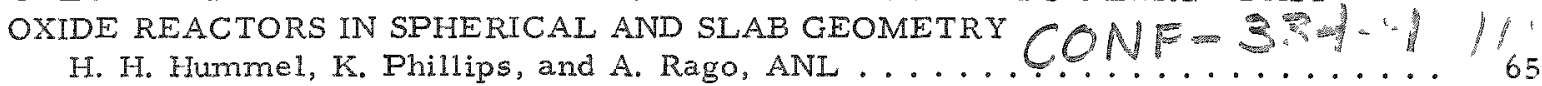

COMPARISON OF DOPPLER AND SODIUM-VOID COEFFICIENTS OF DILUTE FAST REACTORS

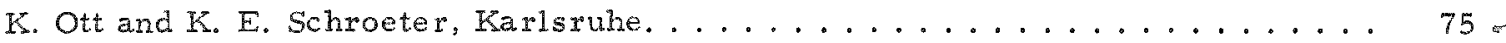

GENERAL DISCUSSION OF SODIUM VOID AND DOPPLER COEFFICIENTS

(excluding details of theoretical methods or experimental techniques for the latter). .

87

\section{SESSION II}

October 7,1963

CORE MELTDOWN AND THE MAXIMUM ACCIDENT

Chairman: D. B. Hall

MELTDOWN STUDIES

C. Dickerman, E. Sowa, L. Robinson, and D. Gopinath, ANL . . . . . . . . . 99

BRISEIS: TRANSIENT REACTOR FOR FAST REACTOR FUEL TESTING COMF- 334-6

Cl. Descamps, E. Fossoul, N. Meyvaert, M. Stiévenart, G. Tavernier, and

J. Van Dievoet, BelgoNucleaire

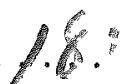

STUDY OF FAST REACTOR MELTDOWN ACCIDENTS USING SIMULANT MATERIALS

P. R. Ifuebotter, APDA. . . . . . . . . . . . . . . . 123

INFLUENCE OF THE DOPPLER EFFECT ON THE MELTDOWN ACCIDENT

B. Wolfe, N. Friedman, and D. Riley, GE-APED . . . . . . . . . . 171

PARAMETER DEPENDENCE OF AFTER-MELTDOWN ACCIDENTS

H. Böhm, Karlsruhe. . . . . . . . . . . . . . . . . . . . . 193 
FAST REACTOR MELTDOWN EXPERIMENTS

J. B. Nims, Jr., APDA. . . . . . . . . . . . . . . . . . 203 .

GENERAL DISCUSSION OF MELTDOWN PROBLEM. . . . . . . . . . . . 227

\section{SESSION III}

October 8, 1963

PERFORMANCE, FUELS AND ECONOMICS

Chairman: S. Law roski

STATUS AND INTERRELATION OF FAST BREEDER PROGRAMS CON -

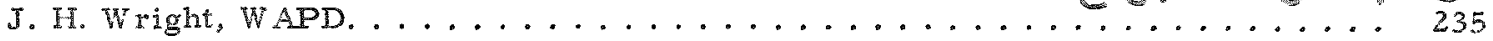

PRINCIPLES AND PROBLEMS OF THE DEVELOPMENT OF A FAST REACTOR

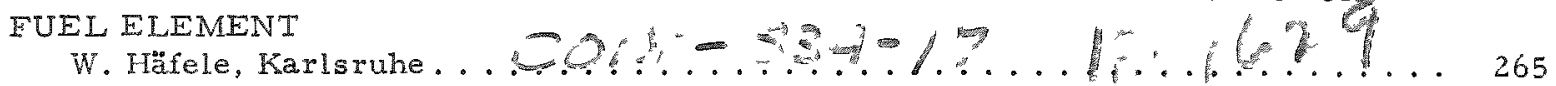

STATUS OF THE URANIUM-PLUTONIUM CARBIDE FUEL DEVELOPMENT

PROGRAM

A. A. Strasser, UNC . . . . . . . . . . . . . . . . . . . . . 297

$P_{U O_{2}}$-SS FUEL IN A FAST REACTOR

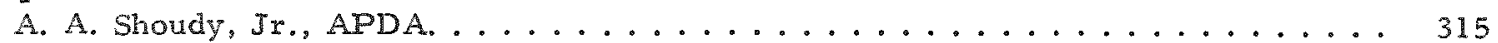

COMPARISON OF THE VALUE OF PLUTONIUM AND URANIUM ISOTOPES IN

COMPARISON OF THE VALUE OF PLUTONIUM AND URANIUM ISOTOPES IN
FAST REACTORS
A. R. Baker and R. W. ROss, UKAEA-Winfith.

EFFECTS OF AXIAL AND RADIAL BLANIET DESIGN ON BREEDING AND ECONOMICS

M. A. Perks and R. M. Lord, UKAEA-Risley ... . . . . 367

UNCERTAINTIES IN FAST POWER REACTOR DOPPLER COEFFICIENTS AND

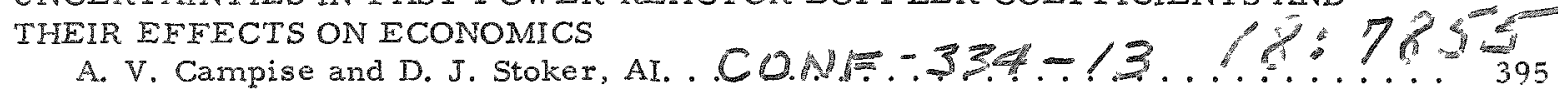

\section{SESSION IV}

October 8,1963

SAFETY AND ECONOMICS

Chairman: Manson Benedict

FAST NEUTRON BREEDER ECONOMICS

R. J. Creagan, WAPD. . . . . . . . . . . . . . . . . . . . 419

ECONOMICS AND SAFETY ASPECTS OF LARGE CERAMIC U-Th FAST BREEDER REACTORS

G. A. Sofer, A. H. Kazi, and J. Bobker, UNC . C.M.N. 
A STUDY OF THE FUTURE ECONOMIC PROSPECTS FOR FAST REACTORS

B. I. Spinrad, L. E. Link, and J. T. Weills, ANL . . . . . . . . . . .

SOME IDEAS ON THE REACTIVITY COEFFICIENTS IN THE FAST LARGE REACTORS

C. P. Zaleski, Euratom-CEA (Cadarache) ...................

REACTOR SAFETY AND CONSIDERATIONS OF FUEL-CYCLE ECONOMICS FOR FAST REACTORS

K. Cohen, P. Greebler, M. J. McNelly,

E. I. Zebroski, GE-APED

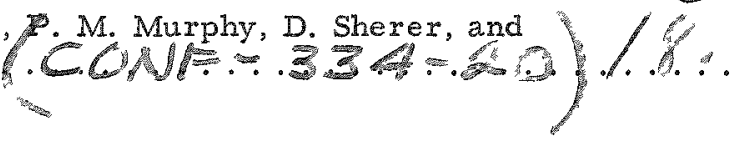

\title{
SESSION $V$
}

October 9, 1963

LESS CONVENTIONAL REACTORS

Chairman: J. R. Dietrich

OPTIMIZATION AND SAEETY OF HELIUM-COOLED FAST BREEDERS

D. Smidt, Karlsruhe. ........................ 515

SEGMENTED CORE DESIGN FOR A SUPERCRITICAL PRESSURE WATER-

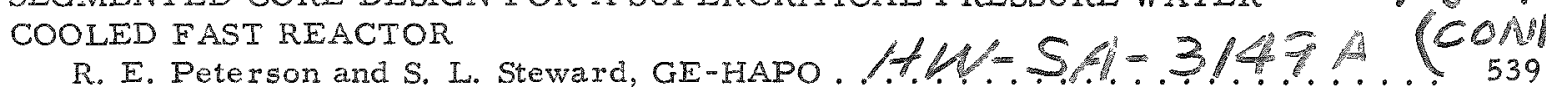

MOLTEN-SALT FAST REACTORS

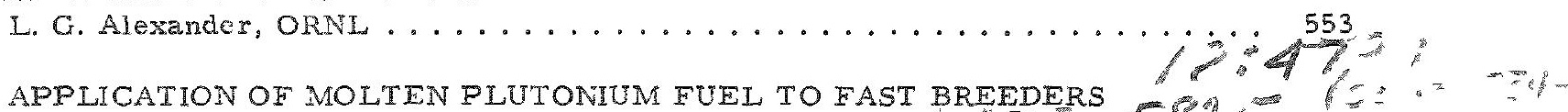

AN APPLICATION OF MOLTEN PLUTONIUM FUEL TO FAST BREEDERS
W. H. Hannum, G. L. Ragan, and B. M. Carmichael, LASL $2.57 \%$

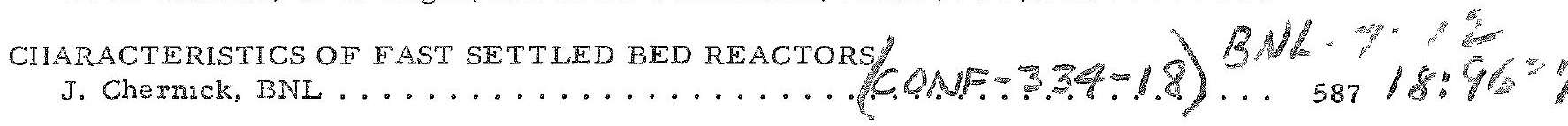

NUCLEAR PARAMETER STUDIES OF THE FAST SETTLED BED REACTOR

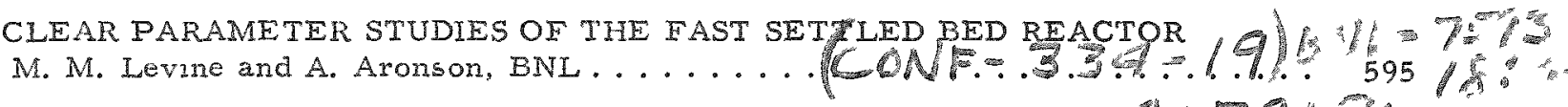

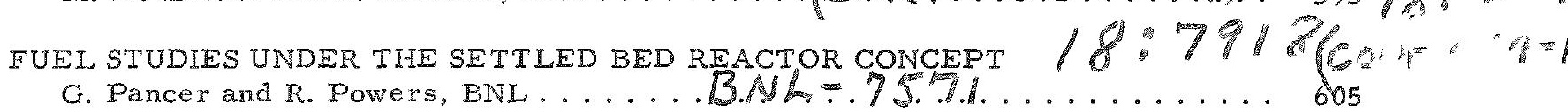

\section{SESSION VI}

October 9,1963

\author{
SAFETY - GENERAL SUMMARY \\ Chairman: D. Okrent
}

COMPARISON OF THE DYNAMIC AND SAFETY CHARACTERISTICS OF FAST AND FAST-COUPLED REACTOR SYSTEMS

A. V. Campise, AI ............................ 617 
NEUTRON CONTROLLED EXPANSION FUEL FOR FAST BREEDER REACTORS

F. M. Heck, WAPD . . . . . . . . . . . . . . . . .

FACTORS INVOLVED IN THE TIME TO START UP FAST REACTORS, INCLUDING

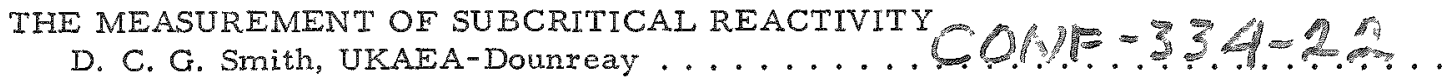

Pareshis 663

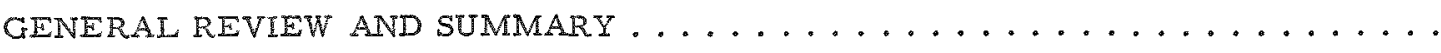

\section{SESSION VII}

October 10,1963

THEORY OF DOPPLER EFEECT

Chairman: P. Greebler

NEUTRON CROSS SECTIONS IN FISSILE ELEMENTS

D. B. Adler and F. T. Adler, University of Jllinois . . . . . . . . . . . . . 695

SOME CALCULATIONS CONCERNING THE INFIUENCE OF RESONANCE OVERLAPPING ON THE DOPPLER EFFECT IN A DILUTE FAST REACTOR

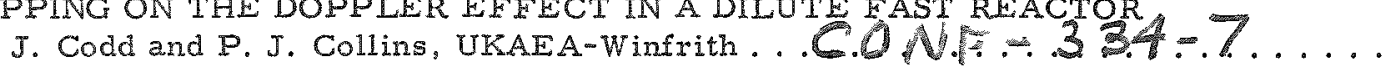

AN IMPROVED METHOD OF DOPPLER-EFFECT CALCULATION FOR FISSILE MATERIALS IN THE INTERMEDIATE ENERGY REGION

R. N. Hwang, ANI................. . . . . . . . .

EFFECT OF PARAMETER VARIATION IN DOPPLER EFFECT CALCULATIONS

H. H. Ifummel and A. L. Rago, ANL . . . . . . . . . . . . . . . . .

DEPENDENCE OF FAST REACTOR DOPPLER COEFFICIENTS ON NUCLEAR DATA UNCERTAINTIES

R. Froelich, K. Ott, and J. J. Schmidt, Karlswhe .............

CALCULATION OF DOPPLER COEFEICIENTS OF DILUTE FAST REACTORS

R. Froelich, K. Ott, and J. J. Schmidt, Karlsruhe . . . . . . . . . . .

METHODS OF EVALUATION OF EFFECTIVE CROSS SECTION FOR DOPPLER COEFFICIENT CALCULATION IN LARGE FAST BREEDER REACTORS

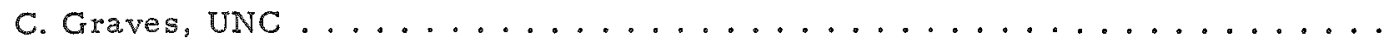

DOPPLER EFEECT IN FAST REACTORS FOR NONUNIFORM HEATING AND TEMPERATURE DISTRIBUTION

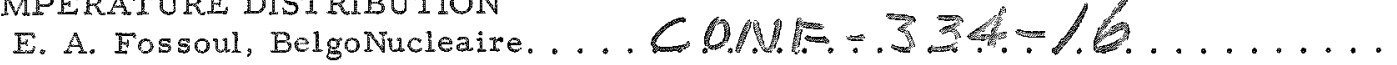


SESSION VIII

October 10,1963

DOPPLER EXPERIMENTS

Chairman: H. H. Hummel

Page

MEASUREMENTS OF THE DOPPLER COEFFICIENT IN LARGE FAST POWER

REACTORS USING A FAST CRITICAL ASSEMBLY AND AN EXPERIMENTAI

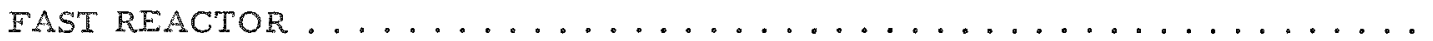

PART I. Theoretical Aspects of the Measurement of the Doppler Coefficient

in a Critical Facility

F. Storrer, A. Khairallah, and J. Ozeroff, Euratom-CEA (Cadarache)

PAR'I II. Use of Rapsodie for the Measurement of the Doppler Coefficient of Fast Power Reactors

J. Ladet, I. Puig, and L. Stevens, Euratom-CEA (Cadarache)

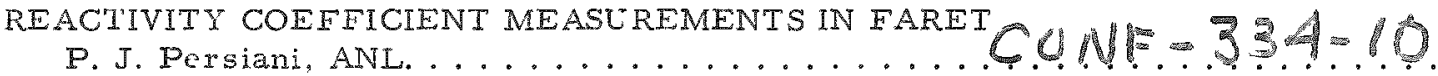

EXPERIMENTAL RESULTS FOR U ${ }^{238}$ DOPPLER MEASUREMENTS IN EAST REACTOR SPECTRA

G. J. Fischer, H. II. Ilummel, J. R. Folkrod, and D. A. Meneley, ANL . . . . . .

FAST SPECTRUM DOPPLER MEASUREMENTS OF Th ${ }^{232}$ U ${ }^{238}$, AND $\mathrm{U}^{235}$ METALS

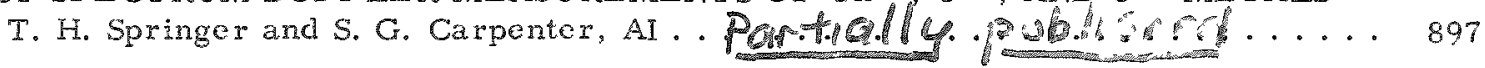

SUMMARY ON DOPPLER EFFECT .................... 907

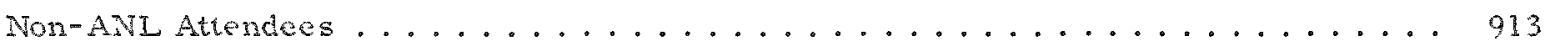




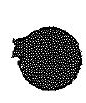




\author{
SESSION I \\ October 7, 1963 \\ NUCLEAR DATA - REACTIVITY COEFFICIENTS \\ Chairman: R. AVERY \\ Argonne National Laboratory \\ Secretary: P. I. Persiani \\ Argonne National Laboratory
}


- 


\author{
A. T. G. Ferguson and N. J. Pattenden \\ UKAEA, Atomic Energy Research Establishment \\ Harwell, England \\ (Paper presented by Mr. Ferguson)
}

Introduction

This paper describes measurements of neutron cross sections made at Harwell in support of the $U$. $K$. fast reactor programme. These meas urements include $\bar{\nu}(E)$, cross soctions for inelastic scattering in the $\mathrm{MeV}$ region, and also total, fission, and capture cross sections in the resonance region. The latter reflects our interest in large reactors with soft spectra with attendant worries over Doppler and other coefficients.

Measurements of $\bar{\nu}(E)$. (M.W. Sowerby, D. W. Colvin)

The most basic measurement to the whole programme is a precision measurement of $\bar{\nu}$. This is being made by Sowerby and Colvin using the Boron Pile, (1) a large block of reactor graphite with $B F_{3}$ counters in the channels (see Fig. 1). A fission chamber is placed at the centre,

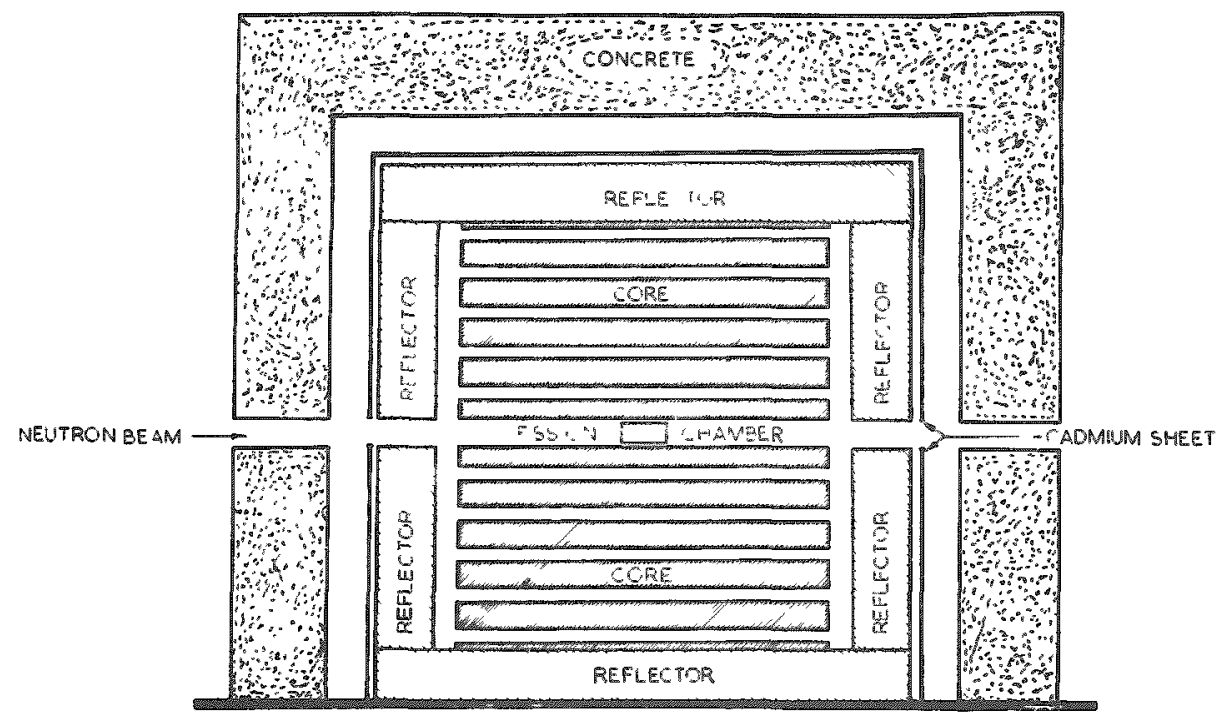

Fig. 1. The "Boron Pile"

*Including work by D. W. Colvin, M. Jo Sowerby, E. Barnard (un leave from South African Atomic Energy Board), I. J. Van Heerden, R. McMurray (both on attachment from Southen University Research Institute, South Africa), C. A. Utiley, F. D. Brooks, M. C. Mosun, and G. D. James. 
and the number of neutron counts associated with a fission pulse is determined. To obtain $\bar{\nu}$, it is necessary to know the efficiency of the device for neutrons of the fission spectrum. The outstanding features of this experiment have been

(a) that a direct experimental measure of the efficiency $\epsilon$ has been made:

(b) all the corrections that are necessary have either been calculated exactly or verified experimentally.

The efficiency has been measured for a number of points between $190 \mathrm{keV}$ and $4.9 \mathrm{MeV}$ and found to be constant. This was done using the photodisintegration of deuterium in an ion chamber placed at the centre of the pile. This process releases a neutron of well-defined energy into the pile and an associated proton in the chamber. The detection of a proton in the chamber opens a gate for $4 \mathrm{~ms}$ during which time most neutrons are captured. After a delay of $15 \mathrm{~ms}$, another 4 -ms gate is opened to count background. Corrections are made for
(a) count rate and overlap;
(b) neutrons captured after the prompt gate;
(c) variation of efficiency with position and direction of emission;
(d) effect of ion chamber, etc, on the efficiency.

Care has been taken over interference and effects of modulation of the Van de Graaff beam used to excite the required $\gamma$ radiation. With these precautions it is estimated that $c$ is known to $\pm 05 \%$.

The final results are being evaluated with an IBM 7090 computer, but results available at present are quoted below. The final values should not be altered appreciably, but errors in the absolute measurements will be reduced.

\begin{tabular}{lc}
\multicolumn{2}{l}{ Absolute Measurements } \\
$\bar{\nu}\left(\mathrm{Cf}^{252}\right)$ & $3.76 \pm 1 \%$ \\
$\bar{\nu}_{\text {th }}\left(\mathrm{U}^{235}\right)$ & $2.42 \pm 1 \%$ \\
Thermal Fission Data & (Ratios) \\
\hline $\mathrm{U}^{233} / \mathrm{U}^{235}$ & $1.020 \pm 0.006$ \\
$\mathrm{Pu}^{239} / \mathrm{U}^{235}$ & $1.182 \pm 0008$ \\
$\mathrm{Pu}^{240} / \mathrm{U}^{235}$ & $0.888 \pm 0.005$
\end{tabular}




$$
\begin{array}{ll}
\mathrm{Cf}^{252} / \mathrm{U}^{235} & 1.554 \pm 0.008 \\
\mathrm{Pu} & 1.206 \pm 0.011
\end{array}
$$

Fast Fission Data [Based upon $\left.\bar{\nu}\left(\mathrm{Cf}^{252}\right)=3.770\right]$

\begin{tabular}{c|c||c|c}
\hline $\bar{\nu}\left(\mathrm{U}^{235}\right)$ & $\begin{array}{c}\text { Neutron Energy } \\
(\mathrm{MeV})\end{array}$ & $\bar{\nu}\left(\mathrm{U}^{235}\right)$ & $\begin{array}{c}\text { Neutron Energy } \\
(\mathrm{MeV})\end{array}$ \\
\hline $2.478 \pm 0.027$ & $0.101 \pm 0.060$ & $2.534 \pm 0.018$ & $0.946 \pm 0.128$ \\
$2.524 \pm 0.045$ & $0.514 \pm 0.054$ & $2.583 \pm 0.017$ & $1.497 \pm 0.109$ \\
$2.511 \pm 0.023$ & $0.571 \pm 0.156$ & $2.668 \pm 0.019$ & $2.123 \pm 0.095$ \\
$2.501 \pm 0.029$ & $0.572 \pm 0.015$ & $2.717 \pm 0.023$ & $2.572 \pm 0.085$ \\
$2.519 \pm 0.023$ & $0.604 \pm 0.053$ & & \\
\hline
\end{tabular}

Elastic and Inelastic Neutron Scattering. (A. T. G. Ferguson, I. J. Van Heerden, R. McMurray, E. Barnard)

A programme of elastic and inelastic scattering is being carried out with our pulsed 3-MeV Vande Graaff (IBIS). This machine produces pulses of protons $\sim \frac{1}{2}-1$ ns long, with a peakcurrent of $\sim 10$ ma. The repetition rate is only $1 \mathrm{M} / \mathrm{C}$ which, though it reduces our average current, avoids over lap problems when neutron spectra extending to low energies are being studied. The neutron detector used has an efficiency extending down to a threshold at $-50 \mathrm{keV}$.

Figure 2 shows the layout of the machine when used for a scattering experiment. Most of the work so far has used neutrons from the

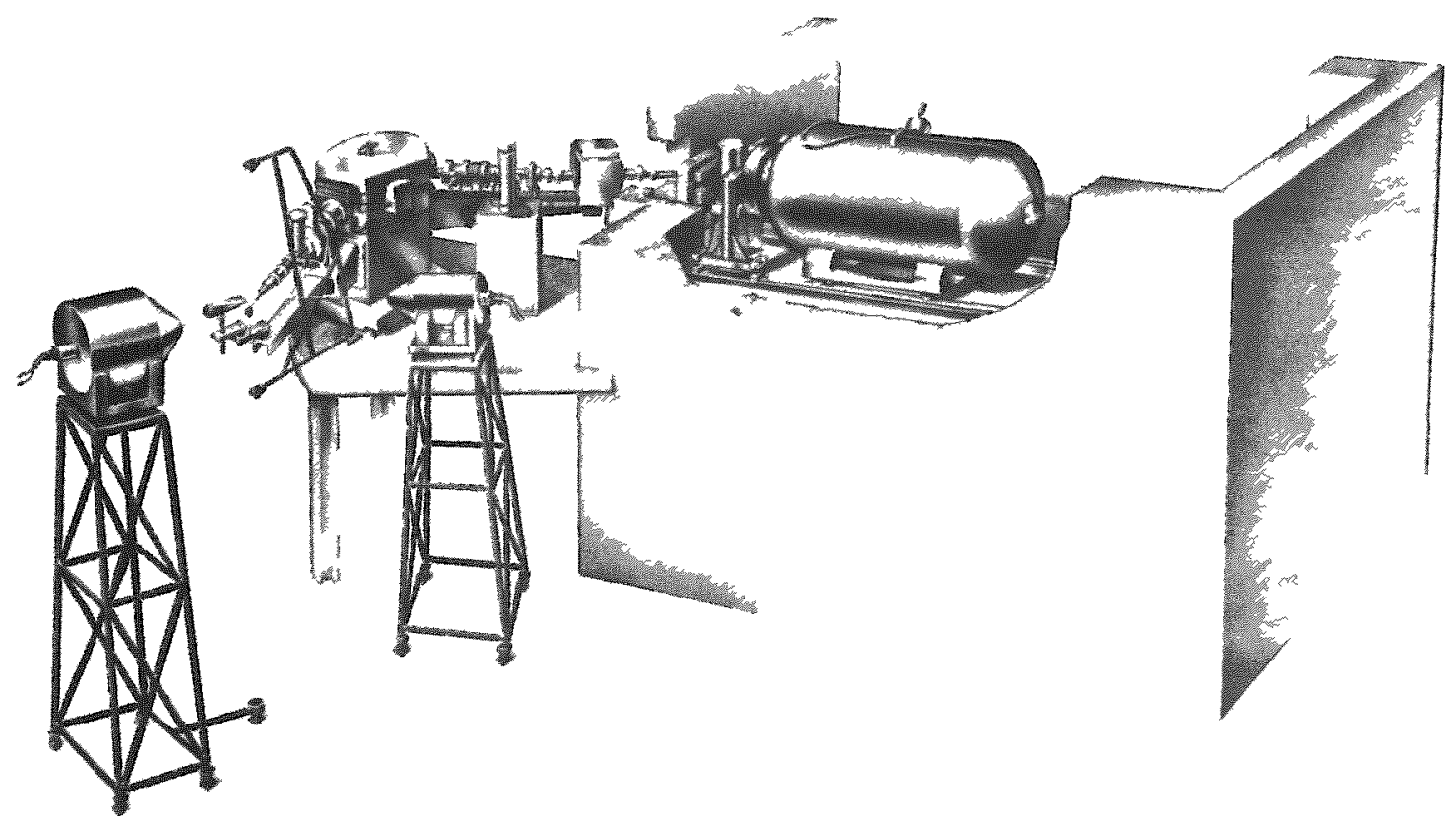

Fig. 2. The Lay out of "IBIS" - $3 \mathrm{MeV}$ Pulsed Van de Graff. A scattering experiment is set up. 
Li $(p, n)$ reaction with targets $\sim 5-8 \mathrm{keV}$ thick. In this work it has been possible to measure the cross sections absolutely by direct observation of the incident and scattered fluxes.

In the case of inelastic scattering the ratio of efficiency at incident energy and at that of the scattered neutrons is required. This has been obtained in a separate series of experiments by comparison of the observed and calculated angular distribution of neutrons scattered from hydrogen (as polythene). This has been cross checked by direct comparison with a calibrated "long-counter." Scattering results are corrected for effects of multiple scattering by the "Maggie" Monte-Carlo programme developed at AWRE by J.B. Parker et al. (2)

A typical application of these techniques has been a study of elastic and inelastic scattering from $U^{238}$ over the energy range from $0.075 \mathrm{MeV}$ to $1.40 \mathrm{MeV}$. Spectra of neutrons scattered from $U^{238}$ at a number of neutron energies are shown in Figs. 3, 4, and 5. In addition to the groundstate rotational band, widely studied $(3,4)$ levels have been observed at 680 and $730 \mathrm{keV}$ corresponding to the 1 - and 3 - levels found in Couloumb excitation. A further weak level has been seen at $832 \mathrm{keV}$, probably the 5 - member of this band. At above $900 \mathrm{keV}$ very many levels have been distinguished and appear consistently at all bombarding energies and angles. These occur at $937,960,1000,1039,1061,1104,1123,1161,1177$, 1215,1266 , and $1306 \mathrm{keV}$, and with less certainty at 1235,1346 , and $1375 \mathrm{keV}$.

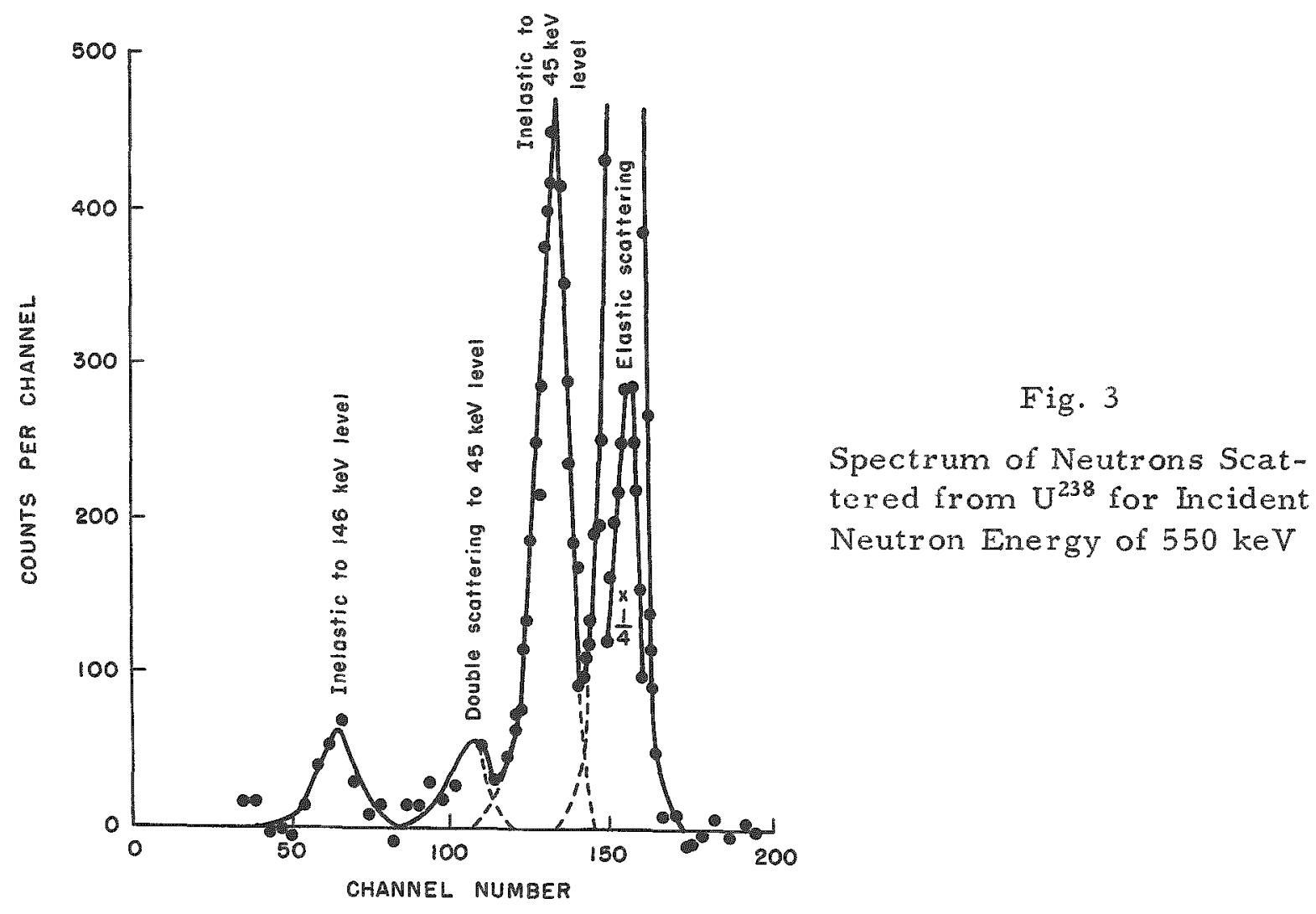




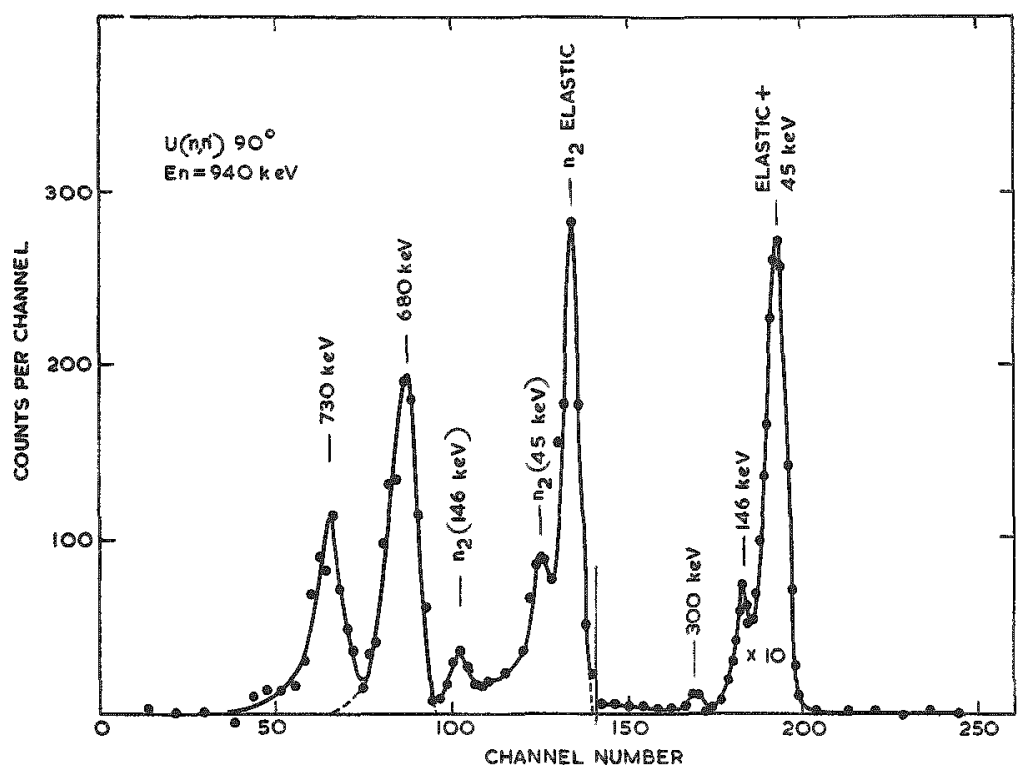

Fig. 4. Spectrum of Neutrons Scattered from $\mathrm{U}^{238}$ for Incident Neutron Energy of $940 \mathrm{keV}$

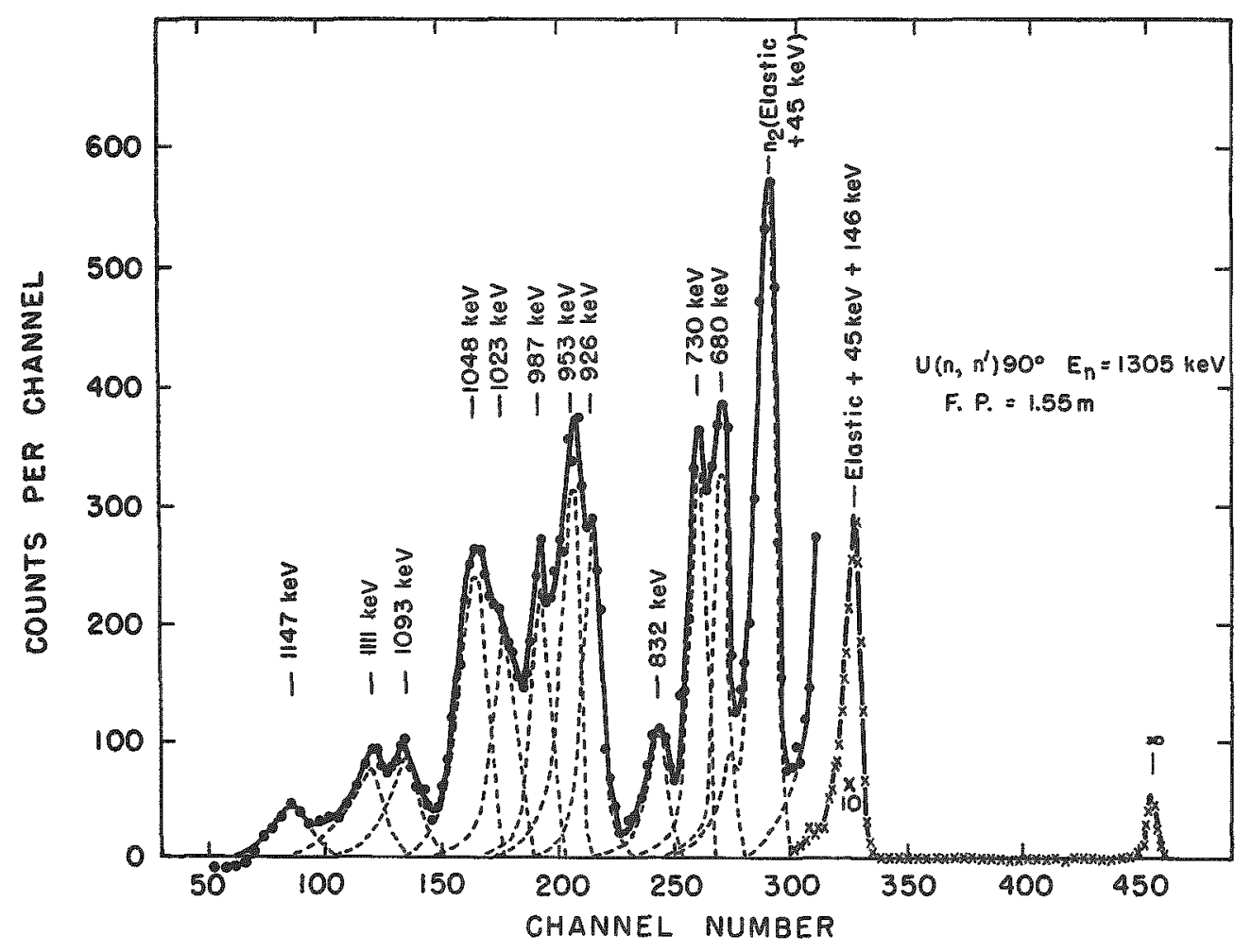

Fig. 5. Spectrum of Neutrons Scattered from $U^{238}$ for Incident Neutron Energy of $1305 \mathrm{keV}$ 
There is some correspondence between many of these levels and those identified with $\beta$ and $\gamma$ vibrational bands in $U^{238}$ by the Couloumb excitation studies of Elbeck et al. (4a) at UCRL, though many additional levels are seen.

Exploiting the low-energy sensitivity of our detector, angulax distributions of elastically scattered neutrons have been measured at 75, 157, 250,405, and $550 \mathrm{keV}$ (see Fig. 6). At $550 \mathrm{keV}$, the Harwell results can be compared with those of both Cranberg(3) and Smith, (4) all three sets agreeing to within $2-3 \%$. These results have been fully corrected for multiple scattering and flux attenuation in the sample though minor corrections may still be made.
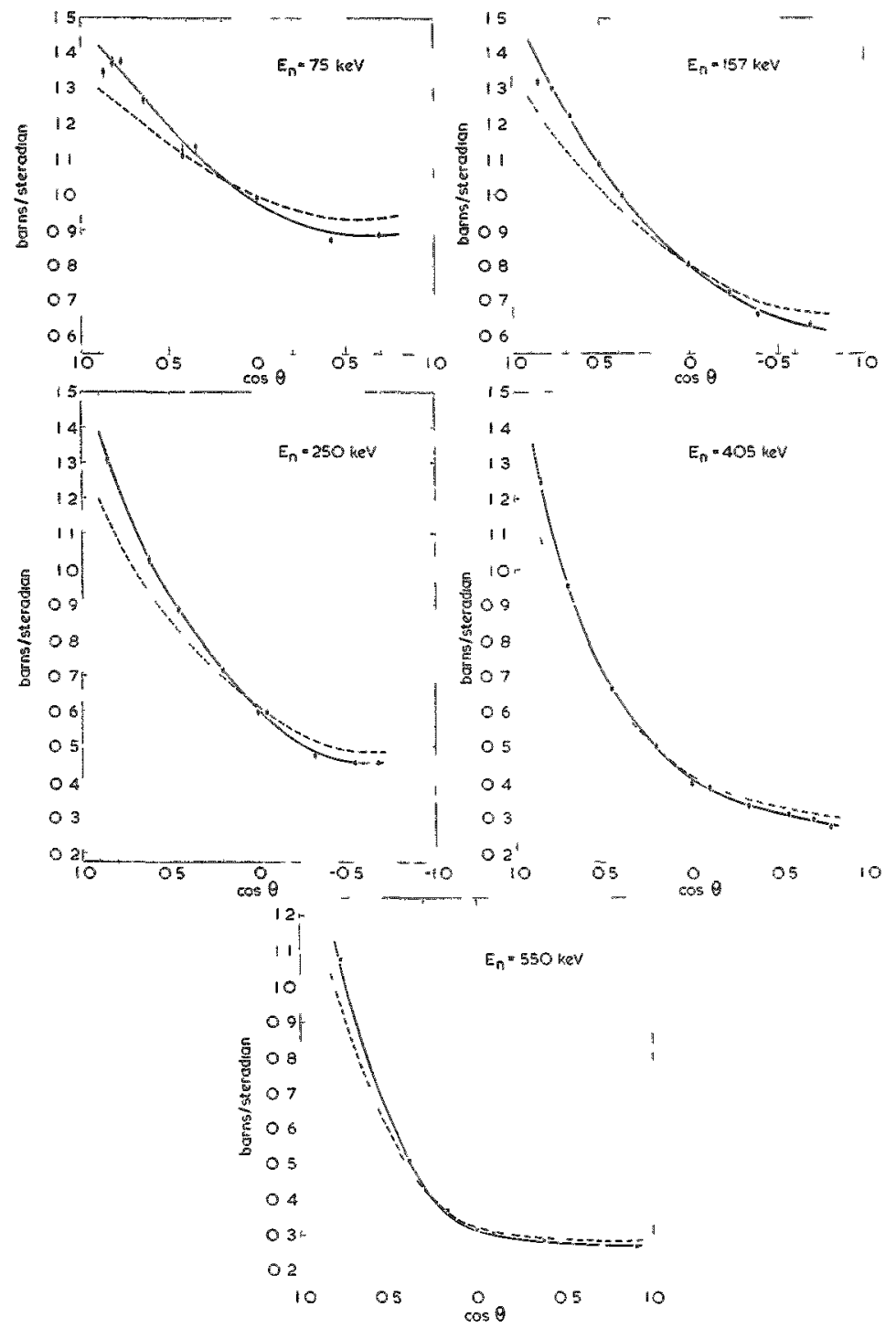

Fig. 6. Angular Distributions of Neutrons Elastically Scattered from $\mathrm{U}^{238}$, Corrected for Multiple Scatterng. The dotted line shows the cross sections before currection. 


\section{Inelastic Scattering}

The $90^{\circ}$ cross sections for excitation of the $45-\mathrm{keV}$ and $146-\mathrm{keV}$ levels have been measured from $120 \mathrm{keV}$ to $700 \mathrm{keV}$. These results, not corrected for multiple scattering, are shown in Fig. 7. The multiplescattering correction varies these values by $\sim 5-10 \%$. At $550 \mathrm{keV}$, detailed angular distributions have been measured. A less detailed angular distribution has been measured at $157 \mathrm{keV}$, which shows isotropy within the accuracy of the experimental results. A small anisotropy at $550 \mathrm{keV}$ agrees in detail with that reported by Smith. (4) When multiple-scattering corrections are applied, our cross sections at $550 \mathrm{keV}$ agree well with those of Cranberg. (3)

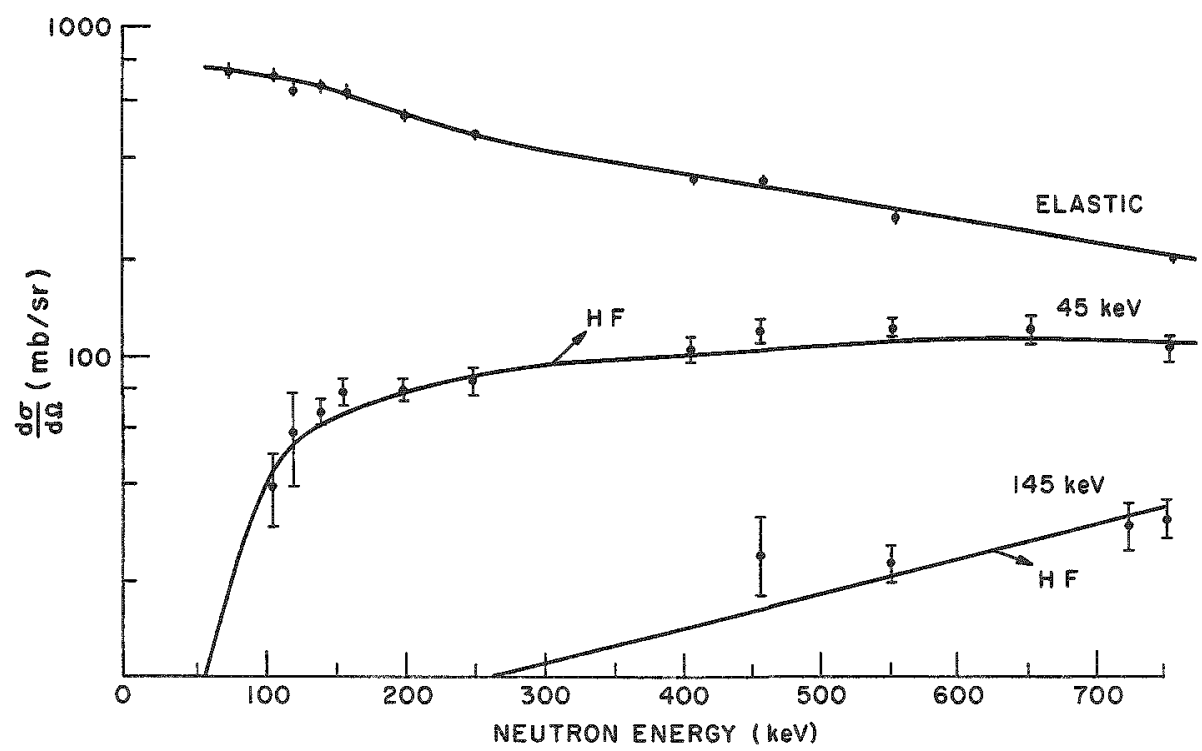

Fig. 7. Preliminary Values of the Differential Cross Section at $90^{\circ}$ (lab) for Inelastic Scattering of Neutrons to the $45 \mathrm{keV}(2+)$ and $146 \mathrm{keV}(4 t)$ Levels in $\mathrm{U}^{238}$. No multiple scattering corrections have been applied to these results.

Inelastic scattering to the higher levels discussed above has been studied in some detail. The cross sections for inclastic scattering through $90^{\circ}$ to these levels have been measured at $\sim 50-\mathrm{keV}$ intervals from $800 \mathrm{keV}$ to $1400 \mathrm{keV}$. In addition, angular distributions have been measured, our preliminary analysis of which shows isotropy of all groups within the errors of the measurements. The results of these measurements are shown in Fig. 8 (not corrected for multiple scattering). It is difficult to compare these more detailed cross sections with these of earlier workers, due to differences in resolution. There is, however, no obvious discrepancy.

A final assessment of the errors of these measurements is still to be made, but it is anticipated that for the elastic and inelastic scattering 
to the ground-state rotational band, they will be $\lesssim 6 \%$; at higher energies and excitations, errors of individual partial cross sections vary with reso-
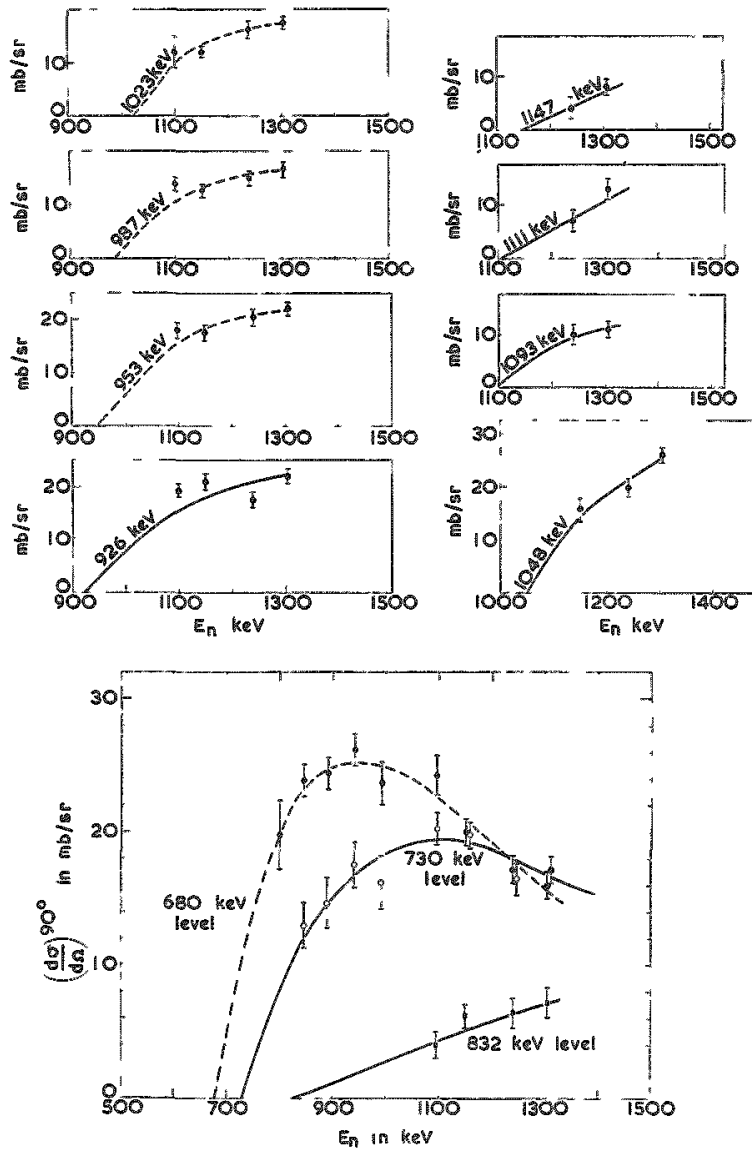

Fig. 8. Preliminary Values of the Differential Cross Sections at $90^{\circ}$ (lab) for Inelastic Scettering of Neutrons to the Higher Levels of $\mathrm{U}^{238}$. These results have not been currected for multiple scattering. lution and intensity, but will give errors for the sum of the partial inelastic cross sections of $\sim 5 \%$.

For the future a major part of our programme will be a series of measurements on similar lines to those with $\mathrm{U}^{238}, \mathrm{U}^{235}$, and $P \mathrm{u}^{239}$.

We had hoped to be able to report measurements which we have made on the spectrum of neutrons from $\mathrm{U}^{238}$ fission, but measurements at incident neutron energies of 2 and $5 \mathrm{MeV}$ have only just been completed and await analysis. For comparison, neutron spectra following fission of $\mathrm{U}^{235}$ and $\mathrm{Pu}{ }^{239}$ by $30-\mathrm{keV}$ neutrons have been measured.

\section{Measurements with the} Harwell Linac

As fast reactors become larger, the importance of the energy region below $70 \mathrm{keV}$ increases, particularly from the point of view of Doppler coefficients.

The Harwell 28-Mev electron linear accelerator, used as a pulsed neutron source, has the main responsibility for satisfying the nuclear data requirements of the UKAEA reactor program for this energy region. The machine has been described by Poole and Wiblin (5) and has provided an electron pulse $200 \mathrm{nsec}$ wide with about $400 \mathrm{~mA}$ in the pulse at a repetition rate of 200 pulses per second, in typical operation over the past year. A subcritical $U^{235}$ assembly with mercury cooling ("booster") is used as target, giving a neutron burst of about $250-n s e c$ width, and a multiplication of about 10 in the neutron yield. The neutrons are partially moderated by about $2 \mathrm{~cm}$ of water. Up to 10 flight paths are available, of lengths from 5 to $300 \mathrm{~m}$, on which different time-of-flight experiments may be performed simultaneously. 
Total Cross Section of $\mathrm{U}^{235}$ below $70 \mathrm{keV}$. (C. A. Uttley)

A flight path of $120 \mathrm{~m}$ was used, giving a resolution of about $3 \mathrm{nsec} / \mathrm{m}$, to measure the total cross section of $\mathrm{U}^{235}$ below $70 \mathrm{keV}$. The transmission of four samples of $U^{235}$ with different thicknesses was measured over a neutron energy range from $100 \mathrm{eV}$ to $70 \mathrm{keV}$. Values of the $s$ - and $p$-wave neutron strength functions and the effective potential scattering cross section have been extracted from the data by average cross section analysis.

The experimental total cross-section points, averaged over suitable energy intervals, are shown in Fig. 9. Also shown are three curves, calculated from the parameters given,
a) being the contribution of the potential scattering cross section;
b) being the sum of potential scattering and s-wave contributions;
c) being the sum of the potential scattering, s-wave and p-wave contributions.

Since the sensitivity of the total cross section to these three quantities varies over the energy range covered, three independent values for the contributions may be obtained by an iterative process. In the region below

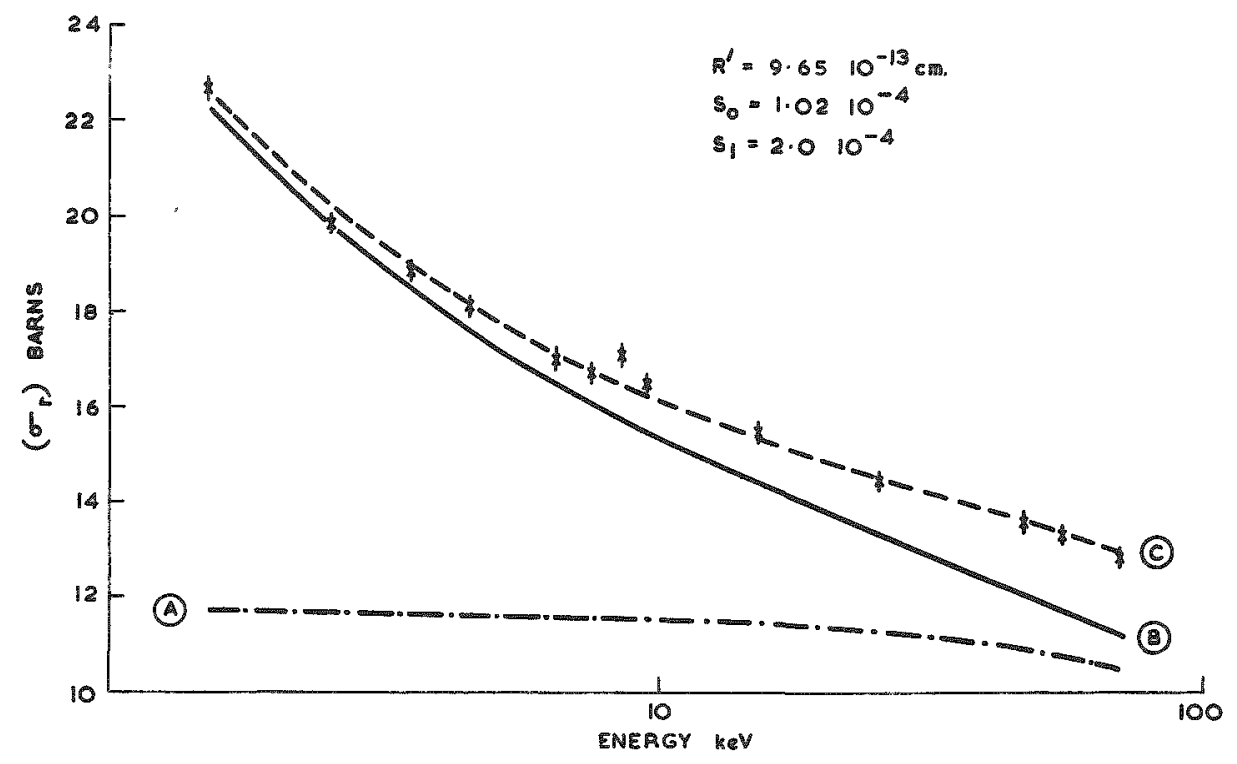

Fig. 9. Average Total Cross Section of $\mathrm{U}^{235}$. Curve A potential scattering cross section. Curve B average total s-wave and potential scattering cross section. Curve C - average total cross section. 
$5 \mathrm{keV}$, the $\mathrm{p}$-wave effect is small and the potential scattering is essentially energy-independent. Thus, initial estimates of $S_{0}$ and $\sigma_{p}$ may be made, which can in turn be used to give an initial estimate of $S_{1}$ in the region of higher energy. This value was then used in the complete expression for total cross section to get new values for $\sigma_{p}$ and $S_{0}$. The degree of sensitivity to these quantities is illustrated in Fig. $10 ; S_{0}$ is varied until the

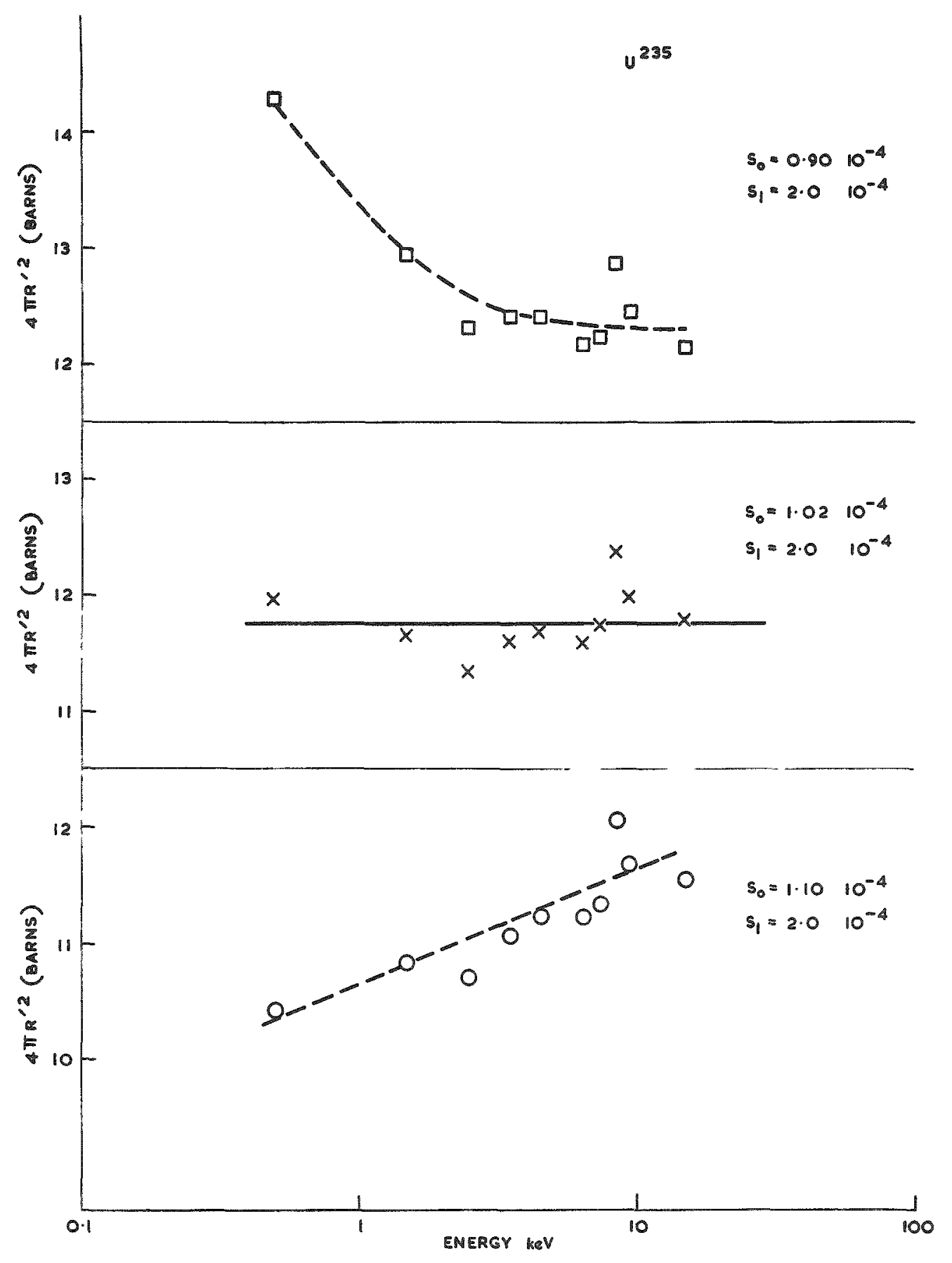

Fig. 10. Determination of Potential Scattering Cross Section and $s$-wave Strength Function 
experimental points, with the strength-function contributions removed, sit on a horizontal straight line. An estimate of the uncertainty for the value of $S_{0}$ obtained in this way was arrived at by looking at the region below $2 \mathrm{keV}$ in $100-\mathrm{eV}$ intervals and determining a variance of $S_{0}$ from the distribution of the separate values. The final results obtained were

$$
\begin{aligned}
& S_{0}=(1.02 \pm 0.03) \times 10^{-4} ; \\
& S_{1}=(2.0 \pm 0.3) \times 10^{-4} ; \\
& \sigma_{p}=(11.7 \pm 0.1) \mathrm{b}
\end{aligned}
$$

The data have been corrected for potential and compound elastic scattering to produce the average absorption cross section. In estimating the compound elastic scattering, the term

$$
\left\langle\frac{\Gamma_{n}^{2}}{D\left(\Gamma_{n}+\Sigma_{j}+F^{2}\right)}\right\rangle
$$

is required, but the better known quantity is

$$
\text { D. } \frac{\Gamma_{n} \nu^{2}}{\left(\Gamma_{n}+\Gamma_{\gamma}+\Gamma_{F}\right)} \text {. }
$$

The ratio of the two is called $S_{j}$, for which values of 1 and 2 have been separately assumed. The two sets of derived average absorption crosssection values have been used together with the Saclay fission crosssection data to obtain values for $\alpha$ in $1-\mathrm{keV}$ steps up to $10 \mathrm{keV}$. These are shown in Fig. 11 and compared with other data. $(6-8)$ The value of 1 ior $\mathrm{S}_{\mathrm{j}}$ seems to be favoured.

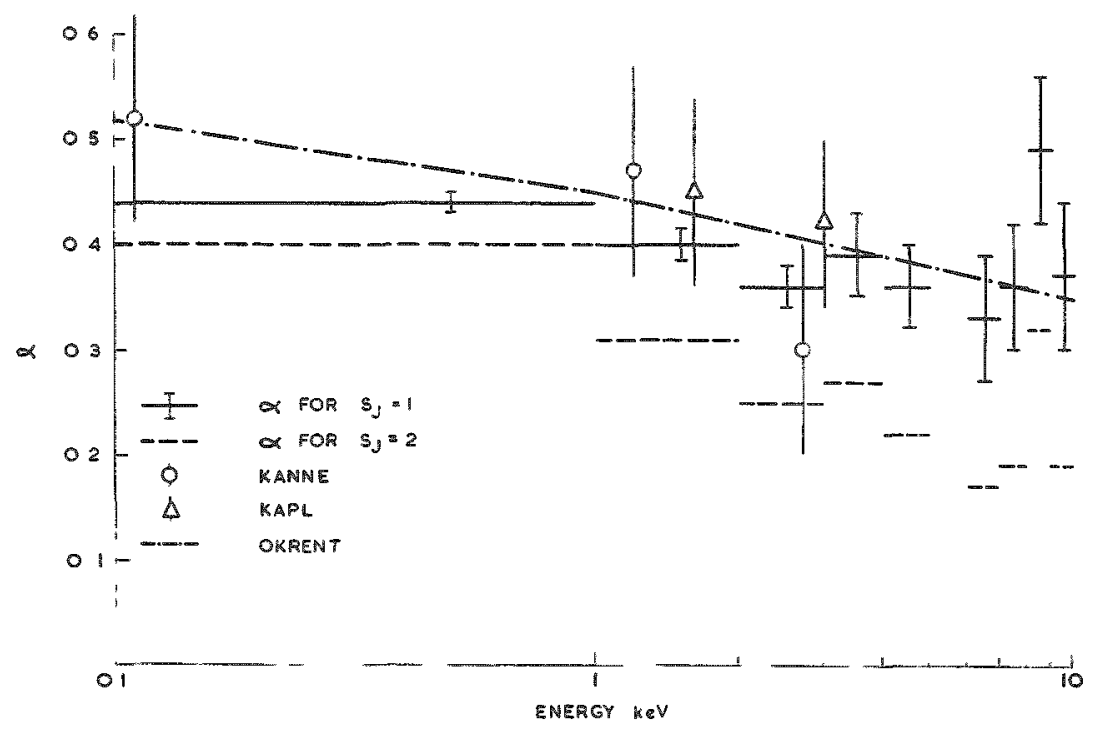

Fig. 11. Variation of a $U^{235}$ with Energy 
Low-energy Eission Cross Section of $\mathrm{U}^{235}$ (F. D. Brooks)

The use of the Saclay fission cross-section data in the above anal$y$ sis underlines the importance of the low-energy (below $10 \mathrm{eV}$ ) region, where the Saclay data agree with those of Shore and Sajlor, (9) but not of Bowman et al. (10) The low-energy part of an experiment to measure $\eta$ and the fission cross section over a wide energy range has been completed. The method uses fission-neutron detection, and four different sample thicknesses have been employed. A flight path of $22.4 \mathrm{~m}$ was used, with a resolution of $44 \mathrm{nsec} / \mathrm{m}$. Corrections are made for self-screening and multiple scattering (with no energy loss) in the sample, and these corrections, together with counting statistics, provide the main uncertainty in the measurement. In the region below $10 \mathrm{eV}$, the data agree closely with those of Shore and Sailor. The total area under the fission cross-section curve between 5 and $10 \mathrm{eV}$ is within $2 \%$ of the similar area of Shore and Sailor: the similar area of the data of Bowman et al. is about $8 \%$ lower.

Fission Cross Section of $\mathrm{Pu}^{239}$ up to $160 \mathrm{keV}$. (G. D. James)

A $15-\mathrm{m}$ flight path was used, giving a resolution of $17 \mathrm{nsec} / \mathrm{m}$. The fission detector was a gas scintillation counter, containing $40 \mathrm{mg}^{\circ}$ P $\mathrm{u}^{239}$ on a foil. The foil was oriented at $78^{\circ}$ to the neutron beam to keep the photomultiplier out of the beam and to give a better pulse-height spectrum. The neutron spectrum was measured with a $B F_{3}$ counter. A correction was made for the deviation of the $B^{10}(n, \alpha)$ cross section from $1 / v$ above $50 \mathrm{keV}$. Background measurements were made at spot energies with "black resonance" filters up to $35.2 \mathrm{keV}$ (Al). The background had a time-dependent component which was fitted by a power law function. It was assumed that, in the region above $35.2 \mathrm{keV}$, the background followed the same function. If this is not true, then an unknown systematic error will be added to the data above this energy.

The resolution of the fission and spectrum detectors were not identical; hence, the fission cross section could be determined for each time channel only in regions where the spectrum varied smoothly, which was below $20 \mathrm{keV}$. Above that energy, it could be determined only over wide energy bands.

The corrected fission counts per time channel were normalized to fission cross section by using the data of Bollinger et al., (11) equating the area under the $7.83-\mathrm{eV}$ resonance in each set of data. The results from 10 to $160 \mathrm{keV}$ are shown in Fig. 12. In the region from 1 to $10 \mathrm{keV}$, the results are in good agreement with those of Bollinger et al. Comparisons are made with other data at higher energies. $(12,13)$ 
Fig. 12

AVERAGE VALUES OF PU ${ }^{239}$ FISSION CROSS SECTION ABOVE $10 \mathrm{keV}$

G. D. James, Harwell

\begin{tabular}{c|c|c|c}
\hline \multirow{2}{*}{$\begin{array}{c}\text { Energy Range } \\
\text { (keV) }\end{array}$} & \multicolumn{3}{|c}{$\sigma_{F}$ (barns) } \\
\cline { 2 - 4 } & $\begin{array}{c}\text { Present } \\
\text { Data }\end{array}$ & BNL-325 & $\begin{array}{c}\text { Allen and } \\
\text { Ferguson }\end{array}$ \\
\hline $10-15$ & $1.8 \pm 0.1$ & 2.35 & \\
$15-50$ & $1.92 \pm 0.1$ & 2.06 & \\
$55-75$ & $2.05 \pm 0.1 *$ & 1.82 & $1.95=0.05$ \\
$50-160$ & $1.8 \pm 0.1 *$ & 1.78 & $1.69 \pm 0.05$ \\
\hline
\end{tabular}

*Excluding possible systematic eror from background assumption.

Capture Cross Sections of Fe. Th, and U from 1 to $30 \mathrm{keV}$. (M. C. Moxon)

A $30-m$ flight path was used, with a resolution of $11 \mathrm{nsec} / \mathrm{m}$. The $\gamma$-ray detector was the Moxon-Rae type. (14) The accuracy of the data produced in such a measuremont depends mainly on the detector-efficiency calibration and the determination of incident neutron flux.

The shape of the neutron spectrum was determined by measuring the capture $\gamma$ rays from $\mathrm{B}^{10}(\mathrm{n}, \alpha) \mathrm{Li}^{7}$. It was assumed that the reaction was $1 / v$ dependent and that the fractional $\alpha$-particle population of the $480-\mathrm{keV}$ $\mathrm{Li}^{7}$ state was constant over the energy range from $1 \mathrm{eV}$ to $100 \mathrm{keV}$.

The detector efficiency is proportional to the $\gamma$-ray energy. Thirtytwo black resonances in different nuclei were used to obtain a best-fit value of efficiency per MeV $\gamma$-ray energy. This is shown in Fig. 13.

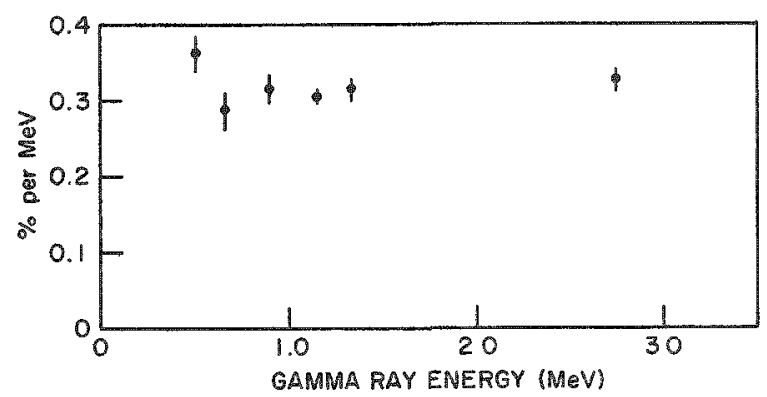

(1)

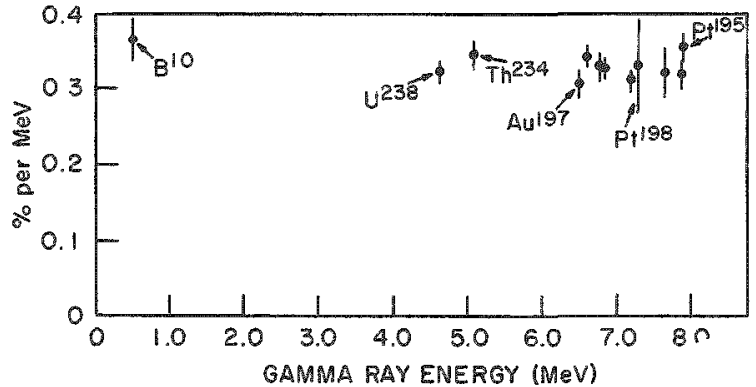

(2)

Fig. 13. (1) Detection Efficiency for Single $\gamma$-ray per MeV.

(2) Detection Efficiency for Neutron Capture Divided by Total Capture $\gamma$-ray Energy, Normalized to $0.36 \%$ per $\mathrm{MeV}$ for $\left(B^{10}+n\right)$. 
For thin samples, the capture cross section can be obtained directly as the flux times the efficiency times the binding energy divided by the atoms per barn. The uncertainties in the measurements mainly come from the uncertainty in the $B^{10}$ cross section and background estimations. The normalization uncertainty is at a minimum of $\pm 3 \%$ between 1 and $100 \mathrm{eV}$, and rises to $\pm 7.5 \%$ at $50 \mathrm{keV}$. In uranium and thorium the background uncertainty gives an error in the cross section of about $\$ 0.16 \mathrm{~b}$ at $1 \mathrm{keV}$, decreasing to $=0.045 \mathrm{~b}$ at $10 \mathrm{keV}$, and rising to about $\pm 0.06 \mathrm{~b}$ at $50 \mathrm{keV}$. The observed capture cross sections of these elements are shown in Figs. 14 and 15. The shapes of the curves are in fair agreement with the Oak Ridge data $(15)$ and the absolute magnitudes at $24 \mathrm{keV}$ are in good agreement with the shell transmission results of Schmitt and Cook $(16)$ and of Belanova.

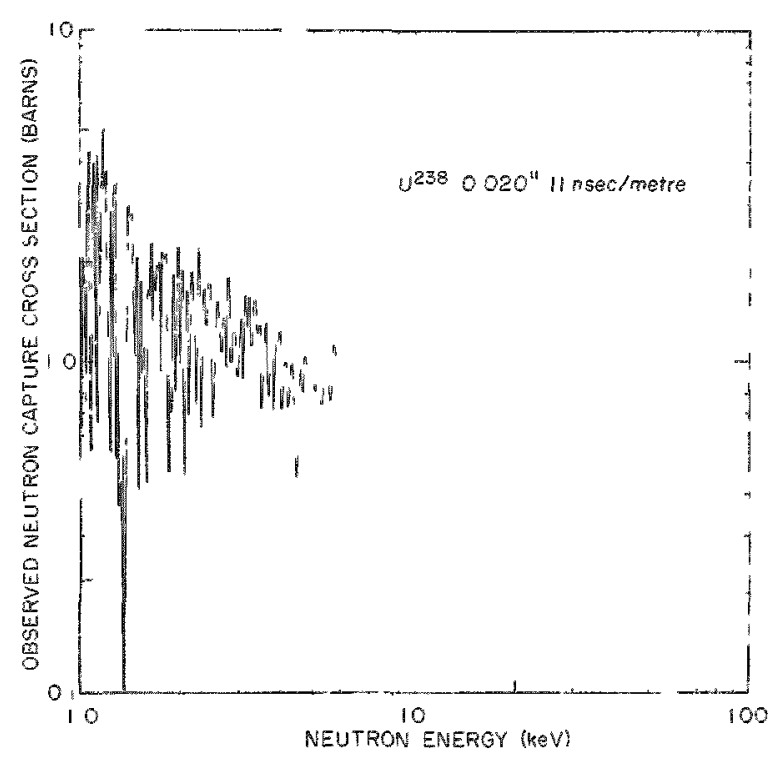

Fig. 14

Observed Capture Cross Section of Uranium

Fig. 15

Observed Capture Cross Section of Thorium

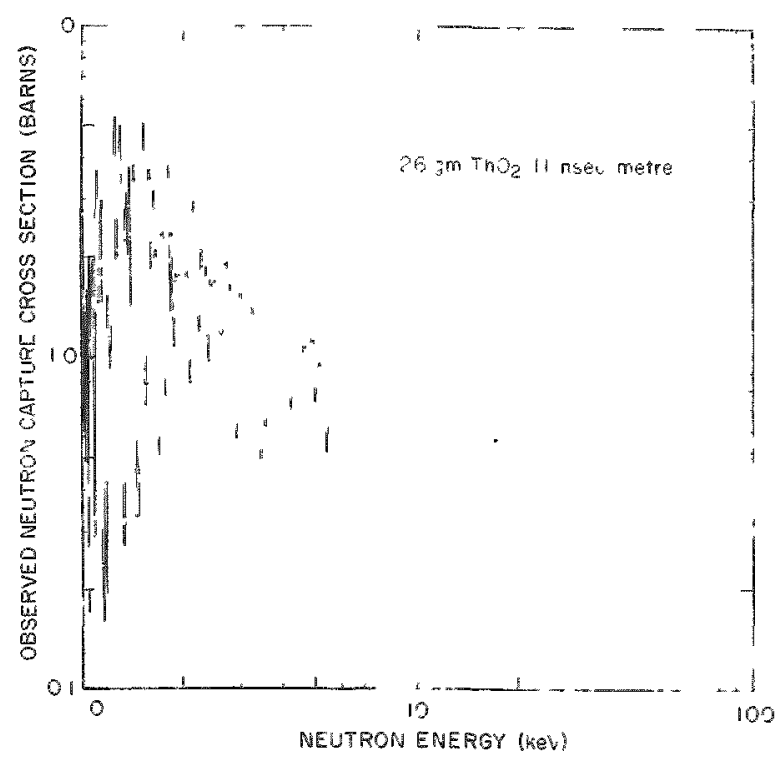


The data for iron are shown in Fig. 16. The level at $1180 \mathrm{eV}$ observed by Isakov et al. (18) was confirmed with an estimated $\Gamma_{\mathrm{n}}$ of $42 \mathrm{MeV}$. An upper limit for $\overline{F_{\gamma}}$ of $1.3 \mathrm{eV}$ is set for the $28 \mathrm{keV}$ level in $\mathrm{Fe}^{56}$.

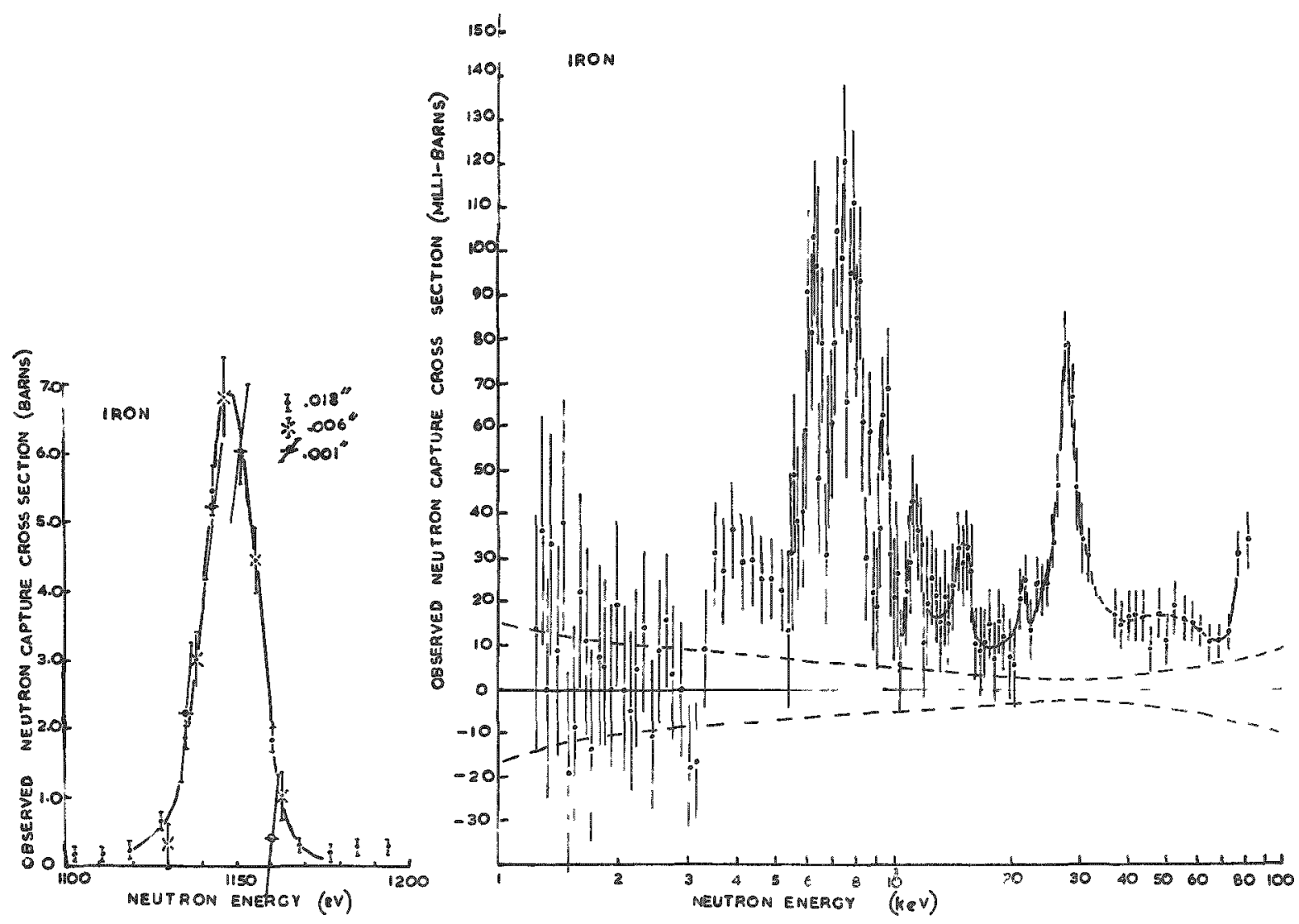

Fig. 16. Observed Capture Cross Section of Iron

\section{References}

1. D. W. Colvin and M. G. Sowerby, Proceedings of the Second United Nations International Conference on the Peaceful Uses of Atomic Energy, Geneva, Switzerland, 16, 121 (1958).

2. J. B. Parker, J. H. Towle, D. Sams, and P. G. Jones, Nuclear Inst. and Methods, 14, 1-12 (1961).

3. L. Cranberg and J. S. Levin, Phys. Rev., 109, 2063 (1958).

4. A. B. Smith, paper submitted to Physical Review.

4a. B. Elbeck, A. Igo, F. S. Stephens, and R. M. Diamond, UCR L-9566, p. 25 . 
5. M. J. Poole and E. R. Wiblin, Proceedings of the Second United Nations International Conference on the Peaceful Uses of Atomic Energy, Geneva, Switzerland, 14, 266 (1958).

6. W. R. Kanne et al., Proceedings of the First United Nations International Conference on the Peaceful Uses of Atomic Energy, Geneva, Switzer land, 4, 315 (1956).

7. Reactor Physics Constants, ANL-5800 (1958).

8. P. A. Moldauer, S. Yiftah and D. Okrent, "Fast Reactor CrossSections," Pergamon Press, Oxford (1960).

9. F. J. Shore and V. L. Sailor, Phys.Rev,, 112, 191 (1958).

10. C. D. Bowman, G. F. Auchampaugh, and S. C. Fultz, Phys. Rev., $130,1482(1963)$.

11. L. M. Bollinger et al., Proceedings of the Second United Nations International Conference on the Peaceful Uses of Atomic Energy, Geneva, Switzerland, 15, 127 (1958).

12. D. J. Hughes and R. B. Schwartz, BNL-325 (1958).

13. W. D. Allen and A. T, G. Ferguson, A.E.R.E. NP/GEN/3.

14. M. C. Moxon and E. R. Rae, Proc. Neutron Time of Flight Methods Symposium, Saclay, 439 (1961).

15. J. H. Gibbons, R. L. Macklin, P. D. Miller, and J. M. Neiler, Phys. Rev., 122, 182 (1961).

16. H. W. Schmitt and C.W. Cook, Nuc. Phys., 20, 202 (1960).

17. T. S. Belanova, Sov. Jour. At. En., 8, 462 (1960).

18. A. I. Isakov, Jr., P. Popov, and F. L. Shapiro, Zh. eksper。teor. Fiz., 38, 989 (1960). 


\section{Discussion of Paper}

Presented by Mr. Ferguson

MR. REDMAN (Argonne):

What method did you use in the determination of $\nu$ to establish the efficiency of your fission counter?

MR. FERGUSON:

There has been a very full report in the Proceedings of the Second United Nations International Conference on the Peaceful Uses of Atomic Energy, Geneva, Switzerland, 16, 121 (1958).

MR. CAMPISE (Atomics Internationa1):

I have two questions, one referring to your data for $\nu$. What is the accuracy with which you know the neutron energy? Have you compared your $\alpha$ data for $\mathrm{U}^{235}$ with the Diven and Hopkins data at higher energies to see whether they dovetail?

MR. FERGUSON:

The accuracy of the neutron energy at $0.1 \mathrm{MeV}$ was $\pm 0.060 \mathrm{MeV}$, giving a quite well-defined neutron energy. Other energies were $572 \pm$ $150 \mathrm{keV}$ and $2.57 \pm 0.085 \mathrm{MeV}$.

I am sorry, I cannot tell you about the comparison with the Hopkins data.

MR.SCHMIDT (Kar1sruhe):

What are the average capture rates you determined for $F e^{56}$ resonances?

MR. FERGUSON:

I do not have this information, but it can be obtained by writing to Dr. Moxon at Harwell.

MR.PERSIANI (Argonne):

Your data on the resonance region of $U^{238}$ are beautiful. Is anybody attempting to assign resonance-parameter levels to these?

MR. FERGUSON:

Dr. Lynn is working on attempting to get resonance parameters in this region. These are not yet available. 


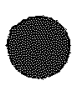




\title{
RECENT CHANGES IN HEAVY ELEMENT CROSS SECTIONS
}

\author{
A. B. Smith \\ Argonne National Laboratory \\ Argonne, Illinois
}

\section{Introduction}

The title of this paper bears a certain connotation of time, energy, and mass. In the quantum sense, time and energy are conjugate variables undefinable simultaneously and absolutely. I will take considerable license with this uncertainty principle and define "recent" as those measurements reported during or subsequent to 1960. Further, this paper will be largely confined to the incident neutron energy interval of $10 \mathrm{keV}$ to $1.5 \mathrm{MeV}$.

This restricted energy range does not imply that other regions are unimportant. However, reactions with neutrons of lower energies are generally of a complex resonance nature and beyond the scope of this paper. The title implies an attention to "heavy" nuclei. I choose to interpret "heavy" as those nuclei above mass number fifty.

In the following, recent progress in the fields of fast neutron fission, capture, and scattering will be cited, and effort will be made to offer a prognosis of what reasonably can be expected in these fields in the coming few years.

\section{The Fission Process}

Fission, of all nuclear processes, is most basically related to reactor systems. Thus it is fitting that we first consider recent changes in fission cross sections, $\bar{\nu}$ values, and delayed-neutron emission.

\section{A. Fission Cross Sections}

The fission cross scctions of even-odd nuclei are summarized in Fig. 1. The curves pertaining to $\mathrm{U}^{235}, \mathrm{U}^{233}$, and $\mathrm{Pu}{ }^{239}$ are representative of the "established" fission cross sections as given in the review of Allen and Henkle. The $\mathrm{Pu}^{241}$ curve represents a composite of recent measurements made at Argonne and Los Alamos. Particular attention should be given to the inset in which the "accepted" $U^{235}$ fission cross section from 0.1 to $1.0 \mathrm{MeV}$ is shown together with recent measurements carried out by White and others at Aldermaston. These new xesults, 6-8\% lower than the "accepted" curve, have a rather far-reaching effect, not only directly on reactor design, but on other important cross sections which have been determined relative to the fission of $\mathrm{U}^{235}$. 


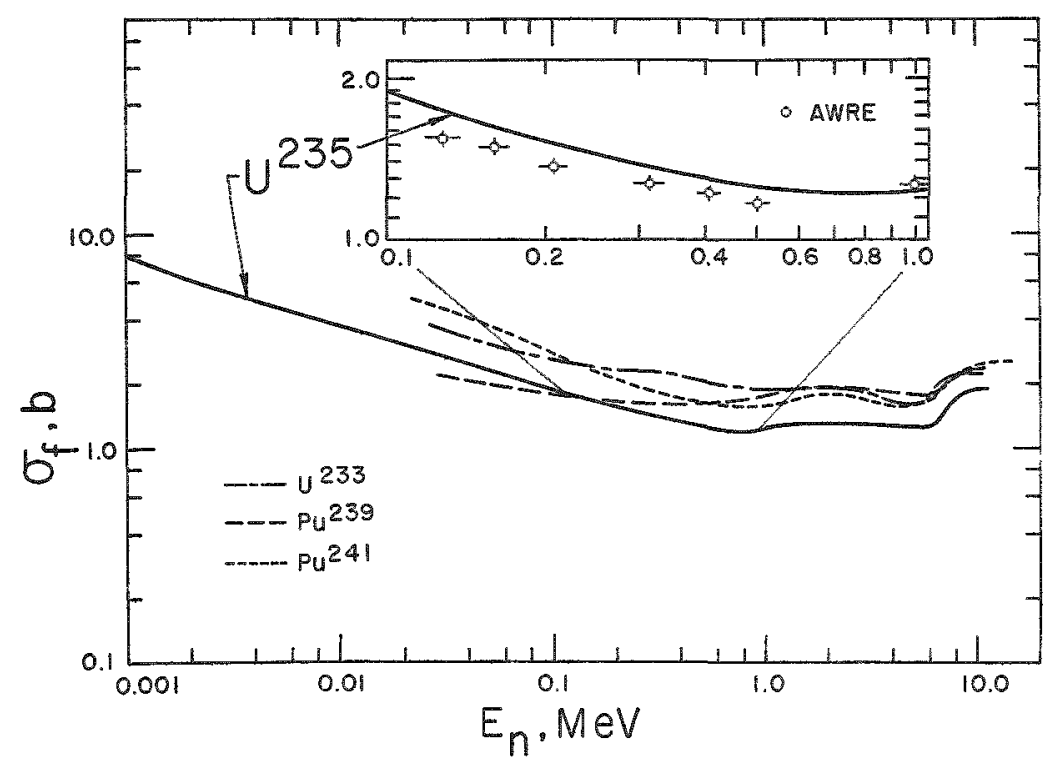

Fig. 1. U $\mathrm{U}^{235}$ Fission Cross Section. Inset shows recent results of White et al. AWRE, Aldermaston.

The current status of even-even fission cross sections is shown in Fig. 2. The fission cross sections of $\mathrm{Pu}^{238}$ and $\mathrm{Pu}^{242}$ are recent additions.

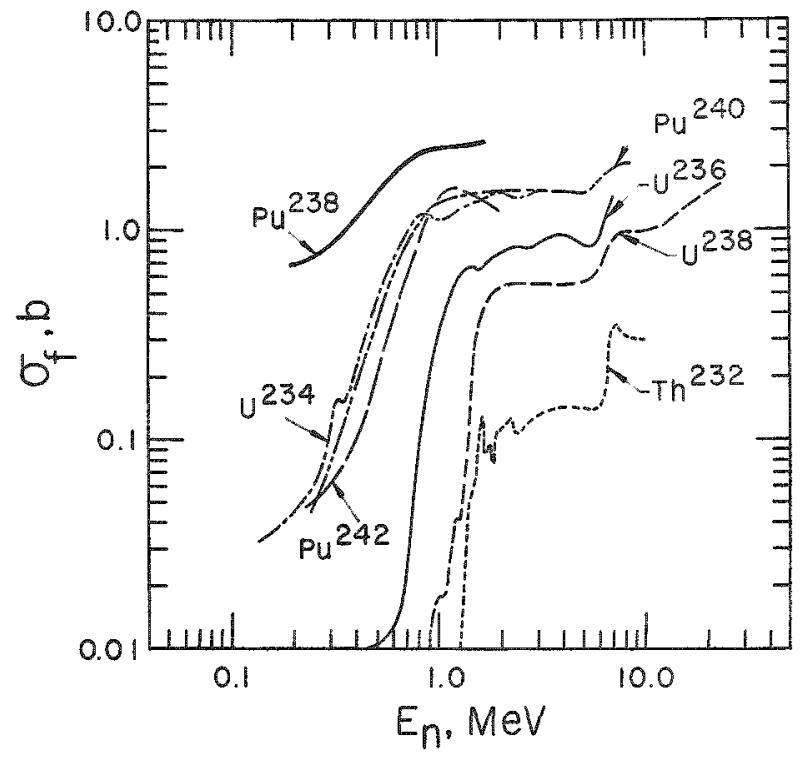

Fig. 2

Summary of Even-Even Fission Cross Sections

It is possible to formulate a systematic correlation of fission cross sections with the mass and charge of the fissioning nucleus, as is shown in Fig. 3. Here fission cross sections at $2.5 \mathrm{MeV}$ are plotted as a function of $Z^{2} / \mathrm{A}$. Clearly, fission in each element follows a smooth curve. These systematic trends permit a semiquantitative prediction of the fission cross sections of unmeasured nuclei. 


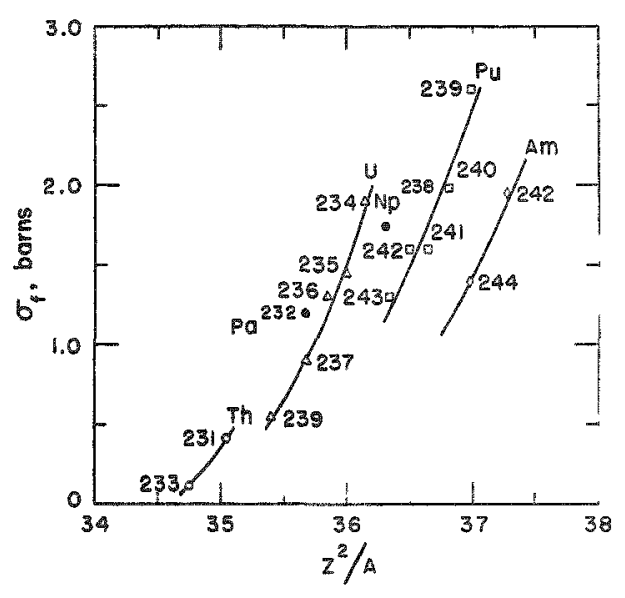

Fig. 3. Systematic Behavior of Fission Cross Sections at $2.5 \mathrm{MeV}$

\section{B. $\bar{\nu}$ Values}

Recent precision measurements have greatly improved our understanding of $\bar{\nu}$ for $U^{235}$, as indicated in Fig. 4. Here $\bar{\nu}$ prompt is shown as a function of incident neutron energy. Note that $\bar{\nu}$ for thermal fission of $U^{235}$ has not appreciably changed. The energy dependence is not linear, but may be described by a cubic expression indicated by the solid curve, the constants of which are shown at the top of the figure. At energies below several MeV the slope of $\bar{\nu}$ for $\mathrm{U}^{235}$ is somewhat less than 0.1 as contrasted with previous estimates of 0.14 or 0.15 .

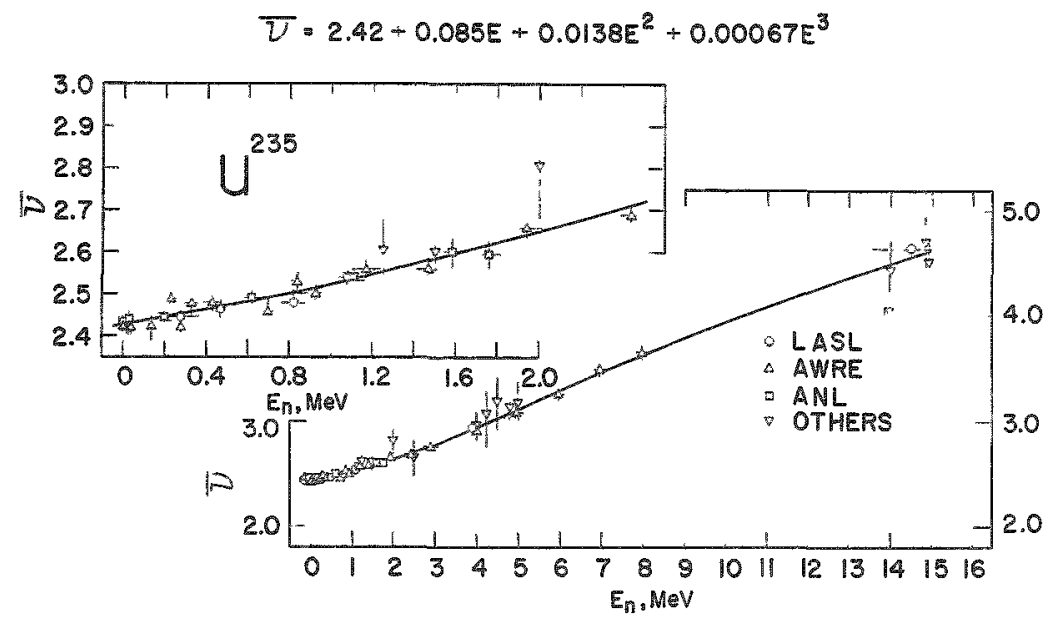

Fig. 4. Values of $\bar{\nu}$ for $U^{235}$. Recent results are indicated by the legend.

Our knowledge of $\bar{\gamma}$ for $\mathrm{U}^{23.3}$ and $\mathrm{Pu}{ }^{239}$ has also considerably improved. The current status is indicated in Fig. 5. Changes in the thermal values have been negligible. Cubic leasi-square fits to the data are shown as both analytical expressions and as solid curves. Qualitatively, the slopes of $\bar{\nu}$ for $U^{233}$ and $P u^{239}$ decrease at lower energies in a manner similar to that for $\mathrm{U}^{235}$.

Recent measurements of $\bar{\nu}$ for $\mathrm{U}^{238}$ and $\mathrm{Th}^{232}$ have tended to subsiantiate the previous results. The current status is indicated in Fig. 6. Quadratic fits to the respective measurements are indicated by solid curves and algebraic expressions. 


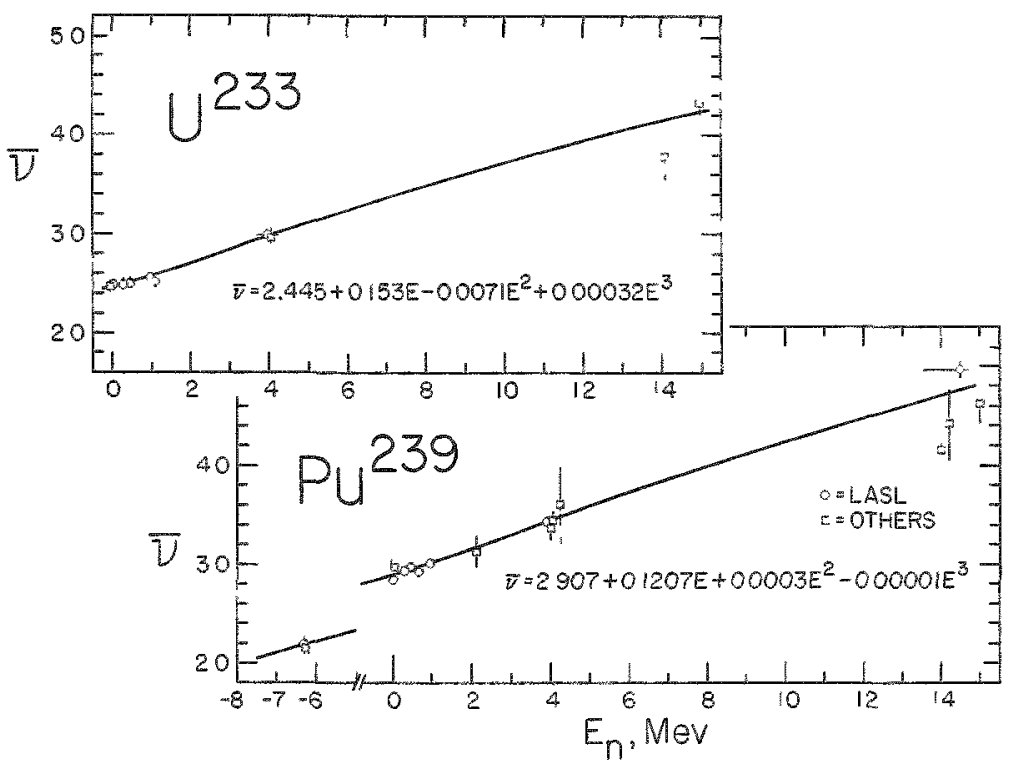

Fig. 5. Values of $\bar{\nu}$ for $P u^{239}$ and $U^{233}$. Analytical expressions were derived by least square fitting procedures.

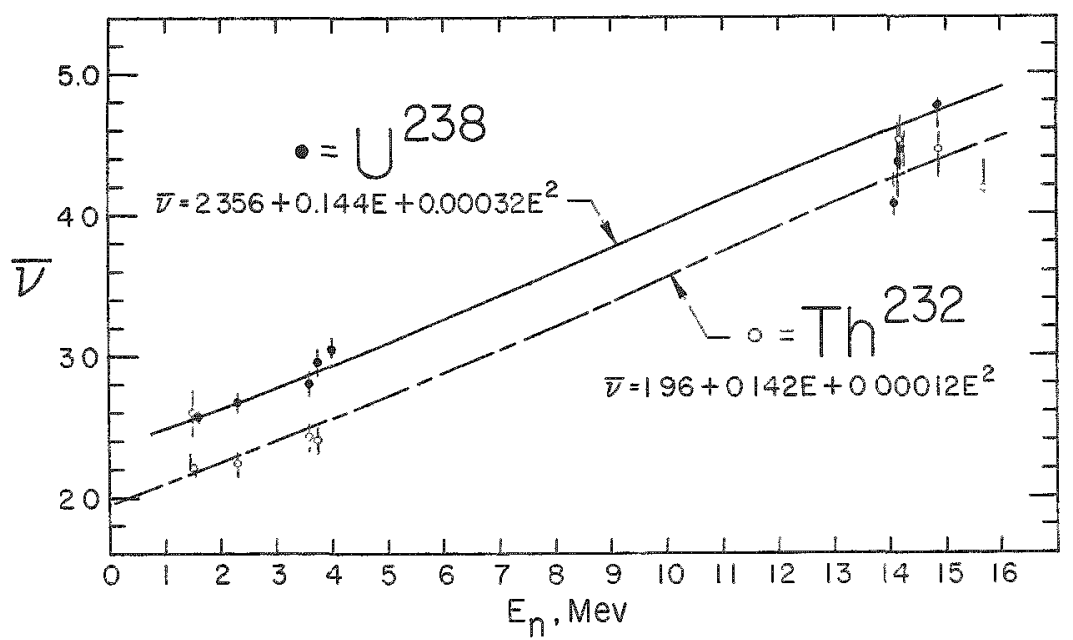

Fig. 6. Values of $\bar{\nu}$ for $\mathrm{Th}^{232}$ and $\mathrm{U}^{238}$

\section{Delayed-neutron Emission}

Recent delayed-neutron studies, devoted to the heaviex plutonium and transplutonium nuclei, have contributed to our understanding of the systematics of delayed-neutron emission, as illustrated in Fig. 7. Here the delayed-neutron yield is given for each of the usual periods as a function of the mass of the fissioning uranium or plutonium nucleus. Smooth trends 
are evident, trends which make it possible to predict the yields of given delayed-neutron groups from the fission of unmeasured nuclei, such as $\mathrm{Pu}^{242}$.
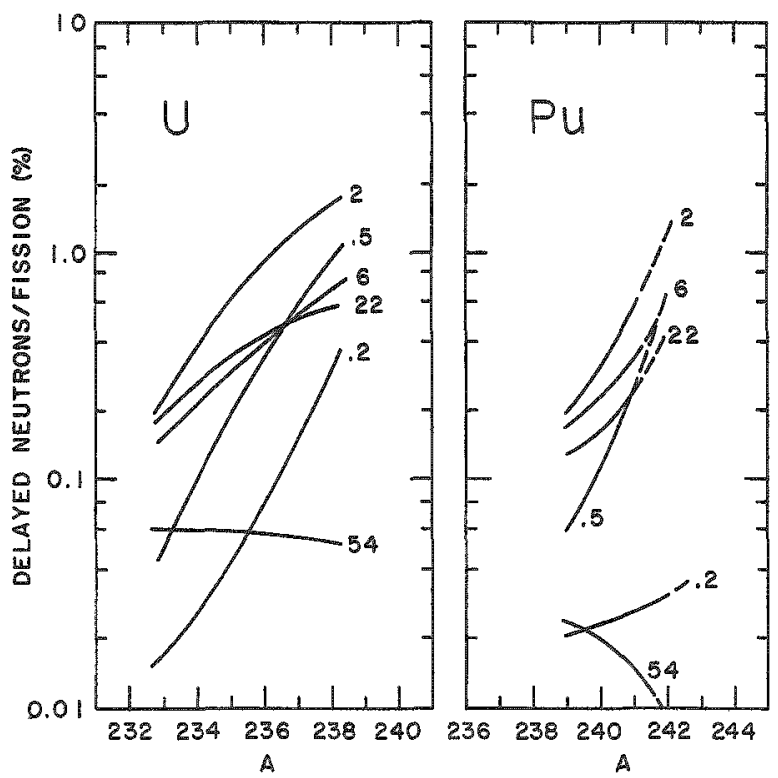

Fig. 7

Systematics of Delayed Neutron Emission from Uranium and from Plutonium. Individual periods are noted.

\section{Fission Summary}

It should be pointed out that several laboratories are now diligently at work on determinations of various fission cross sections relative to that of $U^{235}$ and on an absolute redetermination of the $U^{235}$ cross section itself. Results should be available within the next few years. The value of $\bar{\nu}$ for $\mathrm{U}^{235}$, determined to better than $1 \%$, is possibly the best known of all fast neutron-induced reactions. This is a remarkable thing. I would soon expect to know $\bar{\nu}$ of most other fissile nuclei to a similar precision.

\section{Fast Neutron Capture}

During the past several years a great deal of effort has been expended in the determination of capture cross sections of fast neutrons. One would like to think that this has resulted in an improved understanding of such processes.

\section{A. Fissile Nuclei}

Studies of capture in $\mathrm{U}^{235}$ have taken the form of $\alpha$ measurements (that is, of the ratio of capture to fission). Recent results, indicated in Fig. 8 by the open points, are in reasonable agreement with previously reported measurements. If anything, the newer work tends to drop a bit lower at higher energies. These new values of $\alpha$ for $U^{235}$ give some sustenance to the reliability of previous determinations of $\alpha$ for $\mathrm{Pu}^{239}$ and $\mathrm{U}^{233}$. 


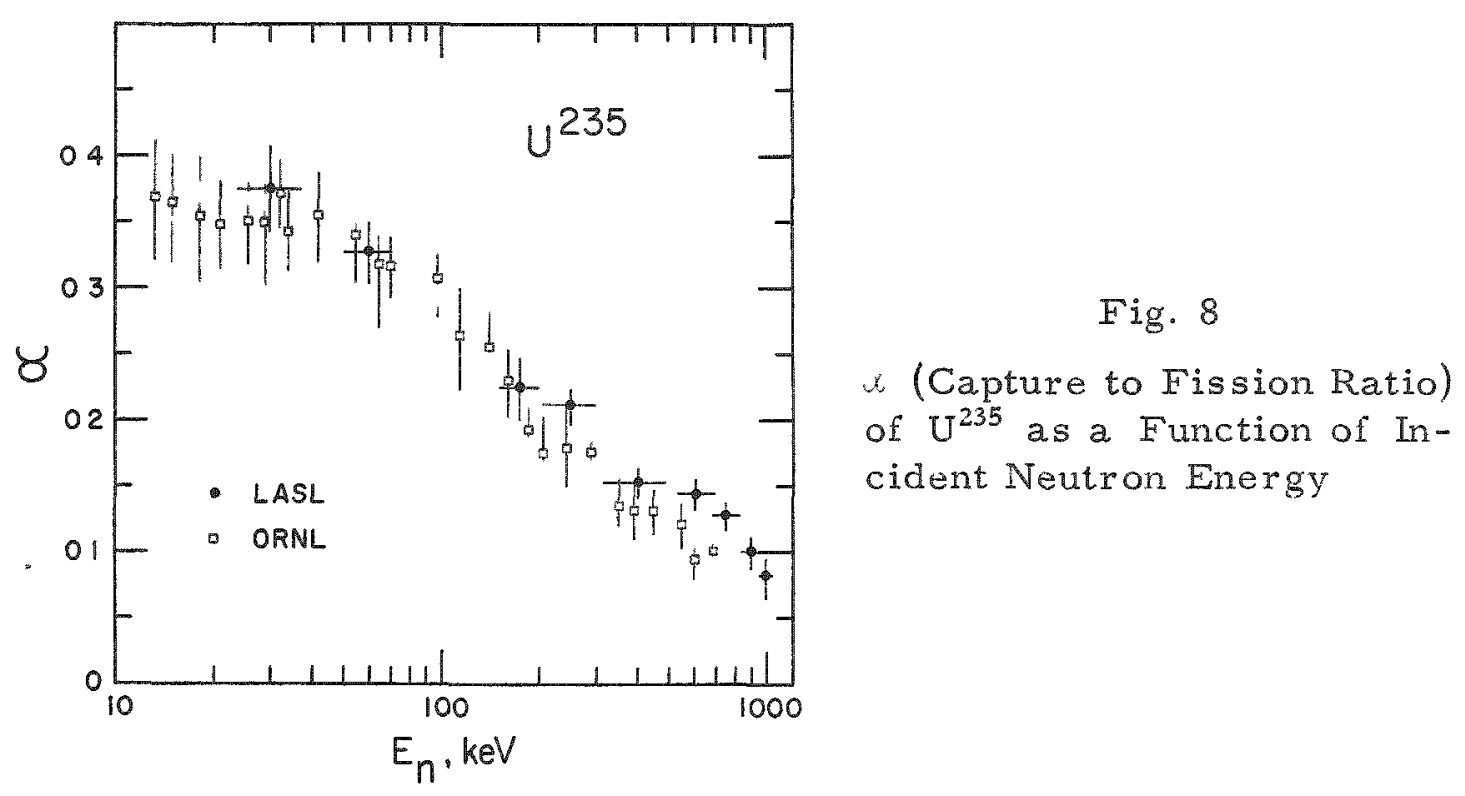

B. Capture in Fertile Materials

Although there have been a number of new measurements of capture in fertile materials, they have not resulted in any significant changes in our knowledge of capture in $\mathrm{U}^{238}$ and $\mathrm{Th}^{232}$. This is illustrated in Fig. 9. A number of measurements of thorium capture near $800 \mathrm{keV}$ have confirmed that some previous low values in this region are anomalous. At lower energies, the newer measurements only tend to substantiate the previous conception of these cross sections.

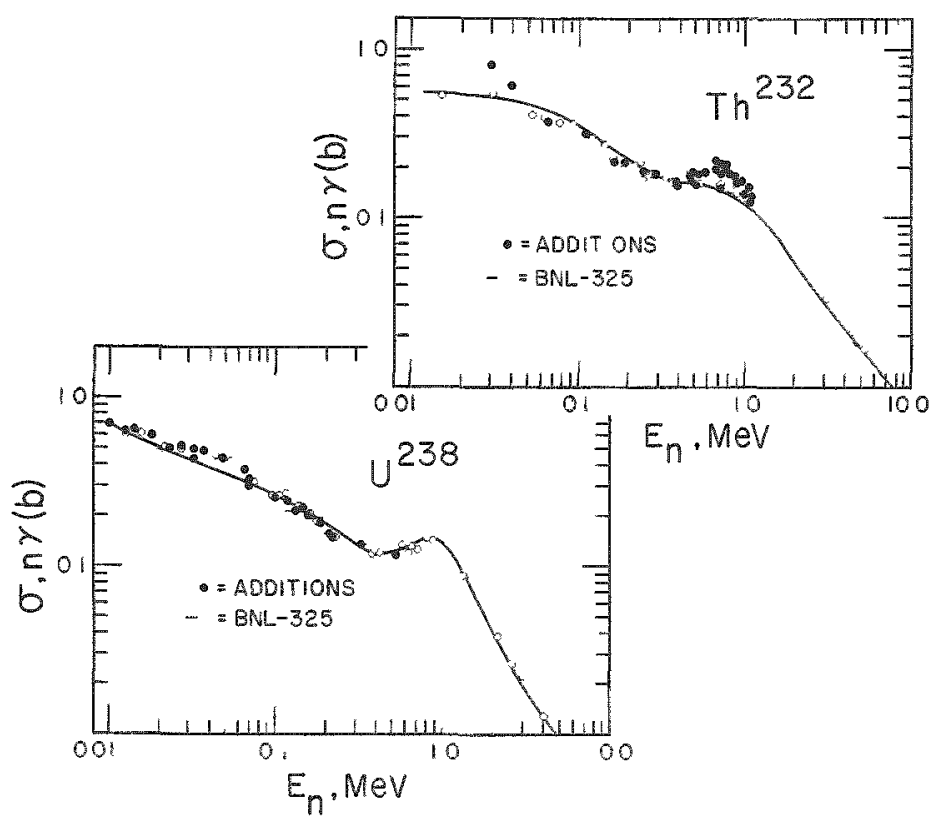

Fig. 9. Capture Cross Sections of $\mathrm{Th}^{232}$ and $\mathrm{U}^{238}$ 


\section{Capture in Structural Materials}

Due to a wide variation in results, one can only hope to illus trate, with some examples, recent capture studies in structural and standard materials. The status of capture in the medium-weight nuclei of $F e$, $\mathrm{Cr}$, and $\mathrm{Nb}$ and in the heavy nucleus of $\mathrm{W}$ is shown in Fig. 10. The curves for iron and chromium illustrate the experimental problem of dealing with complex resonance structure even at relatively high energies. The meas urements for niobium and tungsten average the resonant structure sufficiently to yield a meaningful measurement and to permit theoretical interpretation. However, the individual measurements are not in particularly good agreement.

Perhaps more characteristic of the present disreputable status of fast neutron capture are the four sets of cross sections shown in Fig. 11. Here, a profusion of new measurements of capture in the standard materials I, In, and $\mathrm{Au}$ and in the structural material $\mathrm{Ta}$ is shown. The agreement certainly is not good, particularly in the region from 20 to $150 \mathrm{keV}$. The measurements tend to follow two loci separated by as much as a factor of two.

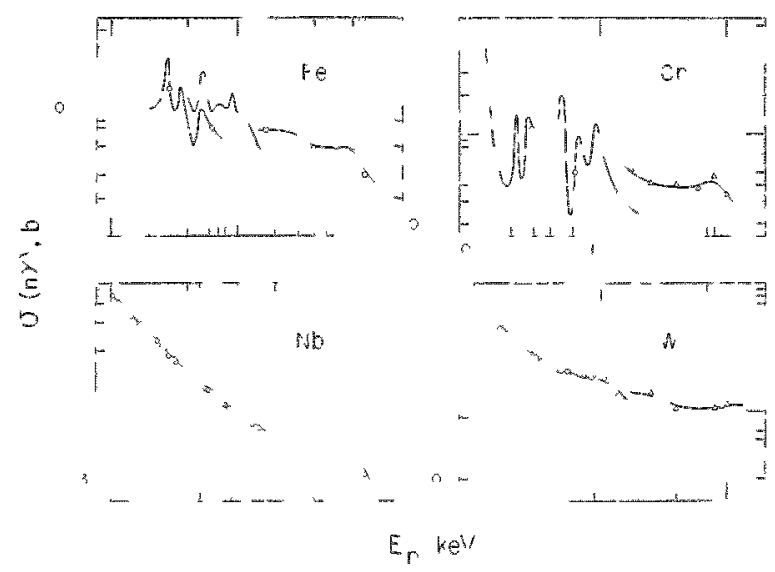

Fig. 10. Capture Cross Sections of Iron, Niobium, Chromium, and Tungsten

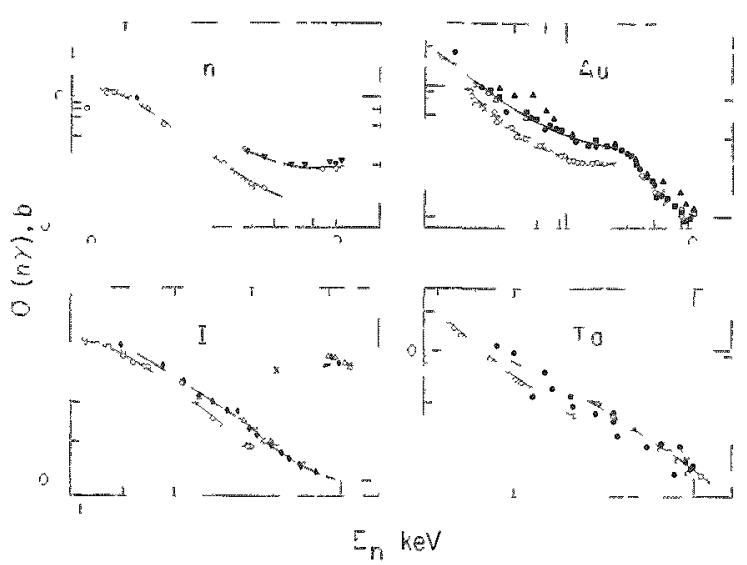

Fig. 11. Capture Cross Sections of Indium, Iodine, Gold, and Tantalum

\section{Fast Neutron Capture - Summary}

The evidence indicates that $\alpha$ measurements for the less exotic fissile materials are improving and that they are converging to a good set of values. For highly active transuranium isotopes such as $\mathrm{Pu}^{242}$ the prognosis for satisfactory $\alpha$ values is not good. Experimentally, the problem is one of intensity. Perhaps the solution lies in the application of a weapon burst to this type of physical measurement. Capture in fertile 
nuclei is probably not known with the precision desired by the reactor theorist, but recent values have improved the situation. Capture in structural and standard materials is embarrassingly confused. What is needed is a careful study of the existing results in order to ascertain, if possible, where the measurements have gone astray. In addition, there is a desperate need for a standard source or cross section in the energy range from 10 to $100 \mathrm{keV}$. A determination of such standards is now underway at several laboratories, but the work will be tedious.

IV. Elastic Scattering of East Neutrons

Contrasted with fast neutron capture, the application of new techniques, that involving time of flight in particular, has recently resulted in

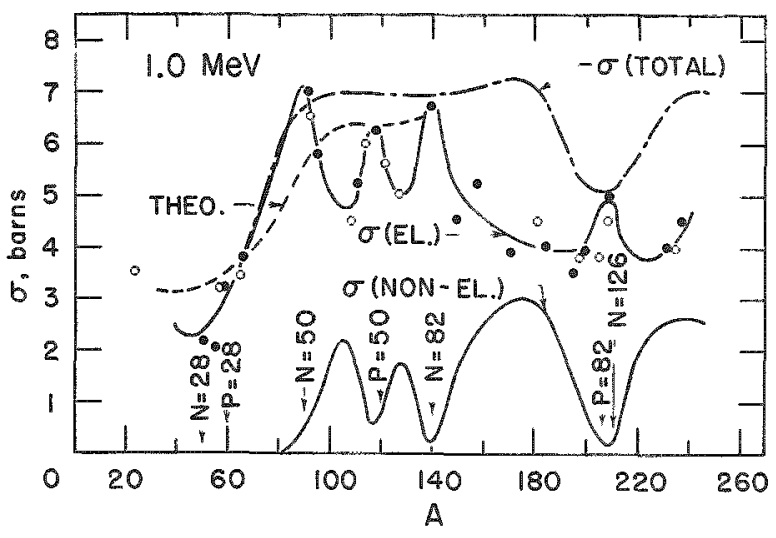

Fig. 12. Elastic Scattering Cross Sections as a Function of Target Mass Number. In cident neutron energy $1.6 \mathrm{MeV}$. a considerably improved knowledge of elastic scattering of fast neutrons.

\section{A. Physical Measurements}

Elastic scattering from forty or more elements is now well known. A typical summary of results at $1.0 \mathrm{MeV}$ is shown in Fig. 12 , when the cross section is plotted as a. function of mass. The effect of closed neutron and proton shells is clearly evident. This shell effect is due to the changes in the density of low-lying nuclear levels as one approaches closed shells. The consequent change in compound elastic

scattering is evident here. The total cross section as given in BNL-325 is indicated in the figure. The difference between the total and elastic cross sections is, by definition, the nonelastic cross section. The directly measured inelastic cross sections are in good agreement with the nonelastic curve shown. The remaining dotted curve represents the theoretical elastic cross sections obtained by Moldauer.

This example of elastic cross sections can be extended to a wide range of energies, as illustrated in the three-dimensional plot of Fig. 13. Here, the elastic cross section is plotted as a function of mass number and incident neutron energy.

The elastic-transport cross section derived from the measured elastic distribution is indicated in Fig. 14. Again, the presentation is three-dimensional in cross section, mass, and neutron energy. Figures 13 and 14 include the data for the fertile nuclei $U^{238}$ and $T h^{232}$ and the fissile nucleus $\mathrm{U}^{235}$ 


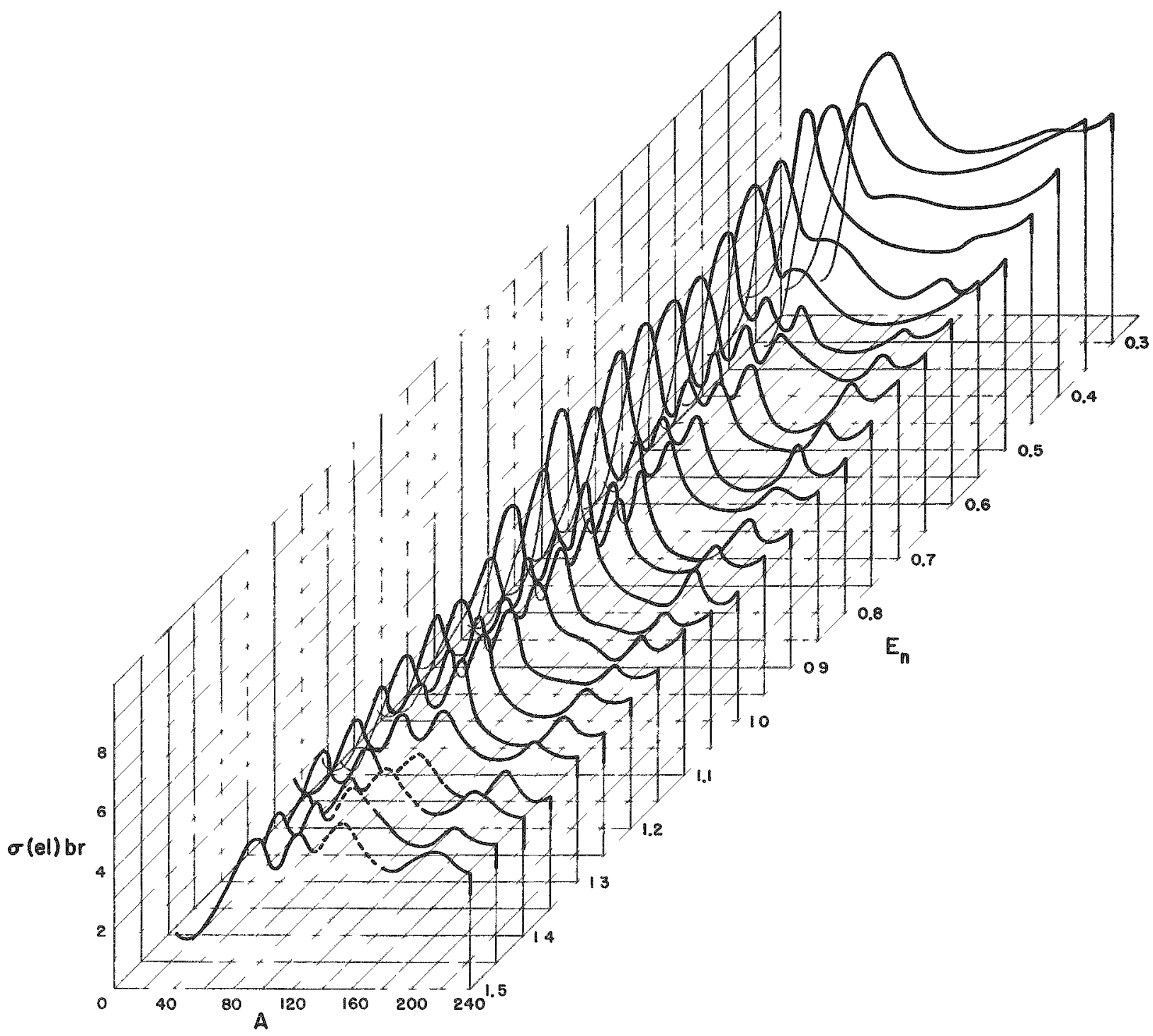

Fig. 13. Three-dimensional Plot of Elastic Cross Sections as Function of Incident Energy and Mass Number 


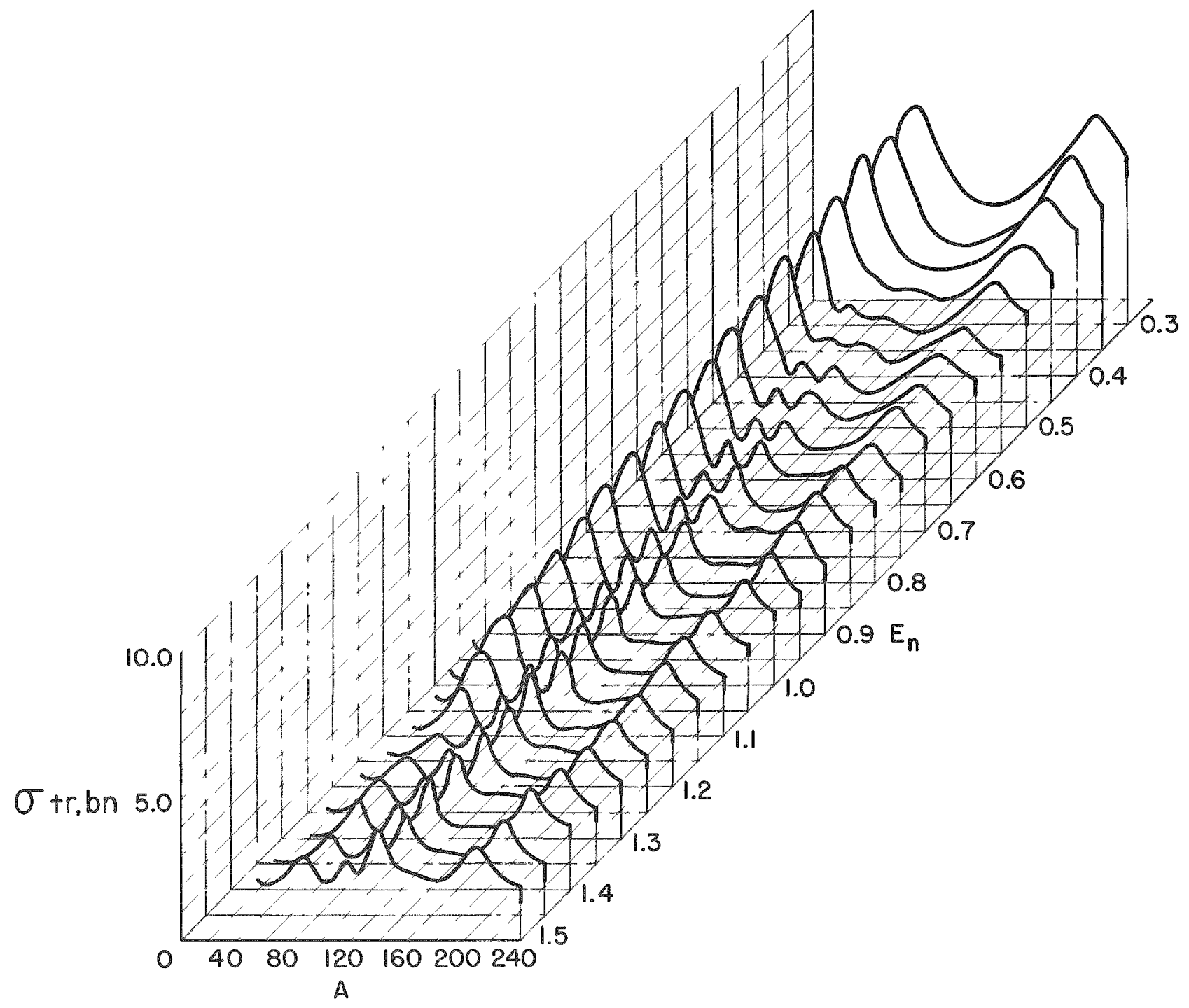

Fig. 14. Transport Cross Section as Function of Mass Number and Incident Neutron Energy 
The experimental results we have just seen characteristically employ incident neutron resolutions of 10-30 keV. Such resolutions average over the resonance structure in most heavy nuclei. However, there are exceptions and the exceptions become the rule as one considers lighter structural materials. In these instances, the resonance structure is neither resolved nor averaged by this experimental resolution. Figure 15 illustrates how drastic this resonance effect can be in the structural material $\mathrm{Fe}$. Here, the total cross section as measured with good resolution is plotted as a function of energy from 500 to $1500 \mathrm{keV}$.
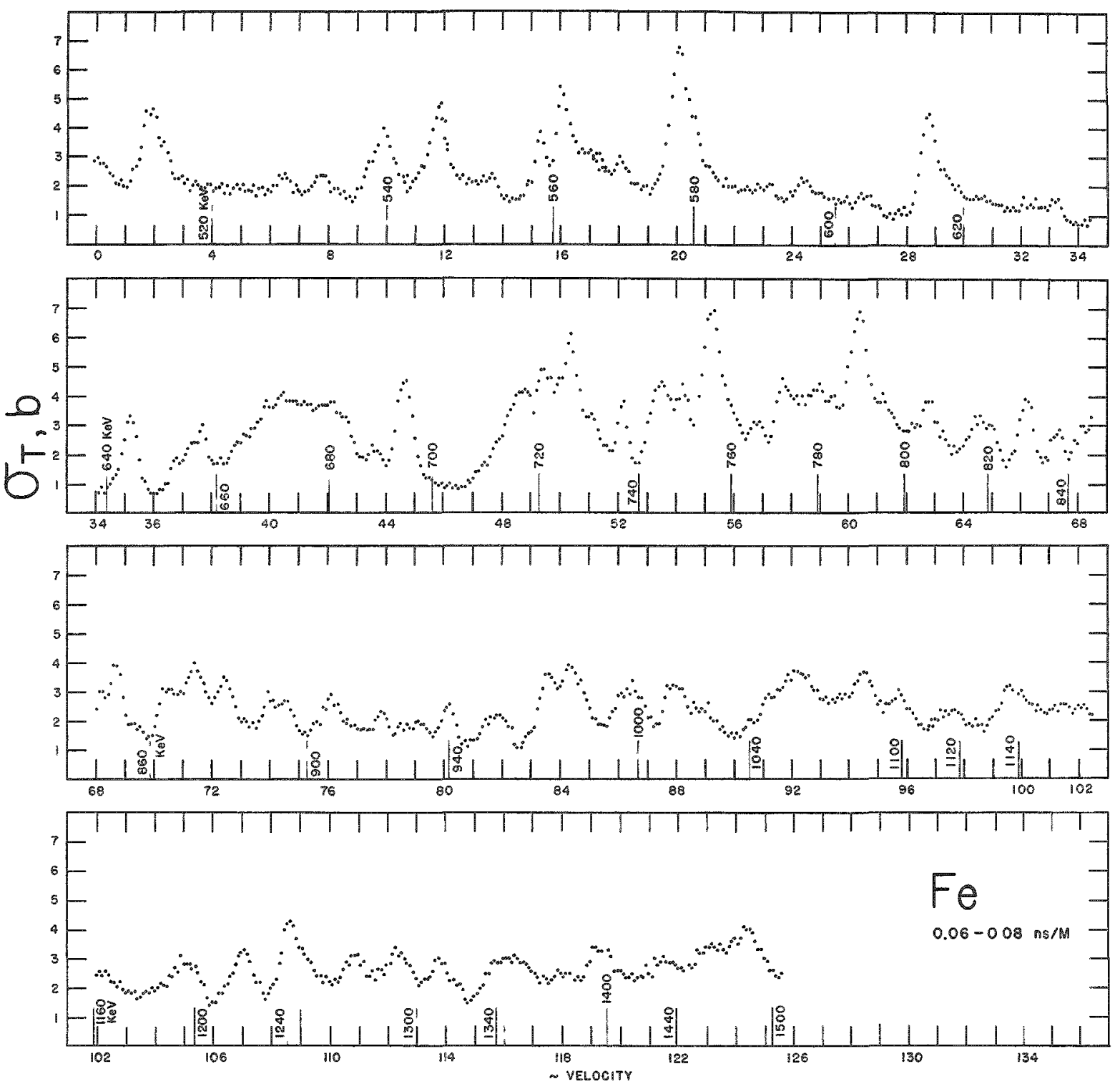

Fig. 15. High Resolution Total Cross Section of Iron 


\section{B. Summary of Elastic Scattering}

Certainly, recent measurements have contributed much to our understanding of elastic scattering from several hundred keV to more than $1.5 \mathrm{MeV}$. At lower energies, inelastic scattering is not usually a factor; where it is, calculations can often be employed to correct the existing wealth of total scattering information for their inelastic content. The resonance structure of medium-weight nuclei, particularly at lower energies, presents a formidable problem which is not so much experimental as it is a matter of physical interpretation and applied usage.

\section{Inelastic Scattering of East Neutrons}

The previous paper described an excellent study of inelastic scattering from a fertile nucleus. The time-of-flight method outlined therein is typical of that allied to a number of similar problems. As a result of the application of this technique what was, not long ago, a largely unknown phenomena is now rapidly becoming familiar.

It would be desirable to review this current knowledge of inelastic scattering systematically. Unfortunately, these reactions are dependent upon individual nuclear structure and thus do not lend themselves to generalization. Due to this complexity the remaining section of this paper will be devoted to isolated examples of inelastic scattering which, it is hoped, will convey the very real improvement in our knowledge of this process.

\section{A. Inelastic Angular Distributions}

The angular distributions of neutrons inclastically scattered to a hundred or more levels in varying nuclei are now known. With a few exceptions, notably in the rare earth region, the inelastic emission is essentially symmetric about $90^{\circ}$ and very nearly isotropic. Two examplas are shown in Fig. 16. The first inelastic group from $\mathrm{U}^{238}$ is slightly peaked about $90^{\circ}$, in agreement with Hauserm Feshbach calculations (denoted by H-E).
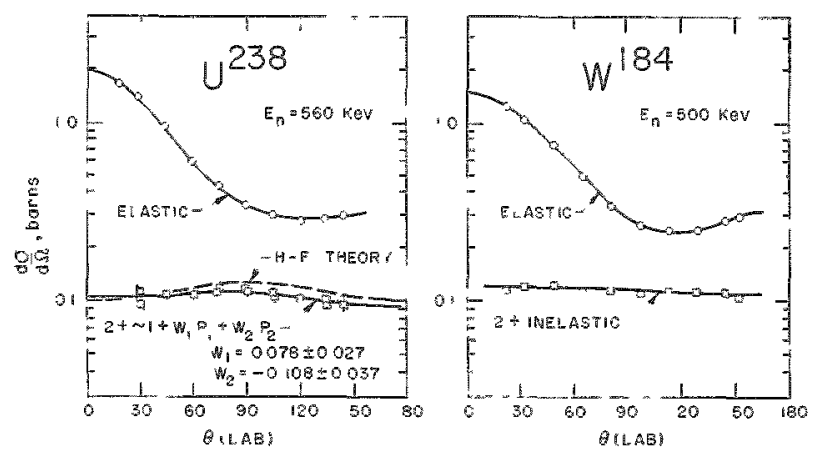

Fig. 16

Differential Elastic and Inelastic Scattering Cross Sections of $U^{238}$ and $W^{184}$ 

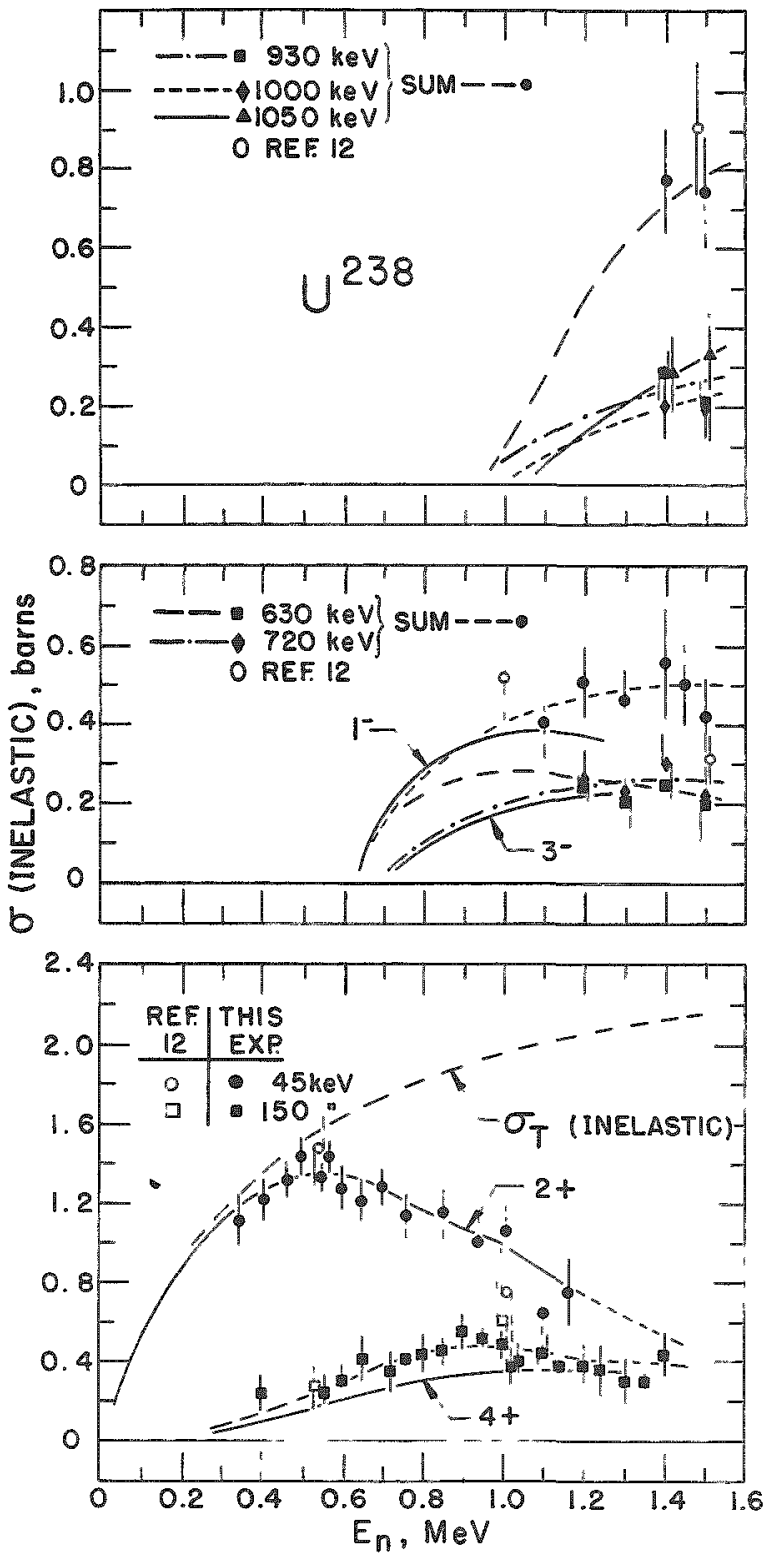

Fig. 17. Inelastic Scattering Excitation Functions of $\mathrm{U}^{238}$. Solid curves are theoretical predictions.
In addition, the distribution is slightly biased toward forward angles. This tendency is undoubtedly due to the direct interaction between the incident neutron and the rotational states of the nucleus.

The distribution of inelastic scattering to a similar rotational level in $W^{184}$ is essentially isotropic, with only a slight indication of a forward bias.

\section{B. Inelastic Scattering from Heavy Element}

In addition to placing emphasis on the large amount of recent experimental information about inelastic scattering, I would also like to indicate the progress in the theoretical interpretation of such processes. In particular, Moore, Auerbach, and Porter have considered, in detail, scattering from $U$, $\mathrm{Th}, \mathrm{W}, \mathrm{Ta}$, and Au. The theoretical fit to the measured inelastic cross sections of $\mathrm{U}^{238}$ is indicated in Fig. 17. The experimental data shown represent two sets of measurements carried out in the US. In the lower portion of the figure, the measured inelastic excitation of the first two rotational states is shown together with the theoretical cross sections indicated by solid curves. The theoretical agreement with the measured excitation of the $2^{+}$state is good. The predicted excitation of the $4^{+}$state is somewhat lower than indicated by experiment. The excitation of the next two prominent levels in the vicinity of $700 \mathrm{keV}$ is shown in the middle portion of the slide. If spin assignments of $1^{-}$and $3^{-}$are made, satisfactory theoretical interpretation of the data is achieved.

Figure 18 shows a fit to the measured inelastic cross suctions of $\mathrm{Th}^{232}$. The agreement between theory and experiment is again reasonable. The spin and parity assignments necessary for these computations 
were derived from a number of sources including Coulomb-excitation experiments and studies of radioactive decay.

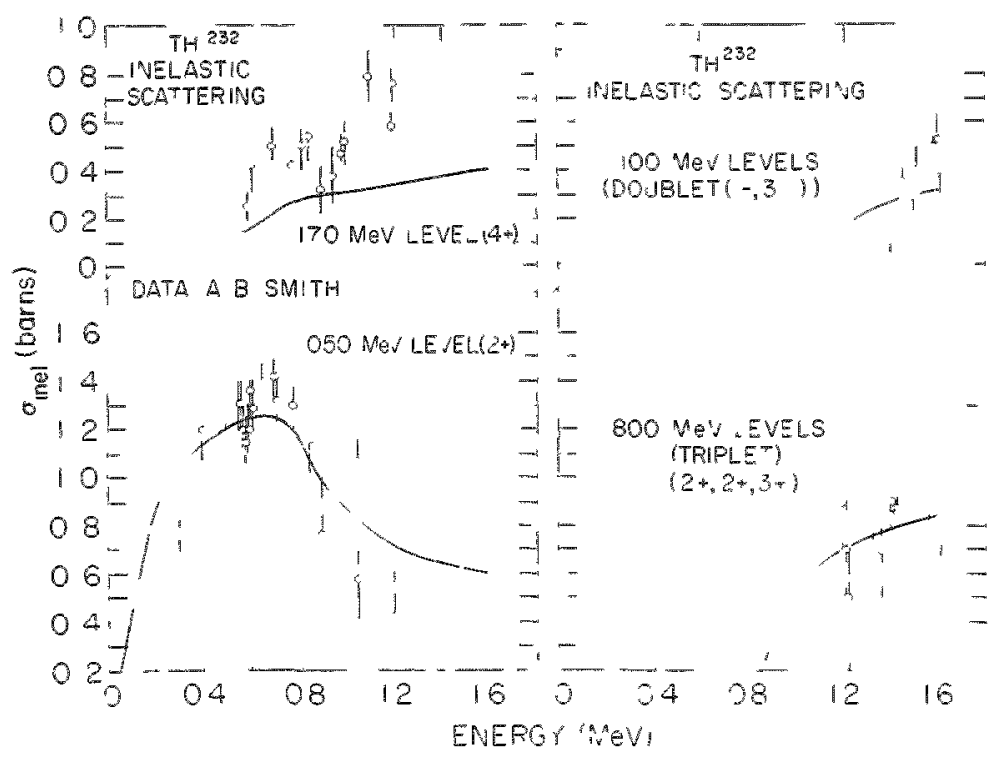

Fig. 18. Inelastic Cross Sections of $\mathrm{Th}^{232}$. Solid curves are theoretical calculations.

Figure 19 shows the experimental and theoretical cross section for inelastic scattering from $\mathrm{Ta}^{181}$. The first excited state in $\mathrm{Ta}^{181}$ is a. $9 / 2^{-}$level at only $6 \mathrm{keV}$. Scattering to this level would be exceedingly difficult to observe with present techniques. However, theory, based on the measured excitation of higher energy states, can predict the magnitude of the scattering to this first level with reasonable precision as indicated.

Figure 20 presents the measured and calculated cross sections for inelastic scattering from Au ${ }^{197}$. Above several hundred $\mathrm{keV}$, the calculated cross section for the first level, at $77 \mathrm{keV}$, is in reasonable agreement with the experiment. Excitation of the doublet at 279 and $268 \mathrm{keV}$ was not experimentally resolved. Thus, the experimental points shown represent the sum of the two theoretical excitation functions. The agreement between theory and experiment for the two higher levels is reasonably good.

The final example of inelastic scattering from heavy elements is shown in Fig. 21. This example was chosen because $\mathrm{W}^{184}$ is known to be a highly distorted nucleus. Thus, one would expect the equivalent spherical potential employed in the previous examples to be less satisfactory. This would seem to be the case as even the experimental excitation of the first rotational $2^{+}$state does not agree particularly well with the theory. 


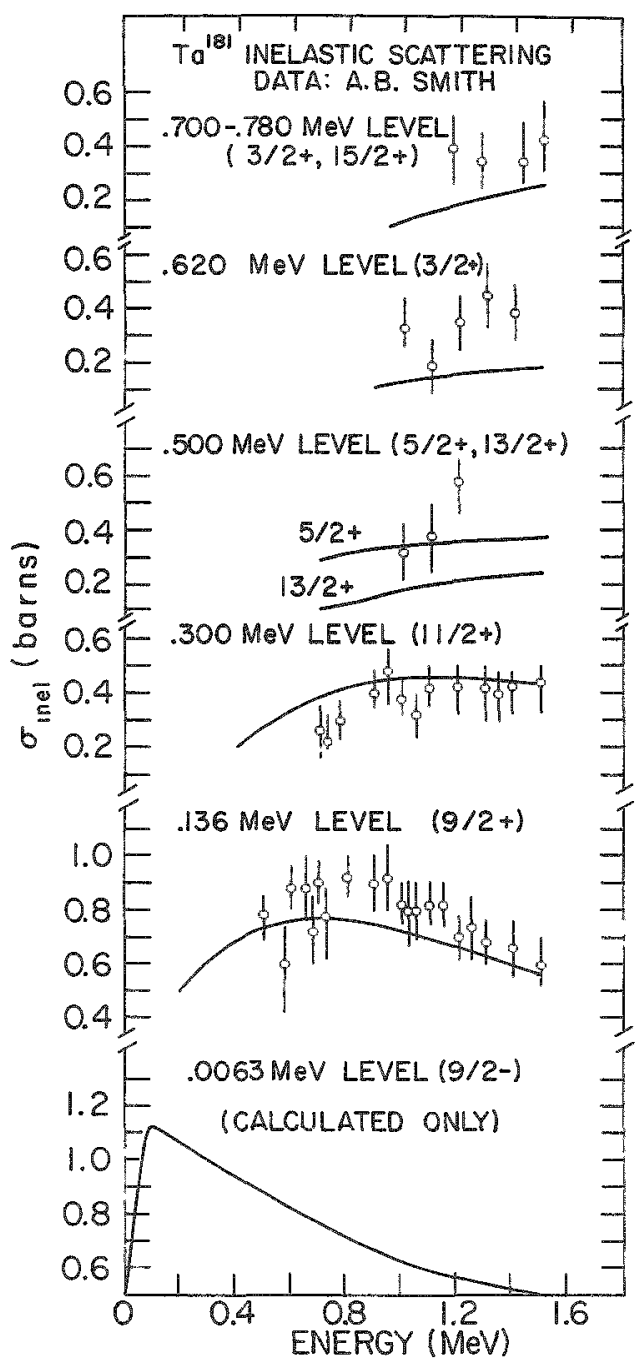

Fig. 19

Inelastic Cross Sections of $\mathrm{Ta}^{181}$. Solid curves represent the predictions of optical model theory.

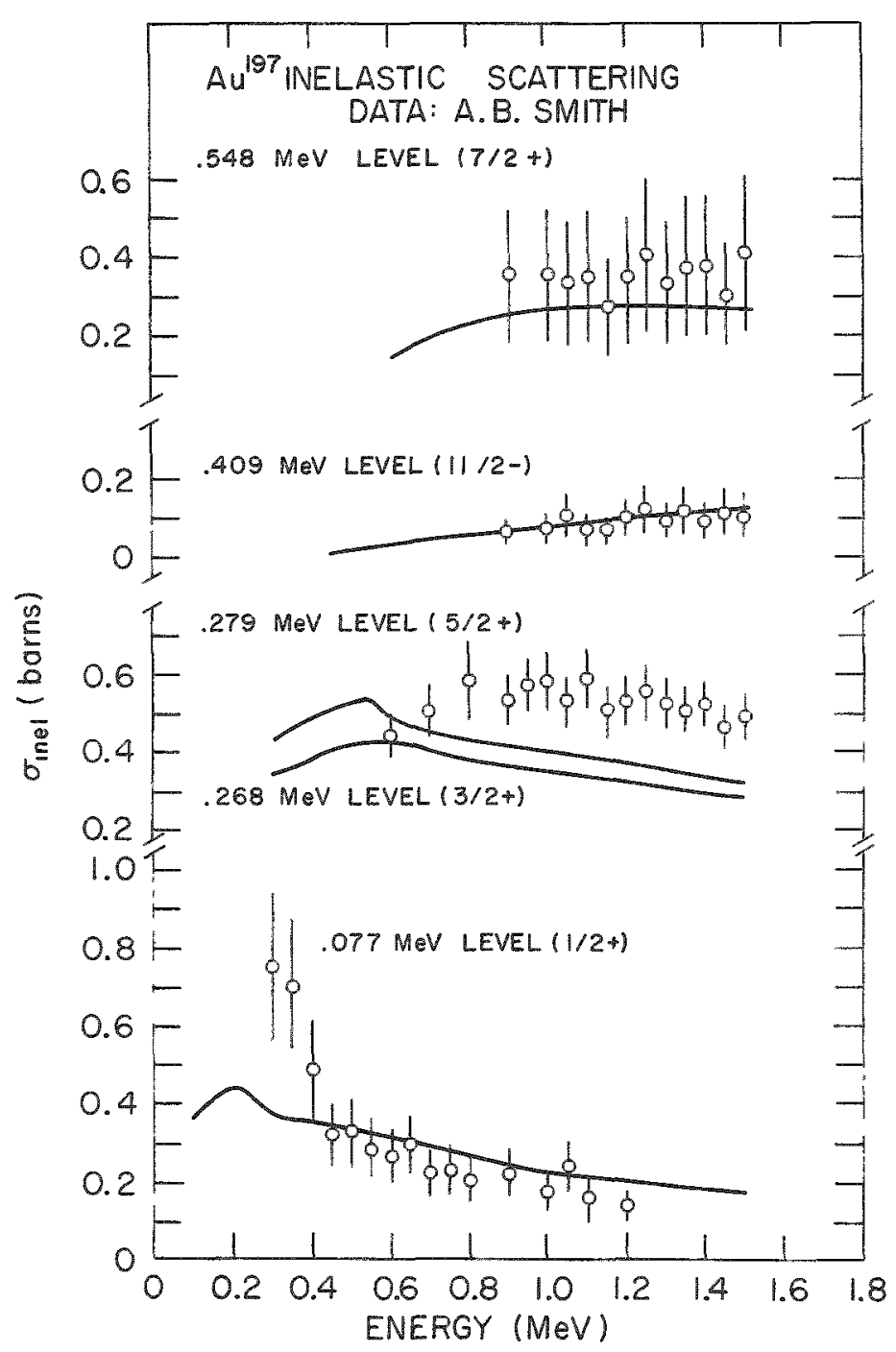




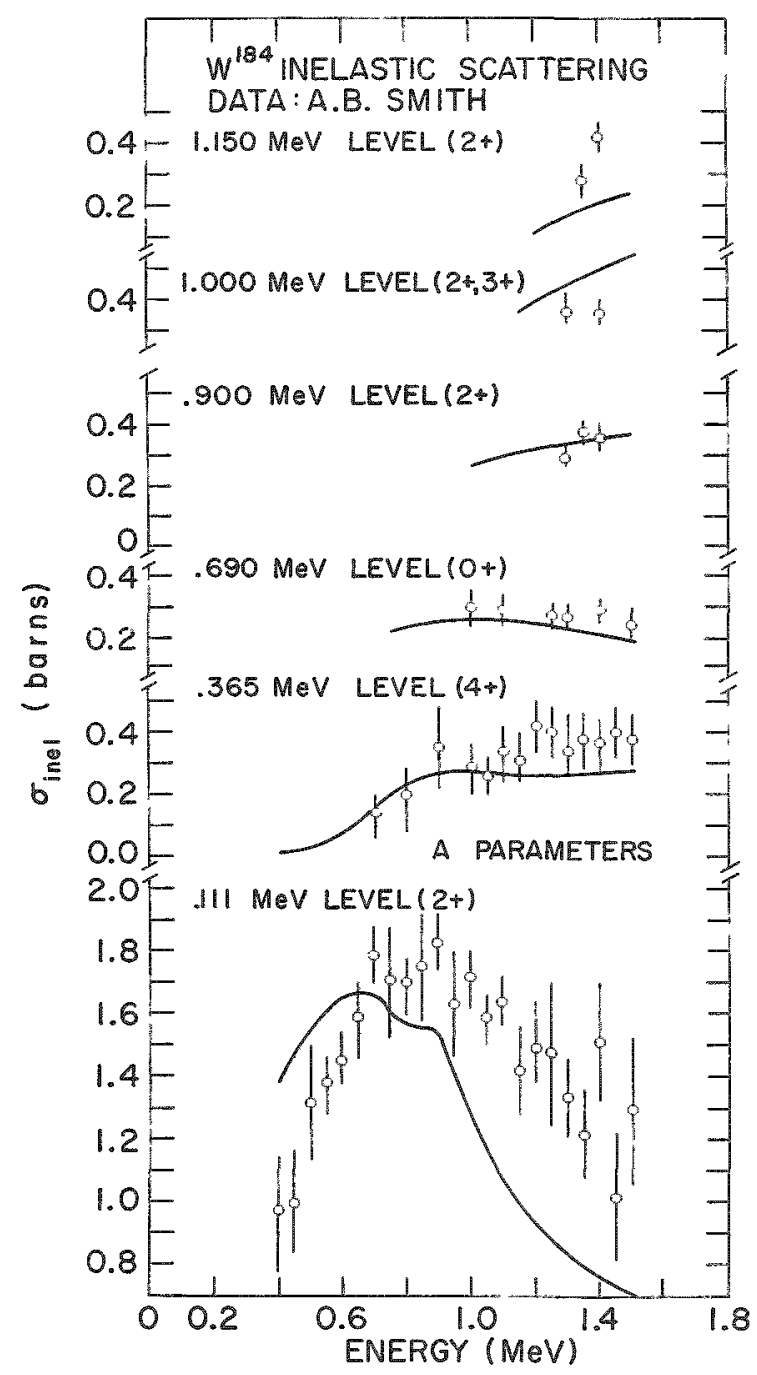

Fig. 21

Inelastic Scattering from $\mathrm{W}^{184}$. Results of calculations based upon a spherical model are indicated by the solid curves.

\section{Inelastic Scattering from Medium-weight Nuclei}

Nuclear structure becomes simpler as the mass decreases. As a result, studies of inelastic scattering from lighter nuclei, experimentally and theoretically, are more definitive. An example of this is scattering from the structural material $\mathrm{Nb}$, shown in Fig. 22. The inelastic excitation of nuclear levels up to $1.0 \mathrm{MeV}$ is given. By means of nuclear systematics and the shell model, the permissible choices of spins and parities are rapidly narrowed to those indicated. With these assignments, Hauser-Feshbach calculations were carried out by Engelbrecht and Moldauer. From the experimental measurements there is no obvious choice between the two sets of level assignments. From the point of view of systematics, the solid curves are favored.

Inelastic scattering from a still lighter nucleus, copper, is shown in Fig. 23. These curves were obtained by an analysis similar to 
that used for niobium. The dotted curves are the result of HauserFeshbach calculations; the solid curves represent predictions incorporating width fluctuation effects. The latter appear to give the better fit with the experimental measurements.

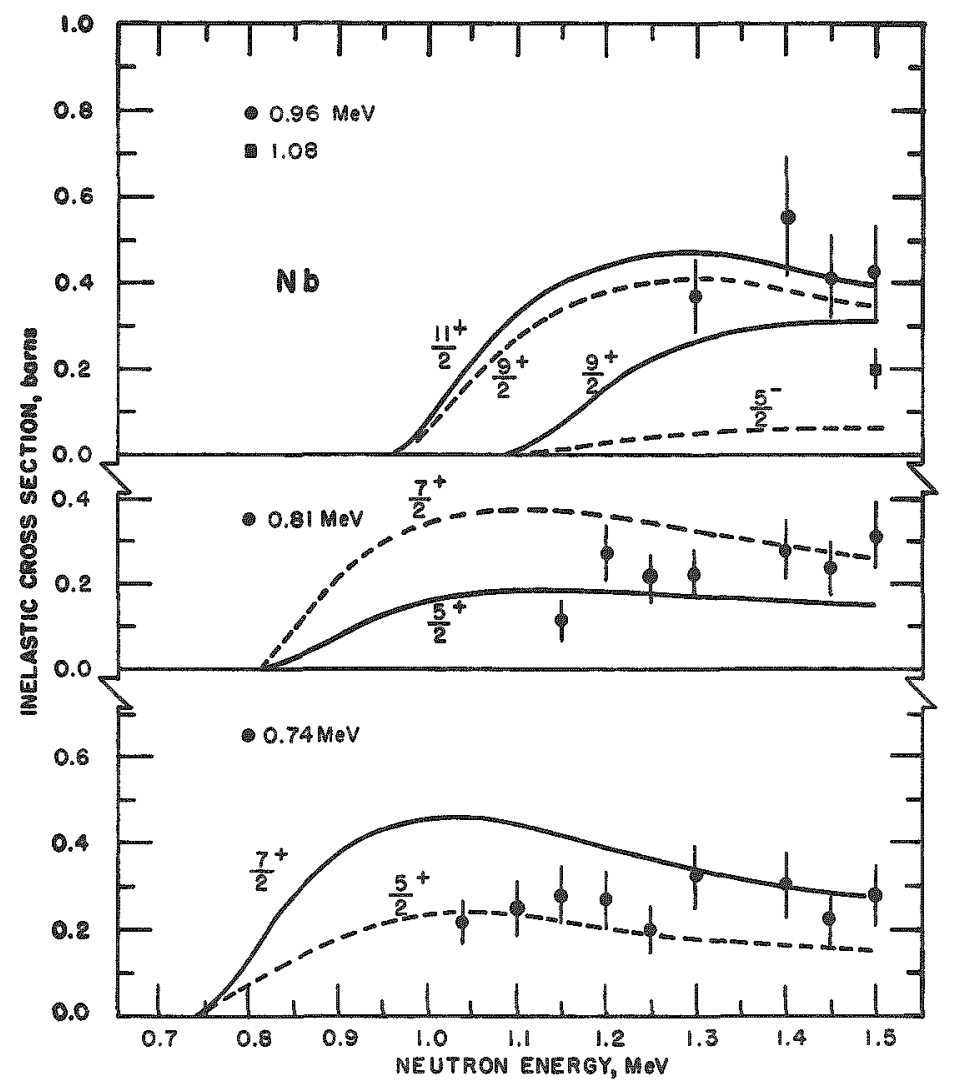

Fig. 22

Inelastic Excitation Functions of Niobium. Alternate choices of level parameters are noted by solid and dashed curves respectively.

Fig. $2: 3$

Inelastic Cross Sections of Natural Copper. Hauser-Feshbach calculations are noted by dashed curves. Solid lines include corrections for width fluctuation effects.

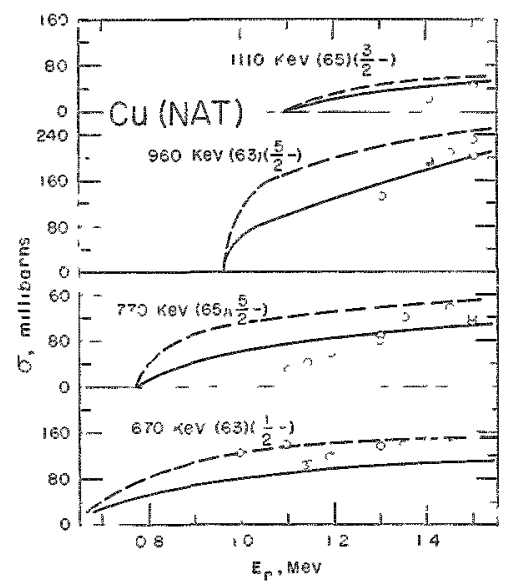

\section{Inelastic Scattering Summary}

The examples we have seen substantiate our initial promise that inelastic scattering is a process peculiar to each individual nucleus. 
The theoretical results are largely qualitative, but they represent a marked improvement over what existed only a short time ago. Much of this improvement is due to the large amount of experimental data now becoming available. I would expect this theoretical and experimental productivity to increase rapidly during the next few years. However, a major obstacle is inelastic scattering from the fissile elements. It will be some time before our knowledge of inelastic scattering from $U^{235}$ is comparable with our present understanding of $U^{238}$. The higher isotopes of plutonium are at least an order of magnitude more difficult. Perhaps theory will be the answer in this region for some time to come.

VI. Conclusion

I have tried to summarize each of the above topics as we have concluded the respective discussions. Thus, I now feel free to philosophize a. bit during these last few moments. First, I believe that the physical theory of chain reactions and basic microscopic data are the ultimate keys to successful reactor physics. This opinion is not unique, as evidenced by the growing interest in nuclear data, particularly in Europe. Macroscopic engineering experiments usually offer only short-term and limited rewards. Secondly, I would like to stress the importance of nuclear-data analysis, storage, and evaluation. At this date, we are still attempting to organize and utilize the available information in the format of the conventional "barn book." I submit that this method is archaic and that a modern system of data analysis, storage, and utilization is a matter of highest priority. Modern nuclear laboratories are capable of producing an enormous amount of information from their highly automated facilities. A way to properly utilize this knowledge must be found

\section{References}

1. W. D. Allen and R. L. Henkle, Fast Neutron Data on the Isotopes of Th, U, and Pu, Progress in Nuclear Energy, Series I, Physics and Mathematics Vol. II (Pergamon Press, London, 1958).

2. D. K. Butler, Fission Cross Section of $\mathrm{Pu}^{238}$, Bull. Am. Phys. Soc. 8, 4 (1963).

3. D. K. Butler, Private Communication.

4. P. White, R. Batchelor, et al. (Private Communication).

5. J. Hopkins and B. Diven, Nuclear Science and Engineering (To be published). 
6. D. S. Mather, P. Fieldhouse and A. Moat (Private Communication). Also, see Ref. 5

7. J. Meadows and J. Whalen, Energy Dependence of Prompt $\bar{\nu}$ for Neutron Induced Fission of $U^{235}$, Phys Rev。 126, 197 (1962).

8. J. Meadows (Private Communication).

9. H. Condé and N. Starfelt, Measurement of $\bar{\nu}$ in Fast Neutron Fission of $\mathrm{Th}^{232}$ and $\mathrm{U}^{238}$ Nuclear Sci. and Eng. 11, 397 (1961).

10. S. A. Cox (Private Communication).

11. D. C. Stupegia, A. B. Smith and K I. Hamm, Fast Neutron Capture in $\mathrm{Th}^{232}$, Journal of Inorganic and Nuclear Chemistry, 25, 627 (1963).

12. J. Barry et al (Private Communication).

13. J. H. Gibbons, W. M. Good, and R. L. Machlin et al. (Private Communication), see also ORNL-2910 (Unpublished).

14. See Ref. 12; also, S. A Cox et al., Neutron Activation Cross Sections for $\mathrm{Br}^{79}, \mathrm{Br}^{81}, \mathrm{Rh}^{103}, \mathrm{In}^{115}, \mathrm{I}^{127}$, and $\mathrm{Ta}^{181}$ ( $\mathrm{To}$ be published)。

15. A. B. Smith. Unpublished results of author.

16. A. B. Smith, Elastic Scattering of Neutrons from U ${ }^{235}$, Nuclear Science and Engineering (In Press).

17. A. B. Smith, The Scattering of Fast Neutrons from Natural Uranium, Nuclear Physics (In Press)

18. A. B. Smith, The Elastic and Inelastic Scattering of Fast Neutrons from $T h^{232}$, Phys。Rev。 126,718 (1962)

19. S. O. Moore, E. H. Auerbach, Optical Model Analysis of Inelastic Scattering of Neutrons by Heavy Nuclei, BNL-818 (T-317) (1963).

20. A B. Smith, The Scattering of Fast Neutrons from W' $W^{124}$, Zeitschrift für Physik (In Press)

21. A. B. Smith, Fast Neutron Scattering from Ta ${ }^{181}$, ANL-6727 (1963).

22. J. A. M. de Villiers and A B. Smith, Fast Neutron Scattering from Gold, Bull Am. Phys Soc, I, 6 (1962)。 
23. D. Reitmann, C. Engelbrecht, and A, B. Smith, The Scattering of Fast Neutrons from Natural $\mathrm{Zr}$ and $\mathrm{Nb}$, Nuclear Physics (In Press).

24. A. B. Smith, C. A. Engelbrecht, and D. Reitmann, Scattering of Fast Neutrons from Copper, Zinc, and Cobalt (To be published).

Discussion of Paper

Presented by Mr. Smith

MR.WEINBERG (Oak Ridge):

Apropos of your last comment concerning the acquisition of data, I wonder if you or some of the people at Brookhaven would be willing to comment on the function of the Sigma Center there and of the Nuclear Constants Center at Argonne?

MR SMITH:

It is perhaps inappropriate that I comment too much on Sigma Center. At Argonne we are trying to coordinate our efforts with Sigma Center; for example. we use the Sigma Center tape code symbolism to write the new request list for nuclear data, so there will be an automated cross check periodically. hopefully at every quarter Perhaps somebody from the Sigma Center would make some comments.

MR. IEVINE (Brookhaven).

The Sigma Center is automating data handling and data storage by means of a magnetic tape that can be interrogated by the computer. The question of how data can be stored so as to be compatible with the use of another laboratory is also being investigated. I believe that this question. is a lot more difficult than it might seem superficially. This isn't a question simply of storing multigroup cross sections so that one code or an other could use them. This is the question of whether you are storing angular distributions together with the resolution of the measurements: whether you are storing cross sections as a function of energy with resolution on the energy and so on

All of this is under study. I believe, at manv other places in addition to Brookhaven. The primary work at Brookhaven right now will soon allow us to have this data interrogation scheme from a magnetically stored library by the computer. 
MR.SMITH:

Yes. I think it is the only way to go. The problem of evaluation also enters in it. The theoretical calculations by Moore, Auerbach, and Porter are also part of the Sigma Center.

MR. LEVINE:

Yes, indeed. This data storage will include not only measurements, but it will also include every category of cross section information that you can think of. That is, there will be a category for calculated cross sections, too. If you ask for all known information, for example, you can get that. If you ask for experimental information, you will get that.

MR. SMITH:

The two do not agree usually.

MR. GREEBIER (General Electric):

Accurate microscopic cross-section data constitute the key to successful reactor physics design. How can we who are involved in reactor design best transmit our needs to you who are doing the measurements and have these needs translated into measurements on a suitable priority basis?

MR. SMITH:

There is a formula for this. It starts with the Office of the Division of Research, and ultimately comes to the cross-section group to be put on a list. Too often, having made this request does not lead to imme diate measurements, however.

There is also the method of personal contact. I feel this is perhaps the best way.

MR. HÄFELE (Karlsruhe):

I would like to comment that European groups forward their requests, including a priority list, to the European Atomic Nuclear Data Committee (EANDC). At frequent meetings, priorities are fixed. This procedure works very well.

MR. SMITH:

New requests for nucleax data coming from the United States crosssectional group are cross referenced with the EANDC list. Where the work 
is being done in Europe, it is so noted. Where the request is a priorityone item, it is so noted.

MR. LOEWENSTEIN (Argonne):

The 6-8\% discrepancy in Fig. 1 between the accepted $U^{235}$ fission cross section and the recent measurements is quite large. Do you have any idea of what currently are the best values between 10 and $100 \mathrm{kV}$, and if you don't, what is the best way of resolving the discrepancy?

MR. SMITH:

I have no answer to that problem. I only know that I looked at the measurements, I talked to the people who did them, and I am reasonably convinced they did a very good job. If you look at all existing data that go back a number of years, it is not too shocking to find out that this dis crepancy exists, because the previous measurements have easily wandered around by more than that. I believe that Los Alamos has ventured on a program of measuring fission cross sections to solve this problem.

MR. PATTENDEN (Harwell):

In addition to the data which was shown by Ferguson (Paper I-1), Moxon at Harwell also has new data on capture cross sections of indium and gold. His results seem to be somewhat lower than yours.

MR. SMITH:

Fine. I wish to emphasize that the data that were shown are not necessarily our data.

MR. FERGUSON (Harwell):

With regard to the fission cross-section problem again, the discrepancy which you mention is of the order of $6-7 \%$. I would take a definition of a discrepancy as when measurements differ outside the errors. The points measured have an error of about $2 \frac{1}{2}-3 \%$. The old measurements, with which I was associated, had an error of about $5 \%$ in the region of $120 \mathrm{keV}$, and an error of about $3 \%$ in the region of $550 \mathrm{keV}$. Thus, the measurements do not really differ too far outside the experimental errors. The line that you showed has no real significance.

MR. SMITH:

Yes, I agree. If all the actual measurements through the years are plotted, a wide band is obtained. The new measurements lie at the lower extremities of the band. 


\title{
RECENT IMPROVEMENTS IN CALCULATIONS OE DOPPLER AND SODIUM REACTIVITY EFFECTS FOR LARGE FAST REACTORS
}

\author{
P. Greebler \\ General Electric Company \\ Atomic Power Equipment Department \\ San Jose, California.
}

Considerable work has been done in the past few years to improve our understanding of the Doppler and the sodium-loss reactivity effects occurring in large, dilute fast reactors. This paper will deal first with the sodium-loss problem, including its effect on the Doppler coefficient, and conclude with a few specific topics of interest to Doppler calculations.

Factors in Sodium-loss Reactivity Calculation

The sodium-loss reactivity problem for a $\mathrm{U}^{238}$ - and plutoniumfueled fast reactor arises mainly from the hardening of the neutron spectrum when sodium atoms are expelled.(1-3) Hardening of the spectrum, in turn, increases reactivity by several processes. These include decreasing the $\mathrm{Pu}^{239}$ capture-to-fission
1. Loss of $\mathrm{Na} \xi \sum_{\mathrm{s}}(+)$
2. Loss of $N a \Sigma_{\text {fr }}(-1)$
3. Loss of Na $\Sigma_{a}(+1$
4. Increase of Reflector Savings $(t)$
5. Reduction of $\xi \Sigma$ of Steel \& Oxygen $(t)$
6. Reduction of U.238 $\Sigma_{a}(t)$
A. Higher Pu Isotopes \& Fission Products $(t)$
B. Pu Buildup in Blanke $1+1$
c. Loss of Doppler with Loss of $\mathrm{Na}$
D. Spatial Dependence of Na Loss Reactivity

Fig. 1. Factors in Sodium Loss $\triangle \mathrm{k}$ Calculations ratio, increasing the fast fission yield in $\mathrm{U}^{238}$, reducing the absorp tion rate in $\mathrm{U}^{238}$ and $\mathrm{Pu}^{240}$ more than the fission rate in $\mathrm{Pu}^{239}$ is reduced, and decreasing the absorption rate in fission products, in control rods, and in other parasitic materials. A compensating factor is the increase in neutron leakage upon loss of sodium atoms, which reduces reactivity. Several of the factors which must be considered in calculating the reactivity change due to partial or total loss of sodium are shown in Fig. 1.

The reactivity gain due to hardening of the spectrum upon loss of the sodium slowing down cross section and the reactivity loss due to increasing neutron leakage upon loss of the sodium transport cross section were discussed above. These are the dominant reactivity effects, but, because they are of opposite sign, and, hence, tend to cancel, several smaller effects take on a considerable importance. One of these is loss of the sodium capture cross section, which occurs mostly in the strong sodium resonance near $3 \mathrm{keV}$. 
This effect is calculated to vary from about 0.3 to $1 \%$ in reactivity for large ceramic-fueled reactors, the larger values corresponding to more degraded neutron spectra. This range is based on an assumed l-ev radiative capture width for the strong sodium resonance (and this $\Gamma_{\gamma}$ value is highly uncertain). There is an appreciable positive reactivity effect due to the increase of reflector savings when sodium is lost, particularly if it is lost only in the core and not in the blanket. This effect tends to reduce the negative reactivity due to increased leakage with loss of the sodium transport cross section. When doing one-dimensional diffusion calculations on cylindrical reactors, the energy-dependent perpendicular bucklings must be reduced to account for the higher reflector savings with loss of sodium. A small positive reactivity effect occurs because of a decrease of the multigroup slowing down cross sections of the lighter isotopes, steel and oxygen, when sodium is removed. This effect was first noted by Hummel et al., in results obtained from calculations with their many-group ELMOE Code $(4,5)$ Another small positive reactivity increment results from the loss of the sodium potential scattering cross section which, in turn, reduces the $U^{238}$ effective resonance integrals and, hence, lowers the $U^{238}$ multigroup absorption cross sections.(6) The sum of these latter two effects is generally less than 0.5 per cent in reactivity. In order to take them into account, however, in diffusion calculations, it is necessary to use a different set of multigroup cross sections for the sodium-out case than for the normal sodium-in condition, or at least a perturbation calculation must be superimposed on the sodium-out condition.

There are some additional factors to be considered when evaluating the potential magnitude and sign of the reactivity change due to sodium loss. First, the presence of the higher plutonium isotopes and of fission products in highly irradiated fuel adds significantly to the positive component of the sodium coefficient. $(7,8)$ In typical cases with oxide fuel at 50,000-MWD/t average burnup, we computed the reduced capture rate in $\mathrm{Pu}^{240}$ and in fission products to contribute almost as much to the positive sodium reactivity effect as did the reduced capture in $U^{238}$. Neutron capture in the se relatively dilute constituents is particularly sensitive to the neutron spectrum and, hence, to sodium loss, because their low-energy resonances are not appreciably self-shielded.

Plutonium buildup in the blanket adds significantly to the positive sodium-loss reactivity effects, typically 0.3 to $1.0 \%$, depending on the specific geometry and the blanket exposure. (A $1.0 \%$ effect was computed for the extreme case of a $38-\mathrm{cm}$-thick core with $50 \mathrm{v} / 0$ sodium when the axial blanket composition changed from a clean condition to one corresponding to the $\mathrm{Pu}^{239}$ distribution after exposure to $50,000-\mathrm{MWD} / \mathrm{t}$ core burnup.)

Hardening of the spectrum by loss of sodium reduces the Doppler coefficient, since the Doppler effect is sensitive to the neutron spectrum. $(9,10)$ This interaction between loss of sodium and the Doppler effect 
makes some reactor compositions having large sodium volume fractions appear unattractive even though the large sodium fraction may render a high neutron leakage and, hence, a negative reactivity change upon total loss of the coolant. (11)

Another important factor is the spatial dependence of the sodiumloss reactivity effect. (12) The positive reactivity, which is associated with the lowering of the rate of neutron absorption relative to neutron production when the spectrum is hardened, makes its highest contribution in the center of the reactor, where the neutron and adjoint fluxes are highest. The negative reactivity effect, which is associated with the increased rate of leakage upon loss of sodium transport cross section, makes its major contribution in the outer portions of the core, where the gradient of the flux is high. If one now considers a process in which the removal of sodium starts in the center of the reactor core and moves outward - a loss-offlow accident could conceivably lead to such a sequence of sodium voidage the reactivity increment would initially be positive and increase to a maximum as the voidage proceeded outward, and then would drop off as the voidage reached the outer portions of the reactor where the leakage effect is dominant.

Figure 2 illustrates this situation. The per cent reactivity increase resulting from removal of all the sodium in a central region of the reactor is plotted against the half-thickness of the region from which sodium has been removed. The two curves are for two different reactor compositions, each with cores of $61-\mathrm{cm}(2-\mathrm{ft})$ thickness and $370-\mathrm{cm}$ diameter for reactor $\mathrm{A}$, of $425-\mathrm{cm}$ for $\mathrm{B}$ (essentially slab reactors).

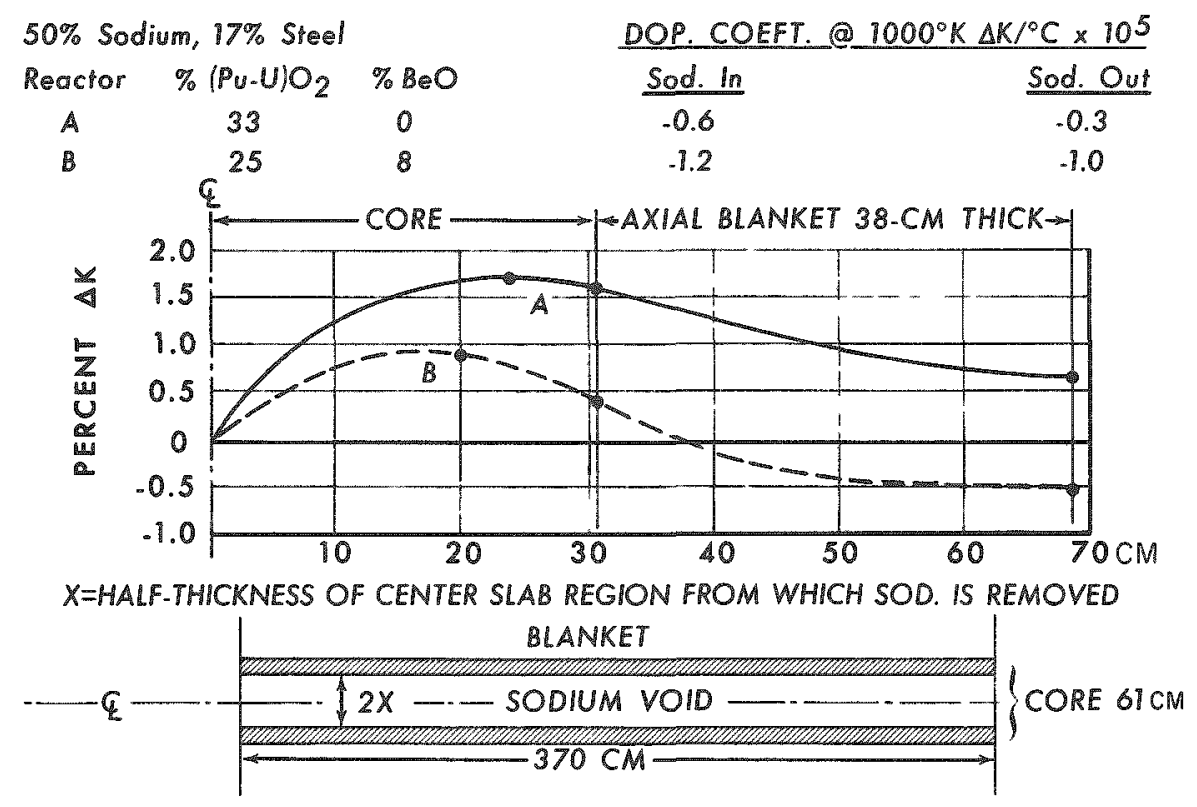

Fig. 2. Sodium and Doppler $\Delta k$ Effects 
Consider the curve for Reactor A. Removal of sodium from a $46-\mathrm{cm}-$ thick slab comprising the center portion of the core (23-cm half-thickness) produces about a $1.7 \%$ positive reactivity. Further removal of sodium in the outer portion of the core reduces reactivity slightly, and sodium voldage from the entire core and blanket produces a net positive reactivity of about $0.7 \%$ (less than half of the maximum value).

Use of Moderating Material to Improve Sodium and Doppler Coefficients

The data in the right-hand corner of Fig. 2 illustrate the interaction between sodium loss and the Doppler effect. For Reactor A, loss of the coolant reduces the Doppler coefficient to about one-half of its original value. In order to increase the magnitude of the Dopplex coefficient and to reduce the interaction between sodium loss and the Doppler coefficient, a study was made of the effect of adding some moderating material to the reactor composition.(13) Reactor $B$ has the same amount of sodium and steel as $A$, but $8 \mathrm{v} / 0$ BeO has been added, replacing some of the fuel. With this small addition of moderating material, several large improvements are realized in the Doppler and sodium coefficients. The Doppler coefficient is appreciably increased as a result of the degradation of the neutron spectrum by the BeO; and the effect of sodium loss on the Doppler coefficient is considerably diminished (at least, on a fractional basis). Since the influence of the sodium on the neutron spectrum has been appreciably lessened, the positive reactivity from sodium voidage in the center of the core is considerably reduced. (For Reactor $B$, the maximum reactivity is $0.8 \%$ corresponding to sodium voidage in a central slab of $20 . \mathrm{cm}$ half-thickness.)*

In the portion of the core near the blanket, the increased leakage due to loss of sodium increases the neutron-moderating effectrveness of the blanket containing BeO, the reby partrally compensating the spectrum hardening due to loss of the sodium Loss of sodium from the entire core gives a $0.46 \%$ positive reactivity for Reactor $B$, and coolant loss from both core and blanket gives a significant negative reactivity $(-0.5 \%)$.

A negative reactivity for total loss of sodium from core and blankets can be achieved without addition of moderating material by increasing the sodium volume fraction to about $60 \%$. This approach is not satisfactory, however, since it gives a large positive reactivity $(\sim 1.5 \%)$ for loss of sodium in the center portion of the core (1.0\% $\Delta \mathrm{k}$ for total core loss) and aggravates the problem of the loss of Doppler coefficient due to loss of sodium. We also found that use of a thinner core $(46 \mathrm{~cm})$ without moderating material does not give a satisfactory solution to the sodium coefficient problem $(1 \% \Delta \mathrm{k}$ for total core loss and $-0.3 \times 10^{-5}$ Doppler coefficient at $1000^{\circ} \mathrm{K}$ ).

*If the coolant voidage is varied in the radial direction as well as axially, the maximum reactivity changes for central voldage are increased to $1.8 \%$ and $1.05 \%$, respectively, for Reactors $\mathrm{A}$ and $\mathrm{B}$. 
Figure 3 shows per cent reactivity as a function of reduction in the sodium density in the central regions of Cores $A$ and $B$, corresponding to the maximum $\triangle \mathrm{k}$ values in Fig. 3 for $100 \%$ reduction of sodium density. The curve of reactivity versus sodium density reduction for Reactor $A$ appears to have a slight negative curvature, that for $B$ a small positive curvature. This would be consistent with Reactor $B$ having (1) a $12 \%$ lower neutron leakage and (2) a greater sensitivity to the highly self-shielded sodium resonance at $2.8 \mathrm{keV}$ (Reactor $B$ has 2.3 times as many fissions below $10 \mathrm{keV}$ as Reactor A). The number of computed data points is too small, however, to attach very much significance to these indicated curvatures.

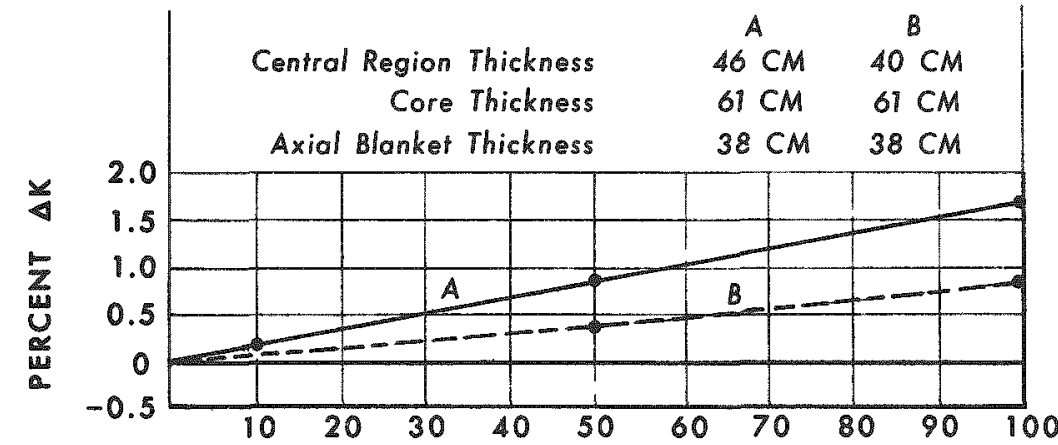

PERCENT REDUCTION OF SODIUM DENSITY IN CENTRAL REGION

Vol. Composition (\%)

Atom \% $P_{u}(239+241)$ in $\left(U+P_{u}\right)$ Core Breading Rotio Total Breeding Rotio
A

No 50, Steel 17, Fuel 33

11.5

0.84

1.34
$B$

Na 50, Steel 17, Fuel 25, BeO 8

14.7

0.71

1.12

Fig. 3. $\Delta k$ vs. Sodium Density Reduction

The safety improvements realized with the use of the moderating material, namely, a larger Doppler coefficient, one which is relatively insensitive to loss of sodium, a much smaller maximum reactivity increment due to central coolant voidage, and a negative reactivity for total coolant voidage from core and blankets are achieved in return for some decrease in the neutron economy. This is shown by the plutonium "enrichments" and breeding ratios listed at the bottom of Fig. 3 for Reactors A and B. I will not comment further on the safety and economics aspects of these problems, as they will be covered in a later paper by Dr. Karl Cohen.

There are several variations in the use of moderating material which can be considered with respect to both geometrical configurations and the type of material used. For example, one would expect that hydrogen would be an ideal material for this purpose (considering only the physics of the situation). This is because it has a large slowing down 
cross section relative to its transport cross section and, hence, lessens the influence of sodium on the spectrum much more than its influence on leakage. Several cases were computed with $Z$ rH as the moderating material; it was found that this material was slightly more effective than BeO for reducing the reactivity increment due to sodium loss. (15) However, the desirable amount of hydrogen did not produce as large a Doppler effect as was obtained with BeO. This is because the hydrogen slows down an appreciable number of neutrons below $250 \mathrm{eV}$ where the contribution to the Doppler effect is considerably less than at energies in the 500-to 2000-eV region. (This is mainly because the change in the $\mathrm{U}^{238}$ capture cross section due to Doppler broadening at energies below $250 \mathrm{eV}$ is a much smaller fraction of the total absorption cross section than at energies in the $500-$ to $2000-\mathrm{eV}$ range.)

Influence of New Cross-Section Data on Sodium and Doppler Calculations

It needs to be emphasized that the accuracy of the aforementioned results for both sodium and Doppler coefficients is limited by the present unsatisfactory status of cross-section data for large, dilute fast reactors. Furthermore, many complex factors are contributing positive and negative reactivities to these coefficients; and it is not at all certain that they are all being adequately treated with the various approximations that must be made in the computational models. We expect that the qualitative trends shown in the comparisons of Reactors $A$ and $B$ above are valid and will withstand expected changes in cross-section data and refinements in computational methods. It would be extremely fortuitous, however, if the quantitative results did not change somewhat as such new information became available. Deficiencies in cross-section data that significantly influence the computed coefficients include the alpha values for all the plutonium isotopes (and especially for $\mathrm{Pu}^{239}$ below $30 \mathrm{keV}$ ) and the capture cross section (or $\Gamma_{\gamma}$ ) in the $2.8-\mathrm{keV}$ sodium resonance.

About two years ago, when the Hopkins and Diven data(16) on the high-energy $\mathrm{Pu}^{239}$ alpha values were released, we learned that the calculated Doppler effect was highly sensitive to changes in cross-section data which affected the energy dependence of the neutron importance. The new $\mathrm{Pu}^{239}$ alpha values made low-energy neutrons less important and, hence, reduced the magnitude of the Doppler effect which is primarily a low-energy process. About the same time, the new YOM cross section set (17) for the higher energies was issued and predicted a considerably harder neutron spectrum for dilute fast reactors than had been computed earlier. Fig. 4 shows the influence of the new data and of improvements in computation methods on the spectrum and on Doppler and sodium reactivity effects and the breeding ratio for a 500-MW(e) fast oxide reactor. Also listed for comparison purposes are the results for Reactors $A$ and $B$ discussed above. We found that the combination of the new YOM cross sections and the new Pu ${ }^{239}$ alpha values reduced the computed Dopplex coefficient of the 
500-MW(e) fast oxide reactor by about 30\%. (18) These changes of cross section also added about $0.6 \%$ positive reactivity in a total-loss-of-sodium accident. The present status of the cross-section data still does not permit a precise prediction of the neutron spectrum in dilute, plutoniumfueled reactors. This is the case even for $\mathrm{U}^{235}$-fueled assemblies, as noted by Davey in his analysis of some of the recent ZPR-III neutronlifetime measurements. (19)

Fig. 4

NEUTRON SPECTRUM AND RELATED PHYSICS PARAMETERS

\begin{tabular}{l|c|c|c|c}
\hline & \multicolumn{2}{|c|}{$500 \mathrm{MW}(\mathrm{e})$} & \multicolumn{2}{|c}{$1000 \mathrm{MW}(\mathrm{e})$} \\
\cline { 2 - 5 } & $\begin{array}{c}\text { Old } \\
\text { Data }\end{array}$ & $\begin{array}{c}\text { New } \\
\text { Data* }\end{array}$ & $\begin{array}{c}\text { Case A } \\
\text { No BeO }\end{array}$ & $\begin{array}{c}\text { Case B } \\
\text { BeO }\end{array}$ \\
\hline Mean Neutron Energy in Power Spectrum(keV) & 180 & 210 & 230 & 100 \\
\% Fissions below 9 keV & 15 & 13 & 12 & 28 \\
Doppler Coefficient at $1000^{\circ} \mathrm{K}\left(\frac{\Delta \mathrm{k}}{\circ \mathrm{C}} \times 10^{5}\right)$ & -1.1 & -0.68 & -0.60 & -1.2 \\
\%o for Total Loss of Sodium in & & & & \\
Core and Blankets & $\sim 0$. & +0.6 & +0.7 & -0.5 \\
Total Breeding Ratio & 1.08 & 1.36 & 1.34 & 1.12 \\
\hline
\end{tabular}

*Includes effects of new YOM cross sections and $\mathrm{Pu}^{239}$ alphas.

In the area of Doppler calculations, one significant cross-section development is the extension of the resolved resonance region for $\mathrm{U}^{238}$ to almost $2000 \mathrm{eV} .(20)$ This means that the major contribution to the Doppler coefficient in a very large fast ceramic reactor no longer involves the uncertainties associated with the statistical distribution of unresolved resonances. Fortunately, the new $\mathrm{U}^{238}$-resonance data, published by Firk, Lynn, and Moxon of Harwell, $(20)$ followed the expected distributions rather closely, and there were no unpleasant surprises so far as the Doppler effect is concerned. It would be very advantageous to the fast reactor programs to have the energy range of resolved $U^{238}$ resonances extended further, and also to have resolved $\mathrm{Pu}^{240}$-resonance data up to at least $1 \mathrm{keV}$.

Resonance Overlap Effect in Doppler Calculations

In the area of Doppler calculation methods, probably the most significant recent development was the study by Codd and Collins of the overlapping of the low energy $\mathrm{U}^{238}$ and $\mathrm{Pu}^{239}$ resonances. (21) Our calculations on the effect of this resonance overlap, using essentially the same method as Codd and Collins, but taking into account the distributions of the resonance parameters, indicated that the negative Doppler coefficient 
contributed by this overlapping was less than that originally reported. (21) Some results of our overlap calculations are shown in Fig. 5.*

Fig 5

U-PU RESONANCE OVERLAP DOPPLER EFEECTS

\begin{tabular}{|c|c|c|c|}
\hline \multirow{2}{*}{$\begin{array}{c}\text { Energy, } \\
e V\end{array}$} & \multicolumn{2}{|c|}{$\Delta \sigma_{\mathrm{a}}$ for $\mathrm{Pu} \mathrm{u}^{239 *}$} & \multirow{2}{*}{$\begin{array}{c}\text { Fuel Temperature } \\
\text { Rise }\left({ }^{\circ} \mathrm{K}\right)\end{array}$} \\
\hline & $\begin{array}{c}\text { Neglecting } \\
\text { Overlap }\end{array}$ & $\begin{array}{c}\text { Including } \\
\text { Overlap }\end{array}$ & \\
\hline 100 & 2.6 & 2.0 & 700 to 1400 \\
\hline 500 & 0.46 & 0.32 & 700 to 1400 \\
\hline 800 & 0.24 & 0.05 & 700 to 1400 \\
\hline $1,000 * *$ & 0.18 & 0.05 & 700 to 1400 \\
\hline $10,000 * *$ & 0.006 & -0.002 & 700 to 1400 \\
\hline $1,000 * *$ & 0.20 & 0 & 1000 to 2250 \\
\hline $1,000 * *$ & 0.18 & $0.13\left(\mathrm{Pu}^{240}\right)$ & 700 to 1400 \\
\hline
\end{tabular}

* For $\sigma_{\mathrm{p}}=430$ per $\mathrm{Pu}^{239} ; \mathrm{U}^{238}$ to $\mathrm{Pu}^{239}=7$.

**For $\mathrm{U}^{238}$ Resonances Unresolved.

The two center columns of data give the calculated increase in the absorption cross section of $\mathrm{Pu}^{239}$ at various energies due to an increase of the fuel temperature (as indicated in the right-hand column). The data at the left were obtained by neglect of the overlap of $\mathrm{U}^{238}$ and $\mathrm{Pu}^{239}$ resonances; that on the right took this overlap into account (except that the last value is for overlap of $\mathrm{Pu}^{240}$ and $\mathrm{Pu}^{239}$ resonances). At energies around $1000 \mathrm{eV}$ (and at higher energies up to $10 \mathrm{keV}$ ) the negative Doppler effect due to the overlap cancelled out most of the positive $\mathrm{Pu}^{239}$ Doppler effect due to its own self-shielding. At lower energies the overlap cancelled out a much smaller portion of the $\mathrm{Pu}^{239}$ Doppler effect. For higher temperatures than the 700 to $1400^{\circ} \mathrm{K}$ fuel-temperature change considered in the upper data of Fig. 5, the overlap effect becomes larger as noted for the 1000 to $2250^{\circ} \mathrm{K}$ fuel-temperature change at $1000 \mathrm{eV}$. For a large fast oxide reactor, such as Reactors $A$ and $B$ described previously, the overlap accounted for about a 10 per cent increase in the magnitude of the negative Doppler coefficient. (The overlap also contributes a small positive reactivity to the sodium coefficient - about $0.2 \% \Delta \mathrm{k}$ for total loss of sodium - since $\mathrm{Pu}^{239}$ cross sections at low energy are depressed relative to those of the $\mathrm{U}^{238}$ by the overlapping.)

Overlap calculations were also made at $1000 \mathrm{eV}$ for $\mathrm{Pu}^{240}$ and $\mathrm{Pu}^{239}$ resonances, and this interaction is seen in Fig. 5 to reduce the $\mathrm{Pu}{ }^{239}$ Doppler

*A computer code RAVE was programmed to carry out the overlap corrections. Details of the code will be published later. 
effect from $\Delta \sigma_{a}=0.18$ to 0.13 . Taking into account all of the overlap effects in a reactor containing interacting resonances of three different absorbers is a formidable task when the resonances are in the unresolved region; it is difficult enough for just one resonance absorber in the energy region of weak or intermediate overlapping. However, now that the $\mathrm{U}^{238}$ resonance parameters have been resolved over the energy range in which most of the Doppler effect occurs in a very large dilute fast reactor, it is not too difficult to calculate cross sections which are directly flux-averaged over the resonances.

We developed a computer code which uses a very fine-energy multigroup scheme to calculate fluxes and use them to average cross sections of resonance matexials over any energy interval which may contain many resonances of that material and also of two additional resonance absorbers. One of the results obtained with this code was an evaluation of the $U^{238}$ selfoverlap correction in the energy region where one generally assumes the resonance integral formulation valid for highly separated resonances.* ( This $\mathrm{U}^{238}$ self-overlap correction is actually just a reduction of the average flux due to Doppler broadening of the $U^{238}$ resonances. When this effect is taken into account, the $\mathrm{U}^{238}$ and $\mathrm{Pu}{ }^{239}$ overlap results change in such a manner as to make part of the overlap appear as an additional negative $\mathrm{U}^{238}$ Doppler reactivity and the reduction of the $\mathrm{Pu}^{239}$ positive Doppler reactivity becomes correspondingly lower.) At $1000 \mathrm{eV}$, we computed that the flux-averaged change in the $U^{238}$ cross section due to the Doppler effect (700 to $1400^{\circ} \mathrm{K}$ ) was $10 \%$ higher than that obtained from the formulation for highly separated resonances. With respect to the $\mathrm{U}^{238}$ and $\mathrm{Pu}{ }^{239}$ overlap, we found that the averaged $\mathrm{Pu}^{239}$ change of cross sections due to the Doppler effect was quite sensitive to the positioning of the $\mathrm{Pu}^{239}$ resonance relative to those of the $\mathrm{U}^{238}$. Even with a random sampling of the spacing distributions in an energy interval containing about $100 \mathrm{Pu}^{239}$ resonances and $14 \mathrm{U}^{238}$ resonances, it was necessary to carry out several runs to get a good statistical average. This indicates that the overlap effect may be significantly larger or smaller than that computed by averaging over all possible spacings and resonance parameter distributions of $\mathrm{U}^{238}$ and $\mathrm{Pu}^{239}$, depending on how nature may actually have chosen to position the various $\mathrm{Pu}^{239}$ resonances relative to those of the $U^{238}$ within a given energy interval.

\section{Conclusions on Other Factors in Doppler Calculations}

Other conclusions that we have reached from our Doppler studies, listed in Fig. 6, are:

1. The effect of heterogeneity of fuel and coolant on the Doppler coefficient of a typical fast oxide reactor is negligibly small. (25)

*This is the formulation utilized in our earlier Doppler studies $(22,23)$, and it is the basis for Nicholson's Method B. (24) 
2. Crystalline binding of the uranium atoms in a $\mathrm{UO}_{2}$ lattice introduces an uncertainty of the order of $25 \%$ in the Doppler coefficient at low fuel temperatures due to an uncertainty of the Debye temperature of the lattice. (26) It appears that crystalline binding is not an important effect at high fuel temperatures representing full power operating conditions.

3. The Doppler effect computed using a multilevel formalism with resonance parameters obtained from a Breit-Wigner single-level model gives essentially the same result as that obtained with a consistent single-level model in the energy region around $1 \mathrm{keV}$. (27)

4. Only the $s$-wave resonances make a significant contribution to the Doppler effect in a large fast ceramic reactor. (28)

CRYSTALLINE INDING

$\sim 25 \%$ Uncertainty in Low Temp. Dop. Coeff. Due to vO 2 Debye Temp. Unceriainty

HETEROGENEITY OF FUEL \& SODIUM

Negligible Effect on Doppler

MULTILEVEL FORMULATION \& P-WAVE RESONANCES

Appear Unimporiant for Doppler of Large Dilute Reactor

Fig. 6. Factors in Doppler Calculations

References

1. J. B. Nims and P. F. Zweifel, Preliminary Report on Sodium Temperature Coefficients in Large Fast Reactors, APDA-135, pp. 6-13 (1959).

2. P. Greebler, B. A. Hutchins, and J. R. Sueoka, Calculation of Doppler Coefficient and Other Safety Parameters for a Large Fast Oxide Reactor, GEAP-3646 (March 9, 1961).

3. D. Okrent, Breeding, Safety and Fuel Cycles, paper presented at the VIII Nuclear Congress, Rome, Italy (June 17-23, 1963).

4. M. G. Bhide and H. H. Hummel, "Reactivity Coefficient of Sodium in Fast Assemblies," Physics of Fast and Intermediate Reactors, Vol. II, 117 (1962), Proceedings of Seminar in Vienna, August, 1961. 
5. H. H. Hummel and A. L. Rago, "An Accurate Treatment of Resonance Scattering in Light Elements in Fast Reactors," Physics of Fast and Intermediate Reactors, Vol. I, 231 (1962), Proceedings of Seminar in Vienna, August, 1961 .

6. P. Greebler and E. Goldman, Doppler Calculations for Large Fast Ceramic Reactors - Effects of Improved Methods and Recent Cross Section Information, GEAP-4092, Table 7, pp. 2-19,2-20 (Dec 1962).

7. S. Yiftah, "Effect of the Plutonium Isotopic Composition on the Performance of Fast Reactors," Physics of Fast and Intermediate Reactors, Vol. II, 257 (1962), Proceedings of Seminar in Vienna, August, 1961 .

8. D. Okrent, "Performance of Large Fast Power Reactors Including Effects of Higher Isotopes," Physics of Fast and Intermediate Reactors, Vol. II, 271 (1962), Proceedings of Seminar in Vienna, August, 1961.

9. M. G. Bhide and H. H. Hummel, Calculations of the Doppler Coefficient of Large Ceramic-fueled Fast Reactors, ANL-6601 (1962).

10. See Ref.6, Table 22, pp. 4-2.

11. See Ref. 2, Fig. 3, pp. 16.

12. See Ref. 1, pp. 13-17.

13. Sodium Cooled Reactors Program, Fast Ceramic Reactor Development Program, Fifth Quarterly Report, GEAP-4158, Section VII, pp.7-10 and 7-11, (October-December 1962).

14. K. P. Cohen, Reactor Safety and Fuel Economics Considerations for Fast Reactors:presented at this conference, Session IV, Safety and Economics.

15. Sodium Cooled Reactors Program, Fast Ceramic Reactor Development Program, Seventh Quarterly Report, GEAP-4300, Section VII (AprilJune 1963).

16. J. C. Hopkins and B. C. Diven, Neutron Capture to Fission Ratios in $\mathrm{U}^{233}, \mathrm{U}^{235}, \mathrm{Pu}^{233}$, Nuclear Science and Engineering, 12, 169 (1962).

17. S. Yiftah, D. Okrent, and P. Moldauer, Fast Reactor Cross Sections, Pergamon Press, New York (1960).

18. See Ref. 6, pp. 1-3;pp. 4-1 and $4-2$.

19. W. G. Davey, A Comparison of Experimental and Calculated Prompt Neutron Lifetimes and Central Reactivity Coefficients in ZPR-III Assemblies and Their Relationship to Other Reactor Parameters, ANL-6682 (June 1963).

20. F.W.K. Firk, J.E. Lynn, and M. C. Moxon, Resonance Parameters of the Neutron Cross Section of U ${ }^{238}$, Nuclear Physics, 41,614-629 (1963). 
21. J. Codd and J. P. Collins, Plutonium-239 and Uranium-238 Resonance Interaction Effects in a Dilute Fast Reactor, E.A.E.S. - Symposium on Advances in Reactor Theory, Karlsruhe (April 23-24, 1963).

22. P. Greebler, "Computation of Doppler Coefficient and Other Safety Parameters for a Large Fast Oxide Reactor " Proceedings of the US/UK Fast Reactor Data Conference at Harwell, TID-7605, US-3 (Sept 26-27, 1960).

23. P. Greebler and B. A. Hutchins, "The Doppler Effect in a Laxge Oxide Reactor - Its Calculation and Significance for Reactor Safety," Physics of Fast and Intermediate Reactors, Vol. III, 212 (1962), Proceedings of Seminar in Vienna, August, 1961. (See also Ref. 2.)

24. R. B. Nicholson, The Doppler Effect in East Neutron Reactors, APDA-139(1960).

25. See Ref. 6, pp. 3-1, 3-8 through 3-12.

26. See Ref. 6, pp. 4-8 through 4-12.

27. Sodium Cooled Reactors Program, Fast Ceramic Reactor Development Program, Seventh Quarterly Report, GEAP-4300, Section VII, 7.2 pp. 7-1 (April-June 1963).

28. See Ref. 6, Tables 12 and 13, pp. $2-25$ and $2-26$.

Discussion of Paper

Presented by Mr. Greebler

MR. FERGUSON (Harwel1):

Dr. Greebler has mentioned his misgivings about our knowledge of the spectrum in the region which greatly affects both sodium and Doppler coefficients. Has he done any analysis which would indicate the sensitivity of the calculated spectrum to the cross sections at higher energies?

MR. GREEBIER:

When we went over to the new YOM cross section, for example, we found that for a given reactor, the number of fissions below $10 \mathrm{keV}$, which is very critical to the Doppler coefficient, dropped from $15 \%$ down to $13 \%$. This is one measure of how much the low-energy spectrum was in fluenced by a change in the cross section.

MR. FERGUSON:

Would a very systematic study of the effect of variation of individual cross sections provide a very useful guide to people who are doing 
cross-section measurements? Most of the studies that have already been done for guidance have been mostly on the basis of reactivity calculations.

MR.GREEBLER:

Yes, I thoroughly agree that this would be very desirable. Virtually all of the studies which have been made, including our own, were integral studies. One could not separate out the effect of any one cross section for even a component cross section.

MR. LEVINE (Brookhaven):

You mentioned that uncertainties in the crystalline binding effects lead to uncertainties in the Doppler effect at low temperatures. What temperatures are low in this respect?

MR。GREEBLER:

Temperatures which are low relative to the Debye temperatures. One of the problems is a very large uncertainty of this value. For example, the literature gives anywhere from 150 up to $900^{\circ} \mathrm{K}$ for $\mathrm{UO}_{2}$. Greater uncertainty exists for mixed uranium-plutonium oxide. The best value appears to be about $600^{\circ} \mathrm{K}$. The Doppler coefficient would be expected to be lower, if one is calculating it for temperatures which are a few hundred degrees lower than that. 
•

64 


\title{
CALCULATIONS OF SODIUM VOID REACTIVITY EFFECT FOR LARGE FAST OXIDE REACTORS IN SPHERICAL AND SLAB GEOMETRY
}

\author{
H. H. Hummel, K. Phillips, and A. Rago \\ Argonne National Laboratory \\ Argonne, Illinois
}

(Paper presented by Mr. Hummel)

The work in this paper was undertaken in order to gain a better understanding of the phenomena involved in sodium removal from the core of a large fast reactor. This study is still under way, so that this paper constitutes a progress report rather than a finished piece of work. Only uniform removal from the reactor core is considered in the cases pre. sented here, but a discussion of the effect of removal from the blanket is also given. Two points that were of special interest are the following:

1. A major difficulty at present in problems of this type is that usually the reactor geometries of interest can be treated properly only with two-dimensional calculations, whereas the use of enough groups to calculate properly has not yet been feasible. If it can be shown that the spectral and capture components of the sodium void effect are not strongly dependent on details of geometry, then accurate calculation of the se is possible with fundamental mode or one-dimensional calculations, perhaps with flux-synthesis techniques. The latter would be appropriate for cores with variable fuel enrichment. The emphasis in two-dimensional calculations can then be placed on calculation of the leakage component, which may not require very many groups. Results obtained so far indicate that this surmise is reasonable, although its validity in all cases of interest is still being studied.

2. Another question considered here is the usefulness of "pan. caking" the core by going to a small length-to-diameter (L/D) ratio as a means of combatting this positive sodium void coefficient problem. Doubts as to the efficacy of this procedure were raised by the results of twodimensional calculations by Chaumont(1) An attempt to study this problem here has been made by calculations with infinite and radially bare slabs. It is concluded that these doubts are probably justified.

Calculations were made for oxide-fueled reactors with sodium contents of 52,59 , and $70 \mathrm{v} / 0$, and with stainless steel structural material. Specifications for these problems are given in Table I. The effect of loss of half and of all of the sodium from the core was calculated to study nonlinearity of $\mathrm{k}$ with sodium removal. In most of the calculations $\mathrm{Pu}^{240}$ was taken as equal to 0.4 of the $\mathrm{Pu}^{239}$ content, fission products corresponded to $2.5 \%$ burnup, and the capture associated with $1.6 \mathrm{v} / 0$ molybdenum was 
added also. The last was intended to represent a refractory metal liner several mils thick for the fuel-pin clad. The reactivity effect of this addition can be estimated from data given in the paper. Although such a liner is more clearly indicated for carbide than for oxide fuel, it is of interest to see what the reactivity effect is. This could also represent an addition of molybdenum to improve fuel conductivity.

Table I

PROBLEM SPECIFICATIONS

\begin{tabular}{c|c|c|c|c|c|c|c}
\hline \multirow{2}{*}{ Sodium } & \multicolumn{2}{|c|}{$\mathrm{v} / \mathrm{0}$} & \multicolumn{3}{c|}{ Atoms/cc $\left(\mathrm{x} 10^{-24}\right)$} & \multicolumn{2}{c}{$\sigma_{\mathrm{p}}(\mathrm{b})$} \\
\cline { 2 - 7 } & $\begin{array}{c}\mathrm{UO}_{2}-\mathrm{PuO}_{2} \\
\mathrm{Fuel}\end{array}$ & $\begin{array}{c}\text { Stainless } \\
\text { Steel (304) }\end{array}$ & Sodium & $\begin{array}{c}\text { Fuel } \\
\text { (Metal Atoms) }\end{array}$ & $\begin{array}{c}\text { Stainless } \\
\text { Steel }\end{array}$ & $\mathrm{Pu}^{239}$ & $\mathrm{U}^{238}$ \\
\hline 52 & 32 & 16 & 0.0114 & 0.00784 & 0.0136 & 300 & 40 \\
59 & 25 & 16 & 0.0130 & 0.00560 & 0.0136 & 400 & 60 \\
70 & 18 & 12 & 0.0154 & 0.00453 & 0.0102 & 400 & 60 \\
\hline
\end{tabular}

Note: There is a slight inconsistency between the $52 \%$ sodium cases and the other two, as a fuel density of $10.9 \mathrm{~g} / \mathrm{cc}$ was assumed in that case and 10.0 in the others.

In the cases in which $\mathrm{Pu}^{240}$ is present, atoms $\mathrm{Pu}^{240}$ /atoms $\mathrm{Pu}^{239}=0.40$.

Fission products when present correspond to $5 \mathrm{a} / \mathrm{o}$ of fuel $(2.5 \%$ burnup).

When added, molybdenum is $0.001011 \times 10^{24}$ atoms $/ \mathrm{cc}$.

Blanket in all cases is $52 \mathrm{~F} / 0$ sodium oxide, of $46-\mathrm{cm}$ extrapolated thickness.

All materials evaluated at $1500^{\circ} \mathrm{K}$.

The use of $\left\langle\Gamma_{\mathrm{n}}^{0}\right\rangle / \Delta$ equal to $I \times 10^{-4}$ instead of $2 \times 10^{-4}$ for $\mathrm{Pu}^{240}$ had little effect on the results.

S-wave Resonance Parameters

Pu $239 \% 2$ channels $/$ fission, $\left\langle\Gamma_{\mathrm{n}}^{0}\right\rangle / \mathrm{D}=1 \times 10^{-4} ;\left\langle\Gamma_{\mathrm{f}}\right\rangle=0.099 \mathrm{eV} ; \Gamma_{\gamma}=0.039 \mathrm{eV}$; $\mathrm{D}_{\mathrm{J}=0}=11.6 \mathrm{eV}, \mathrm{D}_{\mathrm{J}=1}=3.86 \mathrm{eV}$.

$\mathrm{U}^{238}: \quad\left\langle\Gamma_{\mathrm{n}}^{0}\right\rangle / \mathrm{D}=0.94 \times 10^{-4} ; \mathrm{D}=18.5 \mathrm{eV} ; \Gamma_{\gamma}=0.0246 \mathrm{eV}$.

$\underline{\mathrm{Pu}^{240}}:\left\langle\Gamma_{\mathrm{n}}^{0}\right\rangle / \mathrm{D}=2 \times 10^{-4} \mathrm{D}=11.0 \mathrm{eV} ; \Gamma_{\gamma}=0.034 \mathrm{eV} ; \sigma_{\mathrm{p}}=600 \mathrm{~b}$.

The blanket in the present series of calculations was always $52 \mathrm{v} / \mathrm{o}$ sodium, with a 46-cm extrapolated thickness and with no reflector. It was found in an earlier series of calculations that the use of a thicker blanket had little effect on the void effect for the core. Likewise, use of a blanket of higher density, e.g., $60 \mathrm{v} / 0 \mathrm{U}^{238}$ metal, $18 \mathrm{v} / 0$ stainless steel, and $22 \mathrm{v} / 0$ sodium, had little effect on the coefficient. Plutonium buildup in the blanket was not taken into account in any of the calculations.

\section{Cross Sections Used}

ELMOE(2) was used to calculate the macroscopic core cross sections for various full sodium volume fractions at full, half, and no sodium, 
at an enrichment ratio in the range of interest. The enrichment ratio was then adjusted to the desired value by addition or subtraction of $\mathrm{U}^{238}$, $P \mathrm{u}^{239}$, and $\mathrm{Pu}^{240}$. For the blanket the same cross sections were used, all fuel atoms being $\mathrm{U}^{238}$. The blanket was assumed to be $52 \mathrm{v} / \mathrm{o}$ sodium in every case.

The coarse-group cross-section set used was the $22-$ group Set 215 described in Ref.3. Fission and capture cross sections used in the calculations are given in Table A-I in the Appendix. The cross sections derived from ELMOE which were used for the study of the sodium-removal effect by perturbation theory are given in Tables A-II and A-III. There is seen to be little nonlinearity except at the $2.85-\mathrm{keV}$ resonance.

The cross sections for coarse groups 2-12 were based on a finegroup lethargy interval of 0.0087 , and for groups $13-21$ on twice this. The effect of applying the narrow-resonance approximation to the sodium resonance data was checked, with results given in Table A-IV for elastic removal. For the transport cross section the error was negligible. Changes in sodium cross sections are small from one case to another, and the double spacing probably could have been used throughout with adequate accuracy.

$\underline{\text { Result of Calculations }}$

The spectral plus capture components of the sodium void effect for removal of half the sodium from a full sodium core are given in Figs. 1 and 2 for $\mathrm{Pu}^{240}$, fission products and molybdenum either present or not. These results were obtained by a coarse-group fundamental-mode calculation (the HOBO code) based on the full sodium flux.

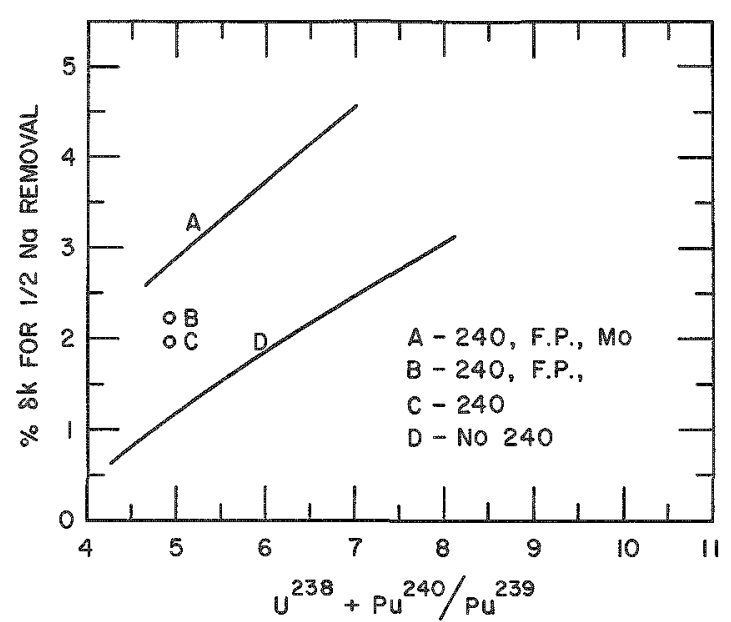

Fig. 1. Spectral Plus Capture $\delta k$ for Sodium Removal, $70 \mathrm{v} / \mathrm{o} \mathrm{Na}$.

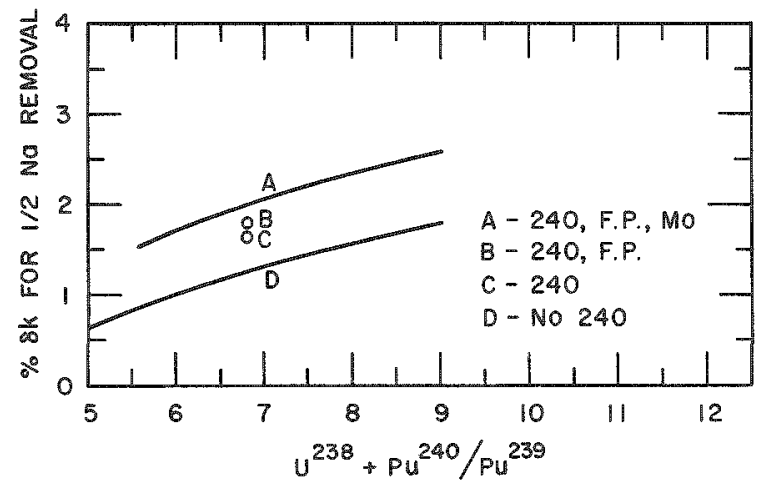

Fig. 2. Spectral Plus Capture $\delta \mathrm{k}$ for Sodium Removal, $52 \mathrm{v} / \mathrm{O} \mathrm{Na}$. 
As a check on the accuracy of use of a fundamental-mode calculation, the PERT code, which performs one-dimensional perturbation-theory calculations, was used for comparison, with results given in Table II. The agreement is seen to be quite close for the spherical cases presented. The PERT code actually handles only 18 groups, so that the contribution of the last 4 groups (neutrons with energy below $1 \mathrm{keV}$ ) had to be neglected. This contribution is small and would reduce the discrepancy between PERT and HOBO calculations of the elastic removal effect.

Table II

COMPARISON OF FUNDAMENTAL-MODE (HOBO) AND SPACE-DEPENDENT (PERT) CALGULATIONS OF SPECTRAL AND CAPTURE COMPONENTS OF SODIUM REMOVAL REACTIVITY EFFECT FROM FULL TO HALF SODIUM

240, F. P., Mo (Full Sodium Flux)

\begin{tabular}{|c|c|c|c|c|c|c|c|c|}
\hline \multirow{2}{*}{$\begin{array}{c}\text { Sodium, } \\
v / 0\end{array}$} & \multirow{2}{*}{$\frac{\mathrm{U}^{238}+\mathrm{Pu}^{240}}{\mathrm{Pu}^{239}}$} & \multirow{2}{*}{$\begin{array}{c}\text { Core } \\
\text { Vol, } \\
\text { (Sphere) }\end{array}$} & \multicolumn{2}{|c|}{ Elastic } & \multicolumn{2}{|c|}{ Inelastic } & \multicolumn{2}{|c|}{ Capture } \\
\hline & & & HOBO & PERT & HOBO & PERT & HOBO & PERT \\
\hline \multirow[t]{2}{*}{70} & 4.66 & 2,500 & 1.68 & 1.75 & 0.72 & 0.77 & 0.19 & 0.19 \\
\hline & 5.62 & 5,000 & 2.27 & 2.33 & 0.89 & 0.92 & 0.23 & 0.22 \\
\hline \multirow[t]{2}{*}{52} & 5.82 & 1,000 & 1.04 & & 0.48 & & 0.09 & \\
\hline & 7.00 & 2,000 & 1.39 & 1.42 & 0.58 & 0.58 & 0.11 & 0.11 \\
\hline \multicolumn{9}{|c|}{ No 240 (Full Sodium Flux) } \\
\hline 70 & 5.62 & & 0.47 & & 0.52 & & 0.31 & \\
\hline 52 & 7.00 & & 0.71 & & 0.43 & & 0.15 & \\
\hline
\end{tabular}

Comparisons have also been made in slab geometry for two cases with $\mathrm{Pu} 240$, fission products, and molybdenum. For $70 \mathrm{v} / 0$ sodium and a $\left(\mathrm{U}^{238}+\mathrm{Pu}^{240}\right) / \mathrm{Pu}^{239}$ ratio of 4.66 (2500-1iter sphere), agreement was close. For $52 \mathrm{v} / 0$ sodium and a ratio of 7.00 , agreement was not very good. Whether this effect is real or due to an error in the calculation has not yet been determined.

It is noted that the spectral and capture effects increase sharply with decreasing enrichment. This is due partly to the fact that the ratio of sodium to fuel increases and partly to the fact that the adjoint flux $\phi_{j}^{+}$ varies more sharply with energy at lower enrichment (see Fig. 3). At a given enrichment the spectral and capture effects also increase with sodium content, mostly because of the increase of the ratio of the sodium to fuel. 


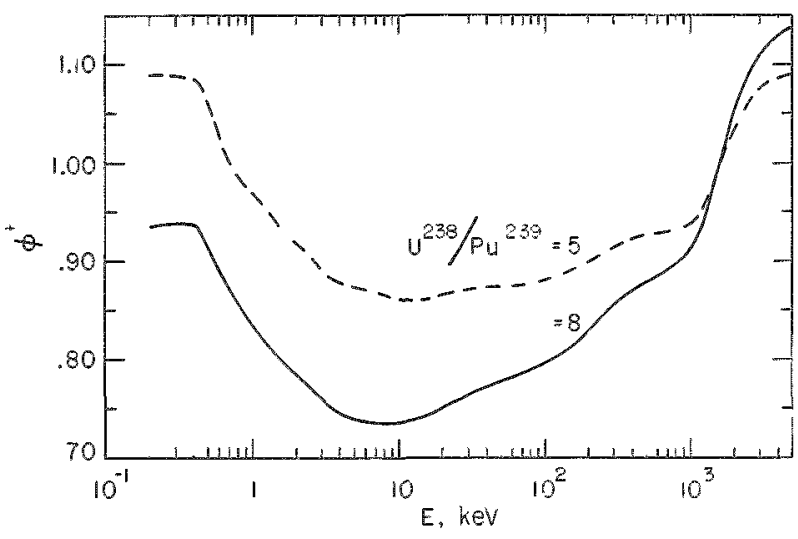

Fig. 3. Adjoint Flux for $70 \mathrm{v} / \mathrm{o}$ Sodium No $\mathrm{Pu}^{240}$
The increase of the spectral component that occurs when $\mathrm{Pu}^{240}$ is added is due mostly to the change in the elastic-moderation component because of the change in adjointfunction variation. There is an inelastic scattering effect because of the low fission threshold of $\mathrm{Pu}^{240}$, which is less important than the elastic effect. The further addition. of capture due to fission products and molybdenum further increases the elastic component because of change in adjoint function (see Figs. 4 and 5). The most critical energy region for this effect is between ten and several hundred keV. Below about $10 \mathrm{keV}$ the elastic effect becomes negative. Because of serious uncertainties in the capture cross sections of the materials involved, these calculations cannot be regarded as very reliable. This constitutes a major uncertainty in sodium roid coefficient calculations and seems to be considerably more important than uncertainties in sodium group cross sections, which probably do not amount to more than a few per cent.

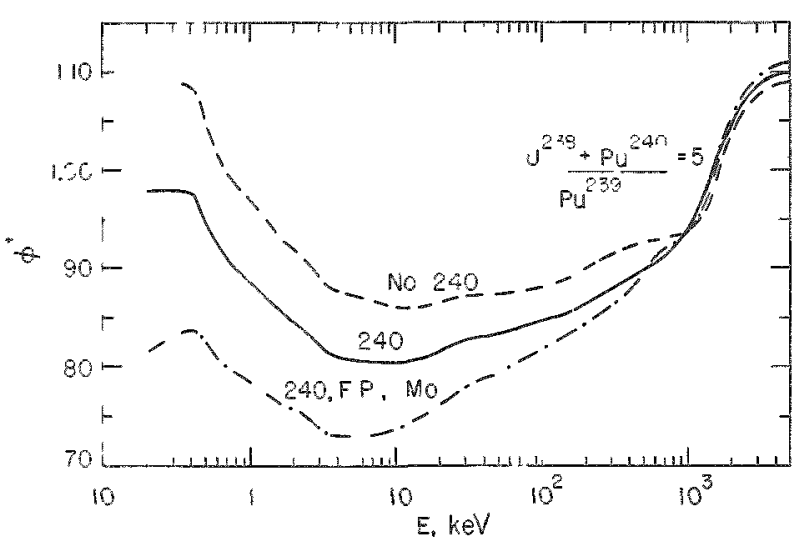

Fig. 4. Adjoint Flux for $70 \mathrm{v} / \mathrm{o}$ Sodium

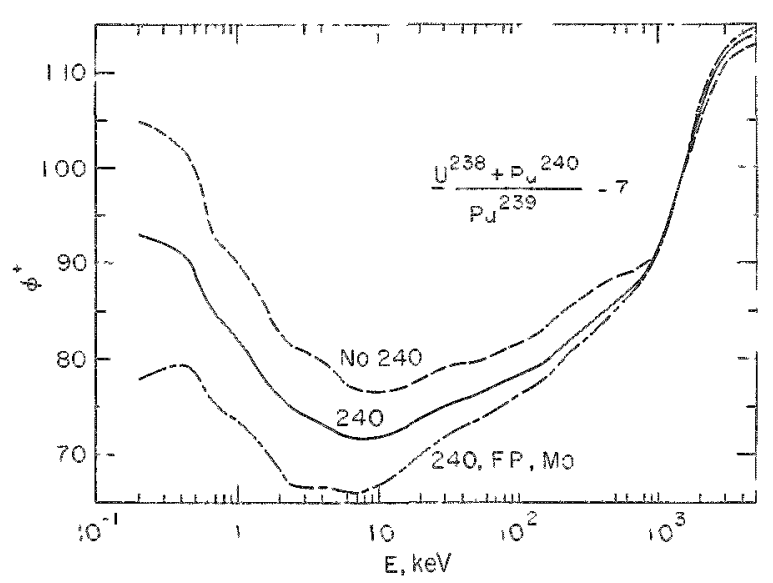

Fig. 5. Adjoint Flux for $52 \mathrm{v} / \mathrm{O}$ Sodium

Leakage components of the roid effect were obtained by subtracting the spectral plus capture effect from the total reactivity effect. Results are given for spherical cores in Table III. The spectral plus capture components for full to half sodium are averages of results with full and with half sodium flux, and the half to no sodium effects are averages of results with half sodium and no sodium fluxes. 
LEAKAGE AND SPECTRAL PLUS CAPTURE COMPONENTS OF SODIUM REMOVAL REACTIVITY EFEECT FOR SPHERES

\begin{tabular}{|c|c|c|c|c|c|c|c|c|c|}
\hline \multirow[b]{2}{*}{ Spheres } & \multirow[b]{2}{*}{$\begin{array}{c}\text { Sodium, } \\
\nabla / 0\end{array}$} & \multirow[b]{2}{*}{ Vol, k } & \multirow[b]{2}{*}{$\frac{\mathrm{U}^{238}-\mathrm{Pu}^{240}}{\mathrm{Pu}^{239}}$} & \multicolumn{3}{|c|}{ \% ck Full-Half } & \multicolumn{3}{|c|}{ \% ok Hali-No } \\
\hline & & & & Total & $\begin{array}{c}\text { Spectral } \\
+ \\
\text { Capture }\end{array}$ & Leakage & Total & $\begin{array}{c}\text { Spectral } \\
+ \\
\text { Capture }\end{array}$ & Leakage \\
\hline $\begin{array}{l}240+ \\
\text { E. P. t Mo }\end{array}$ & $\begin{array}{l}70 \\
59\end{array}$ & $\begin{array}{l}5,000 \\
2,500 \\
5,000 \\
3,000 \\
2,000 \\
3,000 \\
2,000 \\
1,000\end{array}$ & $\begin{array}{l}5.62 \\
4.66 \\
6.56 \\
5.86 \\
5.47 \\
7.62 \\
7.00 \\
5.82\end{array}$ & $\begin{array}{r}1.14 \\
-0.15 \\
1.52 \\
0.76 \\
0.17 \\
1.22 \\
0.76 \\
0.00\end{array}$ & $\begin{array}{l}3.61 \\
2.70 \\
3.10 \\
2.65 \\
2.38 \\
2.34 \\
2.15 \\
1.68\end{array}$ & $\begin{array}{l}-2.47 \\
-2.85 \\
-1.58 \\
-1.89 \\
-2.21 \\
-1.12 \\
-1.39 \\
-1.68\end{array}$ & $\begin{array}{r}0.75 \\
-0.74 \\
1.49 \\
0.53 \\
-0.17 \\
1.04 \\
0.80 \\
-0.11\end{array}$ & $\begin{array}{l}4.00 \\
2.83 \\
3.40 \\
2.85 \\
2.53 \\
2.50 \\
2.27 \\
1.70\end{array}$ & $\begin{array}{l}-3.25 \\
-3.57 \\
-1.91 \\
-2.32 \\
-2.70 \\
-1.46 \\
-1.47 \\
-1.81\end{array}$ \\
\hline $\operatorname{No} 240$ & $\begin{array}{l}70 \\
59\end{array}$ & $\begin{array}{r}6,000 \\
10,000 \\
6,000\end{array}$ & $\begin{array}{l}6.14 \\
6.85 \\
6.98\end{array}$ & $\begin{array}{r}-1.47 \\
0.47 \\
0.42\end{array}$ & $\begin{array}{l}2.20 \\
2.65 \\
2.10\end{array}$ & $\begin{array}{l}-3.67 \\
-2.18 \\
-1.68\end{array}$ & $\begin{array}{r}-1.45 \\
0.19 \\
0.23\end{array}$ & $\begin{array}{l}2.60 \\
3.20 \\
2.35\end{array}$ & $\begin{array}{l}-4.05 \\
-3.01 \\
-2.12\end{array}$ \\
\hline
\end{tabular}

The expected decrease of leakage component with increasing size occurs, and also there is the expected increase in leakage component with sodium content. The spectral component for a given size (and composition series) does not vary much with sodium content because of changes in enrichment ratio. What needs further investigation is why the leakage component for a given size is smaller when $\mathrm{Pu}^{240}$, fission products, and molybdenum are present than when they are not.

There is some nonlinearity, due almost entirely to changes in the flux and adjoint as sodium is removed. In these calculations the dependence of $\sigma_{\gamma}$ on sodium content for the $2.85-\mathrm{keV}$ resonance has not been taken into account. This will underestimate the half to no sodium reactivity change by not more than $0.1 \% \mathrm{k}$ except for the no $\mathrm{Pu}^{240}, 52 \mathrm{v} / 0$ sodium case, in which the error may be as large as $0.2 \% \mathrm{k}$. This error will not appear in the total $\delta \mathrm{k}$ as it was calculated directly with ELMOE cross sections. Some nonlinearity exists in both spectral plus capture and leakage effects. This does not lead to a strong nonlinearity in the void coefficient unless there is a close balance between the opposing effects, so that the total reactivity change on removal of sodium from the core is only plus or minus several tenths per cent.

Infinite slab reactors were also studied because it was believed that they would represent a good approximation to very large pancake cores, which might typically have a height of $2 \mathrm{ft}$ and a diameter of $12 \mathrm{ft}$, corresponding to a volume of about 6000 liters. Although it was believed that one-dimensional calculations were not very trustworthy for obtaining the void effect for a finite reflected cylinder, it was thought that if only a small extrapolation from the infinite slab case was involved the error could not be very serious. Some results of calculations are given in Table IV. In the 
case of the finite slab, the 12 -ft diameter was augmented by the indicated sphere reflector savings. For the $70 \mathrm{v} / 0$ sodium case, the material buckling is small enough that the radial leakage effect on the sodium void coefficient for a fixed composition is still important, mainly because of the change in core height. Extrapolation in this case is regarded as uncertain. For the $52 \mathrm{v} / \mathrm{o}$ sodium case, the extrapolation is small.

Table IV

SODIUM RFACTIVTX EEEECT AND REF LECTOR SAVINGS FOR INEINITE AND FINITE SLABS $240, F . P .$, No

\begin{tabular}{|c|c|c|c|c|c|c|c|c|c|}
\hline \multirow{2}{*}{$\begin{array}{c}\text { Sodium, } \\
\text { vo } \\
\end{array}$} & \multirow{2}{*}{$\frac{\mathrm{U}^{233} \mathrm{Pu}^{24 !}}{\mathrm{I}^{24} \mathrm{u}^{239}}$} & \multirow{2}{*}{ Sodiun } & \multirow{2}{*}{$\begin{array}{l}\text { Sphere } \\
\text { R.S. S. en }\end{array}$} & \multicolumn{3}{|c|}{ Infinta Sab } & \multicolumn{3}{|c|}{ 12. It Dia Slab } \\
\hline & & & & IIt, $: \mathrm{m}$ & $k, 0_{0}$ & R. S., cm & $\mathrm{Ht}, \mathrm{cm}$ & $i k, \pi_{0}^{\prime}$ & R.S., $\mathrm{cm}$ \\
\hline \multirow[t]{2}{*}{70} & 5.22 & $\begin{array}{l}\text { Ful' } \\
\text { IntLi } \\
\text { Noo }\end{array}$ & $\begin{array}{l}23.0 \\
29.4 \\
.40 .1\end{array}$ & 84.6 & $\begin{array}{l}10 \\
2.38 \\
5.42\end{array}$ & $\begin{array}{l}22.7 \\
21.9 .9 \\
34.8\end{array}$ & 102.4 & $\begin{array}{l}0 \\
1.72 \\
3.40\end{array}$ & $\begin{array}{l}23.7 \\
27.3 \\
35.6\end{array}$ \\
\hline & 4.66 & $\begin{array}{l}\text { Fall } \\
\text { Hotif } \\
\text { No }\end{array}$ & $\begin{array}{l}23.6 \\
29.6 \\
40.3\end{array}$ & 63.3 & $\begin{array}{l}0 \\
1.54 \\
3.50\end{array}$ & $\begin{array}{l}22.3 \\
26.6 \\
33.9\end{array}$ & 72.7 & $\begin{array}{l}0 \\
0.85 \\
1.58\end{array}$ & $\begin{array}{l}22.8 \\
27.2 \\
34.5\end{array}$ \\
\hline \multirow[t]{2}{*}{53} & 7.00 & $\begin{array}{l}\text { Full } \\
\text { Hetil } \\
\text { No }\end{array}$ & $\begin{array}{l}17.3 \\
19.0 \\
22.6\end{array}$ & 61.3 & $\begin{array}{l}0 \\
1.47 \\
3.31\end{array}$ & $\begin{array}{l}13.5 \\
15.3 \\
17.9\end{array}$ & 68.6 & $\begin{array}{l}0 \\
1.19 \\
2.70\end{array}$ & $\begin{array}{l}10.9 \\
18.9 \\
21.4\end{array}$ \\
\hline & 2.82 & $\begin{array}{l}\text { Full } \\
\text { Hall } \\
\text { Ne }\end{array}$ & $\begin{array}{l}16.8 \\
19.0 \\
22.0\end{array}$ & $\begin{array}{l}45.1 \\
0.99 \\
2.25\end{array}$ & 0 & $\begin{array}{l}16.8 \\
18.4 \\
20.5\end{array}$ & 49.6 & $\begin{array}{l}0 \\
0.70 \\
1.05\end{array}$ & $\begin{array}{l}10.6 \\
16.01 \\
20.8\end{array}$ \\
\hline
\end{tabular}

From the results in Table IV it appears that a rather thin slab is indicated if it is required that the void effect not be positive for expulsion from the core alone. It is true that the addition of molybdenum has made the situation more pessimistic by about $0.5 \% \mathrm{k}$ for half removal of sodium for $70 \mathrm{v} / 0$ sodium and by about $0.2 \% \mathrm{k}$ for $52 \mathrm{v} / 0$ sodium. Even taking this into account, this criterion appears difficult to meet. It is seen that for $a$. given composition the slab reactor has a more positive reactivity effect than the sphere of the same composition. The reason for this has been given by Davey and Chaumont.(4) Reflector savings increases on withdrawal of sodium from the core, and this effect is more important for the slab than for the sphere of the same composition, even though the change in reflector savings is less for the slab than for the sphere. For reactors of a given size, one can gain an advantage by going to the pancake, but the required thickness may be too small and the enrichment too high for a satisfactory breeding ratio if the specification of a nonpositive reactivity effect on expulsion of sodium from the core alone is to be met.

It may be argued that this criterion is unreasonably restrictive, since it is expected that sodium will also be expelled from the upper blanket. Such expulsion can lead to quite large reactivity losses in some cases. In an 
earlier series of calculations it was found that with a $70 \mathrm{v} / 0$ sodium blanket, $46 \mathrm{~cm}$ thick with no reflector behind it, expulsion from the (total) blanket in spherical or infinite slab geometry give reactivity losses of the order of $5 \% \mathrm{k}$ with a $52 \mathrm{v} / 0$ sodium blanket, losses of about $1.5 \%$ for a 61 -cm-thick blanket (extrapolated) and of about $2 \%$ for a $46-\mathrm{cm}-$ thick blanket, the thickness used in the present series of calculations. Use of a reflector behind the blanket would reduce these effects. If, however, a means can be found of trapping the neutrons passing through the blanket region, perhaps with moderator plus absorber in the reflector, they could be eliminated as far as reactivity is concerned but still contribute to breeding.

The one criterion that clearly must be satisfied under any conditions is that the total power coefficient not be positive. It seems that because of the relatively small magnitude of the available Doppler effect, perhaps $0.5 \% \mathrm{k}$, compared to the positive effects possible with sodium expulsion, a minimum requirement should be that the power coefficient due to sodium heating alone not be positive. This would leave a margin for error in case more unfavorable expulsion of sodium occurred.

\section{References}

1. J. M. Chaumont, unpublished work at ANL.

2. H. H. Hummel and A. Rago, "An Accurate Treatment of Resonance Scattering in Light Elements in Fast Reactors," Proceedings of IAEA Conference on Physics of Fast and Intermediate Reactors, Vol. I, pp. 231-250, International Atomic Energy Agency, Vienna (1962).

3. H. H. Hummel and A, Rago, "Effect of Parameter Variations in Doppler Effect Calculations," This Proceedings, Papex No. 4 of Session VII.

4. W. G. Davey and J. M Chaumont, unpublished work at ANL.

5. P.A. Moldauer, Proceedings on the Conference on the Physics of Breeding, ANL, ANL-6122, October 19-21, 1959, p. 67.

6. P. A. Moldauer, ANI, private communication. 


\section{Appendices}

Table A-I

FISSION ANO CAPTURE CROSS SECTIONS (b) USED IN SODIUM VOID REACTIVITY CALCULATIONS (T - I5OOOK)

\begin{tabular}{|c|c|c|c|c|c|c|c|c|c|c|c|c|c|c|c|}
\hline \multirow{4}{*}{ Group } & \multicolumn{6}{|c|}{ Pu239 } & \multirow{3}{*}{\multicolumn{2}{|c|}{$\frac{1238^{\text {(a) }}}{\sigma \gamma}$}} & \multirow{2}{*}{\multicolumn{3}{|c|}{$\mathrm{Pu} 240 \mathrm{O}_{\mathrm{p}}=600$}} & \multirow{4}{*}{$\frac{M_{0}(6)}{\sigma_{\gamma}}$} & \multirow{4}{*}{$\begin{array}{c}\text { Fission (5) } \\
\text { Products } \\
\text { per Atom } \\
\text { of Product } \\
\sigma_{\gamma}\end{array}$} & \multirow{4}{*}{$\frac{N a}{\sigma \gamma}$} & \multirow{4}{*}{$\begin{array}{c}\begin{array}{c}\text { Stainless } \\
\text { Stee! } \\
304\end{array} \\
\sigma y \\
\end{array}$} \\
\hline & \multirow{3}{*}{$\frac{E_{L}}{\mathrm{keV}}$} & \multirow{3}{*}{$v$} & \multicolumn{2}{|c|}{$\sigma_{p}-300$} & \multirow{2}{*}{\multicolumn{2}{|c|}{$\sigma_{p}-400$}} & & & & & & & & & \\
\hline & & & & & & & & & \multirow{2}{*}{$v$} & \multirow{2}{*}{$\sigma_{f}$} & \multirow{2}{*}{$\sigma_{\gamma}$} & & & & \\
\hline & & & $\sigma_{\hat{f}}$ & $\sigma_{\gamma}$ & $\sigma f$ & $\sigma_{\gamma}$ & $\sigma_{p}=40$ & $\sigma_{p}=60$ & & & & & & & \\
\hline 1 & 3670 & 3.45 & 1.83 & 0.054 & 1.83 & 0.054 & 0.010 & 0.010 & 3.85 & 1.54 & 0.018 & 0.006 & 0.020 & 0.0005 & 0.004 \\
\hline 2 & 2230 & 3.22 & 1.99 & 0.080 & 1.99 & 0.080 & 0.022 & 0.022 & 3.51 & 1.63 & 0.039 & 0.010 & 0.040 & 0.0001 & 0.001 \\
\hline 3 & 1350 & 3.06 & 1.96 & 0.097 & 1.96 & 0.097 & 0.057 & 0.057 & 3.32 & 1.59 & 0.100 & 0.017 & 0.063 & 0.0001 & 0.002 \\
\hline 4 & 825 & 2.97 & 1.81 & 0.104 & 1.81 & 0.104 & 0.129 & 0.129 & 3.21 & 1.39 & 0.226 & 0.028 & 0.090 & 0.0002 & 0.004 \\
\hline 5 & 500 & 2.93 & 1.70 & 0.129 & 1.70 & 0.129 & 0.139 & 0.139 & 3.15 & 0.76 & 0.243 & 0.40 & 0.092 & 0.0003 & 0.005 \\
\hline 6 & 300 & 2.91 & 1.70 & 0.182 & 1.70 & 0.182 & 0.127 & 0.127 & 3.10 & 0.191 & 0.222 & 0.041 & 0.095 & 0.0005 & 0.006 \\
\hline 7 & 380 & 2.90 & 1.70 & 0.100 & 1.70 & 0.190 & 0.16 & 0.16 & 3.07 & 0.041 & 0.282 & 0.044 & 0.110 & 0.0007 & 0.007 \\
\hline 8 & 110 & 2.90 & 1.70 & 0.23 & 1.70 & 0.23 & 0.24 & 0.24 & - & . & 0.397 & 0.059 & 0.125 & - & 0.008 \\
\hline 9 & 67 & 2.90 & 1.80 & 0.26 & 1.80 & 0.26 & 0.31 & 0.31 & - & - & 0.508 & 0.077 & 0.140 & - & 0.010 \\
\hline 10 & 40.7 & 200 & 1.93 & 0.33 & 1.93 & 0.33 & 0.42 & 0.42 & - & - & 0.648 & 0.108 & 0.165 & 0.003 & 0.007 \\
\hline 11 & 25.0 & 2.90 & 2.17 & 0.61 & 2.17 & 0.61 & 0.45 & 0.45 & - & - & 0.788 & 0.157 & 0.200 & - & 0.018 \\
\hline 12 & 15.0 & 2.90 & 2.40 & 1.12 & 2.40 & 1.12 & 0.493 & 0.498 & - & - & 1.179 & 0.213 & 0.250 & - & 0.010 \\
\hline 13 & 9.1 & 2.90 & 2.45 & 1.33 & 2.45 & 1.33 & 0.505 & 0.605 & - & . & 1.430 & 0.299 & 0.33 & - & 0.015 \\
\hline 14 & 4.0 & 2.90 & 2.49 & 1.55 & 2.49 & 1.56 & 0.726 & 0.751 & $\infty$ & - & 1.891 & 0.45 & 0.45 & - & 0.022 \\
\hline 15 & 2.8 & 2.90 & 2.93 & 1.79 & 2.96 & 1.83 & 0.887 & 0.942 & - & - & 2.57 & 0.60 & 0.8 & $0.444^{(b)}$ & 0.016 \\
\hline 16 & 2.0 & 2.90 & 3.39 & 2.06 & 3,42 & 2.10 & 1.014 & 1.061 & - & - & 3.23 & 0.70 & 0.9 & 0.019 & 0.014 \\
\hline 17 & 1.4 & 2.90 & 4.00 & 2.57 & 4.20 & 2.50 & 1.104 & 1.228 & - & - & 3.86 & 0.82 & 1.3 & 0.003 & 0.020 \\
\hline 18 & 1.0 & 2.90 & 4.84 & 3.01 & 5.07 & 3.08 & 1.23 & 1.40 & - & - & 4.79 & 1.00 & 1.7 & 0.003 & 0.044 \\
\hline 19 & 0.55 & 2.90 & 6.31 & 4.46 & 6.43 & 4.03 & 1.26 & 1.62 & - & . & 6.05 & 1.4 & 2.4 & 0.003 & 0.016 \\
\hline 20 & 0.30 & 2.90 & 8.46 & 5.44 & 8.68 & 5.54 & 1.31 & 1.44 & 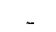 & - & 8.69 & 2.0 & 3.5 & 0.004 & 0.022 \\
\hline 21 & 0.10 & 2.90 & 11.94 & 8.10 & 12.61 & 8.02 & 1.60 & 2.21 & - & - & 12.9 & 3.5 & 9.0 & 0.006 & 0.031 \\
\hline 22 & 0.03 & 2.90 & 16.74 & 11.50 & 18.20 & 11.54 & 2.10 & 2.67 & - & - & 17.9 & 7.2 & 15.0 & 0.010 & 0.059 \\
\hline
\end{tabular}

(a) $v$ andof from Yon

bise Table A-III

Table A-II

EFFECTIVE TRANSPORT AND ELASTIC REMOVAL CROSS SECTIONS $(b)$ OF SODIUM

\begin{tabular}{|c|c|c|c|c|c|c|c|c|}
\hline \multirow{3}{*}{$\mathbf{j}$} & \multicolumn{4}{|c|}{$70 \%$ Oxide } & \multicolumn{4}{|c|}{$52 \%$ Oxide } \\
\hline & \multicolumn{2}{|c|}{$\sigma_{t r, j}$} & \multicolumn{2}{|c|}{ ol.j } & \multicolumn{2}{|c|}{$\sigma_{t x, j}$} & \multicolumn{2}{|c|}{$\sigma_{\mathrm{el}}, \mathrm{j}$} \\
\hline & Full-Half & Half-No & Full-Half & Half-No & Full-Half & $\mathrm{Half}$-No & Full-Half & Half-No \\
\hline 2 & 1.51 & 1.54 & 0.320 & 0.322 & 1.55 & 1.56 & 0.325 & 0.325 \\
\hline 3 & 1.66 & 1.69 & 0.266 & 0.265 & 1.69 & 1.70 & 0.263 & 0.263 \\
\hline 4 & 2.73 & 2.81 & 0.489 & 0.520 & 2.80 & 2.85 & 0.514 & 0.524 \\
\hline 5 & 4.58 & 4.81 & 0.767 & 0.807 & 4.74 & 4.90 & 0.790 & 0.816 \\
\hline 6 & 3.76 & 3.82 & 0.802 & 0.810 & 3.80 & 3.84 & 0.808 & 0.812 \\
\hline 7 & 3.90 & 4.07 & 0.726 & 0.743 & 4.01 & 4.14 & 0.734 & 0.746 \\
\hline 8 & 4.11 & 4.28 & 0.726 & 0.744 & 4.17 & 4.28 & 0.732 & 0.743 \\
\hline 9 & 4.53 & 4.70 & 0.808 & 0.816 & 4.61 & 4.73 & 0.806 & 0.811 \\
\hline 10 & 4.30 & 4.61 & 0.803 & 0.847 & 4.43 & 4.86 & 0.816 & 0.878 \\
\hline 11 & 4.56 & 4.79 & 0.750 & 0.766 & 4.57 & 4.70 & 0.748 & 0.754 \\
\hline 12 & 4.28 & 4.40 & 0.718 & 0.712 & 4.28 & 4.32 & 0.702 & 0.691 \\
\hline 13 & 5.03 & 5.04 & 0.870 & 0.865 & 5.03 & 5.04 & 0.864 & 0.858 \\
\hline 14 & 8.95 & 9.84 & 0.989 & 1.044 & 9.34 & 10.02 & 1.014 & 1.059 \\
\hline 15 & 35.02 & 45.54 & 15.10 & 17.18 & 36.70 & 54.82 & 15.66 & 19.29 \\
\hline 16 & 7.52 & 9.54 & 2.63 & 3.14 & 7.80 & 11.56 & 2.78 & 3.47 \\
\hline 17 & 4.00 & 4.08 & 0.990 & 0.976 & 4.06 & 4.11 & 0.974 & 0.939 \\
\hline 18 & 3.13 & 3.14 & 0.812 & 0.805 & 3.14 & 3.14 & 0.800 & 0.794 \\
\hline 19 & 3.04 & 3.04 & 0.415 & 0.405 & 3.04 & 3.04 & 0.402 & 0.374 \\
\hline 20 & 3.02 & 3.03 & 0.425 & 0.414 & 3.03 & 3.03 & 0.408 & 0.388 \\
\hline 21 & 3.03 & 3.03 & 0.177 & 0.154 & 3.03 & 3.03 & 0.157 & 0.136 \\
\hline
\end{tabular}


EFFECTIVE SODIUM $\sigma \gamma$ (b) FOR GROUP 15 $(2.8-4.0 \mathrm{keV})$ FROM ELMOE

\begin{tabular}{c|c|c|c}
\hline $\begin{array}{c}\text { Sodium, } \\
\mathrm{V} / \mathrm{o}\end{array}$ & $\mathrm{U}^{238} / \mathrm{Pu}^{239}$ & $\begin{array}{c}\text { Full-Half } \\
\text { Sodium }\end{array}$ & $\begin{array}{c}\text { Half-No } \\
\text { Sodium }\end{array}$ \\
\hline 70 & 8.1 & 0.436 & 0.497 \\
59 & 7.0 & 0.432 & 0.533 \\
52 & 7.0 & $0.444(\mathrm{a})$ & 0.670 \\
\hline
\end{tabular}

(a) This value was used in all perturbation calculations. See text of paper for discussion of error involved.

Table A-IV

COMPARISON OF SODIUM ELASTIC REMOVAL CROSS SECTIONS(b) FOR REGULAR ELMOE AND NARROW-RESONANCE APPROXIMATION

\begin{tabular}{|c|c|c|c|c|c|c|c|c|}
\hline \multirow{3}{*}{ j } & \multicolumn{4}{|c|}{$70 \%$ oxide } & \multicolumn{4}{|c|}{$52 \%$ Oxide } \\
\hline & \multicolumn{2}{|c|}{ Full Na } & \multicolumn{2}{|c|}{ Half $\mathrm{Na}$} & \multicolumn{2}{|c|}{ Full Na } & \multicolumn{2}{|c|}{ Half $\mathrm{Na}$} \\
\hline & ELMOE & $\begin{array}{c}\text { Narrow } \\
\text { Resonance }\end{array}$ & ELMOE & $\begin{array}{c}\text { Narrow } \\
\text { Resonance }\end{array}$ & ELMOE & $\begin{array}{l}\text { Narrow } \\
\text { Resonance }\end{array}$ & EMOE & $\begin{array}{c}\text { Narrow } \\
\text { Resonance }\end{array}$ \\
\hline 5 & 0.664 & 0.671 & 0.634 & 0.642 & 0.618 & 0.625 & 0.591 & 0.598 \\
\hline 6 & 0.812 & 0.837 & 0.819 & 0.843 & 0.815 & 0.838 & 0.819 & 0.842 \\
\hline 7 & 0.702 & 0.719 & 0.693 & 0.709 & 0.694 & 0.710 & 0.686 & 0.702 \\
\hline 8 & 0.732 & 0.742 & 0.742 & 0.752 & 0.735 & 0.745 & 0.741 & 0.752 \\
\hline 9 & 0.775 & 0.785 & 0.767 & 0.780 & 0.752 & 0.764 & 0.745 & 0.760 \\
\hline
\end{tabular}

Note: These elastic removal cross sections are the actual values for sodium in the mixture rather than effective values for removal of sodium, and thus do not reflect changes in elastic removal cross sections of other elements that occur when sodium is removed.

\section{Acknowledgment}

Extensive aid with the computations was provided by Mrs. Stella Dean and Miss Joann Zeman.

Discussion of Paper

Presented by Mr. Hummel

There were no questions or comments. 


\section{COMPARISON OF DOPPLER AND SODIUM-VOID COEFEICIENTS OF DILUTE FAST REACTORS}

K. Ott and K. E. Schroeter

Institut für Neutronenphysik und Reaktortechnik

Kernforschungszentrum, Karlsruhe, Germany

(Paper presented by Mr. Ott)

\section{Introduction}

We have calculated the neutron-physics properties of about fifty oxide-fueled, sodium-cooled, fast reactors as a basis for the investigation of safety, economics, and doubling time. Especially for optimization studies, analytical expressions of the characteristic quantities are very helpful. Therefore we have tried to find such relations.

Further, we have investigated the limitations coming from the sodium-void coefficient. The Doppler coefficients for two systems are evaluated, as well as their changes resulting from loss of sodium.

The neutron-physics consequences of a molybdenum addition, to increase the thermal conductivity, is investigated.

The composition of the reactors is given in Table I. Calculations have been made for all these systems with 100,75, 50,25, and $0 \%$ of relative sodium density to get information about the sodium-void coefficient.

$$
\begin{array}{rlr}
\text { Table I } & \\
\alpha= & \text { Volume Fraction of Sodium } & 0.30 ; 0.40 ; 0.50 \\
\beta_{0}= & \text { Fixed Steel Content } & 0.15 \\
\mathrm{x}_{\mathrm{St}}= & \text { Steel Volume/Fuel Volume } & 0.25 ; 0.50 \\
& \text { (fuel = fissile }+ \text { fertile material) } & \\
\mathrm{x}_{\mathrm{Mo}}= & \text { Molybdenum Volume/Fuel Volume } & 0.0 ; 0.15 \\
\mathrm{y}= & \mathrm{U}^{238} \mathrm{O}_{2} \text { Volume } / \mathrm{Pu}^{239} \mathrm{O}_{2} \text { Volume } & \frac{4 ; 5 ; 6 ; 7}{48 \text { reactors }}
\end{array}
$$

For these survey calculations, we have used the multigroup onedimensional diffusion code developed by $W$. Münzner(1) and the 16-groupconstants set of Yiftah, Okrent, and Moldauer(2) with some modifications listed in an internal Karlsruhe report.(3) 
Apart from some special cases, all reactors are calculated in spherical geometries.

\section{Results and Discussion}

\section{Internal breeding ratio (BRint)}

For all systems the internal $B R$ has, with increasing fertileto-fissile ratio $y$, in principle the behavior as shown in Fig. 1, i.e., up to about $y=4$ a slightly increasing slope and a nearly constant slope between $y=4$ and $y=7$. Therefore, in this interval of main interest the internal $B R$ can be represented by a linear function in $y$. Fortunately, the dependence on all other components can be described (see Fig. 2) by the critical mass at $y=5$ only $\left[M_{C r}(5)\right]$ :

$$
B R_{\text {int }}=[0.508+0.135(y-5)]\left(1.32-\frac{2.4}{\sqrt[3]{M_{C 1}(5)}}\right)
$$

The second factor simultaneously gives the change of the constant and the slope in the first bracket. The internal $B R$ increases with increasing critical mass (at $y=5$ ) nearly independently of core composition, i.e., for increasing sodium or steel content, as well as for addition of molybdenum.

This formula describes the calculated results with an accuracy of about 1 to 2 per cent.

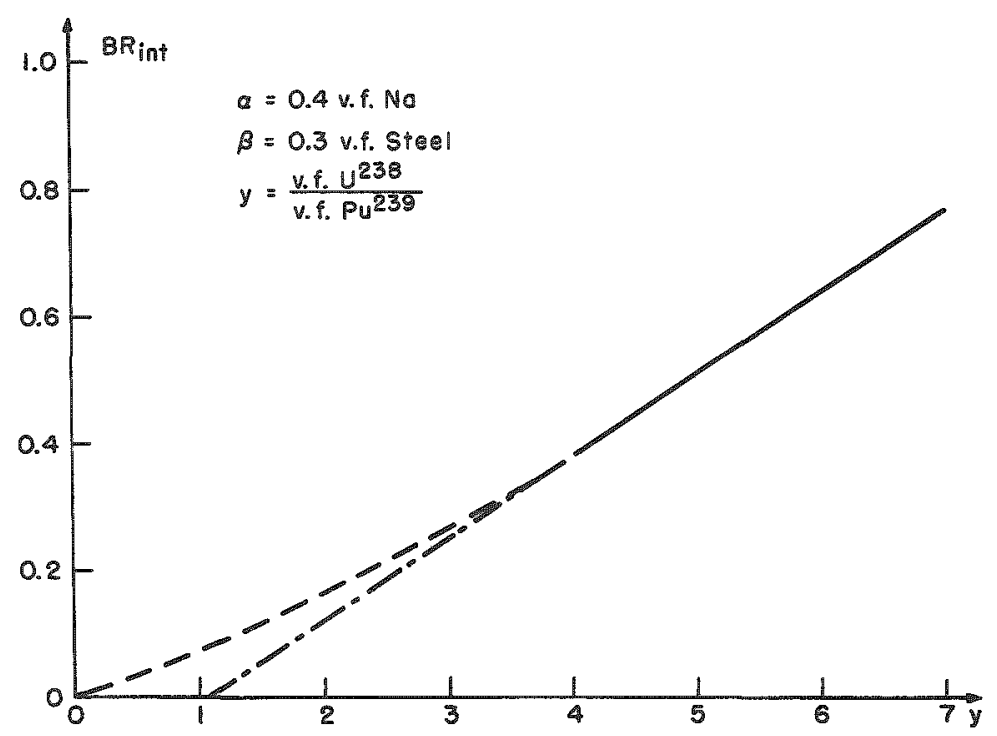

Fig. 1. Internal Breeding Ratio, as a Function of Fertile-to-Fission Ratio $\mathrm{y}$ 


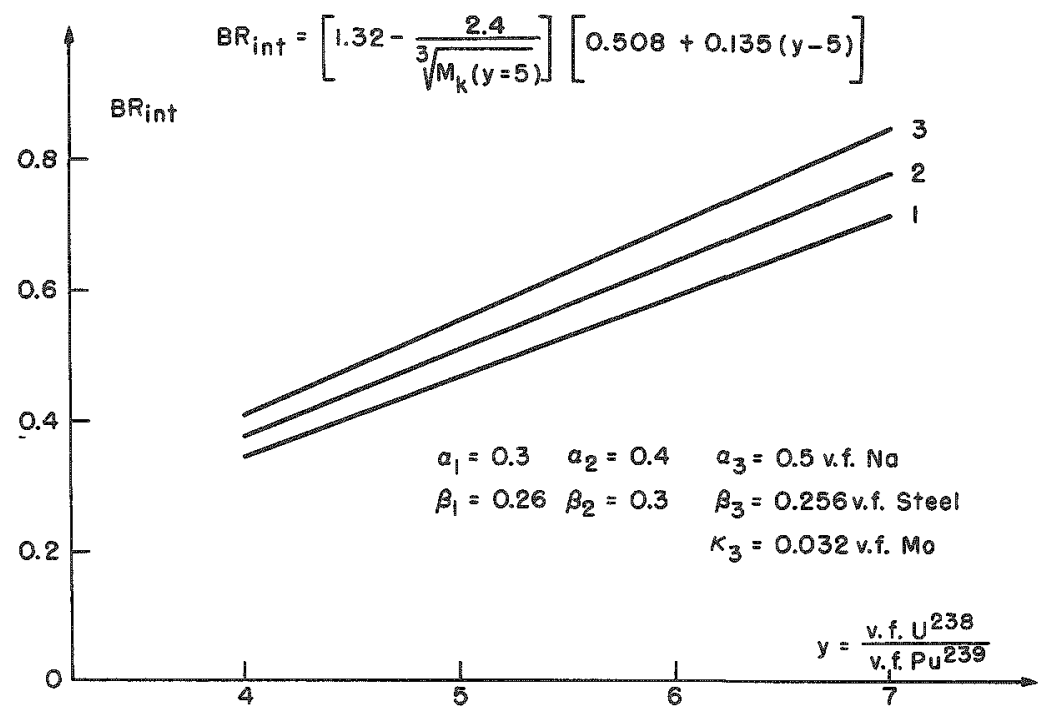

Fig. 2. Dependence of Internal Breeding Ratio for $y=5$ on Critical Mass

\section{External breeding ratio (BRext)}

For all reactors considered we have used a spherical shell of 80 - cm thickness as a blanket. The composition was chosen to represent a mixture of a radial and an axial blankel. The axial blanket (especially the coolant) is influenced by the core:

$$
\begin{aligned}
\alpha(\mathrm{Na}) & =a(\mathrm{Na}-\text { core }) ; \\
\hat{F}_{0} & =\beta_{0}(\text { core }) ; \\
\mathrm{x}_{\mathrm{St}} & =0.25(\text { fixed }) ; \\
\mathrm{U}_{\text {nat }} & =\text { rest. }
\end{aligned}
$$

The radial blanket was kept fixed:

$$
\begin{aligned}
\alpha & =0.3 ; \\
\beta_{0} & =0 ; \\
\mathrm{x}_{\mathrm{St}} & =0.25 ; \\
\mathrm{U}_{\text {nat }} & =\text { rest. }
\end{aligned}
$$

The calculated external BR may be represented by a very simple formula:

$$
\mathrm{BR}_{\mathrm{ext}} \approx \frac{9.1-0.3 \mathrm{y}}{\sqrt[3]{\mathrm{M}_{\mathrm{Cr}}}} ; \mathrm{M}_{\mathrm{CI}} \lesssim 2000 \mathrm{~kg}
$$

with a mean accuracy of about $5 \%$ 
To optimize the thickness $\mathrm{b}$ of the blanket, one must have a formula for the reduction of the external $B R$ with decreasing blanket thick. ness. Fortunately, as can be seen from Fig. 3, in all the cases considered the breeding density has very accurately an exponential shape with a "decay" constant, to a high degree of accuracy, independent of core size and composition. As long as boundary effects are negligible, the reduction of $B R_{\text {ext }}$ may be described by

$$
\begin{aligned}
& B R_{\text {ext }}(b)=B R_{\text {ext }}(\infty)[1-f(b)] \\
& B R_{\text {ext }}(\infty) \simeq B R_{\text {ext }}(80 \mathrm{~cm}) \simeq \frac{9.1-0.3 \mathrm{y}}{3 \sqrt{M_{c x i t}}}
\end{aligned}
$$

and

$$
f(b)=\frac{\int_{R+B}^{\infty} e^{-\lambda r} r^{2} d r}{\int_{R}^{\infty} e^{-\lambda r} r^{2} d r},
$$

where $R$ is the core radius and for all blankets considered

$$
\lambda=0.082 \mathrm{~cm}^{-1} \text {. }
$$

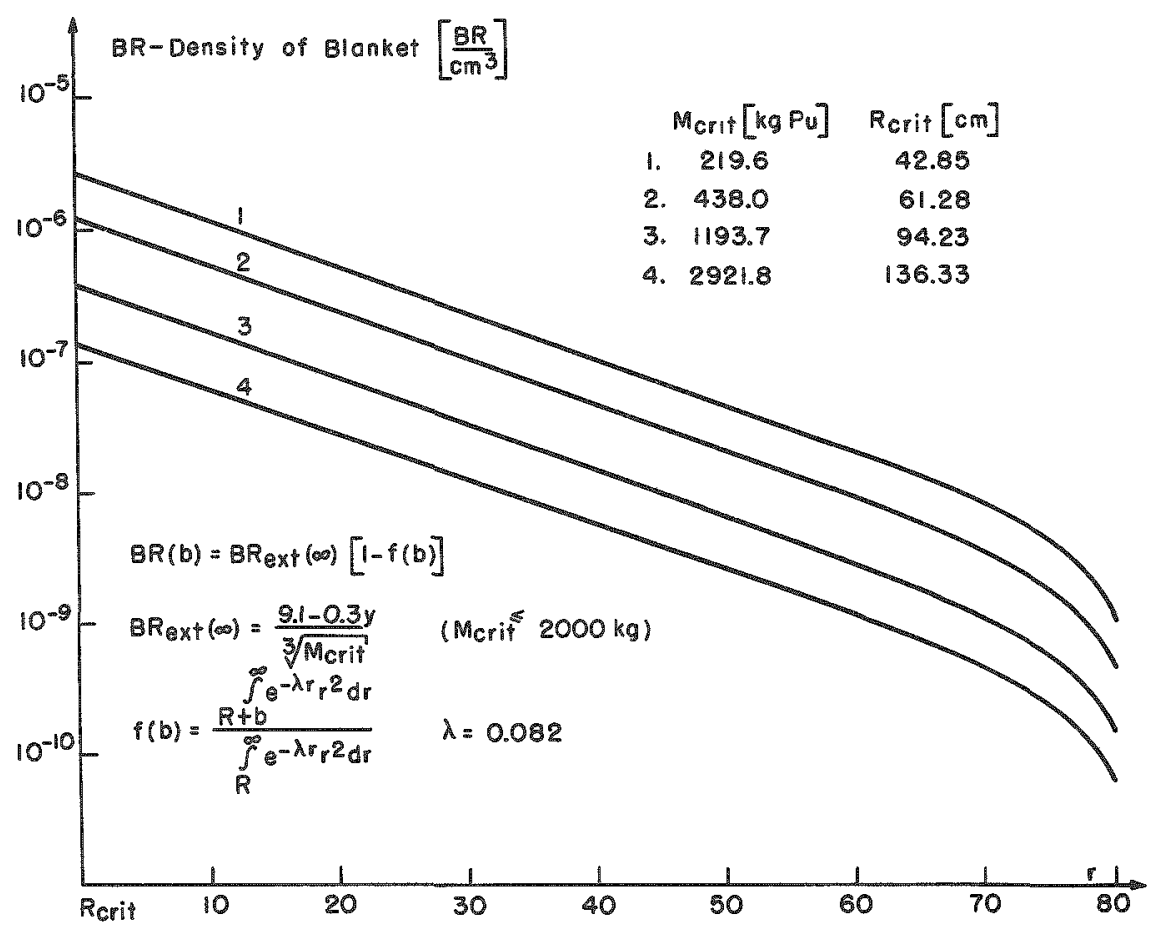

Fig. 3. Variation of Breeding Density with Blanket Thickness 


\section{Sodium-void coefficient and Sodium-loss $\Delta k$}

As can be seen from Figs. 5 and 6 , the sodium-void coefficient depends somewhat on the sodium density. For simplicity, we use the sodium-loss $\Delta \mathrm{k}$ defined as

$$
\Delta \mathrm{k}_{\text {loss }}=\Delta \mathrm{k} \text { for full sodium loss from the core.** }
$$

At first we consider the shape dependence by calculating the sodium-loss $\Delta \mathrm{k}$ for critical reflected cylinders, spheres, and slabs. Fig. 4 shows the result: spheres have the most negative sodium-loss $\Delta \mathrm{k}$, which may be connected with the fact that spheres have the largest surface-tovolume ratio, if one compares critical systems. Therefore in the following we consider spheres only.
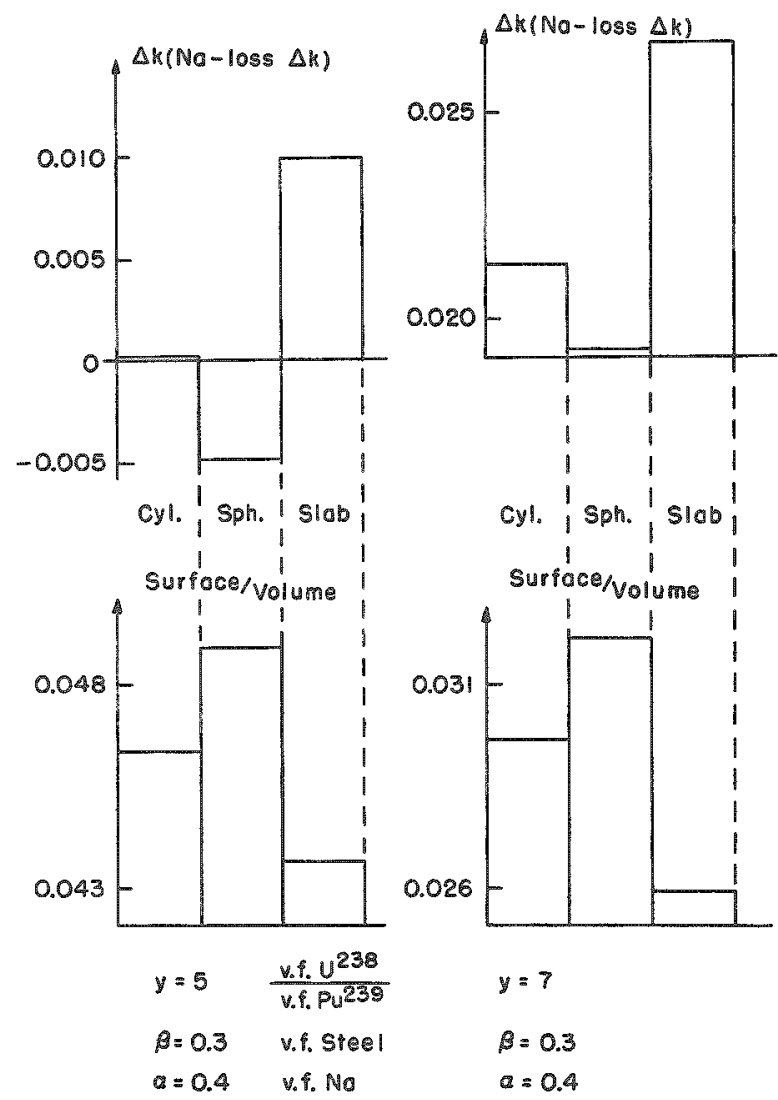

Fig. 4

Sodium Loss Coefficients

Figure 5 shows the multiplication factor ( $k_{e f f}$ ) as a function of the sodium content for small reactors, and Fig. 6 the same for large reactors. From these figures we derive Fig. 7 , in which the sodium-loss $\Delta \mathrm{k}$ is plotted. One can find values of $y$, denoted by $\mathrm{y}^{*}$, for which the sodiumloss $\Delta \mathrm{k}$ is zero. For $\mathrm{y}>\mathrm{y}^{*}$ it is positive. The final results are given in Table II. Figures 8,9 , and 10 show the same curves for medium and large reactors.

** The sodium from the upper axial blanket is also considered as lost. 


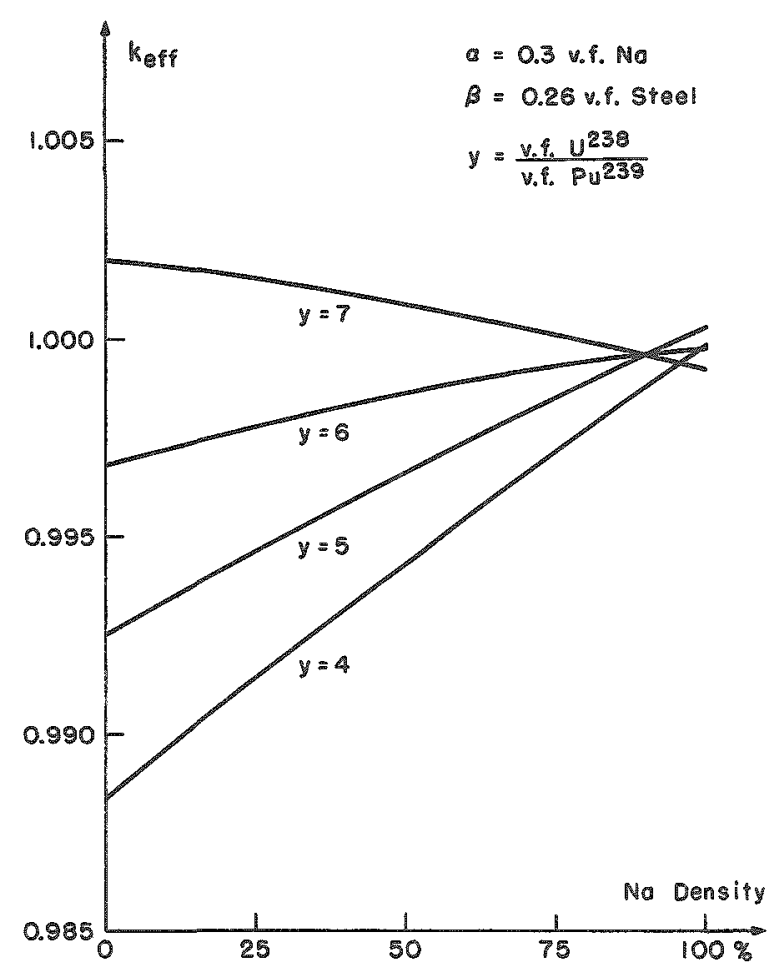

Fig. 5. Multiplication Factor as a Function of Sodium Content for Small Reactors

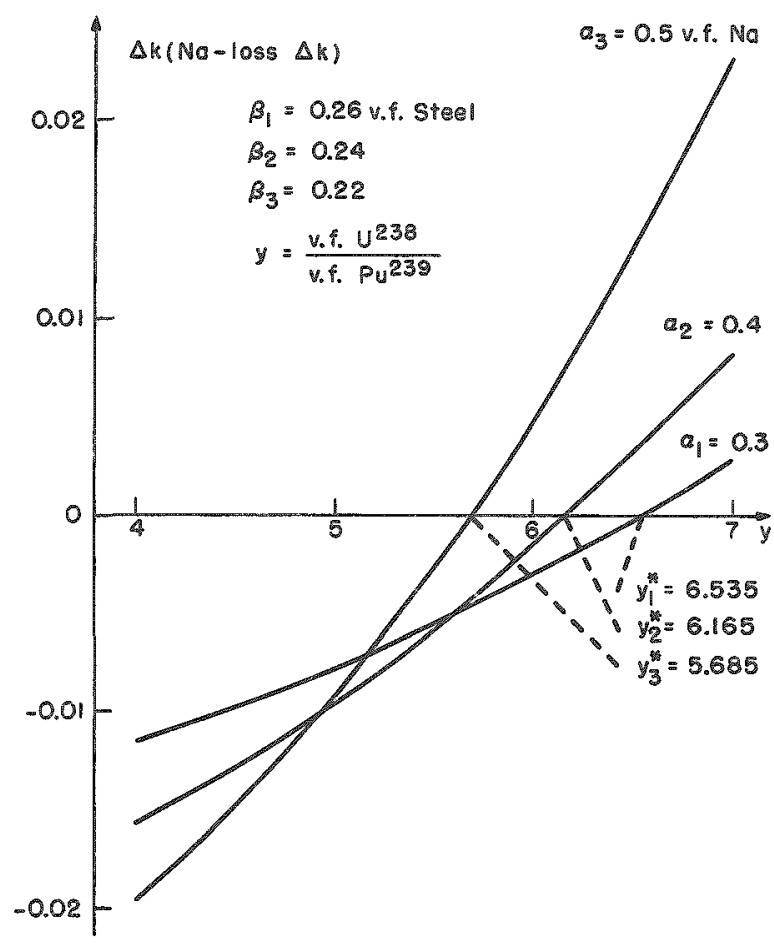

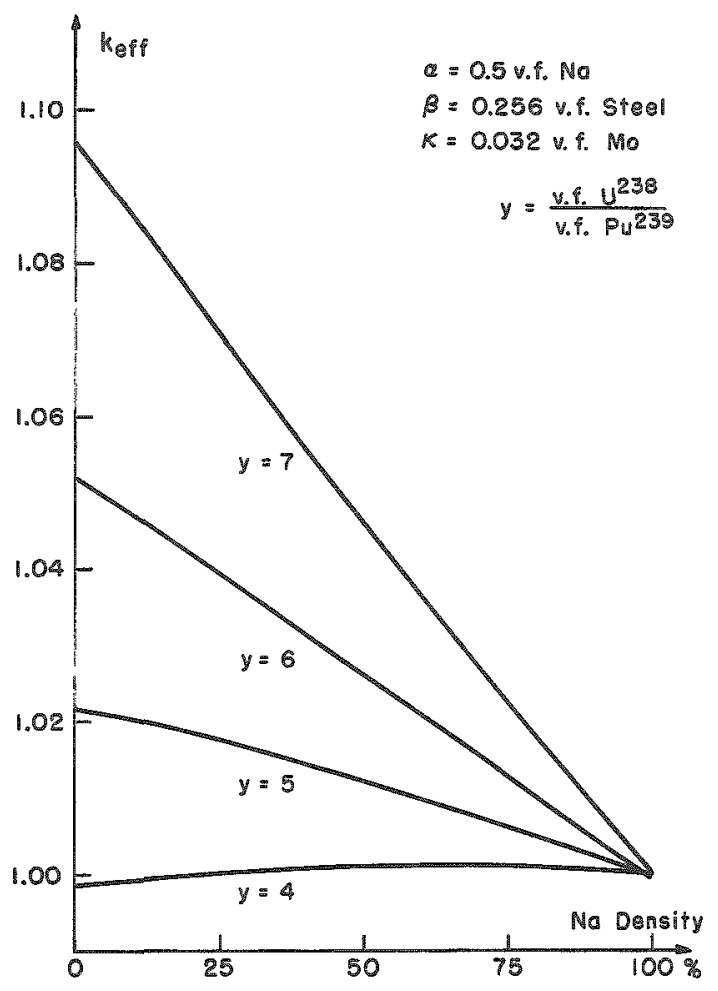

Fig. 6. Multiplication Factor as a Function of Sodium Content for Large Reactors

Fig. 7

Calculated Sodium Loss $\Delta \mathrm{k}$ Values as a Function of $y$ 
Table II

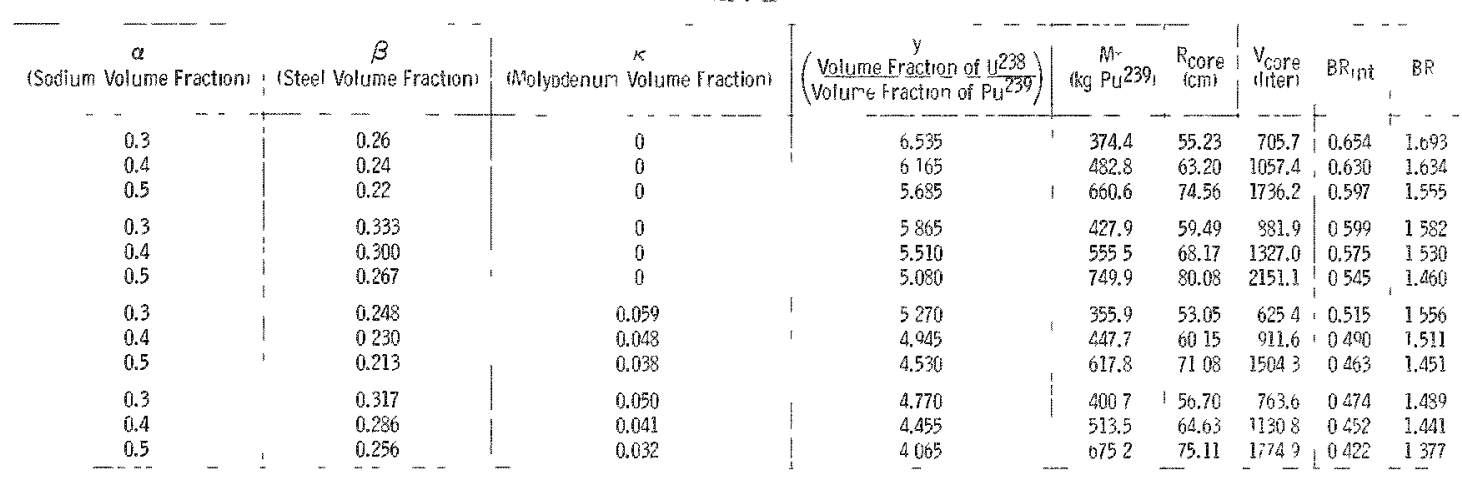

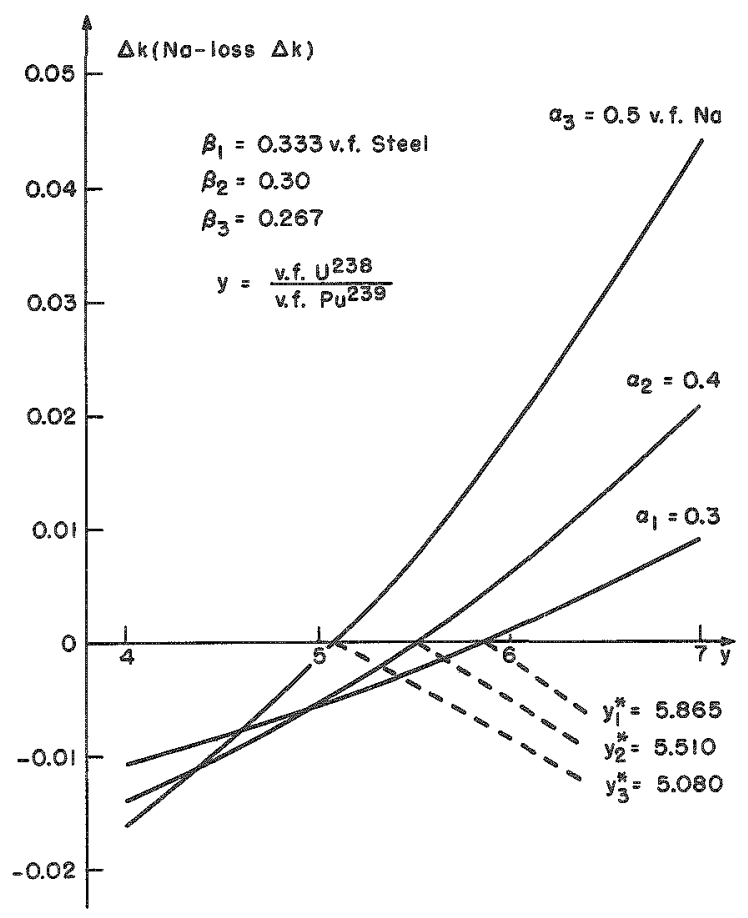

Fig. 8

Sodium-loss $\Delta \mathrm{k}$ Curves for Medium and Large Reactors

Fig. 9

Sodium-loss $\Delta \mathrm{k}$ Curves for Medium and Large Reactors

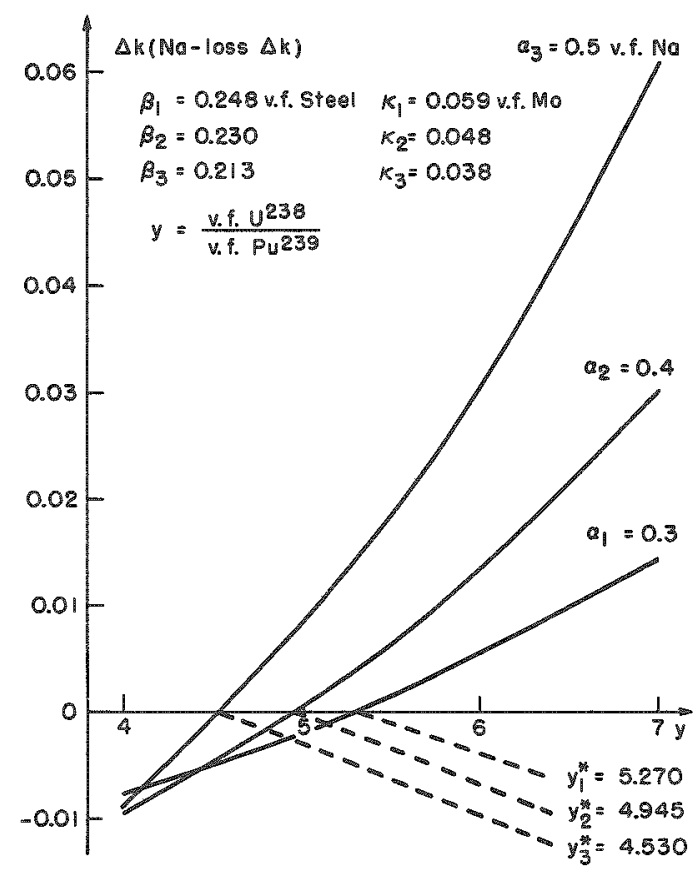




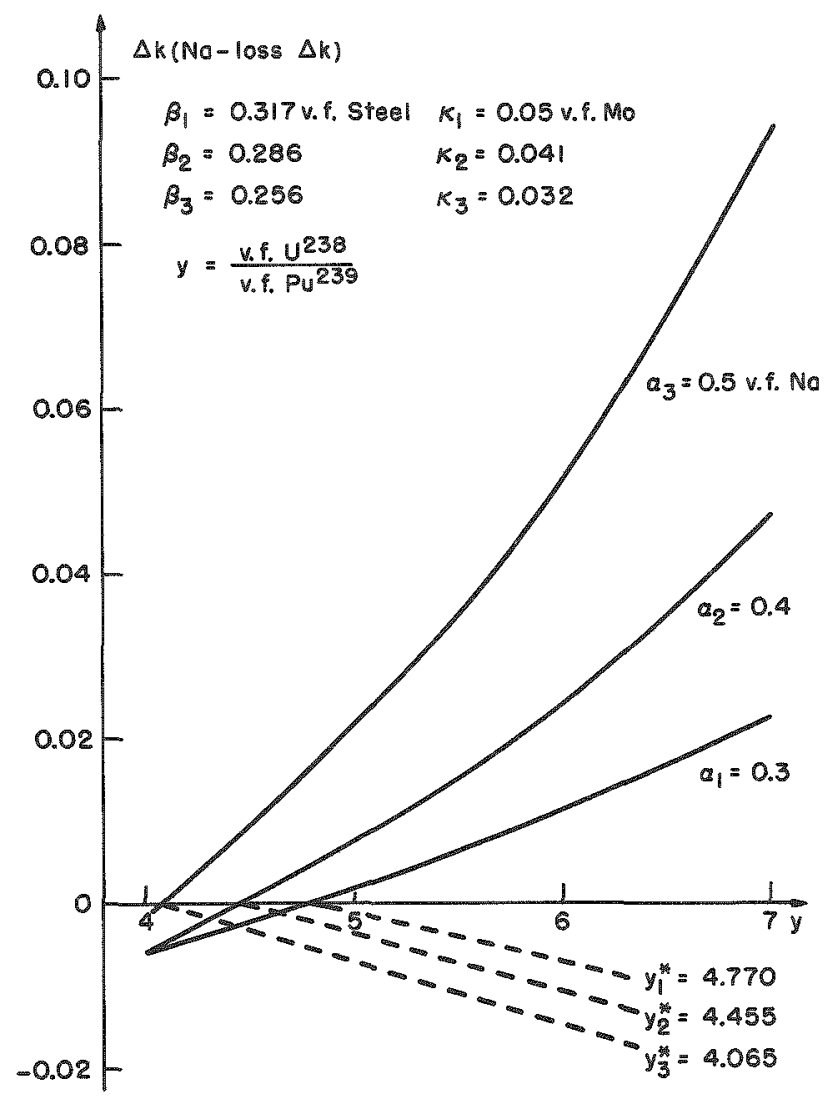

Fig. 10

Sodium-1oss $\Delta \mathrm{k}$ Curves for Medium and Large Reactors

Table II gives critical mass, volume, and internal and total breeding ratios.

Considering spheres with vanishing sodium-loss $\Delta \mathrm{k}$ only, whose properties are listed in Table II, one can draw essentially the following conclusions:

A large critical mass, as wanted for an economic power breeder, can only be achieved at the expense of internal as well as external $B R$. If one wants an internal $\mathrm{BR}$ greater than 0.5 , it seems from $T$ able II that the critical mass cannot exceed about 800 to $1000 \mathrm{~kg} \mathrm{Pu}{ }^{239}$.

An addition of $15 \%$ molybdenum to the fuel, which increases the thermal conductivity by about a factor of two, decreases the limit of the critical mass by about $10 \%$ and lowers the internal BR by about 0.13 .

It appears that an increase of the limits of critical masses calls for other geometrical shapes.

\section{Doppler coefficients}

For two reactors we have calculated the Doppler coefficients depending on temperature and sodium density by means of a method described 
in a paper of Froelich et al. (4) The composition of these reactors is given in Fig. 4. The two values of the fertile-to-fissile ratio were 5:1 and 7:1. We give in Table III the results for isothermal Doppler coefficients only because one can account for the temperature distribution by means of a formula which is given in Ref. 4. The Doppler coefficient can be expressed by a temperature law:

$$
\operatorname{DC}(T)=C\left(\frac{T_{0}}{T}\right)^{\gamma}
$$

where $T$ means a suitable averaged temperature of the fuel. The values of $C$ and $\gamma$ are given in Table III, where $T_{0}$ is chosen to $300^{\circ} \mathrm{K}$.

Table III

\begin{tabular}{c|c|c|c}
\hline$y=7$ & Sodium density & $\mathrm{C}=\mathrm{DC}\left(300^{\circ} \mathrm{K}\right)$ & $\gamma$ \\
\hline & $100 \%$ & $-1.34 \times 10^{-5} /{ }^{\circ} \mathrm{K}$ & 0.89 \\
& $50 \%$ & $-1.28 \times 10^{-5} /{ }^{\circ} \mathrm{K}$ & 0.96 \\
& $0 \%$ & $-1.26 \times 10^{-5} /{ }^{\circ} \mathrm{K}$ & 1.06 \\
\hline$y-5$ & & & \\
\hline & $100^{6 \%}$ & $-5.47 \times 10^{-6} /{ }^{\circ} \mathrm{K}$ & 0.88 \\
& $50 \%$ & $-5.29 \times 10^{-6} /{ }^{\circ} \mathrm{K}$ & 0.98 \\
& $0 \%$ & $-5.28 \times 10^{-6} /{ }^{\circ} \mathrm{K}$ & 1.11 \\
\hline
\end{tabular}

These temperature laws permit the evaluation of the $\Delta \mathrm{k}$ values between the cold reactor and a tolerable fuel melting, on the one hand, and between the operational temperature and the same melting situation on the other. As temperatures which can account for these in a isothermal Doppler coeficient, we choose

$$
\begin{array}{ll}
\mathrm{T}(\text { cold }) & =400^{\circ} \mathrm{K} ; \\
\mathrm{T}(\text { operationa }) & =1400^{\circ} \mathrm{K} ; \\
\mathrm{T}(\text { melting }) & -1800^{\circ} \mathrm{K} .
\end{array}
$$

The temperature laws given above yield for this the $\Delta \mathrm{k}$ values which are given in Table IV.

Table IV

\begin{tabular}{c|c|c|c|c}
\hline $\mathrm{y}=7$ & Sodium density & $\begin{array}{c}\Delta \mathrm{k} \\
400^{\circ} \mathrm{K} \rightarrow 1800^{\circ} \mathrm{K}\end{array}$ & $\begin{array}{c}\Delta \mathrm{k} \\
1400^{\circ} \mathrm{K} \rightarrow 1800^{\circ} \mathrm{K}\end{array}$ & Sodium-10ss $\Delta \mathrm{k}$ \\
\hline & $100^{\circ}$ & $-6.794 \times 10^{-3}$ & $-1.209 \times 10^{-3}$ & $+19.2 \times 10^{-3}$ \\
& $50 \%$ & $-6.118 \times 10^{-3}$ & $-1.112 \times 10^{-3}$ & \\
\hline$y=5$ & $0 \%$ & $-5.349 \times 10^{-3}$ & $-0.864 \times 10^{-3}$ & \\
\hline & & & & \\
& $100 \%$ & $-2.803 \times 10^{-3}$ & $-0.506 \times 10^{-3}$ & $-4.99 \times 10^{-3}$ \\
& $50 \%$ & $-2.443 \times 10^{-3}$ & $-0.411 \times 10^{-3}$ & \\
& $0 \%$ & $-2.127 \times 10^{-3}$ & $-0.333 \times 10^{-3}$ & \\
\hline
\end{tabular}


The prompt Doppler coefficient can overcome only a small delayed sodium-loss $\Delta \mathrm{k}$. Therefore, the sodium-loss $\Delta \mathrm{k}$ should be nonpositive as used in Section 3 .

\section{References}

1. W. Múnzner, Internal Karls ruhe report.

2. S. Yiftah, D. Okxent, and P.A. Moldauer, Fast Reactor Cross Sections, Pergamon Press (1960).

3. J. J. Schmidt and K. E. Schroeter, Internal Karlsruhe report.

4. R. Froelich, K. Ott, and J. J. Schmidt, Dependence of Fast Doppler Coefficients on Nuclear Data Uncertainties, this conference, paper No. VII-5.

$\frac{\text { Discussion of Paper }}{\text { Presented by Dr. Ott }}$

MR. HUMMEL (Argonne):

I do not think I quite understood those Doppler effects versus sodium 1oss. Was an oxide-fueled reactor involved?

MR。OTT:

Yes.

MR. HUMMEL:

You did not show a big change from going from $100 \%$ sodium to no sodium, is that right? It looked like the change involved a factor of 1.3 or 1.4.

MR。OTT:

Yes.

MR. HUMMEL:

That seems to contradict my experience.

MR. OTT:

Yes, it does. 
MR。 HUMMEI:

What was the volume per cent sodium? Only about $30 \%$ ? Maybe that is the reason.

MR。OTT:

We had a forty per cent sodium loss, which is one reason why our effect is smaller than yours. A second reason is that we have considered the interaction between the large sodium resonance and all of the other resonances, as will be described in Paper 6 of Session VII. The fact is that in the neighborhood of the large sodium resonance, the Doppler effect will increase after sodium los because of the very high potential scattering due to sodium. With the sodium in the reactor, the Doppler effect is small. Removal of sodium increases the Doppler effect.

MR. HUMMEL:

I have taken this effect into account in my calculations. Perhaps we can thrash this out on Thursday.

MR. COHEN (General Electric):

I should like to observe that the temperature coefficient of sodium is not the same as the void coefficient. The temperature coefficient is what happens when the sodium expands with temperature. I think it will turn out that in almost all of these reactors, unless you take an extreme case, the sodium coefficient, even if positive, will remain smaller than the Doppler coefficient. 
- 
GENERAL DISCUSSION OF SODIUM VOID AND DOPPLER COEFFICIENTS (Excluding Details of Theoretical Methods or Experimental Techniques for the Latter)

MR. AVERY (Argonne):

At this point I will open the meeting to informal discussions. Perhaps I may make a general comment.

In general, the sodium void coefficient problem seems to be a very depressing factor insofar as very large reactors are concerned. Is there anyone who wishes to give us some rays of hope in this general area?

MR. GREEBLER (General Electric):

It appears to be virtually impossible to have a large sodium-cooled reactor that does not have a positive sodium coefficient somewhere. Whether or not this is depressing depends on how one defines possible accidents.

MR. CAMPISE (Atomics International):

I agree with $\mathrm{Mr}$ 。Greebler that we must understand what accident we are talking about. Now, positive sodium coefficient by itself does no harm. In fact, the SRE and Hallam both operate with a positive sodium coefficient but a very negative power coefficient. The problem is to define what is the maximum credible accident and how does the positive sodium coefficient enter into it?

MR. HÄFELE (Karlsruhe):

I would like to make a provocative statement. If I see the situation correctly, a large pancake-type reactor with sodium cooling of 50 per cent does have the advantage that the reactor concept is open for high powers. that is, is capable of developing $500 \mathrm{MW}$ or even more; there is no upper limit. According to the results presented today, benefit is obtained from the fact that $y$, the ratio of the fertile to fissile material, is comparatively low Both $\mathrm{Mr}$. Hummel and $\mathrm{Mr}$. Ott presented figures for $\mathrm{y}$ of the order of 5 . The benefit of having more sodium in large reactors is paid for by a low internal breeding ratio, one in the neighborhood of 0.5 . But, if in turn you insist on having very high burnups, say, 100,000 MWD/tonne, or even more, this gives a greater reactivity change.

At Karlsruhe, we are concentrating on very large fast breeders with a very high internal breeding ratio because that, after all, gives the whole benefit of the reactor system we are talking about. Today, the only possibility we see to achieve such a goal is the use of gas cooling instead 
of sodium cooling. I apologize for that. But that is the way we see it. Gas cooling is more suitable for very dilute reactors. There are two factors influencing this conclusion. As we move away from metallic fuel and compact reactors, we can use lower fuel densities and lower enrichment, giving higher internal breeding ratios. And low power densities, say 200 kilowatts per liter, seem reasonable. These factors point to the possibility that gas cooling may be a very attractive thing.

I put this provocative statement out in order to get a strong response in behalf of sodium.

MR. PERSIANI (Argonne):

I concur only partially. I do believe that studies I made during the past summer indicate that one should perhaps go back to breeding in the blanket rather than in the core. Since the major contributions to the positive coefficient $w i l l$ be captures in the core, the change to more capture to the blanket will help. On the other hand, in a gas-cooled reactor, you obtain a negative coolant coefficient, perhaps at the expense of the Doppler coefficient, which is more important, I think, since it is a prompt negative feedback. Have you looked at the Doppler coefficients for gas cooled systems?

\section{MR. HAFEIE:}

Yes, we did, with gas cooling when $y$ is large, say in the neighborhood of 7 or even 8. You are then in very good shape to get high negative Doppler coefficients.

MR. CAMPISE:

Let us ask ourselves, why are we worried about the positive sodium void coefficient? It is only in reference to a maximum credible accident in which something very severe happens in the core. We either blow the sodium out or we vaporize it. The question that has to be answered is, what happens when the sodium leaves the core? If the meltdown that follows brings the reactor subcritical, you have suffered and have lost the core. But, that is no reason in itself to sacrifice the economics of an operating fast reactor on the basis of the positive sodium coefficient.

\section{MR. SPINRAD (Argonne):}

I think it is perhaps appropriate here to come to the defense of the sodium reactox by anticipating one of the impressions of my paper to be presented later, to the effect that because the very large-sized reactors do have a very striking positive sodium coefficient, the engineering 
incentive to go to extremely high power densities with sodium cooling is very great. It is a problem for the heat transfer and materials engineers to find out how to get high enough power densities so that the system is small enough.

MR. HAFELE:

Mr. Campise's statement on meltdown accidents applies very much to the helium situation because of the lack of emergency cooling and here it implies the same argument.

With respect to what $M r$. Spinrad said, our evaluations indicate that a helium gas-cooled breeder is capable of achieving specific powers of the order of 0.8 to $1 \mathrm{MW} / \mathrm{kg}$.

MR. WOLFE (General Electric):

Our calculations for gas cooling, and with superheaters for steam cooling, led us to conclude, as $\mathrm{Mr}$. Häfele mentioned, that you can get power densities of 800 to $1,000 \mathrm{~kW}$ per kilogram of fissile material. On the other hand, it looked to us as if you are then striving to attain a limiting value, whereas I do not believe that is the case with sodium. If you go to gas cooling, you are essentially stretching the technology now and not leaving yourself much margin for the future, whereas with the sodium, I would expect the power densities to go much above a $1000 \mathrm{~kW} / \mathrm{kg}$ in the future.

A second point. When we looked at this loss-of-coolant accident in our superheater, it was a serious problem, but we did have a large tank of hot water, which we used as an emergency measure that we flashed when we lost our cooling. Even then one had to open valves very quickly and design the reactor very carefully so that in the second or so it took to melt the fuel you had taken precautionary remedial action. I think in a gas-cooled reactor, when you get up to the power densities you are talking about, any type of accident which affects the gas coolant, a loss-of-power accident, a blower out, and so on, will lead to melting which in consequence can be at least as dangerous as the loss of sodium from a sodium reactor with positive coefficient. I agree with Dr, Campise that the consequences of these various mechanisms have to be examined in detail. You have to look at the accident in detail to see exactly how you would void sodium in one case or lose your gas coolant in another case, or lose your steam in the third case.

MR. HÄFELE:

Let me make a final remark, that we in Karlsruhe have not decided what type of coolant would be the best one. My arguments were presented a little bit in a provocative fashion in order to get the objections raised. 
MR. OKRENT (Argonne):

I would like to ask Mr. Hafele for the value of the helium coefficient for the high-pressure case. Was it of any significance?

MR. HÄFELE:

No significance.

MR. AVERY:

How about when it's steam cooled? Is it bad then?

MR.HÄEELE:

The steam cooling coefficient is much more tricky. You have to do lots of theoretical work. We are not yet ready to give an answer.

MR. KATO (Argonne):

How reliable are the computations for the sodium void coefficient? Secondly, since experiments with large quantities of plutonium are a few years off, can one get a good idea of these coefficients by working, let us say, with $\mathrm{U}^{235}$ in very large dilute systems?

MR. HUMMEL (Argonne):

The big uncertainty is in the spectral effect. I think this can adequately be studied with central danger coefficients and zoned loading. We hope to do this with ZPR-III before long, when they have a couple of hundred kilograms of plutonium available. They can build up a zoned loading and make central danger coefficient measurements with sodium, which I think would greatly reduce the maxgin of uncertainty. I am not perturbed myself about the transport cross sections. The leakage problem is mainly one of handling the two-dimensional geometry adequately.

MR, GREEBLER:

I do believe it is essential to have plutonium in the system in order to get an understanding of the sodium coefficient problem. For example, Mr. Hummel showed curves of neutron importance versus energy. We find that we get a turnover in the neutron importance curve at somewhat lower energies than he showed. I am sure this is entirely a function of what alpha values we may have chosen for the plutonium. Possibly also to a lesser extent there may be a question of how we may have calculated the self-shielding of $U^{238}$. Thus critical measurements in a $U^{235}$ assembly might not be very meaningful. I am sure we will get some useful information from it, but we do need measurements in a plutonium critical. 
MR. THALGOTT (Argonne):

I agree that sodium coefficient measurements can be made in a plutonium-fueled assembly. What worries me somewhat from the figures that I have seen today is that it might appear also to be very sensitive to the plutonium-240 content, and that is going to be quite difficult experimentally.

MR. DAVEY (Argonne):

In partial answer to Mr. Kato, some perturbation calculations of central danger coefficients of sodium in $U^{235}$ fueled ZPR-III assemblies show some considerable discrepancies of up to a factor of 2 with experiment for highly enriched systems. I wouldn't pretend that these are exact, because it is quite possible in these cases there is some sample-size effect. But dis. crepancies do exist.

MR. SOFER (United Nuclear Corp.):

I would like to add this thought. In some other fissile-fertile material systems, such as $\mathrm{U}^{233}$-thorium, there is the prospect of less severe effects coming from the void coefficient in that the eta of $U^{233}$ is not as steep as the eta of plutonium, and the cross section of thorium is such that the effect of hardening of the spectrum does not result in as large a positive void coefficient. It may even permit us to get a negative void coefficient. Of course, in going to the $\mathrm{U}^{233}$ whorium system, one buys other problems which I will discuss tomorrow.

MR. CAMPISE:

With reference to the point that Mr. Greebler brought up about the alpha variations and its effect on neutron importance, in a series of criticals which we ran at Atomics International on the AETR Program, we made measurements with a set of neutron sources We noted that the comparison of calculated and measured neutron importance around $25 \mathrm{kV}$ was very sensitive to the alpha values assumed. Thus it might be very important, in addition to making sodium void coefficient measurements, to also make source measurements in order to get additional data on neutron importance.

MR. CODD (Winfrith):

I imagine that most of the calculations reported this morning concerned homogeneous systems. I wonder if anyone has made calculations for heterogeneous systems and, for example, investigated the effect of neutron streaming on the sodium loss coefficient. 
MR. PERSIANI:

I think there was an experiment performed with ZPR-III, in which the sodium was removed first from a core section, and then from the blanket. When the sodium was removed from the core you got a positive effect, but when it was removed from the axial blanket, that coefficient did become negative.

MR. LONG (Argonne):

I do not know whether the significance of that experiment has been fully analyzed yet.

MR. AVERY:

I believe that certainly we all agree the blanket effects are going to have profound effects on the sodium void coefficient. I do not believe that is relevant to the heterogeneity effect necessarily.

MR. THALGOTT:

We attempted very diligently in ZPR.-III with the early small cores to measure a streaming effect. We were not able to get an effect which could clearly be attributed to streaming.

MR. SPINRAD:

At the time of the commissioning of EBR-I, we discovered that the heterogeneity effect of the lumping of fuel was much moxe significant than the heterogeneity effect of streaming in a well-reflected system. Indeed, EBR-I was considerably less reactive than the critical experiments it was supposedly based on because the reactor had smaller fuel pieces and void segments.

MR. STORRER (EURATOM):

I noticed on the slide given by Mr. Greebler, he said heterogeneity was no problem. I would expect that heterogeneity might have considerable importance on the Doppler coefficient.

MR. GREEBIER:

The heterogeneity I was referring to was that between fuel and coolant, not that due to control rod channels or anything like this. We found that such heterogeneity had very little effect on the Doppler coefficient except in the energy region of the strong sodium resonance and hexe indeed the 
system is heterogeneous. For example, you can calculate the equivalent $\sigma$ pot for homogeneous systems. We found that in the region of the sodium resonance there was indeed a considerably smaller effective potential scat tering cross section when one took into account the heterogeneity. But the Doppler effect is small there anyway, because you are close to infinite dilution in the sodium resonance, that it made very little difference, even though the $\sigma_{\text {pot }}$ did come down considerably there. Elsewhere it made virtually no difference. Again, this was for a 0.25 -in. rod, so it is a very tight system.

Incidentally, we hoped that we might find some negative component for the sodium coefficient by taking into account the streaming, again for the very fine channel. The calculated effect was negligibly small. This does not say there would not be a significanteffect on streaming in larger channels.

MR. AVERY:

I think a very relevant point might be in the attempt to measure the sodium void coefficient in critical assemblies where one is likely to have much larger heterogeneity than in the prototype reactor. There it might involve more correction.

MR. FISCHER (Argonne):

I was partly associated with the ZPR-III experiments when the sodium void measurements were made. Two separate experiments were made. In the one case, they measured a void at the center of the zoned loading, where they tried to mock up something like a spherical bubble in the middle of what was rather a long cylinder. The cylinder mocked up approximately a 5,000-liter uranium monocarbide system which initially had 50 per cent sodium. The objective was to take out the sodium cans, which were a quarter inch thick by two inches long, and some number of inches in length in the drawer and which were distributed rather uniformly through the drawer, and to replace the cans directly with dummy cans. The sodium void coefficients which were measured represented this uniform situation.

The other experiment involved channel streaming, in which we removed sodium cans in the same manner throughout the whole length of this central zone. The two extremes of this large dilute central zone, had an axial blanket which was compatible with the sodium flow in the vertical direction through the standard laxge reactor of this type. Here again they took out the sodium directly in channels and replaced it with dummy cans over a much smaller radius.

As far as the heterogeneity effect is concerned, one experiment was in the driver region, which had most of the reactivity of this zone loading. There was a strong gradient of the flux in the driver zone because it was 
rather short in radius. We were concerned about streaming effects on reactivity. A number of drawers were rearranged from being all in the normal arrangement to being perpendicular to each other. In those directions where there was any streaming the neutrons would be going out through the gaps. No effect of the orientation of the plates parallel or normal to the gradient of the flux was observed.

MR. SMITH (Winfrith):

I would like to go back to a point that was raised by $\mathrm{Mr}$. Thalgott on the question of the experimental determination of sodium coefficients. We have agreed it is necessary to use plutonium. We have seen graphs which show there is a big effect due to plutonium-240 on data which is more or less guess data. How did we get into this difficulty? Do we have to do zeropower experiments with different kinds of plutonium with different amounts of plutonium 240 in it? Or can we rely on nuclear data or nuclear models in the coming years to give us a good enough estimate on what the 240 effect will be?

MR. HUMMEL:

I think probably the most efficient thing would be for somebody to put his back into measuring the capture cross section of $P u^{240}$ as a function of energy. We might feel pretty good about the extrapolation from the $\mathrm{Pu}^{239}$ system to the dirty one, if we could get the cross sections of these higher isotopes.

MR. SCHMIDT (Karlsruhe):

In answer to the question of Dr. Smith's, I would say if one knows for plutonium-240 the s-wave strength functions and the p-wave strength functions, and if one has a rather reliable estimate of the s-wave capture rates, then you can be confident. I think that the $s$-wave and $p$-wave strength functions for plutonium-240 will be rather well known when one has them for uranium-238. I think for $\mathrm{U}^{238}$ in one year it will be possible to have calculations from up-to-the-moment data on one side and from statistical theory fits on the other side. So I would think that this question of nuclear data. for $\mathrm{Pu}^{240}$ will be settled, from a theoretical point of view, in one year.

MR. HÄFEL:

I would like to mention that the French critical facility and the Karlsxuhe critical facility are preparing themselves to use several types of dirty plutonium. Because they require air cooling, there are, unfortunately, technical consequences. 
MR, PERSIANI:

Would not the Einstein characteristic temperature be a little more appropriate than the Debye temperature, and is this known for the uranium oxide?

MR, GREEBLER:

Yes, I think the Einstein formulation is a better one. But it still uses the Debye temperature, does it not? At least we've been using it this way.

MR. PERSIANI:

Well, call it the characteristic temperature, using an Einstein model. In the case of the Einstein model, I think you are in a range where you are involving multi-phonon processes. This gives you a better value of the cross section.

MR. GREEBIER:

This is correct.

MR. FISCHER:

There is one thing that Mr. Kato can do for us with ZPR-VI. The sodium void coefficient also depends on the nature of the cladding one uses. One thing that can be done is mock up the right kind of a spectrum and to study what it means when you have a certain amount of niobium, tantalum, or molybdenum. I think this is extremely important and can be done with ZPR-VI right now. 
- 


\section{SESSION II}

October 7, 1963

CORE MELTDOWN AND THE MAXIMUM ACCIDENT

Chairman: D. B. HALL

Los Alamos Scientific Laboratory 


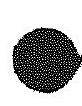



C. Dickerman, E. Sowa, L. Robinson, and D. Gopinath
Argonne National Laboratory
Argonne, Illinois

(Paper presented by Mr。 Dickerman)

\section{Introduction}

One of the key areas in investigations of fast reactor safety has been the meltdown problem. Since typical core designs for fast power reactors contain sufficient fuel to provide a number of critical masses if rearranged compactly enough, and since typical fast power reactor prompt-neutron lifetimes are short, it has been comparatively easy to imagine serious hypothetical meltdown accidents. $(1,2)$ In such accidents, molten fuel is assumed to collect in a more reactive configuration and produce a super-promptcritical excursion with large release of energy.

Attempts to scale down the estimated energy release from such hypothetical accidents have been hampered by lack of knowledge of the mechanisms affecting fuel-element failure and movement of material that could affect reactivity. For example, in a power excursion, it is necessary to know the previous ir radiation history of the fuel, its transient temperature rise, the relevant mechanisms of element failure, whether the element fails in an environment of liquid or boiling coolant, the effects of the coolant on movement of fuel, and the movements of the coolant induced by the fuel.

Because of the comparative lack of understanding of these phenomena, an experimental program to investigate meltdown phenomena has been under taken by Argonne National Laboratory, (3) centering on in-pile experiments in the Transient Reactor Test Facility (TREAT). (4) The goal of the program is to provide information, together with supporting theoretical studies, to assist the safety analyst in assessing potential meltdown hazards for specific core designs or types of fuel. Thus, it is necessary to investigate a given type of fuel from experiments on failure under simple idealized conditions extending on through the interaction between fuel and coolant. (3)

Evaluation of designs for large cores and ceramic fuel has not changed the general scope of the meltdown problem, but has changed emphases somewhat from those associated with small cores containing singlepiece metal-fuel pins.

Changes in effective neutron absorption produced by the Doppler effect become significant in the softer neutron spectra of ceramic cores. The core-averaged sodium void coefficient can be positive for the larger cores, and can be locally positive even if zero or negative when averaged 
over the core.(2) Interaction between the Doppler effect and neutron spectrum shifts caused by loss of sodium(5) also must be considered, and this adds to the complexity of effects involved in meltdown incidents. For example, a large ceramic reactor with $\mathrm{U}^{238}-\mathrm{Pu}^{239}$ fuel, having a zero sodium void coefficient - as calculated with non-Doppler-broadened cross sections - and a negative Doppler fuel coefficient, can show a gain in reactivity upon loss of sodium due to the change in neutron spectrum caused by loss of the moderating effects of the sodium.

Transient pressure surges in coolant are important for the possible influence upon motion of fuel-meltdown products. In addition, transient

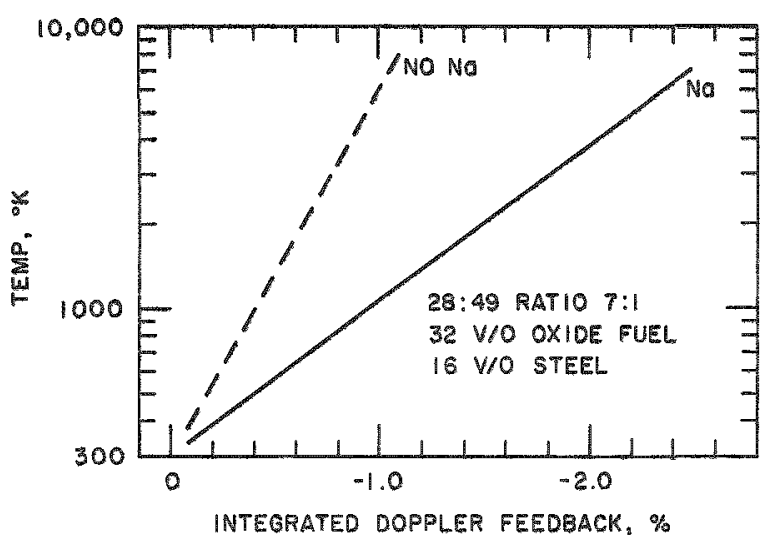

Fig. 1. Integrated Doppler Fuel Reactivity Feedback Curves for a Large Fastoxide Reactor coolant vaporization is a potential source of significant rates of reactivity addition. Figure $l$ is a graph showing integrated feedback curves produced from Doppler feedback data obtained recently by Hummel, who performed fundamental mode calculations by the EIMOE code for the case of a large oxide reactor core with $52 \mathrm{v} / \mathrm{o}$ sodium and $32 \mathrm{v} / \mathrm{o}$ fuel. The figure is typical of results for atom ratios of $U^{238}$ to $P u^{239}$ of $7: 1$ and $9: 1 .(6)$ In the figure, the two integrated Doppler curves are arbitrarily normalized at a fuel temperature of $300^{\circ} \mathrm{K}$. The exact normalization does not affect the following conclusions to be drawn for these compositions from the illustration:

1. Loss of coolant without change in fuel temperature can lead to a significant increase in reactivity although the Doppler coefficient does not change sign.

2. The Doppler coefficient remaining after loss of coolant is less ( one-half) than for coolant present.

3. The integrated Doppler feedback is a logarithmetic function of temperature, becoming less effective as temperature rises.

These results do not apply quantitatively to all large ceramic cores, but they are representative of changes in relevant nuclear safety properties as core sizes increase.

The Doppler broadening of nuclear resonances can play a significant role in shutting off fast reactor excursions before core damage occurs, $(7)$ 
and in decreasing severity of large releases of nuclear energy in maximum accidents. However, as the example in Fig. I indicates, the situation is not simple.

In addition, pre-failure movement of ceramic fuel is more complicated than for the single-piece metallic fuel pins. The ceramic fuels are brittle, and have low thermal conductivity and less than theoretical density. Hence, in addition to possible reactivity changes due to thermal expansion, such fuels can produce reactivity changes as a result of cracking, slumping, or central melting. In compensation, fuel-element bowing becomes less important for the larger cores than in the existing small cores.

II. Experimental Results

\section{A. Previous Studies}

Earlier results have been reported for TREAT experiments run, in the absence of coolant, on Experimental Breeder Reactor-II Mark-I and Fermi Core-A metal samples, $(3,8)$ and $\mathrm{EBR}-\mathrm{II}-\mathrm{sized} \mathrm{UO}_{2}$ specimens. (9) Quantitative measurements of EBR-II-element failure times in-pile have also been made $(10)$ for comparison against the times of penetration measured for steel jacket material in out-of-pile experiments. (11)

\section{B. Stagnant Sodium Environment}

Experiments have been performed in TREAT to study the behavior of single EBR-II elements submerged in stagnant sodium. (12) Each sample was contained in a sodium annulus mocking up the average sodium crosssectional area per pin in an $E B R$-II subassembly. Limited transient temperature data were obtained and checked against calculated temperatures. Figure 2

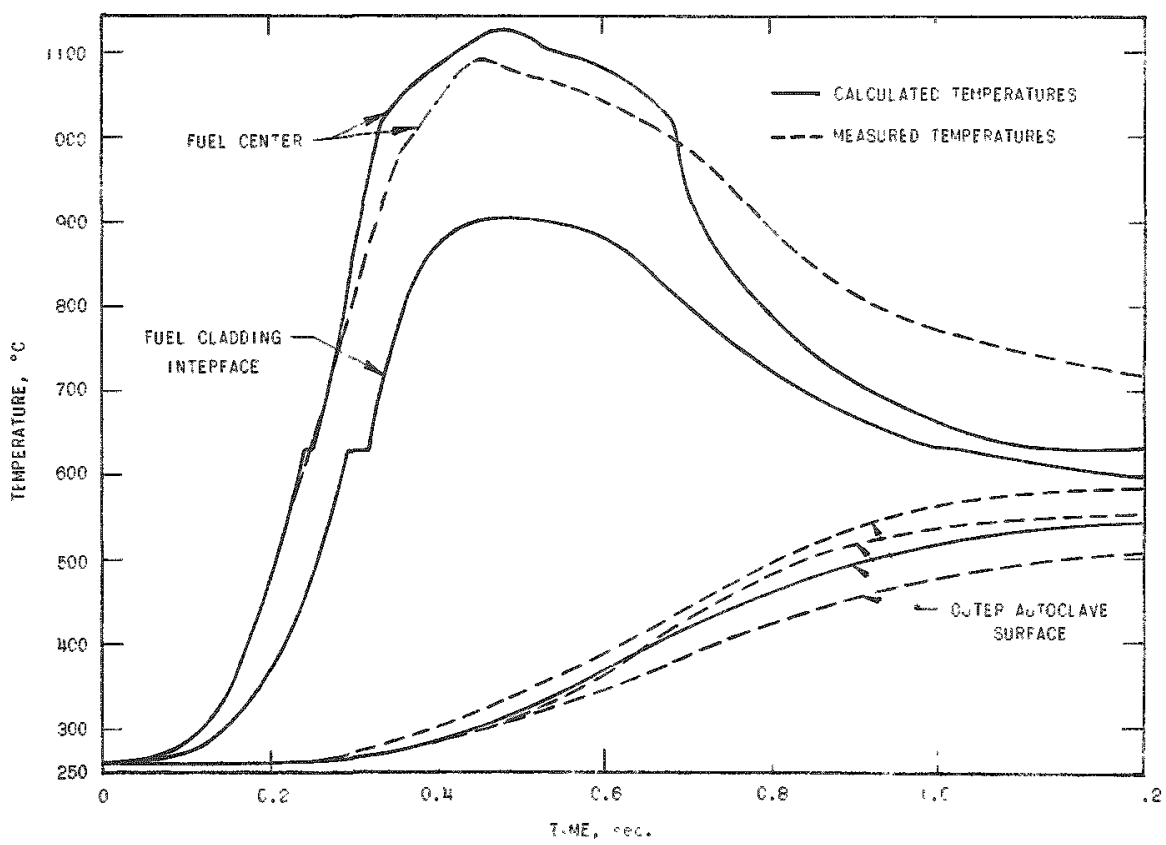

Fig. 2

Comparison of Calculated and Experimental EBR-II Sample Central Fuel Temperatures for a Stagnant Sodium Meltdown Experiment 


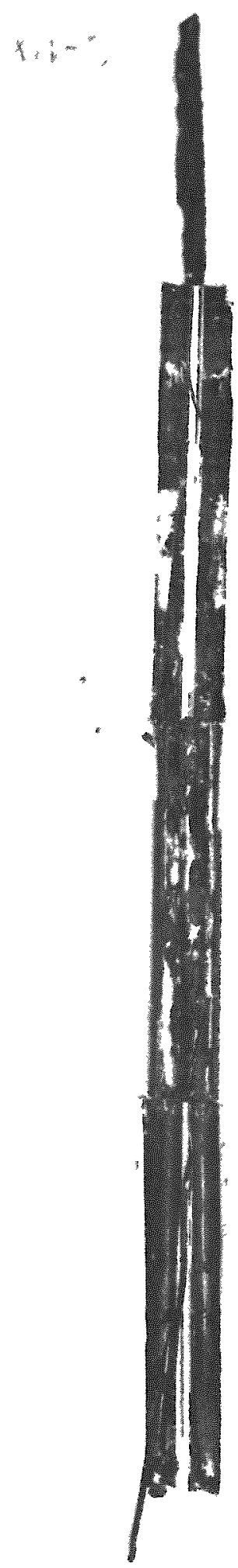

Fig. 3. Post-TREAT Appearance of Two EBR-II Samples Run in Stagnant Sodrum Meltdown Experiments shows calculated and experimental transient centerline temperatures for a transient run with a sample containing an internal, Ta-Mo the rmocouple. Also shown are temperatures for the fuelcladding interface, and the outer surface of the autoclave containing the stagnant sodium. The threshold of failure, modes of failure, and post-experiment distribution of fuel were obtained for experimental conditions including both uniform axial power and axial power shaped to approximate a typical fast power reactor core powex profile. Samples were run with sodium initially at saturation, and with sodium pressurized to inhibit boiling. The results, although somewhat different qualitatively, were consistent with those found previously for EBR-II elements run in the absence of sodium. Figure 3 is a photo of sample remains from the first two stagnant sodium test capsules, after disassembly. The fuel pin on the right, XIV-1, shown after removal of its (outwardly undamaged) cladding, has been attacked by the silvery iron-uranium eutectic. The pin on the left, shown inside the autoclave tube after the tube was cut open for inspection, displays results of cladding failure by dissolution of cladding and expulsion of fuel into the surrounding region.

\section{Fission Gas Effects}

Ir radiated EBR-II and

Fermi A samples have been subjected to transient nuclear heating in TREAT in the absence of sodium using opaque meltdown capsules. (13) Burnup ranged from $0.09 \mathrm{a} / 0$ to $0.9 \mathrm{a} / 0$ for the $\mathrm{EBR}-\mathrm{II}$ pins, and was about $0.25 \mathrm{a} / \mathrm{o}$ for the Fermi elements run. For the burnups studied, prior ir radiation did not have a large effect on the EBR-II sample threshold of failure. Further, the failure mechanism for a very low fission 
product inventory $(\sim 0.09$ a/o burnup) appeared to be essentially the same as that of unirradiated samples. Meltdown residue from two failed samples at the other end of the burnup range had density about half that of unirradiated elements. Figure 4 shows the opened meltdown capsule for a 0.9-a/o-burnup sample. Two "spongy" masses of solidified fuel are visible. Figure 5 is a close-up of the sponge-like fuel remains from the other $0.9-\mathrm{a} /$ meltdown sample, showing more clearly the macroscopic structure. Post-TREAT void contents of the four failed specimens, which
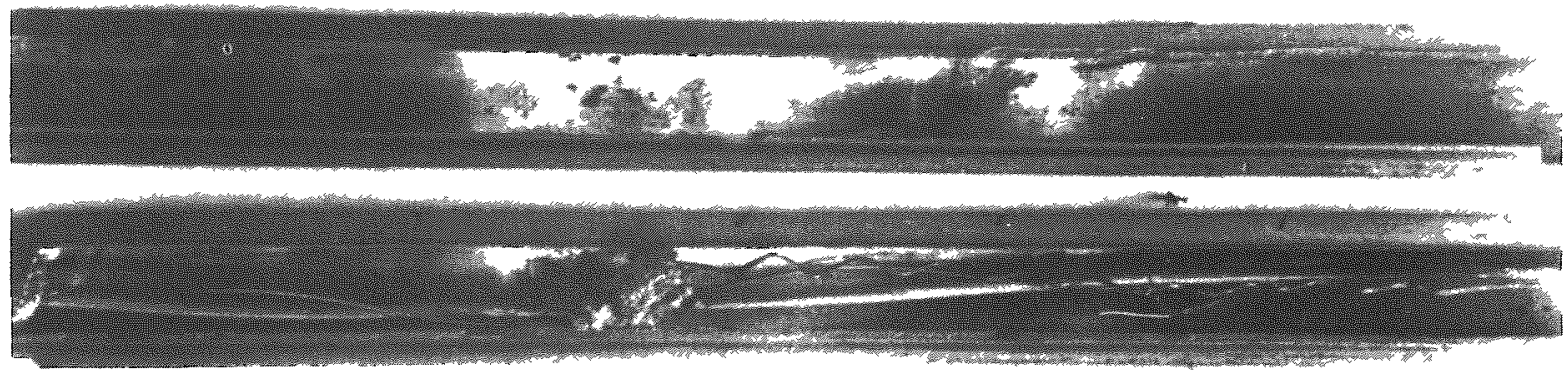

Fig. 4. Post-TREAT Appearance of Failed EBR-II Element with 0.9 a/o Burnup
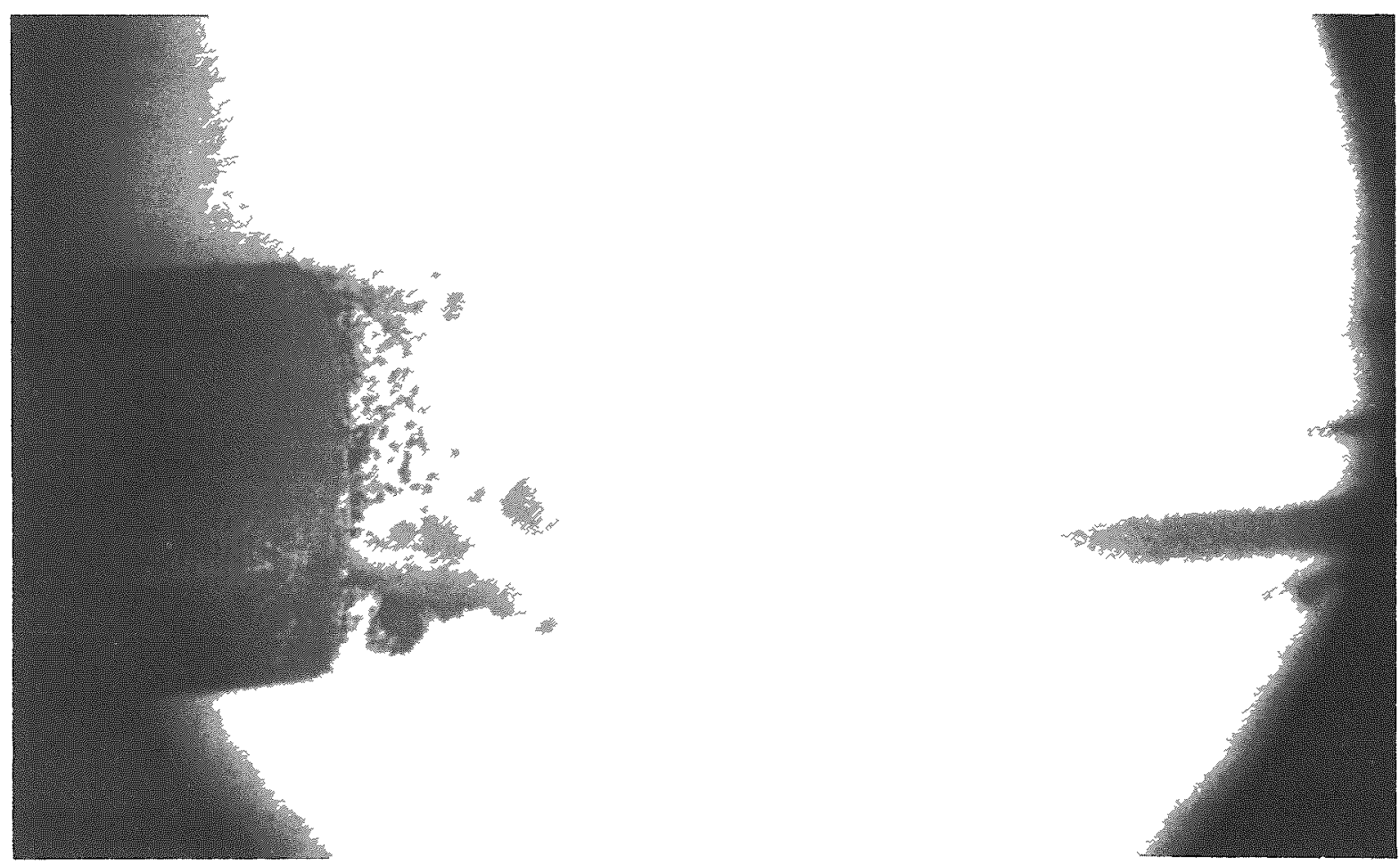

Fig. 5. Close-up of Fuel Remains from Failed EBR-II Element with 0.9 a/o Burnup, after TREAT Experiment 
spanned the range from $0.09-a / 0$ to $0.9-a / 0$ burnup, were approximately proportional to burnup. Fermi samples failed by splitting of cladding caused by swelling of the fuel inside. Solidified clumps of porous fuelmeltdown residue were found outside the split cladding. These results thus confirm the large swelling resulting from short-time heating under meltdown conditions found in mock-up experiments using direct electrical resistance heating(14) and furnace heating.(15) However, they do not give results directly applicable to higher burnups. These large density decreases upon meltdown for samples with appreciable fission gas inventories may play a significant role in limiting possible maximum rates of meltdown-product movement - and thus in permitting definition of limits on severity of resulting nuclear excursions. However, the swelling also displaces sodium that might still be present in the core and could cause a reactivity increase in a reactor with a positive sodium void coefficient.

\section{Uranium Sulfide Transient Tests}

As part of the program for exploring transient characteristics of advanced fuels, transient ir radiations have been performed in TREAT on half-length EBR-II-sized inert gas-bonded, refractory metal-clad uranium sulfide elements. All were exposed in an inert gas atmosphere, rather than a sodium environment. Maximum transient temperatures, as recorded by fast-response tungsten-rhenium-alloy-cladding thermocouples, ranged from $900^{\circ} \mathrm{C}$ to $2300^{\circ} \mathrm{C}$. In contrast with the oxide samples run earlier, (9) the US pellets showed no cracking into shorter lengths, nor central zones of loosely bonded material. However, detectable slumping of fuel was found for maximum cladding temperatures equal to or greater than about $1600^{\circ} \mathrm{C}$. Figure 6 shows typical pellets from the excursion with maximum cladding temperature of about $2300^{\circ} \mathrm{C}$. Note the "cast" appearance of some surfaces, resulting from flow of the sulfide material along and against the inner surface of the cladding. Analysis of the experimental temperatures showed a high-temperature specific heat for US of $0.046 \mathrm{cal} / \mathrm{g}-{ }^{\circ} \mathrm{C}$, which may be compared with the Dulong and Petit relationship prediction of $0.0444 \mathrm{cal} / \mathrm{g}^{\circ}{ }^{\circ} \mathrm{C}$.

\section{Safety Analyses}

As an example of possible use of meltdown information in safety analyses, results will be given for calculations of three hypothetical accidents for an EBR-II-like reactor. The detailed specifications for the three cases are somewhat artificial: however, they do present an interesting range of conditions, running from a case in which the time scale for fuelelement failure is determined by the time for penetration of fuel-element cladding by molten fuel, to a particularly extreme case in which the time of movement of meltdown products is essentially the time during which the fuel is expelled from a single element. 


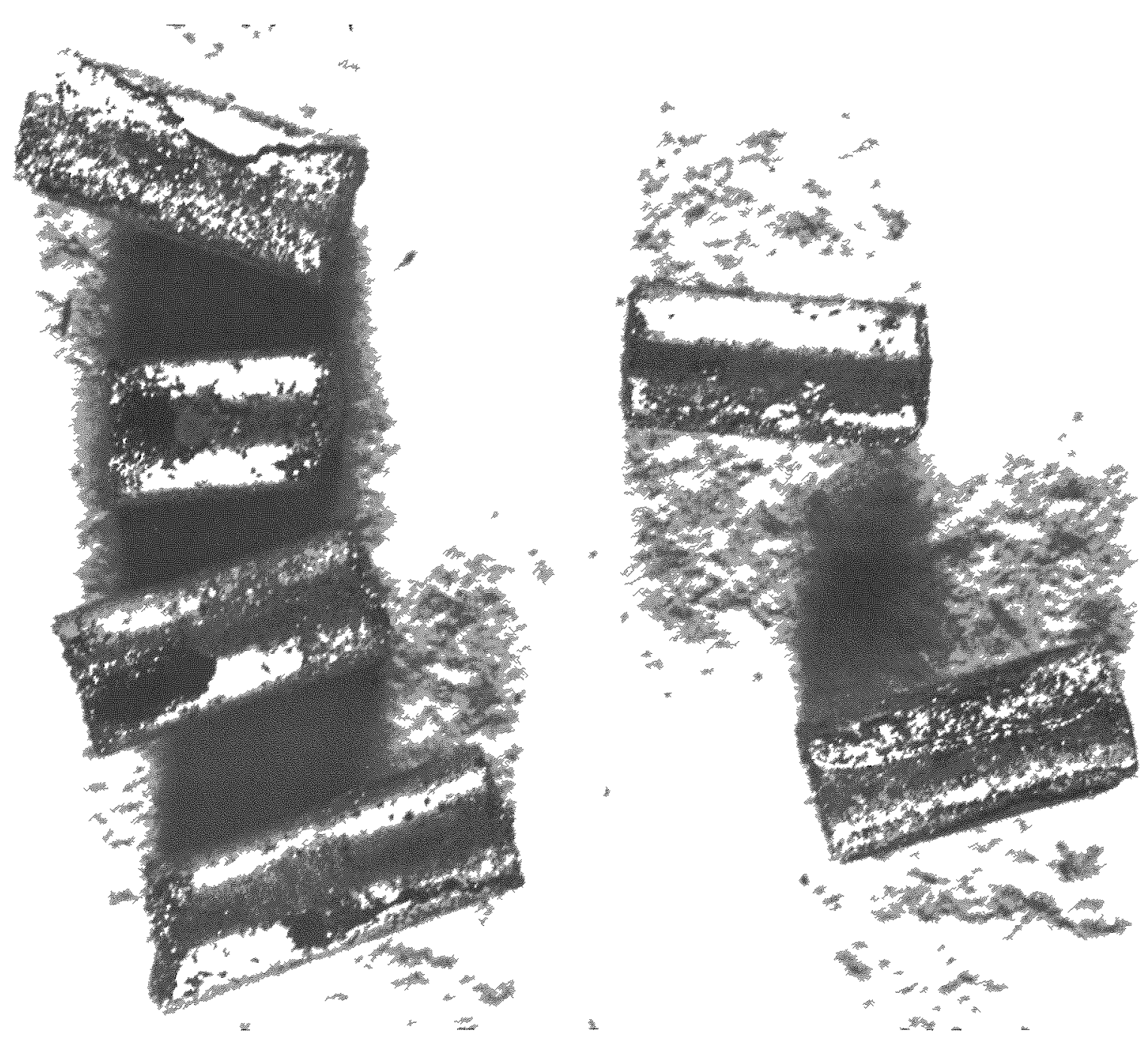

Fig. 6. Post-TREAT Appearance of US Pellets - Maxmum Cladding Temperature of about $2300^{\circ} \mathrm{C}$

In the event of an accident in a fast reactor leading to fuel-clement fallure, the distribution in time of meltdown of different fuel elements determines the rate of reactivity change. In general, the reactivity change would be either negative or positive. Since these changes are not well understood, it will be assumed here that the changes are positive, and lack of coherence in fallure times is desirable.

Case 1. Falure of Pumping Power. The EBR-II reference core consists of 61 subassemblies, each of which contains 91 fuel elements. The subassemblies are grouped into rows, depending on location with respect to the core centerline. Sodium coolant enters the bottom of each subassembly through an orifice, which is pre-set to vary the flow rate from row to row to reduce differences in coolant outlet temperature. Inside a subassembly, elements can be classed as (1) internal pins (which 
have an effective coolant cross-section area of $0.110 \mathrm{~cm}^{2}$ ) and (2) periphe ral pins (which have $0.180 \mathrm{~cm}^{2}$ ). The coolant flow rate decay curve used to describe results of pumping power failure has been given previously. (16) Reactor power is assumed to remain constant throughout the flow transient at design EBR-II level. Five cases were studied by the IBM 704 "ARGUS"(17) transient heat transfer code:

(1) Central row - internal element

(2) Central row - peripheral element

(3) Fourth row - internal element

(4) Fourth row - peripheral element 1 (nearest to core center)

(5) Fourth row - peripheral element 2 (farthest from core center)

In the EBR-II reference design, the central and fourth rows are those of maximum power and maximum coolant outlet temperature, respectively. Figure 7 is a graph of maximum fuel-cladding interface tempera-

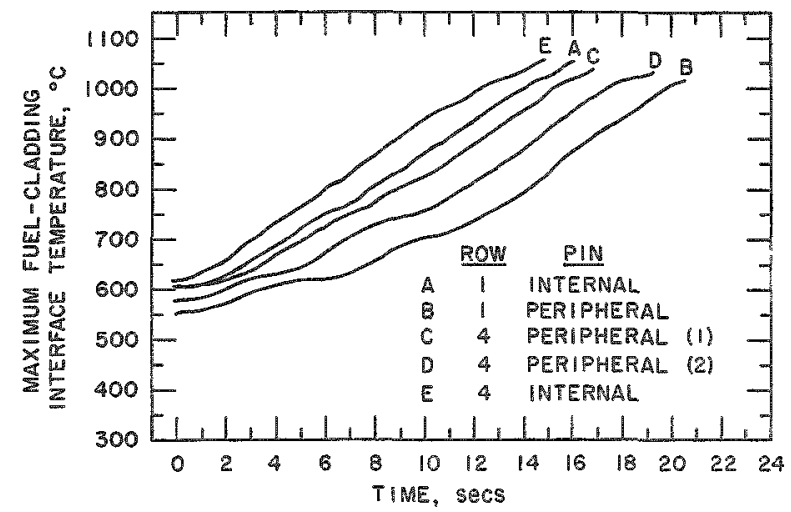

Fig. 7. Maximum Fuel-Cladding Interface Temperatures Calculated for Hypothetical Loss of Pumping Power Incident in EBR-IILike Reactor tures, as a function of time, for these five cases. On the basis of analysis of meltdown experiment results, $(3,12)$ a fuel-cladding interface temperature of $1015^{\circ} \mathrm{C}$ was taken as the threshold for element failure by cladding dissolution, and a temperature of $1200^{\circ} \mathrm{C}$ was taken to be the threshold for failure due to bursting of the cladding. The time for penetration of the cladding by molten fuel was assumed to be of the order of one second. $(10,11)$ Figure 7 shows that the time spread within subassemblies is larger than between subassemblies in rows 1 and 4. Failure of one internal element can "trigger" failure of a nearby peripheral element (or vice versa) by means of molten fuel-cladding eutectic* from the failed pin eating its way into the cladding of the still intact neighbor. Hence, the time scale for element failures in this hypothetical accident is dominated by the cladding-penetration time. The time scale would be of the order of $2 \mathrm{sec}$ for each of the two subassembly locations, and of the order of $3.6 \mathrm{sec}$ for all the elements in both subassembly rows. This situation might be complicated by agitation of sodium produced by boiling and/or natural convection. However, this agitation probably would increase the time spread in failure.

*The low-melting iron-uranium eutectic occurs at $725^{\circ} \mathrm{C}$. 
Case 2. Blocked Orifice. For another set of problems, it was assumed that the flow rate through the subassembly decreased linearly in one second to $1 \%$ of design flow because of an unspecified orifice blockage at time zero. Reactor power was assumed to be constant. The assumption of constant reactor power is not as extreme in this case as in Case 1 , since

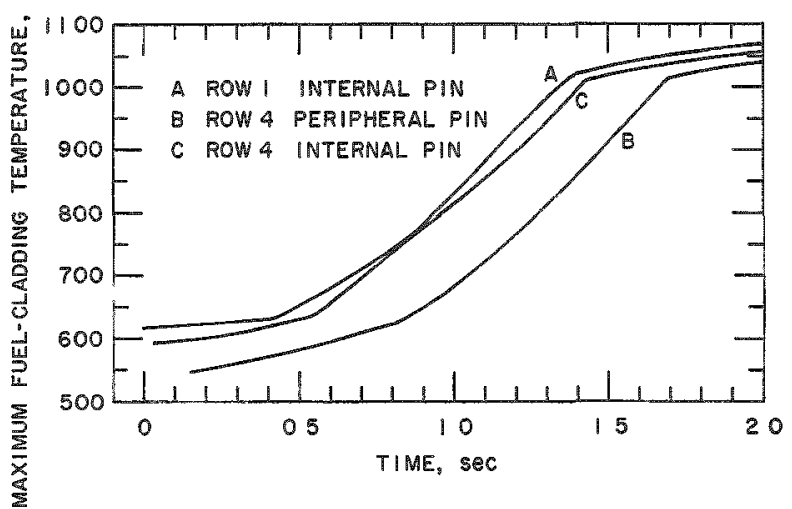

Fig. 8. Maximum Fuel-Cladding Interface Temperatures Calculated for Hypothetical Blocked Onfice Accilent un EBR-II-Like Reactor the blocked-orifice incident is basically a single subassembly event, i.e., the negative reactivity change caused by temperature rise is almost two orders of magnitude smaller here than for a temperature excursion for the reactor as a whole. Figure 8 shows calculated maximum fuel-cladding interface temperatures as a function of time for three element locations:

(1) Central row - internal element

(2) Fourth row - peripheral element (nearest to core center)

(3) Fourth row - internal element.

For the fourth row subassembly, a spread of about 0.26 sec is shown in time of attaining the failure threshold, and this time should be combined with the duration of fuel movement, which is estimated to be of the same order of magnitude. In the event of complete core inlet coolant blockage, the figure shows a falure time spread of about $0.33 \mathrm{sec}$, which must be combined with the duration of fuel movement for one element to obtain an estimate of the total duration of reactivity input. If the central-row peripheral clements and fourth-row peripheral elements farthest from the center are also considered, the time spread would be increased by about a factor of two, but the order of magnitude of the time spread would be the same.

Case 3. Fuel Subassembly Dropped during Loading. An extreme hypothetical loading accident was examined as Case 3 . It is assumed that the reactor is critical at low power, and that, at the worst possible position, the central fuel subassembly is dropped to fall free into position in the core. Reactor fecdback is estimated to be essentially that for adiabatic heating of the fuel, because of the extremely short duration of the excursion. Figure 9 shows the reactor neutron density and time-integrated neutron density calculated by the IBM 704 kinetics code RP $129 \mathrm{H}$. (18) In this case, the reactor becomes prompt critical at about $194 \mathrm{~ms}$. 


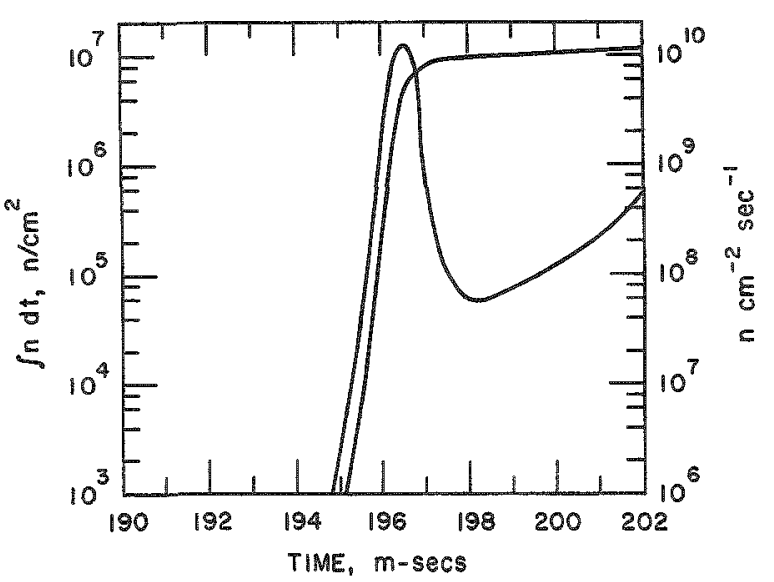

Fig. 9. Neutron Density and Integrated Neutron Density Calculated for Hypothetical Dropped Subassembly Loading Accident

Within about $5.5 \mathrm{~ms}$, the average fuel temperature has $r$ isen to $1200^{\circ} \mathrm{C}$, and the rate of rise at this time is about $160^{\circ} \mathrm{C} / \mathrm{ms}$. If the radial variation in subassembly power is considered, the overall time spread in reaching the failure threshold is estimated to be about $4 \mathrm{~ms}$. Thus, the time duration of reactivity change would be essentially that for fuel movement of one element in Case 3 .

\section{Current Plans}

Within the next few months, it is planned to investigate more thoroughly the effects of fission gases on fuel-element meltdown, and to begin studies of meltdown into a flowing sodium environment.

\section{References}

1. W. J. McCarthy, Jr., R. B. Nicholson, D. Okrent, and V. Z. Jankus, Studies of Nuclear Accidents in Fast Power Reactors, Proc. Second. UN Int. Conf. Peaceful Uses of Atomic Energy, Geneva, Switzerland, 12, 242 (1958).

2. D. Okrent, A Review of the Nuclear Aspects of Fast-Reactor Safety, Physics of Fast and Intermediate Reactors, IAEA, Vienna, III, 155 (1962).

3. C. E. Dickerman, D. Okrent, and E. Sowa, The Fast Reactor Safety Programme in TREAT, Physics of Fast and Intermediate Reactors, IAEA, Vienna, III, 171 (1962).

4. G. A. Freund, H. P. Iskenderian, and D. Okrent, TREAT-A Pulsed Graphite-moderated Reactor for Kinetics Experiments, Proc. Second UN Int. Conf. Peaceful Uses of Atomic Energy, Geneva, Switzerland, 10, 461 (1958).

5. M. G. Bhide and H. H. Hummel, Calculations of the Doppler Coefficient of Large Ceramic-fueled Fast Reactors, ANL-6601 (1962).

6. H. H. Hummel, Dynamics of Large Ceramic-fueled Sodium Cooled Fast Reactors, Presented at 2nd AMU-ASEE Faculty-Student Conference, Argonne, Illinois (1963). 
7. P. Greebler and B. A. Hutchins, The Doppler Effect in a Large Fast Oxide Reactor - Its Calculation and Significance for Reactor Safety, Physics of Fast and Intermediate Reactors, IAEA, Vienna, III, 121 (1962).

8. C. E. Dickerman, G. H. Golden, and L. E, Robinson, In-pile Photographic Studies of EBR-II Mark-I and Fermi Core-A Sample Meltdown, Nuc. Sci. and Eng., 14, 30 (1962).

9. C. E. Dickerman, L. E. Robinson, E. S. Sowa, and S. B. Skladzien, Transient In-pile Experiments on Fast-reactor-type Uranium Uxide Fuel Elements in the Absence of Sodium, Trans. Amer. Nuc. Soc., 6 , No. 1, 104 (1963).

10. C. M. Walter and C. E. Dickerman, TREAT Study of the Penetration of Molten U/5 wt \% Fs Alloy through Type 304 Stainless Steel, Trans. Amer. Nuc. Soc., 6, No. 1, 104 (1963).

11. C. M. Walter and L. R. Kelman, Penetration Rate Studies of Stainless Steel by Molten Uranium and Uranium-Fissium Alloy, J. Nucl. Mat. 6 , 281 (1962).

12. C. E. Dickerman, E.S. Sowa, J.H. Monaweck, and A. Barsell, In-pile Experiments on Meltdown of EBR-II Mark-I Fuel Elements in Stagnant Sodium, Nuc. Sci. ard Eng. (to be published).

13. J. H. Monaweck, C. E. Dickerman, and E. S. Sowa, Behavior of Irradiated Metallic Fuel Elements Exposed to Nuclear Excursions in TREAT, Trans. Amer. Nuc. Soc. (to be published).

14. D. G. Freas, A. F. Leatherman, and J. E. Gates, Meltdown Studies of Irradiated Uranium-10 w/o Molybdenum Fuel Pins, BMI-PRDC-656 (1960).

15. J. H. Buddery and K. T. Scott, A Study of the Melting of Ir radiated Uranium, J. Nuc. Materials, 5,81 (1962).

16. L. J. Koch et al., Hazard Summary Report Experimental Breeder Reactor II, ANL-5719(1957)。

17. D. F. Schoeberle, L. B. Miller, and J. Heestand, A Method of Calculating Transient Temperatures in a Multiregion, Axisymmetric, Cylindrical Configuration. The ARGUS Program 1089/RP 248, Written in Fortran II, ANL-6654 (to be published).

18. RP 129 is the IBM 704 version of the space-independent kinetics code RE 29 which was written for AVIDAC, and is described by R. O. Brittan, Some Problems on the Safety of Fast Reactors, ANL-5577 (1956). 


\section{Discussion of Paper \\ (Presented by Mr. Dickerman)}

MR. ALLIO (Westinghouse):

I did not think your pictures of fuel pellets showed great evidence of deformation. Were the se typical of pellets in which you claimed that slumping had taken place?

MR. DICKERMAN:

These were typical of this particular compound, uranium sulfide. I was a little more extreme in this case. The ones that obtained temperatures close to $1600^{\circ} \mathrm{C}$ showed their deformation mainly by having a somewhat larger diameter, more like a barrel. These actually went past the melting point. When temperatures were a little bit lower, sagging occurred.

MR. de STORDEUR (EURATOM):

There is no doubt there is some inherent limitation in the TREAT reactor as such. I do not think you have actually reached the melting temperature of oxide specimens in TREAT.

MR. DICKERMAN:

We have. However, I was trying to restrict myself today to experiments which we had not previously reported, although our oxide results were reported at the last ANS meeting. In those cases, we did have some failures of tantalum-clad uranium oxide elements in which there was extensive melting of the uranium oxide, and there was no problem of limitation of the reactor as such. These experiments were transients which were run at about one-fourth of the capacity with elements which had 10 per cent enrichment, chosen because we wanted to try to minimize flux-depression effects.

MR. de STORDEUR:

The second question, coming to the inherent limitation of the TREAT reactor with regard to melting of relatively large specimens of several pins, where you wouldn't like to have relatively large depression of the flux within the specimens, and my question is, are you thinking of an improved version of the TREAT reactor for the future?

MR. DICKERMAN:

Well, I personally am thinking more at the moment on grading enrichments for clusters. You have a rather important point; with this 
particular machine one sticks to low enrichments, and in clusters one changes the enrichment from the outside pin to the inside pin, as we actually have done. We have performed about 12 dry cluster experiments in an attempt to study cluster interactions. It is not the nicest way in the world to do it.

MR. OKRENT (Argonne):

I wonder if Mr. Dickerman, or anyone else in the audience, could speculate on whether meltdown in a ceramic-fueled reactor would lead to movement of the fuel in the same way that people now visualize for metal? Should we look for something different when we get into the ceramics, or does one still mentally form the same kind of picture?

MR. COHEN (General Electric):

We have done some speculation in a somewhat different direction. We assumed that such an occurrence would be as bad as has been said about metals. Assume that there is a collapse at the top of the core, and that melting occurs all of the way down. Our calculations, as will be shown later today in a presented paper, indicate that nothing really serious occurs.

MR. HÄFELE ( Karls ruhe):

We have been speculating about this in connection with reactor designs involving suspended fuel elements. The sort of anticipated meltdown accident where the inner part of the core is compressed does not then apply any more because with down-going coolant, the lower part of the core is taken away. In addition, due to the manner of fission gas buildup and release, there is a tendency not to have very dense molten material.

MR. CAMPISE (Atomics International):

You should specify what ceramics you are talking about. The case of oxide leads to a completely different melting situation than in the case of a carbide, which is of superior thermal conductivity. I would expect the carbide to act more like a metal. Would you care to comment on this?

MR. DICKERMAN:

Carbide will act more like a metal, depending on what you do with it.

We have evidence that a large per cent of the fission gases, if not nearly all, as based on steady-state irradiation data, tends to stay in carbide. There is also a rather large set of data from radiation experiments 
with mixed plutonium-uranium oxide by GE which indicated that about half of the fission gases tended to stay in the oxide, even though some were run under rather hard operating conditions. Therefore, one can speculate that spongy masses might happen in a meltdown in which appreciable amounts of fission gas remain in the ceramic

Another point is that the ceramic materials do tend to melt and flow down. There have been experiments at Oak Ridge, on clusters of oxide, out of pile, in which the material can be seen in its final form.

Carbide is a rather complicated material. There are questions about vapor pressure, decomposition upon melting, and perhaps the biggest point about carbide is that you don't know how to consuder it after you have a meltdown. Do you have a uniq de equation of state or do you try some weighting procedure for uranium and for carbon?

The possibility exists, particularly in the case of a sodium-bonded carbide, that we have an element which physically behaves quite a bit like some of the old sodium-bonded metallic pins, although there would be differences because of the high melting point of the carbide

Under some rather 1 dealized conditions, that is with no liquid metal coolant on the outside, just an inert gas, we were able to get failures of our oxide elements, which looked quite a bit like some of the failures that we had obtained with EBR-II metallic pins In other words, there was extensive failure inside the cladding, the cladding falled at rather high temperature, and even the gas pressure build-up of what had originally beer about one cc of argon at atmospheric pressure when the element was put together, was enough to push the uranium oxide outside of the cladding So hypothetically you could have an expulsion-type fallure of ceramic fuel, just as you could with a sodium-bonded EBR-II pin 


\section{BRISEIS:}

TRANSIENT REACTOR FOR FAST REACTOR FUEL TESTING

C1. Descamps, E. Fossoul, N. Meyvaert, M. Stiévenart, G. Tavernier, and J. Van Dievoet

BelgoNucleaire

Brussels, Belgium

(Paper presented by Mr. Tavernier)

\section{Introduction}

During the last few years, a wide experimental program has been going on in various laboratories on testing of fast reactor fuels.

Besides the static tests carried out in high flux material testing reactors and in existing fast reactors like the DFR, a considerable number of experiments have been made in TREAT.

They have been described in various publications and have substantially contributed to the understanding of the mechanisms of fuelelement behaviour and failure during abnormal operation.

TREAT has been designed with a graphite-to-uranium-235 ratio. $\mathrm{C} / \mathrm{U}$, of $10^{4}$, and therefore the neutron flux energy spectrum is mostly thermal.

As known from the beginning by the designers. some limitation to the test could occur because of the thermal neutron flux depression in the samples.

This is the reason why the irradiations have mainly been carried out in the past on single pins of natural or slightly enriched uranium.

The reactor project BRISEIS has been studied with the specific purpose, in mind, to be able to test fast reactor pin clusters or assemblies.

Furthermore, it was postulated from the beginning that the proper plutonium enrichment should be kept in the fuel in order to have valid test results; this enrichment can be anything from $12 \%$ upwards.

This paper gives some of the preliminary results obtained during the first year of study; a larger effort will be devoted to this design in the near future. 
Some word should also be said about another study going on in the ISPRA center of Euratom, where a transient reactor called CE TI is being calculated under somewhat a different line of approach. They consider a graphite loaded with $U^{235}$ with a ratio $\mathrm{C} / \mathrm{U}=2 \times 10^{\frac{4}{2}}$; the impermeable graphite is unclad and a temperature surge of $1700^{\circ} \mathrm{C}$ is assumed. The spectrum is, of course, very well thermalised, but preliminary calculations show that acceptable performance can be hoped within a central test loop surrounded by a borated carbon filter.

\section{Design Philosophy and Basis}

As a starting point, a typical reference loop geometry was defined; this loop is assumed to be located on the axis of the cylindrical transient reactor.

Different types of drive zones were postulated in order to reach a suitable power transient in the test assembly and to be within safe limits in the dxive zone itself.

The fuel located in the loop has to be at the same metallurgical enrichment as it would be in the power reactor; by metallurgical enrichment, we mean the $\mathrm{Pu} /(\mathrm{U}+\mathrm{Pu})$ ratio. However, if the energy input to the fuel were not high enough, some doping with $U^{235}$ can be considered.

As a typical fuel assembly the following geometry and composition were chosen:

Active length:

Fuel pin OD:

Cladding thickness:

Cladding material:

Fuel:

Enrichment:

Volume ratios:

fuel

steel

sodium
$78 \mathrm{~cm}$

$7.15 \mathrm{~mm}$

$0.58 \mathrm{~mm}$

Stainless steel

$\mathrm{PuO}_{2}-\mathrm{UO}_{2}$

$12 \%$

$47 \%$

$30 \%$

$23 \%$

Number of pins in cluster: 19

A typical assembly of 19 pins contains, therefore, $0.52 \mathrm{~kg}$ of plutonium and $3.83 \mathrm{~kg}$ of uranium.

by:

In the calculation results, given below, this assembly is designated

$A_{0}$ when the uranium is $90 \%$ enriched in $\mathrm{U}^{235}$;

$A_{\mathbb{N}}$ when the uranium is natural. 
In the first case, the doping with $U^{235}$ is made to enhance the energy input to the tested fuel.

In some calculations, the assembly was assumed to be made of 37 pins or more, rather than 19, in order to see the influence of this parameter on the expected performance of the reactor. It was believed indeed that some test should be preferably conducted on larger assemblies.

A double-walled loop is assumed, each wall being $5 \mathrm{~mm}$ thick and made of stainless steel. The outer tube has $2 \mathrm{w} / \mathrm{o}$ boron to act as a shield for thermal neutrons. In some cases, the boron was taken as $\mathrm{B}^{10}$ to $\mathrm{im}$ prove the efficiency of this filter.

The drive zone is made of fuel elements of graphite homogeneously loaded with uranium oxide, $90 \%$ in $U^{235}$. The se fuel elements are enclosed in stainless steel boxes and are separated by a thin gap through which air cooling is possible. The volume fractions of the drive zone are:

$\begin{array}{lr}\text { Graphite (density } 1.7 \mathrm{~g} / \mathrm{cm}^{3} \text { ): } & 96 \% \\ \text { Stainless Steel: } & 1 \% \\ \text { Gap: } & 3 \%\end{array}$

The fuel content in the drive zone was one of the variables considered in obtaining acceptable performances.

The fuel concentration is expressed as the atomic $x$ atio $\mathrm{C} / \mathrm{U}$ of carbon to $\mathrm{U}^{235}$. It is known that in TREAT C/U is $10^{4}$ and that the neutron spectrum is well thermalised.

In the BRISEIS design, we reduced this ratio considerably, especially close to the test 100 , in order to harden the spectrum and decrease somewhat the power depression close to and within the tested assembly. $\mathrm{C} / \mathrm{U}$ ratios down to 500 were considered, although a weighted average ratio of 1500 seems to be adequate.

Axial and radial graphite reflector blocks were included for the obvious reasons of decreasing the critical mass and of flattening the power distribution in the drive zone.

The height of the core is taken as $80 \mathrm{~cm}$ in this preliminary set of calculations, and the criticality is achieved by varying the radial loading.

Calculations were carried out in cylindrical geometry by a onedimensional diffusion theory code with the Los Alamos 16-group set of constants and with a suitably averaged thermal group. 
Each radial region of the reactor was treated as homogeneous.

The operation of BRISEIS is basically the same as TREAT: a sudden reactivity insertion brings the reactor over prompt criticality; a transient is developed and self-terminated by the internal negative temperature coefficient.

The energy developed in the drive zone is limited to a maximum value corresponding to a temperature increase in the graphite of approximately 500 to $600^{\circ} \mathrm{C}$.

The air cooling acts only to extract the heat slowly after a power transient.

\section{Survey of the Results of Spatial Power Distribution}

A set of calculations was carried out a year ago bearing on a wide variety of possible core compositions. This allowed the spatial distribution, life time, and temperature coefficients to be estimated and a range of preferred composition chosen.

It appeared that a $\mathrm{C} / \mathrm{U}$ ratio of approximately 1500 would allow the use of stainless steel ( $r$ ather than Zircaloy) as cladding of the drive zone elements and therefore would permit a somewhat larger temperature rise and a corresponding larger specific energy pulse.

The latest set of calculated cases are summarized in Table $I$. The entries of this table are:

In column 1, Ao stands for a $U^{235}$-doped test as sembly and $A_{N}$ for a normal assembly. The number of pins in the assembly is given. For the last cases presented, the boron in the outer loop tube is $\mathrm{B}^{10}$ to filter out the low energy neutrons.

In column 2, the core $\mathrm{C} / \mathrm{U}$ is given; in the fourth case, the drive zone has been split in three with the 600 ratio in the inner ( 0 to $11.28 \mathrm{~cm}), 1500$ ratio in the middle $(11.28$ to $26.32 \mathrm{~cm})$ and 2000 ratio in the outer $(26.32$ to $37.6 \mathrm{~cm})$ regions.

In column 5 is given the ratio of the average power density within the test zone to the average power density in the drive zone.

In column 6 , is given the ratio of the maximum power to the average power within the test zone. It gives an indication of the power distribution and depression in that region. 
Table I

\begin{tabular}{|c|c|c|c|c|c|}
\hline $\begin{array}{c}1 . \\
\text { Test-zone Loading } \\
\text { and Filter }\end{array}$ & $\begin{array}{c}2 . \\
\text { Core } \mathrm{C} / \mathrm{U} \\
\text { Ratio }\end{array}$ & $\begin{array}{l}3 . \\
\text { Critical Drive- } \\
\text { zone Thickness } \\
(\mathrm{cm})\end{array}$ & $\begin{array}{c}\text { C. } \\
\text { Critical Mass } \\
\left(\mathrm{kg} \mathrm{U} \mathrm{U}^{235}\right)\end{array}$ & $\frac{5}{\overline{\mathrm{P}}_{\mathrm{A}}}$ & $\left.\left(\frac{6}{S_{\operatorname{Max}}}\right)_{\mathrm{Av}}\right)_{\mathrm{A}}$ \\
\hline$A_{0}-19$ pins & 3000 & 53.0 & 8.275 & 18.85 & 1.46 \\
\hline$A_{0}-19$ pins & 1500 & 37.6 & 8.704 & 14.50 & 1.41 \\
\hline$A_{0}-19$ pins & r $\begin{array}{l}500 \\
600\end{array}$ & 28.0 & 15.20 & 9.83 & 1.29 \\
\hline$A_{0}-19$ pins & $\left\{\begin{array}{l}1500 \\
2000\end{array}\right.$ & 37.6 & 9.242 & 15.00 & 1.34 \\
\hline$A_{N}-19$ pins & 1500 & 40.0 & 9.76 & 3.81 & 1.25 \\
\hline$A_{0}=19$ pins $-B^{10}$ & 1500 & 40.6 & 10.04 & 9.7 & 1.19 \\
\hline$A_{0}-37$ pins $-B^{10}$ & 1500 & 40.6 & 10.39 & 8.7 & 1.21 \\
\hline
\end{tabular}

In our calculations and presentation of the results, we have preferred to include from the beginning a set of typical test zones and work on the basis of the source distributions (power) rather than to obtain the unperturbed neutron fluxes in a test volume and then estimate their action on a test sample. Doing so is more realistic.

If we assume that during a power transient the energy is accumulated adiabatically and that the average graphite temperature increase can be taken as $600^{\circ} \mathrm{C}$, then the equivalent energy is $310 \mathrm{cal} / \mathrm{cm}^{3}$ of drive zone.

The average energy input per $\mathrm{cm}^{3}$ in the tested fuel is then

$$
\frac{310}{0.47} \frac{\overline{\mathrm{P}}_{\mathrm{A}}}{\overline{\mathrm{P}}_{\mathrm{E}}}=660 \frac{\overline{\mathrm{P}}_{\mathrm{A}}}{\overline{\mathrm{P}}_{\mathrm{E}}} \mathrm{cal},
$$

the factor 0.47 arising from the volume fraction of the fuel in the test zone.

It is known that 1860 cal are necessary to bring the $\mathrm{UO}_{2}$ to its melting point (from $20^{\circ} \mathrm{C}$ ) per $\mathrm{cm}^{3}$ at density $10 \mathrm{~g} / \mathrm{cm}^{3}$ and that the latent heat of fusion is $590 \mathrm{cal}\left(\right.$ per $\left.\mathrm{cm}^{3}\right)$.

Complete melting is therefore obtained with 2450 cal which, according to the above relationship. requires a power ratio

$$
\frac{\overline{\mathrm{P}}_{\mathrm{A}}}{\overline{\mathrm{P}}_{\mathrm{E}}}=\frac{2 \pm 50}{660}=3.7 \text {. }
$$

From the results in column 5 of Table 1 , it would the refore appear that complete melting could be obtained even with a normal type of loaded 
test assembly. Furthermore, the slightest doping with $\mathrm{U}^{235}$ would allow an energy input in the fuel considerably above what is required for the complete melting (see Fig. 1).

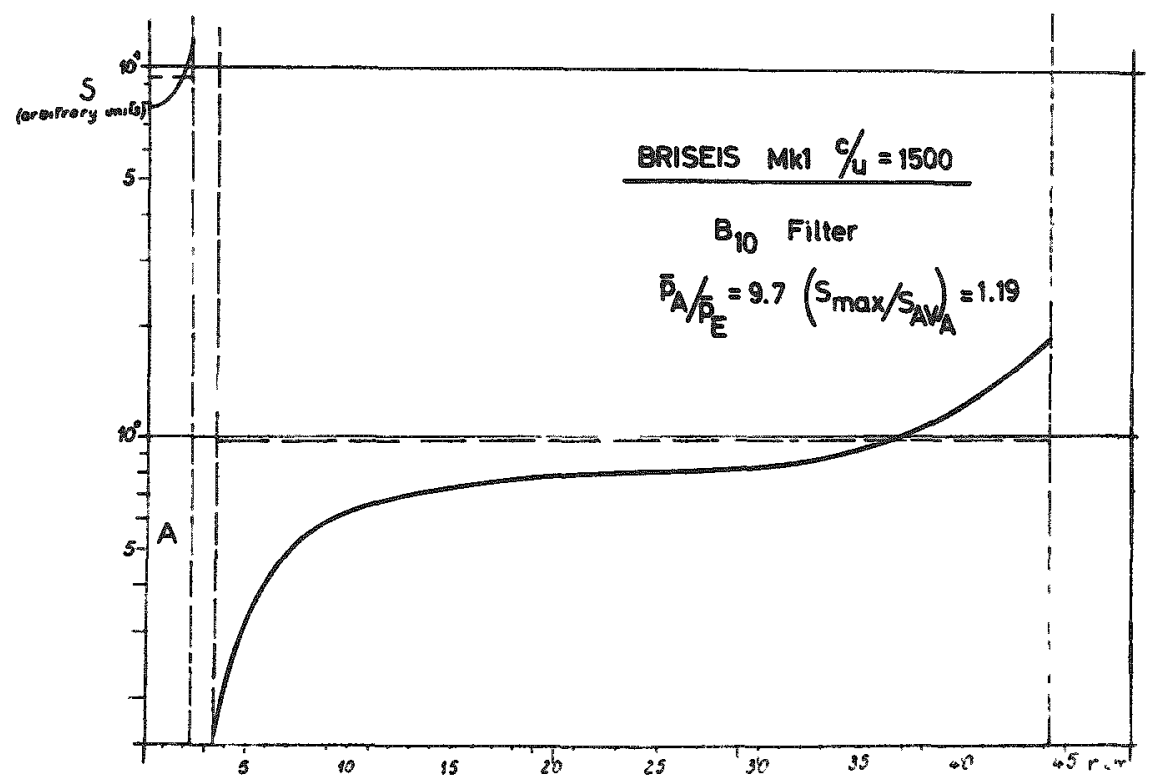

Fig. 1

The power depression (see column 6) is probably acceptable for most experiments; the results indicate that a thicker boron filter would still reduce this depression without decreasing much further the power ratio. This will be studied in more details in later work.

Some other interesting conclusions can also be obtained from Table I and also from the earlier survey work:

1. If it is assumed that a hard epithermal spectrum is necessary in the test zone in order to avoid a too severe power depression, it means that the thermal - and low-energy neutrons must be filtered out from the incident flux.

As was pointed out very clearly by Raievski, any $C / U$ ratio in the drive zone will lead approximately, for the same enthalpy $x$ ise of the graphite in the drive zone, to the same fast and slowing down spectrum and therefore to the same energy input in the tested fuel.

However, the strong low-energy absorption area of the central loop leads to a large thermal neutron flux depression around it, and therefore diminishes the fast-neutron source density around the test zone. 
It seems therefore that a lower $\mathrm{C} / \mathrm{U}$ ratio is favourable for the purpose sought, especially around the loop.

One result obtained by progressive inward loading of the drive zone $(C / U=2000 / 1500 / 600)$ is shown in Table I and in Fig. 2.

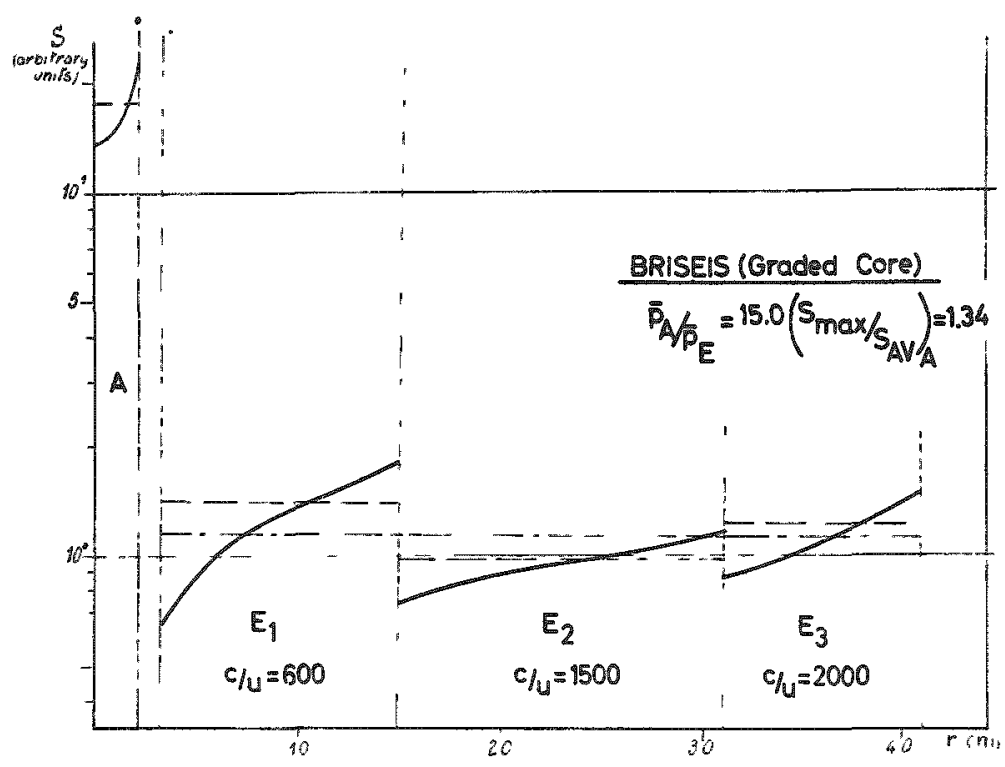

Fig. 2

2. A larger test assembly ( 37 or 61 pins) does not change significantly the results in BRISEIS: power ratio and depression are practically the same as for a 19-pin assembly.

The reactirity worths of the various assemblies or plugs which can be located in the test zone have been studied with the following conclusion for a $1500 \mathrm{C} / \mathrm{U}$ ratio and a natural boron-loaded stainless steel filter:

1. Stainless steel plug in test zone: reactor just critical

2. Sodium-filled loop:

$$
\text { uk }=0.06 \%
$$

3. Graphite plug in loop:

$\mathrm{k}=0.08 \%$

4. 19-pin assembly, type AN:

$k=0.12^{m}$

5. 19-pin assembly, type $A_{0}$ :

$i \mathrm{k}=2.13 \%$

These reactivity values are small and can be easily reduced by a proper shimming prior to a particular experiment. With a $B^{10}$ filter, they appear to be even smaller, showing that the decoupling between the test zone and the drive zone is adequate. 


\section{Transient Characteristics}

Besides the arguments given in Sect. 4 for choosing a hardspectrum drive zone, we were aware of the interest that could be so obtained from the smaller life time and temperature coefficient of the reactor in its transient behaviour.

The life time estimated for the 1500 nominal $C / U$ ratio was

$$
\ell=100 \mu \mathrm{s} .
$$

The temperature coefficient was calculated by reactivity difference between the hot and cold reactor:

$$
\gamma=-4 \times 10^{-5} /{ }^{\circ} \mathrm{C} \text {. }
$$

In a thermal reactor in which $\mathrm{C} / \mathrm{U}$ would be typically $10^{4}$ or larger with Zircaloy cladding, the life time would be at least 6 times larger and the temperature coefficient approximately 10 times larger.

As a first conclusion, the prompt reactivity step necessary to obtain a given energy pulse in the reactor is much smaller, but still sizeable enough to allow a simple control system. A typical value of the reactivity step is $1 \%$ over prompt critical.

As a second conclusion, the rise time $R$ and the energy pulse width $\tau$ are quite sharp for the largest transients; they have typical values of

$$
\begin{aligned}
& \tau=\frac{8 l}{\gamma \Delta \mathrm{T}}=10 \text { to } 50 \mathrm{~ms} ; \\
& R=\frac{1}{4} \tau .
\end{aligned}
$$

The waiting time between the reactivity step and the power transient is of the order of $10 \tau$.

In Fig. 3 is shown a calculated power transient for a reactivity step of $1.1 \%$ over prompt critical and an initial reactor power of $40 \mathrm{~W}$.

The peak power of the drive zone in this case is $30,000 \mathrm{~mW}$.

Shaped transient experiments are also possible and are, indeed, of the utmost importance, for example, for the experimental simulation of a loss of flow or coolant accident at constant or shaped power of the test fuel elements. 


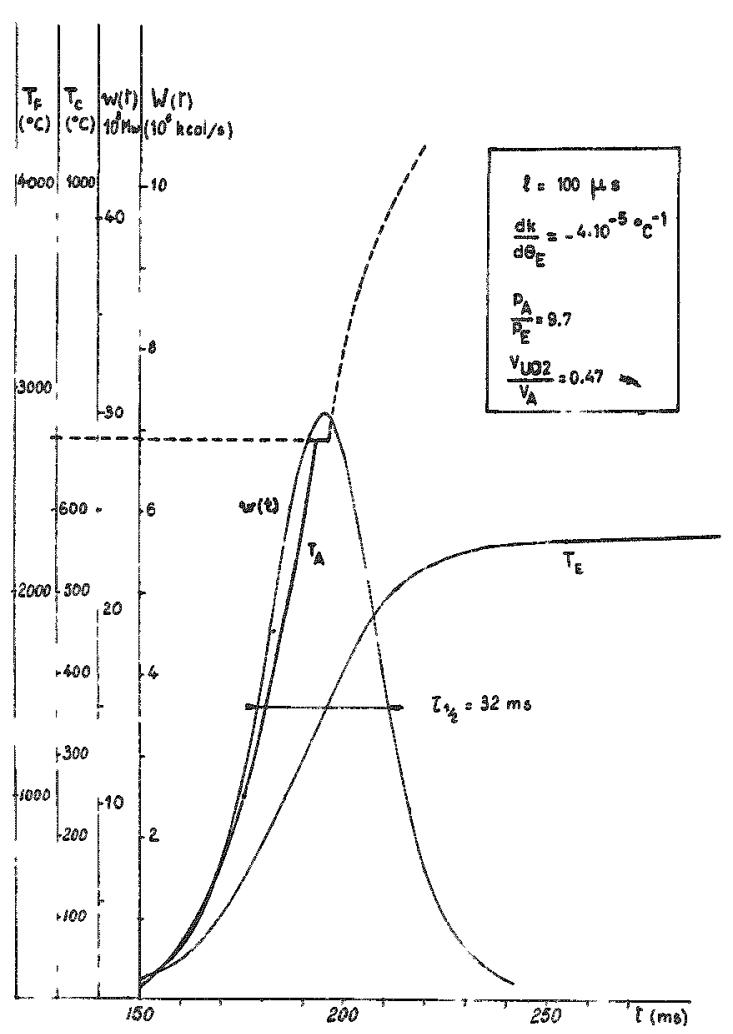

Fig. 3. Power and Temperatures Transient for a 1.1\% prompt critical reactivity step
For a 19-pin assembly operating at a nominal steady power of $1 \mathrm{~kW} / \mathrm{cm}$, BRISEIS can hold the appropriate power level for this during $50 \mathrm{sec}$, which is ample for obtaining an equilibrium temperature within the loop and then provoke a loss of flow or coolant accident.

\section{Conclusions}

In this paper, only the striking neutronic characteristics of BRISEIS have been discussed. It appears from the preliminary calculations that the high thermal capacity of a graphite, homogeneous drive core and the space and time behaviour of the neutrons can be successfully used in the design of a reactor suitable for transient testing of highly absorbing fuel in a loop.

In order to be successful, the design must also take into consideration other facts such as:

ability to store and clean plutonium and fission products contaminated sodium;

ability to load and test to destruction a highly active fuel element already having a certain degree of burn up;

ability, if possible, to follow up the space-time behaviour of the tested fuel during and after the transient through some belino gamma graphic device.

It appears that the demand for experiments in such a reactor would be high during the next decade; we intend therefore to push further the design study of BRISEIS and in particular all the special problems connected with it.

We want to express our gratitude to the many physicists and engineers who have helped us through discussions both in U.S. and in Europe. 
DESCAMPS et al.

Discussion of Paper

Presented by Mr. Tavernier

There were no questions or comments. 


\title{
STUDY OF FAST REACTOR MELTDOWN ACCIDENTS \\ USING SIMULANT MATERIALS
}

\author{
Paul R. Huebotter \\ Atomic Power Development Associates, Inc. \\ Detroit, Michigan
}

\begin{abstract}
The program reported herein was an experimental and analytical effort designed to produce a better understanding of meltdown accidents (and the conditions that could cause them) in a sodium-cooled fast reactor fueled with uranium-alloy pin-type elements. The approach was to perform dynamic mockup tests with lead and water in substitution for uranium alloy and sodium, respectively, and electrical resistance heating to simulate fis sion heating.

Stated broadly, the objectives were to answer the following questions:

1. Under what conditions of power and coolant flow (pressure drop) would a fast reactor be vulnerable to a meltdown accident?

2. Where would the fuel go in the event of a meltdown?

3. How fast would the fuel redistribute itself?

The complete report on this AEC-sponsored effort is given in APDA-155, Study of Fast Reactor Meltdown Accidents Using Simulant Materials, by Paul $R$. Huebottex. (August 31, 1963.)
\end{abstract}

\section{Introduction}

One of the advantages of liquid-metal-cooled reactors is that they can be operated at low pressures. The maximum primary loop pressure is typically less than 100 psia, and the absolute pressure at the core outlet is bayely above one atmosphere. In a coolant starvation accident, however, these conditions can create serious problems. The vaporization of a given weight fraction of coolant would cause, in a typical liquid metal system, an increase in volumetric flow which is one or two orders of magnitude greater than that of the same system operated in the pressure range characteristic of boiling or pressurized water reactors. Since large volumetric flow rates produce large pressure drops, flow would tend to decrease and thus produce still 
more boiling. At the high power densities typical of fast reactors, coolant boiling does not provide adequate heat removal, and fuel melting is a distinct possibility unless power is promptly and drastically reduced.

The foregoing discussion points up the following existing needs in the field of fast reactor safety, which prompted the work described herein:

1. Improved understanding of the power-flow-pressure drop relationships at pressures and in geometries typical of fast reactor cores, with the specific objective of defining, with sufficient accuracy for design purposes, the conditions necessary for the maintenance of stable singlephase coolant flow.

2. Improved understanding of the consequences of boiling and fuel melting.

\section{Similitude Considerations}

When attempting to develop an understanding of an event or process in which phase changes, dynamic forces, metallurgical reactions, etc. interact in a highly complex manner, one would not employ simulant tests unless absolutely necessary. An increase in cost of a factor of ten would probably be justified should such an increase permit the experimenter to use actual materials under conditions of actual interest.

A core meltdown is an event of such complexity that a full understanding may require the construction of an expendable reactor. The obviously high cost of such a facility produces a great incentive for the development of imaginative experiments to explore some aspects of the problem for a much lower cost than that of the "ideal" experiment.

TREAT experiments and certain out-of-pile tests employing electrical resistance heating of irradiated and unirradiated fuels have been extremely useful in revealing mechanisms of meltdown failure. However, the role played by the coolant in causing a meltdown and relocating the fuel following a meltdown is not adequately understood. Early work by $R$. B. Nicholson and J. M. Morelle of APDA showed that useful information on coolant boiling and fuel melting can be obtained with simulation experiments - specifically, ex periments that use water, lead, and resistance heating as simulants for sodium, uranium alloy, and fission heating, respectively.

In the planning stages of this program, it seemed advantageous to identify a specific reactor system as a reference for simulation purposes and, thereby, minimize the danger of selecting parametric values not mutually compatible in actual practice. Because of APDA's familiarity with the Enrico Fermi reactor, this reactor was chosen. However, testing encompassed a sufficiently wide range of the important parameters of power, flow 
rate, and axial blanket geometry that the results should be useful to those whose primary interest is in other fast reactors. Extrapolation to drastically different geometries and materials, however, would not be wise.

The aspects of the Enrico Fermi reactor that influence the similitude criteria are as follows:

Coolant - sodium

Fuel - uranium-10 w/o molybdenum

Nominal coolant inlet temperature $-550^{\circ} \mathrm{F}$

Subassembly outlet pressure - 20 psia

Nominal flow (at $200 \mathrm{MW}$ ) - $155 \mathrm{gpm}$ per fuel subassembly - 0.95 gpm per interior pin

Core $\triangle P$ at nominal flow -31.5 psi

Axial blanket $\triangle P$ at nominal flow (each) - $2.0 \mathrm{psi}$

Core geometry -0.158 in. in diameter, 30.5 -in.-long pins on a $0.199-i n$. square pitch perodically spaced by "honeycomb" grids

Nominal heat generation per pin (avg at $200 \mathrm{MW}$ ) $-11.8 \mathrm{~kW}$

A. Hydrodynamic Similitude

1. Iiquid Coolant Regime

Single-phase hydrodynamic simulation is accomplished by using a test section that is a full-sized model of a four-pin unit cell of the reference core. Nine- and sixteen-pin unit cells were considered, but the benefits did not seem commensurate with the increased cost primarily represented by the power supply. This full-scale modeling establishes the correct relationship between average coolant velocity, volumetric flow per fuel pin, and core residence time. Reynolds Numbers (at a given velocity) will be nearly duplicated because of the similarity between kinematic viscosities of sodium and water over their respective working ranges. Similar Reynolds Numbers should produce similar drag coefficients. Liquid drag forces will be similar due to the density similarity which also tends to duplicate liquid inertial effects, assuming the geometrical factors are duplicated. The inertia. of the circuit was considered when the distance was fized between the upper axial blanket outlet and the free 1iquid surface. Again the Fermi reactor was taken as the standard. 


\section{Two-phase Coolant Regime}

To maintain hydrodynamic similarity as the fluid enters the two-phase regime, it becomes necessary to consider the parameter $\vec{F}$. which is the ratio of specific volume of vapor to specific volume of liquid. To assure comparable expansion effects upon vaporization of unit volumes of water and sodium, $\beta$ in each case should be the same. Figure 1 shows

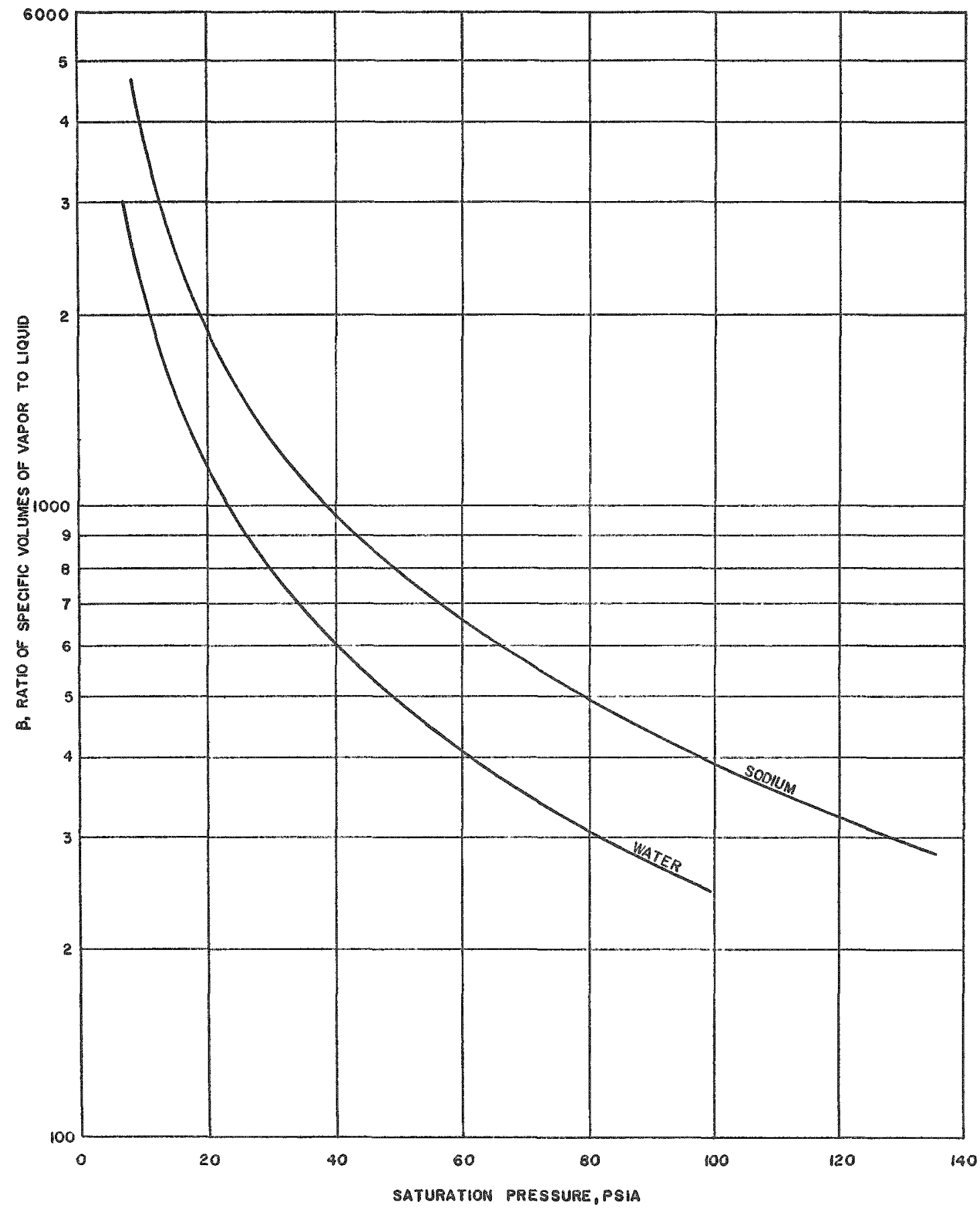

Fig. 1. Ratio of Specific Volumes of Vapor to Liquid as a Function of Saturation Pressure for Water and Sodium 
the value of $\beta$ for both fluids as a function of saturation pressure. At the outlet pressure of the Fermi subassembly (about 20 psia), $\beta$ is about 2000 . The same value of $\beta$ is found on the water curve at 11 psia. Therefore, by designing for an 11 -psia pressure at the test section exit, $\beta$ similitude was satisfied at that point.

To achieve an equal value of $\beta$ at all locations, the test section must have a lower hydraulic resistance than the reference reactor subassembly. If it be assumed that the vapors of sodium and water are ideal gases with equivalent compressibilities over the pressure range of interest, $\beta$ can be duplicated at all elevations by satisfying the following condition:*

$$
\frac{\Delta P_{W}}{\Delta P_{S}}=\frac{P_{O W}}{P_{O S}}=\frac{11}{20}
$$

Since the support grids contribute importantly to the coresection pressure drop, this condition can be satisfied by judicious selection of the number of grids.

\section{B. Thermal Similitude \\ 1. Inception of Coolant Boiling}

At a given volumetric flow rate per pin, the criterion $\mathrm{R}_{2}$ for the inception of coolant boiling can be written thus:

$$
R_{1}=\frac{\rho_{w} C_{p w}\left(t_{B P}-t_{i w}\right)}{\rho_{s} C_{p s}\left(t_{B P}-t_{i s}\right)}
$$

The quantity $R_{1}$ will vary slightly with pressure and will depend upon the value of $t_{i s}$ one chooses to simulate. The parameter tiw is a discretionary parameter, although $32^{\circ} \mathrm{F}$ represents a lower limit. For the condition $t_{i s}=550^{\circ} \mathrm{F}$ (Fermi's inlet temperature), curves of $R_{1}$ vs static pressure were calculated for $t_{i w}=32^{\circ} \mathrm{F}, 60^{\circ} \mathrm{F}$, and $100^{\circ} \mathrm{F}$, and are presented in Fig. 2 . The physical significance of $R_{1}$ can be stated as follows:

$R_{1}$ is the ratio of power generation in the simulant loop to that in a four-pin unit cell of the reference reactor at which the inception of coolant boiling is properly simulated.

\section{Vapor Quality}

Having assured equal expansion effects upon the vaporization of unit volumes of water and sodium, one must also satisfy the ratio of power

* Refer to Appendix I for nomenclature and abbreviations used in this report. 
generations at which equal volume fractions of the two coolants would be vaporized. At a given volumetric flow rate per pin, this ratio, $R_{2}$, can be computed as follows:

$$
R_{2}=\frac{\rho_{w} h_{f_{g_{w}}}}{\rho_{s} h_{f_{g_{s}}}} .
$$

These properties do not vary significantly with pressure, and $R_{2}$ is approximately 0.73 over the entire pressure range of interest (see Fig. 2).

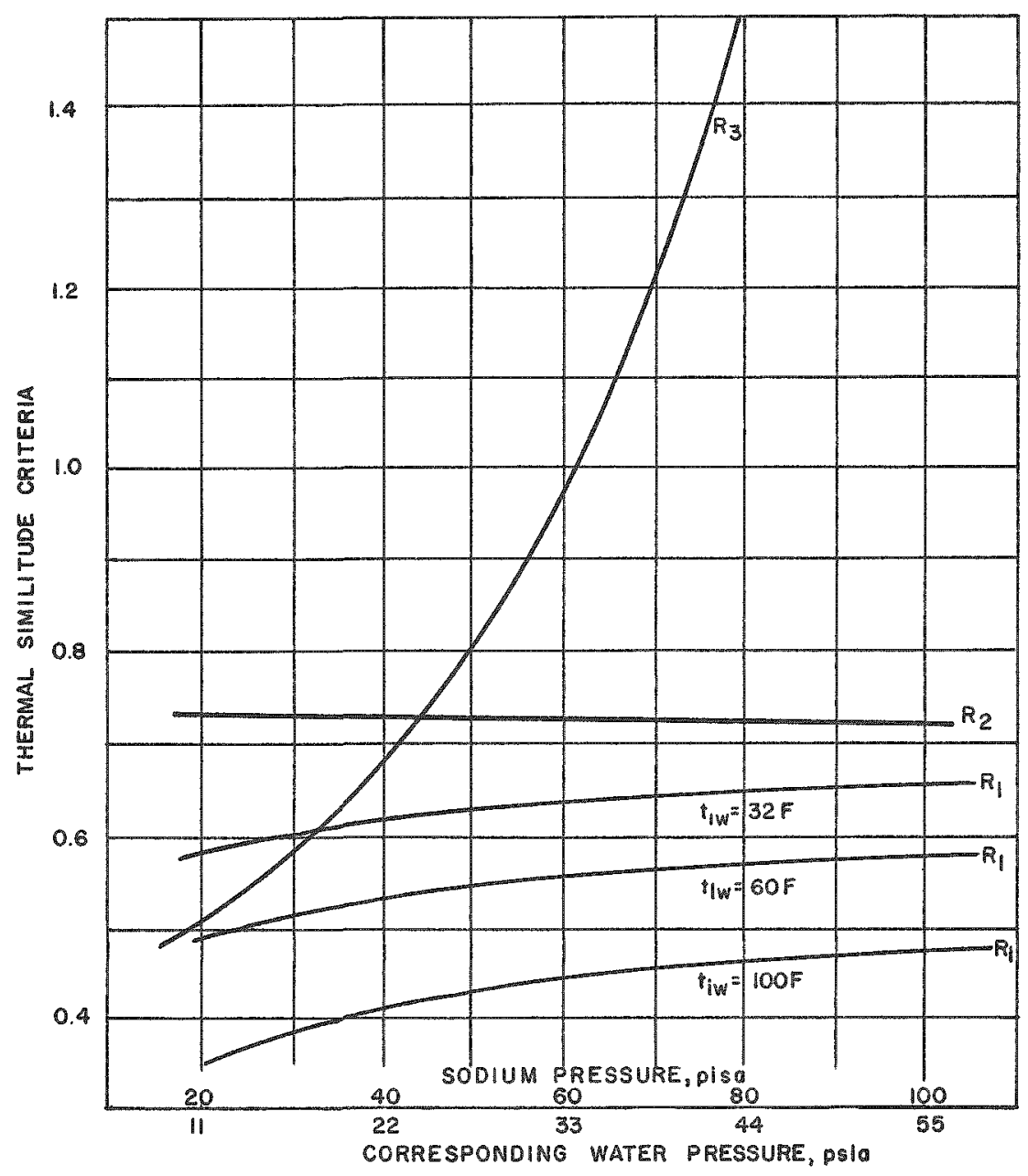

Fig. 2. Similitude Criteria as a Function of Pressure

\section{Fuel Melting}

After coolant boiling begins, a certain time increment will elapse before the fuel begins to melt. This time increment will be duplicated at a particular ratio of power generations, $R_{3}$, which is evaluated as follows: 


$$
R_{3}=\frac{{ }_{\ell} C_{p_{\ell}}\left({ }^{t_{M P}}{ }^{-t_{B P}}\right)}{\rho_{U-M o} C_{P_{U-M o}}\left({ }^{t_{M P}}{ }_{U-M o}{ }^{-t_{B P}}\right)}
$$

This ratio has a rather strong dependence on pressure, as may be seen in Fig. 2.

In this simplified method of calculating $R_{3}$, the assumption is made that the boiling heat transfer coefficients are about the same for water and sodium. On the basis of fragmentary information existing, this is a fair assumption. The characteristics of fast reactors - low pressure, high power density, and relatively small difference between the temperatures at which the coolant boils and the (alloy) fuel melts - are such that coolant boiling would be closely followed by fuel melting. Therefore, even if the boiling heat transfer coefficients were different, the power ratio selected to produce the same time lag between the boiling and melting events would not be greatly affected.

\section{Similitude of Fuel Element Failure}

For maximum simulation, the location of the initial failure and the mode of failure should be duplicated.

In addition to the considerations already discussed, the location of the initial failure depends upon the thermal conductivity of the fuel and the axial power distribution. In a fast reactor, the axial power distribution curve has the appearance of a chopped cosine wave with the maximum occurring at the core midplane. If the fuel had a very poor thermal conductivity, it would first reach its melting point near the midplane, as fuel centerline temperature would be more a function of power than of adjacent coolant temperature.

In metallic fuel elements ( $U-10 \mathrm{w} / \mathrm{O} \mathrm{Mo}$ and its lead simulant) the conductivity is good, and the hot spot moves toward the location of maximum coolant temperature. In the reference reactor, the hot spot normally occurs near the $3 / 4$ elevation. It could move higher or lower in a flow starvation or power excursion accident depending on specific conditions. Unfortunately, the axial power curve in the simulant tests was flat and the hot spot always occurred at the top of the pins. This is a recognized weakness of electrical resistance heating.

No satisfactory method was devised for simulating the cladding, although a copper wire running down the center of each lead pin (to maintain current flow) contributes to its strength and, like the zirconium cladding on the Fermi pin, prevents total collapse of the pin as the melting temperature of the fuel is approached. 


\section{Postfailure Similitude}

The fact that the lead is directly exposed to the flow and enters the coolant stream immediately upon detachment from the pin, probably makes the simulant experiments conservative from the standpoint of degree and rate of fuel relocation in a meltdown accident. The frothiness of uranium alloy expelled from irradiated pins decreases the mobility of the alloy, and since no method was employed to simulate this effect, the simulant tests would be more representative of meltdown behavior at low burnup. This condition, due to better fluidity, is probably the most dangerous in terms of formation of critical masses where they are not wanted.

The ability of molten uranium to dissolve stainless steel components such as the support grids would seem to increase the potential range of fuel migration following a meltdown event. This metallurgical interaction could not be simulated in the present program. Another shortcoming was the fact that resistance heating fails to maintain heat generation in a droplet of fuel following its detachment from the parent pin.

\section{E. Summation}

1. Hydrodynamic similitude is well satisfied by the lead-watex system and the selected parameters of pressure and geometry.

2. The three thermal similitude criteria are fairly consistent among themselves and can therefore be reasonably well satisfied. Referring to Fig. 2, it is clear that for best simulation of the reference reactor the inlet water temperature should be as low as possible and the inlet pressure should be less than 33 psia (which corresponds to 60 psia in the reference reactor). Under these conditions the power density should be about $60 \%$ of the value in the reference core to produce the same thermal effects on coolant and fuel.

3. Simulation is poorest in the following respects:

a. location of initial failure:

b. constraining effect of the cladding;

c. postfailure metallurgical reactions;

d. retention of fuel heat generation following detachment from the pin.

Note: Items $b$ and $c$ would be very difficult to satisfy with confidence. Items a and d could be better simulated through the use of induction heating, which was extensively evaluated at the outset of this program but dropped because of lack of funds. 
4. Although not perfect, the simulation is considered adequate for obtaining semiquantitative data on fast reactor meltdown, and certainly good enough to test whether certain "maximum accident" models are sufficiently conservative.

III. Description of Test Apparatus

\section{A. Loop}

The discharge from the loop (see Fig. 3) flows into an open 55-gal drum, but a float-operated aspirator in the elevated water box maintains a flooded conditon in the test section. The aspirator maintains the pressure at the outlet of the upper axial blanket at one atmosphere less 102 in. of water (11 psia), satisfying the condition for hydrodynamic similitude discussed in Section II.A.2.

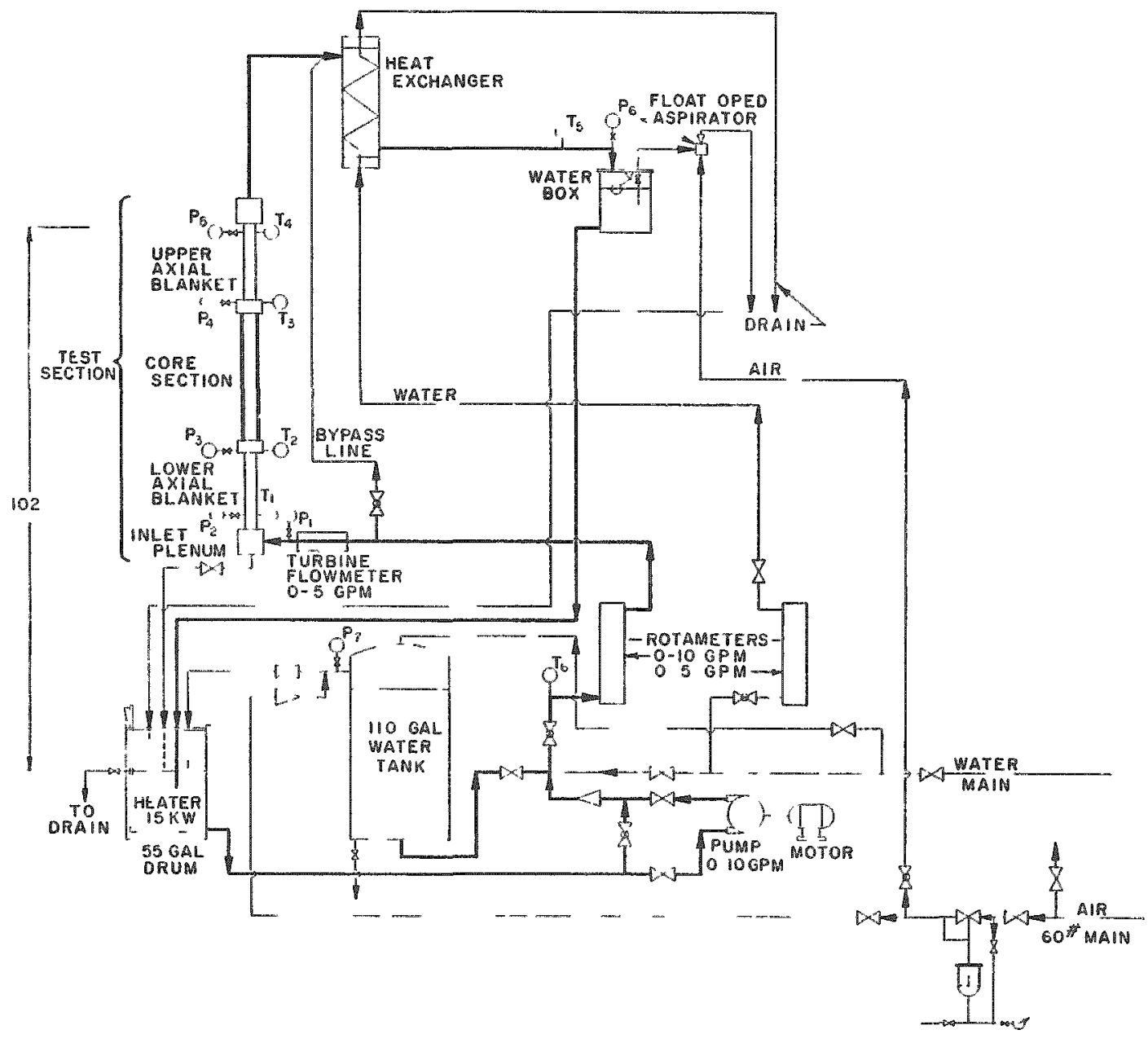

Fig. 3. Schematic Diagram of Loop 
A controllable bypass stream parallels the test section and simulates that portion of the core and/or inner radial blanket not involved in the meltdown. The amount of bypass flow was a variable in some of the test sequences and ranged from 0 to $80 \%$ of total flow.

The loop is equipped with dual feed systems representing the two extremes of feed pump pressure-drop-vs-flow characteristic. The constant-volume feed system utilizes a positive-displacement gear pump driven by a variable-speed, 1-hp motor. Theoretically, this system produces stable flow at all values of power and flow, thus permitting the establishment of test section pressure-drop-vs-flow curves in the twophase regime. The constant-inlet-pressure feed system utilizes a pressurized 110 -gal feed tank and has a characteristic curve similar to that of a centrifugal pump of the type commonly used in fast reactors. This feed system was used to determine the conditions under which flow through the test section would become unstable, and also was used in the meltdown tests.

\section{B. Test Section}

The test section, considered that portion of the loop from the "lower sodium plenum" to the "upper sodium plenum," is shown in Fig. 4. The core section consists of a 30 -in. length of square glass tubing of 1 -cm $\times 1$-cm ID surrounding the four 0.158-in.-diameter stainless steel (or lead) pins. The pins are maintained in a square array on 0.199-in. centers by dimpled metal straps of the type used in the manufacture of the honeycomb support grids used in the Fermi reactor. In this geometry, the flow area associated with each pin is essentially the same as that in the core section of the Fermi reactor. The lead pin bundles contained six support grids, this being the minimum number needed to retain some semblance of rigidity. After it was learned that terminal fixation was sufficient to maintain rigidity of the stainless steel bundle, all spacer grids were removed to minimize pressure drop.

The upper and lower axial blanket sections consist of 15 -in.

lengths of $\frac{3}{4}$-in-OD glass tubes. A range of inside diameters from 0.3093 in. to $0.5805 \mathrm{in}$. Were used in the tests to span a maximum-to-minimum range of blanket hydraulic resistance of about 25. The fact that no heat is generated in the blanket sections is not a serious weakness, since the role played by the axial blankets (particularly the upper blanket) in a reactor meltdown or hydraulic instability is far more a function of hydraulic resistance of the blankets than the relatively insignificant amount of heat genexated therein.

Catchpots simulating the upper and lower reactor plenums are located downstream from the upper blanket and upstrom from the lower blanket, respectively. As illustrated in Fig. 4, these catchpots are split for easy disassembly and contain a wire screen to trap the "fuel" that escapes from the core during a meltdown. 


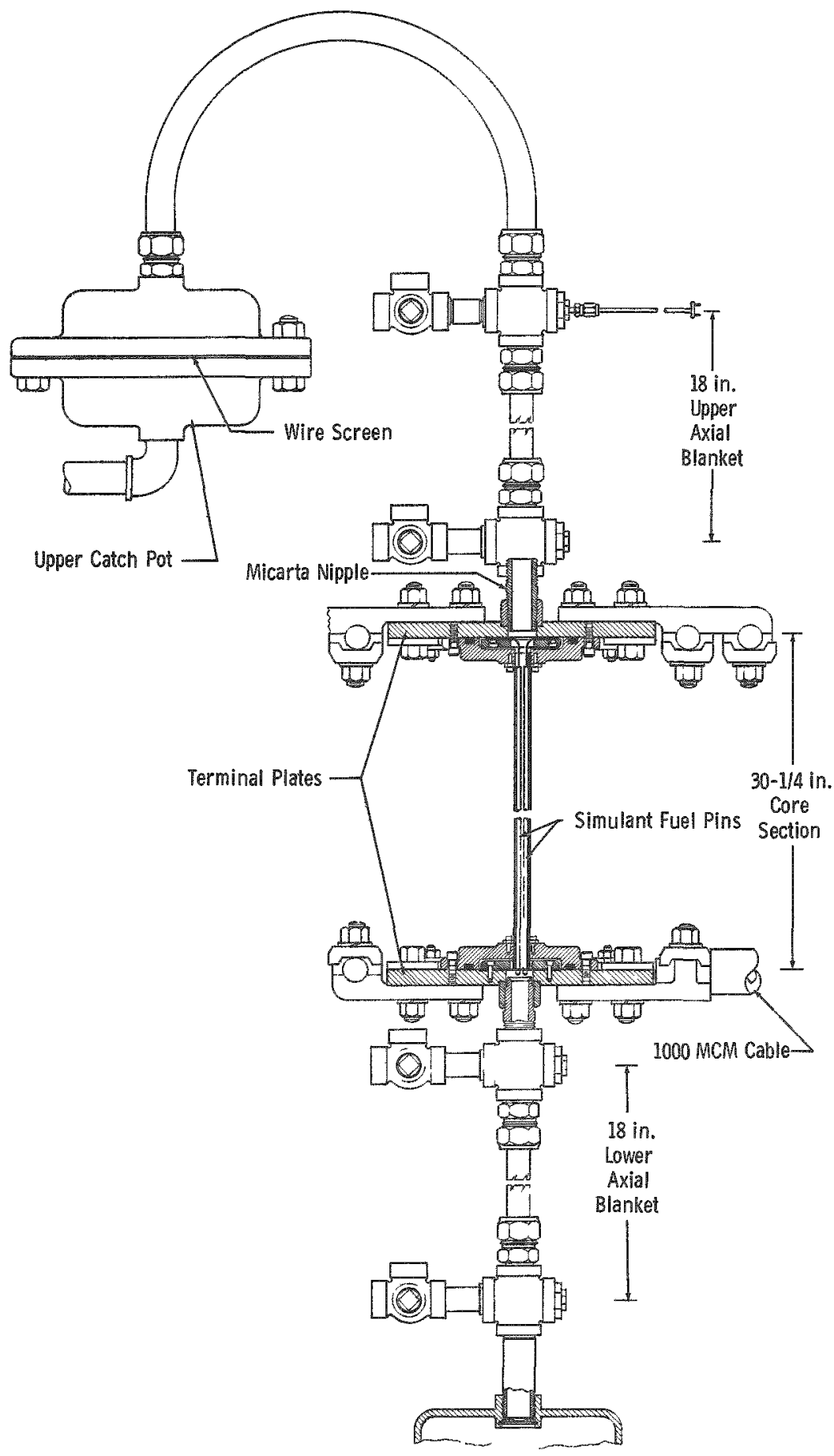

Fig. 4. Test Section Assembly Drawing 


\section{Power Supply}

The principal feature of the power supply is a train of nine specially designed current transformers shown schematically in Fig. 5. The primary side of each transformer is energized at $240 \mathrm{~V}$ through its own circuit breaker in a distribution panel which, in turn, is fed through

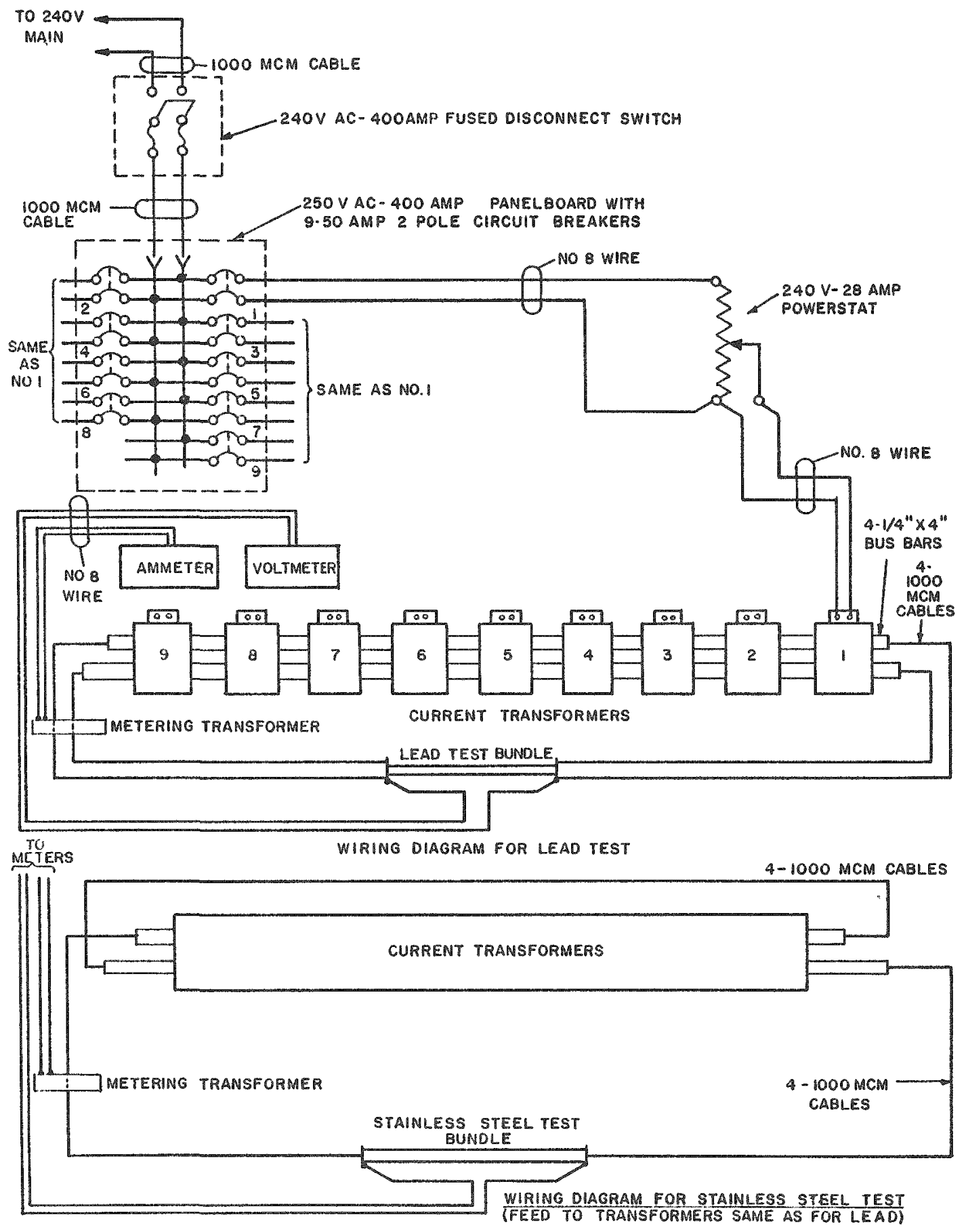

Fig. 5. Schematic Diagram of Power Supply 
a 240-v main disconnect switch of 400-amp capacity. Each current transformer (CT) is connected in a series with a $240-\mathrm{v}, 28$-amp Powerstat. The Powerstat serves two purposes: (1) it provides input voltage control from 0 to $240 \mathrm{v} ;(2)$ it provides a complete path for current flow through the primary winding at all times and eliminates the inductive cancellation of an energized unit by a nonenergized unit.

The test bundle is connected in series with the bus bar that passes through the "window" of each CT and constitutes a common secondary winding. The voltage induced in the secondary loop is a function of the number of CT's energized and the design of the CT (ratio of primary to secondary turns). The power generated in the pin bundle is, of course, a function of the electrical resistance of the bundle and the voltage drop across it.

The power supply was designed for $60 \mathrm{~kW}$ which, with the similitude criteria applied, represents about twice the average power density in the Fermi core. In the actual tests, other restrictions held the maximum power to $27 \mathrm{~kW}$ for the lead pin bundles, although power approached $60 \mathrm{~kW}$ when the stainless steel bundle was in service.

\section{Instrumentation}

The locations at which pressures, temperatures, and flow rates are monitored are shown in Fig. 3. The test-section flow rate, four testsection pressures $\left(P_{2}, P_{3}, P_{4}, P_{5}\right)$, and three test-section temperatures $\left(T_{2}\right.$, $T_{3}, T_{4}$ ) are continuously recorded on an eight-channel Sanborn recorder with a chart speed adjustable up to $100 \mathrm{~mm} / \mathrm{sec}$. Other instruments, including three Bourdon-tube pressure gauges $\left(P_{1}, P_{6}\right.$ and $\left.P_{7}\right)$ and two dial thermometers $\left(T_{5}\right.$ and $\left.T_{6}\right)$, assist in establishing proper test parameters.

Total flow rate (test section plus bypass) is observed with a 1 - to $10-\mathrm{gpm}$ rotometer, and another rotometer monitors the rate of coolant water flow to the tube side of the shell-and-tube heat exchanger located immediately downstream from the test section. Of the signals entering the Sanborn recorder, the temperatures come from iron-constantan sheathed-type thermocouples, the pressures from BLH 0 - to 100 -psia pressure transducers, and the test-section flow from a Cox $0.6-$ to $9-\mathrm{gpm}$ turbine flowmeter.

The power to the test section is computed from the indicated voltage drop across the test section and the signal from a recording ammeter in the secondary loop. Power computed from electrical measurements did not deviate from that calculated thermodynamically by more than $5 \%$ and, typically, by less than $2 \%$. This fact was interpreted as proof of the reliability of the electrical method (the only one feasible under two-phase flow conditions) and as an indication that heat losses to the atmosphere could be neglected. 
IV. Constant-mass-flow Tests

\section{A. General}

In this first series of power tests, stainless steel pins were installed in the test section, and the constant-mass-flow feed system was employed. The attainment of the desired core hydraulic resistance required the removal of all support grids, and the rigidity of the 0.158-in. stainless steel pins, firmly clamped to the terminal blocks at each end of their 30-in. length, permitted this to be done with little sacrifice of geometric ability. In addition to their strength, stainless steel pins offered the advantage over lead pins of being less vulnerable to damage from accidental undercooling.

The purpose of these tests was to determine test-section pressure drop vs mass flow rate as a function of power, inlet temperature, and upper axial blanket hydraulic resistance. In this series of tests, power ranged from 0 to $56.5 \mathrm{~kW}$, inlet temperature from 45 to $160^{\circ} \mathrm{F}$, and the 18-in.-long glass tube simulating the upper axial blanket (UAB) ranged from an ID of 0.3093 to $0.5805 \mathrm{in}$. The lower axial blanket (LAB), also simulated by an 18-in.-long glass tube, had an ID of 0.500 in. throughout the program.

The data obtained in this test series were plotted in the form: core + UAB pressure drop vs flow. In all, the "demand" curves for $50 \mathrm{com}$ binations of power, inlet temperature, and UAB size were determined. Five of them are shown in Fig. 6 .

It is apparent that at the low pressures characteristic of sodiumcooled fast reactors the pressure drop increases sharply as flow rate is decreased to force the ccolant into the two-phase state. The negative slope of that portion of the curve just to the left of the minimum point increases with increasing degrees of subcooling. In fact, at the lowest value of inlet temperature $\left(45^{\circ} \mathrm{F}\right)$, the slope was so great that even the positive displacement pump could not produce stable flow in this region.

As a general rule, flow stability requires the satisfaction of the following inequality:

$$
\left(\frac{\mathrm{dP}}{\mathrm{dW}}\right)_{\text {supply }}<\left(\frac{\mathrm{dP}}{\mathrm{dW}}\right)_{\text {demand }}
$$

B. Correlations

1. Theoretical Background

Before discussing the attempted data correlations, it may be useful to identify the information a reactor designer requires to analyze whether or not a given flow or power transient will produce a meltdown. 


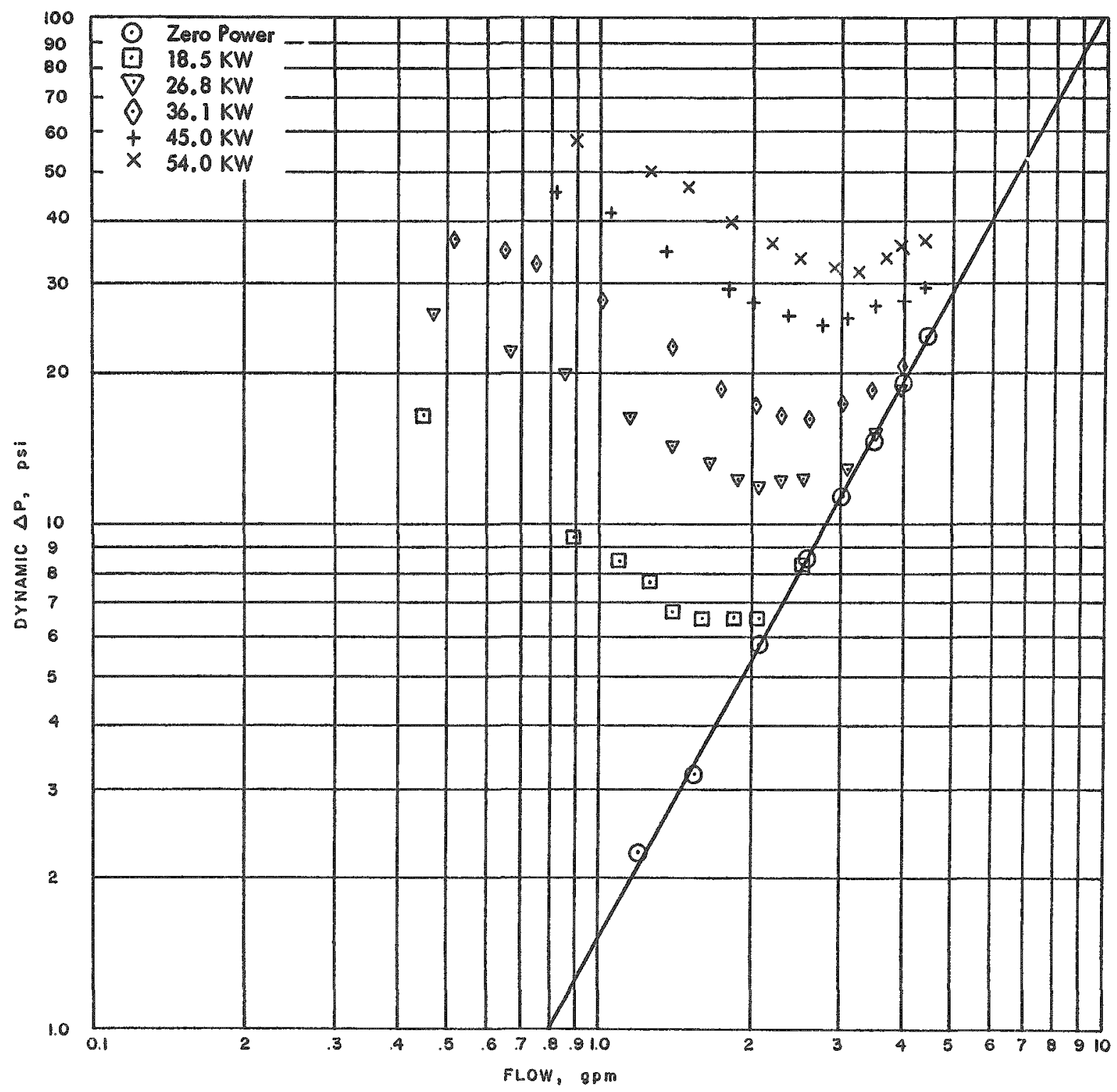

Fig. 6. Core t UAB Pressure Drop vs. Flow. $t_{i}=120^{\circ} \mathrm{F}$ and $\mathrm{UAB}=0.5805 \mathrm{in}$.

Consider Fig. 7, which shows the general relationship between subassembly pressure drop and flow at no power $(Q=0)$ and at power levels of $Q_{1}, Q_{2}, Q_{3}$, and $Q_{4}$. The same inlet coolant temperature applies to all of these "demand" curves. The "supply" curve, $P=C$, represents the constant inlet pressure characteristic of a centrifugal pump operating in a system with little hydraulic resistance upstream from the core. Prior to nuclear startup $(Q=0)$, the pump $(s)$ are turned on, the inlet plenum pressure reaches $P=C$, and flow rate $W_{1}$ is established. When the reactor is brought to the power level represented by $Q_{1}, W_{1}$ is maintained. If the power were increased to $Q_{2}$, some vaporization would occux and the flow would be reduced to $\mathrm{W}_{2}$. Theoretically, however, the flow would remain stable. Should the 
power be increased further though, say to $Q_{3}$ or $Q_{4}$, vaporization would choke the flow severely, and the rate would drop to $W_{3}$. Under conditions generally found in fast reactors, the fuel would soon be molten unless power were quickly and drastically reduced.

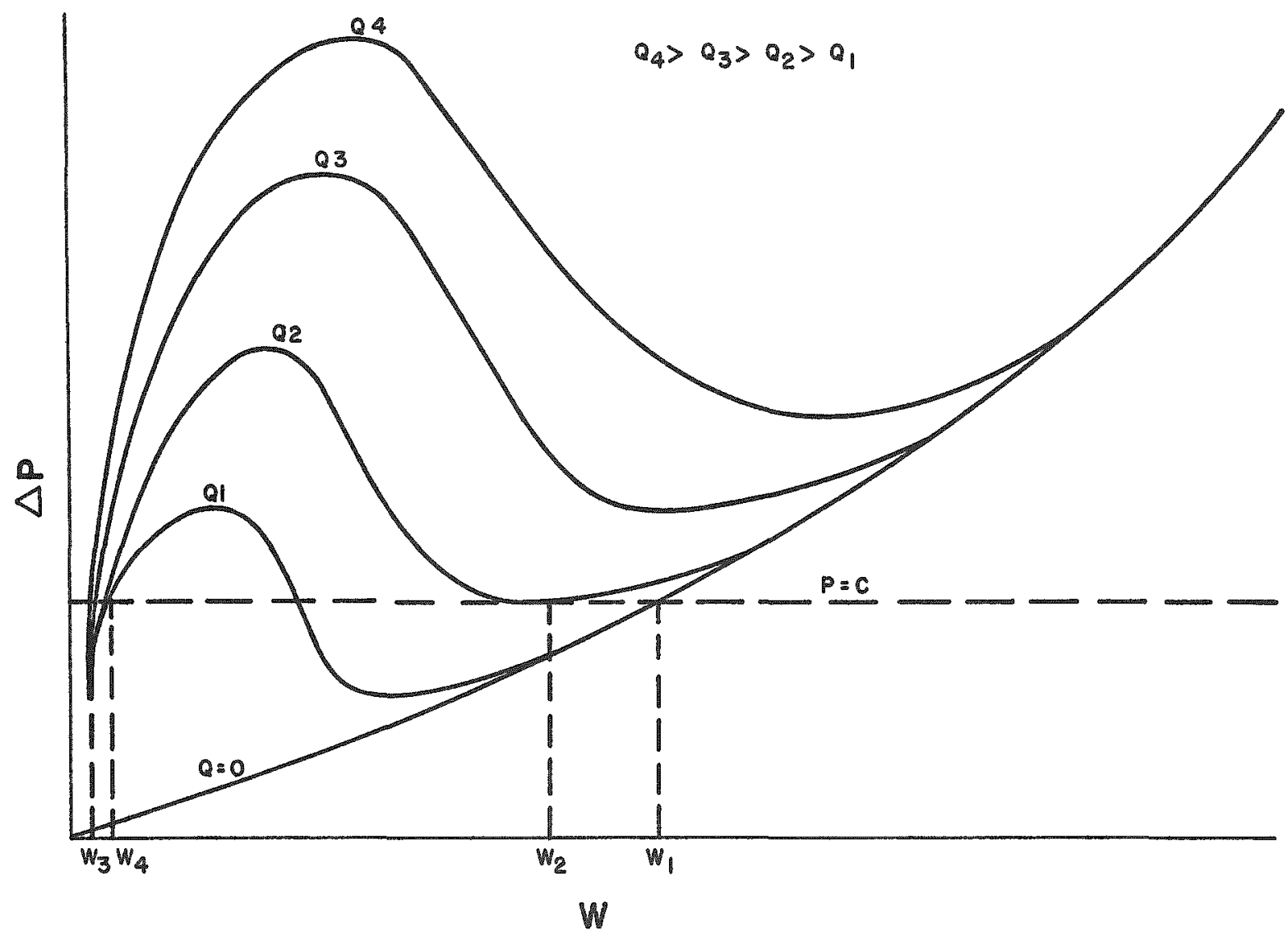

Fig. 7. Power-flow-pressure-drop Relationships in a Typical Unorificed Fuel Subassembly

Just as flow is stable to the right of the demand-curve minimum, so it is to the left of the curve maximum. Therefore, after an excursion in power to a level beyond $Q_{2}$, the coolant flow cannot be restored to $W_{1}$ by reducing power to $Q_{1}$; this reduction merely produces a flow change to $W_{4}$. It is necessary to reduce power to a lesser value whose demand curve peaks at a pressure less than $P=C$. In practice, of course, it would be appropriate to scram the reactor and return to $Q-0$.

The foregoing has been a discussion of a potential meltdown accident initiated by a power excursion. Similar reasoning could be applied to the case of flow reduction caused by complete or partial loss of core-inlet pressure. For whatever the initiating cause, the fuel is in danger of melting whenever the supply curve falls beneath the minimum point on the demand curve. 
With the background theory discussed above, it is clear that the reactor designer would be most interested in the coordinates of the minima of the demand curves, hereafter referred to as the critical flow rate and critical pressure drop.

\section{Critical Flow Rate}

The critical flow rate was often difficult to identify from the experimental data because of the typical flat shape of the demand curves at their minima. (By the same token, however, the abscissa is not of major importance, since the supply curves are quite flat in unorificed systems.) In those cases for which it could be defined with reasonable assurance, the experimental value was compared with the critical flow rate as calculated by heat balance:

$$
W_{c}=\frac{Q}{\bar{h}_{s o p}-\bar{h}_{j}}
$$

The agreement, judging from Figs. 8 and 9 , should be satisfactory for engineering purposes, and the correlation is apparently independent of inlet temperature and UAB size.

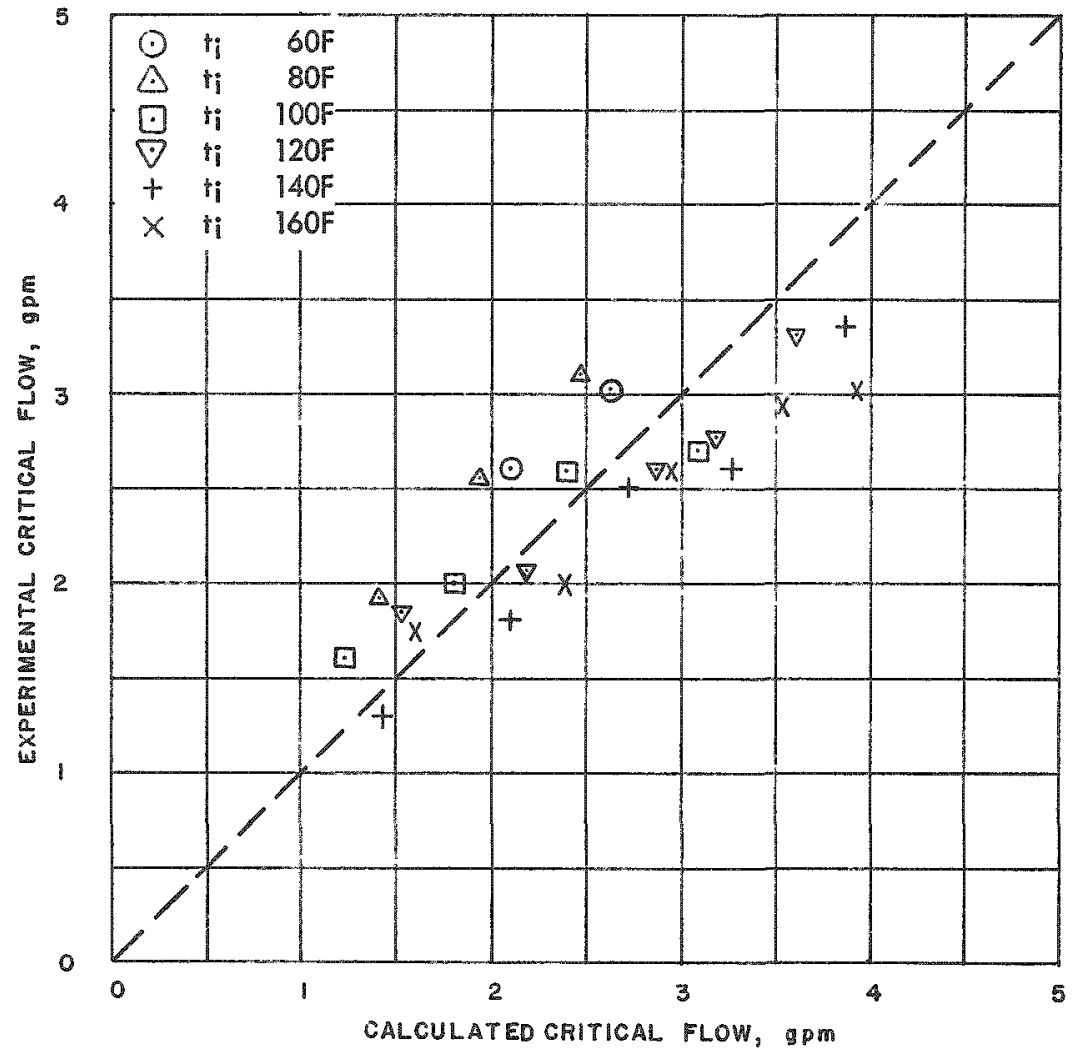

Fig. 8. Calculated vs. Experimental Values of Critical Flow at Various Inlet Temperatures with UAI $=0.5805 \mathrm{in}$. 


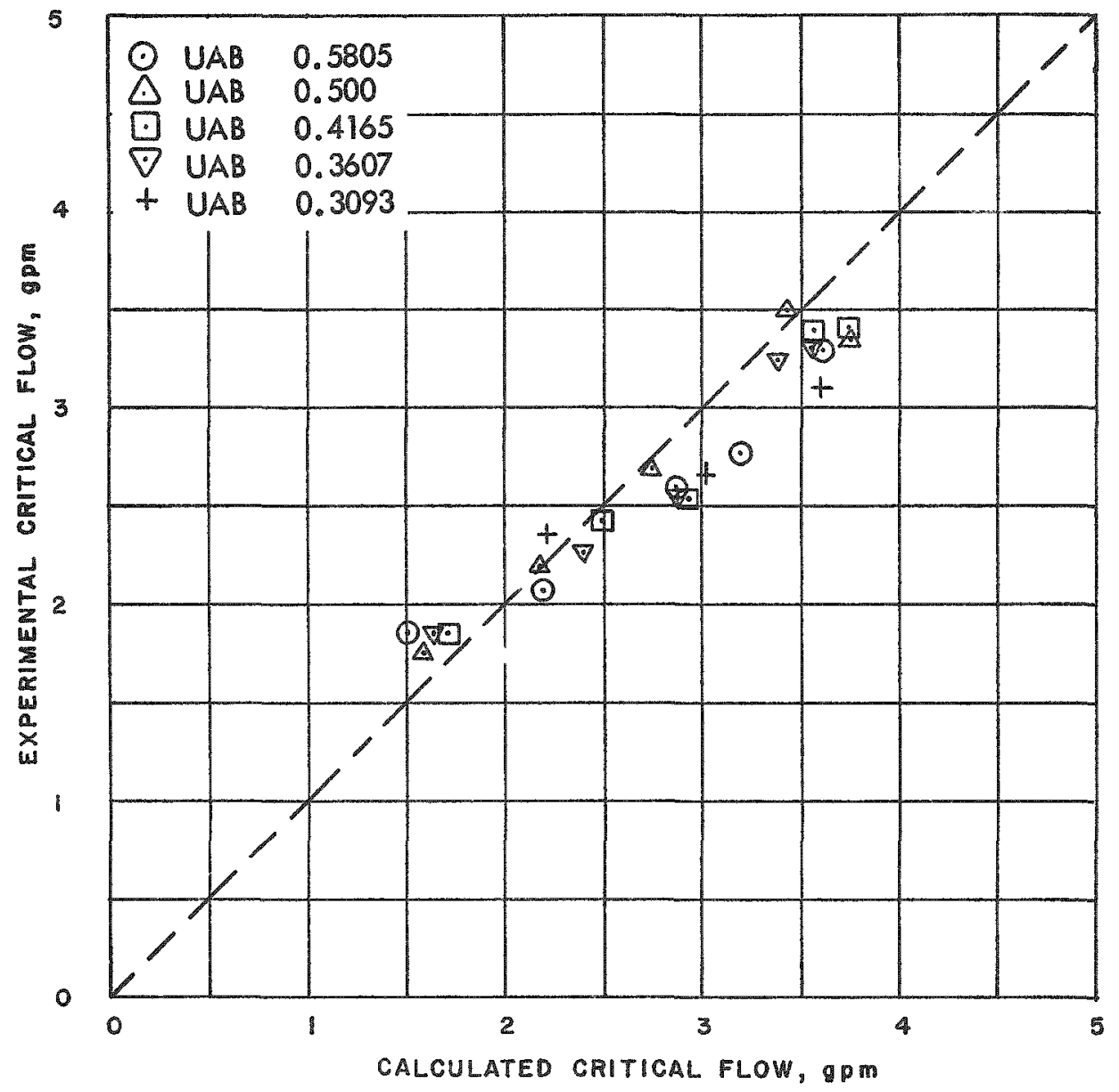

Fig. 9. Calculated vs. Experimental Values of Critical Flow with Various UAB Sizes at $120^{\circ} \mathrm{F}$ Inlet Temperature

\section{Critical Pressure Drop}

The critical pressure drop plays a far more significant role in dictating the initiation of flow instability and, hence, fuel melting, and it was, therefore, the subject of more exhaustive attempts for data correlation.

a. Bulk-boiling Criterion - The first approach was to compare the experimentally determined critical pressure drop (minimum dynamic $\triangle P$ across core and $U A B$ ) with the zexo-powex pressure drop (across the same section) at the calculated value of critical flow. The assumption that would justify this method of correlation is that local boiling does not appreciably affect the demand curve; thus the demand curve, at a given power level, closely follows the single-phase demand curve until bulk boiling begins at the UAB outlet. 
It developed that the experimental value, $\Delta \mathrm{P}_{\mathrm{e}}$, was 30 to $120 \%$ greater than the critical pressure drop as calculated by the bulk-boiling criterion, $\triangle P_{B B}$, and as shown in Figs. 10 and 11 , there is considerable scatter and no apparent trend with inlet temperature or system pressure drop.

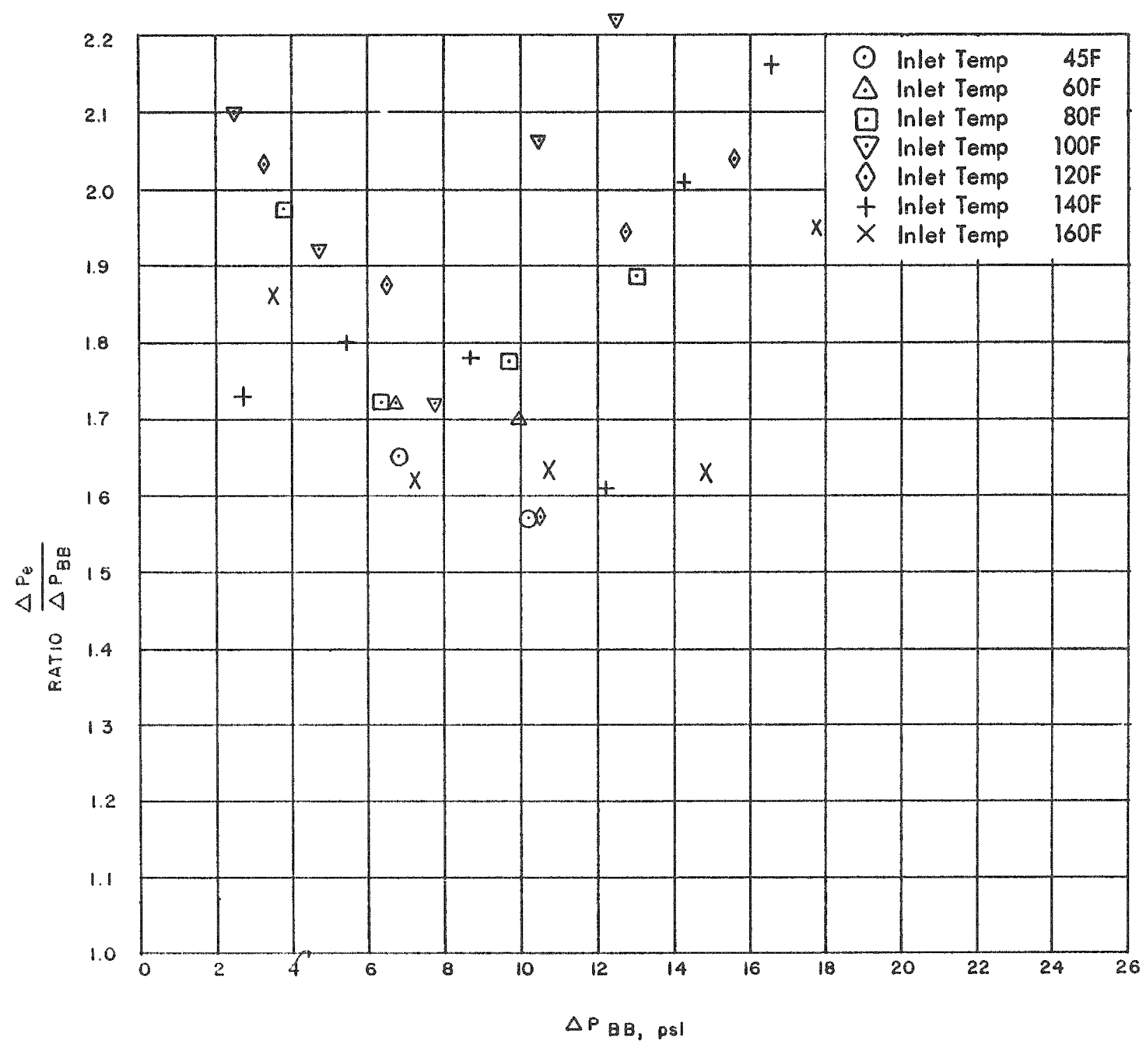

Fig. 10. $\triangle P_{e} / \triangle P_{B B}$ vs. $\triangle P_{B B}$ for Various Inlet Temperatures Using 0.5805 in. UAB 


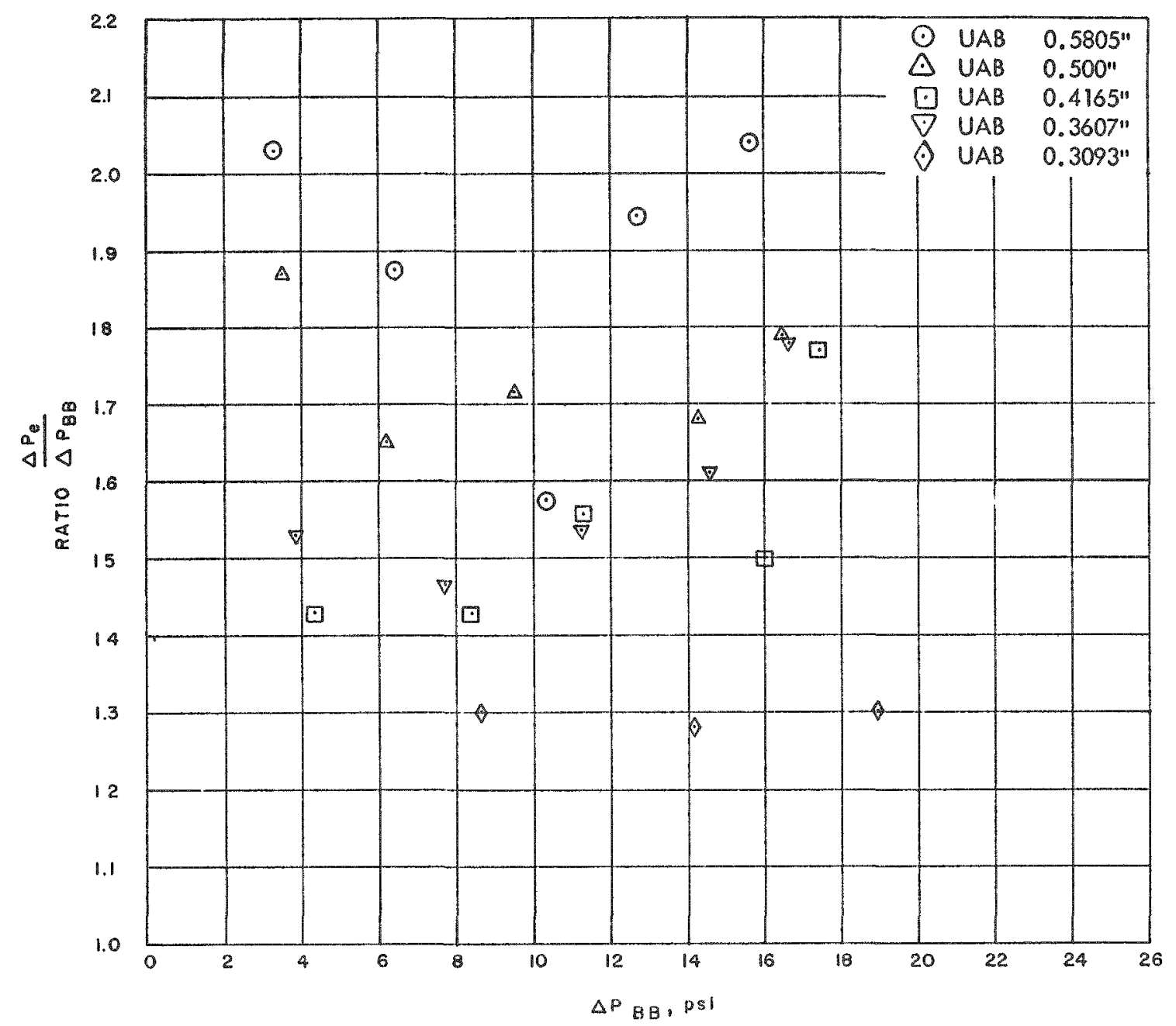

Fig. 11. $\triangle \mathrm{Pe} / \triangle \mathrm{PBB}$ vs. $\triangle \mathrm{PBB}$ for Various $U A B$ Sizes at $120^{\circ} \mathrm{F}$ Inlet Temperature

b. Local Boiling Criterion - A method for conservatively estimating the critical pressure drop is to use the single-phase pressure drop for the lowest value of coolant flow at which the surface temperature of the fuel pin does not exceed the saturation temperature of the adjacent coolant at any point along the pin. The concept is best understood through Fig. 12, which illustrates the simplified case in which heat generation is axially uniform. The method requires the calculation of the unique value of flow rate that produces the intersection between the pin surface temperature curve and the coolant saturation temperature curve at the core outlet. The equation for calculating this flow rate is derived as follows:

When $0.7<N_{P r}<120,10,000 \leqslant N \operatorname{Re}<120,000$, and $\mathrm{L} / \mathrm{D}>60$.

$$
\frac{h D}{k}=0.023\left(\frac{D G}{\mu}\right)^{0.8}\left(\frac{C_{p} \mu}{k}\right)^{0.4} .
$$


Coolant pressure at core outlet $\sim 13$ psia.

Corresponding saturation temperature $\sim 206^{\circ} \mathrm{F}$.

For simplification, as sume average film drop $=40^{\circ} \mathrm{F}$.

Then bulk coolant temperature at core outlet $=166^{\circ} \mathrm{F}$.

$$
\begin{aligned}
\mathrm{k} & =0.394 \mathrm{Btu} / \mathrm{hr}-\mathrm{ft}-{ }^{\circ} \mathrm{F} . \\
\mu & =0.39 \mathrm{cp} \text { or } 0.94 \mathrm{lb} / \mathrm{ft}-\mathrm{hr} . \\
\mathrm{C}_{\mathrm{p}} & =1.0 \mathrm{Btu} / 1 \mathrm{~b}-{ }^{\circ} \mathrm{F} .
\end{aligned}
$$

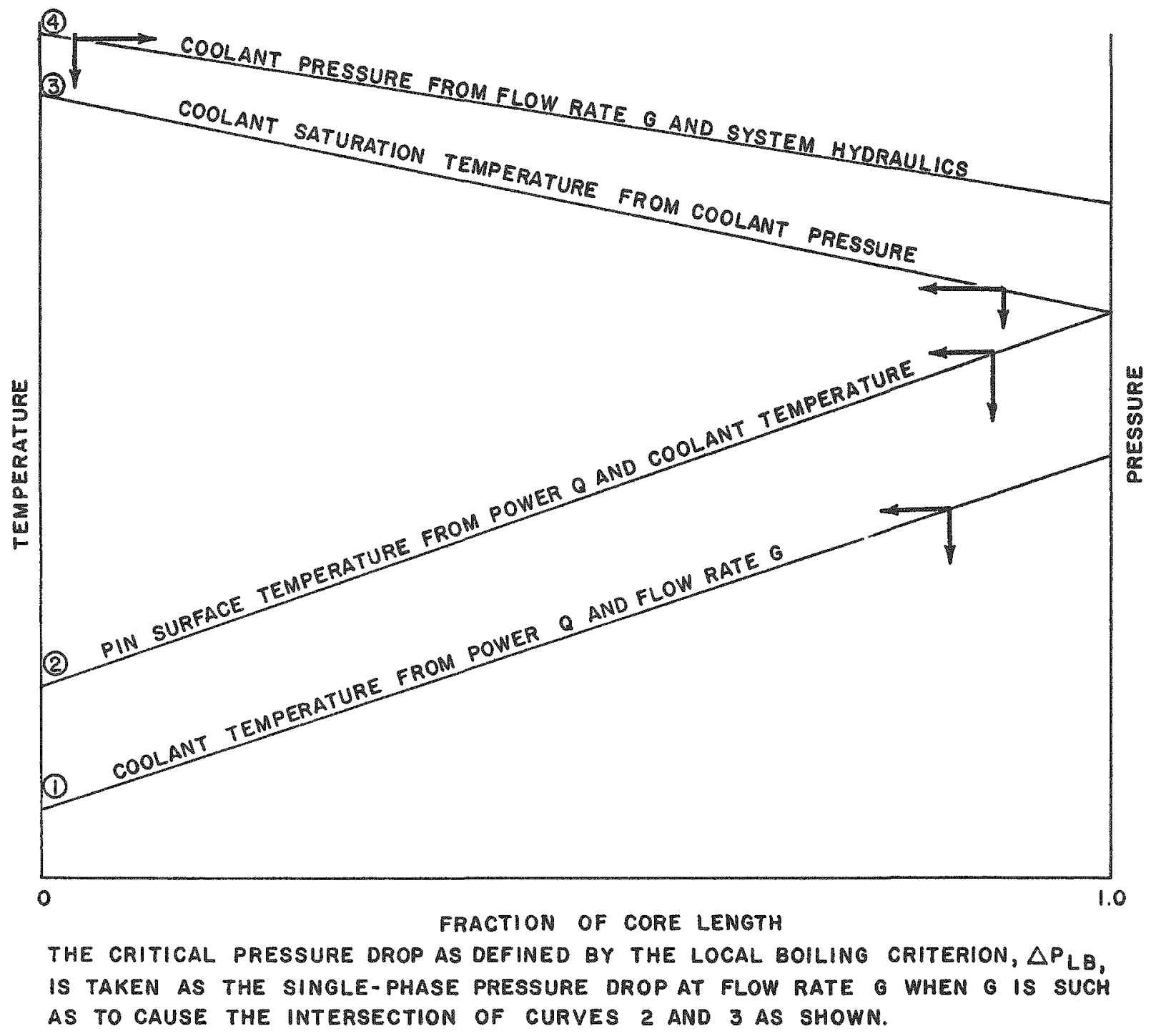

Fig. 12. Diagram of Local Boiling Criterion for Determining Critical Pressure Drop 


$$
\begin{aligned}
& \left(\frac{C_{p} \mu}{k}\right)^{0.4}=\left[\frac{1.0(0.94)}{0.394}\right]^{0.4}=1.417 \\
& A=\frac{1}{(2.54)^{2}}-4 \frac{\pi}{4}(0.158)^{2} \\
& =0.0769 \mathrm{in}^{2} \text {. } \\
& \bar{P}=\frac{4}{2.54}+4 \pi(0.158) \\
& =3.56 \mathrm{in} \text {. } \\
& D=4 A / P \\
& =\frac{4(0.0769)}{3.56}=0.0864 \mathrm{in.}=7.2 \times 10^{-3} \mathrm{ft} .
\end{aligned}
$$

Substitute in equation 7.

$$
\begin{aligned}
h & =\frac{0.023(0.394)}{7.2 \times 10^{-3}}(1.417)\left(\frac{7.2 \times 10^{-3} \mathrm{G}}{0.94}\right)^{0.8} \\
& =1.78\left(7.66 \times 10^{-3} \mathrm{G}\right)^{0.8} \text { with } \mathrm{G} \text { in } 1 \mathrm{~b} / \mathrm{hr}-\mathrm{ft}^{2} \\
& =1.78\left(7.2 \times 10^{3} \mathrm{G}\right)^{0.8} \text { with } \mathrm{G} \text { in } \mathrm{gal} / \mathrm{min} . \\
\mathrm{h} & =\frac{\mathrm{Q}}{\mathrm{A} \Delta \mathrm{t}_{f}} \\
\mathrm{~h} & =\frac{\mathrm{Q}}{\Delta \mathrm{t}} \frac{3413(144)}{\pi(0.158) 120} \\
& =\frac{8250 \mathrm{Q}}{\Delta \mathrm{t}} \text { with } \mathrm{Q} \text { in } \mathrm{kW} .
\end{aligned}
$$

Combining equations 12 and 14 ,

$$
\frac{8250 \mathrm{Q}}{\Delta \mathrm{t}_{\mathrm{f}}}=1.78\left(7.2 \times 10^{3} \mathrm{G}\right)^{0.8}
$$

or

$$
\Delta t_{f}=\frac{3.79 Q}{G^{0.8}} \text { with } Q \text { in } k W, G \text { in gpm, and } \Delta t \text { in }{ }^{\circ} \mathrm{F} \text {. }
$$


At the desired value of flow, $\Delta t_{f}$ is the difference between the bulk coolant temperature at the core outlet and the local saturation temperature of $206^{\circ} \mathrm{F}$.

Therefore

$$
\Delta t_{f}=206-\left(t_{i}+\frac{Q}{W C_{p}}\right) \text { with } Q \text { in Btu/hr and } W \text { in } 1 b / h r
$$

or

$$
\Delta t_{f}=206-\left(t_{i}+\frac{6.84 Q}{G}\right) \text { with } Q \text { in } \mathrm{kW} \text { and } G \text { in gpm. }
$$

Combining equations 16 and 18 ,

$$
206-\left(t_{i}+\frac{6.84 Q}{G}\right)=\frac{3.79 Q}{G^{0.8}}
$$

Reduction to simplest form gives:

$$
G^{0.2}=\left(\frac{206-t_{i}}{3.79 Q}\right) G-1.805
$$

The determination of $G$ for a given combination of $t_{i}$ and $Q$ is done by trial and exror, but is not especially difficult. $G$ was calculated for each of the 50 experimental runs, and in each case the zeropower demand curve corresponding to the appropriate inlet temperature was entered to determine the value of critical pressure drop, $\Delta P_{L B}$, as defined by the local boiling criterion. $\triangle P_{I B}$ was then compared with $\Delta \mathrm{P}_{\mathrm{e}}$ as shown in Figs. 13 and 14. In both figures it is apparent that the correlation gets increasingly better as $\triangle P_{L B}$ is decreased, with the ratio $\triangle P_{L B} / \Delta P_{e}$ approaching 1.0 as $\triangle P_{L B}$ approaches 0 . As would be expected, the ratio $\triangle P L B / \triangle P_{e}$ never falls below 1.0. Figure 13 shows some experimental scatter reflecting the numerous potential sources of errors in the determination of $\triangle P_{L B} / \triangle P_{e}$, but the correlation appears independent of axial blanket size as one would expect. As one would also expect, Fig. 14 suggests a rather strong dependence on inlet temperature. Since the true critical pressure drop must be related to the single-phase demand at some flow rate between those calculated for bulk boiling and local boiling, it is logical that the local boiling method becomes increasingly conservative with decreasing amounts of inlet subcooling.

\footnotetext{
* It should be noted that actual surface boiling requires a superheated surface and the required degree of superheat (a function of heat flux, puriry, amount of agitation, etco) is not factored into the calculation of $G$. This omission necessarily makes $\mathrm{P}_{\mathrm{LB}}$ conservative.
} 


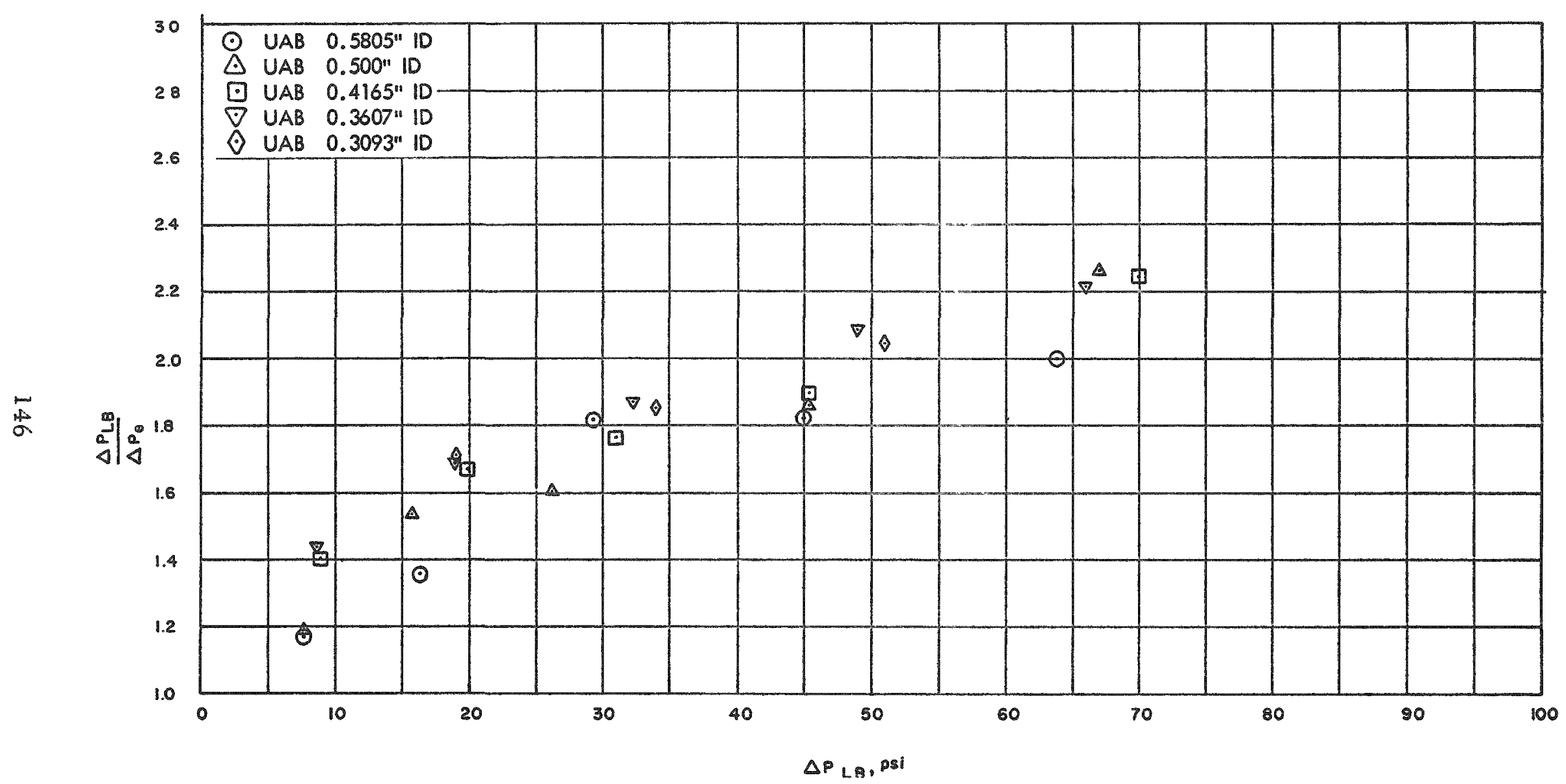

Fig. 13. $\Delta \mathrm{P}_{\mathrm{LB}} / \Delta \mathrm{P}_{\mathrm{e}}$ vs. $\Delta \mathrm{P}_{\mathrm{LB}}$ for Various Upper Axial Blankets with $120^{\circ} \mathrm{F}$ Inlet Temperature 


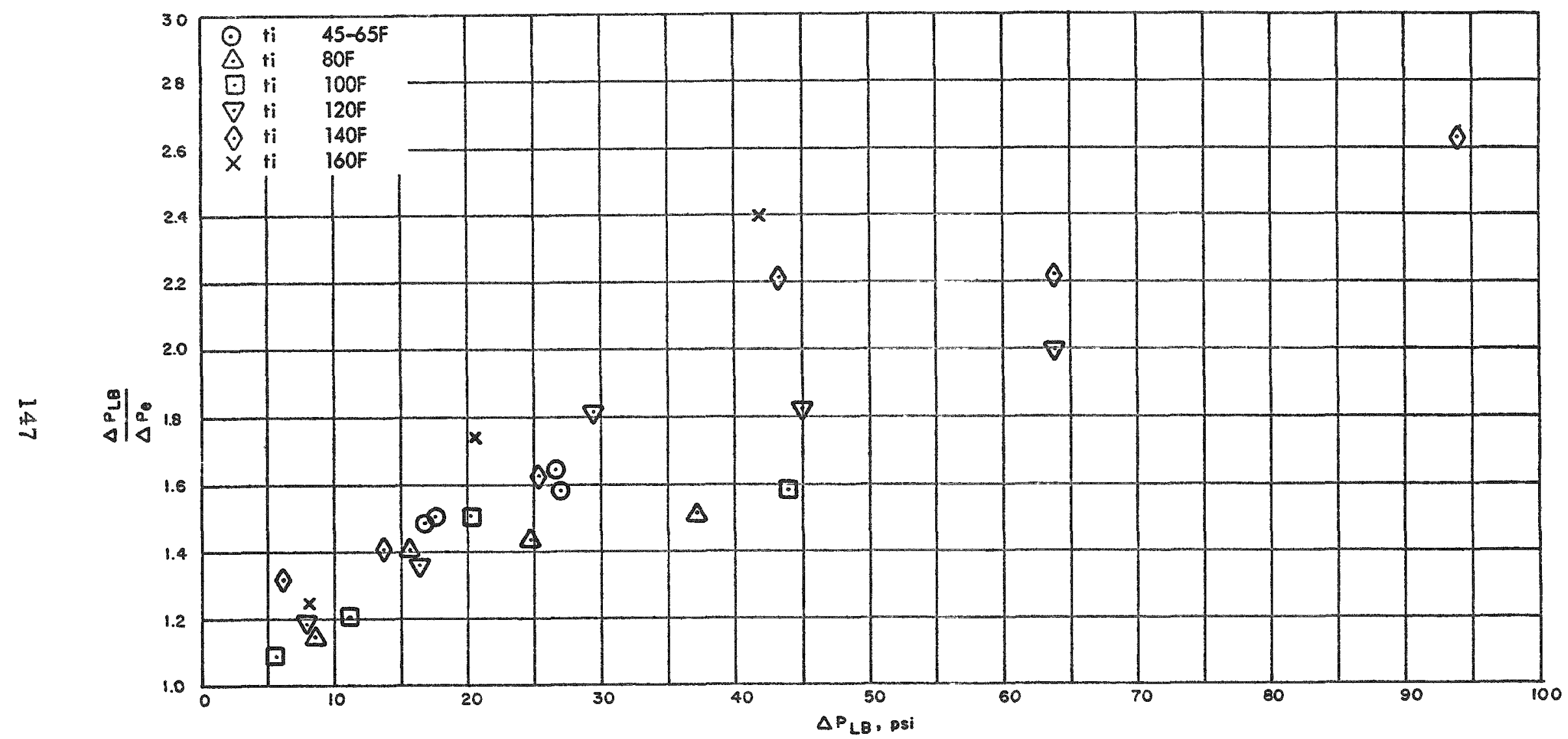

Fig. 14. $\Delta P_{L B} / \Delta P_{e}$ vs. $\Delta P_{L B}$ for Various Inlet $T$ emperatures with $U A B=0.5805$ in. ID 


\section{Application to Sodium Systems}

In terms of experimentally identifying the coordinates of the critical point in the demand curve, water is not the ideal substitute for sodium. Simulation in this important area has two major weaknesses:

1. Thermal Conductivity - With its superior thermal conductivity, sodium will have a much flatter profile for radial temperature. Sodium vapor, therefore, will not appear as quickly in a fast reactor as in the simulant loop.

2. Inlet Subcooling - The film $\Delta t$ in the sodium system occupies a much smaller fraction of the working range of the fluid than is the case with water. Consider, for example, a sodium-cooled reactor whose core inlet temperature is $550^{\circ} \mathrm{F}$. The boiling point at the core outlet pressure is about $1600^{\circ} \mathrm{F}$. Of this $1050^{\circ} \mathrm{F}$ working range, the film $\Delta t$ is perhaps $50^{\circ} \mathrm{F}$. The difference between the critical flow rates as calculated by the two methods discussed herein is only $50 / 1050$ or $4.8 \%$. Since single-phase pressure drop is approximately proportional to flow squared, the difference between the two methods is only about $10 \%$, as compared with factors of 2 to 4 in water. Thus, which of the two methods is used to predict critical pres sure drop makes little practical difference to the fast reactor designer although, as established by the simulant tests, the conservative approach would be to use the local boiling criterion.

The coordinate of lesser practical importance, critical flow rate, can be calculated with good engineering accuracy by heat balance (Equation 6).

\section{Constant-inlet-pressure Tests}

In this second series of power tests, stainless steel pins were used again. The upper axial blanket had an ID of $0.5805 \mathrm{in.}$, and the water feed was supplied from the pressurized tank (see Fig. 3). The purpose of these tests was to verify the importance of the minimum point on the demand curve as the point of transition between stable flow, which produces adequate cooling, and unstable flow, which leads inevitably to fuel meltdown under typical fast reactor conditions.

The general procedure given below was followed:

1. Establish the desired water temperature in the 110-gal feed tank.

2. Set the tank pressure at a value of about $25 \%$ higher than that required by the critical pressure drop in the demand curve for the intended power level. 
3. Initiated flow in the test section at the "full open" setting of the main throttle valve.

4. Open bypass-line throttle valve so that the desired percentage of the total flow (experimental range was 0 to $80 \%$ ) bypasses the core.

5. Establish desired power level.

6. Begin reducing flow with main throttle valve. Pause at each level long enough to permit equilibrium pressures, temperatures, and core section flow rate to be recorded. Continue until flow becomes unstable.

This procedure does not, strictly speaking, produce constantpressure feed since a pressure drop occurs across the throttle valve, Consider Fig. 15. At the full-open throttle valve setting, the supply curve is very close to $S_{1}$ (the tank pressure), and since flow was established before

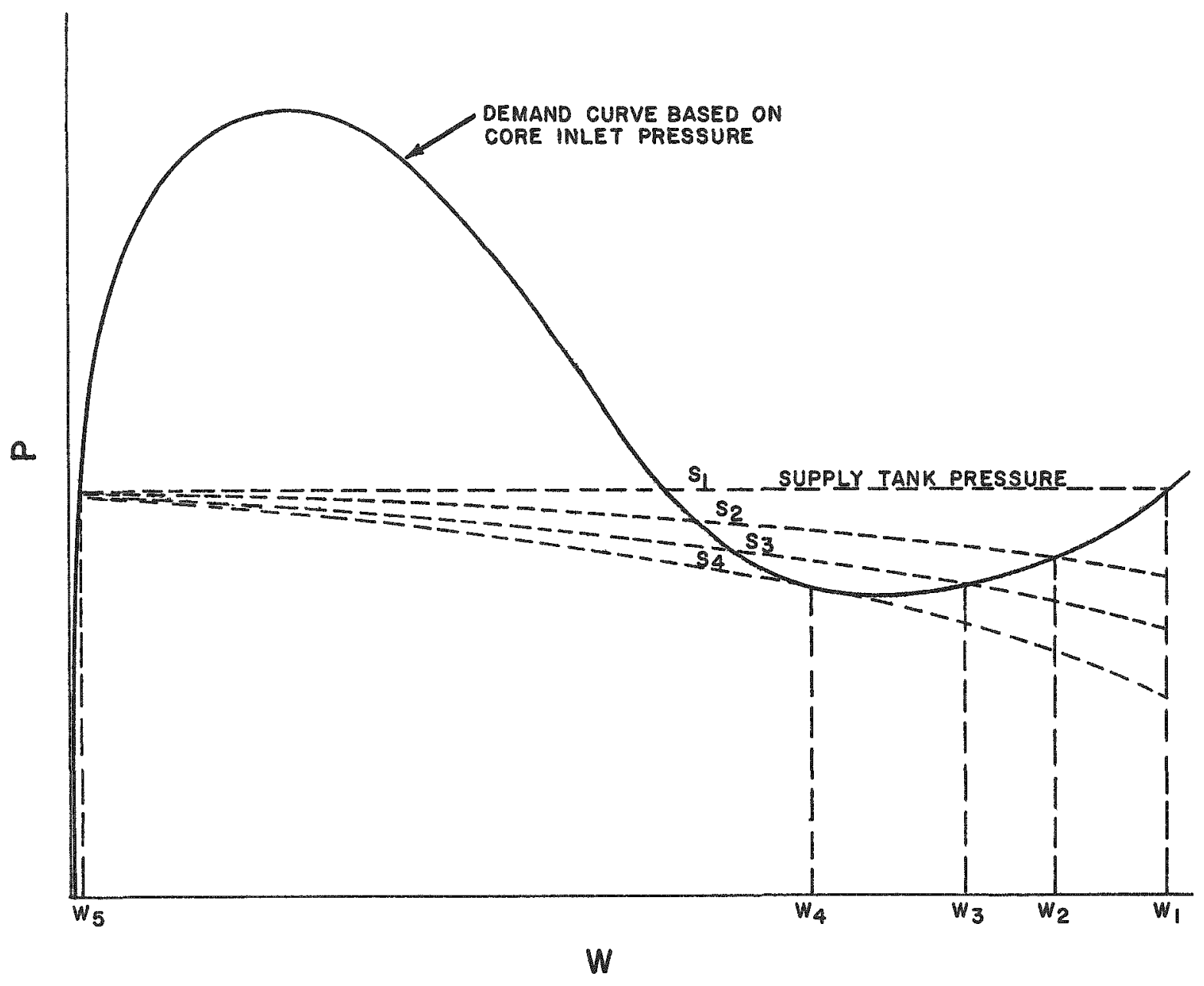

Fig. 15. Supply and Demand Curves in Typical Power Test Using Constant Pressure Feed Source 
power was turned on, the resultant flow rate is $W_{1}$. Upon partial closure of the throttle valve, supply curves $S_{2}, S_{3}$, and $S_{4}$ are produced with the corresponding reduction of flow to $W_{2}, W_{3}$, and $W_{4}$, respectively. Note that supply curve $\mathrm{S}_{4}$ intersects the demand curve to the left of the minimum point. This intersection satisfies the theoretical conditions for flow stability and represents the minimum stable flow since any further throttling would tend to reduce the flow to $W_{5}$, and a fuel meltdown would ensue.

It would seem that the possibility of obtaining flow stability at a point to the left of the minimum point in the demand curve would be greatest when no bypass flow exists. This theory was borne out by the tests summarized in Table I. In all the runs with zero bypass, the critical inlet pressure was greater than the minimum inlet pressure, and the flow rate at the critical inlet pressure was less than that at the minimum inlet pressure. (In this context, critical inlet pressure is the measured static pressure at the core entrance for the minimum obtainable throttle-valve setting.) With bypass flow, however, the critical inlet pressure corresponded to the minimum value, and this critical pressure and flow condition duplicated, within the limits of experimental accuracy, the minimum point obtained in the zerobypass run at the same power level and inlet temperature. The practical significance of the minima of the demand curves is, thexefore, verified. experimentally.

Table I

SUMMARY OF CONSTANT-INLET-PRESSURE TESTS

\begin{tabular}{c|c|c|c|c|c|c|c}
\hline $\begin{array}{c}\text { Run } \\
\text { No. }\end{array}$ & $\begin{array}{c}\text { Inlet } \\
\text { Temp } \\
\left({ }^{\circ} \mathrm{F}\right)\end{array}$ & $\begin{array}{c}\text { Power } \\
(\mathrm{kW})\end{array}$ & $\begin{array}{c}\text { Bypass } \\
\text { Flow } \\
(\%)\end{array}$ & $\begin{array}{c}\text { Minimum } \\
\text { Inlet } \\
\text { Pressure } \\
\text { (psia) }\end{array}$ & $\begin{array}{c}\text { Core Flow at } \\
\text { Minimum Inlet } \\
\text { Pressure } \\
\text { (gpm) }\end{array}$ & $\begin{array}{c}\text { Critical } \\
\text { Inlet } \\
\text { Pressure } \\
\text { (psia) }\end{array}$ & $\begin{array}{c}\text { Core Flow at } \\
\text { Critical Inlet } \\
\text { Pressure } \\
\text { (gpm) }\end{array}$ \\
\hline 1 & 55 & 18.5 & 0 & 20.5 & 0.82 & 21.0 & 0.65 \\
2 & 116 & 19.5 & 0 & 20.5 & 1.75 & 22.5 & 1.25 \\
3 & 70 & 20.5 & 0 & 18.0 & 1.0 & 18.5 & 0.8 \\
4 & 70 & 20.5 & 40 & 18.0 & 1.0 & 18.0 & 1.0 \\
5 & 70 & 20.5 & 81 & 18.5 & 1.05 & 18.5 & 1.05 \\
6 & 55 & 26.5 & 0 & 19.0 & 1.35 & 19.5 & 1.22 \\
7 & 55 & 26.5 & 74 & 19.0 & 1.35 & 19.0 & 1.35 \\
8 & 52 & 35.1 & 0 & 21.5 & 2.05 & 22.5 & 1.65 \\
9 & 52 & 35.1 & 53 & 21.5 & 2.05 & 21.5 & 2.05 \\
10 & 49 & 46.0 & 0 & 25.0 & 2.6 & 26.5 & 2.3 \\
11 & 49 & 46.0 & 37 & 25.0 & 2.6 & 25.0 & 2.6 \\
\hline
\end{tabular}

An interesting phenomenon was observed as the throttle valve was closed beyond the setting which produces tangency between the supply and demand curves. Flow would decrease very rapidly, as expected, and flow reversal would occur, beginning at a point near the core midplane. After a fraction of a second, liquid coolant would re-enter from the bottom, only 
to be expelled again, both upward and downward from a point once again near the core midplane. By this time, the stainless steel pins were turning cherry red above the core midplane, and the power was quickly interrupted to save the test section.

These observations proved to be a preview of events later photographed in the lead-meltdown tests. At this stage, they suggested two things to the experimenters, however:

1. Reactor designers have long been concerned about local coolant starvation caused by entrained solid contaminants being sucked up against a subassembly inlet nozzle. If the observed flow reversals occur prior to meltdown, they could remove the cause of blockage before any damage is done.

2. Expulsion of coolant could have a pronounced effect upon reactivity. depending upon reactor design. If the reactor has a large negative sodium coefficient of reactivity, rapid expulsion of sodium from many subassemblies could be a shutdown mechanism. If, on the other hand, the sodium has a large positive reactivity coefficient, rapid expulsion of coolant from the core could produce a reactivity insertion of perhaps $\$ 20$ to $\$ 50$ per second. Reactors of the latter type are outside the scope of the present study. However, as larger fast reactors are designed, the sodium coefficients are expected to become positive, and this safety problem must be more thoroughly analyzed.

VI. Lead-meltown Tests

\section{A. General}

The objective of all previous effort had been to define the conditions under which fuel melting could occur. Following this, a program for conducting meltdown tests was formulated.

For the first time, lead pins were installed in the core section of the test loop. The lead pins, like the stainless-steel pins previously used, were 30 in. long and had an $O D$ of 0.158 in.; the four pins comprising the test bundle were positioned on a 0.199 -in. square pitch. The lead pins had been made by a process of hot extrusion around a 0.045 -in. copper wire, the purpose of which was to prevent the interuption of current flow upon initial melting of the lead. These lead-copper pins, unlike those of stainless steel, were very weak, and in an effort to maintain the nominal pin spacing six support grids of the same type used in the core of the Enrico Fermi reactor were attached at uniform intervals along the length of the bundle. 
The 0.5805-in.-ID UAB tube was used throughout the meltdown tests, since it offered the best chance of avoiding flow abnormalities caused by the formation of a lead plug above the core.

The pressurized tank was employed as the coolant feed system.

In all, eight meltdown tests were run. The power and flow conditions and the manner in which each meltdown was initiated are summar ized in Fig. 16. The exploration of higher power levels was precluded by difficulties in providing a junction of sufficiently low electrical resistance between the lead ( $\mathrm{Pb})$ pin and the copper lead to the terminal block. Another problem, even at low power levels, was the early burnout of the 0.045-in. copper conductors after their surfaces were bared by local lead melting. Even at $25 \mathrm{~kW}$, fox example, the surface heat flux on the first exposed surfaces of these axially positioned conductors was over $2 \times 10^{6} \mathrm{Btu} / \mathrm{hr}-\mathrm{ft} \mathrm{t}^{2}$, and burnout, particularly at the low pressures near the top of the core, is inevitable. It is fortunate that the bared conductors continued to carry current for 1 to 3 sec, thus allowing more than just local melting in the typical test.

\section{B. Meltaown Test Observations}

Of the eight meltdown tests conducted, three will be described and discussed herein. APDA-155 contains a complete description of all eight.

Test 3

Initial Conditions: Power $=26.3 \mathrm{~kW}$

Core flow $=2.5 \mathrm{gpm}$

Bypass flow $=0 \mathrm{gpm}$

Bypass per cent $=0$

Core inlet temperature $=59^{\circ} \mathrm{F}$

Core and UAB outlet temperature $=132^{\circ} \mathrm{F}$

Core inlet pressure $=31$ psia

UAB outlet pressure $=12.0$ psia

Test Chxonology: The following sequence of events was obtained from correlating the strip chart data with the high-speed $(\sim 350$ frames/sec) movie of the test -

Time

$\underline{(\mathrm{sec})}$

0 Flow transient begins.

1.2 Core outlet temperature begins increasing.

1.6 UAB outlet temperature begins increasing. 


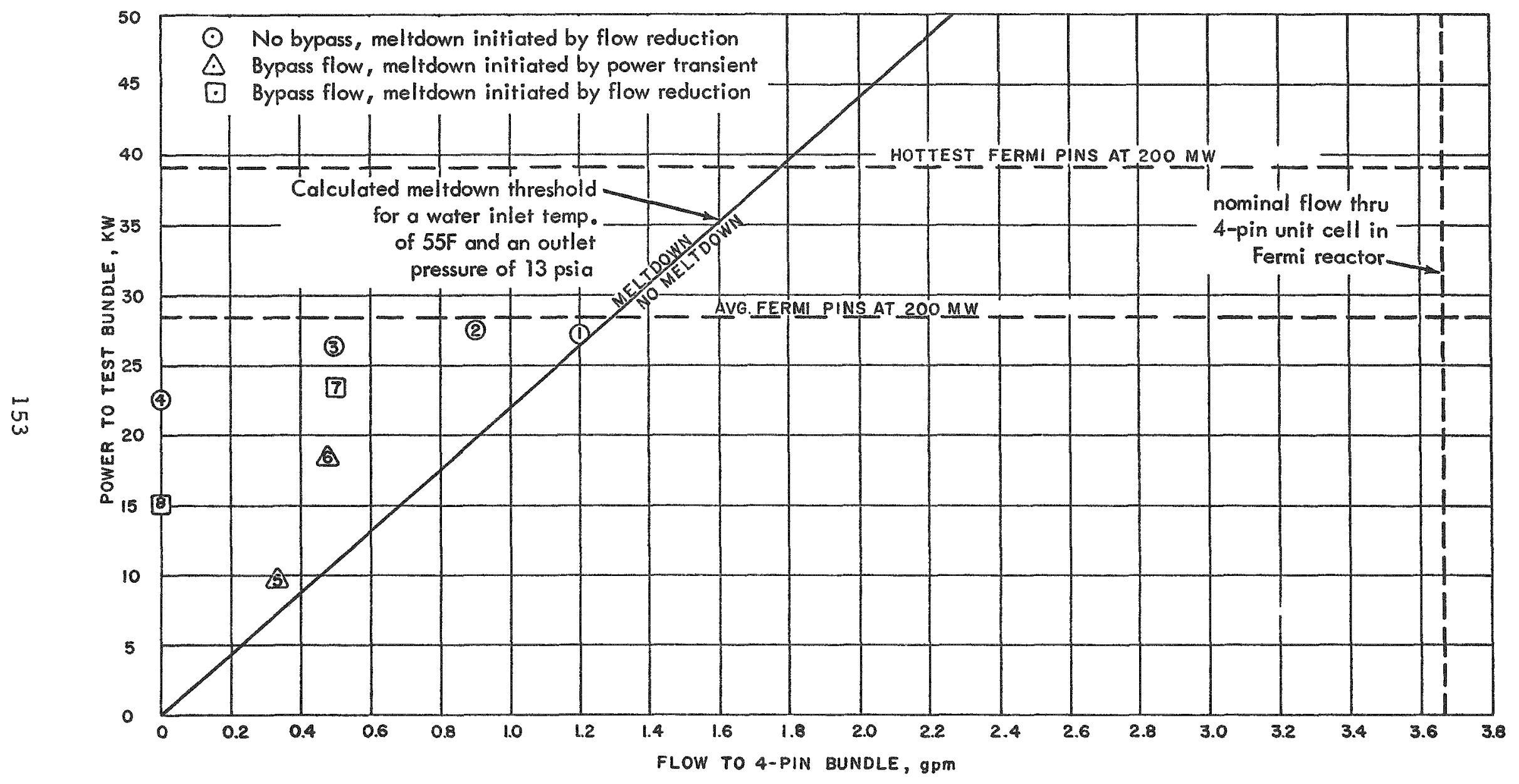

Fig. 16. Test Conditions in Pb Meltdown Test Program 
Time

(sec)

1.88 Flow reaches desired level of $0.5 \mathrm{gpm}$

inlet pressure $=19.0 \mathrm{psia}$

2.3 First boiling seen at 0.9 to $1.0 \mathrm{~h}$.

3.6 Core outlet temperature $=165^{\circ} \mathrm{F}$ :

begins increasing at accelerated rate.

3.68 Core and UAB outlet pressures begin oscillating with approximately 0.3 -sec-period.

4.03 First flow reversal seen at $0.6 \mathrm{~h}$.

4.2 Core inlet pressure begins oscillating with approximately 0.3 -sec period.

4.77 First melting occurs at $0.8 \mathrm{~h}$.

4.97 Entire upper half molten; most of the lead moving upward at speeds up to $6.3 \mathrm{ft} / \mathrm{sec}$.

5.0 Lead at $0.5 \mathrm{~h}$ begins falling at approximately $2.5 \mathrm{ft} / \mathrm{sec}$.

5.63 Lead redistribution essentially complete.

5.8 First arcing occurs at $0.55 \mathrm{~h}$.

7.0 Apparent power failure.

This was one of the better runs in that flow blockage did not occur and the pins did not fail prematurely. By the time power was interrupted by burnout at $0.55 \mathrm{~h}$, the entire upper half of the bundle had melted and most of this material had been carried upward by the coolant stream. Figure 17 shows the final distribution of lead. Other than a concentration at the second spacer grid, practically all of the lead in the uppex half of the bundle had escaped to the upper plenum.

This run simulated a loss-of-pump accident in the Fermi reactor at $200 \mathrm{MW}$. The flow reduction to $0.5 \mathrm{gpm}$ represents the pony-motor output (about $15 \%$ of nominal). It is encouraging to note that when no flow blockage occurs, the net tendency of the fuel is to escape to a zone of lower reactivity, i.e., away from the core midplane. The absence of blockage should be assured in the reactor by retained fuel fluidity although, with the resistanceheating simulation, blockage was avoided purely by good fortune.

The flow behavior was typical, at least in the qualitative sense, of all the tests: (1) The flow rate decreased sharply as net boiling began. (2) Considerable vapor appeared in the upper half of the core. (3) Flow reversal occurred, beginning at a point slightly above the core midplane. 
Before Melidown

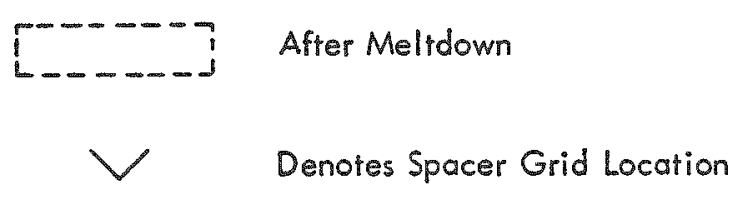

Post-test Inventory Summary

\begin{tabular}{lc} 
Below Core & $0.0 \%$ \\
Above Core & 33.7 \\
In Core & 57.7 \\
Unrecovered & 8.6 \\
\cline { 2 - 2 } & 100.0
\end{tabular}

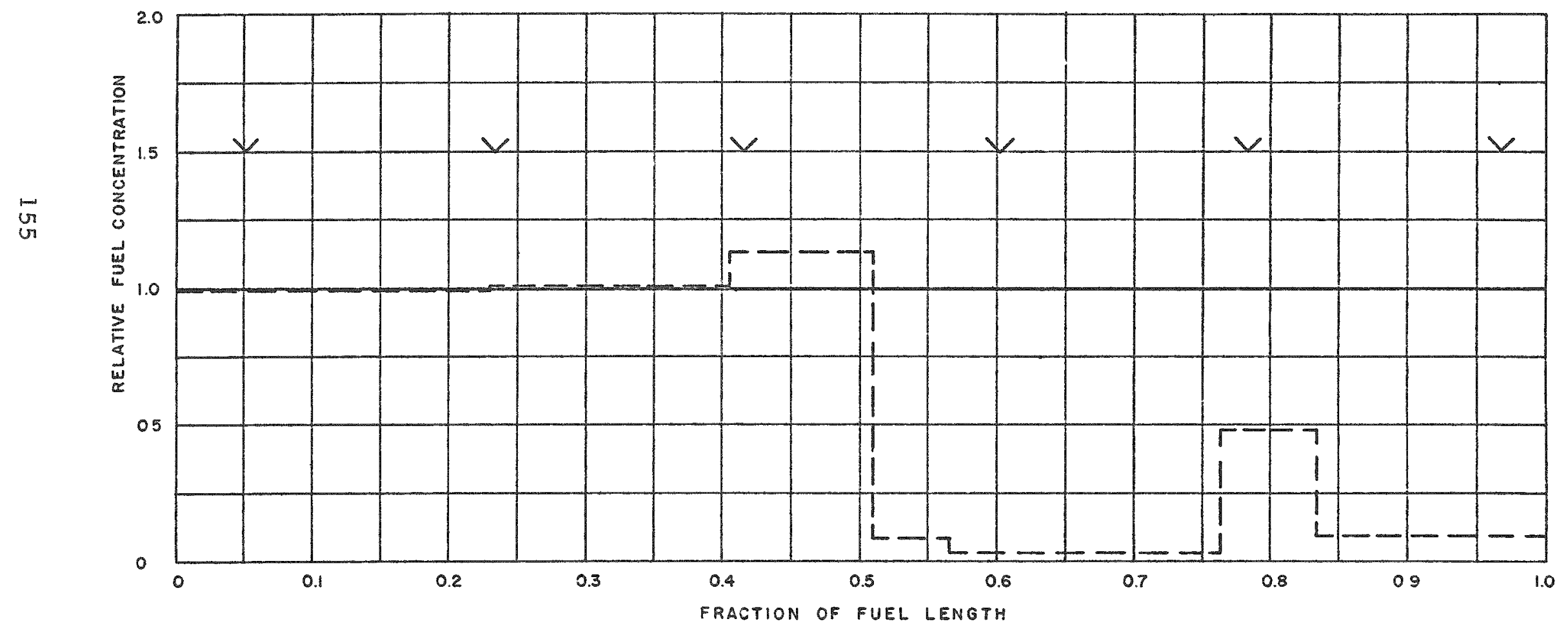

Fig. 17. Fuel Distribution before and after No. 3 Meltdown Test 
(4) "Chugging" (alternate appearance of liquid and vapor) was evident beneath the point of flow reversal for about two full cycles prior to the initial melting.

Test 6

Initial Conditions: Power $=0$

Core flow $=0.47 \mathrm{gpm}$

Bypass flow $=0.38 \mathrm{gpm}$

Bypass per cent $=45$

Core inlet temperature $=60^{\circ} \mathrm{F}$

Core inlet pressure $=19.5 \mathrm{psia}$

$\mathrm{UAB}$ outlet pressure $=16.5$ psia

Test Chronology:

Time

$\underline{(\mathrm{sec})}$

1 Power transient begins.

2.2 Core outlet temperature begins increasing.

2.7 Power reaches desired plateau at $18.5 \mathrm{~kW}$.

4.17 Boiling begins at 0.9 to $1.0 \mathrm{~h}$.

Boiling front slowly moves downward.

4.93 Flow reversals begin at $0.5 \mathrm{~h}$.

5.0 Inlet pressure oscillations begin;

continue with period of $\sim 0.25 \mathrm{sec}$.

6.2 Melting begins at $0.8 \mathrm{~h}$; initial movement is upward and very slow $(<1 \mathrm{ft} / \mathrm{sec})$; molten zone spreads very rapidly until -

6.57 Upper $80 \%$ of bundle generally molten.

6.77 Void appears at $0.65 \mathrm{~h}$; most of lead drops at $\sim 2.5 \mathrm{ft} / \mathrm{sec}$.

6.83 Void appears at $0.35 \mathrm{~h}$; most of lead drops at $\sim 3.75 \mathrm{ft} / \mathrm{sec}$;

lesser amount moves up at slower rate $(<2.0 \mathrm{ft} / \mathrm{sec})$.

6.83 No gross redistribution of lead, but much of it seems

7.23 to be held in a turbulent suspension.

7.23 Lead from $0.75 \mathrm{~h}$ begins to fall past void at $0.65 \mathrm{~h}$ at a speed of $\sim 1.5 \mathrm{ft} / \mathrm{sec}$.

7.5 First arcing occurs at $0.8 \mathrm{~h}$.

7.8 Glass test section explodes violently; power simultaneously terminated. 
Almost the entire bundle participated in this meltdown. The first lead to melt showed a slight tendency to rise, but none escaped the core. Gross coolant flow through the core virtually ceased at about 5.0 sec, apparently because of the preferential bypass path, and downward flow of lead was more evident than in previous tests. Interestingly, the only downward flow of lead at a rate faster than gravity was from a point below the core midplane. Apparentiy this material was caught in one of the several flow reversals which, in this test, centered at approximately $0.5 \mathrm{~h}$. This eventful test culminated spectacularly with the violent explosion of the test section at $7.8 \mathrm{sec}$. The explosion was probably caused by lead plugs forming near the top and bottom of the core, thus trapping a volume of coolant.

Figure 18, consisting of four pictures made from the film sequence, illustrates the highlights of this test.

Test 8

Initial Conditions: Power $=15.1 \mathrm{~kW}$

Core flow $=2.49 \mathrm{gpm}$

Bypas flow $=1.88 \mathrm{gpm}$

Bypass per cent $=43$

Core inlet temperature $=60^{\circ} \mathrm{F}$

Core and UAB outlet temperature $=102^{\circ} \mathrm{F}$

Core inlet pressure $=30$ psia

$\mathrm{UAB}$ outlet pressure $=12.0$ psia

Test Chronology:

Time

$\underline{(\mathrm{sec})}$

0 Flow transient begins.

0.6 General boiling begins and occurs all along the length of the bundle.

0.8 Flow reaches 0 gpm.

1.0 Chugging begins; flow reversals start at $0.55 \mathrm{~h}$.

1.4 Inlet pressure begins to oscillate: period $=0.37 \mathrm{sec}$.

1.42 Core outlet temperature begins increasing (UAB outlet temperature remains at $103^{\circ} \mathrm{F}$ for entire run).

2.8 Melting begins at 0.9 to $1.0 \mathrm{~h}$. Material flows upward at undetermined rate.

3.0 Upper half of bundle generally molten. 


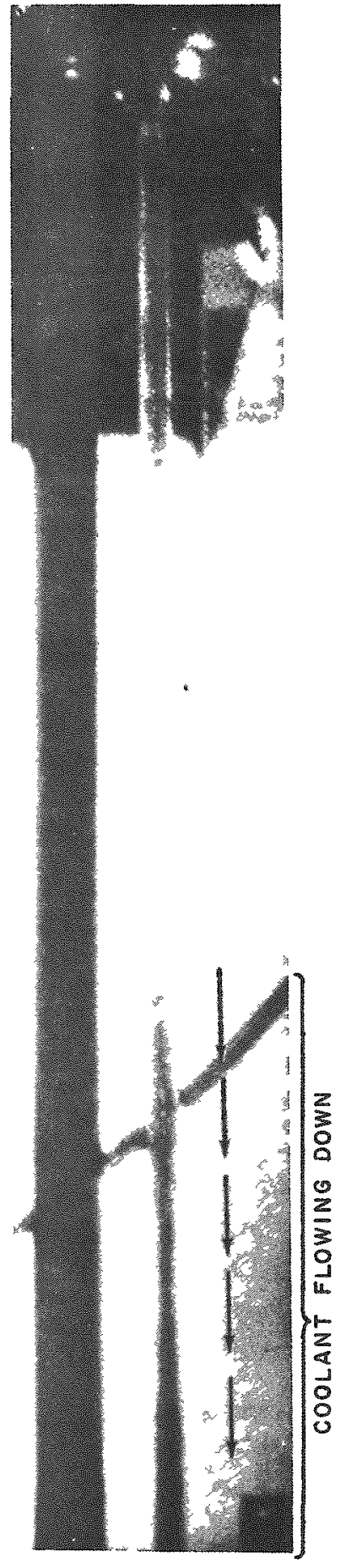

(a)

50 SEC - FIRST FLOW REVERSAL IN PROGRESS
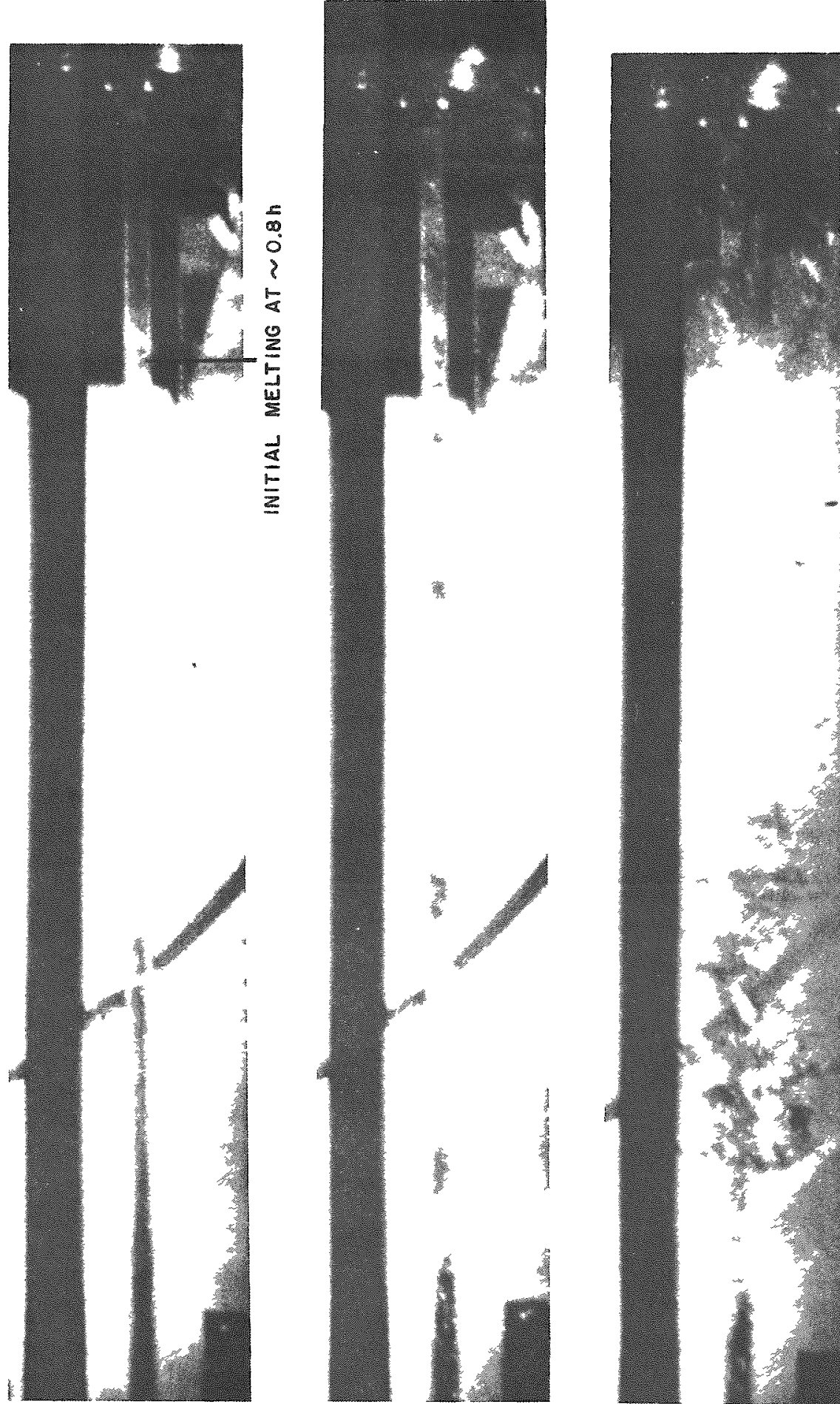

(c)

686-SEC - UPPER $80 \%$ GENERALLY MOLTEN

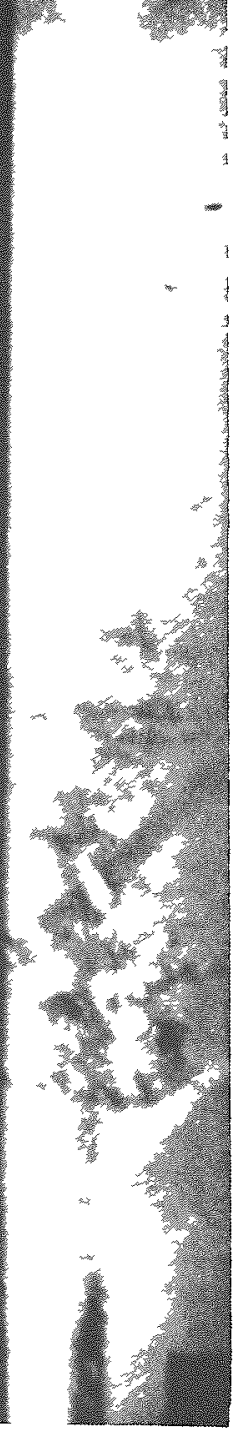

(d)
621 SEC - INITIAL MELTING
78 SEC - TEST SECTION EXPLODES
$-$

Fig. 18. Highlights from Film Sequence of No. 6 Meltdown Test 
Time

$(\mathrm{sec})$

3.2 Void appears at $0.75 \mathrm{~h}$.

Lead above this point flows upward at approximately $2.5 \mathrm{ft} / \mathrm{sec}$;

lead below this point flows downward at approximately $1.5 \mathrm{ft} / \mathrm{sec}$.

Section between 0.75 and $0.8 \mathrm{~h}$ left practically void from

3.5 to 5.1 sec.

5.1 Setting of material from 0.8 to $1.0 \mathrm{~h}$ begins at rate of approximately $2.5 \mathrm{ft} / \mathrm{sec}$.

5.2 Arcing begins.

6.27 Redistribution of fuel es sentially complete.

6.7 Power interrupted.

About $5 \%$ of the original lead was subsequently recovered above the core, probably part of the material initially melted at 0.9 to $1.0 \mathrm{~h}$. The final arrangement of fuel (see Fig. 19) was developed through downward movements of lead from the upper third to the middle third between $t=5.1$ and $t=6.27$ sec. The rate was about $2.5 \mathrm{ft} / \mathrm{sec}$, very close to that for which gravity would be responsible. This run, more so than any other, resembled the model assumed in the calculation of maximum accident studies on the Enrico Fermi reactor (melting of the core and gravitational collapse to a compact configuration in the lower half of the core), except that the upper portion did not fall as a unit but xather as individual particles.

\section{Discussion of Results}

The meltdown behavior of a reactor core, fast or thermal, is the result of a combination of many complex forces and processes. Fully understanding even one of the contributing mechanisms, much less the entixe system, requires far more than a single, modestly financed experimental program. When one considexs the effort that has gone into the study of a one-component, two-phase (boiling water) flow, it is staggering to project the amount of experimental and theoretical work that would be needed to predict, with any degree of accuracy, the sequence of events in a core meltdown - a two-component, four - phase system, not counting the fuel cladding, suppoxt haxdwaxe, and fission gas, all of which are known to affect the rate of migration of fissionable material. Fortunately, the parallel is not a good one. Two-phase flow is, by definition, the normal event in boiling water reactors, and the behavior of the coolant under all realistically possible steady-state and transient conditions must be known before any such reactor can be considered safe. Core meltdowns, on the other hand, require no such level of understanding. The engineer's assignment is to prevent them with evexy tool at his disposal. The field of fast reactor safety has been, and should be, 


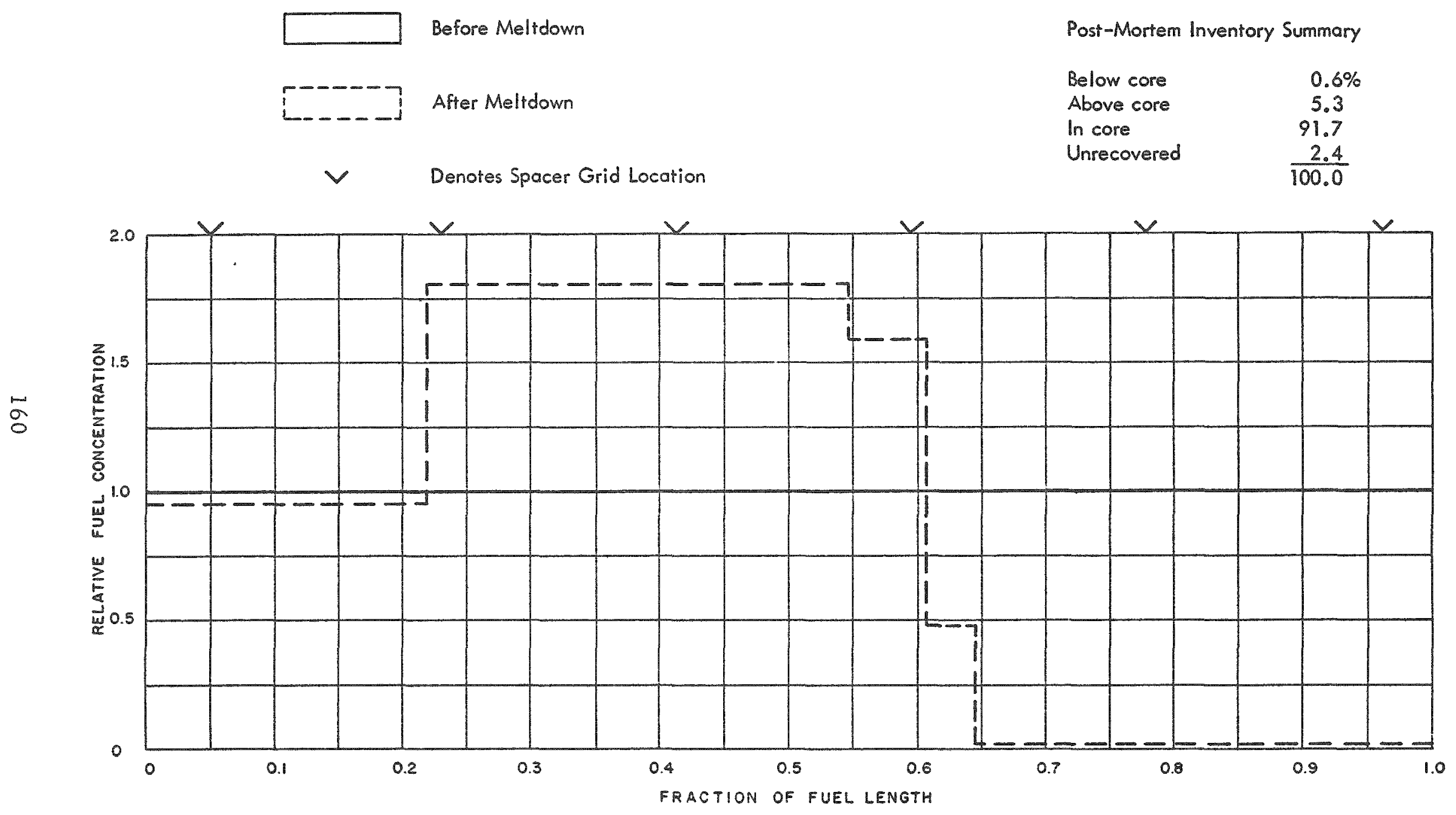

Fig. 19. Fuel Distribution before and after No. 8 Meltdown Test 
essentially geared to this thought. Thus, a considerable portion of this program was directed toward defining experimentally the meltdown threshold over a wide range of the parametric values of power, flow, inlet temperature, and hydraulic resistance downstream from the core.

But those who design and analyze reactor containment still demand, and legitimately so, a model of the "maximum credible accident." Many have been proposed. The analytical model for the study of the "maximum accident" involving the Enrico Fermi reactor anticipates the melting of fuel elements and the subsequent gravitational collapse of the pins onto a fixed core bottom. Downward flow is assumed to occur uniformly and simultaneously (slug flow), and no spacer grid holdup time is considered. This seems to be a good model against which to compare the following meltdown test observations:

1. Downward fuel movements faster than gravity were detected in two tests (6 and 7). In both cases, the lead was originally below the core midplane.

2. Upward fuel movements faster than gravity were detected in three tests (2,3, and 4). In all but one of these cases (Test 4), the fuel involved was originally above the core midplane.

3. Downward fuel movements at a rate equal to gravity were seen in five tests $(2,3,4,6$, and 8$)$. The original material was both above and below the core midplane.

4. Slug flow does not occur; flow is more like that of a slurxy in which individual fuel globules are visible.

In none of the eight tests did the fuel mass compact in a more potentially dangerous manner than that of the analytical model. In general, migration rates exceeding the rate that would result from gravity alone (about $2.6 \mathrm{ft} / \mathrm{sec}$ ) acted in the direction of spreading rather than compacting the fuel mass. In most tests (those where the initial melting was near the top of the bundle), the initial migration was clearly away from the core midplane. In the remainder (those where the initial melting was at the point of flow reversals), the lead usually moved in both directions, but the upward flow was at a greater speed and seemed to involve more material than the downward movement.

In no test did the initial melting occur beneath the point of flow reversal. This is entirely logical since the flow-reversal elevation is deprived of coolant for a longer period between reversal and re-entry than any point beneath it. A point above the reversal elevation may be deprived of coolant for a still longer time derending on whether the re-entry velocities are increasing or decreasing. 
As long as the flow reversal is at or above the core midplane and the initial melting is at or above the point of flow reversal, the initial movement of fuel should result in a decrease in core reactivity. As judged from the test observations, subsequent movements may reintroduce part of this reactivity, but not at a rate faster than the model of gross slumping undex gravity. The final disposition of fuel in all but perhaps the last test (No. 8) appears to be less reactive than the original core. As the dominant tendency of the fuel is to escape from the top of the core, flow areas in the upper axial blanket should be kept as large as possible.

VII. Conclusions

From this study, the following conclusions have been reached:

1. Thermal and hydraulic characteristics of a sodium-cooled fast reactor fueled by uranium-alloy pins can be adequately simulated by water, lead pins, and electrical resistance heating.

2. The simulation is poorest in the following respects: (a) location of initial failure, (b) constraining effect of the cladding, (c) postfailure metallurgical reactions, and (d) retention of fuel heat generation following detachment from the pin.

Note: Items $2 \mathrm{a}$ and $2 \mathrm{~d}$ could be better simulated through the use of induction heating. Items $2 \mathrm{~b}$ and $2 \mathrm{c}$ would be very difficult to satisfy with confidence.

3. At the low pressures characteristic of liquid-metal-cooled reactors, the pressure drop increases sharply as the coolant enters the twophase regime. The negative slope of this portion of the demand curve increases with increasing subcooling.

4. Flow stability, $i . e$, the requisite for avoiding a core meltdown, demands the satisfaction of the following inequality at the point of intersection of the supply and demand pressure drop-flow curves:

$$
\left(\frac{d P}{d W}\right)_{\text {supply }}\left(\frac{d P}{d W}\right)_{\text {demand }}
$$

5. Critical flow rate, i.e., the abscissa of the minimum point on the demand curve, can be calculated by

$$
W_{c}=\frac{Q}{\bar{h}_{s o p}-\bar{h}_{i}} .
$$


6. Critical pressure drop, i.e., the ordinate value of the minimum point on the demand curve, can be conservatively predicted as the singlephase pressure drop for the lowest value of coolant flow at which the surface temperature of the fuel pin does not exceed the saturation temperature of the adjacent coolant at any point along the pin.

7. In a typical sodium-cooled reactor, critical pressure drop as calculated by the local-boiling and bulk-boiling criteria will differ by only about $10 \%$.

8. Fuel-meltdown accidents are precipitated when the supply pressure falls beneath the demand pressure at the critical flow rate.

9. In the geometry tested, initial fuel melting is preceded by a period of coolant chugging and at least two flow reversals at or slightly above the core midplane.

10. With uniform axial heat generation, initial melting of the alloy fuel occurs at or above the point of flow reversal.

11. The initial fuel movement is generally away from the coxe midplane. Subsequent fuel movements can be toward the core midplane, but none were detected at rates faster than gravity.

12. The metallic fuel tends to flow as a slurry rather than as a slug.

13. The dominant tendency of the metallic fuel is to escape from the top of the core; to avoid plugging, flow areas in the upper axial blanket should be kept as large as possible.

14. No mechanism was found to suggest that reactivity increase in an alloy core meltdown could be more rapid than the slug flow-gravitational collapse analytical model. It is believed that this conclusion can be broadened to include cermet fuels but not fuels containing a built-in energy source, e.g., sodium-bonded or bulk ceramic pins. 


\section{Appendix I}

\section{Nomenclature and Abbreviations}

A

$\mathrm{cp}$

$\mathrm{C}_{\mathrm{p}}$

CT

D

G

h

$\bar{h}$

$h_{\mathrm{g}}$

ID

k

LAB

$\mathrm{MCM}$

$\mathrm{P}$

$\overrightarrow{\mathrm{P}}$

$\mathrm{Pb}$

$Q$

$\left.\begin{array}{l}R_{1} \\ R_{2} \\ R_{3}\end{array}\right\}$

RF

논

UAB

W

$\beta$

$\triangle P$

$\Delta t$
Area (in. $\left.{ }^{2}\right)$

Centipoise

Specific heat (Btu/1b- $\left.{ }^{\circ} \mathrm{E}\right)$

Current transformer

Diameter (ft)

Mass flow rate ( $1 \mathrm{~b} / \mathrm{hr}-\mathrm{ft} \mathrm{t}^{2}$ unless otherwise noted)

Surface heat transfer coefficient (Btu/hr $-\mathrm{ft}^{2}{ }^{\circ} \mathrm{F}$ )

Specific enthalpy (Btu/1b)

Specific heat of vaporization (Btu/1b)

Inside dimension(s)

Thermal conductivity $\left(\mathrm{Btu} / \mathrm{hr}-\mathrm{ft}-{ }^{\circ} \mathrm{F}\right)$

Lower axial blanket

1000 circular mils

Absolute pressure (psia)

Wetted perimeter (in.)

Lead

Heat generation rate (Btu/hr unless otherwise noted)

Dimensionless thermal similitude criteria

Radio frequency

Temperature $\left({ }^{\circ} \mathrm{F}\right)$

Upper axial blanket

Mass flow rate (lb/hr)

Dimensionless ratio of specific volume of vapor to specific volume of liquid

Pressure drop (psi)

Temperature drop $\left({ }^{\circ} \mathrm{F}\right)$ 


$$
\begin{array}{ll}
\mu & \text { Viscosity }(\mathrm{Ib} / \mathrm{ft}-\mathrm{hr}) \\
\rho & \text { Density }\left(\mathrm{Ib} / \mathrm{ft} \mathrm{t}^{3}\right)
\end{array}
$$

$\underline{\text { Subscripts }}$

$\begin{array}{ll}\text { BB } & \text { Bulk boiling } \\ \text { BP } & \text { Boiling point } \\ \text { e } & \text { Critical } \\ f & \text { Experimental } \\ \text { i } & \text { Film } \\ \text { l } & \text { Inlet } \\ \text { LB } & \text { Lead } \\ \text { MP } & \text { Local boiling } \\ \text { o } & \text { Melting point } \\ \text { s } & \text { Outlet } \\ \text { sop } & \text { Sodium } \\ \text { U- Mo } & \text { Saturation conditions at outlet pressure } \\ \text { W } & \text { Uranium-molybdenum alloy }\end{array}$


MR. CAMPISE (Atomics International):

Did you have rapidly responding thermocouples in your coolant stream to measure temperature variations? Have you been successful in theoretically understanding this flow reversal, and the conditions required and so forth?

MR, HUEBOTTER:

Although I don't recall their time constants, I think you would consider the thermocouples as rapidly responding. We have not investigated a theoretical interpretation of the flow reversal. The flow reversal is a transient, temporary, partial pressure inversion caused by boiling occurring too fast for the ejection for the normal flow of the coolant.

MR. GOLDMAN (United Nuclear Corp.):

With regard to Fig. 7 , did you say that the critical point was where the trough occurred?

MR. HUEBOTTER:

Yes.

MR. GOLDMAN:

Was there any reason to believe that the incipient condition of bulk boiling in the fluid was not the point at which this whole sequence of events occurred? In other words, is there some part of the regime where bulk boiling can occur, and you will not get this sequence of flow reversal followed by melting?

MR. HUEBOTTER:

If you have an unorificed system, a fairly flat pressure drop versus flow supply characteristic, and you begin to see boiling, I am virtually sure you are in for the meltdown effect. The flow rate coordinate of the trough point was fairly well predicted by simple heat balance.

MR. GOLDMAN:

Just the incipience of the bulk boiling? 
MR, HUEBOTTER:

Yes, but the pressure drop at which that occurred was somewhat highex than the single-phase pressure drop at the calculated critical flow as calculated by heat balance. This is evidently due to local boiling boosting the pressure drop.

\section{MR GOLDMAN:}

Another question: In those cases in which you found a good deal of the fuel deposited above the core and a good portion of the core below the midplane was substantially unaffected, is it possible in the Fermi reactor and other reactors also to accumulate this material in the upper reflector and have the whole upper reflector fall down on the unaffected portion of the core to make a worse sort of hypothetical accident than the one that was assumed?

MR。HUEBOTTER:

I would not be surprised. The fact that the lower portion was unaffected was more due to the manner in which we ran our tests than to anything else. We had a copper wire running up through the center of the lead pins, this being to maintain electrical current flow, after the initial meltdown had occurred. If we didn't have this, of course, the local melting would destroy the current flow and interrupt the test. As soon as the copper conductors are bared, they have a tremendous heat flux, something like 3 or 4 million Btu/ $\mathrm{hr} / \mathrm{ft}^{2}$, and burnout is inevitable. We had burnout of bared copper wires up at the top where the melting started before we had any effect on the lead at the bottom. We would guess ultimately they would all melt if we could preserve the power long enough.

MR GOLDMAN:

What do you think would have happened to the fuel at the bottom then?

MR. HUEBOTTER:

I think that by this stage of the game the gross upward flow is pretty well stopped. If there is an alternative path, the coolant is taking that, and I think you would have the gravity settling of this material. Probably it would end up beneath the core if it had time to do so. The important thing. I think, is that the initial movement was upward, and since the initial melting occurred above the core midplane, the initial flow is away from the core midplane, and thus to a region of lower reactivity. 
MR.STORRER (EURATOM):

Did you estimate to what pressure you should go in order to avoid the circumstance that initial boiling results in flashing your assembly dry? It seems to me that this combined with the positive void coefficient is a drawback of sodium-cooled reactors. Could not this problem be avoided by operating sodium-cooled reactors at high pressure? Of course, nobody would. like to do it.

MR。 HUEBOTTER:

I don't think you would want to operate at high pressure. You throw away one of the principal advantages of the sodium-cooled reactor, namely, that you can operate at low pressures.

MR. STORRER:

Certainly, but when you compare this to a gas-cooled reactor, if you pressurize the liquid you don't accumulate potential energy the way you do when you pressurize gas. This is a provocative statement again. I am not seriously suggesting that you pressurize sodium-cooled reactors.

MR, de STORDEUR (EURATOM):

We heard from Dr. Dickerman about tests which were run in TREAT on both unirradiated or irradiated Fermi pins. No suggestion of an unknown mechanism worse than those previously considered has turned up. You get a similar conclusion from the lead-water tests. Do you think that there is now enough data with the TRE,AT results, and with your results on leadwater tests, to have a real test done in the TREAT reactor, or in an improved TREAT reactor, such as that described by Dr. Tavernier, where you could melt an actual small bundle of Fermi pins, with flowing sodium, perhaps as a loss of flow accident, and to see really what happens when you have irradiated pins?

MR. HALL:

In other words, are the simulation studies such as you are doing here coupled with the single element-type of experiment that can be done in TREAI sufficient, or does one have to go to the integral test? This is a controversial question.

MR. HUEBOTTER:

It is a matter of judgment. The answer may depend on the fuel sys tem being considered. 
MR. WOLFE (General Electric):

First, how does the fact that once the fuel begins to melt and presumably the lead gets away from the hot spots of the element, affect your results? Secondly, with regard to Mr. Storrer's question, I don't see why one is anxious to stop this flow reversal. It seems to me it disperses the core in the right direction. Maybe that is the thing you want. Once you get to the point where you are boiling in a sodium reactor, it is very probable that something drastic is going to happen to the core. If it's going to happen I don't see why you don't take advantage of the possibility that it blows apart. The third question is, what is the stored energy in the sodium-bonded or oxide fuel?

MR. HUEBOTTER:

Let me take your questions in reverse order. With regard to the stored energy, you have pressure building up in the bulk ceramic pin and in the sodium-bonded element in which a breach of the clad occurs. This pressure assists the fuel in relocating itself by squirting it out. I know they have seen this effect a number of times in the TREAT experiments.

With regard to the desirability of flow reversal, we got melting mostly in the upper half of the bundle. This is above the point of flow reversal. If fuel below that point were melting and there was still enough coolant movement to have one of these flow reversals, then it would tend to force the fuel downward, which would also be in the right direction.

I am just worried about the flow reversals from the standpoint of ejecting sodium. I do not know if it would lead to a negative worth or positive worth. If we have a positive void coefficient, then the flow reversals can be a reactivity insertion mechanism.

As regards the reduction in heating, I think that there is a tendency to freeze too soon and possibly hang up on some of these supports. We always seem to get a concentration of lead at those supports. If heat were still being generated so as to maintain the molten state, probably different things would happen.

One of the things we kind of wanted to do at the outset of this program was to use induction rather than resistance heating. This would have been an advantage with regard to the very point that you raise. We would have had an induction field which is quite analogous to a neutron field as long as the fuel stayed within the induction field. It would be molten. It would have made the simulation somewhat better, although I do not think it would have drastically changed the net results. 


\title{
INELUENCE OF THE DOPPLER EFFECT ON \\ THE MELT DOWN ACCIDENT
}

\author{
B. Wolfe, N. Friedman, and D. Riley \\ General Electric Company \\ Atomic Power Equipment Department \\ San Jose, California \\ (Paper presented by Mr. Wolfe)
}

\begin{abstract}
The influence of the Doppler effect in the core-disassembly process following a meltdown accident is examined with a Bethe-Tait-type model in which the Doppler effect, as well as core disassembly, is considered in the reactor shutdown process.
\end{abstract}

By means of a digital computer, the power excursion and reactor shutdown are followed starting at the time that a threshold energy density $Q^{*}$ is achieved at the core center and the reactor is above prompt critical by an excess reactivity $k_{0}$. As in the standard Bethe-Tait treatment, pressures tending towards disassembly are assumed proportional to the energy density above $Q^{*}$. The Doppler effect is taken to have either a $T^{-1 / 2}$ or a $\mathrm{T}^{-3 / 2}$ temperature dependence. The reactor power, Doppler reactivity, and the reactivity reduction produced by disassembly are all followed in time.

In the Bethe-Tait treatment with no Doppler effect, the energy release is a function of one parameter, $X$, which depends upon the core properties and is proportional to

$$
\mathrm{k}_{0}^{3} / \mathrm{l}^{2}
$$

where $l$ is the neutron lifetime.

In the presence of a Doppler effect, the energy release is dependent not only on the parameter $X$, but also directly on $k_{0}$. It is shown that, as the Doppler effect is increased for a given $k_{0}$, the mechanism determining energy release passes through a transition, being determined at one extreme by the core disassembly process and at the other extreme solely by the Doppler effect. In the latter case, significant core disassembly occurs only after the power is reduced to a low value by the Doppler effect. Where the Doppler effect is determining, the energy release depends primarily on $k_{0}$ and not on the reactor parameters. As one result, it is shown that for the case of a constant reactivity insertion rate, in the presence of a strong Doppler coefficient, the energy release is reduced as the neutron lifetime is reduced. 
In the absence of a Doppler effect, strong pressures are required to overcome the inertia of the core, whereas a strong Doppler effect provides time for core disassembly by much lower pressures. Thus, with the Doppler effect, attention must be focused on regions of low-pressure buildup which can normally be ignored, to good accuracy, when no prompt temperature coefficient is present.

As a quantitative example, the worst hypothetical accident described in the Fermi Hazards Report is recalculated as a function of the Doppler effect. Maximum explosive energy releases are reduced from about $6501 \mathrm{~b}$ of high explosive energy with no Doppler effect to less than $20 \mathrm{lb}$ in the case of a Doppler coefficient with

$$
\mathrm{T} \frac{\mathrm{dk}}{\mathrm{dT}}=-0.01
$$

at the vaporization point.

\section{Introduction}

In this paper we will consider a serious fast reactor accident in which the ultimate shutdown of the reactor is accomplished by destructive disassembly of the core. This is an accident of the type generally classed as a "worst conceivable" or "worst hypothetical." We will show that a strong negative Doppler effect can radically reduce the explosive energy released in such an accident.

The Doppler effect influences the course of an accident in two important ways. First, in an accident involving reactivity insertion, the Doppler effect removes reactivity prior to the point of fuel damage. In many cases, the presence of a strong negative Doppler effect can prevent entirely major damage which would otherwise result from a given accident. In his paper tomorrow, Dr. Cohen will discuss such situations. In our opinion, this ability of the Doppler effect to prevent otherwise serious core damage is the major attribute of a strong negative Doppler coefficient.

In the present paper, however, we assume that, due to core melting or other reactivity-insertion mechanisms, the reactor is still above prompt critical at the time that substantial pressure starts to be generated in the core. Further, we assume that all control mechanisms will be inoperative so that, in fact, the ultimate shutdown of the reactor must come from core disassembly.

We shall consider the accident from the point in time when initial pressure is generated in the core. Thus, the reactivity present at this time has already been reduced by the negative Dopplex effect and heat generation up to the point that pressure is generated. 
Under the most pessimistic circumstances, however, there is some justification for ignoring reactivity reduction by the Doppler effect prior to pressure buildup. One can, for example, imagine a reactivity addition which occurred after the molten state had been reached and the fuel temperature had risen to a value just below the threshold value for pressure generation. Secondly, one might consider a reactivity ramp of unlimited extent. In this case, if one ignores delayed neutrons, the presence of a strong negative Doppler effect produces a series of power peaks with the maximum power and maximum reactivity attaining identical values on each peak. Thus, one can imagine that on the Nth peak the threshold temperature for pressure generation is reached. On the $N+1$ st power pulse, therefore, the excess reactivity is the same as that which would be present if there were a reactivity ramp initiated after the reactor were already at the threshold temperature. (In actual fact, the delayed neutrons will damp the power oscillations.) We can consider both of the situations discussed above as limiting and pessimistic, and in general, realize that a given excess reactivity at the time of pressure buildup is equivalent, in the case of a strong negative Doppler coefficient, to a more serious accident if there were no negative Doppler coefficient.

In the analysis to follow, we will make use of the Bethe-Tait formalism as modified by Jankus. $(1-3)$ The Bethe-Tait approximation involves as sumptions regarding the hydrodynamics of the core during the disassembly process. The assumptions have been discussed by McCarthy etal. (1) Stratton etal., (4) Nicholson, (3) and in most detail by Jankus. (2) We will not delve into such matters in this paper. However, on the basis of the Jankus work, (2) it appears that the method is adequate for the work described herein.

A second assumption which is generally made in the Bethe-Tait analysis is that of a threshold equation of state for the materials in the core. Here it is assumed that the pressure buildup is proportional to energy density above a threshold energy density $Q^{*}$, i.e., $P=(\gamma-1)\left(E-Q^{*}\right)$, where $(\gamma-1)$ is a proportionality constant and $E$ has units of energy per unit mass. For reasons discussed below, we will retain this threshold equation of state in the analyses of this paper.

The only published work on the influence of the Doppler effect in a meltdown accident is that of Nicholson, who found, as one might hope, that the Doppler effect does indeed greatly reduce the energy release in the meltdown accident. In order to increase the accuracy of his results, Nicholson abandoned the threshold equation of state and the analytical approach of the Jankus-Bethe-Tait method. Instead, he allowed the pressure to fall off smoothly as the energy censity fell below the threshold value and utilized numerical techniques to follow the excursion. However, as Nicholson noted, this attempt at greater accuracy causes the results to depend upon each of the many parameters of the theory individually. It complicates the treatment to the point at which physical insight is lost and the functional 
dependence of the results on the parameters of the problem is not obtained except by pexforming many individual calculations.

The present study is aimed at a general understanding of the action of the Doppler effect during the core-disassembly process rather than an investigation restricted to the details of a particular reactor. We have found that by retaining the threshold equation of state and modifying only slightly the standard Bethe-Tait analysis, it is possible to express results in terms of a small number of general parameters. We believe that for the purposes of this study, this advantage outweighs the overestimate in energy release obtained for cases having small energy releases in the presence of small Doppler coefficients.

\section{Equations of State}

Although our study is intended to produce results of general interest, we have chosen as a point of reference the Fermi reactor. In the study, we have utilized two different threshold equations of state. We consider a reactor loaded with metallic uranium fuel and have utilized the threshold equation which was suggested for the worst hypothetical accident in the Fermi Hazards Analysis: $\gamma-1=0.98$, and $Q^{*}=0.98 \times 10^{10} \mathrm{ergs} / \mathrm{gm}$. This equation of state (hereafter referred to as $A$ ) assumes that there is no pressure buildup until the temperature reaches about $14,000^{\circ} \mathrm{K}$ in the fuel. All energy densities, i.e., $Q^{*}$, are measured from the liquid state at the melting point. As Nicholson has shown (and as we will show shortly), in the absence of a Doppler effect, pressures corresponding to these high temperatures are generally achieved in serious accidents, since the inertia of the core prevents rapid disassembly until extremely high pressures are achieved. On the other hand, there will be some initial pressure generation at the vaporization temperature, or shortly thereafter, and in the presence of a strong Doppler effect, it is these low pressures which eventually disassemble the core. Thus, we have utilized another threshold equation of state, $B$, which takes the vaporization temperature of uranium as the threshold temperature. We have chosen the pressure-energy slope $(\gamma-1)$ in $B$ so that the pressure in the two equations of state are identical at an energy one and one-half times the threshold energy density of equation $\mathrm{A}$, i.e., at $\mathrm{E}=1.5 \times 0.98 \times$ $10^{10} \mathrm{ergs} / \mathrm{gm}$. For equation $\mathrm{B}_{8} \gamma-1=0.192$ and $\mathrm{Q}^{*}=3 \times 10^{\circ} \mathrm{ergs} / \mathrm{gm}$. The need to consider the lower threshold arises physically from the fact that, with a strong Doppler effect, the excursion is essentially terminated by the Doppler effect, which then provides time for core disassembly by relatively low pressures. It is these relatively low pressures which, in the presence of a strong Doppler coefficient, ultimately disassemble the core. To good accuracy the low pressure buildup can be ignored for serious accidents when there is no prompt temperature coefficient.

The various equations of state are shown in Fig. 1. 


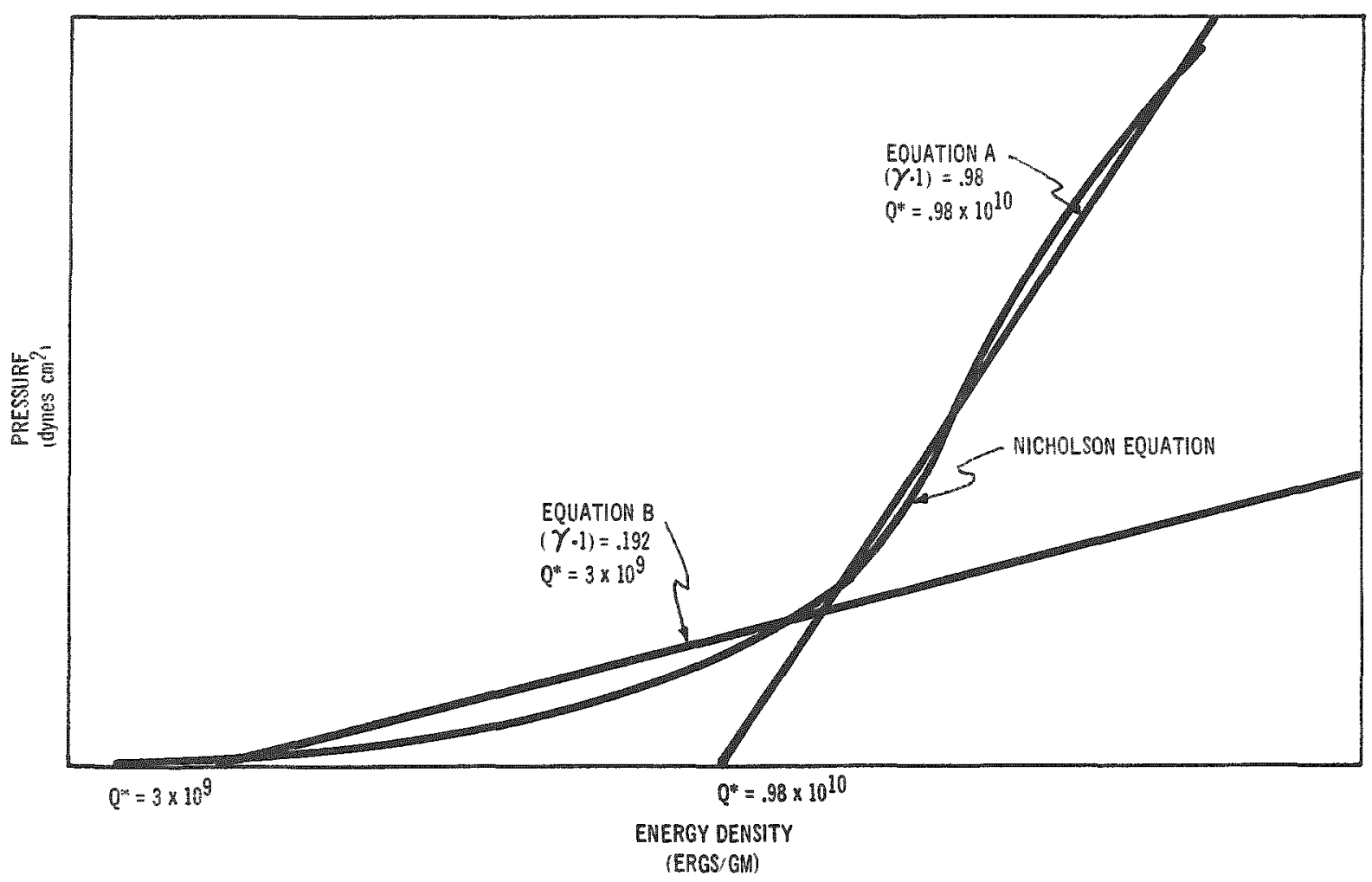

Fig. 1. Equations of State Discussed in This Paper. Equation A is that used in Fermi Hazards Analysis. Equation B approximates the fact that some pressure will be generated from the start of vaporization. Nicholson's (analytic) equation attempts to approximate both the saturation pressures at low energy density and the rapid buildup of pressure after the fuel forms a single phase occupying all available space.

\section{Mathematical Formulations:}

Doppler reactivity reduction

The rate of change of reactivity due to the Doppler effect can be expressed as

$$
\frac{d k}{d t}=-\left(\frac{d k}{d T}\right)_{T_{0}}\left(\frac{T_{0}}{T}\right)^{n} \frac{d T}{d t}
$$

where $-(\mathrm{dk} / \mathrm{dT})_{\mathrm{T}}$ is the Doppler temperature coefficient at temperature $T_{0}$ and $t$ is time. The Doppler coefficient is assumed to decrease in magnitude inversely as the $n^{\text {th }}$ power of the temperature $T$, measured from absolute zero. 
In this work we will consider the course of an accident from the time when the energy density $Q^{*}$ is achieved at the core center. If $T_{0}$ is taken as the temperature at which the energy density $Q^{*}$ is achieved, then (1) can be rewritten as

$$
\frac{d k}{d t}=-\left(\frac{d k}{d T}\right)_{T_{0}} \frac{\left(T_{0}\right)^{n}}{\left(T_{0}+\frac{Q-Q^{*}}{C_{v}}\right)^{n}} \frac{d}{d t}\left(\frac{Q-Q^{*}}{C_{v}}\right) .
$$

In deriving (2), we ignore changes in the volume of the core during the excursion and use the heat capacity at constant volume, $\mathrm{C}_{\mathrm{V}}$. This is in the spirit of the Bethe-Tait approximation although in principle it would cause us to overestimate the Doppler reactivity reduction. Actually, when the Doppler effect is of major significance, we shall show below that the important Doppler reactivity reduction does occur before significant core motion occurs. Thus, the error in our use of $\mathrm{C}_{\mathrm{v}}$ will not be due primarily to a question of principle, but rather to the fact that we do not have good values of $\mathrm{C}_{\mathrm{v}}$ at the temperatures of interest. By algebraic manipulation, (2) can be rewritten as

$$
\frac{d k}{d t}=-\alpha \frac{A^{n-1}}{(A+y)^{n}} \frac{d y}{d t}
$$

where

$$
y=\frac{Q-Q^{*}}{Q^{*}} ; A=C_{V} T_{0} / Q^{*} ; \alpha=-T_{0}\left(\frac{d k}{k T}\right)_{T_{0}} .
$$

Finally, taking $k_{0}$ as the reactivity above prompt critical when $Q^{*}$ is achieved, expression (3) can be put in the following form:

$$
\frac{d \Gamma}{d \tau}=\frac{-\alpha}{k_{0}} \frac{A^{n-1}}{(A+y)^{n}} \frac{d y}{d \tau}
$$

where

$$
\Gamma=\mathrm{k} / \mathrm{k}_{0} ; \tau=\left(\mathrm{k}_{0} / \mathrm{l}\right) \mathrm{t}
$$

and $l$ is the prompt neutron lifetime. The reason for this choice of the form of (4) will become apparent below.

The Bethe-Tait Equations:

Because there are several $(1-3)$ fairly complete treatments of the Bethe-Tait analysis as modified by Jankus, we do not repeat the work here. 
However, throughout this paper we have carried the notation of reference (3) so that the reader may examine the Bethe-Tait analysis and the present work with the convenience of a consistent notation.

The Bethe-Tait equations have been written by Nicholson(3) in the form

$$
\begin{aligned}
& \frac{d^{2} \kappa}{d \tau^{2}}=\frac{y+1}{x}\left[\frac{y}{(y+1)}\right]^{5 / 2} \quad \text { for } 0<y<\frac{q}{1-q} \\
& \frac{d^{2} \kappa}{d \tau^{2}}=-\frac{5 q^{3 / 2}}{2 X}\left[\left(1-\frac{3}{5} q\right) y-\frac{3}{5} q\right] \quad \text { for } y>\frac{q}{1-q} ; \\
& \frac{d^{2} y}{d \tau^{2}}-\kappa \frac{d y}{d \tau}=0
\end{aligned}
$$

where $K$ is the reactivity above prompt critical measured in terms of the initial reactivity $k_{0}$. Thus, $K=1$ at $T=0$, i.e., at the time that $Q^{*}$ is first achieved in the core. Similarly, other boundary conditions are $y=0$ at $\tau=0 ; \mathrm{dy} / \mathrm{d} \tau=1$ at $\tau=0 ;$ and $\mathrm{d} K / \mathrm{d} \tau=0$ at $\mathrm{y}=0$.

Equation (5) describes the change in reactivity due to disassembly of the core, whereas equation (6) is the standard equation (in Nicholson's notation) relating rate of change of power to reactivity. Equations (5) and (6) depend only upon the parameter $\mathrm{X}$ and, weakly, on $\mathrm{q}$.

In deriving (5), the assumption was made that the reactor power shape in the (spherical) core is of the form

$$
P(r)=1-q \frac{r^{2}}{a^{2}}
$$

where a is the core radius and $\mathrm{q}$ is a constant. Similarly, the reactivity worth function of core material was also assumed parabolic and of the form

$$
W(r)=W(0)-\frac{d r^{2}}{2 a^{2}}
$$

The expression for $\mathrm{X}$ is given by

$$
X=\frac{5 q^{3 / 2}}{8 \pi a d(\gamma-1) Q^{*}} \frac{k_{0}^{3}}{l^{2}} .
$$

Thus, for a given reactor, $X$ is proportional only to $k_{0}^{3}$. The value of $y$ achieved at the end of the excursion (i.e., the energy density) increases monotonically with $\mathrm{X}$. 
Jankus (2) has shown that according to one-group theory, the dependence of $\mathrm{d}$ on core size is such that $\mathrm{X}$ increases with increasing core size. Thus, the energy release for a given $k_{0}$ will increase more rapidly than the core mass as the core is made larger assuming $q, b,(\gamma-1)$, and $Q^{*}$ remain unchanged.

The Doppler effect can be included in the above framework by modifying equation (6) to read

$$
\frac{d^{2} y}{d \tau^{2}}-(\kappa+\Gamma) \frac{d y}{d \tau}=0
$$

where $\Gamma$ is obtained from equation (4). Equations (4), (5), and (6a) then constitute the Bethe-Tait equations including a prompt temperature (Doppler) effect. For the convenience of the reader, the equations are repeated below:

$$
\begin{aligned}
& \frac{d \Gamma}{d \tau}=-\frac{\alpha}{k_{0}} \frac{A^{n-1}}{(A+y)^{n}} \frac{d y}{d \tau} ; \\
& \frac{d^{2} \kappa}{d \tau^{2}}=-\frac{y+1}{X}\left(\frac{y}{y+1}\right)^{5 / 2} \text { for } 0<y<\frac{q}{1-q} ; \\
& \frac{d^{2} \kappa}{d \tau^{2}}=-\frac{5 q^{3 / 2}}{2 X}\left[\left(1-\frac{3}{5} q\right) y-\frac{3}{5} q\right] \text { for } y>\frac{q}{1-q} ; \\
& \frac{d^{2} y}{d \tau^{2}}-(\kappa+\Gamma) \frac{d y}{d \tau}=0 .
\end{aligned}
$$

The equations are to be solved with the initial conditions

$$
\mathrm{y}(0)=0 ; \quad \kappa(0)=0 ; \quad \Gamma(0)=0 ; \quad \frac{\mathrm{dy}(0)}{\mathrm{d} \tau}=1 ; \quad \frac{\mathrm{d} \kappa(0)}{\mathrm{d} \tau}=0 .
$$

For the work described in this paper, the above equations have been solved simultaneously on a digital computer. Starting with the initial conditions, the equations were numerically integrated in short-time steps. The integrations continued until the reactor power fell below a preset value, after which the computation was terminated. In all cases discussed herein, the criterion for terminating the calculation was set at dy $/ \mathrm{d} \tau=1 \times 10^{-6}$, i.e., the calculation terminated when the power dropped to one millionth of the initial power level. In all cases it was observed that the energy density $y$ was not changing appreciably during the period prior to termination of the calculation. The value of $y$ at the end of the calculation measures the energy density achieved at the core center and is algebraically related to the total energy release in the excursion. 
In order to make the points of this paper in concrete terms, we have used the Fermi reactor as a model and have used the parameters given in the Fermi Hazards Report. (5) Thus in all cases, except as noted, the following values have been assigned to the various parameters:

$$
\begin{aligned}
& a=45 \mathrm{~cm} ; \\
& d=5.6 \times 10^{-6} \mathrm{~cm}^{-3} \\
& l=1 \times 10^{-7} \mathrm{sec} ; \\
& q=0.8 .
\end{aligned}
$$

For equation $\mathrm{A}$,

$$
\begin{aligned}
Q^{*} & =0.98 \times 10^{10} \mathrm{ergs} / \mathrm{gm} ; \\
(\gamma-1) & =0.98 ; \\
A & =1.6 .
\end{aligned}
$$

For equation $B$,

$$
\begin{aligned}
Q^{*} & =0.3 \times 10^{10} \mathrm{ergs} / \mathrm{gm} ; \\
(\gamma-1) & =0.192 \\
A & =1.9 .
\end{aligned}
$$

$\underline{\text { Results }}$

We have performed a number of calculations to illustrate the effect of a Doppler coefficient on a meltdown accident. Calculations have been performed as a function of the initial reactivity above prompt critical at the threshold energy density and as a function of the Doppler coefficient. We have characterized the Doppler coefficient by the quantity $\alpha$, which is equal to $-\mathrm{T}(\mathrm{dk} / \mathrm{dT})$ at the threshold temperature $\mathrm{T}_{0}$.

In the large (several thousand-liter) cores presently proposed for fast power reactors, ${ }^{(6)}$ the Doppler coefficient exhibits a temperature behavior approximately proportional to $1 / T$, so that $T(\mathrm{dk} / \mathrm{dT})$ is independent of temperature. Greebler (7) and others have calculated values of $T(\mathrm{dk} / \mathrm{dT})$ in excess of 0.01 for power reactors of interest. On the other hand, for smaller reactors and for very high temperatures, $\mathrm{dk} / \mathrm{dT}$ is proportional to $1 / \mathrm{T}^{3 / 2}$. A number of parallel calculations were performed for the two situations $[\mathrm{n}=1$ and $\mathrm{n}=3 / 2$ of equation (4)]. Our calculations indicate that the important quantity is $\mathrm{dk} / \mathrm{d} T$ at $\mathrm{T}_{0}$ and that the temperature dependence has a significant influence only for cases of an intermediate Doppler coefficient; that is, for very small coefficients there is, in any event, little effect on energy release, while for very large coefficients the excursion is terminated before the temperature rises a significant fraction of $T_{0}$. Thus, the temperature dependence of the Doppler coefficient plays its major role in determining the value of the Doppler coefficient at the temperature of initial pressure generation. 
WOLFE et al.

In the case for which the Doppler coefficient is zero $(\alpha=0)$, our work is equivalent to the normal Bethe-Tait analysis and the energy release depends only upon the Bethe-Tait parameter $X$. On the other hand, in the presence of the Doppler coefficient, the energy release depends not only upon $\mathrm{X}$, but also upon the initial reactivity $k_{0}$. Results of a set of calculations are shown in Fig. 2. Hexe we have plotted the parameter $y=\left(Q-Q^{*}\right) / Q^{*}$ against the Doppler coefficient for different values of $X$. For a given reactor, $X$ is a function only of $k_{0}^{3}$. However, in order to gain physical insight, we have for each $X$ value changed $k_{0}^{3}$ by a factor of ten in each direction, while at the same time keeping $X$ constant by making a corresponding change in one of the other parameters in $X$ (it matters not which). The solid line in Fig. I corresponds to the parameters of the Fermi reactor, the dashed lines to parameters modified so that with $k_{0}^{3}$ increased (or decreased) by a factor of 10, X remained unchanged. * The important result shown in Fig. 2 is this:

For small $\alpha$, the energy released depends primarily upon $\mathrm{X}$; how ever, for large $\alpha$, the energy released becomes independent of $X$, but depends only upon the initial reactivity $k_{0}$. This can be seen by noting that for $\alpha=0.01$ the various curves with the same $k_{0}$ asymptotically approach each other. Physically what is happening is that for large $\alpha$ the Doppler coefficient terminates the excursion and reduces the power level to a low value. The pressures generated up to this time, although small, now have time to overcome the inertia of the core and "gently" blow the reactor apart. Thus, in the presence of a strong Doppler coefficient, the energy generated becomes independent of the details of the pressure-energy relationship provided only that enough pressure is generated to eventually disassemble the core. (In the case of a continuing reactivity ramp, enough pressure would have to be generated to disassemble the core and terminate the ramp before another power pulse would occur.)

This point is further illustrated in Table I, which shows the results for a typical case at the time that the excursion has been turned around and the power level has returned to the power level at the threshold condition. It can be easily shown that if the Doppler coefficient were temperature independent and were alone responsible for the reactor shutdown, then at the time when the power level had returned to the initial power level, the Doppler effect would have reduced reactivity by an amount equal to twice the initial excess reactivity. In the case in which the Doppler coefficient decreases with temperature, greater energy release, but less reactivity reduction, occurs. In Table I it can be seen that for $\alpha=0$, the reactivity reduction due to disassembly of the core is about 5 times the initial reactivity for the case considered. If the Doppler coefficient increases in magnitude, the reactivity reduction by disassembly gets smaller while the

*It may make the point clearer to note that in practice when $k_{0}^{3}$ was increased by a factor of 10 , $d$ was also increased to keep $X$ unchanged. The results would have been identical if we had increased $b^{2}$. 


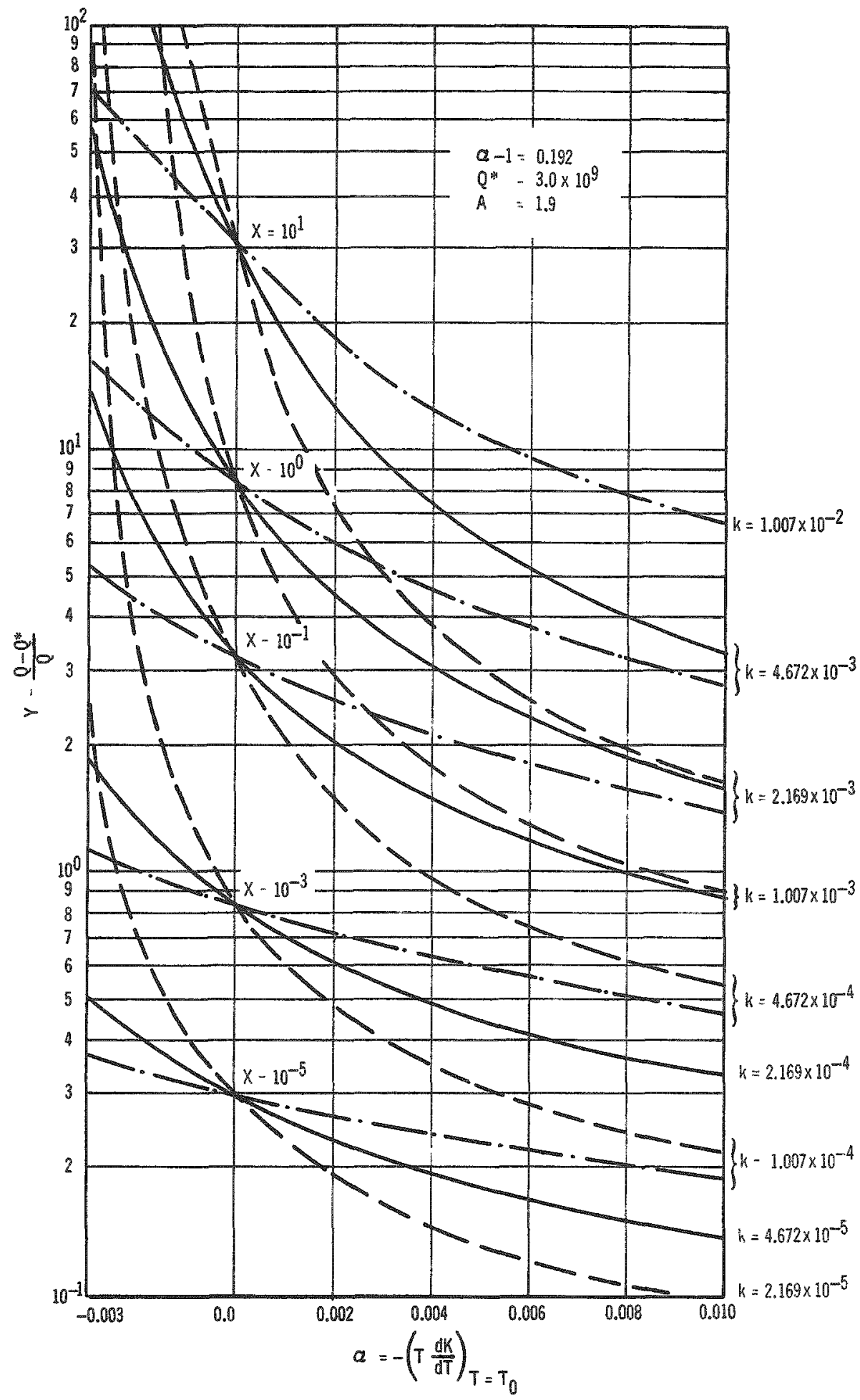

Fig. 2. The Energy Density Achieved at the End of the Excursion as a Function of the Bethe-Tait Parameter X and the Doppler Fffect. The solid line represents the Fermi reactor model as given in the Fermi Hazards Report. $X$ is proportional to $k_{0}^{3}$. The dashed lines were obtained for cases where $k_{0}^{3}$ was increased or decreased by a factor of ten while at the same time one of the reactor parameters was also changed to leave $\mathrm{X}$ unchanged. 
WOLFE et al.

reduction caused by the Doppler effect gets larger. For the large Doppler coefficient, the power reduction can be attributed almost entirely to the Doppler effect.

Table I

REACTIVITY REDUCTION WHEN POWER LEVEL IS REDUCED TO INITIAL POWER LEVEL

$$
\begin{aligned}
\mathrm{k}_{0} & =0.0013 \\
Q^{*} & =3 \times 10^{9} \mathrm{ergs} / \mathrm{gm} \\
A & =1.47 \\
N & =3 / 2
\end{aligned}
$$

\begin{tabular}{c|c|c}
\hline \multirow{2}{*}{$\begin{array}{c}\text { Doppler Effect } \\
\alpha\end{array}$} & \multicolumn{2}{|c|}{ Reactivity Reduction* due to: } \\
\cline { 2 - 3 } & $\begin{array}{c}\text { Core Disassembly } \\
(1-\kappa)\end{array}$ & $\begin{array}{c}\text { Doppler Effect } \\
\Gamma\end{array}$ \\
\hline 0 & 5.23 & 0 \\
0.001 & 3.055 & 0.66 \\
0.005 & 0.104 & 1.74 \\
0.01 & 0.0044 & 1.88 \\
\hline
\end{tabular}

* Measured in units of $k_{0}$

To summarize, then, in the case of a strong Doppler coefficient, the enexgy release becomes essentially independent of the parameters of the problem and depends primarily on the reactivity at the start of pressure buildup and the magnitude of the Doppler effect.

One other point of interest may be noted from Fig. 2. Whereas, with a strong negative Doppler effect, the inserted reactivity, and not the reactor parameters, determines the energy release, the situation is reversed in the case of a positive prompt temperature coefficient. Here the governing reactivity is not that at the start of the excursion, but the reactivity inserted during the excursion. The energy release then depends not so much on $\mathrm{k}_{0}$ as on the other parameters in X. Thus, in Fig. 2, where we have decreased $k_{0}^{3}$ and kept $X$ constant bychanging other parameters, the energy release increases for a positive temperature coefficient. With a positive coefficient, a short neutron lifetime, a low rate of pressure buildup with energy, or any other condition which causes a high temperature before disassembly, will obviously increase the energy release. 
Energy Release in a Hypothetical Accident

To explore the effect of the Doppler coefficient on the meltdown accident further, we have calculated the worst hypothetical accident described in the Fermi Hazards Report as a function of an assumed Dopplex coefficient. In this case, the initial reactivity above prompt critical is taken to be 0.0013 , and again the parameters of the reactor described in the hazards report have been used. We have performed the calculations with the two different threshold equations of state, $A$ and $B$, and have cast the results in several different forms.

Figure 3 shows the excess energy above threshold for the two equations of state as a function of the Doppler coefficient. It can be seen that

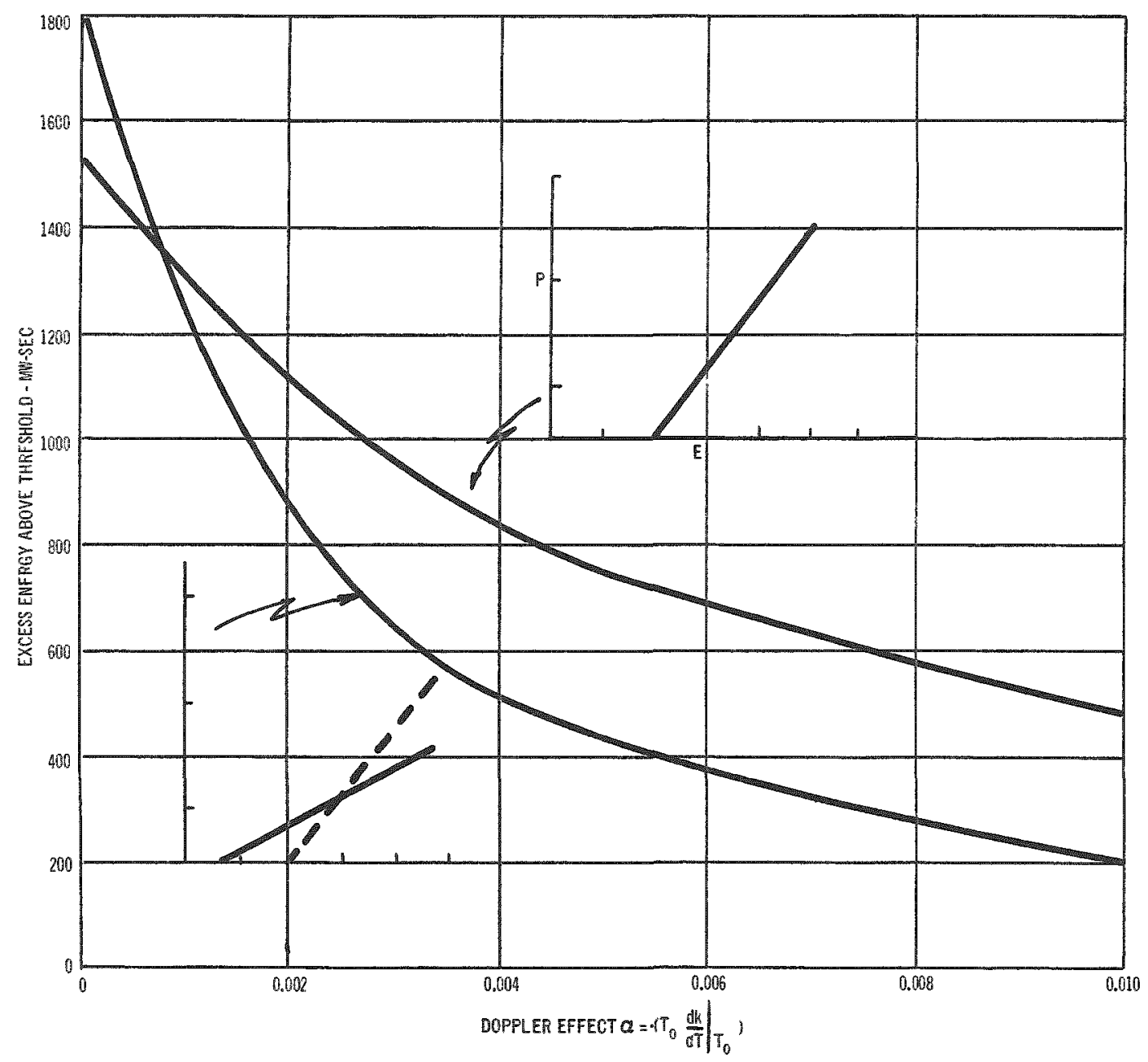

Fig. 3. Excess Energy Release above Threshold for Equation $A$ and Equation $B$ with $k_{0}=0.0013$ and $\mathrm{n}=3 / 2$. In each case, the excess energy is measured from the threshold appropriate to the equation used in the calculation. For small $\%$, equation $A$ is more effective in term nating the excursion because pressure buildup is more rapid once the threshold is exceeded. At large $x$, equation $A$ is less effective because its higher threshold temperature means a lower Doppler coefficient for the same value of $a$. 
for a zero coefficient there is actually greater energy release above threshold for the case of the lower threshold energy. As previously noted, this is due to the fact that with no Doppler coefficient, high pressures must be generated to overcome the inertia of the core. The equation with the high threshold (equation A) involves more rapid pressure generation above the threshold and thus causes the disassembly of the core at a lower value of excess energy. With a large Doppler coefficient, on the other hand, the energy release depends only upon the Doppler coefficient for a given initial reactivity. For a given value of $\alpha$, the Doppler coefficient is smaller for the high-threshold case than for the low-threshold case. This explains the crossover of the curves as $\alpha$ is increased.

The excess energy above threshold does not measure directly the damage potential of the accident since much of the energy produced cannot be converted into work. Jankus (2) has estimated the fractional energy available for work for a threshold equation of state by assuming an isentropic expansion to the threshold energy. We have used Jankus' method to convert the results shown in Fig. 3 into curves of maximum explosive energy as shown in Fig. 4. It must be noted that these are maximum values which in an actual case will be reduced, since the expansions will be isentropic.

The curves of Figs. 3 and 4 measure, in each case, the energy above the assumed threshold values. To assess the situation more realistically, we should, in both cases measure the energy from the same value. This is done in Fig. 5, in which the total energy release is measured from the vaporization temperature for both equations of state. We expect from Nicholson's work that for a zero Doppler coefficient the energy release will be close to that of the upper curve. On the other hand, with a strong Dopplex coefficient, we expect that the actual energy release will be closer to the lower curve. To illustrate this point, we have approximated the true situation by the dashed curve shown in the figure.

Brout(8) has estimated the maximum work available from vaporized uranium under an isentropic expansion, and his results have been interpreted in the form of an analytical curve by the APDA group. (5) We have applied the APDA analytical curve to the results of Fig. 5 in order to obtain an upper estimate to the explosive energy which could be produced in the accident. The results are shown in Fig. 6, where again we have taken the liberty of drawing a dotted line to indicate what we consider to be an approximation to the true situation.

It should be noted that the Brout results were obtained for the very high temperatures resulting for the case with no Doppler coefficient. We have taken an unwarranted liberty in extending them to the much lower temperatures resulting when there is a strong Doppler coefficient. In this case, we would expect that the Jankus method would have more validity, so 


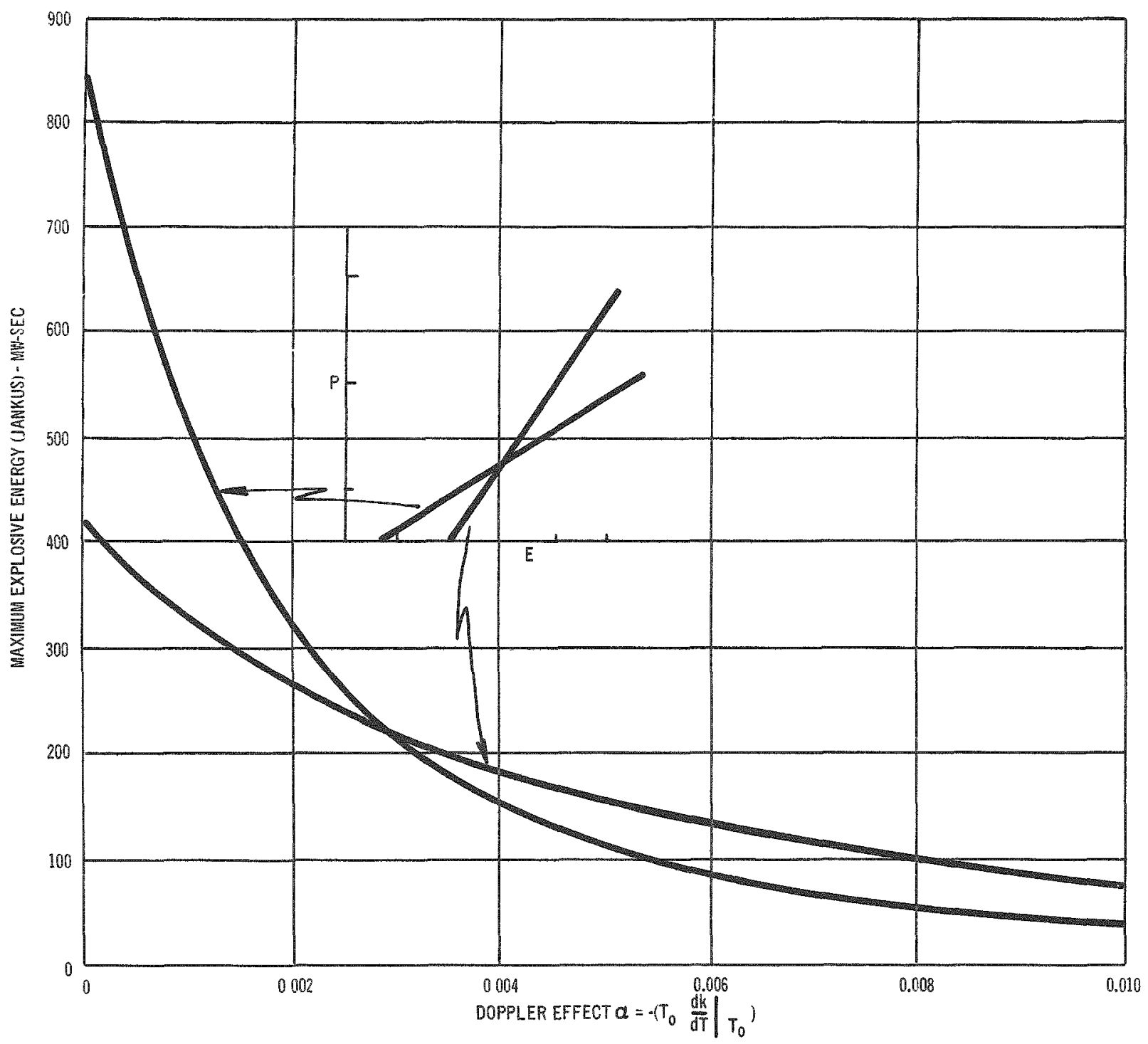

Fig. 4. The Maximum Release of Explosive Energy as a Function of the Doppler Effect for Equations A and B. In each case, the work available from the excess 4 nergy of Fig. 3 has been estimated by assuming an isentropic expansion with the Jankus model. 
WOLFE et al.

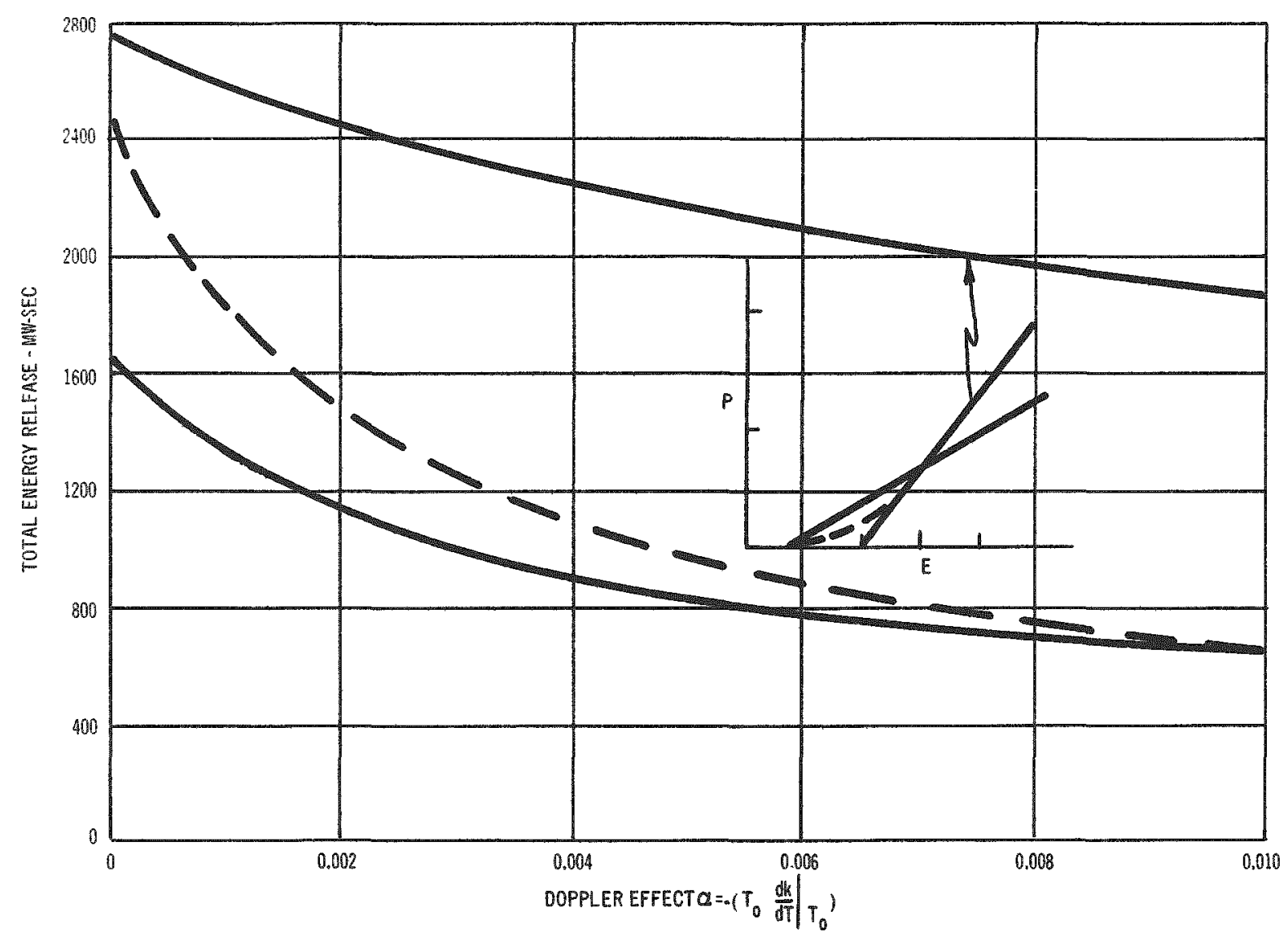

Fig. 5. Total Energy Release Measured from the Melting Point of Uranium for the Fermi Worst Hypothetical Accident, $k_{0}=0.0013$. The upper curve results with equation $A$, the lower with the equation of state $B$. The dashed curve is estimated to be close to the true situation. 


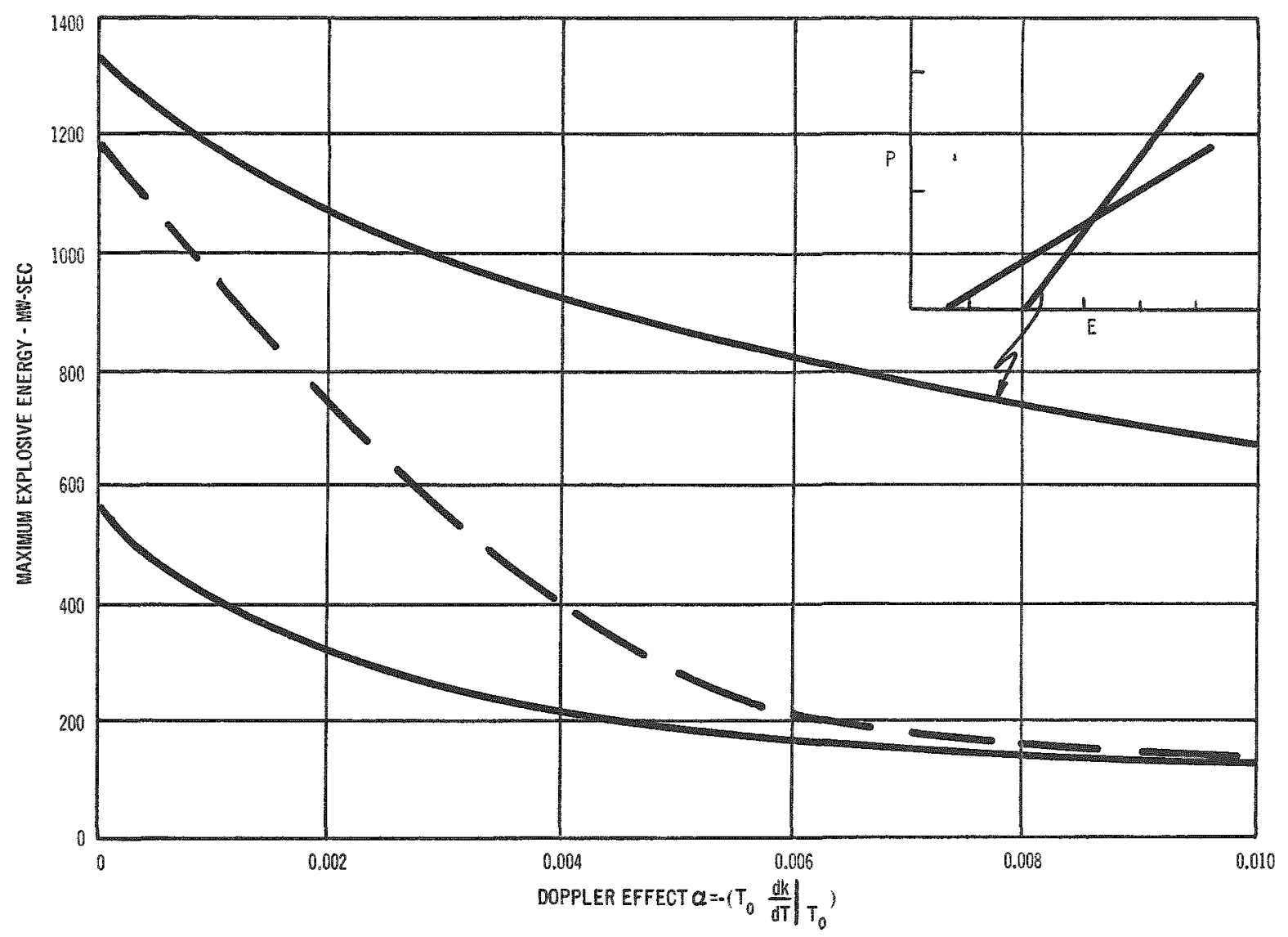

Fig. 6. Maximum Explosive Energy vs. Doppler Effect. The explosive work available from the total energy of Fig. 5 has been estimated by use of Bout's calculations as interpreted by APDA. For the high values of $\alpha$ and equation $B$, the Jankus estimate (Fig. 4 ) is probably more accurate.

that the maximum explosive energy release would be reduced further over the dashed line shown in Fig. 6. Thus, the calculations indicate that the maximum explosive energy release would be reduced from a figure of about $1350 \mathrm{MW}-\mathrm{sec}$ with no Doppler coefficient to a figure close to $35 \mathrm{MW}-\mathrm{sec}$ for a case with a Doppler coefficient having an $a$ value at threshold of 0.01 . Again we note that the actual explosive release would be reduced fusther by the less than ideal expansions involved.

\section{Conclusions}

The Fermi (5) and EBR-II(9) hypothetical meltdown accident studies indicated that (maximum) explosive energies equivalent to several hundred pounds of high explosives could be produced. Studies of these reactors have indicated the feasibility of designing reactor structures to contain such energy releases. However, as indicated in the EBR-II analysis, an energy release in excess of several hundred pounds of high explosives makes 
difficult a design which will assure the integrity of the reactor containment. The Fermi containment is designed to withstand 1,000 lb of high explosive release. Since with no Doppler effect the energy release increases faster than the core mass, containment of large power reactors 10 to 20 times larger than the Fermi reactor poses a serious problem. However, in the present work it has been shown that a negative Doppler effect of the magnitude calculated for these large power reactors can reduce the energy release by factors of 10 or more, so that the containment problem again becomes manageable.

Other conclusions are:

1. In the presence of a strong negative Doppler effect, the energy release per unit mass of core becomes essentially independent of the reactor parameters and depends only on the inserted reactivity at the point where substantial pressure buildup is initiated.

2. In the presence of a strong Doppler effect, the energy release from the meltdown accident can be terminated by a relatively low pressure buildup. The pressure-energy relation must, therefore, be understood for temperatures well below those normally of importance in the absence of a Doppler coefficient.

3. If one relies on the Doppler effect to reduce the energy release, it is advantageous to have a short neutron lifetime. This is because the energy release depends only on the inserted reactivity at the threshold of pressure generation. The inserted reactivity, on the other hand, assuming a reactivity ramp insertion, will increase with increasing neutron lifetime, as shown by McCarthy et al. (1)

4. In the presence of a strong Doppler effect, the energy release is linear with reactor mass. The energy per unit mass does not increase as (McCarthy et al., indicate) would be the case with no Doppler coefficient.

Finally, the authors wish to point out that the analysis does not consider the effect of a positive sodium coefficient. If, in the disassembly process, it were possible to blow out sodium preferentially from regions where positive reactivity would result, this would, in some respects, resemble the situation of an accident with a positive Doppler coefficient and might lead to a greatly increased energy release. 


\section{References}

1. W. J. McCarthy, R. B. Nicholson, D. Okrent, and V. Z. Jankus, Studies of Nuclear Accidents in Fast Power Reactors, Proc. 2nd UN Intn1. Conf. on Peaceful Uses of Atomic Energy, Geneva, Switzerland, 12, 207 (1958).

2. V. Z. Jankus, A Theoretical Study of Destructive Nuclear Bursts in Fast Power Reactors, ANL-6512 (Feb 1963).

3. Richard B. Nicholson, Methods for Determining the Energy Release in Hypothetical Reactor Meltdown Accidents, APDA-150 (Dec 1962).

4. W. R. Stratton, T. H. Calvin, and R. B. Lazarus, Analysis of Prompt Excursions in Simple Systems and Idealized Fast Reactors, Proc. 2nd UN Intnl. Conf. on Peaceiul Uses of Atomic Energy, Geneva, Switzerland, 12, 196 (1958).

5. Power Reactor Development Co., Enrico Fermi Atomic Power Plant, Technical Information and Hazards Summary Report, Volume 7, Section VI - Evaluation of Hazards.

6. K. M. Horst, B. A. Hutchins, F. J. Leitz, and B. Wolfe, Core Design Study for a 500 MWe Fast Oxide Reactor, GEAP-3721 (Dec 1961).

7. P. Greebler, Recent Improvements in Calculations of Doppler and Sodium Reactivity Effects for Large Fast Reactors, presented at this conference.

8. R. H. Brout, Equation of State and Heat Content of Uranium, APDA-118 (1957).

9. L. J. Koch et al. Hazard Summary Report - Experimental Breeder Reactor II, ANL-5719 (1957).

[Editorial Note: Dr. Wolfe's paper as given above was submitted in revised form after the conference and differs somewhat from the oral presentation. In order to provide continuity with the discussion, the following paragraphs are cited from the transcription.]

"With no Doppler effect, there is an energy release of 1,200 megawatt seconds which would be equivalent to about 600 pounds of high explosive; with a Doppler effect of 0.01 , which is about the value quoted for large dilute reactors, the energy release comes down to something like 150 megawatt seconds which is roughly 75 pounds TNT. 


\begin{abstract}
"Now, if I used Jankus" method of estimating the explosive energy available from the total energy release, this value of 75 pounds is further reduced, and I end up with something closer to 20 pounds of high explosive. I believe the latter figure is nearer to the true situation than the curve shown in the slide.

"The conclusion then is that with a strong Doppler effect, the classical meltdown accident can be reduced to a value which is es sentially one which can be handled using normal construction prac tices and with normal amounts of shielding. That is 20,50, or something under 100 pounds of TNT can be accommodated in general without doing very much to the reactor structure one would have with normal shielding. On the other hand, for example, the EBR-II people found that if their accident release reached something over 300 pounds of high explosive, they had difficulty showing their containment could take it. So the conclusion is that with a large negative Doppler effect, one can reduce the magnitude of the accident."
\end{abstract}

Discussion of Paper

Presented by Mr. Wolfe

MR. OKRENT (Argonne):

Do I understand that your calculations were done for a reactor of the size of a Fermi reactor?

MR. WOLFE:

Yes, I used the Fermi numbers directly.

MR. OKRENT:

Well, then, it seems to me that in a sense you have been optimistic because you took the negative Doppler coefficient of a 3,6 or 8,000-1iter reactor, but the dimensions of the small one, and neglected the fact that the yield by the same Bethe-Tait model goes up rapidly with core size, faster than the volume. So that I think that one does not really know what to make of the numbers you present. I am afraid one might generate an undue wave of optimism to offset the undue wave of pessimism we had this morning.

I as sume that the value of -0.01 for $T(d k / d T)$ [see Abstract] is in the presence of sodium or some moderator that maintains this appreciably negative Doppler coefficient. Was sodium present under the conditions you are talking about? 
MR. WOLEE:

I am glad you brought up the first point because I did not point this out, but the effect of the large Dopplex coefficient is to negate to some extent the point you are making. Namely, with the large Doppler coefficient, the energy yield per core mass does not go up with core mass, that is, it becomes independent of the value of the $X$ parameter. Thus it becomes in dependent of lifetime and core mass. The other point you make is a valid one. According to this model, the energy release should be directly proportional to core mass if one assumed that the accident involved the whole core in each case. On the other hand, it is very probable that in these very large reactors the whole core will not be really involved, and it will be a small local part of the core which will in fact melt down and cause the pressure generation.

I did want to make one other point, too, and that is that with a large Doppler effect you attain an independence from neutron lifetime. If one analyzes an accident, he finds that, if it is a ramp accident, you put more reactivity in with a longer lifetime, but the energy release is dependent only on the reactivity put in. It is not of benefit to have a long lifetime if one depends on the Doppler effect. It's a deficiency.

MR。OKRENT:

I did not quite understand. Did you say that in the event of a large Doppler coefficient, assuming that one is present, the yield would be independent of core size?

MR.WOLEE:

The yield per unit mass does not increase with the core size. The yield would go up only as the mass involved in the excursion.

MR. OKRENT:

But one would have a factor of 10 or 20 between core volumes.

MR. WOLFE:

Yes

MR. OKRENT:

400 liters to 4,000 , let us say. 
MR. WOLFE:

Yes. The accidents that one would generally expect to occur would probably involve only a small part of the core in the actual excursion, not a large part. In other words, probably local core meltdown would occur.

MR. MILLER (Argonne):

Recent studies in the physical behavior of metals by Eyring and by von Grosse have shown that some of the equations of state which have been proposed for metals, which had been based on normal materials such as hydrocarbons and the like, will be inaccurate under the conditions that one finds in metals. Generally the net effect is to show that the critical temperature, for instance, which normally is about 50 per cent higher in absolute temperature than the boiling point, in the case of liquid metals will run somewhere between 2.75 to about 5 times the boiling temperature. In general what this means is that the pressures will be somewhat reduced from the ones that had been estimated, using critical properties estimated from non-metallic materials, as was done by Elrod and by Brout.

MR. WOLFE:

Yes, I think that is right. There are some data that suggest that with liquid metals thecritical points will be higher. On the other hand, there is some recent work reported at the Vienna Conference on Metals that indicated that Brout's assumptions might be somewhat pessimistic. I do not want to get into a discussion of the equation of state here. The point I am making is that with the Dopplex effect, one is going to become much more sensitive to the pressure buildup at much lower temperatures and pressures than at 0.8 of the critical temperature, say, which is the usual assumption in the meltdown accidents. 


\section{H. Böhm}

Institut fi̛r $r$ Neutronenphysik and Reaktortechnik

Kernforschungszentrum, Karls ruhe, Germany

(Paper presented by Mr. Ott)

\section{Introduction}

The energy release in after-meltdown excursions is investigated in the framework of the Bethe-Tait model with the use of a refined equation of state. With a sufficient degree of accuracy the equation of state can be approximated as a polynomial in powers of the energy.

The nonlinear system of differential equations describing the time behavior of the neutron flux during the second excursionis solved analytically.

This permits an easy investigation of the dependence of the energy release on all the parameters involved; composition, void fraction, critical mass, ramp rate, initial flux, and uncertainties of the equation of state.

As examples, the dependence on initial flux and ramp rate for a system containing fuel and fertile material in the ratio $1: 5$ are given.

II. Method of Analysis

\section{Simplifying As sumptions}

The energy release of a fast reactor excursion after meltdown is studied with a simple spherical model. As in the basic work of Bethe and Tait, the model is assumed to be a homogeneous mixture of fissionable and nonfissionable materials, as well as of voids. This configuration shall just be in a prompt-critical state.

Reactivity is inserted into the system, initiating the power excursion. Whereas below prompt critical the reactivity will increase by an arbitrary function of time, it is assumed that in a fast reactor the reactivity increase over prompt critical should be sufficiently well approximated by a linear function of time, i.e., a ramp

$$
\alpha(t)=\frac{a}{1} t(\alpha=\text { inverse period })
$$

In the kinetic equation the term for the delayed neutrons is ignored. As Häfele(1) has shown, it is, however, possible to take into account the influence of the delayed neutrons by substituting the real initial flux at prompt critical by a pseudo-flux which is twice the real flux. 
The critical mass of the molten system is strongly influenced by the quality of the reflector, but the active reflector is not well defined. Therefore, the mass of the critical assembly before the second excursion has to be regarded as a parameter within a reasonable range.

For describing the excursion a one-group diffusion equation is applied; effective macroscopic one-group cross sections are determined by 16-group calculations.

The flux is assumed to be separable in space and time, and according to Bethe-Tait, the space distribution is approximated by a parabola:

$$
\begin{aligned}
\phi(r, t) & =\phi(r) n(t) ; \\
n(r) & =n_{0}\left(1-\frac{q}{b^{2}} r^{2}\right),
\end{aligned}
$$

where $\mathrm{b}$ is the core radius and $\mathrm{q}$ a shape parameter.

For integration of the hydrodynamical equations, an equation of state must be known, giving a relation of internal energy and pressure with constant density. As the system contains various materials and the specific heat is not well known, the real equation of state describing the model is fairly uncertain. Therefore it should be adequate to approximate the equation of state by a cubic parabola:*

$$
p(E)=\rho\left(a_{0}+a_{1} E+a_{2} E^{2}+a_{3} E^{3}\right) .
$$

All these simplifications enable one to obtain an analytical expression for the time dependence of the flux.

\section{Hydrodynamical Treatment}

As the reactivity is inserted by a ramp, the flux and the fission energy released increase and cause a rising pressure, which initiates a dynamical expansion of the system. The feedback to the reactivity by the density change is determined by first-order perturbation theory, which is applied to the one-group diffusion equation:

$$
M \phi=\alpha \phi \text {. }
$$

Then the change of the inverse period is given by

$$
\delta \alpha=\frac{\int V^{\phi M \phi d V}}{\int V^{\phi^{2} d V}},
$$

*'Nicholson(2) has applied an exponential type of equation of state, but this permits only a numerical treatment of the problem. 
with

$$
\delta M=v\left[\nabla \delta_{D} \nabla-\delta \Sigma_{a}+\nu \delta \Sigma_{f}\right]
$$

After inserting the flux separation and expressing the density change (in $\delta \Sigma$ ) in terms, of $\delta r$, the change of the coordinate $r$, one finds the following expression after differentiating twice with respect to time:

$$
\begin{aligned}
& \ddot{\alpha}=-\int_{V} \ddot{r} f(r) d r \\
& f(r)=\frac{3 v}{\left(\frac{b}{r q}\right)^{3}(0.0762)}\left[4 D \frac{q^{2}}{b^{2}} r^{2}-\left(\Sigma_{a}-v \Sigma_{f}\right)\left(1-\frac{q}{b^{2}} r^{2}\right)^{2}\right] r .
\end{aligned}
$$

The acceleration of the volume element, $\ddot{r}$, is described by the equation of hydrodynamics:

$$
\rho \ddot{r}+\frac{\partial p}{\partial r}=0 \text { (for spherical geometry). }
$$

In the Bethe-Tait method $\dddot{I}^{2}$ in equation (1) is substituted by the pressure gradient of (2)

$$
\ddot{\alpha}=\int_{V} \frac{1}{\rho} \frac{\partial p}{\partial r} f(r) d r .
$$

The approximation of the equation of state can be meaning ful only for positive pressures. Therefore the total process is subdivided into two phases.

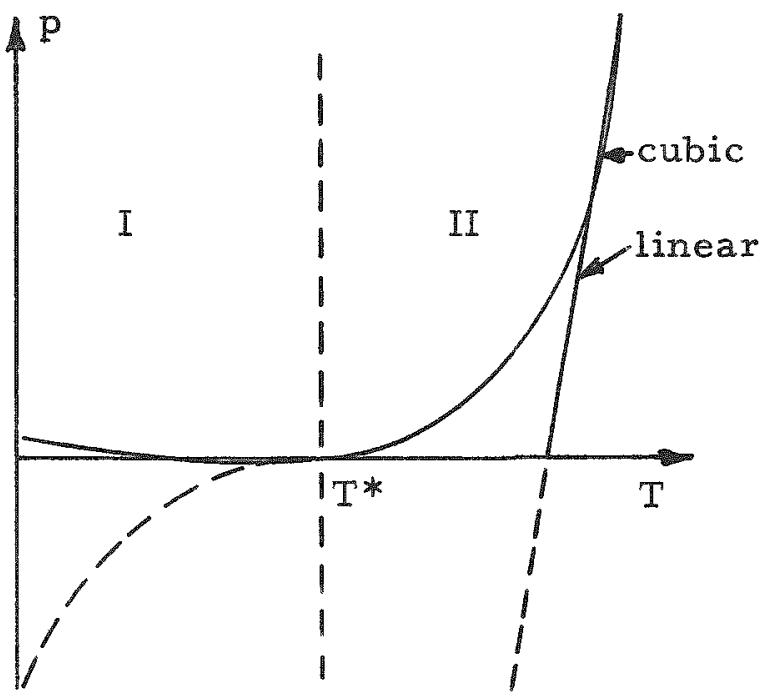

Phase I

During phase I the pressure is arbitrarily set equal to zero, i.e., the feedback Is ignored. When the energy density in the center reaches a threshold, phase II begins and the pressure is computed from the equation of state. In the cubic-polynomial approximation, it is reasonable to choose the threshold energy density slightly above the boiling point of the core material to 
describe a reasonable pressure distribution. In the linear equation of state of the Bethe-Tait method the threshold energy is much higher.

equation

$$
\text { The end of phase I at the time } t=t_{s} \text { is determined by the }
$$

$$
K=\frac{Q^{*}-Q_{0}}{\Sigma_{f}\left(2 \phi_{0}\right)} c \rho=\int_{0}^{t_{s}} e^{(a / z \cdot 1) t^{2}} d t
$$

where $c=$ fissions/erg. For $t_{s}$, Jankus $(3)$ has derived an asymptotic formula:

$$
t_{s}=\left[\frac{1}{a}\left(\ln \frac{a}{1} K^{2}+\ln \ln \frac{a}{l} K^{2}\right)\right]^{1 / 2} .
$$

Phase II

Though the calculations have been made with the cubic type of the equation of state, the method of solving the problem analytically shall be demonstrated with the linear equation of state of Bethe-Tait for sake of simplicity. In this case the pressure gradient is given by the simple expression

$$
\frac{\partial p}{\partial r}=-2 \rho a_{1} \frac{q}{b^{2}} Q(\tau) r
$$

with

$$
Q(\tau)=\frac{\Sigma_{f}\left(2 \phi_{0}\right) e^{(a / 2 \cdot 1) t_{S}^{2}}}{c \rho} I(\tau)+Q^{*} .
$$

Here $I(\tau)$ is the integral over the flux, and the new variable $\tau=t-t_{s}$ has been introduced:

$$
I(\tau)=\int_{0}^{\tau} n\left(\tau^{\prime}\right) d \tau^{\prime}
$$

After inserting this into equation (3), we get together with the kinetic equation, a complete set of two coupled nonlinear differential equations describing phase II of the excursion, in which feedback on reactivity is taken into account:

$$
\begin{aligned}
& \ddot{\alpha}(\tau)=-K_{0}-K_{1} I(\tau) ; \\
& \ddot{I}(\tau)=\alpha(\tau) \dot{I}(\tau) .
\end{aligned}
$$


The unique solution of this set of equations is obtained by the appropriate initial conditions

$$
\begin{aligned}
& \alpha_{0}=\alpha(\tau=0)=\frac{a}{1} t_{s} ; \\
& \dot{\alpha}_{0}=\dot{\alpha}(\tau=0)=\frac{a}{1} ; \\
& I(\tau=0)=0 \\
& \dot{I}(\tau=0)=1 .
\end{aligned}
$$

\section{Dynamical Treatment of Reactor}

To solve the set of equations (4), the first equation is integrated three times with respect to time and the second is integrated once:

$$
\begin{aligned}
& A(\tau)=\int \alpha(\tau) d \tau=-\iiint\left[K_{0}+K_{i} \int \dot{I}(\tau) d \tau\right] d \tau^{3} ; \\
& \dot{I}(\tau)=e^{A(\tau)} .
\end{aligned}
$$

After substituting $\dot{I}(\tau)$, the first equation only depends on the function $A(\tau)$, and this integral equation can be solved by means of iteration:

$$
A(\tau)=-\iiint\left[K_{0}+K_{1} \int e^{A(\tau)}\right] d \tau^{3} .
$$

A first approximation for $A(T)$ is obtained by the assumption that the system expands only under the pressure, built up until the time $t=t_{S}(\tau=0)$. Equation (4) gives for $\ddot{\alpha}(0)$

$$
\ddot{\alpha}_{1}=-K_{0} \text {; }
$$

it then follows from equations (5) and (6) that

$$
A(1)(\tau)=\alpha_{0} \tau+\frac{\alpha_{0}}{2} \tau^{2}-\frac{K_{0}}{3 !} \tau^{3} .
$$

This means that the first three Taylor-series terms are determined by the initial conditions only. Insertion of this first approximation into the righthand side of Equation (7) and expansion of the exponential yields four further correct Taylor-series terms:

$$
\begin{aligned}
\mathrm{A}^{(2)}(\tau)= & \alpha_{0} \tau+\alpha_{0} \frac{\tau^{2}}{2 !}-\mathrm{K}_{0} \cdot \frac{\tau^{3}}{3 !}-\mathrm{K}_{1} \frac{\tau^{4}}{4 !}-\alpha_{0} \mathrm{~K}_{1} \frac{\tau^{5}}{5 !}-\alpha_{0}^{2} \mathrm{~K}_{1} \frac{\tau^{6}}{6 !} \\
& -\left(\alpha_{0}^{3} \mathrm{~K}_{1}-\mathrm{K}_{0} \mathrm{~K}_{1}+\alpha_{0} \alpha_{0} \mathrm{~K}_{1}\right) \frac{T_{7}}{7 !}
\end{aligned}
$$


Normally, $\mathrm{A}(2)(\tau)$ gives a sufficient degree of accuracy. It can easily be seen that each step of iteration brings out four further coefficients in the Taylor expansion for $A(\tau)$, which are exact. This behavior originates from the special type of feedback, in which a $t^{\mathrm{n}}$-influence is fed back in the language of Taylor expansion four powers later, i.e., in the $t^{\text {nt }}$ term: a th reactivity influences the $t^{\text {ntl }}$ term in the flux and this cortributes a $t^{n+2}$ term to the energy expansion. The energy causes, via pressure, $a$ material displacement two t-powers later and so changes the reactivity in the $t^{n+4}$ term.

If $\mathrm{A}(\tau)$ is known, the time dependence of the flux is given by

$$
\mathrm{n}(\tau)=\stackrel{\mathrm{I}}{(}(\tau)=\mathrm{e}^{\mathrm{A}(\tau)}
$$

and the total amount of energy density in the center. released during the excursion, is

$$
E_{\max }=\frac{\Sigma_{\mathrm{f}}\left(2 \phi_{0}\right) \mathrm{e}^{(\mathrm{a} / 2 \cdot 1) \mathrm{t}_{\mathrm{s}}{ }^{2}}}{\mathrm{c} \rho} \int_{0}^{\infty} \mathrm{e}^{\mathrm{A}(\tau)} \mathrm{d} \tau+\mathrm{Q}^{*}
$$

The total energy release is obtained by simple integration over the space distribution.

\section{First Results and Discussion}

As a first example, second excursions of molten spheres containing $48 \mathrm{v} / 0 \mathrm{UO}_{2}, 40 \mathrm{v} / 0$ steel, and $12 \mathrm{v} / 0$ voids have been investigated. The $\mathrm{UO}_{2}$ contains $U^{238}$ and $U^{235}$ in the ratio five to one. The critical assembly was assumed to contain $200 \mathrm{~kg}$ fissionable material representing a farrly wellreflected system.

Because of the lack of an equation of state for this mixture we investigate two limiting cases, between which the true result should lie.

In the first case only the vapor pressure of iron is considered as a cause of the expansion; in the second case, the vapor pressure of $\mathrm{UO}_{2}$ only is taken into account. The other materials only fill the volume. $\mathrm{UO}_{2}$ has a higher vapor pressure than iron, and therefore it is obvious that the real case lies between the two cases mentioned, but at should surely lie nearer to the second one (that with $\mathrm{UO}_{2}$ only).

The total energy release contains, as a background, the energy (denoted by $B$ ) necessary to heat the material (which causes the expansion) up to the point of vaporization (ixon in case 1 and $\mathrm{UO}_{2}$ in case 2). In Table I the energy release exceeding this background value is given: 


$$
B_{\text {iron }}=3.73 \times 10^{3} \mathrm{MW}_{5} ; \quad \mathrm{BUO}_{2}=2.83 \times 10^{3} \mathrm{MW} \text {. }
$$

Shortly after the nuclear excursion the total energy exists as thermal energy. The energy in the system at the point of vaporization (i.e., the background energy) will surely remain as thermal energy, whereas a great deal of the excess energy might be converted into kinetic energy during the further expansion. The exact portion of kinetic energy depends on the surrounding of the critical assembly.

The excess energy can be regarded as an upper limit of the kinetic energy.

Table I

EXCESS ENERGY RELEASE OF SECOND EXCURSIONS

(in units of $10^{3} \mathrm{MW}_{\mathrm{g}}$ )

\begin{tabular}{|c|c|c|c|c|c|c|c|c|}
\hline \multirow[b]{3}{*}{$\begin{array}{ll}\text { Flux } \\
\left(\mathrm{cm}^{-2} \mathrm{~s}^{-1}\right) & \text { Ramp- } \\
\end{array}$} & \multicolumn{8}{|c|}{ Reactivity Feedback from Boiling of } \\
\hline & \multicolumn{4}{|c|}{$\mathrm{Fe}$} & \multicolumn{4}{|c|}{$\mathrm{UO}_{2}$} \\
\hline & 10 & 30 & 100 & $300 \$ / \mathrm{s}$ & 10 & 30 & 100 & $300 \$ / \mathrm{s}$ \\
\hline $10^{4}$ & $\begin{array}{c}5.61 \\
(9500)\end{array}$ & $\begin{array}{c}8.95 \\
(12900)\end{array}$ & $\begin{array}{c}14.46 \\
(18500)\end{array}$ & $\begin{array}{c}22.53 \\
(26750)\end{array}$ & $\begin{array}{c}2.26 \\
(7200)\end{array}$ & $\begin{array}{c}4.33 \\
(10600)\end{array}$ & $\begin{array}{c}7.78 \\
(15000)\end{array}$ & $\begin{array}{c}12.79 \\
(22100)\end{array}$ \\
\hline $10^{6}$ & $\begin{array}{c}4.11 \\
(8000)\end{array}$ & $\begin{array}{c}7.12 \\
(1100)\end{array}$ & $\begin{array}{c}12.09 \\
(16100)\end{array}$ & $\begin{array}{c}19.42 \\
(23600)\end{array}$ & $\begin{array}{c}1.42 \\
(6000)\end{array}$ & $\begin{array}{c}3.12 \\
(8400)\end{array}$ & $\begin{array}{c}6.33 \\
(13000)\end{array}$ & $\begin{array}{c}11.07 \\
(19600)\end{array}$ \\
\hline $10^{8}$ & $\begin{array}{c}2.77 \\
(6600)\end{array}$ & $\begin{array}{l}5.44 \\
(9350)\end{array}$ & $\begin{array}{c}10.0 \\
(14000)\end{array}$ & $\begin{array}{c}16.77 \\
(20900)\end{array}$ & $\begin{array}{c}0.85 \\
(5200)\end{array}$ & $\begin{array}{c}2.04 \\
(6900)\end{array}$ & $\begin{array}{c}4.92 \\
(11000)\end{array}$ & $\begin{array}{c}9.46 \\
(17400)\end{array}$ \\
\hline $10^{10}$ & $\begin{array}{c}1.70 \\
(5550)\end{array}$ & $\begin{array}{c}3.85 \\
(7750)\end{array}$ & $\begin{array}{c}8.04 \\
(12000)\end{array}$ & $\begin{array}{c}14.36 \\
(18500)\end{array}$ & $\begin{array}{c}0.5 \\
(4700)\end{array}$ & $\begin{array}{c}1.22 \\
(5700)\end{array}$ & $\begin{array}{c}3.51 \\
(9000)\end{array}$ & $\begin{array}{r}7.83 \\
(15100)\end{array}$ \\
\hline $10^{32}$ & $\begin{array}{c}1.0 \\
(4800)\end{array}$ & $\begin{array}{c}2.44 \\
(6300)\end{array}$ & $\begin{array}{c}6.09 \\
(10000)\end{array}$ & $\begin{array}{c}12.02 \\
(16100)\end{array}$ & $\begin{array}{c}0.3 \\
(4400)\end{array}$ & $\begin{array}{c}0.7 \\
(5000)\end{array}$ & $\begin{array}{c}2.21 \\
(7100)\end{array}$ & $\begin{array}{c}6.04 \\
(12500)\end{array}$ \\
\hline $10^{14}$ & $\begin{array}{c}0.58 \\
(4400)\end{array}$ & $\begin{array}{c}1.4 \\
(5200)\end{array}$ & $\begin{array}{r}4.11 \\
(8000)\end{array}$ & $\begin{array}{c}9.5 \\
(13500)\end{array}$ & $\begin{array}{c}0.18 \\
(4200)\end{array}$ & $\begin{array}{c}0.4 \\
(4600)\end{array}$ & $\begin{array}{c}1.21 \\
(5700)\end{array}$ & $\begin{array}{r}4.07 \\
(9700)\end{array}$ \\
\hline
\end{tabular}

$M\left(U^{235}\right)=200 \mathrm{~kg}$

$M\left(U^{238}\right)=5 \times 200 \mathrm{~kg}$
$M($ steel $)=925 \mathrm{~kg}$

Void $=12 \mathrm{v} / 0$
()$=$ max temperature, in ${ }^{\circ} \mathrm{K}$, in the center

\section{References}

1. W. Häfele, Nukleonik, 5, 201-208 (1963).

2. R. B. Nicholson, APDA-150 (1962).

3. W. J. McCarthy, R. B. Nicholson, D. Okrent, and V.Z. Jankus, Proceedings of the Second UN Intnl. Conf. on the Peaceful Uses of Atomic Energy, Geneva, Switzerland, 12, 207 (1958). 
Appendix

Equation of State

For the integration of the hydrodynamical equations, an equation of state is necessary, giving a correct $\partial \mathrm{p} / \partial \mathrm{E}$ in the whole energy range. As the model contains voids, the equation ought to describe the range of two phases up to the critical isothermal as well as the real gas range above. The experimental data for uranium dioxide are very scanty and for plutonium dioxide or a mixture of several materials nothing exists at all. Therefore, in the present state of knowledge, only rough approximations are possible. Therefore it should be adequate to approximate the given experimental data on vapour pressure by a cubic polynomial.

To investigate the influence of several materials it seems to be useful to consider two cases. In one case the fuel material and material of cans and subassemblies are slightly inhomogeneous. Only the fuel material is heated and initiates expansion. In the other case, the mixture is extremely homogeneous and expansion is influenced by the vapour pressure of subassembly material, e.g., iron.

For $\mathrm{UO}_{2}$ the following equation of state has been used:

$p=\rho\left(9.921 \times 10^{-23} E^{3}-5.358 \times 10^{-12} E^{2}+9.595 \times 10^{-2} E-5.7 \times 10^{8}\right) \mathrm{dyne} / \mathrm{cm}^{2}$.

For iron the equation of state has been used:

$p=\rho\left(3.056 \times 10^{-23} E^{3}-2.15 \times 10^{-12} E^{2}+5 \times 10^{-2} E-3.831 \times 10^{8}\right) \mathrm{dyne} / \mathrm{cm}^{2}$.

Formulae for the Cubic Equation of State

Whereas the method of analysis has been demonstrated by means of a linear type of equation of state in the preceding chapter, the formulae for the cubic equation of state on which the calculations are based shall now be given. The corresponding equation (4) has now the form:

$$
\alpha=-k_{0}-k_{1} I(\tau)-k_{2} I^{2}(\tau)-k_{3} I^{3}(\tau)
$$

After two steps of iterations, we get for $\mathrm{A}(\mathrm{u})$ with $\mathrm{u}=\alpha_{0} \tau_{\text {: }}$ 


$$
\begin{aligned}
A(2)(u)= & u+s \frac{u^{2}}{2 !}-r \frac{u^{3}}{3 !}-q \frac{u^{4}}{4 !}-(q+2 p) \frac{u^{5}}{5 !} \\
& -\left(b_{2} q+6 p+6 w\right) \frac{u^{6}}{6 !} \\
& -\left(b_{3} q+8 b_{2} p+6 p+36 w\right) \frac{u^{7}}{7 !} \\
& -\left(b_{4} q+10 b_{3} p+20 b_{2} p+60 b_{2} w+90 w\right) \frac{u^{8}}{8 !} \\
& -\left(b_{5} q+12 b_{4} p+30 b_{3} p+20 b_{2 p}^{2}+90 b_{3} w+360 b_{2} w+90 w\right) \frac{u^{9}}{9 !} \\
& -\left(b_{6} q+14 b_{5} p+42 b_{4} p+70 b_{2} b_{3} p+126 b_{4} w+630 b_{3} w+420 b_{2}^{2} w\right. \\
& \left.+630 b_{2} w\right) \frac{u^{10}}{10 !},
\end{aligned}
$$

with

$$
s=\frac{\alpha_{0}}{\alpha_{0}^{2}} ; \quad r=\frac{k_{0}}{\alpha_{0}^{3}} ; \quad q=\frac{k_{1}}{\alpha_{0}^{4}} ; \quad p=\frac{k_{2}}{\alpha_{0}^{5}} ; \quad w=\frac{k_{3}}{\alpha_{0}^{6}} ;
$$

and

$$
\begin{aligned}
& b_{2}=(s+1) \\
& b_{3}=(-r+3 s+1) \\
& b_{4}=\left(-q-4 r+6 s+3 s^{2}+1\right) \\
& b_{5}=\left(-6 q-2 p-10 r-10 s q+15 s^{2}+10 s+1\right) \\
& b_{6}=\left(-16 q s-22 q-30 r-18 p+45 s^{2}+15 s^{3}+15 s+1-6 w\right)
\end{aligned}
$$


Discussion of Paper

Presented by Mr. Ott

MR. TAVERNIER (BelgoNucleaire):

Would not the pressure developed in the accidents considered be very high? I wonder then whether there is not another type of accident to be taken into consideration from the fact that you have compressibility up to the crushing point of the solid surrounding the exploding zones. Does this lead to a secondary nuclear incident?

MR.OTT:

Enlargement of the spherical super-critical mass is fairly small after the nuclear excursion is finished. I think there should be space enough after meltdown that the effect mentioned by you will not be of great importance.

MR. THOMPSON (MIT):

Perhaps this is a generalized question, but I would like to ask if you know how much effect there is by considering that the space and time flux variation is separable. Very often this is used, and I wondered how much of an error you think this makes in the calculation. Does it still make the calculation worthwhile?

MR. OTT:

I think this effect is not very large because higher modes should account for this. Inasmuch as the flux form is only used in a very rough shape, any effect of higher modes cannot be considered. If this rough flux shape can describe the situation, then separability should also be possibly good enough.

MR. WOLFE (General Electric):

I think if there is any effect, it would be to lead to a more conservative energy release because the effect would be to peak the flux more than it was peaked initially. That is at the place where you have added the excess reactivity, you would tend to get a flux peak which would generate pressures sooner and in a more localized area than would be the case if you assumed the flux stayed the same flux shape. Jankus has done some calculations that did in effect take this into account, and he found that if one assumed a uniform reactivity insertion, that for lifetimes something under a few tenths of a microsecond, and reactivity insertions of something like half a per cent or under, as I recall, that he got extremely good agreement between the Bethe-Tait and the more exact AX-1 type calculations. 


\author{
John B. Nims, Jr. \\ Atomic Power Development Associates, Inc. \\ Detroit, Michigan
}

\title{
I. Introduction
}

The problem of meltdown has been the dominating problem in fast reactor safety for many years. The concern over meltdown is based on the fact that fast reactors contain several times a critical mass if the fuel were arranged in a more dense configuration. Because of this, fuel rearrangement presents a potential mechanism for increasing reactivity rapidly and causing an explosive energy release.

The analysis of a fast reactor meltdown accident $(1-5)$ can be considered as a number of separate problems. One of these is the determination of the conditions under which a meltdown will occur. Following this, the next problem consists of calculating the reactivity insertion rate, pres sures generated, fuel movement, and finally the magnitude of the energy release.(1) The final step is to determine how this energy, contained in high pressure uranium gas, is dissipated and to establish whether or not containment would be breached. The entire process is a very complex one and, because of limited information, must be analyzed in an approximate but conservative manner.

The equation of state and thermodynamic properties of uranium(6-10) above $5000^{\circ} \mathrm{K}$ and at pressures of up to several thousand atmospheres are one source of uncertainty in the energy release calculation and in the analysis of the energy dissipation. The critical constants of uranium are estimated from those of other materials rather than from measurements with uranium. The estimates of specific heats of uranium do not include the effect of ionization, which may be important. However, the most difficult part of the determination of the energy release is the prediction of the motion of the fuel, which determines the reactivity insertion rate. To predict this on theoretical grounds would require taking into consideration the failure mechanism of the cladding and the manner in which the fuel escapes from the fuel element; the forces of the coolant and gravity that the fuel is subjected to after it leaves the fuel element; the cooling effect of the coolant vapor or liquid; and the possibility of freezing, sticking, and blockage of the coolant as the fuel moves along the channel contacting subassembly walls, fuel element spacers, support pieces, and blanket rods.

Because of the impossibility of making a realistic theoretical treatment, it has been necessary to postulate hypothetical accidents to use as the basis for analyzing containment. The hypothetical accident, for example, might be based on conservative assumptions such as instantaneous 
melting of the entire reactor core, and gravity collapse of the upper surface of the core while the lower surface remains fixed. While such conservative assumptions and the energy release obtained have made it possible to avoid the necessity of a realistic analysis, this may become an impractical approach in the future. Advanced fast reactor designs have core volumes of several thousand liters as compared with a few hundred liters for contemporary designs. The energy release may be expected to increase roughly in about the same proportion as the volume. In the future it may be possible to show that the Doppler effect is laxge enough to keep the energy release low for those reactor designs which have a sufficiently high $\mathrm{U}^{238}$ concentration and a low energy spectrum component. This will probably not be the case for all fast reactor types. Although the energy release can be expected to increase by a factor of 10 or more, the surface area of the vessel, which is a measure of the volume of the energy absorbent material, will also increase but by a considerably smaller value. The problem of containment can therefore be expected to become a much more difficult task. It appears that a major refinement in the present approach will become necessary.

Another consideration, of increasing importance in the safety of advanced systems, is the positive sodium temperature coefficient.(11) The ability to investigate the reactivity effects of sodium boiling under realistic conditions is becoming most important.

For these and other reasons the study of the technical feasibility of fast reactor meltdown experiments $(12)$ conducted by APDA has special significance.

\section{Experimental Approach}

One way of obtaining the desired information is to build a reactor, initiate a meltdown, and observe the amount of destruction that results. By proper instrumentation, it would be possible to measure the power during the excursion, the pressure generated in the release, and its decay as energy is dissipated by heat transfer, shock effects, mechanical distortion of reactor components, and acceleration of materials by the expanding gas. The major objection to this approach is the cost. The experiment would have to be carried out on a reactor prototype that had all the features of an engineering design, including coolant loops and heat exchangers.

The energy release is undoubtedly very dependent upon the initial conditions and upon details of the design. Certainly, it would be necessary to know that the set of initial conditions which give the maximum energy release had been included in the experiment. Because the worst combination of initial conditions are not known, it would be necessary to perform the experiment many times, varying coolant flow rates, initial power level, burnup of fuel, etc. In addition, to be of real value, the experiments should 
provide the designer with some indication of how the meltdown accident is affected by changes in the detailed design. For example, it may be possible that structures supporting the fuel elements above the core may interfere with the expulsion of fuel, but the consequence of a meltdown could be improved considerably if this structure were modified. Similar examples can be offered for fuel element spacers and axial blanket design.

As an alternative approach, the use of two reactor facilities, each having a different purpose, has been studied. These two facilities have been designated as FARME-A and B (EAst Reactor Meltdown Experiment). In the first facility partial meltdown experiments would be conducted. The reactor, particularly the core region, would be a close approximation of the fast reactor design under consideration. A separate core meltdown region is located in the central part of the core. This region can accommodate capsule experiments of variable size and is designed so that any radioactive contaminants or fuel from meltdown are confined to this loop. In addition, the loop is designed for relatively easy accessibility for changing experiments. The purpose of this facility is to investigate the severity of meltdown as it depends both on the initial conditions and design details by melting a small fraction of a core in each experiment. It is hoped that the set of conditions which give the maximum energy release can be determined from these experiments.

Once these conditions have been obtained for a particular core design, the energy release and the pressure time history during the accident would be determined in the second facility. From the experiments performed in FARME-A, the configuration of the fuel and the rate of reactivity insertion at prompt critical would be deduced if detailed information on fuel motion is obtained. This would permit a simplified mockup in FARME-B. Another possibility is that only the initial conditions yielding the maximum release can be determined in the FARME-A experiments. A detailed mockup in FARME-B would then be necessary,

The FARME-A facility in its preliminary concept could contain a meltdown section of variable size up to about 15 per cent of a core of Fermi Core A dimensions or larger. The limitation on the amount to be melted, other than the physical dimensions of the meltdown region, is the severity of the particular experiment, that is, the proximity of prompt critical during the excursion and the temperature rise in the normal core region. An analysis based on a very pessimistic value of the rate of assembly of fuel in the meltdown section of a metal alloy core indicated that a 4 per cent meltdown could not introduce more than $\$ 1$ in reactivity and that the excursion resulting if the top half of the meltown zone collapsed under gravity into the central region would not damage the normal core. It is probable that larger meltdowns can be tolerated, but this must be determined in the initial experiments. 
A strong effort should be made to obtain direct detailed information on fuel motion. If this can be done successfully, then it should be possible by analytic techniques to predict the reactivity insertion rates for partial and complete meltdowns. The degree of detail with which the fuel motion can be determined is uncertain, and it may be necessary to rely on less direct information.

One of the primary pieces of information that certainly can be obtained is the power trace of the resulting excursion. From this trace, the reactivity variation with time can be determined. This reactivity variation includes the feedback reactivity from the normal core as well as the effect of events taking place within the meltdown capsule. From preliminary experiments, the feedback characteristics of the normal core would be known. From this information and also from temperature sensors located in the normal core, an estimate of the feedback reactivity with time can be determined. The difference between these two is then the reactivity variation due to the fuel motion. By conducting the melting experiment within a core which has the same composition as the one under investigation, the reactivity effect due to the fuel motion requires a minimum amount of interpretation. Of course, this result applies directly only to a meltdown confined to a limited region, and it is necessary to determine what the result would be if an entire core were subjected to the same conditions. From the time-dependent reactivity attributed to the fuel motion and from other data that it may be possible to measure during the experiment, such as the change in power distribution with time, as well as from postirradiation examination of the capsule, a picture could be constructed of what the fuel motion had been, which would then be used to predict what would happen if the meltdown region were enlarged.

A second experiment would then be performed in which a greater amount of fuel is melted. Conceivably, the experiment could be repeated up to the point where 15 per cent of the core had been exposed to melting conditions, with predictions being made of the result to be found in the subsequent step. If the results show that the prediction can be made accurately, then the extrapolation to the full core meltdown may be quite believable.

The results may be less satisfactory. As indicated in the discussion above, it is hoped that a good deal of insight and detailed information on fuel motion during the transient is achievable. Another possibility, less satisfactory but nevertheless quite useful, is that the experiments will yield es sentially only qualitative information. Even though of this nature, if it is possible to distinguish the severity of the different accidents so that at the end of the series of FARME-A experiments on a given design it is possible to state one set of conditions that give the maximum energy release, this would be adequate information. For example, the experiments may show 
that as long as coolant flow is present, the fuel will disperse and shut down the reactor. This information would then eliminate all classes of meltdown accidents except for the loss-of-sodium accident.

Another possibility is that for the complete meltdown, the supercritical configuration that results is a secondary configuration which is quite dissimilar to that represented by the experiment. In this case, the reactivity determinations obtained in FARME-A cannot be extrapolated to an entire core meltdown, and the FARME-A results would be used to determine the time dependent fuel configuration; the reactivity insertion rate at prompt critical would be obtained analytically.

\section{Facility Description}

The purposes of the facility are to allow partial meltdowns of fast reactor cores to be accomplished in a controlled manner and to provide the maximum amount of information on fuel reassembly rates and consequent reactivity changes. The greatest possible flexibility must be incorporated into the facility so that fast reactors of present or future interest can be studied. In practice, this means the ability to accommodate a large size range both of full cores and the partial core being investigated in the meltdown region.

The following ground rules were established:

1. The core sizes to be accommodated would lie in the range from 200 to 3000 liters. The radial blankets will be of minimum size and may even be replaced by reflectors for the large cores.

2. The maximum fuel size of the meltdown region is 15 per cent of the core volume. This value is somewhat arbitrary, but is consistent with the objectives of achieving reactivity control in small cores, of preventing secondary criticality of the molten fuel, and reducing the expense of meltdown experiments in large cores.

3. The meltdown region must be completely isolated from the normal core. This, in effect, means inserting a loop through the center of the reactor, consisting essentially of the meltdown capsule and test fuel, a heat exchanger, pump, and associated piping. The meltdown region must have guaranteed containment to prevent contamination of the normal core. No damage should be suffered by the normal core; this is equivalent to preventing fuel melting or sodium boiling in the normal core.

4. The meltdown region must be readily accessible for handing, as its contents are the major variable in each experiment, resulting in connections to the loop that are easily detachable. 
5. The normal core need have only limited accessibility. Major changes would be undertaken only at the start of a new test series on a different core. The reactivity variation between individual experiments will be minimized by adjusting the amount of fissionable material or absorber in the transition core. Small variations will be handled by the control system.

6. Contamination of the loop from the meltdown region is to be avoided by means of suitable traps and settling tanks.

7. The fuel in the normal core should be the same general type as that being tested in the meltdown region to ensure that the reactivity effect of the fuel motion during the meltdown would be approximately the same as in an actual accident where the melting zone is limited.

8. The hydraulic conditions in the meltdown region need not necessarily be identical with those in the normal core. Flexibility in design of end gaps and axial blankets is thereby achieved, allowing direct investigation of their effect on meltdown. Limited subcooling of the normal core, or overheating of the meltdown region, can also be easily achieved to facilitate the experiment.

The reactor and essential auxiliary equipment would be situated in an exclusion area having a radius of about 1 mile. The inherent hazardous nature of the experiments make an exclusion area imperative. Furthermore, even though the facility may be situated in a remote area, it is advisable to provide containment around the reactor to prevent a large area from becoming contaminated in case of an accident. This is dictated mainly by the possible use of plutonium and irradiated fuel in the reactor.

Operation of the reactor would be conducted from the support facilities located outside the exclusion area. The main facilities are the control room, inspection building, decay and storage area, and laboratory. A railway connects the reactor and support facilities to provide transport of fuel subassemblies, meltdown capsules, and other equipment. The control room would include all the usual features for reactor startup and operation, and, in addition, a wider range of special instrumentation and control devices required for the transient experiments.

The layout of the reactor and meltdown loop are shown in Figs. 1 and 2. The sodium system for only the meltdown loop is shown; that for the normal core is straightforward and is assumed to pose no unusual problems. The power generated will be dissipated to cooling water or the atmosphere. 


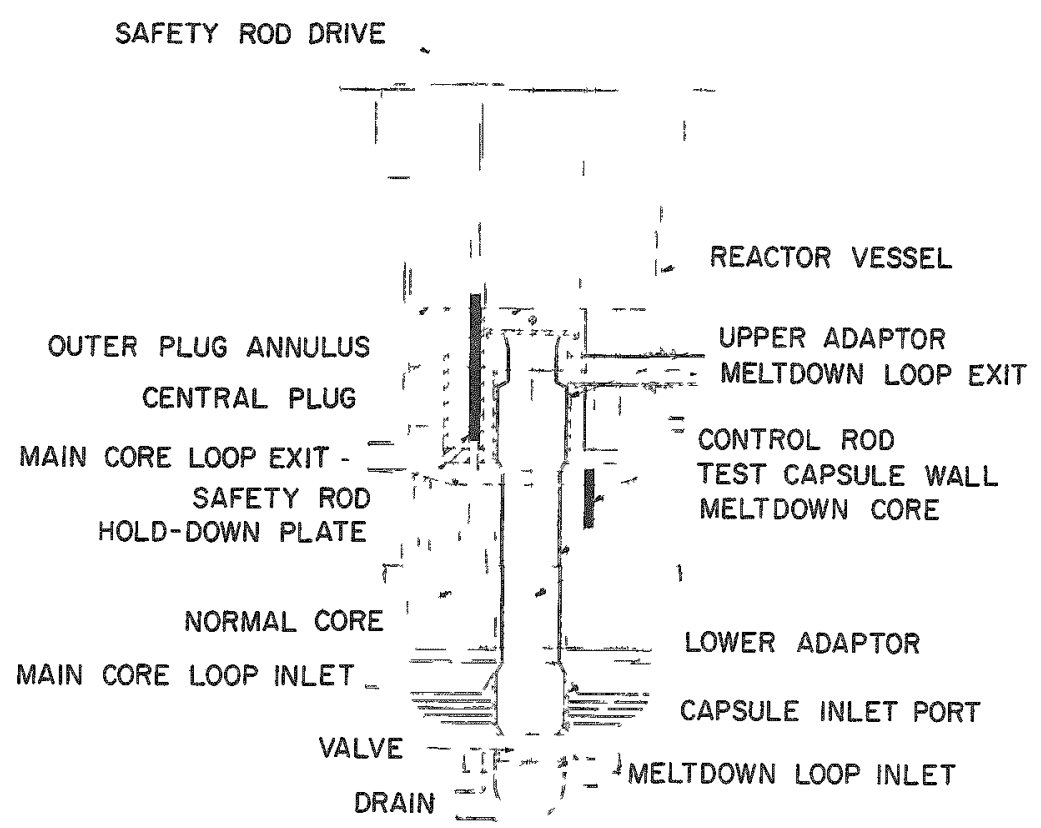

Fig. 1. Reactor Details

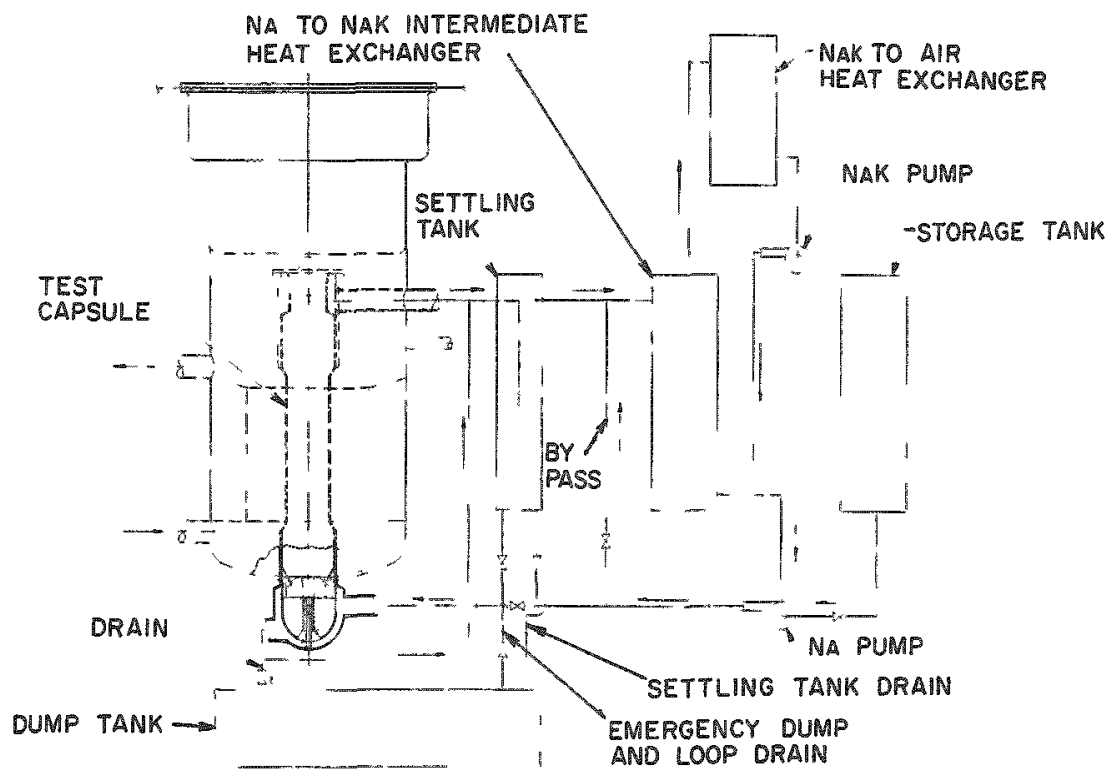

Fig. 2. Schematic Diagram of Meltdown Loop

The reactor building contains an inner and outer containment vessel. The latter serves the usual containment purpose, whereas the former, positioned directly over the reactor, serves as additional containment and also as an enclosed space in which an inert atmosphere can be maintained during operations requiring removal of the reactor vessel cover. 
The reactor is contained in a pressure vessel of a size that will accommodate the largest core to be used in the experiments. It will be designed to adapt to smaller cores also. A typical core would be composed of a normal core which surrounds a meltdown region and, in most experiments, a transition core. Separate loops supply sodium coolant to the normal core and meltdown region. Coolant to the transition core comes from the loops that supply the normal core.

In the center of the core is an interchangeable capsule that contains the part of the core to be subjected to melting conditions. The capsule is slipped into the core region vertically and, when in place, physically separates the portion to be melted from the remaining core with a $\frac{1}{2}-$ in.-thick tantalum wall. Located in the lower end of the capsule is a sliding valve which moves upward as the capsule is positioned in the lower bonnet that extends below the reactor vessel. This action uncovers holes in the capsule wall through which the coolant flows. As the capsule is removed, the valve slides downward and covers these holes so that particulate matter cannotescape from the capsule into the normal core region.

Holes in the capsule wall near the upper end complete the coolant flow circuit through the capsule. No valve is provided here because the capsule will be handled in a vertical position, as shown on Fig. 2 .

It is unnecessary that the subassemblies in the meltdown capsule contain any features not having an influence on the meltdown. Thus, it is conceivable that these subassemblies can be built to less stringent tolerances than standard core subassemblies. Attached to the capsule wall is the surrounding transition core which must be used whenever the diameter of the meltdown capsule is less than the maximum permitted by the upper and lower adaptors. These adaptors, which are supported from the reactor vessel, position the capsule while providing a seal between the meltdown loop and the main core loops. Provision may be made for changing the upper adaptor to permit the use of capsules of a different diameter in case the range of core sizes makes this necessary.

The normal core for a given series of experiments consists of subassemblies which are supported by a pair of lower structural plates. Above, a removable mechanical hold-down is provided to offset hydraulic lifting forces on the subassemblies. This hold-down structure is attached to a removable shield plug. Because the hold-down plate cannot be continuous and still be removable, owing to the suspension structure for the upper adaptor and the lateral pipe of the meltdown loop, subassemblies below these components will possibly be interlocked with neighboring subassemblies that fall under the hold-down fingers. These fingers extend downward from the hold-down plate. 
In addition to providing cooling capacity of the meltown region in the steady state, it is essential that during the transient the sodium flow in the loop simulates the hydraulic conditions in an actual reactor meltdown. Its essential feature is that in most meltdown accidents, in which a small portion of the core first begins to melt, the pressure drop across the subassemblies is relatively unaffected by the meltdown, at least initially, because of the large parallel flow path in the nonmelting core. The melting fuel is then subjected to hydraulic forces corresponding to this pressure drop. It is imperative that these conditions be simulated in the meltown loop.

The most direct simulation is to provide a bypass flow across the meltdown region. At first sight, it may appear necessary to provide a total flow in the combined meltdown plus bypass loop equal to that for the reactor size and power level being tested. It has been established, how ever, that the flat characteristic curves typical of liquid metal pumps allow considerable relaxation of this condition. In fact, the bypass flow need be no greater than that in the meltdown region.

Another, but less important, hydraulic condition to be simulated is the free surface at the sodium pool of most fast power reactors, which will be essentially provided by the free surface at the exit pipe from the capsule. Another free surface is provided in the setting tank which is available in case the one in the meltdown capsule is eliminated by a rapid transient. The settling tank provides the further function of retaining any particulate matter, such as molten fuel, carried over during a transient. It is proposed to $r$ un the inlet pipe to the bottom of the settling tank and place the outlet at a higher level so that the fuel and cladding particles will settle down at the bottom of the settling tank as a result of their weight. It is also proposed to drain the settling tank after one or more meltdown accidents, depending on the quantity of uranium which may be allowed to accumulate in the settling tank. The concept of a settling tank is preferred to that of a filter because of the unavailability of a suitable material for a filter and because of difficulties associated with the removal and replacement of filter elements.

The remaining major features of the primary meltdown loop consist of the heat exchanger and pumps, which do not pose any special problems. A nominal initial heat-removal capacity of $300 \mathrm{MW}$ and a maximum melt down region size of 15 per cent of the core volume has been chosen for the facility. The flow in the meltown region is $4500 \mathrm{gpm}$, with an equal flow in the bypass.

Minor features of the primary meltdown loop are cold traps, plugging indicators, a dump tank, and piping for draining and purging the meltdown region. A storage tank is provided to supply clean make-up sodiun to the loop. 
The secondary system of the meltdown loop can be quite conventional, with the heat being dissipated to the most readily available sink.

\section{Experimental Program}

There are four basic types of fast reactor accidents to be studied in FARME-A. The first of these is a failure of the primary system flow. This event, with the simultaneous failure to scram while the reactor is at power, could lead to gross melting of fuel. The power density and decay rate of the flow will determine when melting starts. If melting occurs when there is still a significant amount of coolant, it is important that this be simulated correctly, because the hydraulic forces will have an effect on the movement of fuel. At the other extreme, melting may not occur until the coolant flow has stopped completely. Experiments should be performed at different power densities and flow decay rates to determine the effect of hydraulic forces on the motion of the molten fuel and the corresponding reactivity variation.

Another situation that requires investigation is the initiation of meltdown by means of an unplanned reactivity insertion. This could happen as a result of a control rod being driven out at runaway speed or by the dropping of a core subassembly. In most actual fast reactors, these possibilities are far-fetched circumstances. Therefore, it may be difficult to specify an actual design accident to simulate. A parametric set of experiments may be desirable in which the rate of reactivity insertion, the total amount inserted, the initial power level, and the flow rates are the important variables.

Another type of accident is loss of sodium. Such an occurrence is believed to be incredible in reactors based on present-day designs because of the double containment specified. It would be useful in future designs to know the seriousness of a loss-of-sodium accident, because if it is not serious, the cost savings to be realized in eliminating double containment could be appreciable.

Different ruptures in the primary system could be simulated by draining the meltdown capsule at different rates. In this accident, fuel melts as the sodium level drops and flows down to the new sodium level, where it probably freezes. What happens in detail is not certain. The fuel may run out the bottom of the subassembly, it may stick to the cooler surfaces it encounters below the sodium level and act as a plug to further fuel movement, or it may pile up at some lower elevation and eventually form a supercritical assembly.

A fourth type of accident is the blockage of flow in one or, at most, a few subassemblies. The flow blockage could be caused by the plugging action of some extraneous piece or pieces of material in the primary 
system. Another possible cause is localized excessive fuel swelling, which might eventually result in melting and additional restriction of flow by the transfer and freezing of fuel in the coolant passages above or below the location of the melting. In this case, it is important toduplicate the hydraulic forces of the coolant and to include the provision for flow to bypass the plugged or partially restricted channel, as would be possible in the actual case because the motion of the fuel is dependent on the force exerted on it by the coolant. For example, if the fuel tries to refreeze and plug, and if bypass is not possible, a large pressure increase will occur and exert a greater force on the fuel than in the case with bypass. With bypass, the pressure exerted would be just the inlet pressure in the inlet plenum instead of a pressure approaching the shut-off head of the pump.

The possibility of fuel element failure in one subassembly spreading to neighboring subassemblies will also be investigated in this type of experiment. It is possible that the molten fuel in a subassembly that had its coolant supply cut off could, by melting, work its way through the subassembly walls to adjacent subassemblies which were being adequately cooled and operating satisfactorily. The entry of the molten fuel could then cause blockage in these previously unaffected subassemblies and induce melting in them. If this spreading mechanism can actually happen, a relatively mild event then becomes quite serious.

In addition, this facility offers a unique capability for investigating the transient effects of positive sodium void coefficients. The processes of void formation and of void distribution with time in a given channel and in neighboring channels are very complicated and cannot be analyzed accurately. The sequence of events is important in a boiling accident for which the core sodium coefficient is positive. Voids will form first in parts of the core and shortly thereafter in the axial blanket before the core section is completely voided. A sodium void in the blanket will tend to compensate for positive effects in the core. Thus, the timing of the void formation from one channel to the next and the distribution of the void space within a given channel are important.

\section{Intexpretation of the Experiments}

Following a typical experiment conducted in the meltdown facility FARME-A, the data available for interpretation are at least the reactivity inserted through control rod motion, the total reactor power as a function of time, temperature measurements in the outer zone, and the final accounting of the distribution of the fuel which was melted during the transient. In addition, there would be experimental information on the transfer function of the core from previous experiments without melting. This information would be used to establish the reactivity effects from the outer unmolten part of the core so that the reactivity effects from meltdown and sodium boiling can be determined by subtraction. It is, therefore, assumed that 
the reactivity effects produced in the experiments by meltdown are also part of the available data It will be evident in the discussion that follows that additional information which would better define location of the fuel throughout the entire transient would greatly aid the interpretation.

Three separate approaches to interpretation of the experiments are discussed: (1) a direct approach in which the results of the meltdown experiments are assumed to give directly, or by extrapolation, the results of a hypothetical meltdown accident, (2) an indirect approach in which the experiments are assumed to provide information on the motion of the fuel but in which the reactivity effects and the energy release, if any, are predicted theoretically from the observed fuel motion; (3) a completely indirect or differential approach in which even the fuel motion is predicted theoretically for the hypothetical meltdown accident, and che experuments are used only to check the reliability of the calculation and to improve the theoretical model Each of the three methods of interpretation has its advantages and disadvantages: which one is best suited to a particular situation depends upon the experimental results obtaned

\section{A Direct Approach}

The direct approach is probably the most convincing and would be used whenever possible There are two restrictions on the experiments that limit the application of this approach to certain classes of experimental results One is the maximum size of the meltown zone, which is no more than $15 \%$ of the core The second restriction is the limitation of the severity of the excursion so that the outer cone of the core is not damaged. If a severe cxcursion begins to develop, it us reminated by the reactor scram system

The direct approach could be applied, for example, to cases in which there is a largo negative reactrinty effect produced by the fuel rearrangement subsequent to meltdown such that if it is certain that the entire core had been included in the meltdown zone, then the result would still have been qualitatively the same, i e, no secondary critical assembly and, therefore, no excursion Another situation that permits a partial direct interpretation is one in which there is a defunte increase in reactivity upor meltdown of a small part of the core and in which the positive reactiviry effect becomes monotonically stronger as successive experiments are run with larger and larger meltdown zones in such cases, one can conclude directly chat a secondary criticality hazard cxists and the rate of reactivity increase can be estimated by extrapolation. There can, of course, be no direct determination of the energy release because of the requirement that there be no damage to the outer zone of the core The energy release must be determined esther by calculation or by further experiments in the second facility, FARME-B, which would be designed to permit complete reactor meltown accidents to $\mathrm{I}$ un their course without interference from the control system. 
It might appear at first glance that the restrictions imposed by the facility prevent most meltdown experiments from being interpreted by the direct approach. There is some possibility that this will be the case, but it seems more likely that the majority of the situations encountered would result in definite strong reactivity changes that would permit a direct interpretation even with the limitation of $15 \%$ of the core in the meltdown section. It is not possible to determine this definitely with the present state of knowledge about the behavior during meltdown, and therefore less direct methods of interpretation must also be considered.

\section{B. Indirect Approach}

The indirect method must be applied to situations in which there are no immediate strong reactivity changes even when the entire $15 \%$ of the core is included in the meltdown section. It could happen, for cxample, that when the first material in the core melts, there is just rearrangement within the confines of the original core because of effects of refreezing and plugging. Then, perhaps, most of the subassemblies within the meltdown section would be at least partially molten before a large amount of fuel actually drained out of the core to reduce the reactivity by a large amount; it would be necessary to estimate the extent to which the outer region of the core would have influenced the results had it also been allowed to melt. There is really no reliable way of making such an estimate unless something is known about the motion of the fuel after it melts. Conversely, if the motion of the fuel is known, then it is possible to make reasonable estimates of the resulting behavior by theoretical means. Therefore, the indirect approach necessarily involves a determination, or at least an estimate, of the actual motion of the fuel subsequent to melting. The development of experimental equipment to observe fuel motion will require a substantial amount of further research.

Even without equipment to observe the fuel motion, some progress can be made by inferring the fuel motion from the observed reactivity changes. There are, of course, many different arrangements of the fuel which could produce a given reactivity and this greatly complicates the problem. Several possibilities must be considered and the ones that are completely unreasonable eliminated. Then, a determination is made of which of the remaining possibilities is the most unfavorable in terms of producing a secondary criticality accident and power excursion when the behavior of the total core meltdown is predicted. If the conservative prediction is severe, then perhaps it would be necessary to proceed with further testing in the FARME-B facility.

In detail, the indirect approach is necessarily quite complex; it is not by any means a simple extrapolation of the behavior from the results of the tests to a full-core meltdown. The procedure, in concept, is as follows: From the reactivity effects observed, the motion of the fuel 
in single subassemblies must be estimated as a function of the power history to which the subassemblies are subjected from the time of initial melting. Also, from the experiments, the time at which melting will begin in each subassembly must be deduced. Once this information is known, it is possible to attempt to synthesize the behavior during total core meltdown. The conditions of power level, reactivity, and temperature are, of course, known at the time when sodium boiling and fuel melting first begins. But as the boiling and melting propagates radially outward from the core center, the associated reactivity effects influence the power and in this way also influence the course of future melting. In the indirect approach these reactivity effects must be calculated by means of two-dimensional multigroup diffusion theory and digital computers. Then the power is, in turn, calculated from the reactivity by the reactor kinetics equations; and the power influences on the melting and movement of uranium are determined indirectly from the experiments.

It is not clear at the present time that the indirect approach can really be useful, i.e., that it can lead to predictions of the behavior that can be accepted with confidence. The weakest link in the procedure is the prediction of the actual movement of fuel from observed reactivity changes. If some additional instrumentation, such as the gamma-ray camera, can be developed which would give more direct information on the material movement, then the indirect approach would have a greater chance of success. It is, of course, not necessary that the end result be an accurate prediction of the energy release. A great deal is accomplished if the experiments in FARME-A are only successful in screening out the majority of the possible combinations of conditions so that only a small number of tests are required in the FARME-B facility.

\section{Indirect or Differential Approach}

The completely indirect or differential approach utilizes the experiments in FARME-A mainly as a check on theoretical calculations of the meltdown. It would be required if a case should arise in which it is impossible to effect an accurate simulation of the accident conditions in FARME-A, e.g., of the coolant-flow conditions. The procedure would be to perform experiments as near to the desired conditions as possible; then to develop calculational techniques that predict the experimental results accurately; and finally to use the same techniques to calculate the results of the hypothetical meltown accident.

The required calculations in the differential approach involve the heat transfer from fuel to coolant, the transient two-phase flow that exists as the sodium is boiled out of the core, the melting of the fuel, the movement of the fuel under the forces of gravity and the forces exerted by the coolant, the reactivity effects from sodium boiling and fuel movement, and the reactor kinetics. The problem is indeed a formidable one. 
The effects are sufficiently complex so that it is not clear that the differential approach can ever be applied with confidence except in situations that result in the clearcut predictions that the fuel is expelled from the core as it melts, thus preventing the formation of a secondary critical assembly. Certainly, the present state of knowledge is not sufficiently detailed to permit reliable predictions by this method.

Each of the three methods of interpretation has advantages and disadvantages, which are summarized below:

The direct approach is the most reliable and convincing. However, it requires that the experimental conditions closely match the conditions of the meltdown accident under investigation. The restrictions upon the facility probably prevent the application of this method in some cases of interest.

The indirect approach is less reliable than the direct approach, but it can be applied to cases in which the latter is ruled out by the limitation of the meltown to only 15 per cent of the core. The indirect approach also requires that the accident conditions be closely matched by the experiment. The reliability of this method can be substantially improved if techniques for directly detemining the motion of the fuel during meltdown can be developed.

The differential approach is too complex to be considered reliable at the present time except perhaps in special cases that lead to clear predictions that there is no secondary critical configuration formed. It has the advantage that if the state of the technology can be advanced to the point where the method can be applied with confidence, then only a few experiments are required and the conditions of the experiment need not accurately match the conditions of the hypothetical meltdown accident.

\section{Conclusions}

In order to establish some limit on the environmental extremes in which the required instrumentation must perform and to estimate the size of the meltdown that can be allowed if pessimistic assumptions are used regarding fuel motion, a crude kinetics analysis has been made of the meltdown transient in an alloy core. It is assumed that a coolant perturbation initiates the meltown and that the power level remains constant until meltdown begins. Upon melting, this fuel is assumed to escape from the clad and move under gravity toward the core center, but never fall below the centerline, the fuel below centerline remaining in place. The reactivity effect of such fuel motion is determined by perturbation theory. This reactivity is used as input for a transient analysis utilizing the CASEY code, a one-dimensional space time kinetics code that solves the point neutron kinetics equations utilizing feedback produced by one-dimensional space-and 
time-dependent coolant, fuel, and clad temperatures. The assumption that the geometry change of the meltdown region does not affect the feedback of the reactor and that the only reactivity effect of the meltown region is due to fuel motion is implicit.

The resulting reactor power and maximum fuel and coolant temperatures experienced in the normal core, as functions of time after shutdown, are shown in Fig. 3 for a transient involving 4 per cent of the core in the meltdown capsule. Figure 4 portrays the same initial conditions,

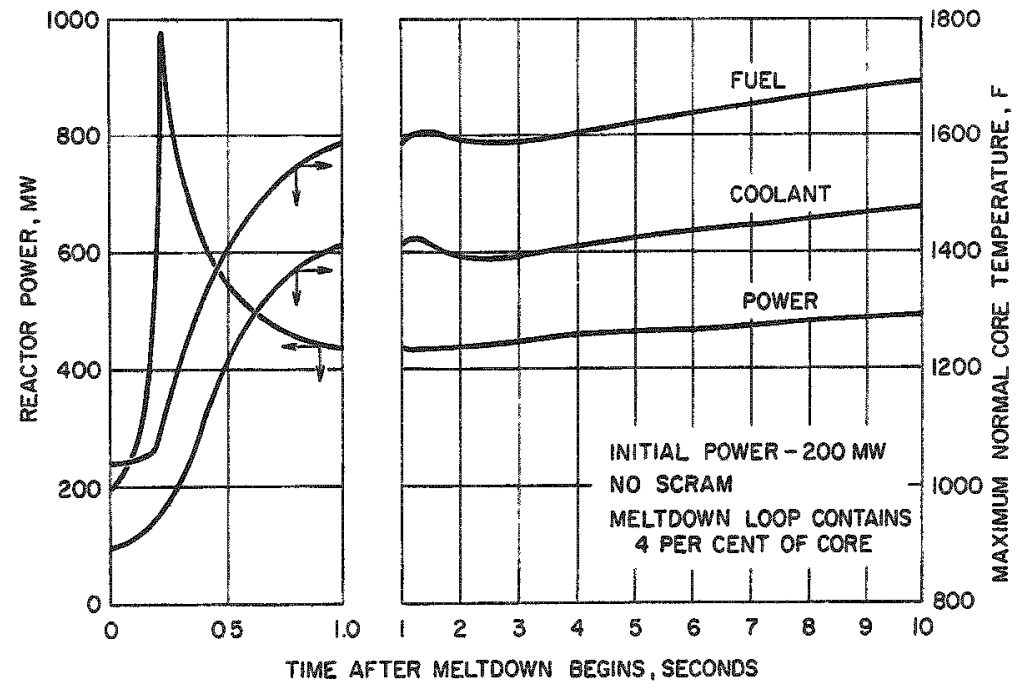

Fig. 3. Reactor Power and Temperature Response for Gravity Collapse Alloy Core Meltdown Experiment

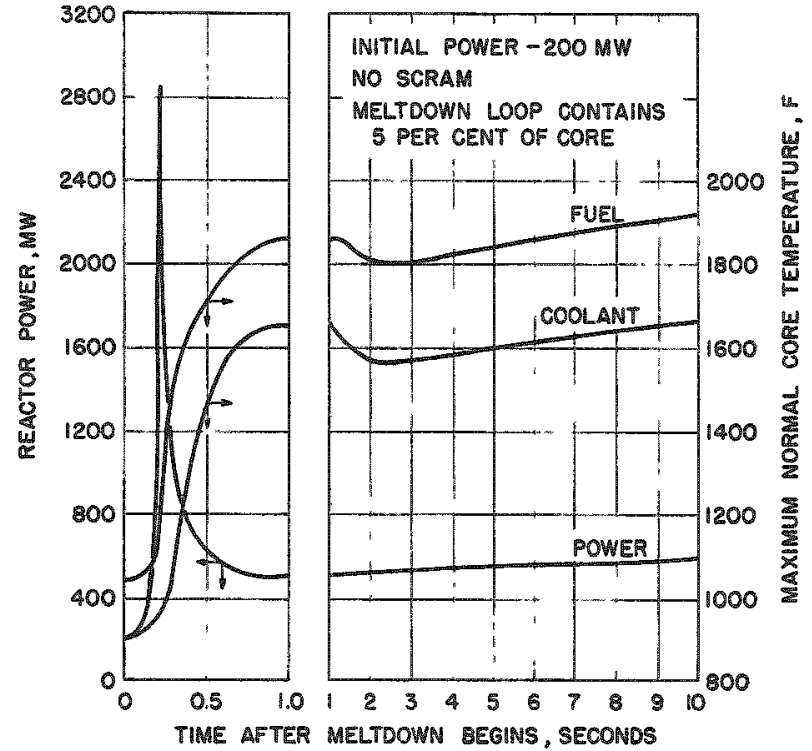

Fig. 4

Reactor Power and Temperature Response for Gravity Collapse Alloy Core Meltdown Experiment 
but with 5 per cent of the core in the capsule. Note that the sodium boiling point of $1630^{\circ} \mathrm{F}$ is exceeded for this case. Figure 5 shows the result for the case when the capsule again contains 5 per cent of the core but in which the initial power level is lowered from $200 \mathrm{MW}$ to $1 \mathrm{MW}$. Figure 6 repeats the conditions of Fig. 4 but adds an $\$ 8.00$ scram at the time of complete collapse (0.22 sec).

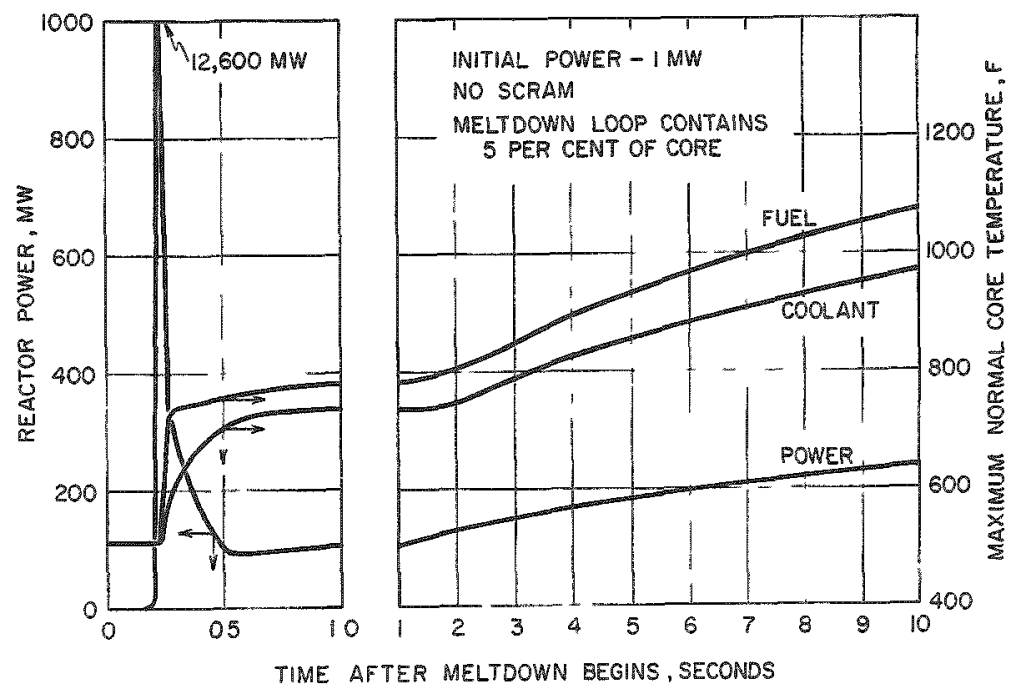

Fig. 5. Reactor Power and Temperature Response for Gravity Collapse Alloy Core Meltdown Experiment

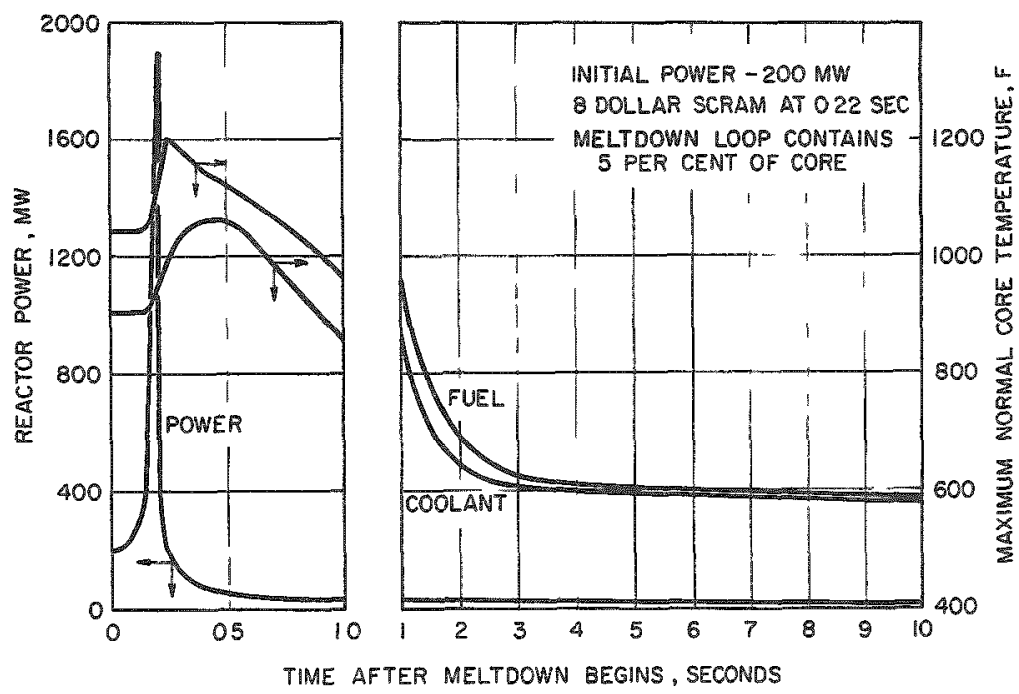

Fig. 6. Reactor Power and Temperature Response for Gravity Collapse Alloy Core Moltdown Experiment 
Table 1 summarizes the major variables for the transients of greatest interest that were computed. It can be seen that the maximum amount of fuel that can be allowed in the meltdown capsule if the gravity collapse model is assumed with no scram action is from 4 per cent to 5 per cent of the core, depending on the initial power level.

Table I

GRAVITY COLLAPSE TRANSIENTS IN ALLOY CORES

\begin{tabular}{cccccccc}
$\begin{array}{c}\text { Size of } \\
\begin{array}{c}\text { Meltdown } \\
\text { Region } \\
(\% \text { of Core) }\end{array}\end{array}$ & $\begin{array}{c}\text { Initial } \\
\text { Reactor } \\
\text { Power } \\
(\mathrm{MW})\end{array}$ & $\begin{array}{c}\text { Safety } \\
\text { Rod } \\
\text { Action }\end{array}$ & $\begin{array}{c}\text { Peak } \\
\text { Power } \\
(\mathrm{MW})\end{array}$ & $\begin{array}{c}\text { Peak } \\
\text { Reactivity } \\
(\$)\end{array}$ & $\begin{array}{c}\text { Min } \\
\text { Positive } \\
\text { Period } \\
(\mathrm{msec})\end{array}$ & $\begin{array}{c}\text { 10 Sec } \\
\text { Energy } \\
\text { Release } \\
(\text { kcal })\end{array}$ & $\begin{array}{c}\text { Max } \\
\text { Coolant } \\
\text { Temp } \\
(F)\end{array}$ \\
\hline 4 & 200 & None & 970 & 0.82 & 50 & 1100 & 1475 \\
5 & 200 & None & 2850 & 0.84 & 27 & 1320 & 1655 \\
5 & 200 & Scram & 1750 & 0.80 & 27 & 88 & 1060 \\
5 & 1 & None & 12600 & $\sim 1$ & 1 & 480 & 970 \\
10 & 200 & None & 510000 & $\sim 1$ & 1.25 & - & $>3000$ \\
10 & 1 & None & 550000 & $\sim 1$ & $<1$ & - & $>1900$
\end{tabular}

It is upon these limiting transients that the instrumentation requirements were based. It would appear that present technology would, in principle, allow the necessary measurements to be made of reactor power and temperature in the normal core for these transients. Some additional development work would be necessary in the area of in-core the rmocouple mounting.

Although there are definite limitations to the experimental approach outlined here, it would supplement the other fast reactor programs now underway, and it possesses a number of advantages which are listed below.

1. There is sufficient fuel involved in the meltown to permit a direct measurement of the reactivity effect, a measurement not attempted in any existing facility in the fast reactor safety program.

2. Transient situations involving sodium boiling in systems having positive sodium temperature coefficients can be studied.

3. Meltdowns of up to 15 per cent of a core can be investigated for those situations which warrant it, provided that safety and the normal core are not jeopardized.

4. The axial and radial power distributions at the time melting occurs are the actual distributions, with no special measures necessary to reproduce the axial distribution or to account for radial and axial flux depression. 
5. The heating in individual fuel particles will not be changed radically once meltdown begins. In the case of a the rmal meltdown facility, this heating will change with changes in fuel location due to the necessary use of different fuel enrichments.

6. The experiments can be performed over a wide range of fuel types, power levels, and hydraulic conditions of the sodium coolant at a cost far less than required if an equivalent number of reactors were purposely subjected to complete meltdown.

7. The refractory capsule with independent cooling makes the facility a suitable one for irradiation testing of fast reactor fuel elements to destruction.

However, to establish the technical feasibility of the approach outlined above, additional effort is needed to show that the following requirements can be met.

1. The molten fuel must be contained within the meltdown capsule of FARME-A. Preliminary calculations, together with the available data on the properties of tantalum, indicate that a $\frac{1}{2}$-in.-thick wall of tantalum will provide the containment. However, the computations are crude and the data on tantalum are fragmentary so that containment is not a certainty.

2. A fuel element must be designed for the normal core that will withstand the temperature transients and possess the desired physics characteristics.

3. The particular set of conditions most favorable with respect to producing a secondary critical assembly and power excursion in complete core meltdown accidents must be deduced from the experiments on partial core meltdown. Success in this area will depend partially upon the results of an instrumentation $R$ \& $D$ effort, and on the nature of the experimental results themselves. Further, it should be stated that the criteria for such interpretive success is not clear, so that the final feasibility of this experimental approach must include a weighting of its several advantages against its cost. A fairly detailed cost estimate for the meltdown facility FARME-A is fifty-five million dollars, including the first core and research and development. FARME-B would range between two million and fifty million, depending on the required complexity of the facility. 


\section{References}

1. R. B. Nicholson, Methods for Determining the Energy Release in Hypothetical Reactor Meltdown Accidents, APDA-150 (Dec 1962).

2. W. J. McCarthy, Jr., R. B. Nicholson, D. Okrent, and V. Z. Jankus, Studies of Nuclear Accidents in Fast Power Reactors, Proceedings of the Second United Nations International Conference on the Peaceful Uses of Atomic Energy, Geneva, Switzerland, 12, 207 (1958).

3. H. A. Bethe and J. H. Tait, An Estimate of the Order of Magnitude of the Explosion When the Core of a Fast Reactor Collapses, UKAEA-RHM (56)/113 (1956).

4. V.Z. Jankus, A Theoretical Study of Destructive Nuclear Bursts in Fast Power Reactors, ANL-6512 (1962); also see Chapter IV.4, Safety Problems, Volume III of Physics of Fast and Intermediate Reactors, Proceedings of the Seminar on the Physics of Fast and Intermediate Reactors (Vienna, 1962).

5. W. R. Stratton et al., Analysis of Prompt Excursions in Simple Systems and Idealized Fast Reactors, Proceedings of the Second United Nations International Conference on the Peaceful Uses of Atomic Energy, Geneva, Switzerland, 12, 196 (1958).

6. R. H. Brout, Equation of State and Heat Content of Uranium, APDA-118 (1957).

7. H. Elrod, Estimation of the Critical Temperature and Pressure of Uranium, NDA-14-122 (1956): also see H. Elrod, Revisions and Extensions of Estimated Saturation Properties of Uranium, NDA-14-139 (1956); also see W. R. Stratton et al., Ref. 5.

8. R. D. Cowan, On the Equation of State of Uranium at Low Densities and Temperatures, Los Alamos Scientific Laboratory, unpublished.

9. E. G. Rauh and R. J, Thorn, The Vapor Pressure of Uranium, J. Chem. Phys. 22, 1414 (1954),

10. P. Marne11, J. DeFelice, and J. Barker, Some Thermodynamics Properties of Uranium, Molybdenum, Iron and Zirconium at High Temperatures and Pressures, Nuclear Technology Corporation. (Feb 10, 1962).

11. J. B. Nims and P. F. Zweifel, Preliminary Report on Sodium Temperature Coefficients in Large Fast Reactors, APDA-135 (Nov 1959). 
12. T. A. Doyle, R. B. Nicholson, J. B. Nims, E. M. Page and J. T. Hagstrom, Two Facility Approach to Investigations of Fast Reactor Meltdowns, APDA-151 (Jan 15, 1963).

\section{Discussion of Paper}

Presented by Mr. Nims

MR. HALL (Los Alamos):

I am glad you added the words that a few engineering details remain to be worked out.

MR. NIMS:

They are minor, the $\$ 50,000,000$ is the big problem.

MR. HALL:

Is that the estimated cost?

MR. NIMS:

We believe the first facility has to be essentially the Fermi-type reactor, and this costs $\$ 50,000,000$. We really haven't looked in much detail at the second facility, and I don't think it makes much sense to do so until you have a fairly good idea what you can get out of the first facility. It may turn out that you can use a very simplified approach in the second. one. On the other hand, the first facility may not be that good, and you may need a very detailed approach, in which case your cost may go up another $\$ 50,000,000$.

MR, FISCHER (Argonne):

It seems to me that in some of the proposed experiments you are losing a lot of information, for instance, if your fuel is slumping or melting. You are not sure how it is slumping or melting, and what your reactivity effects are due to. Would not a good zero-power reactor and a sequence of measurements in which in the course of time you shifted fuel in known ways, taking out sodium and moving fuel as you choose, provide more useful information?

MR。 NIMS:

I think that approach is complementary. It could be that more than one configuration would give the same type of behavior. I think you might 
want to operate a zero-power facility along with this to check out what sort of configuration would give you an identical reactivity history. Secondly, I did not emphasize enough, I guess, that you should have a very strong instrumentation-development program along with this to obtain as much information as possible on the fuel motion during the experiment.

MR. deSTORDEUR (Euratom):

How did you select the 15 per cent core volume to be reproduced in such a facility?

MR. NIMS:

We selected 15 per cent because it is a lot less than 100 per cent, and in order to save money you should make the volume small. Also, it seemed to fit in with a number of requirements. You have to worry about going critical when you are melting down; 15 per cent would not lead to a worry about secondary criticality problems when you are pulling out the meltdown capsule. Further, about 15 per cent of the core is occupied with the meltdown experiment, there is still room to get in enough safety rods and control rods to control the reactor. It seemed to work out also with one or two engineering or practical considerations.

MR. deSTORDEUR:

Do you think that such a facility would be necessary if you could have a good visualization of the meltdown or meltup of a fast reactor as sembly in another type of reactor? I am thinking, of course, of Dr. Tavernier's BRISEIS and his visualization system. Suppose that you could have in a single subassembly a good way of visualizing where the molten material goes as a function of time when you are reproducing an accident, which is itself characteristic of a fast reactor. Do you think there is then a need for such a reactor?

MR. NIMS:

I would say that if you could get some good information on how fuel moves so you knew this quite precisely as a function of power level, flow rates, pressure drops, in enough detail so that you could synthesize a meltdown accident, then you could determine where the fuel was at every given instant of time with reasonable accuracy. I think you would have gone a long way towards the goal. This would be a big step and a very satisfactory one, I think. I do not know whether you can do this in BRISEIS. 
MR. HETRICK (University of Arizona):

First I have a general comment about reactor dynamics experiments. On the one hand, one can do an integral experiment with the entire reactor under consideration, which has certain practical value. But a disadvantage is that you learn very little about the individual physical problems involved. Or you can do basic experiments on each one of the phenomena at a time. That has disadvantages too. I am just a little afraid that your experiments may have, primarily, the disadvantages of both kinds because it is not the entire reactor nor is it basic. I would be concerned that you would have such little knowledge of the individual physical processes that are important and at the same time no real knowledge of what the entire reactor would be doing.

MR。NIMS:

Well, there is one way to check. Start out with a one per cent meltdown experiment. You then predict what is going to happen if you go to two per cent or three per cent. If you next do this experiment, you can get a very good check on how well you can calculate things.

Another point is that the first facility can be used to select the one experiment in which to do a complete meltdown and get the whole picture.

MR. HETRICK:

I want to bring up a more general point. Perhaps it is more relevant to some of the earlier speakers this afternoon. The question is, in the event that the high-pressure pulse is localized to a small volume of a fast reactor, what is the risk of this pulse then compressing or changing the geometry in the remaining part of the reactor in such a way as to give a large reactivity increase simply due to that compression, that change of geometry or lattice spacing or what have you?

MR. HALL:

That is a very broad question. I will throw this open to the general discussion. 
226 


\section{GENERAI DISCUSSION OF \\ MELTDOWN PROBLEM}

MR. HALL (continuing):

Anyone who feels brave enough to try to answer this question of: Is there an autocatalytic reagent or agent in this that will feed into a compressible area?

MR. NICHOLSON (University of Wisconsin):

I think the question probably arises as a result of some of the things that were presented with respect to the APDA experiments with molten lead and water, in which it was indicated there was a local high-pressure area produced which caused flow reversal. I would like to point out that that was really an extremely mild pressure pulse which would be totally incapable of any major rearrangement of the core. If it was that particular experiment that led to the question. I don't think there is any problem.

My belief is that such high local pressures, if they could be produced anywhere in the reactor, would be produced at the location where the power density is the highest, and generally this is also the location where the reactivity worth of the materials are highest. Therefore the pressure would tend to disperse the core rather than to assemble it in any way. I cannot say that I have any definitive answers to the question, however. About all I can say is that we have looked for such a possible mechanism for producing high rates of reactivity increase and have not found any.

MR. BUMP (Argonne):

Right now we have a pretty generally accepted mechanism which I think consists of assuming the top half of the core drops with the speed generated by the acceleration of gravity toward the bottom half. I am wondering if we did spend $\$ 100,000,000$ on experiments which failed to show that such a thing were possible, would people still throw up this present mechanism as being possible? In other words, would you have gained anything?

MR. HALL:

I think that is a very valid comment. I am not sure that we know what the answer is.

MR. CAMPISE (Atomics International):

In reference to this comment, I would like to ask a more provocative question of the group. Maybe Dr. Hall will be kind enough to answer 
it. I admit that the Doppler coefficient is an operational parameter enabling us at least to minimize accidents if they are going to occur. My question is: at what point in time do we believe we will start to accept the engineering and instrumentation that we put into our reactor power plants? We keep talking about accidents that might happen. The question is: how do they occur and can we actually engineer around them?

MR. HALL:

I hope you do not expect an answer to that. I realize it is a plea from the applicant.

MR. WEINBERG (Oak Ridge):

In that connection, there is a somewhat broader, perhaps related, problem to the question. Listening a little from the outside to a discussion this morning, and to some extent this afternoon, one cannot help but get a little of the impression that a double standard is being imposed on the reactor business. One is concerned very much with the consequence of a loss-of-coolant accident in fast reactors, and the impression I have is that one does not subject some of our old thermal reactors to the same kind of scrutiny. Now we have an awful lot of kilowatts as you know in this country and in Canada, I guess, which are really subjected to very much the same kind of difficulties as were proposed here. If the water at Hanford stopped, things would be very bad. If the water at Chalk River stopped, things would be very bad. How is it really that one requires, at least implicitly, that with fast reactors one must always have the sodium going through the reactor, whereas with reactors like Hanford and possibly Chalk River this requirement is not made in quite as stringent a fashion?

MR. HALL:

I cannot answer for Chalk River. I think the requirement concerning the loss of the coolant as one of the accident considerations has been the result of the very high power densities in the fast reactors, and the resulting fuel melting. The fuel melting in the thermal reactor does not lead to a secondary criticality, but it does put restraints on the confinement and the containment of the system. It is the same thing in the fast reactor, except that some people have been able to imagine that the fast reactor can, in its secondary criticality, release so much energy that containment becomes a very difficult problem. I think that in all of the accident analyses we try not to concern ourselves with what happens internally. If you lose a reactor, this is an industrial accident, and it is a catastrophe as far as the management goes. It does not affect the public. 


\section{A. VOICE:}

Secondary criticality is the answer.

MR. HALL:

Yes.

A VOICE:

It is a good answer.

MR. TAVERNIER (BelgoNucleaire):

I would like to come back now to the point made by $\mathrm{Mr}$. deStordeur and to the communication of names. The point is this, in the type of transient reactor that we proposed, we think that we would be able to visualize roughly the motion of the fuel in case of a meltdown within the assembly. There would be mainly mechanical information available, the behavior of the fuel-element assembly within its coolant, with practically no reactivity feedback to the dry zone. This I didn't say earlier this afternoon, but the de-coupling between the test zone and the dry zone is very good in this case. In other words, our proposal has limitations but possibly some advantage to be able to forsee, or calculate later on with better information, the starting point of some accident into a reactor produced by various causes. Now in the reactor experiment proposed by Nims, we must see here something which is much more relevant, and which actually gets to the actual secondary criticality accident. In other words, how the things do really form, the way in which this could be exploded, and the type of and the amount of energies generated, etc. In other words, I feel that we are talking here about possibly two different types of facilities and experimental results with, of course, the logical coupling between these experiments.

MR. COHEN (General Electric):

I do not want to buy a hundred million dollar program today. I think one point about fast reactors, which is very difficult to duplicate, should be remembered, and that is that they are very fast pulses. If one is talking about fuel slumping, I am not at all sure that one sees anything about fuel slumping in a pulse. For example, in TREAT, which is quite slow compared with what would actually happen in a real sharp pulse (which takes about a millisecond or two), things would not have time to get moving. I think one of the simulation problems you have with any thermal reactor compared with a fast reactor is that you are going to have great difficulty in getting pulses which are sharp and fast, characteristic of what we fear if not expect in fast reactors. 
MR. TAVERNIER:

Coming to this point, I agree that the fast accident in the fast reactor could have a lifetime which is fairly short. But do you think that a simulated accident on a facility which would still have a lifetime of a few milliseconds would lead to highly different results, being given the fact that, for the first part of the accident anyway, the time constant to be considered was possibly one hundred to a thousand times larger? I admit possibly that if there were afterwards, say, a grain of molten fuel going into the sodium and formation of small frozen particles within the sodium, the thermal time constants are much smaller, but this should come after the pulse.

MR.WOLFE (General Electric):

I would like to clarify one point. In the analysis that I did, I as sumed that the only feedback mechanism after the pressure built up was the Doppler coefficient. Now, with regard to the sodium in the core, the assumption, therefore, is that in the accident, once you start vaporizing and disassembling the core, the fuel moves out at least as fast as the sodium does. If you can imagine an accident where somehow the fuel vaporizes and pushes out the sodium without pushing out the fuel, then, of course, these accidents would increase in magnitude.

\section{MR. THOMPSON (MIT):}

I just wanted to add a note here regarding Weinberg's question. First of all, in the fast reactor the time scale is very much shorter, and one has to worry a little bit about an accident which really occurs and is almost essentially complete before the instrumentation even knows it is started. This is quite a serious problem. There is also the problem of the progressive failure of fuel in which a single fuel element or section is blocked off, and this causes melting in the axea which then progressively goes through the core. I think somebody should lay that ghost some day, one way or the other. There are a number of such questions for which there is really no analogue in the thermal reactor. There are some differences which have to be considered, and the technology of fast reactors is a bit behind that of the thermal reactors. I wanted to ask the last speaker what provisions he has taken to differentiate clearly between the meltdown zone and the ordinary reactor. How is he sure that the melting down will stay in the meltdown zone?

MR. NIMS:

You have some benefit of power distribution, of course; also, you can use different enrichments in the meltdown zone. Furthermore, you 
can use different temperature conditions of the coolant and you can use different flows. These things you would all have to do and somehow prevent melting in the normal zone.

A VOICE:

Are you not changing your spectrum?

MR. NIMS:

Changing enrichment might change it slightly. Changing temperature conditions and changing flow conditions would not have very much effect on spectrum. 


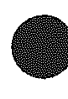

232 


\section{SESSION III}

October 8, 1963

PERFORMANCE, FUELS AND ECONOMICS

Chairman: S. LAWROSKI

Argonne National Laboratory

Secretary: C. E. Dickerman

Argonne National Laboratory 
234

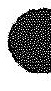




\title{
STATUS AND INTERRELATION OF FAST BREEDER PROGRAMS
}

\author{
J. H. Wright \\ Westinghouse Atomic Power Division \\ Pittsburgh, Pennsylvania
}

Introduction

The basic aim of the atomic energy programs which have been initiated in all parts of the world is to increase the available energy supplies of the world and of the energies available to each individual country. The first objective is to develop nuclear power technology to the point where it is economically competitive with conventional or fossil fuel sources of power. The last few years of progress in nuclear reactor technology have seen atomic power become economically competitive in the United States and in many other areas of the world. Thus, the first major milestone of the world-wide program in atomic energy is being accomplished. A second major objective is to continue the development of nuclear power to the point where it can effectively replace large quantities of the fossil-fuel energy sources available. It is a clear certainty that in order to accomplish this second objective over a long period, several centuries or more, that a successful program in breeding reactors must be conducted.

The purpose of this paper is to present a summary of the fast breeder programs being conducted in each of the major nuclear development countries of the world and to compare these programs with regard to content, adequacy, objectives and schedule.

\section{Program Objectives}

In considering the objectives for breeder reactor programs of the nuclear development countries, it is important to note that the factors affecting the degree of urgency for such a program vary widely from country to country. Significant among these factors are as follows:

1. available reserves of fossil-fuel resources;

2. competitive position of other than fast breeder reactor types currently available;

3. independence from foreign sources of enriched uranium;

4. economics of fast reactor systems not necessarily producing breeding.

These factors do not affect the basic objective which requires breeding eventually in all countries, but affect the required rate of progress 
which these countries should strive for. One extreme case is the Canadian program, which is based solely on the large amount of available fossilfuel reserves and natural uranium reserves to be found in Canada; hence, the heavy water-cooled-and-moderated reactor systems are now programmed to supply all the incremental nuclear power deemed necessary for the next several decades. By virtue of this position, there is no active fast breeder program in the Dominion of Canada.

Italy represents an opposite extreme, in which it has sought contracts for foreign reactor designs for three large plants which are nearing completion. It is not particularly well endowed with fossil-fuel energy resources, nor does it have a domestic availability of uranium and thorium. Furthermoxe, the Italians do not now have the technology to build large nuclear electric plants of Italian design.

The program for nuclear power in Italy is widely diversified. Italy is the only country which has under construction large reactor plants of three different foreign types: the Calder type, the closed-cycle water reactor, and the boiling water reactor. Their program in fast breeder development has not yet been fully formulated, but the enthusiasm so far exhibited would indicate that it is high on the priority for domestic development. The use of a paste-type fuel with fertile material of thorium seems to separate this program to some extent from the others being conducted in Europe.

Most of the European programs are based on reactor technology which will eventually provide independence from sources of enriched uranium.

The program of nuclear power development in United Kingdom has been reasonably well disclosed and consists of three distinct phases:

1. The first phase was based on an apparent shortage of fossil fuel and called for the immediate construction of $5000 \mathrm{MW}$ of the Calder Hall-type power reactors. The Wilfra plant constituted the last step in completing this program.

2. The second phase is currently underway. Present indications are that the advanced gas-cooled reactor AGR is the preferred choice for the next $5000 \mathrm{MW}$ of power produced for that country. They are also considering Canadian heavy water reactors and American light water reactors.

3. The ultimate aim of the British program is to attain most of their power through the application of fast breeder reactors, probably commencing in quantity in the late $1970^{\prime} \mathrm{s}$. 
Fossil-fuel reserves in England are now estimated to be somewhat more plentiful. This factor plus the developing technologies in other competitive nuclear types indicate no great cause for an accelerated crash program in fast breeder development.

The program in France is both very active and widely diversified. France is participating with Belgium in the construction of a large water reactor and in addition has programs for the development of natural uranium reactors (both heavy water and graphite-moderated, gas-cooled reactors) and fast breeder reactor development. The preponderance of their domestic research effort is oriented to reactors of high neutron economy (either natural uranium or breeders) with the current intention of maintaining independence toward foreign sources of enriched uranium.

The program in Germany is oriented similarly to that of the French, but is on a somewhat later time scale. Recent agreements to share gascooled technology with the French, as well as joint arrangements with United States on fast breeders, will assist in closing the lag which now besets them. The German program has also included the construction of large American water reactors, and additions of this type are currently being considered. The domestic program is, however, directed primarily at natural uranium and fast breeder development for reasons common to other European countries.

The Civilian Power Program in the USSR has proceeded on a very broad basis, somewhat similar to our own, except that emphasis has not been placed on the construction of large quantities of nuclear kilowatt capacity to date; rather, the Russians are obtaining first hand experience on all the different types considered important. Work will undoubtedly continue in fast breeder technology, but the driving force of limited fuel resources apparently does not exist. It is also presumed that enriched reactors can be amply supplied with enriched material from within the Soviet Union.

The position of the United States is well known and adequately reported in Dr. Seaborg's report to the President. (1) In summary, the objective of this program is to supply nuclear energy for the production of electric power on an economical basis. Consistent with this objective, fast breeder reactors are necessary both for the replenishment of fis sionable material and for conserving other sources of energy supplies.

Fast Breeder Program Criteria

In order to formulate a basis for comparing program content, adequacy, and schedule, it is important to set forth criteria for these features. If large-scale fast breeder reactor development is to follow the general 
pattern already established by economically successful reactors of today, it is to be reasonably expected that a broad program encompassing physics, materials, fuels, and systems and component development must be undertaken and demonstrated in total systems through increasing plant sizes. Figure 1 illustrates an outline of the criteria of adequacy for the development program. These criteria attempt to define in general terms the research and development required and the reactor plants which are necessary to demonstrate principles and achieve final objectives.

Fig. 1

PROGRAM EVAIUATION CRITERIA FOR LARGE BREFDER DEVEIOPMENT

I. Large Plant Research and Development
A. Core design, fuel cycle and safety studies.
B. Fuel and materials development and testing.
C. Physics rusearch, including large scale criticals.
D. Large plant designs.
F. Large components development.

II. Reactor Plants
A. Hxperimental fast breeder reactors.
B. Special purpose fast reactors.
C. Power demonstration reactors.
D. Prototype power reactors.
F. Economic large scale breeders.

Fuel Cycle - A study of any new system, particularly one so complex as the fast breeder, must incorporate detailed attention to the reactor core design and fuel-cycle analysis together with its parameters, with particular regard for their effect on the safety of the overall system. Indeed, one of the unsolved riddles to date is how to obtain an economic fast reactor fuel which has a very high breeding ratio and excellent safety characteristics. From the studies made thus far, it is apparent that compromises of one or more of these objectives will be in order. It is also apparent that a large core is greatly different in neutron economy, distribution of power and local conversion ratio, and in safety characteristics from a small-sized fast neutron core. Core design and fuel-cycle studies relating to large cores, of 1500-liter volume or more, are considered necessary criteria for a fast breeder program.

Fuel and Materials Development and Testing - Fast fuels and materials, which must bc used to attain a large, economic fast reactor, have either not been developed or else have not been sufficiently developed for the severe requirements seen in a long-burnup fast reactor. From a consideration of the fuel cycles and safety studies, il appears that the ceramic 
fuels offer the best hope for good economics as well as for reasonably good breeding and adequate safety requirements. Therefore, work in these areas is required for economic fuel development and testing. There are numerous related problems of materials development, mass transfer, fuel element-clad selection, and sodium-purification technology, which are far from resolved for the present and require significant additional development and in-pile testing in order to proceed with confidence to the construction of a fast reactor prototype.

Physics Research - The physics of fast reactors, even at this date, is known only in limited detail, particularly with respect to 5000 - or 6000-liter cores. There are many unanswered questions regarding neutron cross sections, particularly with regard to Doppler coefficients in fast systems, especially where higher isotopes of plutonium are present. With regard to plutonium, for example, there is an extensive amount of information in the classified literature on weapons-grade plutonium, but a rather limited amount of experimental information on equilibrium plutonium found in the discharge of water reactors; scarcely any data at all exists on the equilibrium plutonium values associated with the equilibrium cycle in a fast breeder reactor. Only large-scale criticals will provide information of importance to the solution of the physics problems. Therefore, critical assemblies larger than 1500 liters and having plutonium capability have been selected as the criteria in physics research for large breeder systems.

Large-plant Designs - In order to determine the preferred type of fuel to be developed for breeder reactors, it has been necessary to under take core-design studies and fuel-cycle analyses to serve as a means of evaluating various alternatives and schemes. A similar requirement is mandatory for the development of large-plant components and systems. It will be necessary to undertake a reasonably detailed design of fast breeder reactor plants in order to understand the complex economic and technical problems associated with optimizing the total plant design and layout. Typical problems requiring deeper insight are:

1. optimum sodium outlet temperatures;

2. minimizing the number of heat transfer loops;

3. preferred plant arrangement and layout to alleviate safety problems and minimize costs:

4. optimum steam conditions;

5. incremental advantages of hot leg vs. cold leg pump locations;

6. refueling concepts for high plant availability; 
7. differential costs of vented fuel and "dirty sodium" vs uncontaminated primary coolant;

8. feasibility of eliminating secondary sodium heat transport loop.

Such design studies must be conducted in order to give valuable aid and direction to the component development programs listed in the next paragraph. Plant-design studies of large systems of 1000-1500 MWe are selected as essential criteria for large breeder development.

Large-component Development - From the direction given by the large-plant design studies, programs for the development and testing of large components should be initiated. An operating reactor is a poor technical or economic choice for a test bed for vital sodium systems and components. Reliability cannot be designed into sodium systems, but must be developed through prototype designs and testing. Of particular interest among these large components aresteam generators, heat exchangers, liquid metal pumps. reactor vessel, and the fuel-handling and control and safety rod drives. Supplemental development and testing in these areas is considered vital to a program for economic and reliable large fast breeders

Plant-construction Programs - In order to be meaningful, the solution to the interrelated problems must be experimentally tested as a complete system through various stages of design size and purpose Typical of the types of plants that are required to support an overall program are those listed below:

1. Experimental Fast Breeder Reactors: A first experiment in fast reactor technology provides as much value in training people as in the actual experiments.

2 Special Purpose Reactors. In a complete program it is apparent that there will be certain needs for special purpose reactors. Examples of these are:

a reactors for testing fast fuel power coefficients,

b. reactors for demonstrating complete fuel-cycle technolog $\mathrm{y}^{\circ}$

c. a fast fuel-testing reactor facility.

The difficulties associated with trying to obtain pertinent information on fast reactor technology from thermal reactor tests are formidable.

3. Power Demonstration: At one time it seemed advisable in the US programs to have power demonstration reactors of various types Thesereactors were not prototype reactors, inasmuch 
as they were not small versions of future, large, economic plants, but were reactors usually in the small or intermediate range of size for the purpose of technically demonstrating feasibility of that particular type of nuclear power plant. The advantage of such power demonstration reactors is sometimes questionable, inasmuch as they often are not prototypes of future large plants, and they usually represent a severe economic burden for as long as the plant is operated.

4. Prototype Power Reactors: A prototype power reactor is necessary in some intermediate size ranging from $\frac{1}{5}$ to $\frac{3}{10}$ the size of the ultimate economic plant to be built. This reactor may not be economic in itself and can be justified only if it adequately and accurately represents a prototype of the design features of future large economic plants.

5. Economic Large-scale Breeders: It is quite apparent that any program must have as its ultimate objective the construction and operation of economic large-scale breedex reactors.

\section{Comparisons of Fast Breeder Programs}

The last two years have been quite eventful with regard to world wide progress in fast breeder development. Germany and Italy have each recently announced their progressive interest in fast breeder programs. The programs in the UK and France have taken more definitive shape with regard to orienting toward future large economic plants, and the US program has been re-evaluated so that a re-emphasis is being made on the importance of breeding to the future national economy.

A comparison in the scope of large fast breeder development programs of six nations is shown in Fig. 2. $(2-9)$ This comparison shows that most of the countries are active in the area of core designs, fuel-cycle studies, and safety evaluation for plants of larger size. It is also impor tant to note that five of these countries have chosen essentially the same fuel material, namely, mixed uranium and plutonium oxide or mixed uranium-plutonium carbides. A notable exception is Italy, which is working on a thoria-urania paste in sodium. Other fuels are also being developed but have secondary importance with regard to first-generation economic feasibility. These include the metallic and the cermet fuels.

It is also interesting to review the potential of two other fuels not currently considered as strong candidates for first-generation economic breeders. One is the liquid plutonium fuel being developed and demonstrated at Los Alamos (LAMPRE), and the other is the packed pebble-bed fuel currently being tested in-pile by Brookhaven National Laboratory. 
Fig. 2

\section{SCOPE OF LARGE FAST BREEDER DEVELOPMENT}

\begin{tabular}{|c|c|c|c|c|c|c|}
\hline & United States & Uunited Kingdom & France & Germany & Vialy & USSR \\
\hline Date of Program Intiation & 1948 & $1854-?$ & 1957 & 1960 & 1962 & $1950 ?$ \\
\hline \multicolumn{7}{|l|}{ 1. Large Plant a } \\
\hline A. Core Design. Fuel Cycle \& Safety Studies & Active & Active & Active & Active & - & Active \\
\hline 8. Fuel, Material Development. Testing & $\mathrm{MOO}_{2}, \mathrm{UC}$ & MOZ.MC & MO2. UC & $\mathrm{MO}_{2}$ & Sodium Paste & $\mathrm{MO}_{2}$ \\
\hline c. Large Scale Criticals & $\begin{array}{c}\text { 1961-AL } \\
(1963-2 \mathrm{PR}-1 \mathrm{~V} \text { 1500) } \\
(1967-2 \mathrm{PPR}-5000)\end{array}$ & ZEEMA-3000 & (1965 MASUACA.5000) & (1965 SNEAK-2000) & - & BF \$.6000.9 \\
\hline D. Large Scaie Plant Designs & - & $300 . \$ 40.1000$ & 500 & - & - & $500+?$ \\
\hline \multicolumn{7}{|l|}{ E. Large Component Development } \\
\hline 1. Steam Generator & (2-30 Mำ & - & - & - & - & $?$ \\
\hline 2. Heat Exchanger & - & - & - & - & - & $?$ \\
\hline 3. Liquid Metal Pumps & {$[60.80,000 \mathrm{GPM}]$} & {$[8,500 \mathrm{GPA}]$} & - & - & - & ? \\
\hline 4. Reactor Vessel Fuel Handling & - & Active & - & - & Active & ? \\
\hline 5. Control and Safety Mods & - & - & - & Actuve & - & ? \\
\hline
\end{tabular}

(1) Under Construction

[1 Planned

With regard to physics development and the use of large-scale criticals, five of the six countries either have in operation or are building one or more criticals having sizes of 2000 liters or larger. Many of these criticals will use plutonium as it becomes available, either from other reactor sources or on lease agreements with other countries.

At the moment, large-scale plant studies have been reported in only two countries: the United Kingdom, where prototypes of sizes of 300-500 MW have been investigated with ultimate plant sizes $(6)$ to $1000 \mathrm{MW}$, and in the USSR, where sizes from 250 to $1000 \mathrm{MW}$ have been reportedly investigated. (4) Recent additions to the French program will include largescale $(500-\mathrm{MWe})$ design studies.

There is an appalling lack of development work in the six countries in the area of large-component development. The lack of reliability of sodium components and systems to date has constituted all the delays or downtime in present fast breeder reactors. Component sizes must be increased to reduce cost, and their quality must be improved to the point where reliable components and systems can be designed and built. Two countries at the present time, the United States and France, have shown their interest in steam-generator development. In France construction of a facility for testing 5-MW steam generators will begin in early 1964 . In the United States a Sodium Components Test Installation will soon be in operation at Canoga Park, where 30-MWt steam generators of advanced design can be tested. 
Two countries have already expressed interest in proceeding with the development of large-scale sodium pumps required for the future economic breeders, the US and UK. The US program, currently under consideration, suggests the development of sodium pumps in the $60-80,000-\mathrm{gpm}$ range. It is important to note the recent interest that the UK has shown in large sodium pumps. In the Dounreay Fast Reactor a decision was made to use numerous small pumps, which led to very complex piping problems and contributed significantly to the oxide contamination problem experienced during the first two years of the DFR operation. (6) The UK program is constructing a pump test loop for testing sodium pumps with capacities as large as $8500 \mathrm{gpm}$.

Reactor vessel design with particular emphasis on the fuel-handling system is a problem of significant importance. Not only is it necessary to develop systems which can reliably handle large quantities of fuel which are not available for direct observation by the operator, but they must be able to do so rapidly with minimum downtime in order to attain high availability factors and at minimum cost in the transfer and storage equipment.

Fast-acting control and safety rods may also be of considerable benefit, particularly in large plant sizes, in order to overcome disadvantageous factors associated with large, fast neutron cores. The only work on this component which has been reported to date is the work in the German program for the very fast acting control rods.

Development Schedules

The schedule for these development programs, giving a time comparison for reaching various research and development objectives for the six countries, is presented in Fig. 3. Preliminary fuel-cycle and coredesign studies were completed two or three years ago in the United States, (10) the United Kingdom, France, (11) and the USSR. Of more particular significance, however, is that fuel testing of mixed oxide fuel has been conducted up to $100,000 \mathrm{MWD} / \mathrm{T}$ in both the United States and Russia. (5) Burnups of lesser magnitude have also been experienced in France and the United Kingdom.

This schedule also shows that large critical facilities are now operating in the United States, the United Kingdom, and in Russia. The French and German facilities, MASURCA and SNEAK, will both be operational in 1965 and are programmed to use a common source of $300 \mathrm{~kg}$ plutonium. (7) The schedule also points out that the UK and USSR are both completing large-plant design studies at the present time. It is hoped that the US program will include large-plant studies in the near future, and these have been indicated as proposed on Fig. 3. The schedule for delivery of heat exchangers for the SCTI is shown as 1963 and 1966 with a suggested additional advanced sodium steam generator for 1968 or 1969. A suggested 
pump-development program is also shown in this chart with the US programs. The 10-MWt component test loop at Cadarache, France, has not been included on this schedule because its purpose is the proof testing of Rapsodie I rather than the prototypes of large components. It may, however, eventually be used to test prototypes of larger components.

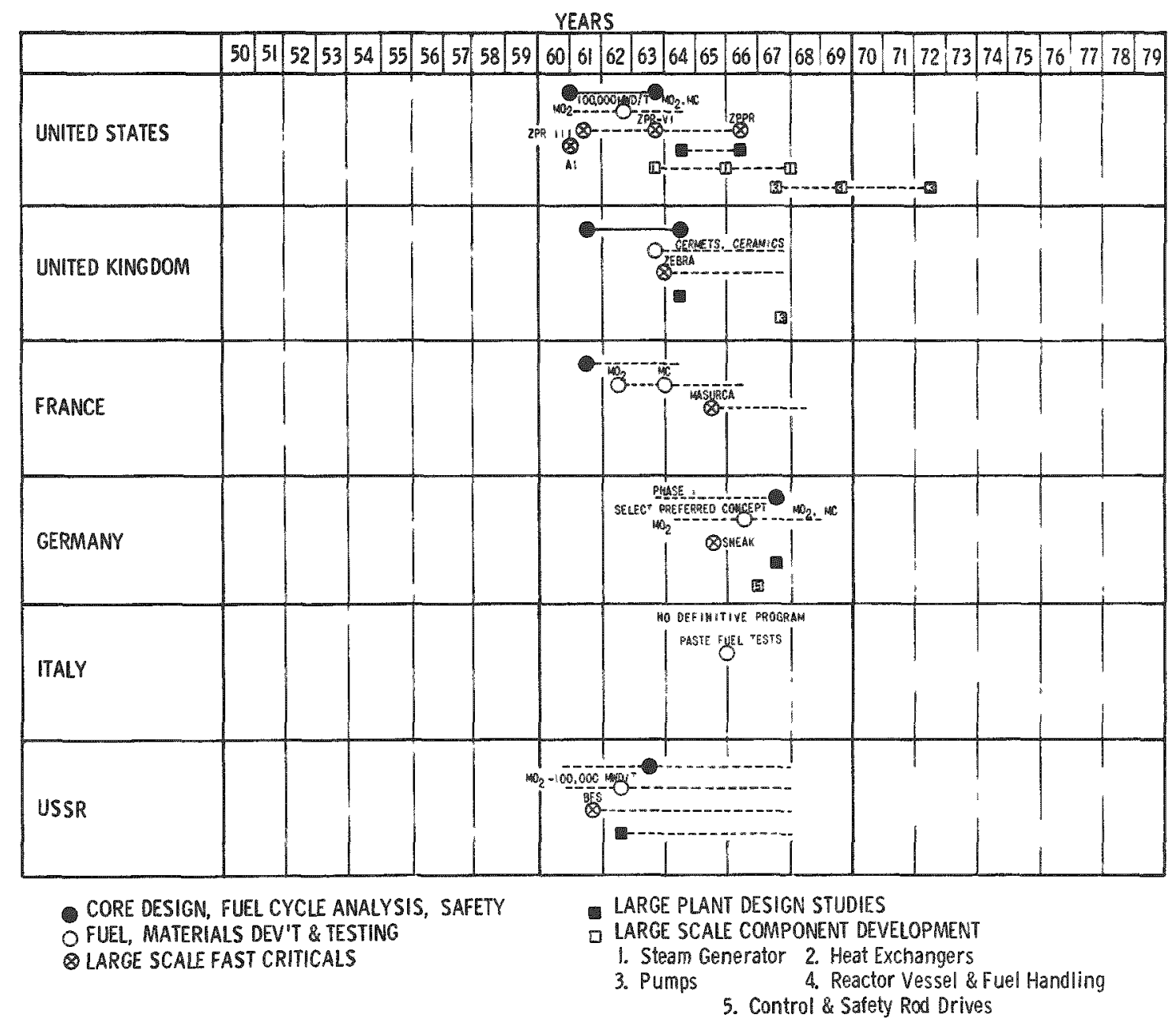

Fig. 3. Large Plant Development Programs (Operating Dates)

It is apparent from this schedule that the US, the UK, and the USSR have actually accomplished considerable amounts of physics and experimental materials testing to date and have avery definite lead in these areas.

Fast Breeder Plant Schedule

The fast breeder plants required to fulfill the program objectives are listed in Fig. 4. The plant descriptions surrounded by parentheses indicate plants that are currently under construction. Plants surrounded by brackets indicate those plants which are in a planned program but are 
not actually under construction. It can be noted here that there are only seven reactors critical or operational in the world today, and over half of these are in the US. Fast reactor experiments are operating at Los Alamos and NRTS. The EBR-II and Enrico Fermi Fast Breeder reactors are both critical at this time. The DFR and $B R-5$ are operating in the $U K$ and the USSR, respectively.

Fig. 4

\section{FAST BREEDER PLANTS}

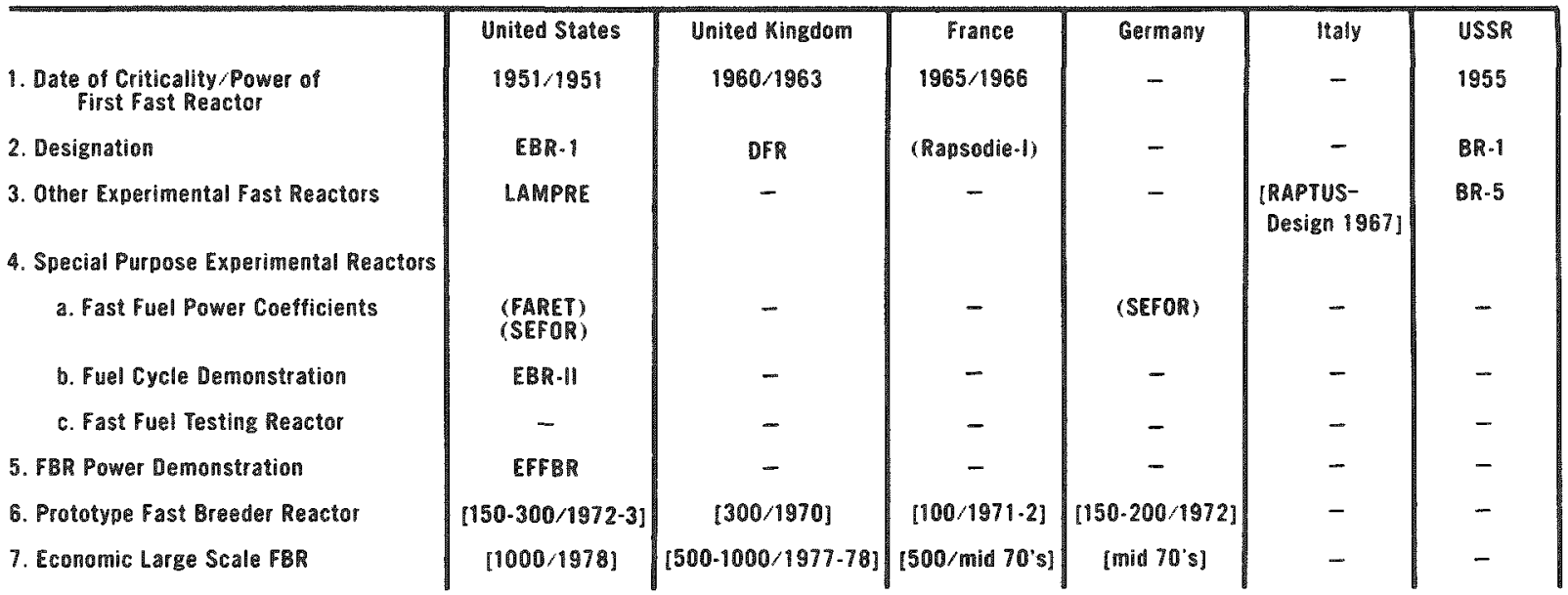

() Under Construction

[] Planned

The schedule for future plant operation is shown in Fig. 5. This schedule shows that the BR-1, EBR-I, and BR-5 were the only reactors started up prior to this decade. Of these, the BR-I has been dismantled and the EBR-1 is scheduled to be dismantled during the next calendar year. The DFR first went critical in 1960 but became operational only in 1963, owing to problems of maintaining sodium purity in the complex piping arrangement.

With regard to special-purpose reactors, there are three in the United States. Two of these, FARET and SEFOR, have as their primary objective the obtaining of physics information on ceramic fuel at highpower and-temperature operation. It should be noted that SEFOR is a joint project between the US and Germany. The EBR-II is a special purpose reactor for the demonstration of a completely integrated fuel cycle in a single plant.

A separate reactor for the special purpose of fast fuel testing does not now exist in any of the six programs. This vital need might be filled in the US program by converting EBR-II or EFFBR to that objective. Some 
technical difficulties will doubtlessly be encountered with regard to limitations in core size and other test variables, but their present existence and availability are reasons for extensive investigation.

YEARS

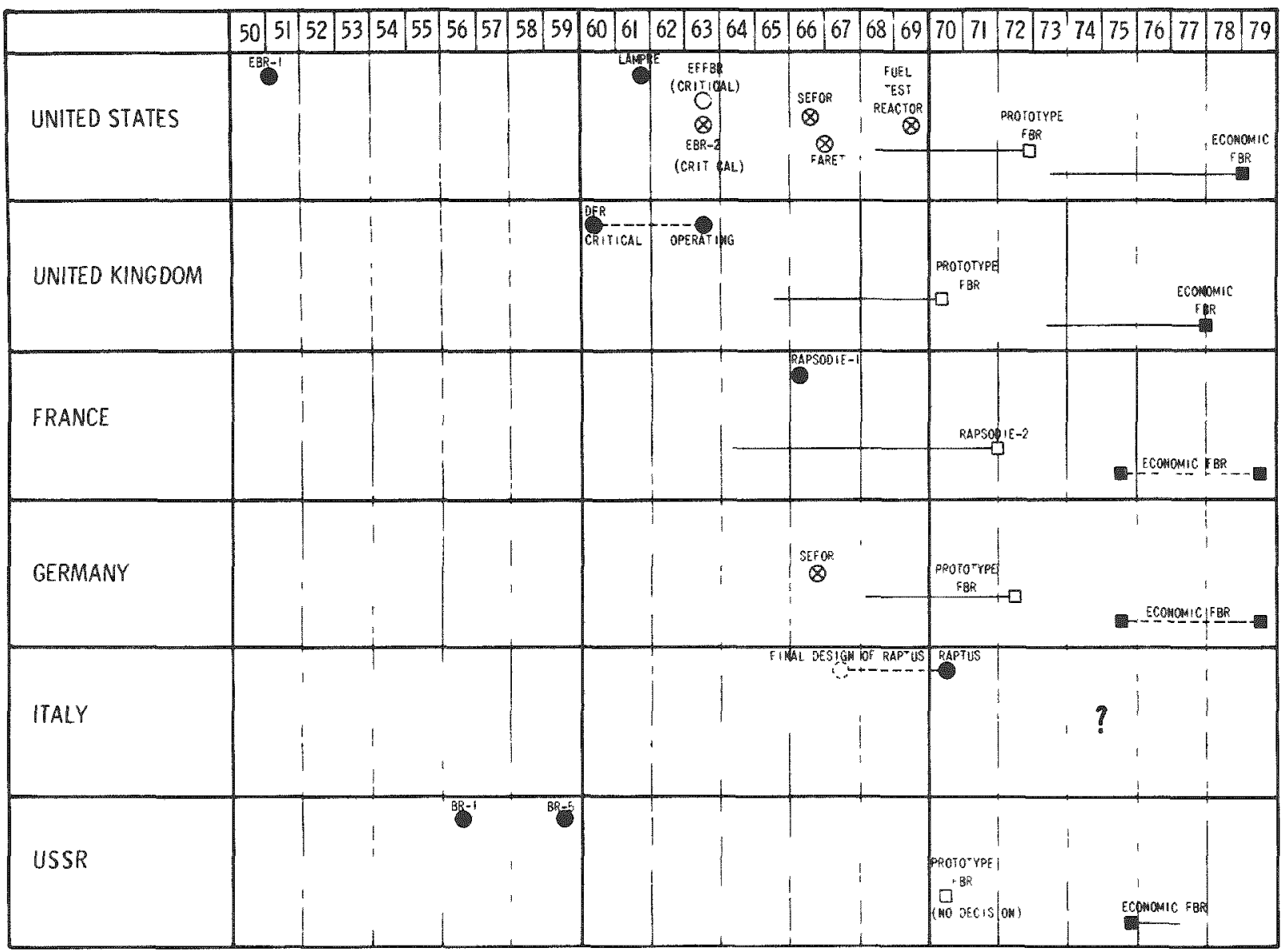

FIRST REACTOR EXPERIMENT ODMONSTRATION REACTOR

Q SPECIAL PURPOSE EXPERIMENTAL REACTOR
D PROTOTYPE OF COMMERCIALFBR

- ECONOMIC LARGE SCALE REACTOR

Fig. 5. Fast Breeder Plant Schedules (Operating Dates)

The prototype fast breeder reactors are being scheduled in each of four countries: US, UK, France, and Germany. From the schedule, one interesting fact may be noted, namely, no matter when you start fast reactor breeder development, you have an economic fast reactor running between 1975 and 1980 .

Comments on Programs

In reviewing the programs of these different countries against the criteria of the complete breeder development program, it may be concluded that nocountry has at the present time a really complete program. 
The US program is the most nearly complete, but additional effort in several areas would insure the progress to keep pace with the scheduled objectives. Among the areas deserving additional effort are:

1. increase of capacity for fuel-element irradiation in fast fluxes;

2. large-plant (1000-5000 MWe) design studies;

3. developing and testing of fuel-handling systems;

4. developing and testing prototype systems and components (sodium pumps, a third steam generator, etc.);

5. development effort in advanced fuel materials and concepts.

The program in Europe emphasizes experimental and analytical physics, and then proceeds in diminishing coverage through fuel development, fuel-cycle analysis, and safety, with only spot coverage of component development. Extensions of the existing program will include 500-MWe plant designs with industrial participation.

The European programs tend to appear on the optimistic side with regard to schedule for economic breeders. The lack of industrial participation in the "hardware" of fast breeder design may eventually limit the rate of progress and degree of success. The capacity for testing fast fuel is severely limited and will remain so for many years. A question might be raised regarding the overall benefit of each country having strong physics programs while maintaining so limited a participation in other areas.

It is difficult to see in the UK program sufficient attention to all the plant problems to the extent required to deliver an economic fast breeder reactor by the mid 70's. It is assumed that other development programs must be planned but not yet disclosed in order to meet this schedule.

The French program, based primarily on the two Rapsodie reactors, seems very optimistic, especially with regard to plant schedule dates. It is doubtful if a large economic fast breeder reactor can be operating in France by 1975, particularly considering the lack of component development and fuel development and testing experience.

The German program, although started very recently, is oriented primarily axound physics and safety considerations. The hardware aspects of the German program are totally lacking, and it, therefore, will be quite difficult to meet an early 1970 schedule for a prototype fast breeder. 
The Italian program in fast breeder development has clearly not yet been formulated. The advanced design on which they are working seems fraught with greater perils and problems than the other fast systems, but the eventual payoff may justify its selection.

The USSR decisions on fast reactor plants present some interesting food for thought. Although the BR-5 has been operating since 1959 and has had certain difficulties, these difficulties have been overcome; the reactor is now a safe and reliable system used quite extensively for fuel testing and other development work. (5) Recent design studies done by USSR on large fast breeder plants showed that a prototype could by built by 1970 and that an economic fast breeder could be operating(3) by 1973 or 1974. But no firm decision has been made to start construction of the plant for delivery in 1970. (4) This may be generally consistent with the Russian position to do active development work in several areas, but not to commit appreciable blocks of power to any one type.

\section{Schedule Considerations for the US Program}

A consideration of the US schedule for fast breeder development leads to many questions:

1. Is it fast enough?

2. Is it too fast?

3. Is it really required at all?

4. Is it logically oriented?

Although such questions have been asked and answered many times before, a few general thoughts on schedule would be in order at this time. In the first place, if we presume that the program should proceedas rapidly as is feasible consistent with schedule requirements, the question should then be asked: "What are the constraints?" The constraints are:

1. money:

2. trained people:

3. required facilities (especially required is capacity for fast fuel testing):

4. availability of plutonium for criticals and experiments;

5. definitive program.

For example, there are indications that there will not be sufficient plutonium produced from civilian reactors to supply the plutonium requirements in fast breeder criticals and in various thermal and fast fuel test experiments. Figure 6 indicates a probable deficiency when the 


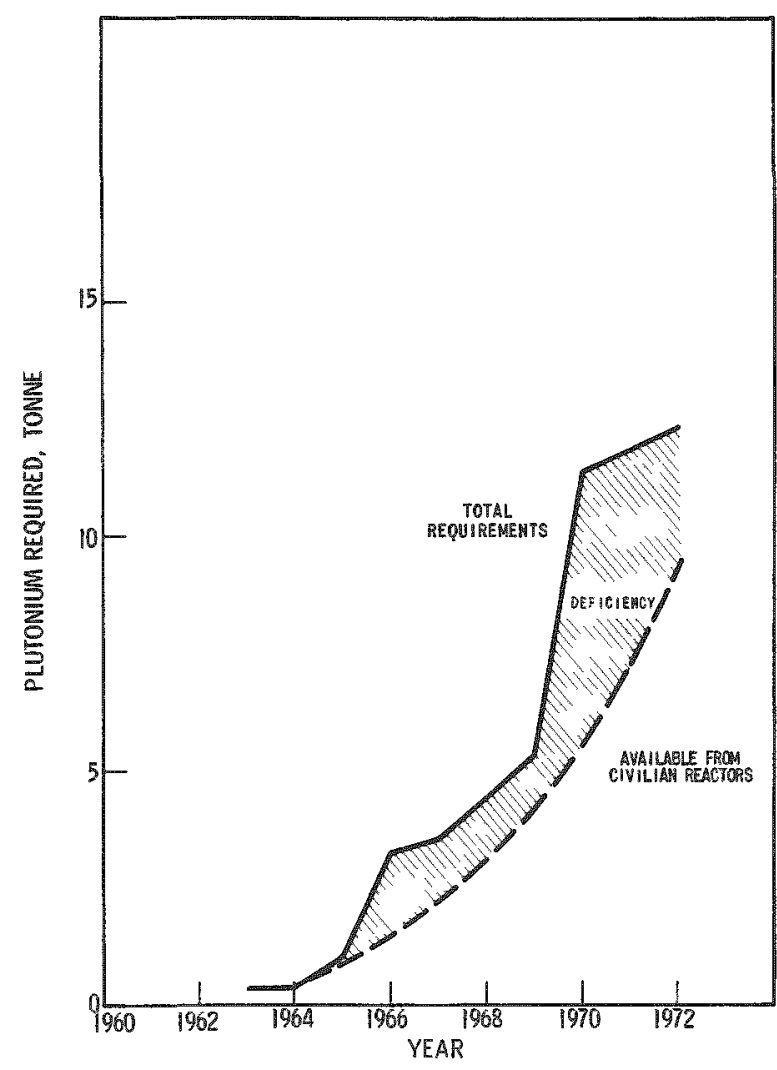

Fig. 6. Plutonium Requirements (Cumulative), Total civilian reactor produced plutonium is compared to the total plutonium requirements. $(12,18)$ However, this deficiency could be overcome by making available a relatively small quantity of plutonium from the weapons program, if the need should definitely develop.

It is important that the fast breeder program be continued at a logical and fruitful rate. The next major milestone in this program is to have a $150-\mathrm{MWe}$ prototype fast reactor operating in 1972 or 1973. This date is set in part by the time effort required to determine the ultimate objective plant from which the prototype will be scaled, in part by the predicted rate of technological development and, finally, by long-range requirements of fast breeders.

It may be technically feasible to undertake a prototype some years earlier, but whether or not that reactor would be truly a prototype or just another power demonstration reactor is quite questionable. Projects undertaken on a crash basis and not well integrated into the overall plan usually end up in partial to total economic chaos.

A case in point might be the Superheat program undertaken by the AEC some four years ago. This program was instigated on the part of the nuclear industry to try to answer requests by utilities for modern steam conditions. The resultant programstarted with a high degree of enthusiasm, which was interpreted erroneously as technical confidence. The program initially provided for the development of low-pressure, superheated steam rather than truly modern high-pressure, high-temperature steam. Neither the efficiency nor the component cost of these low-pressure systems justify taking on the greatly increased problems. Nevertheless, within a reasonably short space of time, three different power-producing superheat reactors were undertaken, having only modest variations in basic concept, and all without benefit of even preliminary fuel tests. These plants, by virtue of their small size and questionable technical feasibility, will become economic liabilities either to the USAEC or to utilities, and certainly to the public as a whole, for the balance of their operating lifetime. 
Construction projects undertaken on a crash basis, without sufficient research and development to point up the critical problem areas and without sufficient long-range thinking to determine the objectives that such

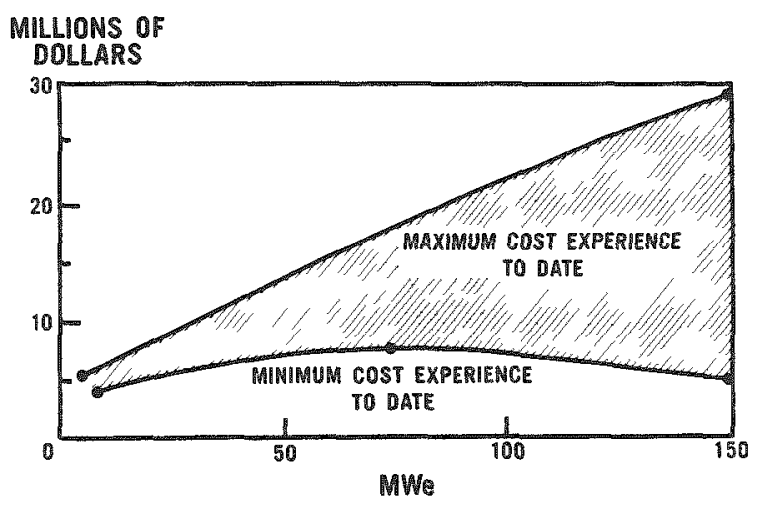

Fig. 7. Annual Net Loss tor Prototype Operation

a. small plant should try to meet in order truly to pave the way for future improvement almost invariably result in aborted effort.

The cost of operating experimental or demonstration reactors bears consideration. A survey of some 12 to 15 power reactors was made regarding the annual net cost of operating the plant. This information is presented in Fig. 7. It is important to note that the annual cost may vary between 2 and 20 million dollars, depending on the size of the plant and the degree to which it fails to meet the competition of other fuel sources in that area. The truly disconcerting thing about these numbers is that these are annual numbers, and some budget, AEC or industry, continues to suffer as a result. Dollars vital to research and development programs are diverted to sustaining a plant for which the incremental informational value may be quite marginal.

There are many cases, of course, in which the construction and operation of power demonstration reactors have provided valuable experience and knowledge upon which future programs and designs have continued to progress. These costs emphasize not just the high cost of sustaining a power demonstration reactor, but also that a power demonstration reactor should not be built until it is really required to fit into an overall schedule of an economically competitive program.

In summary, there are three reasons why a power demonstration or prototype reactor should not be built until it logically fits into an overall program:

1. The power demonstration reactor must be a true prototype of what is to follow, in terms of components, systems, and fuel.

2. The power demonstration reactor should not be built before technology generally can define the requirements of the economic power reactor to follow.

3. Money spent on advanced programs considerably ahead of the point of economic fruition simply increases "economic entropy" 
and can be quite costly if the utility procedure of "present worthing" is applied to the whole program. The present worthing principles simply illustrate the point of lost return on money invested prematurely.

The writer believes that the program proposed to the AEC for a prototype startup in 1972 or 1973 and a commercial plant startup in 1978 is entirely consistent with a reasonable and sound program for fast breeder reactor development and reaches its objectives in sufficient time to meet the long range objectives of energy conservation.

\section{Future Objectives for Nuclear Power}

In order to test the adequacy of the present program, let us consider a more specific set of objectives which the program should meet. A suggested list of objectives to be pursued in a total energy balance is as follows:

Objectives for the Year 2000

1. Economics:

a. Conserve fossil fuel.

b. Increase nuclear power participation in electricity production.

2. Minimize net consumption of fissionable material.

3. Develop a complex of fast-thermal fuel cycles which is selfsustaining with regard to fissionable materials.

\section{$\underline{\text { Economics }}$}

The first test of any nuclear power program is that it must be economically competitive with conventional fuel or other nuclear systems. In a system of free enterprise, it can scarcely be hoped that nuclear power will be used at the expense of the fossil-fuel industry unless the actual cost of nuclear power is cheaper than the fossil fuel.

To illustrate the difficulty of the economic target that the fast breeder must reach, a graph is presented in Fig. 8 of the fuel costs from a fast breeder reactor plotted as a function of burnup. (10) On the same curve is the estimated fuel costs from a dry and saturated steam, closedcycle water reactor (13) and a direct-cycle supercritical reactor. (14) The sensitivity of each of these fuel costs to changes in present-day ground rules, such as fuel inventory charges going from $4 \frac{3}{4} \%$ to $10 \%$ and the value of the uranium increasing as much as $50 \%$ of its present price, with a 
corresponding increase in plutonium value, axe also shown. Because of its somewhat higher capital cost, it is quite apparent that the fast reactor must have a very low fuel-cycle cost to be competitive with water-cooled systems. To achieve these very low fuel-cycle costs, the curves show breeders should have burnups approaching 100,000 MWD/T, which means maximum burnups in the range of 160,000 to $200,000 \mathrm{MWD} / \mathrm{T}$. This point further emphasizes the need for intensive development in fuel testing before another power generating fast breeder reactor is undertaken.

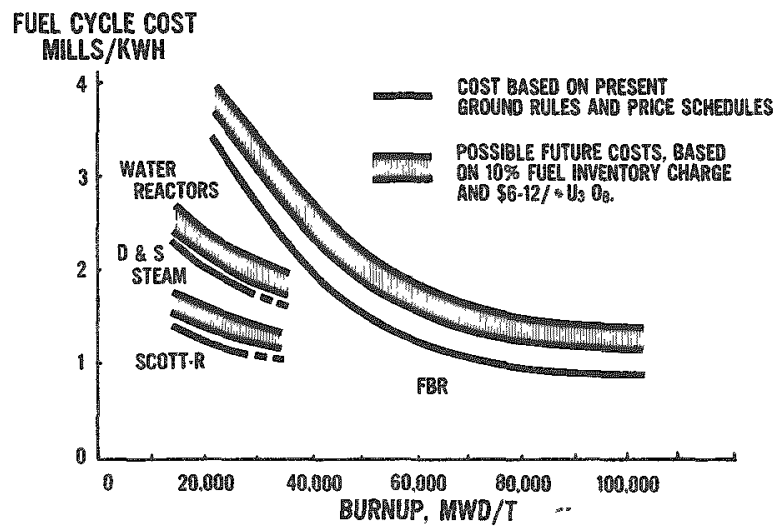

Fig. 8

Effect of Burnup on Fuel Cycle Costs

\section{Minimize Net Consumption of Fissionable Material}

In considering the second objective for the year 2000 , the following points are of interest:

1. Estimates of the various rates of energy consumption are plotted in Fig. 9, which shows the integrated consumption of energy by year. The AEC curve (1) intersects a total energy consumption of about 5 "Q" units by the year 2000. These 5 "Q" units can be compared with the 6 "Q" units in known recoverable reserves of fossil fuel at present prices and an additional 28 to 30 "Q" units available at 10 to $15 \%$ above present fossil fuel prices. An additional 124 " $Q$ " units is shown to be in reserve at higher than present-day prices. (15)

2. Estimates of the total energy value in " $Q$ " units vs. cost of the natural uranium found in the United States is shown in Fig. 10. Four different curves are presented for consideration. The upper three curves $(1,16,17)$ of the total uranium found in the United States are based on estimates de$r$ ived at a time when there was little or no exploration for new uranium reserves going on in the United States. For the past five years the uranium industry in this country has been quite slow, and there has been little or no incentive for exploration and discovery in development of new resources. Many similar references have been made regarding the depletion and rising costs of petroleum products and coal in this country, but costs have not varied significantly for many decades. The presumption is, 
therefore, that the quantities of uranium reserves shown by three references would be found quite conservative if uranium were really in demand. Uranium will probably be found within the US or available from other countries on a much more liberal rate as suggested by the lower curve. The lower curve would include imports from Canada, and other areas of free trade in uranium. It is important to note that the $Q^{\prime}$ of this abscissa represents the quantity of energy which could be recovered from nonbreeding thermal reactors.

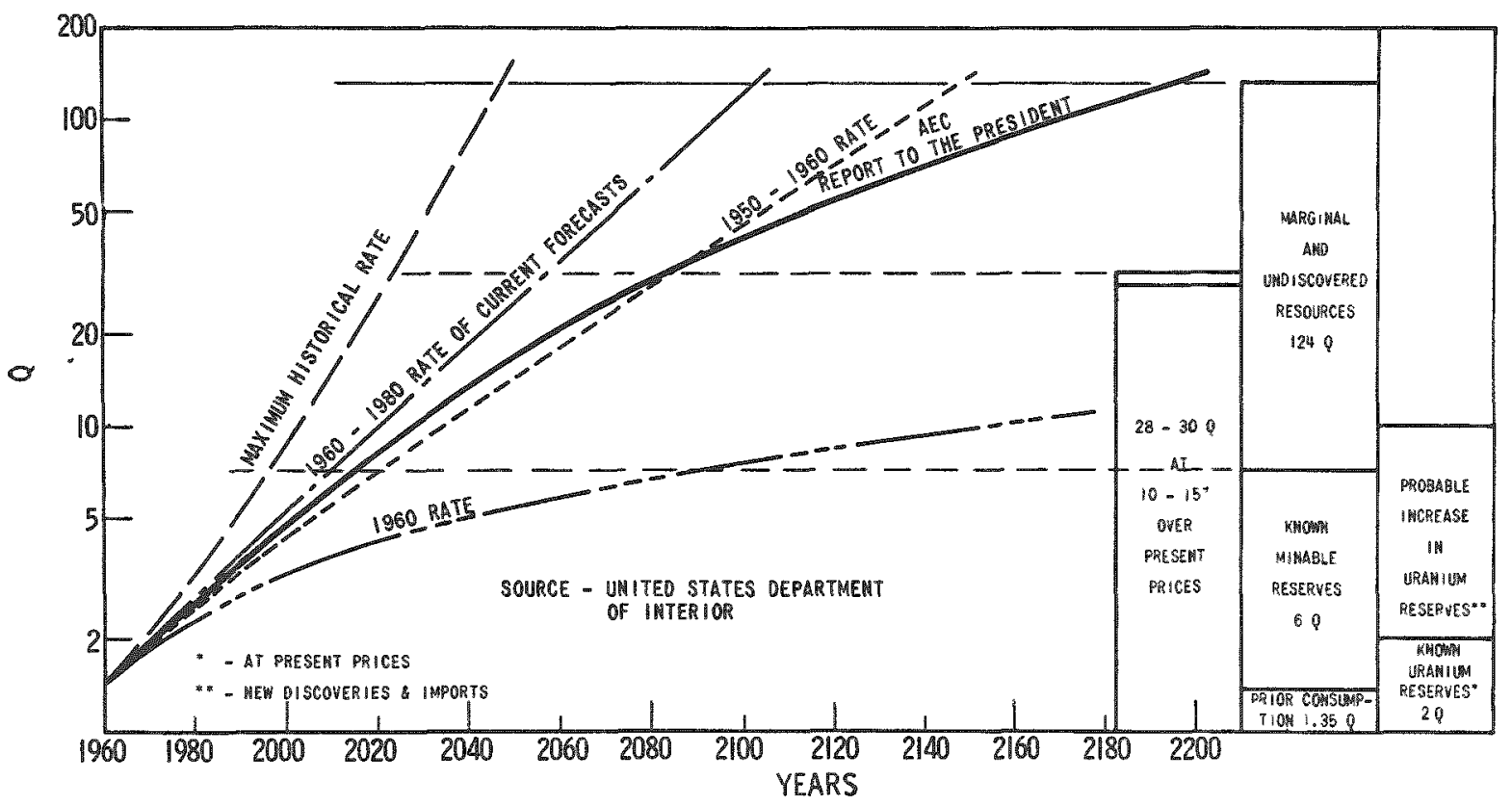

Fig. 9. Hypothetical Cumulative Use of Energy in the United States Compared to the Life of Presently Envisioned Resources of Fossil Fuels

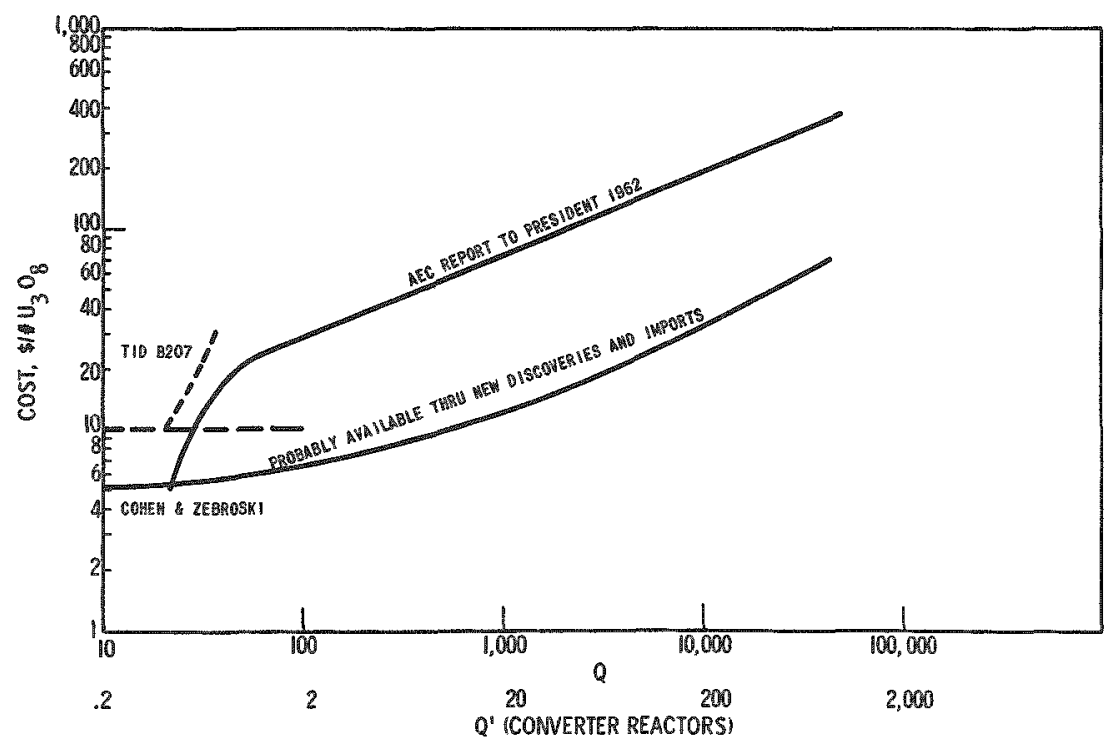

Fis. 10

Estimates of Uranium Reserves

EMERGY RESERVES AT OR BELOW A GIVEN COST 
3. Inasmuch as it seems likely that the principal use of nuclear energy will be for the production of electric power, Fig. 11 shows the same curve of estimated total energy requirements previously shown in Fig. 9, with the added line of that energy required for the electrical utility industry. This shows that a total of 2 " $Q$ " units will be consumed by electrical generation by the year 2000 .

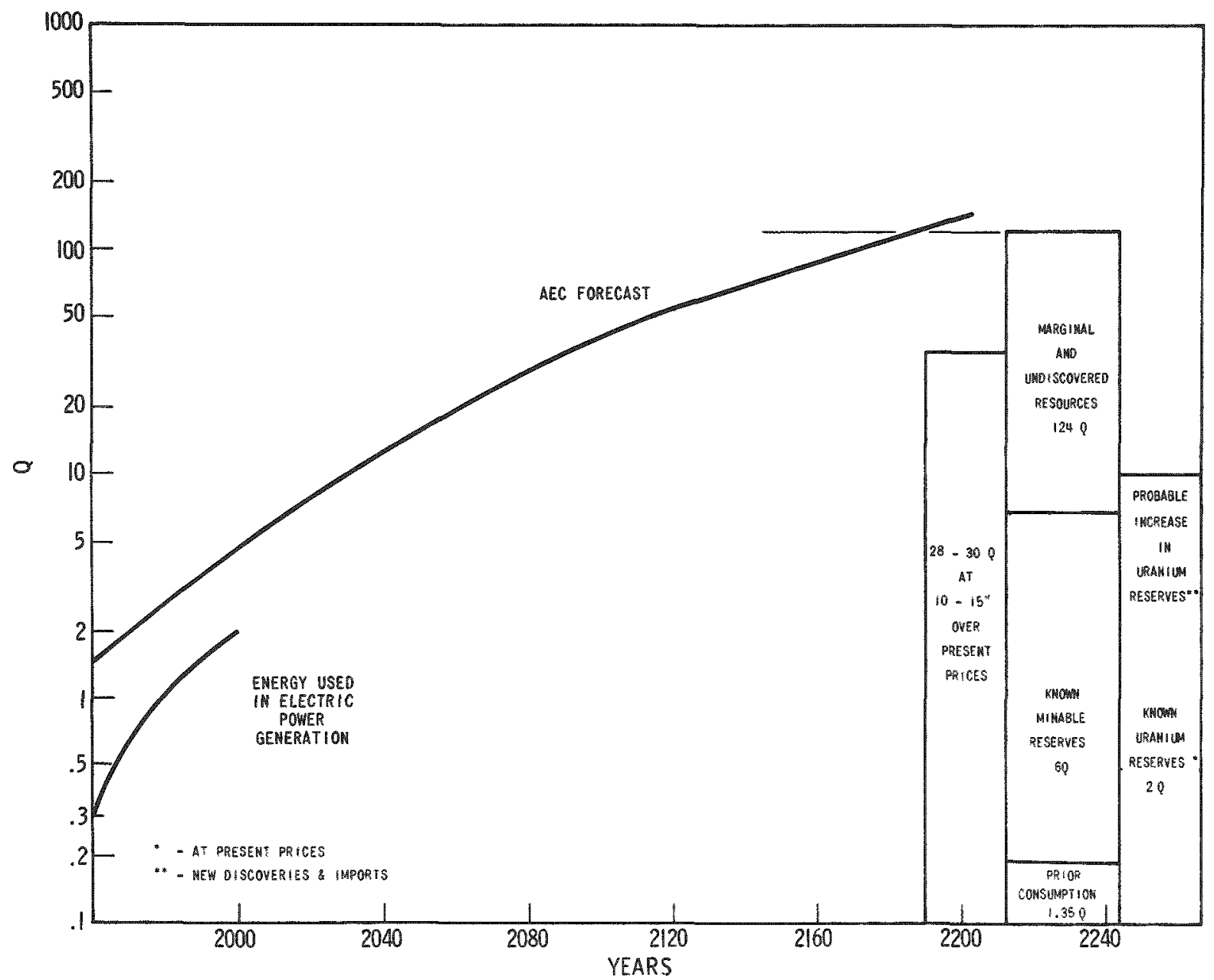

Fig. 11. Hypothetical Cumulative Use of Energy in the United States Compared to the Life of Presently Envisioned Resources of Fossil Fuels

4. Figure 12 shows the total rate of energy consumption by the electrical industry and its distribution among the nuclear and fossil energy sources. (1) The integrated energy under the nuclear curve is equal to about 0.3 "Q" unit by the year 2000, a number quite below the most conservative estimate of uranium reserves even if used entirely in thermal reactors. If a total of 0.3 " $Q$ " unit is utilized by the year 2000 out of the total resource of between 2 and 10 "Q" units available to thermal reactors, 
it does not seem apparent that fast breeders will be required to supplement energy availability at least to the year 2000.

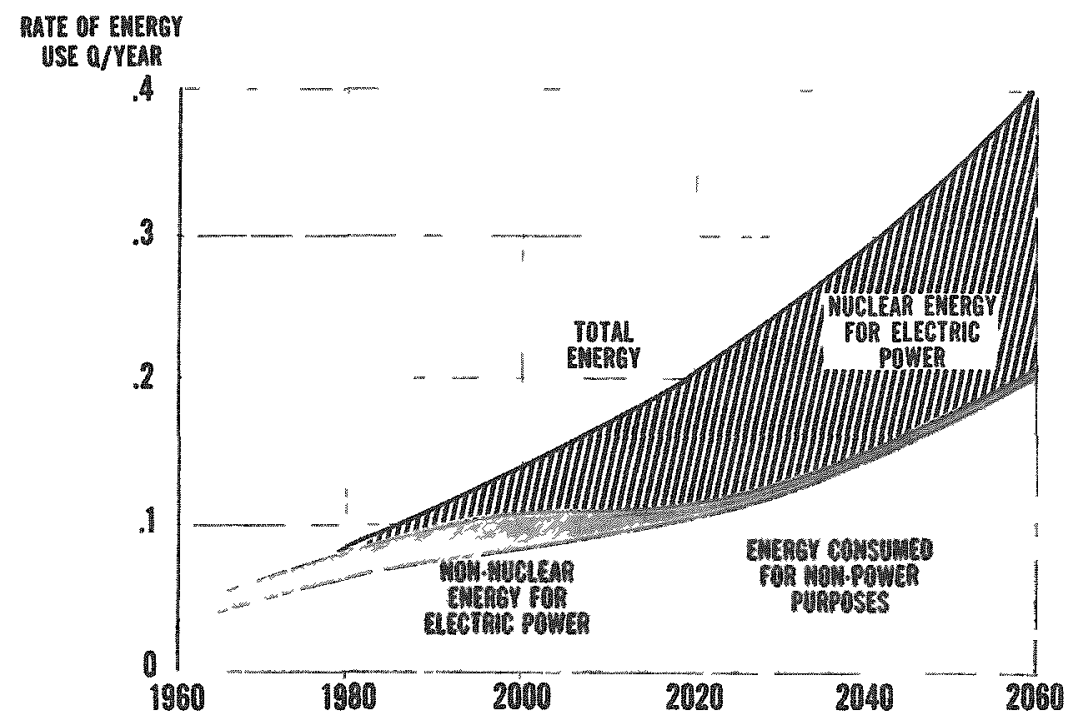

Fig. 12. Effect of Nuclear Power on

Fossil Euel Consumption

5. An alternative and, perhaps, more pressing need for fast reactor technology is to develop a moans of efficiently utilizing plutonium, either as breeder or as burner fuel. Consideration should now be given to the distribution of energy consumption of the 0.3 "Q" unit in nuclear power between thermal converters and fast breeder reactors.

6. The next point to be considered is the rate of fast breeder development and the degree to which it may be expected to supplement thermal converter power. In order to resolve this question, it is necessary to consider what quantity of fast reactor capacity will be on the line as a function of time. The rate of nuclear kilowatts from fast breeder reactors is subject to three constraints:

(1) the zero point at which the first major block of fast breeder power can be technically and economically added;

(2) an interim period during which the rate of fast breeder plant construction will depend upon its ability to attract low-risk capital investment in competition with other means of producing power;

(3) the ultimate rate of fast breeder addition will depend upon the availability of plutonium. first from water reactors and, eventualiy, from the excess plutonium created by the breeders. 
The first constraint of initial large plant startup in 1978 has been previously discussed. For the interim period an estimated rate of fast breeder additions, beginning in 1978, is shown in Fig. 13. This curve indicates that breeders might be added at a rate of about 1 every 2 years until 3 or 4 have been added, after which time the rate of addition could be increased considerably owing to the confidence level which would be developed in this type of system. Utilities would then be willing to invest larger sums of capital investment in a fast breeder type of plant, such that by the year 1988 , the rate of breeder additions would be so great as to be consuming totally the quantity of plutonium produced in thermal converter reactors; thus, the third constraint would be reached.

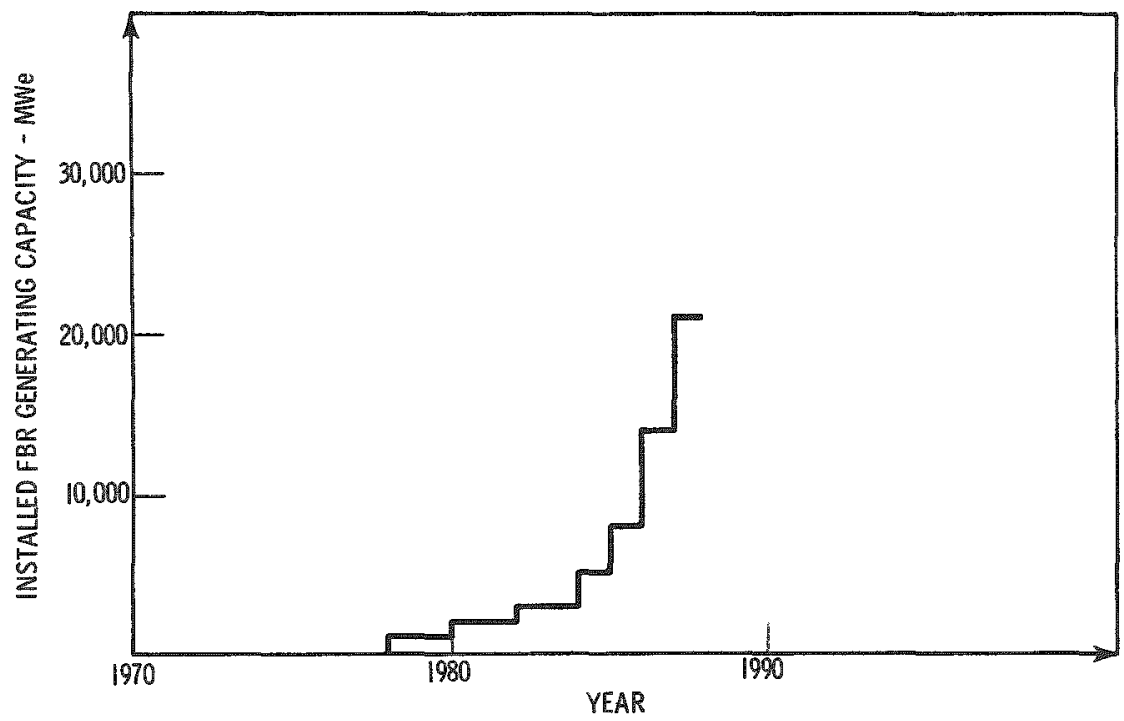

Fig. 13. Estimate of USA Installed Fast Breeder Reactor (FBR) Generating Capacity

The total nuclear picture would then be as presented in Fig. 14, where the total amount of installed nuclear $\mathrm{kW}$ is plotted vs. time up to the year 2000. There are three points on this curve worthy of note:

1. The rate of fast breeder installation is allowed to rise after 1988 at a rate equal to the amount of plutonium available to start them up.

2. The shaded portion of the middle of the curve indicates that this added amount of fast breeder capacity will be obtained through a breeding ratio of 1.4 .

3. The lower area indicates the amount of water-reactor plutonium which would be recycled through fast breeder reactors.

4. Finally, the last point is that by the year 2000 the total nuclear capacity would consist of approximately $\frac{1}{2}$ fast breeder capacity. 
This graph, incidentally, shows that most of the plutonium in fast reactors is probably water-reactor-grade plutonium. This would indicate that, for the next 30 years, water-reactor plutonium, having lower isotopic quantities of plutonium-240 and -241 , will be predominant in all operating fast reactors in this country. The integrated quantity of fast breeder energy supplied, illustrated in Fig. 14, is approximately 0.06 "Q" unit, which means that of the total of 0.3 " $Q$ " unit from nuclear power, 0.24 "Q" unit would still be derived from thermal converter reactors.

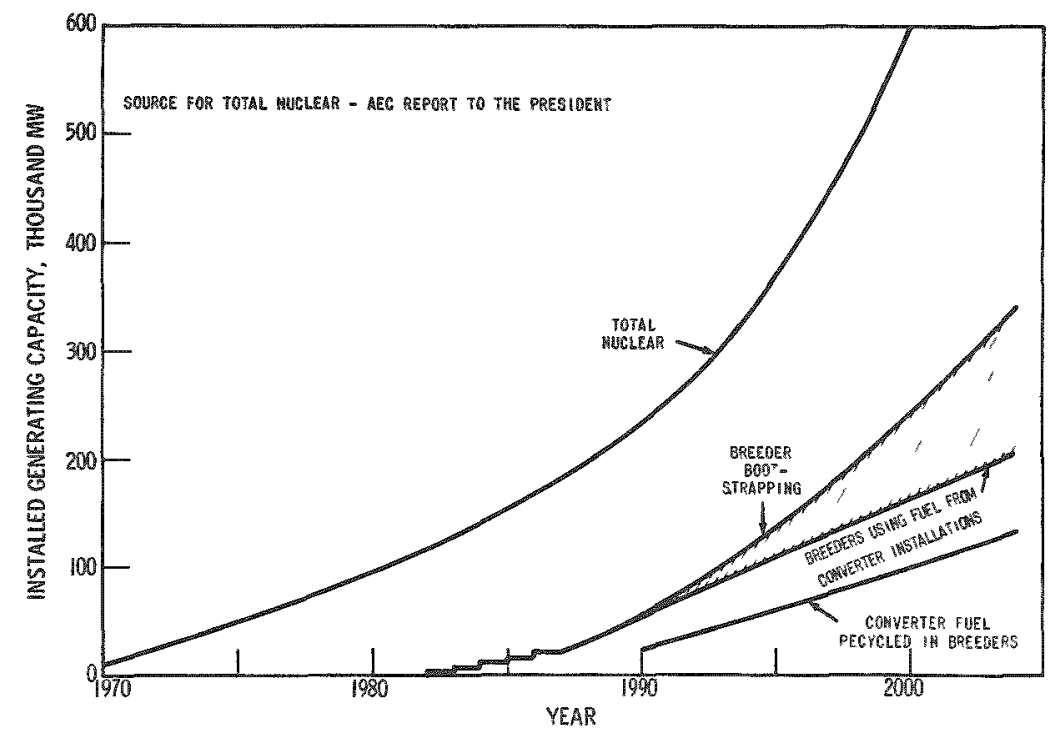

Fig. 14

Breceder Capacity in a Mixed Economy

Effect of Weapons Plutonium Availability - In Fig. 15 an alternative assumption is made for the purpose of accelerating the fast breeder program at a fast rate. This curve presumes that as soon as fast breeder reactors have demonstrated themselves to be clearly tochnically and

Fig. 15

Breeder Capacity in a Mixed Economy

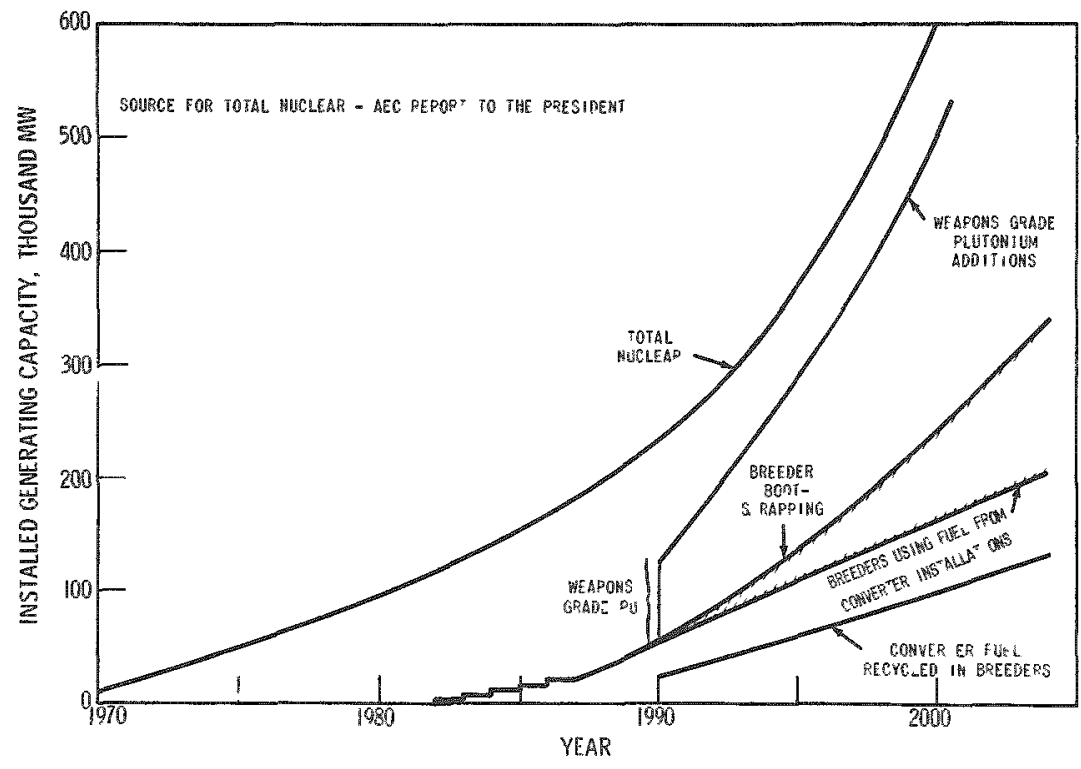


economically feasible and reliable, a large quantity of incremental plutonium is made available to the fast reactor growth from the weapons program. It might be suggested that a bold step of adding 50 tons of plutonium could be taken. A decision to add could be made in 1985 on the basis of performance to that date. Actual plant construction could begin at that time for operation in 1990. The curve then shows a step change upward in the quantity of fast breeder capacity and continues upward at a still faster rate owing to the still presumed breeding ratio of 1.4 . This curve shows that by the year $2000,90 \%$ of the installed nuclear capacity could be fast breeder capacity. In such a case the integrated quantity of energy produced by fast breeders would be approximately 0.10 " $Q$ " unit rather than the 0.06 produced under the earlier assumption. In such a case there would still be required a 0.2 "Q" unit from thermal converter reactors.

It is apparent from these two examples that the quantity of total power which could be obtained from fast breeders between now and the

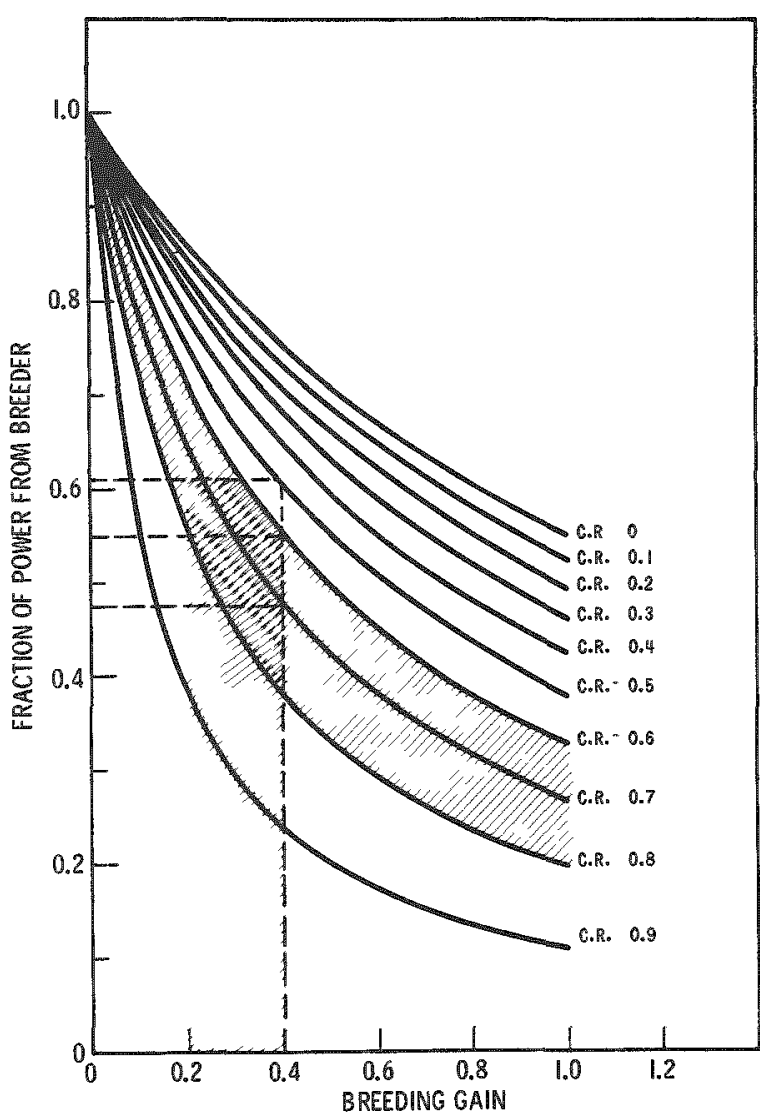

Fig. 16. Alsed Breeder-Converter Nuclear System (Limitung Case, Zero Losses) year 2000 will be small with regard to the total produced by nuclear power: in one case, $20 \%$, and in the case of adding weapons plutonium, $33 \%$.

Fast-Thermal Mixed Fuel Economy

The third objective for the year 2000 is to enter the millenium with a matrix of fast and thermal breeder reactor capacity such that it would be nearly self-sustaining with respect to fissionable material. Figure 16 shows an overall plan of "peaceful coexistence" * between thermal converters and fast breeders for a fixed inventory of fissionable material. Breeder reactors are suggested to have conversion ratios in the range of 1.2 to 1.4 , and thermal converter reactors are suggested to have conversion ratios in the range of 0.6 to 0.8 . The required percentage of fast breeder capacity is equal to between 45 and $65 \%$ of the total installed capacity.

*(Credit for this gem goes to Aristarkhov from the IAEA meeting, 1963.) 
This means that the mixture of fast and thermal reactors which would result from breeder additions at the rate of plutonium availability would be quite adequate to build a mixed fuel economy by the year 2000 .

\section{Conclusions}

Thus, it would appear that the three objectives for the year 2000 can be readily met by the presently planned Commission program for operating the first large breeder reactor by the year 1978. It is, further, important to note that the addition of prototype reactors or power demonstration reactors at too early a point not only serves to abort technical manpower but also to divert dollar-funding badly needed for the long-range development of economic breeder reactors. From these results, it would appear that the rate of progress suggested for the AEC program is both adequate and necessary.

A brief review of the fast breeder reactor program in six major countries has been presented, and their relative scopes, objectives, and schedules reported. This review has shown that none of the programs in any of the countries is completely adequate at the present time.

The US program is most nearly complete, but appears to lack effort in the imposing areas of plant-design technology and in some major component developments; it also would appear that a serious need exists in the US program for fast reactor fuel-testing capacity. In final comment on the US program, one additional question could be raised, namely, the necessity for two special-purpose experimental power reactors for the purpose of investigating fast fuel power coefficients when fuel-test capacity is so vitally needed.

The UK program has been dedicated primarily to getting the DFR running well, and, now that the objective has been achieved, attention is being focused on the long-range objectives of power prototypes and economic breeder reactors. Much work is required, particularly in the area of fuel testing and plant, component and systems design, development, and testing.

The French program, started approximately six years ago, is progressing reasonably well for its near-term objective, the construction and operation of Rapsodie I. The remainder of their program for a power prototype and an economic breeder reactor appears to be incomplete at this time, and schedule slippage is very likely, indeed.

The German program, although only very recently begun, seems to be very thorough in the area of fast reactor physics and power coefficient 
testing. It would appear to be advisable to have projects in Germany where experience in fabricating and testing vital sodium components and systems can begin.

The USSR program has proceeded at a satisfactory rate and now appears to be constrained by administrative decisions regarding the relative importance of fast breeder development and construction compared to other projects in that country. It can only be presumed that this relative importance is justified for the economics of that country.

It would appear that, either through EURATOM or the European Common Market, a more reasonable method of joint programs in developing fast breeders in Europe could be conducted. At the present time, the general European program in fast breeder development appears to have large amounts of wasteful duplication, particularly in the areas of fast reactor physics, while other areas critical to their mutual needs, such as large fast sodium components, and a fast fuel test facility, seem a.most neglected.

\section{Acknowledgements}

The writer wishes to express appreciation to Mr. E. E. Smith for his many calculations and other contributions to this paper.

\section{References}

1. Seaborg, G. T., Civilian Nuclear Power - A Report to the President 1962, USAEC, Washington (Nov 20, 1962).

2. Giambusso, A., and Wensch, G. W., The U.S.A. Fast Breeder Program, VIII Congresso Nucleare, Rome, Italy, 1963.

3. Pursel, C. A., Trip Report to IAEA Conference on Operating Experience with Power Reactors, July 4-8, 1963.

4. Seaborg, G. T., Atomic Energy in the Soviet Union (May 1963).

5. Leipunskii et al., Operational Experience with the BR-5 Reactor, IAEA, Vienna, Austria, 1963.

6. Laithwaite, J. M., The UK Fast Reactor Programme, VIII Congresso Nucleare, Rome, Italy (1963). 
7. de Stordeur, A., The European Community Activities in the Fast Breeder Field, VIII Congresso Nucleare, Rome, Italy, 1963.

8. Staff Paper - ANL and DRD, Fast Breeder Reactor Program, USAEC (July 1963).

9. Gunson, W. E., Boyd, W. S., and Smith, E. E., Status Report on Fast Reactor Programs as of December 1962, WCAP-2171 (Dec 1962).

10. Zebroski, E. L., et al., Plutonium Fuel Fabrication and Reprocessing for Fast Ceramic Reactor, GEAP-2876 (Feb 1962).

11. Kania, A., et a1., Physics of Fast and Intermediate Reactors, pp. 301-356, IAEA, Vienna (1962).

12. Smith, E. E., and Wright, J. H., Near-term Plutonium Requirements and Availability, WCAP-2339 (Aug 1963).

13. Malay, F. L., 1000 MWe Pressurized Water All Nuclear Power Plant Study, Contract AT(30-1)-3070 (March 1963).

14. Keyfitz, I. M., et al., $1000 \mathrm{MWe}$ Supercritical Pressure Nuclear Reactor Power Plant, SCOTT-R Design Study, WCAP-2040 (May 1963),

15. Energy Policy Staff, Supplies, Costs and Uses of Fossil Fuels, U.S. Department of Interior (Feb 1963).

16. Cohen, K., and Zebroski, E. L., Uranium Utilization in the Twentieth Century, Atomic Industrial Forum (1962).

17. Kuhn, D. W., et a1., Energy from Uranium and Coal Reserves, TID-8307 (May 1960).

18. Plutonium Survey - 1963, Edison Electric Institute, New York, New York (Sept 1963). 


\section{Discussion of Paper}

Presented by Mr. Wright

MR. LANE (Oak Ridge):

It seems to me that there is a little dilemma about getting enough plutonium into the fast breeder business fast enough without going to military grade. Did you look at, say, building a number of natural uranium heavy water reactors as a better way of getting the additional amount of plutonium?

MR. WRIGHT:

The intent of the paper was to show that there is not a dilemma in this regard. The plutonium coming from other converter reactors, water reactors for example, will supply plutonium sufficiently fast to be consumed by the fast breeder capacity that can legitimately attract competitive capital.

MR. DIETRICH (General Nuclear Engineering Corp.):

Is the real question not how much plutonium you get from the hypothetical water reactors, but how much uranium they burn up in the meantime?

MR. WRIGHT:

Well, I think there is an interesting point here. This is brought out in the paper. It says that of the total of 2 " $Q$ " units to be used for electricity between now and the year 2000, only 0.3 of a " $Q$ " unit will be derived from nuclear power. The one approach not using weapons grade plutonium means this distribution will be 0.06 " $Q$ " units of fast breeders, and 0.24 " $Q$ " units of thermal power.

There are at least 2 " $Q$ " units available from low conversion, thermal reactors, and 2 " $Q$ " units compares very favorably with the 0.24 " $Q$ " units that are anticipated to be burned. So this does not constitute any particular problem at all.

MR. de STORDEUR (EURATOM):

I noticed several errors in Fig. 2 concerning the programs in Germany, France, and Italy.

Also, you started with a comparison of the different programs in European countries, Russia, and the United States. However, the rest of 
the discussion was based on an American economy-type of system, be cause in the plutonium-deficiency curves which you presented, you did not take into account the fact that a good part of the European, EURATOM, and UKAEA, power system is based on the natural uranium-graphite reactors.

MR. WRIGHT:

Yes, that is right.

MR. de STORDEUR:

What I would like to know is do you draw any conclusions about the world situation in your paper?

MR. WRIGHT:

Yes. I doubt that Germany will have a prototype running in the early 1970's because they are not developing any heavy industry with which to supply their equipment. I think it is somewhat questionable that many of the other places in Europe are going to have economic breeder reactors running in 1975. I state this in the paper. I think theseareoverly optimistic objectives which will not be met.

MR. LAWROSKI:

One more question.

MR. COHEN (General Electric):

I would like to make merely one comment on a particular slide, that is, concerning the cost estimates which show that the fast breeder reactor fuel cycle cost is greater than one mill per kilowatt hour. Also, the projection of what is going to happen to the uranium prices. Let me say I do not believe the fuel cost for a fast breeder reactor will be as high as one mill per kilowatt hour.

MR. WRIGHT:

The chart showed $0.8 \mathrm{mill}$ on present ground rules.

MR. COHEN:

I don't think it will be as high as 0.8 , and I don't think the ground rules are going to evolve the way you believe they will. 
MR. WRIGHT:

Thank you. We will have to wait and see.

MR. MENKE (United Nuclear):

Using a fast conversion factor given me by Mr. Lane, that 50 "Q" is $10^{6}$ tons of $U_{3} O_{8}$. I think you are optimistic on how much uranium we see today. As you said, we have not been looking for five years; but the amount of uranium we can see right now is very much less than you indicated.

MR. WRIGHT:

We11, actually, the curve shown contains three different postulates of what the uranium content is. One of them is TID-8201. One is the contribution in the Report to the President. The third was made by our colleague, Dr. Cohen. These were shown on the curve, and on that basis I think it is probable.

MR. MENKE:

Let me say that among those three, there are no uranium miners: and we speak as one. 


\title{
PRINCIPLES AND PROBLEMS OF THE DEVELOPMENT OF A FAST REACTOR FUEL ELEMENT
}

\author{
Wolf Håfele \\ Institut für Angewandte Reaktorphysik \\ Kernforschungszentrum, Karlsruhe, Germany
}

\section{Introduction}

For the development of a fuel element of a large fast oxide breeder reactor it is necessary to recognize the underlying principles and problems as well. The natural way to operate a laxge fast oxide breeder is to use a closed plutonium fuel cycle. The fact that the fuel cycle is closed creates an interdependence of the fuel element with regard to reprocessing and refabricating. A straightforward fuel-cycle cost analysis is an excellent way to investigate this interdependence. It is possible to improve the fuel heat conductivity by addition of ingredients (such as molybdenum and BeO). Several ways to use the improved heat conductivity must be considered.

In addition there is the question whether the performance of the fuel is compatible with the performance of the cladding material. Finally, there is the problem of inherent safety. Large fast oxide reactors rely on the Doppler coefficient, and this has to be a prompt-acting coefficient. These are the points of view to establish principles for a fuel-element development of a large fast oxide breeder.

II. The Parameter Dependence of the Costs of a Closed Fuel Cycle for a Fast Breeder Reactor.

We now list the parameter dependence of the costs for a closed fuel. cycle. The involved algebra is simple and self-explanatory. We assume in oux formulas that the blanket does not contxibute to the power production, which, of course, is not exact. But a proper adjustment of the effective thermal efficiency can take care of this.

First, we consider the costs for the plutonium inventory. They are given by the following expression:

$$
\mathrm{K}_{\text {interest }}=\frac{\alpha \beta\left(1+\frac{\mathrm{tw}}{\mathrm{tsT}}\right)}{8.7 \delta \mathrm{b} \mu} \quad\left[\frac{\mathrm{mills}}{\mathrm{kWhe}}\right] \text {, }
$$

where $\alpha$ is the price of plutonium in $\$ / g$ of fiss $P u, \beta$ is the interest rate, tw is the number of days required to recycle plutonium from the fast reactor core back to the fast reactor core, $t_{\mathrm{ST}}$ is the average irradiation time of the fast reactor in days, $\delta$ is the load factor, $\mu$ the effective thermal efficiency, and $\mathrm{b}$ is the rating in MWt per $\mathrm{kg}$ of fissionable plutonium. The 
factor $\left[1+\left(\mathrm{tw} / \mathrm{t}_{\mathrm{ST}}\right)\right]$, therefore, is the ratio of the total plutonium inventory to the in-pile plutonium inventory. For $\mathrm{t}_{\mathrm{ST}}$ we find the following expression:

$$
\operatorname{tST}_{S T}=\frac{a_{M}(1+y)}{10^{3} b \delta} \quad[\text { days }]
$$

where aM is the average burnup in MWD/tonfuel, and $\mathrm{y}$ is the ratio of fertile $\left(U^{238}+P_{u^{240}}\right)$ to fissile $\left(P_{u^{239}}+P_{u^{241}}\right)$ material in the fuel. Combination of (2) and ( 1 ) shows that $K_{\text {interest }}$ is independent of $b$.

$$
K_{\text {interest }}=\frac{\alpha \beta}{8.7 \delta \mathrm{b} \mu}+\frac{\alpha \beta \mathrm{tw} \times 10^{3}}{8.7 \mu \mathrm{a} \mathrm{M}(1+\mathrm{y})} \text {. }
$$

We will call the first term of $(1 \mathrm{a}) \mathbb{K}_{\text {interest }}^{\prime}$ and the second term $K_{\text {interest: }}^{\prime \prime}$

$$
\begin{aligned}
K_{\text {interest }}^{\prime} & =\frac{\alpha \beta}{8.7 \delta \mathrm{b} \mu} ; \\
K_{\text {interest }}^{\prime \prime} & =\frac{\alpha \beta t w \times 10^{3}}{8.7 \mu a_{M}(1+y)} .
\end{aligned}
$$

The next cost to be considered is that due to refabrication, which can be given either in terms of $(\$ / \mathrm{kg}$ of fuel $)\left(Z_{1}\right)$ or in terms of $(\$ /$ pin $)\left(k_{1}\right)$. In case of a fast reactor the fuel pins are of a small diameter, and fabrication costs for one fuel pin are somehow independent of the diameter. The two quantities $Z_{1}$ and $k_{1}$ are related as follows:

$$
Z_{1}=\frac{k_{1} \times 10^{3}}{H\left(\pi R^{2}\right) \rho_{F}} \quad\left[\frac{\$}{k g \text { fuel }}\right] \text {, }
$$

where $H$ is the core length in $\mathrm{cm}, \mathrm{R}$ is the radius of the pin in $\mathrm{cm}$, and $\rho$ is the fuel density in $\mathrm{g} / \mathrm{cm}^{3}$. In general, both $Z_{\mathbb{I}}$ and $k_{\mathbb{I}}$ will depend on the radius $R$ of the pin. By means of the relation

$$
\pi R^{2}=\frac{\chi(1+y)}{\left(b \times 10^{3}\right) \rho_{F}} \quad\left[\mathrm{~cm}^{2}\right]
$$

where $\chi$ is the average power per $\mathrm{cm}$ pin length $[\mathrm{W} / \mathrm{cm}]$, a dependence of the rating $b$ is found. Whereas for a detailed cost analysis it is important to explore the exact dependence of $k_{1}$ (or $Z_{1}$ ) on radius, we will here assume that $k_{1}$ is a constant.

The number of pins, $N_{\text {pin }}$ in the core is 


$$
N_{\text {pin }}=\frac{\mathrm{bM}_{\text {core }}^{\text {fiss }} \times 10^{6}}{H \chi} .
$$

The number of pins to be fabricated in one year, $\mathrm{n}_{\text {pin }}$, is

$$
\mathrm{n}_{\text {pin }}=\frac{\left(3.6 \times 10^{11}\right) \mathrm{M}_{\text {core }}^{\mathrm{fiss}} \mathrm{b}^{2} \delta}{\mathrm{H} \times \mathrm{aM}(1+\mathrm{y})}
$$

where $\mathrm{M}_{\mathrm{Core}}^{\mathrm{fiss}}$ is the core inventory of fissionable material in $\mathrm{kg}$.

We now find

$$
\mathrm{K}_{\mathrm{refabr}}=\frac{\left(10^{8}\right) \mathrm{k}_{1} \mathrm{~b}}{(2.4) \mathrm{H} \mu \mathrm{aM} X(1+\mathrm{y})} \quad\left[\frac{\mathrm{mills}}{\mathrm{kW}-\mathrm{he}}\right]
$$

or, in terms of $\mathrm{Z}_{1}$;

$$
\mathrm{K}_{\mathrm{refabr}}=\frac{\mathrm{Z}_{1}\left(10^{2}\right)}{(2.4) \mathrm{aM}_{\mathrm{M}}^{\mu}} \quad\left[\frac{\mathrm{mills}}{\mathrm{kW}-\mathrm{he}}\right] \text {. }
$$

Now, $k_{1}$ are the costs in $\$ / p i n, Z_{1}$ the costs in $\$ / \mathrm{kg}$ of fuel. The costs for reprocessing are easy to calculate. Suppose that the reprocessing of $1 \mathrm{~kg}$ of fuel costs $Z_{2} \$$; we then find

$$
\mathrm{K}_{\text {repro }}=\frac{\mathrm{Z}_{2}\left(10^{2}\right)}{(2.4) a_{\mathrm{M}} \mu}\left[\frac{\mathrm{M}}{\mathrm{M}_{\text {Core }}^{\text {fiss }}(1+\mathrm{y})}\right]\left[\frac{\text { mills }}{\mathrm{kW}-\mathrm{he}}\right],
$$

when $M_{\text {core }}^{\text {fiss }}(1+y)$ is the amount of total fuel in the core in $\mathrm{kg}$, $M$ is the amount of fuel to be processed with the core, also in $\mathrm{kg}$. In most cases it will be necessary to process the axial blanket together with the core because the one pin contains the two axial blankets together with the core. So we have in this particular case

$$
\frac{M}{M_{\text {core }}^{\text {fiss }}(1+y)}=1+\frac{M_{\text {Blanket, ax }}}{M_{\text {core }}^{\text {fiss }}(1+y)} .
$$

The first core capitalization is also easy to calculate. One finds

$$
K_{\text {first core }}=\frac{k_{1} \beta\left(10^{3}\right)}{H \chi \delta \mu(8.7)} \quad\left[\frac{\text { mills }}{\mathrm{kW}-\mathrm{he}}\right] \text {. }
$$

To be complete we also note the expression for the contribution of the capital costs to the fuel cycle, although we are not going to use it. Assume that the installed electrical $\mathrm{kW}$ costs $\mathrm{A} \$$, then we find 


$$
K_{I}=\frac{A \beta}{8.78} \quad\left[\frac{\mathrm{mills}}{\mathrm{kW}-\mathrm{he}}\right]
$$

We now concentrate on the plutonium gain in the blanket. At the end of each core cycle the following amount of fissionable plutonium must be added to the core in order to reach criticality again:

$$
\operatorname{Miss}_{\text {Core }} \frac{\operatorname{aM}(1+y)}{10^{6}}\left(1-B . R \cdot{ }^{a}\right) \quad[\mathrm{kg}]
$$

where B.R. ${ }^{a}$ is the sum of the internal (core) breeding ratio and the breeding ratio due to the axial blanket. It is helpful to realize that $a_{M}(1+y) / 10^{6}$ is the burnup in per cent of initial fissionable atoms (using: I $g$ fiss mat = $1 \mathrm{MWD}$ ). The plutonium buildup in the radial blanket after one core cycle is

$$
M_{\text {core }}^{\text {fiss }} \frac{a_{M}(1+y)}{10^{6}}(B . R . g e s-B . R . a) \quad[k g]
$$

In case the radial blanket is not processed at the same time with the core rather than after $P$ core cycles, a plutonium holdup in the radial blanket, and therefore an intexest loss, takes place. In that case one has to buy the fraction (1-B.R.a) of (14) after each core cycle, say at $\alpha[\$ / g$ of fiss $\mathrm{Pu}]$ and interest rate $\beta$. It also would have been possible to enjoy the interest income due to the fraction (B.R.ges - 1) of (14) in case we had not waited for $P$ core cycles. The sum of these two interest losses gives the following loss of dollars:

$$
\frac{P(P-1)}{2} \frac{{ }^{t} S T}{365} \alpha \beta\left(10^{3}\right) M_{\text {Core }}^{\text {fiss }} \frac{a_{M}(1+y)}{10^{6}}(B . R . g e s-B . R . a) \quad[k g] .
$$

We realize that the refabrication of the radial blanket rods costs $Z_{3}[\$ / \mathrm{kg}]$ and the reprocessing costs $Z_{A}[\$ / \mathrm{kg}]$, and further that during $P$ core cycles

$$
\text { PtSTM } \text { Core }_{\text {fiss }} \text { bo }\left(24 \times 10^{3}\right) k W-\text { he }
$$

were produced. We then find the following net effect of the bred plutonium:

$$
\begin{aligned}
-K_{P u}= & \frac{\alpha(B \cdot R \cdot g e s-1)}{24 \mu} \\
& -\frac{(P-1) \frac{t_{S T}}{730} \alpha \beta(B \cdot R \cdot g e s-B \cdot R \cdot a)}{24 \mu} \\
& -\frac{\left(Z_{3}+Z_{4}\right)\left(10^{3}\right)}{24 a_{M}{ }^{\mu}} \frac{M_{B l a n k e t, r a d}}{M_{\text {core }}^{\text {fis }}(1+y)} \frac{1}{P}\left[\frac{\text { mills }}{k W-h e}\right]
\end{aligned}
$$


Looking into the expressions for the partial costs (1) - (15), one finds two ways to optimize the costs.

$\mathrm{K}_{\text {refabr }}$ is proportional to $\mathrm{b}$ and $\mathrm{K}_{\text {interest }}$ is proportional to $1 / \mathrm{b}$. In the same way, (15) contains a term proportional to $p$ and a term proportional to $1 / \mathrm{P}$. Therefore we have to look for an optimum value for $\mathrm{b}$ as well as for $P$. For $P$ we find the following optimum value

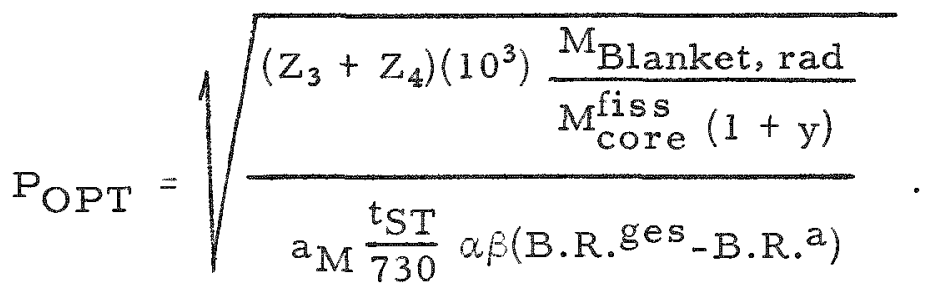

For the optimum value of $-\mathrm{K}_{\mathrm{Pu}}$ we therefore find the following:

$$
\begin{aligned}
& -\left(\mathrm{K}_{\mathrm{Pu}}\right)_{\mathrm{OPT}}=\frac{\alpha(\mathrm{B} \cdot \mathrm{R} \cdot-1)}{24 \mu}+\frac{\frac{\mathrm{t}_{\mathrm{ST}}}{730} \alpha \beta(\mathrm{B} \cdot \mathrm{R} \cdot \mathrm{ges}-\mathrm{B} \cdot \mathrm{R} \cdot \mathrm{a})}{24 \mu} \\
& -\frac{2}{24 \mu} \sqrt{\frac{\left(\mathrm{Z}_{3}+\mathrm{Z}_{4}\right) \frac{\mathrm{M}_{\text {Blanket, rad }}\left(10^{3}\right)}{\mathrm{M}_{\mathrm{core}}(1+\mathrm{y})}}{\mathrm{a} \mathrm{M}} \frac{\mathrm{tST}}{730} \alpha \beta(\text { B.R.ges - B.R.a })\left[\frac{\mathrm{mills}}{\mathrm{kW}-\mathrm{he}}\right]}
\end{aligned}
$$

The optimum value of $b$ is found in the same way by putting $K_{r e f a b r}=$ $\mathrm{K}_{\text {interest: }}$ :

$$
b_{O P T}=\sqrt{\frac{\alpha \beta H_{M} \times(1+y)}{\left(10^{8}\right)(3.6) k_{1} \varepsilon}} .
$$

Using (6), we find

$$
R_{\mathrm{OPT}}=\sqrt{\frac{1}{\pi \rho_{\mathrm{f}}} \sqrt{\frac{\left(3.6 \times 10^{2}\right) k_{1} \delta \times(1+y)}{\alpha_{\mathrm{fHa}} \mathrm{H}}}}
$$

and

$$
K_{\text {interest }}^{\prime}+K_{\text {refabr }}=\frac{0.440}{\mu} \sqrt{\frac{\alpha \beta k_{1}\left(10^{8}\right)}{\operatorname{Ha}_{M} \chi(1+y) \delta}} .
$$

The optimized sum of the partial costs can be written as follows: 


$$
\begin{aligned}
\left(K_{\text {total }}\right) \text { OPT } & =\left(K_{\text {interest }}^{\prime}+K_{\text {refabr }}\right) \text { OPT }+\left(K_{\text {repro }}+K_{\text {interest }}^{\prime \prime}\right) \\
& +K_{\text {first core }}-\left(K_{P u}\right) \text { OPT }
\end{aligned}
$$

It is interesting to note that the first texm varies like $\sqrt{1 / a_{M}}$, the second term like $1 /$ aM, the third term like $1 / X$, whereas the fourth term has no such clear parameter dependence. It is also interesting to see from (19) that $R O P T$ varies slowly with most of the parameters.

It is worthwhile to note that the parameter dependence of the fuelcycle costs is quite different for optimized and nonoptimized reactors. Take, for example, the dependence of the plutonium price $\alpha$. In the first case we consider a reactor which is already built. The optimization has taken place at $\alpha=\alpha_{0}$, and the parameter dependence is therefore as follows:

$$
K=a+b \alpha
$$

( $a$ and $b$ are constants with respect to $\alpha$ ). Suppose further that the reactor was optimized for a plutonium price $\alpha=\alpha_{0}$. If the price now changes from $\alpha_{0}$ to $\alpha_{1}$, the change of the fuel-cycle costs is in accordance with (2la).

In the second case we consider a reactor which has to be built after the plutonium price has changed from $\alpha_{0}$ to $\alpha_{1}$. Here the fuel-cycle costs are as follows ( $c$ is also a constant):

$$
\begin{aligned}
K_{\mathrm{OPT}} & =c+\sqrt{\alpha} \frac{2(a-c)}{\sqrt{\alpha_{0}}}-\alpha\left(\frac{a-c}{\alpha_{0}}-b\right) \\
& =c+b \alpha+(a-c)\left(2 \sqrt{\frac{\alpha}{\alpha_{0}}}-\frac{\alpha}{\alpha_{0}}\right) .
\end{aligned}
$$

We have

$$
\begin{aligned}
& \mathrm{KOPT}_{\mathrm{OP}}\left(\alpha_{0}=\mathrm{K}\left(\alpha_{0}\right)\right. \\
& \left(\frac{\mathrm{dKOPT}}{\mathrm{d} \alpha}\right)_{\alpha=\alpha_{0}}=\left(\frac{\mathrm{dK}}{\mathrm{d} \alpha}\right)_{\alpha=\alpha_{0}} ; \\
& \mathrm{K}_{\mathrm{OPT}}(\alpha)<\mathrm{K}(\alpha) \quad \text { for } \alpha \neq \alpha_{0}
\end{aligned}
$$

It is often stated that the fuel-cycle costs of fast breeders do not depend strongly on the value of $\alpha$. Such a statement, however, implies that the reactor is already built. 
III. Numerical Examples for Cost Figures

For general orientation purposes it is useful to give numerical examples. We concentrate our attention to large fast oxide reactors and assume the following figures for a reference reactor:

$$
\begin{aligned}
& M_{\text {core }}^{\text {fiss }}=1200 \mathrm{~kg} ; \\
& M_{\text {fuel }}=M_{\text {core }}^{\text {fiss }}(1+y)=9600 \mathrm{~kg}\left(\text { this means } y=7 \text { or } \frac{1}{1+y}=12.5 \times 10^{-2}\right) ; \\
& \mathrm{M}_{\text {Blanket, ax }}=7660 \mathrm{~kg} \text {; } \\
& \mathrm{M}_{\text {Blanket, rad }}=21140 \mathrm{~kg} \text {; } \\
& \delta=0.8 ; \quad \mu=0.4 ; \quad H=100 \mathrm{~cm}\left(\text { this means } \frac{H}{D} \approx 0.6\right) ; \\
& \left.a_{M}=10^{5} \frac{M W D}{\text { ton }} ; \quad \chi=300 \frac{W}{\mathrm{~cm}} \text { (this means } \chi_{\mathrm{MAX}} \approx 600 \frac{\mathrm{W}}{\mathrm{cm}}\right) ; \\
& \rho_{F}=10 \frac{\mathrm{g}}{\mathrm{cm}^{3}} ; \quad \beta=0.11 ; \quad \alpha=6.5 \frac{\$}{\mathrm{gPu}} ; \quad \mathrm{k}_{1}=\frac{60 \$}{\mathrm{pin}} ; \\
& \text { B.R.ges }=1.45 ; \quad \text { B.R. } a=0.9 ; \quad Z_{2}=120 \frac{\$}{\mathrm{~kg}} ; \quad Z_{3}=Z_{4}=50 \frac{\$}{\mathrm{~kg}} .
\end{aligned}
$$

The analysis of a great number of multigroup calculations of our Karlsruhe group shows that for the class of considered large oxide reactors the value of $B . R{ }^{a}$ is mostly between 0.85 and 0.9 . The value of $Z_{2}$ is highly uncertain; the values of $Z_{3}$ and $Z_{4}$ are in agreement with AEC cost compilations (1); the value of $k_{1}$ is in accordance with the estimate of other authors. $(2,3)$

First, evaluation of ROPT and bOPT gives

$$
R_{\text {OPT }}=0.275 \mathrm{~cm} ; \quad b_{O P T}=1 \frac{\mathrm{MW}}{\mathrm{kg} \text {, fiss }}
$$

We also get

$$
t_{S T}=10^{3} \text { days }
$$

Assuming tw $=200$ days, we get

$$
\left(1+\frac{t w}{t_{S T}}\right)=\frac{M_{\text {total }}}{M_{\text {inpile }}}=1.2 .
$$


This leads to the following break down:

$$
\begin{aligned}
& K_{\text {interest }}^{\prime}=0.261 \mid-\left(K_{\mathrm{Pu}}\right) \mathrm{OPT}=0.132 \text { for } \mathrm{POPT}=2 \\
& \mathrm{~K}_{\text {refabr }}=0.261 \\
& K_{\text {interest }}^{\prime \prime}=0.052 \\
& K_{\text {repro }}=0.230 \\
& K_{\text {first core }}=\frac{0.070}{0.874} \mid\left(K_{\text {total }}\right) \text { OPT }=0.74\left[\frac{\text { mills }}{\mathrm{kW}-\mathrm{he}}\right]
\end{aligned}
$$

In case such a reactor is already built, we find the following $\alpha$-dependence:

$$
\mathrm{K}=0.676+0.0093 \alpha\left[\frac{\mathrm{mills}}{\mathrm{kW}-\mathrm{he}}\right]
$$

In case the reactor is to be built, the $\alpha$-dependence is

$$
\mathrm{K}_{\mathrm{OPT}}=0.300+0.295 \sqrt{\alpha}-0.048 \alpha\left[\frac{\mathrm{mills}}{\mathrm{kW}-\mathrm{he}}\right]
$$

In Fig. $1 \mathrm{~K}$ and KOPT are plotted as a function of $\alpha$.

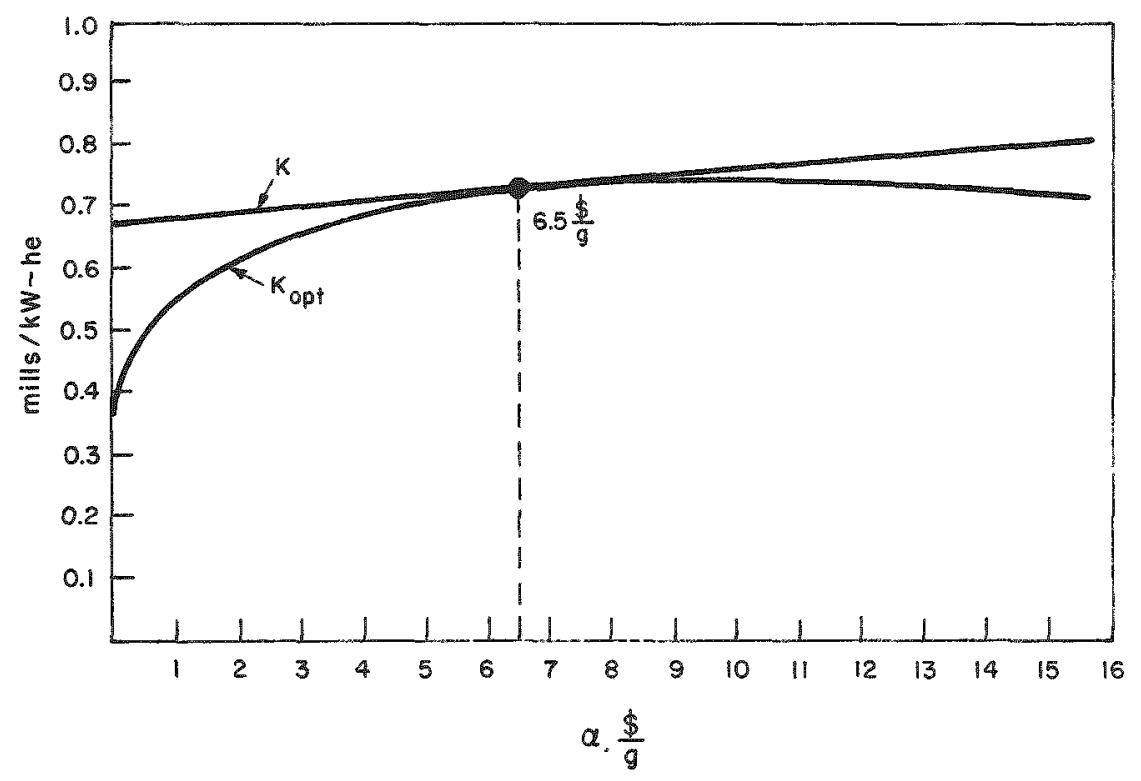

Fig. 1. The Dependence of the Fuel-cycle Cost $K$ and $K_{O P T}$ on the Plutonium Value $\alpha$

$$
\begin{aligned}
\mathrm{K} & =0.676+0.0093 \alpha\left[\frac{\mathrm{mills}}{\mathrm{kW}-\mathrm{he}}\right] \\
\mathrm{K}_{\mathrm{OPT}} & =0.300+0.295 \alpha^{1 / 2}-0.048 \alpha\left[\frac{\mathrm{mills}}{\mathrm{kW}-\mathrm{he}}\right]
\end{aligned}
$$


It is very instructive to repeat the numerical evaluation for a burnup of $\mathrm{a}_{\mathrm{M}}=30,000 \mathrm{MWD} / \mathrm{ton}$. Here we find

$$
\begin{aligned}
& \mathrm{R}_{\mathrm{OPT}}=0.37 \mathrm{~cm} \\
& \text { bOPT }=0.58 \mathrm{MW} / \mathrm{kg} \text {, fiss: } \\
& t_{S T}=518 \text { days: } \\
& K_{\text {interest }}^{\prime}=0.493 \mid-\left(K_{\text {Pu }}\right) \text { OPT }=0.035 \text { for } P_{\text {OPT }}=5 \\
& \mathbb{K}_{\text {refabr }}=0.493 \\
& \mathrm{~K}_{\text {interest }}^{\prime \prime}=0.156 \\
& \mathbb{K}_{\text {repro }}=0.770 \\
& K_{\mathrm{fixst} \text { core }}=\frac{0.07}{1.982} \quad K_{\text {total }}=1.947\left[\frac{\mathrm{mills}}{\mathrm{kW}-\mathrm{he}}\right]
\end{aligned}
$$

It is interesting to see that for $a_{M}=30,000 \mathrm{MWD} /$ ton the reprocessing costs are already the largest term, whereas for a $\mathrm{M}=100,000 \mathrm{MWD} /$ ton the reprocessing costs are reasonably low, so that a burnup of $100,000 \mathrm{MWD} /$ ton seems to be essential for the concept of large fast oxide breeders.

More generally, we conclude that at a burnup of $100,000 \mathrm{MWD} /$ ton fuel-cycle costs in the neighborhood of $0.8 \mathrm{mill} / \mathrm{kW}$-he seem likely. This was stated before.(4) If a fuel element is available which meets the assumptions (22), most of the development effort should then be used to lower the capital costs. For $A=165 \$ / \mathrm{kW}-\mathrm{h}$ we find from (13) and (22),

$$
K_{I}=2.6 \mathrm{mills} / \mathrm{kW}-\mathrm{he}
$$

for $\beta=0.14$ the value is $3.3 \mathrm{mills} / \mathrm{kW}$-he instead. So $K_{I}$ is by far larger than the fuel-cycle cost ( $\left.K_{\text {total }}\right)$ OPT.

IV. The Influence of the Out-of-Pile Part of the Fuel Cycle to the FuelElement Design

The study of our two numexical examples shows that, in particular for high burnups, the costs contribution of the term (Kinterest + $K_{\text {refabr }}$ OPT in (21) is the largest. This term varies like $\sqrt{a b k_{1} / a M^{\prime}}$ But this means that the step of refabricating $\left(k_{1}\right)$ influences strongly the fuel and reactor design [see (18) and (19)]. The step of reiabricating depends in particular on the question whether remote refabricating is necessary or not [see also Ref. 7]. 
In case of a closed plutonium core cycle, one adds only the amount

$$
M_{\text {core }}^{\text {fiss }} \frac{a_{M}(1+y)}{10^{6}}\left(1-B \cdot R^{a}\right)\left[\frac{\mathrm{kg}}{\text { core cycle }}\right]
$$

of plutonium in order to reach criticality for the next cycle again. The complementary fraction of the plutonium bred in the radial blanket, namely,

$$
M_{\text {core }}^{\text {fiss }} \frac{a_{M}(1+y)}{10^{6}}(B . R . g e s-1)\left[\frac{\mathrm{kg}}{\text { core cycle }}\right] \text {, }
$$

will be used for sale. The plutonium of the radial blanket is clean $\mathrm{Pu}^{239}$ almost. Such a closed core cycle leads to the following isotope composition, denoted as $\mathrm{Pu}^{\infty}$ :

$\begin{array}{lll}\text { Isotope } & & \text { PerCent } \\ 239 & & 47.76 \\ 240 & & 43.28 \\ 241 & & 5.58 \\ 242 & & 3.38\end{array}$

These figures were calculated by $\mathrm{K}$. Ott and J. Jansen.(5) In the paper of Zebroski, Alter, and Collins $(3,6)$ for the same case the follow-

\begin{tabular}{|c|c|}
\hline Isotope & Per Cent \\
\hline 239 & 43.1 \\
\hline 240 & 40.0 \\
\hline 241 & 5.9 \\
\hline 242 & 10.5 \\
\hline
\end{tabular}
ing composition is given:

In view of the uncertainty of the nuclear data involved this means that the composition of $\mathrm{Pu}^{\infty}$ is the same. Our own calculation of the neutron dose rate associated with $\mathrm{Pu}^{\infty}$ led to the same result as presented by Zebroski, Alter and Collins.(3) We therefore cite simply Table II-3 of their paper:

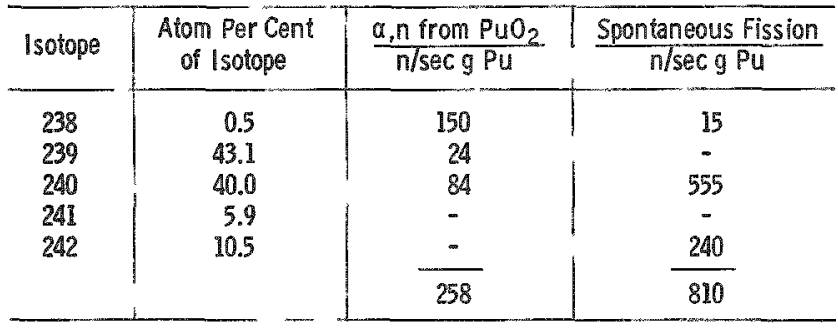

$258+810=1068$ 
To evaluate the neutron dose rate we realize that

$$
7.7 \frac{\mathrm{n}}{\mathrm{cm}^{2}-\mathrm{sec}}=1 \frac{\mathrm{mrem}}{\mathrm{hr}}
$$

Having agreed with Zebroski, Alter, and Collins for their Table II-3, we disagree with their Table II-4, where neutron dose rates are given. First: the figures for the tolerance doses in Germany are different. We have

$$
100 \frac{\text { mrem }}{\text { week }} \text { for the body }
$$

and

$$
\frac{15 \text { rem }}{13 \text { weeks }}=\frac{60 \text { rem }}{52 \text { weeks }}=1.15 \frac{\text { rem }}{\text { week }} \text { for hands, feet etc. }
$$

Secondly, the figures for the case of one pin are too large by a factor of ten (by printing error). Thirdly, the case of one assembly needs more discussion.

\begin{tabular}{|c|c|c|c|c|}
\hline \multirow{3}{*}{ Source } & \multicolumn{2}{|c|}{$\begin{array}{c}\text { Neutron Dose Rate } \\
{[\text { mrem }]}\end{array}$} & \multicolumn{2}{|c|}{$\begin{array}{l}\text { Time Limits } \\
{\left[\frac{h x}{\text { week }}\right]}\end{array}$} \\
\hline & \multicolumn{2}{|c|}{ Distance } & \multicolumn{2}{|c|}{ Distance } \\
\hline & $1 \mathrm{~cm}$ & $100 \mathrm{~cm}$ & $1 \mathrm{~cm}$ & $100 \mathrm{~cm}$ \\
\hline One Pellet $(0.22 \mathrm{~g}$ Pu) & 2.5 & $2.5 \times 10^{-4}$ & no & no \\
\hline $\begin{array}{l}\text { One Pin (46 g Pu, } \\
3.5 \mathrm{ft} \text { long) }\end{array}$ & 14.5 & 0.046 & 80 & no \\
\hline Sphere of $46 \mathrm{~g} \mathrm{Pu}$ & 510 & $5.1 \times 10^{-2}$ & 2.25 & no \\
\hline
\end{tabular}

So we give a table for one pellet, one pin, and a sphere of $46 \mathrm{~g} \mathrm{Pu}$, using our new figures:

The last example indicates that arrangements for a proper fuel geometry in the gloveboxes are vital. Zebroski, Alter, and Collins consider also one subassembly of 127 pins. There is again a printing error of a factor of ten. The dose rate at 1 -cm distance is about $372 \mathrm{mrem} / \mathrm{hr}$, leading to an exposure time of $3.1 \mathrm{hr}$. But it is easy to calculate that a hand put around such an assembly (about $7 \mathrm{~cm} \times 7 \mathrm{~cm}$ ) gets a dose of about $1250 \mathrm{mrem} / \mathrm{hr}$. The upper limit there is certainly the value $127 \times 14.5=$ $1840 \mathrm{mrem} / \mathrm{hr}$. So we have: 


\begin{tabular}{|c|c|c|c|c|}
\hline \multirow{3}{*}{ Source } & \multicolumn{2}{|c|}{$\begin{array}{c}\text { Neutron Dose Rate } \\
{[\text { mrem }]}\end{array}$} & \multicolumn{2}{|c|}{$\begin{array}{l}\text { Time Limits } \\
{\left[\frac{\text { hrs }}{\text { week }}\right]}\end{array}$} \\
\hline & \multicolumn{2}{|c|}{ Distance } & \multicolumn{2}{|c|}{ Distance } \\
\hline & $1 \mathrm{~cm}$ & $100 \mathrm{~cm}$ & $1 \mathrm{~cm}$ & $100 \mathrm{~cm}$ \\
\hline $\begin{array}{l}\text { One Subassembly } \\
\text { (127 pins) }\end{array}$ & $\begin{array}{l}372 \\
1250^{*} * \\
1840^{+}\end{array}$ & $5.8 *$ & $\begin{array}{l}3.1 \\
0.9 \\
0.6\end{array}$ & 200 \\
\hline
\end{tabular}

*Calculated as $0.046 \times 127=5.8$

* *Hand around one subassembly at $1-\mathrm{cm}$ distance

tTheoretical maximum $=127 \times 14.5$

To be complete one should calculate the number of subasssemblies to be fabricated per week(for one reactor). Using (8) and (22) we find that about 3 subassemblies have to be fabricated per week.

Without having enough practical experience it is hard to judge whether about $1 \mathrm{hr} /$ week is enough to produce three subassemblies. But it seems that some sort of remote refabrication bridging a distance of $50-100 \mathrm{~cm}$ is necessary. Once this point is accepted, a variety of possibilities to arrange things has to be considered. Certainly one would not be ready to use true hot cell procedures, but small and thin shielding walls may be easily acceptable. It may be that the se walls can be even transparent.

The neutron dose can be brought down by a factor of 10 and $35 \mathrm{~cm}$ with $20 \mathrm{~cm}$ of paraffin.

Zebroski, Alter, and Collins also bring out another point. They claim the decontamination factor for fission products should be such that the dose due to the $\gamma$ activity should not be larger than the dose due to the neutron emission. For this a decontamination factor of about $3 \times 10^{7}$ is necessary. But, having admitted that a comparatively thin wall might be acceptable, a thin shield of lead to shield the $0.7-\mathrm{MeV} \gamma$ of the fission products should also be considered. $10 \mathrm{~cm}$ of lead give a shielding factor of about $2 \times 10^{-5}$ for $0.7-\mathrm{MeV} \gamma$.

Therefore, decontamination factors of $3 \times 10^{7} \times 10^{-5}=3 \times 10^{2}$ are suddenly under discussion. To reach a decontamination factor of $3 \times 10^{2}$ by wet reprocessing is very easy; more than that, new techniques may be acceptable. The simplest improvement might be to use only one wetreprocessing step, or, in particular, one simple precipitation step. This could certainly bring the high reprocessing costs of $Z_{2}=120 \$ / \mathrm{kg}$ down to perhaps $70 \$ / \mathrm{kg}$ or even to $50 \$ / \mathrm{kg}$. 
It is interesting to realize that a completely different core-blanket management might be interesting. It is one of the most striking features of the large fast oxide reactors that the average core irradiation times are unusually large. For our reference reactor we had found 1000 days, whereas in former times irradiation cycles of about 100 days were under discussion. An irradiation period of 1000 days is now large enough to be compared with the time required to build up the marginal plutonium in the radial blanket. Suppose, therefore, the core and the blanket will be processed in batches simultaneously. The most interesting change is the change of the plutonium concentration in the fuel to be processed. In a common reprocessing we find the following plutonium concentration:

$$
\frac{\text { Pufiss }}{\text { Putotal }+U}=\frac{M_{\text {Core }}^{\text {fiss }}}{M \text { ges }} \begin{aligned}
& \text { (neglecting the effect of plutonium burnup in } \\
& \text { the core and plutonium buildup in the blanket); }
\end{aligned}
$$

M ges $=$ Mcore $_{\text {fiss }}(1+y)+$ MBlanket,ax + MBlanket, rad.

For our reference reactor (22), we find

$$
\frac{\text { Pufiss }}{\text { Putotal }+U}=\frac{1,200}{37,200}=3.25 \times 10^{-2} \text {. }
$$

Let us assume that this sort of reprocessing costs $Z_{2}^{\prime} \$ / \mathrm{kg}$ fuel. We can ask for that value of $Z_{2}^{\prime}$ for which the same cycle costs appear as in case of a separate reprocessing. We will denote this value as $\left(Z_{2}^{8}\right)_{0}$, which is easy to calculate.

We find the following condition:

$$
\begin{aligned}
\frac{\left(Z_{2}^{\prime}\right)_{0} \times 10^{2}}{2.4 a M^{\mu}} \frac{M g e s}{M_{\text {core }}^{\text {fiss }}(1+y)}= & \frac{Z_{2} \times 10^{2}}{2.4 a_{M}^{\mu}}\left(1+\frac{M_{B l a n k e t, a x}}{M_{\text {Core }}^{\text {fiss }}(1+y)}\right) \\
& +\frac{Z_{4} \times 10^{2}}{2.4 a_{M}^{\mu}} \frac{M_{B l a n k e t, r a d}}{M_{C o r e}^{f i s s}(1+y)} \\
& -\left[\left(-K_{P u}\right)_{\left.P=P_{O P T}-\left(-K_{P u}\right)_{P=1}\right] .}\right.
\end{aligned}
$$

The last term describes the loss in the term $-\left(K_{P u}\right)$ if the radial blanket is not processed after the optimum number of core cycles. Common reprocessing of radial blanket and core means $P=1$. For our reference reactor (22) we find 


$$
\left(\mathrm{z}^{2}\right)_{0}=66 \mathrm{~s} / \mathrm{kg} \text {. }
$$

If the reprocessing costs for the common treatment of core and blanket can be lower than $66 \$ / \mathrm{kg}$, then there is an economical advantage in the cycle costs.

But it is worthwhile to note that the asymptotic isotope composition (5) of the plutonium is also different in this case. For a total breeding ratio B.R.ges $=1.434$, Ott and Jansen have calculated the following composition

\begin{tabular}{|c|c|}
\hline Is otope & Per Cent \\
\hline 239 & 76.03 \\
\hline 240 & 21.46 \\
\hline 241 & 2.11 \\
\hline 242 & 0.41 \\
\hline
\end{tabular}

This gives a neutron dose rate of about

$$
400 \frac{n}{\sec , g \text { of } P u}
$$

for the plutonium of (32). Therefore, the permitted handling times are larger by a factor of 2.5 and this means $2.5 \mathrm{hr} /$ week. This might bring acceptance of the usual glovebox technique.

The last point is the question of reasonable burnup. The numerical examples of the last paragraph show that for $a_{\mathrm{M}}=100,000 \mathrm{MWD} /$ ton the core-reprocessing costs are smaller than either $\mathrm{K}_{\text {interest }}$ or $\mathrm{K}_{\text {refabr. }}$. whereas in case of burnup of $a_{M}=30,000 \mathrm{MWD} /$ ton the term $\mathrm{K}_{\text {repro }}$ is already the leading one.

As long as the term $K_{\text {refabr }}$ is not unreasonably large or small, it makes sense to ask for that burnup ( $\left.a_{M}\right)_{0}$ which gives equal reprocessing and refabricating costs. The equation

$$
\mathrm{K}_{\text {repro }}=\mathrm{K}_{\text {refabricating }}
$$

is, of course, strictly heuristic, and one should be caxeful in drawing conclusions from it, but it is on the other hand illuminating to evaluate (34) by using (20) and (11). One finds the following:

$$
\left(\mathrm{a}_{\mathrm{M}}\right)_{0}=\frac{\mathrm{Z}_{2}^{2} \mathrm{H} \chi(1+\mathrm{y})}{\alpha \beta \mathrm{k}_{1}} \frac{\delta}{0.28 \times 10^{4}}\left[\frac{\mathrm{M}}{\mathrm{M}_{\text {COYe }}^{\mathrm{fiss}}(1+\mathrm{y})}\right]^{2} .
$$


For our reference reactor (22) we find

$$
\left(a_{M}\right)_{0}=75,000 \mathrm{MWD} / \text { ton. }
$$

It is important to realize that ( $\left.a_{M}\right)_{0}$ varies with the square of $Z_{2}$, and that the value of $Z_{2}$ is highly uncertain. Should it be larger than $120 \$ / \mathrm{kg}$, for example, $200 \$ / \mathrm{kg}$, burnups of about $200,000 \mathrm{MWD} /$ ton are necessary to satisfy equation (34). On the other hand if the fuel cycle costs as a whole, (21), are sufficiently low, equation (34) loses its meaning.

V. The Artificial Improvement of the Heat Conductivity of the Fuel

It is possible to improve the heat conductivity of the fuel by adding ingredients. $B$. Liebmann et al. (8) reported on the basis of development work which was done by NUKEM for the Karlsruhe Fast Breeder Project, that it is possible to improve the fuel heat conductivity by a factor of about 2.5 at $1000^{\circ} \mathrm{C}$ if $15 \mathrm{v} / 0$ of molybdenum are added to the fuel. Figures 2 and 3 show the effect of molybdenum on the heat conductivity.

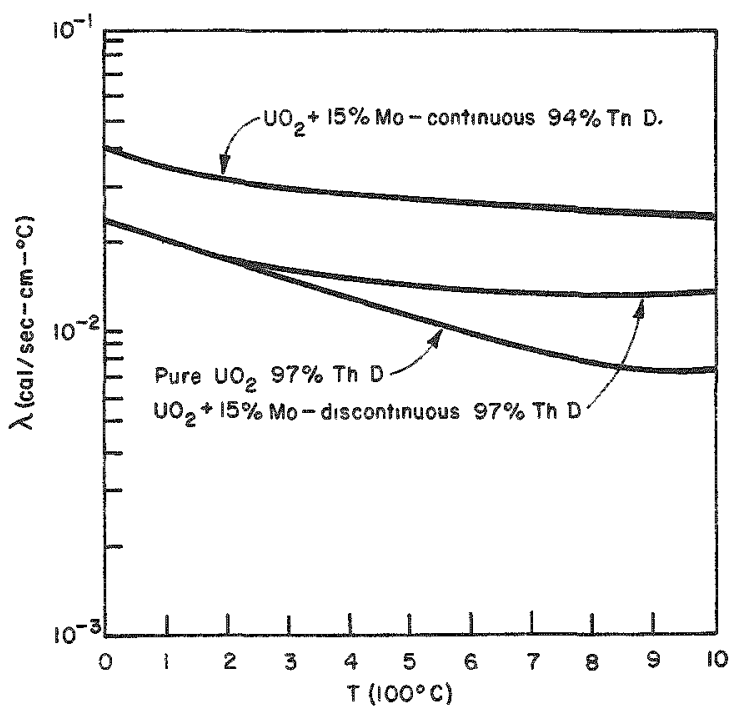

Fig. 2. Thermal Conductivity of Sintered Pellets between 20 and $1200^{\circ} \mathrm{C}$.

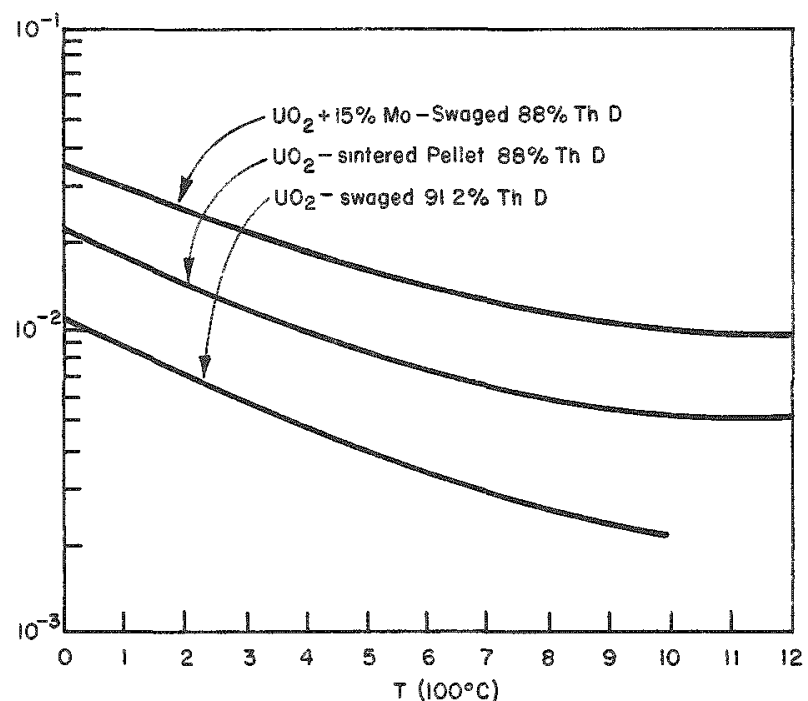

Fig. 3. Thermal Conductivity of Packed Powder Fuel between 20 and $1200^{\circ} \mathrm{C}$.

The molybdenum content lowers, of course, the total breeding ratio. Therefore a number of multigroup calculations were performed by the Karlsruhe group in order to explore the effect of the added molybdenum on the core characteristics, in particular to the partial breeding ratios. Here only a few representative results are reported. 
Assume that the fraction of coolant in the core is $\alpha$, the iraction of fixed structural material (besides of cladding) is $\beta_{0}$; further, let us assume that $\mathrm{X}$ is the volume ratio of a material (cladding or ingredient) to the fuel and $y$ is, as before, the volume ratio of fertile to fissile material. It was assumed:

Steel as cladding and fixed structural material

$\left(\mathrm{Pu}^{239}\right) \mathrm{O}_{2}+\left(\mathrm{U}^{238}\right) \mathrm{O}_{2}$ as fuel

Molybdenum as ingredient

Sodium as coolant.

The considered reactors were spheres with an $80-\mathrm{cm}$ blanket. With $F_{0}=0.15$ we found the results given in Table 1 for $\alpha=0.4$. It is interesting to see that the internal breeding ratio is almost independent of the molybdenum added. Only a small increase (but not decrease!) due to the decreascd leakage into the blanket can be observed. Each core absorber takes the neutrons it needs, and only the blanket experiences a reduction of the bieeding ratio due to the added molybdenum. The change of the total breeding ratio, therefore, is almost independent of the steel content of the core. We find

$$
\Delta(\text { total breeding ratio })-\left\{\begin{array}{l}
0.22 \text { for } y=6 \\
0.26 \text { for } y=7
\end{array}\right.
$$

Table 1

\begin{tabular}{|c|c|c|c|c|c|c|c|}
\hline $\mathrm{X}_{\mathrm{Mo}}$ & $\mathrm{x}_{\text {steel }}$ & $y$ & $\begin{array}{c}\text { Core Radius } \\
(\mathrm{cm})\end{array}$ & $\begin{array}{c}\text { Breeding Ratio } \\
\text { of the Core }\end{array}$ & $\begin{array}{c}\text { Breeding Ratio } \\
\text { of the Total } \\
\text { Blankes }\end{array}$ & $\begin{array}{c}\text { Total } \\
\text { Breeding Ratio }\end{array}$ & $\begin{array}{c}\text { Critical Mass } \\
(\mathrm{kg})\end{array}$ \\
\hline 0 & 0.25 & 6 & 61 & 0.608 & 1.035 & 1.643 & 447 \\
\hline 0 & 0.25 & 7 & 73 & 0.740 & 0.851 & 1.591 & 680 \\
\hline 0 & 0.5 & 6 & 75 & 0.639 & 0.858 & 0.497 & 682 \\
\hline 0 & 0.5 & 7 & 94 & 0.779 & 0.652 & 1.430 & 1194 \\
\hline 0.15 & 0.25 & 6 & 76 & 0.628 & 0.789 & 1.417 & 770 \\
\hline 0.15 & 0.25 & 7 & 100 & 0.763 & 0.564 & 1.327 & 1542 \\
\hline 0.15 & 0.5 & 6 & 95 & 0.656 & 0.622 & 1.278 & 1291 \\
\hline 0.15 & 0.5 & 7 & 140 & 0.789 & 0.368 & 1.166 & 3559 \\
\hline
\end{tabular}

The increased heat conductivity of the fuel now can be used for three different purposes (or combinations of them):

1. to decrease the average fuel temperatuxe;

2. to increase only the radius $R$ of the pin;

3. to decrease the optimized costs; this in turn implies a simultaneous increase of the rating $b$ and the radius $R$ of the pin. 
Factor 1 is a way to improve safety. Factor 2 is a way to make the construction of the subassembly easier (including refabricating). Factor 3 is a way to improve the economy.

The influence of a decreased fuel temperature on safety is not easy to express. In case of an accidental ramp reactivity insertion, one has to ask for the delay time of the counteracting shut-off rods and their imposed negative ramp rate. If the accidental ramp rate is such that prompt criticality cannot be reached, the power excursion does not show the narrow peak behavioux which takes place if superprompt criticality stages can be reached. (9) To reach the superprompt stage, high accidental insertion rates are necessary. But this, in turn, means that the well-known narrow peak tends to appear periodically until the negative ramp rate of the shutoff rods comes into action [see (9)]. So it is not very typical for the safety situation to evaluate the quantity

$$
1+\frac{(\Delta k) \text { Doppler }}{2} \text { (in terms of dollars), }
$$

which refers only to the termination of one single prompt supercritical excursion after a reactivity step.

In the context of this paper we therefore give the change of the maximum fuel temperature instead. Assuming a can temperature of $500^{\circ} \mathrm{C}$ and a heat transfer of $0.6 \mathrm{~W} / \mathrm{cm}^{2}{ }^{\circ} \mathrm{C}$ over the gap between fuel and can, and further assuming a maximum heat flux of $300 \mathrm{~W} / \mathrm{cm}^{2}$, we obtain

$$
\begin{aligned}
\mathrm{T}_{\text {can }}= & 500^{\circ} \mathrm{C} ; \\
T_{\text {fuel surface }}= & 1000^{\circ} \mathrm{C} ; \\
\mathrm{T}_{\mathrm{MAX}}= & 2500^{\circ} \mathrm{C} \text { for } X_{\mathrm{MAX}} \approx 600 \mathrm{~W} / \mathrm{cm} \text { and a heat conduc }- \\
& \text { tivity of about } 0.08 \mathrm{cal} / \mathrm{sec}-\mathrm{cm}-{ }^{\circ} \mathrm{C} .
\end{aligned}
$$

If the heat conductivity is larger by a factor of 2.5 , the maximum temperature in the fuel goes down to

$$
T_{M A X}=1600^{\circ} \mathrm{C}
$$

In addition to the aid for safety, this helps to lower the fission gas release, which can be very important.

For this benefit one has to pay the price of the lowered breeding gain, of course. Because of the unchanged value of $X$, the only term of (21) which changes is $-K_{P u}$. Using (17) and (37) and our first numerical example of paragraph II, according to (22) we find that for a breeding gain of 1.19 the term - $(\mathrm{KPu})$ OPT goes down to zero. This gives us a fuel-cycle cost 


$$
\mathrm{K}_{\text {total }}=0.87 \mathrm{mill} / \mathrm{kW}-\text { he }(\text { in case of safety) }
$$

Compared with the value from (23), namely, $0.74 \mathrm{mill} / \mathrm{kW}$-he, this is an increase of $0.134 \mathrm{mill} / \mathrm{kW}-\mathrm{he}$, and this is therefore the price for the improved safety situation. One can say that $100^{\circ} \mathrm{C}$ temperature gain in the fuel center costs $0.015 \mathrm{mill} / \mathrm{kW}$-he.

The second possibility to use the improved conductivity is to in crease only the radius. According to (6) this gives the factor $\sqrt{2.5}=1.58$ for $R$. In the first numerical example of paragraph III we found a radius of $0.275 \mathrm{~cm}$. We now have $0.275 \times 1.58=0.435 \mathrm{~cm}$. Already this gives a considerable fuel pin diameter and therefore, perhaps, a simple and cheap construction of the subassembly. But one must realize also that the heat flux at the surface of the fuel pin is increased by a factor $\sqrt{2.5}$. For a maximum power per pin length of $600 \mathrm{~W} / \mathrm{cm}$ (unimproved heat conductivity). one finds the following heat flux q":

$$
\ddot{q}=x / 2 \pi R
$$

Using the values of the first numerical example, we find

$$
\ddot{q}=350 \mathrm{~W} / \mathrm{cm}^{2}
$$

Thus, this value is increased to $350 \sqrt{2.5}=550 \mathrm{~W} / \mathrm{cm}^{2}$, and this means that sodium is really necessary as a coolant, because a strong gas cooling goes only up to about $300 \mathrm{~W} / \mathrm{cm}^{2}$. Besides, one has to be concerned about the temperature step in the gap between fuel and cladding. Usually this leads to too high temperatures in the fuel center.

A radius of $0.435 \mathrm{~cm}$ is probably already large enough to make the assumption of the radius-independent refabrication costs per pin $\left(k_{1}\right)$ fail. In spite of this, we will employ the formerly used set of formulas. If only the radius is changed and not the rating $b$ according to (18) and (19), we do not have optimum fuel-cycle costs. We have the following breakdown instead:

$$
\begin{aligned}
& R=0.435 \mathrm{~cm} ; \\
& \mathrm{b}=1 \mathrm{MW} / \mathrm{kg} \text {, fiss; } \\
& \text { tST } \sim 10^{3} \text { days } \\
& \text { Kinterest }=0.261 \mid-\left(K_{\mathrm{Pu}}\right) \text { OPT } \cong 0 \\
& K_{\text {refabr }}=0.104 \\
& K_{\text {interest }}^{\prime \prime}=0.052 \\
& K_{\text {repro }}=0.230 \quad K_{\text {total }}=0.675 \mathrm{mill} / \mathrm{kW} \text {-he } \\
& \text { Kirst core }=0.028 \text { (for the case oi enlarge- } \\
& \text { ment of only the radius) }
\end{aligned}
$$


In this example there is a lower fuel-cycle cost although the term -(KPu)OPT $\cong 0$. Compared with the fuel-cycle cost for the case without molybdenum, we have a gain of $0.065 \mathrm{mill} / \mathrm{kW}$-he, besides the benefit of the larger diameter. The assumption of a constant refabrication cost per pin, however, tends to give too good a picture. Therefore the gain of $0.065 \mathrm{mill} / \mathrm{kW}$-he is not realistic and the fuel-cycle cost probably remains unchanged. But this means that one has to pay no price for the benefit of the larger pin diameter.

The third possibility is to use again a design which is strictly optimized with respect to the fuel-cycle costs. Here we find according to $(18)-(20)$ :

$$
\begin{aligned}
& R=0.346 \mathrm{~cm} \\
& \mathrm{~b}=1.58 \mathrm{MW} / \mathrm{kg} \text { fiss: } \\
& t_{S T}=630 \text { days } \\
& \begin{array}{ll|l}
\mathbb{K}_{\text {interest }}^{\prime} & =0.166 \\
\mathbb{K}_{\text {refabr }} & =0.166
\end{array} \mid-\left(K_{\text {Pu }}\right) \text { OPT }=0.018 \\
& K_{\text {interest }}^{\prime \prime}=0.052 \\
& \mathrm{~K}_{\text {repro }}=0.230 \\
& \mathrm{~K}_{\text {first core }}=\frac{0.028}{0.642} \mid \begin{array}{l}
\mathrm{K}_{\text {total }}=0.624 \text { mills } / \mathrm{kW}-\text { he } \\
\text { (for the case of optimized fuel } \\
\text { cycle costs) }
\end{array}
\end{aligned}
$$

The term - (KPu) OPT here is notzerobecause of the shorter cycle period. The radius has increased only by a factor $\sqrt{\sqrt{2.5}}=1.26$. This makes the assumption of a constant value of $k_{1}$ somewhat more valid. We find against the case without molybdenum (23) a cost decrease of $0.116 \mathrm{mill} /$ $\mathrm{kW}$-he. This is the benefit for having molybdenum in the fuel.

One tends to say that perhaps the increase of the fuel pin diameter is the most attractive thing. Fuel-cycle cost changes of the order of $0.10 \mathrm{mill} / \mathrm{kW}$-he don't look very attractive if the capital costs are in the neighborhood of $3 \mathrm{mill} / \mathrm{kW}$-he (see the end of paragraph III).

Let us recall that these conclusions are drawn as general guide lines; the numerical figures are not supposed to be true cost figures.

In the scope of this paper it is also impossible to take into account all effects which may come from the molybdenum. There is, for example, an effect on the chemical reprocessing costs. 
Molybdenum is not the only additive capable of improving the fuel heat conductivity. Lately it was reported, that $15 \mathrm{v} / 0 \mathrm{BeO}$ added to $\mathrm{UO}_{2}$ fuel (10) gives an increase of the heat conductivity by a factor of 2 at $1000^{\circ} \mathrm{C}$. The multigroup calculation of the Karlsruhe group indicate that the associated loss of the breeding gain is smaller by a factor of about 0.67 than in case of the addition of molybdenum. This leads to about the same improvement of the fuel cycle cost as for molybdenum (42). But here it is necessary to realize, that about 9000 neutrons/sec-g Pu will be emitted by the $\mathrm{BeO}-\mathrm{PuO}_{2}-\mathrm{UO}_{2}$ fuel due to $(\alpha, n)$ reaction of $\mathrm{Be}^{9}$. This is nine times more than in case of the original fuel without BeO [see (26)]. One therefore is reluctant to use BeO for the improvement of the heat conductivity.

\section{The Problem of Fission Gas Release and the Performance of the Cladding Material}

It is recognized that for the concept of a large fast oxide breeder burnups of $100,000 \mathrm{MWD} /$ ton are essential. For $y=7$ this means that $80 \%$ of the original fissionable atoms of a fuel element are burned. For such high burnups one has to expect that virtually all of the fission gases are released. Due to a serious lack of experimental information it is not cleax whether such an assumption is too pessimistic, but here we think it is reasonable, except the average fuel temperature is remarkably lowered (see paragraph V). Engelmann has calculated the fission gas pressure buildup in fuel elements. (11) He assumed a uniform fuel with 90, 80, and $70 \%$ of the theoretical density. The larger the porous volume, the smallex is the fission gas pressure. For $80 \%$ of the theoretical density he concluded that about 500-atm pressure buildup is to be expected. Therefore one is persuaded to look for a fuel of about $80 \%$ theoretical density. But it turns out that the fuel after some ten thousand MWD/ton is no longer uniformly distributed: the well-known central void formation has taken place. Leitz has reported experimental results for long-irradiated mixed $\mathrm{UO}_{2}$ $\mathrm{PuO}_{2}$ fuel. (12) He gives photographs of fuel cross sections with the central void formation. The swaged fuel had originally $75 \%$ theoretical density, the pelleted fuel about $90 \%$. Stoll(13) now has recognized that according to the size of the central void in the photographs of Leitz's paper, the swaged fuel was displaced in such a way that after the void formation the density has increased from $75 \%$ to $90 \%$, and in the case of the pelleted fuel from $90 \%$ to $94 \%$.

If the above-mentioned $80 \%$ theoretical density fuel concentrates its homogeneously distributed voids into the one large central hole, one has to be concerned about possible slumping through that hole if long cylindrical rods are used as fuel elements. The use of spherical fuel elements does not lead to such a problem. In case of cylindrical rods, therefore, a density of about $90 \%$ is reasonable, because the fuel tends to approach that density anyway. The central hole should be just large enough to act as a chimney for the fission gas release, but too small for the slumping of fuel. This, 
of course, implies that the fission gas shall be given to a plenum. In such a case, usually the gas has to travel over large distances, passing the axial blanket before a plenum at the end of the fuel element can be reached. It is not clear whether this is possible. Condensation of cesium at coldex parts of the axial blanket, for example, could inhibit that.

In case the idea works, it is self-evident that the axial blanket and the core must be in one pin; separate pins for the axial blankets are then impossible. In paragraph IV (influence of the out of pile part of the fuel cycle to the fuel element design) we had already assumed one pin for the axial blanket and core.

In case this scheme of fission gas release fails, the problem of the strength of the cladding material is suddenly emphasized. The fission gas pressure buildup gives a stress $\sigma_{1}$ in the cladding annulus:

$$
\sigma_{1}=(R / d) P
$$

where $R$ is the radius of the fuel in the pin and $d$ is the thickness of the cladding annulus. The heat flux passing the wall of the cladding material produces a stress $\sigma_{2}$ for which we find

$$
\sigma_{2}=\frac{E \alpha}{2} \frac{X d}{2 \pi R \lambda}
$$

where $E$ is Young's modulus, $\alpha$ is the expansion coefficient, and $\lambda$ is the heat conductivity of the clad. Both stresses sum up to the total stress

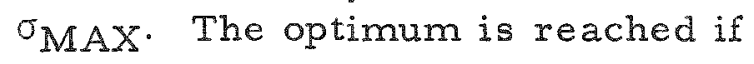

$$
\sigma_{1}=\sigma_{2}=\frac{1}{2} \cdot \sigma_{\mathrm{MAX}}
$$

Thus we find

$$
\left(\frac{\mathrm{d}}{\mathrm{R}}\right)_{\mathrm{OPT}}=\begin{gathered}
4 \pi \mathrm{P} \lambda \\
\operatorname{E\alpha } \alpha \chi
\end{gathered}
$$

If (46) is inserted into (43) and (44), we obtain values for $\sigma_{1}$ and $\sigma_{2}$ which are too large for the envisaged cladding material. Then the cladding material is not compatible with the assumed values for $P$ and $X$ of the fuel. In order to be compatible with the requirements of the fuel, the cladding material has to fulfill the following condition [multiplying (43) with (44) and using (45)]:

$$
\left(\sqrt{\frac{\pi \sigma_{\mathrm{MAX}}^{2}}{\alpha \mathrm{E}}}\right)_{\text {cladding }} \geq(\sqrt{\chi P})_{\text {fuel. }}
$$


The remarkable feature of (47) is that on the left side there are only cladding properties, whereas on the right side there are only properties of the fuel. The meaning of the term $(\sqrt{\chi P})_{\text {fuel }}$ almosi coincides with the meaning of the term $\sqrt{\chi_{M}}$ which we used quite often in discussing fuelcycle costs. We will call the term $\sqrt{\chi_{\mathrm{a}}}$ the performance of the fuel. We can then say that the performance of the cladding material must be consistent with the (corresponding) performance of the fuel.

In Fig. 4, we reproduce the plot of equation (47) by Ritz and Hübschmann(14) for various materials. One starts with an assumption of the fission gas pressure, say $400 \mathrm{~atm}$, and goes down into the lower diagram to the line of the required power per pin length, $\chi$. The quantity $P X$ must be equal to $\left(\pi \sigma_{\mathrm{MAX}}^{2} \lambda\right) / \alpha \mathrm{E}$. We therefore go parallel to the plotted curve representing $\left(\pi \sigma^{2} \mathrm{MAX} \lambda\right) / \alpha E$ in order to see for which temperature equation (47) is fulfilled. The upper diagram represents equation (43) for $\sigma_{1}=\frac{1}{2} \sigma_{\mathrm{MAX}}$

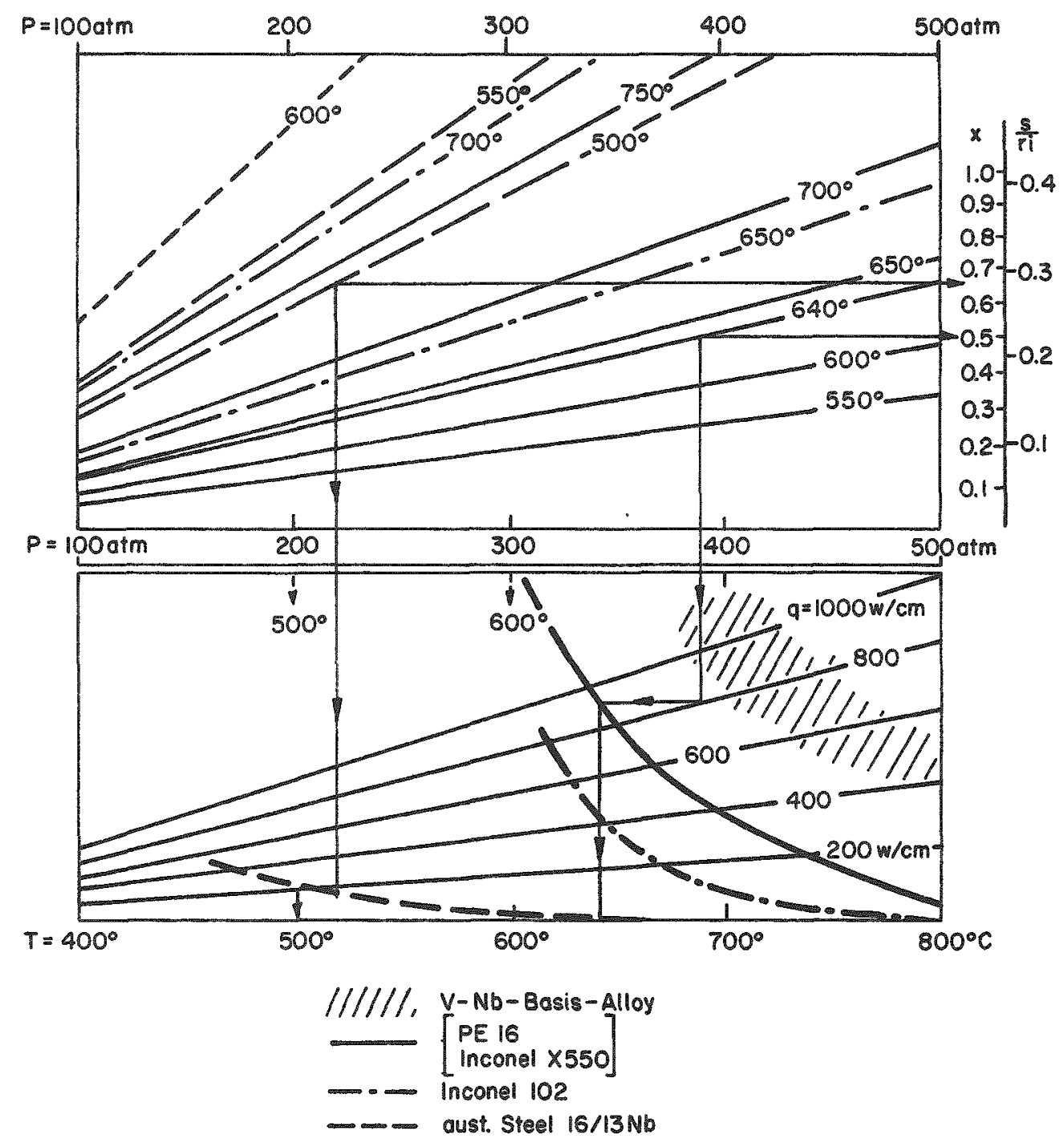

Fig. 4. Performance Diagram for Cladding Materials 
and helps to determine the value of $(d / R)$ OPT ( $X$ is, as before, the ratio of cladding to fuel volume; see paragraph V). From Fig. 4 one realizes that steel can only be used if the fission gas pressure release really works. Otherwise alloys like Inconel must be used. But here the question of radiation damages comes up. Robertshaw and Collins (15) report on a substantial reduction for the stress rupture strength of $\mathrm{A}-286$ and Inconel $\mathrm{X}$ at $660^{\circ} \mathrm{C}\left(1200^{\circ} \mathrm{F}\right)$ and, to a lesser degree, at $550^{\circ} \mathrm{C}\left(1000^{\circ} \mathrm{F}\right)$. The involved neutron doses were varying from $2.8 \times 10^{18} \mathrm{n} / \mathrm{cm}^{2}$ to $7 \times 10^{20} \mathrm{n} / \mathrm{cm}^{2}$, but for the values as low as $1.2 \times 10^{16} \mathrm{n} / \mathrm{cm}^{2}$ it was still possible to observe a measureable reduction in the rupture strength.

All this imposes serious problems in case the fission gas pressure release in a plenum fails. Especially the problem of the radiation damages seems to be bad. We can easily calculate the fast neutron doses per core cycle. If $D$ is the neutron dose in $\mathrm{n} / \mathrm{cm}^{2}$, we find the following:

$$
D=10^{18} \mathrm{aM}_{\mathrm{M}}(1+\mathrm{y}) / \hat{o}_{\mathrm{f}} \mathrm{n} / \mathrm{cm}^{2},
$$

where $\hat{\sigma}_{f}$ is the average fission cross section in barns. For $\hat{\sigma}_{f}=2$, $a_{M}=10^{5}$, and $y=7$, we find

$$
D=4 \times 10^{23} \mathrm{n} / \mathrm{cm}^{2} \text {. }
$$

This is certainly a high neutron dose. For a thermal reactor ( $\mathrm{y} \approx 50$ with $\mathrm{aM}=10,000 \mathrm{MWD} /$ ton and $\hat{\sigma}_{f}=500 \mathrm{~b}$, we find, instead of (49),

$D=10^{21} \mathrm{n} / \mathrm{cm}^{2}$ (for a thermal reactor).

Thus the problem of the cladding material is very serious. Steel can be used only if the fission gas release really works. In case of some pressure buildup, strong materials of about $50-\mathrm{kg} / \mathrm{mm}^{2}$ creep strength or more are necessary. Inconel has such properties, but shows strong radiation damages. Therefore it is very likely that new alloys, possibly with a vanadium basis, have to be developed. Large-scale in-pile testing using fast neutrons is vital.

\section{The Prompt Doppler Coefficient}

The stability of large fast reactors relies on the prompt-acting Doppler coefficient. In order to do so the mixture between $\mathrm{UO}_{2}$ and $\mathrm{PuO}_{2}$ has to be an intimate one: separation of the $\mathrm{PuO}_{2}$ component would lead to a prompt-acting positive effect accompanied by a larger delayed negative $\left(\mathrm{U}^{238}\right) \mathrm{O}_{2}$ effect. Two ways of separation seem possible. Macroscopic accumulation of one component at the outer parts of the fuel-element radius or microscopic separation, where only small coagulations of $\mathrm{PuO}_{2}$ in $\mathrm{UO}_{2}$ take place, such that the overall density of $\mathrm{PuO}_{2}$ is unchanged and independent of the position. In case the macroscopic accumulation of $\mathrm{PuO}_{2}$ 
(for example) does not change, the effect of the local value of $y$ is probably not very serious. The microscopic separation can be eventually more serious.

The degree of the homogeneity of a $\mathrm{PuO}_{2}-\mathrm{UO}_{2}$ mixture can be described by a parameter $r_{2}$, which is the radius of a $\left(U^{238}\right) \mathrm{O}_{2}$ sphere which includes a smaller $\mathrm{PuO}_{2}$ sphere of a radius $r_{1}\left(r_{2}>r_{1}\right)$ such that the ratio of the volume of the $\left(\mathrm{U}^{238}\right) \mathrm{O}_{2}$ shell to the volume of the $\mathrm{PuO}_{2}$ sphere equals $y$. The larger is $x_{2}$, the larger is the heterogeneity; for $r_{2} \rightarrow 0$ we have a strictly homogeneous mixture. Although this is a model averaging over a spectrum of spheres $w$ ith different radii, it helps to evaluate quantitatively the influence of the heterogeneityof the $\mathrm{PuO}_{2}-\left(\mathrm{U}^{238}\right) \mathrm{O}_{2}$ mixture.

Fraude has recently completed a study of such a model.(16) The time behaviour of a power excursion is governed by a set of eigenvalues $t_{V}$. The larger is $t_{1}$, the larger is the time delay of the negative $\left(U^{238}\right) O_{2}$ coefficient. We have

$$
t_{v}=\frac{\rho c}{\lambda}\left(\frac{\mathbb{1}_{2}}{X_{V}}\right)^{2}
$$

where $\rho c$ is the heatcapacity of the fuel and $\lambda$ the heat conductivity. The values $X_{V}$ are solutions of the following equation:

$$
\mathrm{X}_{\mathrm{V}}=\operatorname{tg} \mathrm{X}_{\mathrm{V}}
$$

It is difficult to arrive at general criteria for permitted values of $r_{2}$. The special features of the safety system, the reactivity insertion, and the permitted temperature increase must be taken into account. Fraude investigated a number of special examples. It seems that $r_{2}<300 \mu$ is a reasonable condition.

We mention this problem of the fuel homogeneity only for the sake of completeness. Much work needs to be done, and its is not sufficient to consider only equations (50) and (51). Such detailed work has to include, besides the heterogeneity parameter $r_{2}$, all the other dynamic features of the considered reactor system. It is beyond the scope of this paper.

\section{Conclusions}

The considerations of this paper lead to the following conclusions:

1. For a large fast oxide breeder fuel cycle costs in the neighborhood of 0.7 to $0.8 \mathrm{mill} / \mathrm{kW}$-he seem likely.

2. If the costs for reprocessing of the core are as high as $120 \$ / \mathrm{kg}$, a burnup of $100,000 \mathrm{MWD} /$ ton is necessary to have the fuel-cycle costs in the above-mentioned range and to have the reprocessing costs at the same level as for the other items. 
3. The fission gas release associated with a burnup of $100,000 \mathrm{MWD} / \mathrm{ton}$ makes it necessary to have either a complete fission gas venture or a strong can material.

4. The fuel density has to be low enough in order to permit fission gas venting, and it has to be large enough in order to prevent slumping. The optimum density is supposed to be in the neighborhood of $90 \%$ theoretical density.

5. Strong can materials with creep strength of $50 \mathrm{~kg} / \mathrm{mm}^{2}$ at $600^{\circ} \mathrm{C}$ or more (gas cooling), capable of withstanding fast-neutron doses of about $4 \times 10^{23} \mathrm{n} / \mathrm{cm}^{2}$, are not yet available. This might be the most serious problem. Alloys with a basis of vanadium or niobium might perhaps bring the solution.

6. It is possible to improve the heat conductivity by a factor of about 2.5 if $15 \mathrm{v} / 0 \mathrm{Mo}$ are added. This can be used to bring the central fuel tempexature down to $1600^{\circ} \mathrm{C}$, with a simultaneous increase of the fuel cycle costs of $0.13 \mathrm{mill} / \mathrm{kW}-\mathrm{hr}$. Or it can be used to have rod diameters of about $9 \mathrm{~mm}$, with the fuel-cycle costs being about constant. Or, finally, it can be used to decrease fuel-cycle costs. A gain of $0.12 \mathrm{mill} /$ $\mathrm{kW}$-he seems possible.

7. It is necessary to explore the conditions for the reprocessing of core fuel with a high plutonium content and to establish a realistic price for it. This price should, hopefully, be not larger than $120 \$ / \mathrm{kg}$.

8. In case of a separate core and radial blanket reprocessing, it is important to experience the conditions of fuel refabrication under slight neutron doses. Handling times for subassemblies of $1 \mathrm{hr} /$ week appear to be possible with standard glovebox technique. If some sort of remote handling technique is accepted, a simple wet reprocessing with a decontamination factor as 1 ow as $10^{3}$ becomes interesting and might lead to considerably lower reprocessing costs.

9. A combined core-blanket reprocessing leads to plutonium concentrations in the reprocessing plant which are as 10 as $3 \%$. But in order to compete with the separate treatment, reprocessing costs must be as low as about $60 \$ / \mathrm{kg}$ fuel. The combined core-blanket reprocessing also leads to a more favorable isotope composition of the equilibrium plutonium. For this it is necessary that the total breeding ratio is clearly larger than one. For a breeding ratio of 1.45 the equilibrium plutonium isotope composition is such that during the refabrication step of the subassemblies handling times of about $2.5 \mathrm{hr} /$ week are permitted. This is 2.5 times more than in case of the separate reprocessing. But, of course, the plutonium for sale becomes dirty. 
10. The mixture of $\mathrm{PuO}_{2}-\mathrm{UO}_{2}$ has to be of a reasonable homogeneity. $\mathrm{PuO}_{2}$ should not be separated more than probably $300 \%$. This value is uncertain, and depends on the dynamic and safety features of the involved reactor system.

\section{Acknowledgement}

The author is obliged to a large number of members of the Karlsruhe team. In particular to the following gentlemen:

Mr. Schroeter (for carrying out the necessary multigroup calculations), Mr. Wittek (for shielding considerations), Dr. Vogg (for discussions on reprocessing), Dr. Ott, Dr. Kummerer and Dr. Beisswenger (for principal discussions). Dr. Liebmann (NUKEM) (for discussion on the fuel), and Mr. Ritz (for discussions on the cladding problem).

\section{$\underline{\text { References }}$}

1. Costs of Nuclear Power, TID-8531 (Jan 1961), pp. 26-29.

2. A. Kania, P. Moinereau, G. Vendryes, and C. P. Zaleski, Quelques Etudes de Piles Rapides de Puissance, Proceedings of the Seminar on the Physics of Fast and Intermediate Reactors, Vienna, 3-11 Aug 1961, p. 331 .

3. E. L. Zebroski, A. W. Alter, and G. D. Collins, Plutonium Fuel Fabrication and Reprocessing for Fast Ceramic Reactors, GEAP-3876 (Feb 1, 1962).

4. C. Cohen and B. Wolfe, Development of the Fast Ceramic Reactor, Nuclear News, ANS (Feb 1963), pp. 11-15.

5. K. Ott and A. Jansen, Long Time Behaviour of Fast Breeder, Proceedings of the Seminar on the Physics of Fast and Intermediate Reactors, Vienna, 3-11 Aug 1961, pp. 213 ff.

6. B. A. Hutchins, Plutonium Isotopic Compositions in FOR Design Fuel, A Revision of GEAP-3087, Memorandum, November 10, 1961.

7. W. Häfele, Closed Plutonium Fuel Cycle in Fast Reactors, Enlarged. Symposium of the European Atomic Energy Society, Baden-Baden, September 9-14, 1963. 
8. H. Beisswenger, H. Huschka, B. Liebmann, N. Müller, and O. Neumann: Fuel Development for a High Temperature. Fast Reactor, Enlarged Symposium of Fuel Cycles for Power Reactors, European Atomic Energy Society, Baden-Baden (Sept 1963).

9. See, for example, W. Håfele, Prompt ůberkritische Leistungsexkursionen in schnellen Reaktoren, NUCLEONIK (Springer Verlag) Band 6. Heft 5, Seite 201 f.f. (1963).

10. Herstellung von $\mathrm{UO}_{2}$ und $\mathrm{UO}_{2}-\mathrm{BeO}$ Mischpellets, EURATOM. 116.d (1962).

11. P. Engelmann, Fission Gas Pressure Buld Up and Fast Breeder Economy, Proceedings of the Seminar on the Physics of Fast and Intermediate Reactors, Vienna, 3-11 Aug 1961, p. 229 if.

12. F.J. Leitz, "Plutonium as a Fuel for the Fast Ceramic Reactor." ANS Topical Meeting Plutonium as a Power Reactor Fuel, Richland, Washington (Sept 14, 1962).

13. W. Stoll, NUKEM, Private Communication

14. L Ritz and W. Hưbschmann, Internal Karlsruhe Report (1963)

15 F.C. Robertshaw and C. G. Collins, The Effect of Neutron Irradia = tion on the Stress-Ruptuxe Strength of A-286 and Inconel X. Am Soc Metals, Trans, Quart., 55, pp. 565-579 (1962): see the references there also.

16. A. Fraude, Institut fur Neutronenphysik und Reaktortechnik, Kernforschungszentrum Karlsruhe, Untersuchungen über die Wirkung ron zwei Temperatur-koeffizienten der Reaktivitat die aus der Entmischung eines ursprünglich homogenen Brennstoffes resultierer. to be published.

$\frac{\text { Discussion of Paper }}{\text { Presented by Mr.Häfele }}$

MR.ZEBROSKI (General Electric):

I have a few comments on the cost calculations which, I think, make one fairly important omission. That is the effect of scaling law on a number of the parameters, to wit, the reprocessing cost, the fuel fabrication cost. and the fuel diameter. If these are taken into account, the conclusion that the cost goes up sharply at $30,000 \mathrm{MWD} /$ tonne is soitened considerably. I do not think that the scaling law whych extrapolates the zero cost of infinite 
size is the appropriate one to use always, but there is some dependence surely which should be taken into account.

\section{MR. HAFELE:}

Yes. On the other hand, if I may comment on this, $\$ 120$ per kilogram is a guess at the cost of reprocessing. Nobody has yet demonstrated that reprocessing of high-plutonium content, high-burnup fuel can be done for that price. If one accepts that refabrication costs are equal to the reprocessing costs, then the necessary burnup from this equation is proportional to the square of the dollars per kilogram reprocessing cost. Suppose it is not $\$ 120$ but $\$ 200$. Then you need about $300,000 \mathrm{MWD} /$ tonne burnup to meet this fuel cycle cost. Thus, because the reprocessing cost for this sort of fuel is unknown, the amount of burnup necessary to have the se low fuel-cycle costs is still not really established.

MR. HALL (Los Alamos):

I would like to add one small comment on this reprocessing cost. We recently negotiated for the reprocessing of plutonium-rich fuels of not very high burnup, and the price was $\$ 2,000$ a kilogram.

\section{MR. ZALESKI (EURATOM)}

Is Dr. Häfele speaking about mean value of the burnup, or maximum value of the burnup?

MR. HÅFELE:

The maximum burnup possible for the fuel, obtained by fuelshuffling, by the average number of the rods.

MR. ZALESKI:

So it is the average mean burnup?

MR HÄFELE:

Indeed.

\section{MR ZALESKI:}

If that is the case, I think that perhaps the value of $100 \mathrm{MWD} /$ tonne is a little ambitious, and I am not sure it is quite necessary to achieve it I think that even 60,70 , or $80 \mathrm{MWD} /$ tonne as a mean value for all fuel would be reasonably good. Of course, it is closely related to refabrication and reprocessing costs. 
MR. SHOUDY (Atomic Power Development Associates):

With regard to the fuel-cycle cost versus plutonium price for a plant already built, did the fuel cycle cost increase with plutonium?

MR. HÄFEL:

Yes, slightly, but it depends on the rating. You can easily produce decreasing cost if the rating is high enough.

MR. SHOUDY:

For a fixed plant, it would seem to me the only variable then is your income from the breeding ratio.

MR. HÄFELE:

Yes.

MR. SHOUDY:

For the fixed plant should the fuel-cycle cost decrease with the increase in plutonium?

MR. HAFELE:

Your earnings from the sale of plutonium are about just as high as the interest you have to pay for the plutonium.

MR. SHOUDY:

At 10 per cent?

MR. HÄFELE:

At 10 per cent, yes, depending on the breeding ratio. I assumed a breeding ratio of 1.45. Now, if you have a high enough rating, you decrease the interest charges and you may have a net gain from the plutonium.

MR. SHOUDY:

There is another point I would like to make. Somewhere along the line you assume that the fuel cycle costs are so low that we should really concentrate on the capital equipment cost. 
MR. HÄFELE:

Indeed.

MR. SHOUDY:

I think this is true in the assumptions made. But, it has been pointed out that the reprocessing today is a couple of thousand dollars as against 160. I think fabrication costs at today's technology are quite a bit higher than the assumptions made.

MR. HÄFELE:

That is precisely the reason why at Karlsruhe reprocessing studies. refabricating studies, and studies for capital costs are made. Provided that these targets can be met, it only makes sense to lower the capital cost, but only provided that the targets can be met.

MR. SHOUDY:

Finally, you concluded on the basis of the GE test that you might as well start out with a 90 per cent dense pin. Don't you feel there is an advantage in a 70 per cent dense pin from burnup standpoint, that you already have a built-in voidage for fission gas release? I think it has been demonstrated that a lower density oxide does have higher burnup capability than a higher density oxide.

MR. HAFELE.

We started with that sort of contemplation at Karlsruhe, but we don't follow it any longer. After some 30,000 MWD/tonne, the amount of original voidage is reflected only in that diameter of the chmmey. But the density is around 90 per cent anyway, and to have a somewhat larger chimney makes no sense and gives no advantage From this we conclude that it should be around 90 per cent, where we have a small chimney, just large enough to have sufficient gas release.

MR. PUECHL (Nuclear Materials and Equipment Corp.).

I would like to make one general comment about refabrication and reprocessing costs. I think we all tend to forget the time scale As Dr. Wright pointed out, the amount of plutonium available that will be refabricated or reprocessed will only be appreciable after 1980. We cannot compare such costs for plutonium today or in the next five years with refabrication and reprocessing costs for uranium, because industry cannot afford to build the multi-ton-a-day plant in the case of fabrication on uranium; and for plutonıum we are still talking about kilograms-a-day 
plants until the $1980^{\prime} \mathrm{s}$. Thus the initial cost for both reprocessing and refabrication of plutonium will be quite high compared to uranium. When the plutonium industry is on its feet, when the re is enough plutonium available, then the cost differential between plutonium and uranium will be relatively minor.

MR. HÄFELE:

Our idea was to study the implications; therefore, the parameter study to see how strongly this uncertainty you are talking about has an impact on the core design. 


\title{
STATUS OF THE URANIUM-PLUTONIUM CARBIDE FUEL DEVELOPMENT PROGRAM
}

\author{
A. A. Strasser \\ United Nuclear Corporation \\ White Plains, New York
}

This paper summarizes the property data obtained to date on the uranium-plutonium carbide fuel development program, sponsored by the USAEC and USAEC-Euratom at the United Nuclear Corporation. The objectives of the program are to develop a fabrication technique, measure the out of pile properties and study the irradiation behavior of the fuel. Much of the work is still in progress and this should be considered a status report.

Fabrication $(1,2,3)$

Carbon reduction of the oxide followed by cold pressing and sintering of the carbide powder was chosen as an economical carbide-fabrication process. United Nuclear Corporation with the Carborundum Co. as subcontractor has been fabricating various dense bodies of (UPu)C for over two years by this method:

The individual oxides are mechanically mixed with carbon, pelletized, and reacted at about $1625^{\circ} \mathrm{C}$ in a helium atmosphere to form a (UPu)C clinker. The reaction is

$$
4 \mathrm{UO}_{2}+\mathrm{PuO}_{2}+15 \mathrm{C} \rightarrow 5\left(\mathrm{U}_{0.8} \mathrm{Pu}_{0.2}\right) \mathrm{C}+10 \mathrm{CO} .
$$

The use of a stoichiometric amount of carbon always produced a second phase of a sesquicarbide. Ninety-five percent of stoichiometric carbon produced single-phase monocarbide powdex.

The (UPu)C clinker is milled to a fine powder. The powder is cold pressed and sintered in helium. Densities $96 \%$ of theoretical (average) are achieved by sintering at $1550^{\circ} \mathrm{C}$ with $0.1 \%$ nickel sintering aid, and $94 \%$ of theoretical (average) by sintering at $1925^{\circ} \mathrm{C}$ without sintering aid. Typical structures obtained are shown in Figs 1 and 2 The material made without sintering aid is single phase or has traces of dicarbide. The material made with sintering aid always has a second phase of sesquicarbide. The nickel is in uniform solid solution in the carbide.

The carbon content of both types of material can be held to $\pm 0.1 \mathrm{w} / \mathrm{O}$ carbon or better. Plutonium homogeneity is within the sensitivity of microprobe analysis. 

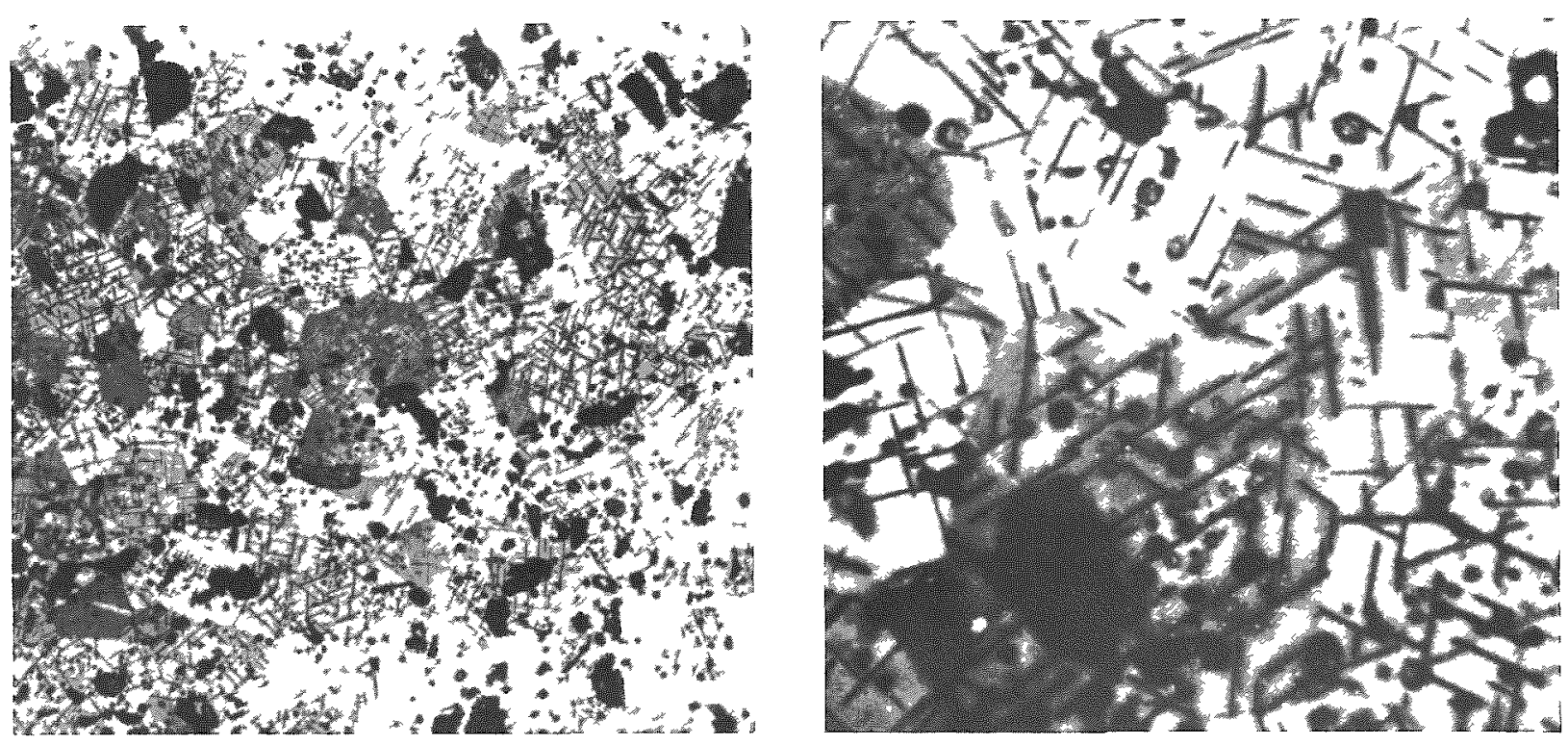

$150 \mathrm{X}$

$600 \mathrm{X}$

Fig. 1. (U $\left.\mathrm{U}_{0.8} \mathrm{Pu}_{0.2}\right) \mathrm{C}_{0.95}$ - Typical Structure of Irradiation Specimen. Etchant: nitric acid-acetic acid-water.
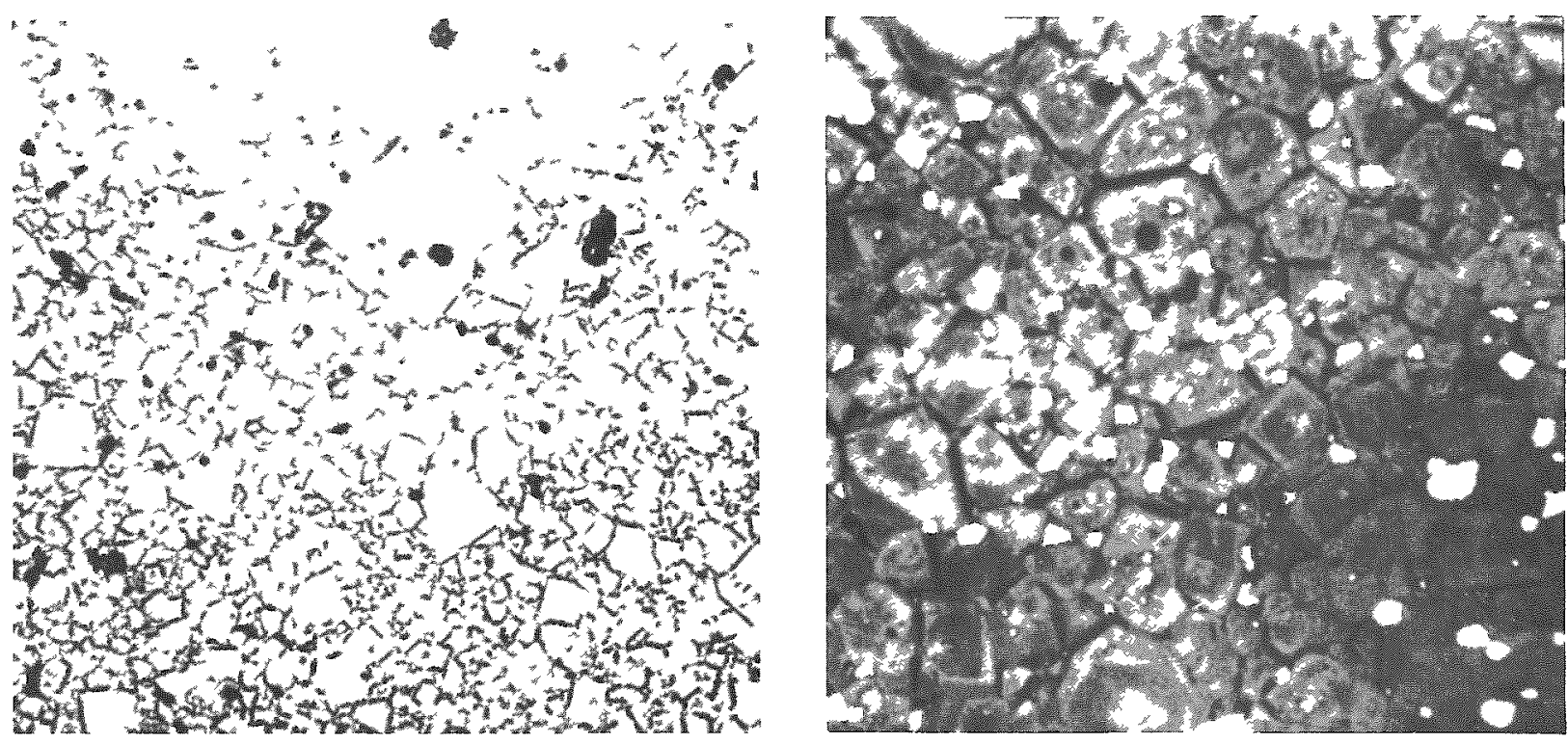

Fig. 2. $\left(\mathrm{U}_{0.8} \mathrm{Pu}_{0.2}\right) \mathrm{C}_{0.95}+0.1 \mathrm{Ni}$ - Typical Structure of Irradiation Specimen. Etchant. nitric acid-acetic acid-water.

Pellets produced by this process were ground to OD tolerances of $\pm 0.0005 \mathrm{in}$. They have also been drilled and ground to produce various shapes of test sample.

The advantages of this process are: 
1. adaptability to economical mass production;

2. low-cost raw material;

3. ease in making small- as well as large-diameter pellets:

4. relatively small amounts of plutonium handled at one location, as compared with large amounts in arc casting, particularly skull melting;

5. good control of carbon content:

6. good plutonium homogeneity:

7. good dimensional control in the as-sintered condition with the possibility that grinding may be omitted even with a helium fuel-clad "bond." (Arc-cast rods require considerable grinding or a liquid metal bond with its associated compatibility problems.)

The disadvantage is:

The necessity of handling large quantities of very fine particle size, easily oxidizable, pyrophoric powders. This requires high purity and inert handling atmospheres.

The sintered product differs from arc-cast material in several ways. Whether these differences are advantageous or disadvantageous remains to be seen:

1. higher level of oxygen impurity in the sintered material (2000-4000 $\mathrm{ppm}$ ) than the arc cast material (100 ppm):

2. smaller grain size in the sintered material;

3. somewhat lower achievable density for sintered material.

Properties

\section{Melting Point}

The melting point for $\left(\mathrm{U}_{0.8} \mathrm{Pu}_{0.2}\right) \mathrm{C}_{0.95}$ as measured is in the range $2430-2480^{\circ} \mathrm{C}$. The lower temperature indicates the solidus and the upper temperature complete melting. 
The measurements were made optically with 0.20 -in. diameter pellets with a black-body hole placed in the center of a tubular tantalumresistance olement. The crucible used was internally carburized tintalum, which pretented fucl-crucible reactions.

Cocficient of Expansion $(3,4)$

The coefficients of expansion were measured to $1400^{\circ} \mathrm{C}$, with the results shown in Table 1. The values are similar to those for pure UC. Changes in the plutonium content or the prosence or absence of the nickel sintering aid does not appear to affect the expansion coefficients significantly. The expansion curves are smooth and do not show any transformations.

Table 1

COEFFICIENT OF THERMAL EXPANSION $\left(10^{-6} /{ }^{\circ} \mathrm{C}\right)$

\begin{tabular}{|c|c|c|c|c|c|}
\hline $\begin{array}{c}\text { Tempexature } \\
\text { Range, } \\
{ }^{\circ} \mathrm{C}\end{array}$ & $\begin{array}{l}\text { Arc-cast } \\
\text { UC }\end{array}$ & $\begin{array}{c}\text { Sintered } \\
\left(U_{0.95} P_{0.05}\right) C_{0.98}\end{array}$ & $\begin{array}{c}\text { Sintexed } \\
\left(\mathrm{U}_{0.95} \mathrm{Pu}_{0.05}\right) \mathrm{C}_{0.98} \\
+0.1 \mathrm{Ni}\end{array}$ & $\begin{array}{c}\text { Sintered } \\
\left(\mathrm{C}_{0.8} \mathrm{Pu}_{0.2}\right) \mathrm{C}_{0.95}\end{array}$ & $\begin{array}{c}\text { Sintered } \\
\left(\mathrm{U}_{0.8} \mathrm{Pu}_{0.2}\right) \mathrm{C}_{0.95} \\
+0.1 \mathrm{Ni}\end{array}$ \\
\hline $25-200$ & 8.9 & 9.0 & 8.6 & 8.6 & 9.0 \\
\hline $25-400$ & 10.2 & 10.0 & 10.2 & 9.7 & 9.8 \\
\hline $25-600$ & 11.3 & 10.8 & 10.9 & 10.5 & 10.5 \\
\hline $25-800$ & 12.0 & 11.5 & 11.4 & 21.1 & 11.0 \\
\hline $25-1000$ & 12.2 & 12.0 & 11.9 & 11.6 & 11.5 \\
\hline $25-1200$ & 12.4 & 12.4 & 12.1 & 11.9 & 11.9 \\
\hline $25-1400$ & 12.6 & 12.6 & 12.2 & 12.2 & 12.3 \\
\hline
\end{tabular}

The measurements were made by a diferential expansion dilatomoter, with polycrystalline alumina elements in vacuum. A dial gage and a diferential transformer measured expansion simultaneously.

Coeficients of expansion obtained by Los Mlamos(5) and Marwell(o) on (UPu) C of $13-15 \%$ PuC are similar to these values.

Comparibility $(3.4)$

The compatibility of the solid solution carbides is being studied at $593^{\circ} \mathrm{C}\left(1100^{\circ} \mathrm{F}\right)$ and $810^{\circ} \mathrm{C}\left(1500^{\circ} \mathrm{F}\right)$ for 1000 and $4000 \mathrm{hr}$. Results to date are shown in Table 2 .

The results with (LPu) C are similar to those obtained previously with UC. There were no significant reactions between fuel and Type 316 stainless steel, $2 \frac{1}{4} \mathrm{Cr}-1$ Mo steel, niobium, and niobium-10 zirconium alloy. A few isolated reactions were seen which penetrated about $0.0002 \mathrm{in}$. into the cladding. Representative photographs are shown in Figs. 3,4 and 5. A possible slight reaction was noted with ranadium. Attempts to identify the "reaction product" by etchants indicated it was neither 
uranium, plutonium, nor carbide. More significant reactions were noted with Inconel $\mathrm{X}$ and Zircaloy-2. Differences in plutonium level, the presence or absence of nickel sintering aid, or the presence of sesquicarbide did not make any difference in the results.

Table 2

(UPU)C COMPATIBILITY TEST RESULTS

(Test Conducted in Helium in Differential Expansion Capsule)

\begin{tabular}{|c|c|c|c|c|}
\hline & Material & Temp, ${ }^{\circ} \mathrm{C}\left({ }^{\circ} \mathrm{E}\right)$ & $\begin{array}{l}\text { 1000-hr Tests. } \\
\text { Results by } \\
\text { Metallographic } \\
\text { Exammation }\end{array}$ & $\begin{array}{l}\text { 4000-hr Tests. } \\
\text { Results by } \\
\text { Metallographic } \\
\text { Examination }\end{array}$ \\
\hline Type 316 & $\begin{array}{l}\mathrm{vs}\left(\mathrm{U}_{0.8} P \mathrm{u}_{0.2}\right) \mathrm{C}_{0.95} \\
\left(\mathrm{U}_{0.8} P \mathrm{u}_{0.2}\right) \mathrm{C}_{0.95}+0.1 \mathrm{Ni} \\
\left(\mathrm{U}_{0.95} \mathrm{Pu}_{0.05}\right) \mathrm{C}_{0.98} \\
\left(\mathrm{U}_{0.95} \mathrm{Pu}_{0.05}\right) \mathrm{C}_{0.98}+0.1 \mathrm{Ni}\end{array}$ & $820(1500)$ & $\begin{array}{l}\text { No reaction* } \\
\text { No reaction } \\
\text { N }\end{array}$ & No reaction \\
\hline $2 \frac{1}{4} \mathrm{Cx}-1 \mathrm{Mo}$ & $\begin{aligned} v s & \left(U_{0.8} P_{0.2}\right) C_{0.95} \\
& \left(U_{0.95} \mathrm{Pu}_{0.05}\right) C_{0.98}\end{aligned}$ & $\begin{array}{l}820(1500) \\
820(1500)\end{array}$ & 1 & $\begin{array}{l}\text { No reaction } \\
\text { No reaction }\end{array}$ \\
\hline $\mathrm{Nb}, \mathrm{Nb}-1 \mathrm{Zr}$ & $\begin{aligned} &\left(\mathrm{U}_{0.8} \mathrm{Pu}_{0.2}\right) \mathrm{C}_{0.95} \\
&\left(\mathrm{U}_{0.8} \mathrm{Pu}_{0.2}\right) \mathrm{C}_{0.95}+0.1 \mathrm{Ni} \\
&\left(\mathrm{U}_{0.95} \mathrm{Pu}_{0.05}\right) \mathrm{C}_{0.98} \\
&\left(\mathrm{U}_{0.95} \mathrm{Pu}_{0.05}\right) \mathrm{C}_{0.98}+0.1 \mathrm{Ni}\end{aligned}$ & $820(1500)$ & No reaction & $\begin{array}{c}- \\
- \\
\text { No reaction } \\
\text { No reaction }\end{array}$ \\
\hline V & $\mathrm{vs}\left(\mathrm{U}_{0.8} \mathrm{Pu}_{0.2}\right) \mathrm{C}_{0.95}$ & $\begin{array}{l}590(1100) \\
820(1500)\end{array}$ & $\begin{array}{l}\text { No reaction } \\
\text { No reaction* }\end{array}$ & $\begin{array}{l}\text { Possible slight } \\
\text { reaction }\end{array}$ \\
\hline Zircaloy-2 & $\begin{array}{l}\mathrm{vs}\left(\mathrm{U}_{0.8} \mathrm{Pu}_{0.2}\right) \mathrm{C}_{0.95} \\
\left(\mathrm{U}_{0.95} \mathrm{Pu}_{0.05}\right) \mathrm{C}_{0.98}\end{array}$ & $\begin{array}{l}590(1100) \\
820(1500) \\
590(1100) \\
820(1500)\end{array}$ & Reaction noted & $\begin{array}{l}- \\
- \\
-\end{array}$ \\
\hline Inconel X & $v s\left(U_{0.8} P_{0.2}\right) C_{0.95}$ & $\begin{array}{l}590(1100) \\
820(1500)\end{array}$ & $\begin{array}{l}\text { No redction } \\
\text { Reaction noted }\end{array}$ & - \\
\hline
\end{tabular}

* One sample in each case showed possible reaction to a depth of 0.0002 m. mas.

Interface with fuel

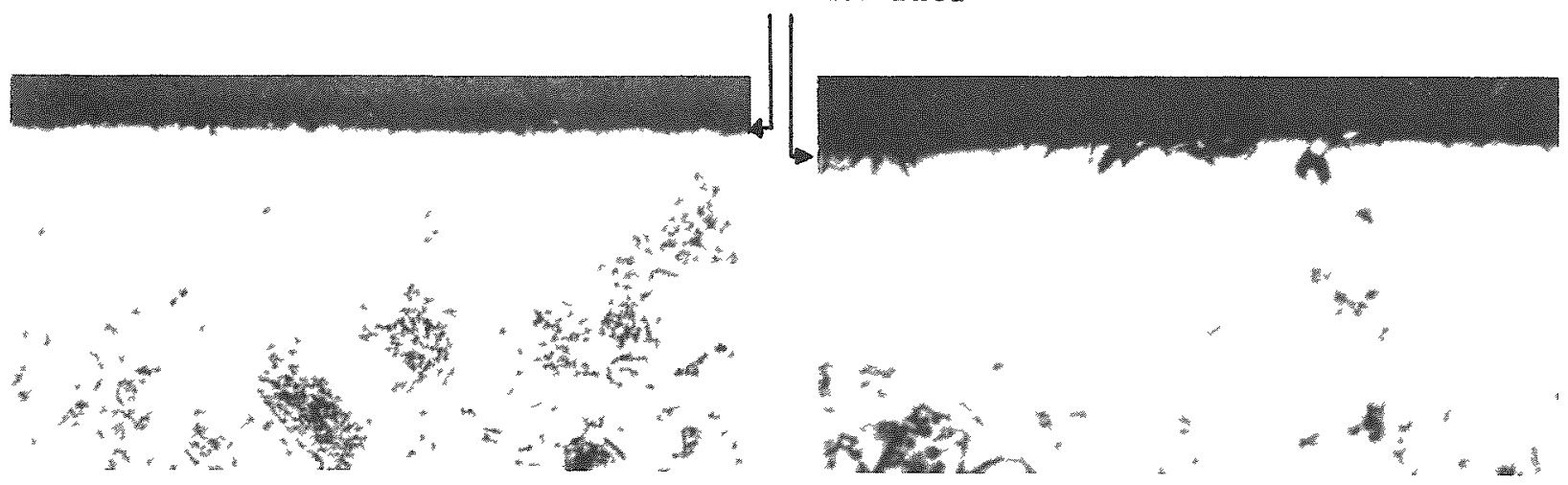

$150 \mathrm{X}$

$600 X$

Fig. 3. Type 316 Stainless Compatibility Test vs $\left(\mathrm{U}_{0.8} \mathrm{Pu}_{0.2}\right) \mathrm{C}_{0.95}+0.1 \mathrm{Ni}$, $816^{\circ} \mathrm{C}\left(1500^{\circ} \mathrm{F}\right), 4000 \mathrm{hr}$ 
Interface with fuel

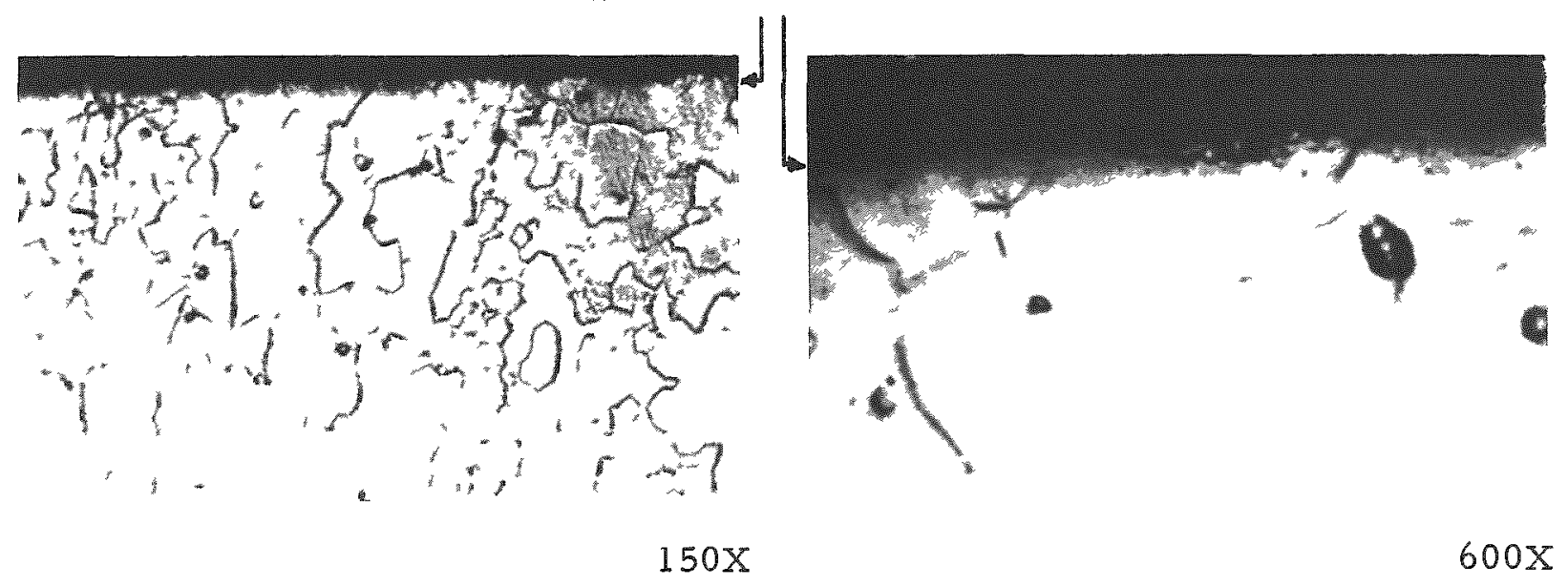

Fig. 4. Niobium from Compatibility Test with $\left(U_{0.95} P_{u_{0.05}}\right) C_{0.98}$, $816^{\circ} \mathrm{C}\left(1500^{\circ} \mathrm{F}\right), 4000 \mathrm{hr}$

Interface with fuel

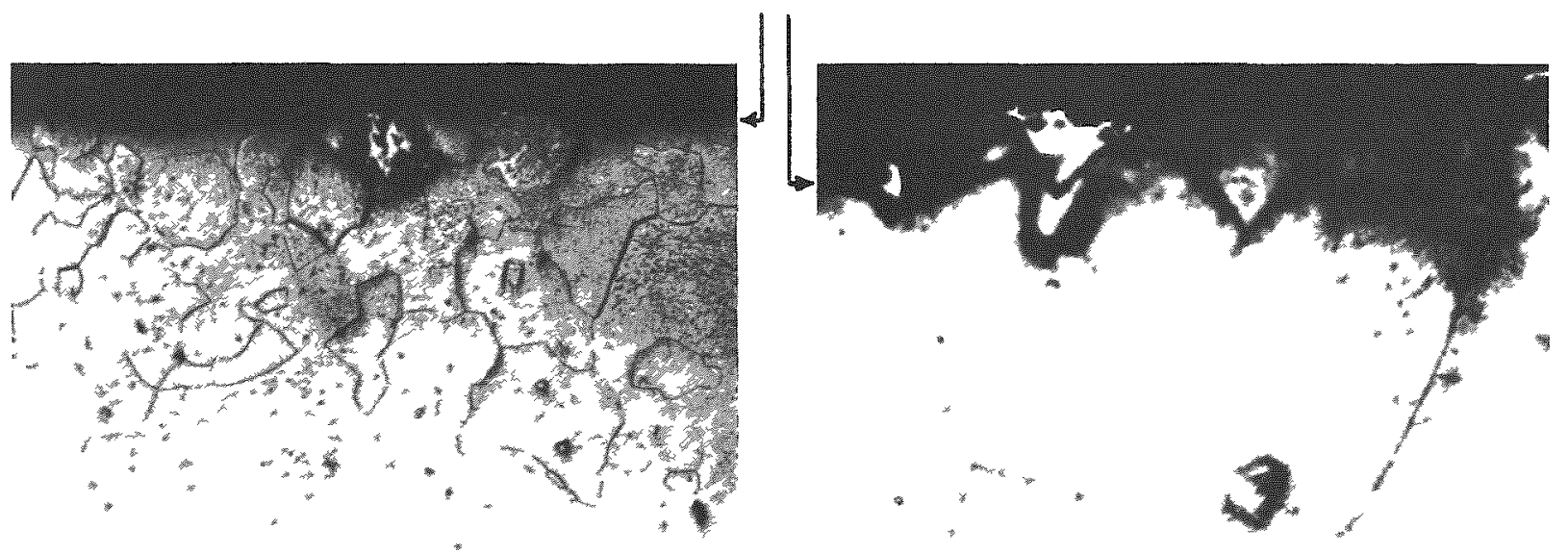

$150 x^{-}$

Fig. 5. Vanadium from Compatibility Test with $\left(\mathrm{U}_{0.8} \mathrm{Pu}_{0.2}\right) \mathrm{C}_{0.95}$, $816^{\circ} \mathrm{C}\left(1500^{\circ} \mathrm{F}\right), 4000 \mathrm{hr}$

The tests were carried out in helium in differential expansion capsules of the type shown in Fig. 6.

Irradiation Tests $(1-4)$

Twelve clad specimens of the solid solution carbides, $\left(\mathrm{U}_{0.8} \mathrm{Pu}_{0.2}\right)$ $\mathrm{C}_{0.95}$, are being irradiated in GETR. Each specimen consists of 16 fuel pellets, $0.191 \mathrm{in}$. in diameter, for a 3 -in. fueled length, clad in stainless steel or niobium. The void space is filled with helium. There are two specimens per capsule, one containing fuel fabricated without sintering aid, and one with sintering aid. 


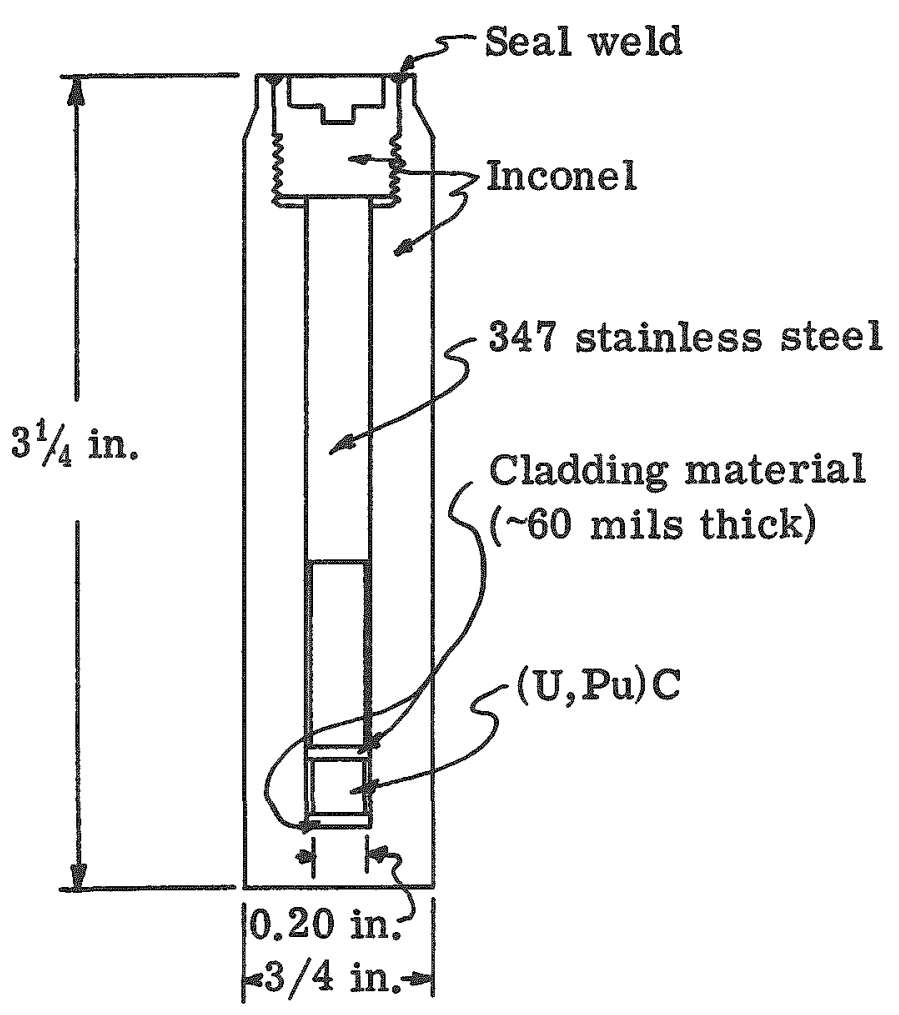

Fig. v. Compatibility Capsule
The irradiation conditions for the twelve specimens are given in Table 3 . The specimens designed to operate at less than central fuel temperatures of $1040^{\circ} \mathrm{C}\left(1900^{\circ} \mathrm{F}\right)$ (Capsules 61, 62, 63) have a niobium central thermocouple well for temperature measurement, and for this reason are also clad with niobium. The specimens for operation at higher temperature (Capsules $64,65,66$ ) do not have provision for fuel center temperature measurement. These specimens are clad with Type 316 stainless steel, because this is potentially one of the most economical fuel clads for a fast-neutron. central-station, power plant.

Table 3

SIMPLIEIED TABLE OF AVERAGE IRRADIATION CONDITIONS FOR $\left(U_{0.8} \mathrm{Pu}_{0.2}\right) \mathrm{C}_{0.95}$ SPECIMENS

\begin{tabular}{|c|c|c|c|c|c|}
\hline $\begin{array}{c}\text { Capsule } \\
\text { No. }\end{array}$ & $\begin{array}{l}\text { Number of } \\
\text { Specimens }\end{array}$ & $\begin{array}{c}\text { Clad Surface } \\
\text { Temperature, } \\
{ }^{\circ} \mathrm{C}\left({ }^{\circ} \mathrm{F}\right)\end{array}$ & $\begin{array}{c}\text { Fuel Center } \\
\text { Temperature. } \\
{ }^{\circ} \mathrm{C}(\mathrm{F})\end{array}$ & $\begin{array}{c}\text { Power } \\
W / \mathrm{cm}(\mathrm{kW} / \mathrm{ft})\end{array}$ & $\begin{array}{l}\text { Burnup, } \\
10^{20} \text { fissions } / \mathrm{cm}^{3} \\
(\mathrm{MWD} / \mathrm{T})\end{array}$ \\
\hline $\begin{array}{l}61 \\
62 \\
63\end{array}$ & $\left.\begin{array}{l}2 \\
2 \\
2\end{array}\right\}$ & $600(1110)$ & $1000(1830)$ & $370(11.3)$ & $\begin{array}{r}7.9(21,500) \\
12.5(34,000) \\
24.9(70,000)\end{array}$ \\
\hline $\begin{array}{l}64 \\
65 \\
66\end{array}$ & $\left.\begin{array}{l}2 \\
2 \\
2\end{array}\right\}$ & $560(1040)$ & $1220(2230)$ & $520(15.9)$ & $\begin{array}{r}5.9(16,000) \\
12.5(34,000) \\
24.9(70,000)\end{array}$ \\
\hline
\end{tabular}

An approximate in-pile the rmal conductivity of the fuel was obtained by measurement of the temperature drop from fuel center to clad surface, and knowledge of heat-generation rate in the specimen. Since this measurement will give the combined fuel t helium gap conductivity, an estimate of gap conductivity is needed to deduce the fuel conductivity. The gap conductivity was obtained from the irradiation of UC, which has a relatively well-known thermal conductivity. Clad UC specimens, identical with the (UPu) C specimens were irradiated, and fuel thelium gap 
conductivities calculated. Assuming the range of known conductivity values for $\mathrm{UC}$, the helium gap conductances were calculated. The results are given in Table 4 The gap conductance does not vary greatly with variations of UC conductivity A $1.00 \mathrm{~W} / \mathrm{cm}^{2}-{ }^{\circ} \mathrm{C}\left(1740-\mathrm{Btu} / \mathrm{hr}-\mathrm{ft}^{2}-{ }^{\circ} \mathrm{F}\right)$ value for the gap conductance is reasonable.

Table 4

FUEL AND HELIUM GAP CONDUCTIVITIES FOR UC IRRADIATION SPECIMENS

\begin{tabular}{|c|c|c|c|c|c|}
\hline & \multicolumn{5}{|c|}{ Helium Gap Conductance, Btu/hr-fth ${ }^{\circ} \mathrm{E}$} \\
\hline $\begin{array}{l}\text { Burnup, MWD } / \mathrm{T} \\
\text { Specimen }\end{array}$ & $\begin{array}{l}\text { Startup } \\
\text { Type } 316 * *\end{array}$ & $\begin{array}{l}\text { Startup } \\
\mathrm{Nb}\end{array}$ & $\begin{array}{l}2500 \\
\mathrm{Nb}\end{array}$ & $\begin{array}{l}3800 \\
\mathrm{Nb}\end{array}$ & $\begin{array}{c}6500 * \\
\mathrm{Nb}\end{array}$ \\
\hline $\begin{array}{c}\text { UC Thermal } \\
\text { Conductivity, } \\
\text { Btu/hr-ft }{ }^{\circ} \mathrm{F} / \mathrm{ft}, \\
\text { (Assumed) } \\
10 \\
12 \\
14\end{array}$ & $\begin{array}{l}2200 \\
1950 \\
1800\end{array}$ & $\begin{array}{l}1900 \\
1700 \\
1600\end{array}$ & $\begin{array}{l}1850 \\
1700 \\
1600\end{array}$ & $\begin{array}{l}1850 \\
1650 \\
1550\end{array}$ & $\begin{array}{l}2300 \\
2050 \\
1900\end{array}$ \\
\hline
\end{tabular}

*Central thermocouple in niobium clad specimen failed shortly after specimen achieved $6500 \mathrm{MWD} / \mathrm{T}$. preventing further calculations of gap conductance for this specimen

**Central thermocouple in stainless steel clad specimen falled shortly after startup, preventing further calculations of gap conductance for this specimen

If we use the $1.00 \mathrm{~W} / \mathrm{cm}^{2}-{ }^{\circ} \mathrm{C}$ value for the helium gap conductance of the $\left(\mathrm{U}_{0.8} \mathrm{Pu}_{0.2}\right) \mathrm{C}$ specimens, the $\left(\mathrm{U}_{0.8} \mathrm{Pu}_{0.2}\right) \mathrm{C}$ thermal conductivity $1 \mathrm{~s}$ an average of $0.046 \mathrm{cal} / \mathrm{sec}-\mathrm{cm}^{2}-{ }^{\circ} \mathrm{C} / \mathrm{cm}\left(11 \mathbb{1} \mathrm{Btu} / \mathrm{hr}-\mathrm{ft}^{2}-{ }^{\circ} \mathrm{F} / \mathrm{ft}\right)$ The $\mathrm{range}$ of values was $0.029-0.059 \mathrm{cal} / \mathrm{sec}-\mathrm{cm}^{2}{ }^{\circ} \mathrm{C} / \mathrm{cm}$ in the temperature range $700-1010^{\circ} \mathrm{C}\left(1290-1850^{\circ} \mathrm{F}\right)$

This is approximately $84 \%$ of the value for $\mathrm{UC}$ and is in the range of out $=$ of - pile thermal conductivity values for (UPu) C recently reported by Los Alamos (7) for temperatures up to $400^{\circ} \mathrm{C}$ British(8) roomtemperature thermal conductivity values for (UPu) C are somewhat lower, about $70 \%$ of UC.

The irradiation of four of the specimens is complete and the postirradiation examination is in progress at Battelle Memorial Institute. The specimens were contained in Capsules 61 and 64 Their 1 rradiation conditions and results of the post-irradiation examination to date are given in Table 5. 
Table 5

SUMMARY OF IRRADIATION CONDITIONS AND

HOT LABORATORY EXAMINATION RESULTS

OF CAPSULES 61 AND 64

\begin{tabular}{|c|c|c|c|c|}
\hline \multirow{2}{*}{ Specimen } & \multicolumn{2}{|c|}{ Capsule 61} & \multicolumn{2}{|c|}{ Capsule 64} \\
\hline & Top & Bottom & Top & Bottom \\
\hline Fuel & $\begin{array}{l}\left(U_{0.8} P_{u_{0.2}}\right) C_{0.95} \\
+0.1 \mathrm{Ni}\end{array}$ & $\left(\mathrm{U}_{0.8} \mathrm{Pu}_{0.2}\right) \mathrm{C}_{0.95}$ & $\left(\mathrm{U}_{0.8} \mathrm{P}_{4_{0.2}}\right) \mathrm{C}_{0.95}$ & $\begin{array}{l}\left(\mathrm{U}_{0.8} \mathrm{Pu}_{0.2}\right) \mathrm{C}_{0.95} \\
+0.1 \mathrm{Ni}\end{array}$ \\
\hline $\mathrm{Clad}$ & $\mathrm{Nb}$ & $\mathrm{Nb}$ & Type 316 & Type 316 \\
\hline Fuel-clad "bond" & He & He & $\mathrm{He}$ & He \\
\hline Burnup, (fissions $\left./ \mathrm{cm}^{3}\right) \times 10^{-20}$ & 8.0 & 7.8 & 5.8 & 6.0 \\
\hline $\begin{array}{l}\text { Fuel centex, }{ }^{\circ} \mathrm{C} \\
\text { Maximum } \\
\text { Average }\end{array}$ & $\begin{array}{l}1305 \\
1030\end{array}$ & $\begin{array}{l}1280 \\
1015\end{array}$ & $\begin{array}{l}1315 \\
1125\end{array}$ & $\begin{array}{l}1415 \\
1175\end{array}$ \\
\hline $\begin{array}{l}\text { Clad suxiace, }{ }^{\circ} \mathrm{C} \\
\text { Maximum } \\
\text { Average }\end{array}$ & $\begin{array}{l}795 \\
625\end{array}$ & $\begin{array}{l}770 \\
615\end{array}$ & $\begin{array}{l}740 \\
655\end{array}$ & $\begin{array}{l}795 \\
690\end{array}$ \\
\hline $\begin{array}{c}\text { Power, } \mathrm{W} / \mathrm{cm} \\
\text { Maximum } \\
\text { Average }\end{array}$ & $\begin{array}{l}450 \\
355\end{array}$ & $\begin{array}{l}440 \\
350\end{array}$ & $\begin{array}{l}470 \\
380\end{array}$ & $\begin{array}{l}510 \\
395\end{array}$ \\
\hline Clad OD changes & $\begin{array}{l}\text { Negligible } \\
(+0.000 \% \text { in. })\end{array}$ & $\begin{array}{l}\text { Negligible } \\
(+0.000 \mathrm{z} \text { in. })\end{array}$ & $\begin{array}{l}\text { Negligible } \\
(+0.000 \mathrm{x} \text { in. })\end{array}$ & $\begin{array}{l}\text { Negligible, except } \\
\text { for } \frac{3}{8} \text { in. long } \\
\text { molten section }\end{array}$ \\
\hline $\begin{array}{l}\text { Number of pellets, } \\
\text { Whole } \\
\text { Cracked }\end{array}$ & $\begin{array}{r}4 \\
12\end{array}$ & $\begin{array}{l}9 \\
7\end{array}$ & $\begin{array}{l}\text { Shattered with } \\
\text { light handling }\end{array}$ & $\begin{array}{l}\quad 7 \\
4(4 \text { in failed } \\
\text { section) }\end{array}$ \\
\hline $\begin{array}{l}\text { Pellet dimension changes, } \\
\text { OD, } \% \\
\text { Length, } \%\end{array}$ & $\begin{array}{l}+1.3 \rightarrow+1.8 \\
-2.0 \rightarrow+3.7\end{array}$ & $\begin{array}{l}+1.2 \rightarrow+2.6 \\
+1.1 \rightarrow+3.9\end{array}$ & $+1.6(1 \mathrm{pellet})$ & $\begin{array}{l}+1.4 \rightarrow+1.9 \\
+1.5 \rightarrow+4.0\end{array}$ \\
\hline $\begin{array}{l}\text { Fission gas release, } \\
\text { \% of total } \\
\text { (Based on } \mathrm{Ka}^{85} \text { ) }\end{array}$ & 0.12 & 0.11 & 0.23 & 12.9 \\
\hline $\begin{array}{l}\text { Glad bend tests }\left(180^{\circ}\right. \\
\text { on } \frac{1}{2} \text { in. radius) }\end{array}$ & Ductile & Ductile & Ductile & $\begin{array}{l}\text { Less ductile than } \\
64 \mathrm{~T} \text {. Broke } \\
\text { before } 110^{\circ}\end{array}$ \\
\hline Clad metallography & None yet & None yet & $\begin{array}{l}\text { No fuel-clad } \\
\text { reaction }\end{array}$ & Incomplete \\
\hline Fuel metallography & As betore & $\begin{array}{l}\text { As before with- } \\
\text { out dicarbide }\end{array}$ & $\begin{array}{l}\text { As before with- } \\
\text { out dicarbide }\end{array}$ & $\begin{array}{l}\text { As before, except } \\
\text { in failed section }\end{array}$ \\
\hline
\end{tabular}

The four specimens are shown in Fig. 7. Specimens $61 \mathrm{~T}, 61 \mathrm{~B}$, and $64 \mathrm{~T}$ appeared as before irradiation. There were no significant changes in the cladding OD. Specimen 64B, which was clad in stainless steel, had melted and ruptured in a $\frac{3}{8}$-in.-long section near the specimen center. The failure was highly localized, and the remainder of the specimen was not affected by the failure. The $O D$ of the cladding away from the failure did not change. Figure 8 shows an enlarged view of the failure. The cause of the failure has not been determined yet. It could have been a local hot spot or a local fuel-clad interaction. 


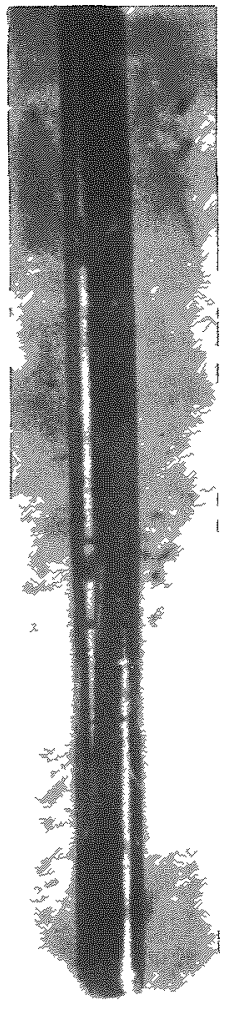

$61 \mathrm{~T}$

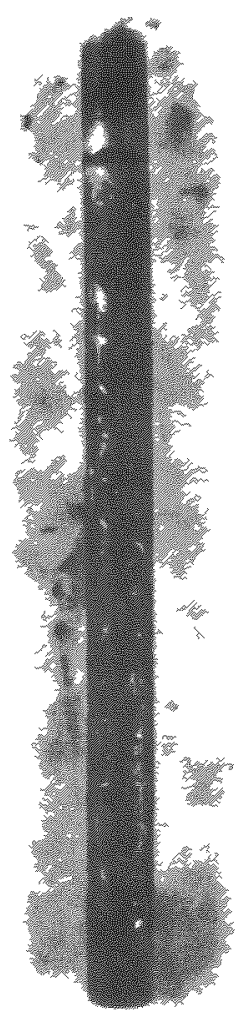

$61 \mathrm{~B}$

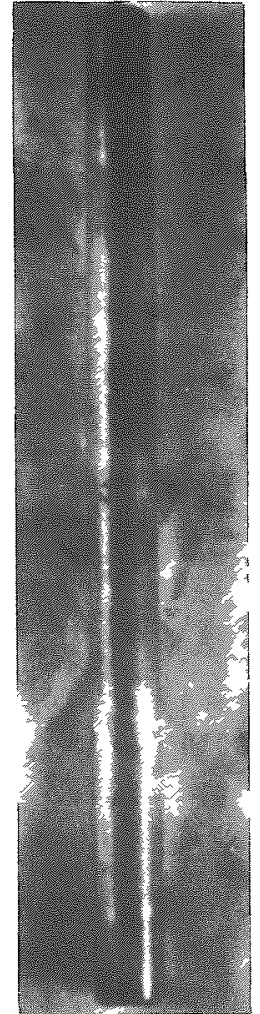

$64 \mathrm{~T}$

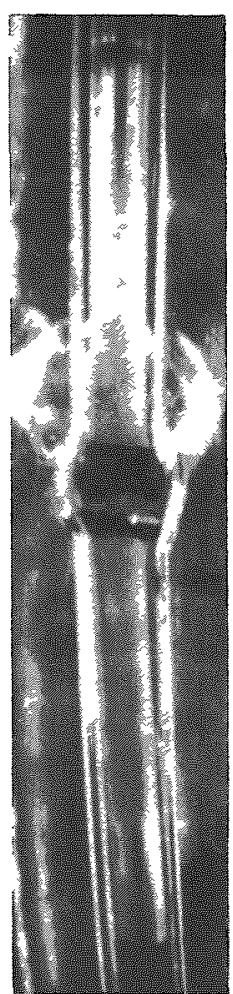

$64 \mathrm{~B}$

Fig. 7. Clad $\left(\mathrm{U}_{0.8} \mathrm{Pu}_{0.2}\right) \mathrm{C}_{0.95}$ Fuel Specimens after Irradiation

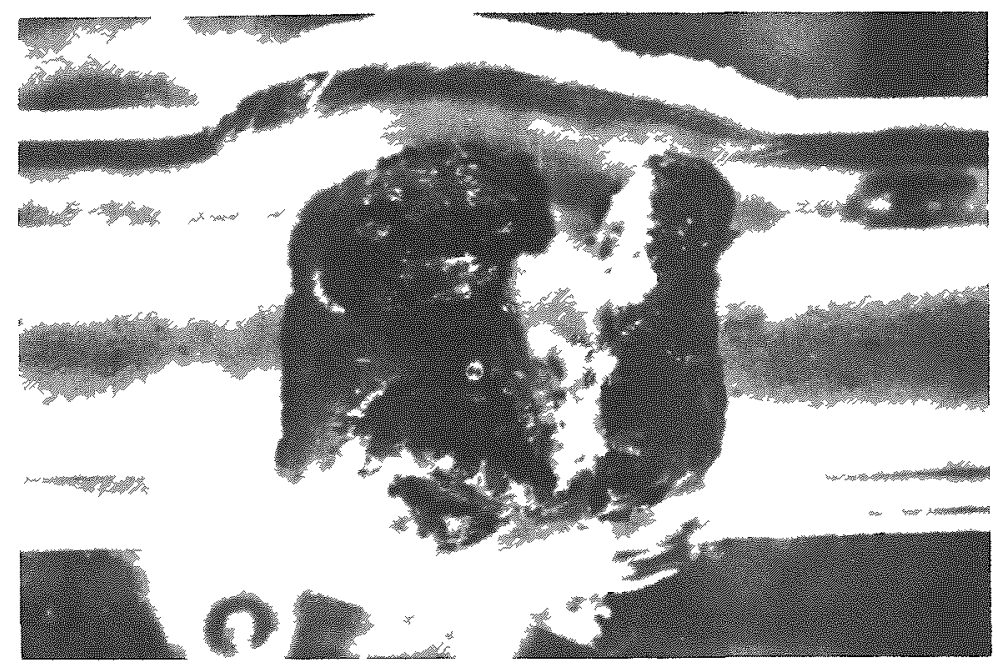

Fig. 8. Magnification of Failed Section of Specimen $64 \mathrm{~B}$

The normal operating temperatures of the stainless cladding was a peak of $845^{\circ} \mathrm{C}\left(1550^{\circ} \mathrm{F}\right)$ and an average of $730^{\circ} \mathrm{C}\left(1340^{\circ} \mathrm{F}\right)$, temperatures at which stainless steel would be marginal for power reactors on the basis of strength considerations. 
The fission gas release from the three intact specimens was low, about the amount expected from recoil. The fissiongas released to the inner shell of Capsule 64 by the ruptured Specimen $64 \mathrm{~B}$ was $13 \%$ of the theoretical total, or $100 \%$ of the fission gas in the two pellets contamed in the failed section.

The pellets were removed from all specimens by slitting the cladding longitudinally. Figure 9 shows pictures of representative pellets. As shown by Table 5, a large fraction of the pellets were intact, including all the pellets in Specimen $64 \mathrm{~B}$ which were not drectly in the failed section. The majority of the pellets were broken in a few large chunks. The pellets were quite brittle after irradiation, and even light handling tended to cause cracking. Thus many of them could have been cracked during disassembly.
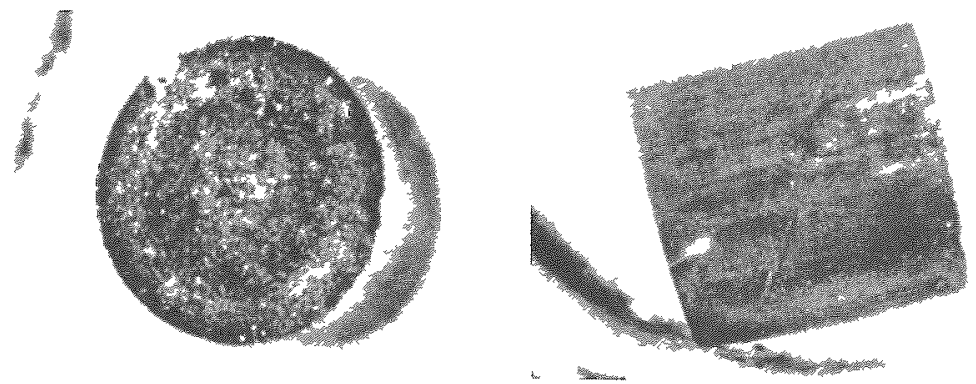

Fig. YB

Typical Cracked $\left(\mathrm{U}_{0.8} \mathrm{Pu}_{0.2)}\right.$ Cu. 95 Pellets atter Irradiation (from Specimen 61T)
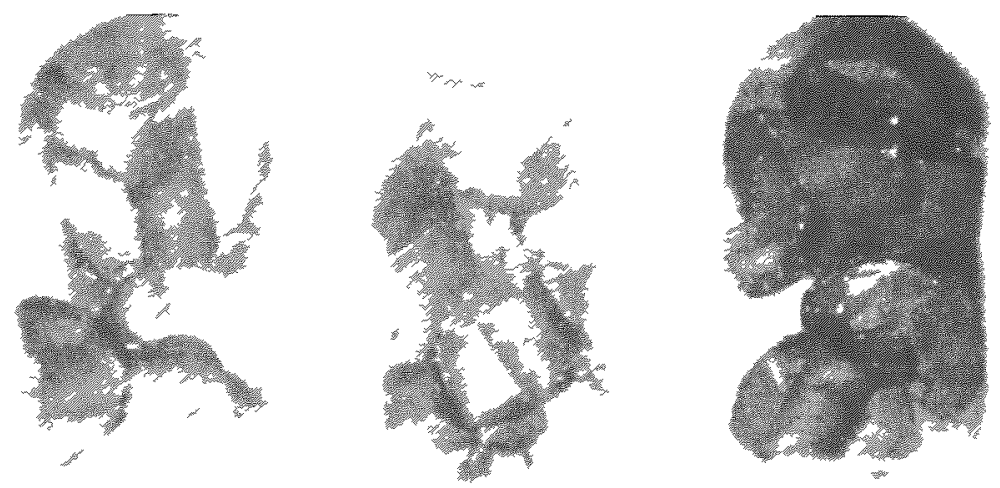
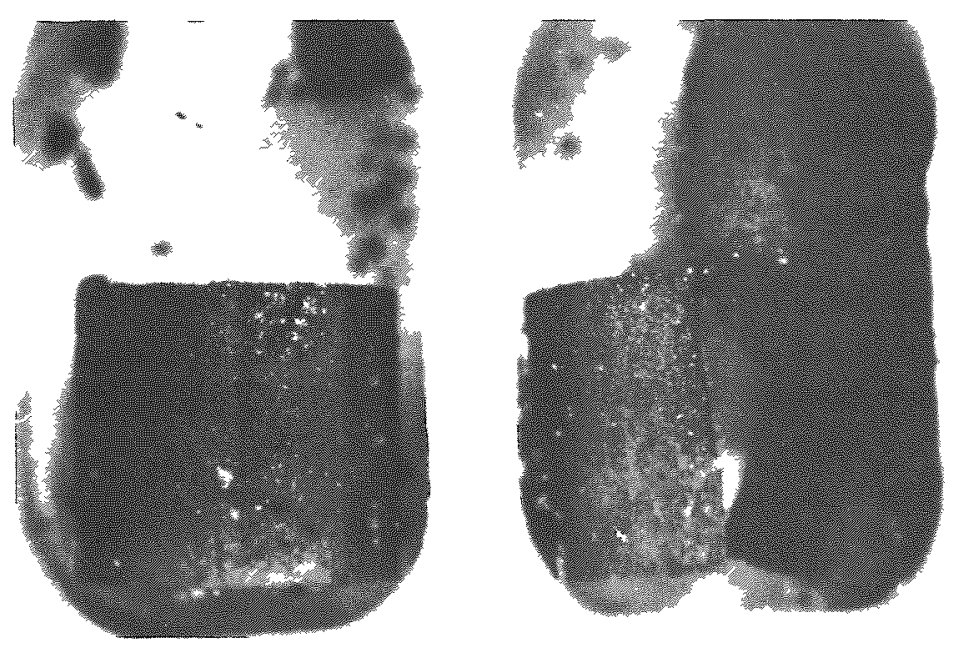

Fig. 9A

Typical Whole Pellets. Irra Jated $\left(\mathrm{U}_{0.8} \mathrm{P}_{40.2}\right) \mathrm{C}_{0.95}+0.1 \mathrm{~N} 1$ Specimen $61 \mathrm{~T}$
Fig. 9C

Typical Whole and Cracked $\left(U_{0.8} P^{P_{0} .2}\right) C_{0.95}$ Pellets after Irradiation (from Specimen 61B) 
Dimensional changes of whole pellets increased an average of 0.002 -in. OD over on an original 0.191-in. OD, and an average of $0.003 \mathrm{in}$. in length over on a variable original length of $0.185-0.192 \mathrm{in.}$

Mctallography of the fuel showed no significant changes in microstructure due to irradiation. Typical structures of fuel with and without sintering aid are shown in Figs. 10 and 11 . The trace of dicarbide structure present in some of the unirradiated control pellets was not found in any of the irradiated pellets examined. The grain size was the same in the pellet centers as at the pellet edges showing that the temperatures of irradiation did not cause any grain growth. It is significant to note that at $\int_{T_{S}}^{T_{C}} k d \theta$ ratings of these specimens $(\sim 30-40 \mathrm{~W} / \mathrm{cm})$, one would get col umnar grain formation with oxides.

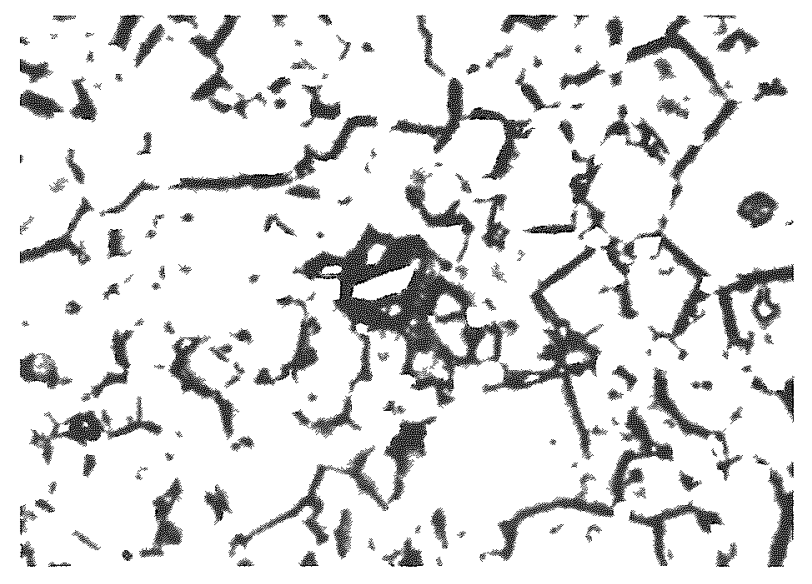

Pre-irrdiacion

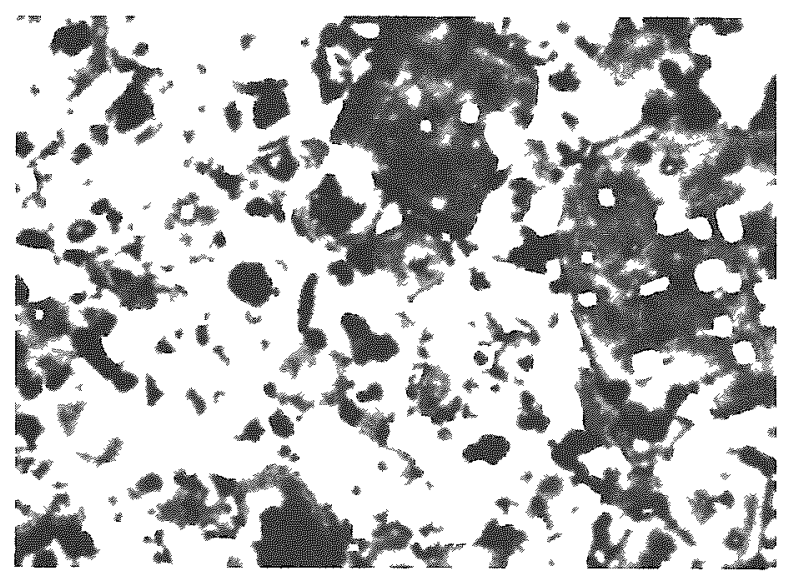

Post-irradiation $(-21,000 \mathrm{MWD} / \mathrm{T})$

$600 \mathrm{X}$

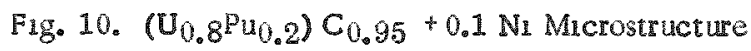

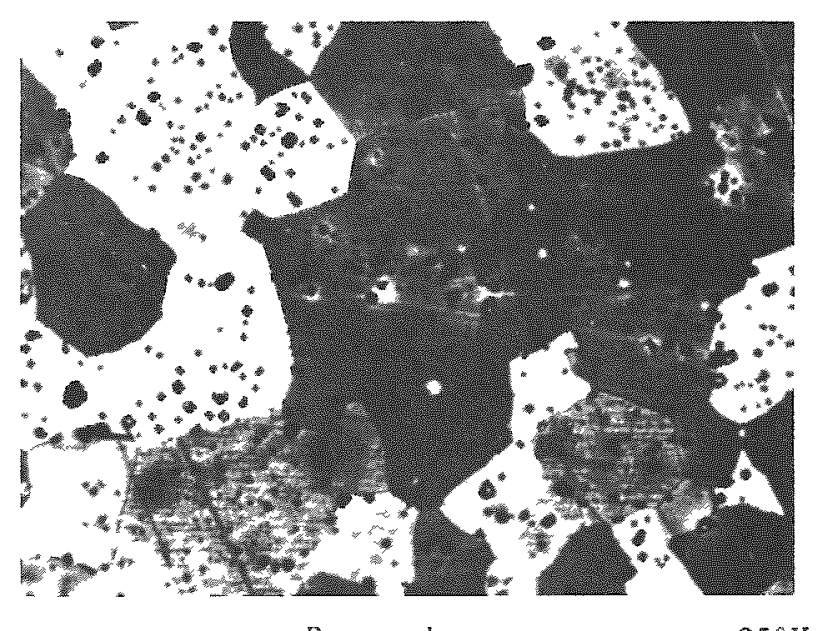

Pre-irradianon

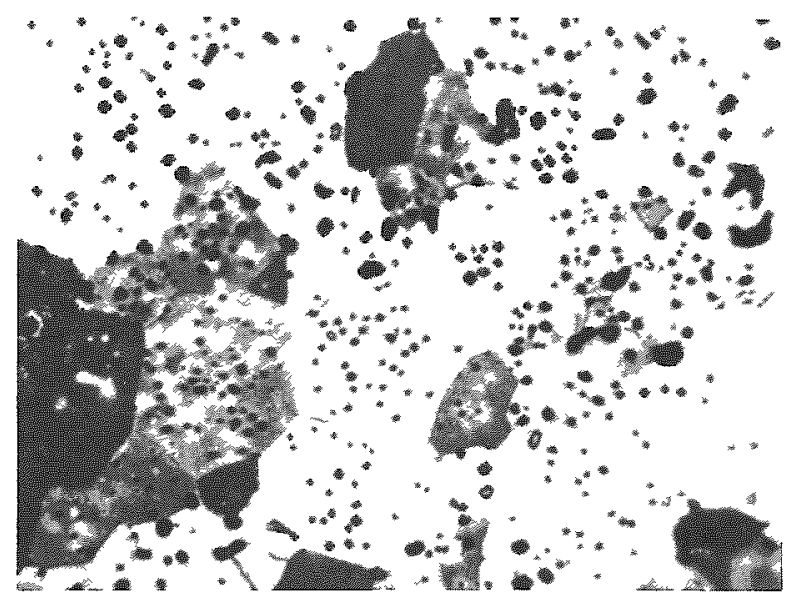

Post-imadiation $(\omega 16,000 \mathrm{MWD} / \mathrm{T})$

Fig. 11. $\left(\mathrm{U}_{0.8} \mathrm{Pu}_{0.2}\right) \mathrm{C}_{0.95}$ Microstructure 
Bend tests of samples of niobium from Specimens $61 \mathrm{~T}, 61 \mathrm{~B}$, and stainless steel from Specimen $64 \mathrm{~T}$ showed the cladding to be ductile. The stainless steel cladding from Specimen $64 \mathrm{~B}$ was less ductile than that from $64 T$.

The only clad metallography to date has been the examination of the Type 316 stainless steel from Specimen $64 \mathrm{~T}$. No evidence of fuel-clad reactions was found. A typical clad ID surface that was in contact with fuel at a peak $830^{\circ} \mathrm{C}\left(1530^{\circ} \mathrm{F}\right)$ and average $730^{\circ} \mathrm{C}\left(1350^{\circ} \mathrm{F}\right)$ for about $2000 \mathrm{hr}$ is shown in Fig. 12. The calculated temperatures at the stainless clad ID of Specimen $64 \mathrm{~B}$ were a peak of $895^{\circ} \mathrm{C}\left(1640^{\circ} \mathrm{F}\right)$ and an average of $765^{\circ} \mathrm{C}\left(1410^{\circ} \mathrm{F}\right)$.

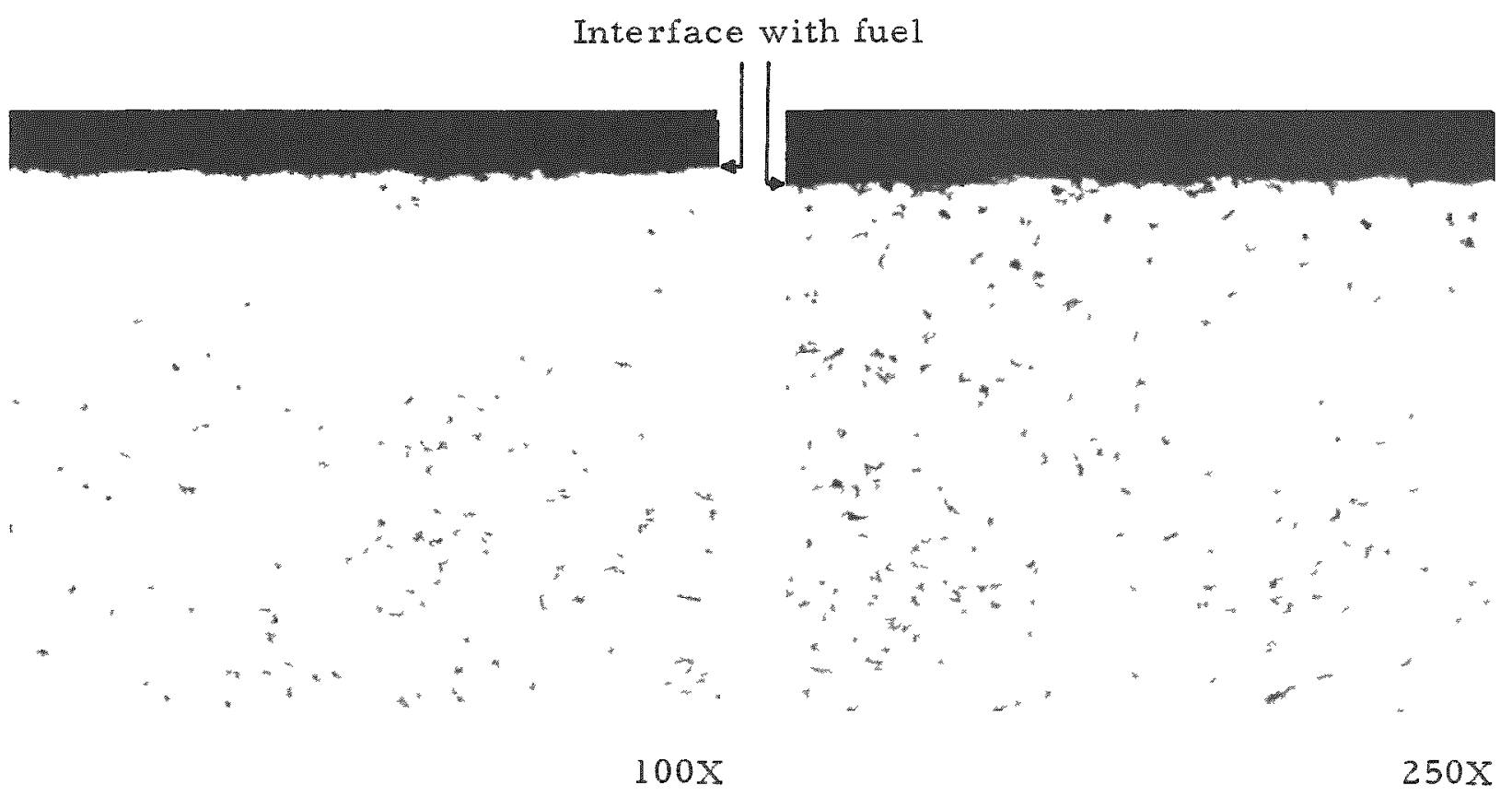

Fig. 12. Type 316 Stainless Cladding from Specimen $64 \mathrm{~T}$ Operated in Contact with $\left(\mathrm{U}_{0.8} \mathrm{Pu}_{0.2}\right) \mathrm{C}_{0.95}$ for $\sim 2000 \mathrm{hr}$ at $730^{\circ} \mathrm{C}\left(\sim 1350^{\circ} \mathrm{F}\right)$ to $840^{\circ} \mathrm{C}\left(\sim 1550^{\circ} \mathrm{F}\right)$

The Harwell $(9)$ irradiation of arc cast $\left(\mathrm{U}_{0.9} \mathrm{Pu}_{0.1}\right) \mathrm{C}$ to burnups similar to those of Capsules 61 and 64 at slightly lower temperatures indicated similar low fission gas release, small dimensional changes, no significant microstructural changes in the fuel, and compatibility with stainless steel cladding.

Our examination is continuing to determine the cause of Specimen $64 \mathrm{~B}$ failure, plutonium distribution, burnup, structure by $\mathrm{X}-\mathrm{ray}$ diffraction and stability of all metallic specimen components by metallography. 
The work reported is the result of the efforts of a hard-working, enthusiastic group of people: specifically, J. Cihi, R. Jaroszeski, W. Sheridan, D. Stahl, and O. Sullivan of the United Nuclear Corporation; K. Taylor and J. Anderson of the Carborundum Corporation; and V. Storhok of Battelle. They are all supported by a capable group of technicians.

\section{$\underline{\text { References }}$}

1. A. Strasser, W. Sheridan, K. Taylor, and J. Anderson, Carbide Fuel Development, Phase III Report, NDA 2162-5 (Sept. 1961).

2. A. Strasser, K. Taylor, and J. Anderson, Carbide Fuel Development, Phase IV Report, UNC-5055 (March 1963).

3. A. Strasser, D. Stahl, K. Taylor, and J. Anderson, Carbide Euel Development Progress Report, UNC-5056 (May 1963).

4. A. Strasser, D. Stahl, K. Taylor, and J. Anderson, Carbide Fuel Development Progress Report, UNC-5068 (Sept. 1963).

5. A. Ogard, C. Land, and J. Leary, The Thermal Expansion of PuC and PuC-UC Solid Solution, LA-2768 (1962).

6. L. Russell, The Structure and Properties of U-C and (UPu)-C Alloy, AERE-R4330 (May 1963). (Presented at the IAEA Conference on New Nuclear Materials Technology, Prague, July, 1963).

7. Quarterly Status Report on Solid Plutonium Fuels Program, LAMS-2949 (July 1963).

8. B. Frost, P. Mardon, and L. Russell, "Research on the Fabrication, Properties and Irradiation Behavior of Plutonium Fuels for the U.K. Reactor Programme," HW-75007 p. 41, Plutonium as a Power Reactor Fuel, ANS Topical Meeting, September, 1962.

9. B. Bradbury, J. Cole, B. Frost, and J. Lambert, Post Irradiation Examination of Stainless Steel Clad UC, UC-UFe 2 and PuC-UC Fuel Pellets, AERE-R4323 (May 1963). 
Discussion of Paper

Presented by Mr.A.A.Strasser

MR. JENS (Atomic Power Development Associates):

Could you elaborate on the cracks in the pellets? Is it true pellets in the center were the ones that cracked up?

MR. STRASSER:

No, they all cracked. There were cracked pellets in other parts of the specimen, but they did get fairly rough handling, because you open the 30 -mil cladding, with a milling cutter, and you bend the cladding back with manipulators. You can crack these pellets just by handling them with tongs.

MR. JENS:

NaK?

Was the capsule that these were irradiated in filled with sodium or

MR. STRASSER:

It was filled with sodium.

MR. KUMMERER (Karlsruhe):

Have you any idea about the fabrication cost of this carbide type, or maybe a comparison between oxide type and carbide type?

MR. STRASSER:

Absolute numbers are not too meaningful on such a relatively small fabrication effort as we have had. One can only get good absolute numbers after one has made a core loading of such a material. But comparative numbers are meaningful, and $\mathrm{Dr}$. Kazi is giving a paper on this comparing carbide and oxide at the next ANS meeting.

MR. ALLIO (Westinghouse):

You explained your variation in thermal conductivity by postulating an increase in gap conductance. Wouldn't it be more reasonable to predict a decrease in gap conductance because of fission product poisoning and an increase in the conductivity of the fuel because of sintering and densification? 
MR. STRASSER:

This would be certainly one trend, but an overriding trend might be a slight swelling of the fuel. We measured all of the pellets that were still in one piece, and they all increased from a diameter of $191 \mathrm{mils}$ to 193 mils. So this is probably an overriding effect on the gap conductance.

MR. WRIGHT (Westinghouse):

Were any uranium eutectics formed with your clad material? With regard to your gas bonding, our analyses indicated that the carbide does not hold much value over oxide where you use a conductance of even 2,000 in a gas bond. Do you believe that eutectic formation is real or is it real only for sodium-bonded capsules? Secondly, I would like to know your opinion of what happens to the carbon content on high burnup. Do you end up with excess carbon or a carbon deficiency? I have seen this handled both ways.

MR. STRASSER:

Eutectic formation occurs if you have free metal. We do not start off with material which has free metal. We prefer higher carbides. By some fluke you might make free metal during irradiation, and this would be very undesirable.

MR. WRIGHT:

With sodium-bonded capsules as well?

MR. STRASSER:

I think you might make it more easily with sodium bonding, because the sodium is a good carbon transfer agent, and if you reduce the carbon content you are left with free metal.

MR. WRIGHT:

What happens to carbon content as a function of time?

MR. STRASSER:

We don't know. As far as these irradiations are concerned, the microstructure does not seem to change radically. 
MR。BUMP (Argonne):

May I ask what the initial gaps were for both the irradiation samples and the compatibility samples, and also the operating gaps?

MR. STRASSER:

The gap is about one mil to start with. The tolerance on the pellets are $\pm 0.5 \mathrm{mil}$ and on the ID of the tubing also \pm 0.5 mil. We don't know what the operating gap is. I think it changes with irradiation. In one experiment with UC, we varied the theoretical gap by using two claddings of different coefficients of expansion and measuring the center temperature. We had one specimen with a stainless steel cladding and one with niobium cladding, the coefficient of expansion of stainless being greater. We measured the center temperature at the same flux and the same capsule, and found no difference.

MR。 LEIT Z (General Electric):

Irradiations were carried out under rather conservative power densities, it would seem, for a carbide fuel. Do you plan irradiations at higher ratings?

MR. STRASSER:

The maximum power we intend to go to in this irradiation is about 20 kilowatts per foot. The reason we did not go any higher to start with is because we want to find out what happens at lower powers first. 
.

314

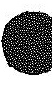




\title{
$\mathrm{PUO}_{2}-\mathrm{SS}$ FUEL IN $\triangle$ FAST REACTOR
}

\author{
A. A. Shoudy, Jr. \\ Atomic Power Development Associates, Inc. \\ Detroit, Michigan
}

Introduction

One of the major objectives in the development of economic fast reactors is to develop a plutorium fuel system that is satisfactory from the standpoint of economics and safety. However, the fuel developed for the initial cores in EBR-II and the Fermi reactor (EFFBR) is based on the use of $U^{235}$ as the fissionable material. Several years ago Argonne National Laboratory began developing the plutonium-uranium-fissium alloy fuel for the EBR-II. They have found that this alloy lacks the radiation stability of the uranium-fissium alloy, (1) and consequently the utilization of plutonium fuel in EBR-II may be delayed for some time. In addition, the re currently is no active program to develop a plutonium fuel for EFFBR. The only other major plutonium fuel program for fast reactors in the $U$. S. is being conducted by General Electric under an AEC contract. This fuel is the mixed plutonium-uranium oxide fuel clad in stainless steel. Although the mixed oxide fuel is attractive from an economic point of view, $(2-4)$ the safety characteristics of the oxide fuel have been questioned for fast power reactors. (5) It appears doubtful if the oxide could be used in an existing United States fast reactor before considerable test work, which would involve long-term irradiation tests, has been conducted. Since these tests have not been started, the use of plutonium as the fuel in a large fast reactor does not appear imminent.

One fuel system that has not been considered in any great detail in the United States for use in fast reactors is $\mathrm{PuO}_{2}$ dispersed in stainless steel. It does offer the possibility for the early use of plutonium in a fast reactor such as EFFBR. The British had for some time considered it as the reference fuel for their fast reactor prototype plant. Their selection of the cermet was based on the experience in the United States with the cermet fuel system consisting of $\mathrm{UO}_{2}$ particles dispersed in a matrix of stainless steel.

To obtain a positive breeding gain and high thermal efficiency, the British selected a fuel composition and operating temperature outside the range of predictable good performance. As a result, their initial ir radiation results show excessive swelling. However, by reducing the operating temperature and volume loading of oxide, improved radiation stability is possible. This prediction is based on a large amount of radiation-stability data available from many United States reactor programs. These data were obtained with uranium oxide; however, there is no reason to believe that it does not also apply to $\mathrm{PuO}_{2}$ dispersed $1 n$ stainless steel. These data have been 
compiled and evaluated at several fuel-development laboratories. $(6,7)$ A summary of the burnup potential is shown in Fig. 1. Along with this radiation test work, there has also been considerable fabrication development of processes for making both fuel plates and pins in the past, and HAPO is presently working on $\mathrm{PuO}_{2}$ cermets for the Phoenix fuels. (8)

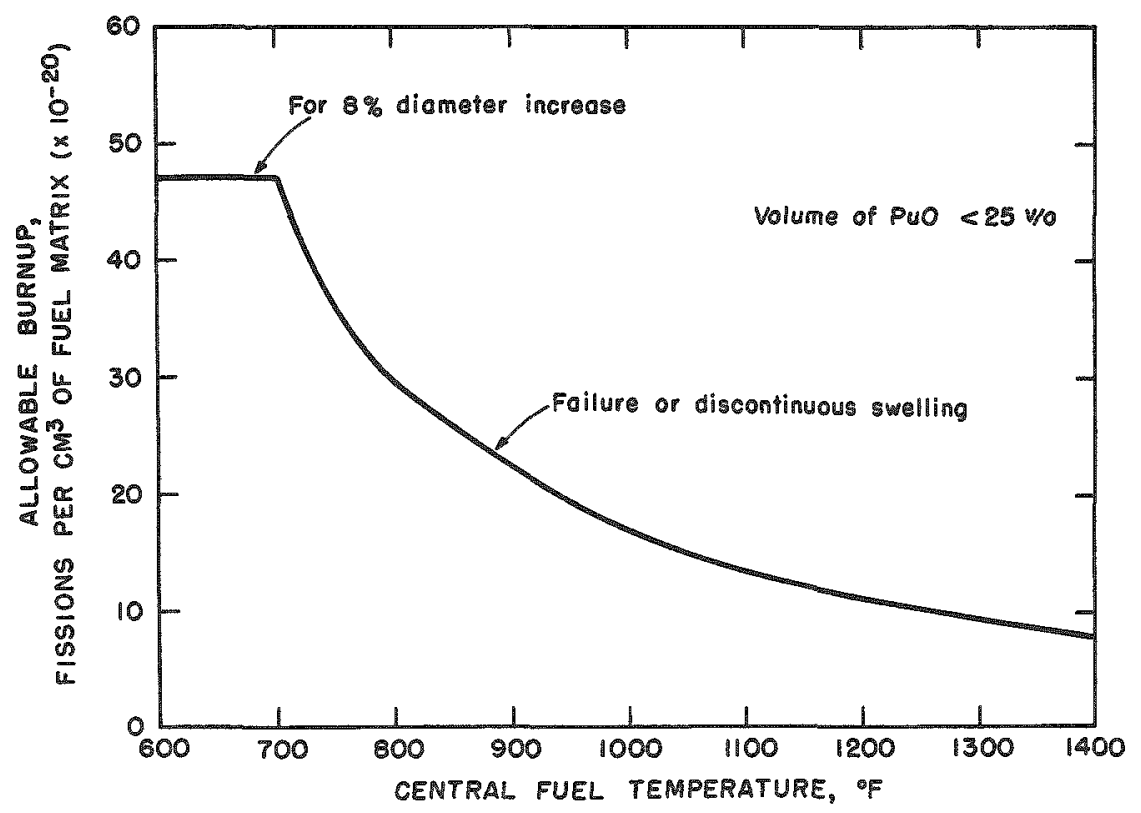

Fig. 1. Allowable Burnup of $\mathrm{PuO}_{2}$ Dispersed in Stainless Steel

The fabrication experience and high-burnup capability of the cermet were the prime factors leading to consideration of this fuel as in interim core loading for the Fermi reactor, with $U^{235}$ as the fissionable material. A core design with the cermet in the form of plates is now essentially complete; only out-of-pile testing is still to be completed. The purposes of this cermet core are to reduce fuel-cycle costs, to provide a core that could be used to test the plant at full power, and to provide the environment to irradiate promising breeder fuel elements. Since the core was needed within a short period of time, $U^{235}$ was selected as the fissile material to enable commercial fabrication of the core.

In 1960, APDA briefly evaluated $\mathrm{UO}_{2}$-stainless steel and $\mathrm{PuC}-\mathrm{U}$ $15 \mathrm{w} / \mathrm{O}$ Mo cermets $(4)$ and found them to be quite attractive. In particular, the PuC in the fertile metal matrix appeared comparable, economically, to a $\mathrm{PuO}_{2}-\mathrm{UO}_{2}$ fuel system evaluated in the same study. In 1962, APDA also made a preliminary evaluation of $\mathrm{PuO}_{2}$ in stainless steel. At that time, the plate-type design as it existed for the Fermi reactor was considered with the $\mathrm{UO}_{2}$ being replaced with $\mathrm{PuO}_{2}$. An evaluation of such a substitution from the standpoint of fabricability and radiation stability was made, and it appeared that the $\mathrm{UO}_{2}$ technology would be applicable. The 
fuel-cycle costs indicated that the $\mathrm{PuO}_{2}$-stainless steel cermet fuel was sufficiently attractive when compared with a $\mathrm{PuO}_{2}-\mathrm{UO}_{2}$ fuel design to warrant further consideration. The prime advantages of the $\mathrm{PuO}_{2}$-stainless steel cermet fuel appeared to be:

1. good radiation stability;

2. utilization of plutonium in a big fast power reactor at the earliest possible date. Only a relatively small research and development effort would be required, since the substitution of $\mathrm{PuO}_{2}$ for $\mathrm{UO}_{2}$ appears to involve no feasibility problems;

3. development of the $\mathbf{P u O}_{2}$-stainless steel cermet would be a direct step toward the development of the attractive $\mathrm{PuO}_{2}$-fertile metal matrix cermet. The low breeding ratio of the stainless steel cermet would be markedly improved with a fertile metal matrix.

Two major areas needed additional study before development of the plutonium cermet fuel would be justificd: economics and safety. The purpose of the study carried out by APDA was to determine the relative fuelcycle costs of the $\mathrm{PuO}_{2}$-stainless steel cermet as compared with the mixed oxide fuels and to evaluate the safety of the cermet core with regard to the Doppler temperature coefficient of reactivity. To make a more valid comparison, a pin design was used for the cermet as well as the mixed oxide. Three reactor sizes (to yield 100,300, and $500 \mathrm{MWe}$ ) were used in this study. A summary of the principal parameters for these three reactors is given in Table 1 .

Table 1

REACTOR PARAMETERS USED IN STUDY

\begin{tabular}{l|c|c|c}
\hline & EFFBR & PFFBR & FOR $(2) *$ \\
\hline Power (MWe), nominal & 100 & 300 & 500 \\
Power (MWt), nominal & 300 & 800 & 1395 \\
Tin $^{\circ} \mathrm{F}$ ) & 550 & 550 & 550 \\
Tout $^{\circ} \mathrm{F}$ ) & 800 & 985 & 815 \\
Total Flow (106 lb/hr) $_{\text {Core Pressure Drop (psi) }}$ & 85 & 25 & 60 \\
Subassembly Shape & Square & 100 & 70 \\
Dimensions (Inside Can)(in.) & 2.464 & 3.485 & 4.08 \\
Number of Subassemblies & 130 & 200 & 320 \\
\hline
\end{tabular}

*It is pointed out that certain parameters of the FOR used in this study do not duplicate those used by $G E$. One case in point is that of the sodium temperatures used. 
For each reactor size, a cermet and mixed oxide fuel design was developed. The details of these designs are shown in Table 2.

In general, the cermet designs were optimized for optimum burnup, and the oxide designs were based on a fuel thermal loading $\int k d \theta$ of $5000 \mathrm{Btu} / \mathrm{hr}-\mathrm{ft}$.

The critical mass, breeding ratios, and the important temperature coefficients of reactivity are summarized in Table 3.

In examining the results, it can be seen that the critical masses for the cermetfuel are generally about the same as the oxide fuel for core designs of similar power except for the large reactor. This is probably because the cermet cores do not contain $\mathrm{U}^{238}$, a fairly strong neutron absorber, whereas the oxide cores do.

Since reactors of smaller size require that the available fuel volume be used entirely to meet reactivity requirements, it was arbitrarily decided in the study to use only plutonium and no $\mathrm{U}^{238}$ in all the cermet reactors studied. However, in the two larger cermet cores, $U^{238}$ could have been included without affecting burnup. If this were done, the breeding ratio and economics would be somewhat improved. In the $100 \mathrm{MWe}$ size, the cermet core has a total breeding ratio slightly greater than 1.0 . Since the bulk of the breeding is done in the blanket, as core size is increased and neutron leakage to the blanket reduced, the total breeding ratio falls off to considerably less than 1.0. The addition of $\mathrm{U}^{238}$ to the core in the larger sizes would tend to keep the breeding ratio higher. The improvement in the breeding ratio would probably be of the order of 0.1 or 0.2 .

Although there are numerous other reactivity effects due to temperature changes, the coefficients evaluated are usually the largest in magnitude and the most important. As expected, the Doppler coefficients of the cermet cores are small and positive. The positive Doppler components of the $\mathrm{Pu}^{239}$ and $\mathrm{Pu}^{241}$ are significantly compensated by the negative Doppler component of the $\mathrm{Pu}^{240}$. Even without the $\mathrm{Pu}^{240}$ contribution, the Doppler coefficient is no greater than $1.1 \times 10^{-6}$. Therefore the small positive Doppler coefficients should not constitute a safety problem, even if the $\mathrm{Pu}^{240}$ component were overestimated. Furthermore, the addition of small amounts of $\mathrm{U}^{238}$ could even eliminate the positive Doppler coefficient if this were necessary. The axial fuel-expansion coefficients for all three cermet cores are negative and considerably larger than the Doppler coefficients. In most transients, feedback due to fuel expansion and Doppler broadening would be indistinguishable as far as time response is concerned, and the negative fuel-expansion effect would completely overshadow the small 
Table 2

SUMMARY OF CORE ANALYSIS

\begin{tabular}{|c|c|c|c|c|c|c|c|c|c|c|c|c|c|c|}
\hline $\begin{array}{l}\text { Fuel } \\
\text { System }\end{array}$ & Reactor & $\begin{array}{l}\text { Core } \\
\text { Power } \\
\text { (MWt) }\end{array}$ & Fuel & $\begin{array}{c}\text { No. of } \\
\text { Subs \& Shape }\end{array}$ & $\begin{array}{l}\text { Flow Rate } \\
\text { (lb/hr/sub) }\end{array}$ & $\begin{array}{l}\text { Core } \\
\text { Pressure } \\
\text { Drop \& } \\
\text { Support } \\
\text { Structure }\end{array}$ & $\begin{array}{l}\text { Fuel } \\
\text { Height } \\
\text { (in.) }\end{array}$ & $\begin{array}{l}\text { No. of } \\
\text { Pins/Sub. } \\
\text { \& Inerts }\end{array}$ & $\begin{array}{l}\text { Fuel } \\
\text { Pin } \\
\text { OD (in.) } \\
\text { Cladding }\end{array}$ & $\begin{array}{c}\text { Pellet } \\
\text { op lin.) } \\
\text { Cold } \\
\text { Clearance }\end{array}$ & $\begin{array}{l}\text { Coolant } \\
\text { Inlet } \\
\text { Temp } \\
\text { pop }\end{array}$ & $\begin{array}{l}\text { Power } \\
\text { Ratios }\end{array}$ & $\begin{array}{l}\text { Fuel Temp (OF) } \\
\text { Specific } \\
\text { Power }\end{array}$ & $\begin{array}{c}\text { Average } \\
\text { Thruput Burnup }\end{array}$ \\
\hline Cermet & EFFBR & $\begin{array}{l}270 \\
(300)\end{array}$ & $\begin{array}{l}26.4 \text { vio } \\
0.85 \text { Dense } \\
\text { PuO } 0_{2} \text { in } \\
0.93 \text { Dense } \\
\text { Matrix }\end{array}$ & $\begin{array}{l}130 \mathrm{Sq} . \\
2.646-\mathrm{in} . \mathrm{OD} \\
0.107-\mathrm{in} . \text { Wall }\end{array}$ & 110,000 & $\begin{array}{l}85 \\
\text { Obl Grid }\end{array}$ & 48 & $\begin{array}{r}117 \\
4\end{array}$ & $\begin{array}{l}0.192 \\
0.007\end{array}$ & - & $\begin{array}{l}550 \\
250\end{array}$ & $\begin{array}{l}\text { Ax. } 1.33 \\
\text { Rad } 1.5 \\
\text { H.C. } 1.25\end{array}$ & $\begin{array}{l}4.4 \mathrm{~kW} f \mathrm{ft} \text { avg } \\
11.1 \mathrm{~kW} \text { ft max }\end{array}$ & $9.4 \times 10^{20} 0_{f / c c}$ \\
\hline $\mathrm{PuO}_{2}-\mathrm{HO}_{2}$ & : & " & Pu $\left\{\begin{array}{l}70 \% 239 \\
25 \% 240 \\
5 \% 241\end{array}\right.$ & $"$ & " & 3 & $\begin{array}{l}39.4 \\
60-\text { in. Tube }\end{array}$ & $\begin{array}{r}140 \\
4\end{array}$ & $\begin{array}{l}0.176 \\
0.010\end{array}$ & $\begin{array}{l}0.153 \\
0.003 \\
93 \% \text { Dense }\end{array}$ & " & $\begin{array}{l}\text { "1 } \\
\text { "1 } \\
\text { P.0. } 1.5\end{array}$ & $\begin{array}{l}\text { Below mett max } \\
4.52 \mathrm{~kW} \text { ft avg } \\
16.95 \mathrm{max}\end{array}$ & $100,000 \frac{M W D}{\text { Ton }}$ \\
\hline Cermet & PFFBR & $\begin{array}{l}720 \\
(800)\end{array}$ & $\begin{array}{l}\text { 14.1 v/o } \\
0.85 \text { Dense } \\
\mathrm{PuO}_{2} \text { in } \\
0.93 \text { Dense } \\
\text { SS Matrix }\end{array}$ & $\begin{array}{l}200 \text { Sq. } \\
\text { 3.675-in. OD } \\
\text { 0.095-in. Wall" }\end{array}$ & 110,000 & $\begin{array}{l}100 \\
\text { Dbl Grid }\end{array}$ & 60 & $\begin{array}{r}221 \\
4\end{array}$ & $\begin{array}{l}0.190 \\
0.007\end{array}$ & - & $\begin{array}{l}550 \\
435\end{array}$ & $\begin{array}{l}\text { " } \\
\text { " }\end{array}$ & $\begin{array}{l}1174 \text { avg max } \\
3.26 \mathrm{~kW} f \mathrm{ft} \text { avg } \\
8.15 \mathrm{~kW} \text { ft max }\end{array}$ & $8.8 \times 10^{20} \mathrm{f} / \mathrm{CC}$ \\
\hline $\mathrm{PuO}_{2}-\mathrm{UO}_{2}$ & " & " & $\begin{array}{l}\mathrm{Pu}, 70 \% 239 \\
25 \% 240 \\
5 \% 241\end{array}$ & " & " & $"$ & $\begin{array}{l}39.4 \\
60 \text {-in. Tube }\end{array}$ & $\begin{array}{r}221 \\
4\end{array}$ & $\begin{array}{l}0.198 \\
0.010\end{array}$ & $\begin{array}{l}0.175 \\
0.003 \\
93 \% \text { Dense }\end{array}$ & " & $\begin{array}{c}" \\
\text { P. } 0.1 .5\end{array}$ & $\begin{array}{l}\text { Below melt max } \\
4.96 \mathrm{~kW} \text { ft avg } \\
18.6 \mathrm{~kW} \text { if max }\end{array}$ & $100,000 \frac{M W D}{\text { Ton }}$ \\
\hline Cermet & FOR & $\begin{array}{r}1200 \\
(1395)\end{array}$ & $\begin{array}{l}15.0 \text { vo } \\
0.85 \text { Dense } \mathrm{PuO}_{2} \\
\text { in } 0.93 \text { Dense } \\
\text { SS Matrix }\end{array}$ & $\begin{array}{l}320 \\
\text { Hex } \\
4.16 \text {-in. Flats oD } \\
0.040 \text {-in. Wall }\end{array}$ & 175,200 & $\begin{array}{l}70 \\
\text { Dbl Grid }\end{array}$ & 69 & $\begin{array}{r}265 \\
6\end{array}$ & $\begin{array}{l}0.200 \\
0.007\end{array}$ & - & $\begin{array}{l}550 \\
265\end{array}$ & $\begin{array}{l}\text { Ax. } 1.33 \\
\text { Rad } 1.5 \\
\text { H.C. } 1.25\end{array}$ & 928 & $15.8 \times 10^{20} 9 / \mathrm{cc}$ \\
\hline $\mathrm{PuO}_{2}-\mathrm{UO}_{2}$ & : & : & 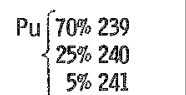 & " & " & $\begin{array}{l}70 \\
\text { Spiral }\end{array}$ & $\begin{array}{l}39 \\
69-\text { in. Tube }\end{array}$ & 127 & $\begin{array}{l}0.250 \\
0.015\end{array}$ & $\begin{array}{l}0.220 \\
0 \\
93 \% \text { Dense }\end{array}$ & $"$ & $\begin{array}{l}\text { Ax. } 1.2 \\
\text { Rad } 1.7 \\
\text { H.C. } 1.5\end{array}$ & $\begin{array}{l}\text { Below melt max } \\
13.2 \mathrm{~kW} \text { fi avg } \\
39.6 \mathrm{~kW} \text { ft max }\end{array}$ & $100,000 \frac{M W D}{\text { Ton }}$ \\
\hline
\end{tabular}

"Internal Wrapper Tube Support Structure reduces wall thickness 
positive Doppler effect. Therefore, no stability problems should arise because of the positive Doppler coefficient. The Doppler coefficient in a $\mathrm{U}^{235}-$ fueled cermet similar to the Fermi-size plutonium cermet was calculated to be $+0.3 \times 10^{-6} / \mathrm{C}$. This compares with a value of $+0.7 \times 10^{-6} / \mathrm{C}$ for $\mathrm{Pu}{ }^{239}$. (The Doppler coefficient given in Table 3 is composed of $0.7 \times 10^{-6}$ and $-0.3 \times 10^{-6}$ for $\mathrm{Pu}^{239}$ and $\mathrm{Pu}^{240}$, respectively.) Thus it appears that the $\mathrm{Pu}^{239}$ Doppler effect for this particular composition and spectrum is larger by about a factor of 2 than that for $U^{235}$. Although there are several differences involved, the larger value of $\nu$ and wider spacing of the resonances of $\mathrm{Pu}^{239}$ are probably responsible for the larger Doppler effect of Pu ${ }^{239}$.

Table 3

SUMMARY OF PHYSICS RESULTS

\begin{tabular}{|c|c|c|c|c|c|c|}
\hline & \multicolumn{2}{|c|}{ EFFBR } & \multicolumn{2}{|c|}{ PFFBR } & \multicolumn{2}{|c|}{ FOR } \\
\hline & Cermet & Oxide & Cermet & Oxide & Cermet & Oxide \\
\hline $\begin{array}{l}\text { Equilibrium Critical Mass } \\
\text { (kg Pu) }\end{array}$ & 553 & 552 & 1022 & 1050 & 1973 & 1410 \\
\hline \multicolumn{7}{|l|}{ Breeding Ratio } \\
\hline Core & 0.09 & 0.47 & 0.09 & 0.73 & 0.12 & 0.73 \\
\hline Radial Blanket & 0.92 & 0.68 & 0.55 & 0.36 & 0.37 & 0.26 \\
\hline Axial Blanket & 0.09 & - & - & - & - & 0.23 \\
\hline Total & 1.10 & 1.15 & 0.64 & 1.09 & 0.49 & 1.22 \\
\hline \multicolumn{7}{|l|}{$\begin{array}{l}\text { Reactivity Coefficient } \\
\left(\Delta \mathrm{k} / \mathrm{k} \text { per } C \times 10^{-6}\right)\end{array}$} \\
\hline Doppler & +0.43 & -0.7 & +0.30 & - & - & -3.8 \\
\hline Sodium Expansion & -7.7 & -7.7 & -8.1 & -7.0 & -4.0 & +4.0 \\
\hline Fuel or Clad Axial Expansion & -7.8 & -9.5 & -8.7 & -8.4 & -9.6 & -7.9 \\
\hline Core Radial Expansion & -13.4 & -17.0 & -15.0 & -16.8 & -18.9 & -17.4 \\
\hline
\end{tabular}

The sodium temperature coefficients of reactivity for all cermet cores calculated are negative. This coefficient has a tendency to become less negative in the larger cores as is evident from comparing the $300-$ and 500-MWe cases. Surprisingly, this is not the case in going from the 100- to the 300-MWe size, where little difference occurs. The sodium danger coefficient decreases smoothly in absolute magnitude and the mass of sodium increases almost linearly with core volume. The sodium temperature coefficient, which is proportional to the product of the se two quantities, is not monotonic, but apparently peaks in the intermediate volume range. The 100 - and 300-MWe cores apparently lie on either side of this peak. 
The radial core-expansion temperature coefficient of reactivity is based on the assumption of a tight core design. As the core subassembly cans are heated, the core expands according to the thermal-expansion properties of the can material, which is stainless steel. The calculated coefficients are similar in magnitude and sign to those calculated for Fermi Cores $A$ and $B$.

The oxide-fueled cores contain substantial amounts of $U^{238}$. The fertile-to-fissile atom ratios range from about 3 in the 100-MWe design up to almost 6 in the large oxide core. Therefore these cores have significant internal breeding ratios and negative Doppler coefficients. The overall breeding ratio increases with size because of the increasing core $\mathrm{U}^{238}$ concentration. The two smaller cores would have higher breeding ratios than the large core if axial blankets had been included. It is estimated this would add 0.1 to 0.2 to the breeding gain for these cases.

The Doppler temperature coefficient of reactivity increases in magnitude from -0.7 to $-3.8 \times 10^{-6} / \mathrm{cc}$ from the small to the large cores. These coefficients are not substantially different from the Doppler coefficients estimated for metal cores, such as Fermi Core A, despite the softer spectrum and higher ratios of fertile to fissile atoms. This is primarily because of the compensating effect of higher fuel temperatures in the oxide core.

The clad-expansion coefficient is based on the assumption that the oxide attaches itself to the clad material. As the clad heats and expands, fuel is displaced uniformly outward, thus giving a negative reactivity effect. The amount of fuel displacement is determined by the thermal-expansion properties of the clad material and the temperature rise in the clad. The coefficient, therefore, is smaller than that of the direct fuel expansion and is delayed. The calculated coefficient does not include any effect due to the thermal expansion of the oxide material itself, which probably will occur during initial startup. Because of the dufferential expansion of the clad and oxide materials, it is expected that the oxide elements will develop horizontal cracks or gaps along the length of the element after operation and. shutdown. Once these gaps axe developed, the expansion effects of the oxide probably will follow the assumption made in this study. If the oxide should expand to cause a positive temperature coefficient due to the crack formation, a serious problem could develop. Under these conditions, the expansion coefficient conceivably could be large, positive, and prompt. There is an additional problem if the gaps do exist. Collapse of the oxide to fill the gaps as a result of vibration, or a minor transient, could cause a substantial reactivity increase. Therefore, the temperature-coefficient calculations have ignored one of the very important and serious aspects of the oxide element. This problem can probably be reduced substantially by design. How ever, the design requirements for prevention of oxide movement would undoubtedly increase complexity of manufacture and, therefore, fabrication cost. Also, should a reduction of oxide loading be required, the fuel-cycle costs would increase and core life would be reduced. 
The sodium coefficient in the small oxide core is a fairly large negative coefficient, but becomes more positive in the large cores because of the lesser importance of core neutron leakage and the larger $\mathrm{U}^{238} \mathrm{con-}$ centrations. In the 500 MWe core, the coefficient is calculated to be $+4 \times 10^{-6} / \mathrm{C}$. Because the spectral and leakage components are compensating, this coefficient is quite sensitive and subject to large uncertainty.

The results of the core fuel-cycle cost study comparing the two fuel systems are shown in Table 4.

Table 4

RESULTS OF CORE FUEL-CYCLE COST ANALYSIS

\begin{tabular}{|c|c|c|c|c|c|c|c|}
\hline \multirow{4}{*}{ Item } & \multicolumn{7}{|c|}{ Mills/kW-hr } \\
\hline & \multicolumn{2}{|c|}{ EFFBR } & \multicolumn{2}{|c|}{ PFFBR } & \multicolumn{3}{|c|}{ FOR } \\
\hline & \multirow[b]{2}{*}{ Cermet } & \multirow[b]{2}{*}{ Oxide } & \multirow[b]{2}{*}{ Cermet } & \multirow[b]{2}{*}{ Oxide } & \multirow[b]{2}{*}{ Cermet } & \multicolumn{2}{|c|}{ Oxide } \\
\hline & & & & & & $\mathrm{A}$ & $\mathrm{B}$ \\
\hline Fabrication & 0.52 & 0.38 & 0.51 & 0.33 & 0.32 & 0.23 & 0.36 \\
\hline Reprocessing & 0.70 & 0.44 & 0.36 & 0.23 & 0.21 & 0.18 & 0.29 \\
\hline $\begin{array}{l}\text { Plutonium } \\
\text { Depletion }\end{array}$ & 0.91 & 0.44 & 0.93 & 0.24 & 0.91 & 0.24 & 0.24 \\
\hline Use Charge & 0.77 & 0.63 & 0.52 & 0.43 & 0.47 & 0.33 & 0.55 \\
\hline Total & 2.90 & 1.89 & 2.32 & 1.23 & 1.91 & 0.98 & 1.44 \\
\hline
\end{tabular}

The major factors affecting fuel-cycle economics are the fabrication and reprocessing of fuel, fissionable material depletion charge, and use charge. Since one of the prime objectives of this study was to estimate the relative economics of the cermet and the mixed oxide fuel for long-range fast reactor applications, the same unit prices and ground rules used in the Fast Oxide Reactor Program $(2,9)$ were used. The basis for the fuel-cycle costs in this study together with the unit costs and values of the important economics parameters and the methods used to arrive at these values relative to those used in the FOR study are discussed in detail in APDA-154.

To limit the scope of the study to fast reactor cores, it was assumed that the net blanket fuel costs were zero in all cases. Basically, this assumption infers that the fabrication, reprocessing, inventory, and handling costs are exactly offset by the sale of plutonium recovered from the blanket. If it should be possible to add revenue in the blanket cycle, the relative fuel-cycle costs are probably still valid. Since the external 
breeding ratio is not too different for each fuel system, and since the blanket designs could be very similar, the effect of obtaining a net revenue from the blanket fuel-cycle cost would be to lower the overall fuel-cycle cost about the same amount for each system. Although ignored in this study, at some point blanket fuel-cycle costs must be considered to optimize both systems. At present (1963) blanket fabrication costs, it is doubtful whether the recovery of blanket plutonium at $\$ 6.50 / \mathrm{g}$ would be economically justified. The optimization of blanket cycles is complex and constitutes an important area that warrants development when the price of plutonium is low. The economic recovery of blanket plutonium is fundamental to the development of the fast breeder reactor and should be studied separately.

As can be seen in Table 4, the oxide cores show a slight cost advantage in each case studied. This cost advantage basically reflects two major factors. The first, and the most significant in the case of the 100-MWe design, is the difference in allowable burnup used for each fuel system. The lower burnup used for the cermet material at the coolant temperatures used in this study results in increased fabrication, reprocessing, and plutoniuminventory charges. In the case of the 500-MWe design, this was partially overcome by the ability to design the cermet fuel to operate at lower temperatures and thereby achieve high burnups. Under these conditions, the fabrication, reprocessing, and plutonium-inventory costs of the cermet are nearly comparable with those of the oxide. To further evaluate the effect of burnup, cost studies were made where the burnup of the oxide cores was $50,000 \mathrm{MWD} / \mathrm{T}$, case $\mathrm{B}$ of Table 4. Under these conditions, the fuel-cycle costs of both systems are very nearly the same and certainly within limits of accuracies of a long-range cost study of this type. As discussed in APDA-154, under certain conditions $\mathrm{UO}_{2}$ has been found to swell extensively beyond $50,000 \mathrm{MWD} / \mathrm{T}$. This was the basis of case-B burnup.

The second most important factor favoring the oxide fuel-cycle cost is the core breeding ratio. This is especially noticeable for the 300 - and 500-MWe designs. It is believed that this could be partially offset by the introduction of uranium, in the form of $\mathrm{UO}_{2}$, in the cermet fuel Due to the low-volume per cent loading of $\mathrm{PuO}_{2}$ in the 300- and 500-MWe cermet designs, $\mathrm{UO}_{2}$ could be introduced without reducing the allowable burnup. This could reduce the fuel-cycle costs of the large cermet cores about a $0.1 \mathrm{mill} / \mathrm{kW}-\mathrm{hr}$.

\section{Summary and Conclusions}

1. The Doppler coefficient for the cermet cores is small and positive. It is slightly less than $+\frac{1}{2} \times 10^{-6} / \mathrm{C}$ for an assumed equilibrium isotopic plutonium composition for reactors that yield 100 and $300 \mathrm{MWe}$, and is easily overriden by the axial fuel-expansion coefficient, which is about $-8 \times 10^{-6} / \mathrm{C}$ in this reactor-size range. In the $100-\mathrm{MWe}$ reactor size, the Doppler coefficient of $U^{235}$ is $+0.3 \times 10^{-6} / \mathrm{C}$, as compared with $+0.7 \times 10^{-6} / \mathrm{C}$ for $\mathrm{Pu}^{239}$. 
2. In reactors of smaller size, the mixed oxide Doppler coefficient is negative but small. If any undesirable reactivity effects occur due to longitudinal movement of the oxide, they probably would not be overridden by the negative Doppler coefficient.

3. In the 500-MWe reactor, the Doppler temperature coefficient of reactivity is negative and about one-half of the value of the temperature coefficient of reactivity due to clad axial expansion of fuel for isothermal changes. As power coefficients, the Doppler effect is the larger of the two. It is possible in this case that undesirable longitudinal fuel movements could be overridden by the Doppler effect, but this will require considerable analysis and experimentation. For this large reactor, however, the sodium reactivity coefficient is positive. The undesirable effects of this positive coefficient were not evaluated in this study. In the 500-MWe cermet core, the sodium coefficient has about the same magnitude as in the mixed oxide core but is negative. Therefore, it does not constitute a problem in this fuel system.

4. If long-range projected costs of fabrication and plutonium price are used, the core fuel-cycle costs for the plutonium cermet fuel system in fast reactors from 100 to 500 MWe range from 3 to $2 \mathrm{mills} / \mathrm{kW}-\mathrm{hr}$.

5. In each reactor size, the oxide designs show slightly better cconomics, amounting to about $1 \mathrm{mill} / \mathrm{kW}-\mathrm{hr}$. This results from the lack of internal breeding in the cermet core and the assumed allowable burnup for the two systems. It appears that the internal breeding of the cermet core could be increased by the addition of $\mathrm{UO}_{2}$ without adverse effect on the allowable burnup. The uncertainties associated with burnup of the mixed oxides when in the form of long fuel pins, can only be resolved by radiation testing. The uncertainties in design can be resolved by making a detailed design with full recognition of the safety and performance requirements.

In general, uncertainties associated with the assumptions used in the fuel-cycle cost estimates, in the burnup, and in design details to meet the requirements of safety could more than offset the differences in fuel-cycle costs between the two fuel systems.

6. In both fuel systems, the core fuel-cycle costs decrease with incrcasing reactor size. In the case of the oxide fuels, this reflects improved core-breeding ratios and lower plutonium inventory. In the case of the cermets, this results from improved burnup achieved by a more optimized design.

7. Although the cermet appears to suffer a small economic penalty, it has no major safety problems that would not permit its early 
application to a fast reactor. It also would be a logical step in the development of fertile metal matrix cermet, which is one of the ideal fast breeder fuel materials.

\section{$\underline{\text { References }}$}

1. Horak, J. A., Kittel, J. H., and Dunworth, R. J., The Effects of Irradiation on Uranium-Plutonium-Fissium Fuel Alloys, ANL-6429 (July 1962).

2. Horst, K. M., Hutchins, B. A., Leitz, F. J., and Wolfe, B., Core Design Study for a 500 Mwe Fast Oxide Reactor, GEAP-AEC-3721。

3. A Plutonium-fueled. Fast Breeder Atomic Power Plant, APDA-129 (April 1960).

4. Fast Reactor Core Design Parameter Study, APDA-133 (March 1960)。

5. Dickerman, C. E., and Okrent, D., More on Fast-Ceramic Reactor Safety, NUCLEAR NEWS-ANS, pp. 20-21 (March 1963).

6. Keller, D. L., Predicting Burnup of Stainless $-\mathrm{UO}_{2}$ Cermet Fuels, Nucleonics, 19 (June 1961).

7. Haynes, V. O., Neill, F. H, and Schaffer, L. D., Summary of $\mathrm{UO}_{2}$ Stainless Steel Dispersion Element Irradiation Experiments, ORNLCF-58-2-71 (March 18, 1958).

Also, Thurber, W. C., "Private Communication."

8. Green, J. $K$, and Brown, J. E, Unclassified Research and Development Programs Executed for the Division of Reactor Development and the Division of Research - February, 1963, HW-77011 (March 26, 1963).

9. Enrico Fermi Atomic Power Plant, APDA-124 (Jan 1959).

10. Collins, G.D., "Fabrication Cost Estimate for $\mathrm{UO}_{2}$ and $\mathrm{Mixed} \mathrm{PuO}_{2}$ $\mathrm{UO}_{2}$ Fuel, "GEAP-AEC-3824, January 24, 1962.

Discussion of Paper

Presented by Mr. Shoudy

MR. HÄFELE (Karlsruhe):

How do you control your reactor? If you do it by poison, do you take into account the reduction in breeding gain by such sort of control, especially with respect to the very low internal breeding ratio (for you have almost zero internal breeding ratio)? 
MR. SHOUDY:

I will call on John Nims, the head of our physics section.

MR. NIMS (Atomic Power Development Associates):

You are right. There is a large reactivity loss with burnup. You would have to build in the excess reactivity to start with, or you might start out with a smaller core size than your equilibrium core, and as you burn out reactivity, gradually add two or three subassemblies until you build it up to the maximum size. Fifty thousand MWD/tonne, about 5 per cent of your mass, corresponds to about $2 \frac{1}{2}-3$ per cent reactivity. It is not an impossible thing to build into the system.

MR. WOLFE (General Electric):

I wonder if you might consider that positive Doppler coefficient a Little more seriously, that is, whether it might be more serious if you looked at the worst hypothetical type of accident. In the paper I gave yesterday, on one of the slides there was the effect of a positive coefficient on the worst hypothetical accident. Nicholson also calculated this, and it makes a substantial difference in the maximum energy release, more than, say, a factor of about 4 with the magnitude of the positive Doppler you showed there. If you used the same hypothetical accident the Fermi containment might not hold in that case.

MR. NIMS:

We did not look at maximum accident problems of this core. Certainly the Doppler would contribute to this and make it a little bit more severe than if we had a negative Doppler. In this case, I think it was a very small Doppler coefficient. It is within the accuracy of calculation. One doesn't know whether it is a small positive or a small negative number. Before we went to the extent of actually loading one of these things, this would have had to have been gone into in a lot of detail.

MR.SMITH (Dounreay):

Coming back to this Doppler coefficient of +0.43 that you mention, this being the difference between $\mathrm{Pu}^{239}$ and $\mathrm{Pu}^{240}$, did you also consider the possible negative contribution from the iron? Would this have helped you at all?

MR. SHOUPY:

It was not considered. 
MR. deSTORDEUR (EURATOM):

How does the case of the pin-type cermets compare with the platetype cermets which was considered for Fermi core B, at least as far as the total amount of fuel material and total amount of $\mathrm{U}^{238}$ in the core, allowing for a high volume fraction of oxide in your cermet?

MR. SHOUDY:

We can get a slightly greater loading with a plate design. The plate design, however, is more difficult from the standpoint of the rmal stress. Basically, we went to a pin design in an effort to keep the cost analysis on as close a relative basis as we could. By choosing or selecting the fabrication process that we did, we were in relatively good agreement with the oxide as far as projecting costs for fabrication. I think we could not have done this too readily if we tried to project on the basis of a plate-type element and its fabrication costs. But basically, the only reactor where we did have any problem so far as getting enough material into the core would be in the Fermi, or something smaller, so there would be no real desire to go to the plate type design.

MR. CAMPISE (Atomics International):

Would you not expect some advantage from the axial expansion coefficient of your fuel in this particular case to minimize the positive Doppler? MR. NIMS:

That is right. We were not concerned about the safety of this core from, let's say, the credible accident standpoint. There was no question about a net positive prompt coefficient. It did have good axial expansion, more than enough to overcome the Doppler effect.

MR. WRIGHT (Westinghouse):

You mentioned that you could load up the cermet with about enough fertile material to raise your conversion ratio about 0.2 . You did not mention how much that might be worth on your fuel cycle, how much that might diminish the mills per kilowat hour difference between that and the ceramic. Also, I think it is really important to take a look at the thoriumplutonium cermet, you might say odd fuel cycles, because the whole reactor program, particularly in this country, will be balanced between the rmal and fast reactors for a very long time. You do not have to build them all like EBR-II, where they have a complete fuel cycle within themselves. 
MR. SHOUDY:

I do not have a real number for the effect on fuel-cycle cost, but I think it would be equivalent to something like $0.1-0.2 \mathrm{mill}$, or on that order. I certainly agree we should consider some of the odd-ball fuels, if you can call it that. I think most studies we have made on a cermet containing either the carbide or the oxide always look extremely attractive. Perhaps they look so attractive because we don't know how much they are going to cost to make. But from most aspects they are quite superior to the other type of fuels that we look at. But at this stage of development fabrication is a real problem.

MR. GREEBLER (General Electric):

I would like to pursue the Doppler question just one more step if I may. You minimize the influence of the positive Doppler because it is such a small value. Now, when you go to the larger reactor, $300-$ or 500-MW size, the positive Doppler coefficient will no longer be so small. Also, the negative axial expansion coefficient will not be as large as it now is. Will this not be a serious safety question?

MR. SHOUDY:

As regards the axial expansion, we calculated for the small reactor with the cermet, -7.8 , and for the large reactor, -9.6 . We did not calculate the Doppler for the large reactor because we ran out of time and money in this study of six weeks' duration. This work was done by the entire group at APDA. It has been reported in APDA-154. 


\title{
COMPARISON OF THE VALUE OF PLUTONIUM AND URANIUM ISOTOPES IN FAST REACTORS
}

\author{
A. R. Baker and R.W. Ross \\ Atomic Energy Establishment \\ Winfrith, England \\ (Paper presented by Mr.R.D. Smith)
}

Abstract

Upper limits are obtained for the values of $\mathrm{Pu}^{239}$ and higher isotopes by estimating those values which will make fuel costs the same as for enriched uranium fuel. When the value of the excess bred plutonium is neglected, $\mathrm{Pu}^{239}$ is valued at 1.4 times the value for $\mathrm{U}^{235}$ in highly enriched uranium. The excess bred plutonium is taken into account, later, in a study of converter reactors and breeders started up on $\mathrm{U}^{235}$. The value of $\mathrm{Pu}^{239}$ is increased to 1.6 times the value of $U^{235}$. The paper describes a new treatment of breeding based on a new parameter, the breeding gain, which is shown to be more useful than the breeding ratio usually used. There are also some new calculations of the approach to equilibrium composition for cores fueled initially with $\mathrm{Pu}{ }^{239}$ or $99 \%$ $\mathrm{U}^{235}+1 \% \mathrm{U}^{23 \frac{4}{*}}$

1. Genera.I Introduction

In the long-term future when fast reactor power stations may be in widespread use, plutonium will be a marketable commodity with a price determined by the laws of supply and demand. This paper deals with the upper limit which a fast reactor owner could afford to pay in the particular case that the nearest competitor has a similar fast reactor, fueled with enriched uranium instead of plutonium. The value for plutonium is worked out which makes the price of electricity the same for either plutonium or enriched uranium fuel, on the assumption that these fuels would be interchangeable in a fast reactor station without change in capital or operating costs. Plutonium values derived on the basis of this limited approach are of value when considering the economics of a power station network based on a mixture of reactor types.

Section 2 describes the physical principles of the detailed calculations making up the background to the economic studies of Section 3 . The treatment of breeding is based on a new parameter, the breeding gain $G$, which is discussed and justified in Sections 2.2 and 2.3. In Section 2.4, the differential equations governing the concentrations of fuel isotopes 
are solved numerically with the proper restraints for conservation of total fuel concentration and reactivity. The cases studied are the approach to equilibrium for breeder reactors fueled initially with Pu ${ }^{239}$ and with $U^{235}$. The 16-group cross sections of Yiftah, Okrent and Moldauer(1) are used whenever available. Some supplementary data and the one-group cross sections used for the approach-to-equilibrium studies are discussed in Section 2.1. In Section 2.5, some studies on the optimising of blankets are described.

2. Physical Studies

\subsection{Models and Cross Sections}

Calculations have been performed for a cermet-fueled reactor and for carbide-fueled reactors. The main properties of these reactors are given in Table $I$. The carbide reactors chosen for study are those given by Okrent and Yiftah. (2)

Table I

PROPERTIES OF THE REACTORS STUDIED

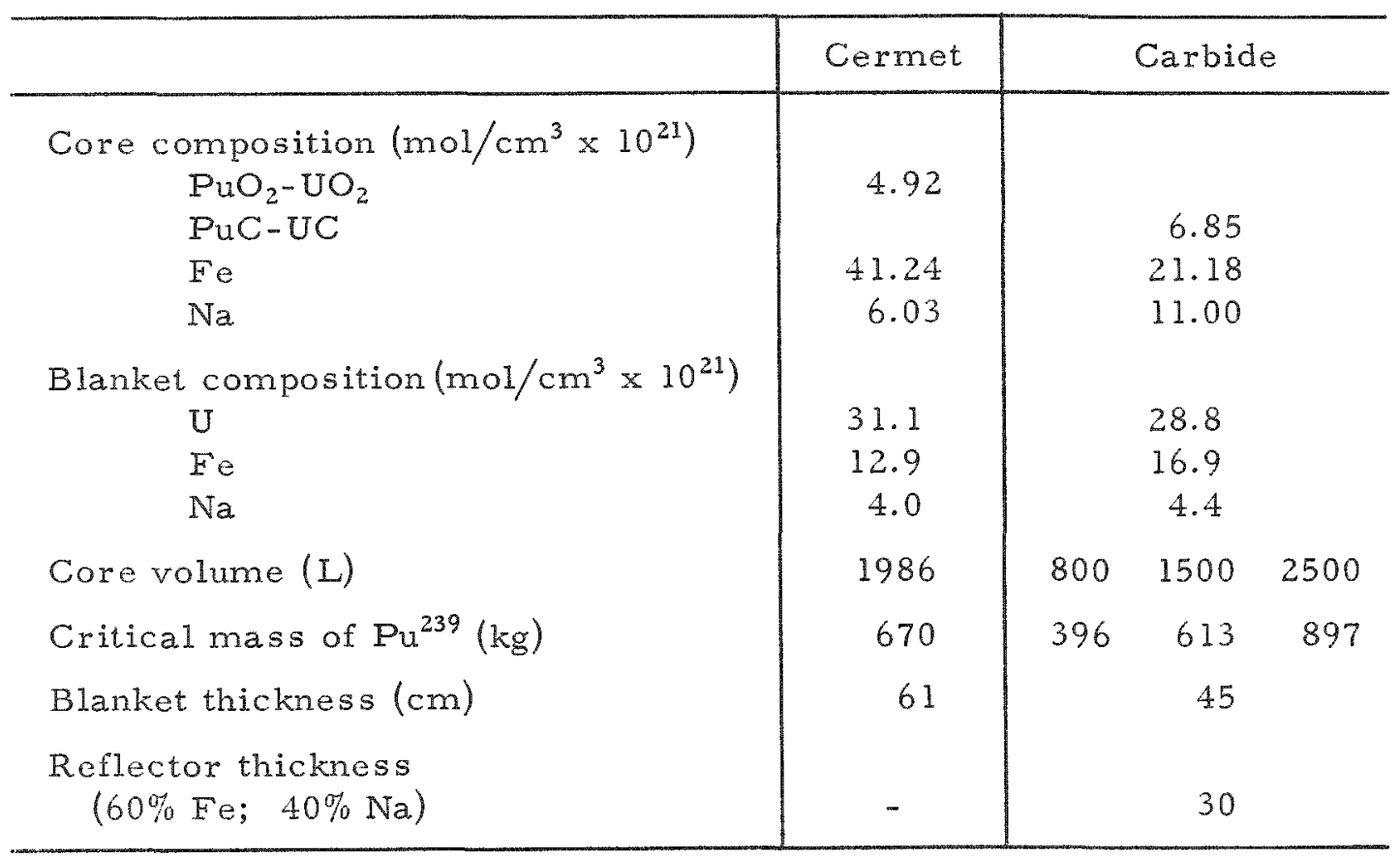

The plutonium in the cermet reactor has composition $81 \% \mathrm{Pu}^{239}$, $19 \% \mathrm{Pu}^{240}$.

The uranium contains $0.4 \% \mathrm{U}^{235}$.

The plutonium in the carbide reactor is $100 \% \mathrm{Pu}^{239}$.

The uranium is $100 \% \mathrm{U}^{238}$. 
The cross-section set of Yiftah, Okrent, and Moldauer(1) is used for all calculations. The values used for $\mathrm{U}^{234}$ and $\mathrm{U}^{236}$ are given in Table II. The values of $\nu$ for $U^{234}$ and $U^{236}$ were estimated by J. S. Story from the systematics of neighbouring nuclides. The values of $\sigma_{f}$ were based on the curves in BNL-325. (3) The values of $\sigma_{c}$ for $U^{236}$ were based on experiments at Aldermaston. (4) A curve of the same general shape as that for $U^{238}$ was drawn through the preliminary experimental data. The values of $\sigma_{C}$ for $U^{234}$ were assumed to be the same as those for $U^{236}$.

Table II

SIXTEEN-GROUP CROSS SECTIONS FOR $\mathrm{U}^{234}$ AND $\mathrm{U}^{236}$ (in b)

\begin{tabular}{|c|c|c|c|c|c|c|}
\hline \multirow{2}{*}{ Group } & \multicolumn{3}{|c|}{$\mathrm{U}^{234}$} & \multicolumn{3}{|c|}{$U^{236}$} \\
\hline & $\nu$ & $\sigma_{f}$ & $\sigma_{c}$ & $\nu$ & $\sigma_{1}$ & $\sigma_{c}$ \\
\hline 1 & 2.99 & 1.57 & 0.027 & 2.93 & 1.5 & 0.027 \\
\hline 2 & 2.76 & 1.483 & 0.057 & 2.85 & 0.916 & 0.057 \\
\hline 3 & 2.61 & 1.460 & 0.138 & 2.73 & 0.788 & 0.138 \\
\hline 4 & 2.53 & 1.196 & 0.333 & 2.63 & 0.470 & 0.333 \\
\hline 5 & 2.47 & 0.863 & 0.307 & 2.60 & 0.080 & 0.307 \\
\hline 6 & 2.44 & 0.236 & 0.208 & & & 0.208 \\
\hline 7 & 2.42 & 0.061 & 0.258 & & & 0.258 \\
\hline 8 & 2.41 & 0.034 & 0.363 & & & 0.363 \\
\hline 9 & 2.40 & 0.022 & 0.164 & & & 0.464 \\
\hline 10 & & & 0.592 & & & 0.592 \\
\hline 11 & & & 0.720 & & & 0.720 \\
\hline 12 & & & 0.832 & & & 0.832 \\
\hline 13 & & & 0.963 & & & 0.963 \\
\hline 14 & & & 1.056 & & & 1.056 \\
\hline 15 & & & 1.488 & & & 1.488 \\
\hline 16 & & & 3.200 & & & 3.200 \\
\hline
\end{tabular}

The effective one-group cross sections used in the fuel-cycle studies are given in Table III. The sixteen-group values were reduced to one group by use of the average core spectrum for $\mathrm{Pu}^{239}$ fuel as a weight. The numbers in Tables II and III are not, of course, accurate to the number of figures quoted, but they are given to so many figures so that calculations of interest can be reproduced.

It is assumed in the approach-to-equilibrium analysis of Section 2.1 that, for a system initially just critical, the reactivity is maintained zero provided that in all subsequent changes 


$$
\sum_{i} N_{i} x_{i}=\text { constant }
$$

where $N_{i}=$ atomic density of heavy element $i$ in the fuel,

$$
x_{i}=\text { value of }\left(\nu \sigma_{f}-\sigma_{f}-\sigma_{c}\right) \text { for element } i
$$

and the sum is taken over all heavy elements. The same assumption was made by Okrent. (5) It can be justified by perturbation theory, provided the total number of heavy nuclei is unaltered by all changes and the scattering properties of all heavy nuclei can be assumed identical. For the approachto-equilibrium calculations of Section 2.4, the first proviso is rigorously true since fission products and capture products heavier than $\mathrm{U}^{236}$ or $\mathrm{Pu}^{242}$ are assumed removed continuously and the core is assumed replenished continuously. It can be seen from the YOM set( 1 ) that the second is approximately true.

Table III

\begin{tabular}{|c|c|c|c|c|c|c|c|c|c|}
\hline & & $\mathrm{U}^{234}$ & $\mathrm{U}^{235}$ & $U^{236}$ & $\mathrm{U}^{238}$ & $\mathrm{Pu}^{239}$ & $P u^{240}$ & $\mathrm{Pu}^{241}$ & $\mathrm{Pu}^{242}$ \\
\hline \multirow{3}{*}{$\begin{array}{l}800 \mathrm{~L} \\
\text { Carbide }\end{array}$} & $\int \nu \sigma_{f}$ & 1.039 & 4.356 & 0.394 & 0.150 & 5.586 & 1.326 & 7.573 & 1.289 \\
\hline & $\sigma_{f}^{2}$ & 0.404 & 1.757 & 0.143 & 0.053 & 1.889 & 0.404 & 2.501 & 0.393 \\
\hline & $\sigma_{c}^{1}$ & 0.409 & 0.423 & 0.409 & 0.240 & 0.423 & 0.418 & 0.282 & 0.418 \\
\hline \multirow{3}{*}{$\begin{array}{l}1500 \mathrm{~L} \\
\text { Carbide }\end{array}$} & $\nu \sigma_{f}$ & 0.958 & 4.530 & 0.356 & 0.134 & 5.661 & 1.218 & 7.858 & 1.186 \\
\hline & $\sigma_{f}$ & 0.373 & 1.832 & 0.130 & 0.047 & 1.918 & 0.371 & 2.599 & 0.362 \\
\hline & $\sigma_{c}^{b}$ & 0.436 & 0.461 & 0.436 & 0.258 & 0.462 & 0.450 & 0.308 & 0.450 \\
\hline \multirow{3}{*}{$\begin{array}{l}2500 \mathrm{~L} \\
\text { Carbide }\end{array}$} & $\nu \sigma_{f}$ & 0.906 & 4.657 & 0.332 & 0.124 & 5.720 & 1.148 & 8.062 & 1.119 \\
\hline & $\sigma_{f}$ & 0.353 & 1.886 & 0.121 & 0.044 & 1.940 & 0.350 & 2.670 & 0.342 \\
\hline & $\sigma_{\mathrm{c}}$ & 0.456 & 0.489 & 0.456 & 0.272 & 0.491 & 0.473 & 0.326 & 0.473 \\
\hline \multirow{3}{*}{ Cermet } & $\nu \sigma_{f}$ & 0.811 & 4.832 & 0.281 & 0.102 & 5.783 & 1.018 & 8.380 & 0.992 \\
\hline & $\sigma_{f}$ & 0.317 & 1.963 & 0.102 & 0.036 & 1.966 & 0.312 & 2.780 & 0.304 \\
\hline & $\sigma_{c}$ & 0.484 & 0.530 & 0.484 & 0.290 & 0.530 & 0.502 & 0.354 & 0.502 \\
\hline
\end{tabular}

EFFECTIVE ONE-GROUP CROSS SECTIONS

The accuracy of this approximation is demonstrated in Table IV. The direct calculations, with two exceptions, are published by Okrent and Yiftah; $(2)$ the calculations for $U^{235}$ and equilibrium plutonium for the $2500 \mathrm{~L}$ carbide core were performed on mercury with Hassitt's diffusion theory code. The results in the first column, which are obtained from the results for $\mathrm{Pu}^{239}$ in each case, assuming that the critical mass is inversely proportional to $\left(\bar{x}-x_{8}\right)$, are in good agreement. The discrepancy between direct and indirect calculation is always less than $1 \%$ in critical mass. 
Table IV

RELATIONSHIP BETWEEN CRITICAL MASS AND $\left(x-x_{8}\right)$

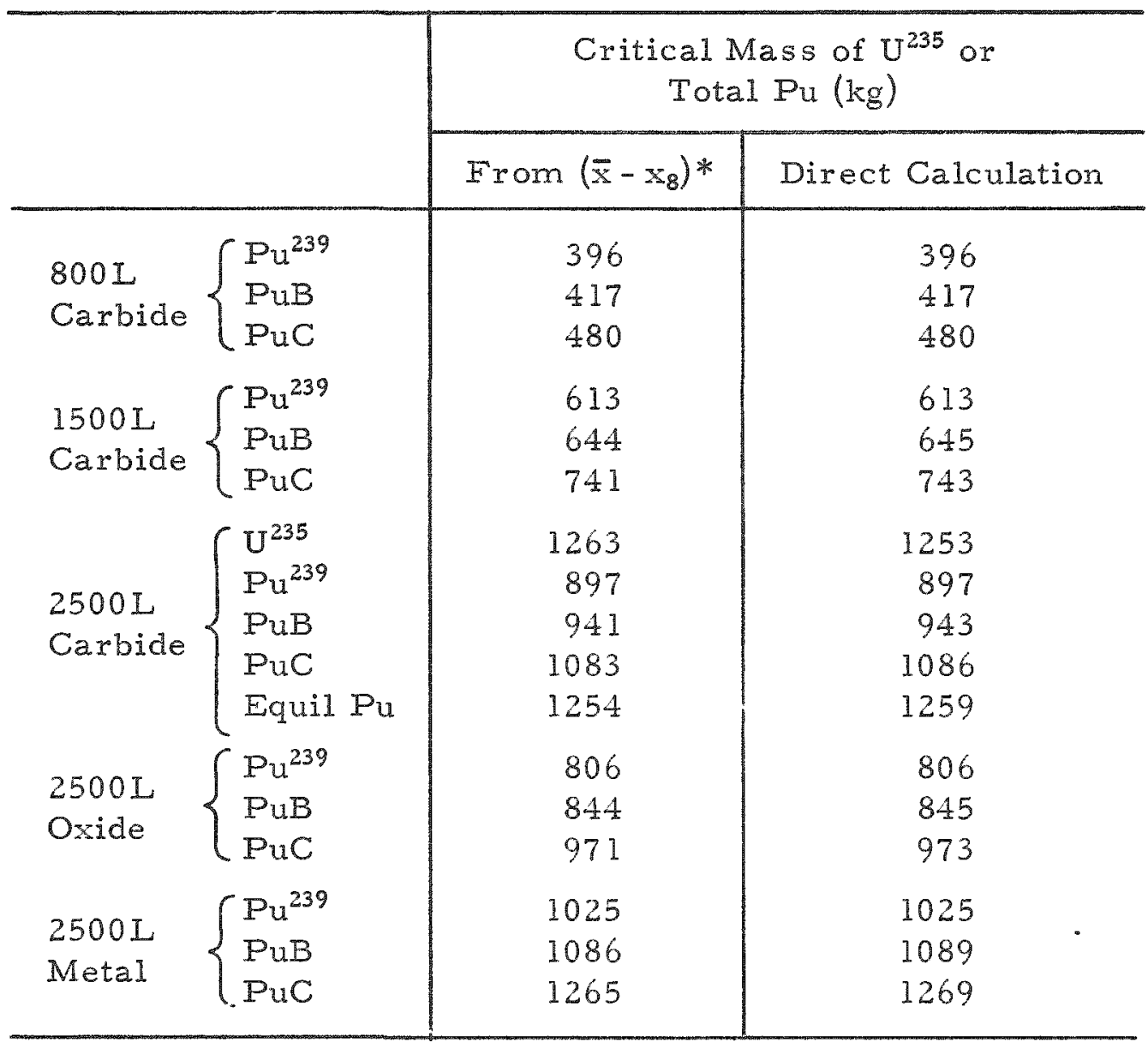

$* \mathrm{M}=\left(\right.$ critical mass for $\left.\mathrm{Pu}^{239}\right) /(\mathrm{w}$ for fuel specified),

where

$$
w=\frac{\left(\bar{x}-x_{8}\right) \text { for fuel specified }}{\left(x-x_{8}\right) \text { for } P u^{239} \text { fuel }}
$$

It is, therefore, possible to define a quantity $w$, the worth of any isotope or mixture in forming a critical mass, as

$$
w=\frac{\bar{x}-x_{8}}{x_{9}-x_{8}},
$$

where $\overline{\mathrm{x}}$ is the average value of $\nu \sigma_{\hat{f}}-\sigma_{\mathfrak{f}}-\sigma_{c}$ for the mixture of isotopes under consideration as fuel. As defined,

$$
w=1 \text { for } \mathrm{Pu}^{239} \text {. }
$$


For all combinations of isotopes i which make a certain core critical,

$$
\sum_{i} M_{i} w_{i}=\text { constant }
$$

where $M_{i}$ is the mass of isotope $i$ in the core.

Values of worth $w$ for several isotopes and mixtures for various reactors are given in Table $V$. It can be seen that $\mathrm{w}$ is approximately constant for all large cores, so that it may form the basis of a monetaryvalue scheme in Section 3.

\section{Table V}

\section{RELATIVE VALUES W OF URANIUM AND PLUTONIUM ISOTOPES AS FAST REACTOR FUELS}

\begin{tabular}{|c|c|c|c|c|c|c|}
\hline & \multicolumn{3}{|c|}{ Carbide } & \multirow{2}{*}{$\begin{array}{l}\text { Oxide } \\
2500 \mathrm{~L}\end{array}$} & \multirow{2}{*}{$\begin{array}{l}\text { Metal } \\
2500 L\end{array}$} & \multirow{2}{*}{ Cermet } \\
\hline & $800 \mathrm{~L}$ & $1500 \mathrm{~L}$ & $2500 \mathrm{~L}$ & & & \\
\hline $\mathrm{U}^{233}$ & & 1.190 & & & & 1.243 \\
\hline$U^{234}$ & 0.108 & 0.093 & 0.083 & 0.072 & 0.104 & 0.067 \\
\hline$U^{235}$ & 0.679 & 0.697 & 0.710 & 0.725 & 0.660 & 0.730 \\
\hline$U^{236}$ & -0.004 & -0.011 & -0.016 & -0.019 & -0.008 & -0.023 \\
\hline $\begin{array}{l}\text { Equil } U^{234}+ \\
U^{235}+U^{236 *}\end{array}$ & 0.378 & 0.375 & 0.373 & 0.373 & 0.366 & 0.367 \\
\hline $\mathrm{Pu}^{239}$ & 1.000 & 1.000 & 1.000 & 1.000 & 1.000 & 1.000 \\
\hline $\mathrm{Pu}^{240}$ & 0.189 & 0.165 & 0.148 & 0.132 & 0.175 & 0.122 \\
\hline$P_{u}{ }^{241}$ & 1.444 & 1.484 & 1.511 & 1.539 & 1.403 & 1.558 \\
\hline $\mathrm{Pu}^{242}$ & 0.182 & 0.158 & 0.142 & 0.126 & 0.168 & 0.117 \\
\hline Equil $\mathrm{Pu} *$ & 0.750 & 0.729 & 0.715 & 0.702 & 0.745 & 0.693 \\
\hline
\end{tabular}

*The values for equilibrium mixtures correspond to one atom divided as given in Table $\mathrm{X}$.

The phrase "equivalent $\mathrm{Pu}^{239 "}$ occurs throughout this paper. It means "the mass of isotope $i$ multiplied by $w_{i} . "$ It is a useful concept when expressing ratings because the rating for a given reactor expressed in MW per $\mathrm{kg}$ of total heavy atoms is constant, for different isotopic mixtures, provided that the rating in $\mathrm{MW} / \mathrm{kg}$ equiv $\mathrm{Pu}^{239}$ is constant. 


\subsection{Definition of Breeding Gain G}

A new parameter, the breeding gain $G$, is introduced in this paper. It is proposed that the breeding gain be used in future instead of breeding ratio, defined in its various ways. The breeding gain $G$ is defined as the excess $\mathrm{Pu}^{239}$ produced per fission above that required to maintain reactivity and therefore may be written as

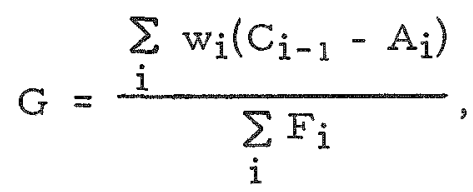

where

$w_{i}$ is the relative value of nuclide $i$ as a fast reactor fuel as given in Table $\mathrm{V}$;

$i$ is a suffix that can take the values $4,5,6,8,9,0,1$ and 2 , representing $\mathrm{U}^{234}, \mathrm{U}^{235}, \mathrm{U}^{236}, \mathrm{U}^{238}, \mathrm{Pu}^{239}, \mathrm{Pu}^{240}, \mathrm{Pu}^{241}$, and $\mathrm{Pu}^{242}$, respectively. The sum in the denominator is over all $i$ present in the system; the sum in the numerator is over all $i$ except 8 for $U^{238}$;

$C_{i}$ is the capture rate for element $i_{;} A_{i}$ is the absorption rate for element $i$;

$C_{i-1}-A_{i}$ is therefore the net rate of production of element $i$ and $F_{i}$ is the fission rate for element $i$

When the rates $C_{i}, A_{i}$, and $F_{i}$ refer to the whole system, then $G$ represents the total breeding gain. When the rates $C_{i}$ and $A_{i}$ in the numerator refer to the core only, then the expression is to be called GI, the internal breeding gain. An example of the calculation of $G$ is given in Table VI. Values of breeding gain for various reactors are given in Table VII.

The new parameters $G$ and $G_{I}$ are preferable to the various breeding ratios currently in use for two reasons. First, the parameters $G$ and $G_{I}$ have exactly the same meaning for reactors fueled with $\mathrm{Pu}^{239}$, $\mathrm{U}^{235}$, or higher isotopes of uranium or plutonium. If the blanket production is neglected, or otherwise disposed of, then $\mathrm{G}_{I}$ atoms of $\mathrm{Pu}^{239}$ are available for disposal after maintaining criticality. In most cases,

$$
\mathrm{G}_{\mathrm{I}}<0,
$$

which implies a continuous feed to the core to maintain criticality. If this feed is supplied by $\mathrm{Pu}^{239}$ produced in the blanket, then $\mathrm{G}$ is the number of atoms of $\mathrm{Pu}^{239}$ per fission available for disposal from the systemas a whole. 
Table VI

TYPICAI BALANCE SHEET FOR 1 FISSION: I500L CARBIDE WITH PUB

\begin{tabular}{|c|c|c|c|c|c|c|}
\hline & & Fissions & Captures & $\begin{array}{c}\text { Net } \\
\text { Change }\end{array}$ & & $\begin{array}{l}\text { Equiv } \\
\text { Change } \\
\text { in } \mathrm{Pu}^{239}\end{array}$ \\
\hline Core & $\begin{array}{l}\mathrm{U}^{238} \\
\mathrm{Pu}^{239} \\
\mathrm{Pu}^{240} \\
\mathrm{Pu}^{241} \\
\mathrm{Pu}^{242}\end{array}$ & $\begin{array}{l}0.1151 \\
0.6575 \\
0.0173 \\
0.1477 \\
0.0045\end{array}$ & $\begin{array}{l}0.6269 \\
0.1585 \\
0.0211 \\
0.0175 \\
0.0056\end{array}$ & $\begin{array}{r}-0.1891 \\
0.1201 \\
-0.1441 \\
0.0074\end{array}$ & $\mid$ & $\begin{array}{r}-0.1891 \\
0.0198 \\
-0.2138 \\
0.0012\end{array}$ \\
\hline Blanket & $\begin{array}{l}\mathrm{U}^{238} \\
\mathrm{Pu}^{239}\end{array}$ & $\begin{array}{c}0.0579 \\
0\end{array}$ & $\begin{array}{c}0.9891 \\
0\end{array}$ & 0.9891 & $\begin{array}{c}\mathrm{G}_{\mathrm{I}}= \\
\mid \\
\mathrm{G}=\end{array}$ & $\begin{array}{r}-0.3819 \\
\frac{0.9891}{0.6072}\end{array}$ \\
\hline
\end{tabular}

Table VII

COMPARISON OF BREEDING GAIN AND BREEDING RATIOS

\begin{tabular}{|c|c|c|c|c|c|}
\hline & \multirow{2}{*}{ Fuel } & \multicolumn{3}{|c|}{ Carbide } & \multirow{2}{*}{ Cermet } \\
\hline & & $800 \mathrm{~L}$ & $1500 \mathrm{~L}$ & $2500 \mathrm{~L}$ & \\
\hline $\begin{array}{l}\text { Breeding } \\
\text { gain G }\end{array}$ & $\begin{array}{l}\mathrm{U}^{235} \\
\text { Equil U } \\
\text { Pu } \\
\text { PuB* } \\
\text { PuC* } \\
\text { Equil Pu }\end{array}$ & $\begin{array}{l}0.66 \\
0.66 \\
0.66 \\
0.77\end{array}$ & $\begin{array}{l}0.49 \\
0.36 \\
0.61 \\
0.60 \\
0.57 \\
0.74\end{array}$ & $\begin{array}{l}0.45 \\
0.31 \\
0.58 \\
0.57 \\
0.52 \\
0.70\end{array}$ & 0.48 \\
\hline $\begin{array}{l}\text { Physicist's } \\
\text { breeding } \\
\text { ratio } B_{\mathrm{p}}\end{array}$ & $\begin{array}{l}\mathrm{U}^{235} \\
\text { Equil U } \\
\mathrm{Pu}^{239} \\
\text { PuB* } \\
\text { PuC* } \\
\text { Equil Pu }\end{array}$ & $\begin{array}{l}1.62 \\
1.73 \\
1.90 \\
1.84\end{array}$ & $\begin{array}{l}1.15 \\
1.04 \\
1.56 \\
1.67 \\
1.82 \\
1.80\end{array}$ & $\begin{array}{l}1.12 \\
1.00 \\
1.52 \\
1.62 \\
1.77 \\
1.74\end{array}$ & 1.44 \\
\hline $\begin{array}{l}\text { Chemist's } \\
\text { breeding ratio } \\
\text { neglecting } \mathrm{Pu}^{242} \\
\text { capture, } \mathrm{B}_{\mathrm{C}}\end{array}$ & $\begin{array}{l}\mathrm{Pu}^{239} \\
\mathrm{PuB} * \\
\mathrm{PuC} * \\
\text { Equil Pu }\end{array}$ & $\begin{array}{l}1.99 \\
2.00 \\
2.00 \\
1.93\end{array}$ & $\begin{array}{l}1.94 \\
1.95 \\
1.96 \\
1.89\end{array}$ & $\begin{array}{l}1.91 \\
1.92 \\
1.93 \\
1.84\end{array}$ & 1.70 \\
\hline $\begin{array}{l}\text { Chemist's } \\
\text { breeding ratio } \\
\text { with Pu } 242 \\
\text { capture, } B_{C}^{\prime}\end{array}$ & $\begin{array}{l}P_{u}^{239} \\
P u B * \\
\text { PuC* } \\
\text { Equil Pu }\end{array}$ & $\begin{array}{l}1.99 \\
2.00 \\
1.94 \\
1.93\end{array}$ & $\begin{array}{l}1.94 \\
1.95 \\
1.89 \\
1.88\end{array}$ & $\begin{array}{l}1.91 \\
1.92 \\
1.85 \\
1.84\end{array}$ & 1.70 \\
\hline
\end{tabular}

*The compositions are as assumed by Okrent, (5) namely,

$\begin{array}{lllll} & P u^{239} & P u^{240} & P u^{241} & P u^{242} \\ \text { PuB } & 0.747 & 0.102 & 0.124 & 0.027 \\ \text { PuC } & 0.4 & 0.1 & 0.25 & 0.25\end{array}$


Secondly, the new parameter $G$ is directly involved in formulae used in economic studies. The contribution of plutonium credit to the cost per unit of electricity sent out from a power station is given by

$$
\delta W=0.0106 \frac{G V}{E} d / \text { u.s.o. }
$$

where all the symbols are as defined in Appendix I. (The numerical constant does not contain any assumption about the reactor.) For example,

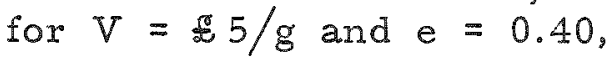

$$
\delta W=0.13 \mathrm{G} \mathrm{d} / \mathrm{u} . \mathrm{s} .0 \text {. }
$$

The breeding gain $G$ is related to the doubling time for fuel in the fuel cycle by the following simple formula:

$$
\text { Doubling time }=\frac{1.8}{R G} \mathrm{yr} \text {, }
$$

where $R$ is the average heat rating of fuel in MW/ kg, taking account of load factor and out-of-pile inventory. The coefficient is equal to

$$
\frac{H \ln 2}{A} \text {. }
$$

\subsection{Comparison of Breeding Gain with Various Breeding Ratios}

The relationship between the breeding gain $G$ and breeding ratio defined in various ways will now be demonstrated. The so-called "physicist's breeding ratio" may be defined, for plutonium fuels only, as

$$
B_{p}=\frac{\text { production of } \mathrm{Pu}^{239} \text { or } \mathrm{Pu}^{241}}{\text { destruction of } \mathrm{Pu}^{239} \text { or } \mathrm{Pu}^{241}}=\frac{\mathrm{C}_{8}+\mathrm{C}_{0}}{\mathrm{~A}_{9}+\mathrm{A}_{1}} \text {. }
$$

The "chemist's breeding ratio" (so-called because chemists cannot distinguish plutonium isotopes) may be defined either as

$$
B_{C}=\frac{\text { production of plutonium }}{\text { fission of plutonium }}=\frac{C_{8}}{F_{9}+F_{0}+F_{1}+F_{2}}
$$

or as

$$
B_{C}^{1}=\frac{\text { net production of plutonium }}{\text { fission of plutonium }}=\frac{C_{8}-C_{2}}{F_{9}+F_{0}+F_{1}+F_{2}} .
$$


The last two definitions differ only in the exclusion or inclusion of capture in $\mathrm{Pu}^{242}$ in the numerator.

For plutonium containing $100 \% \mathrm{Pu}^{239}$, the formulae reduce to

$$
\begin{aligned}
B_{p} & =\frac{C_{8}}{F_{9}+C_{9}} ; \\
B_{C} & =B_{C}^{1}=\frac{C_{8}}{F_{9}} ; \\
G & =\frac{F_{9}+C_{9}}{F_{q}+F_{8}}\left(B_{p}-1+\frac{w_{0} C_{9}}{F_{9}+C_{9}}\right) \sim B_{p}-1 .
\end{aligned}
$$

It can be seen from the above formula that the breeding gain $G$ is a quantity closely related to a breeding ratio less unity. It has the great advantage over all definitions of breeding ratio in that there is a natural extension to higher isotopes of plutonium and to $U^{235}$ fuel. There is also a more precise meaning, namely, for $G=0$ a reactor is exactly selfsustaining. There is only such a simple explanation for $B_{C}^{1}$ and in one special case only. For equilibrium plutonium fuel, the reactor is self sustaining if $B_{C}^{1}=1$.

All definitions of breeding ratio $\left(B_{p}, B_{c}\right.$, or $\left.B_{c}^{l}\right)$ suffer from the drawback that they do not correctly take account of the varying worths of different fuel isotopes. This is illustrated by Table VII. Both $B_{p}$ and $B_{C}$ suggest that breeding is superior with $\mathrm{PuC}$ than with pure $\mathrm{Pu}^{239}$ whereas, in fact, the high concentration of $\mathrm{Pu}^{242}$ worsens breeding as shown by $\mathrm{G}$ and $B_{C}^{1}$. On the other hand, equilibrium plutonium is a better fuel than pure $\mathrm{Pu}^{239}$. The opposite is suggested by $\mathrm{B}_{C}$ or $\mathrm{B}_{\mathrm{C}}^{1}$, but it is demonstrated by $G$ and $B_{p}$. Only $G$ gives the correct indication in all cases.

\subsection{Approach to Equilibrium}

The approach to equilibrium fuel composition was studied in two cases: a $1500 \mathrm{~L}$ carbide core starting with plutonium containing only $\mathrm{Pu}^{239}$ initially, and the same starting with enriched uranium consisting of $1 \% \mathrm{U}^{234}$ and $99 \% \mathrm{U}^{235}$ instead of $\mathrm{Pu}^{239}$. The remainder of the heavy atoms in the core were $U^{238}$ in each case. The core was assumed to be replenished with pure $\mathrm{Pu}^{239}$ from the blanket in each case. The equations given by Okrent $(5)$ for plutonium isotopes were solved numerically. The analytical solution was obtained for the corresponding equations for $U^{234}, U^{235}$, and $U^{236}$. The solutions were obtained subject to the same two constraints as used by Okrent, namely, 


$$
\begin{aligned}
& \sum_{i} N_{i}=\text { constant; } \\
& \sum_{i} N_{i}\left(\nu \sigma_{f}-\sigma_{i}-\sigma_{c}\right)_{i}=\text { constant. }
\end{aligned}
$$

The sums were taken over all heavy isotopes in each case.

The first constraint maintained the total number of heavy atoms constant, which implied that fission products and isotopes of mass 237 and 243 were continuously removed. It was assumed that $\mathrm{Pu}^{239}$ was formed immediately on capture of a neutron in $\mathrm{U}^{238}$; reactions of the intermediate products $\mathrm{U}^{239}$ and $\mathrm{Np}^{239}$ were neglected. Capture of a neutron in $\mathrm{U}^{236}$ or $\mathrm{Pu}^{242}$ was assumed to produce immediately $\mathrm{Np}^{237}$ or $\mathrm{Am}^{243}$, respectively, which were removed continuously; reactions of the intermediate products $\mathrm{U}^{237}$ and $\mathrm{Pu}^{243}$ were neglected.

A continuous feed of $\mathrm{U}^{238}$ and $\mathrm{Pu}^{239}$ was necessary to satisfy the first constraint. The second constraint maintained criticality and determined the proper ratio of $\mathrm{U}^{238}$ to $\mathrm{Pu}^{239}$ in the feed. This constraint is justified in Section 2.1.

The results of the calculations are given in Table VIII and Figs. 1 and 2. The symbols shown on the curves are included to indicate the actual points calculated. A rather wide mesh was necessary because the calculations were performed with a desk computer. The rise of the $\mathrm{Pu}^{239}$ to a shallow maximum for $\mathrm{U}^{235}$ startup is genuine. The initial rise is made necessary by the burnout of the $U^{235}$, but there is a slight fall, giving the maximum, when eventually the $\mathrm{Pu}^{241}$ builds up.

Table YIII

APPROACH TO EOULLIBRIUW FOR A 1500L CARBIDE CORE FUELED INITIALLY

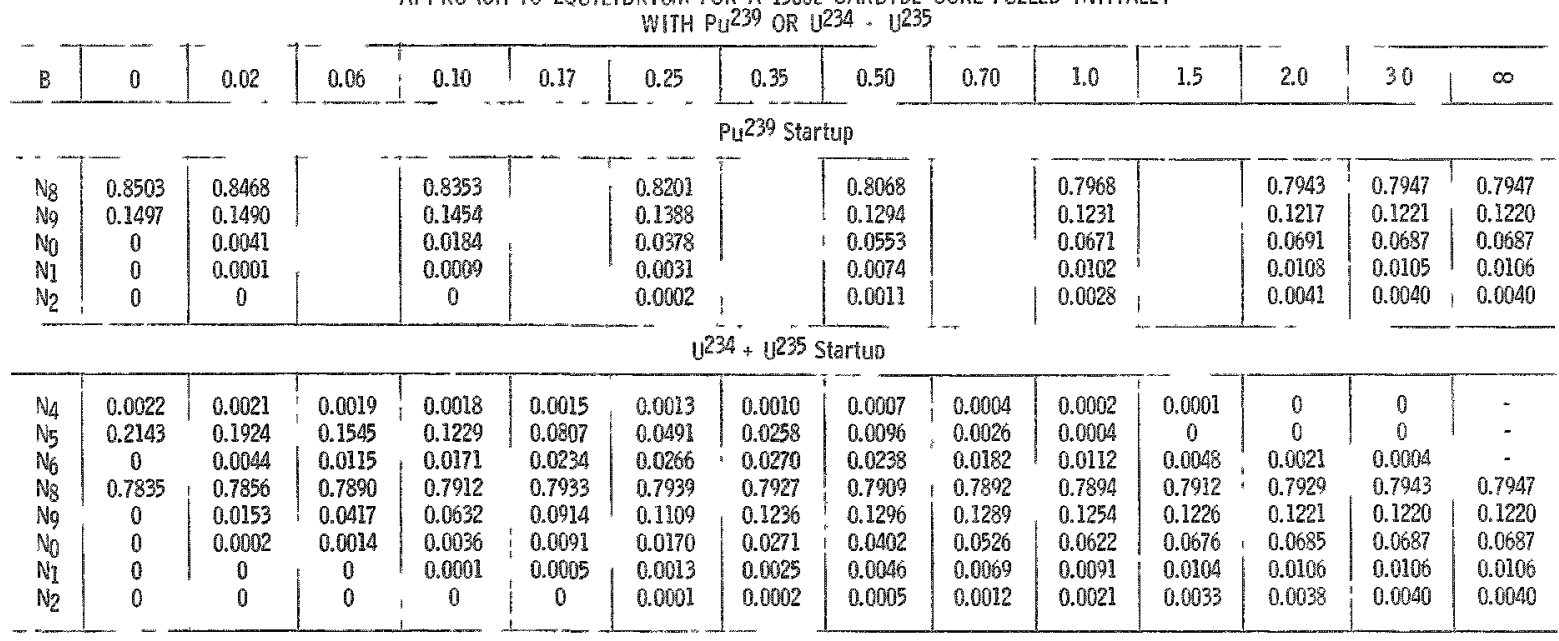




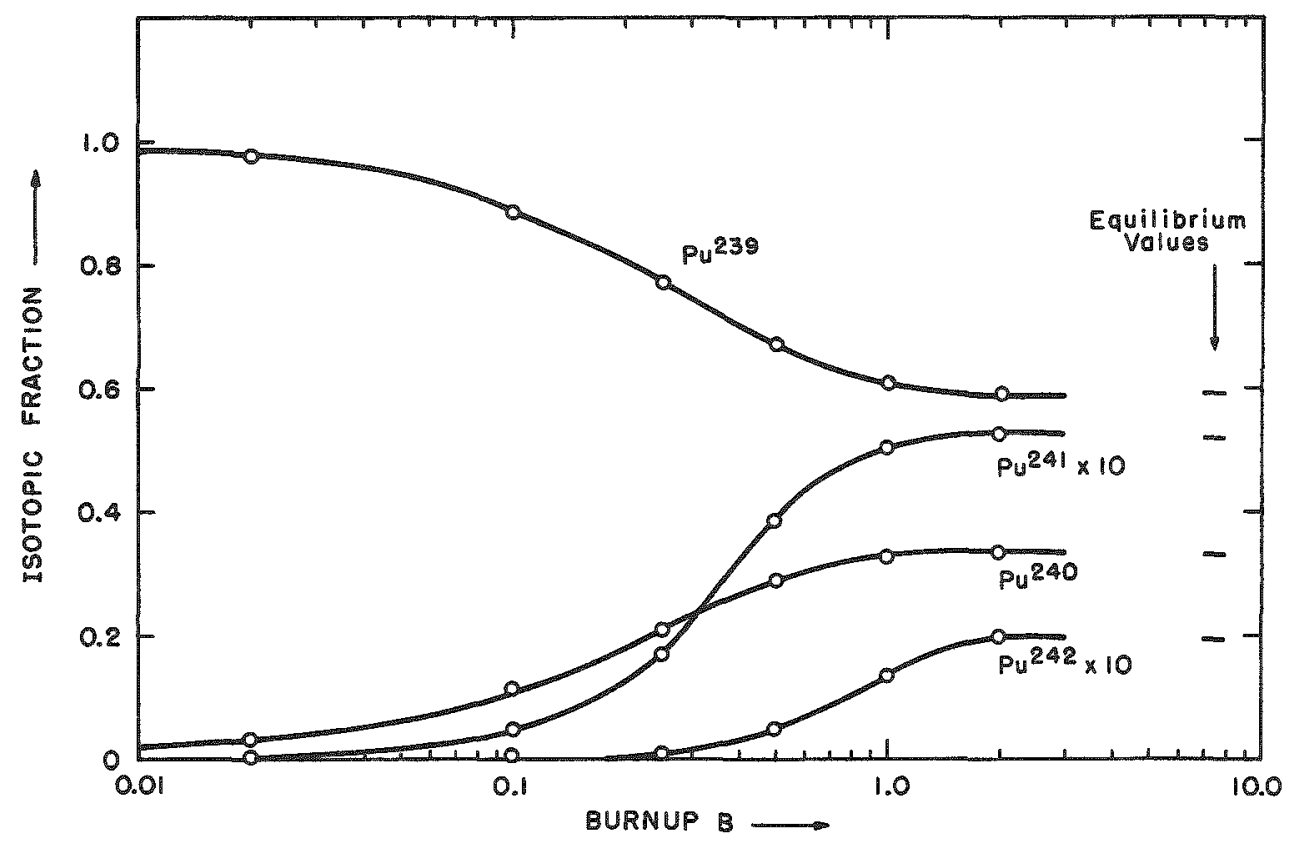

Fig. 1. Approach to Equilibrium for 1500L Carbide Core for $\mathrm{Pu}^{239}$ Initial Charge and $\mathrm{Pu}^{239} \mathrm{Feed}$

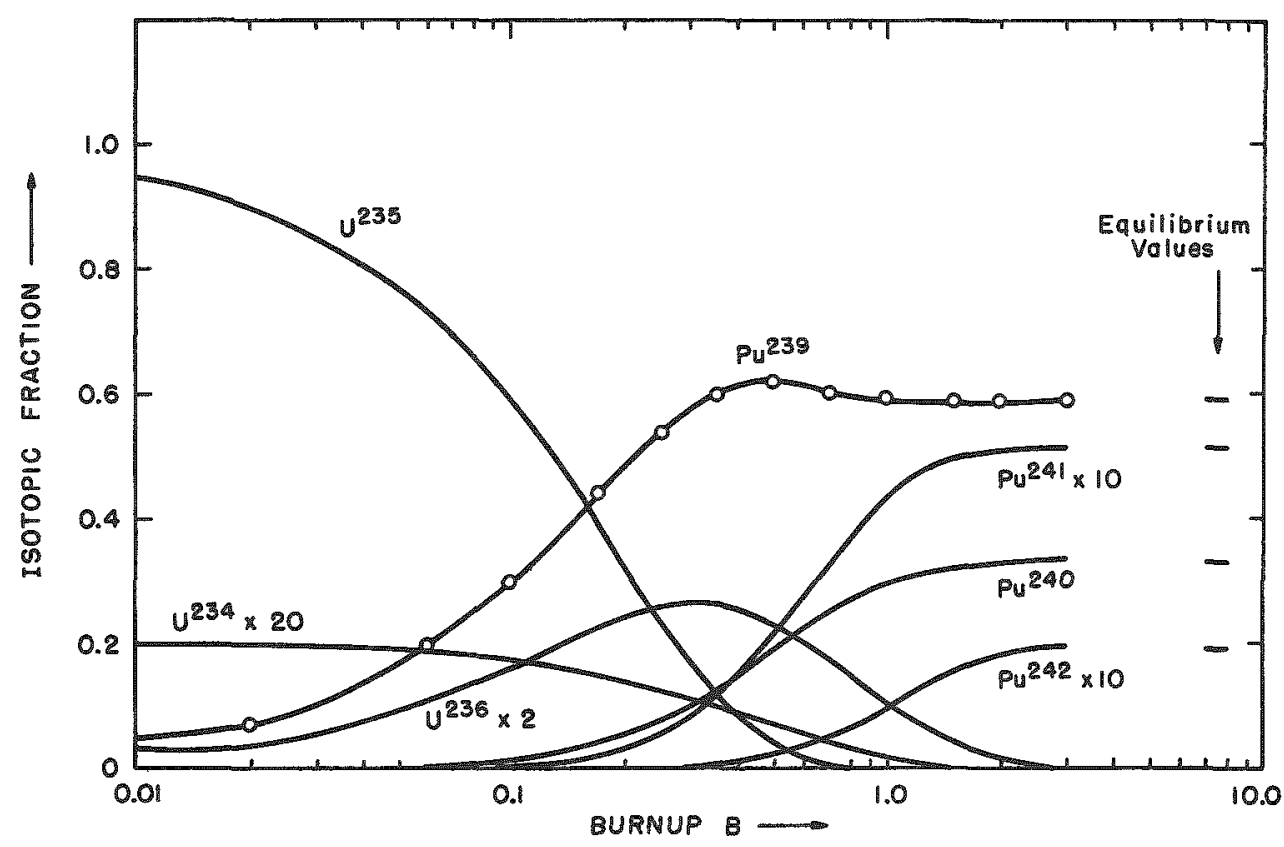

Fig. 2. Approach to Equilibrium for $1500 \mathrm{~L}$ Carbide Core for Initial Charge of Enriched Uranium and $\mathrm{Pu}^{239}$ Feed

Although these calculations were performed for a particular $1500 \mathrm{~L}$ carbide reactor, the results will apply approximately to other dilute fast reactors provided that dose or burnup of equivalent $P_{u^{239}}, B^{*}$, is 
regarded as the independent variable. The burnup of total heavy atoms, $B$, is not satisfactory for this purpose because it is dependent on the ratio of $\mathrm{Pu}^{239}$ to total heavy atoms which varies for different designs of fast reactor.

When a fast reactor is started up on $\mathrm{U}^{235}$, there is a loss of byproduct $\mathrm{Pu}^{239}$ because of the lower breeding gains with $\mathrm{U}^{235}$ fuels, although there may be an overall gain in plutonium supplies since electricity has been generated from the consumption of $\mathrm{U}^{235}$ rather than the consumption of plutonium. The cumulative loss is calculated as a function of dose $D$ or of burnup in Table IX. All the numbers were based on a single computer calculation for $100 \% \mathrm{Pu}^{239}$ fuel with empirical extrapolation for other fuels. For example, the integrated flux in the blanket was assumed to be $7 \%$ higher, for $U^{235}$ fuel, based on calculations for the 2500 Lcarbide. The cumulative loss at any burnup does not depend on the reactor rating, but, for a given rating, dose is proportional to the number of years that the reactor has operated. The operating period, in years, corresponding to the assumed cumulative doses are also given in Table IX for a typical overall fuel rating, $0.5 \mathrm{MW} / \mathrm{kg}$ of equivalent $\mathrm{Pu}^{239}$ in the system. The values in Table IX are used in the economic studies of Section 3.

Table IX

Pu ${ }^{239}$ LOST IF FIRST LOADING OF BREEDER REACTOR IS ENRICIED URANIUM (1500I CARBIDE)

\begin{tabular}{|c|c|c|c|c|c|c|c|}
\hline $\begin{array}{l}\text { Irradiation dose } \mathrm{D} \\
\quad\left(\mathrm{MWD} / \mathrm{g} \text { equiv } \mathrm{Pu}^{239}\right)\end{array}$ & 0 & 0.18 & 0.91 & 1.82 & 3.65 & 7.30 & $\infty$ \\
\hline Burnup of equiv $\mathrm{Pu}^{239}, \mathrm{~B} *$ & 0 & 0.23 & 1.14 & 2.28 & 4.56 & 9.12 & $x$ \\
\hline Burnup of total heavy atoms, B & 0 & 0.034 & 0.171 & 0.341 & 0.682 & 1.364 & $w 2$ \\
\hline $\begin{array}{l}\text { Breeding } \\
\text { gain } G\end{array}\left\{\begin{array}{l}\mathrm{Pu}^{239} \text { startup } \\
\mathrm{U}^{235} \text { startup }\end{array}\right.$ & $\begin{array}{l}0.611 \\
0.488\end{array}$ & $\begin{array}{l}0.623 \\
0.500\end{array}$ & $\begin{array}{l}0.065 \\
0.553\end{array}$ & $\begin{array}{l}0.696 \\
0.614\end{array}$ & $\begin{array}{l}0.718 \\
0.681\end{array}$ & $\begin{array}{l}0.730 \\
0.722\end{array}$ & $\begin{array}{l}0.730 \\
0.730\end{array}$ \\
\hline$\Delta G$ & 0.123 & 0.123 & 0.112 & 0.082 & 0.037 & 0.008 & 0 \\
\hline $\begin{array}{l}\frac{\text { Cumulative } 10 \mathrm{ss} \text { of } \mathrm{Pu}^{239}}{\text { Mass of equiv Pu }}{ }^{239} \text { in system } \\
\text { For } \mathrm{R}=0.5 \mathrm{MW} / \mathrm{kg} \text { only: }\end{array}$ & 0 & 0.024 & 0.116 & 0.210 & 0.319 & 0.395 & 0.415 \\
\hline Operating period t $(y r)$ & 0 & 1 & 5 & 10 & 20 & 40 & $\infty$ \\
\hline$\frac{\text { Ammual loss of } \mathrm{Pu}^{239}}{\text { Mass of equiv Pu }{ }^{239} \text { in system }}$ & 0.0238 & 0.0238 & 0.0216 & 0.0159 & 0.0072 & 0.0015 & 0 \\
\hline
\end{tabular}

When the reactivity of a core is maintained as burnup proceeds by continual replenishment with the same type of fuel, the concentrations of all isotopes approach a steady state. Equilibrium compositions for enriched uranium cores replenished with enriched uranium and plutonium cores replenished with $\mathrm{Pu}^{239}$ are given in Table $\mathrm{X}$. 
Table X

EQUIIIBRIUM ATOMIC COMPOSITION FOR REACTORS FED WITH $1 \% \mathrm{U}^{234}$ AND $99 \% \mathrm{U}^{235}$ OR $100 \% \mathrm{Pu}^{239}$

\begin{tabular}{ll|l|l|l|l}
\hline & \multicolumn{3}{|c|}{ Carbide } & \multirow{2}{*}{ Cermet } \\
\cline { 2 - 5 } & $\mathrm{U}^{234}+\mathrm{U}^{235}$ feed & $800 \mathrm{~L} *$ & $1500 \mathrm{~L} *$ & $2500 \mathrm{~L}$ & \\
\hline $\mathrm{U}^{235}$ & 0.015 & 0.016 & 0.016 & 0.016 \\
$\mathrm{U}^{236}$ & 0.558 & 0.542 & 0.533 & 0.517 \\
& 0.427 & 0.442 & 0.451 & 0.467 \\
\hline $\mathrm{Pu}^{234}$ & 0.617 & 0.594 & 0.579 & 0.556 \\
$\mathrm{Pu}^{240}$ & 0.318 & 0.334 & 0.345 & 0.361 \\
$\mathrm{Pu}^{241}$ & 0.048 & 0.052 & 0.054 & 0.058 \\
$\mathrm{Pu}^{242}$ & 0.017 & 0.020 & 0.022 & 0.025 \\
\hline
\end{tabular}

*The plutonium equilibrium mixtures for $800 \mathrm{~L}$ and $1500 \mathrm{~L}$ cores are in agreement with values given by Okrent, (3) who also gives values for metal and oxide fuels for these sizes.

\subsection{Blanket Discharging Studies}

The variations of fission and capture rates with distance into a typical blanket were investigated in order to provide data for economic studies. If, for example, there were no market for excess plutonium, the economics of the system could be improved by reducing the thickness of the breeder.

The relative reaction rates for $P u^{239}$ and $U^{235}$ fission and $U^{238}$ fission and capture for the $U^{238}$ blanket of the $2500 \mathrm{~L}$ carbide core are given in Table XI. The fuel in the core is equilibrium plutonium. The rates refer to reactions per $\mathrm{kg}$ of isotope and are normalised to unity at

Table $\mathrm{ZI}$

REACTION RATES IN THE BLANKET OF A $2500 L$ CARBIDE REACTOR

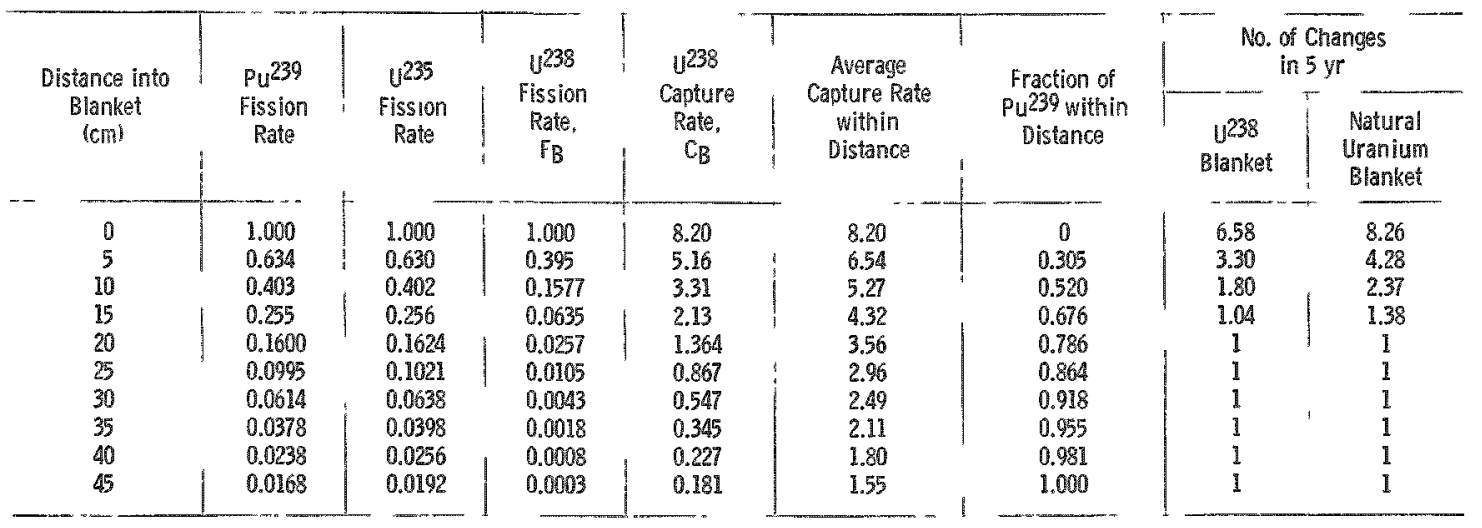


the inner boundary for the fission rates. The normalisation for capture in $\mathrm{U}^{238}$ is the same as that for fission in $\mathrm{U}^{238}$. Because the concentration of $\mathrm{Pu}^{239}$ in the blanket falls off rapidly with increasing distance, the actual fission rate in $\mathrm{Pu}^{239}$ falls off more rapidly than the relative rate which refers to $1 \mathrm{~kg}$.

It can be seen that the relative reaction rates in Table XI are typical of any large fast reactor from the following information: The ratio of total $U^{238}$ captures to total $U^{238}$ fissions, from the calculations of Okrent and Yiftah, (2) is 15.4 for an $800 \mathrm{~L}$ carbide core and 18.2 for a $2500 \mathrm{~L}$ core. The principal change with core size and composition will be in the rating of innex blanket elements. For an average in-core rating of $1 \mathrm{MW} / \mathrm{kg}$ equivalent $\mathrm{Pu}^{239}$ and load factor 0.75 , the average rating of innermost blanket elements is $0.0058 \mathrm{MW} / \mathrm{kg} \mathrm{U}^{238}$ for the $2500 \mathrm{~L}$ carbide core studied. In the remainder of this section a round number, $0.005 \mathrm{MW} / \mathrm{kg} \mathrm{U}^{238}$, is assumed.

The actual blanket computed contained no $\mathrm{U}^{235}$. If it be assumed that the flux and spectral distribution were unaltered by the replacement of $\mathrm{U}^{238}$ by natural uranium, the contribution from $\mathrm{U}^{235}$ was calculated. Although this approximate treatment of the effect of $\mathrm{U}^{235}$ will underestimate the flux in the outer parts of a natural uranium blanket, it should be of sufficient accuracy for the exploratory studies of this section. The average power generation in the bred plutonium was also calculated. At the core-blanket boundary, the average power was $0.0085 \mathrm{MW} / \mathrm{kg} \mathrm{U}^{238}$, assuming replacement of elements at $0.2 \%$ burnup, made up as follows:

$\begin{array}{ll}\text { from } U^{238} & 0.0050 \\ \text { from } U^{235} & 0.0021 \\ \text { from } P u^{239} \text { (average) } & 0.0014\end{array}$

Table XI also includes columns which give the number of blanket-element changes necessary in $5 \mathrm{yr}$ if the burnup is not allowed to exceed $0.2 \%$ of total heavy atoms. Values are given for a $U^{238}$ blanket and also for a natural uranium blanket.

The throughput of uranium in the processing plant and the average $\mathrm{Pu} / \mathrm{U}$ ratio of the discharged blanket elements was considered under alternative schemes for both a $\mathrm{U}^{238}$ and a natural uranium blanket. The results are given in Table XII. In the first scheme, it was assumed that the complete charge of blanket elements was sent for processing when the innermost elements reached a burnup of $0.2 \%$ by atoms. Fission in $\mathrm{U}^{235}$ and $\mathrm{Pu}{ }^{239}$ was taken into account when calculating the burnup.

It can be seen from Table XII that the $U^{235}$ present in a natural uranium blanket would reduce the $\mathrm{Pu} / \mathrm{U}$ ratio that could be achieved in any 
element for a given burnup by about $20 \%$ compared with a $\mathrm{U}^{238}$ blanket. If blanket performance is determined by the burnup achievable, then depleted uranium should be used.

Table XII

MASS OF URANIUM TO BE PROCESSED AND Pu/U RATIO IN ALTERNATIVE PROCESSING SCHEMES FOR A 2500L REACTOR.

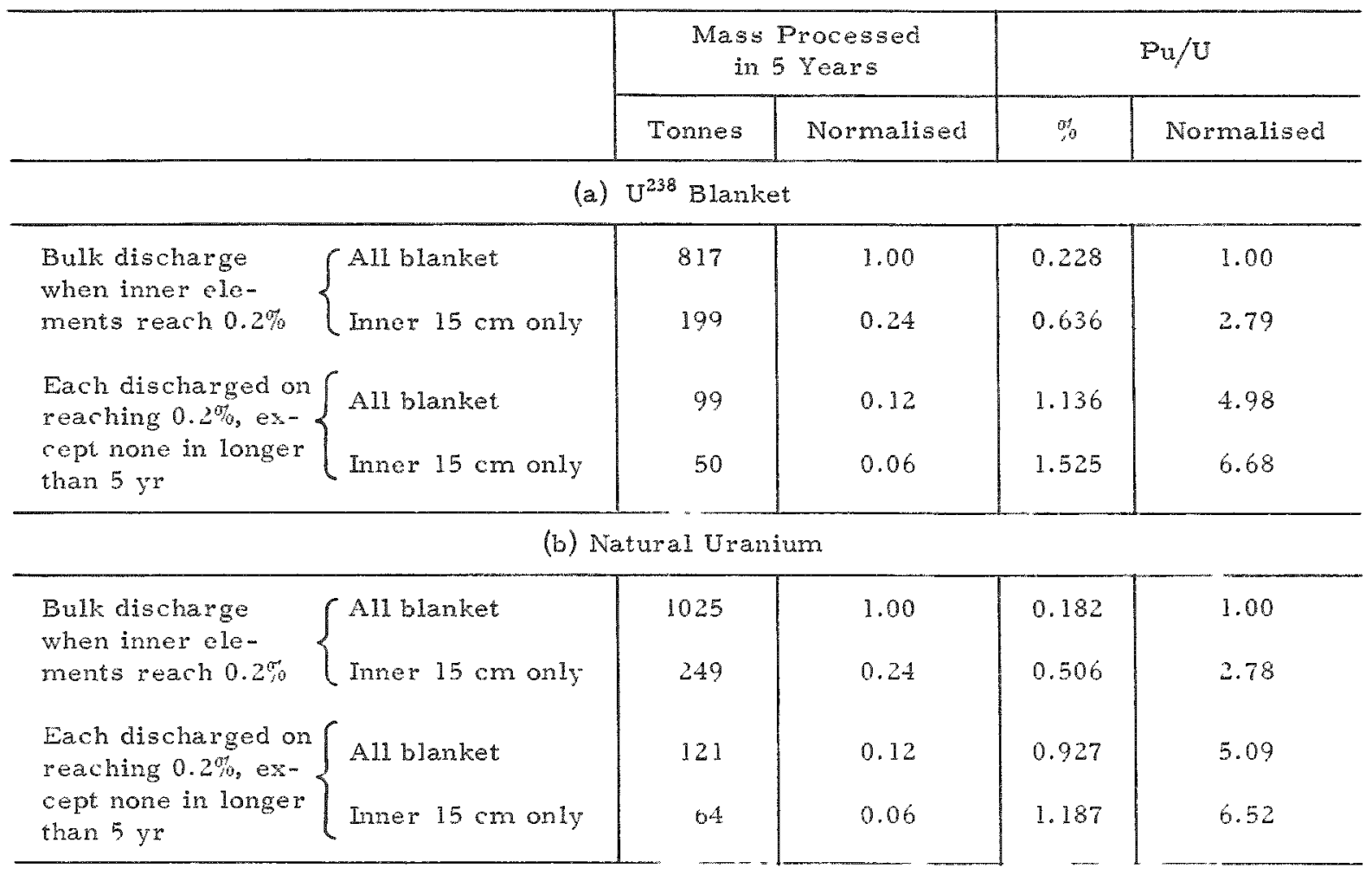

It can also be seen that if the reactor operator only requires two-thirds of the blanket plutonium, then the blanket thickness can be reduced from 45 to $15 \mathrm{~cm}$. The throughput of the processing plant would be reduced by a factor of 4 , and the $\mathrm{Pu} / \mathrm{U}$ ratio increased by a factor of 3 .

In the second discharge scheme, each element is left in the blanket until it reaches a burnup of $0.2 \%$ up to a maximum of $5 \mathrm{yr}$. It can be seen from Table XII that this reduces the throughput as compared with the basic first scheme by a factor of 8 and increases the $P u / U$ ratio by $a$ factor of 5 . For a $15-\mathrm{cm}$ blanket, the reduction in throughput is by a factor of 15 and the increase in Pu/U ratio by a factor of 7 .

\section{Economic Studies}

\subsection{Introduction}

It is no simple matter to answer the question, "What can a fast reactor afford to pay for its fuel?" One might consider an idealized 
situation, with plutonium always available as feed at a certain price and with a market always available for plutonium produced at the same fixed price. Fast reactors with a short fuel-inventory doubling time, would then benefit from a very high plutonium price. Alternatively, a future system of fast reactor power stations might be considered separately from other reactor systems, except for the initial plutonium supplied from thermal reactors. In this case, credits for bred plutonium will correspond to bookkeeping transactions within the system, but payments for plutonium fed from other reactors will increase the cost of fast reactor electricity,

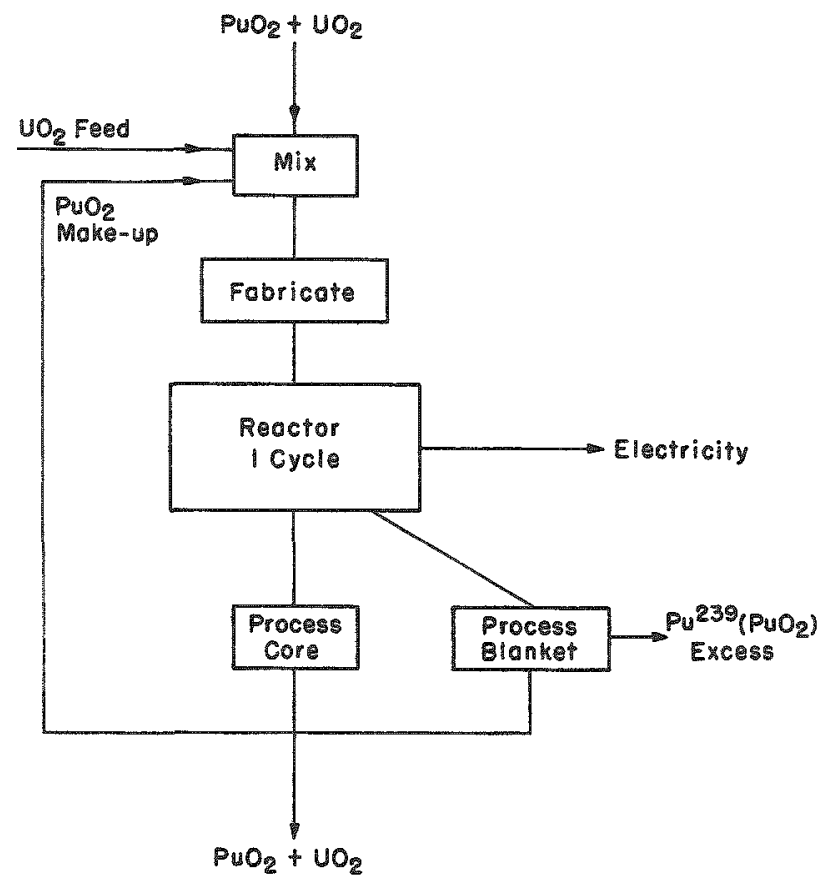

Fig. 3. Cycle for Fast Breeder and fast reactor designers would require the lowest price for plutonium that would ensure the production of the necessary quantities of plutonium. Whether or not a continual feed from thermal reactors will be required depends on the relationship between the doubling time for the fast reactors installed and the growth rate of the power system as a whole.

A typical fast reactor cycle is shown in Fig. 3. The fuel illus trated is the mixed oxides of plutonium and $\mathrm{U}^{238}$, but might equally well have been metal or carbide. It is most important to note that the input and output material is assumed to have the same physical form. It would be reasonable to assign the same value to both, except that the purchase of the initial charge and the sale of bred fuel are separated in time by 10 yr or more. In some parts of this paper, bred plutonium is assumed to have the same value as the plutonium in the initial charge, but a lesser value and zero value are also considered. There are three variants of the cycle shown in Fig. 3:

(i) Converter reactors. The feed for all cycles is enriched uranium. The plutonium produced in the core and blanket is separated periodically and sold.

(ii) Breeder reactors with $U^{235}$ startup. The initial charge is enriched uranium but the core is replenished with plutonium bred in the blanket. 
(iii) Purifier reactor. The feed of the core for all cycles is "dirty" plutonium of high heavy-isotope content. The near-pure $\mathrm{Pu}^{239}$ produced in the blanket is separated and sold. At present there is no known demand which would justify operating on this cycle.

Since $\mathrm{U}^{235}$ can be used as a substitute for $\mathrm{Pu}^{239}$, an upper Iimit for the price of $\mathrm{Pu}^{239}$ is set by the production cost of enriched uranium. The different cycles described above, except the last, are looked at in order to estimate the limit.

The following three contributions to fuel costs are considered in this paper:

(i) interest and capital repayment charges on the initial charge of fuel;

(ii) debit for fuel consumed;

(iii) credit for fuel produced by breeding - a negative charge.

Other charges, for example for chemical processing and fabrication, are not considered in these comparative studies, on the assumption that they should vary little with the cycle chosen. It should be pointed out, however, that if breeding were unimportant, then the thickness of breeder could be reduced with consequent reductions in capital and other costs. This subject is treated briefly in Section 3.2 .

In Section 3.2, only the first contribution is considered. It is assumed that the reactor produces sufficient luel to replenish itself but no value is assigned to the excess bred plutonium or to the core at the end of the life of the reactor. Converter reactors are considered in Section 3.4. Since it is probable that bred fuel will be worth something, even if less than the fuel in the initial charge, plutonium is worth somewhat more when compared with $U^{235}$ than Section 3.2 would suggest because of the larger output of excess plutonium. Reactors started up with $U^{235}$ but replenished with plutonium are considered in Section 3.5.

\subsection{Relative Values Based on Capital Charges Only}

At the beginning of a fast reactor programme we may consider only the charges on the capital invested in the first charge of fuel. In a properly designed fast reactor there should be an excess of plutonium produced, but it may be unwise to assign any value to this excess until its future usefulness is assured. The monetary value of each isotope is as sumed to be proportional to its contribution to criticality, that is, proportional to the values of $w_{i}$ given in Table $V$. With this assumption, any 
core that would make that reactor critical would have the same value. The capital charge will therefore not vary with composition.

When the investment charges are the only ones considered, it is a simple matter to calculate the contribution to the cost of electricity, in pence per unit sent out, due to fuel cost for any assumed value for $\mathrm{Pu}^{239}$. The value of other isotopes are related to the value of $P^{239}$ by $T$ able $V$. In Table XIII the opposite approach is used:

The price of $\mathrm{Pu}^{239}$ corresponding to a given margin in $\mathrm{d} / \mathrm{u} . \mathrm{s} .0$. is given. The price of $\mathrm{Pu}^{239}, \mathrm{~V}$ in $\mathrm{E} / \mathrm{g}$, is related to the margin, $W$ in $\mathrm{d} / \mathrm{u} . \mathrm{s.o.}$, by the following equation:

$$
V=r W
$$

where $r$ is a reduced form of rating, defined by

$$
\begin{aligned}
& r=\mathrm{REA} / 240 \mathrm{I}_{\mathrm{a}} \\
& I_{a}=\text { interest and amortisation rate, typically } 0.0837 \text { (5 } \frac{1}{2} \% \mathrm{written} \\
& \text { off over } 20 \mathrm{yr} \text { ); } \\
& \mathrm{R}=\text { average thermal rating of } \mathrm{Pu}^{239} \text { in the system in } \mathrm{MW} / \mathrm{kg} \text {. } \\
& \text { taking account of load factor and out-of-pile inventory; } \\
& \mathrm{E}=\text { thermal efficiency of station, } 0.40 \text {; } \\
& A=\text { number of hours in } 1 \text { year, } 8.76 \times 10^{3} \text {. }
\end{aligned}
$$

For the typical values mentioned above

\begin{tabular}{|c|c|c|c|c|c|c|}
\hline Fuel Cost & $\begin{array}{l}\text { Rating of } \mathrm{Pu}^{239} \\
(\mathrm{MW} / \mathrm{kg})\end{array}$ & 0.25 & 0.5 & 1.0 & 1.5 & 2.0 \\
\hline & $x$ & 43.6 & 87.2 & 174 & 262 & 349 \\
\hline 0.01 & & 0.4 & 0.9 & 1.7 & 2.6 & 3.5 \\
\hline 0.02 & & 0.9 & 1.7 & 3.5 & 5.2 & 7.0 \\
\hline 0.05 & & 2.2 & 4.4 & 8.7 & 13.1 & 17.4 \\
\hline 0.10 & & 4.4 & 8.7 & 17.4 & 26.2 & 34.9 \\
\hline 0.15 & & 6.5 & 13.1 & 26.2 & 39.2 & 52.3 \\
\hline
\end{tabular}

$$
r=174 R \text {. }
$$

By combining Tables $V$ and XIII, the value of any isotope can bo estimated on this basis.

Table XIII

VALUE OF $P^{239}$, IN $\$ / g$, TO A FAST REACTOR FOR A GIVEN FUEL COST IN d/u.s.o., ASSUMING NO VALUE FOR THE NET Pu ${ }^{239}$ PRODUCED 
From Table $V$, it can be seen that the value of $\mathrm{Pu}^{239}$ is 1.4 times the value of $U^{235}$. Since it is likely that $U^{235}$ can be purchased from diffusion plants at $\underset{2}{2} 5 / \mathrm{g}, \mathrm{Pu}^{239}$ is worth no more than $\mathbb{L} 7 / \mathrm{g}$. It can be seen from Table XIII that $\mathrm{U}^{235}$ or $\mathrm{Pu}^{239}$ at the above prices would give a fuel cost of about $0.08 \mathrm{~d} / \mathrm{u} . \mathrm{s} .0$. for $\mathrm{R}=0.5 \mathrm{MW} / \mathrm{kg}$, a possible value for civil fast reactors.

It is not strictly accurate to assume equal processing costs for plutonium and uranium fuels when taking account only of capital costs for fuel. A plutonium-fueled reactor produces more excess fuel than a $\mathrm{U}^{235}$-fueled reactor. If this excess had no value, the reactor operator would only process enough blanket material to replenish the core. Suppose that a certain reactor when fueled with enriched uranium were just self-replenishing, taking into account processing and other losses. The breeding gain for the same reactor fueled with plutonium would be higher by about 0.3 (from Table VII). The plutonium-fueled reactor would also be just self-replenishing if only the inner $15 \mathrm{~cm}$ of the blanket were processed (from Table VI). It can be seen from Section 2.5 that the throughput of the blanket processing plant might be reduced by a factor of two in this way (second scheme). There might also be a reduction in capital costs if the reactor vessel, etc., could be made smaller by reducing the blanket thickness. The effect of breeding on the comparison of the values of $\mathrm{Pu}^{239}$ and $\mathrm{U}^{235}$ is considered quantitatively in the next sections.

\subsection{Breeder Reactors}

In this section, the credit for the excess plutonium produced is taken into account. In the equations to follow, the plutonium in the core is considered as $100 \% \mathrm{Pu}^{239}$, but they also apply to any mixture provided that concentrations, ratings, etc., are expressed in terms of "equivalent Pu" "39" $^{\prime}$ as defined in Section 2.1. By this treatment of higher isotopes, the ratios of worths of isotopes of plutonium in Table $V$, based on critical mass alone, axe still maintained. Since the breeding gain $G$ varies only slightly with isotopic variations, as shown by Table VII, this is a good assumption.

When the excess Pu ${ }^{239}$ produced by a breeder reactor is as signed some value, then the equation of the previous section linking the plutonium and fuel costs must be modified as follows:

$$
r W=V_{i}-q r G V_{0}
$$

where

$$
\begin{aligned}
& V_{i}=\text { value of initial charge of } \mathrm{Pu}^{239} \text { in } \frac{6}{2} / \mathrm{g} ; \\
& V_{0}=\text { value of excess } \mathrm{Pu}^{239} \text { produced in } \mathrm{f} / \mathrm{g} ;
\end{aligned}
$$




$$
\begin{aligned}
G= & \text { number of atoms of } \mathrm{Pu}^{239} \text { produced per fission in excess of } \\
& \text { the number required to maintain criticality; } \\
\mathrm{H}= & \mathrm{kWh} \text { (heat) per } \mathrm{g} \text { fissioned, } 2.26 \times 10^{4} ; \\
\mathrm{q}= & 240 / \mathrm{HE}=0.0266 \text { for } \mathrm{E}=0.40 .
\end{aligned}
$$

Values of W are given in Table XIV as a function of $r$ and $G$ as calculated from the formula with $q=0.0266$ and with the same value for input and output plutonium.

\section{Table XIV}

CONTRIBUTION OF RAW MATERIAL COSTS TO ELECTRICITY COST IN d/u.s.o. PER \&/g OF Pu ${ }^{239}$ PRICE AS A FUNCTION OF RATING AND BREEDING GAIN G

\begin{tabular}{r|r|r|r|r|r}
\hline$G$ & \multicolumn{1}{|l|}{50} & 100 & 200 & 500 & $\infty$ \\
\hline-1.0 & 0.047 & 0.037 & 0.032 & 0.029 & 0.027 \\
-0.8 & 0.042 & 0.032 & 0.027 & 0.024 & 0.022 \\
-0.6 & 0.036 & 0.026 & 0.021 & 0.018 & 0.016 \\
-0.4 & 0.031 & 0.021 & 0.016 & 0.013 & 0.011 \\
-0.2 & 0.025 & 0.015 & 0.010 & 0.007 & 0.005 \\
0 & 0.020 & 0.010 & 0.005 & 0.002 & 0 \\
0.2 & 0.015 & 0.005 & 0.000 & -0.003 & -0.005 \\
0.4 & 0.009 & -0.001 & -0.006 & -0.009 & -0.011 \\
0.6 & 0.004 & -0.006 & -0.011 & -0.014 & -0.016 \\
0.8 & -0.002 & -0.012 & -0.017 & -0.020 & -0.022 \\
1.0 & -0.007 & -0.017 & -0.022 & -0.025 & -0.027 \\
\hline
\end{tabular}

It can be seen from Table VII that for a typical large plutonium carbide-fueled fast reactor in the early years of its life, $G$ is about 0.6. This value is a potential value, not achievable in practice. Suppose that the core is reprocessed after a number of fissions equal to $20 \%$ of the $\mathrm{Pu}^{239}$ originally present, equivalent to about $5 \%$ burnup of heavy atoms, and $1 \%$ is lost in processing. Then $G$ is reduced by 0.05 . Further, suppose only $80 \%$ of the potential blanket breeding is actually achieved because of engineering compromises in a practical design, because of processing losses, and because the outermost blanket elements are not worth processing. Then $G$ is further reduced by about 0.15 . Thus a realistic value of $G$, taking account of losses, is 0.4 .

For currently proposed reactor designs, the overall rating $\mathrm{R}$ is about $0.5 \mathrm{MW} / \mathrm{kg}$, which corresponds to $r=87$. A good round number representing current designs is, therefore, 


$$
r=100 .
$$

Typical values for fuel costs, assuming

$$
r=100 \text { and } 200
$$

and

$$
V=\$ 5 / g
$$

are given in Table XV.

Table XV

FUEL COSTS FOR A LARGE CARBIDE-FUELED BREEDER*

\begin{tabular}{l|c|c|c}
\hline \multirow{2}{*}{$\begin{array}{c}V_{0} \\
\left(\frac{K}{2} / g\right)\end{array}$} & $r$ & \multicolumn{2}{|c}{ Fuel Cost $(\mathrm{d} /$ u.s.o. $)$} \\
\cline { 3 - 4 } & & No Losses & With Losses \\
\hline 5 & 200 & -0.055 & -0.030 \\
5 & 100 & -0.030 & -0.005 \\
2.5 & 100 & +0.010 & +0.025 \\
\hline
\end{tabular}

*Assumptions:

$$
\begin{aligned}
G= & 0.6 \text { for no losses, } 0.4 \text { including losses (losses } \\
& \text { include those due to blanket design - see text). } \\
V_{i}= & \frac{\pi}{2} 5 / g .
\end{aligned}
$$

The importance of rating and of losses is well demonstrated. The los ses considered appreciably reduce the credit due to fuel contributions.

Values may also be obtained from Table XIV for cases for which the input and output prices are different by reading off values corresponding to an adjusted value of $G$. For example, the values in Table XV for $V_{0}=\mathscr{t} 2.5 / \mathrm{g}$ were obtained by reading from Table XIV the values corresponding to half the actual values of $G$. Reducing the price of output plutonium increases fuel costs in Table $\mathrm{XV}$, as expected.

\subsection{Converter Reactors}

The same reactor was considered fueled with either plutonium or enriched uranium in order to obtain an upper limit for the ratio of the values of $\mathrm{Pu}^{239}$ and $\mathrm{U}^{235}$. The treatment may be generalised by expressing higher isotopes in terms of "equivalent $\mathrm{Pu}^{239}$ " or "equivalent $\mathrm{U}^{235}$ "from Table $V$. It has already been shown in Section 3.2 that, if only the initial 
capital charges are taken into account, $\mathrm{Pu}^{239}$ is worth about 1.4 times the value of $U^{235}$ (from Table $V$ ). In this section the superior breeding for the plutonium system is taken into account, and it is shown that $P^{239}$ is, therefore, worth approximately 2.0 times the value of $\mathrm{U}^{235}$ for present estimates of achievable overall rating.

This section is included for completeness. For reasons given in the next section, the somewhat smaller ratio $\mathrm{V} / \mathrm{U}$ obtained from a consideration of breeder reactors started up with $\mathrm{U}^{235}$ is to be preferred.

The ratio of values of $\mathrm{Pu}^{239}$ and $\mathrm{U}^{235}$ was calculated which made the fuel cost the same for a reactor used as a breeder or as a converter. From Section 3.3, the fuel cost in d/u.s.o. for a breeder reactor is given by

$$
r W=V-q r G V
$$

if it may be assumed that the same price exists for $\mathrm{Pu}^{239}$ initially and throughout the life of the reactor. The fuel cost for the same reactor fueled with $\mathrm{U}^{235}$ instead of $\mathrm{Pu}{ }^{239}$ is given by

$$
r^{\prime} W=U+q r^{\prime}\left(G_{1} U-G_{2} V\right)
$$

where

$$
\begin{aligned}
& \mathrm{G}_{1}=\text { number of atoms of } \mathrm{U}^{235} \text { consumed per fission, and } \\
& \mathrm{G}_{2}=\text { number of atoms of } \mathrm{Pu}^{239} \text { produced per fission. }
\end{aligned}
$$

Since the rating of each fuel element is to be invariant and the critical mass of $U^{235}$ is larger than that of $P u^{239}$ by a factor $y=1 / W_{5}(=1.4$ approximately, from Table V):

$$
x^{\prime}=x / y,
$$

and the equation may be rewritten as

$$
r W=y U+s\left(G_{1} U-G_{2} V\right)
$$

where

$$
\mathrm{s}=\mathrm{q} r=\mathrm{AR} / \mathrm{HI}_{\mathrm{a}}=0.388 \mathrm{R} / \mathrm{I}_{\mathrm{a}} \text {. }
$$

On making $W$ the same for breeders and converters, the two equations reduce to

$$
\frac{v}{U}=\frac{y+s G_{1}}{1+s\left(G_{2}-G\right)}
$$


Now, if

$\mathrm{G}_{\mathrm{u}}=$ breeding gain for the converter reactor, and

$\Delta G=$ difference between values of $G$ for breeder and converter,

then

$$
\begin{aligned}
G_{u} & =G_{2}-\frac{G_{1}}{y} ; \\
\Delta G & =G-G_{u} .
\end{aligned}
$$

Thus,

$$
\frac{V}{U}=y /\left\{1-\frac{\Delta G}{\frac{1}{s}+\frac{G_{1}}{y}}\right\} .
$$

The above equation is exact. The quantity $G_{1} / y$ is very nearly a constant for reactors of interest. Thus, the equation may be rewritten approximately as

$$
\frac{V}{U}=y /\left\{1-\frac{\Delta G}{\frac{1}{s}+0.8}\right\} .
$$

This equation is evaluated in Table XVI as a function of $\mathrm{s}$ and $\mathrm{G}$.

\section{Table XVI}

RATIO OF VALUES $V / U$ FOR Pu ${ }^{239}$ AND U ${ }^{235}$ FOR EQUAL FUEL COSTS FOR A BREEDER AND SIMILAR

CONVERTER REACTORS

\begin{tabular}{l|c|c|c|c}
\hline$\Delta G$ & 1 & 2 & 5 & 10 \\
\hline 0 & 1.40 & 1.40 & 1.40 & 1.40 \\
0.1 & 1.48 & 1.52 & 1.56 & 1.57 \\
0.2 & 1.57 & 1.65 & 1.75 & 1.80 \\
0.3 & 1.68 & 1.82 & 2.00 & 2.10 \\
0.4 & 1.80 & 2.02 & 2.33 & 2.52 \\
0.5 & 1.94 & 2.27 & 2.80 & 3.15 \\
\hline
\end{tabular}


For the same typical values as in the previous section, $R=0.5 \mathrm{MW} / \mathrm{kg}$ and $I_{a}=0.0837$, then

$$
s=2.3
$$

From Table VII, it can be seen that $\Delta G$ is about 0.1 for the comparison of $\mathrm{Pu}^{239}$ with $\mathrm{U}^{235}$, but rises to about 0.4 for equilibrium mixtures. From Table XVI,

$$
\mathrm{V} / \mathrm{U}=1.5 \mathrm{rising} \text { to } 2.0
$$

If the overall rating could be doubled, the margin would be increased to

$$
\mathrm{V} / \mathrm{U}=1.55 \text { rising to } 2.3
$$

The latter figures in each case are the better ones to represent a practical system of fast reactor power stations.

\subsection{Breeder Reactors Started Up with $\mathrm{U}^{235}$}

In the previous section, it was difficult to account properly for the change in the margin, $\Delta G$, with time after startup. The treatment given there would apply to a system containing a large number of reactors most of which had reached equilibrium. In this section a problem more appropriate for the early stages of a power programme is considered. The ratio $\mathrm{V} / \mathrm{U}$ was calculated which made the electricity cost the same for a breeder reactor fueled with plutonium throughout and the same reactor started up with enriched uranium and replenished with its bred plutonium thereafter.

The loss of plutonium product by this course of action has been evaluated in Table IX. The value of $G$ has been worked out as a function of irradiation dose for startup with $100 \% \mathrm{Pu}^{239}$ or $99 \% \mathrm{U}^{235}+1 \% \mathrm{U}^{234}$, with replenishment with $\mathrm{Pu}^{239}$ from the blanket in each case. The irradiation dose is defined as the average thermal energy in MWD generated per unit mass of equivalent $P u^{239}$ (from Table $V$ ) in $g$ in the core. The average irradiation dose $D$ is defined by

$$
D=0.365 R t \quad\left(M W D / g \quad \text { equiv } P^{239}\right),
$$

where

$$
t=\text { operating interval in years. }
$$

The second to the last line in Table IX gives the operating interval $t$ corresponding to the doses at the head of the Table for an overall rating 
$\mathrm{R}=0.5 \mathrm{MW} / \mathrm{kg}$. The plutonium product lost by the choice of $\mathrm{U}^{235}$ for initial loading is given by

$$
\text { Loss }=\frac{\mathrm{A}}{0.365 \mathrm{H}} \int_{0}^{\mathrm{D}} \Delta \mathrm{G} d \mathrm{D} \mathrm{kg} \text { of } \mathrm{Pu}^{239} / \mathrm{kg} \text { of equiv } \mathrm{Pu}^{239} \text { in system, }
$$

where $A$ and $H$ are numerical constants defined previously. Thus,

$$
\text { Loss }=1.06 \int_{0}^{D} \Delta G \mathrm{dD} \text {. }
$$

This formula illustrates the fact that the loss is dependent only on the irradiation dose $D$ and the difference between corresponding values of $G$, namely, $\Delta \mathrm{G}$.

It is much easier, however, to visualise the course of events if time is used as variable as follows:

$$
\begin{aligned}
\frac{\text { Annual loss of } \mathrm{Pu}^{239}}{\text { Mass of equiv } \mathrm{Pu}^{239} \text { in system }} & =(\mathrm{A} / \mathrm{H}) \mathrm{R} \Delta \mathrm{G} \\
& =0.388 \mathrm{R} \Delta \mathrm{G} .
\end{aligned}
$$

This quantity is given in Table IX for a typical value, $R=0.5 \mathrm{MW} / \mathrm{kg}$ of equiv $\mathrm{Pu}^{239}$. Since the burnup variable is time, this row of values refers to the explicit choice of $\mathrm{R}=0.5 \mathrm{MW} / \mathrm{kg}$. The cumulative loss given in Table IX is correct for any combination of $R$ and $t$ which leads to the value of $D$ at the head of the Table.

In order to evaluate this system, account must be taken of the different points in time of the various charges and credits. This can be done by "discounting back" the charge to a previous time, in the sense used by C. E. Iliffe. Suppose that the credit for $M g$ of $\mathrm{Pu}^{239}$ was $\mathrm{MV}_{0}$ at $t$ years after startup. This is equivalent to a credit at startup of

$$
M V_{0} \exp (-I t)
$$

where $I$ is the annual interest rate, not including amortisation.

The total loss of plutonium production, discounted back to the date of startup, is therefore given by

$$
L=1.06 \int_{0}^{\infty} \Delta G \exp (-D / Q) d D
$$


where

$$
\begin{aligned}
Q & =0.365 \mathrm{R} / \mathrm{I} \\
& =6.64 \mathrm{R}(\text { for } I=0.055) \\
& =6 \frac{2}{3} \mathrm{R} \text { very nearly. }
\end{aligned}
$$

The above integral is evaluated in Table XVII by use of the data in Table IX.

\section{Table XVII}

RELATIVE VALUES OF Pu ${ }^{239}$ AND U U 235 FROM A COMPARISON

\begin{tabular}{|c|c|c|c|c|c|}
\hline \multirow{2}{*}{$\begin{array}{c}\text { Overall } \\
\text { Rating } \\
\mathrm{R}(\mathrm{MW} / \mathrm{kg})\end{array}$} & \multirow{2}{*}{$Q$} & \multirow{2}{*}{$\begin{array}{l}\text { Discounted } \\
\text { Loss I }\end{array}$} & \multicolumn{3}{|c|}{ Ratio $V_{i} / U$} \\
\hline & & & $\begin{array}{c}\text { For } \\
\mathrm{V}_{0}=\frac{1}{2} \mathrm{U}\end{array}$ & $\begin{array}{c}\text { For } \\
\mathrm{V}_{0}=U\end{array}$ & $\begin{array}{c}\text { For } \\
V_{0}=V_{i}\end{array}$ \\
\hline 0.25 & 1.67 & 0.162 & 1.48 & 1.56 & 1.67 \\
\hline 0.5 & 3.33 & 0.234 & 1.52 & 1.63 & 1.83 \\
\hline 1.0 & 6.67 & 0.296 & 1.55 & 1.70 & 1.99 \\
\hline 1.5 & 10.00 & 0.324 & 1.56 & 1.72 & 2.07 \\
\hline$\infty$ & $\infty$ & 0.415 & 1.61 & 1.82 & 2.40 \\
\hline
\end{tabular}
OF A BREEDER REACTOR AND THE SAME REACTOR STARTED UP WITH ENRICHED URANIUM

It is now possible to write an equation which must be satisfied if the breeder reactor is to have the same fuel cost for $\mathrm{Pu}^{239}$ or $\mathrm{U}^{235}$ startup, namely,

$$
V_{i}=y U+L V_{0}
$$

where $y$ is set equal to 1.400 . This equation for $V_{i} / U$ is evaluated in Table XVII for three degrees of optimism about the market for plutonium, namely, that byproduct plutonium will have

(i) half the current value of $U^{235}$ (pessimistic);

(ii) the current value of $U^{235}$;

(iii) the same value as plutonium of the initial charge (optimistic).

It may be concluded from Table XVII that consideration of the improved breeding of plutonium-fueled reactors increases the value of $\mathrm{Pu}^{239}$ as compared with $\mathrm{U}^{235}$ by about $15 \%$. 
This is a smaller margin than that derived from a study of converter reactors in the previous section. In the present case, for the first time, the economic penalty is taken into account due to the delay in receiving the credit corresponding to bred plutonium. It can be seen from Table XVIII that for lower ratings or higher interest rates (that is, lower Q), the margin between the values of $\mathrm{U}^{235}$ and $\mathrm{Pu}^{239}$ becomes smaller.

Table XVIII

MONETARY VALUES FOR PLUTONIUM AND URANIUM ISOTOPES*

\begin{tabular}{l|c|c}
\hline & $\begin{array}{c}\text { Based on Capital } \\
\text { Charge Only } \\
(\mathbb{E} / \mathrm{g})\end{array}$ & $\begin{array}{c}\text { Breeding Gains } \\
\text { Also Considered } \\
(\mathrm{E} / \mathrm{g})\end{array}$ \\
\hline $\mathrm{U}^{233}$ & 8.5 & - \\
$\mathrm{U}^{234}$ & 0.5 & 0.5 \\
$\mathrm{U}^{235}$ & 5.0 & 5.0 \\
$\mathrm{U}^{236}$ & $\mathrm{Nil}$ & $\mathrm{Ni1}$ \\
$\mathrm{U}^{238}$ & $\mathrm{Ni1}$ & $\mathrm{Nil}$ \\
$\mathrm{Pu}^{239}$ & 7.0 & 8.0 \\
$\mathrm{Pu}^{240}$ & 1.0 & 1.2 \\
$\mathrm{Pu}^{241}$ & 10.5 & 12.0 \\
$\mathrm{Pu}^{242}$ & 1.0 & 1.2 \\
\hline
\end{tabular}

*The values are based on $5 / g$ for $U^{235}$. If plutonium can be obtained more cheaply than the figures given above, it becomes cheaper to start up fast reactors with $U^{235}$.

\subsection{Discussion and Conclusions}

(i) Fast reactors may be either net producers or consumers of plutonium, and the only completely satisfactory way to determine the price of plutonium is to consider the overall economics of a powerproducing network. However, by considering some special limited cases, some indications of the range of possible prices can be obtained. In particular, relative values for plutonium and $\mathrm{U}^{235}$ can be obtained by consideration of the relative merits of these prices in fast reactors.

(ii) If fast reactors can be made with such short doubling times that the net plutonium credit outweighs the inventory charges, designers may be tempted to favour a high plutonium price to increase the fuel credit. This advantage is an illusion because payments made for fuel 
at the start of a programme must be recouped at some time during the life of the system. Designers should favour the lowest price that will ensure adequate production of plutonium.

(iii) If the price of fuel is not determined by studying the economics of the nearest competitor, a price of plutonium may be obtained by comparison with the price of enriched uranium from diffusion plants. If the price of plutonium is set above a certain maximum, it becomes cheaper to start up fast reactors with $U^{235}$. If this maximum price is determined by the investment charges on the initial loading, the values of each isotope become as in Table XVIII. The monetary values are based on an assumed price for $U^{235}$ in highly enriched uranium of $55 / g$.

(iv) If the increased breeding with plutonium fuel is taken into account, the value of plutonium is increased by about $15 \%$, giving the values listed in Column 2 of Table XVIII. These figures can only be approximate and depend on the simplifying assumptions made on the future costs of plutonium and enriched uranium. When breeder reactors are started up with $\mathrm{U}^{235}$ but replenished with their own $\mathrm{Pu}^{239}$, the loss of plutonium product at an overall electrical rating of $0.2 \mathrm{MWe} / \mathrm{kg}$ equivalent $\mathrm{Pu}^{239}$ (taking account of out-of-pile inventory and load factor), would be $1.05 \mathrm{~kg}$ per installed MWe in the first $10 \mathrm{yr}, 0.55 \mathrm{~kg}$ in the next $10 \mathrm{yr}$, and a further $0.48 \mathrm{~kg}$ if the reactor life could be extended to infinity.

\section{References}

1. S. Yiftah, D. Okrent, and P. A. Moldauer, Fast Reactor Cross Sections, Pergamon Press (1960).

2. S, Yiftah and D. Okrent, Some Physics Calculations on the Performance of Large Fast Breeder Reactors, ANL-6212 (1960).

3. BNL-325, Neutron Cross Sections, Revised (1958).

4. J. F. Barry, J. I. Bunce, and J. L. Perkins, The Radiative Capture Cross-Section of $\mathrm{U}^{236}$ for Neutrons in the Energy Range 0.3 to $4.0 \mathrm{MeV}$, Proc. Phys. Soc., 78, 801 (1961).

5. D. Okrent, Performance of Large Fast Reactors Including Effects of Higher Isotopes, Recycling and Fission Products, Seminar on the Physics of Fast and Intermediate Reactors. SM-18/41 (1961). 


\section{Appendix 1}

\section{List of Symbols}

A Number of hours in 1 year, $8.76 \times 10^{3}$

$A_{i}$ Absorption rate in element $i$ in the reactor

B Burnup in $\mathrm{kg}$ fissioned per $\mathrm{kg}$ of total heavy atoms in the system

$\mathrm{B} *$ Burnup in $\mathrm{kg}$ fissioned per $\mathrm{kg}$ of equivalent $\mathrm{Pu}^{239}$ in the system

$B_{p} \quad$ Physicist's breeding ratio $=\frac{C_{8}+C_{0}}{A_{9}+A_{0}}$

$B_{C} \quad$ Chemist's breeding ratio neglecting $P^{242}$ capture $=\frac{C_{8}}{F_{9}+F_{0}+F_{1}+F_{2}}$

$B_{C}^{\prime} \quad$ Chemist's breeding ratio including $P^{242}$ capture $=\frac{C_{B}-C_{2}}{F_{9}+F_{0}+F_{1}+F_{2}}$

$C_{i}$ Capture rate in element $i$ in the reactor

D $\quad 0.365 \mathrm{Rt} \mathrm{MWD} / \mathrm{g}$ equiv $\mathrm{Pu}^{239}$

E Thermal efficiency of the fast reactor station, typically 0.40

$F_{i} \quad$ Fission rate in element $i$ in the reactor

$\mathrm{G} \quad$ Breeding gain, defined as $\mathrm{Pu}^{239}$ produced per fission in excess of that required to maintain criticality (see Section 2.2)

$\mathrm{H} \quad \mathrm{kWh}$ (heat) per $\mathrm{g}$ fissioned, $2.26 \times 10^{4}$

I Annual interest rate, typically 0.055

$I_{\mathrm{a}}$ Annual interest rate including amortisation, typically 0.0837

i Suffix taking the values $4,5,6,8,9,0,1$, and 2 for $U^{234}, U^{235}, U^{236}$, $\mathrm{U}^{238}, \mathrm{Pu}^{239}, \mathrm{Pu}^{240}, \mathrm{Pu}^{241}$, and $\mathrm{Pu}^{242}$, res pectively.

L Discounted loss defined in Section 3.5

$\mathrm{N}_{\mathrm{i}} \quad$ Atomic density of element $i$ in the core

Q $\quad 0.365 \mathrm{R} / \mathrm{I}$

q $240 / \mathrm{HE}$ 
$R$ Average rating, taking account of load factor and out-of-pile inventory, in $\mathrm{MW} / \mathrm{kg}$ of equiv $\mathrm{Pu}^{239}$

r REA $/ 240 I_{2}$

s $q r$

$t \quad$ Operating time of reactor in years

U Value of $\mathrm{U}^{235}$ in $\frac{\pi}{2} / \mathrm{g}$, typically $\frac{\pi}{2} 5 / \mathrm{g}$

$\mathrm{V}_{i} \quad$ Value of input $\mathrm{Pu}^{239}$

$\mathrm{V}_{0} \quad$ Value of output $\mathrm{Pu}^{239}$

W Contribution of fuel costs to electricity cost in d/u.s.o.

$w_{i} \frac{\bar{x}_{i}-x_{8}}{x_{9}-x_{8}}$

$\mathrm{x}_{\mathrm{i}} \quad$ Value of $\left(\mathrm{v} \sigma_{f}-\sigma_{f}-\sigma_{c}\right)$ for isotope $\mathrm{i}$

y $\quad 1 / w_{5}$, usually rounded off to 1.400 
Appendix 2

Conversion to Values in Dollars

In order to make comparison with American fuel prices easier, some of the calculations in this report have been repeated to give costs in American dollars.

Relative Values Based on Capital Charges

Following the argument of Section 3.2, the price of $\mathrm{Pu}^{239}, \mathrm{~V}$ in $\$ / \mathrm{g}$, is related to a given margin $\mathrm{W}$, in mills $/ \mathrm{kWh}$, by

$$
V=r W
$$

where $r$ is a reduced form of rating defined by

$$
r=R E A / 1000 I_{a}
$$

$\mathrm{I}_{\mathrm{a}}=$ interest and amortisation rate, typically 0.0837 ( $5 \frac{1}{2} \%$ written off after $20 \mathrm{yr}$ );

$R=$ average thermal rating of $\mathrm{Pu}^{239}$ in the system in $\mathrm{MW} / \mathrm{kg}$, taking account of load factor and out-of-pile inventory;

$E=$ thermal efficiency of station, 0.40

$A=$ number of hours in 1 year, $8.76 \times 10^{3}$.

For the typical values mentioned above

$$
r=41.9 R
$$

and, through combination of Tables $\mathrm{V}$ and XIII, the value of any isotope can be estimated. The results are shown graphically in Fig. 4, which gives data corresponding to that in Table XIII in the original report.

\section{Breeder Reactors}

The data given in Section 3.3 can also be converted to dollars.

We have marginal cost

$$
W=\frac{V_{i}}{r}-q G V_{0} \text {. }
$$


$V_{1}=$ value of initial charge in $\$ / g$,

$V_{0}=$ value of excess $P^{239}$ produced in $\$ / g_{\text {g }}$

and other symbols have the same meaning as before, then

$q=1000 / H E$

$=0.111$ for $E=0.40$

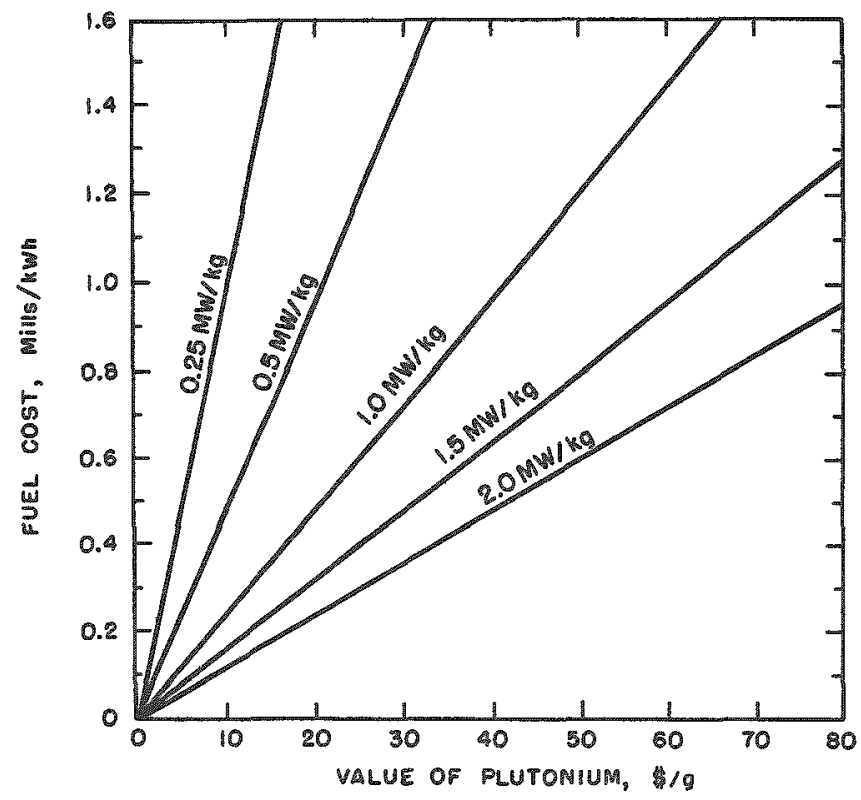

Fig. 4

Value of Plutonium to Fast Reactor Considering Only Investment Charges

ASSUMING THAT A GIVEN MARGIN OF COST CAN BE ALLOCATED TO INVESTMENT CHARGES ON THE FUEL, THIS GRAPH GIVES THE RESULTING VALUE OF PLUTONIUM IN $\$ g$.

IT IS ASSUMED THAT THE REACTOR IS SELF-SUSTAINING BUT NO CREDIT IS GIVEN FOR ANY SURPLUS PLUTONIUM PRODUCED. THE RATING IS AN OVERALL RATING INCLUDING LOAD FACTOR AND OUTOF-PILE INVENTORY. THE INTEREST AND AMORTISATION RATE IS 5-1/2\% WRITTEN OFF OVER 20 YEARS AND A THERMAL EFFICIENCY OF $40 \%$ IS ASSUMED.

The contribution of raw material costs to electricity costs for various breeding gains can then be evaluated in terms of mills/ $\mathrm{kWh}$ per $\$ /$ gram of $\mathrm{Pu}^{239}$ price.

The resulis corresponding to Table XIV are shown graphically in Fig. 5. Typical values for fuel costs for a large carbide-fueled fast reactor are given in Table XIX. 


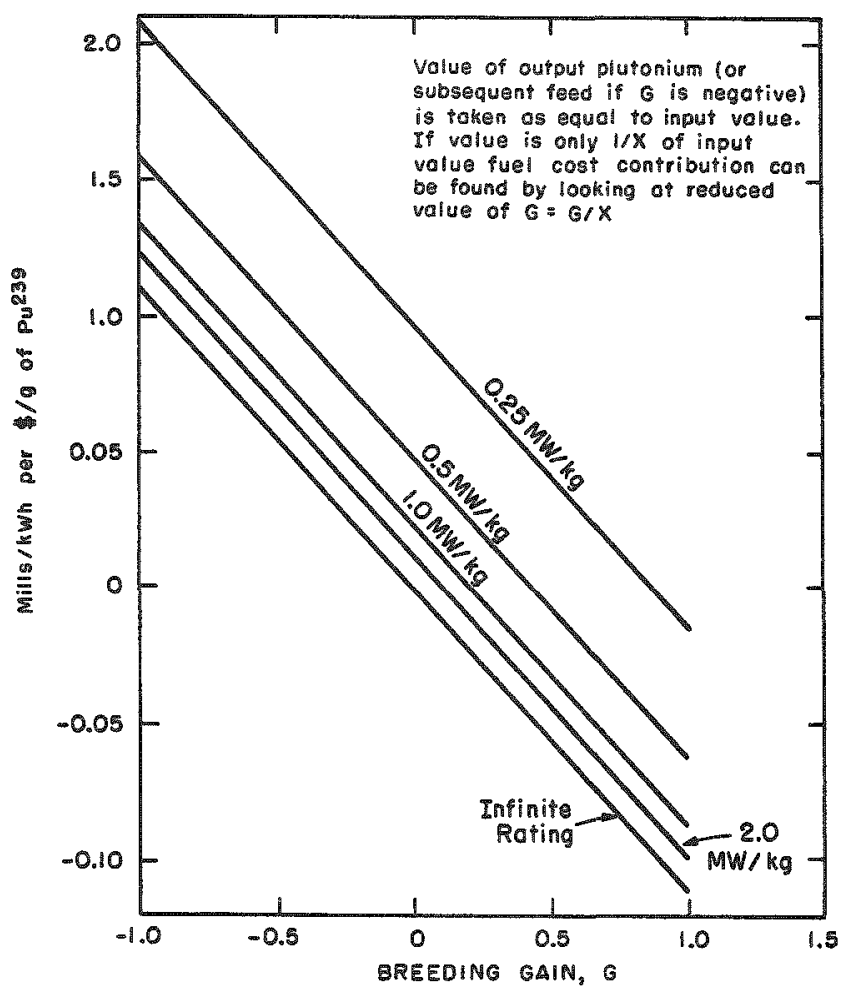

Fig. 5

Contribution of Fissile Material Costs in Mills/kWh per $\$ / \mathrm{g}$ Cost of $\mathrm{Pu}^{239}$

Table XIX

FUEL COSTS FOR LARGE BREEDER

\begin{tabular}{|c|c|c|c|c|}
\hline \multirow{2}{*}{$\begin{array}{c}\text { Value of } \\
\text { Initial Charge } \\
\text { of Plutonium } \\
(\$ / g)\end{array}$} & \multirow{2}{*}{$\begin{array}{l}\text { Value of } \\
\text { Excess } \\
\text { Plutonium } \\
\text { Produced } \\
(\$ / \mathrm{g})\end{array}$} & \multirow{2}{*}{$\begin{array}{l}\text { Rating } \\
(\mathrm{MW} / \mathrm{kg})\end{array}$} & \multicolumn{2}{|c|}{ Fuel Cost (mills/kWh) } \\
\hline & & & $\begin{array}{c}\text { No Losses: } \\
G=0.6\end{array}$ & $\begin{array}{l}\text { With Losses: } \\
\qquad G=0.4\end{array}$ \\
\hline 43 & 43 & $\frac{1}{2}$ & -0.78 & +0.17 \\
\hline 43 & 10 & $1^{2}$ & +0.34 & +0.58 \\
\hline 10 & 10 & 0.5 & -0.18 & +0.04 \\
\hline 10 & 5 & 0.5 & +0.15 & +0.26 \\
\hline 10 & 5 & 1.0 & -0.10 & +0.02 \\
\hline
\end{tabular}

Breeder Reactors Started Up with $U^{235}$

In Section 3.5 by comparing two similar reactors, one started up with $\mathrm{U}^{235}$ and one with $\mathrm{Pu}^{239}$, it was possible to arrive at comparative values for uranium and plutonium. Startup with $U^{235}$ results in a net loss of plutonium production, and the value of this plutonium is estimated by "discounting back" to the date of startup. Values obtained in this way can only be regarded as approximations owing to the assumptions made about the 
rate of surplus plutonium, but it can be seen from Table XVII that Pu ${ }^{239}$ will be worth at least 1.6 times $U^{235}$ in a typical reactor with a rating of about $0.5 \mathrm{MW} / \mathrm{kg}$. The suggested monetary values deduced on this basis and given in Table XIX have been converted to dollars per gram relative to a value of 12 dollars per gram $U^{235}$ and are given in Table XX.

Table XX

MONETARY VALUES FOR PLUTONIUM AND

URANIUM ISOTOPES RELATIVE TO A

VALUE OF $12 \$ / \mathrm{g}$ FOR U $\mathrm{U}^{235}$

\begin{tabular}{l|c|c}
\hline & $\begin{array}{c}\text { Based on Capital } \\
\text { Charges Only } \\
(\$ / g)\end{array}$ & $\begin{array}{c}\text { Breeding Gains } \\
\text { Also Considered } \\
(\$ / g)\end{array}$ \\
\hline $\mathrm{U}^{233}$ & 20 & - \\
$\mathrm{U}^{234}$ & 1.2 & 1.2 \\
$\mathrm{U}^{235}$ & 12 & 12 \\
$\mathrm{Pu}^{239}$ & 17 & 19 \\
$\mathrm{Pu}^{240}$ & 2.5 & 2.9 \\
$\mathrm{Pu}^{241}$ & 25 & 29 \\
$\mathrm{Pu}^{242}$ & 2.5 & 2.5 \\
\hline
\end{tabular}




\section{Discussion of Paper}

Presented by Mr. Smith

MR. GUERON (EURATOM):

Let me say first that this method of reduced variables is very attractive, and it seems very clear. It is, on the other hand, rather surprising to see the figures you arrive at, especially if you compare them with those which Kronberger has been advertising for the last year or so.

MR. SMITH:

Let me make clear those were relative values, but it shows that $\mathrm{Pu}^{241}$ is a valuable material for fast reactors. It is not, of course, such a valuable material for thermal reactors; this is why the other figures are lower.

MR. ZALESKI (EURATOM):

We have made some studies about the utilization of $\mathrm{U}^{235}$ for fast reactor programs, and we think that one important aspect of this utilization is the long term reactivity evolution. For oxide fuels in large reactors we have found that internal breeding can be about 0.8 or 0.9 . For this internal breeding we found that perhaps 50 to $100,000 \mathrm{MWD} /$ tonne is possible with little reactivity loss. In comparison with plutonium-fueled fast reactors with similar internal breeding ratios, $U^{235}$ has an advantage, since it is replaced by plutonium atoms of more reactivity worth. We think it is not unreasonable to start with $U^{235}$, at least from the point of view of the evolution of reactivity.

MR. SMITH:

There is one difficulty that arises if you do this transition. The reactivity worth of a $U^{235}$ mixture as it changes itself into plutonium does not change in quite the same way as the power output worth, as you put it into a constant flux. If you try to mix up elements which contain different amounts of $U^{235}$ and plutonium, you may get the reactivities $x$ ight but you will get the heat production by the individual elements wrong. Now, this applies particularly to $U^{235}$. It so happens that with the plutonium isotopes the difference is not marked. In fact, it is very small, so that you can fuel the reactor with elements having different mixtuxes of plutonium isotopes without getting into this difficulty; but if you put in $U^{235}$ elements, you may be in some difficulty in adjusting the individual ratings. 
MR, ZALESKI:

I agree quite completely with $D r$. Smith that there is a problem with adjusting of heat generation between the one and the other fuel subassemblies. But I think this problem exists even in a plutonium-fueled reactor, and that you must allow for some modification of heat generation in design.

MR. ZEBROSKI (General Electric):

I think as Dr. Häfele noted in his paper, there are quite sizable differences in the reaction rates that you calculate for different models and different fast cross sections. I think the nice table of relative values should have this uncertainty in the cross sections factored in. 


\title{
EFFECTS OF ANIAI AID RADIAL BLANRET DESIGN ON BREEDING AND ECONOMICS
}

\author{
M. A. Perks and R. M. Lord \\ U. K. Atomic Energy Authority \\ Risley, England
}

(Paper presented by Mr。J.E. Mann)

\section{Introduction}

In initial studies of fast power reactors, attention tends to be concentrated somewhat on aspects of core performance. In this paper consideration is given to variations of axial and radial blanket design, separately and in combination for a fixed core configuration. The effects of these variations on derived static parameters of the systemareconsidered, and the economic implications of the changed parameters are discussed.

A criterion for optimum radial blanket burnup for maximum economic benefit from blanket in terms of plutonium value, reprocessing cost, and nuclear cross sections is derived.

Results of some detailed calculations for a carbide blanket are presented and considered in the light of the derived formulae.

2. Calculations for a $500 \mathrm{MW}$ (e) Oxide-fuelled Reactor

Blanket variations have been investigated for a 3,400-litre 500-MW(e), two-zone, power-flattened core of reduced-density oxide fuel with sodium coolant and stainless steel canning and structure, and with the volume fractions and dimensions as in Table 1 . The effects of such variations on critical mass, core power form factor (maximum core power density/average core power density), breeding ratio (defined as (captures in $\left.U^{238}\right) /($ all fissions in plutonium in the system)], and the sodium-loss coefficient were examined. The effect of the radial and axial blankets were treated separately, the radial blankets by using a one-dimensional cylindrical model (see Section 3 below) and the axial blankets by using a one-dimensional slab model (see Section 4 below). The best individual cases were selected and combined in twodimensional models (see Section 5 below).

All calculations were done on the basis of multigroup diffusion theory (Ref. 1) with the cross sections of Hansen and Roach (Ref. 2). The full 16 groups were used in the one-dimensional cases, and in general a 5-group reduction of the 16 groups was used in the two-dimensional models. 
Table 1

\section{CORE SPECIEICATIONS}

Volume fractions

$\begin{array}{lll}\text { Fuel } & -0.308 & \\ \text { Coolant } & =0.364 & \text { (Sodium) } \\ \text { Structure and Canning } & =0.250 & \text { (Stainless Steels) } \\ \text { Void } & =0.078 & \end{array}$

Enrichments

Inner Core

Outer Core

$\frac{235}{238-235}$

$\frac{240}{240+239}$

Dimensions

Height

Diameter

Flattened Radius

Height/Diameter

Powex

Total Power

Max Rating $\left.\begin{array}{l}=0.175 \\ =0.1293\end{array}\right\} \frac{P u}{U+P u}$

$=0.004$

$=0.160$

$=47 \mathrm{in}$

$=75.4 \mathrm{in}$.

$=25.3 \mathrm{in} .=0.67$ Flattened

$=0.62$

$=1200 \mathrm{MWh}$ (includes $\simeq 50 \mathrm{MW}$ from breeders)

$=500 \mathrm{MWe}$

$=500 \mathrm{~W} / \mathrm{cc}$

The appropriate resonance self-shielding corrections were inserted as indicated by Ref. 2. An estimate for the $3-\mathrm{keV}$ sodium capture resonance, allowing for self-shielding, of $7 \mathrm{mb}$ in groups 7 and 8 was added in some of the cases (see Sections 3 and 4). Altogether, ten separate blanket material mixtures were considered, and the compositions are given in Table 2. Each region is identified by code letters for easy reference. Regions Bl-B3 are typical breeder regions with depleted uranium oxide fuel. Regions GI and BG consist mainly of graphite; G2 and G3 contain mainly of sodium and graphite, and GS is a graphite-stainless steel mixture.

Table 2

BLANKET REGION COMPOSITIONS (Volume Fractions)

\begin{tabular}{|c|c|c|c|c|c|c|c|}
\hline Region & Code & $\begin{array}{c}\mathrm{UO}_{2} \\
\text { (depleted) }\end{array}$ & $\begin{array}{c}\text { Stainless } \\
\text { Steel }\end{array}$ & Sodium & Graphite & $\begin{array}{c}\text { Boron } \\
\text { (Natural) }\end{array}$ & Void \\
\hline Radial Breeder & $\mathrm{B} 1$ & 0.466 & 0.266 & 0.216 & & & 0.052 \\
\hline Radial Breeder & $\mathrm{B} 2$ & 0.65 & 0.20 & 0.15 & & & \\
\hline Axial Breeder & B3 & 0.310 & 0.148 & 0.542 & & & \\
\hline Radial Graphite & G1 & & 0.10 & 0.08 & 0.82 & & \\
\hline Axial Graphite & G2 & & 0.143 & 0.489 & 0.368 & & \\
\hline Axial Graphite & G3 & & 0.250 & 0.364 & 0.308 & & 0.052 \\
\hline Graphite Steel & GS & & 0.51 & 0.08 & 0.41 & & \\
\hline Borated Graphite & $\mathrm{BG}$ & & 0.10 & 0.08 & 0.787 & 0.033 & \\
\hline Anial Void & $\mathrm{V}$ & & 0.250 & 0.364 & & & 0.386 \\
\hline Sodium & NA & & 0.1 & 0.9 & & & \\
\hline
\end{tabular}




\section{Radial Blanket Variations}

In the radial blanket variations only six of the regions detailed in Table 2 were considered. Of the breeder regions, BI is the standard reduced-density oxide breeder and $\mathrm{B} 2$ is one with a higher oxide fraction probably the maximum which could be achieved. Regions GI and GS are similar to each other except that $50 \%$ of the graphite in Gl has been replaced by stainless steel in GS. The borated graphite BG is included solely as a shielding region.

The combinations of radial blanket regions considered are given in Table 3. The total blanket thickness was maintained at 2 ft with a region of sodium, 3 ft thick outside this. Case 1 is the reference case which is of

Table 3

RADIAL BLANKET VARIATIONS

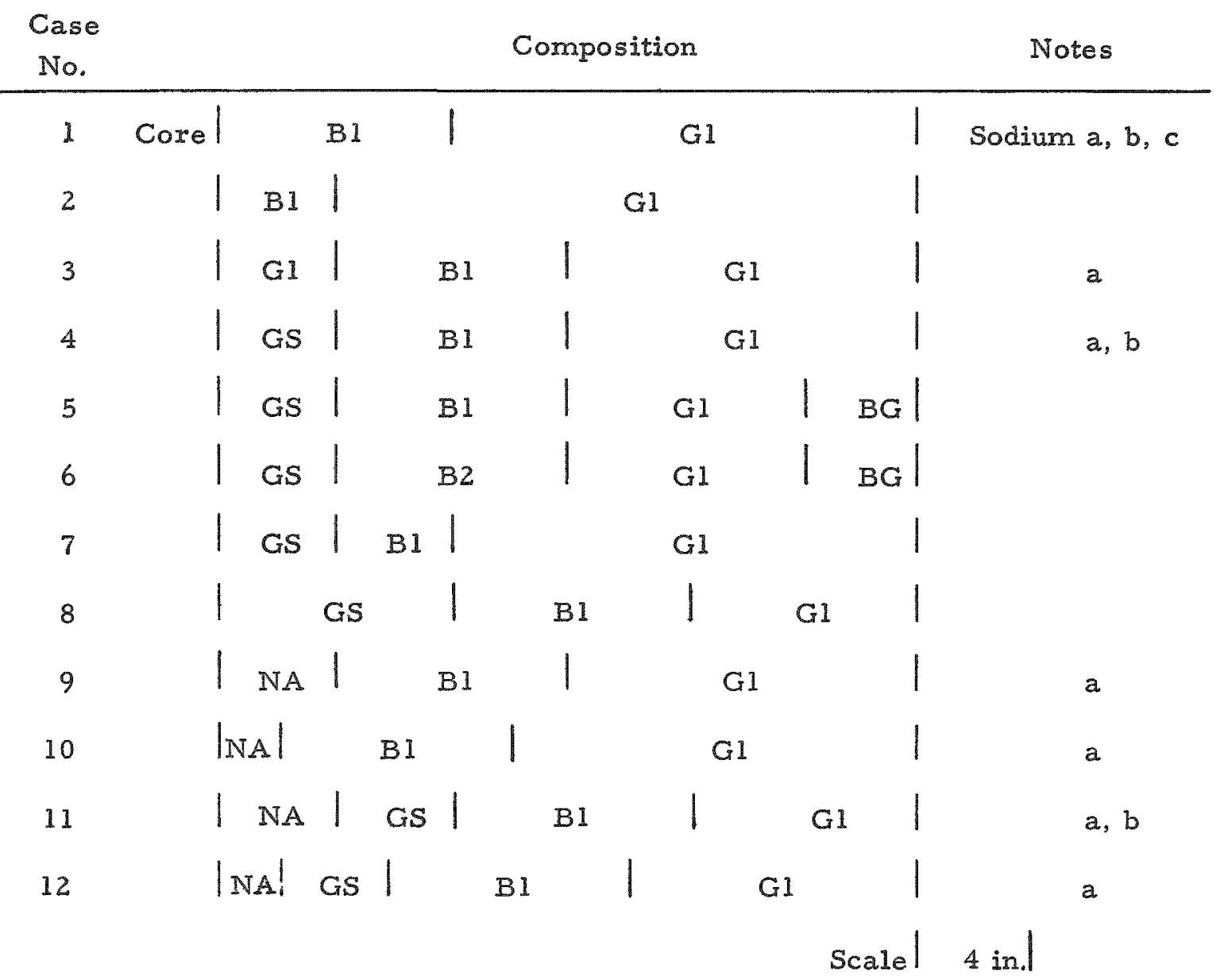

Notes: a - corresponding total sodium-loss case also calculated.

b - case done with allowance for $3-\mathrm{keV}$ sodium resonance.

c - sodium-loss case done with allowance for change in self-shielding. 
a conventional design with 8 -in. breeder adjacent to the core, followed by a graphite shield region. In case 2 the breeder thickness is reduced to 4 -in. Cases 3 and 4 have 4 in. of graphite and graphite-steel, respectively, between the 8 -in. breeder and the core. In case 5 the last 4 in. of graphite shield have been replaced by 4 in. of borated graphite (containing $4 \%$ natural boron). In case 6 the high-density breeder is considered. Cases 7 and 8 consider varying the graphite and breeder thicknesses of case 4. In cases 9 to 12 a region of $90 \%$ sodium is introduced next to the core. Variations in thickness of this region of 2 to 4 in. with both breeder and graphite following are considered.

The model adopted for the radial blanket calculations was an infinite cylinder with a constant axial buckling to simulate the actual finite height of the reactor. This buckling was determined with the radial blanket system of case 4 of Table 3 and the core composition and radius of Appendix A. The programme used will determine the value of buckling required to make the system critical. Sixteen equally spaced mesh-points were used in the inner enxichment zone of the core and 12 in the outer. Mesh-points at 1-in. intervals were used in the blankets.

For each of the 12 cases of Table 3 the value of $k$ eff was determined. Neutron flux, power density distributions, and neutron balance were also obtained. (Breeding ratios could be found directly from the neutron balance.) Of these results only case 4 had keff exactly unity, since this was the case for which the axial buckling was determined. It was, therefore, necessary to correct the other cases to obtain critical masses and breeding ratios. A calculation was done on the system of case 1 to find the fraction of plutonium in the oxide required to make keff unity. From this result a relationship between reactivity and mass of plutonium $\delta \mathrm{k} / \mathrm{k}=0.52 \delta \mathrm{M} / \mathrm{M}$ was found which could be used to correct other cases. (This treatment should be satisfactory for the order of changes involved - maximum $1.8 \%$ in $\mathrm{k}$.) The resulting critical masses are given in Table 4. The enrichment search described above gave in addition the critical breeding ratio for that case. This could also be determined by using the enrichment change found and applying it to the neutron balance of the original case. These two methods gave identical results and hence using enrichment changes to correct breeding ratios in the other cases was adopted as satisfactory. It was also possible to check that these enrichment corrections would have very little effect on the power form factors found in the $k$ calculations.

Results for all 12 cases are given in Table 4. The second column gives critical mass (inTe of plutonium metal). Case 3 has the lowest mass because of the effect of the graphite reflector. Cases 4 to 8 with graphitesteel reflectors also have appreciably smaller masses than the conventronal case 1 (by $30 \mathrm{~kg}$ ). Next, in Table 4, are given the core, breeder, and total breeding ratios. The first conclusion to be drawn from these results is that replacing $4 \mathrm{in}$, of breeder adjacent to the core by graphite or graphite-steel 
does not necessarily produce a large decrease in the breeding ratio. The effect on the core breeding ratio is in fact to increase it by 0.02 . This is due to marked softening of the spectrum at the edge of the core. The breeder itself generally has its breeding ratio reduced, but again spectrum softening offsets to some extent the inevitable decrease arising from moving the breeder out 4 in. In particular, case 3 has an overall ratio very close to the conventional case 1. With the GS region a significant loss (0.036) results, although this could be restored by using the more dense breeder B2. Case 7 which has 4 in. of breeder outside $4 \mathrm{in.}$ of graphite-steel has a total breeding ratio very close to case 2 with 4 in. of breeder adjacent to the core. Of the cases with a sodium region adjacent to the core, only case 10, with 2 in. sodium before the breeder, suffers a reasonably small loss in total breeding ratio.

Table 4

RADIAL BLANKET CASES - RESULTS

\begin{tabular}{|c|c|c|c|c|c|c|}
\hline \multirow{2}{*}{$\begin{array}{l}\text { Case } \\
\text { No. }\end{array}$} & \multirow{2}{*}{$\begin{array}{c}\text { Critical Mass } \\
(\mathrm{Te} \mathrm{Pu})\end{array}$} & \multicolumn{3}{|c|}{ Breeding Ratio } & \multirow{2}{*}{$\begin{array}{l}\text { Form Factor } \\
\text { (overall radial) }\end{array}$} & \multirow{2}{*}{$\begin{array}{c}\text { Sodium-loss } \\
\text { Reactivity Change } \\
(\%)\end{array}$} \\
\hline & & Core & Breeder & Total & & \\
\hline 1 & 1.611 & 0.995 & 0.341 & 1.336 & 1.234 & -0.67 \\
\hline 2 & 1.611 & 0.994 & 0.246 & 1.240 & 1.230 & \\
\hline 3 & 1.573 & 1.017 & 0.311 & 1.328 & 1.159 & -0.45 \\
\hline 4 & 1.582 & 1.014 & 0.286 & 1.300 & 1.181 & -0.51 \\
\hline 5 & 1.582 & 1.014 & 0.286 & 1.300 & 1.181 & \\
\hline 6 & 1.583 & 1.015 & 0.326 & 1.341 & 1.182 & \\
\hline 7 & 1.583 & 1.014 & 0.224 & 1.238 & 1.181 & \\
\hline 8 & 1.575 & 1.017 & 0.194 & 1.211 & 1.172 & \\
\hline 9 & 1.624 & 0.987 & 0.298 & 1.285 & 1.255 & -3.56 \\
\hline 10 & 1.619 & 0.992 & 0.319 & 1.311 & 1.247 & -2.40 \\
\hline 11 & 1.604 & 0.999 & 0.236 & 1.235 & 1.199 & -3.71 \\
\hline 12 & 1.595 & 1.006 & 0.261 & 1.267 & 1.193 & -2.46 \\
\hline
\end{tabular}

The next quantity derived in Table 4 is the overall radial power form factor. Case 3 has the closest value to unity and shows a considerable improvement (0.07) over the conventional case 1 . This is due to the rise of power density at the edge of the core with the softened spectrum from the graphite reflector. Cases 4 to 8 with graphite-steel reflectors also show significantly better form factors than case 1.

The last item in Table 4 is the change in reactivity due to complete sodium loss from the reactor. These results were obtained by direct calculation for the system with no sodium present. In all the cases the change is negative. Although the absolute magnitude of the reactivity changes provided by one-dimensional estimates will be in error (because of the large change in leakage associated with sodium loss), the order of the effects shown should be meaningful. 
For cases 1, 4 , and 11, the effect of the $3-\mathrm{keV}$ resonance in the sodium capture cross section (which is not included in the standard HansenRoach set was calculated. By making allowance for this as indicated in Section 2 , it was found that this decreased keff by $0.1 \%$ for cases 1 and 4 and by $0.7 \%$ for case 11 . Hence, the estimated sodium-loss reactivity changes in Table 4 should be increased by about $+0.1 \%$ for cases 1,3 , and 4 by about $+0.7 \%$ for cases 9 to 12 .

For case 1 , only the effect of the change in self-shielding on the $U^{238}$ capture cross sections upon loss of sodium was considered. This was found to be small - increasing the sodium loss reactivity change by only $+0.04 \%$.

\section{Axial Blanket Variations}

The variations treated for the axial blankets are detailed in Tables 5 and 7. Five of the regions listed in Appendix $B$ were used in these variations: B3, G2, GS, sodium, and void (V).

Table 5

\section{SYMMETRIC AXIAL BLANKET CONFIGURATIONS}

Case

No.

Notes

\begin{tabular}{|c|c|c|c|c|c|c|c|}
\hline 1 & Core & & & & $\mathrm{V}$ & $\mathrm{G}_{2}$ & $b$ \\
\hline 2 & & B3 & & & $V$ & G2 & $a$ \\
\hline 3 & & & $V$ & & B3 & $G 2$ & \\
\hline 4 & & GS & B3 & & V & $G 2$ & a. \\
\hline 5 & & B3 & GS & & $\mathrm{V}$ & $\mathrm{G} 2$ & \\
\hline 6 & & GS & & & $\mathrm{V}$ & $\mathrm{G} 2$ & \\
\hline 7 & & NA & B3 & & V & $\mathrm{G} 2$ & $a, b$ \\
\hline 8 & & $\mathrm{NA}$ & $\mathrm{G} 2$ & $\mathrm{B3}$ & $\mathrm{V}$ & $\mathrm{G} 2$ & $a$ \\
\hline
\end{tabular}

Notes: a - Corresponding total sodium loss case calculated.

b - Case done with allowance for the $3 \mathrm{keV}$ sodium resonance calculated. 
B3 is a typical axial breeder having the same fuel fraction as the core, G2 is a graphite region having $36.8 \%$ of graphite, $V$ is a void region and has the same coolant and stainless steel fractions as the core and NA is a sodium region that has $90 \%$ sodium and $10 \%$ structure. The use of region GS as an axial blanket is perhaps unrealistic (in view of the coolant fraction), and in the 2 -dimensional cases this was changed to $G 3$, which has the same coolant, structure, and canning fractions as the core.

The core was treated as a slab, 47 in. thick, with the average core composition of the two-zone core and the critical radial buckling determined for case 1 (see Table 5). This radial buckling was used throughout the axial variations. The othere cases (cases $2-14$ ) were thus not exactly critical. The breeding ratios were corrected for this and the critical mass obtained as indicated in Section 3 above. Twenty-four equally spaced mesh parts were used in the core and mesh points at 1 -in. intervals used in the blankets.

The same trends axe shown for the axial blankets as for the radial blankets but in general the changes are smaller.

\section{4(a) Symmetric Cases}

The second column of Table 6 gives the critical mass. The conventional case (case 1) has a critical mass of 1.582 tonne. Halving the breeder thickness increases the critical mass by $3 \mathrm{~kg}$, and interchanging the void and breeder regions (see case 3 ) increases it by $7 \mathrm{~kg}$.

Table 6

SYMMETRIC AKIAL BIANKE' CONHCURA HOAS - RESULTS

\begin{tabular}{|c|c|c|c|c|c|c|}
\hline \multirow{2}{*}{$\begin{array}{l}\text { Case } \\
\text { No }\end{array}$} & \multirow{2}{*}{$\begin{array}{c}\text { Critical Mass } \\
(\mathrm{T} \Theta)\end{array}$} & \multicolumn{3}{|c|}{ Brosding Ratio } & \multirow{2}{*}{$\begin{array}{c}\text { Power } \\
\text { Form Eactor }\end{array}$} & \multirow{2}{*}{$\begin{array}{c}\text { Sodiun-loss } \\
\text { Reactivity Change } \\
\text { (") }\end{array}$} \\
\hline & & Core & Bredier & Oxeall & & \\
\hline 1 & 1.582 & 1.004 & 0.182 & 1.186 & 1.277 & - \\
\hline 2 & 1.585 & 1.013 & 0.1074 & 1.1087 & 1278 & -1.5 \\
\hline 3 & 1.589 & 0.997 & 0.1003 & 1.097 & 1.284 & - \\
\hline 4 & 1.562 & 1.025 & 0.112 & $1.13-18$ & 1235 & -1.0 \\
\hline 5 & 1.578 & 1.008 & 0.1337 & 1. $1 . \frac{1}{1} 14$ & 1269 & - \\
\hline 6 & 1.562 & 1.026 & 0.1525 & 1.1550 & $1.23 \cdot \frac{1}{3}$ & - \\
\hline 7 & 1.538 & 0.9996 & 0.0977 & 10073 & 1.281 & -3.9 \\
\hline 8 & 1578 & 1009 & 0.1035 & 1.11 .0 & 1.257 & -3.9 \\
\hline
\end{tabular}

Cases 4-6 show the effect of introducing a 4 -in. region of graphite-stainless steel near to the core. The mass is reduced by $20 \mathrm{~kg}$ when this region is adjacent to the core, but is hardly affected when this region is placed after the breeder.

Cases 7-8 show the effect of introducing a sodium region adjacent to the core. Case 7 shows the effect on the conventional case (case 1) of replacing the first $4 \mathrm{in}$. of breeder by sodium and gives an increase of $6 \mathrm{~kg}$ in the critical mass. Case 8 , which shows the effect on a case with a graphite region (cf. case 4), gives an increase of $16 \mathrm{~kg}$. 
Columns $3-5$ of Table 6 give the breeding from core and breeder, and the overall breeding ratio. The conventional case (case 1) has an overall breeding ratio of 1.186: 1.004 from the core and 0.182 from the breeder. Halving the breeder thickness (see case 2) approximately halves the contribution from the breeder and reduces that from the core slightly. Interchanging the void and breeder regions in case 1 (see case 3 ) reduces the breeding from the core by 0.0069 and again approximately halves that from the breeder.

Cases $4-6$, cores with graphite regions, show an improvement in the core breeding of about 0.02 when the graphite region is adjacent to the core and of about 0.004 when the graphite is placed after the breeder. However, the breeding from the breeder is reduced from 0.02 to 0.07 in the se cases, and the net result is that the overall breeding ratios are 0.001 (case 6), 0.045 (case 5), and 0.051 (case 4) smaller than case 1 - the reference case.

Cases 7 and 8 , cases with sodium regions, show an overall reduction in breeding compared to case 1 - in case 7 both the core and breeder breeding are reduced and in case 8 the core breeding is increased by 0.0054 (due to the presence of the graphite region) and the breeder breeding reduced by 0.08 .

Column 6 of the table gives the power form factor of the core. The conventional case (case 1) gives a figure of 1.277 for the form factor. Halving the breeder thickness (see case 2) makes little change, but interchanging the void and breeder (see case 3) increases the form factor by 0.007 .

Cases 4 and 6 show the significant improvement in form factor obtained by the inclusion of a graphite region, case 6 giving an improvement of 0.043 .

Cases 7 and 8 show the effect on the form factor of a sodium region adjacent to the core. The form factor is poorer compared with the corresponding case without the sodium region, but case 8 still yields an improvement of 0.02 over the conventional case.

Column 7 of the table gives the change in reactivity for complete loss of sodium from the system for the cases in which it was calculated. Cases 7 and 8 show that the inclusion of a sodium region leads to an improvein sodium-loss coefficient of about $-2 \%$. The correction to the sodium cross section to allow for the $3-\mathrm{keV}$ resonance (see Section 3 above) when used in cases 1 and 7 reduced the reactivity by $0.1 \%$. 


\section{4(b) Asymmetric Cases}

Practical reasons may dictate the use of an asymmetric configuration of the blankets. In particular, it may be desirable to have all the void at one end of the core. Cases 9-14 of Table 7 show various arrange ments with $24 \mathrm{in}$ of void and $4 \mathrm{in}$. of graphite at one end, and differing blanket configurations at the other end.

Table 7

ASYMMETRIC AXIAL BLANKET CONFIGURATIONS

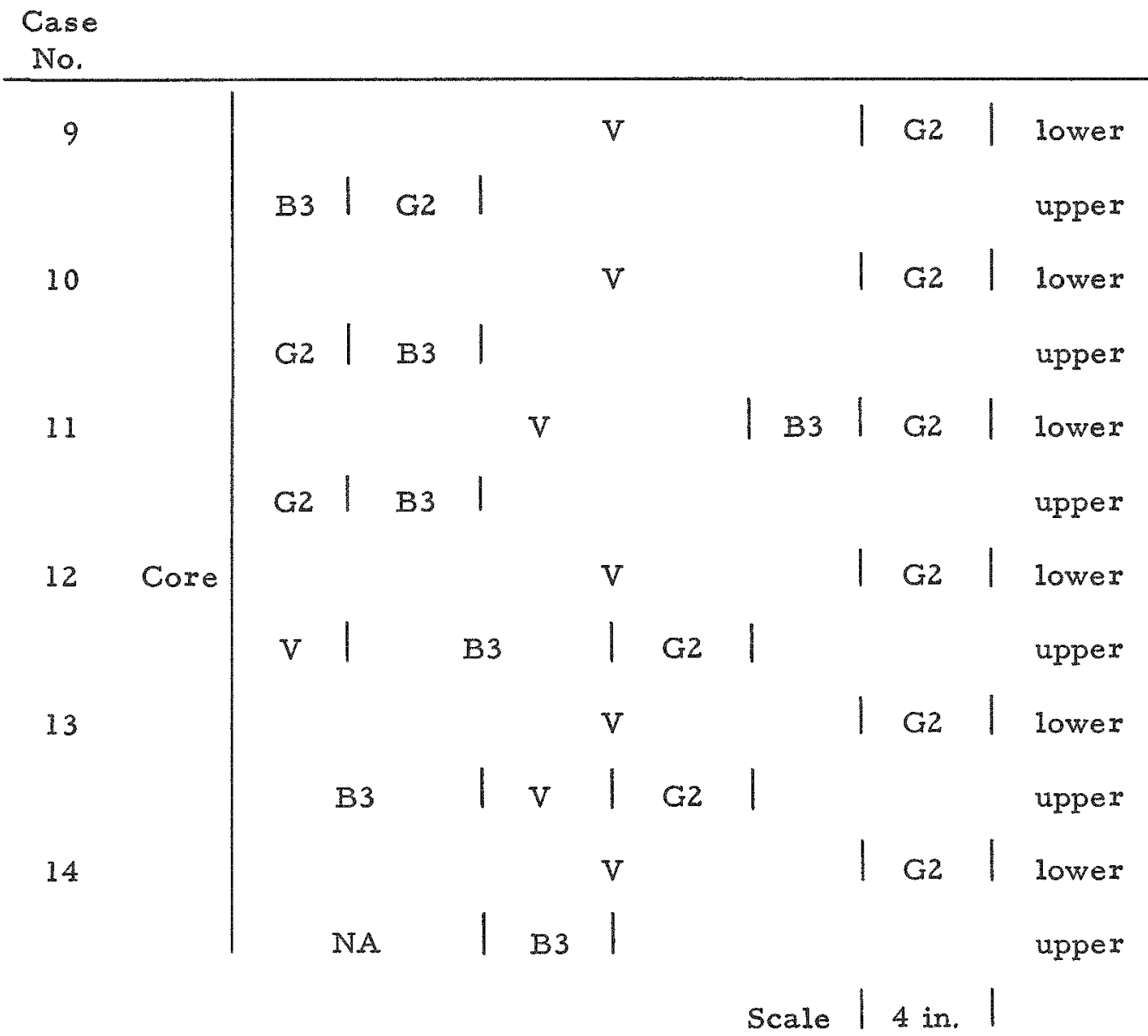

These cases (see Table 8) all show poorer overall performance compared with the symmetric cases of Section 2.21, which is to be expected since effectively only one end of the core is being used.

The effects are of the same size, but down by about a factor of two from those for the corresponding symmetric cases. Case 14 shows the effect of putting all the sodium of case 7 at one end; this reduces the sodiumlos coefficient by $+0.2 \%$. 
Table 8

ASYMMETRIC BLANKET CONEIGURATIONS - RESULTS

\begin{tabular}{c|c|c|c|c|c|c}
\hline \multirow{2}{*}{$\begin{array}{c}\text { Case } \\
\text { No. }\end{array}$} & $\begin{array}{c}\text { Critical Mass } \\
\text { (Te) }\end{array}$ & \multicolumn{3}{|c|}{ Breeding Ratio } & \multicolumn{1}{c|}{$\begin{array}{c}\text { Power } \\
\text { Form Factor }\end{array}$} & $\begin{array}{c}\text { Sodium-loss } \\
\text { Reactivity Change } \\
\text { (\%) }\end{array}$ \\
\cline { 2 - 6 } & Core & Blanket & Overal1 & & - \\
\hline 9 & 1.600 & 0.9867 & 0.0372 & 1.024 & 1.303 & - \\
11 & 1.596 & 0.9911 & 0.0210 & 1.0119 & 1.293 & - \\
12 & 1.596 & 0.9916 & 0.0383 & 1.0299 & 1.291 & - \\
13 & 1.591 & 0.995 & 0.074 & 1.069 & 1.288 & - \\
14 & 1.589 & 0.997 & 0.082 & 1.079 & 1.286 & -3.67 \\
\hline
\end{tabular}

\section{Two-dimensional Cases}

Cases selected for further consideration are numbers $1,3,4$, and 11 of the radial variations, and numbers $1,6,8$, and 14 of the axial variations.

A reference case, an example of conventional design, is provided by case 1 (see Table 9), which is a combination of the one-dimensional reference cases (numbers 1).

Table 9

TWO-DIMENSIONAL CORES

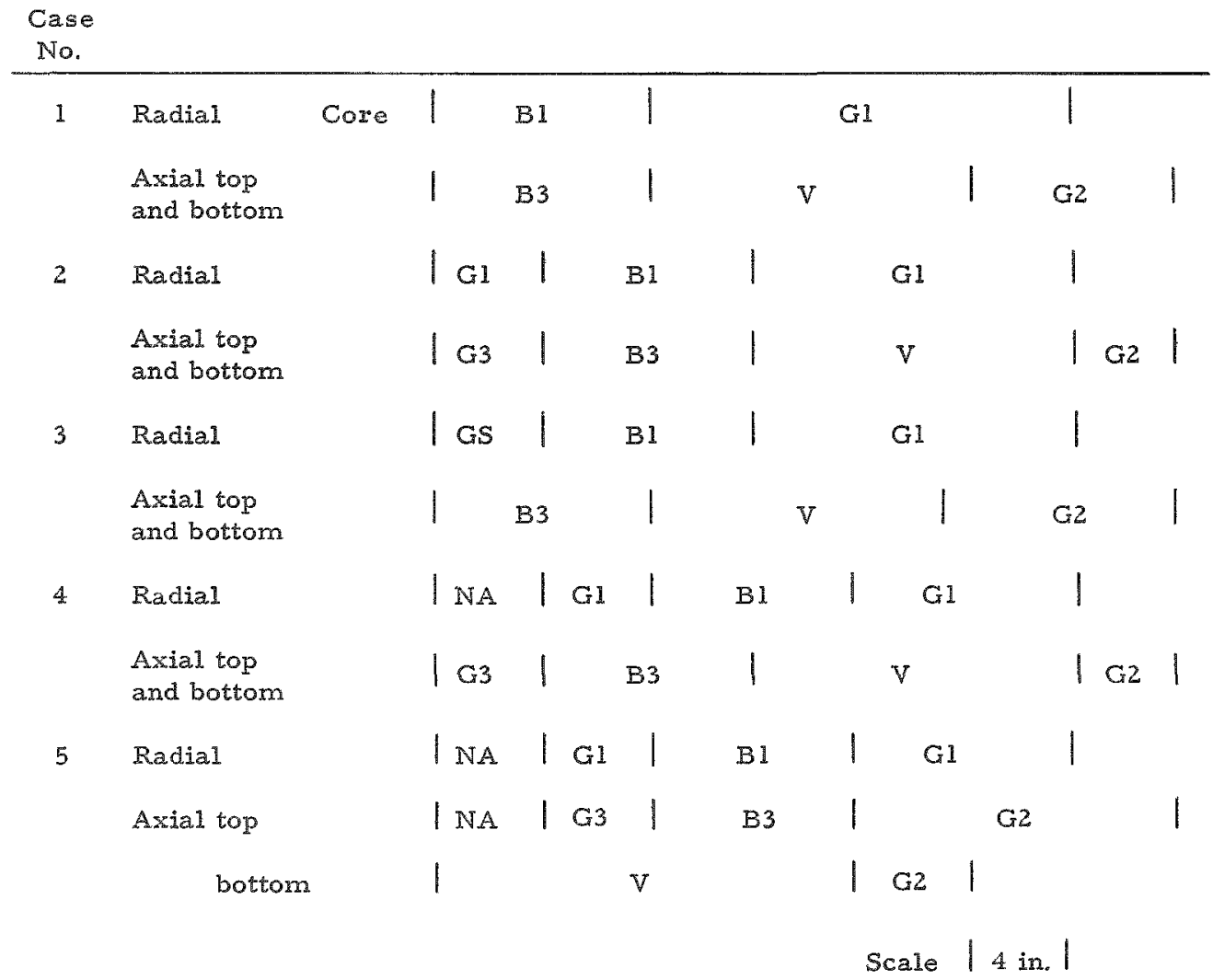


Case 2 of Table 9 was obtained by combining case 3 of the radial cases and case 6 of the axial cases, which give lowest critical mass and best form factors.

Case 3 is a combination of the conventional axial breeder (case 1) and the radial case 4 , which also had a low critical mass and form factor.

Case 4 is a combination of axial case 6 and radial case 11 (with GS replaced by G1), chosen because of the large negative sodium-loss coefficient.

Cases 1 through 4 are symmetric. One 2 -dimensional asymmetric case, case 5, was obtained by combining the radial case 11 (with G5 replaced by G1) with an upper axial blanket similar to axial case 8 (but with 8 in. of breeder and no void) and the bottom axial blanket as in axial case 14 .

The dimensions and configurations of these cases are given in Fig. 1. Evenly spaced meshes were used in the inner core, outer core, and blankets. The radial mesh consisted of 7 points in the

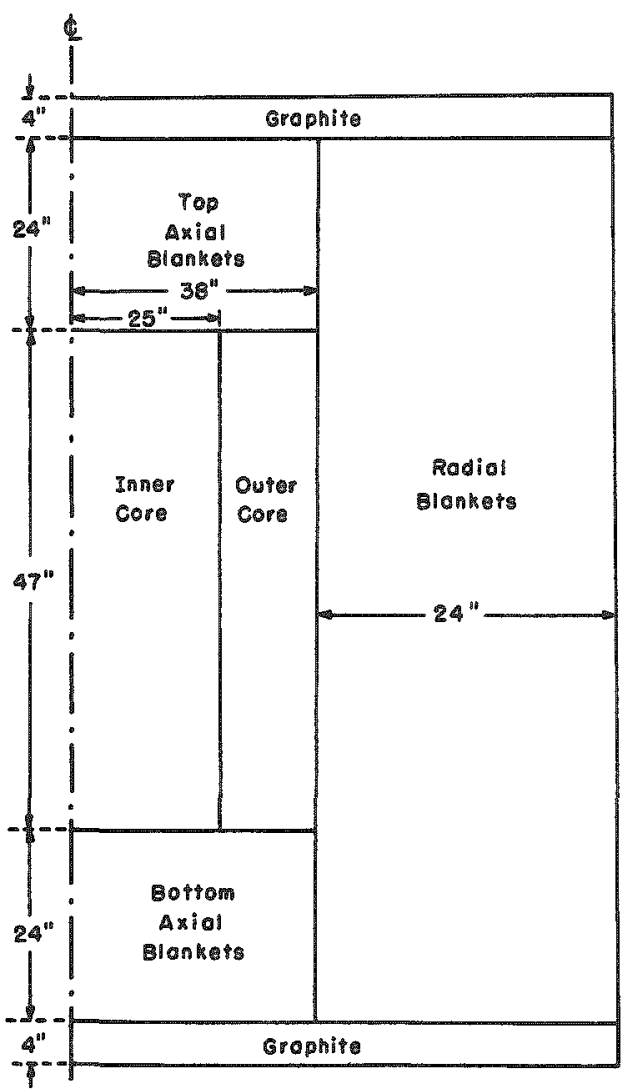

Fig. 1. Cross Section of System inner core, 3 points in the outer core, and 6 points in the blankets. The axial mesh consisted of 12 points in the core and of 16 points in the blanket regions.

In case 2 another calculation was done to investigate the effect of mesh length on the calculations. Twice the number of points (in each direction) as given above (case $2 / D$ ) were considered.

In the two-dimensional calculations, the Roach set was condensed to 5 groups by combining groups 1 to 3,4 to 6,7 to 9,10 and 11 and 12 to 16 . The condensing spectra used were taken from the appropriate onedimensional 16-group case. In case 2, a calculation with the full 16 groups was performed in addition to the 5 -group calculation (case 2/16).

For each of these two-dimensional cases the value of $k_{\text {eff }}$ was determined. The critical masses and breeding ratios were obtained by using the same relationship between $\delta \mathrm{k}$ and $\delta \mathrm{m}$ as in the one-dimensional cases. The axial, radial, and overall power form factors wexe also calculated for each case. The change in reactivity on complete removal of sodium from the reactor was determined directly in all cases except case $2 / D$. 
The results of these two-dimensional calculations are given in Table 10; comparison with Tables 4,6 and 8 show a very satisfactory agreement with the corresponding axial and radial calculations except for sodium-loss values. For example, the value obtained for the critical mass in each of the two-dimensional cases lies between those of the two corresponding one-dimensional cases.

Table 10

TWO-DIMENSIONAL CASES - RESULTS

\begin{tabular}{|c|c|c|c|c|c|c|c|c|c|}
\hline \multirow{2}{*}{$\begin{array}{l}\text { Case } \\
\text { No. }\end{array}$} & \multirow{2}{*}{$\begin{array}{c}\text { Critical Mass } \\
\text { (Te) }\end{array}$} & \multicolumn{4}{|c|}{ Breeding Ratio } & \multicolumn{3}{|c|}{ Power Form Eactors } & \multirow{2}{*}{$\begin{array}{c}\text { Sodium-loss } \\
\text { Reactivity Change } \\
(\%)\end{array}$} \\
\hline & & Core & Radial & Axial & Total & Radial & Axial & Overall & \\
\hline 1 & 1.602 & 1.015 & 0.419 & 0.188 & 1.622 & 1.235 & 1.263 & 1.560 & +0.15 \\
\hline 2 & 1.569 & 1.072 & 0.344 & 0.173 & 1.589 & 1.168 & 1.241 & 1.449 & -0.15 \\
\hline $2 / D$ & 1.577 & 1.069 & 0.366 & 0.178 & 1.613 & 1.181 & 1.245 & 1.470 & $\infty$ \\
\hline $2 / 16$ & 1.548 & 1.020 & 0.338 & 0.168 & 1.526 & 1.125 & 1.233 & 1.387 & +0.25 \\
\hline 3 & 1.582 & 1.042 & 0.309 & 0.204 & 1.555 & 1.178 & 1.274 & 1.501 & -0.14 \\
\hline 4 & 1.589 & 1.056 & 0.297 & 0.181 & 1.534 & 1.210 & 1.240 & 1.500 & -2.2 \\
\hline 5 & 1.589 & 1.055 & 0.321 & 0.080 & 1.456 & 1.200 & 1.248 & 1.497 & -2.8 \\
\hline
\end{tabular}

Table 10A

$\left(\delta u / 10^{-6} \mathrm{C}\right)$ AS A FUNCTION OF $Q / \mathrm{C}$

FOR THE REACTORS OF TABIE 10

(changes relative to case 1)

\begin{tabular}{|c|c|c|c|}
\hline $\begin{array}{l}\text { Cas } \\
\text { No. }\end{array}$ & 0.1 & 0.05 & 0.025 \\
\hline 2 & 0.00035 & 0.00040 & 0.00043 \\
\hline 3 & 0.00136 & 0.00134 & 0.00132 \\
\hline 4 & 0.00161 & 0.00167 & 0.00170 \\
\hline 5 & 0.00329 & 0.00332 & 0.00334 \\
\hline
\end{tabular}

The value of the core breeding ratio obtained in the two-dimensional calculations is always greater than the values obtained in the corresponding one-dimensional axial and radial cases. This is because in the onedimensional cases the beneficial effect of spectrum softening on breeding ratio at edges of the core is obtained in one direction only. This effect is particularly noticeable in the cases which have graphite regions included in the blankets. The same effect occurs in the blanket regions. It is the only reason why the two-dimensional value of the breeding ratio for the axial blanket is larger than in the corresponding one-dimensional axial calculation. In the radial breeder the difference is much greater and is due mainly to the fact that the radial blanket in the two-dimensional cases is twice the length of the core (see Fig. 1).

Both axial and radial form factors agree very well with the corresponding one-dimensional case. As indicated in Section 3 , the magnitude 
of the sodium-loss coefficient cannot be anticipated by a combination of results for the corresponding one-dimensional case. An attempt was made to allow for the change in buckling in the one-dimensional cases on loss of sodium through use of leakages calculated from the two-dimensional case 3 with and without sodium. This reduces the discrepancy between the onedimensional and two-dimensional sodium-loss results; for example, application of this correction to radial case 1 gave $+0.01 \%(=0.67 \%$ without the correction), which is now in much better agreement with the two-dimensional case (see Table 11).

Table 11

ONE-DIMENSIONAI SODIUM-LOSS CASES ALLOWING FOR CHANGE IN LEAKAGE

\begin{tabular}{|c|c|c|c|c|}
\hline Case & $\begin{array}{l}\text { Buckling }\left(\mathrm{Bn}^{2}\right) \\
\text { With Sodium }\end{array}$ & $\begin{array}{l}\text { Buckling }\left(\mathrm{Bo}^{2}\right) \\
\text { Without Sodium }\end{array}$ & $\begin{array}{c}\text { Reactivity Change } \\
\text { For Sodium Loss }(\%) \\
\text { Using } \mathrm{Bn}^{2}\end{array}$ & $\begin{array}{l}\text { Reactivity Change } \\
\text { For Sodium Loss }(0) \\
\text { Using } \mathrm{Bn}^{2} \text { And } \mathrm{Bo}^{2}\end{array}$ \\
\hline $\left.\begin{array}{l}\text { Radial case } 1 \\
\text { Radial case } 11\end{array}\right\}$ & 0.0004085 & 0.0003863 & $\begin{array}{l}-0.67 \\
-3.71\end{array}$ & $\begin{array}{l}+0.01 \\
-2.88\end{array}$ \\
\hline $\left.\begin{array}{l}\text { Axial case } 4 \\
\text { Axial case } 8\end{array}\right\}$ & 0.0005193 & 0.0004973 & $\begin{array}{l}-1.0 \\
-3.9\end{array}$ & $\begin{array}{l}-0.38 \\
-3.13\end{array}$ \\
\hline
\end{tabular}

As anticipated, case 2 gives the lowest critical mass and form factor - due to increased reflection and spectrum softening from the graphite regions $G 1$ and $G 3$. Cases $3-5$ also have lower critical masses and form factors than case 1 .

Case 1 has the best overall breeding ratios, but cases $2-5$ all have higher core breeding ratios. For case 2, however, the loss in overall breeding is small compared with case 1 .

Case $2 / D$ shows that the effect of halving the mesh lengths in the calculations is small - the main effect is in the radial direction (radial breeder breeding ratio and radial form factor).

Case 2/16 shows the effect of using the full 16 groups in the calculation. This reduces the critical mass, breeding ratio, and form factors all these effects are due to the true spectrum of $2 / 16$ being softer than the condensing spectra used for the core in case 2.

Although cases 2 and $2 / 16$ show that the sodium-loss coefficient is rather sensitive to the group condensation adopted, cases 1 to 3 show the coefficient is, however, small.

Cases 4 and 5 show (as in the one-dimensional cases) that the incorporation of a sodium region adjacent to the core makes the sodium-loss coefficient substantially negative. 


\section{Economic Effects}

The economic effects of these changes have been estimated in terms of the static parameters of the system on the basis of an assigned value of plutonium. Let

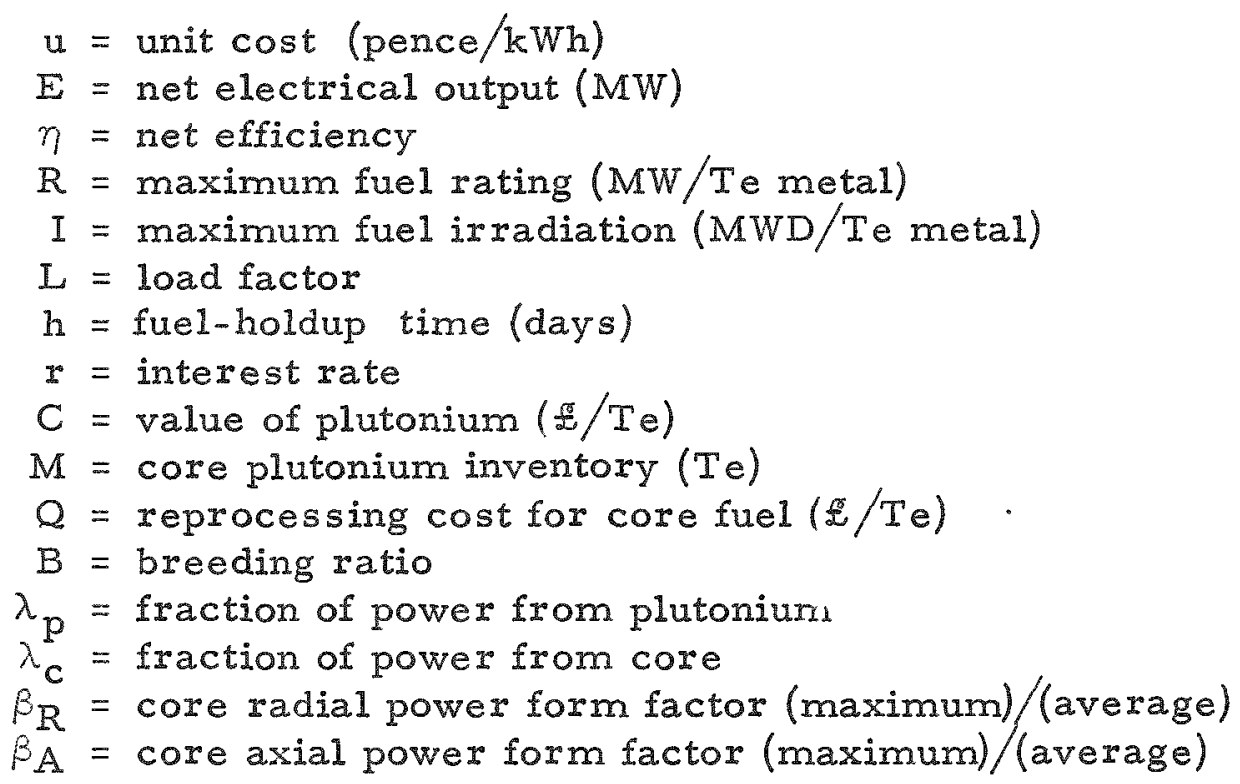

Then

$$
\begin{aligned}
\delta u= & (2.74) 10^{-5}\left\{\delta M \frac{C r}{E L}+\delta M \frac{C r}{E}\left(\frac{h R L}{I \beta_{R}}\right)+\delta \beta_{A} \frac{C r}{E}\left(\frac{M h R L}{I \beta_{R} \beta_{A}}\right)\right\} \\
& +\left(\delta \beta_{A}\right) 10^{-2} \frac{Q \lambda_{c}}{\eta I}-\delta B \frac{C \lambda_{p}}{\eta}(1.07) 10^{-8}
\end{aligned}
$$

where the terms represent, respectively, the effect of the change in critical mass on fuel-inventory charge and fuel-holdup charge, the effect of change in axial form factor on fuel-holdup charge and fuel-replacement charge, and the effect of change on breeding ratio on fuel-replacement charge.

The third term arises because in this formula no account has been taken of improved form factors to increase the power output of the system, so that steam-raising and electrical-generating plant costs are unaffected.

The doubling time of the system, D (years), is approximately proportional to the ratio of total plutonium inventory (core inventory plus holdup) to the breeding gain $(B-1)$. Therefore the effect on doubling time of small changes in the parameters considered is given by 


$$
\frac{(\delta D)}{D}=\frac{\delta M}{M}-\frac{\delta(B-1)}{(B-1)}+\frac{\delta \beta_{A}}{\beta_{A}\left(1+\frac{I \beta_{x}}{h L R}\right)} .
$$

For the basic system considered,

$$
\begin{aligned}
E & =500 \mathrm{MW}(\mathrm{e}) \\
\eta & =0.42 \\
R & =176 \mathrm{MW} / \mathrm{Te} \\
\mathrm{M} & =1.6 \mathrm{Te} \mathrm{Pu} \\
B & =1.62 \\
\lambda_{\mathrm{p}} & =0.83 \\
\lambda_{c} & =0.95 \\
\beta_{R} & =1.235 \\
\beta_{A} & =1.263 .
\end{aligned}
$$

Thus with

$$
\begin{aligned}
\mathrm{L} & =0.75 \\
\mathrm{~h} & =180 \text { days } \\
I & =47,000 \mathrm{MWD} / \mathrm{Te} \\
\mathrm{r} & =0.06,
\end{aligned}
$$

the formulae reduce to

$$
\begin{aligned}
\delta u= & (\delta \mathrm{M})(0.006)\left(10^{-6} \mathrm{C}\right)+\delta\left(\beta_{\mathrm{A}}\right)\left\{(0.002) 10^{-6} \mathrm{C}+(0.048) 10^{-5} \mathrm{Q}\right\} \\
& -\delta(B)(0.021)\left(10^{-6} \mathrm{C}\right)
\end{aligned}
$$

and

$$
\frac{\delta D}{D}=\frac{\delta M}{M}-\frac{\delta B}{B-1}+\frac{0.3\left(\delta \beta_{A}\right)}{\left(\beta_{A}\right)}
$$

For the system considered in Table 10, application of these formulae give the results shown in Table 10A.

The results given are values of $\left(\delta \mathrm{u} / 10^{-6} \mathrm{C}\right)$ as a function of $\mathrm{Q} / \mathrm{C}$ relative to case 1 . It can be seen that the results are fairly insensitive to $Q / C$. This is because the main effects on unit cost are due to changes in mass and breeding ratio rather than in form factor. In terms of actual effect on unit cost, it appears that case 2 is only very little worse than the reference case. For cases 3 and 4, the effect is no longer negligible, but case 4 also has an enhanced sodium-loss reactivity change. For case 5, the effect is appreciable. In general, the results indicate that the change of a conventional blanket arrangement by placing graphite regions between the core and 
breeders would have little effect on unit cost. Also, the inclusion of a radial sodium region adjacent to the core can make the sodium-loss coefficient substantially negative without a great rise in unit cost. However, placing a void region adjacent to the core should be avoided if possible.

In the above treatment, only effects directly resulting from changes in core parameters have been considered, and no advantage has been taken of improved form factors to reduce core dimensions. Other effects which are involved in radial blanket variations are concerned with time-dependent properties of the blanket (e.g., blanket power output, which would require consideration in a complete analysis) and with the influence of blanket composition on shielding requirements for the reactor. Although these effects have not been discussed directly, the original choice of cases was to some extent influenced by these considerations.

\section{Time-dependent Effects}

The equations governing the time-dependent concentration of isotopes at a point are well-known and will not be restated here.

For sufficiently small values of $\sigma_{a} x$, where $x$ is the flux-time variable $\phi t$, the exponential solutions may be expanded giving, to third ordex in $x$,

$$
\begin{aligned}
& N_{5}=N_{5}^{0}\left\{1-\sigma_{a 5} x+\frac{\left(\sigma_{a 5} x\right)^{2}}{2 !}-\frac{\left(\sigma_{a} x\right)^{3}}{3 !} \cdots\right\} \\
& \mathbf{N}_{8}=\mathbf{N}_{8}^{0}\left\{1-\sigma_{28} \mathrm{x}+\frac{\left(\sigma_{a_{8} \mathrm{x}}\right)^{2}}{2 !}-\frac{\left(\sigma_{a_{8} \mathrm{x}}\right)^{3}}{3 !} \ldots\right\} \\
& N_{9}=N_{9}^{0} \sigma_{c 8}\left\{x-\left(\sigma_{a 9}+\sigma_{a 8}\right) \frac{x^{2}}{2 !}+\left(\sigma_{a 9}^{2}+\sigma_{a 9} \sigma_{a 8}+\sigma_{a 8}^{2}\right) \frac{x^{3}}{3 !} \ldots\right\} \\
& \mathbf{N}_{0}=\mathbf{N}_{8}^{0} \sigma_{\mathrm{c} 8} \sigma_{\mathrm{cq}}\left\{\frac{\mathrm{x}^{2}}{2 !}-\left(\sigma_{\mathrm{a} 0}+\sigma_{\mathrm{ag}}+\sigma_{\mathrm{ag}}\right) \frac{\mathrm{x}^{3}}{3 !} \ldots\right\} \\
& N_{I}=N_{8}^{0} \quad \sigma_{C 8} \quad \sigma_{C g} \sigma_{C 0} \frac{x^{3}}{3 !} .
\end{aligned}
$$

The notation is obvious, and the blanket is as sumed to be free of plutonium at $\mathrm{x}=0$.

The buildup of plutonium concentration $P$ is then given by 


$$
\begin{aligned}
P= & \frac{N_{8}}{N_{H}}=\frac{N_{8}^{0}}{N_{H}} \sigma_{c 8}\left\{x-\left[\sigma_{a 9}+\sigma_{a 8}-\sigma_{c 9}\right] \frac{x^{2}}{2 !}+\left[\sigma_{a 9}^{2}+\sigma_{a 9} \sigma_{a 8}+\sigma_{a 8}^{2}\right.\right. \\
& \left.\left.-\sigma_{c 9}\left(\sigma_{a 0}+\sigma_{a 9}+\sigma_{a 8}\right)-\sigma_{c 9} \sigma_{c 0}\right] \frac{x^{3}}{3 !} \cdots\right\}
\end{aligned}
$$

where

$$
N_{H}=N_{5}^{0}+N_{8}^{0} \text {. }
$$

Similarly, if burnup $y$ is defined as the ratio of the integrated number of fissions to the number of heavy atoms originally present,

$$
\begin{aligned}
y=\left\{\frac{N_{5}^{0} \sigma_{f_{5}}}{N_{H}}+\frac{\sigma_{f_{8}} N_{8}^{0}}{N_{H}}\right\} x+\left\{\frac{N_{8}^{0}}{N_{H}}\left(\sigma_{f_{9}} \sigma_{c_{8}}-\sigma_{f_{8}} \sigma_{a 8}\right)-\frac{N_{5}^{0}}{N_{H}} \sigma_{f_{5}} \sigma_{25}\right\} \frac{x^{2}}{2 !} \\
+\left\{\frac{N_{5}^{0}}{N_{H}} \sigma_{f_{5}} \sigma_{a_{5}}^{2}+\frac{N_{8}^{0}}{N_{H}}\left[\sigma_{f_{8}} \sigma_{a 8}^{2}-\left(\sigma_{f_{9}} \sigma_{c 8}\right)\left(\sigma_{a 9}+\sigma_{a 8}\right)+\sigma_{f_{0}} \sigma_{c_{8}} \sigma_{c_{9}}\right]\right\} \frac{x^{3}}{3 !} .
\end{aligned}
$$

If $S$ is the fractional increase in fission power per unit volume, then $S$ is given by

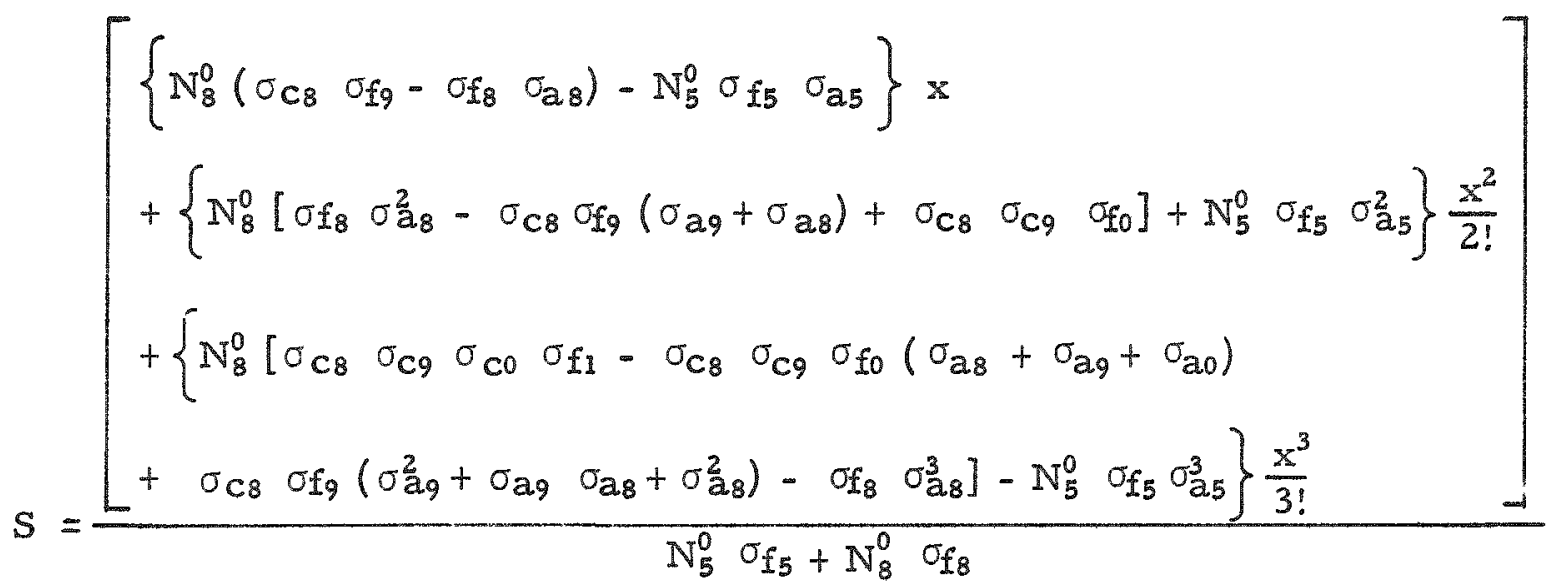

Because the buildup of plutonium is not linear with time, there is an optimum irradiation time for blanket material and, therefore, an optimum burnup for maximum economic credit from blanket operation.

If $P(t)$ represents the plutonium mass per unit mass of fertile material originally present, $C$ is the value ascribed to plutonium, and $F$ the reprocessing cost per unit mass of fertile material, then the credit, per unit sent out, is proportional to

$$
(\mathrm{CP}-\mathrm{F}) / \mathrm{t} \text {. }
$$


The maximum credit is obtained when

$$
\frac{C \dot{P}}{t}=\frac{C P-F}{t^{2}}
$$

i.e.,

$$
t_{\text {opt }}=\frac{C P-F}{C P} .
$$

The introduction of other variables, for example chemical plant losses or an interest charge on plutonium buildup in the blanket modifies the formulae but does not affect the general argument.

If it is assumed that for sufficiently small time $P$ may be represented by a quadratic function, i.e.,

$$
P=a x-b x^{2} ;(a, b>0),
$$

then

$$
x_{\text {opt }}=\sqrt{\mathrm{F} / \mathrm{Cb}}
$$

and

$$
t_{\text {opt }}=\frac{1}{\phi} \sqrt{\frac{F}{\mathrm{Cb}}}
$$

The maximum credit is thus proportional to

$$
\phi a C\left(1-\sqrt[2]{\frac{F b}{C a^{2}}}\right)
$$

To the same degree of approximation burnup is given by

$$
y=g x+h x^{2} ; g, h>0,
$$

then

$$
y_{\text {opt }}=(\mathrm{g} \sqrt{\mathrm{F} / \mathrm{Cb}})+(\mathrm{h} F / \mathrm{Cb}) .
$$

Thus, increasing $C$, for fixed $F$, increases the maximum credit and reduces both the optimum irradiation time and the optimum burnup. Increasing $F$, for fixed $C$, reduces the maximum credit and increases both optimum ir radiation time and the optimum burnup. If both $F$ and $C$ are increased by the same factor $p$, optimum burnup and irradiation time are unchanged while the credit is increased by the factor $p$. 
The optimum irradiation time topt will be a function of radial position because of the variation of flux with radius, but the optimum burnup is independent of radial position. The optimum credit, per unit of electricity sent out from the system, also diminishes as radius increases in inverse proportion to the change in topt.

In practice, owing to the general method of blanket subassembly construction, there will be a variation in plutonium produced per unit volume along the length of a fuel pin and across the width of a subassembly. This latter variation may be mitigated during operation by rotation of subassemblies. Axial subdivision of elements and axial shuffing would also be possible but would be more complicated operationally.

An approximation to the axial average plutonium concentration in a pin may be obtained if it is assumed that the flux may be represented axially by $\phi=\phi_{0} f(z)$ with $f(z)=1.0$ for $z=0$ and

$\gamma_{A}=$ axial average/maximum of flux;

$\gamma_{A}^{x}=$ axial average/maximum of the square of the flux.

Then the axial average plutonium concentration $\bar{P}_{z}$ is given by $\bar{P}_{z}=a \gamma_{A}\left(\phi_{0} t\right)-b \gamma{ }_{A}^{x}\left(\phi_{0} t\right)^{2}$

The average-to-maximum concentration ratio is

$$
\frac{a \gamma_{A}\left(\phi_{0} t\right)-b \gamma_{A}^{x}\left(\phi_{0} t\right)^{2}}{a \phi_{0} t-b\left(\phi_{0} t\right)^{2}}=\gamma_{A}\left[1+\frac{b}{a} \phi_{0} t\left(1-\frac{\gamma_{A}^{x}}{\gamma_{A}}\right)+\ldots\right] .
$$

Thus, the average-to-maximum concentration improves during the early stages of irradiation due to the preferential burnup of plutonium produced in the regions of higher flux.

The total credit from blanket operation is then given by

$$
\frac{X}{E} \int_{R_{i}}^{R_{0}} 2 L \frac{(C P-E)}{t(x)} P_{H} 2 \pi r d r
$$

where 
$\mathrm{X}$ is a conversion factor

$E$ is electrical output

$P_{H}$ is heavy-atom density in blanket ( $\mathrm{Te} / \mathrm{cc}$ )

$R_{i}$ is inner radius of blanket $(\mathrm{cm})$

$R_{0}$ is outer radius of blanket $(\mathrm{cm})$

$2 \mathrm{~L}$ is length of blanket $(\mathrm{cm})$.

For maximum burnup independent of radius

$t(r)=\frac{(\phi t)_{r=R_{i}}}{\phi_{r}}$

where the suffix $R_{i}$ denotes the value at the inner edge of the blanket, and the above expression reduces to

$$
X M_{H} \frac{\left[C\left\{a^{\prime}(\phi t)_{R_{i}}-b^{\prime}(\phi t)_{R_{i}}^{2}\right\}-F\right]}{E(t) R_{i}} \gamma_{R},
$$

where

$$
\begin{aligned}
M_{H} & =\text { heavy-atom mass in blanket ( } \mathrm{Te}) \\
\gamma_{\mathrm{R}} & =\text { radial flux form factor (av/max) in blanket } \\
\mathrm{a}^{\prime} & =\gamma_{\mathrm{a}} \\
\mathrm{b}^{\prime} & =\gamma^{*} \mathrm{~b} .
\end{aligned}
$$

The maximum credit is then

$$
\frac{X M_{H}\left(C a^{i}-2 \sqrt{F C b^{\prime}}\right) \gamma_{R} \phi_{R_{i}}}{E} .
$$

A change in electrical output does not affect the credit since $\phi_{R_{i}} / E$ remains constant.

Increasing the thickness of the radial blanket will always increase credit, although at a reducing rate with increased radius. Increasing the length of the blanket, assuming that it is longer than the core initially, will reduce the credit because the product $L \gamma$ will be reduced.

The time-averaged rate of plutonium production in the blanket is

$$
\int_{R_{i}}^{R_{0}} 2 L P_{H} \frac{\bar{P}}{t(r)} 2 \pi r d r(T e / s e c)
$$


For uniform burnup with radius, the plutonium concentration at irradiation is independent of radius and

$$
t(r)=\frac{\phi_{i} t_{i}}{\phi(r)}
$$

so that the above expression reduces to

$$
\frac{\mathrm{M}_{\mathrm{H}} \overline{\mathrm{P}}_{\mathrm{i}}}{\mathrm{t}_{\mathrm{i}}} \gamma_{\mathrm{R}}
$$

and is a decreasing function of time.

If $t_{i}$ is chosen for optimum economic gain, the expression becomes

$$
M_{H} \phi_{i}\left(a^{\prime}-\sqrt{\frac{F b^{\prime}}{C}}\right) \gamma_{R} \text {. }
$$

The initial rate of plutonium production is

$$
\int_{R_{i}}^{R_{0}} 2 L P_{H} a^{\prime} \phi(r) 2 \pi r d r=M_{H} \gamma_{R} a^{\prime} \phi_{i} ;
$$

therefore, the ratio of actual production to initial production is

$$
\begin{aligned}
\frac{\text { Actual production }}{\text { Initial production }} & =1-\frac{b^{\prime}}{a^{\prime}}(\phi t)_{i} \\
& =1-\frac{1}{a^{\prime}} \sqrt{\frac{F b^{\prime}}{C}} .
\end{aligned}
$$

when the blanket is operated for maximum economic gain, and this translates into an effect on the doubling time of the system.

Some calculations have been performed to investigate some of the assumptions which are frequently made in the treatment of radial blankets, such as constancy of cross section and of neutron flux. The particular system considered was of $1000 \mathrm{MW}(\mathrm{h})$ output and consisted of a cermet core surrounded by a $\mathrm{U}^{238} \mathrm{C}$ radial reflector.

Figure 2 shows the $\mathrm{U}^{238}, \mathrm{Pu}^{239}$, and $\mathrm{Pu}^{240}$ concentrations as functions of time for a point at the axial midplane of the system and just beyond the core reflector interface. Figure 3 shows fuel rating and burnup as functions of time for the same position. 

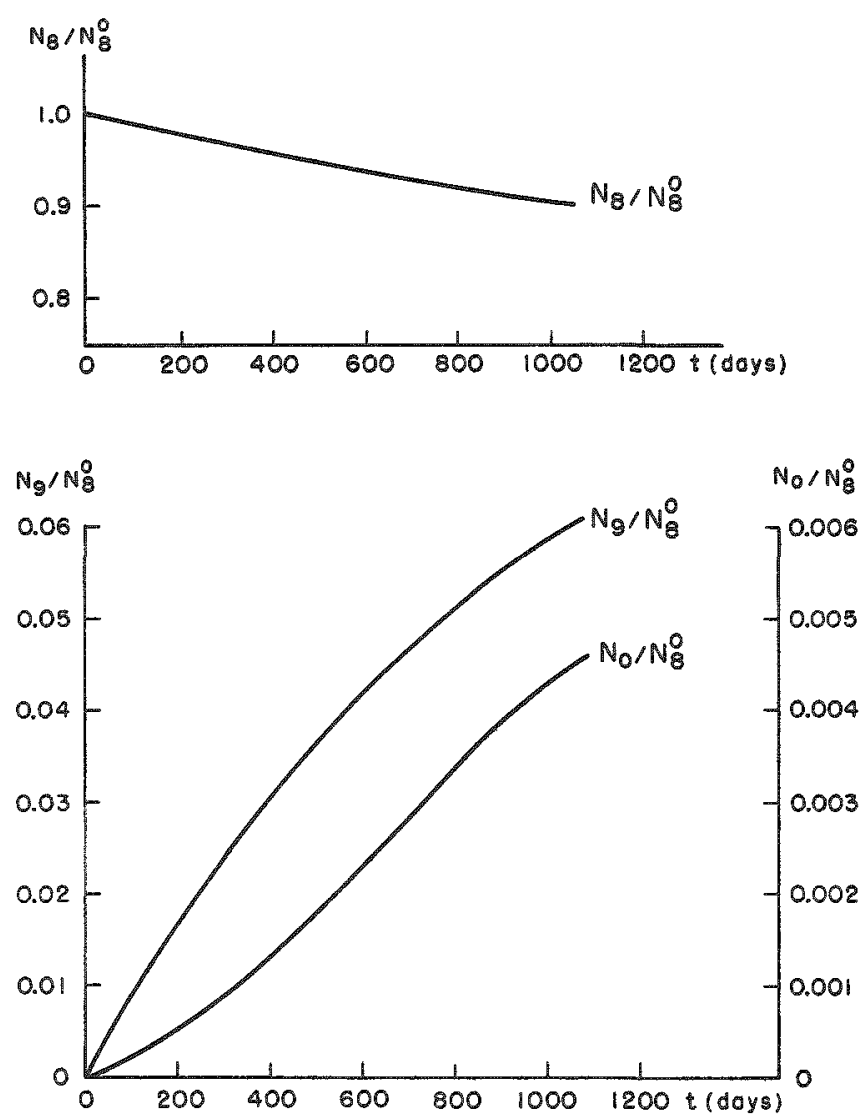

Fig. 2. Isotopic Concentrations $\frac{N_{8}}{N_{8}^{0}} \frac{N_{9}}{N_{8}^{9}}$ and $\frac{N_{0}}{N_{8}^{0}}$

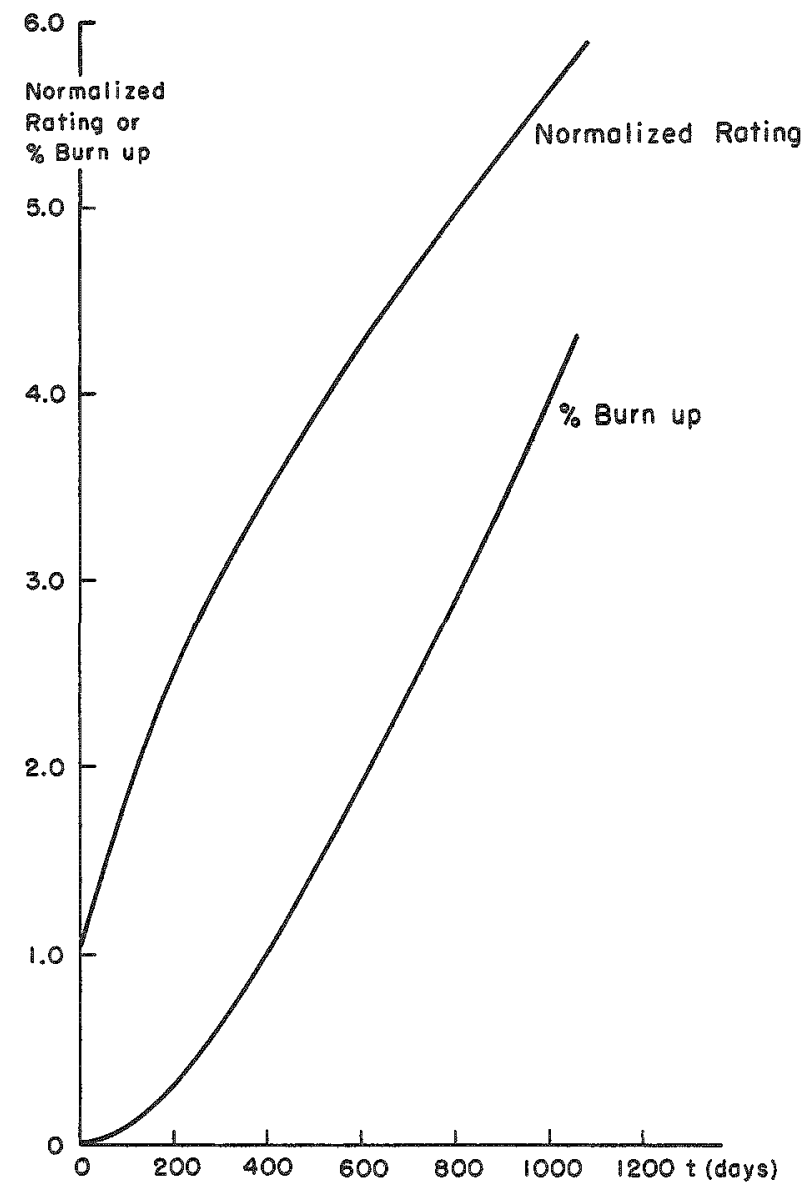

Fig. 3. Normalised Rating and Burnup

The calculations were performed with use of the Roach cross-section set. To obtain cross sections which were shielded appropriately to the concentration of isotopes present, the calculation was carried out for a number of small time intervals. At each time step the flux was recomputed, making allowance for the buildup of plutonium in the blanket, and cross sections were re-evaluated.

Figure 4 shows the time dependence of $\mathrm{Pu}^{239}$ concentration at four radial positions on the axial midplane of the blanket. The figure also includes the points obtained by compressing the curves for the outer points to a time scale given by $\mathrm{T}=t_{0} \phi_{0} / \phi \mathrm{R}$, where $t_{0}$ is the time attaching to the first point, and $\phi_{0}$ and $D R$ are the total fluxes at time $t=0$.

The degree of agreement between the latter curves suggests that it may not be necessary to perform detailed burnup calculations throughout the blanket in order to estimate the economics of blanket operation. 


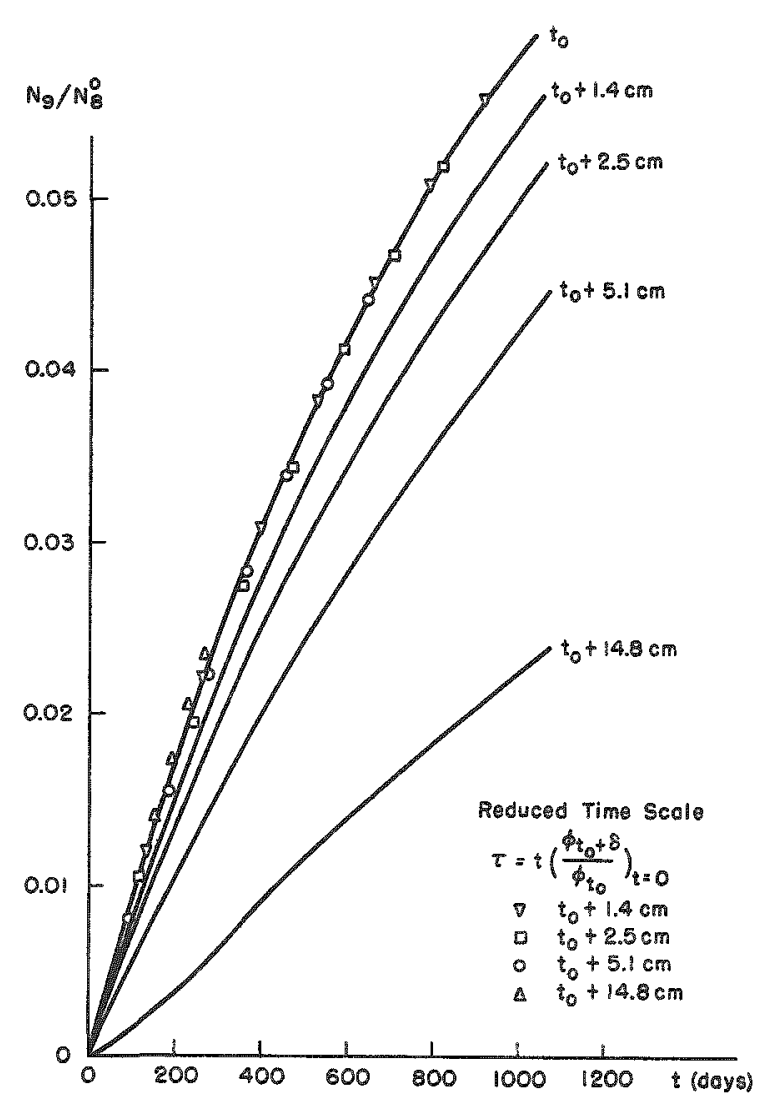

Fig. 4. $\mathrm{Pu}^{239}$ Concentration as a Function of Radial Position

require modification for points near the outer boundary of the reflector whereflux changes, both as to spectrum and absolute value, with plutonium buildup may be more significant.

Results from this example are plotted in Fig. 6, in which the normalised credit is plotted for value of $\mathrm{F} / \mathrm{C}$. The maxima are rather flat, as is to be expected from the shape of the $P(t)$ curve (and even flatter if plotted on a burnup scale).

In considering both the analysis and the above example, the effect of the increase in blanket power output with time has been ignored. Another possible variation might be
The results of calculations for the first radial point by means of different assumptions are given in Fig. 5. Curve (a) refers to the original calculation, taking account of flux and cross-section changes. Curve (b) refers to constant cross sections but time-dependent flux. Curve (c) refers to constant flux but time dependent cross sections, and curve (d) assumes both flux and cross sections independent of time.

From these results it would appear that cross-section variation is more important than flux variation, at least near the core reflector interface. It is of interest to note that the assumption of constant flux and cross sections underestimates $\mathrm{Pu}^{239}$ content by less than $10 \%$ after a 24 -month ir radiation. These conclusions might

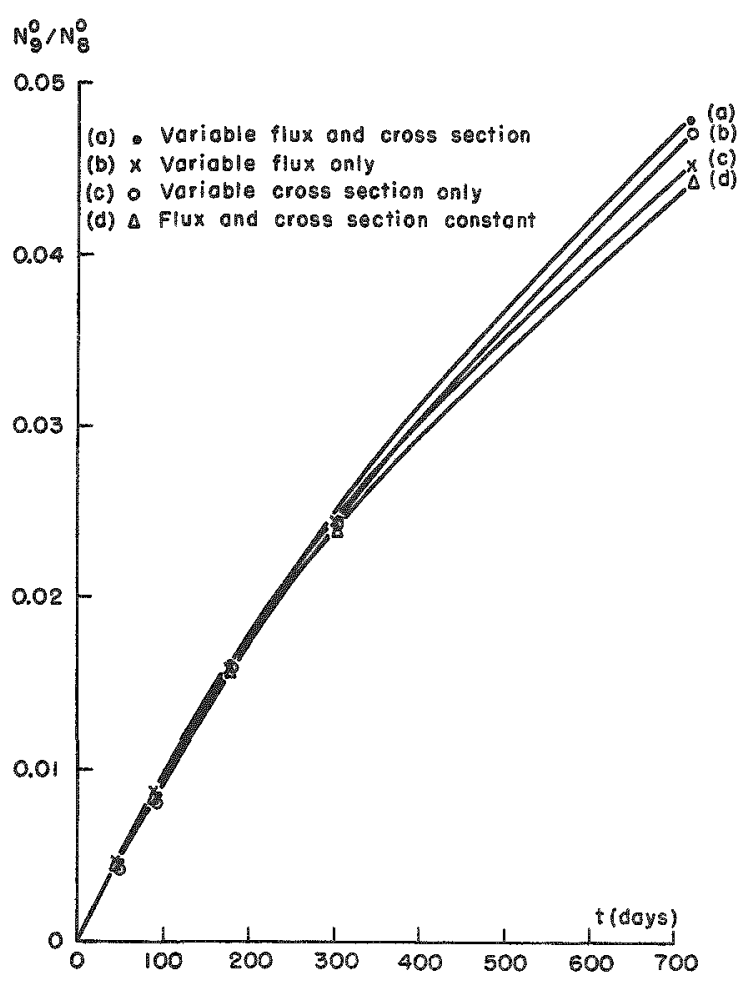

Fig. 5. $\mathrm{Pu}^{239}$ Concentration for Different Assumptions 
the dependence of $F$ on plutonium concentration due to increasing activity of the fuel at higher burnup. If allowance was made for these effects, optimum burnup would be reduced since the inclusion of $F$ as $F(P)$ would be to reduce the credit progressively with buxnup, and because any penalty incurred through the time variation of blanket power would also increase with burnup.

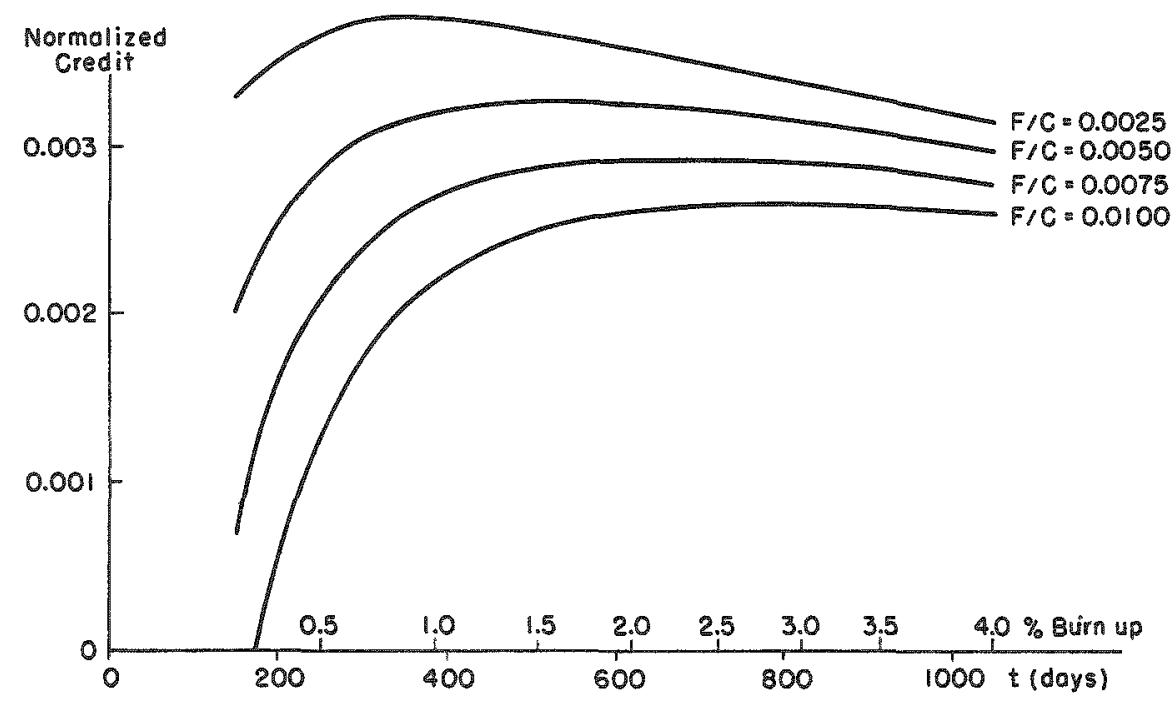

Fig. 6. Normalised Credit as a Eunction of $\mathrm{F} / \mathrm{C}$

\section{Refexences}

1. A. Hassitt, A Computer Programme to Solve the Multigroup Diffusion Equations, T.R.G.Report 229(R).

2. G. E. Hansen and W. H. Roach, Sixteen Group Cross Sections, N-2-753 - Revision I.

$$
\frac{\text { Discussion of Paper }}{\text { Presented by Mr. Mann }}
$$

MR. OTT (Karlsruhe):

You mentioned an internal breeding ratio of about one. How have you defined the breeding ratio and what was your fertile-to-fissile ratio in the core?

MR. MANN:

The breeding ratio was simply defined as the ratio of the capture rate in $\mathrm{U}^{238}$ to the fission rate in plutonium; an arbitrary definition if you 
like. The enrichment figures for the core were as follows: the inner core region had a plutonium to plutonium plus uranium ratio of 0.129 , and the outer region had a ratio of 0.175 .

MR。OTT:

What was your sodium content?

MR。MANN:

The sodium content in the core was 36 per cent.

MR。OTT:

I wonder how you get such large internal breeding ratios.

MR。MANN:

Put it down to the cross sections.

MR. FURET (CEA, Paris):

We have made some similar calculations and found the same breeding ratios. What kind of geometry was used for the one dimensional calculation, cylindrical or plane, and how did you define the buckling?

MR. MANN:

The radial calculations were cylindrical with a buckling in the $z$ direction. For considerations of the sodium coefficient, the one-dimensional calculations may be misleading in that in the case without sodium you ought to change the buckling for a one-dimensional calculation. This, in fact, was done in some cases, and this improved considerably the agreement between the one-dimensional and two-dimensional sodium reactivity calculation.

MR. OKRENT (Argonne):

What was the thickness of your radial blanket?

MR.MANN:

The basic thickness was 8 in.

MR. LOEWENSTEIN (Axgonne):

Could you elaborate on this choice of the 8 -in. blanket? 
MR。 MANN:

We11. I think like Topsy it just grew. It is more or less an arbitrary figure.

MR, LOEWENSTEIN:

The reason I asked the question is because you get very healthy total breeding ratios, and I suspect this is a reflection of these particular cross sections. If you were to take another set of constants you might get very low numbers which would be enhanced somewhat by using a thicker blanket, or perhaps a better moderator in the reflector.

MR. MANN:

One should consider the results only in a relative sense. I know Dr. Loewenstein is aware of the difficulties in choosing a cross-section set to do calculations which are meant to look at more than one aspect of a problem. For example, I did mention that some of this work was extended to provide starting fluxes for shielding calculations, in which case we wanted the lower energy groups, which are not too well-represented in the YOM set.

MR. FISCHER (Argonne):

In our studies, where we have used the Yiftah set, we also see that as the reactor gets larger and larger, the importance of the blanket falls off rather severely. So that you can use even a very thin blanket, such as used here, and still get a very nice breeding ratio. We get core breeding ratios for reactors of this size which are not quite as good as yours, but quite respectable.

I would say that one must be very careful in studying these radial and axial blankets in asking what the total consequence is to the total reactor system. For instance, by the time you get anything like a 2 per cent burnup in the radial blanket, you have a tremendous plutonium buildup, and a huge power shift. Either you have to give up and put in a big sodium fraction, or else you lose thermodynamic efficiency by pumping cold sodium through initially, and this will really hurt. You really cannot go as far as you seem to be going here without looking at the engineering difficulties, which I think would be extreme.

One last point which is quite important: you must not look at a radial blanket simply as a breeder of plutonium, but you must ask how it participates in the total production of electricity, and how it affects the total cost of electricity. 
MR, MANN:

Yes, I agree. The latter subject, of course, introduces us to the question of detailed cost. One is not always after tremendously high burnups in the blanket, or so it might appear. One does not lose a great deal in total credit by reducing the burnup somewhat, provided it is not reduced too far. 


\title{
UNCERTAINTIES IN FAST POWER REACTOR \\ DOPPLER COEFFICIENTS AND THEIR \\ EFFECTS ON ECONOMICS
}

\author{
A. V. Campise and D. J. Stoker \\ Atomics International \\ Canoga Park, California \\ (Paper presented by Mr. Stoker)
}

Nomenclature

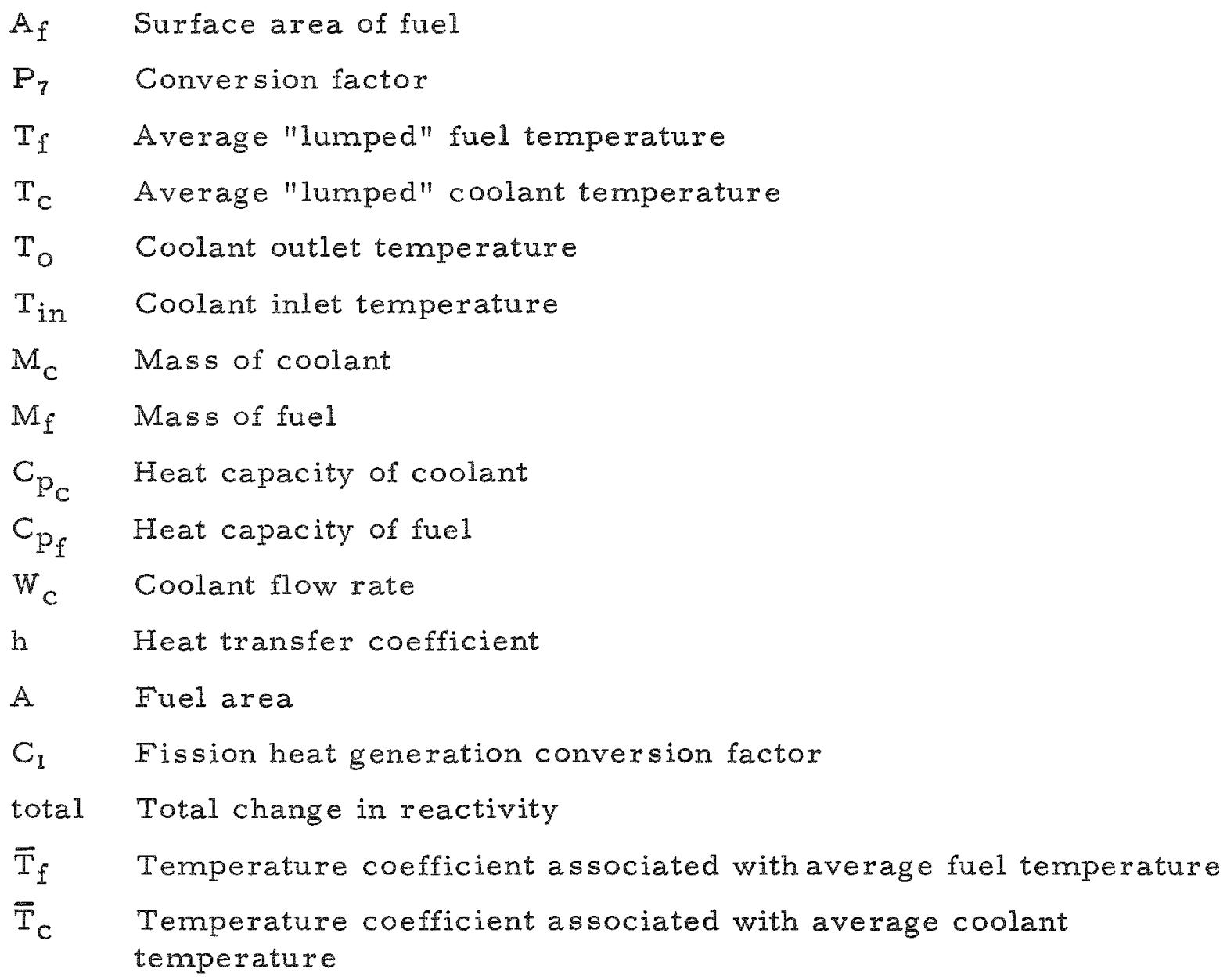

\section{Introduction}

Inherent stability of fast sodium-cooled reactor systems must be thoroughly demonstrated before application of this basic concept is made in the power industry. Stability of fast sodium-cooled reactors is dependent on the many reactivity feedbacks associated with power operation. These feedbacks, which result from temperature changes in the reactor 
core and structure, must be understood in evaluating the inherent stability and safety of proposed advanced fast sodium-cooled reactor systems. The nature of these feedbacks may establish design criteria which will limit the potential economic attractiveness of fast reactor systems.

Because of their potential ability to sustain long irradiation exposures, ceramic fuels are being strongly considered for use in fast sodium-cooled reactor systems. Due to physical changes occurring in this type of fuel element during exposure, it has been postulated that any axial expansion coefficient normally reliable in metal-fueled systems may be unreliable in fast reactor cores utilizing ceramic fuel. For this reason, dependence upon the Doppler coefficient as a reliable inherent negative feedback characteristic appears to be a potential design criteria. The purpose of this paper is to illustrate how the present uncertainty in prediction of Doppler coefficients can influence the economics of fast reactor systems if the Doppler coefficient is used as a design criterion.

\section{Importance of Doppler Coefficient}

The importance of the Doppler coefficient can be understood by an examination of the analysis of the dynamic power coefficient of fast reactor cores. Many feedbacks are present in fast reactors. If a structurally rigid reactor assembly is assumed, the primary reactivity feedbacks in a fast reactor core are due to the Doppler effect in the fuel and the sodium reactivity coefficient. To further understand the relative importance of Doppler and sodium reactivity feedbacks, a brief discussion of the power coefficient of fast reactor cores is presented.

A. The kinetic description of the reactor fuel and coolant temperatures required to study the closed-loop temperature behavior of fast reactor cores was obtained on the basis of the following assumptions:

1. Constant coolant flow is maintained in the reactor core at all times.

2. Reactor kinetic equations are represented in a linearized form.

3. No heat is generated in the coolant.

4. Partial differential equations describing space-dependent heat transfer are described in a one-node model.

5. Coolant and fuel temperature rises are linear.

6. Heat is transferred from a lumped average fuel temperature to a lumped average coolant temperature. 
The heat balance in the fuel element is given by

$$
M_{f} C_{p_{f}} \frac{d \bar{T}_{f}}{d t} \approx P_{T} N(t)-h A\left(\bar{T}_{f}-\bar{T}_{C}\right)
$$

The heat balance in the coolant is assumed to be given by

$$
\mathrm{M}_{c} C_{P_{C}} \frac{d \bar{T}_{C}}{d t} \approx h A\left(\bar{T}_{f}-\bar{T}_{c}\right)-W_{C} C_{P_{C}}\left(T_{O}-T_{i n}\right) .
$$

Reactivity changes associated with temperature changes in the fuel and coolant are given by

$$
\Delta \rho_{\text {total }} \approx \alpha_{f} \Delta \bar{T}_{f}+\beta_{c} \Delta T_{c}+\Delta \rho_{\text {ext }} .
$$

we have

$$
\text { Simplifying the above equations and collecting the terms, }
$$

\section{Fuel:}

$\dot{\overline{\mathrm{T}}}_{f} \approx \gamma_{1} \overline{\mathrm{T}}_{f}+\gamma_{2} \overline{\mathrm{T}}_{\mathrm{c}}+\gamma_{3} N(t)$

Coolant:

$\dot{\bar{T}}_{c} \approx \delta_{1} \overline{\mathrm{T}}_{\mathrm{C}}+\delta_{2} \overline{\mathrm{T}}_{f}+\delta_{3}\left(\mathrm{~T}_{\mathrm{O}}-\mathrm{T}_{\mathrm{in}}\right) ;$

Reactivity:

$\Delta \rho_{\text {total }} \approx \bar{\alpha}_{\mathrm{f}} \Delta \overline{\mathrm{T}}_{\mathrm{f}}+\beta_{\mathrm{c}} \Delta \overline{\mathrm{T}}_{\mathrm{c}}+\Delta \rho_{\mathrm{ext}}$.

Taking the LaPlace transforms of the above equations and linearizing yield the following sets of equations for the fuel, coolant, and reactivity.

Fue1:

$s \Delta \bar{T}_{f}(s) \approx \gamma_{1} \Delta \bar{T}_{f}(s)+\gamma_{2} \Delta \bar{T}_{c}(s)+\gamma_{3} \Delta N(s) ;$

Coolant:

$s \Delta \overline{\mathrm{T}}_{\mathrm{C}}(\mathrm{s}) \approx \delta_{1} \Delta \overline{\mathrm{T}}_{\mathrm{C}}(\mathrm{s})+\delta_{2} \Delta \overline{\mathrm{T}}_{f}(\mathrm{~s})+\delta_{3} \Delta \mathrm{T}_{\mathrm{in}} ;$

Reactivity:

$$
\Delta \rho_{\text {total }}(s) \approx \bar{\alpha}_{f} \Delta \overline{\mathrm{T}}_{f}(s)+\beta_{c} \Delta \overline{\mathrm{T}}_{\mathrm{c}}(\mathrm{s})+\Delta \rho_{\text {ext }} .
$$


The power coefficient is easily discernible as shown below by collecting terms and using a feedback diagram as a visual aid.

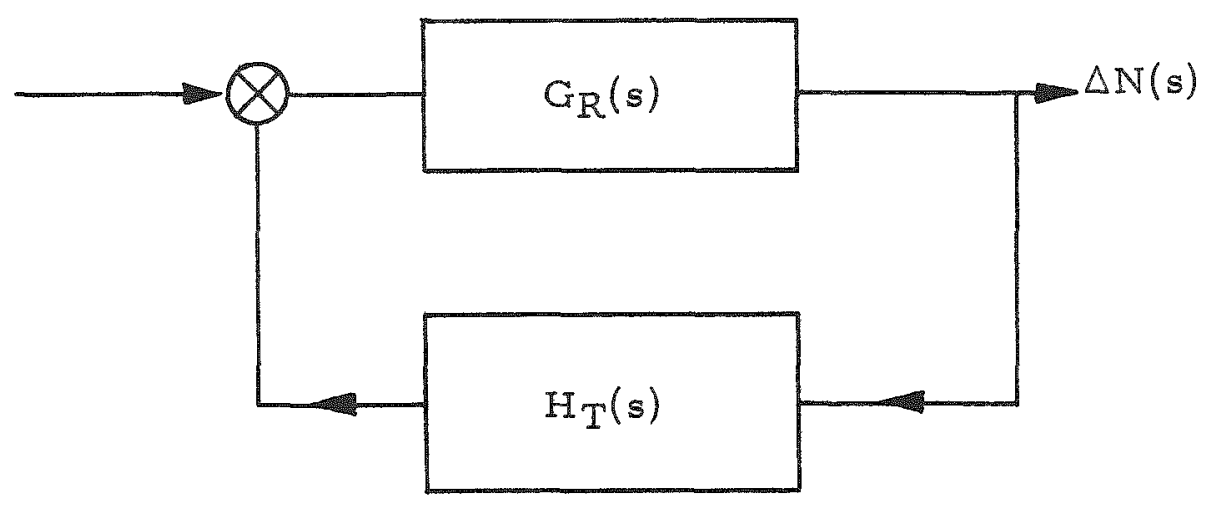

Thus, the power coefficient is defined as

$$
H_{T}(s) \approx \frac{\Delta \rho(s)}{\Delta N(s)} \approx \frac{\left(\gamma_{3} / \gamma_{2}\right)\left\{\bar{\beta}_{c}+\left[\bar{\alpha}_{f}\left(s-\delta_{1}\right) / \delta_{2}\right]\right\} G_{8} G_{7}}{1-G_{8} G_{7}}
$$

Applying the final-value theorem to Equation (10), we can now compute the steady-state power coefficient by setting $S=0$, so that

$$
\mathrm{H}_{\mathrm{ss}=0} \approx \frac{\left(\gamma_{3} / \gamma_{2}\right)\left\{\bar{\beta}_{\mathrm{c}}-\left(\bar{\alpha}_{\mathrm{f}} \delta_{1} / \delta_{2}\right)\right\} \gamma_{2} \delta_{2} / \gamma_{1} \delta_{2}}{1-\left(\delta_{2} \gamma_{2} / \delta_{1} \gamma_{1}\right)}
$$

or

$$
H_{s s=0} \approx \beta_{c}\left(\frac{\gamma_{3} \delta_{2}}{\gamma_{1} \delta_{1}-\gamma_{2} \delta_{2}}\right)-\alpha_{f}\left(\frac{\gamma_{3} \delta_{1}}{\gamma_{1} \delta_{1}-\gamma_{2} \delta_{2}}\right)
$$

We note that the steady-state power coefficient is given by a linearized combination of the fuel temperature coefficient and the coolant temperature coefficient. Each of the se temperature effects are multiplied by a coefficient which is a complicated function of the heat transfer char acteristics of the core. With a plutonium-fueled fast reactor core as a reference, the coefficient of reactivity for Doppler and sodium effects are of opposite signs, and the effects of the coefficients are noted below:

Setting the fuel coefficient $=-0.33 \times 10^{-5} \delta \mathrm{k} /{ }^{\circ} \mathrm{F}$ and the coolant coefficient $=+0.872 \times 10^{-6} \delta \mathrm{k} /{ }^{\circ} \mathrm{F}$, we have, with a sodium flow rate of $9.11 \times 10^{3} \mathrm{lb} / \mathrm{sec}$ and a fuel heat capacity = $0.076 \mathrm{Btu} / \mathrm{b} /{ }^{\circ} \mathrm{F}$, the value of the steady-state power coefficient for this set of conditions:

$$
\mathrm{H}_{\mathrm{SS}} \mathrm{s}_{\mathrm{s}} \approx \alpha_{\mathrm{f}}(0.947)+\beta_{\mathrm{c}}(0.174) \text {. }
$$


As noted in Equation (13), the coefficient multiplying the fuel coefficient is much larger and is dominating in its contribution to the total steady-state power coefficient. Therefore, when considering the power coefficient of fast reactor cores, the effect of the sodium coefficient is negligible; however, in accident conditions this coefficient may play an important part. To evaluate the effects of uncertainties in the cross-sectional data, core conditions, and theoretical techniques involved in computing both the fuel temperature coefficient and the coolant temperature coefficient, the following assumptions were made to obtain conditions for positive power coefficients and therefore unstable reactor conditions:

In all of the following cases a flow rate of $9.11 \times 10^{3} \mathrm{lb} / \mathrm{sec}$ and $a \mathrm{C}_{\mathrm{p}}$ of $0.076 \mathrm{Btu} / 1 \mathrm{~b} /{ }^{\circ} \mathrm{F}$ were used so that the basic equation for the steady-state power coefficient is given by

$$
H_{S S} \approx \bar{\alpha}_{f}(0.947)+\beta_{c}(0.174) \text {. }
$$

In the first case we assume the fuel coefficient $=-0.33 \times 10^{-5} \delta \mathrm{k} /$ ${ }^{\circ} \mathrm{F}$. Then, computing the coolant coefficient necessary for an unstable reactor condition, we have that

$$
\beta_{c}(0.174) \geq 3.14 \times 10^{-6} \text {. }
$$

so that the coolant coefficient must be equal to or greater than

$$
\beta_{\mathrm{C}} \approx 18 \times 10^{-6} \delta \mathrm{k} /{ }^{\circ} \mathrm{F} \text {. }
$$

Reversing the situation, we note that if the coolant coefficient be taken as $\beta_{\mathrm{c}}=0.0872 \times 10^{-5} \delta \mathrm{k} /{ }^{\circ} \mathrm{F}$, the fuel coefficient must be equal to or smaller than

$$
\bar{\alpha}_{f} \approx-0.177 \times 10^{-6} \delta \mathrm{k} /{ }^{\circ} \mathrm{F}
$$

to yield an unstable reactor condition. Since the Doppler coefficient in fast reactor cores is composed of two competing terms, namely, the $\mathrm{U}^{238}$ or thorium negative contribution and the $\mathrm{Pu}^{239}$ or $\mathrm{U}^{233}$ possibly positive contribution, it might be postulated that an overall positive Doppler coefficient could exist. However, the contribution due to the fertile material is always negative and is, by and large, the predominant factor in the Doppler feedback. A negative Doppler coefficient will always result in cores with large fertile to fissile ratios, even though its magnitude may be greatly reduced due to uncertainties in nuclear parameters.

III. Theory Used in Calculation of Doppler Coefficient

The basic theoretical technique for computing resonance cross sections is based on the well-known single-level Breit-Wigner formula 
with the assumption of no interference between neighboring resonances. This basic method is contained in the ARES program(1) and is essentially that described by J. Chernick of Brookhaven National Laboratory and $R$. Vernon of Atomics International. $(2,3)$ An additional improvement is contained in the program for treating the intermediate resonances, a method was developed by $E$. R. Cohen and R. Goldstein. (4)

A brief description of the techniques used in this ARES program is now presented.

\section{A. Treatment of Resolved Resonances}

Different formulations are available in the ARES program for treating the resolved resonance-energy range. First is the well-known narrow-resonance (NR) approximation. The narrow-resonance infinitemass approximation (NRIM), which has been widely used in the treatment of resonances in the resolved energy range, is the second formulation. The third approximation is a nonlinear combination of the NRIM approximation and the NR without potential scattering interference. The last formulation available in the ARES program is the narrow resonance without potential scattering interference formulation, mentioned above. The nonlinear combination of the NRIM and the NR approximation is due to Cohen and Goldstein, (5) and is used for intermediate resonances.

\section{B. Unresolved Resonances}

That portion of the resonance integral due to unresolved resonances is calculated by the NR approximation. Numerical values of the average resonance parameters used in this computation can be input data or data computed by the program based on resonance parameters given in the basic ARES library. The contribution of the $L=0$ neutrons is computed. As an additional option, the contribution of $L=1, J=\frac{1}{2}$ and $\frac{3}{2}$ neutrons can be obtained. A correction is made in the program for statistical distribution of nuclear widths according to the Porter-Thomas distribution.

\section{Doppler Broadening Correction}

The effect of Doppler broadening is applied both to the resolved and unresolved resonances. The technique is to first make the computation for $0^{\circ} \mathrm{K}$ and then to apply the Doppler correction for temperature. The method used is that developed by Dresner(6) based on tables prepared by Adler and Nordheim.(7) 


\section{Calculation of the $1 / \mathrm{V}$ Contribution}

Two methods are used for computing the contribution to the resonance integral by the $1 / \mathrm{V}$ portion of the resolved resonance. The first uses the $2200-\mathrm{m} / \mathrm{sec}$ value of the absorption cross section and assumes a $1 / \mathrm{V}$-energy dependence. The second method, sometimes referred to as the resonance parameter method, uses the resonance parameters exclusively.

\section{E. Multigroup Cross Sections}

Finally, a multigroup cross-section set is formed by summing all the resolved resonances lying in a given neutron group, adding to this the contribution of the unresolved resonances in the group, and finally factoring in the $1 / \mathrm{V}$ contribution to this particular energy group.

\section{F. Reactivity Calculation}

Modified Diffusion Theory techniques contained in the AIM-6 reactor program are used in computing the neutron flux spectrum characterizing the fast reactor core being studied. If the temperature profile both in the reactor and fuel rod are properly accounted for, multigroup resonance cross sections are used to compute the reactivity of various fast reactor cores at various averaged core temperatures. It has been shown by Hummel( 8 ) at Argonne National Laboratory that this crosssection group treatment (coarse-group structure) for computing the Doppler coefficient is in good agreement with the more accurate calculations of Doppler coefficients obtained by the ELMOE program with a fine-group structure.

IV. Characteristics of Basic Reactor Cores and Fuel Cycles

In an effort to achieve attractive fuel-cycle economics for fast sodium-cooled reactor systems, it is desirable to operate at high fuel temperature as well as to increase the average burnup limit. Basically two types of ceramic fuels, oxide and carbide, and two fuel mixtures, $\mathrm{U}^{238} / \mathrm{Pu}^{239}$ and $\mathrm{Th}^{232} / \mathrm{U}^{233}$, are now being proposed for fast sodium-cooled reactor systems. In obtaining the thermal pexformance of various fast reactor cores, two types of fuel arrangements were studied: gas-bonded oxides and sodium-bonded carbides. Rod sizes were chosen in each case so as to yield equivalent core diameters regardless of fuel material.

The basic characteristics of these cores are given in Table I. They are all unmoderated cores, with high specific powers. They are not optimized cores from the viewpoint of obtaining maximum Doppler effect; rather they represent present expectations as to performance of future ceramic-fueled fast reactors. 
CHARACTERISTICS OF EQUILIBRIUM FAST CORES

\begin{tabular}{|c|c|c|c|c|}
\hline & \multicolumn{2}{|c|}{$\begin{array}{c}\text { Gas-bonded } \\
\text { Oxide }\end{array}$} & \multicolumn{2}{|c|}{$\begin{array}{c}\text { Sodium-bonded } \\
\text { Carbide }\end{array}$} \\
\hline & $T h-U^{233}$ & $\mathrm{U}^{238}-\mathrm{Pu}$ & $T h-U^{233}$ & $U^{238}-P u$ \\
\hline Nominal Core Power (MWt) & 1,160 & 1,160 & 1,160 & 1,160 \\
\hline Core Diameter $(\mathrm{ft})$ & 5.10 & 3.25 & 5.10 & 3.25 \\
\hline Core Height (ft) & 5.45 & 6.50 & 5.45 & 6.50 \\
\hline Blanket Thickness (ft) & 1.25 & 1.25 & 1.25 & 1.25 \\
\hline Fuel Slug OD (in.) & 0.21 & 0.21 & 0.35 & 0.35 \\
\hline Radial Peak/Average Power & 1.60 & 1.60 & 1.60 & 1.60 \\
\hline Axial Peak/Average Power & 1.28 & 1.28 & 1.28 & 1.28 \\
\hline Maximum Rod Power, $\mathrm{kW} / \mathrm{ft}$ & 17.4 & 17.4 & 41.6 & 41.6 \\
\hline Core Loading ( $\mathrm{kg}$ fuel) & 10,400 & 10,200 & 13,100 & 12,800 \\
\hline Cumulative Burnup (MWD $/ \mathrm{T})$ & 100,000 & 100,000 & 100,000 & 100,000 \\
\hline Fissile Enrichment $(\%)$ & 15.5 & 14.6 & 11.7 & 10.9 \\
\hline Euel Management Out-in & 3 zone & 3 zone & 3 zone & 3 zone \\
\hline Cycle Time (full-power days) & 263 & 258 & 358 & 350 \\
\hline$\Delta k / k / c y c l e$ & 0.048 & 0.039 & 0.038 & 0.028 \\
\hline
\end{tabular}

V. Computed Doppler Coefficient

The Doppler coefficients of ceramic-fueled, thorium, and $U^{238}$ fast reactor systems were computed from two basic multigroup libraries. The 16-group cross-section set used was that of Yiftah, Okrent, and Moldauer (YOM). This compilation is based on experimental differential crosssection data and basic theoretical work using nuclear models. No adjustments have been made in this cross-section set for integral experimental measurements obtained with critical assemblies. The second set of cross sections used in this analys is is the Los Alamos Scientific Laboratory (LASL) 18-group compilation. This basic set, as published by C. B. Mills of Los Alamos, has been further refined, corrected, and up-dated by recent analysis of the multiregion criticals conducted with the AETR critical assembly machine. The Los Alamos set includes corrections obtained from the analysis and interpretation of integral experiments.

Uncertainties in computed Doppler coefficients are considered highly probable in reference to the basic inaccuracies in the nuclear cross sections and nuclear theory used in obtaining the Doppler coefficient of reactivity in this neutron-energy range. A verification of the theoretical techniques has been obtained in only two limited cases, that due to the thorium and $U^{238}$ Doppler effects in a fast reactor spectrum in the ECEL critical assembly. Further verification is needed before uncertainties 
can be adequately understood and their magnitudes completely assessed. A few basic uncertainties in the cross-sectional data referred to are as follows:

1. Experimental data for radiative capture cross section of $\mathrm{U}^{238}$ in the energy range of $10 \mathrm{kV}$ with a $95 \%$ confidence interval is not known to better than $\pm 60 \%$ (reference: H.W. Newson, ORNL-2309).

2. Low-level variation in the capture and fission resonance widths and the cross sections are not constant within $50 \%$, so that a mean value assumed at higher energies can be grossly in error if low-level crosssectional data are used.

3. The alpha variations for $P \mathrm{u}^{239}$ and $\mathrm{U}^{233}$ in the vicinity of $30 \mathrm{kV}$ is not known to better than 20\% (reference: Diven and Hopkins, Los Alamos).

Various parameters were investigated in computing the uncertainty in the Dopplex coefficient of ceramic-fueled fast reactor systems. These parameters are listed below:

1. variations in neutron spectra caused by using different multigroup cross-section sets;

2. the effect of sodium density in the reactor core;

3. geometric effects (i.e., homogeneous or heterogeneous).

Figures 1-5 depict the type of results obtained in the evaluation of uncertainties. The extremes for all cases considered are outlined in

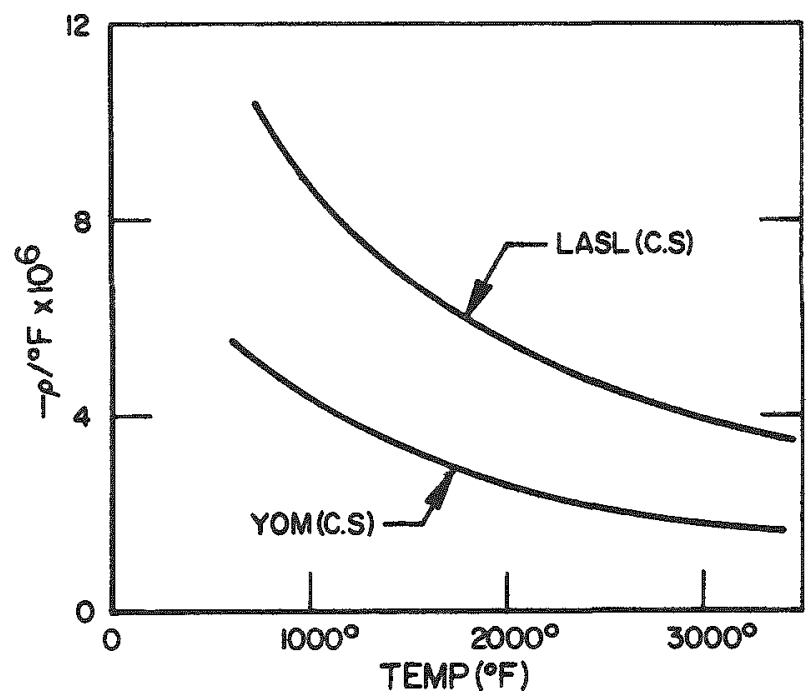

Fig. 1. U238 Doppler Coefficient(Core full of sodium)

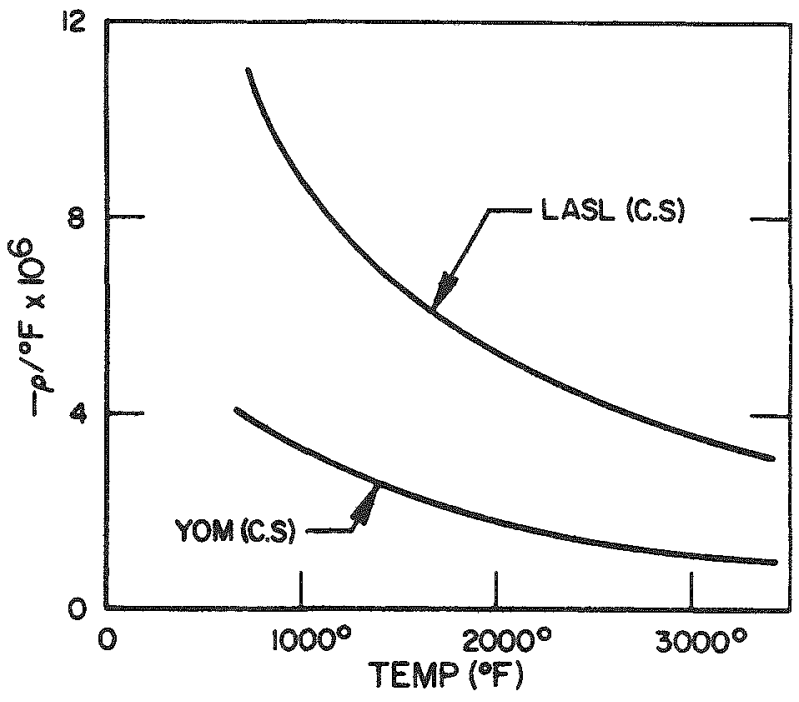

Fig. 2. $\mathrm{Th}^{232}$ Doppler Coefficient(Core full of sodium) 


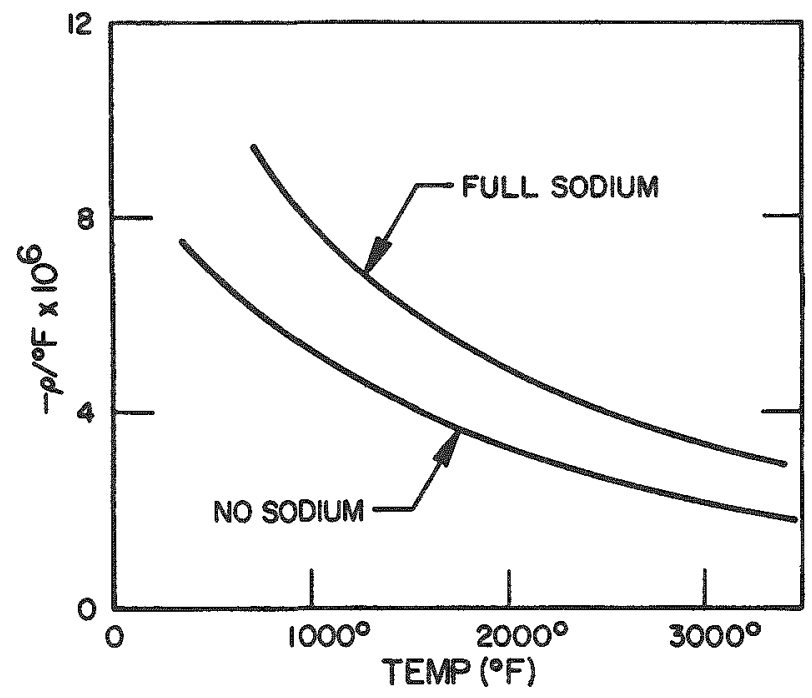

Fig. 3. $\mathrm{Th}^{232}-\mathrm{U}^{233}$ Doppler Coefficient (LASL C.S)

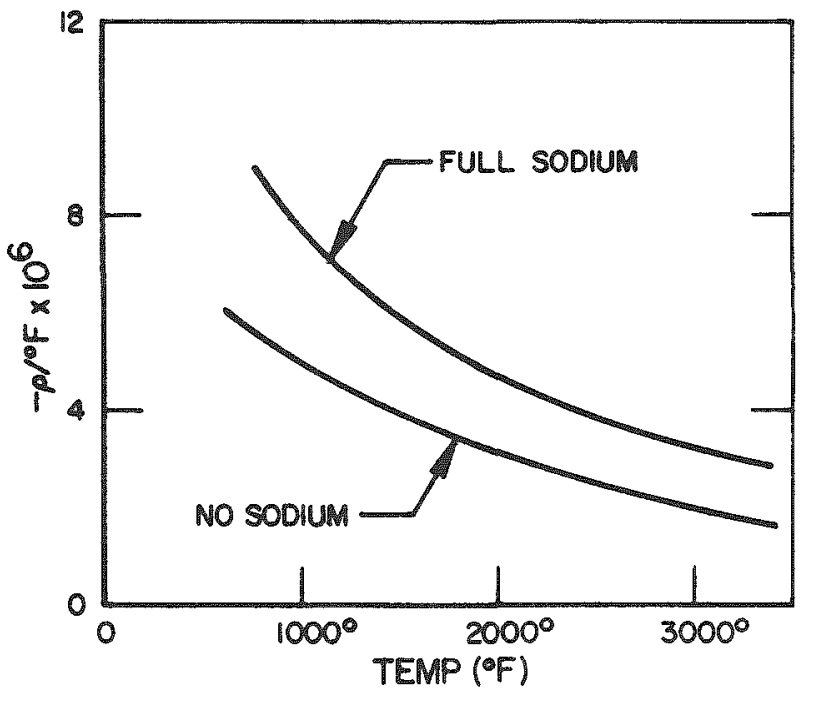

Fig. 4. U $\mathrm{U}^{238}-\mathrm{Pu}^{239}$ Doppler Coefficient (LASL C.S)

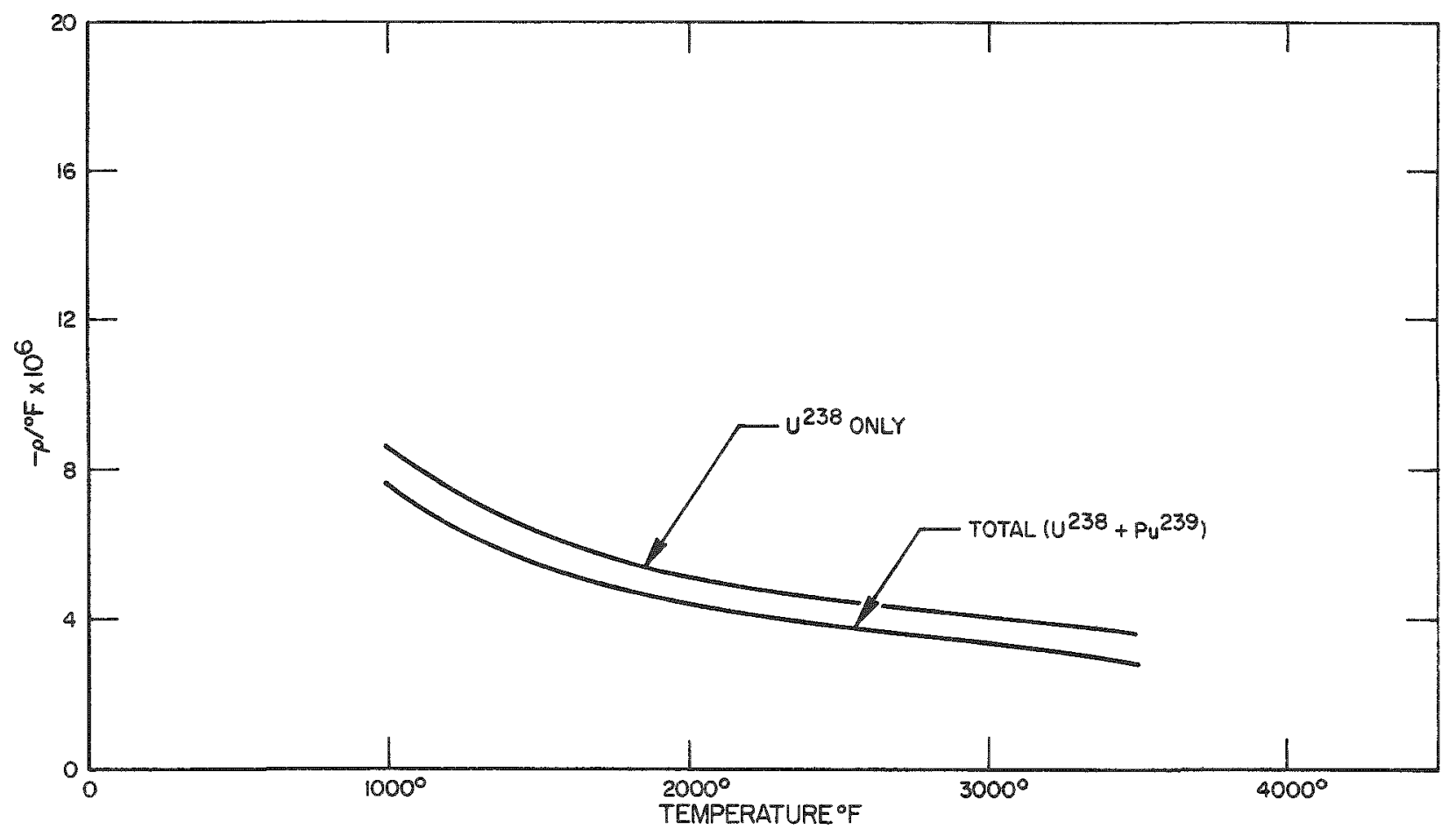

Fig. 5. $\mathrm{U}^{238}-\mathrm{Pu}^{239}$ Doppler Coefficient

Table II. Typical results are as shown in Figs. 1 and 2, obtained for oxide-fueled cores. The largest uncertainty uncovered is the effect of using different multigroup libraries in computing the $\mathrm{U}^{238}$ and thorium Doppler coefficients. The basic effect is the hardness of the neutron flux spectrum. The YOM set yields a harder neutron flux spectrum than the Los Alamos set, thus producing smaller Doppler coefficients. 
Table II

DOPPLER COEFFICIENT OF FAST CORES

Units: $10^{-6} \Delta \mathrm{k} /{ }^{\circ} \mathrm{F}$

\begin{tabular}{l|c|c}
\hline & Gas-bonded Oxide & $\begin{array}{c}\text { Sodium-bonded } \\
\text { Carbide }\end{array}$ \\
\hline \multicolumn{3}{c}{ U-Pu } \\
\hline LASL (Full Na) & 3.8 & 4.9 \\
LASL (No Na) & 2.4 & 3.3 \\
YOM (Full Na) & 1.8 & 2.4 \\
YOM (No Na) & 1.3 & 1.7 \\
\hline \multicolumn{2}{|c}{} \\
\hline LASL (Full Na) & Th-U 233 & 5.0 \\
LASL (No Na) & 2.9 & 2.9 \\
YOM (Full Na) & 1.3 & 1.6 \\
YOM (No Na) & 0.9 & 1.10 \\
Temp Range & $1880-3250^{\circ} \mathrm{F}$ & $1050-2400^{\circ} \mathrm{F}$ \\
\hline
\end{tabular}

The second largest effect is due to the sodium density in the core. This effect is important when considering the safety characteristics of fast reactor cores. Under violent nuclear transients the possibility of voiding the core of sodium must be considered; therefore, consideration must be given to the magnitude of the computed Doppler effect for that case in which the core contains little or no sodium. The effect of sodium voiding in a $\mathrm{U}^{233} / \mathrm{Th}^{232}$ oxide-fueled fast reactor system (see Fig. 3 ) is slightly less than the effect of voiding sodium in a similar plutonium-uranium system (see Fig. 4). This is reasonable since the $\mathrm{U}^{233} /$ thorium system runs at a median fission energy of approximately $140-150 \mathrm{kV}$, whereas the $\mathrm{U}^{238} /$ plutonium system has a median fission energy of $200 \mathrm{kV}$. Also, there are less fissions in thorium, yielding a smaller fast-fission contribution.

The assumptions made as to geometry had a smaller effect than the other considerations previously discussed. Figure 5 illustrates the range of uncertainties as calculated for thorium. In the fuel lump case, the surface-to-mass ratio $(S / M)$ is taken into consideration (i.e., the size of the fuel rod and composition of fuel type). In the homogeneous case the fuel, coolant, and structure are assumed to be a homogenized material with no definite geometry.

The accuracy of the calculational method and the Los Alamos crosssection set has been partially demonstrated in the agreement obtained with the measured Doppler coefficient of thorium and $\mathrm{U}^{238}$ with the ECEL 
critical assembly.(9) These results have been presented by Dr. Carpenter at this conference.

VI. Doppler Coefficient as a Design Criterion and Its Effect on Economics

Using these data, we may now discuss the effect that uncertainties in the Doppler coefficient have on the relative economy of fast reactor cores. The basic assumption will be made that the Doppler coefficient is used as a design criterion limiting the amount of reactivity that may be inserted into the core at any given time in the fuel cycle. If all available excess reactivity in the core is suddenly exposed, the Doppler coefficient will act to return the reactivity to zero.

A second assumption will be that transient fuel temperatures in the center of the hottest fuel pellet in the core can exceed the melting point of the fuel without causing catastrophic failure. There are experimental data available for a limited number of tests with $\mathrm{UO}_{2}$ fuel where temperatures approaching $\sim 7000^{\circ} \mathrm{F}$ are calculated to have occurred during single power transients without loss of mechanical integrity of the clad.(10)

Specifically, for the purposes of this paper it will be assumed that the average temperature in the hottest oxide rods will reach the melting point of the fuel. For the carbide it was assumed that the hottest point in the hottest rod would reach the melting point. The difference in philosophy is due to the sodium bonding for the carbide, which will probably result in a failure of the clad during the temperature transient. If this occurs, but only a small portion of the fuel is near molten, the results should not be catastrophic from the standpoint of secondary criticality. This certainly represents, in our opinion, the maximum-temperature assumptions that could be made in connection with Doppler effect.

The minimum and maximum Doppler coefficients calculated for the temperature ranges of interest are listed in Table II. Based on those values, the ranges of allowable excess reactivity as set by the Doppler criterion are shown in Table III for the various cases considered. The sodium-bonded carbide fuel, operating at the lowest average temperature, permits the greatest excess reactivity. This is because (1) the Doppler coefficient increases as temperature is lowered, and (2) a larger fuel temperature rise may be tolerated when starting at a lower temperature.

For the calculated values of excess reactivity the minimum and maximum number of full power days that would result are shown in Table IV. The plutonium systems have a higher breeding gain, particularly the UC-PuC system; hence, the daily reactivity depletion is considerably smaller than for uranium-233 systems. As may be noted, the minimum 
refueling periods for oxide fuel are too short to be practical for a commercial power-generation unit. If the most optimistic values are utilized, shutdowns for refueling would still be quite frequent.

Table III

ALLOWABLE EXCESS REACTIVITY SET

BY DOPPLER COEFFICIENT

Units: $\varnothing$

\begin{tabular}{|c|c|c|}
\hline & $\begin{array}{c}\text { Gas-bonded } \\
\text { Oxide }\end{array}$ & $\begin{array}{c}\text { Sodium-bonded } \\
\text { Carbide }\end{array}$ \\
\hline \multicolumn{3}{|c|}{$\mathrm{U}-\mathrm{Pu}$} \\
\hline $\left.\begin{array}{c}\text { Lowest } \\
\text { Highest }\end{array}\right\}$ To melt fuel & $\begin{array}{r}45 \\
130\end{array}$ & $\begin{array}{r}58 \\
165\end{array}$ \\
\hline \multicolumn{3}{|c|}{$\mathrm{Th}-\mathrm{U}^{233}$} \\
\hline $\left.\begin{array}{l}\text { Lowest } \\
\text { Highest }\end{array}\right\}$ To melt fuel & $\begin{array}{r}41 \\
178\end{array}$ & $\begin{array}{r}50 \\
225\end{array}$ \\
\hline
\end{tabular}

Table IV

EFFECT OF DOPPLER COEFFICIENT ON REFUELING

\begin{tabular}{l|c|c|c|c}
\hline \multirow{2}{*}{} & \multicolumn{2}{|c|}{$\begin{array}{c}\text { Cycle Time - } \\
\text { Full-power Days }\end{array}$} & \multicolumn{2}{c}{$\begin{array}{c}\text { Refueling Times } \\
\text { per Year* }\end{array}$} \\
\cline { 2 - 5 } & Oxide & Carbide & Oxide & Carbide \\
\hline U-Pu & $11.9-34.4$ & $28.9-82.4$ & $8.5-24.5$ & $\begin{array}{l}3.5-10.1 \\
\text { Th-U } 233\end{array}$ \\
\hline
\end{tabular}

*Assuming an 0.8 load factor or 292 equivalent full-power days.

Some idea of the economic penalty that such shutdowns would incur can be calculated. If we as sume a $500-\mathrm{MWe}$ unit at a total cost of $\$ 175 / \mathrm{kW}$ and $14 \%$ capitalization charges, a capital charge of $\$ 37,300$ per day of shutdown must be absorbed by power-production days. In addition, this plant will normally represent a low-cost incremental unit in a system; consequently, higher fuel cost units have to be incurred whenever the plant is not operating. An incremental cost at least 2.0 mills $/ \mathrm{kWh}$ higher would 
not be unlikely, and this would add another $\$ 21,000$ per day to system costs. It can thus be seen that the shutdown for refueling of a unit such as this power plant represents would cost a utility over $\$ 20,000 /$ day in outof-pocket costs and add a total of about $\$ 60,000 /$ day to system powergeneration costs, without contributing any revenue. Ovex and above these costs is that of spinning reserve or spare capacity which would have to be available at the frequent shutdowns.

The maximum number of times per year a utility can economically afford to take a nuclear plant out of service for refueling depends on many things, including system characteristics, amount of time required for refueling, cost of powex from unit, and spare capacity. Generally, to date, utilities have preferred to have refueling intervals not less than once per year, but this, of course, is predicated to some extent on time required for refueling.

The number of shutdowns required for refueling to keep reactivity within the limits of the Doppler effect will dictate the number of fuel zones. If the fuel-management scheme utilized is one where no shuffling is involved, but only replacement, then the time penalty associated with shutdown will just be the time required for shutdown and startup. The time for actual fuel handling will total the same for a core whether it is changed all at once or in multiple zones. However, if zonal shuffling is employed, actual fuel-handling time per core will depend on number of zones.

If we consider that the time for replacing a fuel element as 60-90 min (figure based on Hallam experience with over-shield fueling machine) then a core with 300 elements and a 2.5 -yr life will require an average of $120-180 \mathrm{hr} / \mathrm{yx}$ if only fuel replacement is assumed. Shuffling of a complete core each shutdown would require as much as 300 additional hours. In addition, about $24 \mathrm{hr}$ will be required to shut down and bring a plant back on the line, over and above time for refueling. Thus the penalty for each shutdown can vary from $24 \mathrm{hr}$ in the case of straight replacement to about $300 \mathrm{hr}$ for complete shuffling.

If each day of shutdown adds $\$ 60,000$ to energy-generation costs, then 6-7 days/yr would add a penalty of $0.1 \mathrm{mill} / \mathrm{kWh}$ to yearly power costs. This is the equivalent $6-7$ shutdowns/yr for strict replacement or less than one per year if complete shuffling occurs. The minimum number of refueling times as set by the Doppler criterion for plutonium oxide fuels is calculated to add $0,13 \mathrm{mill} / \mathrm{kWh}$ to costs for strict replacement only without considering system costs for spare capacity which would be required every three weeks. Anything approaching the maximum number of times would be impractical. For carbide fuels the minimum would be a penalty of about $0.05 \mathrm{mill} / \mathrm{kWh}$, and the maximum would be about $0.15 \mathrm{mill} /$ $\mathrm{kWh}$ for strict replacement of plutonium fuels. 
Reshuffling would, of course, add significantly to costs - about $0.2 \mathrm{mill} / \mathrm{kWh}$ per yearly reshuffling cycle. In this case the carbide fuels, particularly of $U-P u$, are much superior in terms of minimum cost penalties as may be seen in $T$ able $V$, which summarizes calculated penalties for oxides and carbides for replacement and reshuffling modes of fuel management.

Table V

APPROXIMATE ECONOMIC PENALTIES BECAUSE OF SHUTDOWN FOR REFUELING AS DICTATED BY AVAILABLE DOPPLER (Mills/kWh)

\begin{tabular}{l|c|c|c|c}
\hline & Fuel Management & \multicolumn{2}{c|}{$\begin{array}{c}\text { Strict } \\
\text { Replacement }\end{array}$} & \multicolumn{2}{c}{$\begin{array}{c}\text { Complete } \\
\text { Reshuffling }\end{array}$} \\
\hline Range of Uncertainty & Min & Max & Min & Max \\
\hline U-Pu oxide & 0.13 & Not Practical & 1.5 & Not Practical \\
Th-U 233 oxide & 0.15 & 1 & 1.8 & $"$ \\
U-Pu carbide & 0.05 & 0.15 & 0.6 & 1.8 \\
Th-U $U^{233}$ carbide & 0.09 & Not Practical & 1.0 & Not Practical \\
\hline
\end{tabular}

VII. Conclusions

Restriction of available reactivity in a fast reactor core to that which can be overridden by the Doppler effect can result in significant economic penalties depending on the fuel type used and the fuel-management program employed. The current range of uncertainty in the Doppler effect results in a uncertainty factor of about three in refueling intervals for $\mathrm{U}-\mathrm{Pu}$ fuel materials and of about five for $\mathrm{Th}-\mathrm{U}^{233}$ fuels. The higher thermal conductivity of carbide results in lower fuel temperatures, so that a larger integrated Doppler effect is available. In addition, the carbide cores have higher breeding gain and, hence, lower core reactivity depletion rates than comparable oxide cores. This also lengthens refueling intervals. Minimum Doppler values for oxide fuels and thorium car bide result in impractical systems (refueling intervals of 1-2 weeks) when following Doppler design criteria.

The U-Pu systems appear to be somewhat superior to $\mathrm{Th}-\mathrm{U}^{233}$ cycles. Although Doppler coefficients for the two systems are comparable, the $T h-U^{233}$ systems have a higher burnup rate ( $\Delta \mathrm{k} / \mathrm{day}$ ) which results in shorter allowable refueling intervals than $\mathrm{U}-\mathrm{Pu}$ systems.

Fuel handling at power, as a means of meeting Doppler design criteria with no economic penalty, appears highly desirable, particularly when considering oxide fuels or a fuel-management program employing 
extensive reshuffling of fuel. Even with the most optimistic values for carbide fuels, maximum refueling intervals of less than four months are calculated. Shutting down a large plant to refuel this often may well present reserve requirements not compatible with the ultimate users' system even if shutdown is only for replacement of fuel.

Lower specific power or addition of moderating material in the core are other means, not examined in this paper, for increasing the Doppler effect, thereby reducing shutdown penalty. The possible fuel-cycle economic penalty of these changes must be evaluated; however, it is obvious that rather drastic measures must be taken, particularly in the case of oxide fuel if fuel shuffling is to be considered with the Doppler effect as a design criterion.

What really appears necessary is not a firm and absolute knowledge of exact Dopplex values, but whether dependence on Doppler feedback as a design criterion is really necessary. If it can be shown that partial meltdown of a core actually is the final inherent shutdown mechanism, rather than a mechanism causing secondary criticality, then the Doppler effect as an ultimate design criterion will not be required. This seems to the authors to be the answer that analysis and experiments should seek.

\section{References}

1. AMTD-130, ARES Resonance Integral Code.

2. I. Chernick and A. R. Vernon, Some Refinements in the Calculation of Resonance Integrals, Nucl. Sci. and Eng., 4, 649 (1956).

3. A.R. Vernon, Calculation of the Effective Resonance Integral of $\underline{U^{238}}, \underline{i b i d}, \underline{7}, 252(1960)$.

4. E. R. Cohen and R. Goldstein, Neutron Resonance Absorption Integrals, Trans. Amer. Nucl.Soc.3-1,232 (1960).

5. E. R. Cohen and R. Goldstein, Neutron Resonance Absorption. II., Trans. Amer. Nucl. Soc. (1962).

6. L. Dresner, The Effective Resonance Integrals of $\mathrm{U}^{238}$ and $\mathrm{Th}^{232}$, Nucl. Sci. and Eng., 1, 68 (1956).

7. F. T. Adler and I. W. Nordheim, GA-377 (1958).

8. M.G. Bhide and H. H. Hummel, Calculations of the Doppler Coefficient of Large Ceramic-fueled Fast Reactors, ANL-6601. 
9. Thorium and $U^{238}$ Doppler Coefficient Measurement in ECEL.

10. F.J. Leitz, Fast Ceramic Reactor Development Report, OctoberDecember 1962, GEAP-4158 (Jan 1963).

Discussion of Paper Presented by Mr.Stoker

MR. PERSIANI (Argonne):

I am wondering if in survey studies of this type one should not compare systems of equal power rather than equal size. This would bring in a merit factor of the fuel that is used. Have you made a study of this sort, equating power rather than size? Maybe you would be pushing the capability of the carbide fuels, at the same time. You might not find the Doppler effect as a significant parameter since you would be operating at higher temperatures in the carbide. I believe that it is the difference between the Doppler effect in carbide and oxide, am I right?

MR.STOKER:

This is true. The temperatures affect the allowable excess reactivity. There is the other point that the breeding gain in the carbide sys tems was higher. Consequently, you have a smaller daily loss of reactivity under full power, and this does extend the intervals between refueling. We did choose these, as you note, on the same total reactor power and physical size. We used, obviously, different rod powers for the oxide and carbide.

MR. OTT (Karlsruhe):

Can you say what is the main reason for the fairly large error in the Doppler coefficient? You have a factor of 3.

MR. STOKER:

I will ask Mr. Campise to answer that.

MR. CAMPISE (Atomics International):

The main difference that we noted between the two cross-section sets is in the neutron flux spectrum. By and large, the neutron flux spectrum, you get from the Yiftah set is higher than what you get from the Los Alamos cross-section set. The softer spectrum gives you a larger 
flux in the intermediate energy range. This is by and large the effect that you are looking at here.

MR. STERN (Combustion Engineering):

I believe you charged for the shutdown time twice, if I understand it right: once the time for shutdown and capitalization on the original equipment, and once the fuel cost for a fossil-fired reactor. I think that only one of these charges should be used.

MR.STOKER:

What you are saying is, we were trying to indicate not just the outof-pocket cost, but the differential. You said, if I shut down once a year or three times a year, what does it mean to me. The out-of-pocket cost is only the excess or the higher incremental fuel cost. The more you are down, the less power you generate from the system, and the greater is going to be the capital contribution to the power generated.

MR.STERN:

I agree. I still think that you are charging the utility twice for the same expense.

MR.STOKER:

I don't believe we are.

MR. ZALESKI (EURATOM):

I think that one possibility is to try to obtain an internal breeding ratio of unity for the reactor. I would say for plutonium 1.1 is necessary for the initial breeding ratio, if there is to be no reactivity change for a very large period of time. Despite some uncertainty on the cross section our calculation gives, for 50 per cent oxide-type fuel of a density of about $9 \mathrm{gm} / \mathrm{cm}^{3}$, this kind of breeding ratio for large fast reactors producing a thousand electric megawatts in a core of 8000 -liter or so. Perhaps, in this case it would be less important to have the Doppler effect as large as you mention.

MR. STOKER:

We have considered other means of holding down reactivity, such as a fixed poison column, so that you can load a substantial amount of excess reactivity, and yet hope that the amount that the core is seeing at any one time is small. But you still have to come back to the question of what happens if all of the excess reactivity is introduced? 
MR。ZALESKI:

I think it is very important to split the accident study into two parts, the maximum credible accident and the probable accident. If you have a probable accident, you wish not to destroy the reactor. In the maximum credible accident, the problem is different. You accept the fact the reactor will be destroyed or partially destroyed. What you are looking at is the magnitude of this accident. In this case, it seems to me the absolute value of this reactivity is not very important. It is much more the insertion rate that is important. I would also like to ask if you have used the Hansen-Roach cross-section set with the resonance correction?

\section{MR。CAMPISE:}

The multigroup cross-section set that we have used is that of C. B. Mills, an 18-group set, modified by our efforts on the AETR program, where we ran a set of criticals ranging from very fast cores to very epithermal cores. The resonance cross sections now are again modified by our resonance program called Aries as originated by Chernick et al.

MR. SMITH (Dounreay):

I detect an attitude at this conference that we should consider the effects of an accident without paying any regard as to how the accident condition could arise. The only way that the available excess reactivity could be an embarrassment is if the reactivity is added in an uncontrolled manner. If burnup compensation is provided by a depletion rod which may only add reactivity at a very low rate, and I mean a very low rate, then under these conditions this reactivity should not be considered as being available as an accidental fast ramp addition.

MR.STOKER:

I am not sure that present thinking in the United States will allow this.

MR. CAMPISE:

The question is when do we start taking credit for our ability to engineer and to instrument reactors? The present philosophy is not to explain how the accident starts, but, assuming the accident has started, what is the result?

MR.EDWARDS (Dounreay):

I should like to ask whether these Doppler calculations are isothermal ones or whether they apply to the temperature distribution 
corresponding to power. It seems to me if they are isothermal, then they are less appropriate than those involving power. In the latter case you must ask whether the thermal conductivity or the disposition of the fuel within a pin might change the burnup, and whether this would alter your temperature distribution.

MR.STOKER:

If I am correct, we did these Doppler coefficients at power, did we not? These are not is othermal.

MR. CAMPISE:

Yes.

MR. OKRENT (Argonne):

It seems to me that the main point is not what exactly are the uncertainties for a particular reactor, because we are not really going to build this particular reactor. As I understand the work under discussion, they did some calculations of a crude sort to provide some estimate of the uncertainty, and then did a simple analysis to show that if you apply this criterion, and if you cannot assure yourself of very large negative Doppler coefficients, you are in trouble. I think it poses the issue which we really have not met yet at this meeting, namely, what are the criteria for safety that we should apply to design. This is not only the question of how negative should our Doppler coefficient be, but also what do we do with the sodium void effect.

MR. DAVIDSON (Allis-Chalmers):

I think the key conclusion of the paper is the fifth one, which questioned the validity of the first starting assumption. I wonder if we could impose on Dr. Thompson for an off-the-cuff opinion.

MR.THOMPSON (MIT):

I do not think that the ACRS has ever set up any definite criterion of this sort. Such a criterion would probably be vigorously fought by the ACRS and many other groups, because you would then have seriously limited the areas in which you can develop reactors. You have to look at each reactor, and you have to study what the implications are: the addition of reactivity, how you add it, how it is used, and what could happen in reasonable and pertinent circumstances, and from that base you have to make a decision. You cannot generalize into any patent set of rules that define exactly what the answer should be. We are far too early in this business to make this sort of generalization. 
MR.WOLEE (General Electric):

It seems to me there are two accidents involved here. One is the maximum credible accident, one is the worst hypothetical, worst conceivable accident. In the maximum credible accident, you look for a credible mechanism. Having excess reactivity does not necessarily mean you can find a credible way to put it in. The water reactors, for instance, have 20 per cent excess reactivity. In the worst conceivable accident, that accident is determined not by the maximum excess reactivity, but something different, such as the maximum reactivity you can put in during a meltdown with maximum rate at which the core can compact, or some other mechanism of this type. I think the criterion which General Electric used two years ago, when you really get down to looking at details of reactor design is not a valid one.

MR.CAMPISE:

Our paper was based upon operational philosophy. We were more stringent in our requirements of the inherent feedback. We require that no damage occur to the core. 
•

416 


\section{SESSION IV}

October 8, 1963

SAFETY AND ECONOMICS

Chairman: MANSON BENEDICT

Massachusetts Institute of Technology

Secretary: C. E. Dickerman

Argonne National Laboratory 


\title{
FAST NEUTRON BREEDER ECONOMICS
}

\author{
Robert J. Creagan \\ Westinghouse Electric Corporation \\ Atomic Power Division \\ Pittsburgh, Pennsylvania
}

I. Introduction

The EBR-I demonstrated that fast neutron reactors are technically feasible for the production of power and fissionable material.

The motivation for further development is based on the desire to obtain larger amounts of energy from uranium and thorium ores and independence from sources of enriched uranium. Fast neutron economics permit all uranium with the exception of processing losses to be converted into energy. The excess fissionable material produced can be utilized to convert uranium and thorium into energy in either fast or thermal neutron reactors.

There can be no doubt as to the ultimate desirability of fast neutron reactors because the number of neutrons produced per neutron absorbed in plutonium-239 is less than two for thermal neutron fissions as compared with a value as high as 2.7 in a fast neutron spectrum. Thus, for the uranium-plutonium cycle, breeding is impossible except in a fast neutron spectrum.

Despite the excellent neutron economy, the fast breeder must compete on the basis of dollar economics with either conventional or water reactor power plants.

It is the purpose of this paper to discuss the economic environment concerning fast breeder reactor power plants as compared with conventional and water reactor power plants and to illustrate what goals must be met in order to make the fast breeder plant competitive.

If fast reactor power plants can compete economically in the near future, another strong motivation exists for early development which may justify a faster national program than now planned.

The economic factors which will be discussed are market, competition, availability of materials, potential advantages, problem areas, and research and development. 
II. Maxket

One of the most important aspects of the fast reactor program planning is a consideration of the potential markets. The United States electrical utility industry spends approximately $\$ 2$ billion per year on new generating capacity. The trend has been to purchase increasing unit sizes of conventional power-generating equipment. The increase in equipment size has accelerated recently, and it is interesting to explore the reasons.

Figure 1 shows the electrical generating capacity by year of the United States as predicted by the Electrical World forecast. The nuclear generating capacity is seen to be a very small percentage of the entire market, but that fraction could change drastically if nuclear generating power costs were reduced radically. Present nuclear generating capacity is approximately $10^{6} \mathrm{~kW}$; however, nuclear plants committed within the last twelve months have added an additional 1,500,000 kW. Fast breeder units would constitute $10^{6} \mathrm{~kW}$ out of $25 \times 10^{6} \mathrm{~kW}$ in 1978 .

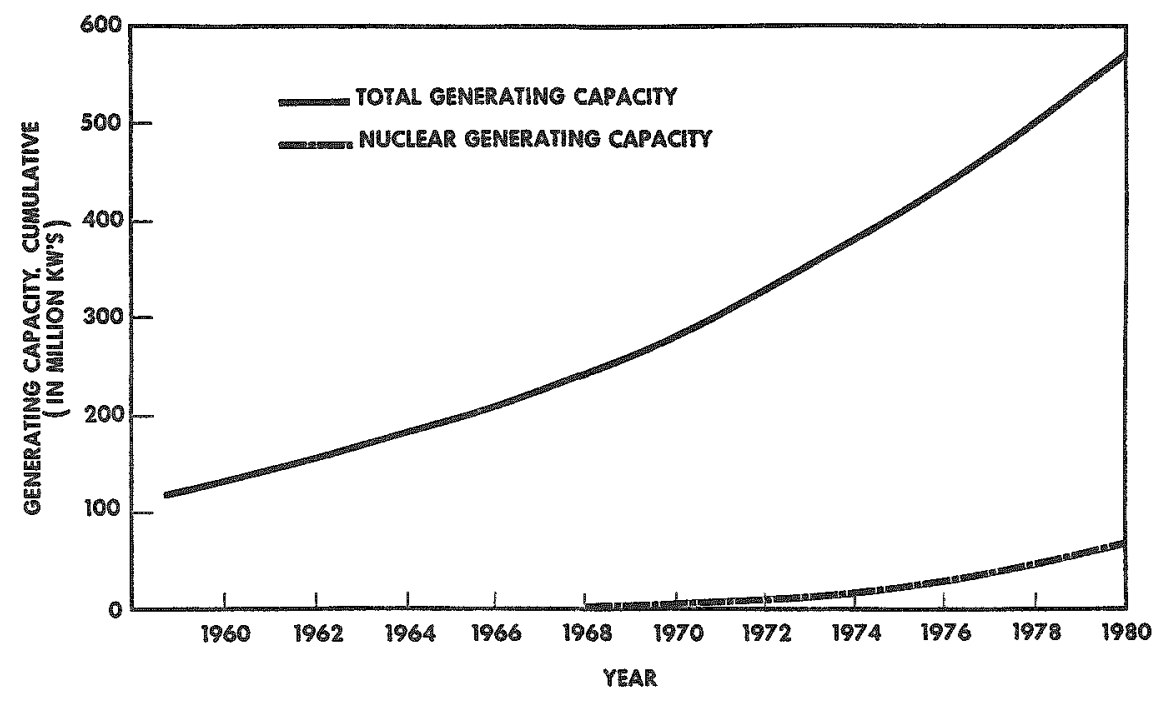

Fig. 1. Electrical World Forecast of Electrical Generating Capacity

The last three nuclear generating units committed are approximately $400,000,500,000$ and $500,000 \mathrm{~kW}$. It is interesting to note why these very large units in comparison with the very small units which were typical a few years ago have been sold.

Figure 2 indicates that in 1955 the electrical utility industry consisted of 370 investor-owned utilities, 1800 municipal, 980 co-ops, and 30 federal, state, and other agencies. These utility companies purchase generating capacity in proportion to their size. The average capacity is only $150 \mathrm{MW}$, or approximately the size of the Yankee Atomic Electric Company nuclear power plant. 
IN 1955-

TME ELCTRIC UTLLTY IMUUSTPY COMSISTED OF:

- 370 Investor Owmed

- 1800 municopals

980 Co-operatives

30 Foderal, state 8 Other Ageney

Fig. 2. Electric Utility Industry in 1955
However, utility power pooling is occurring. Figure 3 indicates the interconnected power pools in the United States. Their average capacity is about ten times that of the utility companies as they existed and operated in 1955. Thus, while the corporate structure of the utilities has not changed significantly in the 10-yr period, the system requirements have. As a result of the much larger interconnected capacity, much larger generator units are acceptable. This occurs because of the greater capac-

ity available if a unit goes off the line and also because the larger interconnected load grows at a rate justifying a larger incremental addition of power-generating capacity.

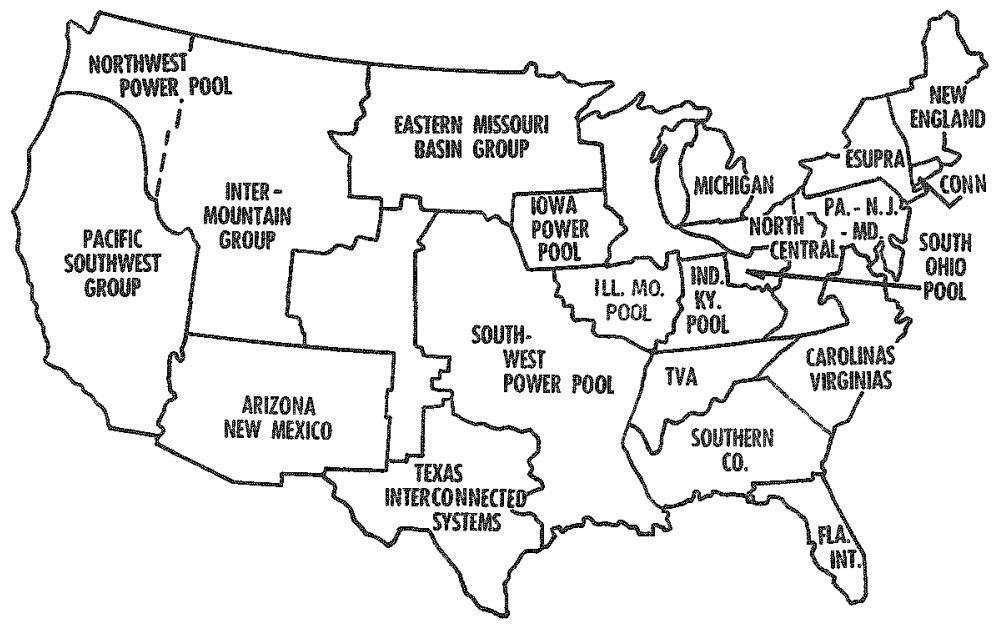

Fig. 3. Pooling Alignment - 1962

Figure 4 indicates the result of the power pooling previously described and is pertinent to the fast breeder planning for the future. It indicates that very large-sized units are being ordered and will be ordered in even larger sizes in the future.

The solid line beginning at the left-hand side of Fig. 4 shows the increase in average unit sizes over the years. Normally, the projection of the average unit size would have been expected to proceed along the dotted line. However, the trend to power pooling has resulted in a jump in average unit sizes which will be required. These increases in unit sizes reduce capital cost but do not change the fuel costs, which are a function of material costs, location, and shipping costs. 


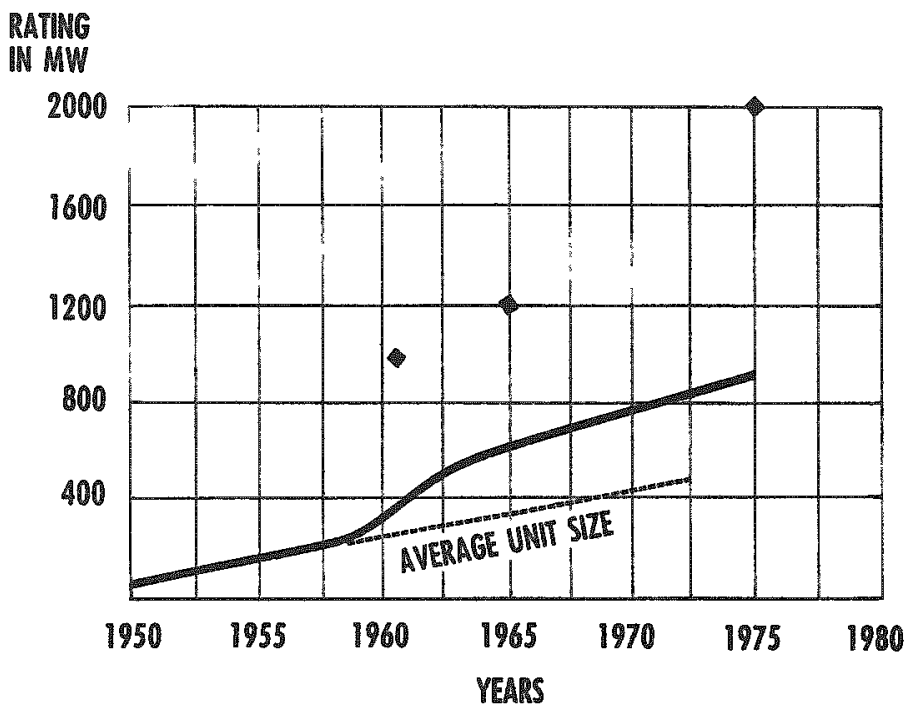

Fig. 4

Unit Size Ordered

Average unit size ordered as a function of time. Dashed curve shows projection without pooling, solid curve with pooling. Diamond points indicate maximum unit size.

The specific units of much larger size than the average are the 1000-MWe unit for Consolidated Edison (Ravenswood No. 3) and the 800-MWe unit for TVA.

As unit size of generating capacity increases, there is a greater possibility for the nuclear power plant to be competitive economically. Because the fast breeder is scaled up in size to reduce costs, this is a distinct advantage.

III. Competition

The competition which the fast breeder faces is from conventional power plants as well as from the reactor types which have become competitive, such as water reactors.

Figure 5 indicates a comparison between power costs vs unit size for nuclear and fossil-fuel power plants with fuel costs of $25 \notin$ to $35 \notin /$ $10^{6} \mathrm{BTU}$. Both the East and the West Coasts have high fuel costs. Thus, already nuclear power is competitive in these regions, as is indicated by the nuclear power plants that have been purchased for installation.

The fast breeder must be competitive eventually with these power costs or it will not gain acceptance as an economic commodity. Because it does offer the possibility of being economically competitive, that fact may supply more incentive for development on a shorter time scale than the consideration of conservation of natural resources which is the basic objective, but which might allow development of the fast breeder reactor over a longer period of time if that were the only consideration. 


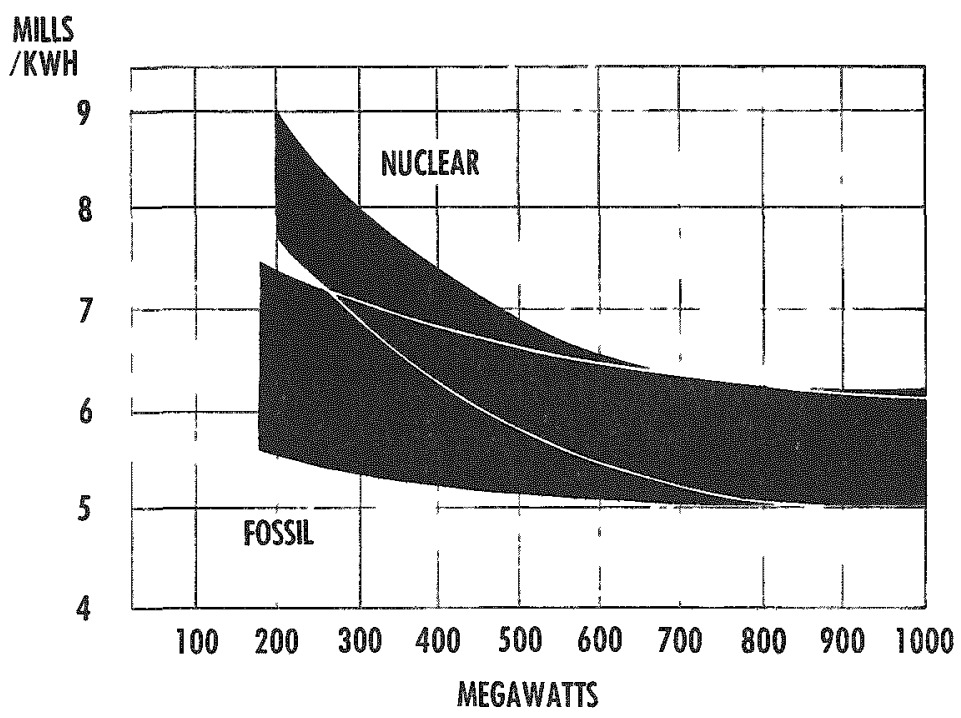

Fig. 5

Power Cost vs. Unit Size

Figure 6 indicates a comparison of 1000-MWe power plants of a conventional, fossil-fuel variety; a water reactor; and a fast breeder reactor. As noted in the comparison, the capital cost for the nuclear power plants is higher, but this is offset by the lower fuel cost. Because of the very great variety of conventional power plants which are purchased, the $\$ 125 / \mathrm{kW}$ is a nominal figure and varies over the United States. The capital costs cited for the water and fast reactor plants are nominal, and

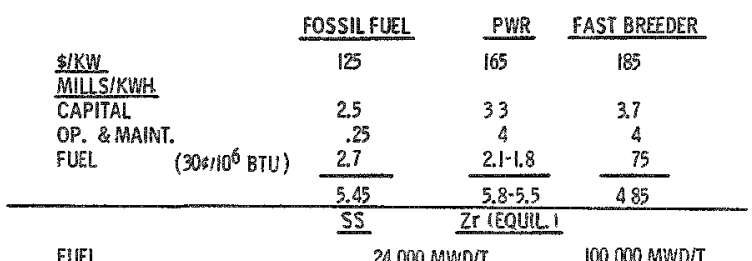
FUEL 24000 MWOT 100000 MWDT FARRICATION DEPLETION USE CHARGE REPROCESSING SPENT FULI SHIPPING PIUTOMUM CREDTT

\begin{tabular}{|c|c|c|}
\hline \multicolumn{2}{|c|}{ 24.000 MwOT } & $100000 \mathrm{MWD}$ \\
\hline .69 & 78 & .60 \\
\hline 1.35 & 1.09 & - \\
\hline .20 & .16 & .35 \\
\hline 26 & .24 & .20 \\
\hline .08 & .07 & .05 \\
\hline$(.65)$ & $(.48)$ & (25) \\
\hline 2.13 & 183 & 75 \\
\hline
\end{tabular}

Fig. 6. Power Costs (1000 MWe) will be influenced by the economic forces of future development and of competition.

The transformation to mills per kilowatt hour is based on an 80 percent plant capacity factor and 14 percent annual charges on capital.

Operation and maintenance is based on a 1000-MWe unit and includes, in addition to operation, charges for insurance against nuclear liability. The fuel costs cited are based on 30 cents per million BTU fossil fuel. The fuel costs are based on the breakdown which is given below.

Present commercial arrangements for fuel procurement based on 2.1 or $2.3 \mathrm{mills} / \mathrm{kWhr}$ for smaller size reactors are available depending upon the scope of the service. The lower fuel costs result from expanded development efforts which will result in lower fabrication costs and longer burnups. 
The $0.75-\mathrm{mill} / \mathrm{kWhr}$ fast breeder fuel cost is based on many calculations, and the breakdown is given below. Other estimates have run as low as $0.5 \mathrm{mill} / \mathrm{kWhr}$, but it is believed that the 0.75 figure may be $x$ ealistic based on a 100,000-MWD/T fuel burnup and the fabrication charge of $\$ 400 / \mathrm{kg}$ of fuel. Although one may quibble about the individual numbers used, the important fact is that these approximate cost figures must be realized for a fast breeder power reactor or it will not be competitive in the United States' economic environment.

Figure 7 shows a cost comparison between a water reactor and a fast breeder reactor as a function of fuel burnup. Again, these are nominal costs and are cited to illustrate trends rather than to establish a com-. mercial selling position. As is noted on the curve, the benefit to the fast breeder reactor in going to $100,000-\mathrm{MWD} / \mathrm{T}$ fuel burnup is such that development objectives should be set at that level and the necessary work performed to insure that such burnups are feasible and consistent with safe performance.

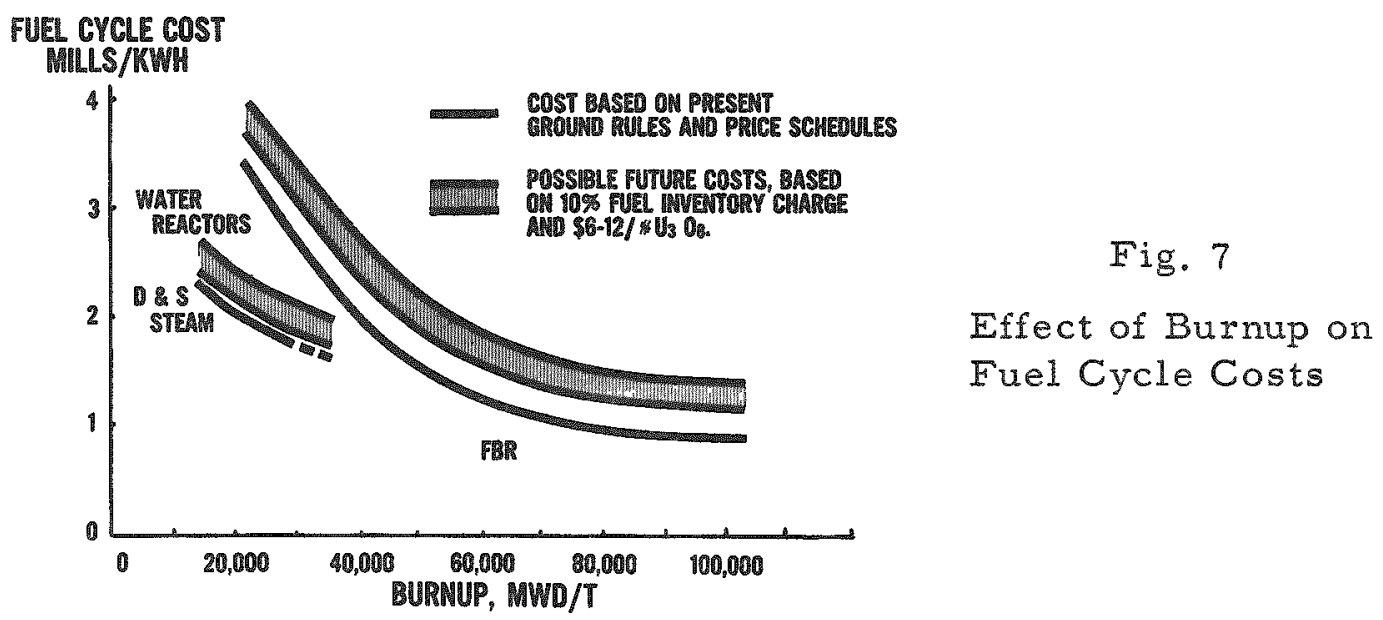

The size of fast breeder reactor to be considered in this paper will be $1000 \mathrm{MWe}$. By the time fast breeders are more fully developed, a capacity of $1000 \mathrm{MWe}$ or larger in a single unit will be the accepted size and may be purchased.

In addition to the United States market described above, foreign countries will purchase fast breeder reactors. The reasons for such purchases will be freedom from diffusion-plant requirements and less dependence on the cost of ore. This will allow foreign countries to be autonomous with respect to the United States enriched fuel supply and also will place them in a better position with respect to ore requirements. Because of the lower ore requirements, the balance of cash flow for the individual countries may be helped by using higher labor costs within a country to mine ore which would not be economically desirable with a reactor requiring more ore per kilowatt hour generated. 
The United States may sell a first nuclear power breeder reactor in a country and thereafter possibly will sell "know-how" and some components through licensees. There is the possibility that as increases in sizes occur, the first of a particular unit size will also be produced in the United States. This will occur because of capital investment required to build a larger component and costs required in doing the necessary research, development, and engineering to increase the individual unit sizes. For these reasons, there may be an appreciable foreign market for many years to come for the fast breeder reactor. As excess plutonium may be used for enriching natural uranium or thorium, foreign countries may use their excess plutonium for assisting power generation in thermal reactors.

IV. Availability of Materials

The availability of the necessary construction materials for a fast breeder reactor is excellent with respect to components and piping. Or dinary austenitic steel is quite adequate for the temperatures external to the reactor core. Reactor vessel material and materials for pumps, valves, and other components are acceptable and available. Improvements will undoubtedly be made in material for components such as the boiler, or it may be that Incoloy -800 will eventually be designed into this application. The complexity of materials problems in the boiler is indicated when one considers that the material contains sodium on one side of the tube and water or steam on the other side. In addition, in the event of failure of the tube, sodium hydroxide will be created on the sodium side as the steam or water side will be the high-pressure side. If sodium hydroxide is produced on the sodium side, design must be such that dilution will occur and that caustic embrittlement is not caused by concentration of sodium hydroxide before the break is corrected.

In addition to the requirements of conventional building equipment, the fuel requirements are such that energy-production requirements can easily be met by the available uranium ore resources. The AEC letter to the President indicates that at present prices of less than 10 dollars per pound of oxide, approximately 50 Q of energy are available in the estimated total resources. Such an energy availability, as indicated in Fig. 8, would make available power resources as required up through the year 2150 . This is verified by Fig. 9, also taken from the AEC letter to the President. These figures indicate that no problem exists with respect to the availability of uranium ore.

With respect to availability of uranium-238, the tailings from dif fusion plants have been collecting over the years and are available in large quantity. There is a real economic motive for using the material since it serves little useful purpose with the exception of counterbalance weights on airplanes and possible paperweights. An estimate of the amount of uranium-238 which exists as tailing from the diffusion plant might be made 
by referring to the Nucleonics article of January, 1958, by Karl Cohen. On p. 68, he indicates that approximately 85 short tons of 90 percent enriched uranium are produced per year. If one assumes that only 0.5 percent of the uranium-235 is taken out of natural uranium, then the tailings which would accumulate over a 10 -yr period would be 16,000 tons. This would be a useful source of material for the fast breeder reactor program and might be increased considerably by production reactor waste uranium.

Fig. 8

FISSION ENERGY CONTENT OE DOMESTIC NUCLEAR RESOURCES*

\begin{tabular}{c|c|c|c|c}
\hline \multirow{2}{*}{$\begin{array}{c}\text { Cost Range, } \\
\text { per Pound } \\
\text { of Oxide }\end{array}$} & $\begin{array}{c}\text { Energy in } \\
\mathrm{U}^{235}, Q\end{array}$ & \multicolumn{2}{|c}{$\begin{array}{c}\text { Total Energy Content, } \\
\text { in Uranium, } \mathrm{Q}\end{array}$} \\
\cline { 2 - 5 } & $\begin{array}{c}\text { Assured } \\
\text { Resources }\end{array}$ & $\begin{array}{c}\text { Estimated } \\
\text { Total } \\
\text { Resources }\end{array}$ & $\begin{array}{c}\text { Reasonably } \\
\text { Assured } \\
\text { Resources }\end{array}$ & $\begin{array}{c}\text { Estimated } \\
\text { Total } \\
\text { Resources }\end{array}$ \\
\hline $0-10$ & 0.16 & 0.4 & 22 & 50 \\
$10-30$ & 0.17 & 0.3 & 24 & 40 \\
$30-100$ & 5.0 & 10.0 & 700 & 1,400 \\
$100-500$ & 220 & 900 & 30,000 & 120,000 \\
\hline
\end{tabular}

*Civilian Nuclear Power... A Report to the President - 1962.

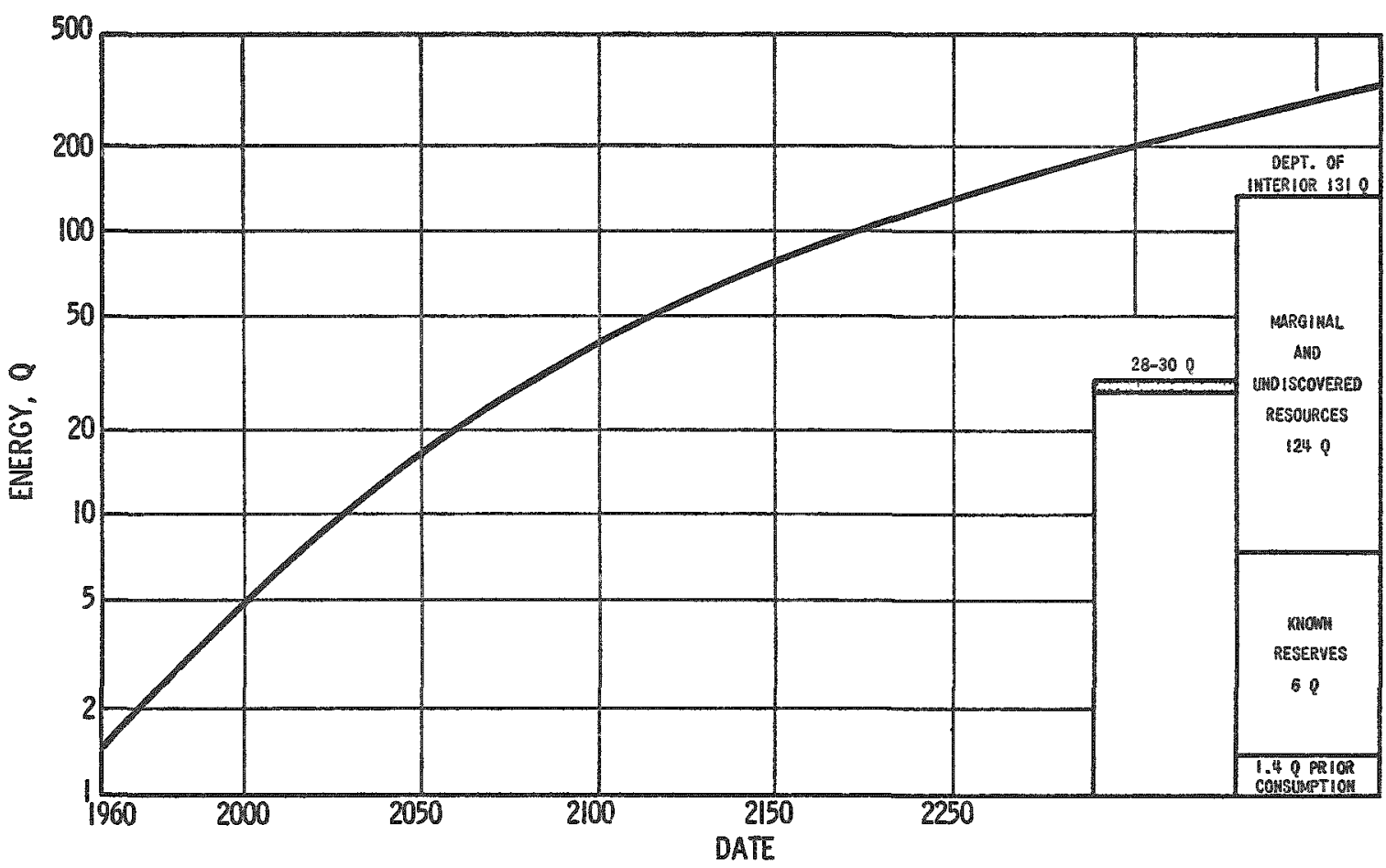

Fig. 9. Cumulative Energy Consumption and Fossil Fuel Resources for the United States. (From Civilian Nuclear Power...A Report to the President - 1962.) 
The probable availability of plutonium from civilian reactors is indicated in Fig. 10. The curve indicates the scarcity of plutonium which will exist for some time; from the curve, subtraction must be made of plutonium required for critical experiments and for commitments other than fast breeder reactors. If the plutonium required for a 1000-MWe breeder reactor is $2500 \mathrm{~kg}$ of plutonium, it would be difficult to provide enough fuel plutonium from civilian reactors before 1970 if one deletes what seems to be reasonable for other requirements. The conclusion to be reached is that plutonium availability will be one of the problems faced by fast breeders through 1970. Thereafter, production rates anticipated are high enough so that the necessary civilian-grade plutonium will be available for fast breeder reactors.

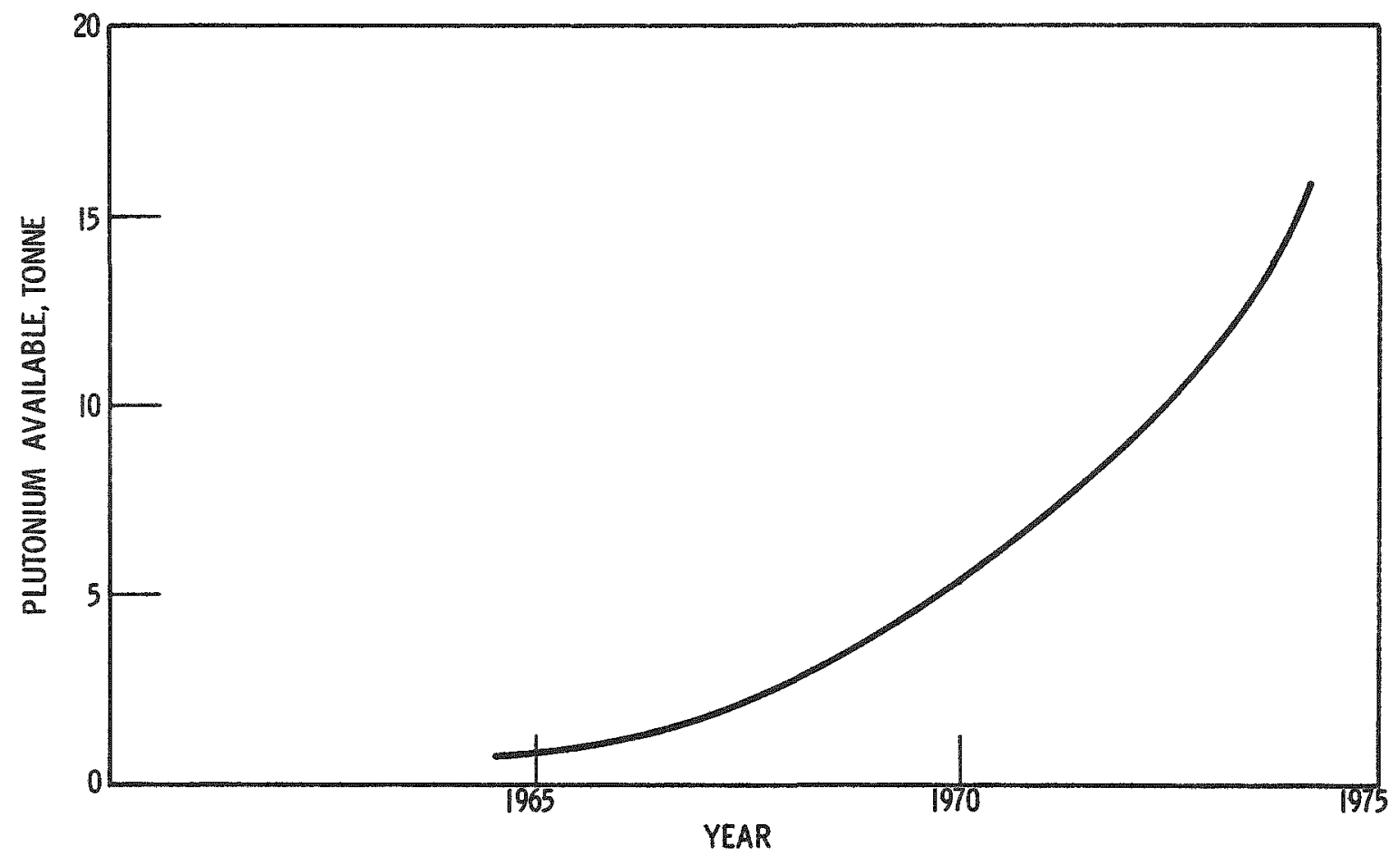

Fig. 10. Plutonium Available (Cumulative) from Civilian Power Reactors

One conclusion to be drawn from the availability of plutonium is that it may be desirable to operate some fast breeders on uranium -235 in order to develop plant characteristics in advance of the availability of large quantities of plutonium. One advantage of this course of action would be the larger amount of delayed neutrons from uranium-235 and the greater ease of fabrication in conventional facilities. Less desirable aspects would be the lower breeding ratio with uranium-235 and the lack of the ability to determine the nuclear characteristics of plutonium with respect to transient characteristics. This would be specifically pertinent to the coefficients important for stability, such as the Doppler coefficient and the sodium void coefficient. 
The facilities now available for plutonium handling seem quite adequate for the present industry. The facilities which do exist are not in full use, and the development of fast breeder reactors should not be impeded for lack of plutonium development facilities. The excellent plutonium developmental facilities here at Argonne are examples of the capabilities existing in the United States. These can be used to explore the material aspects of plutonium or to produce fuel elements in pilot-plant quantities.

\section{Potential Advantages}

The potential advantages of the fast breeder reactor are interrelated with the conservation of natural resources; therefore, it is a very important reactor for a nation to develop. Of more immediate interest to the potential vendor of fast reactors and the potential customers for such reactors is the fact that lower power costs may be available because of lower fuel costs. This may occur because of (1) the high thermal efficiency of the fast breeder, (2) the fact that it will scale up to large sizes in an economical manner as compared with smaller sizes, and (3) that the net production of fissionable material may help in reducing overall power costs. The utilization of more conventional turbine-generator equipment may reduce the cost of the turbine as much as $\$ 10 / \mathrm{kW}$ of capital.

With respect to the efficiency of converting fissionable atoms to electrical energy, the fast neutron breeder is without peer. High thermal efficiency (over 40\%) can be obtained with modern steam conditions at $1000^{\circ} \mathrm{F}$ and whatever pressure is most economic. In addition, more (a factor of 1.4 for plutonium-239) BTU's of heat energy are obtained per gram of fissionable material destroyed in a fast rather than in a thermal neutron spectrum because of the lower nonfission neutron captures in fissionable fuel. Another factor is the greater fraction of energy which comes from fast fission of uranium-238 in a fast (10\% in Fermi and $16 \%$ in a large uranium carbide core) rather than in a thermal neutron spectrum ( $7-9 \%$ in Yankee type reactors). This means that the thermal efficiency of a slow neutron reactor would have to be larger by a factor of 1.5 for plutonium-239 and by 1.09 for uranium -235 to produce as many kilowatt hours of electrical energy per gram of fissionable material destroyed. Thus, even disregarding breeding, the fast neutron reactor is a more efficient burner of fissionable material.

Table I gives the kilowatt hours of electrical energy obtained per gram of fissionable fuel destroyed assuming $40 \%$ efficiency for conversion of heat energy to electrical energy, the same energy release per fission, effective alphas as listed, $14 \%$ uranium -238 fast fissions in a fast reactor, and $8 \%$ in a slow neutron reactor. 
Table I

\begin{tabular}{c|c|c|c|c}
\hline & \multicolumn{2}{|c|}{ Fast Neutron Reactor } & \multicolumn{2}{c}{ Slow Neutron Reactor } \\
\cline { 2 - 5 } & Effective $\alpha$ & $\mathrm{kWhr} / \mathrm{gm}$ & Effective $\alpha$ & $\mathrm{kWhr} / \mathrm{gm}$ \\
\hline $\mathrm{Pu}^{239}$ & 0.17 & 9000 & 0.64 & 6000 \\
$\mathrm{U}^{235}$ & 0.23 & 8600 & 0.26 & 7800 \\
$\mathrm{U}^{233}$ & 0.09 & 9700 & 0.12 & 8800 \\
\hline
\end{tabular}

VI. Potential Problem Areas

Potential problems of the fast reactor which must be resolved for the economic as sumptions to be worthwhile result from the many unknowns which still remain despite the development programs that have been performed. The fact that the neutron-generation time is of the order of $0.1 \mu \mathrm{sec}$ and the fact that the value of beta for plutonium is only one-third that of uranium- 235 have been quoted as reasons for increased care needed for the control of fast reactors. The study of these various effects has been carried out and will be continued to be studied. The Fermi reactor has placed upon itself the maximum $\Delta \mathrm{k}$ available of $\$ 1.00$; however, this would not seem to be a realistic limitation desirable for an economic power plant in the future. The beta value for plutonium referred to above is helped somewhat by fast fissions in the uranium-238, which increase the effective beta value for the fast reactor.

To obtain $1000^{\circ} \mathrm{F}$ steam conditions may require $1400^{\circ} \mathrm{F}$ maximum metal surface temperature in the fuel. At such temperatures, masstransfer phenomena may occur. It is then a question of demonstrating what the limiting temperatures are that can be utilized with typical austenitic steels. The use of more costly cladding materials will probably not be justified.

The effects of a sodium-water reaction can be anticipated, and engineering safeguards can be incorporated; the recent steam-generator sodium-water reaction at Fermi indicated that the "Kapow" plate operated in design fashion. Although the equipment performed as designed, it is not desirable to have this interaction occur because of economic problems resulting in the boiler.

Requirements of high specific power because of inventory costs of the large plutonium investment may require new developments for the fast plutonium reactor not required for the the rmal variety. This high specific power will also cause higher fuel fabrication costs and therefore an economic compromise must be reached. 
The most encouraging aspect of the problems of fast breeder reactors is that the EBR-I has operated successfully; even when an experiment was performed which caused it to fail, the failure was in such a manner that the difficulty was confined to the core of the reactor.

The operation of fast breeder reactors has indicated the feasibility of the use of the various materials involved; however, such reactors have not been operated at the temperatures at which it is desirable to operate large economic fast breeders. It is this increase in temperature which will accentuate some of the difficulties which have already been encountered.

Thermal stress will continue to be one of the important engineering considerations which must be designed into a reactor which has a large $\Delta \mathrm{T}$. The large temperature gradient is caused by the low specific heat of sodium and the desirability of reducing pumping-power requirements.

In summary, there are no problems which appear to be insurmountable for the fast breeder reactor; the question is one of economic optimization rather than the solution of a "go" or "no-go" type of problem.

VII. Research and Development

Figure 11 illustrates the 1962 expenditure for research and development by the Reactor Development Division of the AEC. The pertinent

Fig. 11

\section{REACTOR DEVELOPMENT COSTS}

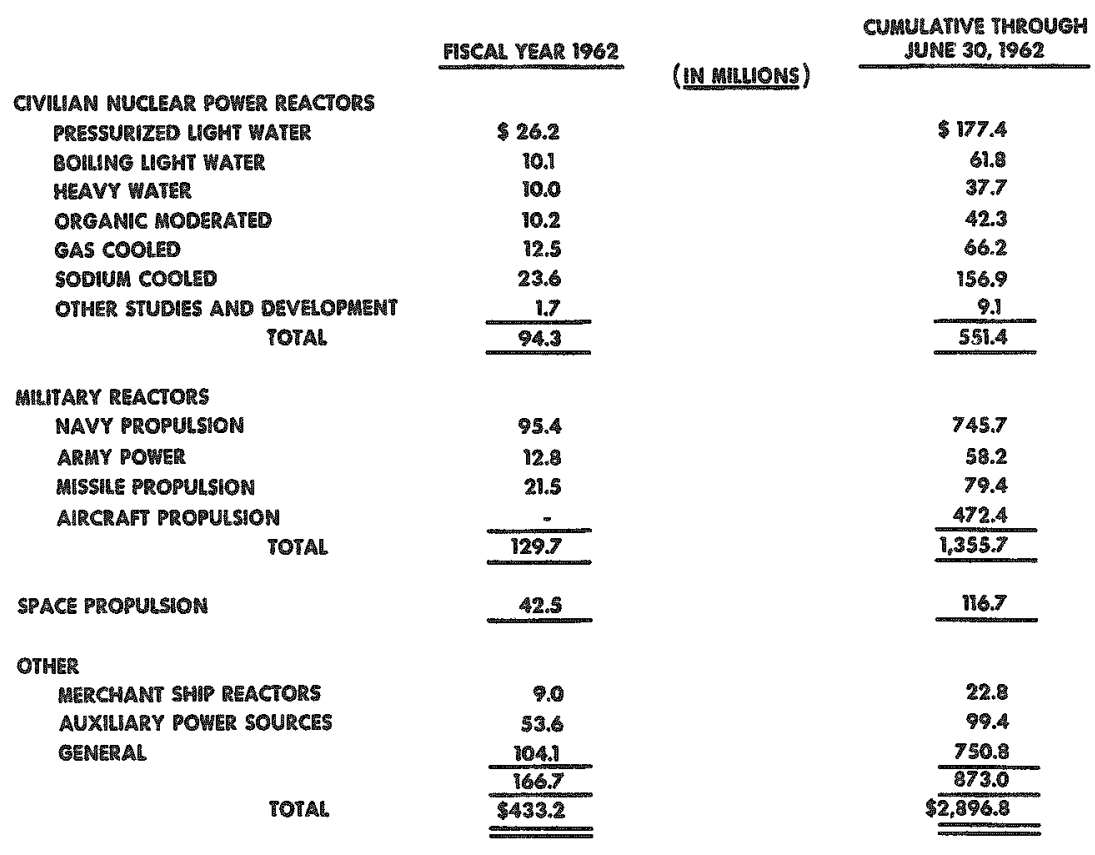


comparison to note is that approximately as much research and development costs have been incurred for sodium-cooled reactors as for any other single type. This is an indication of the basis on which present designs are made. It indicates that enough developmental work has been carried out to date so that many of the problems have been analyzed and confirmed experimentally. This is not to say that many problems do not continue to exist; rather, this illustrates the fact that sodium reactors have come a long way in the civilian program, and this does not include the development effort expended on SIR submarine land-based prototype and sea-going version.

The research and development required must solve the problems indicated earlier in this paper. To indicate some of them:

\section{Containment}

The study of containment must be made to establish (1) whether or not the typical type of containment is as suitable for fast breeder reactors as it is for the water-cooled variety, and (2) whether or not economics will be affected by engineering safeguards into containment particularly fitted to the fast breeder needs. The study of containment is listed because it may be pertinent to the site cost and the transmission cost of electrical power. If a suitable containment can be developed, there will be no site or transmission problems.

2. Long-burnup fuel is a basic requirement of the fast breeder reactor. At present, the mixed oxide (plutonium oxide-uranium oxide) appears to have the necessary properties for a $100,000 \mathrm{MWD} / \mathrm{T}$ burnup and can be considered as a realistic fuel for this application. Because of the properties of mixed carbides, this type of fuel should also be developed; the necessary irradiation experiments should be performed to establish whether or not the carbides are acceptable as fast reactor fuels. They may be less costly than the mixed oxide fuels. The metallic fuels developed to date do not appear to be suitable because of the temperature limitations and distortion upon long-term irradiation. The cermet fuels do not contain the promise for breeding; hence, Great Britain has eliminated them from consideration, because they do not achieve the basic purpose for which the reactor is designed. Other types of fuel concepts, such as the Brookhaven pellet reactor and the molten plutonium reactor at Los Alamos, also have potential merit and should be investigated further.

3. Mechanisms for decreasing nuclear reactivity for the fast reactor must be studied and thoroughly understood. These mechanisms include the Doppler effect, the sodium coefficient, mechanical expansions, and the possible application of fuses. The study in this area is needed for stability requirements with respect to both operation and the accident type of transients. Mechanisms must be investigated not only for terminating 
the operational type of transient, but to establish that an accident type of transient will eventually terminate in a safe manner.

4. The slow-fast reactor should eventually be investigated as a potential compromise of fast and thermal reactor spectrums. The development work done to date indicates that such a reactor can be built and that the calculational methods exist which will predict the neutronics to a reasonable degree of accuracy.

5. Components should be developed to the point at which they are reliable and have demonstrated the complete applicability of the materials to the operational environment. In addition to the materials aspects, the components must be able to be scaled up to large sizes so that a favorable economic position may be achieved. In passing, it is worth noting that increases in size are one of the most fruitul sources of decreasing costs for reactor power plants.

6. Heat transfer considerations must be investigated with respect to power disturbances and distribution of power and coolant throughout the core, both in steady state and in transient conditions. Orificing of a fixed type and a variable type should be developed further.

7. Maintenance of sodium components and the system should be studied and developed to a point where it can be done economically with minimum capital equipment. One example of a general device which should be developed is the under-sodium scanning device of APDA. Experience has shown the advantage of being able to see through water during reactor maintenance and reloading periods. The ability to observe operations under sodium would be highly desirable for the fast breeder program.

8. The entire reloading and fuel-handling scheme for fast breeder reactors is worthy of much development effort. Under-sodium and dryrefueling methods should be investigated. The mechanics and economics should be established as well as the safety aspects.

The timing of the development program now planned by the AEC indicates that a prototype of the fast breedex reactor might be committed in 1968 or 1969, and that a 1000-MW reactor might be committed in the middle or late $1970^{\prime} \mathrm{s}$. My first reaction to this schedule is that it certainly should not be slowed down. The degree of urgency of fast breeder reactor development can be argued, but the ultimate development is undoubtedly required. The necessary industry to support a fast breeder economy will have to develop without the benefit of the nuclear navy that helped the water reactor industry to develop. The water reactor industry was helped by multiple purchase of nuclear components. For this reason alone, the development of components will suffer unless the AEC continues to support 
these efforts. The knowledge derived from work on other reactor types will enable the industry to develop the fast breeder more expeditiously than in the past.

One item which has not been mentioned with respect to the urgency of development which can be evaluated better by the United States Government is the benefit of having an advanced technology available as an export to foreign countries and also as a negotiating tool in interchanging information with other countries.

In addition to the reasons cited and the conservation of natural resources which has been indicated in the AEC letter to the President, we believe that the fast breeder reactors are worth developing for the potential economic benefit to the United States.

\section{Discussion of Paper Presented by Mr. Creagan}

MR. HALL (Los Alamos):

The conclusion of your paper escaped me. Are you saying that the fast reactor is a good thing to do for some particular reason?

MR. CREAGAN:

The point I was making was that to conserve natural resources and to help us keep away from running out of energy is not a motive which one could use to expedite the fast reactor program, and that the economic merits of the system in the future would be a better basis on which to hang our hats as far as developing the system. 


\section{ECONOMICS AND SAFETY ASPECTS OF LARGE CERAMIC U-Th FAST BREEDER REACTORS
G. A. Sofer, A. H. Kazi, and J. Bobker
United Nuclear Corporation
White Plains, New York \\ (Paper presented by Mr. Sofer)}

\section{General}

The $\mathrm{U}^{233}$ - Th cycle has received increasing attention in recent years. Continued interest in this cycle is due to the following:

1. Thorium deposits represent about $20 \%$ of the world's potential fissionable and fusionable energy resources,(1) and 3 times the potential energy available from uranium alone. Countries such as India, Canada, and Brazil (2) possess abundant thorium supplies which could play an important role in meeting future power requirements.

2. The nuclear properties of $U^{233}$ favor breeding over a wider range of neutron energies than do those of $\mathrm{Pu}^{239}$ or $\mathrm{U}^{235}$.

3. More physics data(3) have become available for $U^{233}$ and thorium, thus permitting a better assessment of the potential of the $U^{233}-T h$ breeder systems.

4. The Report to the President by the USAEC(4) has placed important emphasis on the utilization of the $\mathrm{U}^{233}-\mathrm{Th}$ cycle as a means of extending the energy producing capabilities of the world's fissionable and fertile material resources.

Analytical and experimental studies of the $\mathrm{U}^{233}-\mathrm{Th}$ cycle have concentrated in the past on homogeneous thermal breeders and, more recently, on the prospect of achieving breeding or near-breeding conditions in heterogeneous water-cooled reactors. The most efficient utilization of the $\mathrm{U}^{233}-\mathrm{Th}$ cycle, however, lies ultimately in fast spectrum reactors which permit the highest breeding ratio for the $U^{233}-T h$ system.

In 1962, United Nuclear completed a study of $\mathrm{U}^{233}-\mathrm{Th}$ fast breeder reactors, and selected $\left(\mathrm{U}^{233}-\mathrm{Th}^{232}\right) \mathrm{C}$ as the optimum fuel for a $315-\mathrm{MWe}$ sodium-cooled reactor. (5) A breeding ratio of 1.31 and an overall system doubling time of 23 y $r$ was estimated for this reactor, based on a maximum fuel burnup of $50,000 \mathrm{MWD} /$ tonne.

The present paper discusses operational and safety characteristics of fast $\mathrm{U}^{233}-\mathrm{Th}^{232}$ sodium-cooled breeder reactors, updates the economics 
of the reference design in terms of current economic ground rules, and presents conceptual characteristics of a 1000-MWe reactor.

\section{Operation and Safety Characteristics of $\mathrm{U}^{233}-\mathrm{Th}^{232}$ Fast Reactors}

The use of $\mathrm{U}^{233}-\mathrm{Th}^{232}$ fuel in a fast reactor gives rise to several special problems of safety and operation. The buildup of $\mathrm{Pa}^{233}$ increases the excess reactivity to be controlled for burnup, and the decay of $\mathrm{Pa}^{233}$ to $\mathrm{U}^{233}$ causes reactivity to increase following shutdown. The buildup of radioactive daughters of $\mathrm{U}^{232}$ in reprocessed fuel renders the gamma activity of fresh fuel elements too high to permit direct handling. There is need for special care regaxding the kinetic behavior of a fast $\mathrm{U}^{233}-\mathrm{Th}^{232}$ breeder, because the delayed fraction of fission neutrons from $U^{233}-\mathrm{Th}^{232}$ is smaller than the delayed fractions from other fuel systems.

It is concluded that the se characteristics of the $\mathrm{U}^{233}-\mathrm{Th}^{232}$ reactor do not materially affect the operation and safety of the reactor.

The relatively mild variation of $\eta_{233}$ with neutron energy, and the low fission cross section and high energy threshold for $\mathrm{Th}^{232}$ permit the design of large $U^{233}-T^{232}$ fast breeders without serious design restraints arising from the need to avoid a positive void coefficient.

\subsection{Increased Control Requirements Due to $\mathrm{Pa}^{233}$ Buildup}

\subsubsection{Increased Reactivity Change Due to Burnup}

$\mathrm{U}^{233}$ is bred from thorium through the following chain of nuclear reactions:

$$
\mathrm{Th}^{232}(\mathrm{n}, \gamma) \mathrm{Th}^{233} \frac{\beta^{-}}{23.3 \min } \mathrm{Pa}^{233} \frac{\beta^{-}}{27.4 \text { days }} \mathrm{U}^{233} \text {. }
$$

Because $\mathrm{Pa}^{233}$ has a rather long half-life (27.4 days), this isotope tends to build up in irradiated fuel until the rate of its formation nearly equals its rate of decay to $\mathrm{U}^{233}$. Before this condition is attained, the actual rate of $U^{233}$ formation is less than that expected from the breeding ratio and the rate of destruction of $U^{233}$ atoms. The change in reactivity due to burnup is therefore greater than that which would occur if $\mathrm{Pa}^{233}$ had a negligibly small half-life.

Various $\mathrm{U}^{233}-\mathrm{Th}^{232}$ fast breeder designs were studied to determine the increased initial excess reactivity requirements due to protactinium decay. The results in Table I indicate that reactivity requirements are about 1.5 times as large as those which would be required if $\mathrm{Pa}^{233} \mathrm{de-}$ cayed instantly to $\mathrm{U}^{233}$. 
Table I

INCREASE OF EXCESS REACTIVITY REQUIRED FOR BURMUP AND SHUTDOWN CONROL REQUIREMENTS DUE TO Pa233 BUILDUP (855 MWWI)

\begin{tabular}{|c|c|c|c|c|c|c|}
\hline & $\begin{array}{l}\text { Core } \\
\text { Specific } \\
\text { Power } \\
\text { Mutukg U233) }\end{array}$ & $\begin{array}{c}\text { Avg } \\
\text { Core } \\
\text { Plux } \\
\text { nols my }\end{array}$ & $\left(\frac{\Delta k}{k}\right)_{\text {actua }}$ burnup & $\begin{array}{c}\left(\frac{\Delta k^{8}}{\Delta k^{1}}\right) \text { bupmup } \\
\text { no Pa233 } \\
\text { delay }(\text { pro }\end{array}$ & $\left(\frac{\Delta k}{\Delta k^{\prime}}\right)^{*}$ & $\begin{array}{c}\text { Maximum Reactivity Rise } \\
\text { after Shutdown } \\
\triangle \mathrm{kk} \text { (\%) }\end{array}$ \\
\hline U-Th Metal to.17-in.-00 rods) & 1.073 & 4.79 & 0.91 & 0.64 & 1.4 & 1.42 \\
\hline U-Th Metal (0.255-in.-OD rods) & 0.621 & 2.75 & 0.45 & 0.29 & 1.6 & 1.17 \\
\hline U-Th Metal 10.34 -in,-OD rods) & 0.390 & 1.72 & 0.15 & 0.09 & 1.6 & 0.87 \\
\hline U-Th Carbide $0.255-\mathrm{in}$. 00 rods) & 1.500 & 6.19 & 2.63 & 1.77 & 1.4 & 1.68 \\
\hline U-Th Oxide $(0.255-i n .-00$ rods $)$ & 0.610 & 1.18 & 1.18 & 0.89 & 1.3 & 1.07 \\
\hline
\end{tabular}

Ratio of the actual change in reactivity due to burnup between reivelings to the change in reactivity which would result if protactinium had a negligibly small half-life.

\subsubsection{Control for $\mathrm{Pa}^{233}$ Decay after Shutdown}

$A \mathrm{U}^{233}-\mathrm{Th}^{232}$ reactor containing $\mathrm{Pa}^{233}$ undergoes a slow rise in reactivity after shutdown because the protactinium decays to the more reactive $\mathrm{U}^{233}$. These reactivity increases vary with the concentrations of $\mathrm{Pa}^{233}$ at shutdown, which are generally near the equilibrium values. Equating the rates of formation and destruction of $\mathrm{Pa}^{233}$ atoms yields the following expression for the equilibrium $\mathrm{Pa}^{233}$ concentration:

$$
C^{13}=\frac{\sigma_{c}^{02} C^{02}}{\left(\lambda^{13} / \phi\right)+\sigma_{a}^{13}}
$$

where the superscripts 02 and 13 denote $\mathrm{Th}^{232}$ and $\mathrm{Pa}^{233}$, respectively, $\mathrm{C}$ represents concentration, and $\lambda^{13}$ equals $2.9 \times 10^{-7} \mathrm{sec}^{-1}$. For the flux levels of interest here, the equilibrium concentration varies almost directly with neutron flux $\phi$. $\mathrm{U}^{233}-\mathrm{Th}^{232}$ breeders of high power density, and hence of high neutron flux, have the highest $\mathrm{Pa}^{233}$ concentrations and shutdown control requirements.

The last column in Table I lists the maximum reactivity increase after shutdown for the several fuel materials considered, based upon the transformation of all $\mathrm{Pa}^{233}$ present at end of life to $\mathrm{U}^{233}$. The reactivity rise ranges from $0.87 \% \Delta \mathrm{k} / \mathrm{k}$ for a $300-\mathrm{MWe}$ reactor fueled with 0.34 -in. metal rods to $1.68 \% \Delta \mathrm{k} / \mathrm{k}$ for $0.255-\mathrm{in}$. rods of $\left(\mathrm{U}^{233}-\mathrm{Th}^{232}\right) \mathrm{C}$.

The decay of $\mathrm{Pa}^{233}$ to $\mathrm{U}^{233}$ following shutdown or plant load reduction does not present a safety problem because of the slow rate at which the decay occurs. For the reference 315-MWe reactor (see Table $\mathrm{V}$ below), an increase in reactivity of one dollar $(\Delta \mathrm{k} / \mathrm{k}=0.29 \%)$ oc curs 4.5 days after shutdown. The decay of $\mathrm{Pa}^{233}$ may be viewed as a reverse xenon effect, with the important difference that the $P \mathrm{a}^{233}$ has a half-life 70 times longer than that of $\mathrm{X} \mathrm{e}^{135}$. 


\subsection{Buildup of $\mathrm{U}^{232}$ in the Fuel}

$U^{232}$ forms in thorium-uranium fuel principally by the following nuclear reactions, the first process contributing over $95 \%$ of the $U^{232}$ formed:
(a) $\operatorname{Th}^{232}(n, 2 n) \operatorname{Th}^{231} \frac{\beta-}{25 h r}$
$\mathrm{Pa}^{231}(\mathrm{n}, \gamma) \mathrm{Pa}^{232} \frac{\beta-}{1.3 \text { days }} \mathrm{U}^{232}$
(b) $U^{233}(n, 2 n) U^{232}$.

The decay of $U^{232}$ produces $T^{228}$, which begins a long chain of nuclides emitting gamma rays of energies between 0.04 and $2.6 \mathrm{MeV}$ :

$$
\begin{aligned}
& \mathrm{U}^{232} \frac{\alpha, \gamma}{73 \mathrm{yr}} \operatorname{Th}^{228} \frac{\alpha, \gamma}{1.90 \mathrm{yr}} \operatorname{Ra}^{224} \frac{\alpha, \gamma}{3.64 \text { days }} \operatorname{Rn}^{220} \\
& \underset{54 \mathrm{sec}}{\longrightarrow} \operatorname{Po}^{216} \frac{\alpha}{0.16 \mathrm{sec}} \mathrm{Pb}^{212} \frac{\beta, \gamma}{10.6 \mathrm{hr}} \mathrm{Bi}^{212}
\end{aligned}
$$

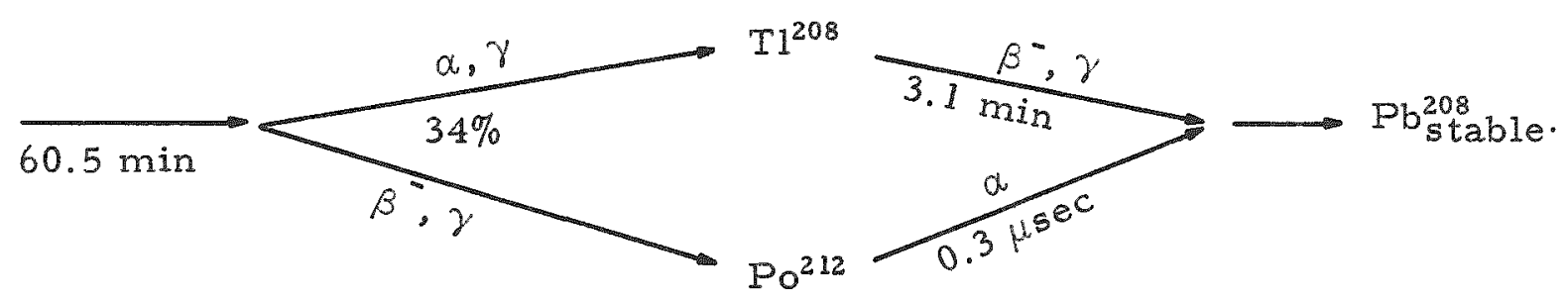

The following one-group relation was used to estimate equilibrium $\mathrm{U}^{232}$ concentrations:

$$
C^{22}=\frac{\sigma_{n, 2 n}^{23}}{\sigma_{a}^{22}} C^{23}+\frac{\sigma_{n, \gamma}^{11} \gamma}{\sigma_{a}^{11}} \times \frac{\sigma_{n, 2 n}^{02}}{\sigma_{a}^{22}} C^{02}
$$

In this equation, $C$ represents concentration, and the superscripts 22,02 , 11 , and 23 denote $\mathrm{U}^{232}, \mathrm{Th}^{232}, \mathrm{~Pa}^{231}$, and $\mathrm{U}^{233}$, respectively. The effect of fast neutron and spontaneous fissions on the equilibrium concentration of $\mathrm{U}^{232}$ has been neglected, leading to a conservative estimate of $\mathrm{U}^{232}$ activity.

Average $(n, 2 n)$ cross sections were obtained by flux averaging experimental measurements (6) with a fission spectrum from the reaction threshold energy to $15 \mathrm{MeV}$. For $\mathrm{U}^{233}, \bar{\sigma}_{\mathrm{n}^{2} \mathrm{n}}=0,000,840 \mathrm{~b}$; for $\mathrm{Th}^{232}$, it is $0.001,627 \mathrm{~b}$. In the absence of adequate cross-section data for $\mathrm{Pa}^{231}$, the ratio $\sigma_{\mathrm{n},}^{11} \gamma / \sigma_{\mathrm{a}}^{11}$ is taken equal to the corresponding ratio for $\mathrm{Pa}^{233}$, namely, 0.809 .

Average absorption cross sections of $\mathrm{U}^{232}$ were determined by extrapolating the absorption cross sections of $U^{238}, U^{236}$, and $U^{234}$. A 
dependence on absolute flux could have been introduced by adding $\lambda^{22} / \bar{\phi}$ core to $\sigma_{\mathrm{a}}^{22}$. This additional term would account for destruction of $\mathrm{U}^{232}$ in the core by radioactive decay. For the reactors considered, $\lambda^{22} / \bar{\phi}_{\text {core }}$ is equivalent to $\sim 0.05 \mathrm{~b}$, which is of the order of the uncertainty in $\sigma_{a}^{22}$, taken as $0.21 \mathrm{~b}$. Here $\lambda^{22}$ equals $3.0 \times 10^{-10} \mathrm{sec}^{-1}$. The extra term was therefore neglected in the calculations.

The necessity for remote handling of recycled fuel is a unique requirement of uranium-thorium reactors, resulting from the buildup of $\mathrm{U}^{232}$ during irradiation. In the chemical processing of irradiated fuel, uranium is isolated from other elements, but $\mathrm{U}^{232}$ remains mixed with $\mathrm{U}^{233}$ and other uranium isotopes. If $\mathrm{U}^{232}$ is present in sufficient quantities, decay daughters from this isotope make remote handling necessary a short period after chemical separation.

The equilibrium concentration of $\mathrm{U}^{232}$ in uranium is estimated at $5700 \mathrm{ppm}$ for the reference $315-\mathrm{MWe}\left(\mathrm{U}^{233}-\mathrm{Th}^{232}\right) \mathrm{C}$ fast breeder. Although equilibrium concentration is not reached until after $\sim 100$ yr of fuel irradiation, shielding requirements for a 10-yr recycling period are not substantially smaller. The gamma dose at the surface of a sphere of uranium carbide containing $1 \mathrm{~kg}$ of uranium and the equilibrium concentration of $\mathrm{U}^{232}$ was calculated as a function of time after chemical separation. After one day, the dose is $4 \mathrm{r} / \mathrm{hr}$, chiefly due to the $1.87-\mathrm{MeV}$ gamma of $\mathrm{Bi}^{212}$ decay and the $2.62-\mathrm{MeV}$ gamma of $\mathrm{T} 1^{208}$ decay. After 100 days, the dose rate rises to $5,000 \mathrm{r} / \mathrm{hr}$. The equilibrium concentration of $\mathrm{U}^{232}$ in fast $U-T h$ reactors is expected to be substantially higher than in thermal U-Th reactors because of a much larger capture cross section $\left(\sigma_{2}^{22}\right.$ thermal $\simeq 300 \mathrm{~b})$ and a substantial thermal fission cross section $\left(\sigma_{f}^{22}\right.$ ther$\mathrm{mal} \simeq 80 \mathrm{~b})$.

The shielding requirements for the 91 -rod cluster of the 315-MWe reference design were calculated on the basis of the following $U^{232}$ concentrations: (1) an equilibrium concentration of 5700 ppm in uranium obtainable after 100 yr of continuous irradiation, (2) 1700 ppm accumulated after $20 \mathrm{yr}$ of core cycling, and (3) 720 ppm accumulated after 10 yr of core cycling.

A four-zone, inward radial-shift scheme of refueling has been selected for the reference design. Each element has a 440-day residence in the reactor core, and is shifted at 110-day intervals. Twenty years of cycling are equivalent to 11.4 yr of in-pile irradiation; ten years of cycling are equivalent to $5.7 \mathrm{yr}$ of in-pile irradiation.

Table II lists required shield thicknesses at periods of one week, one month, and one year following chemical separation. The $\mathrm{Bi}^{212}$ and $T 1^{208}$ decay gammas constitute most of the gamma dose and control the shielding requirements. 
Table II

SHIELDING REQUIREMENTS FOR A CLUSTER OF (U ${ }^{233}-T^{232}$ ) C FUEL RODS (315-MWe REEERENCE DESIGN)

\begin{tabular}{|c|c|c|c|c|c|c|}
\hline \multirow{3}{*}{$\begin{array}{c}\text { Time after } \\
\text { Chemical Sepaxation }\end{array}$} & \multicolumn{6}{|c|}{ Required Shield Thicknesses, itk* } \\
\hline & \multicolumn{2}{|c|}{ Equilibrium U $\mathrm{U}^{232}$} & \multicolumn{2}{|c|}{ Aftex $20 \mathrm{Yx}$ of Cycling ${ }^{t}$} & \multicolumn{2}{|c|}{ After $10 \mathrm{Yx}$ of Cyclingt } \\
\hline & Lead & Concrete* & Lead & Concrete* & Lead & Concrete* \\
\hline One week & 0.45 & 1.5 & 0.36 & 1.2 & 0.31 & 1.0 \\
\hline One month & 0.59 & 2.0 & 0.51 & 1.8 & 0.45 & 1.6 \\
\hline One year & 0.75 & 2.6 & 0.67 & 2.3 & 0.62 & 2.1 \\
\hline
\end{tabular}

*Barytes

* Based on gamma dose of $2.5 \mathrm{mr} / \mathrm{hr}$ at centerplane 6 in. from shield surface.

tBased on in-core ir adiation equal to $57 \%$ of cycling time.

Table II indicates that shielded fabrication facilities are required, with shield thicknesses not substantially affected by increased irradiation time.

\subsection{Sodium Void Coefficient}

The expulsion of sodium coolant from the reactor leads to the following influences on reactivity:

a) A reduction in scattering increases the transparency of the reactor to neutrons, and hence leads to an increased core-to-blanket leakage which tends to decrease reactivity.

b) A reduction in slowing down hardens the spectrum and leads to an increase in the ratio of fission to capture in the fissile and fertile materials, which tends to increase reactivity.

c) A reduction in captures in low-energy resonances of $\mathrm{Th}^{232}$, which follows from the hardening of the spectrum, tends to increase reactivity.

d) A reduction in neutron captures in sodium has a small but positive effect on reactivity.

In small fast reactors, neutron leakage from the core is large, and the net effect of sodium expulsion from the core is a decrease in reactivity.

With increasing core sizes, neutron leakage from the core is diminished, with the result that the first influence becomes small, and the net effect of coolant expulsion could be an increase in reactivity. 
To avoid a positive void coefficient, pan-caked cores with low height-to-diameter (H/D) ratios have been proposed for 500- and 1000-MWe (Pu-U) $\mathrm{O}_{2}$-fueled fast breeders.

The $\mathrm{U}^{233}-\mathrm{Th}^{232}$ system offers an advantage in this respect because of a rather mild increase of the ratio of fission to capture in both fissile and fertile matexials with hardening of the spectrum, and because of the lower fuel density. Pancaking for the purpose of avoiding a positive void coefficient is not necessary for $\mathrm{U}^{233}-\mathrm{Th}^{232}$ reactors as large (5800-liter core volume) as to provide $1000 \mathrm{MWe}$.

Table III gives a comparison of the effect of voiding $50 \%$ and $100 \%$ of the coolant in $\left(\mathrm{U}^{233}-\mathrm{Th}^{232}\right) \mathrm{C}$ and $\left(\mathrm{Pu}^{239}-\mathrm{U}^{238}\right) \mathrm{C}$ reactors of equal volume and core composition. These numbers are based on 10-group diffusion-theory calculations. The sign and magnitude of the net reactivity effect is seen to depend on the difference between the spectral and leakage effects. These results indicate that, although a $315-\mathrm{MWe}\left(\mathrm{Pu}^{239}-\mathrm{U}^{238}\right) \mathrm{C}$ breeder could have positive coolant voiding effects, the voiding effects of the reference $\left(\mathrm{U}^{233}-\mathrm{Th}^{232}\right) \mathrm{C}$ design are decidedly negative.

Table III

COMPARISON BETWEEN EFFECTS OF SODIUM VOIDING IN (U233-Th 232 ) C* AND $\left(\mathrm{Pu}^{239}-\mathrm{U}^{238}\right)$ C FAST BREEDER REACTORS

Coro Composition: Fuel $36 \mathrm{v} / 0$, Steel $17 \mathrm{v} / 0$, Sodiumi $47 \mathrm{v} / \mathrm{o}$ ***

\begin{tabular}{|c|c|c|c|}
\hline \multirow{3}{*}{$\begin{array}{l}\text { Fuel Enrichment, }{ }^{1} \% \mathrm{U}^{233} \text { or } \mathrm{Pu}^{239} \\
\text { Voiding Effects: }\end{array}$} & \multicolumn{2}{|c|}{$\begin{array}{l}\mathrm{U}^{233} \mathrm{C}-\mathrm{Ih}^{232} \mathrm{C} \\
\text { Breeder }\end{array}$} & $\begin{array}{c}P^{239} \mathrm{C}-\mathrm{U}^{238} \mathrm{C} \\
\text { Breeder }\end{array}$ \\
\hline & \multicolumn{2}{|c|}{12.2} & 10.2 \\
\hline & $\begin{array}{l}\Delta \mathrm{k} / \mathrm{k}, \% \\
50 \% \text { void }\end{array}$ & $\begin{array}{l}\Delta \mathrm{k} / \mathrm{k}, \sigma_{0} \\
100^{\circ}, \text { roid }\end{array}$ & $\begin{array}{l}\Delta \mathrm{k} / \mathrm{k}, \% \\
50 \% \text { roid }\end{array}$ \\
\hline Increase in $\bar{y}$ of fissionable isotope & +0.25 & $\div 0.66$ & +0.69 \\
\hline Increase in fast fissions in fertile isotope & +0.35 & +0.78 & +0.94 \\
\hline Decrease in resonance captures in fertile isotope & +1.14 & +2.34 & +0.32 \\
\hline Increase in steel capture & -0.004 & -0.01 & -0.04 \\
\hline Decrease in sodium captures & \pm 0.08 & \pm 0.16 & +0.21 \\
\hline Total of spectral effects & +1.82 & +3.93 & +2.12 \\
\hline Increase in core to blanket leakage & -2.79 & -6.22 & -1.93 \\
\hline Total of all effects & -0.97 & $\overline{-2.29}$ & +0.19 \\
\hline
\end{tabular}

$*\left(P u^{233}-T h\right) C: 10 \mathrm{~g} / \mathrm{cm}^{3} ;(P u-U) C: 13 \mathrm{~g} / \mathrm{cm}^{3}$.

* Correspond to core volume and composition of the reierence $315-M W$ W (U-Th) C reactor.

Based on criticality with $\mathrm{U}^{233}$ or $\mathrm{Pu}^{239}$, with no higher isotopes.

With $\left(\mathrm{Pu}^{239}-\mathrm{U}^{238}\right) \mathrm{C}$ the largest positive spectral effect of voiding is due to the increase in $U^{238}$ fast fissions. This is not the case with $\left(\mathrm{U}^{233}-\mathrm{Th}^{232}\right) \mathrm{C}$. $\mathrm{Th}^{232}$ has both a smaller fast fission cross section $(0.17 \mathrm{~b}$ $\mathrm{vs} 0.58 \mathrm{~b}$ for $\mathrm{U}^{238}$ ) and a higher threshold ( 1.25 MeVvs $\sim 1.0 \mathrm{MeV}$ for $\left.\mathrm{U}^{238}\right)$. The data of Table III indicate that the most important positive effect with $\mathrm{U}^{233}-\mathrm{Th}^{232}$ results from the decrease in $\mathrm{Th}^{232}$ resonance captures. 
An additional effect which has not been included in the calculations summarized in Table III is the presence of higher isotopes of the fissile element. In the case of plutonium, both $\mathrm{Pu}^{240}$ and $\mathrm{Pu}^{242}$ have a lower threshold energy for fission and a much higher cross section $(\sim 1.5 \mathrm{~b})$ than $\mathrm{U}^{238}$. (7) In the case of $\mathrm{U}^{233}$, the isotopes $\mathrm{U}^{234}\left(\sim 1.5 \mathrm{~b} ; \mathrm{E}_{\mathrm{T}} \sim 0.3 \mathrm{MeV}\right)$ and $\mathrm{U}^{236}\left(\sim 0.8 \mathrm{~b} ; \mathrm{E}_{\mathrm{T}} \sim 0.75 \mathrm{MeV}\right)(8)$ also have much higher fission cross sections and lower threshold energies than $\mathrm{Th}^{232}$.

The presence of these higher isotopes increases the positive spectral void effect, and the overall reactivity void effect becomes more positive over the duration of a reactor cycle.(7) Indications to date are that this change-of-void effect with buildup of higher isotopes is less severe in the case of $\mathrm{U}^{233}$ than it is with $\mathrm{Pu}^{239}$, because of the smaller relative concentration of $U^{234}$ and $U^{236}$.

It is also seen from Table III that the greater leakage effect obtained with the less dense $\left(\mathrm{U}^{233}-\mathrm{Th}^{232}\right) \mathrm{C}$ fuel contributes significantly toward its large, negative coolant void effect. The increase in leakage obtained with $T h C$ relative to that obtained with $U C$ is almost inversely proportional to the ratio of the densities of these materials $\left(10 \mathrm{~g} / \mathrm{cm}^{3}\right.$ for $\mathrm{ThC}$ and $13 \mathrm{~g} / \mathrm{cm}^{3}$ for $\mathrm{UC}$ ).

The above characteristics of $\left(U^{233}-\mathrm{Th}^{232}\right) \mathrm{C}$ permit the design insofar as coolant void effects are concerned of a $1000-\mathrm{MWe}$ reactor (5800-1iter core) with an $H / D$ of 1 . Table IV summarizes results of calculations on a 315-MWe design, two 1000-MWe cores, and a 315-MWe $(\mathrm{Pu}-\mathrm{U}) \mathrm{C}$ core, performed with a 16-group transport-theory code (DTF).

The cross sections, geometric model, and core compositions are slightly different from those used in deriving Table III. In the DTF calculations, the $\mathrm{U}^{233}$ capture cross section above $1 \mathrm{MeV}$ is higher than that used in the diffusion-theory calculations. The geometrical model used in the DTF calculation consists of a finite cylinder with 1.33-it axial and radial blankets. It is concluded that from a coolant void point of view, a $1000-M W e\left(U^{233}-T^{232}\right) C$ reactor $c a n$ be designed with reasonable $H / D$. This is not feasible with $\left(P u^{239}-U^{238}\right) C$, as indicated by the results in Tables III and IV, as well as from other studies. For example, a $\left(\mathrm{Pu}{ }^{239}-\mathrm{U}^{238}\right) \mathrm{O}_{2}$ 1000-MWe reactor has been reported as requiring an $\mathrm{H} / \mathrm{D}$ ratio of $0.086(\mathrm{H}=1.25 \mathrm{ft}, \mathrm{D}=14.5 \mathrm{ft}$, core volume $=5840$ liters $)$ in order to avoid a positive void coefficient. (9)

\subsection{Delayed Neutron Fraction}

Representative values of the delayed neutron fraction $\beta$ for $\mathrm{Pu}^{239}-\mathrm{U}^{238}, \mathrm{U}^{235}-\mathrm{U}^{238}$, and $\mathrm{U}^{233}-\mathrm{Th}^{232}$ fast reactors are $0.004,0.007$, and 0003 , respectively. The $\beta$ of $\mathrm{U}^{233}-\mathrm{Th}^{232}$ is the lowest because of the small proportion of $\mathrm{Th}^{232}$ fissions relative to the high importance of $\mathrm{U}^{238}$ fissions with the other fuel types. 
Table IV

SODIUM VOID EFFECT IN 315 - AND $1000-M W e\left(U^{233}-T^{232}\right) C$ AND $\left(\mathrm{Pu}^{239}-\mathrm{U}^{238}\right)$ C FAST BREEDER REACTORS

Core Composition: Fuel, $37 \mathrm{v} / 0$, Sodium, $46 \mathrm{v} / 0$, Iron, $17 \mathrm{v} / 0$.

Radial Blanket Composition: Fuel, $70 \mathrm{v} / 0$, Sodium, $15 \mathrm{v} / 0$, Iron, $15 \mathrm{v} / 0$.

\begin{tabular}{|c|c|c|c|c|}
\hline Number & 1 & 2 & 3 & 4 \\
\hline Power, MWe & 315 & $\sim 1000$ & $\sim 1000$ & $\sim 315$ \\
\hline Fuel & $\left(U^{233}-T h^{232}\right) C$ & $\left(U^{233}-T h^{232}\right) C$ & $\left(U^{233}-T^{232}\right) C$ & $\left(P u^{239}-U^{238}\right) C$ \\
\hline Core Volume, $l$ & 2300 & 5800 & 5800 & 2760 \\
\hline Height, ft & 4.60 & 6.90 & 4.35 & 4.60 \\
\hline Diameter, it & 4.74 & 6.90 & 8.70 & 5.20 \\
\hline Radial Blanket, st & 1.33 & 1.33 & 1.33 & 1.33 \\
\hline Axial Blanket, ft & 1.33 & 1.33 & 1.33 & 1.33 \\
\hline $\mathrm{H} / \mathrm{D}(\operatorname{core})$ & 0.97 & 1.0 & 0.5 & 0.885 \\
\hline Fissionable Isotope & & & & \\
\hline Loading, $\mathrm{kg}$ & 1020 & 2440 & 2660 & 1280 \\
\hline Enxichment (core) & & & & \\
\hline (a/o fissile isotope) & 12.75 & 9.95 & 10.75 & 10.2 \\
\hline$\Delta \mathrm{k} / \mathrm{k}$ Upon $50 \%$ Loss of & & & & \\
\hline Total Sodium, \% & -1.72 & - & - & +0.11 \\
\hline $\begin{array}{l}\Delta \mathrm{k} / \mathrm{k}, 100 \% \text { Void, } \% \\
\text { Exces }\end{array}$ & -3.90 & -1.63 & -2.12 & $\div 0.28$ \\
\hline $\begin{array}{l}\text { Unvoided, } \Delta \mathrm{k} / \mathrm{k}, \% \\
\text { Breeding Ratio }\end{array}$ & 4.56 & 1.96 & 5.89 & 3.34 \\
\hline Core & 0.77 & 1.08 & 0.95 & - \\
\hline Blanket & 0.54 & 0.28 & 0.40 & \\
\hline Total & 1.31 & 1.36 & 1.35 & \\
\hline
\end{tabular}

The small value of $\beta$ for the $U^{233}-T^{232}$ fast breeder does not affect importantly the consequences of very severe accidents involving high reactivity-insertion rates and resulting core meltdowns. In such accidents, the reactor becomes prompt critical and the delayed neutrons have negligible importance. The low value of $\beta$ is important, however, in determining the consequences of accidents, in the range below prompt critical, since in this case the low value of $\beta$ tends to reduce the reactor period and increase the power level obtained for a given ratio of reactivity insertion and initial power level. However, with proper design and operational safeguards, such as low maximum limits on control rod-withdrawal rates, the likelihood of such accidents can be reduced to a negligible level. Thus, the safety of the $\mathrm{U}^{233}-\mathrm{Th}^{232}$ fast breeder is not expected to be materially different than that of othex fast reactors.

\section{Description of 315-MWe Reference Design}

The characteristics of the 315-MWe reference design are summarized in Table $V$. The net overall efficiency is $36.5 \%$. The total breeding $x$ atio of 1.31 is obtained under equilibrium cycle conditions, taking into account the effect of higher uranium isotopes and the buildup of $\mathrm{Pa}^{233}$ and fission products. The nuclear parameters were determined by means of 10-group diffusion-theory calculations with a cylindrical model of core and blanket. 


\section{SUMMARY OF PLANT CHARACTERISTICS OF REFERENCE REACTOR}

1. Cycle Characteristics

Net plant power, MWe 315

Total reactor power, MWt

865

Turbine inlet pressure, psig

Turbine inlet temperature, ${ }^{\circ} \mathrm{F}$

870

Feedwater temperature, ${ }^{\circ} \mathrm{F}$

2. Reactor Vessel

Inside diameter, ft

10

Inside height, ft

Wall thickness, in.

1.0

Material

304 SS

Design pressure, psia

150

Design temperature, ${ }^{\circ} F$

1100

\section{Core Characteristics}

Thermal power (equilibrium), MW

Avg fissionable material loading,

975

$\mathrm{kg}$ of $\mathrm{U}^{233}+\mathrm{U}^{235}$

Total U loading, $\mathrm{kg}$

1319

$\mathrm{U}+\mathrm{Th}$ loading, $\mathrm{kg}$

7637

Height, it

Diameter, ft

4.6

Volume, liters

Power density, MWt/liter core

4.6

Specific power, MWt/kgU $\mathrm{U}^{233}$

Overall composition, $v / o$

$\mathrm{UC}-\mathrm{ThC}$

2165

0.39

0.91

Stainless steel

36.6

Sodium

17.0

46.4

Equilibrium fresh fuel composition, a/o $\mathrm{U}^{233}$

$\mathrm{U}^{234}$

12.76

$\mathrm{U}^{235}$

3.75

$\mathrm{U}^{236}$

0.50

$\mathrm{Th}^{232}$

0.63

82.36

Number of fuel subassemblies

Total heat transfer surface, $\mathrm{ft}^{2}$

5200

Max heat flux (including hot spot factor),

$1.5 \times 10^{6}$

$\mathrm{Btu} / \mathrm{hr}-\mathrm{ft}^{2}$

Average heat flux, Btu/hr-ft

Overall max/avg heat flux ratio

$0.56 \times 10^{6}$

1.95

Total coolant flow area, $\mathrm{ft}^{2}$

6.95

Sodium coolant

Inlet coolant temp, ${ }^{\circ} \mathrm{F}$

Outlet coolant temp, ${ }^{\circ} \mathrm{F}$

650

Coolant flow rate, $\mathrm{lb} / \mathrm{hr}$

1000

$27.7 \times 10^{6}$

Coolant inlet pressure, psia

100 
Table V (Contd.)

4. Core Fuel Element

Fuel material

Clad material

Fuel rods per assembly

Fuel rod dimensions, in.

Fuel meat, OD

Clad thickness

Fuel rod, $O D$

Ligament spacing

Maximum coolant velocity, it/sec

Maximum fuel central temperature, ${ }^{\circ} \mathrm{F}$

Fuel management

Average fuel burnup, $\mathrm{MWD} /$ tonne

Maximum fuel burnup, MWD/tonne

5. Blanket Characteristics

Thermal power (fresh blanket), MW

Thorium carbide loading, $\mathrm{kg}$

Radial blanket thickness, ft

Axial blanket thickness (each section), ft

Overall composition of radial blanket, $v / 0$

$\mathrm{ThC}$

Stainles steel

Sodium

Number of radial blanket assemblies

Number of axial blanket as semblies

6. Radial Blanket Element Design

Blanket material

Cladding material

Rods per assembly

Rod dimensions, in.

Thorium carbide rod, $O D$

Clad thickness

Rod, OD

Ligament spacing

7. Axial Blanket Element Design

Blanket material

Cladding material

Rods per assembly

Rod dimensions, in.

Thorium carbide rod, OD

Clad thickness

Rod, OD

Ligament spacing

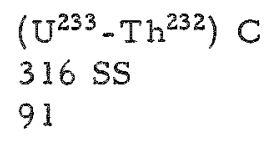

0.300

0.015

0.330

0.090

35

2100

4-zone

inward radial shift

38,700

50,000

6.0

40,100

1.33

1.33

70

15

15

224

144

ThC

316 SS

37

0.585

0.020

0.625

0.033

ThC

316 SS

37

0.475

0.020

0.515

0.143

8. Nuclear Characteristics

Neutron balance (start of equilibrium cycle, inward radial shift refueling) 
Table V (Contd.)

Productions per neutron absorbed in $\mathrm{U}^{233 *}$

\begin{tabular}{ll}
$\mathrm{U}^{233}$ & 2.381 \\
$\mathrm{U}^{234}$ & 0.083 \\
$\mathrm{U}^{235}$ & 0.043 \\
$\mathrm{U}^{236}$ & 0.005 \\
$\mathrm{Th}^{232}$ & 0.049 \\
$\mathrm{~Pa}^{233}$ & 0.002 \\
\multicolumn{1}{c}{ Total } & 2.563
\end{tabular}

Removals per neutron absorbed in $U^{233 * *}$

\begin{tabular}{|c|c|}
\hline$\tau^{233}$ & 1.000 \\
\hline$U^{234}$ & 0.038 \\
\hline$U^{235}$ & 0.007 \\
\hline$U^{236}$ & 0.004 \\
\hline$T_{h}^{232}$ & 0.758 \\
\hline $\mathrm{Pa}^{233}$ & 0.004 \\
\hline $\mathrm{Fe}+\mathrm{Na}+\mathrm{C}$ & 0.02 \\
\hline Fission prod. & 0.018 \\
\hline Core leakage & 0.631 \\
\hline Control & 0.07 \\
\hline Total & \\
\hline
\end{tabular}

Blanket leakage/ $\mathrm{U}^{233}$ absorption

Core breeding ratio (beginning of cycle)

0.755

Blanket breeding ratio (beginning of cycle)

Total breeding ratio (beginning of cycle)

1.284

Core breeding ratio (average of cycle)

0.770

Total breeding ratio (average of cycle)

1.313

Median fission energy, $\mathrm{MeV}$

0.21

Median flux energy, MeV

0.28

Overall system doubling time, yr

In-pile inventory doubling time, yr

Net $U^{233}$ production (average of cycle), $\mathrm{kg}$

23.9

Doppler temperature coefficient, ${ }^{\circ} \mathrm{K}^{-1}$

9. Control Requirements

Burnup between refuelings, $\% \Delta \mathrm{k} / \mathrm{k}$

*Net production $(\nu-1) \frac{\Sigma_{f}^{i}}{\Sigma_{a}^{233}}$, except for $U^{233}, \nu \frac{\Sigma_{f}^{233}}{\Sigma_{a}^{233}}$.

**Removals are $\frac{\Sigma_{c}^{i}}{\Sigma_{a}^{233}}$, except for $U^{233}$ and leakage terms. 
A power of $855 \mathrm{MWt}$ is produced in the core, corresponding to a power density of $0.39 \mathrm{MWt} /$ liter of core. The maximum heat flux at the center of the core is $1.5 \times 10^{6} \mathrm{BTU} / \mathrm{hr}-\mathrm{ft}^{2}$, corresponding to a maximum heat generation rate of $38 \mathrm{~kW} / \mathrm{ft}$. The reactor design is based on a maximum fuel central temperature of $2100^{\circ} \mathrm{F}$ and a thermal conductivity for (U-Th) C fuel of $12.1 \mathrm{BTU} / \mathrm{hr}-\mathrm{ft}-{ }^{\circ} \mathrm{F}$. The melting points of $\mathrm{UC}$ and $\mathrm{ThC}$ are 4190 and $4760^{\circ} \mathrm{F}$, respectively. Therefore, a large margin with respect to central fuel melting exists. A plant cycle diagram and reactor elevation are shown in Figs. 1 and 2.

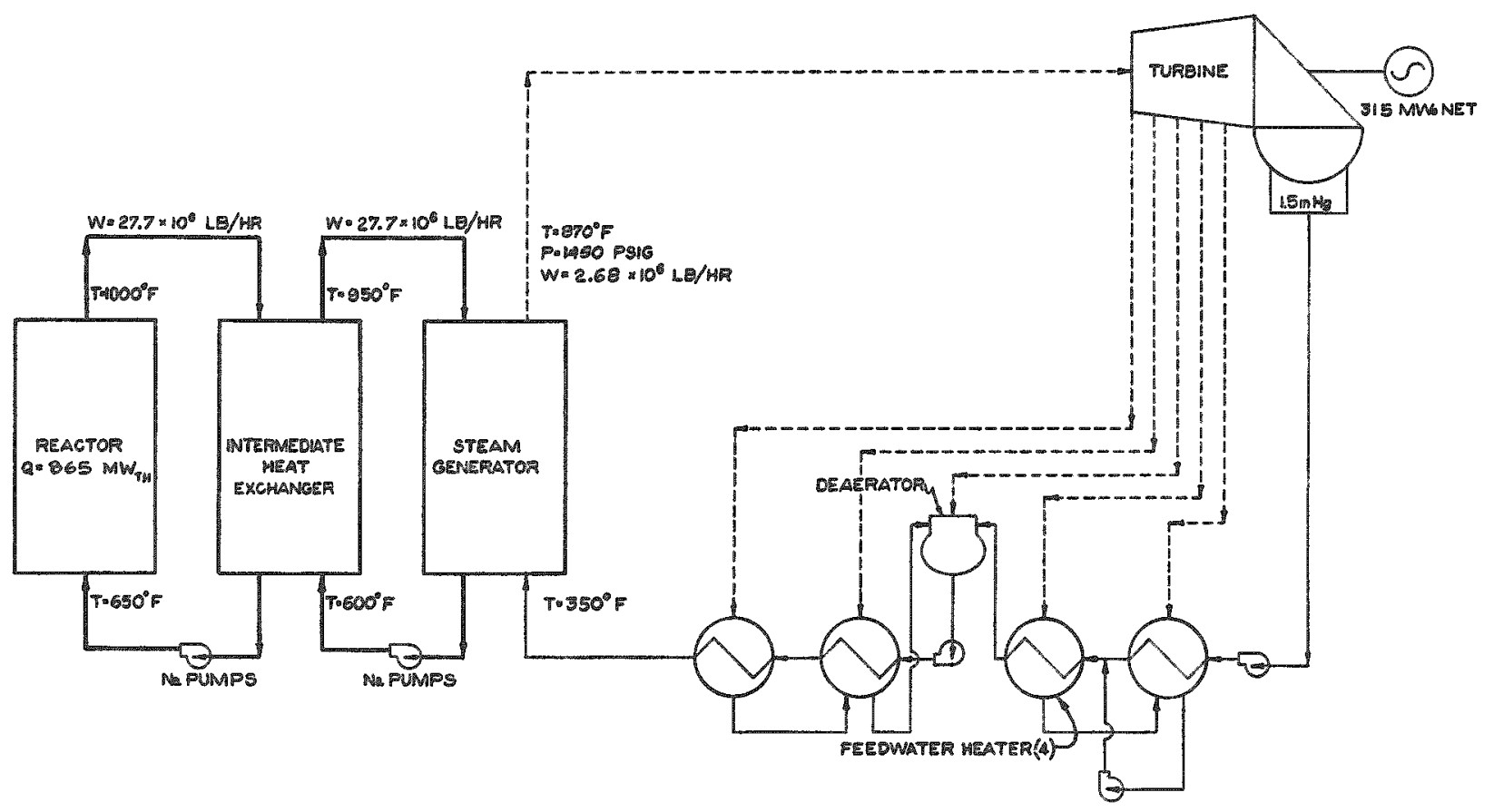

Fig. 1. (Pu-U)C Fast Breeder Reactor - Coolant Flow Diagram

\section{Power-Generation Costs}

Total power-generation costs for the 315-MWe reference design are estimated at $9.01 \mathrm{mills} / \mathrm{kWh}$ based on a $4 \frac{3 .}{4} \%$ AEC use charge and a price of $\$ 14$ per $\mathrm{kg}$ of $\mathrm{U}^{233} .(10)$ Total fixed charges at a $80 \%$ load factor are $5.59 \mathrm{mills} / \mathrm{kWh},(5)$ operation and maintenance are $0.63 \mathrm{mill} / \mathrm{kWh},(5)$ and fuel cycle costs are $2.79 \mathrm{mills} / \mathrm{kWh}$ as estimated in Table VI. These are based on the fuel-cycle cost data of Table VII. (5) The cost of ThC is taken as $\$ 67$ per $\mathrm{kg}$ of contained Th. 
SOFER et al.

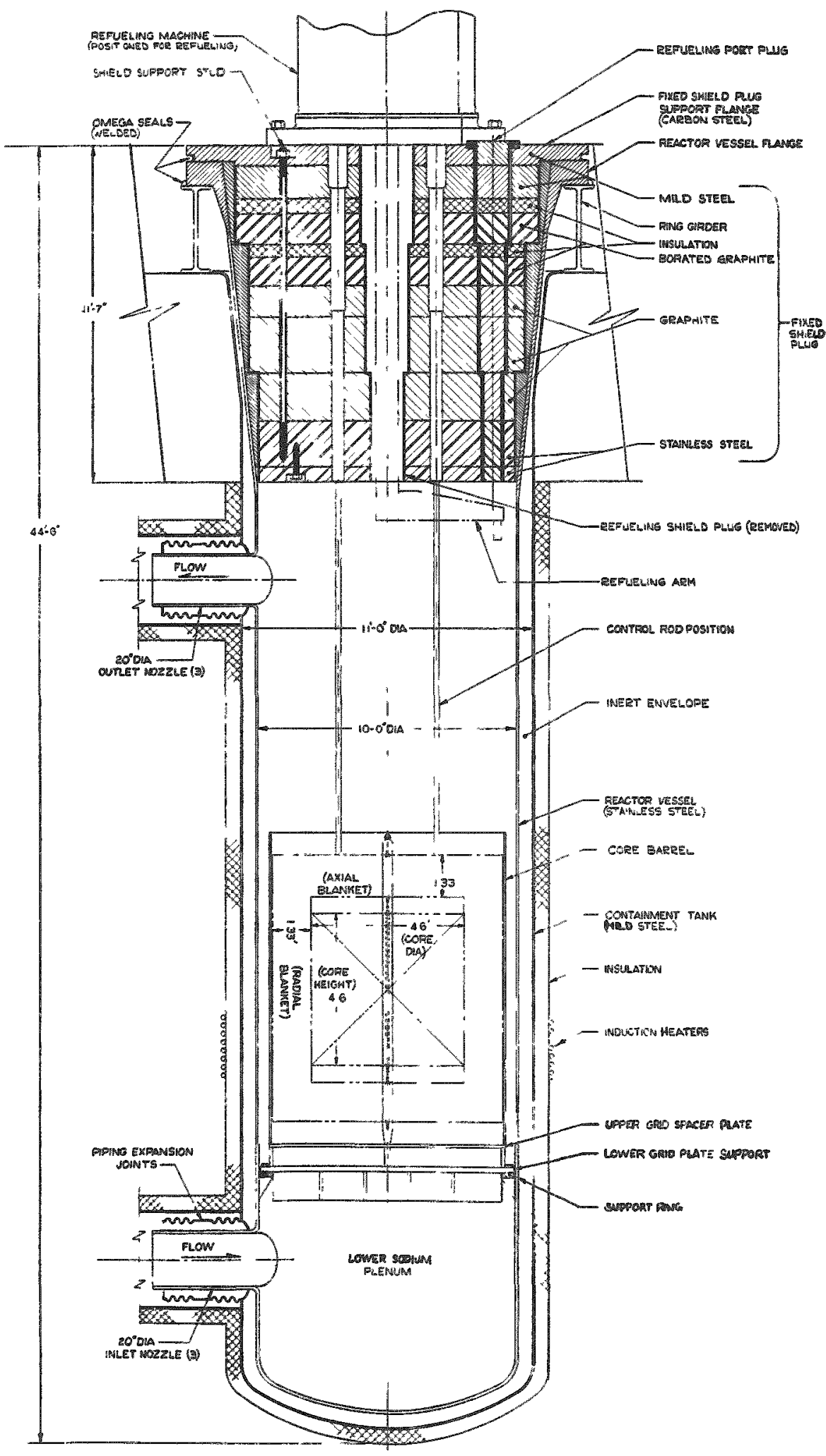

Fig. 2. (Pu-U)C Fast Breeder Reactor - Conceptual Design. Reactor Elevation. 
Table VI

ESTIMATE OF FUEL-CYCLE COSTS

(Maximum Burnup of 50,000 MWD/tonne)

\begin{tabular}{l}
$\$ / \mathrm{kg}$ \\
Core Blanket \\
\hline
\end{tabular}

Fabrication:

Cost of conversion, $\mathrm{UO}_{2}$ to $\mathrm{UC}$

8.8

Cost of fissionable material lost in conversion

Cost of fabrication including scrap recovery

Cost of fissionable material lost in fabrication

Use charge during conversion

Use charge during fabrication

Cost of shipping new fuel

Cost of Thorium

Lease charge during shipping new fuel

Spent Fuel Shipping:

Depletion of $\mathrm{U}^{233}$ :

Reprocessing:

Separation costs

Conversion of UNH to $\mathrm{UO}_{2}$

Reprocessing losses

AEC Use Charge:

Use charge during spent fuel storage, shipping, and processing

Use charge during in-core residence

Use charge for spare fuel on hand

Total fuel cycle cost, $\$ / \mathrm{kg}$

Total annual fuel-cycle cost

Unit fuel-cycle cost, $\mathrm{mill} / \mathrm{kWh}$

Net total fuel-cycle cost, $\mathrm{mill} / \mathrm{kWh}$

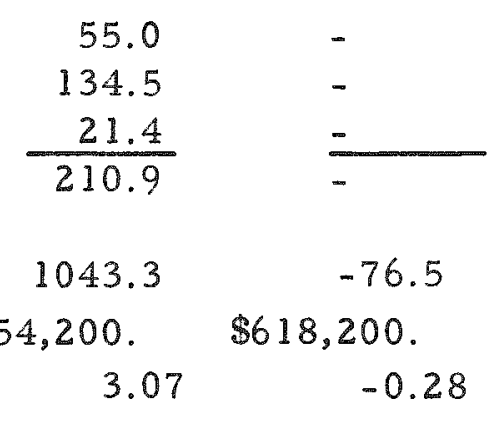

2.79 
FUEL-CYCLE COST DATA (U'233-Th) C REFERENCE REACTOR (Maximum Fuel Burnup 50,000 MWD/tonne)

\begin{tabular}{lll} 
& \multicolumn{1}{c}{ Core } & Blanket \\
\cline { 2 - 2 } Average Burnup, MWD/tonne & 38,600 & 1450 \\
Replacement Batches per Core & 4 & 4 \\
Initial Fuel Loading, * kg & 1958 & 10,100 \\
Initial U Content, \% & 0.1764 & 0 \\
Final U Content,**\% & 0.1583 & 0.0188 \\
Conversion Charge, \$/kg of U & 50 & 0 \\
Fuel Fabrication Price, \$/kg & 210 & 60 \\
Shipping and Insurance, New Fuel, \$/kg & 8 & 3 \\
Total Recoverable Scrap, \% & 10 & 10 \\
Irrecoverable Loss in Fabrication, \% & 1 & 1 \\
Average Fuel-throughput Rate, kg/month & 135 & 168 \\
Chemical Reprocessing Batch Size, kg & 7833 & 10,100 \\
Chemical Separation Rate, kg/day & 249 & 1000 \\
Total Reprocessing Time, days & 128 & 55 \\
Core Residence Time, yr & 1.21 & 5.0 \\
Spare Fuel on Hand, kg & 294 & 1515
\end{tabular}

* Per replacement batch.

**Based on initial fuel loading.

For a 1000-MWe design, at an average burnup of 100,000 MWD/tonne, an overall plant efficiency of $40 \%$, and a breeding ratio of 1.35 (core $=0.95$, blanket $=0.40)$, the total estimated fuel cycle costs are $0.93 \mathrm{mill} / \mathrm{kWh}$. This refers to a core with $H / D=0.5$. A practical system involves a core breeding ratio very close to but below unity so that core life can depend on other than burnup considerations, and with a minimum of excess reactivity.

\section{References}

1. A. M. Weinberg, Conference on the Physics of Breeding, Oct 1959, Proceedings: ANL-6122, pp. 10-22.

2. D. R. Mash and A. Ottenberg, Status and Future Requirements for the $U^{233}$ Power Reactor Program, ASAE-S-4 (1957).

3. B. C. Diven and J. R. Hopkins, Reports to the AEC Nuclear Cross Sections Advisory Group, Los Alamos Scientific Laboratory, $\alpha$ for $\mathrm{U}^{233}, \mathrm{U}^{235}$, and $\mathrm{Pu}^{239}, \mathrm{WASH}-1031$ (Feb. 1961). 
4. Civilian Nuclear Power, a Report to the President - 1962, USAEC.

5. R. Hankel et al., An Evaluation of $\mathrm{U}^{233}-$ Thorium Fast Breeder Power Reactors, NDA 2164-3 (April 15, 1962).

6. M. H. Kalos and E. S. Troubetzkoy, Fast Neutron Cross Sections of Thorium-232, Protactinium-233, Uranium-233, Beryllium, Sodium, and Iron, NDA 2134-2 (Sept, 1, 1960).

7. D. Okrent and F.W. Thalgott, The Physics of Plutonium in Fast Reactors, HW-75007 (Dec。1962), p. 14.1.

8. H. Etherington, ed., Nuclear Engineering Handbook, McGraw-Hill Book Co., Inc., New York (1958) pp. 2-8.

9. Sodium Cooled Reactors Program, Fast Ceramic Reactor Development Program, Sixth Quarterly Report, January-March 1963, GEAP4214 (Apr。1963).

10. AEC Release (May 28, 1963).

Discussion of Paper

Presented by Mr. Sofer

MR. EGGEN (Atomics International):

The conclusions drawn by Mr. Sofer are correct as far as they go. I would like to add from our study on a similar system. We compared the $\mathrm{U}^{233}$ and the plutonium systems quite extensively during the AETR program and concluded that within free market economics, the economics of the plutonium-cycle and $U^{233}$-cycle for fast systems were about equivalent quite comparable. The power coefficients for $\mathrm{U}^{233}$ systems appear to be always negative, that is, although the Doppler coefficient is smaller, the sodium coefficients are definitely negative. There is design flexibility because of the values of eta, which allows more flexibility in working to obtain higher Doppler coefficients by dropping the energies, perhaps by using other materials and more coolant. The increase in amount of coolant in Mr. Sofer's reference design would have softened the spectra and given a larger Doppler coefficient. It does have a smaller total breeding ratio than the plutonium system. The greatest points of concern for the program were the availability of $U^{233}$ and the amount of technology available for thorium-based fuels. 
MR. GREEBLER (General Electric):

I am somewhat surprised at the large breeding ratio of 1.3 that you quoted. Was this based upon an equilibrium cycle? With a practical engineering design we found it touch and go as to obtaining breeding.

MR. SOFER:

The breeding ratio is an equilibrium breeding ratio. It does allow for the fission products. The cross sections we used to get this breeding ratio would give a higher breeding ratio for the plutonium-uranium system.

MR. OKRENT (Argonne):

We have done a few calculations on thorium-uranium systems, and if you have a reasonably efficient blanket, I think one can expect breeding ratios in that vicinity. Certainly closer to 1.2 than to 1 , I would say.

MR. EDLUND (Babcock and Wilcox):

Where are you going to get the $\mathrm{U}^{233}$ as a feed? If you start with $\mathrm{U}^{235}$ as feed material, you do not reach those equilibrium values. I think this constitutes a real practical problem.

MR. SOFER:

I agree. One might consider developing concurrently the utilization of the uranium-thorium cycle in the thermal systems as well as in the fast. The seed and blanket reactor is particularly interesting to me in that a thorium blanket produces $U^{233}$ that is not diluted with $U^{235}$, so one does not have to cycle many, many times before you get enough $U^{233}$ that you can use in fast reactors. Another approach is to use uranium-thorium in $\mathrm{D}_{2} \mathrm{O}$ reactors. This gives a somewhat larger conversion ratio, of the order of 1 to 1.05. The development of fast as well as thermal uraniumthorium systems is feasible, in my opinion. They tend to help each other.

MR. DAVIDSON (Allis-Chalmers):

Another cycle would be to use plutonium as the fuel in the core in order to get started. This allows a start with a good breeding $r$ atio and allows you, if you wanted to, to sell your breeding gain in the form of $\mathrm{U}^{233}$, which would be useful for thorium thermal reactors. Have you looked at this type of cycle? 
MR. SOFER:

No. This perhaps needs to be given some consideration.

MR. BENEDICT (MIT):

Wouldn't you get back to a positive void coefficient?

MR. SOFER:

Not necessarily, because a large part of the sodium coefficient comes from what happens to the captures in the fertile material. It needs to be studied in detail.

MR. SPINRAD (Argonne):

I noticed that in your comparison of reactivity effect due to the sodium voidage, the overriding factors seem to be leakage to the blanket, both in the U-Pu and the Th-U cases. Would it be correct to assume that thorium in the blanket is a poorer reflector and has a less positive effect on sodium voidage, and that therefore thorium might be a desirable blanket in that sense even for a U-Pu system?

MR. SOFER:

I think the main difference is due largely to the density of uranium carbide, $13.6 \mathrm{gm} / \mathrm{cc}$ versus that of thorium carbide, $10.7 \mathrm{gm} / \mathrm{cc}$. Our calculations are based on obtaining 94 per cent of the theoretical density in both systems. Thus, we are comparing a uranium density of about $13 \mathrm{gm} / \mathrm{cc}$ for the uranium case, and a thorium density of about $10 \mathrm{gm} / \mathrm{cc}$. I think a large part of that leakage is probably due to that effect.

Another fairly important fact is the resonance capture in fertile isotopes. There is a release of 1.14 per cent in reactivity, in the thorium case, just from that. However, that number is based on a fairly highly lumped group for the tail of neutrons in the thorium. I think that when one goes to more groups, that number may be significantly different.

MR. EDLUND:

Is your core to blanket leakage the net leakage taking into account the fast neutron production in the blanket?

MR. SOFER:

It is the net leakage. 
SOFER et al.

MR. EDLUND:

Wouldn't part of this be explained by the fact that the fast fission production in the thorium in the blanket is much lower than in the case of uranium?

MR. SOFER:

This is true.

MR. BENEDICT:

Is there enough known about the irradiation characteristics of bas ically thorium carbide fuel compared with basically uranium carbide fuel to be able to say you would be better off or worse off with this system?

MR. SOFER:

As far as I know, there is very little known on thorium carbide. This is something that needs to be worked out. 


\title{
A STUDY OF THE FUTURE ECONOMIC PROSPECTS FOR FAST REACTORS
}

\author{
B. I. Spinrad, L. E. Link, and J. T. Weills \\ Argonne National Laboratory \\ Argonne, Illinois
}

(Paper presented by Mr. Spinrad)

Introduction

Because there is now a need for major activity in development of fast reactors, stemming from an ultimate requirement for their application and current uncertainty as to the time scale required; and because the primary problem is one of fuel cycle, for which a large number of alternative materials, processes, and designs may be postulated; we have concluded that we must now make a judgment as to which fuel cycles merit the most attention. The mechanism which we chose was to make an economic forecast of the future of those fast reactor fuel cycles that are typical and for which sufficient information exists to make reasonable prognoses. To do this properly, an entire nuclear industry must be postulated and estimated; for the various factors leading to nuclear power costs are very closely coupled.

Consequently, we assembled a team of over thirty staff members of Argonne National Laboratory from the various Divisions of the Laboratory. During the months of May and June of this year, a very large mass of infor mation bearing on extrapolated fast reactor economics was assembled. We are now engaged in processing, correlating, and evaluating this information. The presentation here is therefore fundamentally a progress report.

The target of this series of studies was to be a series of normalized optimal reactor configurations, operating conditions, secondary-system criteria, and fuel cycles based upon four different fast reactor types: oxide-, carbide-, and metal-fueled all-fast reactors; and a metal fastthermal system. These normalized optima could be themselves variable, based upon the need for using ranges of technological assumptions; but at least it was hoped that a reasonably definitive translation of the assumptions into extrapolated cost of power could thereby be obtained. These translations could then be used both to isolate problems which have a particularly significant bearing on the economic feasibility of the various types; and to provide input data to assist in determining programpriorities.

We believe there is value in presenting two types of results which have emerged so far. These are: first, the flow of information in an ideal study; and second, some of the particular technological facts which have come to light so far. 
The first task in this type of analysis is the preparation of a master plan. We begin with basic restrictions to the field of inquiry. In the current study, the following conditions were imposed:

1. Consider only the U-Pu cycle.

2. Sodium is the primary coolant.

3. There is an intermediate sodium system.

4. The intermediate sodium is used to raise steam, which is the working fluid of the power-conversion system.

5. The fast reactor fuel and blanket must be reprocessed when discharged from the reactor.

These conditions are arbitraxy, intended to simplify the inquiry. We hoped that they did not prejudice the desired fuel-cycle comparisons. There is no reason why the first four of them should, and the fifth, at worst, will distort the blanket-management picture.

The expected era for application was taken to be 1985 for the fuel cycle and 1975 for the reactor. The reactor is built at the beginning, but the fuel cycle can be varied over reactor life, and is typified at about mean life. The primary date was 1985, a time when the Commission's 1962 Report indicates start of a truly large-scale nuclear power industry. Figure 1 , a reproduction of a famous figure from that report, illustrates the significance of the date by breaking the nuclear power era into two: one, pre-1985, in which nuclear power is in a basically experimental phase; and another, post-1990, in which nuclear power grows explosively to dominate the electrical generation industry.

These dates were in turn the source of two further conditions:

6. Individual reactor plants are to turn out 1000 MWe net power.

7. Individual fabrication and reprocess plants are to be scaled to support 10 reactors of $1000 \mathrm{MWe}$ each.

Three final conditions are necessary, but numerical values were adopted on a subjective basis.

8. Breeding gain should be appreciable. We have defined "appreciable" as 1.2 or greater, computed on total fissile atom content for a steady-state cycle. 
RATE OF ENERGY USE QNR.

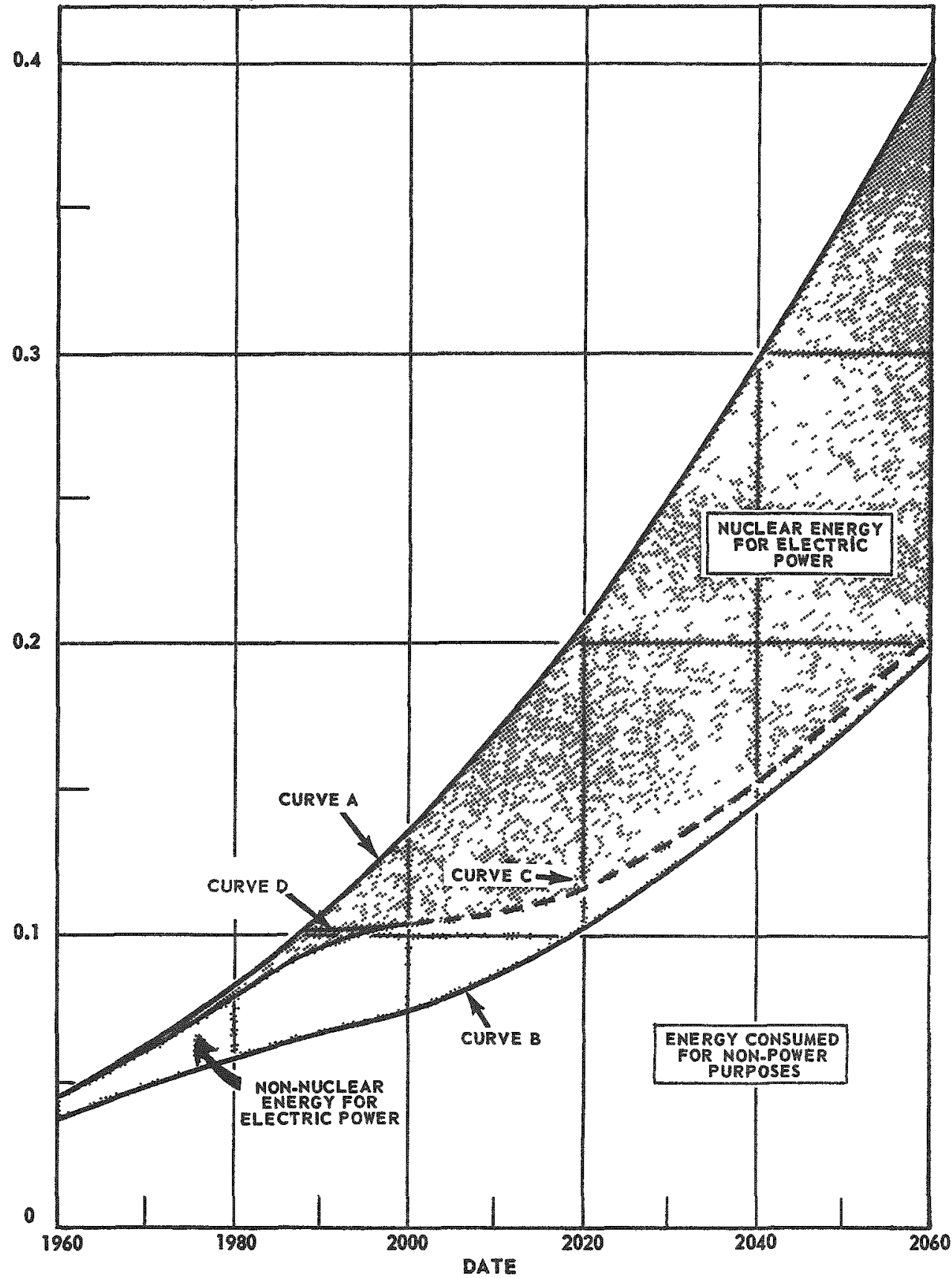

Fig. 1. Effect of Nuclear Power on Fossil Fuel Consumption (from Civilian Nuclear Power...a Report to the President - 1962).

Curve A - Total Energy Consumed.

Curve B - Energy Consumed for Purposes Other Than Central Station Power.

Curve $C$ - Energy Consumed in Orher Ways Besides Nuclear Central Station Power (1.e., Curve A minus Curve C = Nuclear Power).

Curve D - Back Extrapolation of Smoothed Curve $C$ to Illustrate Era of Rapid Penetration of Nuclear Systems into Central Station Power Industry. 
9. No intrinsic positive power-reactivity coefficients are tolerated. Since Doppler and expansion coefficients are intrinsically negative, this means practically that we shall consider designs with either a negative sodium-void coefficient or with a trivial reactivity change between voided-and flooded-core reactivities.

10. The reactor plants are on stream $80 \%$ of the time (effectively).

Together with these basic rules and restrictions, it is necessary for a cost analysis to be standard. The following costing rules have been adopted:

1. Real estate is capitalized at $13 \%$ per year (this is recommended AEC practice).

2. Depreciating assets are capitalized at $14.5 \%$, unless they are shorter lived than $30 \mathrm{yr}$; if so, their capitalization rate is $13 \%$ plus an amortization allowance computed from a sinking fund retirement rate.

3. Working inventory is capitalized at $10.5 \%$ of value. For reactors, this value is that of fabricatedfuel; for fabrication plants and reprocessing plants, the value is that of the purified materials.

4. Nonworking inventory, defined as extra inventories stocked for reasons of managerial prudence rather than technological need, are financed at $7 \%$ of value. This is analogous to the coal pile in a coal-fired plant.

Only the working-inventory capitalization rate requires discussion. Such an inventory is essentially nondepreciating (unless its residence time approaches the lifetime of the plant). Therefore, it would be financed at $13 \%$ as a capital asset. It would be financed at $7 \%$ if it were considered as a short-term recoverable asset. We have taken an intermediate value, about $3 / 5$ of the way between, which is our rough weighting as to the relative validity of the two points of view.

The values of two materials have been standardized: natural or depleted uranium at $\$ 20 / \mathrm{kg}$ of $\mathrm{U}$; and plutonium at $\$ 12 / \mathrm{g}$, regardless of isotopic content.

For uranium, the xeally cheap supplies which have driven the world uranium price down recently will probably have been depleted by 1985 . Large quantities of good ore will still be available at a price, about $\$ 20 / \mathrm{kg}$, about the same as it was before the current price competition. Appreciable stocks of depleted uranium will, of course, be then available, but we consider it unwise to peg designs to that situation being permanent.

For plutonium, the value selected is largely for convenience; $\$ 12 / \mathrm{g}$ is the current value of $\mathrm{U}^{235}$. In fast reactors, mixed plutonium isotopes at equilibrium are generally of comparable value (per gram) with $\mathrm{U}^{235}$. 
We are now ready to describe the plan of attack. Figure 2, a flow diagram of the analytical process, shows the flow of information between fuels and energy states (indicated within circles or ovals) and plants (rectilinear boxes).

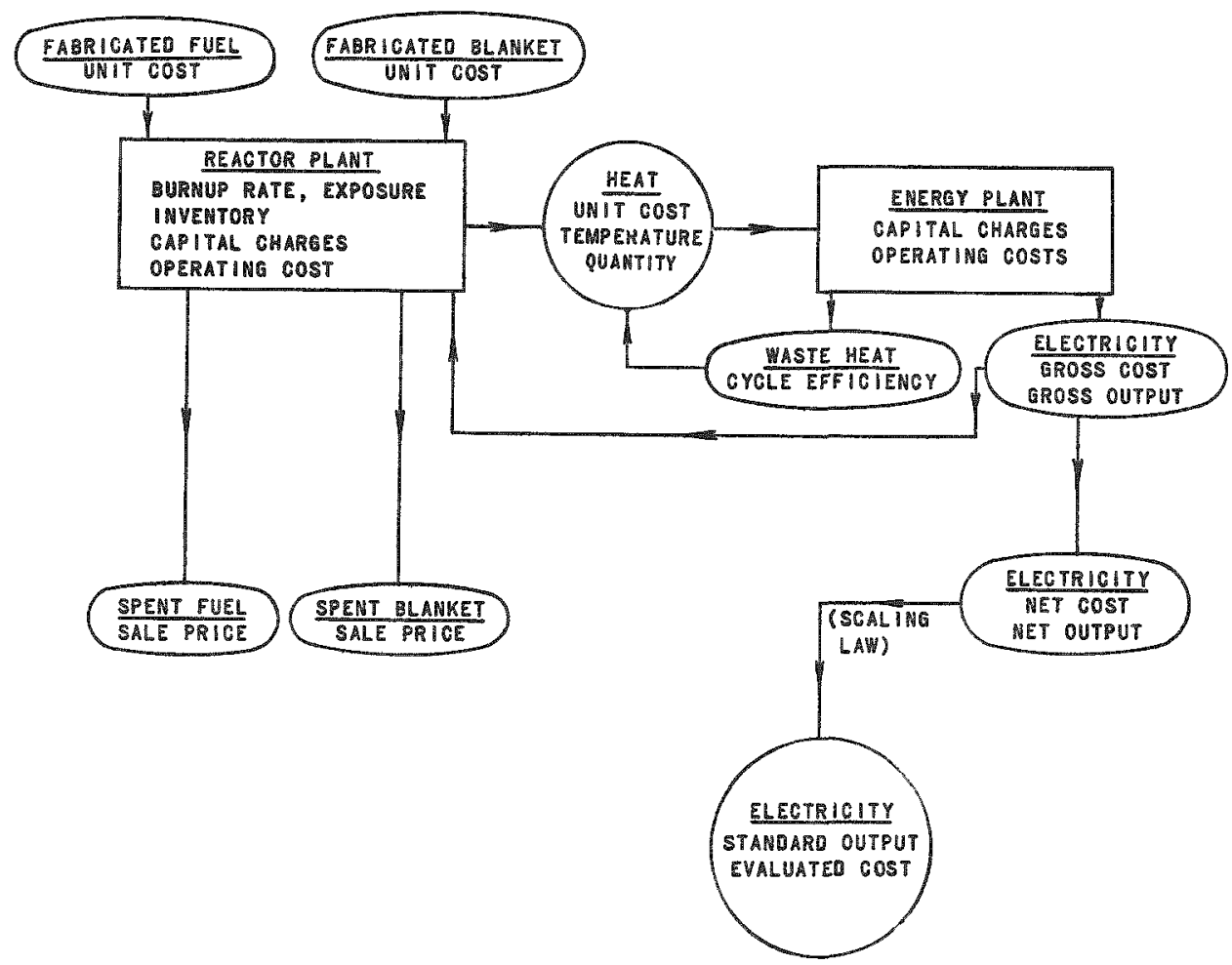

Fig. 2. Economic Evaluation: Reactor and Power Plant

For the reactor and power plant, the input information is the unit cost of purchasing fresh fuel and blanket material, and the price the reprocessor will pay for spent fuel and blanket. The output information is the price of electricity.

The designer of the reactor plant must determine his fuel, capital, and operating costs. The output of the reactor plant is heat, and the reactor designer must associate with this heat three items of information: cost, quantity, and temperature level.

Given this information, the designer of the energy plant must determine his capital and operating charges. His design produces as its output gross electricity and waste heat. Associated with this waste heat is a cycle efficiency, which feeds back to the quantity of heat needed to produce gross output.

For first approximation, we may eliminate the feedback of gross electricity to the reactor plant, and assume we can make small cost 
adjustments by a scaling law to arrive at our standard output. Then the flow of information permits us to define an optimization task.

\section{Task I}

Given hot primary sodium emerging from the intermediate heat exchanger at a given temperature, to be returned to the exchanger at any temperature above $150^{\circ} \mathrm{C}$ (at the discretion of the energy plant designer); and taking cost of heat as an adjustable parameter (i.e., varying over an appreciable range the cost of heating the sodium from reactor inlet to reactor outlet temperature); design an optimum energy conversion plant consisting of:

1. secondary sodium system;

2. sodium-to-steam evaporators, superheaters, and reheaters as appropriate;

3. turbine, condenser, and generator.

The optimum plant is defined as the one which, given the initial reactor outlet temperature and heat cost numbers, will produce $1050 \mathrm{MW}(\mathrm{e})$ at minimum cost.

The technique for solving this problem exists. It consists of laying out an initial guessed design ("standard practice"). Then each design variable is individually varied, and the effect of this variation displayed as a changed cost and changed output. If there is an unbalanced design feature, it $w i l l$ be indicated by a maximum incremental cost change per incremental output. This feature is then varied, changed output being compensated by changed capacity, until a minimum power cost (i.e., a nonlinearity) is observed.

The process is then repeated around this altered design. In gen. eral, it converges. Exceptions to convergence may result from practical discontinuities (for example, we cannot have negative reheat - we get the desired effect by crossing over to feedwater heating), and the process must be cognizant of these matters. Normally there is no trouble. Mathematically, it is a problem of "steepest descents."

The major effort for this problem consists of: first, defining the cost-capacity-performance curves for the items of equipment which are included; and second, programming the optimization for a computer. The first is necessary; the second, highly desirable.

The result of this study would be a two-parameter table. For each unit heat cost and outlet coolant temperature there is a lowest cost of electricity; the reactor designer would not need an energy plant design to tell him whether power cost is high or low; and he would be assured that 
the merits of reactor concepts are not prejudiced by design assumptions which are (to him) irrelevant.

\section{Task 2}

An estimate of fuel fabrication costs is needed as a necessary economic input to reactor cost evaluation. The fabricator's variables are:

1. fuel type (chemical and mechanical form);

2. fuel diameter;

3. bonding options;

4. cladding material;

5. cladding dimensions;

6. enrichment;

7. subassembly size (weak variation);

8. fabrication method: i.e., pelletizing, powder compaction, casting, etc.

His task is further complicated by the fact that his plant capacity is determined by fuel burnup per pass and by reactor thermal capacity, so that for any given fuel-clad-bond combination, he is asked to provide unit costs as a function of:

1. enrichment;

2. fuel diameter;

3. cladding thickness;

4. plant throughput.

The problem of variation with throughput is somewhat ameliorated by the very large size of plant which even high burnups would require: $10 \%$ burnup of $20 \%$ fuel at $40 \%$ net efficiency would still require $50 \mathrm{~kg} /$ day throughput of plutonium, or $250 \mathrm{~kg} /$ day of fuel, for $10,000 \mathrm{MWe}$. At these throughputs, it is expected that common industrial plant-scaling laws can be applied; indeed, replication of parallel lines would be mandatory.

For the rest, the fabricator must estimate:

1. building and equipment needs and capital charges;

2. power, labor, and fuel needs and cost;

3. inventory costs and/or material residence times;

4. charges and inventories for cladding and bonding materials;

5. process efficiency in terms of

a) internal plant recycle requirements;

b) reprocess plant recycle requirements;

c) unrecoverable losses. 
An estimate is needed of the price that the fuel reprocesser will pay to remove spent fuel (and blanket) from the reactor premises. We stip. ulate that the processor must have a large plant (appropriately capitalized) in which plutonium and uranium are separated from fission products for sale. The material sold is U-Pu mixture appropriate for reactor fabrication and uranium suitable for blanket fabrication.

Since the processor's output is determined by reactor parameters and his throughput is dependent on reactor power and burnup, the study must be parametric in the variables: throughput; fission product-Pu-U ratios; and U-Pu ratios in output. The study must consider various fuels and various types of process cycles, selecting the appropriate process cycle for the fuel.

Shipping costs have been considered within the processing cycle as indicated above. It is also assumed that waste disposal is included in the process.

For the rest, the processor's estimates must include essentially the same items as the fabricator (substituting "reagents" for "cladding and bonding materials," and assuming internal plant recycle only). It is generally expected that reprocess plant losses are cycle losses, since the fabricator's waste is submitted for chemical recycling; and the unrecoverable losses therefore loom larger in the process plant economics.

\section{Task 4}

Except for the primary heat transfer system and the primaryatosecondary heat exchanger, pieces of equipment in the reactor plant have a cost which is essentially independent of temperature level; even reactor vessels and internals do not show costs highly sensitive to temperature.

Consequently, it is possible to estimate the equipment in the reactor plant by cost projection against total thermal (rather than electrical) output.

The reactor design evaluation task is then a problem of taking a fuel and blanket, fabricated at a given cost, and determining an optimum in-reactor fuel cycle. The designer may, within the boundaries previously set, vary fuel length and diameter, reactor composition, coolant temperature, and power density.

Knowing that maximum fuel burnup per pass is almost always an economy, he devises fuel-management schemes which will permit the 
highest average burnup at discharge commensurate with the limits which fuel type, down time due to fuel handling, and temperature impose on this quantity.

Basically, then, this task requires:

1. Evaluating fuel-cycle costs on an exposure-inventory basis. The input data (presumably supplied) are unit fabrication costs, unit sale value of discharged material, and temperature-exposure relations. These are augmented by the reactor designer's results, which include, as most significant parameters, critical properties, breeding gain, power density, and internal fuel temperature.

2. Preparing a standard design for each of a number of choices of the preceding variables. These variables, through heat transfer correlations, set core inlet and exit temperatures.

3. From a first approximation to thermal capacity required, evaluating capital charges and refine critical calculations.

4. Design and estimate a "first approximation" primary heat exchange system, including pumps, piping, and heat exchanger.

5. From temperature and cost data, determining tentative power cost from the results of Task 1 . With this power cost is associated a required thermal output and a (secondary) coolant entrance temperature and mass flow rate.

Readjustment of primary output and of coolant flow characteristics should be a convergent process. The inclusion of the primary heat exchanger system as a reactor design variable should assure this; but some caution is necessary in asserting this as a fact, and considerable subjective judgment should be used in iterating the process.

The above process closes a loop, enabling the reactor designer, for given ex-reactor fuel-cycle cost relations, to find an optimum design under parametric assumptions concerning fuel diameter, power density, and core height. He must then undertake a steepest-descents approach to find the optima of these parameters.

The inclusion of variations in blanket design and management further complicates the problem.

Since it is the reactor designer's task to provide the optimum ultimate system, this task cannot be initiated until the three previous ones are completed. In the interim, reactor design groups must prepare by evaluating: 
1. reactor plant capital cost projections;

2. primary heat transfer circuit costs;

3. correlation between core shape, power density, and temperatures;

4. physics and safety guides for core design.

Status of Study and Preliminary Results

The design of the reactors, with their appropriate optimum fuel cycles, reactor operating conditions, and as sociated power costs are the chief goals of this study. This task cannot be completed until all of the information from the other three tasks is available. The status of these tasks is as follows:

\section{Energy Plant}

Costs of standard items have been assembled. "Good practice" designs for heat exchange and turbine-generator equipment have been illustrated and priced. The results show a spectacular decrease of capital cost with increasing sodium temperature. This equipment, including intermediate heat exchanger, steam generator, superheater, reheators, and turbogenerator, is estimated to cost $87.5 \times 10^{6}, 65 \times 10^{6}$, and $52 \times 10^{6}$ dollars for net $1,000-\mathrm{MWe}$ generation at sodium temperatures of 800,1000 , and $1200^{\circ} \mathrm{F}$, respectively. The numbers cited here are not, however, final.

These numbers indicate a very great advantage for hightemperature cycles and reactors which can supply them.

Some indications exist that "good practice" is not optimum for low heat cost. Since the low-temperature cycles appear to have the most margin for capital cost improvement as heat costs decrease, the optimization process may (and we think will) lead to a less severe conclusion. For this reason, the optimization problem, which has not yet been started, should be undertaken and concluded.

More as a guess than as an inference, it is possible that the great cost advantage of high efficiency is dependent upon the very large output of the energy plant. The greater compactness of equipment to handle high-pressure steam and high-enthalpy fluids is likely to be more significant at the $1000 \mathrm{~mW}$. rating, where capacity to manufacture very large, bulky equipment could be limiting.

\section{Fuel Fabrication}

Estimates have been prepared for material and labor costs associated with a number of different types of fuel fabrication. These are: vibratory compaction or pressing and sintering for oxide or carbide fuels; and injection casting for metal fuels. 
The ceramic-process steps were evaluated for plants to feed a single 1,000-MWe reactor. In so doing, it was determined that the economics of centralized fabrication for a 10,000-MWe complex were very great. For such plants, there is little sensitivity of unit cost to the type of process employed, or to the particular fuel (oxide or carbide). The major sensitivity is to rod diameter and cladding type. For $300-\mathrm{kg}$ (total fuel)/day plants, for example, the unit cost (cost $/ \mathrm{kg}$ of contained fuel) varies approximately as the minus 1.5 power of the rod diameter between $1 / 8$ and $1 / 2$ in., with a $50 \%$ penalty for using duplex, refractory-lined clad. Typical costs number under these circumstances are:

Fuel Diameter, Type

$$
\begin{aligned}
& \text { 1/8-in.-diam, stainless clad } \\
& 1 / 8 \text {-in.-diam, duplex refractory clad } \\
& 1 / 2 \text {-in.-diam, stainless clad } \\
& 1 / 2 \text {-in.-diam, duplex refractory clad }
\end{aligned}
$$

Direct Labor and Materials, $\$ / \mathrm{kg}$

62

91

8.0

11.4

The fabrication costs for metal fuel have not been analyzed in the same way. However, results to date indicate that the expected lower costs of fabrication of metal fuel do not lead to significant decreases in power cost; largely, this is due to the low extrapolated cost of fabricating ceramics. The process analyzed was injection casting.

This task, to be completed, must include the following analyses:

1. For ceramic fuels, plant capital equipment costs, amortization rates, and process efficiencies. The costs quoted as typical above were for $100 \%$ efficient flow sheets.

2. For metallic fuels, investigation of extrusion as a preparatory method. This may turn out to be a very cheap process.

Tentatively, unless further information is forthcoming, we may conclude that there will be little difference between fabrication cost for carbide or oxide pins of the same size. Metal fuel has not been demonstrated to require significantly lower fabrication costs.

\section{Reprocessing}

Estimates of the extrapolated economics of fuel-reprocessing plants have been prepared. As with the fabrication plants, the analysis showed that extensive economies exist in central processing for an industry, as opposed to plants scaled to a single reactor. This holds true even when shipping costs are considered under current shipping 
restrictions. Although the evaluation covered both types of operation, I shall discuss here only conclusions based on large plants.

First of all, capital and operating costs for all processes were relatively small, and only mildly dependent on fuel burnup.

Process inventory tends to be a comparable item of cost per fuel burnups less than one-half of the fissile material content of the fuel. In many cases, the material in the plant is worth more than the plant. Inventory charges are highest for aqueous processes and tend to put them at a disadvantage relative to fluoride volatility or pyrometallurgy.

Waste disposal and shipping costs add up to a contribution to reprocessing cost comparable with capital and operating costs and with inventory charges. They are somewhat lower for volatility processes than for the pyro and aqueous processes.

The evaluation indicates a favorable position for volatility processes, particularly for ceramic fuels. The aqueous process is burdened with larger inventory costs because of long cooling and process times.

Total reprocessing costs contribute a small, but appreciable, amount to power cost of -0.3 to $1 \mathrm{mill} / \mathrm{kW}-\mathrm{hr}$, depending on burnup.

The differences among the processes are not large in terms of mills/kW-hr, and therefore will probably not be the deciding factor in the choice of reactor fuel. However, the annual volume of business in a central plant is so great that small differences add up to millions of dollars per year.

Finally, the loss of $1 \%$ of the plutonium per processing cycle does not much affect breeding ratio or processing charges, except when burnups are quite low (less than $2 \%$ ).

\section{Task 5}

Reactor design optimization cannot really be performed until inputs from the other tasks are available. In the interim, however, much work can be done to prepare input of directuse in the design and designoptimization process.

A. Reactor plant capital costs have been estimated by cost projection of previous evaluations. The final projection includes:

containment vessel;

reactor vessel and internals; 
primary and reactor control system;

shielding, cooling, and gas-handling system;

waste handling and miscellaneous.

It does not include the primary heat exchange equipment.

The items covered should have costs quite insensitive to coolant temperature level, and the projection ignores the parameter. The relation obtained is $C=0.65 \mathrm{P}^{0.48}$, where $C$ is the cost in millions of dollars and $P$ is thermal output in megawatts. Over a power range of $2500-$ $5000 \mathrm{MWt}$, projected costs range from 28 to 39 million dollars.

B. Fuel burnup-temperature relations have been established, primarily by subjective discussion and compromise. Fuel-clad combinations are characterized by burnup-temperature relationships according to our current opinion as to the mechanism of radiation damage. We believe that fuel-element deterioration is primarily due to the internal pressure of fission gases, and that the cladding can be an appropriate pressure tube for containing these. The temperature limits reflect strength of materials or the probability of surface reaction at various temperatures between fuel and cladding.

Therefore, metal fuels with thick stainless steel claddings (ca. 25 mils for a 1/4-in.-diameter pin) may be expected to resist exposure up to $10 \%$ burnup, provided that the Pu-Fe eutectic temperature is not approached; closer approach to the eutectic is permitted if a thin layer of refractory metal is used as a liner (duplex tube); and higher temperatures are feasible if the entire cladding is a refractory metal. Ceramic fuels may achieve burnups well above $10 \%$ at temperatures $>650^{\circ} \mathrm{C}$ with steel cladding, or at even higher temperatures with duplex or refractory cladding.

C. Physics calculations were performed primarily to explore the usefulness of various multigroup cross-section sets and to determine the limits of core geometries set by the negative core-sodium-voidcoefficient criterion. The results can best be summarized by the following:

1. The "pancake-core" approach essentially failed to produce negative coefficients whenever core radius was laxge. This is attributable to the reflection characteristics of slab blankets. Unless a different type of criterion is adopted, right-cylindrical cores are more promising.

fuels.

This result was obtained for metal, oxide, and carbide 
It should be noted, however, that this analysis, which has been separately reported by Hummel at this Conference, did not examine extremes of "pancaking" as might result from taking core heights less than $60 \mathrm{~cm}$.

2. The use of high sodium volumes (70\%) produced tolerable negative coefficients for reasonably large oxide and carbide cores, but not for metal cores. If both a high sodium volume fraction and a high power density can be achieved, single ceramic cores with a negative coefficient can be utilized to get $1000 \mathrm{MWe}$.

However, to get high power densities with ceramic cores will require possibly uneconomic subdivision of the fuel, or operation with molten centers.

3. For metal fuels, even high sodium fractions did not produce an acceptable void coefficient in large sizes.

It will therefore be necessary to consider all-metal reactors, and some ceramic ones as complexes of small cores imbedded in matrices of blanket material. Annular cores would appear to be equivalent. It is thus very desirable to develop our computational schemes for such geometries.

4. In running cycle analyses of various large systems, it became apparent that blanket management was a very severe problem. It has been recognized for some time that radial blankets should be cycled out-in to permit high burnup at discharge; that they should not be too thick; and that the outer reaches should probably be backed up by moderator to improve catching efficiency per blanket element. It appears now that equally close attention must be paid to axial blankets: they must be designed to be managed and reused.

The axial blanket management problem would favor cores of large length-to-diameter (L/D) ratio, if these were otherwise usable.

D. Heat transfer studies were run both to determine standard correlations and to examine some effects of varying design philosophy from standard practices. From these latter studies, it was determined that power densities could be increased sharply above what are presently considered to be limiting values if central melting were permitted for fuels containing a conducting bond. Power-density increases by factors of $2-5$ were obtained from $80 \%$ molten fuel.

If one accepts a fixed upper limit for sodium velocity, power density may also be increased by going to cores of higher temperature rise 
and smaller sodium content than typical of current practice. Cores with sodium fractions as low as 0.25 may be attractive because the decrease in core volume might compensate for the more positive sodium coefficient of low-sodium systems.

There seems to be no overriding reason for limiting core coolant-temperature rise. This is an emergency criterion basically, and the thermal shock of sudden shutdown may be avoided by pump programming or other control means.

\section{Impressions}

Because of the incomplete nature of the study, firm conclusions are impossible at this time. Nevertheless, the following impressions of the analysis can be recorded.

1. In general, fuel fabrication and process changes are not determining economic factors for burnups greater than 5\%. For lower burnups, carbide fuels are cheaper than oxide ones because they can be used in somewhat larger rod sizes. Metal fuels have not been shown to have large advantages as regards fabrication cost.

2. Design for maximum sodium outlet temperature is an advantage, and this favors the ceramics.

3. A large incentive for high power density exists in the desire to make reactor cores of small-enough size to have a negative sodium coefficient. This works against employment of high sodium fraction for the same goal. Whether or not the sodium criterion is too restrictive may still be discussed; meanwhile, the means of satisfying it is not clear.

Emphasis on sodium coefficient suggests that annular designs or coupled all-fast cores be investigated.

4. Even for large cores, the influence of blanket design and management on fuel-cycle costs is great. Such concepts as the use of the blanket as an "enrichment factory" for thermal reactor fuels therefore have a strong economic incentive. This consideration may ultimately favor coupled reactor concepts.

Finally, as a much more general conclusion, the experience of undertaking this study has taught us a great deal about the way in which problems of reactor design interact with each other; it has particularly impressed us with the subjective nature of forecasts based on practice, and illustrated the need for severely questioning standard practice whenever it is the major reason for a design choice. 
MR, CAMPISE (Atomics International):

In view of your extrapolation to the year 2000, why do you continue to include an intermediate sodium loop in your plant?

MR. SPINRAD:

The extrapolation for the plant was to plants built in 1975. The fuelcycle costs were evaluated at something close to expected midlife of the plant, 1985. We did not take all the advantage we might have of technological growth. The main purpose of this study was really to see what sort of conditions would be desirable in the fast reactor economy, at what appears to $u s$ to be the take-off point of that economy.

MR. STERN (Combustion Engineering):

In your evaluation of the cost of the energy plant, did you keep your steam and sodium conditions fixed? If so, where did the large change in those costs come in?

MR. SPINRAD:

The biggest single change is due simply to the variation of thermal efficiency as the result of taking standard temperature drops from sodium. In the less efficient case, you have to handle a great deal more steam and consequently need a larger turbine. The size effect, as I mentioned, seems on first blush to be very severe. We do expect that this very large slope of capital cost for the energy plant with sodium temperature will be somewhat decreased by an optimization procedure, primarily because standard practice is usually based upon a fairly efficient plant, and lower temperatures should deviate somewhat from it.

MR. SHANSTROM (General Atomic):

Did I understand that your arguments for high-power density cores were from safety considerations rather than from economic?

MR. SPINRAD:

It goes around the daisy chain. We imposed as a safety limit that we should have a negative coefficient from voiding the core only. As I have mentioned, this is a dubious one and you can argue all night about it. But within that limit, which may be a typical safety limit, it was impossible to get certain of the reactors to function at $1,000 \mathrm{MWe}$ or 3,000 to $4,000 \mathrm{MWt}$ 
as single units without having a positive sodium coefficient or a most peculiar geometry. Therefore, a high power density which diminishes core sizes and enables you to get a single reactor to do the job will indeed be an economic thing. 


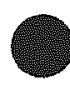


SOME IDEAS ON THE REACTIVITY COEFFICIENTS

IN THE FAST LARGE REACTORS

\author{
C. P. Zaleski \\ EURATOM CEA (Cadarache) \\ Bouches du Rhone, France
}

\title{
I. Introduction
}

In the large, fast power reactors, there are three principal mechanisms through which temperature affects the reactivity:

variation of the fuel cross sections with temperature;

variation of the number of sodium atoms per unit volume of the reactor with the sodium or structure material expansion;

variation of the number of fuel atoms per unit volume of the reactor with the fuel or structure material expansion.

It would be very nice to study these mechanisms separately, but, in fact, they act simultaneously and their sum determines the reactor behavior, at least as long as we neglect the external control system, which is what we shall do in this study.

The temperature or power reactivity feedback mechanism is very important for the reactor design in general, and particularly for fast power reactors design. This problem must be met even when preliminary plans are being made.

One method we consider logical and realistic would be, on the one hand, to make general studies concerning the elementary effects mentioned above by using a very simplified reactor model. On the other hand, we may examine on a more realistic model the simultaneous actions of the different effects in the various transient states of the reactor.

To perform these last studies, we need a reactor model, determined essentially by technology and economics criteria. We hope at the same time that this model is realistic from the point of view of dynamical behaviox.

We shall analyze the behavior of this model in the case of normal operation (stability) and in the case of accidents. The latter can be of two kinds: either probable and if so, they have to be nondestructive; or very improbable, allowing the destruction of the reactor but being without danger to the exterior of the reactor or of its building or even of a certain exclusion zone. 
After such a study we can decide whether the reactor is well designed from the dynamics point of view and what are the requirements concerning the different elementary effects. We may conclude that we are not satisfied with the state of knowledge of some of these effects.

\section{Elementary Effects}

\section{a. Fuel Displacement}

Among the three elementary effects mentioned above, the change of the number of fuel atoms per unit volume is the most trivial problem, at least from the neutronics point of view. However, it is more difficult to calculate exactly the fuel motion for a given change of temperature or power. This is due to the lack in knowledge of the behavior of the irradiated fuel (fuel stuck to the can or not; temperature to which we shall relate the expansion), as well as to the difficulty of calculating precisely the actual clearances between different components of the core structure.

\section{b. Doppler Effect}

It is to be hoped that the theoretical and experimental work on the Doppler coefficient now going on will give a more precise knowledge of this effect in the near future.

Recently (Ref. 1), the method and the approximations used here in the calculation of the Doppler effect were given. The uncertainties in the calculations are due, on the one hand, to an insufficient knowledge of resonance parameters and cross sections for fissile and fertile isotopes, and, on the other, to the imperfections of the calculation methods.

For example, Codd and Collins (Ref. 2) have recently pointed out an important correction due to interference between the plutonium and uranium resonances, which till now was neglected. Other corrections may be necessary to take into account the fine structure of the importance function and perhaps also the utilization of perturbed flux. Even the macroscopic distributions of neutron flux and importance of neutron as function of energy are not well known.*

*Concerning the temperature dependence of the Doppler effect, we suggested at Rome a $T^{-3 / 2}$ law, which seems to be correct for fissile isotopes, but which is probably wrong for the fertile $\mathrm{U}^{238}$, for which a $\mathrm{T}^{-1}$ law is more appropriate; in any case, the law $\mathrm{T}^{-3 / 2}$ is probably a pessimistic one. Let us note that some uncertainties remain concerning this last point. 
Finally, in the actual status of knowledge it is not unreasonable to think of very important uncertainties in the total calculated Doppler effect (e.g., as much as a factor of 2 ).

\section{c. Sodium Effect}

The sodium coefficient may be split into three components, due, respectively, to modification in absorption, in leakage, and in degradation. As stated by Codd et al. (Ref. 3), this coefficient, and particularly the degradation component, is very sensitive to the self-shielding effects of the resonances.

We have performed some calculations for a spherical reactor, the composition of which is identical to that of the reference reactor (reactor A), which we define in Section III. We have used both the normal constants and corrected ones (corrected for the self-shielding effect of resonances): i.e., 16 Hansen and Roach groups (Ref. 4), infinite dilution; 16 Hansen and Roach groups (Ref. 4) corrected for finite dilution.

Results are summarized in Table I and in Figs. 1, 2, 2a, 3, and 3a. A very strong variation of the total sodium coefficient is observed when we take into account the self-shielding effect of resonances. This is essentially due to the variation of the degradation component. We note that there is always a positive central part and a negative peripheral one, but the amplitude and the volume over which each one extends in the integration are very different. We found by some other calculations that the variation of the leakage component, due to the variation of volume in cases of interest to us (3000 and 5000 liters), is not very important. Thus, the above results are representative of self-shielding effect of resonances on the sodium coefficient.

However, let us note, that the breeding ratio decreased by 0.15 and that if we want to readjust this parameter (which is important from the point of view of economics, long-term changes in reactivity, and fuel cycles; see Ref. 5), we must modify the sodium coefficient, making it probably more positive.

Table I

PERFORMANCE OF CORE CALCULATED WITH AND WITHOUT SELF-SHIELDING CORRECTIONS

\begin{tabular}{c|c|c|c|c|c|c|c}
\hline \multicolumn{1}{c|}{ Sets } & $\begin{array}{c}\text { Radius, } \\
\mathrm{cm}\end{array}$ & $\begin{array}{c}\text { Internal } \\
\text { Breeding }\end{array}$ & $\begin{array}{c}\text { Total } \\
\text { Breading }\end{array}$ & \multicolumn{3}{|c}{ Sodium Coefficient, $10^{-5} \Delta \mathrm{k} / \mathrm{k} /{ }^{\circ} \mathrm{C}$} \\
\hline $\begin{array}{l}\text { 16 Groups, Hansen, } \\
\text { Infinite Dilution }\end{array}$ & +108.83 & +1.02 & +1.34 & +1.045 & -0.014 & +0 & +1.031 \\
\hline $\begin{array}{l}\text { 16 Groups, Hansen, } \\
\text { Finite Dilution }\end{array}$ & +95.46 & +0.88 & +1.30 & +0.161 & -0.026 & +0 & +0.135 \\
\hline
\end{tabular}




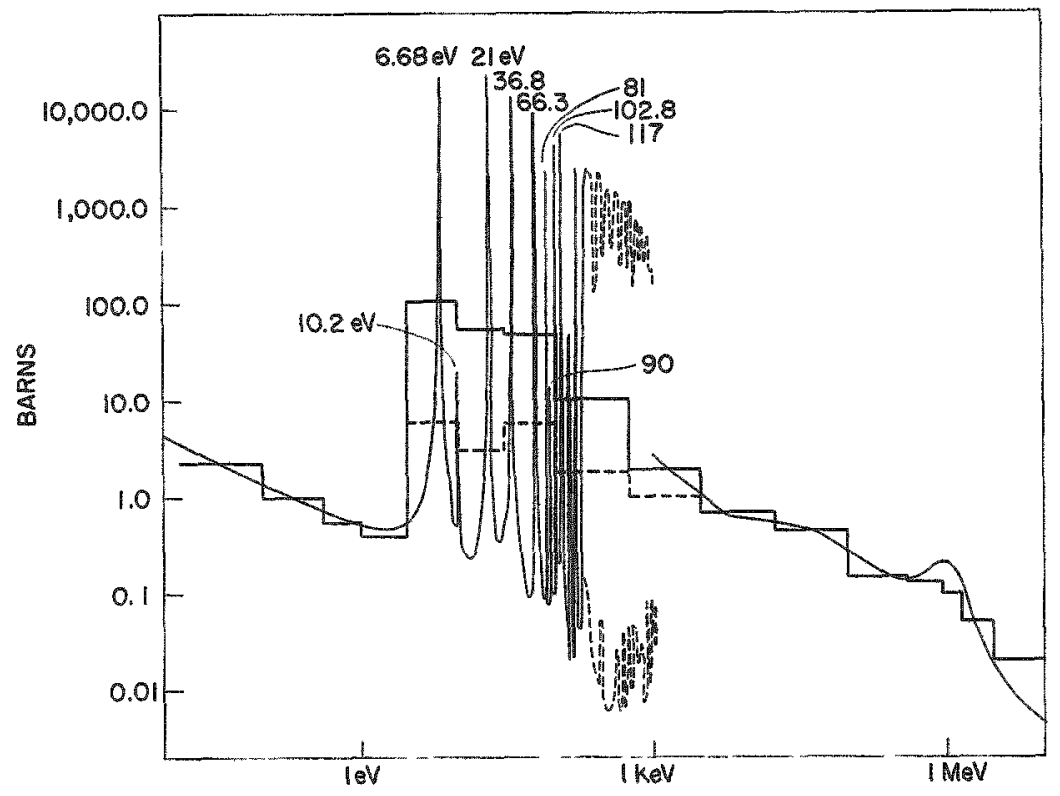

Fig. 1. U $\mathrm{U}^{238}$ Capture Cross Section

Hansen and Roach 16 Group Set

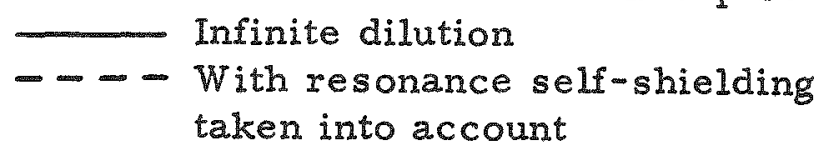
taken into account

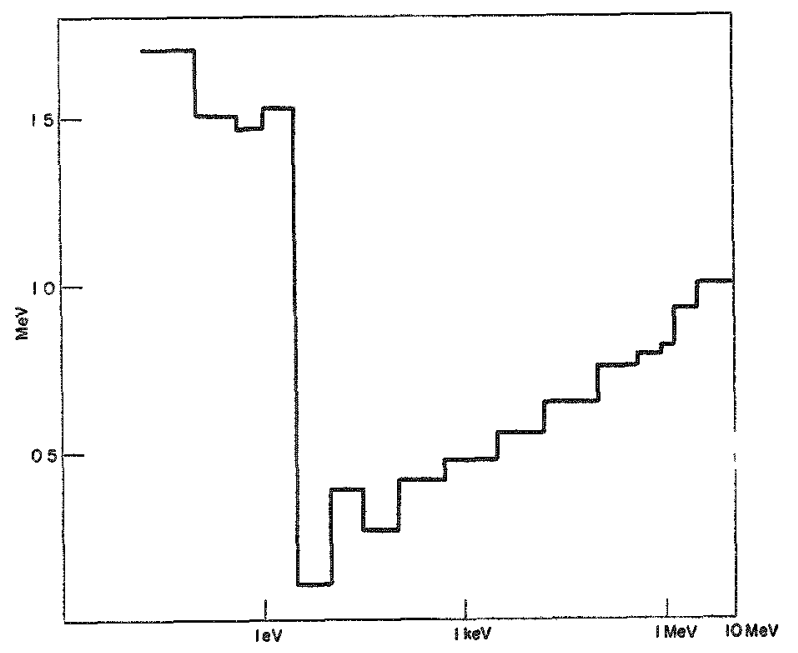

Fig. 2. Importance Function (Normalized to 1 at $10 \mathrm{MeV}$ ) 16-Group Hansen and Roach Set. Infinite Dilution.

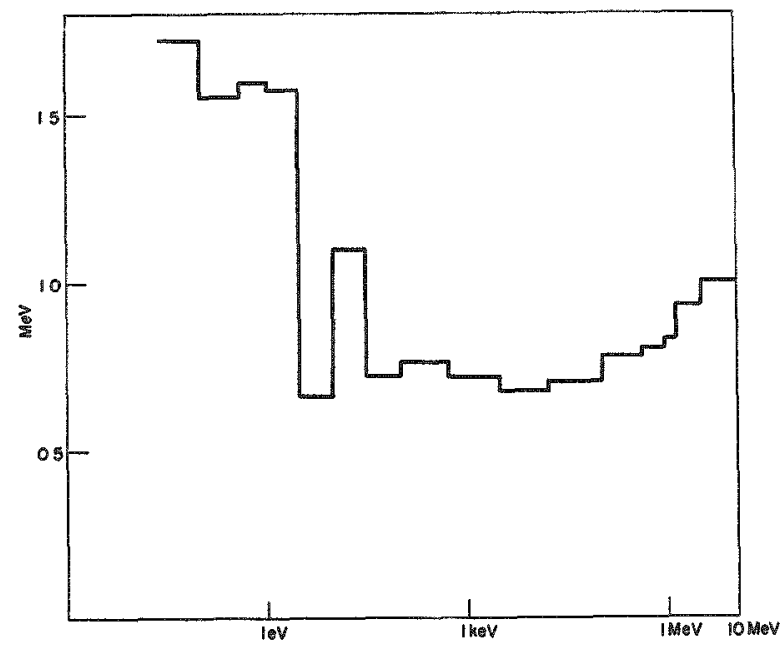

Fig. 2a. Importance Function (Normalized to 1 at $10 \mathrm{MeV}$ ) 16-Group Hansen and Roach Cross-section Set with Resonance Self-Shielding Taken into Account 


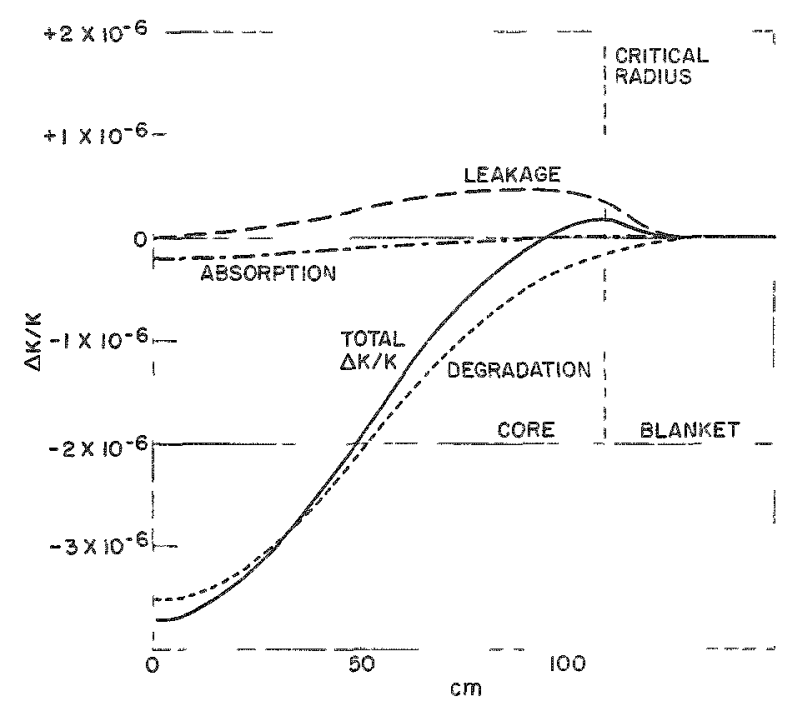

Fig. 3. Sodium Danger Coefficient $\left(\frac{\Delta \mathrm{k}}{\mathrm{k}}\right.$ for $\left.38 \mathrm{~g}\right)$

16-Group Hansen and Roach Set. Infinite Dilution.

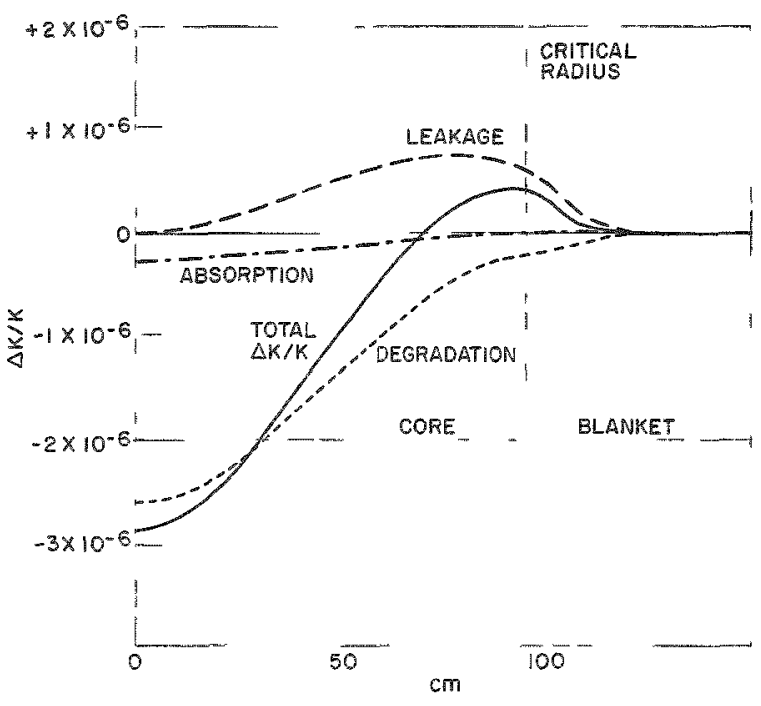

Fig. 3a. Sodium Danger Coefficient $\left(\frac{\Delta \mathrm{k}}{\mathrm{k}}\right.$ for $\left.38 \mathrm{~g}\right)$

16-Group Hansen and Roach Set with Self-shielding Correction.

Moreover we have investigated the influence of different fuels on the sodium coefficient. Calculations have been performed keeping a constant critical volume (5400 liters), the same volume composition, and adjusting the fuel enrichment (fissile/fertile). The constants used were those of Ref. 4 for infinite dilution; thus, results have only a comparative significance. Tables II, III, IV, and $V$ give these results.

Table II

\begin{tabular}{|c|c|c|c|c|c|}
\hline \multirow{2}{*}{ Fuel } & \multirow{2}{*}{$\frac{\mathrm{N} \text { Fissile }}{\mathrm{N} \text { Fertile }}$} & \multicolumn{4}{|c|}{ Sodium Coefficient, $10^{-5} \Delta \mathrm{k} / \mathrm{k} /{ }^{\circ} \mathrm{C}$} \\
\hline & & Core & Blanket & $\begin{array}{l}\text { Steel } \\
\text { Shield }\end{array}$ & Total \\
\hline $\mathrm{U}^{238} \mathrm{O}_{2}-\mathrm{PuO}_{2}$ & +0.157 & +1.045 & -0.014 & +0 & +1.031 \\
\hline $\mathrm{U}^{238} \mathrm{O}_{2}-\mathrm{U}^{235} \mathrm{O}_{2}$ & +0.199 & +0.136 & -0.176 & +o & -0.040 \\
\hline $\mathrm{U}^{238} \mathrm{O}_{2}-\mathrm{U}^{233} \mathrm{O}_{2}$ & +0.117 & +0.425 & -0.161 & to & +0.264 \\
\hline $\mathrm{Th}^{232} \mathrm{O}_{2}-\mathrm{PuO}_{2}$ & +0.183 & +0.813 & -0.182 & to & +0.631 \\
\hline $\mathrm{Th}^{232} \mathrm{O}_{2}-\mathrm{U}^{235} \mathrm{O}_{2}$ & +0.238 & -0.379 & -0.227 & +0 & -0.606 \\
\hline $\mathrm{Th}^{232} \mathrm{O}_{2}-\mathrm{U}^{233} \mathrm{O}_{2}$ & +0.140 & +0.256 & -0.216 & to & +0.040 \\
\hline
\end{tabular}


Table III

\begin{tabular}{c|c|c|c|c|c} 
& \multirow{2}{*}{ Fuel } & $\mathrm{N}_{\mathrm{U}^{238}}$ & \multicolumn{3}{|c}{ Sodium Coefficient, $10^{-5} \Delta \mathrm{k} / \mathrm{k} /{ }^{\circ} \mathrm{C}$} \\
\cline { 3 - 6 } & $\mathrm{N}_{\mathrm{Pu}}$ & Core & Blanket & $\begin{array}{c}\text { Steel } \\
\text { Shield }\end{array}$ & Total \\
\hline $\mathrm{U}^{238} \mathrm{O}_{2}-\mathrm{PuO}_{2}$ & +6.9 & +1.045 & -0.014 & +0 & +1.031 \\
$\mathrm{U}^{238} \mathrm{C}-\mathrm{PuC}$ & +7.75 & +1.127 & -0.012 & +0 & +1.115 \\
$\mathrm{U}-\mathrm{Pu}-\mathrm{MO}$ & +9.73 & +1.534 & -0.003 & +0 & +1.531 \\
\hline
\end{tabular}

$N=$ atom number per cc.

Table IV

\begin{tabular}{c|c|c|c|c}
\hline \multirow{2}{*}{$\% \mathrm{Pu}^{240}$} & \multicolumn{3}{|c|}{ Sodium Coefficient, $10^{-5} \Delta \mathrm{k} / \mathrm{k} /{ }^{\circ} \mathrm{C}$} \\
\cline { 2 - 5 } & Core & Blanket & $\begin{array}{c}\text { Steel } \\
\text { Shield }\end{array}$ & Total \\
\hline 10 & +1.193 & -0.009 & +0 & +1.184 \\
20 & +1.250 & -0.008 & +0 & +1.242 \\
40 & +1.406 & -0.008 & +0 & +1.398 \\
\hline
\end{tabular}

Table V

\begin{tabular}{c|c|c|c|c}
\hline \multirow{2}{*}{$\% \mathrm{Pu}^{241}$} & \multicolumn{3}{|c|}{ Sodium Coefficient, $10^{-5} \Delta \mathrm{k} / \mathrm{k} /{ }^{\circ} \mathrm{C}$} \\
\cline { 2 - 5 } & Core & Blanket & $\begin{array}{c}\text { Steel } \\
\text { Shield }\end{array}$ & Total \\
\hline 0 & +1.250 & -0.008 & +0 & +1.242 \\
5 & +1.170 & -0.009 & +0 & +1.161 \\
10 & +0.929 & -0.009 & +0 & +0.920 \\
\hline
\end{tabular}

We notice that for the sodium coefficient:

$\mathrm{U}^{235}$ and $\mathrm{U}^{233}$ are much better than $\mathrm{Pu}$;

$\mathrm{Th}^{232}$ is much better than $\mathrm{U}^{238}$;

carbides and the U-Pu-Mo (10\% Mo) metal alloy are not as good as oxides; 
an increasing percentage of $\mathrm{Pu}^{240}$ has a bad effect;

an increasing percentage of $\mathrm{Pu}{ }^{241}$ has a favorable effect.

Finally, there remain important uncertainties on the total sodium coefficient.

III. Description of the Reference Model

The reference model is that we have defined in Ref. 1 (reactor 1000 A). The main characteristics axe given in Table VI.

Table VI

\section{PERFORMANCES AND DATA DESIGN OF 1000-MWe REACTOR*}

Fuel type

Average specific power

Net plant efficiency

Outlet temperature of sodium

Temperature drop across the reactor

Core height

Upper and lower blanket height (each end)

Radial blanket thickness

Core reactor volume

Core diameter

Critical mass of plutonium

$\mathrm{Pu} /(\mathrm{U}+\mathrm{Pu})$

Core composition, $v / o$ fuel

$$
v / 0 \text { steel }
$$

$v / 0$ coolant

Fuel pin diameter

Breeding ratio: internal

total

$\mathrm{PuO}_{2}-\mathrm{UO}_{2}$
$0.75 \mathrm{MW} / \mathrm{kg} \mathrm{Pu}$
$40 \%$
$600^{\circ} \mathrm{C}$
$150^{\circ} \mathrm{C}$
$120 \mathrm{~cm}$
$50 \mathrm{~cm}$
$50 \mathrm{~cm}$
81501 iters
$147 \mathrm{~cm}$
$3590 \mathrm{~kg}$
$0.166 * *$
30
16
54
$0.632 \mathrm{~cm}$
1.025
1.34

*Without control rods.

** Pu with about $80 \%$ of fissile isotopes.

Concerning the internal breeding ratio, we can see in Table I that the use of the probably best constants decreases the internal breeding ratio by 0.15 ; furthermore, studies of the long-term reactivity changes (Ref. 5) show that an internal breeding ratio of 1.1 would probably be preferable to 1. These two facts should logically lead us to increase the fuel volume fraction to about $50 \%$. We think, however, that the model 1000 A remains sufficiently representative, of large, fast mixed oxide reactors. 
IV. Study of Dynamics of Reactor $1000 \mathrm{~A}$

\section{a. Stability}

The operational stability of the reactor is strongly connected with the power coefficient, i.e., with the change in reactivity associated with a change of power (taking into account the elementary effects, their distribution over the whole reactor, and the change of the different temperatures associated with the change in power).

It seems interesting to investigate the total isothermal coefficients and the power coefficients with nominal flow. These coefficients are given in Table VII where we have taken:

2 values for the sodium coefficient $\left(-0.6\right.$ and $+1 \times 10^{-5} \Delta \mathrm{k} / \mathrm{k} /{ }^{\circ} \mathrm{C}$ being two arbitrary values for this parameter thought to bracket the real coefficient);

3 values for the Doppler coefficient calculated without any correction $\left(-0.7 \times 10^{-5} \Delta \mathrm{k} / \mathrm{k} /{ }^{\circ} \mathrm{C}\right.$, and, dividing and multiplying this value by 2 : -0.35 and -1.4 );

pessimistic hypotheses for the fuel displacement (no radial expansion, and axial expansion bound to the fuel can expansion).

Table VII

TEMPERATURE COEFFICIENTS

\begin{tabular}{|c|c|c|c|c|c|c|c|}
\hline \multicolumn{8}{|c|}{ Isothermal semperature coefficients: $10^{-5} \mathrm{\Delta k} / \mathrm{k} /{ }^{\circ} \mathrm{C}$} \\
\hline \multicolumn{2}{|c|}{ Sodium expansion } & +1.00 & +1.00 & +1.00 & -0.60 & -0.60 & -0.60 \\
\hline \multirow{2}{*}{ Canning } & Radial expansion & +0.10 & +0.10 & +0.10 & -0.06 & -0.06 & -0.06 \\
\hline & Axial expansion & +0.12 & +0.12 & +0.12 & +0.12 & +0.12 & +0.12 \\
\hline \multirow{2}{*}{$\begin{array}{l}\text { Fuel } \\
\text { axial } \\
\text { expansion }\end{array}$} & $\begin{array}{l}\text { The fuel is in contact } \\
\text { with the canning }\end{array}$ & -0.38 & -0.38 & -0.38 & -0.38 & -0.38 & -0.38 \\
\hline & $\begin{array}{l}\text { The fuel is free in } \\
\text { the canning }\end{array}$ & -0.21 & -0.21 & -0.21 & -0.21 & -0.21 & -0.21 \\
\hline \multicolumn{2}{|c|}{ Core radial expansion } & -0.92 & -0.92 & -0.92 & -0.92 & -0.32 & -0.92 \\
\hline \multicolumn{2}{|c|}{ Doppler effect } & -0.35 & -0.70 & -1.40 & -0.35 & -0.70 & -1.40 \\
\hline \multicolumn{2}{|c|}{$\begin{array}{l}\text { Total ischermal with: } \\
\text { - zero core radial expansion } \\
\text { - fuel in contact will the } \\
\text { canning }\end{array}$} & +0.49 & +0.14 & -0.56 & -1.39 & -1.74 & -2.44 \\
\hline \multicolumn{8}{|c|}{ Power coefficients: $10^{-7} / \mathrm{MW}$} \\
\hline \multicolumn{2}{|c|}{ Sodium expansion } & +3.00 & +3.00 & +3.00 & -1.80 & -1.80 & -1.80 \\
\hline \multirow{2}{*}{ Canning } & Radial expansion & +0.54 & +0.54 & +0.54 & -0.32 & -0.32 & -0.32 \\
\hline & Axial expansion & +0.59 & +0.59 & +0.59 & +0.59 & +0.59 & +0.59 \\
\hline \multirow{2}{*}{$\begin{array}{l}\text { Fuel } \\
\text { axial } \\
\text { expansion }\end{array}$} & $\begin{array}{l}\text { The fuel is in contact } \\
\text { with the canning }\end{array}$ & -1.88 & -1.88 & -1.88 & -1.88 & -1.88 & -1.88 \\
\hline & $\begin{array}{l}\text { The fuel is free in } \\
\text { the canning }\end{array}$ & -8.05 & -8.05 & -8.05 & -8.05 & -8.05 & -8.65 \\
\hline \multicolumn{2}{|c|}{ Core radial expansion } & -2.76 & -2.76 & -2.76 & -2.76 & -2.76 & -2.76 \\
\hline \multicolumn{2}{|c|}{ Doppler effect } & -14.20 & -28.40 & -96.80 & -14.20 & -28.40 & -56.80 \\
\hline \multicolumn{2}{|c|}{$\begin{array}{l}\text { Total power coefficient with: } \\
\text { - zero core radial expansion } \\
\text { - fuet in contad with the } \\
\text { canning }\end{array}$} & -12.0 & -26.2 & -54.6 & -17.6 & -31.8 & -60.2 \\
\hline
\end{tabular}


The power coefficient is strongly negative in all the cases considered above. This means that the reactor is very stable even with a

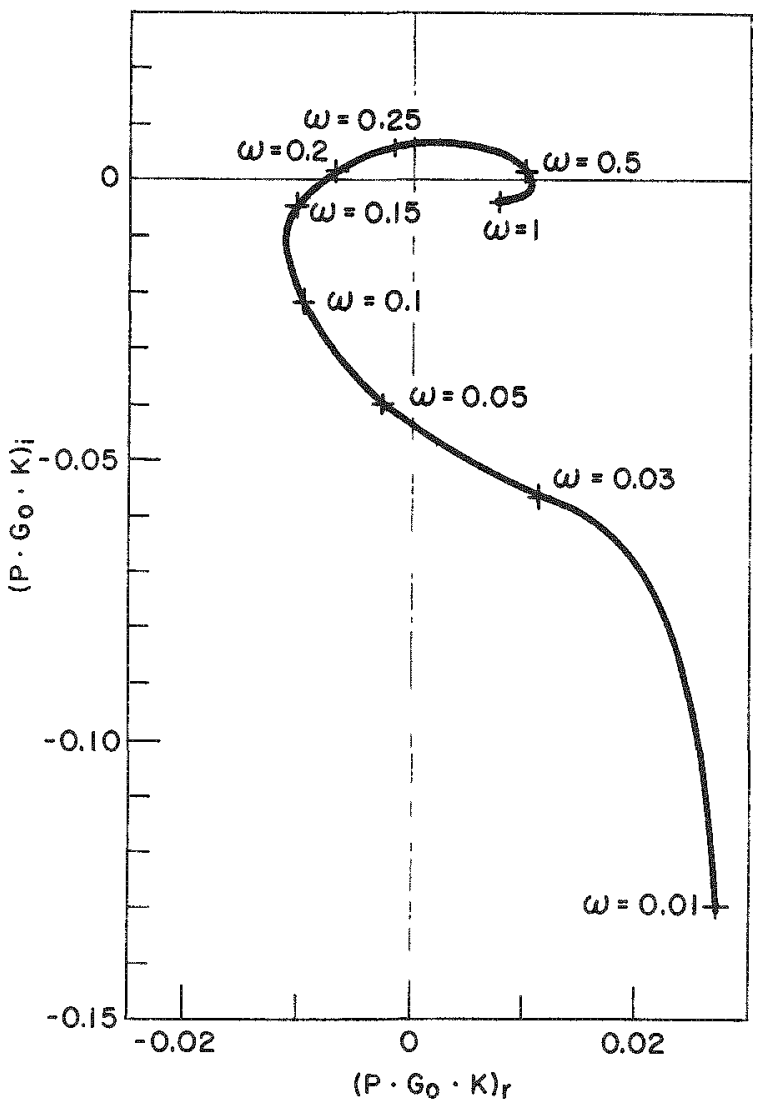

Fig. 4. 1000 MWe Reactor. Temperature drop across the reactor $150^{\circ} \mathrm{C}$, power $100 \mathrm{MW}, \mathrm{G}_{0}(\mathrm{i} \omega)$ zero power transfer function, $K(i \omega)$ feedback coefficient. relatively small flow. This is illustrated by Fig. 4 where, applying the Nyquist criterion, we note that for a flow equal to $1 / 25$ of the operating flow, the reactor is stable up to a relatively high power.

Hence, we can say, that from the stability point of view, the large oxide reactors show a very good behavior and that we can probably be satisfied with our actual knowledge (relatively imprecise) of the elementary coefficients of reactivity.

\section{b. Accident of a Certain Probability}

Here we consider two types of accidents; (1) a more or less strong insertion ramp of reactivity corresponding for instance to a bad operation (noncorrected) with one regulating rod or shim rod: (2) a strong decrease of the sodium coolant flow.

For the first type of accident, starting at norninal power and with a nominal flow leads us to define the maximum reactivity which can be inserted for a certain ramp and for a given margin of fuel temperature before melting occurs.

This maximum excess of reactivity depends strongly on the power coefficient and, consequently, on the Doppler effect. Figure 5 gives us these values for reasonable ramps. The reactor coefficients used are the following:

\begin{tabular}{c|c|c|c|c|c}
\hline Case & $\begin{array}{c}\text { Radial } \\
\text { Expansion } \\
\text { of the } \\
\text { Core }\end{array}$ & $\begin{array}{c}\text { Isothermal } \\
\text { Sodium } \\
\text { Coefficient, } \\
10^{-5} \Delta \mathrm{k} / \mathrm{k} /{ }^{\circ} \mathrm{C}\end{array}$ & $\begin{array}{c}\text { Isothermal } \\
\text { Doppler } \\
\text { Coefficient, } \\
10^{-5} \Delta \mathrm{k} / \mathrm{k} /{ }^{\circ} \mathrm{C}\end{array}$ & $\begin{array}{c}\text { Isothermal, } \\
\text { Total, } \\
10^{-5} \Delta \mathrm{k} / \mathrm{k} /{ }^{\circ} \mathrm{C}\end{array}$ & $\begin{array}{c}\text { Power } \\
\text { Coefficient, } \\
10^{-7} \Delta \mathrm{k} / \mathrm{k} / \mathrm{MW}\end{array}$ \\
\hline 1 & 0 & +1.10 & -1.36 & -0.50 & -0.53 \\
2 & 0 & +1.10 & -0.68 & +0.18 & -0.25 \\
33 & 0 & +1.10 & -0.34 & +0.52 & -0.11 \\
\hline
\end{tabular}




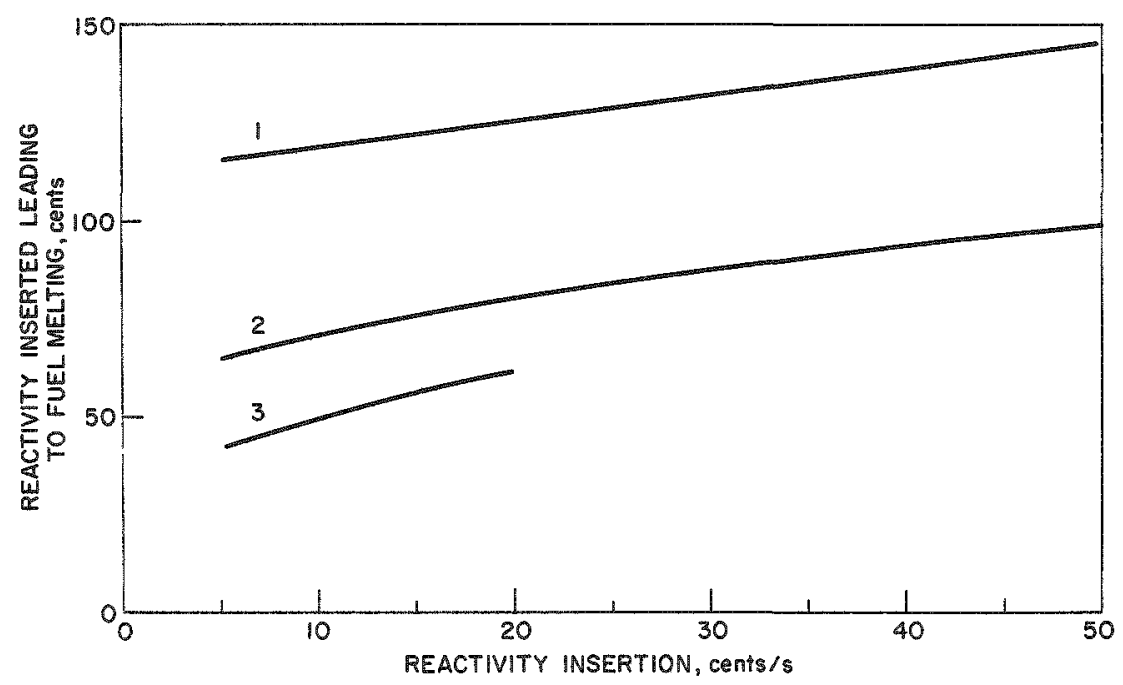

Fig. 5. Insertion of a Reactivity Ramp

If the initial flow and power are much smaller than the nominal values, sodium boiling occurs before melting of the fuel. Since we want to avoid the boiling of sodium, we shall not accept reactivity insertion larger than the given figure which depends:

on the ramp;

on the reactivity coefficient;

on the initial flow and power.

Figure 6 gives the amount of reactivity which can be inserted before boiling as a function of the ramp. Initial flow and power are equal to $1 / 10$ of the nominal flow and power, and the reactivity coefficients (very pessimistic) are as follows:

\begin{tabular}{c|c|c|c|c}
\hline $\begin{array}{c}\text { Radial } \\
\text { ofpansion } \\
\text { Core }\end{array}$ & $\begin{array}{c}\text { Isothermal } \\
\text { Sodium } \\
\text { Coefficient, } \\
10^{-5} \Delta \mathrm{k} / \mathrm{k} /{ }^{\circ} \mathrm{C}\end{array}$ & $\begin{array}{c}\text { Isothermal } \\
\text { Doppler } \\
\text { Coefficient, } \\
10^{-5} \Delta \mathrm{k} / \mathrm{k} /{ }^{\circ} \mathrm{C}\end{array}$ & $\begin{array}{c}\text { Isothermal, } \\
\text { Total, } \\
10^{-5} \Delta \mathrm{k} / \mathrm{k} /{ }^{\circ} \mathrm{C}\end{array}$ & $\begin{array}{c}\text { Power } \\
\text { Coefficient, } \\
10^{-7} \Delta \mathrm{k} / \mathrm{k} / \mathrm{MW}\end{array}$ \\
\hline 0 & +1.10 & -0.68 & +0.18 & -0.20 \\
\hline
\end{tabular}

The amount of reactivity always depends strongly on the power coefficients, but it is to be noted that the importance of the sodium coefficient in the power coefficient is here amplified by a factor of 10 in comparison with the case of the nominal flow (inverse of the flow ratio).

Therefore, the sodium coefficient becomes almost as important as the Doppler coefficient. One can note that, concerning the ramptype accidents, the reactor designer disposes of more flexibility in the 
definition of the reactivity which can be inserted as well as in its insertion rates when the sodium and Doppler coefficients of the fuel are more negative.

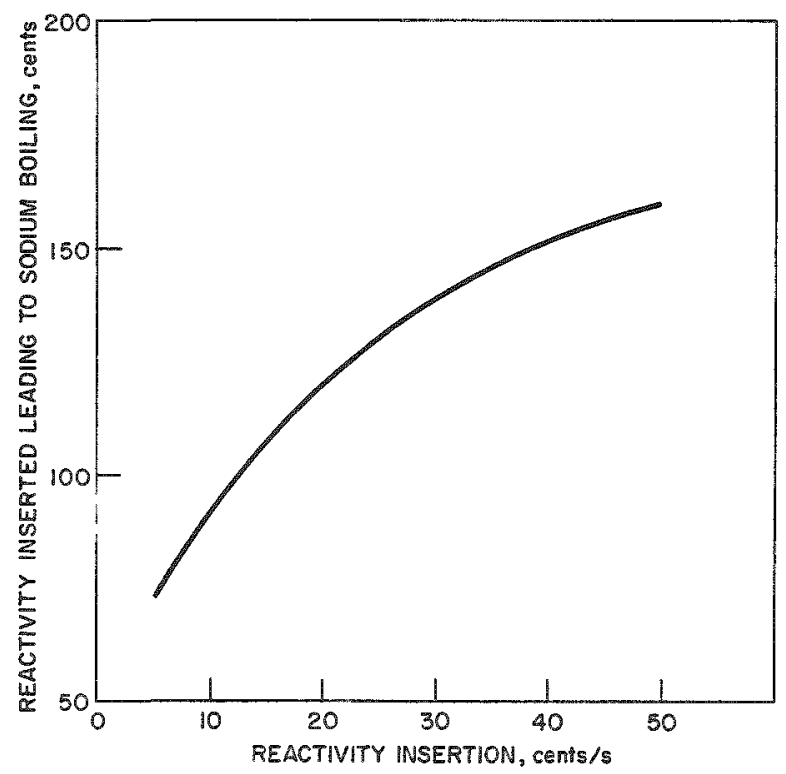

Fig. 6

Insertion of a Reactivity Ramp

Second type of accidents: flow coastdown

following:

The problems we are trying to examine here are the

Which flow coastdown may we accept so that boiling of sodium never occurs?

Which flow coastdown may we accept so that even if there is boiling, the latter does not have serious consequences and, in particular, may be stopped before burnout?

How does our choice (if any) depend on the reactivity coefficients?

To answer these questions we have devised one digital code which deals with the problem with simplified hypotheses:

We assume a flow law which is not modified by the pressure drop.

Boiling occurs at the atmospheric pressure.

We performed the calculations in the 5 following cases: 


\begin{tabular}{|c|c|c|c|c|c|c|}
\hline Case & $\begin{array}{c}\text { Fadial } \\
\text { Expansion } \\
\text { of tho } \\
\text { Core, } \\
10^{-5} \mathrm{Lk} k \mathrm{C}\end{array}$ & $\begin{array}{c}\text { Doppler } \\
\text { Coeficient. } \\
10^{-5} \mathrm{kik} \text { \& } \mathrm{C}\end{array}$ & 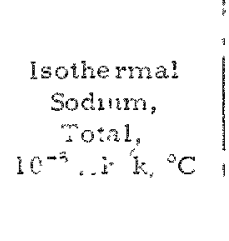 & 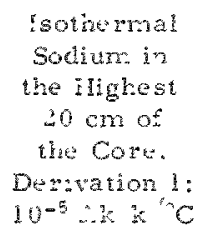 & $\begin{array}{l}\text { "sothermal } \\
\text { Sodsurn Gap. } \\
n^{-n}: k \leq B\end{array}$ & 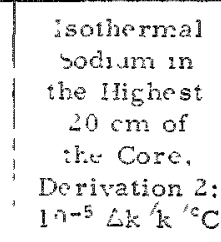 \\
\hline 1 & -0.9 & $-1 . \frac{1}{2}$ & +1 & -0.027 & $-0.0=\frac{1}{1}$ & -0.027 \\
\hline 2 & 0 & $-1 . \frac{1}{2}$ & +1 & -0.027 & $-0.0=1$ & -0.027 \\
\hline 3 & 0 & 0 & +1 & -0.027 & $-0 . \mathrm{c}^{\circ}$ & -0.027 \\
\hline 4 & 0 & -0.7 & 0 & 6 & $\sqrt{3}$ & 0 \\
\hline 5 & 0 & -0.7 & \pm 1 & -0.017 & $-11.0 ; 5$ & -0.008 \\
\hline
\end{tabular}

In all these cases the flow coastdown follows the following law:

$$
\frac{\text { flow }}{\text { initial flow }}=0.06+0.94 \mathrm{e}^{-\mathrm{t} / 5} \text {. }
$$

Figures 7, 8, and 9 represent, respectively, the variation of the reactivity feedback, of the power, and of the sodium density in the upper part of the core, as a function of time. Considering these studies we can conclude the following:

The time and the power at which boiling appears are strongly dependent on the isothermal total coefficient of the reactor.

The power decrease after boiling has started and the eventual stop of boiling depend only on the isothermal sodium coefficient in the upper part of the core and in the transient space.

Other studies with less severe flow coastdown* seem to show that it should be possible to arrive at a reasonably pessimistic flow coastdown which do not lead to sodium boiling or lead to a boiling which can be stopped provided there is a sufficiently strong negative isothermal coefficient (the sodium reactivity coefficient in the upper part of the reactor has to be strongly negative).

reactor.

These conditions are probably satisfied in our reference

Let us note that in the flow accidents, we deal only with perturbation of the total flow. Certainly, local perturbations (plugging) are very important. However, we think that the evolution of such accidents

* The law $e^{-t / 5}$ corresponds, approximately, to a stoppage of all the circuits on pump inertia and the supplying groups. Less severe laws can certainly be considered sufficiently pessimistic if certain precautions in the reactor design (number of circuits, safety on power supply, etc.) are taken. 
does not depend strongly on reactivity coefficients. Indeed, even a large variation of temperature or density, in one or two channels will probably be compensated by a small variation in the hundreds of other channels.

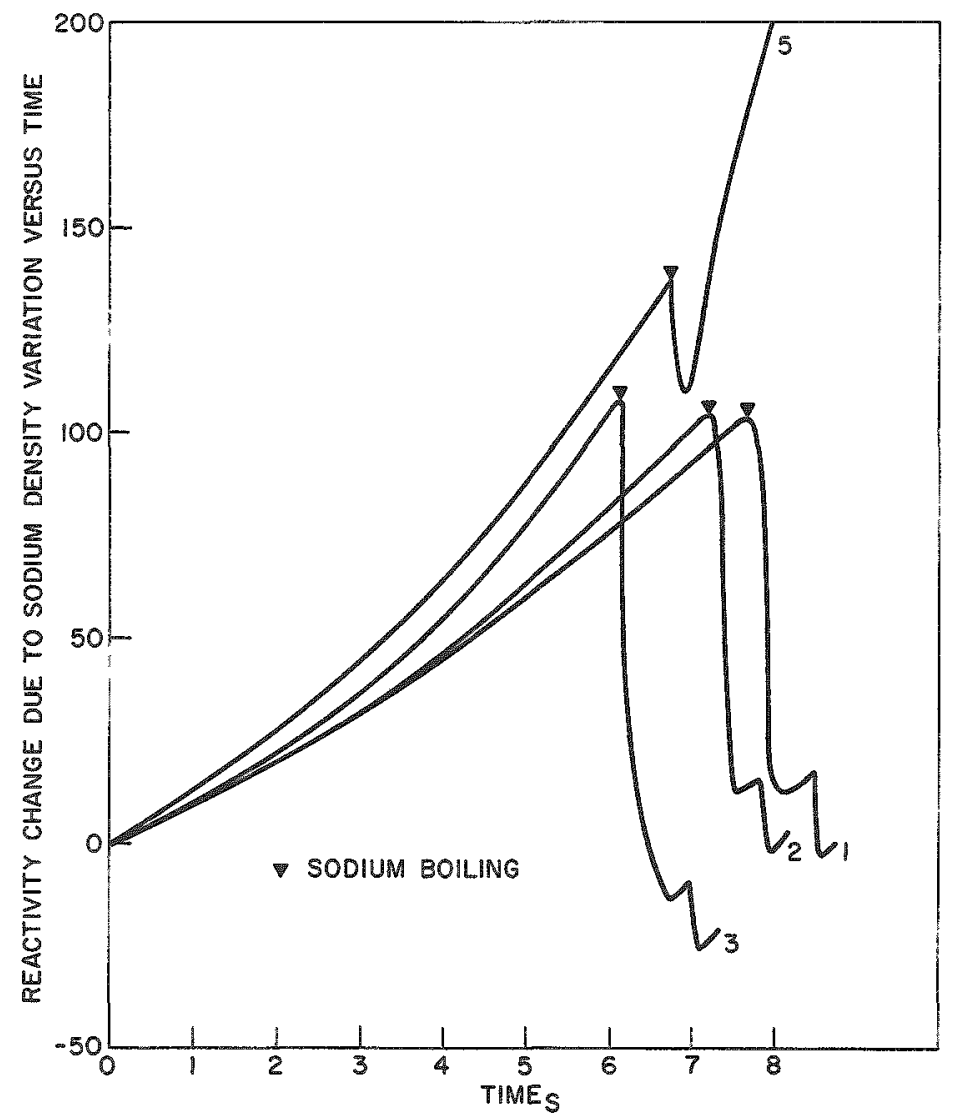

Fig. 7

Cooling Loss Leading to Sodium Boiling

Fig. 8

Cooling Loss Leading to Sodium Boiling

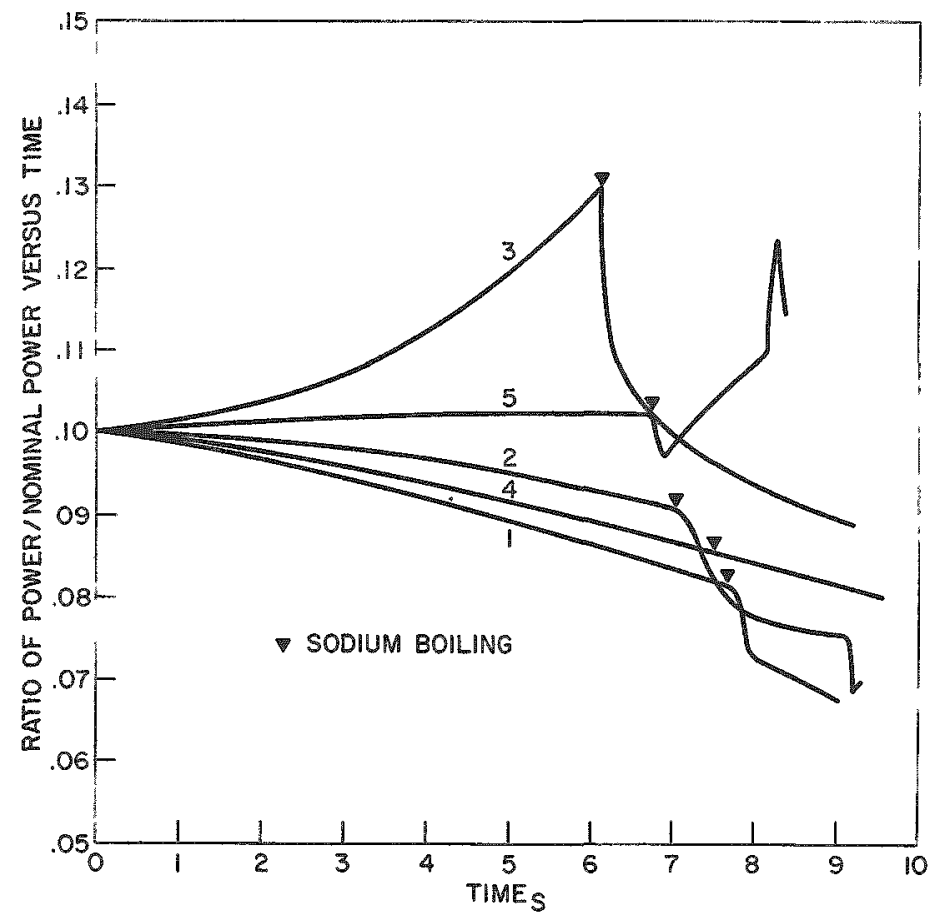




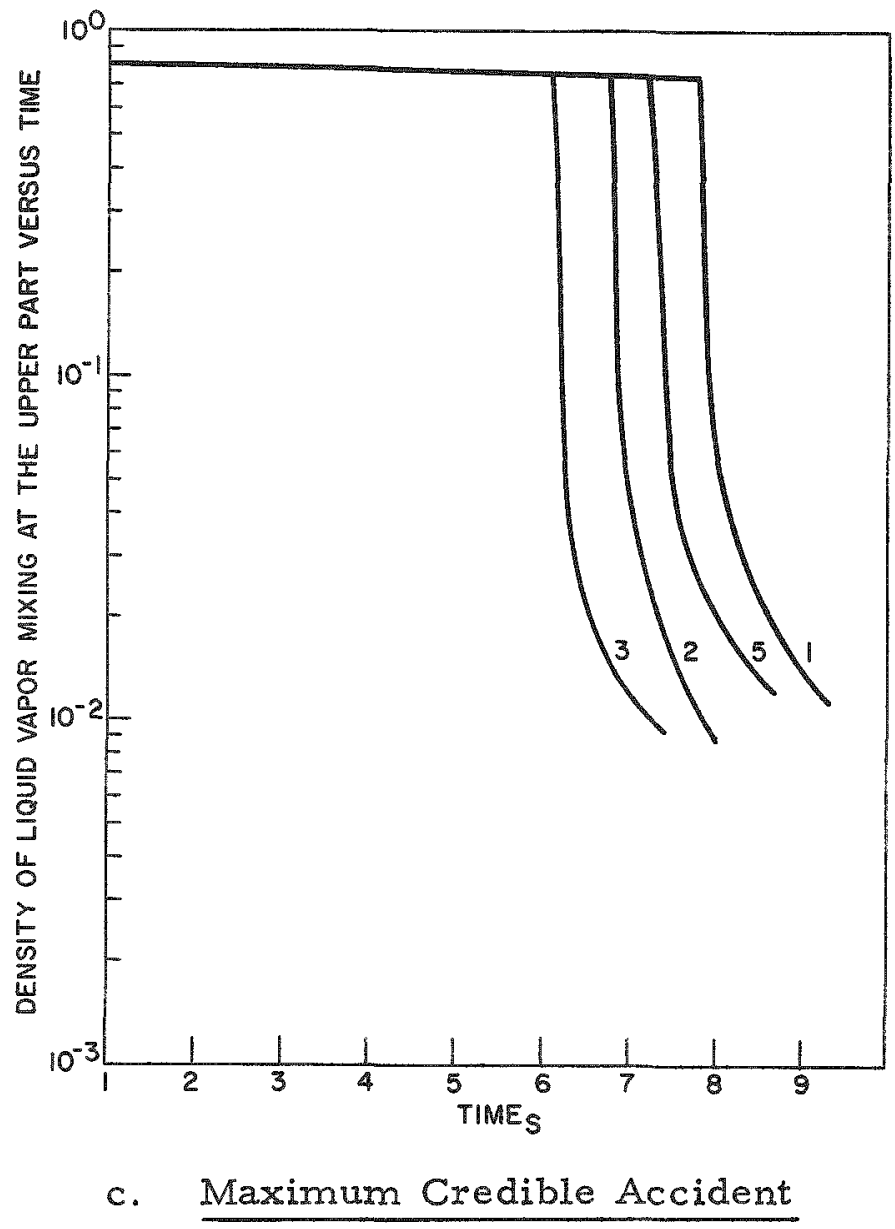

Fig. 9

Cooling Loss Leading to Sodium Boiling

We want to study here the influence of the Doppler and sodium coefficients on the energy released in a maximum credible accident.

We have looked at a hypothetical maximum accident similar to that defined for EFEBR, EBR-II, or RAPSODIE. If we start with an initial temperature of $1300^{\circ} \mathrm{K}$, a reduction by a factor of about 2 in the energy release is to be noticed with a Doppler coefficient of 0.3 to $0.7 \Delta \mathrm{k} / \mathrm{k} /{ }^{\circ} \mathrm{C}$.

It seems that the Doppler coefficient may contribute to a reduction of such an accident. However, we have to be rather skeptical about these estimates because, on the one hand, the hypothesis of $1300^{\circ} \mathrm{K}$ is quite unlikely, and on the other hand, the method of calculation does not apply well to the kind of accident we are considering (maximum rate of reactivity change $\leq 50$ dollars/sec).

Moreover, the contribution of the Doppler effect at high temperature, especially above $3000^{\circ} \mathrm{K}$, is not well known (we have adopted the pessimistic $T^{-3 / 2}$ law). 
Let us note that the maximum hypothetical accident as it is defined for RAPSODIE must be reconsidered for the large reactors. Indeed, Fig. 10 shows that it is difficult to obtain large negative reactivities necessary for this kind of accident with the large reactors.

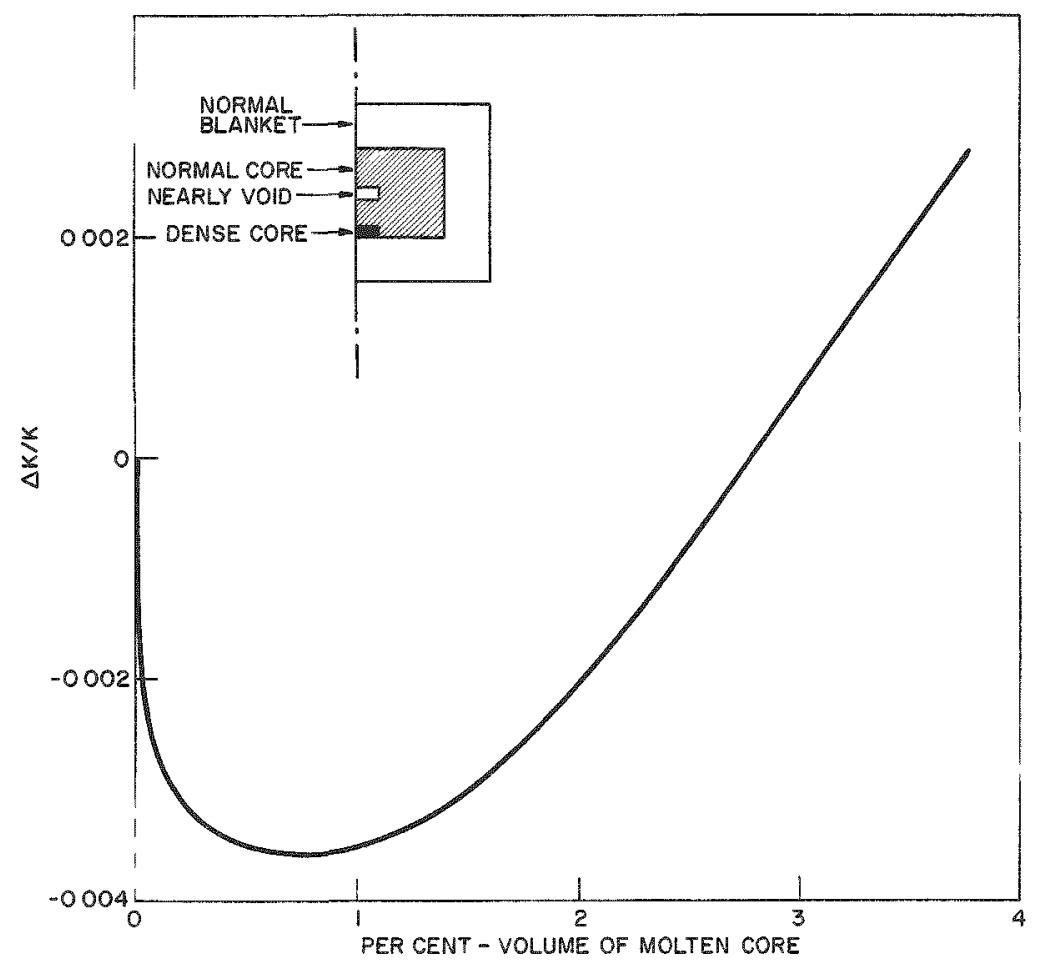

Fig. 10. Variation of Reactivity with Fraction of Core Melted

One may say that a negative and large Doppler coefficient is beneficial, but that one must probably reconsider the mechanisms of the hypothetical maximum accident.

Another aspect of the problem is the following: since the total, or at least the central, sodium coefficient may be positive, can we expect important accidents due to this coefficient? We have examined two cases:

1. sodium removal by leakage;

2. sodium boiling starting from the core center.

Figures 11 and 12 show the changes of reactivity in these two cases. Calculations have been made with constants which are not corrected for the self-shielding effect. Thus, they are very pessimistic. We can see in Fig. 11 that we introduce a maximum of $65 \times 10^{-5} \Delta \mathrm{k} / \mathrm{k} / \mathrm{cm}$ due to sodium removal, so that we require a sodium drain of $46 \mathrm{~cm} / \mathrm{sec}$ to obtain a reactivity insertion of 10 dollars/sec. Assuming this rate constant, we would have to empty the whole core in $2.6 \mathrm{sec}$, which is very unlikely. 


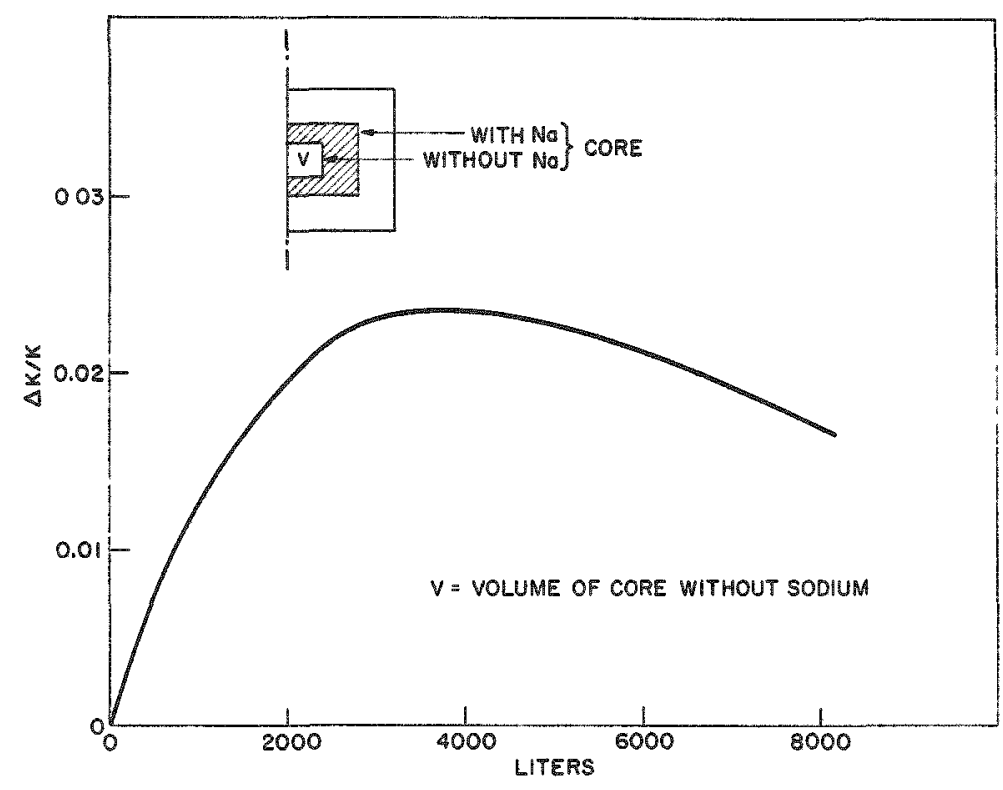

Fig. 11. Sodium Drain in Core of 1000-MW Reactor

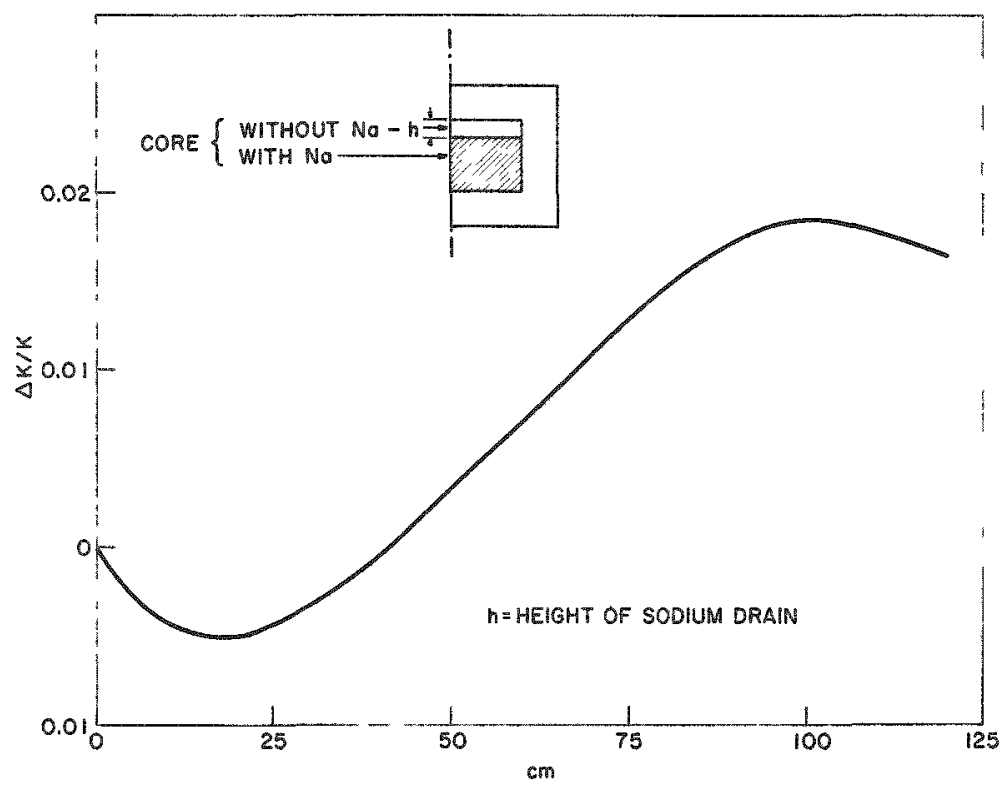

Fig. 12. Boiling of Sodium in Core of 1000-MW Reactor

Concerning the boiling, we have a maximum insertion of $1.26 \times 10^{-5} \Delta \mathrm{k} / \mathrm{k}$ per liter of the core, i.e., for an insertion of 10 dollars/sec, a vaporization rate of about 2600 liters in 1 sec. The reactor should then provide the sodium with about $10,000 \mathrm{MW}$ of heat. Obviously the core would have melted long before.

To conclude, we can say that the rate of reactivity insertion due to the boiling or to the leakage of sodium is not very large in 
comparison with what is admitted in the definition of the hypothetical maximum credible accidents for the actual fast reactors (several hundreds of dollars/sec). We must note, however, that the maximum rates of reactivity insertion for the large reactors will be smaller (see remark concerning the influence of the Doppler effect on the maximum credible accidents). This restricts the preceding conclusion.

Moreover, the influence of the positive sodium coefficient (even if it appears only in the center of the core) must be studied carefully together with other sources of reactivity insertion (fuel displacement) and of negative feedback (Doppler effect).

This complex study requires, first, a definition of the hypothetical maximum credible accidents more suitable for large reactors.

\section{Conclusions}

Our study is not complete. It must be considered as an attempt to make the requirements to be met by a reactor designer concerning the reactivity coefficients more precise. Our first impressions are the following:

1. Our reference reactor is stable if the Doppler effect is negative and not too small in absolute value (larger or equal to about $25 \%$ of the effect calculated now). We can accept the actual values with large uncertainties for sodium and fuel movement coefficients.

2. For the evolution of possible accidents with nonnegligible probability, the influences of the Doppler coefficient, of the sodium coefficient, and, particularly, of the distribution of the latter are important. The influence of the fuel-motion coefficient may be important, but we must be sure of the mechanical hypothesis used. However, we can probably accept the actual values and uncertainties of reactivity coefficients, even if the better knowledge of these coefficients is helpful for the reactor designer.

3. Concerning the maximum hypothetical accident, it does not seem that the sodium coefficient alone can produce a very high enexgy release. The Doppler coefficient, if strongly negative, may have favorable influence, but it has to be specified more precisely, as well as the maximum hypothetical accident itself.

Anyway, more detailed studies of the maximum hypothetical accident and of the influence of the reactivity coefficients on energy release in large reactors are necessary. These studies may, perhaps, increase the precision required for the different reactivity coefficients. 
Moreover, it seems very important to us to use relatively realistic criteria in the definition of probable accidents. Otherwise one takes the risk of increasing very strongly the cost of construction by improper requirements, or even of making a compromise which does not agree with the true safety. I am referring here to the compromise which one may make between the Doppler coefficient, which becomes more negative as the ratio of fissile to fertile material decreases, and the sodium coefficient, which becomes more positive.

It may happen that a too simple criterion as, for instance, a total sodium coefficient equal to zero, or a stopping of a power excursion by the Doppler effect after an insertion of $X$ dollars per second ( $X$ being arbitrary and large), does not lead to optimal solutions.

I wish to thank all the physicists and engineers of the SEPR who helped me in the preparation of this report. I thank especially Messrs. Abdon, Chaumont, Jallade, Ladet, Martin, and Ravier.

\section{References}

1. C. P. Zaleski, Coefficient de réactivité dans les grandes piles de puissance, Symposium in Rome, June 1963.

2. J. Codd and P.J. Collins, $P u^{239}$ and $U^{238}$ Resonance Interaction Effects in a Dilute Fast Reactor, EAES Symposium, Karlsruhe, April 1963.

3. J. Codd et al., Some Calculations of Sodium Reactivity Effects in a Dilute Fast Reactor, AEEW-M 225 (April 1963).

4. G. Hansen and Roach, Multigroup Cross Sections, LAMS-2543.

5. Mr. Vendryes, L'utilisation du Plutonium dans les réacteurs à neutrons thermiques et dans les réacteurs a neutrons rapides, Baden-Baden, Sept 1963.

Discussion of Paper

Presented by Mr. Zaleski

MR. THALGOTT (Argonne):

I would like to make one comment which is not pertinent to Dr. Zaleski's paper alone, but I think to most of the papers we have heard. I think these calculations are very instructive and show trends, and certainly show ways one might proceed to better our state whether it be happy 
or sorry. But I believe that relating any of these calculations to a particular reactor now might be a little fraught with danger. I think our ability to calculate the neutron energy spectrum in these reactors is extremely uncertain, and to my knowledge we have no measurement which indicates that we can correctly calculate the spectrum. Indeed, I think we have some measurements that say we cannot calculate the spectrum. So I think that actually tying these things into a particular reactor, when you want to design one, will require some more work.

MR. HÄFELE (Karls ruhe):

I am deeply concerned about the discrepancy in the calculation of the internal breeding ratio which came out at least today. We all agree that it is very important that the internal breeding ratio is close to 1 , and the paper of this morning submitted by AI concerning the reactivity margin, and the frequency of loading and unloading, also emphasized this point. I think the art to calculate such reactors should be developed to the extent that the internal breeding ratio calculated by the different groups should not be too much different. But an enrichment of $Y=6.7$, which you presented today, gives in your calculation an internal breeding ratio in the neighborhood of 1, whereas, in our case, the internal breeding ratio is between 0.65 and 0.75 , depending on the composition of the reactor. I think this discrepancy is too large and we really ought to have some action to clarify it.

MR. ZALESKI:

I wish to mention that the ratio of 6.4 that I mentioned in my table is for uranium to total plutonium, not just $\mathrm{Pu}^{23}$.

Secondly, as I mentioned, we have now modified the constants used. We now think that the corrected constant gives an internal breeding ratio smaller by 0.2 . So I am not sure that the discrepancy is really so large.

MR. HÄEELE:

If you were the only ones, then perhaps arguments like this presented by you could overcome the difficulty. But, today we had recitals from the British on internal breeding ratios also in the neighborhood of 1. So this is not the only recital of that sort.

MR. SMITH (Dounreay):

Did you say that the Doppler effect had very little influence on the flow rundown accident? We have done some studies on this accident and we find that the Doppler coefficient is very important in the rundown 
case. In fact, we came to what was originally a very curious result. When you think about it, however, it is quite reasonable. Namely, the reactor is safer if you have a positive Doppler effect, for this particular type of accident. It does reinforce what other speakers have said, you must consider each accident and each reactor on its merits.

MR. ZALESKI:

Perhaps I can explain further what I have discussed. When I consider the stopping of the flow. I prefer to have a negative Doppler coefficient and a negative sodium coefficient, if I wish to avoid the boiling. But after this, when the boiling starts, then I say that the Doppler is of no importance in respect to the magnitude of the sodium void effect. If only one channel is blocked, of course, conditions are different.

MR.SMITH (Dounreay):

Let's get the record straight by saying I am not using this as an argument for positive Doppler coefficient.

MR. SMITH (Winfrith):

All I wanted to do was withdraw from the internal breeding ratio competition in the sense that the data we used is comparatively old data.

MR. OKRENT (Argonne):

I thought I detected another discrepancy between papers given here. It seems to me there was an 8,000 liter reactor with which shielded resonances and even 35 or 50 per cent of fuel, still give a negative sodium coefficient. Is this correct?

MR. ZALESKI:

It was a spherical reactor, 5,400 liters. This core composition corresponds to the 8,000 cylindrical reactor with some gaps, some axial blankets, and some controls. We keep the composition fixed for such studies. We have proved by other studies that the leakage component of the sodium coefficient varies not very much between 5,000 and 8,000 liters.

MR. OKRENT:

Is this with $\mathrm{Pu}^{240}$ and fission products? 
MR. ZALESKI: of $\mathrm{Pu}^{240}$.

This was without fission products and with about 20 per cent, I think, MR. OKRENT:

Now, I would like to ask Mr. Hummel if this is compatible with the calculations that he has been doing.

MR. HUMMEL (Argonne):

It strikes me there was a discrepancy there. I stated that my $\mathrm{Pu}^{240}$ cross sections were pretty much guesses, and perhaps the Hansen and Roach cross sections are in that category also. Of course, the Hansen and Roach cross section set has rather broad energy groups in the region of particular interest for the elastic removal component of sodium void coefficient. This might make some difference.

MR. GREEBLER (General Electric):

You reported an increase in the Doppler coefficient due to the overlapping effect. I believe you said that the $\mathrm{Pu}^{239}$ positive Doppler contribution went to a negative value one and one-half times greater in magnitude, which would give you something like a 60 per cent increase in the magnitude of the overall Doppler coefficient perhaps. We find that we get only about a 10 per cent effect from this, which is a rather sizable discrepancy. 


\title{
REACTOR SAFETY AND CONSIDERATIONS OF FUEL-CYCLE ECONOMICS FOR FAST REACTORS
}

\author{
K. Cohen, P. Greebler, M. J. McNelly, \\ P. M. Murphy, D. Sherer, and E. L. Zebroski \\ General Electric Co. \\ Atomic Power Equipment Department \\ San Jose, California
}

(Paper presented by $\mathrm{Mr}$. Cohen)

Since reactor safety means different things to different people, before going into today's discussion, some definitions may be in order. Let us distinguish in the first place between operating incidents and accidents. An operating incident is an abnormal occurrence or sequence of events which can be mastered by the reactor control system, with at most only minor local damage to the reactor. An accident is an event which causes major system damage. We may have accidents without nuclear excursions (for example, after-heat meltdown by loss of coolant), and we may have nuclear excursions without accidents (for example, a prompt critical excursion in a reactor with a strong negative Doppler coefficient). A characteristic of an accident is that the control system, although it may moderate the severity of the accident, is unable to prevent major damage. In fast reactors, in particular, the control system may be limited in its effect on accidents because of the rapidity of the nuclear events, or because there is inertia in heat generation and release which it cannot affect.

The course of the accident depends largely on the inherent properties of the system. Thus the design criteria which we establish to obtain reactor safety must cover three areas:

1) securing desirable operating characteristics so that operating incidents do not generate into accidents;

2) securing inherent reactor characteristics such that accidents are not self-compounding;

3) building in inherent safety devices to limit the energy release in core disassembly.

The importance of the prompt negative Doppler feed back in limiting the energy release in a terminal accident was discussed yesterday by Dr. Wolfe. (1) I shall discuss the design criteria required for desirable operating characteristics and to avoid self-compounding of accidents.

It was appreciated in the earliest scoping studies of the fast ceramic reactor, $(2-4)$ that besides a strong negative Doppler coefficient, a negative sodium power coefficient was desirable. These together lead to 
desirable operating characteristics. As economic studies showed the incentive for large fast ceramic reactors:(5) the design was investigated in more detail. One of the areas investigated was local sodium reactivity coefficients, and in GEAP-4080(6) the actual values (some positive) of the local coefficients were reported for the reference 500-MWe core, which had an overall negative sodium coefficient. Detailed calculations were then undertaken to evaluate the significance of this result during accident conditions. In this investigation the sodium void coefficients as well as the expansion coefficients were examined. (7) Sodium voiding, unless very localized, is itself an accident condition in a fast reactor (no matter what the coefficients) because the heat-transfer properties of sodium vapor are so much below those of liquid sodium. The void coefficients are, however, of concern because, if positive, they might conceivably cause a mild accident to become a severe one.

During this period the new data on high-energy cross section( $(8)$ and improved calculational methods $(9-13)$ for calculating both the Doppler and the sodium void coefficient became available. The changes in cross section resulted in a hardening of the spectrum for a given reactor configuration and composition, and had as a first consequence a vastly improved breeding ratio. (14) However, other results were a reduction in the calculated Doppler coefficient, and the overall and local sodium coefficients became more positive (see Table I).

\section{Table I}

\section{EFFECT OF NEW DATA AND METHODS ON} THE 500-MWE REFERENCE DESIGN

\begin{tabular}{lccc} 
& Old & New \\
\cline { 2 - 3 } Mean energy of power spectrum & (keV) & 180 & 210 \\
Fraction of fis sions below $9 \mathrm{keV}$ & 0.15 & 0.13 \\
Total breeding ratio & 1.08 & 1.36 \\
a/o Pu $(239+24 \mathrm{l})$ & 12.8 & 11.6 \\
Doppler coefficient $\left(\mathrm{T} \frac{\mathrm{dk}}{\mathrm{dT}}\right)$ & -0.011 & -0.0068 \\
$\Delta \mathrm{k}$ for sodium loss from core and blankets & 0 & +0.0063
\end{tabular}

We therefore decided to see whether we could not establish a new reference design point with the same characteristics as the old - assuming of course that the newer calculational procedure more closely represented reality than the preceding.

In view of the current interest in larger unit reactor sizes for utility application, studies for an up-dated reference design were centered on a 
1000-MWe unit. Methods development and general scoping calculations were begun in the middle of 1962 as part of our continuing development program on the Fast Ceramic Reactor. (15) With the beginning of the 1000-MWe Core Design Study this effort has been stepped up and particularized to a specific geometrical configuration. Particular attention has been paid to the local reactivity coefficients, safety criteria, accidents analyses, and economics. The 1000-MWe study is unfortunately not yet complete. The results reported today will accordingly consist of design considerations rather than the exposition of a particular design.

The first direction of design, through use of the newer cross section and methods, was an attempt to find a new balance of characteristics by purely geometric devices, i.e., by exploring the sodium: fuel ratio and varying the height-to-diameter ratio of the core ("pancaking").(15) Large sodium volumes did produce a negative sodium power coefficient and a negative reactivity effect when all the sodium was removed, but was accompanied by undesirable side effects, such as increased core volume, large positive sodium coefficient for voiding of sodium in the center of the core, and increased $\mathrm{Pu} / \mathrm{U}$ ratio. In our earlier work (4) it had been remarked, in agreement with observations of other workers, (3) that the increased leakage from pancaked cores resulted in smaller sodium coefficients. Table II shows the results of some exploratory calculations on pancaking. It will be observed that the available Doppler $\Delta \mathrm{k}$ is small compared with the potential maximum $\Delta \mathrm{k}$ from selective sodium 10 s $\mathrm{s}$ in all cases, and that proceeding to extreme pancaking, by hardening the spectrum, reduces the available Doppler reactivity to unacceptably low values. The sodium-loss case shown in Table II is not quite the worst conceivable situation: as we shall see shortly, a smaller void cylinder whose height is about $\frac{2}{3}$ the core height and whose radius is about $\frac{2}{3}$ of the core radius would give slightly higher $\Delta k^{\prime} s$. Of course, the actual void sequence in a reactor accident will not, in general, traverse the void pattern which gives maximum reactivity, but it appears that we have here a disparity between

Table II

EFFECT OF PANCAKING ON CORE COEFEICIENTS

\begin{tabular}{l|c|c|c|c}
\hline $\begin{array}{c}\text { Height } \\
(\mathrm{ft})\end{array}$ & $\begin{array}{c}\text { Diameter } \\
(\mathrm{ft})\end{array}$ & $\begin{array}{c}\% \text { Fissile } \\
\text { Plutonium }\end{array}$ & $\begin{array}{c}\text { Available } \\
\text { Doppler } \Delta \mathrm{k}\end{array}$ & $\begin{array}{c}\text { Sodium Loss } \\
\text { from Core } \\
\text { Only, } \Delta \mathrm{k}\end{array}$ \\
\hline 1.25 & 14.5 & 15.7 & -0.0014 & +0.0100 \\
2.0 & 11.8 & 11.5 & -0.0027 & +0.0155 \\
3.25 & 9.4 & 10.4 & -0.0036 & +0.0230 \\
\hline
\end{tabular}

Average core composition @ 50,000 MWD/T: $33 \mathrm{v} / 0$ fuel, $50 \mathrm{v} / 0$ sodium, $17 \mathrm{v} / 0$ steel. 
the available Doppler and the available sodium void reactivities. We therefore investigated the use of $\mathrm{BeO}$ in the core to achieve to simultaneous improvements:

1) decrease the dependence of the spectrum on sodium, thus reducing the sodium loss coefficients;

2) softening the spectrum, thus increasing the Doppler coefficients.

We looked in some detail at the behavior under accident conditions of two 1000-MWe pancake cores, one with and one without BeO (see Tables III and IV). In both cases the prompt power coefficient is negative. In Case $B$ the sodium power coefficient itself is negative, which means that Case B (the BeO case) will follow load. Case $A$ is also operable, and in fact there have been water reactors seriously proposed with similar characteristics. (16)

Table III

COMPARISON OF HARD AND SOFT SPECTRUM CORES FOR 1000-MWE FAST OXIDE REACTORS

\begin{tabular}{c|c|c} 
& Case A & Case B \\
\hline Volume \% $\mathrm{BeO}$ & 0 & 8.3 \\
$\mathrm{UO}_{2}-\mathrm{PuO}_{2}$ & 33.3 & 25 \\
Steel & 16.7 & 16.7 \\
Sodium & 50 & 50 \\
Core height $(\mathrm{ft})$ & 2.0 & 2.0 \\
Core diameter $(\mathrm{ft})$ & 11.8 & 13.7 \\
Axial blankets $(\mathrm{ft})$ & 1.25 & 1.25 \\
Radial blankets $(\mathrm{ft})$ & 1.0 & 1.0 \\
Core power $(\mathrm{MWt})$ & 2150 & 2150 \\
Total power (MWt) & 2500 & 2500 \\
\hline
\end{tabular}

Table IV

COMPARISON OF HARD AND SOFT SPECTRUM CORES

\begin{tabular}{l|c|c}
\hline & Case A & Case B \\
\hline Mean energy of power spectrum (keV) & 230 & 100 \\
Fraction of fissions below $9 \mathrm{keV}$ & 0.12 & 0.28 \\
Total breeding ratio & 1.34 & 1.12 \\
a/o Pu (239+241) & 11.5 & 14.7 \\
Doppler coefficient $\left(\mathrm{T} \frac{\mathrm{dk}}{\mathrm{dT}}\right)$ & -0.006 & -0.012 \\
$\Delta \mathrm{k}$ for sodium loss from core and blankets & +0.0070 & -0.0051 \\
Sodium power coefficient $\Delta \mathrm{k} / 1 \% \Delta \mathrm{P}$ at full power & $+0.04 \phi$ & $-0.03 \phi$ \\
Doppler power coefficient $\Delta \mathrm{k} / 1 \% \Delta \mathrm{P}$ at full power & $-0.8 \phi$ & $-1.8 \phi$ \\
\hline
\end{tabular}


A large reactor will almost certainly be built of a large number say a few hundred - of fuel bundles in individual channels. These channels will be orificed to even out the coolant temperature rise, and considerable attention will be paid to continuity of coolant flow. Nevertheless, it is not improbable that a condition will arise in which flow in an individual channel is blocked. Because of the subdivision of the core, the reactivity changes from voiding single channels will turn out to be small compared with the available Doppler coefficient in both Cases A and B. Nobody will enjoy the consequences of burning out a single channel, but it will not propagate to the rest of the core in any reasonable thermal-hydraulic design.

To see serious effects from the potential sodium reactivity coefficients, we will have to consider accidents which involve large regions of the core. These might occur by

1) loss of coolant flow;

2) overpower transients initiated by other causes;

3) loss of coolant.

The basic curves for interpreting the consequences of such accidents are shown in Figs. 1 and 2 .

It is realized that the calculation of the sodium void coefficient is a very imperfect art. The numerical values in the figures are, in particular, subject to considerable uncertainties, perhaps as much as $100 \%$. However, the general trend of the curves is probably real. The conclusions which will be drawn depend more on the general aspect of the curves than on the particular values.

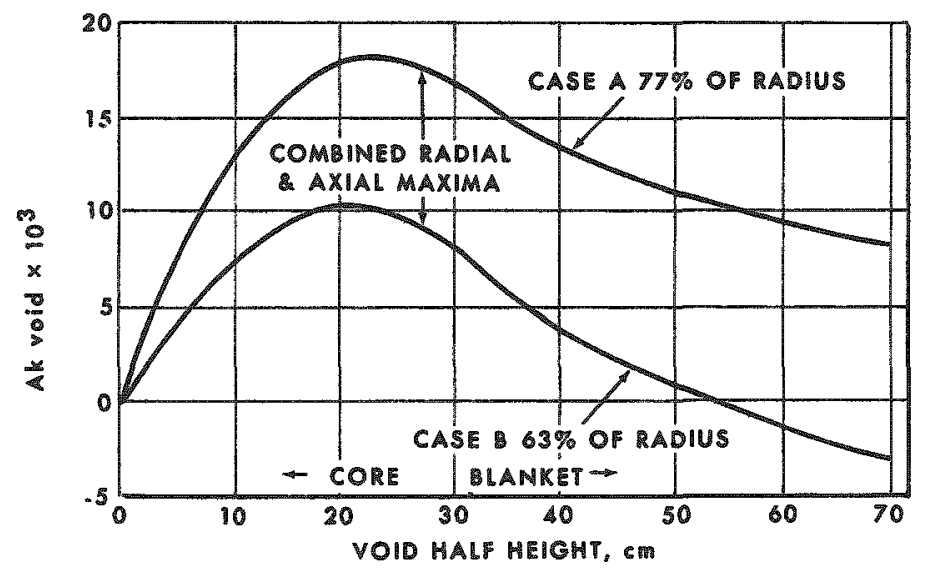

Fig. 1. Spatial Dependence of Sodium Loss and $\Delta k$, with Axial Removal of Sodium from Central Cylinder 


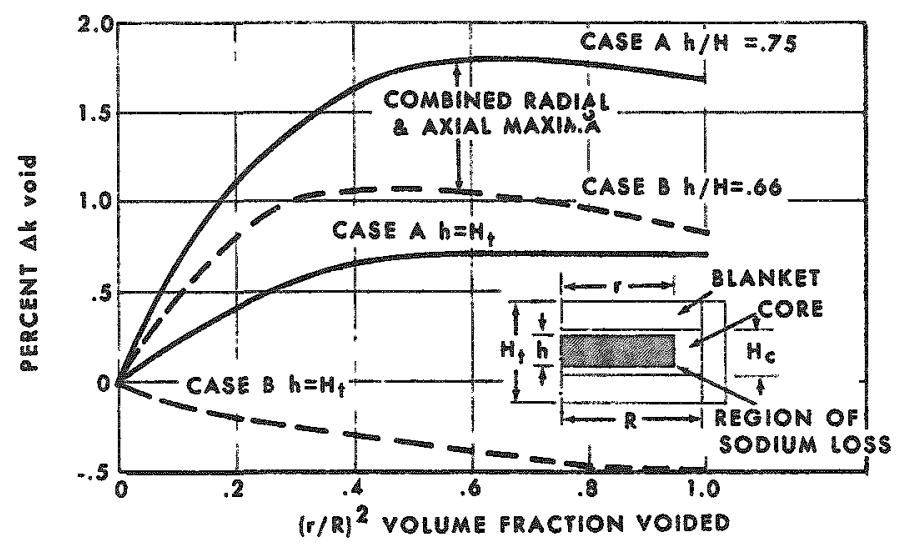

Fig. 2. Spatial Dependence of Sodium Loss $\Delta k$

Figure 1 shows the reactivity changes associated with progressive axial voiding of the core about the midplane. Voiding the outer radial blanket and outer periphery of the core creates a negative reactivity contribution, so in Fig. I these are supposed not voided. Since these are the low-power regions of the reactor, this hypothesis is prudent. It will be noticed that in either case the reactivity peaks when the core is partially voided and then decreases as the blankets are voided. Thus voiding the upper part of the core and the upper blanket gives a negative reactivity contribution. (It may not be obvious that the reactivity change from voiding the blanket on an empty core is the same as voiding the blanket on a full core, but to the degree of approximation of our calculations we have verified this point.)

Figure 2 shows the reactivity changes associated with progressive radial voiding of the core. The solid curves represent the case where the void extends through the core and both blankets; the dotted curve represents a case where the void only extends through that central part of the core (approx $\frac{2}{3}$ ) which gave a peak in the previous figure. From these curves we can deduce approximately the reactivity traverses for particular patterns of sodium voiding.

In general, the result of a mild transient or a mild loss of flow would at most cause boiling to occur in the upper blanket, tending initially to shut either reactor down. Let us investigate more drastic occurrences.

Consider, first, loss of flow with failure to scram on the loss-offlow signal. Initially every channel has (by orificing) essentially the same subcooling at each point along the channel. No coast down was assumed for the calculation. In this case, the hottest channel gives the most heat to the stagnant sodium. Therefore boiling will begin at the central channel. The center of the hottest channel will reach zero subcooling first, and the sodium void will appear in the center of the core. (For finite coast down, the initial void will be displaced towards the top of the core and the time for initial voiding delayed.) Figure 3 shows the ensuing 
pattern and time scale for sodium voiding for Core A. Figure 4 gives the same patterns for Core $B$. Although the actual void pattern will be sensitive to the details of the coast down rate and to the hydraulic assumptions, in any event the void pattern after only a small volume of core is voided will closely resemble progressively expanding radial voids with both core and blankets empty. The reactivity traverses will thus follow quite closely the lower curves of Fig. 2, with an initial reactivity increment in both cases (see Fig. 5). Reactor B tends to shut itself down. The peak reactivity insertion in Reactor $A$ is about $\$ 2$ at $500 \mathrm{~ms}$ after the first voiding. For Reactor A under these assumptions, even assuming a $400-\mathrm{ms}$ scram initiated at the first reactivity increment, a power excursion and fuel temperature of $10,000^{\circ} \mathrm{F}$ would be added to whatever meltdown problems loss of flow itself creates.

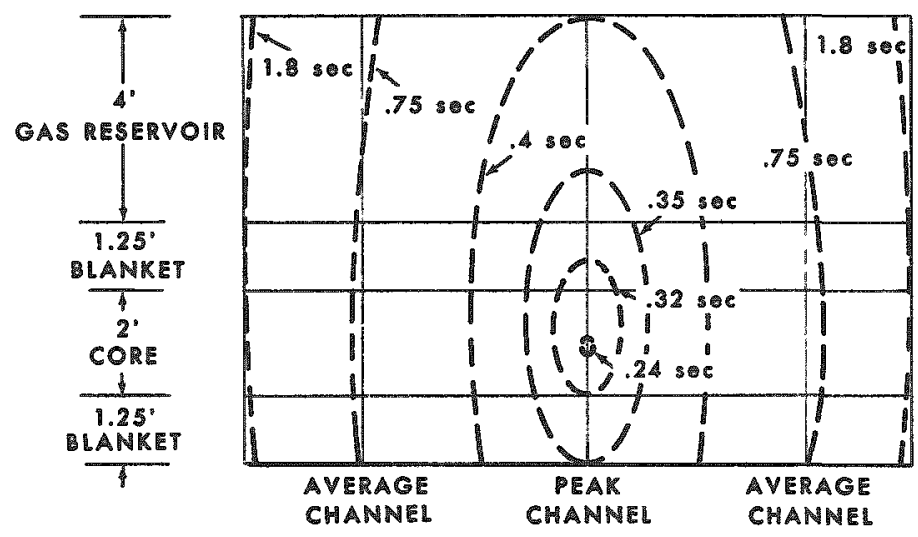

Fig. 3. Case-A Pattern of Sodium Voiding for Instantaneous Loss of Flow At Full Power

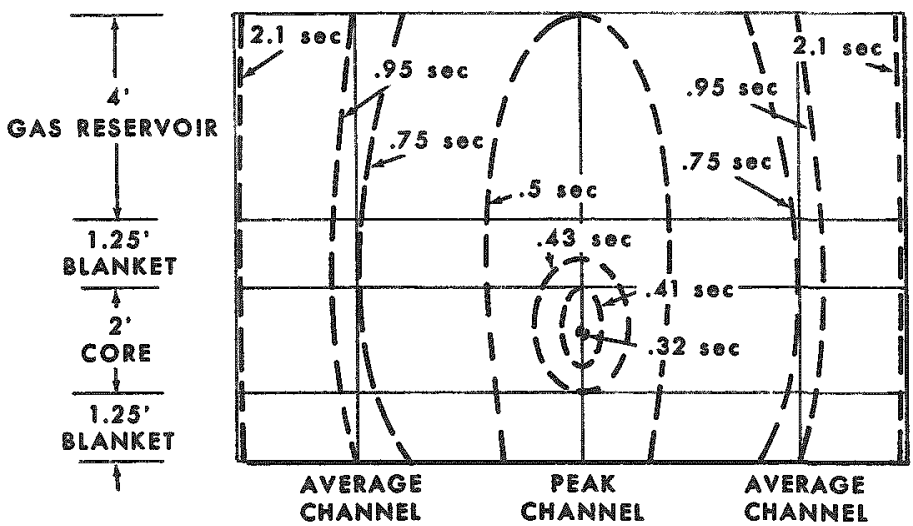

Fig. 4. Case-B Pattern of Sodium Voiding for Instantaneous Loss of Flow at Full Power 


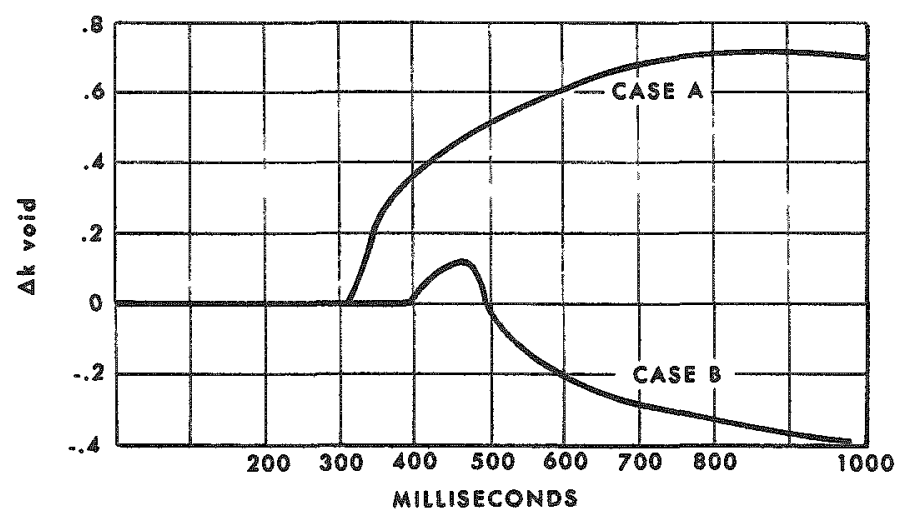

Fig. 5. Reactivity Change Following Loss of Flow

A second accident which we can consider is an excursion consisting of the addition of $\$ 1.50$ worth of reactivity in $10 \mathrm{~ms}$. Normal coolant circulation is assumed to continue. The sudden expulsion of two control rods might lead to such an excursion, although a $50-\mathrm{ms}$ ramp would require forces of the order of $25 \mathrm{~g}$. The transient power profile is shown for Case $A$ in Fig. 6. The energy release in the pulse is about $2000 \mathrm{MW}$ sec. In Case B, which has twice the Doppler coefficient, the release is about $450 \mathrm{MW}-\mathrm{sec}$ (see Fig. 7). The transient fuel temperatures for both cases are shown in Fig. 8.

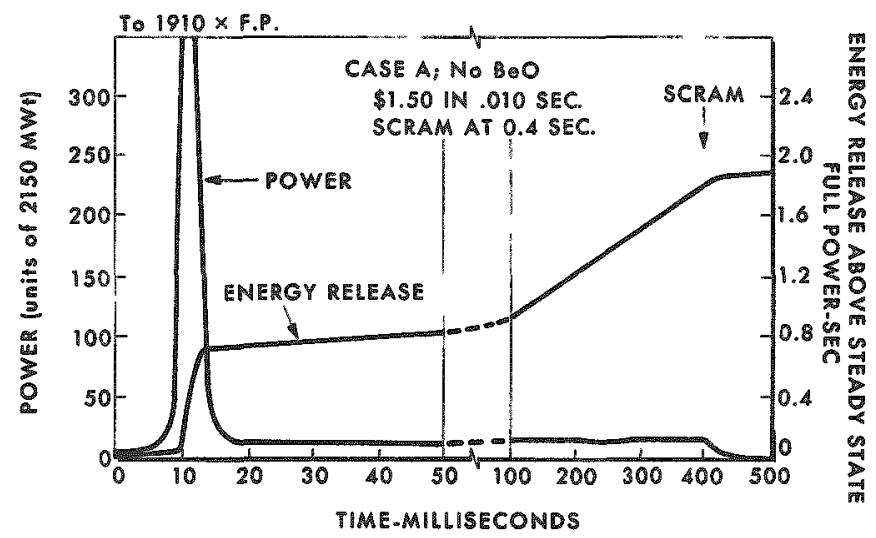

Fig. 6

Case-A Transient Power and Energy Release

Fig. 7

Case-B Transient Power and Energy Release

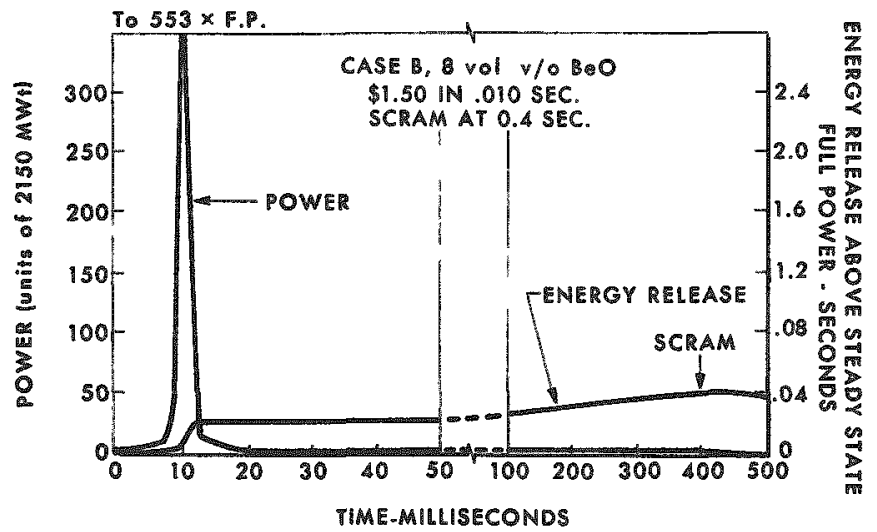




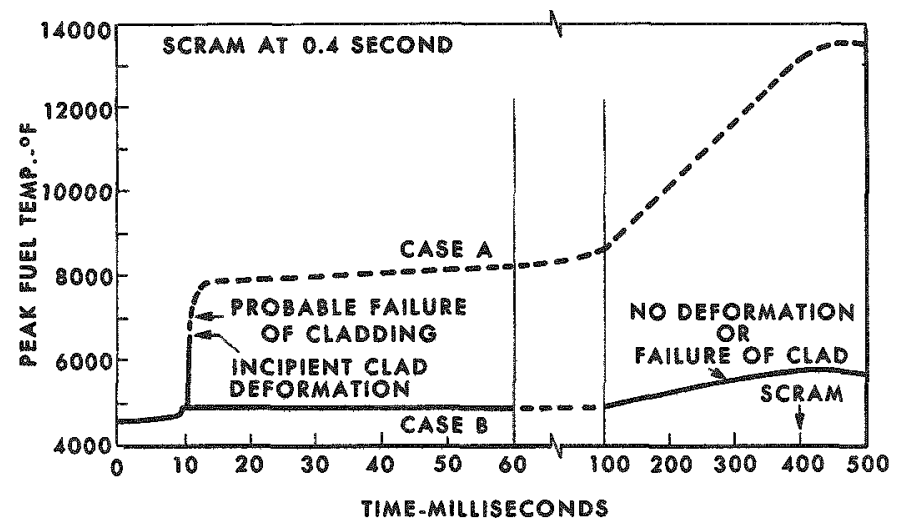

Fig. 8. Transient Fuel Temperatures. 1.5 Dollar, 10 Millisecond Ramp, Inserted at Full Power

In Case A there will probably be clad deformation and perhaps clad failure. If fuel should squirt into the sodium, reactivity would be added rapidly, both by cooling of the fuel and vaporization of the sodium. In this eventuality, Reactor A would be destroyed before the control system could react. If, however, the fuel stayed intact despite the high temperatures and pressures calculated, then the sodium temperatures would rise according to the curve in Fig. 9. All channels would reach boiling at the same time. It will be observed that a scram at $400 \mathrm{~ms}$ would begin before boiling.

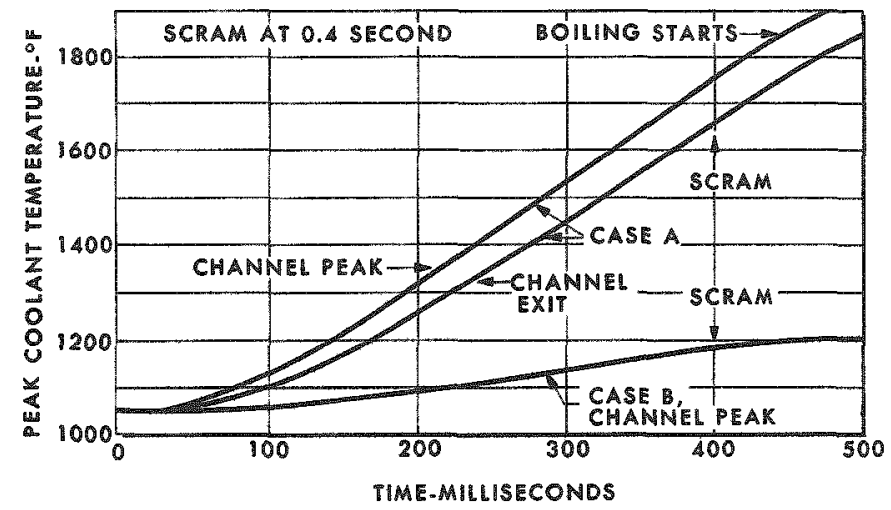

Fig. 9. Transient Sodium Temperatures 1.5 Dollar, 10 Millisecond Ramp, Inserted at Full Power

The approximate void patterns which would ensue are shown in Fig. 10. The peak heat flux in sodium from intact oxide fuel in this case is about $5 \times 10^{6} \mathrm{Btu} / \mathrm{hr} / \mathrm{ft}^{2}$. The delay before boiling is due to the initial subcooling of the sodium. The initial boiling occurs at $480 \mathrm{~ms}$ at the top of the core and is a negative effect. This rapidly reverses, and a very sharp positive peak of $1.5 \% \Delta \mathrm{k}$ occurs at $550 \mathrm{~ms}$ (see Fig. 11). This might or might not be significant, depending on the rate of scram insertion. 


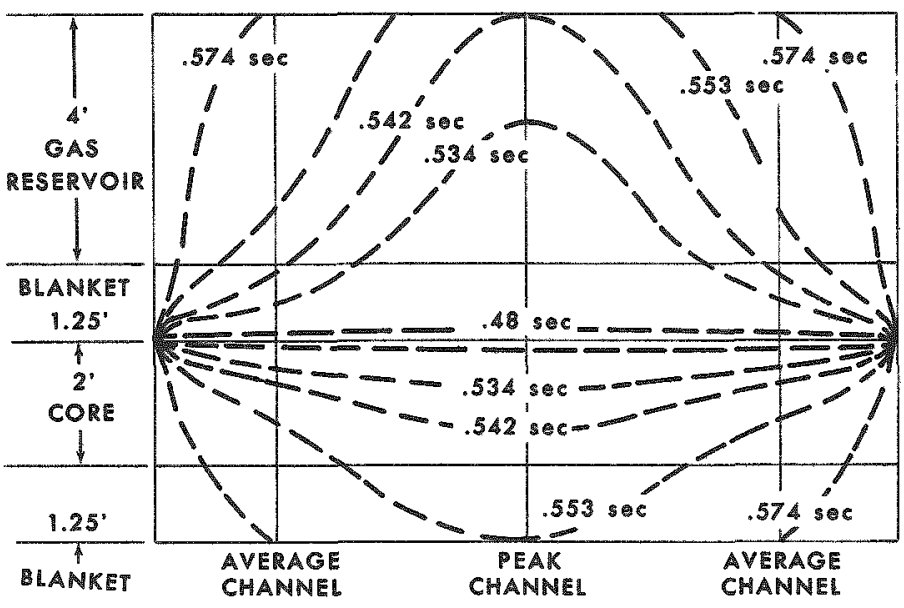

Fig. 10

Possible Pattern of Sodium Voiding Due to Severe Excursion. Core A

Fig. 11

Case-A Reactivity Change

from Void-producing

Power Excursion

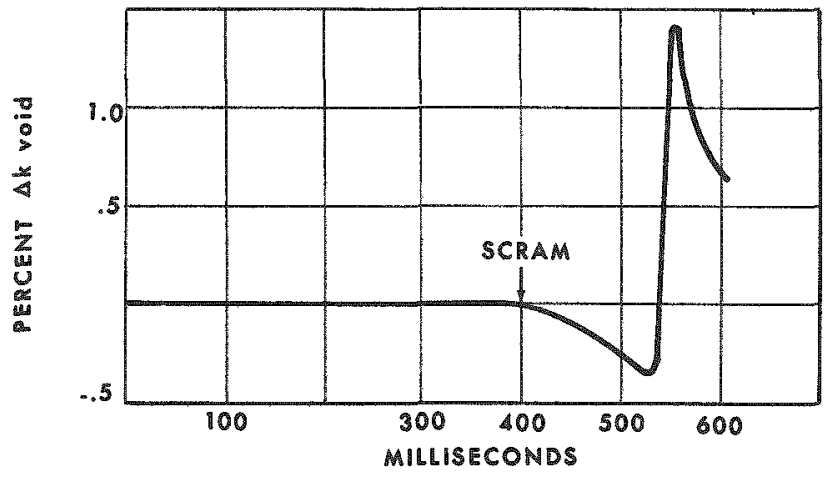

To sum up, for this accident Core $\mathrm{A}$ would almost certainly be destroyed and Core $B$ would almost certainly survive. It is interesting to note, however, that the essential difference between the behavior of these two cores is the difference in Doppler coefficients. The more positive sodium void coefficients of Core A are unlikely to play a decisive part in the accident, although obviously they are not helping.

A third accident which can be considered is progressive voiding of the core from the top down. This might occur from a rupture of the reactor vessel. In this case the liquid level could drop rapidly. The top of the core might be reached in 1 sec and the core voided in $40 \mathrm{~ms}$. The reactivity traverse is shown in Fig. 12. In both cases los of sodium from the upper blanket reduces reactivity initially. As the core is voided, reactivity increases; voiding the lower blanket decreases the reactivities. If by any chance the reactors have not been scrammed, Case A undergoes a reactivity pulse of $\$ 3.50$ in $10 \mathrm{~ms}$, or a ramp reactivity addition of $\$ 350 / \mathrm{sec}$. This is the kind of reactivity increment usually considered possible only by melting down and compacting the core. Case B would have no prompt excursion. The loss-of-coolant accident just considered may appear far-fetched, but there is a class of accidents involving reduced flow which could involve a progressive boiling boundary starting at the top of the blanket and gradually extending into the core. The boundary 
would dip downward in the center of the core. The reactivity changes for these cases would be, if anything, slightly greater (see Fig. 12) than the case of vessel rupture, although the time scale would be different.

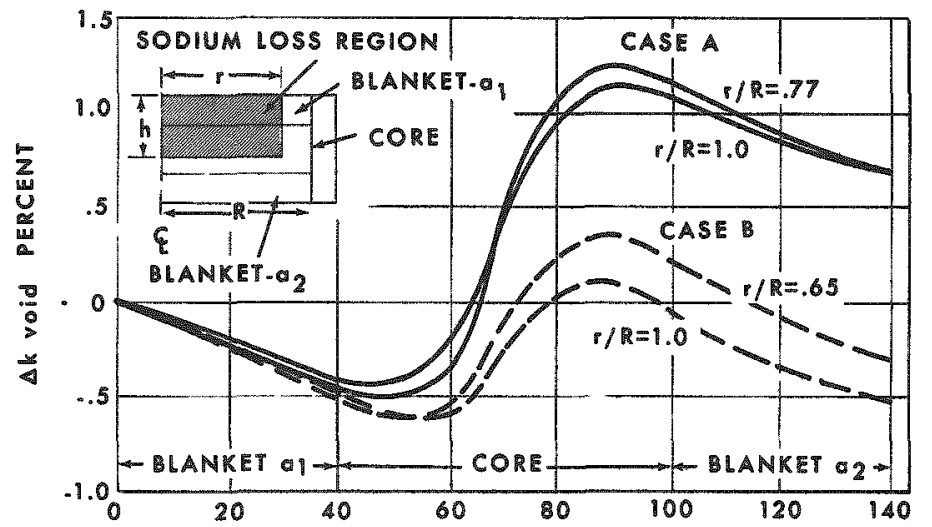

h, THICKNESS OF LOSS REGION, cM FROM TOP OF BLANRET O,
Fig. 12

Reactivity Sequence for Sodium Loss (Starting at the Top of the Reactor)

From these considerations it does not follow that a reactor with the general characteristics of Case $\mathrm{A}$ would not be safely operable. In particular, it might be possible to direct the course of voiding appreciably in a favorable sense by orificing. However, it is clear that the introduction of BeO gives larger Doppler coefficients and more negative sodium coefficients. These together provide a desirably large margin of safety for a wide variety of potential accident-initiating mechanisms.

The next point to be considered is the economic consequences of a softened spectrum core. It is a commonplace that economic comparisons are highly sensitive to the economic assumptions which are used in making them. We have therefore compared the $A$ and $B$ cases on two economic bases. The first basis is the 1963 AEC ground rules for plutonium value and inventory charges. (17) The fuel is assumed to be reprocessed in the AEC multipurpose reprocessing plant, which processes both low-enrichment and high-enrichment fuels at a fixed per diem rate. We have also examined the economics on the basis of our own projections of the economic environment of 1975. Instead of the multipurpose reprocessing plant, the single-purpose fast reactor processing plant $(18,19)$ is assumed. Table $V$ summarizes the economic ground rules. The fuel throughput of 44 tons/yr corresponds to an installed fast reactor capacity of only $1000 \mathrm{MWe}$. Table VI gives the basic fuel-cycle data; at the bottom is a summary of the economic analysis. There is about a $0.3-\mathrm{mill} / \mathrm{kWh}$ penalty against Case $B$ on either economic basis. Case $A$ also has a more seductive breeding ratio. The value of 1.12 for Case $B$ is very close to the 1.08 value which was initially reported by us for the $500-\mathrm{MWe}$ reference design. (5) A likely pattern of development would appear to be to build early reactors more like Case B than Case A. As one gains confidence in the operation of these reactors and has been persuaded that the complete spectrum of accidents has been investigated, the reactors will gradually be optimized to have fuel cycles intermediate between Case $B$ and Case A. 
ECONOMIC BASIS FOR FUEL CYCLE

\begin{tabular}{|c|c|c|}
\hline & 1963 AEC Basis & 1975 (Assumed) \\
\hline Plutonium value, $\$ / g-(239+241)$ & 10.00 & 6.60 \\
\hline$(240+242)$ & - & 1.10 \\
\hline Reprocessing cost; core/blanket; $\$ / \mathrm{kg}$ & $88.70 / 47.40$ & 37.00 \\
\hline Shipping; $\$ / \mathrm{kg}$ & 16.00 & 7.50 \\
\hline Inventory interest rate; \% p.a. & 4.75 & 10.0 \\
\hline Fabrication capital charge, $\%$ & - & $14 \%$ \\
\hline Volume throughput, tons/yr & - & 44 \\
\hline Fabrication cost; core $\$ / \mathrm{kg}$ & \multicolumn{2}{|c|}{264} \\
\hline axial blanket $\$ / \mathrm{kg}$ & \multicolumn{2}{|c|}{$\begin{array}{l}37 \\
62\end{array}$} \\
\hline radial blanket $\phi / \mathrm{kg}$ & \\
\hline
\end{tabular}

Table VI

FUEL CYCLE DATA AND ECONOMICS

\begin{tabular}{|c|c|c|c|c|c|c|}
\hline Fuel Data & B. R. & $\begin{array}{c}U+P u \\
(\mathrm{~kW} / \mathrm{kg})\end{array}$ & $\mathrm{MWD} / \mathrm{kg}$ & $\frac{\text { Batches }}{\text { Cycle }}$ & $\begin{array}{c}\text { Initial } \\
(239+241)\end{array}$ & $\begin{array}{c}\text { Pu a/o } \\
(240+242)\end{array}$ \\
\hline Case A & 1.34 & 125 & 110 & 5 & 12.46 & 5.87 \\
\hline Case B & 1.12 & 125 & 110 & 5 & 10.66 & 5.77 \\
\hline
\end{tabular}

\begin{tabular}{|c|c|c|c|c|c|c|c|}
\hline \multirow[t]{6}{*}{ Economics } & 1963 AEC Basis & $\mathrm{D}$ & $\mathbf{E}$ & I & $R$ & W & $\begin{array}{c}\text { Total } \\
\text { Mills } / \mathrm{kWh}\end{array}$ \\
\hline & Case A & $(0.40)$ & 0.27 & 0.17 & 0.13 & - & 0.17 \\
\hline & Case B & $(0.16)$ & 0.31 & 0.22 & 0.16 & - & 0.53 \\
\hline & 1975 Basis (Assumed) & & & & & & \\
\hline & Case A & $(0.27)$ & 0.27 & 0.34 & 0.09 & 0.13 & 0.56 \\
\hline & Case B & $(0.09)$ & 0.30 & 0.39 & 0.09 & 0.14 & 0.84 \\
\hline
\end{tabular}

\section{References}

1. B. Wolfe, D. R. Riley, and N. Friedman, Influence of the Doppler Effect on the Meltdown Accident, This Conference.

2. P. Greebler, P. Aline, and J. Sueoka, Parametric Study of $300 \mathrm{MWe}$ Reactor Core, GEAP-3287 (Nov 15, 1959).

3. J. B. Nims and P.F. Zweifel, Preliminary Report on Sodium Temperature Coefficients in Large Fast Reactors, APDA-135 (1959).

4. P. Greebler, B. A. Hutchins, and J.R. Sueoka, Calculations of Doppler Coefficient and Other Safety Parameters for a Large Fast Oxide Reactor, GEAP-3646 (March 23, 1961). 
5. K. M. Horst, B. A. Hutchins, F. J. Leitz, and B. Wolfe, Core Design Study for a $500 \mathrm{MWe}$ Fast Oxide Reactor, GEAP-3721 (Dec.28, 1961).

6. F.J. Leitz (ed), Fast Ceramic Reactor Development Program, Fourth Quarterly Report, July-Sept 1962, GEAP-4080.

7. The significance of local sodium void coefficients has also been pointed out by C. E.Dickerman and D. Okrent, Nuclear News, March 1963, p. 21. Detailed calculations of the phenomenon were reported at the Rome Nuclear Congress (June 1963) by C. P. Zaleski.

8. J. C. Hopkins and B. C. Diven, Neutron Capture to Fission Ratios in $\mathrm{U}^{233}, \mathrm{U}^{235}, \mathrm{Pu}^{239}$, Nuclear Science and Engineering, 12169 (1962).

9. S. Yiftah, D. Okrent, and P. Moldauer, Fast Reactor Cross-Sections, Pergamon Press (1960).

10. J.M. Stevenson and A.R. Baker, A Comparison of Calculated and Experimental Cross-section Ratios at the Center of Zephyr, AEEWM184(Dec.1961).

11. H. H. Hummel and M. G. Bhide, Reactivity Coefficient of Sodium in Fast Assemblies, Proceedings of the Seminar on the Physics of Fast and Intermediate Reactors (Vienna August 3-11, 1961) Vol II, 177, IAEA, Vienna (1962).

12. H. H. Hummel and A. L. Rago, An Accurate Treatment of Resonance Scattering in Light Elements in Fast Reactors, ibid., Vol. II, p. 257.

13. D. Okrent, Performance of Large Fast Power Reactors Including Effects of Higher Is otopes, ibid., Vol. II, p. 271.

14. P. Greebler and E. Goldman, Doppler Calculations for Large Fast Ceramic Reactors. Effects of Improved Methods and Recent Cross Sections, GEAP-4092 (Dec. 1962).

15. F. J. Leitz (ed), Fast Ceramic Reactor Development Program, Seventh Quarterly Report, April-June 1963, GEAP-4300.

16. Westinghouse Electric Corporation for N.Y. Operations Office AEC, 1000 MWe Closed Cycle Water Reactor Study (March 1963).

17. U.S. AEC TID-7025 (Vol 1) Guide to Nuclear Power Cost Evaluation (March 15, 1962); TID-7025 Supp 1 (Sept.30, 1962).

18. E. L. Zebroski, H. W. Alter, and G. D. Collins, Plutonium Fuel Fabrication and Reprocessing for Fast Ceramic Reactors, GEAP-3876 (Eeb. 1, 1962).

19. H.W. Alter, A Fuel Reprocessing Plant for Fast Ceramic Reactors, GEAP-4028 (Feb 1, 1962). 


\section{Discussion of Paper}

Presented by Mr. Cohen

MR. CAMPISE (Atomics International):

For Case $A$, if you assume the sodium void coefficient to be zero or negative, would you lose the core with this kind of reactivity insertion? MR. COHEN:

For the rapid insertion which did not have a big enough Doppler, I believe so, yes.

MR. CAMPISE:

Even with the Doppler coefficient you have in Case A?

MR. COHEN:

The Doppler coefficient in Case A was fairly small. In Case B, you would not have any trouble at all. It would just override it.

MR. HALL (Los Alamos):

I was not clear on one point. Did you also include the expansion coefficients of the fuel in your reactivity excursions? If you did, is not the expansion coefficient greatly reduced by the use of the pancake design?

MR. COHEN:

I think that may be true. Our basic point of view is that mechanical expansion coefficients in fuel which has suffered a great deal of irradiation, be it ceramic, cermet, or metal, is a fairly delicate balance of a lot of mechanical constraints, and since we are not quite sure what it will do in any case, we tend to neglect it.

We consider it prudent to neglect it. In short, it is something we do not feel we can count on.

MR. DIETRICH (General Nuclear Engineering Co.):

Can you quote doubling times for those two casès?

MR. COHEN:

I think the doubling times are something like $20 \mathrm{yr}$ for Case $\mathrm{A}$ and 40 yr for Case $B$. 
MR. JENS (Atomic Power Development Associates):

How did you take into account the hydrodynamics of the sodium leaving the core? Was the inertia of the amount of sodium above the core taken into account?

MR. COHEN:

Yes, the dynamic effects were taken into account. But much more remains to be done in this area.

MR. HALL:

It is my impression that when you get liquid metal boiling of this kind you get very extreme overheating, with the result that you get a violent expulsion of the metal. This has been true of liquid metal boiling in other applications. Was this the model that you used?

MR. COHEN:

All of the calculations that I showed were based on the assumption that we were trying to look at the effect of the void in the core with the core staying together as it was. Therefore, for example, in Case A, it was very likely the core would have disintegrated; however, we carried through the calculations as if the fuel had stayed together.

MR.STATHOPLOS (United Nuclear Corp):

Did you state that voiding in a single channel does not propagate in the rest of the core, if you use proper thermal and hydraulic design? MR. COHEN:

Presumably what you are afraid of is that you get a positive reactivity in the one channel which then raises the power in all of the others; they then begin to go in sexies. On the other hand, we see no reason why this should happen because the channels are such a small fraction; the Doppler effect of the whole surrounding region can take care of it. I don't think what I said was any deeper than that.

MR.STATHOPLOS:

I was a little more concerned not with reactivity changes but just with blocking of the coolant in the channels surrounding the one that was in trouble. 
MR. COHEN:

You must then design the channels with that in mind.

MR. JENS:

Mx. Tavernier yesterday brought up an important point that the hydraulic pressure created in this type of an accident tends to force the fuel in the outer parts of the core into a more compact annulus, and thereby increase reactivity. Did you calculate these pressures in the boiling accidents?

MR. COHEN:

Yes, we did; the pressures did not get very high. Actually, the pressure drop across the whole fuel element is something like 20 or $30 \mathrm{psi}$, the pressures that you got from the transient things before boiling got to be something like 70 .

MR. OKRENT (Argonne):

Would you expect anything worse or at least of the same magnitude but more probable from the reversal of flow that Mr. Huebotter mentioned?

MR. COHEN:

We do have reversal of flow. When you have a void extending downward, you are not getting flow through there. We have reversal of flow.

MR. OKRENT:

He showed a rapid voiding phenomenon. Is this what occurs? In other words, could this then provide a mechanism whereby you voided from about the middle of the core down to near the bottom of the core during a certain time period, but not in the blanket regions, because the top had already been voided? So therefore in one part of the oscillatory cycle you would create a void only in the region that contributed positive reactivity.

MR. COHEN:

In order to get a positive reactivity you have to get right down to the center of the core. Here is where you get the plusses. In the outer regions there is a very considerable negative reactivity. You also have to consider the time it takes for these things to happen, and the reaction of the control system. Remember, I talked about an accident as something 
where the control system either could not function or that it would not get a signal. I think in all of these cases, there would be substantial signals, red lights all over the place. The time scale of fairly slow accidents is such that you would expect the controls to catch them. 


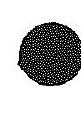

512 


\section{SESSION $V$}

October 9,1963

\section{LESS CONVENTIONAL REACTORS}

Chairman: J.R. DIETRICH

General Nuclear Engineering Corporation

Secretary: B. J. Toppel

Argonne National Laboratory 
3

514

。 


\author{
Dieter Smidt \\ Technische Abteilung \\ Kernforschungszentrum, Karlsruhe, Germany
}

\title{
1. Primary Advantages and Disadvantages of Gas Cooling
}

The following considerations led to the study to determine if a gascooled fast breeder and power reactor is technically feasible:

a. It seemed possible that the technology of the cooling circuit will be simpler if gas is used instead of sodium. The corrosion problems are minimized, especially by the choice of a noble gas. An intermediate cooling circuit is not necessary. The problem of thermal shocks is simplified. Possibly much simpler reactor vessels can be built compared with the very complicated vessels, for instance, of Dounreay or Enrico Fermi.

b. The positive void coefficient of large reactors will be much smaller for non-hydrogen-containing gases compared with sodium. Practically it will be negligible.

c. The breeding gain will possibly be higher because of the small absorption and moderation of the coolant.

d. The radioactivity of the coolant is small compared with that of sodium, especially in the case of helium.

e. A chemical explosion of the coolant will be impossible. Therefore there is no environmental danger in connection with $d$. This is of importance with respect to the special conditions in the densely populated Federal Republic.

f. Loading and unloading of fuel may be done under water.

On the other hand, there exists a number of primary disadvantages of gas coolants:

a. Gases are poor coolants insofar as regards their heat capacity per volume unit and their attainable heat transfer numbers. Therefore gases must be used under high pressure.

b. In case of a leakage in the cooling circuit, the coolant will be lost and the removal of the decay heat will be impossible. Core melting may result. 
c. In order to obtain a reasonable heat transfer, the coolant velocity must be high. Therefore, there will be a high pressure drop and the heat transfer number will react very sensitively to small changes of the coolant channel geometry. This leads to the danger of hot spots.

These considerations in 1961 led to the decision to make a more detailed study, the results of which will be discussed in the following sections.

\section{Cooling and Pumping Power with Helium}

Of the non-hydrogen-containing gases, helium has a very small pumping ratio and does not give corrosion problems by itself.(1) It has to be shown that the attainable power density will be large enough and the pumping power will be small enough if helium is to be used as a coolant. Therefore a large number of parametric studies were made. The fundamental design was based on cylindrical fuel elements areanged as rods to permit parallel flow. Inconel canning was assumed for the $\mathrm{UO}_{2}-\mathrm{PuO}_{2}$ fuel.

The relations of heat transfer and coolant flow and the critical equation have been brought into an analytically soluble form and programmed for the IBM 7070 computer. The properties of the secondary system, especially the thermal efficiency, are described by statistical mean values and expressed in a convenient interpolation formula. The derivation and solution of the resulting system of four equations will be published elsewhere. (2)

The symbols used in the following are

$\theta_{1}\left({ }^{\circ} \mathrm{C}\right) \quad$ Coolant inlet temperature of the core

$\theta_{2}\left({ }^{\circ} \mathrm{C}\right)$ Coolant outlet temperature

$x \quad$ Volume ratio of canning/oxide mixture

y Volume ratio of $\mathrm{UO}_{2}$ to $\mathrm{PuO}_{2}$

$\eta \quad$ Total efficiency of the plant

a $(\mathrm{cm}) \quad$ Outer radius of fuel rods

f Friction factor

$t_{\text {max }}\left({ }^{\circ} \mathrm{C}\right)$ Maximum cladding surface temperature

Bo Volume ratio for structure material except for cladding/core

$\mathrm{p}(\mathrm{atm}) \quad$ Gas pressure

$\mathrm{h} / \mathrm{R} \quad$ Ratio of height to radius of the core

$\mathrm{q}_{\mathrm{spec}} \quad[\mathrm{MW} / \mathrm{kg} \mathrm{Pu}]-$ specific power of the core 
Figure la shows the specific power as a function of $\theta_{1}$. All other parameters are fixed. There exists a flat maximum for $\theta_{1} \approx 230^{\circ} \mathrm{C}$. The separation of the fuel rods is chosen such that the necessary gas velocity for getting the wanted maximum fuel surface temperature is reached.

Therefore there is a variation of critical mass with temperature, because there is a variation of $\alpha$, here defined as the coolant volume fraction of the core:

$$
\alpha=\text { coolant volume/core volume. }
$$

This is shown in Fig. 1b, where the increase of critical mass $\mathbb{M}_{K}$ with $\theta_{3}$ is shown. The smaller the temperature difference between entrance and exit, the more coolant gas has to be pumped, and the larger must be the hydraulic diameter of the cooling channels. Accordingly, the critical mass rises.

The maximum shown in Fig. Ia is relatively flat, and its position is barely influenced by the choice of $\theta_{2}$ and the other parameters. In order to reduce the number of variables for the following studies the value $\theta_{1} \approx 230^{\circ} \mathrm{C}$ was chosen. This gives with good accuracy the optimum value for the specific power.

\section{Dependence on $\theta_{2}$}

There is a maximum of the specific power as a function of $\theta_{2}$, as shown in Fig. 2a, which gives a cross plot through Fig. la for $\theta_{1} \approx 230^{\circ} \mathrm{C}$. For large $\theta_{2}$, the temperature difference for the heat transfer $\left(t_{\max }-\theta\right)$ becomes small and $\mathrm{q}_{\mathrm{spec}}$ is lowered. For small $\theta_{2}$, the thermal efficiency and the temperature difference between entrance and exit are lowered. The position of this maximum depends strongly on the values of other parameters. The critical mass decreases with rising $\theta_{2}$. The cooling channels have to be more narrow, because the heat transfer number and, therefore, the coolant velocity have to $r$ ise for this reason, and also because a rising temperature difference means a smaller mass flow.

Figures $3 a$ and $b$ show the maximum values of a large number of curves of the type of the preceding figures. Abscissa is a. The printed numbers show the corresponding critical mass in $\mathrm{kg}$. The heavier curves show the specific power that would be possible when one considers the heat conductivity of the oxide. The shaded area considers higher numbers for the conductivity integral $\int_{T_{1}}^{T_{2}} k d T$, when $k$ is the thermal conductivity of the fuel, $T$, the outer temperature, and $T_{2}$ the central temperature of the fuel. 
$q_{\text {spec }}$

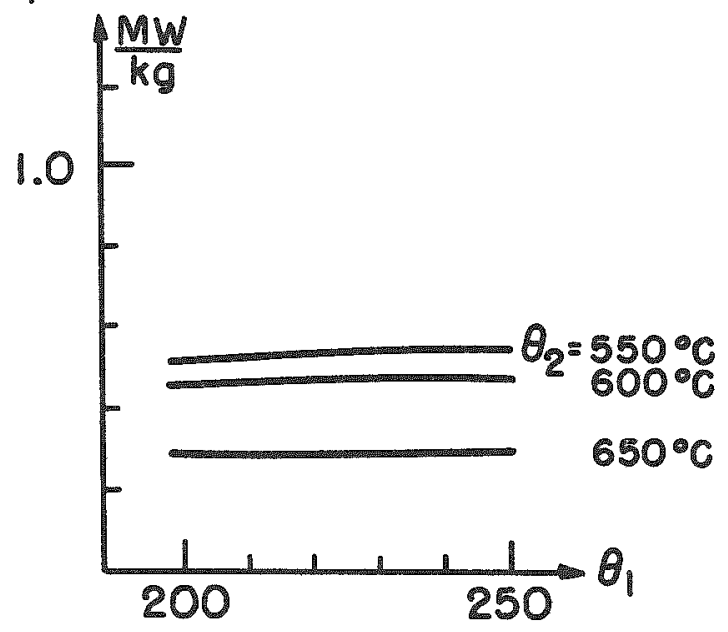

Fig. la. Specific Power as a Function of $\theta_{1}$

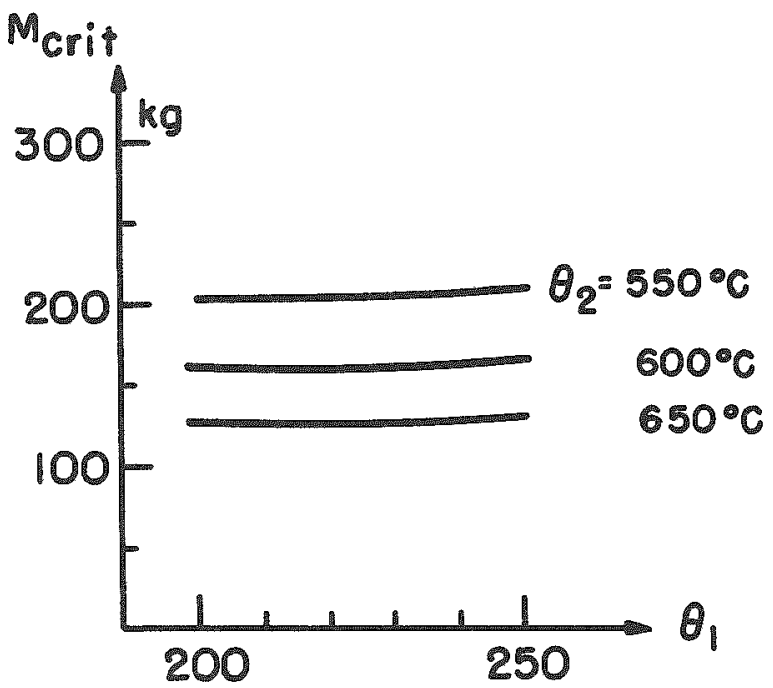

Fig. 1b. Critical Mass as a Function of $\theta_{1}$

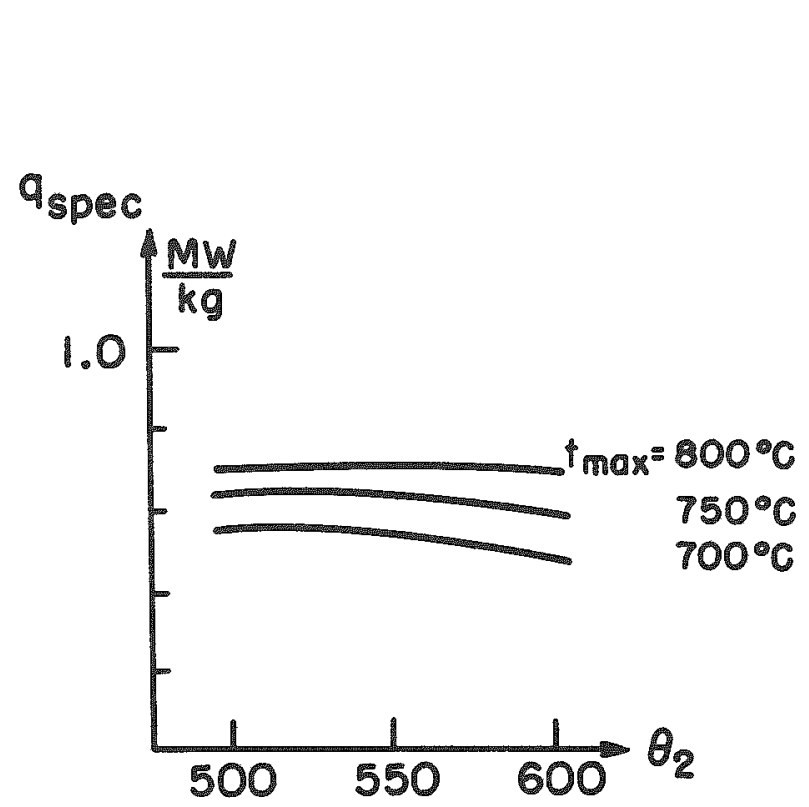

Fig. 2a. Specific Power as a Function of $\theta_{2}$

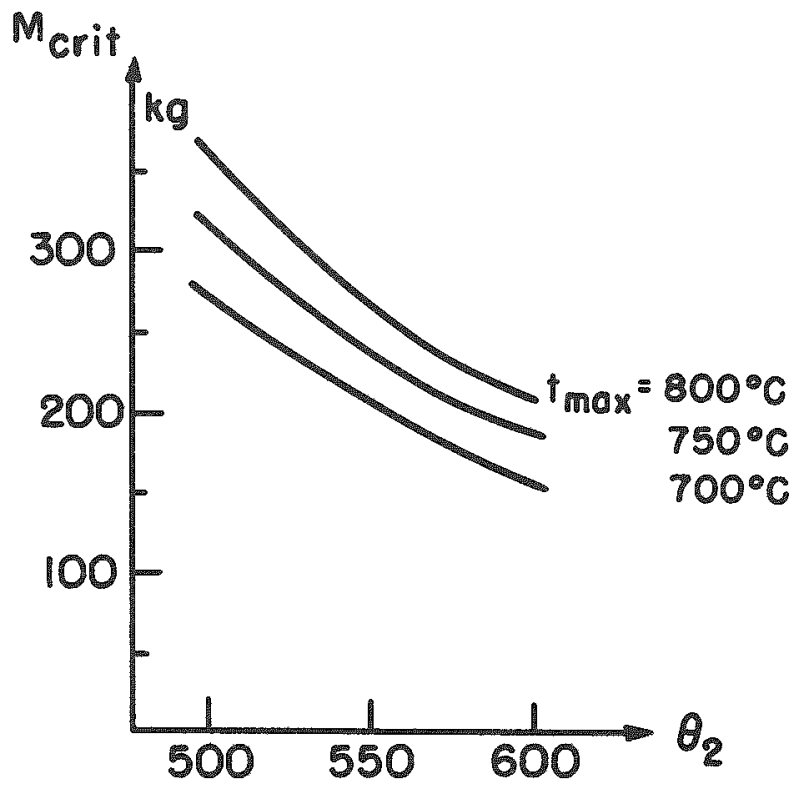

Fig. 2b. Critical Mass as a Function of $\theta_{2}$

For Figs. 1 and 2, the following data are valid unless specified otherwise:

$$
\begin{aligned}
& \theta_{1}=230^{\circ} \mathrm{C} ; \quad \mathrm{x}=0.5 \text {; } \\
& \eta=0.30 ; \\
& \alpha=0.3 \mathrm{~cm} \text {; } \\
& f=0.04 \\
& t_{\max }=700^{\circ} \mathrm{C} ; \quad y=2 \text {; } \\
& \beta_{0}=0.15 \text {; } \\
& \mathrm{p}=50 \mathrm{~atm} ; \\
& \mathrm{h} / \mathrm{R}=1.2
\end{aligned}
$$

(if not given differently). 


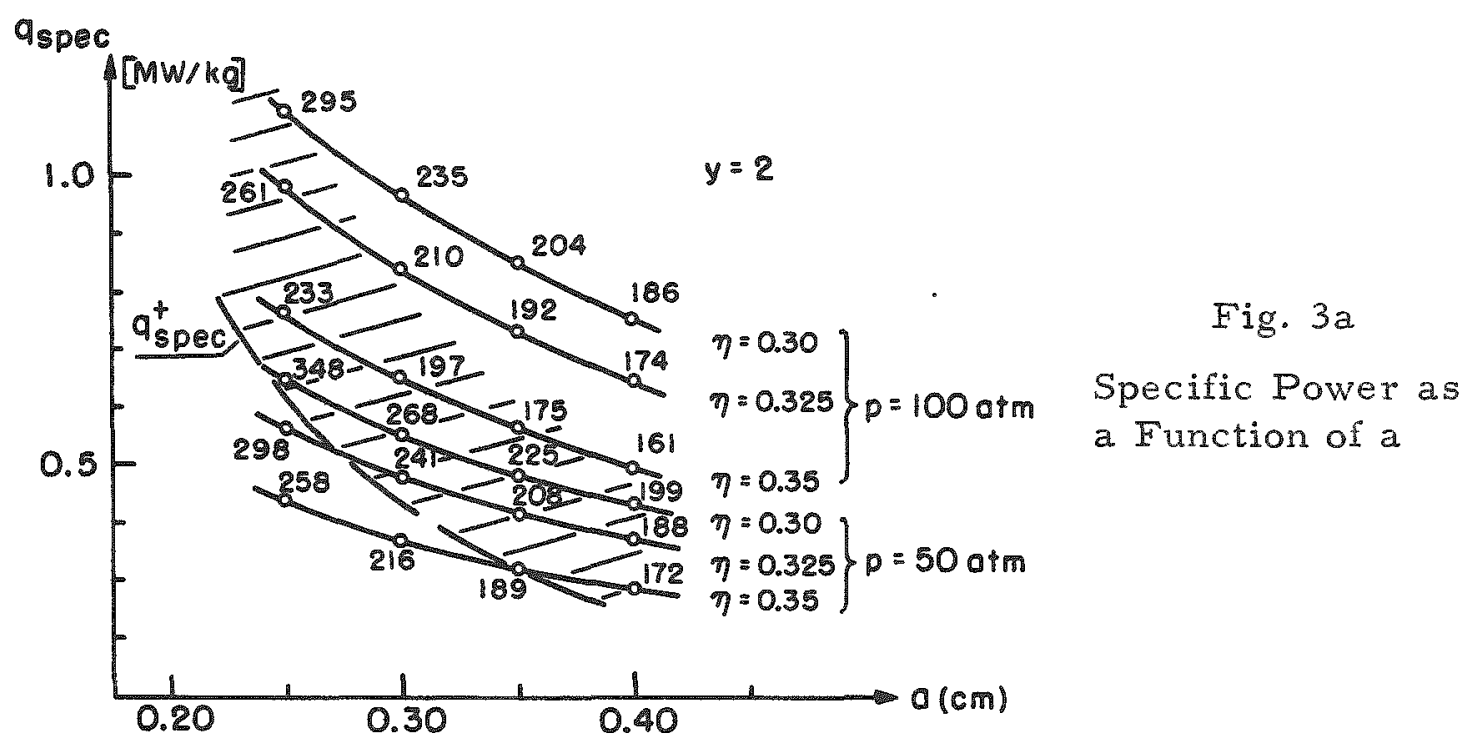

Fig. $3 b$

Specific Power as a Function of a

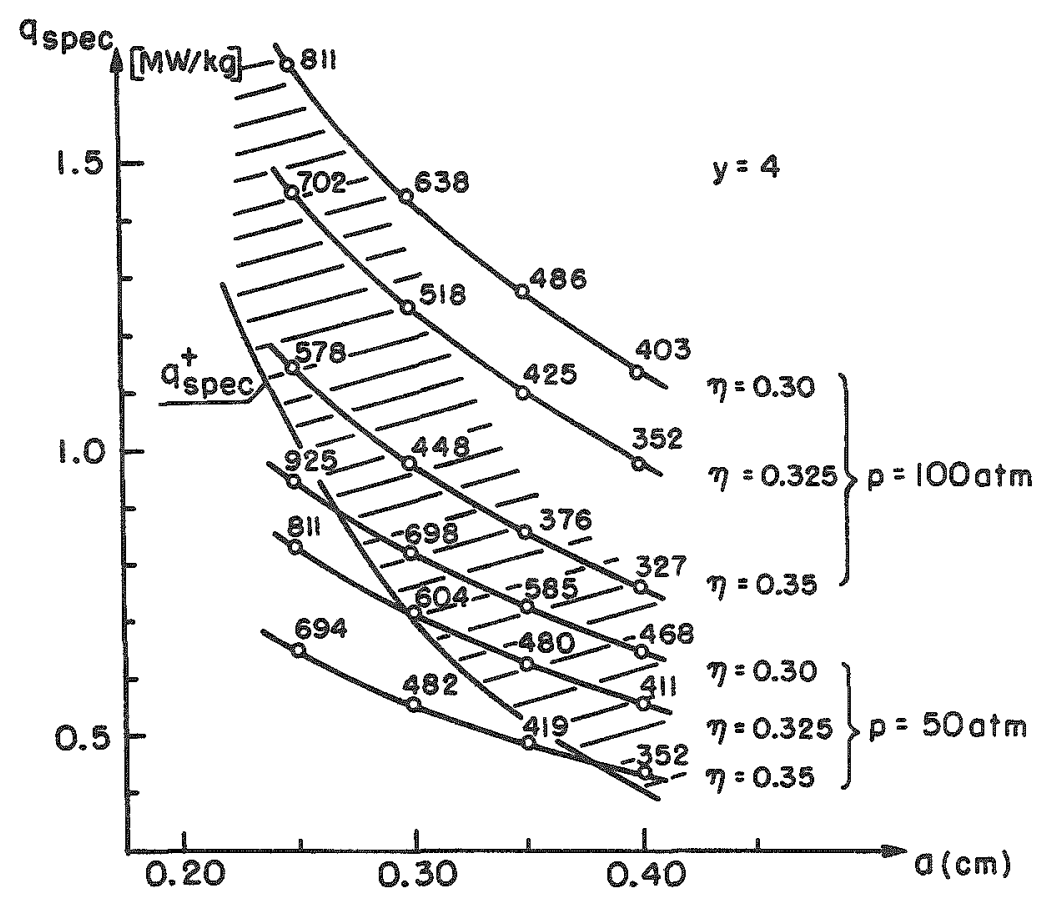

Main Results

With a gaseous coolant it is possible to remove the maximum power which can be generated with respect to the conductivity of an oxide fuel. Gas pressures are of the order of 50-100 atm. With a reasonable central temperature, the plant efficiency is $30 \%$ or higher.

The optimum temperature for gas entry lies around $230^{\circ} \mathrm{C}$, quite independent of the other parameters. With Inconel or Hasteloy canning, to provide a maximum canning temperature of $700^{\circ} \mathrm{C}$ (theoretical), the optimum gas exit temperature is around $550^{\circ} \mathrm{C}$. 
The study of turbulence promoters, for instance cross-flow fins, is of special interest. Measurements of heat transfer and pressure loss have been made by Koch(3) and Sams. (4) The conditions have been studied for some optimum cases (fin height:fin distance $\approx 1: 10$ ). The curves of the type of Figs. 2a and $\mathrm{b}$ show higher maxima of $\mathrm{qspec}$ combined with larger optimum values of $\theta_{2}$. Because of the increased friction, the distance between the fuel rods must be larger than for smooth rods at the same surface temperatures and plant efficiency. Therefore critical masses are considerably higher and breeding ratios lower. We thus concentrated our work on smooth rods.

\section{The Loss-of-coolant Accident}

There are three ways to make the loss-of-coolant accident less probable:

a. The whole system is enclosed within a secondary pressure containment filled with inert gas. This measure is safe but expensive. Also, difficult maintenance problems for machinery arise. The safety container may be of pre-stressed concrete.

b. The pressure vessel itself is of pre-stressed concrete and may contain the whole primary system (integral plant). For loading and unloading the vessel can be filled with water. There also should be mentioned a proposal of Fortescue. (5) The vessel inner diameter is of the order of $5 \mathrm{~m}$ and the length over $40 \mathrm{~m}$ if the primary system is enclosed.

Main problems: Openings for refueling, steam pipes, etc., handling difficulties for distances up to $40 \mathrm{~m}$.

c. By some simple considerations the primary circuit can be divided into two regions. The consequences of leakage are of different danger for the two regions.

Consider Fig. 4a. In region A between blower and core entrance a leakage acts as an instant short circuit for the core. Cooling will be reduced instantaneously. Less dangerous will be a leakage in region $B$ between core exit and blower entrance. A leakage even promotes the core flow. A danger is created only by the continuous depressurization of the whole system. For a leak of $120 \mathrm{~cm}^{2}, 20 \mathrm{sec}$ are needed for a pressure reduction to $10 \% ; \mathrm{sec}$ for a leakage of $300 \mathrm{~cm}^{2}$.

The base philosophy for the design is the following:

A leakage in region $A$ is excluded by double walls. A leakage in zone $B$ will lead to one or more of the following measures: 
There will be a scram.

The leaky pipes or heat exchangers are closed off by fastacting valves.

An emergency cooling reservoir of steam and water under saturation temperatures will be opened (for instance, by rupture discs). The reactivity coefficient for a large reactor is negative.

Figure $4 b$ shows a design in which the coolant flow enters the core from above and leaves it below. The pressure vessel itself has a double wall only above the upper flange with the control rod housing. For the smooth cylindrical part of the pressure vessel, sufficient safety may be obtained by a multilayer vessel.

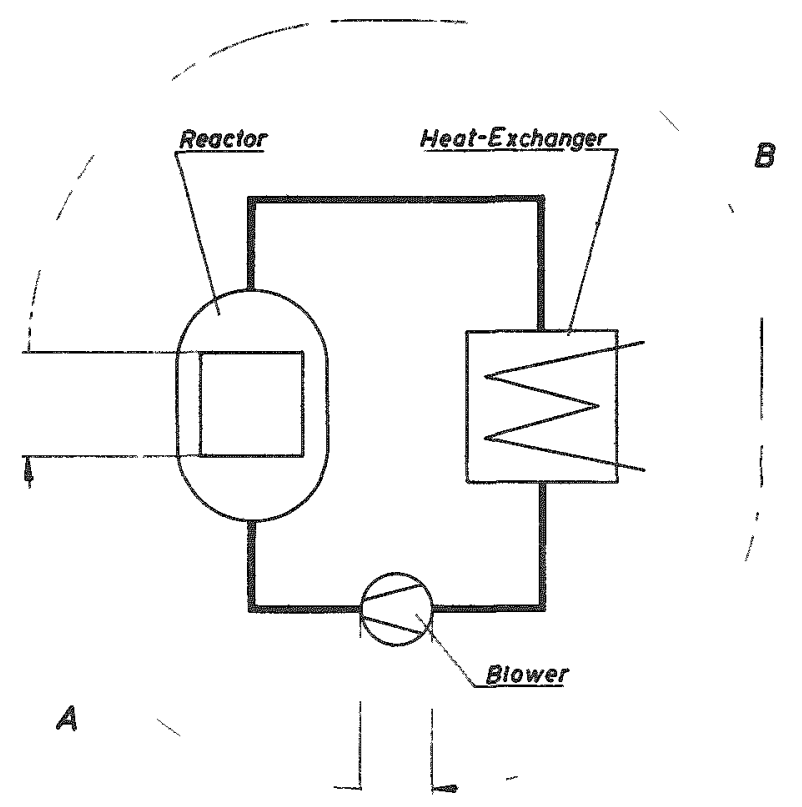

Fig. $4 \mathrm{a}$

Zone of Different Ribs in Case of a "Loss-of-coolant Accident"

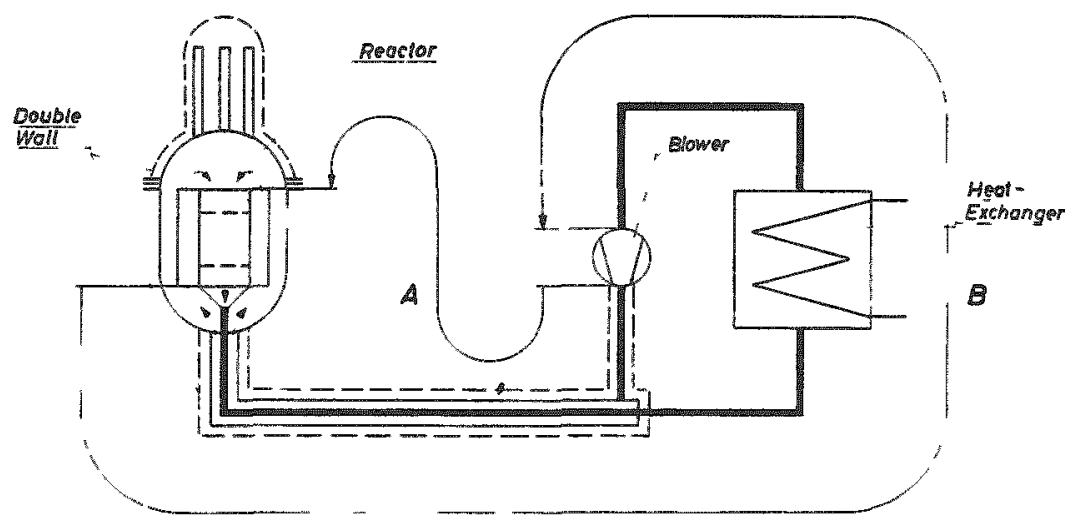

Fig. 4b. Double-wall Design for Region with High Risk in Case of a "Loss-of-coolant Accident" 
It still has to be shown whether this method, which seems to be the most economic, can be used. Detailed studies have to be made to get enough safety which depends on the functioning and reliability of the outer safety system.

4. Hot Spots, Depending on Changes in Geometry

A strong dependence of local temperatures on deformations of the geometry of the cooling channels is to be expected because of the strong dependency of the heat transfer number on velocity. Deformations can be caused by bowing or swelling of the fuel rods. There are only very few measurements on the dependence of the heat transfer numbers on the separation a of the fuel rods. Therefore, the calculations of Deissler and Taylor (6) were used. They employed a simplified model, neglecting especially the existence of secondary flow. Therefore their calculations give an upper limit. Their curves are interpolated by an analytical expression. If the fuel rods are held at distance by longitudinal fins along their whole length, each channel is isolated from the neighbouring one. Then the reaction on fuel dislocations is different from the open case in which no fins are used. Then one gets for small displacements of the rods

$$
\frac{d T_{\max }}{d s}=\frac{2 \Delta \theta}{\pi D}\left[(1.5+1.2 b) \frac{L^{*}}{L}-a+\frac{b c \pi D}{1.28 r}\right]
$$

for isolated channels (longitudinal fins) in the region of the dislocations:

$$
\frac{d T_{\max }}{d s}=\frac{2 \Delta \theta}{\pi D}(1.5+1.2 b) \frac{L^{*}}{L}
$$

for isolated channels in the undisturbed region of the channel; and

$$
\frac{d T_{\max }}{d s}=\frac{2 \Delta \theta}{\pi D}\left[\frac{9}{4 \phi_{a x}} \frac{L^{*}}{L}+0.2 b+\frac{b c \pi D}{1.28 r}\right]
$$

for interconnected channels (no fins) at the place of the disturbance. In these relations the symbols are:

$T_{\max } \quad$ Maximum can temperature

s Displacement

L Channel length

L* Displaced length of rod

$\Delta \theta \quad$ Coolant temperature rise to place of $T_{\max }$ 
For large dislocations the expressions become very complicated. Therefore, we here restrict ourselves to a typical example to show the order of magnitude. For a reactor with $y=4$ and $q_{s p e c}=0.65 \mathrm{MW} / \mathrm{kg}$ with a maximum heat flux of $170 \mathrm{~W} / \mathrm{cm}^{2}$, we assume that two rods touch each other for $10 \%$ of their length. Then we get the following rise of maximum temperatures:

Isolated channels, in the region of the dislocation: $\Delta T=+19^{\circ} \mathrm{C}$

Isolated channels, in the undisturbed region: $\Delta \mathrm{T}=+182^{\circ} \mathrm{C}$

Interconnected channels, at the dislocation: $\Delta \mathrm{T}=+239^{\circ} \mathrm{C}$.

For small changes of geometry, independent cooling channels as they are formed by longitudinal fins are preferable. Here the pressure drop at a displaced part of the channel gives especially good cooling of this part. For interconnected cooling channels, as it is without longitudinal fins, all neighbouring channels will be a short circuit for the narrow path and give an especially hot spot.

\section{Design of the Subassembly} design:

The preceding considerations lead to the following subassembly

a. The subassembly is made of smooth cylindrical rods with an outer diameter of approximately $6 \mathrm{~mm}$ (wall thickness of $0.55 \mathrm{~mm}$ ). This choice followed from pressure-drop considerations resulting from the parametric studies.

b. Longitudinal fins are used as the most reliable spacers. They prevent changes of geometry more or less completely and give a favorable hot spot behaviour. This follows from the discussion in Section 4.

c. To get a small fin height, the fuel rods are assembled in a quadratic array. For an equal void fraction $\alpha$ the separation of fuel rods is small compared with a triangular (hexagonal) array.

d. The use of longitudinal fins as spacers raises the problem of putting and fixing fin to fin for the whole length of the rod. Welding together 
neighbouring fins leads to manufacturing difficulties; also, the strain resulting from the temperature gradients across the subassemblies is too large. One solution of this problem is to use short fuel rods. The short fuel sections are connected by plugs. The plugs (see Fig. 5) are fixed by intermediate grids. By these, the individual rods are positioned and oriented with respect to their fins. The intermediate grids can be made of several layers to give a certain elasticity with respect to distortions of the subassembly box. Measurements of pressure loss with a mock-up of this fuel element gave values of not more than $50 \%$ above the values for smooth tubes.

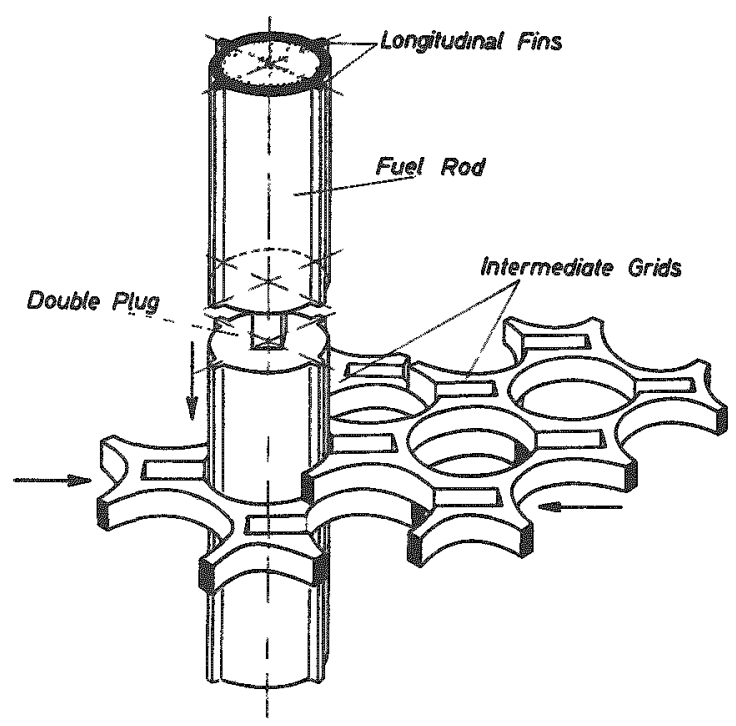

Postiven of Grids Durng the insertron of the Fuel Rods

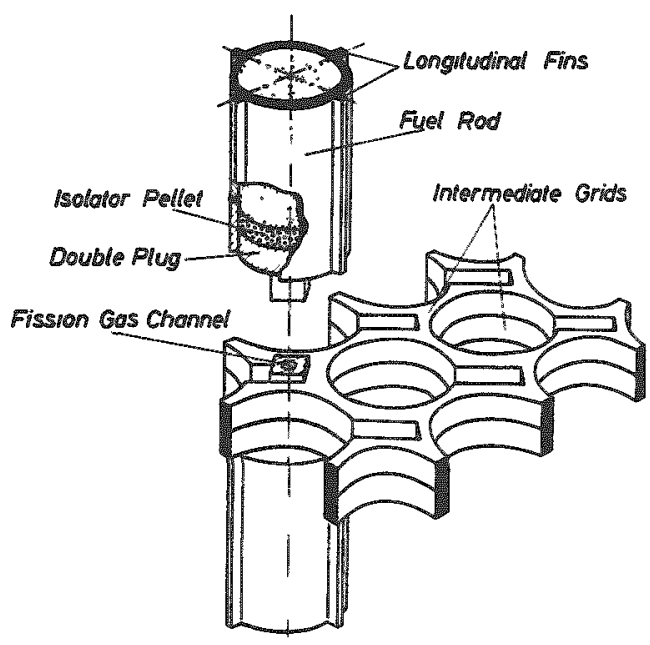

Final Position of Grids

Fig. 5. Intermediate Support of Rods

e. The use of short rods has the consequence that compacting of the oxide mixture by vibrating or swaging seems to be not very attractive. Therefore, probably pellets must be used, which is considered to be a serious disadvantage.

f. The axial upper and lower blanket is a part of the subassembly. There is much less neutron scattering by the gaseous coolant than from. sodium and, therefore, a larger leakage. From the standpoint of breeding gain, the coolant volume fraction $\alpha$ of the blanket: $\alpha=$ coolant volume/ blanket volume has to be small. To combine this with a low pressure drop, the blanket rods must have a comparatively large diameter (coarse blanket). For the same $\alpha$ there are less blanket rods, and the coolant channels have therefore a larger equivalent diameter. Since the pressure loss varies with $L / D$, this is favorable.

g. Because of the requirements resulting from the discussion in $f$ a fission gas plenum in every fuel rod is difficult to design. If there is no 
definite advantage from canning materials usable with helium as compared with sodium with respect to possible fission gas pressure and thermal racheting, the concept of longitudinal fins and a coarse blanket may not be very favorable. Then a larger pressure drop cannot be avoided.

6. Core Design

The basic principle for the core design is the entrance of the coolant flow into the core from above. This flow direction is acceptable because natural convection is too weak under any condition. Then a holddown for the subassemblies is not necessary. Refueling machines and control rods also have to work from above, from the cold side. If the fuel should melt, the subassemblies shall not be compacted by the force of the coolant low. Therefore they are suspended at their upper ends within a quadratic tube. Hanging subassemblies seem to us to be an important safety feature of the downward coolant flow, that is, of the gas-cooled reactor.

Three ways of subassembly support have been studied:

a. The supporting grid of the core is at the upper cold end. The subassemblies are put into it from above. Disadvantages: Because of the necessary grid strength, there is some separation between the subassemblies. There is a large, useless void fraction. Also, the pressure drop tends to bow the support grid (see Fig. 6) in such a manner that less coolant flow leads to more reactivity. But this effect can be kept small.

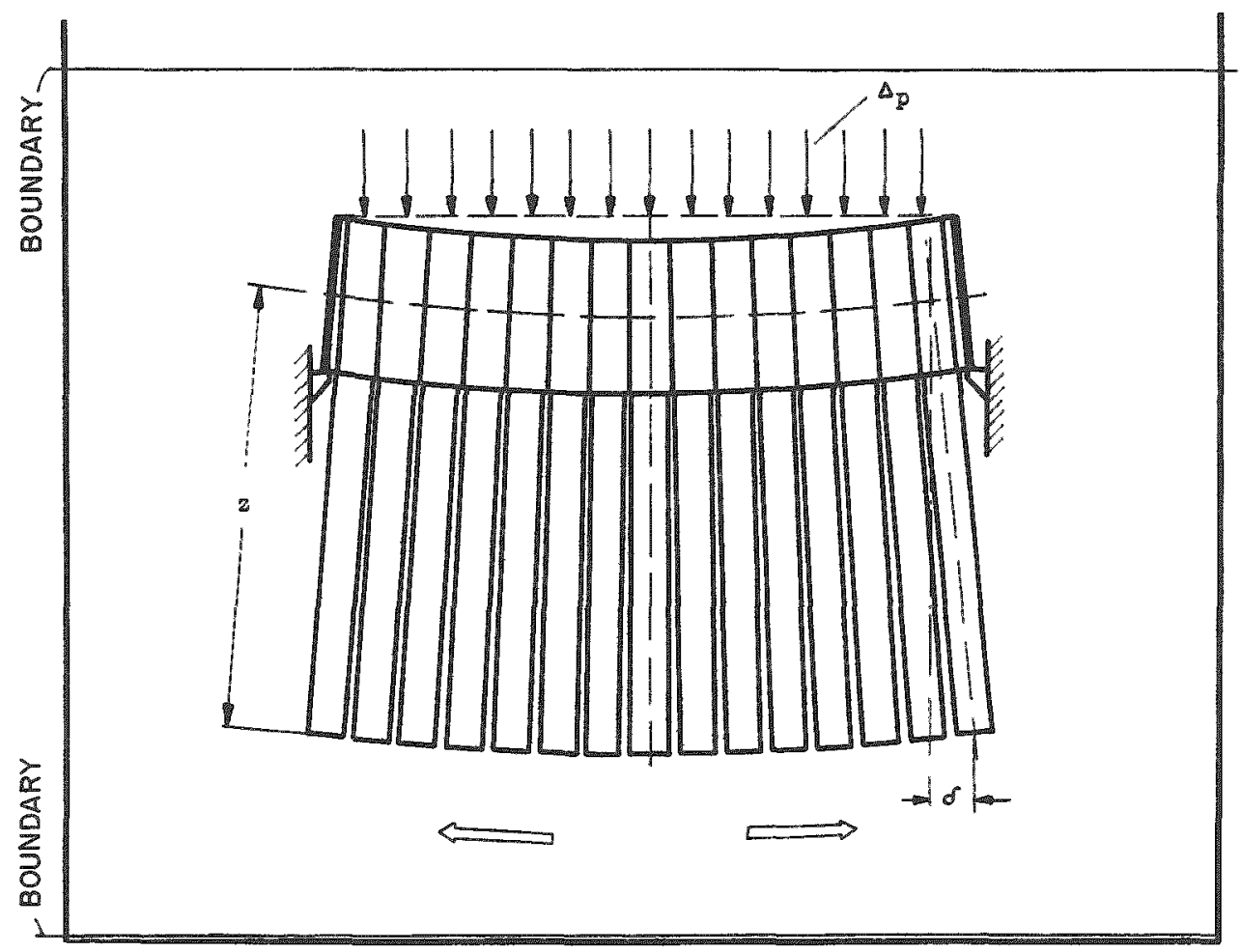

Fig. 6. Bowing of the Support Grid from Coolant Pressure Drop 
b. Subassemblies are put into the upper support grid from below. This avoids the first disadvantage of a, but makes necessary a series of complicated movements. This method has been used for refueling SNEAK, the fast zero-energy assembly at Karlsruhe.

c. Subassemblies are put into a supporting grid below the core. The grid is isolated from the hot gas. The core deformation must be restricted. Figure 7 shows the principal design and Fig. 8 some of the possible core deformations. To avoid too large stresses, we use a ball fitting at the lower end of the subassembly box. The inner radial blanket elements are of the same design as the core element. The outer blanket consists of metallic rods of a larger diameter. The coolant flows upward through the outer blanket, then downward through the core and inner blanket.

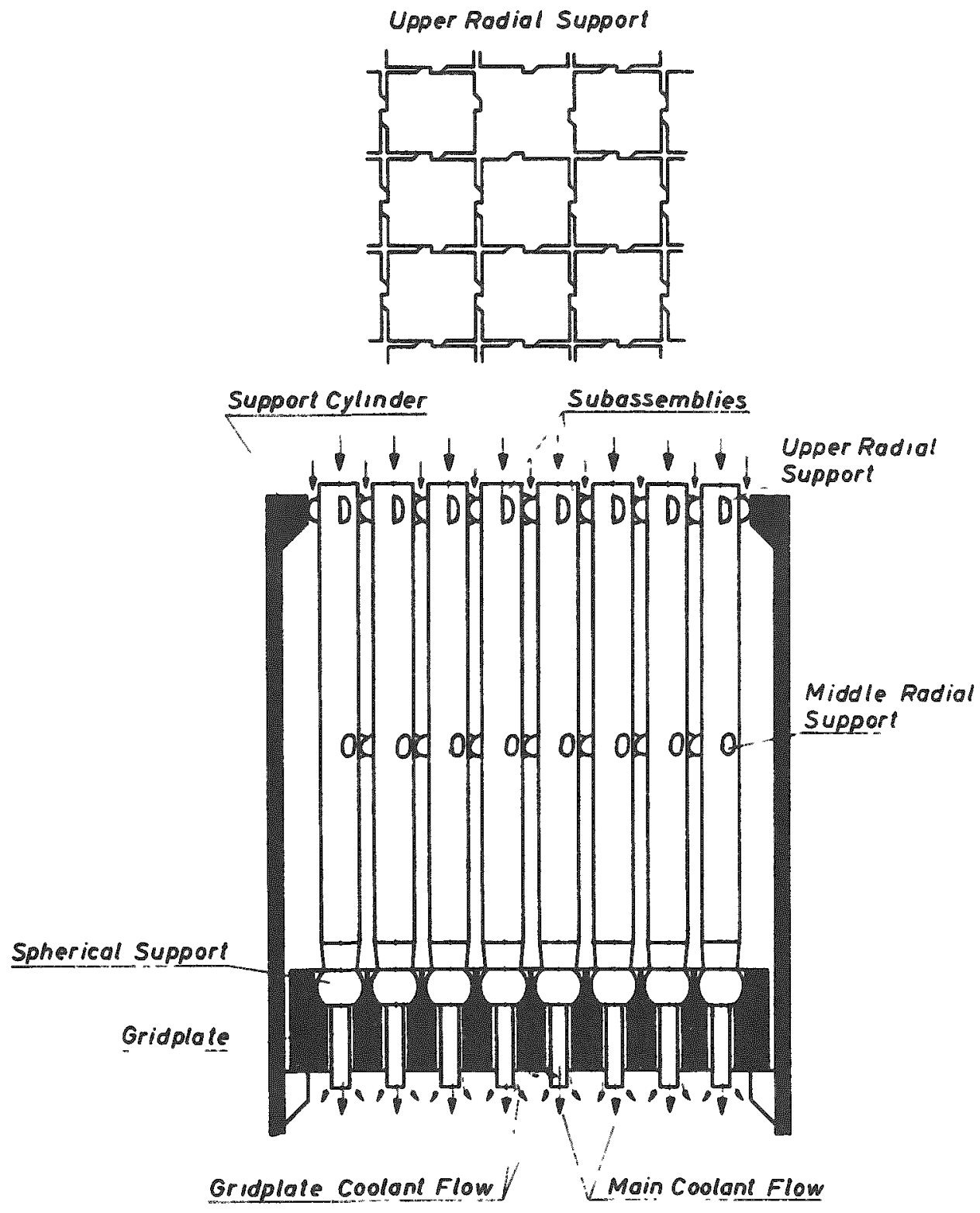

Fig. 7. Core and Inner Blanket Assembly 


\section{Estimated Rodial Thermal Gradient}

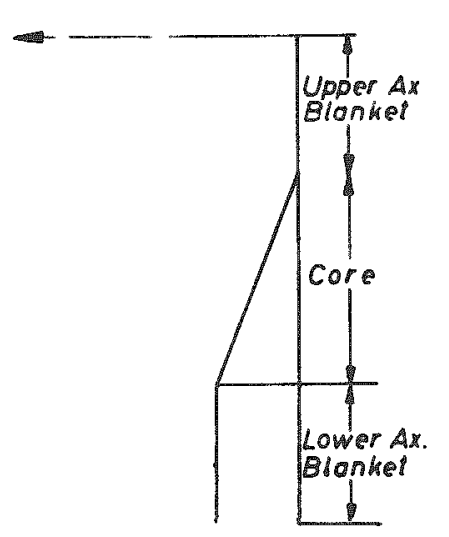

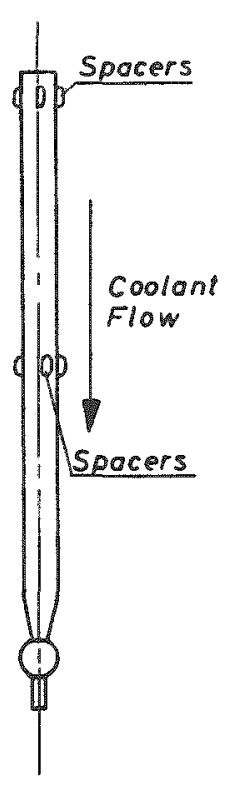

without Spocers

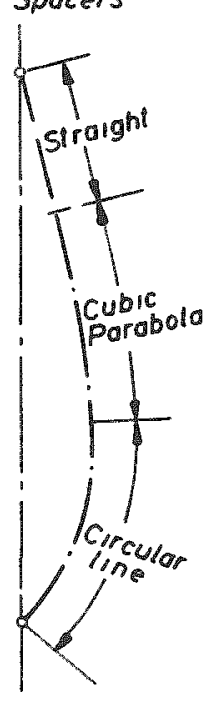

win Spocers

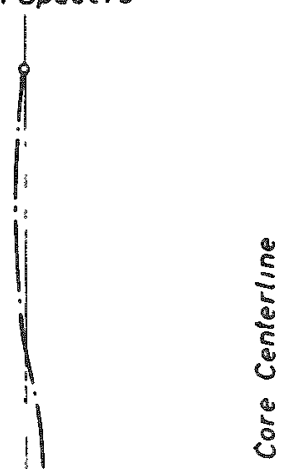

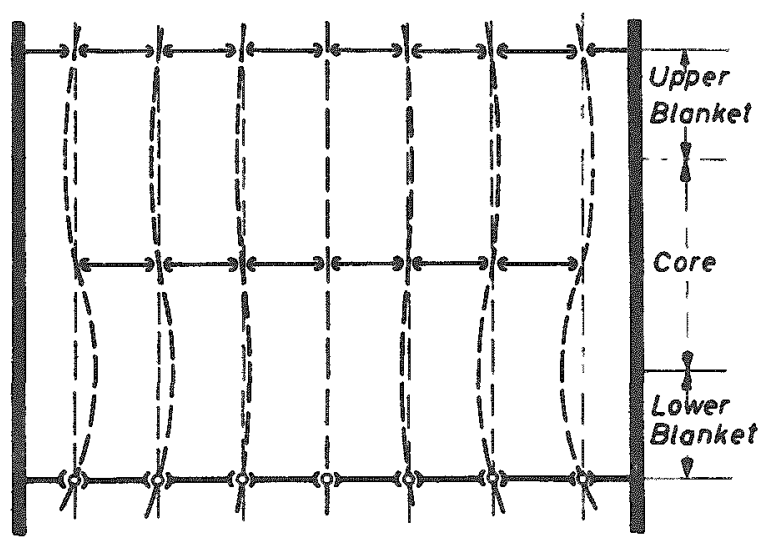

Fig. 8. Thermal Deformations of the Subassemblies and the Core

Figure 9 shows the pressure vessel with its internals as a reference design. The coolant enters by the outer one of two concentric tubes [7] and leaves by the innex one [6]. Below the core is a cone of borated graphite with a liquid metal emergency-cooling system. Refueling is done with the reactor shut down but under pressure. The charge machine puts the elements directly into the airlock [26], from where they are discharged into the upward direction [28]. Studies have been made of the accuracy of positioning the charging machine in the narrow core space. The charging machine can be removed into the shielded stand pipe [19] when the reactor is at power. Replacement is possible. The control rods [20] are arranged around the center stand pipe. They have to be disconnected during refueling operations. A special scram rod [15] in the center position is available at all times for emergency purposes. The stand pipes for the charge machine and the control rod drives are enclosed by a second container hat [25] which stays under inert gas pressure. 


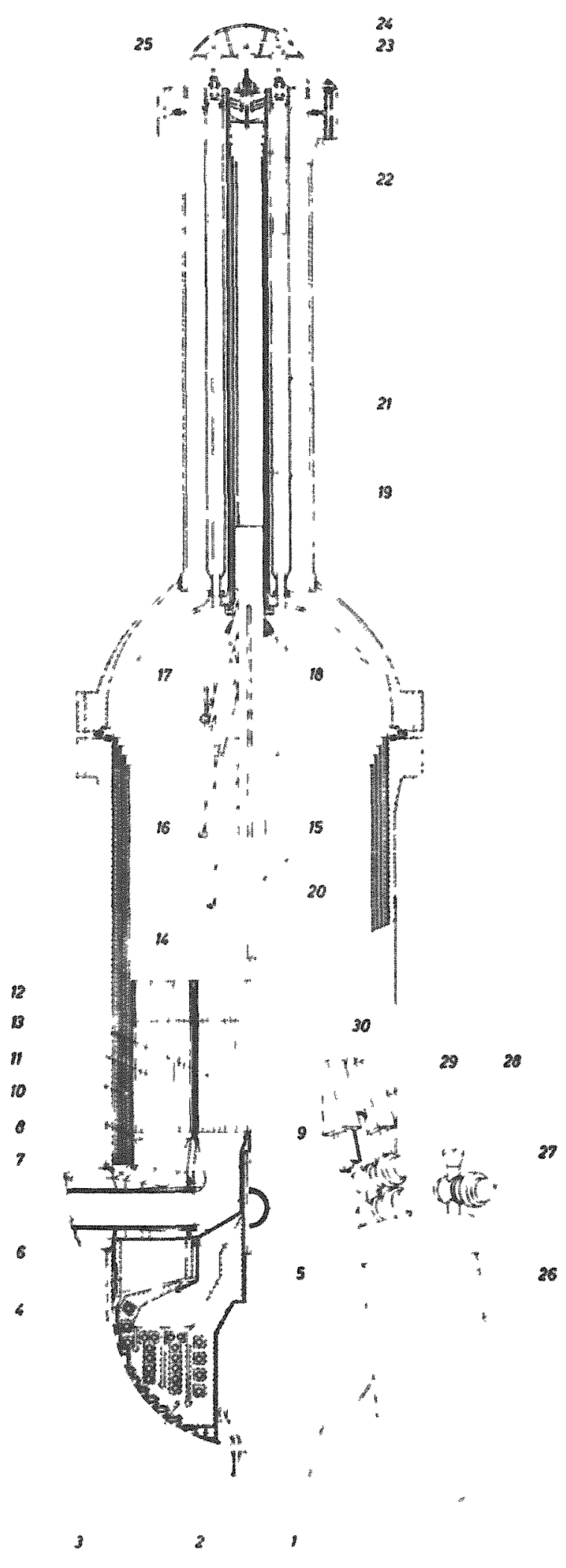

Fig. 9. Pressure Vessel with Internals

\section{Primary Circuit and Heat Exchangers}

Four primary loops are used, each consisting of the steam generator, blower, and fast-acting stop valves for isolating each loop in an emergency case. The size of the heat exchangers, of the oncethrough type, is enormous compared with the core. This is typical for the gas-cooled breeder for three main reasons:

a. The temperature drop from surface to coolant is much smaller than it is in the core. A logaxithmic mean value of $50^{\circ} \mathrm{C}$ must be compared with $150^{\circ} \mathrm{C}$ for the core.

b. Because of the large influence of the pumping power on the plant efficiency, the pressure drop of the primary gas in the heat exchanger must be kept small, smaller than for the core. Therefore heat transfer and power density are smaller too.

c. The diameter of the heat exchanger tubes is large compared with the diameter of the fuel rods. Experience must be gained for tubes of smaller diameter. Development in this direction is underway at several places and is of interest also to thermal gas-cooled reactors.

Figure 10 shows the reactor and one of the four heat exchangers. The tube diameter is about $5.1 \mathrm{~cm}$, and the pressure drop about $7 \%$ of the core pressure drop. Then each heat exchanger is about the size of the core pressure vessel. Higher gas pressures do not change the size 
of the heat exchanger very much. Therefore, the main variables for cost optimization are the wall thicknesses of the different vessels and the pumping power. The evaluation has given an optimum gas pressure between 50 and 100 atm.

Thermal Output. Number of Steam generator He-Inlet-Temperature He-Outlet-Temperature Steam Pressure Steam Temperature
$600 \mathrm{MW}$
4
$550^{\circ} \mathrm{C}$
$180^{\circ} \mathrm{C}$
$64 \mathrm{~atm}$
$530^{\circ} \mathrm{C}$
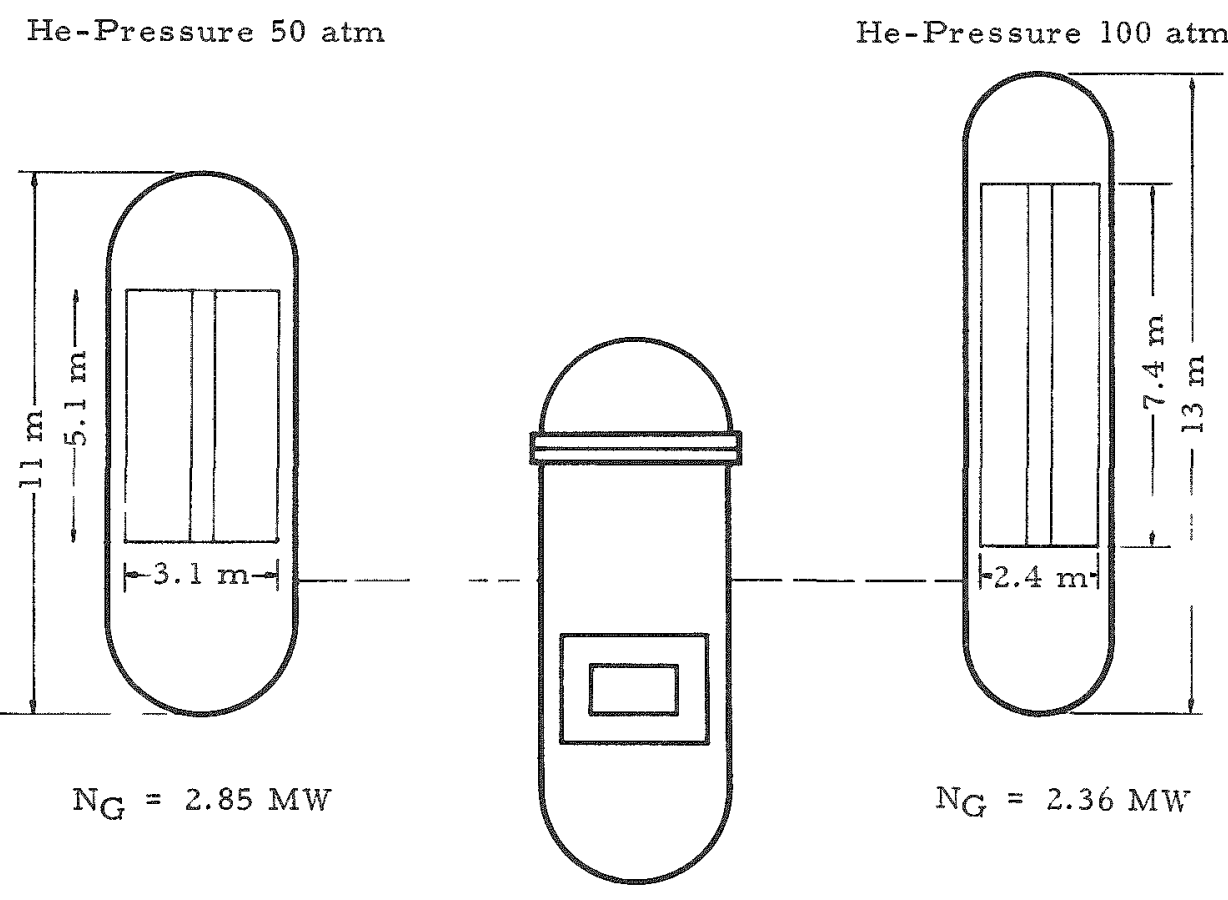

$N_{G} \rightarrow$ Pumping Power for Primary Pressure Drop in Four Steam-Generators

Identical Tube Geometries in Both Concepts

Fig. 10. Size of Steam Generators

The tubes of the heat exchanger are helically wound around a large center tube which forms an inner flow baffle. The innermost layer has the lowest rising; the rising increases to the outside. Feed water enters from above, hot gas from below through the inner concentric tube.

The blowers are of the face-sealed type with a controlled leakage. They are enclosed in a safety container. Figure 11 shows the plant layout. We looked at sizes for yielding 35 and $150 \mathrm{MWe}$. In another design, blower containment and heat exchanger are an integrated unit. 


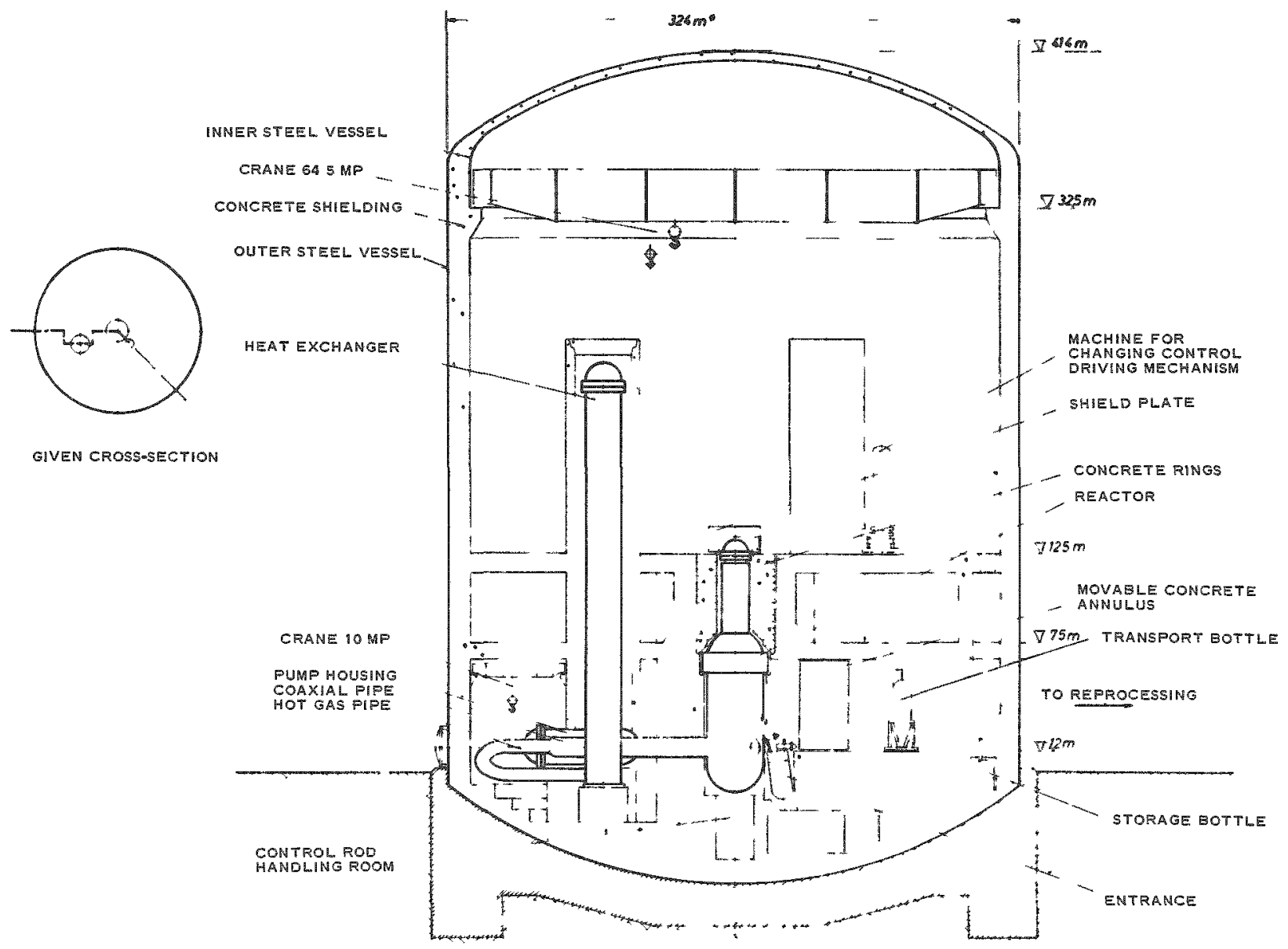

Fig. 11. Reactor Plant

\section{Conclusions}

Table 1 gives some of the important data for two designs of different size.

Some of the main conclusions are:

a. The possible specific power rises with the reactor size. It has an economically reasonable value compatible with the thermal conductivity of the oxide fuel. Cooling becomes easier with reactor size.

b. There exist optimum coolant temperatures.

c. Efficiency is $30 \%$ or slightly above.

d. The dependence of hot spot temperatures on fuel geometry seems to be tolerable.

e. An important safety feature of gas cooling is a downward flow direction. But there are difficulties in subassembly support. 
f. Smooth fuel surfaces are the most efficient ones for a fast reactor.

g. The loss-of-coolant accident can be avoided by several measures, the costs of which have to be studied in much more detail.

h. Because of their size, the heat exchangers play an important part in all economic considerations. Studies are underway to work this out in more detail.

Table 1

REFERENCE DATA FOR A GAS-COOLED FAST BREEDER

REACTOR OF SMALI AND LARGE CRITICAL SIZE

\begin{tabular}{|c|c|c|c|}
\hline \multicolumn{4}{|c|}{$\begin{array}{ll}\text { Fuel: } & \text { Pu-U Oxide } \\
\text { Coolant: } & \text { Helium } \\
\text { Gas Pressure: } & 50 \text { atm }\end{array}$} \\
\hline & & Sma11 Reactor & Medium Reactor \\
\hline Thermal power & $M W$ & 117 & 520 \\
\hline Electrical power & $\mathrm{MW}$ & 35 & 156 \\
\hline Efficiency & 落 & 30.0 & 30.0 \\
\hline Critical mass & $\mathrm{kg} / \mathrm{Pu}$ & 192 & 800 \\
\hline Breeding ratio & & 3.51 & 1.27 \\
\hline Specific power & $\mathrm{MW} / \mathrm{kg}$ & 0.01 & 0.82 \\
\hline Height of core & $\mathrm{cm}$ & 50 & 100 \\
\hline Diameter of core & $\mathrm{cm}$ & 80 & 170 \\
\hline Mean power density of core & $\mathrm{MW} /$ liter & 0.46 & 0.23 \\
\hline Diamoter of huel pin & $\mathrm{cm}$ & 0.0 & 0.0 \\
\hline $\begin{array}{l}\text { Fertile/fissionable } \\
\text { material ratio }\end{array}$ & - & 2 & 4 \\
\hline Canning/fuel material ratio & - & 0.5 & 0.5 \\
\hline Mean surface power & $\mathrm{W} / \mathrm{cm}^{2}$ & 216 & 140 \\
\hline Mas surface power & $w / \mathrm{cm}^{2}$ & 268 & 170 \\
\hline $\begin{array}{l}\text { Max surface temperature } \\
\text { of can }\end{array}$ & ${ }^{\circ} \mathrm{C}$ & 700 & 700 \\
\hline Void percentage & $\%$ & 32.8 & 45.0 \\
\hline Max coolant velocity & $\mathrm{m} / \mathrm{sec}$ & 263 & 150 \\
\hline Pressure drop & $\operatorname{atm}$ & 4.23 & 5.0 \\
\hline Inlet lemperature & ${ }^{\circ} \mathrm{C}$ & 220 & 220 \\
\hline Outlet temperature & ${ }^{\circ} \mathrm{C}$ & 550 & 575 \\
\hline
\end{tabular}

At present we are making a similar study for a sodium-cooled station to get a detailed comparison with respect to economics and safety. 


\section{References}

1. D. Smidt, Nukleonik 3,257 (1961).

2. D. Smidt, to be published in Nukleonik,

3. R. Koch, VDI-Forschungsheft 469 (1958).

4. E. W. Sams, TID-7529 (Pt. 1) Book 2, p. 390 (1957).

5. GAE-17.

6. R. G. Deissler and M. F. Taylor, TID-7529 (Pt. 1) Book 2, p. 416 (1957).

\section{Acknowledgment}

The author greatly acknowledges the work of all members of the breeder design team.

Discussion of Paper

Presented by Mr. Smidt

MR. OKRENT (Argonne): reactor?

In Table 1, why was the breeding ratio smaller for the larger

MR. SMIDT:

Because you have more structural material. It is the total breeding ratio, not the internal breeding ratio.

MR. OKRENT:

What is it that introduces this additional structure in the larger ones? If the composition stayed the same, and the enrichment got less, you might expect a higher breeding ratio. But the trend was very strong the other way. The medium reactor was not very large, about $500 \mathrm{MWt}$. If you went to 2,000 MWt, there would be no breeding if that trend continues. Would you comment?

MR. ENGELMANN (Karls ruhe):

Maybe I can comment on your question. We don't use steel, which is a relatively low absorbing material, as canning material, but rather a 
higher absorbing material like Inconel. With a large core volume, and a high number which would correspond to a high internal breeder, many of the neutrons are absorbed in this structural material. So you lose in overall breeding ratio.

MR. OTT (Karlsruhe):

I should also like to comment. In these reactors, the steel content was kept proportional to the fuel, which is fertile plus fissile material. For the larger reactor there is more $U^{238}$ and less plutonium, and therefore the poison cross section per fissile atom is larger. If one goes to very large reactors, it is not necessary to keep this relation fixed; one can reduce the steel content. Therefore it is not correct to extrapolate the two values simply.

MR. REDMAN (Argonne):

You mentioned you had considered the possibility of loading and unloading under water. Do the safety problems and other complications offset the advantages of this scheme?

MR. SMIDT:

The study is still under way. At present we are thinking of having resonance absorbers in the fuel. By this means it is possible to use water and not get supercritical. There are some difficulties with loading under water especially with an integrated plant, for you have to work over long distances.

MR. OKRENT:

How practical is it really to avoid meltdown in the event of a rapid loss of coolant? You mentioned that you would have steam or mixed steam and water, but I would appreciate hearing what some of the difficulties are that face one in accomplishing this.

MR. SMIDT:

The main point of our study was to see whether it is possible to avoid the loss-of-coolant accident, rather than what might happen if there is a meltdown. We hope that with downward coolant flow there is enough force to push the core apart, but this naturally has to be studied in much more detail. 
MR. OKRENT:

I am afraid my question was not clear. I would like to know whether you think you can avoid meltdown, if you do lose coolant flow, by emergency flooding or by some kind of secondary system. I got the impression that such measures were considered possible.

MR. SMIDT:

Yes. We contemplated such measures as the use of valves and rupture disks which could provide emergency steam cooling. These would be connected with the steam vessel filled with saturated water at saturation temperature.

MR. OKRENT:

Would this have to continue for many minutes or for hours?

MR. SMIDT:

It would have to continue for a long time if you want to avoid decay heat meltdown. Therefore, we did not consider a reservoir of helium, but rather of water.

MR. DIETRICH:

How about loss of coolant flow, particularly with downflow?

MR. SMIDT:

The only measure one can take in this direction is to have more than one cooling circuit. We choose to have four. One also has auxiliary pumps with an independent power supply. One cannot rely on natural convection.

MR. CHARAK (Argonne):

I was interested in the speaker's opening comment, that the system might lead to cheaper reactor vessels. Was this based on the prestressed concrete concept?

MR. SMIDT:

I listed only the preliminary considerations that might lead to this result. For instance, one consideration is that you can use an ordinary 
steel vessel, and have no trouble with stainless steel. These are first thoughts. The study is not completed yet.

MR. SCHLUDERBERG (Babcock \& Wilcox):

Did you show a thermal efficiency of 30 per cent for the plant?

MR. SMIDT:

We11, it depends. The efficiency might be as high as 34 per cent.

MR. SCHLUDERBERG:

Could you give us an idea of what kind of steam cycles you are considering and what sort of pumping powers you anticipate?

MR. SMIDT:

The steam cycle is quite ordinary, single pressure. We did not take the trouble to look into other steam cycles. The pumping power is of the order of 10 to 12 per cent.

MR. SHANSTROM (General Atomic):

Originally you looked at a large number of coolants. Did you narrow the field just to helium?

MR. SMIDT:

Steam and carbon dioxide also seem to be interesting. Steam allows more favorable pumping power and you need not have a heat exchanger, which increases efficiency. But on the other hand, steam has a very large positive power coefficient for a large reactor. This may be much more dangerous than the sodium void coefficient. Carbon dioxide also is favorable under the special condition that you have very narrow fuel and very narrow cooling channels because of its higher heat capacity. However, the corrosion problems which exist with $\mathrm{CO}_{2}$ have to be considered in more detail.

MR. DIETRICH:

What would be the loss of carbon dioxide reactivity coefficient? 
MR. SMIDT:

It wouldn't be large because it is even less a moderator than helium, and it would be used at about the same pressure. I don't think it is too much different.

MR. TAVERNIER (BelgoNucleaire):

My question may be more appropriate after the next paper, but in any case I'Il put it now. Some time ago we had worked out a steam-cooled fast reactor, and we did not have the impression that a large positive coefficient for steam removal was inevitable if the design was properly made. So your statement a few minutes ago surprised me somewhat.

Also, it seems to me that steam cooling can have much better prospects in the loss of coolant accident because you can have circuits which make it easier to handle.

MR. DIETRICH:

I believe Dr. Häfele wants to reply to that.

MR. HÄFELE (Karlsruhe):

With respect to the question of the power coefficient, the situation is that a very rough investigation indicated a temperature coefficient for steam in the order of $+1 \times 10^{-5}$. It is a very tricky business because the whole effect takes place in the resonance area, and one has to improve the whole mathematics and theory in order to be sure that this is the proper coefficient. The Doppler coefficient is $-1 \times 10^{-5}$. The ratio is then one to one. There is a need for greater accuracy of computation. But the statement of Dr. Smidt is quite correct in that at present for large volume reactors there is a good chance that the power coefficient of steam comes out to be too large.

MR. EDLUND (Babcock \& Wilcox):

From our investigations of steam cooling it is possible to design large reactors that have very strong negative coolant coefficients. As you go down in energy into the resonance region, the fission cross section increases relatively much faster than the fertile material cross section. You may have something like 10 per cent of the neutrons in this region ordinarily, and this contributes a very strong negative coefficient as the coolant is expelled and the spectrum moves up. 
MR. HÄFELE:

I agree that the coefficient is strictly negative if the core is small enough. Our calculations showed a switchover from a negative to a positive coefficient at volumes of the order of 300 liters, and if you have $D_{2} \mathrm{O}$ it is as high as 500 liters. I doubt that your statement is true for 1000 or 2000 liters.

MR. EDLUND:

It is true for several thousand liters. 


\title{
SEGMENTED CORE DESIGN FOR A SUPERCRITICAL PRESSURE WATER-COOLED FAST REACTOR
}

\author{
R. E. Peterson and S. L. Stewart \\ General Electric Company \\ Hanford Atomic Products Operation \\ Richland, Washington \\ (Paper presented by Mr. Peterson)
}

I. Introduction

The use of water as a coolant in fast reactor design is generally regarded as unsatisfactory from a nuclear point of view. Nevertheless, the engineering advantages are sufficiently attractive that some effort has been expended in attempting to utilize water at a reduced density, especially in oxide-fueled reactors, in which the neutron spectrum is already somewhat degraded by the fuel. Steam cooling has been inveseigated in conceptual studies of fast reactor cores of more or less conventional design as well as in nuclear superheaters.(1,2) Results of these studies have shown that breeding ratios significantly above unity can be attained, fuel inventories are not excessive, and reasonably good economics can be achieved. It should be noted, however, that these designs are much more limited in size and composition by positive flooding and voluing reactivity coefficients than for similax sodium-cooled reactors.

Supercritical pressure water is currently being considered for use in thermal reactors $(3,4)$ and has been utilized over the past decade in several fossil-iueled steam powex plants. A high heat transfer coefficient in the vicinity of the critical point, a relatively low density, and high the rmal efficiency achievable with this fluid makes supercritical water an interesting prospect as a fast reactor coolant also. (5) Therefore, a conceptual design and economic evaluation of a fast reactor employing steam coolant above the critical pressure was undertaken to better evaluate feasibility and economic incentives. (6) This concept is referred to as the Fast Supercritical Pressure Power Reactor (FSPPR).

\section{Reactar Core Design}

Previous design studies of steam-cooled fast reactors indicate a potentially laxge reactivity effect associated with changes in coolant density. Because of this and the deleterious effect of water on the breeding ratio, the coolant inventory must be minimized. The high heat transfer coefficient attainable with supercritical water is consistent with this objective. Containment of the high-pressure fluid at operating temperatures is also a major problem. Fortunately, the best available high-temperature alloys may be 
employed for in-core hardware in the fast spectrum with nearly negligible effect on neutron economy. Therefore, this conceptual design is based on a reactor composed of massive fuel elements pierced by small, highpressure coolant tubes. The fuel element is shown in Fig. 1. Mixed

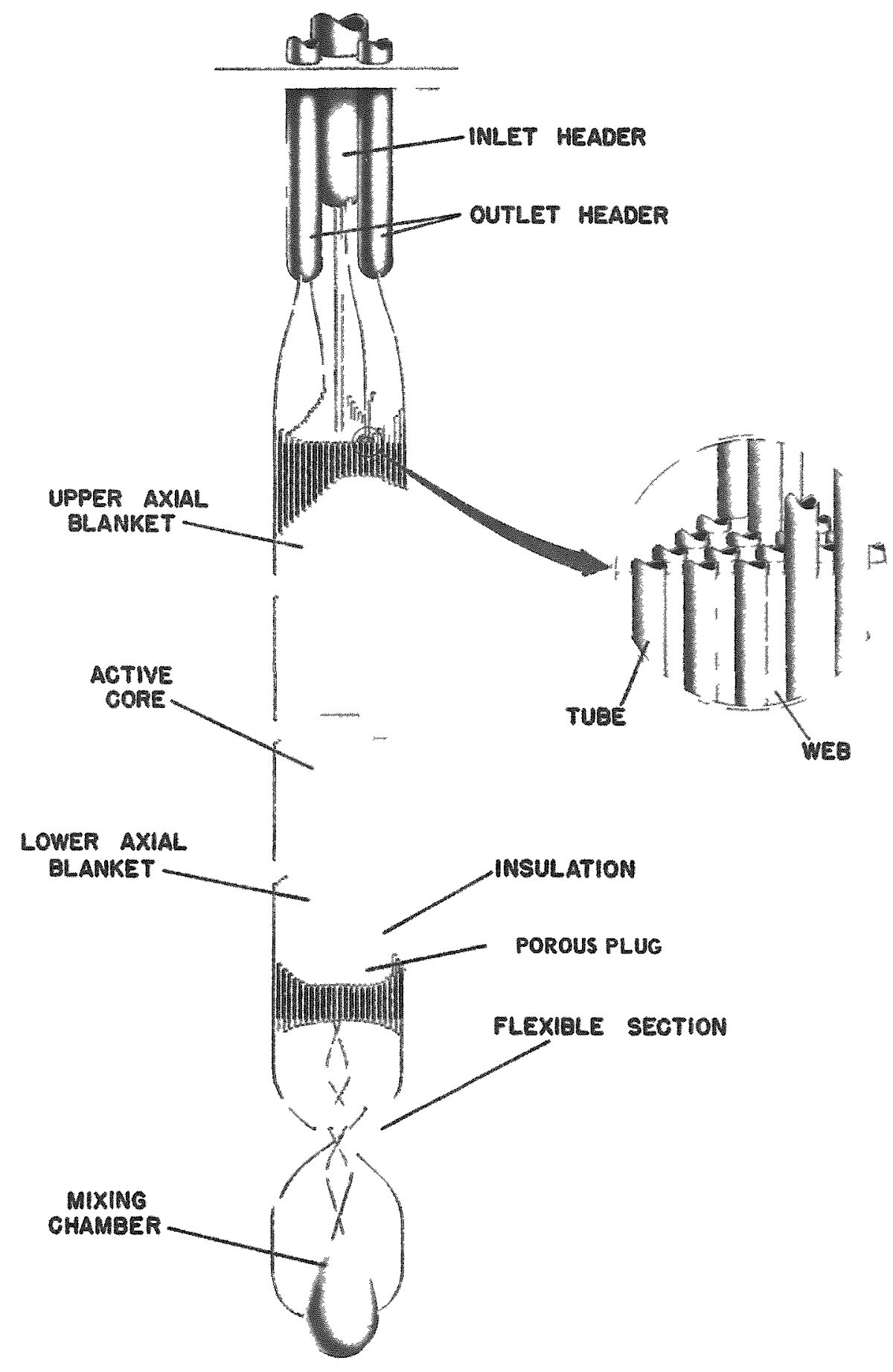

Fig. 1. Fast Supercritical Pressure Power Reactor (FSPPR) Fuel Element Assembly 
plutonium-uranium oxide fuel is utilized to take advantage of the better neutron economy of plutonium in the fast neutron spectrum and to permit an economical burnup, potentially attainable with oxide fuels. The coolant tubes are made of Rene' 41, a nickel-base alloy with excellent high-temperature properties. Fabrication of thes large fuel pieces by vibrational compaction techniques appears entirely feasible.

The reactor core design represents a departure from the more conventional fast reactor concepts, first because of the fuel element design de. scribed above, but more importantly, because of measures taken to counteract a strongly positive coolant reactivity coefficient. The latter arises because of neutron-energy degradation caused by scattering collisions with the coolant. This problem is not restricted to $\mathrm{H}_{2} \mathrm{O}$, but occurs in liquid metalcooled core designs as well, although not as severely. (7) Energy degradation increases the capture-to-fission ratio in the fuel, increases resonance capture in fertile isotopes, decreases fast fission in fertile isotopes, and decreases the average number of neutrons released per fission. For these reasons, a decrease in coolant density from the normal operating value may result in an increase in neutron multiplication unless the corresponding increase in neutron leakage is large enough to counteract these positive effects. Two of the more common methods which have been incorporated in large fast reactor designs to alleviate this problem are (1) to decrease the length-to-diameter (L/D) ratio and/or (2) to increase the coolant volume fraction, both of which enhance the leakage effect. An increase in water density, on the other hand, such as might occur on core flooding, can also increase reactivity if the water-to-fuel volume ratio is large enough to allow sufficient neutrons to escape $U^{238}$ resonance capture and be absorbed in the $0.3-\mathrm{eV}$ plutonium resonance.

Initial calculations for the FSPPR core indicated a substantial decrease in reactivity upon flooding, because of the relatively small coolant volume, but the computed void effect was found to be close to $4 \% \Delta \mathrm{k} / \mathrm{k}$, positive. As a possible solution to this potentially serious safety problem, a new "moderator segmented" coxe design was devised and investigated. In this concept, the core is subdivided by a relatively thin layer of moderating material. This creates an internal boundary which degrades the energy of neutrons crossing it. A layex of absorbing material ( $\mathrm{U}^{238}$ ) is placed on either side of the moderator to form an annular flux trap between fast core regions. A cross section of the core utilizing this arrangement is shown in Fig. 2. When coolant is lost, a larger fraction of neutrons diffuse out of the core regions and are either absorbed in $\mathrm{U}^{238}$ resonances or are degraded in energy. The leakage effect is thus enhanced internally. Yttrim or zirconium hydride is the moderating material selected for this reactor design, although a greater thickness of graphite or beryllium would serve similarly. The $\mathrm{U}^{238}$-absorber region is incorporated in fuel elements on either side of the moderator. Because of the high resonance flux created in the flux trap, 
a high initial rate of formation of plutonium results. It is desirable, therefore, to enrich slightly the depleted uranium with plutonium to avoid large changes in both reactivity and specific power as the plutonium builds up to its equilibrium composition.

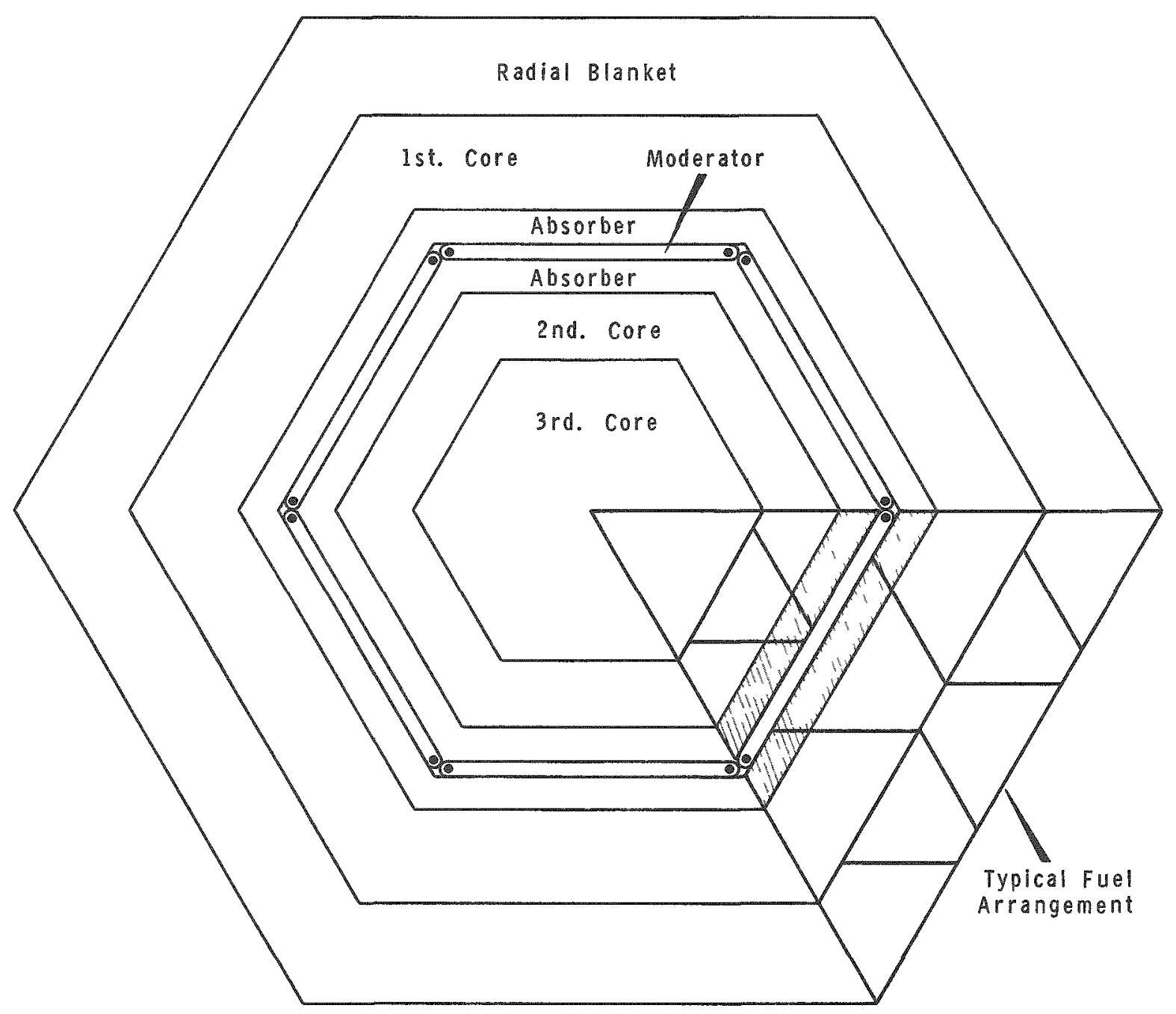

Fig. 2. Core Arrangement - $300 \mathrm{MWe}$ ESPPR

A summary of final design data for this conceptual study is shown in Table I. Because of the nuclear safety of this core against flooding, water shielding may be employed to a large extent, with subsequent savings in capital costs. The core is contained in a stainless steel tank extending far enough below the core to permit charge-discharge from beneath. Typically, the tandem arrangement shown in Fig. 3 might be employed with a batch fuel cycle to provide for preparation of a second core as the first core nears its goal exposure. 
Table I

300-MW(e) FSPPR DESIGN SUMMARY

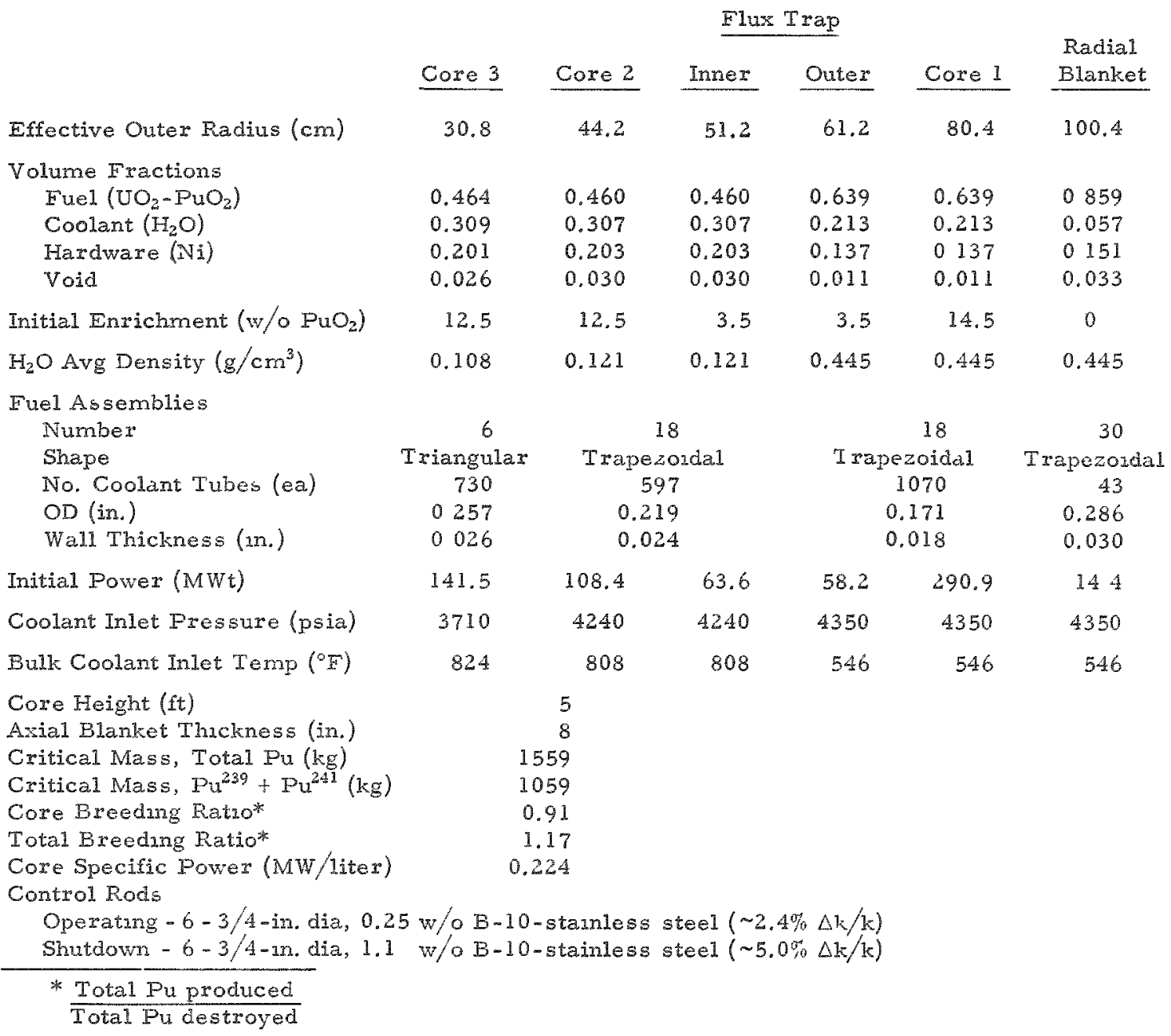

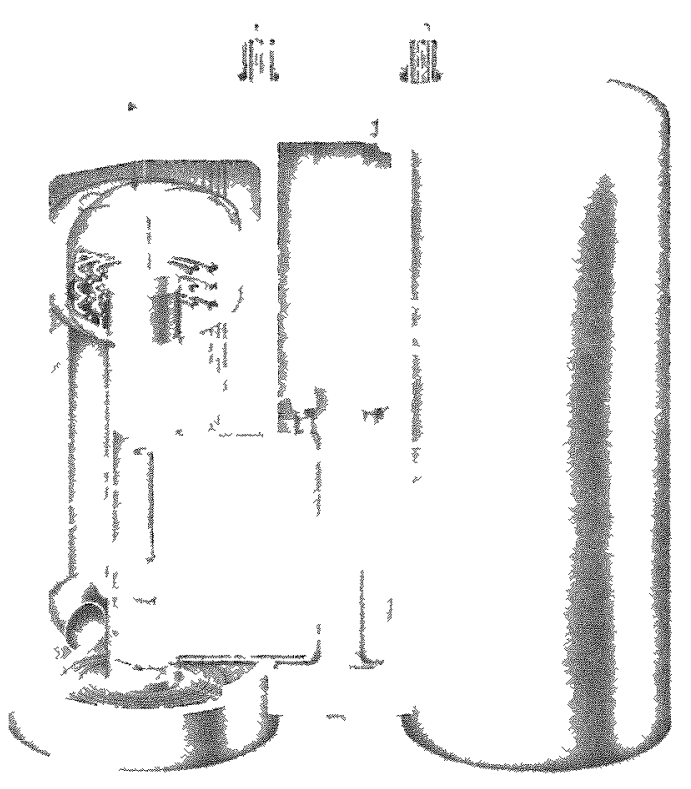

Fig. 3

FSPPR Reactor 


\section{Reactor Operation}

Batch operation appears to be preferable to a graded fuel cycle in this reactor because the massive fuel elements do not permit a finely divided core with facilities for frequent charge-discharge. An average goal exposure of $100,000 \mathrm{MWD} /$ ton was selected as the probable maximum capability of the fuel and represents approximately six full-power operating years. A loss in breeding gain inevitably occurs as a result of the spectral degradation caused by the water coolant. However, the calculated total breeding ratio is 1.17, about equivalent to that quoted for other steamcooled conceptual studies of this size. Although the "fast" core breeding ratio $(\sim 0.91)$ is less than unity, the reactivity loss due to core burnup is more than compensated by plutonium buildup in the flux-trap region. The high statistical weight of this region tends to amplify the reactivity worth of the plutonium formed relative to that in the fast core regions. Thus, a initial rise in reactivity is obtained as the plutonium builds up to its equilibrium. The reactivity then begins to decrease as $U^{238}$ depletion in the flux trap becomes significant. This behavior is shown in Fig. 4. The reactivity burnup characteristic can be altered significantly by varying the initial enrichment of the absorber region between zero and its equilibrium composition. It appears desirable to set this enrichment at a value which minimizes the control requirement. A value of $3.5 \mathrm{w} / \mathrm{OPuO} \mathrm{Pused}_{2}$ in the present core design results in a maximum burnup control span of $1.3 \% \Delta \mathrm{k} / \mathrm{k}$ to reach the goal exposure of 100,000 MWD/ton.

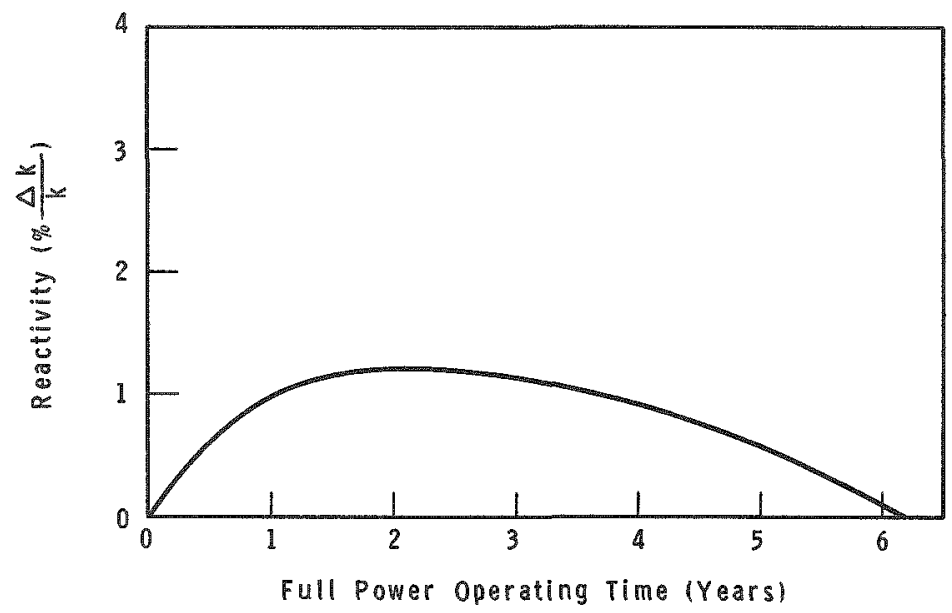

Fig. 4. Reactivity Change Versus Fuel Exposure - Batch Operation

The high statistical importance of the moderator region also makes it an excellent location for control rods. Some variation in the coolant void coefficient might be expected as a result of placing an absorber in this location. However, the variation in plutonium composition and fission product formation being compensated by the control rods tends to maintain the net 
neutron-absorption constant. As a result, only a minor variation in the void coefficient occurs between beginning and end of core life.

As expected, the higher fraction of neutrons in the resonance energy region results in a stronger negative fuel temperature coefficient than is typical of sodium-cooled, fast oxide systems. The fuel coefficient due to Doppler broadening only is computed to be $-2 \times 10^{-5} /{ }^{\circ} \mathrm{C}$ at operating conditions and $-8 \times 10^{-6} /{ }^{\circ} \mathrm{C}$ without coolant. Fuel and cladding expansion effects are estimated to be only a small fraction of these values.

IV. Safety

The coolant reactivity void coefficient is zero at the operating point and is negative for either flooding or voiding. The total reactivity change for a change in coolant inventory from the operating value is shownin Fig. 5 . Voiding of the individual core regions also results in a slightly larger loss in reactivity than does total coolant loss. Both void and flooding coefficients retain nearly their same values throughout core life.

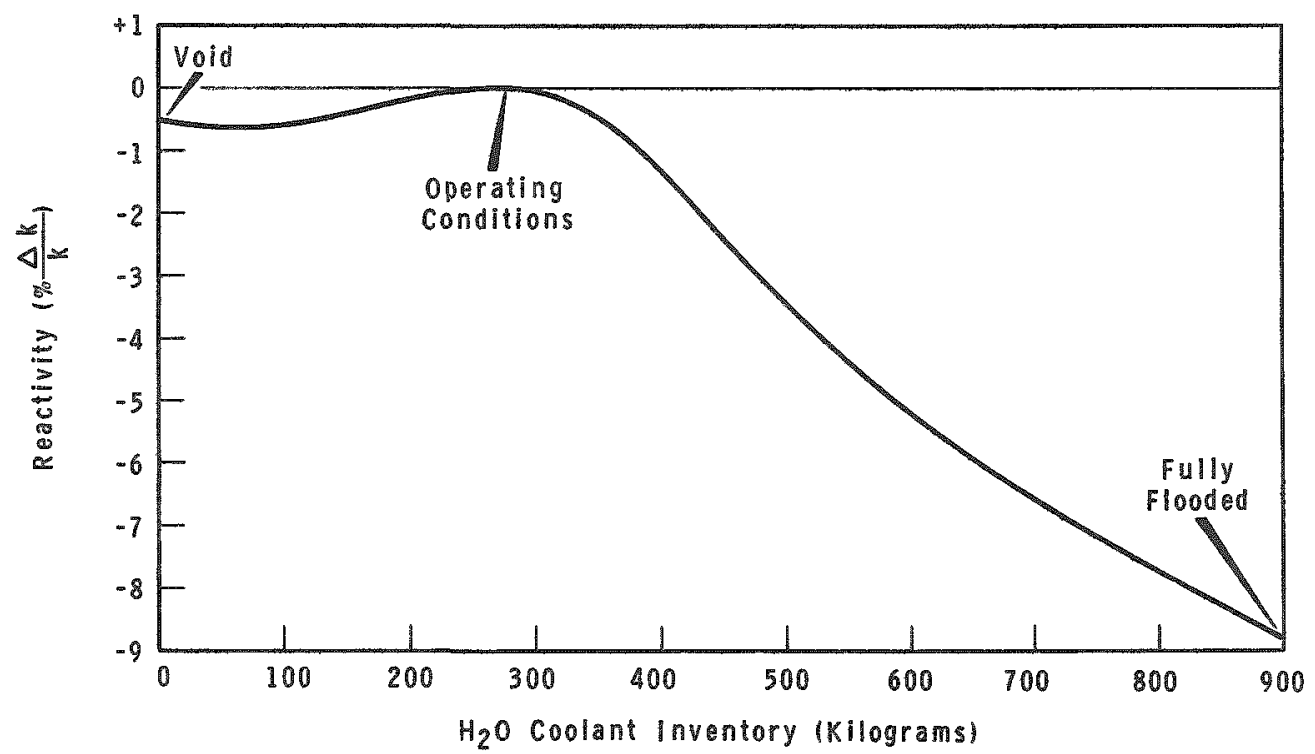

Fig. 5. Reactivity Change Versus Coolant Inventory

As an extreme condition, the loss of moderator from the flux trap is also considered. Results for varying amounts of hydrogen loss are shown in Fig. 6. A loss in reactivity accompanies loss of moderator until nearly 80 per cent of the hydrogen is expelled. With operating control rods fully inserted in the moderator region, this is reduced to about 30 per cent. The coolant coefficients also remain negative beyond this point. This substantial margin of safety is due to the importance of low-energy plutonium fissions adjacent to the moderator. Initial loss of hydrogen decreases the moderation and shifts more neutrons into $\mathrm{U}^{238}$ capture resonances. The 
nuclear safety of this core design thus appears to be adequate. In the event that shutdown rods fail to penetrate the core, flooding the moderating region with borated water would also effect an emergency shutdown.

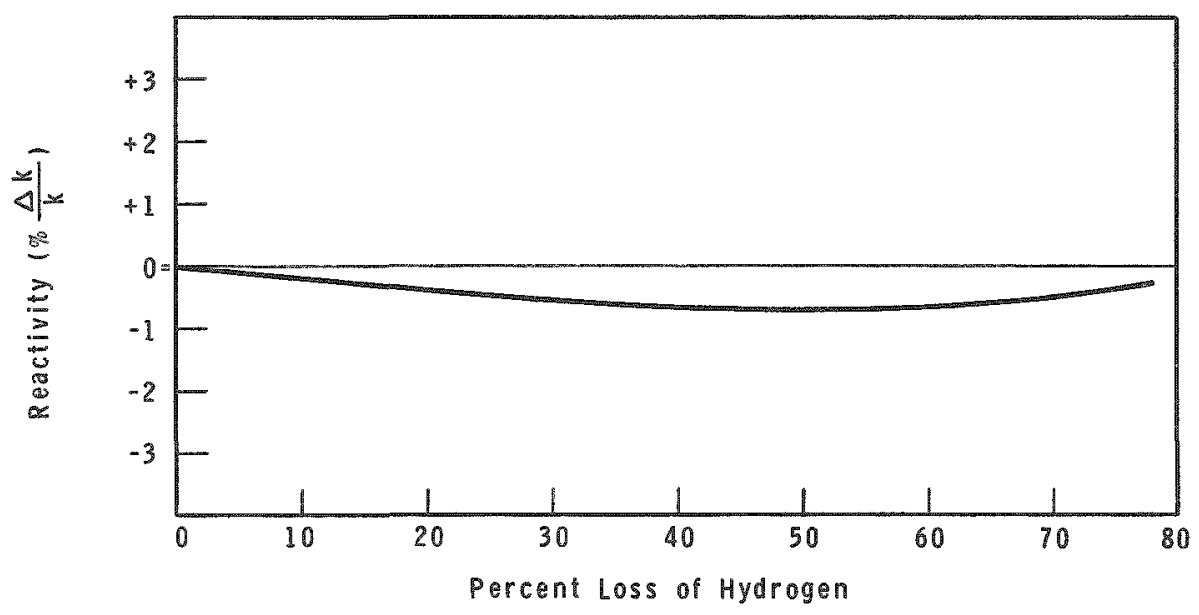

Fig. 6. Reactivity Change as a Function of Hydrogen Loss from Moderator Region

V. Conclusions and Technological Problems

A more detailed comparison of the segmented-core design with alternative methods of solving the coolant coefficient problem in large fast reactors is now being carried out. Preliminary indications are that economic penalties are not significantly greater than for other core designs. It should be pointed out, however, that for the FSPPR design concept, the usual methods of counteracting the void coefficient are unsatisfactory. Increasing the coolant volume to increase core leakage results in an unacceptable degree of spectral degradation and introduces a positive flooding coefficient. Decreasing the length-to-diameter ratio ("pancaking") within an acceptable range is not sufficient to counteract fully the positive coefficient and strongly increases both fuel and capital costs.

The effect of the moderator region on the fast core regions is similar to that of the external blanket. This can be seen in Fig. 7, where the mean fission energy is shown as a function of core radius. Spectraldegrada. tion is confined to a relatively small volume on the surface of the fast core regions. It is also estimated that the segmented-core design increases the total fissile plutonium inventory by about $84 \mathrm{~kg}$ over that for the same core without this feature.

A final consideration is the effect of the moderating region on the distribution of core power. A small fraction of low-energy neutrons originating in the moderator penetrates the absorber region to the fast core surfaces and causes a slight increase in local power. However, a more 
serious problem is the power gradient across the absorber region, which can vary significantly, depending upon the plutonium concentration. Since plutonium builds up rapidly in this region, it is desirable to enrich the uranium with plutonium initially to avoid a large change in power distribution as the equilibrium composition is approached. The initial power distribution for the FSPPR conceptual study is shown in Fig. 8. The desirability of decreasing the large power gradient adjacent to the moderating region is evident. Use of graphite or beryllium rather than a metallic hydride moderator has been found to improve the power distribution considerably. It is thus apparent that the design of a segmented core of this type is a relatively complex compromise between safety and factors which influence economics.

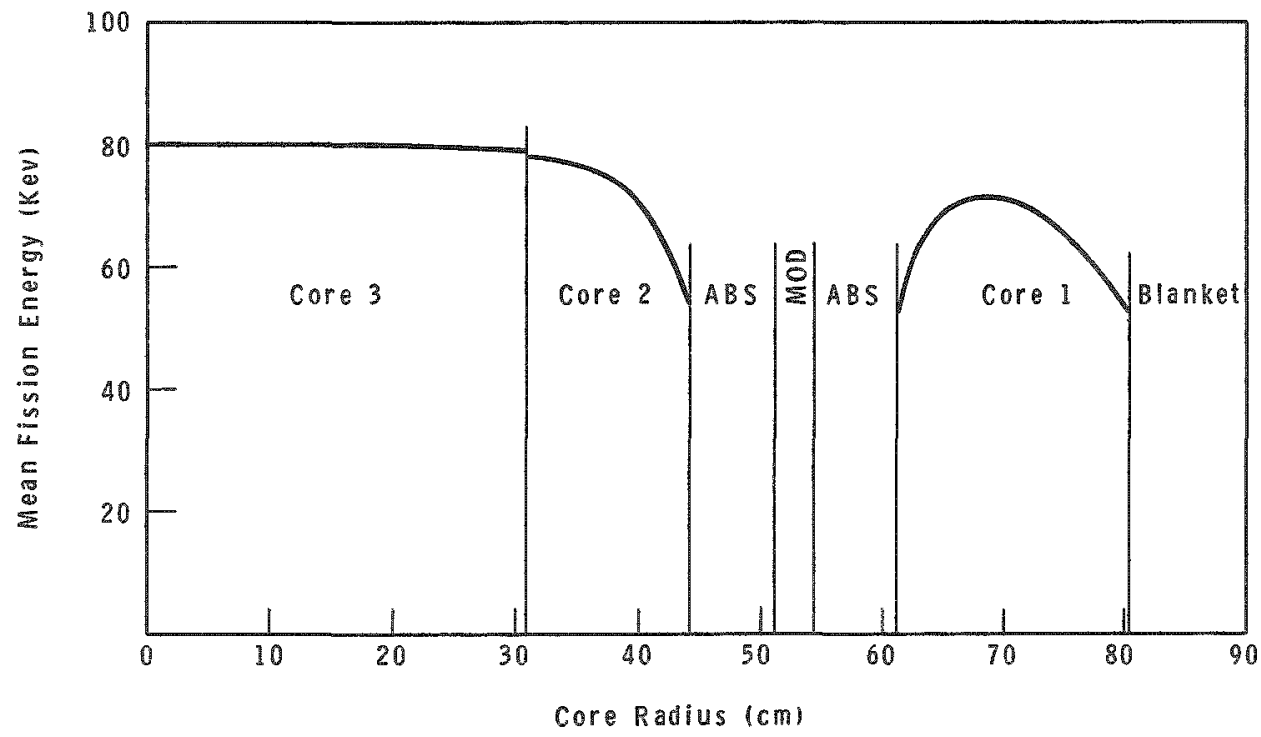

Fig. 7. Mean Fission Energy Distribution

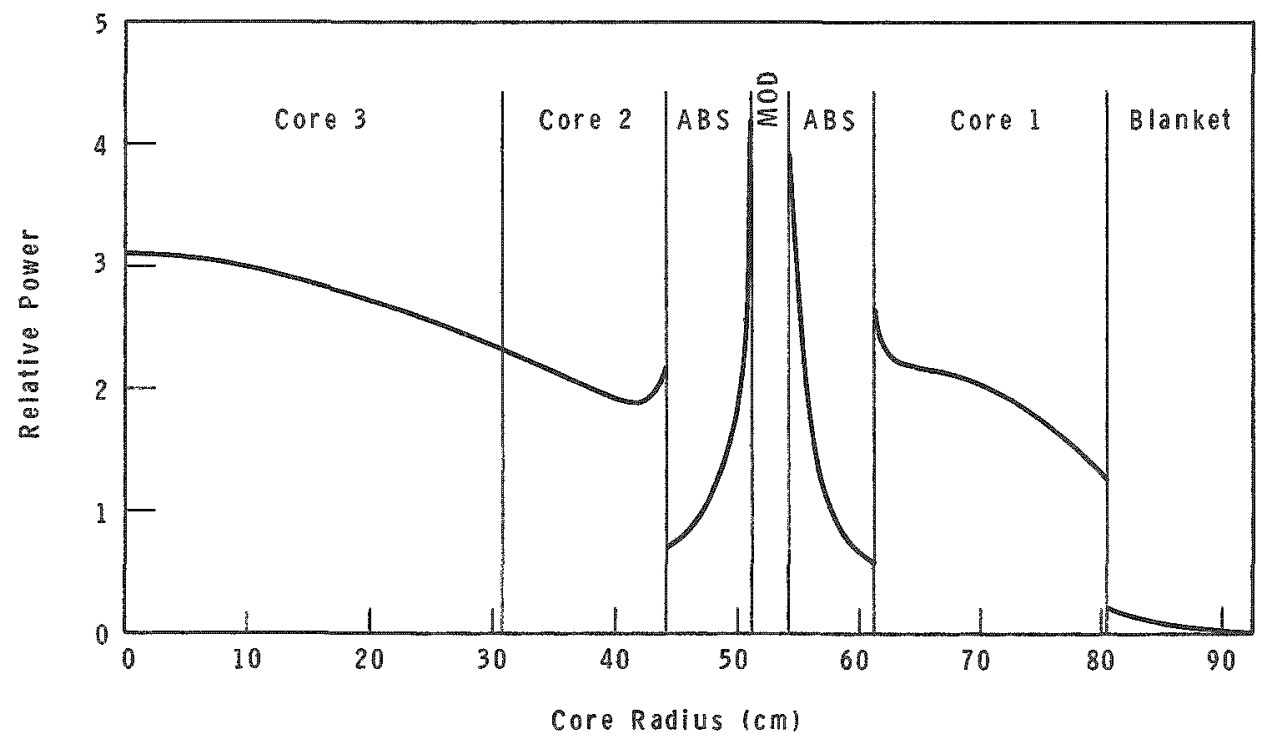

Fig. 8. Power Distribution 
For cores larger than the reference design, additional subdivision may be utilized to maintain safe coolant kinetics. There appears to be no size limitation as in the case for designs which employ more conventional solutions to the coolant-coefficient problem. Application of moderator segmenting to sodium-cooled reactor designs would appear attractive in size ranges of $1000 \mathrm{MW}(\mathrm{e})$ or larger, where the "pancake" design becomes limited because of high axial leakage and decreased Doppler coefficient.

It is clear that the use of supercritical pressure water as a fast reactor coolant results in an extreme degree of spectral degradation. Although this condition brings about a decrease in breeding ratio, the resulting power costs are not unattractive if a reliable fuel design can be achieved. The development of an internally cooled fuel element of the type proposed rests largely on the capability of the coolant tubes to retain their high strength throughout the core life. The second major design problem, that of achieving safe coolant reactivity coefficients, has been effectively solved.

VI. Acknowledgements

The contributions of D. T. Aase, R. J. Hennig, and K. G. Toyoda, Design Analysis Operation, Hanford Laboratories, to this study are gratefully acknowledged.

\section{$\underline{\text { References }}$}

1. G. Sofer et al., Conceptual Design and Economic Evaluation of a Steamcooled Fast Breeder Reactor, NDA-2148-5 (Nov 15, 1961).

2. G. V. Brynsvold et al., Conceptual Design for a $75 \mathrm{MWe}$ Mixed Spectrum Superheating Reactor Power Plant, GEAP-4016 (Feb 25, 1962).

3. H. Harty et al., Economic Evaluation of a 300-MW(e) Supercritical Pressure Power Reactor, HW-68420 Rev. (June 1961).

4. S. N. Tower et al., $1000 \mathrm{MWe}$ Supercritical Pressure Nuclear Reactor Plant Study, WCAP-2042 (July 1962).

5. A. A. Bishop, L. E. Efferding, and L. S. Tong, A Review of Heat Transfer and Fluid Flow of Water in the Supercritical Region and during "OnceThru" Operation, WCAP-2040 (Dec 1962).

6. Staff of Design Analysis Operation, Economic Evaluation of a $300 \mathrm{MW}(\mathrm{e})$ Fast Supercritical Pressure Power Reactor, HW-78953, to be published.

7. H. H. Hummel et al., Using Plutonium in Fast Reactors, Nucleonics, 2I(1) (Jan 1963). 


\section{Discussion of Paper \\ Presented by Mr. Peterson}

MR。OKRENT (Argonne):

We have heard that as the reactor got bigger, the presence of nickel hurts the breeding ratio appreciably. You were concerned with only a 300-MW electrical reactor, I believe.

MR, PETERSON:

That is right. We have not made size comparisons.

MR. OKRENT:

Your breeding ratio was only about 1.1, so one might guess that in the thousand megawatt electrical class the breeding gain would disappear.

MR. PETERSON:

I am afraid I cannot say for sure. The particular core design is a rather complex arrangement, and it is a very difficult thing to make extrapolations based on just the one case. We are looking into this, however.

MR. WOLFE (General Electric):

I would like to comment on Mr. Edlund's comment on the previous paper regarding the loss of coolant. We have been working on a mixedspectrum superheater which has in the center a steam-cooled fast reactor. We agree with Mr. Hafele and the present speaker on the effect of loss of coolant. We find that if you get too big and if you lose your coolant, you do get a positive reactivity change. If you flood, then we find Edlund's effect. Due to the high fission cross section of plutonium, we get a positive flooding effect. Our solution was to use epithermal poisons.

In this mixed spectrum superheater, we found it quite cumbersome to maintain power shapes throughout life. I noticed that in the segmented core described by the speaker that the biggest powex spikes seemed to be right at the edge of the moderator region. Could Mr. Peterson comment please?

MR。PETERSON:

Yes. This need for minimizing the power gradient is probably the most serious objection to the core design discussed. 
MR, WOLFE:

Not only the power gradient, but the actual magnitude.

MR, PETERSON:

Yes, the peak to average is very poor, too.

MR. TOWER (Westinghouse):

Would you elaborate on the primary coolant water density and temperature conditions?

MR. PETERSON:

The inlet pressures are about $4,000 \mathrm{psi}$, at an inlet temperature of around $800^{\circ} \mathrm{F}$. It is a direct-cycle system with two stages of reheat.

MR. MacMILLAN (Babcock \& Wilcox):

Your 3-zone core is a rather complex core arrangement. Were you driven to this in ordex to have both a negative coolant coefficient in the operating range and a negative reactivity associated with the flooding of the core?

MR. PETERSON:

For our particular volume fractions, flooding never was a problem, but voiding certainly was. The sole reason for going to this design was the void coefficient problem.

MR. MacMILIIAN:

I think you mentioned that you had looked at the possibility of increas ing volume fraction of water, and had passed this up because of the problem of very definite positive reactivity associated with it.

MR. PETERSON:

That is right.

MR. EDLUND (Babcock \& Wilcox):

I noticed that in your inner region you had a median fission energy of about $80 \mathrm{kV}$. I made a rough calculation here: if you increase your water by roughly 30 to 50 per cent, then this median fission energy goes down quite quickly to around 30 to $40 \mathrm{kV}$. That relatively small shift in the spectrum does produce the rather strong negative coolant coefficient. I think really it is a function of the design of the lattice and how you play with the spectrum. You can get any of these effects. I think everybody is right. 
MR, KAZI (United Nuclear):

I would like to know what kind of calculations were done?

MR. PETERSON:

We used 18-group diffusion theory calculations with the resonance region treated in a little more detail than the very fast part of the spectrum. We did verify some of the diffusion theory results with transport theory. We were particularly concerned when we got down to very small thicknesses of the flux trap in the absorber regions. The transport theory results, in general, seem to indicate that we were underestimating the multiplication of the system, but this is a strong function of cross section.

MR. KAZI:

Have you looked at local voiding effects?

MR. PETERSON:

To some degree. We have not carried them out nearly to the extent that we have seen in the papers on the sodium-cooled systems, particuiarly the work that APED is doing. But it appeared that voiding the smaller core regions, the subregions, did not introduce positive effect.

MR. KAZI:

We have also looked at steam cooled void effects. In general we agree with Dr. Edlund that apparently one can go to fairly large volumes and still have a negative power coefficient.

MR. WOLEE:

Thexe were a couple of small points that we uncovexed in this mixedspectrum study that might be of interest. Refueling under water, which is an advantage, does pose some difficulties. For example, if you pull out one of the fast elements. you leave a water hole which can give you reactivity additions of as much as 5 or 6 per cent $\Delta \mathrm{k} / \mathrm{k}$ under certain conditions. We got around that by making the can around the fuel element with cadmium or boron, but it was an extra complication.

Also, when you refuel under water, you are essentially refueling in the dark in terms of the reactivity of the operating core. If you have a situation such as Mr. Peterson showed where you are heavily subcritical when you $1100 d$, as you unflood you are increasing reactivity. And there is no way that you can easily test to see if you have loaded to the desirable excess reactivity, when it is unflooded. 
MR, PETERSON:

Yes, this is certainly right. It appears that one would certainly have to take the refueling method into account, particularly here where fuel elements are quite massive. 


\section{MOLTEN-SALT FAST REACTORS}

I. G. Alexander

Oak Ridge National Laboratory

Oak Ridge, Tennessee

Thorium, plutonium, and uranium chlorides and fluorides are soluble in mixtures of the halides of Li, Be, $\mathrm{Na}, \mathrm{K}, \mathrm{Mg}$, and other metals. Their fluoride solutions, at least, are compatible with INOR (an alloy consisting primarily of nickel). They are also compatible with graphite, a structural material as well as a moderator having many desirable properties for high-temperature application. Thus, many embodiments are possible for molten-salt reactors, ranging from simple one-fluid, oneregion systems externally cooled to complex internally cooled, tworegion, two-fluid systems. The capabilities of only a few of the more obvious systems have been studied so iar.

The nuclear and economic potentials of several thermal reactors have been evaluated heretofore, $(1-3)$ and recently a limited program for the preliminary evaluation of fast molten-salt reactor concepts was insituted. Appropriate background studies were performed: (1) a survey was made of data available about the thermal and physical properties of molten fluoride and chloride salt mixtures, (2) the compatibilities of selected reactor materials with various reactor coolants and with moltensall fuels were studied, (3) potential procossing methods for irradiated molten fluoride and chloride reactor fuels were reviewed, and (4) data available for the nuclear properties of chlorine, nickel, and other nuclides or interest wore reviewed.

The compositions and physical properties of typical molten-salt reactor fuels in various reactor concepts are compared in Table 1.

Table 1

COMPARISON OF TVPICAL. MOLTEN-SALT RLACTOR FUELS

\begin{tabular}{|c|c|c|c|}
\hline & $\begin{array}{l}\text { Thermal Conserter } \\
\text { Reactor }\end{array}$ & $\begin{array}{l}\text { Thermal Breedier } \\
\text { Reactor }\end{array}$ & $\begin{array}{l}\text { Fast Breeder } \\
\text { Reactor }\end{array}$ \\
\hline Regions & 1 & 2 & 2 \\
\hline Fluiw streams & 1 & 2 & 2 \\
\hline Moterator & Graphite & Graphite & None \\
\hline \multicolumn{4}{|l|}{ Fissile mixture } \\
\hline Components, m/o & $\begin{array}{l}67 \mathrm{LiF}, 23 \mathrm{BeF}_{2} \\
9 \mathrm{ThF}_{4}, 1 \mathrm{UF} 4\end{array}$ & $\begin{array}{l}63 \mathrm{LiF}, 36 \text { BeF2, } \\
\text { I UF }\end{array}$ & $\begin{array}{l}45 \mathrm{NaCl}, 25 \mathrm{KCl} \\
30 \mathrm{IPu}^{2} \mathrm{U}^{238} \mathrm{Cl}\end{array}$ \\
\hline Liquidus temperature, ${ }^{\circ}$ & 897 & 849 & $11000=500^{2}$ \\
\hline Density, $1 \mathrm{~b} / \mathrm{ft}^{3}$ at $1200^{\circ}$ & 190 & 120 & $1192 \div 10 \%$ \\
\hline $\begin{array}{l}\text { Heat capacity at } 1200^{\circ} \text {. } \\
\text { Btu/b- }{ }^{\circ} \text {. }\end{array}$ & 0.4 & 0.5 & $10.3=0.033^{\circ}$ \\
\hline $\begin{array}{l}\text { Thermal conductivily at } 1200^{\circ} \text {, } \\
\text { Btu/hr-ft }{ }^{-2} \text { F }\end{array}$ & 2.9 & 3.5 & $\left(\begin{array}{c}0.4+0.4 \\
-0.1\end{array}\right)$ \\
\hline 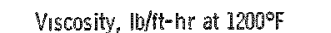 & 21 & 20 & $\left(16 \div 27^{\circ}\right.$ \\
\hline
\end{tabular}

*Values estimated by semitneoretical correlations and by mierenas from measurements on homologous systems. 
Molten-salt Reactor Technology

A molten-salt reactor program was initiated at the Oak Ridge $\mathrm{Na}-$ tional Laboratory in 1957 to exploit, for purposes of economic civilian power, the technology of molten-salt fuels developed at ORNL in connection with the Aircraft Nuclear Propulsion (ANP) project. Molten-salt fuels were conceived originally as a means of satislying the requirements for very high temperature and extremely high power density necessary for aircraft propulsion, and a large amount of work on the physical, chemical, and engineering characteristics of uranium-and thorium-bearing molten fluorides was carried out as part of the ANP program.

The technology of molten-salt reactors was first introduced into the open literature in 1957 by Briant and Weinberg. (4) Bettis et al. (5,6) and Ergen et al. (7) reported on the Aircraft Reactor Experiment, a berylliummoderated reactor fueled with $\mathrm{UF}_{4}$ dissolved in a mixture of the fluorides of sodium and zixconium, and contained in Inconel. The reactor was successfully operated in 1954 for more than $90,000 \mathrm{kWhr}$ without incident at thermal powers up to $2.5 \mathrm{MW}$ and temperatures as high as $1650^{\circ} \mathrm{F}$.

For thermal power reactor fuels, fluorides of $\mathrm{Li}^{7}$ and beryllium are preferred because of their low parasitic neutron capture rate. Mixtures of these with fluorides of thorium and uranium (in the range from 5 to $15 \mathrm{~m} / \mathrm{o}$ ) have liquidus temperatures below $1000^{\circ} \mathrm{F}$, have good heat transfer properties, and are nearly inert with respect to INOR, the preferred container material.

In fast reactors, sodium and potassium may be used in place of $\mathrm{Li}^{7}$ and beryllium, which are costly. Fluorine may be a suitable anion for use with $\mathrm{U}^{233}$ in a fissile stream, but for plutonium fuels, chloride mixtures are required to obtain sufficiently high concentrations of plutonium at temper. atures below $1000^{\circ} \mathrm{F}$. Another important advantage of chlorine is that it has considerably less moderating power than fluorine. On the other hand, $C 1^{35}$ exhibits a disadrantageous $(n, p)$ reaction, and it is necessary to use the separated isotope $\mathrm{Cl}^{37}$ contaminated with less than $5 \% \mathrm{Cl}^{35}$. Since it is not so important to obtain a hard spectrum in the blanket and plutonium concentrations a never high there, fluoride mixtures are satisfactory for the fertile stream.

The potential usefulness of molten-salt fuels for civilian power was recognized from the start. The features that attracted attention were the high temperature at which the fuel could be used (permitting use of modern stream technology and attainment of high thermal efficiency), combined with a low vapor pressure, the unsurpassed stability of halide salts under radiation, and the usual advantages that a fluid fuel provides. These include a negative temperature coefficient of reactivity, absence of the need for initial excess reactivity and its wastage in control elements, no 
limitation to burnup by radiation damage or loss of reactivity, the absence of a complicated structure in the reactor core, removal of the heat-transfer operation from the core to an external heat exchanger, and the potential for a low-cost fuel cycle.

The ORNI molten-salt reaclor program(8) has comprised a reactor evaluation program for selecting the most promising concepts for civilian power and for pinpointing specific development problems; an extensive ma terials development program for fuels, containers, and moderators; an equally extensive program for the development of components, especially pumps, valves, and flanges suitable for extended use with molten salts at $1300^{\circ} \mathrm{F}$; a modest program for the discovery of supplementary chemical processes for recovering valuable components (other than uranium) from spent fuel; and a program for the development and definitive demonstration of the feasibility of maintenance of molten-salt reactor systems.

These programs have substantially reached their initial goals, (1) and the work is now mainly devoted to the design and construction of a molten-salt reactor experiment, $(9,10)$ which will demonstrate the utility of the developments achieved and resolve remaining areas of uncertainty. The MSRE will produce up to $10 \mathrm{MW}$ of heat in a fuel consisting of a solution of highly enriched $\mathrm{U}^{235} \mathrm{~F}_{4}$ dissolved in a mixture of the fluorides of lithium (99.990\% $\mathrm{Li}^{7}$ ), beryllium, and zirconium, having a liquidus temperature of $842^{\circ} \mathrm{F}$. Construction and installation of the entire system are scheduled for completion in mid-1964, and criticality is planned for early in 1965.

System Maintenance

Because of fission-product contamination and induced activity in components and piping, the fuel-containing portions of molten-sall reactors cannot be approached for direct maintenance even after draining and llushing. Semidirect maintenance with long-handled tools through a shield plug is possible for many items, however, and completely remote tools and methods can be employed where needed.

Semidirect maintenance procedures for circulating-fuel reactors were used with success in the repair and maintenance of HRE-2. (11) The techniques of fully remote maintenance were demonstrated at ORNL(12) in the Molten-salt Reactor Remote-maintenance Development Facility, which was a mockup comprising a reactor vessel, heat exchangers, pumps, valves, flanges, heaters, instruments, etc., for a $20-\mathrm{MW}$ reactor. All pieces of equipment in the facility were removed, replaced, and tested remotely both before and after circulation of salt through the system at $1200^{\circ} \mathrm{F}$. 
Chemical Processing of Fluid Fuel

The use of fluid fuels in nuclear reactors provides an opportunity for continuously removing fission products and replacing fissile isotopes at power. Thus, it may be possible to hold losses of neutrons to fissionproduct poisons at low levels, on the one hand, and yet not be forced to waste neutrons by capture in control rods, such as those used in solid-fuel reactors to compensate for initial excess reactivity. Volatility processing, (13) wherein the relative volatilities of the halides of the highvalent states of uranium and plutonium are used to effect separation, is uniquely suited to molten salts. The associated fluorination process is in an advanced stage of development, and a pilot plant for general application is now in operation at ORNL. (14) Volatilization and recovery of chlorides of plutonium and uranium have been studied by Naumann, (23) who proposed a complete process starting with oxide fuels.

Although the fluoride-volatility process was not developed specifically for use with molten-salt fuels, it has been verified in laboratory experiments conducted at ORNL that uranium may be recovered from fluoride mixtures containing $\mathrm{ThF}_{4}$. In this process, elemental fluorine, perhaps diluted with an inert gas, is bubbled through the salt. The UF 4 is converted to $\mathrm{UF}_{6}$, which is volatile at the temperature of operation $\left(500-700^{\circ} \mathrm{C}\right)$ and passes out of the contactor, to be absorbed reversibly in a bed of NaF. The off-gas is cooled and passed through charcoal beds, where fission-product gases are absorbed. The fluorides of a few of the fission products are also volatile, but these are irreversibly absorbed in the NaF beds. Thus, by heating the beds, $\mathrm{UF}_{6}$ is removed in a very pure state, with losses less than $0.1 \%$.

The $\mathrm{UF}_{6}$ may be reduced to $\mathrm{UF}_{4}$ in a hydrogen-fluorine flame and collected as a powder in a cyclone separator backed up by gas filters. This process is quantitative, the losses characteristically being smaller than random errors in the assays, and it has been used successfully for many years in the manufacture of enriched $U^{235}$ from natural uranium in the production plants at Oak Ridge.

Plutonium is not volatilized until all of the uranium has been removed, and large volumes of fluorine are required to bring it over because of an unfavorable equilibrium condition. (24) However, plutonium has been recovered successfully $(25)$ from salt mixtures containing as little as 2 ppm of plutonium.

The recovery of $\mathrm{Cl}^{37}$ from salt mixtures by fluorination has not been studied heretofore; however, fluorine readily replaces chlorine in ionic compounds, and the recovery and separation of $\mathrm{Cl}^{37}$ from excess fluorine does not appear to present any unusual difficulties. 
Reactor Container

The development of nickel-molybdenum base alloys (INOR series) for containment of molten fluorides was conducted jointly by ORNL and several subcontractors. In addition to resistance to corrosion, these alloys have good-to-excellent mechanical and thermal characteristics (superior to those of many ausienitic stainless steels), and they are virtually unaffected by long-term exposure to fluoride salts or to air at $1300^{\circ} \mathrm{F}$. (15) Exhaustive tests at ORNL have shown that the tensile properties, ductility, creep strength, and cyclic fatigue strength (both thermal and mechanical) are adequate formolten-salt reactor applications when judged in accordance with criteria used in the ASME Boiler Code. INOR alloys are weldabie by conventional techniques with welding rods of the same composition as the base metal, and a gold-nickel alloy suitable for remote brazing of reactor components has been developed at ORNL. INOR alloys have been made by several major manufacturing companies. These alloys are presently available on a limited commercial basis in the form of tubing, plates, bars, forgings, and castings.

Although the compatibility of INOR with chlorides remains to be established, it is believed that corrosion will not be severe provided the trichlorides of plutonium and uranium are used, and temperatures do not exceed $1300^{\circ} \mathrm{F}$.

Fast Mollen-salt Reactors

The use of a fluid fuel makes possible a number of economies in fuel utilization and reactor operations. Fabrication consists in compourid. ing the raw materials and purifying these at the reactor site; the cost is small. Fresh fuel may be added and spent fuel withdrawn without interruption of power; thus, plant availability is determined only by the maintenance schedule and not by the fuel cycle. Valuable components of spent fuel may be recovered on-site, simply and economically, by volatilization with chlorine or fluorine. This makes rapid processing attractive and obviates the necessity, found in solid-fuel reactors, of oblaining high incore breeding ratios in order to extend the fuel life. Thus, the fluid-fuel reactors have an additional degree of freedom in design, and as much of the breeding may be accomplished in the blanket as appears desirable. This need not be accomplished by a high blanket inventory of fissile isotopes, however, since a thorium-fluoride blanket may be stripped continuously of bred material by volatilization with fluorine. No additional processing is required, and the stripped salt may be recycled indefinitely. Since the fissile isotope concentration is very low, fission-product accumulation is negligible. It should be emphasized that these characteristics of $\mathrm{ThF}_{4}$ solutions confer an enormous fuel-cycle cost advantage, which, taken with the simplicity of the processing cycle for recovery of uranium or 
plutonium and $\mathrm{Cl}^{37}$ from the fuel stream by volatilization with fluorine, will, it is believed, result in negligibly small processing costs in on-site processing plants.

Continuous processing results in steady-state operation: the power load does not shift back and forth between the blanket and the core, and the power distribution in the core does not vary with time. Hence, all parts of the reactor may be designed for maximum performance over the life of the reactor.

In regard to safety, the fast fluid-fuel reactors have the advantage of an inherently negative contribution to the temperature coefficient of reactivity resulting from thermal expansion of the fuel. Also, since the fuel is already in a molten state, "fuel slumping" in the sense associated with solid fuels is impossible. In a loss-of-coolant accident, the possibility exists that the fuel can be drained away to dump tanks where the reactivity may be controlled and the afterheat removed conveniently.

That molten salts are not difficult to contain has been adequately demonstrated at ORNL, where a highly successful record of handling these materials at high temperatures in radiation fields has been established. Of course, hazards arising from the mobility of the fuel, such as the accidental accumulation of a supercritical mass of fuel, must be considered in the detailed design of fluid-fuel reactors.

A fastmolten-chloride reactor was proposed by Goodman el al. (16) in 1952, and the chemical problems involved in the use of such fuels were reviewed by Scatchard et al. (17) Also, a design study and evaluation of a molten-chloride fast breeder was prepared by Bulmer et al. (18) at the Oak Ridge School of Reactor Technology in 1956. Bulmer chose chloride mixtures in preference to fluorides because of the higher moderating power of the latter, but he noted that enrichment in $\mathrm{Cl}^{37}$ would be required because of the strong $(n, p)$ reaction in $\mathrm{Cl}^{35}$. More recently, the suitability of various molten plutonium salts for use in fast breeders was reviewed by M. Taube (19-21) of the Institute of Nuclear Research, Warsaw. Taube's principal conclusion was that $\mathrm{Cl}^{37}$ is the only anion that satisfies all the rerequirements for fast plutonium-fueled molten-salt reactors. On the other hand, it is believed at ORNL that fluoride salts may prove to be advantageous for use with $U^{233}$, in spite of the extra moderation, and that the use of ThF ${ }_{4}$ dissolved in a mixture of alkali fluorides would have outstanding advantages in the blankets of fast reactors.

Both internally and externally cooled systems are being studied at ORNL. The first kind avoids the disadvantage of having appreciable fuel inventories "idling" outside the reactor; the second avoids cluttering the core with heat-transfer surfaces. 
Structural Material. The leading candidates are INOR-8 (an alloy of nickel with molybdenum, iron, and chromium), niobium, and $\mathrm{Mo}^{92}$ (a separated isotope). The order of listing reflects both availability and cost. Preliminary calculations indicate that niobium will probably satisfy both the nuclear and thermal requirements; INOR may also, with some sacrifice in nuclear performance, tue to the (possibly) higher parasitic resonance capture in nickel.

Choice of Coolant. Although sodium is compatible with these metals, it reacts strongly (though not explosively) with fuel salt, yielding metallic plutonium and uranium. For this reason, and also because of its moderating effect and because of the difficulties associated with positive void coefficients of reactivity, etc., sodium was passed over in favor of helium at $1000 \mathrm{psia}$ and $1100^{\circ} \mathrm{F}(\mathrm{max})$ for cooling. The principal disadvantage is the high pressure required, which increases the probability of loss of coolant by rupture of the gas system. However, the mobility of the fuel contributes to the abatement of the loss-of-coolant hazards, since it may be possible to drain the fuel to cooled ciump tanks.

Configuration. In order to shorten the flow path of cooling gas, the arrangement illustrated in Fig. I was chosen. The fuel salt is contained in a calandria, 1-1/2 to $5 \mathrm{ft}$ thick and $5 \mathrm{fthigh}$, and as long as

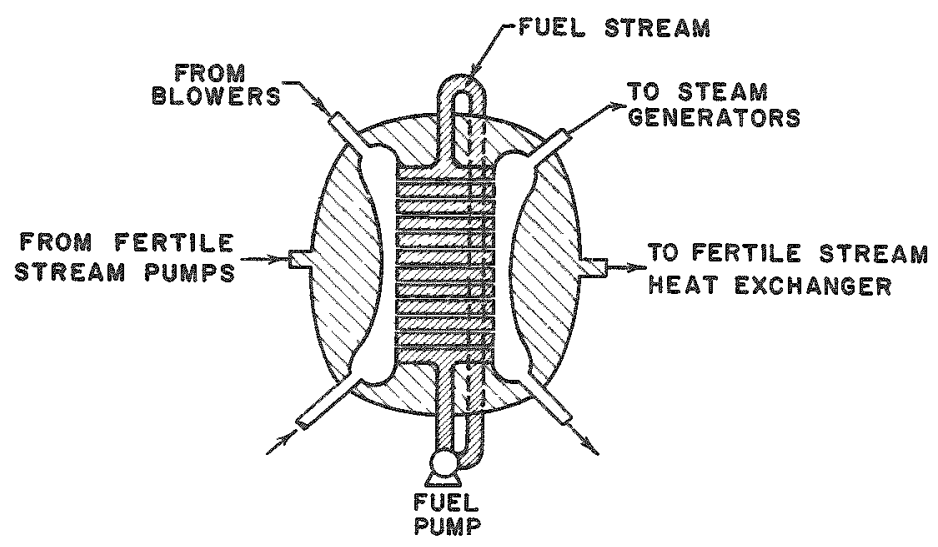

Fig. 1. Internally Cooled Fast Moltensalt Reactor required to obtain the desired power capability. The calandria is penetrated laterally by gas passages (tubes or flat plates), $1 / 4$ to $1 / 2 \mathrm{in}$. wide, and fuel passages, 0.10 to 0.20 in. thick with walls $0.025 \mathrm{in}$, thick, between adjacent passages. Since flow of heat in the salt by conduction alone would result in temperatures exceeding $2000^{\circ} \mathrm{F}$, convection is achieved by circulating the salt in turbulent flow by means of an external pump.

Fuel Stream. A mixture of $\mathrm{NaCl}$ and $\mathrm{KCl}$ with 30 to $50 \mathrm{~m} / \mathrm{o}$ $\left(\mathrm{Pu}, \mathrm{U}^{238}\right) \mathrm{Cl}_{3}$ was chosen. The liquidus temperature is expected to be less than $1000^{\circ} \mathrm{F}$ and the density about $3.1 \mathrm{~g} / \mathrm{cc}$ at $1200^{\circ} \mathrm{F}$. The anion is $95 \%$ $\mathrm{Cl}^{37}$. Isotopes of plutonium, uranium, and $\mathrm{Cl}^{37}$ are recovered by fluorination; the residue contains fission products and is discarded. 
Fertile Stream. The ternary eutectic of the fluorides of sodium, potassium, and thorium is circulated through the blanket region. Bred $\mathrm{U}^{233}$ is removed by fluorination with a cycle time of 10 days. The fertile stream is recycled inderinitely without other treatment.

Status of the Study. The limiting values or ranges given above for several parameters were tentatively established from prior experience and probable inference. The operating conditions selected were a maximum metal temperature of $1300^{\circ} \mathrm{F}$ (to limit corrosion rates and maintain mechanical properties), a maximum salt temperature of $2000^{\circ} \mathrm{F}$ (to avoid thermal shock and mitigate hot-spot conditions), a minimum inlet gas temperature of $600^{\circ} \mathrm{F}$ (to maintain thermal efficiency in the energy conversion cycle and to avoid thermal stress and reduce likelihood of freezing iuel salt near the gas inlet of the core), a maximum ratio of work expended in pumping gas through the reactor core to electrical output of 0.06 , and a maximum pressure drop of 25 psi across the core. Thermal and nuclear calculations are in progress to find the optimum combination of core thickness, volume fraction of fuel, composition of fuel, inlet gas temperature, etc. The primary figure-of-merit is the fuel-cycle cost, with subsidiary figures being the doubling time and its components: breeding ratio and specific power.

The heavy-metal atom density in molten salts suitable for fast $r e=$ actor use is lower than in oxide fuels by a factor of 4 . The moderating power, as defined in Table 2, of the materials in the core is an important quantity, as this determines the spectrum of neutrons and the effective $\eta$ obtainable. In this connection, molten salts for fast reactors may be handicapped by heat-transfer requirements (limited metal and salt temperature) which, in gas-cooled reactors, may force the use of relatively large heat-transfer surfaces and thus large ratios of atoms of structural material to fuel atoms. Thus, it may be difficult to maintain a hard spectrum and obtain a high $\eta$ in an optimized gas-cooled fast molten-salt reactor. A corollary consequence of the soft spectrum is that the para. sitic absorption in carrier salt and structural materials is more difficult to control, especially since nickel, the major component in the preferred alloy for containing molten salts, appears to have some extremely noxious resonances in the range of neutron energies lying between 10 and $100 \mathrm{keV}$. However, the degradation of the spectrum may be no more deleterious than that suffered in sodium-cooled reactors when provision is made to reduce the sodium void coefficient and preserve the Doppler effect by the addition of BeO, for example.

Externally Cooled Reactors

An important advantage of fluid fuels is that the heat-transfer oper ation may be performed outside the reactor by circulating the fuel to an external heat exchanger. Although this permits designing to meet the 
nuclear and thermal requirements independently, it has the disadvantage of having at least half the inventory of fuel materials outside the core. It is easy to see, by reference to Table 2, that the breeding ratio of an externally cooled fast reacto $r^{\circ}$ core will be good, even in the presence of atoms of carrier salt, and that the problems are those of designing for stable flow in the core and adequate heat transfer in the heat exchanger to obtain favorable specific powers.

Table 2

COMPARISON OF MODERATING POWER IN

SODIUM-COOLED AND OXIDE-FUELED

AND MOLTEN-SALT REACTORS

$N=$ atomic density of listed element

$\mathrm{N}_{0}=$ atomic density of $\mathrm{Pu}+\mathrm{U}$

\begin{tabular}{l|c|c|c}
\hline Element & $N / N_{0}$ & $\xi_{S}$ & $N \sigma_{S} / N_{0}$ \\
\hline \multicolumn{3}{c|}{ Sodium-cooled Oxide-fueled Reactor } \\
\hline Oxygen & 2.1 & 0.34 & 0.71 \\
Sodium & 1.8 & 0.26 & 0.47 \\
Stainless steel & 2.1 & 0.10 & 0.21 \\
$\quad$ Total & & & 1.39 \\
\hline
\end{tabular}

Externally Cooled Molten-salt Reactor

\begin{tabular}{l|l|l|l}
\hline $\mathrm{Cl}^{37}$ & 5.3 & 0.11 & 0.59 \\
Sodium & 1.5 & 0.26 & 0.39 \\
Potassium & 0.8 & 0.10 & $\underline{0.08}$ \\
$\quad$ Total & & & 1.06 \\
\hline
\end{tabular}

Since in-core breeding is not required to obtain long fuel-life, fertile materials may be excluded from the core (unless required to maintain a negative temperature coefficient). This lowers the required concentration of fissile isotopes, as does also the absence of structural material and coolant. Thus, the inventory in the circulating system may be quite low in relation to the power level and high specific powers attainable.

The same components in the fuel and fertile streams are used as for the internally cooled reactor, and the processing cycles are the same. The core, of course, contains no structure, and INOR is probably suitable for separating the core from the blanket. The fuel stream is circulated through an external shell-and-tube heat exchanger. 
Should a "conventional" exchanger require too great a volume of fuel salt and result in inventories too high to be economically attractive, E. S. Bettis (26) has proposed to cool and pump the fuel stream by spraying lead or mercury, which are chemically and nuclearly compatible with the fuel salt, into downcomers located outside the blanket. This arrangement is similar to that proposed for LAMPRE by Hammond et al. (22) and hopefully will result in total inventories no more than twice the in-core inventories and specific powers of $1 \mathrm{MW} / \mathrm{kg}$ or more.

The study is presently concerned with establishing upper limits on the power density in the core and the external circuits. With these in hand, the optimization of the core thickness, uranium loading, etc., will proceed.

\section{Conclusions}

In summary, the advantages claimed for molten-salt fast reactors are: (1) simplicity of the reactor core and the semicontinuous fuel handling apparatus, which lead to low capital costs and increased plant availability; (2) simplicity and continuous nature of fissile and fertile stream processing methods, which lead to negligible fuel-cycle costs in on-site plants; (3) improved safety resulting from the negative contribution to the temperature coefficient of reactivity inherent in the thermal expansion of the fuel;

(4) competitive nuclear performance of the internally cooled reactor and superior performance of the externally cooled reactor.

\section{Acknowledgemenis}

This report was based on contributions from E. S. Bettis, W. I. Carter, J. I. Wantland, R. E. Thoma, T.W. Pickel, and others. The comments and suggestions of $\mathbb{M}$. W. Rosenthal, A. M. Pexry, J. A. Lane, R. B. Briggs, S. E. Beall, and H. G. MacPherson are gratefully acknowledged.

\section{References}

1. J. A. Lane, H. G. MacPherson, and F. Maslan (Eds.), Chapters 11-17 in Eluid Fuel Reactors, Addison-Wesley, Reading, Mass. (1958).

2. L. G. Alexander et al., Thorium Breeder Reactor Evaluation, PartI Fuel Yield and Fuel Cycle Costs in Five Thermal Breeders, ORNL CF-61-3-9 and Appendices (March 1961).

3. I. G. Alexander et al., Cost of Power from a 1000-MWe Molten-salt Converter Reactor, report in preparation, Oak Ridge National Laboratory. 
4. R. C. Briant and A. M. Weinberg, Molten Fluorides as Power Reactor Euels, Nucl. Sci. Eng., 2, 797 (1957).

5. E. S. Bettis et a1. The Aircraft Reactor Experiment - Operation, ibid, 2, $841(1957)$.

6. E. S. Bettis et al., The Aircraft Reactor Experiment - Design and Construction, ibid. 2,804 (1957).

7. W. K. Ergen et al., The Aircraft Reactor Experiment - Physics, ibid., $2,826(1957)$.

8. H. G. MacPherson, Molten Salts for Civilian Power, ORNL CF-57-10-41 (Oct 1957).

9. A. L. Boch, "The Molten-Salt Reactor Experiment," Power Reactor Experiments, Vol. I, pp. 247-292, International Atomic Energy Agency, Vienna (1962).

10. S. E. Beall et al. Molten-salt Reactor Experiment Preliminary Hazards Report, ORNL CF-61-2-46 (Add. 1 and 2) (Feb 1961).

11. J. A. Lane, H. G. MacPherson, and F. Maslan, op. cit., Chapters 1-10.

12. W. B. McDonald and C. K. McGlothlan, Remote Maintenance of Moltensalt Reactors, ORNL-2981 (in preparation).

13. D. O. Campbell and G. I. Cathers, Processing of Molten-salt Power Reactor Fuels, Ind. Eng. Chem, 52, 41 (Jan 1960).

14. W. H. Carr, S. Mann, and E.C.Moncrief, Uranium-Zirconium Alloy Fuel Processing in the ORNL Volatility Pilot Plant, ORNL CF-61-7-13 (July 10, 1961).

15. I. H. DeVan and R. B. Evans, III, Corrosion Behavior of Reacior Materials in Fluoride Salt Mixures, ORNI TM-328 (Sept 19, 1962).

16. C. Goodman et al., Nuclear Problems on Non-aqueous Fluid-fuel Reactors, MIT-5000 (Declassified) (Oct 1952).

17. G. Scatchard et al. Chemical Problems of Non-aqueous Fluid-fuel Reactors, USAE $\bar{C}$ Report MIT-5001 (Declassified) (Oct 1952).

18. J. J. Bulmer et al., Fused Salt Fast Breeder, ORNL CF-56-8-204 (Declassified) (Aug 1956). 
19. M. Taube, Plutonium Fused Salts Fuels for Fast Breeder Reactor: Nuclear and Chemical Criterion, Nukleonika, 6, 565 (1961).

20. M. Taube, Molten Plutonium and Uranium Chlorides as Fuels for Fast Breeder Reactors," Proceedings of a Symposium on Power Reactor Experiments, Vienna, 1961, Vol. 1, pp. 353-363 (1962); see also USAEC Translation AEC-tr-5161.

21. M. Taube, Stopione Chlorki Plutonu i Uranu Juko Paliwo Dla Predkich Reaktorow, Report No, 414/V, Instytut Badan Judrowych, Warszawa, Poland (March 1963).

22. R. P. Hammond et al., Mobile Fuel Plutonium Breeders, LA-2644 (Dec 1961).

23. D. Naumann, Laborstudie zur chlorierenden Aufarbeitung neutronenbestrahlter Urankernbrennstoffe, Kernenergie, 2, 5, 118 (Feb 1962).

24. Oak Ridge National Laboratory, Chemical Technology Division Annual Progress Report for Period Ending May 31, 1963, ORNL-3452 (Sept 1963).

25. G. I. Cathers and R. I. Jolley, Recovery of PuF 6 by Fluorination of Fused Fluoride Salts, ORNL-32 98 (Oct 1962).

26. E. S. Bettis, Oak Ridge National Laboratory, personal communication to L. G. Alexander, 1957.

$\frac{\text { Discussion of Paper }}{\text { Presented by Mr. Alexander }}$

MR. HALL (Los Alamos):

What is your concentration of fissile atoms in the fuel? Is it 50 per cent in the salt?

MR. ALEXANDER:

We believe we can operate in the range of 30 to 50 mole per cent, and this gives a concentration of about $0.004 \times 10^{24}$ atoms/cc of heavy elements in the salt.

MR. HALL:

Is there a strong temperature dependence of solubility in the sali? 


\section{MR, ALEXANDER:}

The range that I gave you, 30 to 50 per cent, means that the liquidus temperature is below $1,000^{\circ}$ for mixtures having that composition. If you want to increase it, then the liquidus temperature goes up.

MR. HALI:

In the case of the external heat exchangers, what fraction of the total fuel would have to be out-of-pile inventory?

MR. ALEXANDER:

Not enough to go critical in the heat exchanger, we hope. Also we hope the external inventory would be no greater than the internal inventory.

MR.DIETRICH:

If there is no further discussion of this paper, let us take this op-portunity for further discussion of yesterday afternoon's session, which was cul a little short.

MR. DAVEY (Argonne):

This pertains to the discussion that Dr. Häfele and Dr. Zaleski (Papex IV-4) were having on the discrepancies in calculations of breeding ratio. That discussion may not be 100 relevant, since there are discrepancies of the order of 25 per cent between the calculated and measured breeding ratios in ZPR. III fast assemblies. There is a large discrepancy beiween the ratio of capture in $U^{338}$ to fission in $U^{235}$. This remains 10 be resolved belore one disputes about computational techniques.

The other point I wish to present pertains particularly to Doppler coefficient, and perhaps to sodium coefficient, and is really a remphasis of the point made by Mr. Thalgott yesterday. It is the question of the knowledge of the neutron spectrum. It has long been known that for the ZPR-III fast assemblies, there are quite strong diferences between the calculated and measured lifetimes. Recently, I analyzed quite a large number of these assemblies. The striking fact that emerges is that the prompt neutron lifetimes as calculated are uniformly about three-quarters of those that are measured. The same is true for the central reactivities of boron-10 samples. Both of these are approximately $1 / v$ spectral indices. These results support each other; and, they both indicate that the experimental spectra are appreciably harder than those calculated using a basically Yiftah, Okrent, Moldaucr set. So I just wish to emphasize that we possibly do not know the low energy spectra with any high degree of accuracy. 
MR. OKRENT (Argonne):

I just wonder if I understood the statement. Did you say that the calculated lifetime is three-quarters of the measured?

MR. DAVEY:

The discrepancy is such that the experimental spectrum is softer than the calculated values.

MR, OKRENT:

I think you used the wrong word.

MR. DAVEY:

This is quite correct. Thank you.

MR. DIETRICH:

Are there any other comments?

MR. FISCHER (Argonne):

When we heard rumors that the $U^{235}$ fission cross section should be reduced, we did some experimental calculations where we purposely fudged the $U^{235}$ fission cross sections in the regions we heard they were to be reduced. The consequence was that the lifetime did go up in just the right direction, but not enough. When we put the $U^{235}$ fission cross sections into the 25 to 28 ratios, however, they got worse.

MR. CAMPISE (Atomics International):

One question that I would like to put to the audience - is it surprising that we cannot calculate the prompt neutron lifetime? For instance, in Yiftah, Okrent, and Moldauer's tabulation on cross sections, they used their cross sections to try and calculate the $\mathrm{BBC}$ experiments. They were unable to reproduce the results, indicating a discrepancy in the inelastic slowing down which would degrade the spectrum and give a larger prompt neutron lifetime. Maybe Dr. Okrent can comment on this.

MR。OKRENT:

Were you commenting on the fission ratio? I missed one or two words. 
MR. CAMPISE:

Yes.

MR。OKRENT:

Well, I have two brief statements on other people's work. First, a recent report from the Idaho Division of Argonne describes remeasurement of the fission ratio in a few assemblies. They became suspicious that inelastic scattering in the counters was changing the spectrum measured from the spectrum in the reactor, and with thin wall chambers they found a shift of about 10 per cent, as I recall, toward what I would categorize as a harder spectrum; in other words, the fission ratios of $\mathrm{U}^{238}$ to $\mathrm{U}^{235}$ would go up. This was just about the discrepancy that had been observed between the $16 \mathrm{group}$ set and measurement, so the agreement suddenly looks perfect. Too perfect.

The other piece of information is that Hummel in his calculation by ELMOE of elastic moderation cross sections, finds that these are really quite poor in the 16 group set because of their admittedly poor treatment of resonances. This correction will increase the amount of low energy flux, so that calculation once again disagrees with measurement. And in the other direction.

MR. CAMPISE:

Maybe I should clarify my statement. I was referring to the Bethe, Beyster and Carter experiments, where they measured inelastic removal from above the $\mathrm{U}^{238}$ and the $\mathrm{Np}^{237}$ threshold to below. In your tabulation, you showed disagreement between these measurements and your inelastic cross sections. One would think that if you were able to obtain agreement, we might get a softer spectrum.

MR。OKRENT:

I do not think we can base too much on that series of measurements. They are interesting, but I would really like to see data between one and one-half and ten $\mathrm{MeV}$, like we saw from 100 kilovolts to one and onehalf MeV. That is what Al Smith refers to as neutron microscopy, where they actually measure the excitation of each individual level. That is really what is needed. The Bethe, Beyster, and Carter measurementswere very handy back in the wilderness, but we are no longer there.

MR, DIETRICH:

I would like to make a more general comment which is that I think that some of us who have been wasting our time on thermal reactors were beginning to be afraid that the reactor physicist had worked himself out of 
a job. If this conference has not been reassuring on anything else, I think we can rest easy on that point. (Laughter.)

MR. HÄELE (Karlsruhe):

To some extent this is a similar remark. We should recognize where we are in the whole business. I think it is the first time that second order effects will decide the whole reactor concept. In reactor theory up to now first order calculations decided on a concept. For example, in the study by General Electric (paper IV-5) yesterday putting in a little bit of beryllium oxide changed the prospect of the whole reactor type completely. Second order effects, that means the lower parts of the spectrum in the resonance region, with its effects on Doppler coefficient and sodium void coefficient, decide the whole situation. There are other examples. Discrepancies in lifetime calculations mostly come from the lower energy; the cross sections are increasing, the neutron velocity is decreasing, and it gives the greatest contribution to lifetime. The remark of Mr. Edlund's about the steam coefficient also emphasizes the resonance region. I think the multigroup concept was created and designed to deal with critical mass questions for hard spectra. If one considers the time and the effort necessary to deal with the resonance region for thermal reactors, in order to predict the $p$ factor properly, and if one compares that with the requirements for fast reactors, then one can really see that a factor of 10 is still ahead of us. I think that $10 \mathrm{groups}$ in the resonance region, with each group prepared with the same care as the $\mathrm{p}$ factor in the thermal reactor, is necessary to resolve these difficulties. What we need is a second order reactor theory for the resonance region. Otherwise we can't resolve these difficulties.

MR. EDWARDS (Dounreay):

If the Chaiman permits me, I would like to go back to the first. paper we had yesterday morning (paper III-1). This concerned particularly the optimum size for a prototype. The comment from Dr. W ${ }^{\circ}$ ight, I think, was that a prototype should be a true prototype and a true model. I should like to ask what the characteristics of such a prototype should be. This exercise has engaged us in Britain on more than one occasion. We know we are interested in a reactor of a thousand or more megawatts thermal. We know we want to build a prototype before this is built. The argument then goes something as follows: So far as kinetics measurements are concerned, we are not al all convinced that measurements on a substantially smaller reactor will be of any value at all in predicting the properties of a prototype. So far as physics goes, many of the properties we should measure on a zero energy reactor, such as Zebra, and not on a prototype power reactor.

So far as fuel element testing goes, it is arguable that fuel elements can be adequately tested on quite a small reactor by using the $U^{235}$ for $U^{238}$ 
substitution. And so far as large scale engineering goes, many things can be tested in large loops out of the reactor.

I repeat the first question, therefore, what is the optimum size for a prototype?

MR. CREAGAN (Westinghouse):

Dr. Wright's comments were based to some extent with our experience on the CVTR water reactor that we have already built. Our experience with that device, which was to be a prototype for a large, natural-uranium, heavy water reactor, illustrated to us the difficulties of prototyping, and incorporating even the mechanical fuel elements and othex flow characteristics of the coolant into the device which would then be pertinent to the later building of a large device. Not only the size change, but the very development in which you are involved changes the eventual device which would be desirable built. I am sure that were we to now build a natural-uranium heavy water reactor, the CVTR would not serve as its prototype.

In the case of a fast reactor, we also have the questions: we do not have the answer.

MR. THOMPSON (MIT):

Everyone is talking about a thousand megawatt device, and talking $100,000 \mathrm{MW}$ days/tonne. These are nice round numbers. But is this irradiation goal proven in a last flux? I wonder, aren't you proposing a great leap forward? Is it really necessary? Isn't there some corc size, like 300 or $500 \mathrm{MW}$, for which the physics problems are much reduced?

MR. COHEN (General Electric):

I think the question is a little bit addressed to me perhaps. The reason we are studying a 1000 megawatt fast reactor now, is that the Atomic Energy Commission has asked us to do so. I think we will all agree that there are many steps required before we get there. No one is seriously proposing to build a thousand megawatt fast reactor as the next great leap forward. The next question is, when do we expect to find out that the fuel has the properties that we believe? I think we have concluded from our experiments on thermal radiations of small samples of mixed oxide fuel that this was a promising concept. I don't think we ever said much more.

MR. BUMP (Argonne):

It seems to me that it may tum out that to produce a thousand electrical megawatts, one finds he is a lot better off using multiple 
reactors rather than one single reactor. This would, in a sense, solve a great many of our problems.

MR. HAFELE:

This is to try to answer the statement of Mr. Thompson. The primary difficulty we see in going from a thermal neutron ir radiation test to a fast neutron irradiation test is the cladding problem. So far as we can see, it is not so much the fuel which suffers from the fast neutron flux, but the cladding material. The dose rate goes up from $10^{21} \mathrm{n} / \mathrm{cm}^{2}$, up to about 5 times $10^{23}$, and that is a very high neutron dose rate of fast neutrons. Our concern is that the cladding material cannot stand this.

MR. DIETRICH:

I think we should take our coffee break now. 


\title{
AN APPLICATION OF MOLTEN PLUTONIUM FUEL TO FAST BREEDERS
}

\author{
W. H. Hannum, G. L. Ragan, and B. M. Carmichael \\ Los Alamos Scientific Laboratory \\ Los Alamos, New Mexico \\ (Paper presented by Mr. Hannum)
}

Introduction

Molten plutonium has been shown by more than two years of operating experience in the Los Alamos Molten Plutonium Reactor Experiment I (1) (LAMPRE I) to be a feasible reactor fuel。 Large breeder systems with molten plutonium as a driver* fuel can be expected to have a favorable breeding potential, and to have certain inherent economic and safety features which may be of significant value

The general concept considered here is one of the more conservative of the approaches being considered for utilizing molten plutonium. An alloy of plutonium of low melting point is contained in tantalum capsules, supported in a lattice configuration, and cooled with liquid sodium. The lattice is surrounded with a blanket and serves as the driver for the blanket. The reactor is then composed of a sufficient number of such modules, or cells, to produce the desired power level. The driver of this assembly is a direct extrapolation of LAMPRE I cores.

The present Los Alamos breeder (with plutonium fuels) development program includes continuing LAMPRE I operations, extensive outof-pile experiments, calculational studies, in-pile tests in the Omega West Reactor (OWR), (2) and design and construction of a 20-MWt Fast Reactor Core Test Facility (FRCTF). $(3,4)$

I. LAMPRE Resuits

LAMPRE I is a small fast reactor that operates at powers up to $1 \mathrm{MWt}$ The reactor is used primarily for testing materials which are mvolved in the development of molten plutonium fuel elements In prescnt LAMPRE I cores (see Fig. 1), $23 \mathrm{~kg}$ of molten $P u=F e$ fuel is containcd in about 140 sodium-cooled tantalum capsules of approximately 0.4 -in $O D$. The entire active core region is approximately $6 \mathrm{in}$. in diam. by $6 \mathrm{in}$. high. Space is provided at the top of the fuel elements for the accumulation of fission product gases.

*The terminology used is defined in Appendix I. 


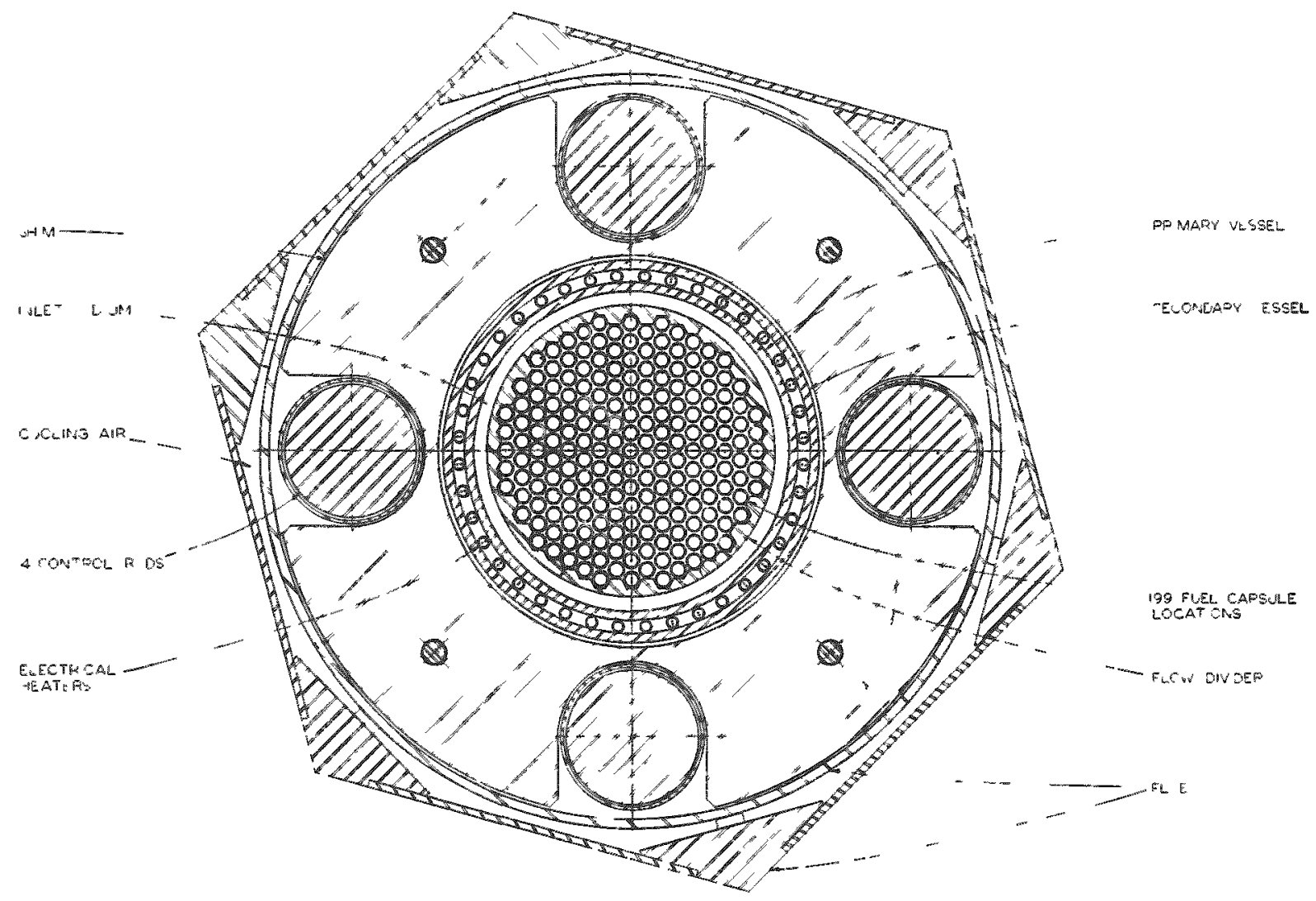

Fig. 1. Horizontal Cross Section through Reactor Core

Power operation with the first (Mark I) core began in April 1961. The results of operation of this core have been reported elsewhere. (5) Operations with the second (Mark II) core in LAMPRE I were completed in June 1963. The Mark-II core was operated at powers up to I MWt for a total of $105 \mathrm{MWD}(\sim 1 / 2 \%$ depletion). During this operation, two capsules failed. Out-of-pile experiments indicate that failures of the Mark-II fuel elements can be expected when the tantalum temperature exceeds $650^{\circ} \mathrm{C}$ $\left( \pm 25^{\circ} \mathrm{C}\right)$. This temperature is consistent with the observed incidence of failures in the Mark-II core. Even though the two failures resulted in the escape of $\sim 75$ and $30 \mathrm{~g}$ plutonium into the sodium coolant, no operational difficulties were experienced.

Reactivity measurements performed during power operation indicate that almost all of the fission gas accumulates in the 2-in. gas space above the fuel in the capsule. It is estimated that full retention of the gases as bubbles in the fuel would cause a reactivity loss rate of about $16 \phi$ $(0.03 \% \Delta \mathrm{k})$ per MWhr. In the $105 \mathrm{MWD}$ of operation of the Mark-II core, however, the total reactivity loss of about $\$ 12(2.5 \% \Delta \mathrm{k})$ corresponds to an average loss rate of only $0.5 \phi / \mathrm{MWh}$. Post-irradiation measurements of the amount of gas in the gas space above the fuel also confirm the release of fission gas from the body of the fuel. 
The large thermal expansion coefficient of the liquid fuel in IAMPRE I provides a negative fuel temperature coefficient of the order of $2 \% /{ }^{\circ} \mathrm{C}\left(4 \mathrm{x} 10^{-5} \Delta \mathrm{k} /{ }^{\circ} \mathrm{C}\right)$. Estimates of the response of LAMPRE I to large transients indicate that reactivity insertion rates of up to $\sim 100 / \mathrm{sec}$ $(20 \% \mathrm{Ak} / \mathrm{sec})$ could be tolerated by the system without exceeding the static stress limit of the tantalum. In a fuel containing fission gas bubbles, the effective compressibility of the fuel is greatly increased. Because of this. the effective fuel expansion will be retarded relative to the no-bubble case in large transients. This causes energy releases and temperature rises to be greater, for given reactivity insertion rates, than for the case without bubbles. On the other hand, the cushioning effect of the bubbles is such that, even with the greater energy release, the pressure rises would actually be less than when no bubbles are present. Thus, it is expected that even larger reactivity insertion rates could be tolerated with bubbles present.

The Mark-III core, which is currently being fabricated, will include a test of a new fuel alloy, Pu-Co-Ce, and of its compatibility with tantalum. In the Pu-Co-Ce fuel, the plutonium content may be varied from 0 to $13 \mathrm{~g} \mathrm{Pu} / \mathrm{cc}$ without significantly altering the melting point of $\sim 450^{\circ} \mathrm{C}$. Preliminary tests indicate that this alloy (at least for plutonium contents below $8 \mathrm{~g} / \mathrm{cc}$ ) may be significantly better with respect to compatibility with tantalum containment than is the Pu-Fe alloy. (4)

Another observation available from LAMPRE I experience is that it is quite feasible to calculate the criticality of LAMPRE I in a number of configurations with acceptable accuracy.(6) These results indicate that the general neutron balance of this type of system can be adequately calculated.

\section{Breeding Potential of a Large Molten Plutonium System}

The two factors which determine doubling-time capability are breeding ratio, as a measure of neutron economy, and specific power. Tantalum, as used in this concept, is the component which limits both neutron economy and specific power.

First, tantalum is a significant nuclear poison for fast neutrons: this indicates the need for a high plutonium density to minimize the parasitic capture by tantalum. Secondly, pure tantalum has an allowable creep strength of $\sim 10,000$ psi and a corrosion resistance which appears to decrease severely above $675^{\circ} \mathrm{C}$. These factors limit the power per capsule, so that, in general, higher specific powers are obtained with lower plutonium densities. The capability of a tantalum-capsule type of molten plutonium system, as constrained by tantalum parasitic captures and by tantalum stress and temperature limitations, is the main subject of this discussion. 
With a breeding ratio well above unity, it is convenient to consider the neutron economy in the driver and in the blanket separately.* Breeding ratio, as used here, ** can be calculated as

$\begin{aligned} & \text { Breeding } \\ & \text { Ratio }\end{aligned}=\frac{\text { Plutonium Produced }}{\text { Plutonium }+U^{235} \text { Atoms Destroyed }}$

$$
\mathrm{BR}=\frac{\text { Net Leakage into Blanket } \times \frac{\text { Plutonium Produced }}{\text { Net Leakage into Blanket }}}{\text { Plutonium Fissioned in Driver }+\mathrm{U}^{235} \text { Destroyed in Blanket }}
$$

Further: Net Leakage into Blanket = (Leakage from Driver to Blanket Leakage out of Blanket).

The neutron leakage rate from driver to blanket must be the excess of neutrons produced and not absorbed in the driver, i.e.,

$$
\left(\eta_{D}-1\right) A_{D}
$$

The plutonium destruction (fission) rate in the driver is

$$
\eta_{\mathrm{D}} \mathrm{A}_{\mathrm{D}} / \nu_{\mathrm{D}}
$$

where $\eta_{D}$ and $\nu_{D}$ are the number of neutrons per absorption and per fission in the driver. Both $\eta_{D}$ and $\nu_{D}$ are determined by the driver spectrum and thus are relatively insensitive to blanket properties. Plutonium produced per net blanket leakage is a property of the blanket and leakage spectra, and thus is relatively insensitive to driver properties. Leakage from the blanket (reactor leakage) and the $\mathrm{U}^{235}$ destruction rate are small correction terms that can be estimated without detailed reference to driver and blanket properties. The multiplication in the blanket is determined from calculations emphasizing blanket properties. Neutron balance may be used to determine the relative absorption rates in the driver (AD) and in the blanket.

All calculations discussed here are two-dimensional $(R Z), S_{4}$ approximations, (7) with 7 neutron energy groups.

Limitation of the present discussion to beginning-of-life (clean) configurations is a specification based largely on convenience, in that it

*The type of analysis used here is similar to that used by Kiehn $(8)$ in a. preliminary survey of the molten plutonium breeder concept.

** Only the beginning of life situation is considered here. This definition is not appropriate to the equilibrium case. 
avoids the necessity of considering a detailed fuel cycle. Doubling times are calculated as if beginning-of-life breeding ratios and specific powers apply throughout life. This is optimistic in some respects and conservative in others. Some penalty in breeding ratio may be encountered in an equilibrium cycle relative to beginning of life, in that the available difference in plutonium and uranium spectra is decreased. However, the specific power may well be greater in the equilibrium cycle, in that the plutonium specific power will, in general, be higher in the blanket than in the driver. Since the blanket power density is not expected to be limiting, this may permit a substantial increase in reactor specific power. Further, the driver neutron economy and specific power in an equilibrium cycle would be estimated in the same mannex used here, i.e., for a typical environment. Thus, although the blanket used here is hardly characteristic of an equilibrium blanket, the calculated driver performance characteristics could be considered as first estimates for equilibrium drivers.

The materials and lattice parameters considered here for the driver regions include:

Fuel: $8.0,5.7,3.0 \mathrm{~g} \mathrm{Pu/cc}$ in $\mathrm{Co}-\mathrm{Ce}$

Capsule wa11: 0.025 in.

Capsule diameter: $1.0-\mathrm{cm} O D$

Diameter/pitch: $0.75,0.85$, and 0.9

Height/diameter: 1.0 .

Some characteristics of these driver lattices are given in Table I. For neutron economy, it is clear from the data of Table I that the principal effect of the variations of the driver lattice parameters appears as changes in tantalum absorptions per driver absorption ( $f_{\mathrm{Ta}}$ ). The specific power limits shown are based on the following set of thermal limits:

Sodium velocity $\leq 30 \mathrm{ft} / \mathrm{sec}$

Max Pu-Ta interface temp - inlet temp $\leq 175^{\circ} \mathrm{C}$

Thermal stress $\leq 8 \times 10^{3}$ psi

Table I

TYDICAL DRIVER PROPERTIES

\begin{tabular}{|c|c|c|c|c|c|c|c|c|c|c|c|c|}
\hline \multicolumn{2}{|l|}{ Case } & \multicolumn{3}{|c|}{ Volume Fractons } & \multicolumn{2}{|c|}{ Atom Denstues } & \multirow{2}{*}{$\begin{array}{l}\text { Drver } \\
\text { Padius } \\
\text { (cm) }\end{array}$} & \multirow{2}{*}{$\begin{array}{c}\text { Loding } \\
\text { Mlodute } \\
\text { ikg: }\end{array}$} & \multirow{2}{*}{$\begin{array}{l}\text { Speculc } \\
\text { Power }\end{array}$} & \multicolumn{3}{|c|}{ Veutron Balance } \\
\hline $\begin{array}{c}\text { Fuel } \\
\text { lg Pu/ce in Co-col }\end{array}$ & Diam/nitch & Fuel & Tantalum & Sodutum & Talpu & No/ & & & & $r o$ & $\widehat{T}_{2}$ & zoak/fission \\
\hline 8 & 0.9 & 0.56 & 0.17 & 0.27 & 0.85 & 0.60 & 9.1 & 35 & 180 & 2.30 & 0.15 & 1.7 \\
\hline 5.7 & 0.9 & 0.50 & 0.17 & 0.27 & 1.19 & 0.93 & 133 & 54 & 310 & 216 & 0.19 & 1.6 \\
\hline 5.7 & 0.85 & 0.50 & 0.16 & 034 & 1.19 & 1.28 & 15.1 & 70 & 410 & 2.15 & 0.20 & 1.6 \\
\hline 5.7 & 0.85 & 0.39 & 0.12 & 0.49 & 1.19 & 2.21 & 20.7 & 130 & 410 & 2.11 & 0.21 & 1.5 \\
\hline 3.0 & 0.9 & 0.56 & 0.17 & 0.27 & 2.24 & 1.75 & 34.6 & 194 & 400 & 3.82 & 0.31 & 1.3 \\
\hline
\end{tabular}


Two blanket fuels, $\mathrm{UC}_{2}$ and $\mathrm{UC}$, have been considered. In both cases, the composition is that of a lattice with

$0.85 \mathrm{diam} / \mathrm{pitch}$

1 -in. $O D$ of rod

$0.020 \mathrm{in}$. of stainless steel clad

$0.3 \%$ enrichment

$95 \%$ dense.

These materials and lattice parameters are selected to illustrate the potential of molten plutonium driver systems with conservatively designed blankets. The driver radius is that required for criticality. The drivers are separated by $\sim 12$ in. of blanket.

As a typical result, 1.2 to 2.3 plutonium atoms are produced per net neutron introduced into the blanket. Estimated breeding ratios and doubling times for several illustrative cases are shown in Table II. Two estimates of the reactor leakages for cores of $1000 \mathrm{MWt}$ are included. Among these cases, two generalizations may be observed with respect to doubling time.

1. UC shows some superiority to $\mathrm{UC}_{2}$.

2. The lattice selection requires a distinct compromise between breeding ratio and specific power (the optimum case may be outside the range considered).

Table II

BRERDING FACTORS

\begin{tabular}{|c|c|c|c|c|c|c|}
\hline \multicolumn{2}{|c|}{ Case } & \multicolumn{2}{|c|}{$\begin{array}{c}\text { Breeding } \\
\text { Ratio }\end{array}$} & \multicolumn{2}{|c|}{$\begin{array}{c}\text { Doubling } \\
\text { Time (UC) } \\
\text { (yr) }\end{array}$} & \multirow{2}{*}{$\frac{\mathrm{DT}_{(\mathrm{v} x)}^{\left(\mathrm{UC}_{2}\right)}}{\mathrm{B}}$} \\
\hline$(g \mathrm{Pu} / \mathrm{cc})$ & Diam/pitch & $A^{*}$ & $B *$ & A & $\mathrm{B}$ & \\
\hline 8 & 0.9 & 1.65 & 1.75 & 29 & 25 & 27 \\
\hline 5.7 & 0.9 & 1.49 & 1.62 & 23 & 18 & 20 \\
\hline 5.7 & 0.85 & 1.44 & 1.60 & 19 & 14 & 15 \\
\hline 5.7 & 0.75 & 1.36 & 1.56 & 23 & 15 & 17 \\
\hline 3.0 & 0.9 & 1.19 & 1.27 & 46 & 32 & 42 \\
\hline
\end{tabular}

* A: Reactor leakage used is a pessimistic estimate $(6-9 \%)$.

B: Reactor leakage used is an optimistic estimate $(\sim 3 \%)$.

Somewhat more detailed calculations have been done for the following case:

Driver: Capsule size: $1-\mathrm{cm}$ OD

Capsule wall: 0.025-in. tantalum

Diameter/pitch: 0.85

Plutonium content: $5.7 \mathrm{~g} / \mathrm{cc}$
Blanket: Fuel: UC

Blanket thickness: 6 in. 
A conceptual reactor layout is shown in Fig. 2. Drivers of this type are among the cores which are to be tested in the FRCTE. These experiments will provide confirmation of driver neutron balance, specific power, and lifetime capability. In the sketch shown, allowance is made for removal of volatile fission products through a vent hole in the top of the capsule. It may be feasible to consider systems in which fission products are vented directly to the sodium coolant, or in which heat transfer is accomplished by directly contacting the coolant and the fuel material. These further developments are not considered in this discussion.

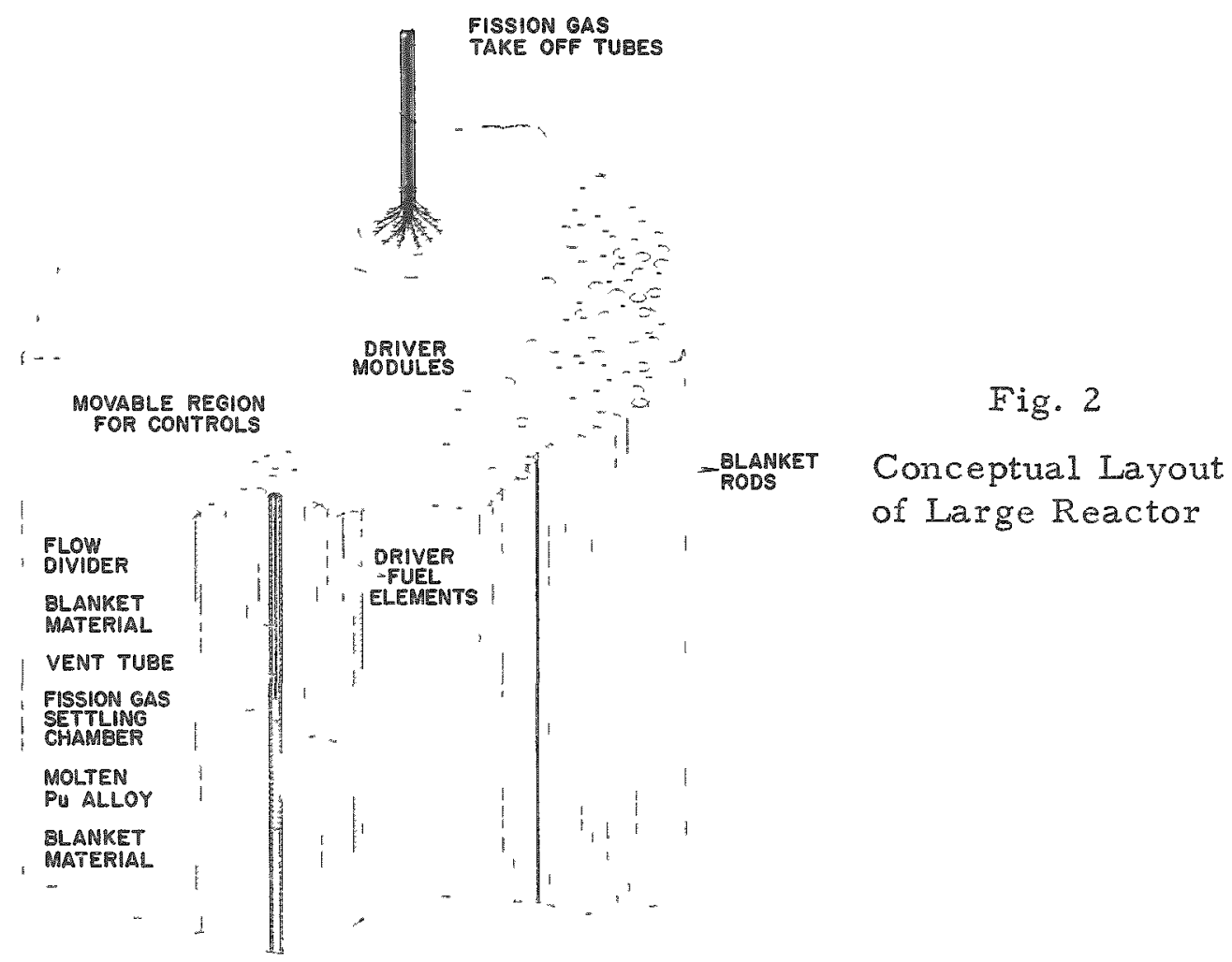

The fertile blanket fuel is shown as uranium carbide. As has been stated, this is intended to be a conservative specification. Other blanket concepts have been suggested (such as a paste blanket)(9) and may prove attractive, but are also beyond the scope of the present discussion.

Typical perfomance properties for this case are given in Table III. More favorable performance could be obtained by increasing the blanket volume, by increasing reactor size, by any of several blanket design changes, or by assuming somewhat less conservative thermal limits in the driver, reactor leakage, etc. Even with the conservative assumptions used, however, adequate performance is indicated.

Some further leatures of this design may be of interest. As indicated, control is exercised here by means of moving fuel. Total control worth is estimated at $\sim 10 \%(\$ 50)$. The temperature coefficient is calculated 
to be $-1 \times 10^{-5} \Delta \mathrm{k} /{ }^{\circ} \mathrm{C}\left(0.5 \% /{ }^{\circ} \mathrm{C}\right)$ from expansion of the fuel only, based on measurements of the expansion coefficient for a Co-Ce alloy (10) and for $3 \mathrm{~g} \mathrm{Pu} / \mathrm{cc}$ alloy. (11) The driver sodium reactivity coefficient of expansion is $\sim-1 \times 10^{-6} \Delta \mathrm{k} /{ }^{\circ} \mathrm{C}$. The sodium coefficient of the blanket is essentially zero. No credit is taken for the blanket Doppler coefficient. The driver Doppler coefficient is nil, in that the neutron flux in the resonance range is quite small.

Table III

\section{CONFIRMATORY CAICULATION}

Descriptive Parameters

Driver: Fuel

Diameter/pitch

Capsule OD
$5.7 \mathrm{gPu} / \mathrm{cc}$

0.85

$1.0 \mathrm{~cm}$

Physical

Driver Radius

Plutonium Loading/Module

Neutron Balance

$$
\begin{aligned}
& \eta \mathrm{D} \\
& \text { fTa } \\
& \text { Leak/fission }
\end{aligned}
$$

Specific Power

Breeding Ratio

$$
\begin{aligned}
& \text { Optimistic Leakage (est) } \\
& \text { Pessimistic Leakage (est) }
\end{aligned}
$$

$14.8 \mathrm{~cm}$

68. $\mathrm{kg}$

Doubling Time

Optimistic Leakage (est)

Pessimistic Leakage (est)
2. 12

0.22

1.4

$410 \mathrm{~W} / \mathrm{g} \mathrm{Pu}$

From a safety point of view, it may first be noted that there is no meltdown problem in the usual sense. The principal accident potential lies in a major loss of capsule integrity. LAMPRE-I experience has indicated that minor capsule failures are not a hazard. Obvious precautions are indicated to prevent nuclear accidents in case of a major loss of capsule integrity by avoiding laxge undiluted regions in which the plutonium alloy could assemble. All accident analyses to date indicate that the thermal expansion of the molten plutonium is adequate to prevent a major nuclear excursion. 
The economic potential of this type of system is, at this state, speculative. However, there is the possibility of significantly extended use of the tantalum capsules In most systems, fuel elements must be refabricated because of one or more of the following.
a. structural irradration damage to the fuel
b. fission product release endangering the containment or
c. reactivity loss

If a liquid or molten fuel material is feasible, these factors noed not limit fuel element life. Being fluid, the fuel can physically adapt to ir radiationinduced changes, volatile fission products can be removed in any of several ways; and even refueling without refabrucalion is conceivable. There may also be economic advantages in the reprocessing of metallic fuels, relative to oxides or carbides. Some studies $(8)$ have indicated that these savings could resuit in a fuel. cycle cost reduction of greater than $1 \mathrm{mill} /$ $\mathrm{kW}$-hr relative to a solid fuel plant

IV. Summary

1. Successful power operation of LAMPRE I during the past two years supports the promise of molten plutonium as a fast reactor fuel. No significant operational dificuities were experienced

2 A thermal limitation and the parastic absorption due to tantalum are determinng tactors in the compromise between specific power and breeding ratio.

3. With consorvative assumptions. favorable doubling times are predicted. If long driver tuel element lifetime is feasibie as anticipated, favorable fuel cycle costs should be availabse.

4. A strong negative temperature coefficient due to fuel expansion is anticipated.

Alhough the reactor whrch has been considered in this discussion is based on a straightforward extension of the LAMPRE-I facility, it should be recalled that there are several features which aro extrapolations from proven technology"

1. The fuel alloy assumed is Pu-Co-Ce The properties, including gas retention, of this allow after high ir iadiation, are not yet established. An alloy in the range of plutonium content considered is presently being prepared for insertion into LAMPRE I. Further, irradiation tests 


\section{HANNUM et al.}

in OWR are planned, in addition to the many out-of-pile tests which are in progress. The FRCTF first core design is currently predicated on the use of Pu-Co-Ce. This will constitute a major integral test of this material.

2. Tantalum integrity, after long ir radiation, is not yet established. The results of current investigations of Ta-W alloys, and the OWR and FRCTF experiments, are required to establish this capability. 


\section{Appendix I}

Definition of Terms

Since there is some ambiguity as to the usage of terms appropriate to a large fast reactor system, the following set of specific terms has been selected for this discussion:

Reactor System: The complex of items including reactor, vessel, primary coolant system up to the heat exchangers, and all subsystems therein.

Reactor: All components of the reactor system inside of the vessel which have a substantial direct effect on the neutronics of the system. In this discussion, the reactors considered are composed of driver, blanket, and reflector.

Driver: That region (or regions) of the reactor which includes a high fissile material content. The driver is characterized by a high neutron multiplication.

Blanket: That region (or regions) of the reactor which includes a high fertile material content. The blanket is characterized by a low (but, in practice, a nonzero) neutron multiplication.

Reflector: Regions on the periphery of the reactor which serve to reflect neutrons that would otherwise leave the reactor. A reflector contains no fissile or fertile material. By definition, reflector regions are contained within, and do not include, the reactor vessel.

Fissile Material. Material composed of nuclides which, on absorption of a single neutron, have a high probability of undergoing fission, independent of the incident neutron energy. This includes $\mathrm{Pu}^{239}$ " $\mathrm{Pu}^{241}$, $\mathrm{U}^{233}$, and $\mathrm{U}^{233}$

Fertile Material: Material composed of nuclides which have a high probability of becoming fissile upon absorption of a single neutron and subsequent radioactive decay processes This includes $\mathrm{U}^{238}{ }^{2} \mathrm{Pu}^{240}$ and $\mathrm{Th}^{232}$.

Breeding Ratio (for U-Pu systems). The instantaneous rate of production of plutonium from fertile material $\left(\mathrm{U}^{238}\right)$, divided by the rate of destruction of plutonium (fission only) and $U^{235}$ (fission plus capture). Note that this is only one of several equally reasonable definitions. 
HANNUM et al.

\section{References}

1. Swickard, E。 O., The Los Alamos Molten Plutonium Reactor Experiment No. 1, Trans. ANS 2, 147 (1959): LAMPRE I Final Design Status Report, ILA-2833 (1963).

2. Jurney, E. T., A Hazards Summary for the Proposed Los Alamos Omega West Reactor, LA-1648 (1954).

3. Fast Reactor Core Test Facility Safety Analysis Report, LA-2735 (1962): A Preliminary Study of a Fast Reactor CTF, LA- 2332 (1959).

4. Quarterly Status Report on LAMPRE Program, LAMS-2915.

5. Carmichae1, B. M., and Ragan, G. L., Startup of the First Molten Plutonium Reactor, Trans.ANS 4, 364-365 (1961): Quarterly Status Report on LAMPRE Program, LAMS-2531, 2564, 2620,2647, 2681.

6. Best, G. H., Carmichael, B. M., and LaBauve, R. J., Two-dimensional

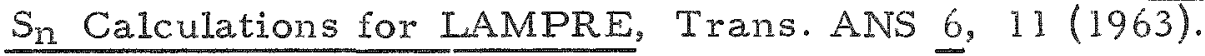

7. Carlson, B., et al., The DSN and TDC Neutron Transport Codes, LAMS-2346 (Feb 12, 1960).

8. Kiehn, R. M., Breeding: Internal or Externa1:, ANL-6122, p. 101 (1959).

9. Hammond, R. P., Stanford, R. E. L., and Humphreys, J. R., Jr., Mobile Fuel Plutonium Breeders - A Study of Economic Potential, $\operatorname{LA}-2644$ (1961).

10. Private Communication from L. D. Kirkbride, LASL.

11. Mound Laboratory Progress Report for August 1962, MLM-1140. 
Discussion of Paper

Presented by Mr. Hannum

MR. DICKERMAN (Argonne):

I wonder if you would care to comment on the possibility that your reactivity coefficients $x$ esult in a great part from the decision to have rather small drivers, compared with the large cores that have been discussed previously, so that perhaps this state of affair might apply to a more conventional system that was divided into separate critical systems.

MR. HANNUM:

This is not the reason we have gone conceptually to this type of approach, although it does undoubtedly contribute to it. The reason we have gone to the multiple driver arrangement is that without internal breeding, you have to keep the neutron economy consistent with the leakage out of the core. If you penalize your neutron economy so that you can tolerate only a few per cent leakage, then you have only a few per cent leakage to make plutonium relative to all of the plutonium destruction in the driver.

MR. OKRENT (Argonne):

Related to the same thing, in your artist's concept, I assume there were several nearly critical cores.

MR. HANNUM:

That is right.

MR. OKRENT:

Is there any problem in instrumenting something like this? Can one core be critical by itself, and you not know what is going on?

MR. HANNUM:

There are obviously some engineering considerations here. The intent would be to construct each driver module so that it could not go critical by itself. Then, there is the question, which we don't feel to be serious, as to the stability between several drivers.

MR. CARMICHAEL (Los Alamos):

I had a comment on the temperature coefficient of the liquid as compared with the solid fuel elements. Since the tantalum expansion 
coefficient is quite small in the liquid, you get the full benefit of its volume expansion on the temperature coefficient compared with the solid element where you get the linear expansion effect on the temperature coefficient.

MR. ZAGOTTA (IIT Research Institute):

I wondered why you chose tantalum, and if you had considered any other things.

MR. HANNUM:

We have looked at a number of other things. Tantalum is the only one we have found so far that seems to do the job. Molten plutonium is very corrosive. The containment is a serious problem.

MR. ZAGOTTA:

Did you consider vanadium?

MR. HANNUM:

Yes.

MR. HECK (Westinghouse):

I think this is one type of concept for which we might be able to prototype a big reactor in a small reactor.

MR. HANNUM:

Very much so. As a matter of fact, the fast reactor core test facility that we are constructing presumably will be used to test modules of this sort.

MR. HECK:

How well do you think you can mock up on a single or a few module basis the performance of a full core?

MR. HANNUM:

The power distribution and so on in the blanket obviously will be wrong. There will be out-leakage from the blanket which is not compensated by in-leakage from the adjacent module. But the driver properties should be fairly well investigable. 
MR.CHERNICK (Brookhaven):

In talking about doubling time, what fuel are you responsible for?

MR. HANNUM.

Assuming that there is some way to remore excess plutonium continuously, we took doubling time to be the length of time operating at 80 per cent utilization in which we would produce enough excess plutonium to fuel an identical reactor.

MR. JAYE (General Atomic).

I wonder if you would comment about the power sharing problems between the modules and the blanket?

MR. HANNUM:

If we begin with a depleted uranium blanket of the order of 0.3 per cent $U^{235}$. We get about 15 per cent of the entire reactor power from the blanket. At equilibrium $x_{e} \in$ might be talking about a 50-50 power split.

MR. KUMMERER (Karisruhe):

The fuel element consists of the plutonium alloy in the core region, and of an axial blanket which is solid. and then you have a vent tube. How do you prevent that vent tube from being blocked by the fission products?

MR. HANNUM:

I would class that all, I am afraid, as an engineering detail.

MR NELSON (Argone)

I wonder about the burnup that you would expect from this fuel and what would limit the burnup. Would it be the concentration of solid fission. products in the fuel?

MR IIANNUM

Again, I can only speculate The concern 25 that the fucl element lifetime will be limited by the tantalum lifetime Our hope is that that would not be limiting, and that we could go to a reactivity limit which, if we were lucky: might approach 100 per cent depletion of the plutonium. 
MR. NELSON:

Under those conditions, you would have a very high concentration of solid fission products in the fuel elements, and the heat transfer would be reduced. I would also like to point out you would have to increase the rate of blanket processing under these conditions, because all of your plutonium is being produced in the blanket and none of it in the fuel.

MR. HANNUM:

Right. The definition of the equilibrium cycle, if we are fortunate with the tantalum containment in the driver, and if we stick with a solid element in the blanket, would probably imply more blanket processing than driver processing. Presumably, at that point we would begin looking seriously at what we could do in the blanket.

MR. TOWER (Westinghouse):

It strikes me that this encapsulation design is quite a retreat from the original concept of being able to flow fuel in and out of this machine. What are your thoughts about the feasibility of the original concept?

MR. HANNUM:

We are still considering it. 


\title{
CHARACTERISTICS OF FAST SETTLED BED REACTORS
}

\author{
Jack Chernick \\ Brookhaven National Laboratory \\ Upton, L. I., New York
}

The program of the Nuclear Engineering Department at Brookhaven emphasizes fundamental studies in nuclear technology rather than reactor projects. Nevertheless, we have found that programmatic work on advanced reactor types tends to unite the different disciplines of the Department and to sharpen our goals. Our general interests in advanced power reactors lie in breeders as opposed to converters, in mobile fuels as opposed to rigld fuels, and in high-temperature systems. For many years the Liquid Metal Fuel Reactor (LMFR) served to focus the work of the Department on liquid metal fuel technology. Since the LMFRE project was cancelled in 1959, our interests have moved in the direction of mobile, particulate fuels. (1-3)

The Fast Settled Bed Reactor is an example of a high-temperature, particulate-fueled, breeder concept on which preliminary studies are being carried out at Brookhaven. The reactor may be regarded basically as a pot of small diameter, densely packed fuel lumps through which a liquid metal is pumped downward for heat removal. During reactor shutdown, the flow is reversed and the bed is fluidized to facilitate removal, reshuffling or reprocessing of the fuel and fertile material. Preliminary design studies $(2,3)$ have been based on the use of UC-PuC spheres of $\frac{1}{4}$ - to $\frac{1}{8}$-in. diameter cooled by liquid sodium at velocities of about $2 \mathrm{ft} / \mathrm{sec}$. Because of the substantial pumping power required to obtain such velocities in large power reactors, some compromises in core geometry have been studied.

Figure 1 shows an axial flow reactor with core height equal to $60 \%$ of the core diameter. The large reduction in core height results not only in a reduction in pumping power requirements, but also in the magnitude of the sodium-density coefficient, which tends to be positive in these reactors. The high packing density of the fuel ( $60 \%$ by volume) in these cores leads to a harder spectrum than is usual in fast breeders, with a resulting large last fission factor and an initial breeding gain close to unity for unclad, monocarbide fuels in sodium.

Figure 2 shows an alternative reactor design in which the coolant is brought in axially but flows radially through an annular core. Neutron leakage losses through the large-diameter duct and neutron absorptions in sodium and structural components reduce the overall breeding gain to about 0.8 . However, the pumping power requirements are decreased, the power density and specific power of the core are greatly increased, and the sodium-density coefficient becomes negative because of the short radial dimension of the annular core. 


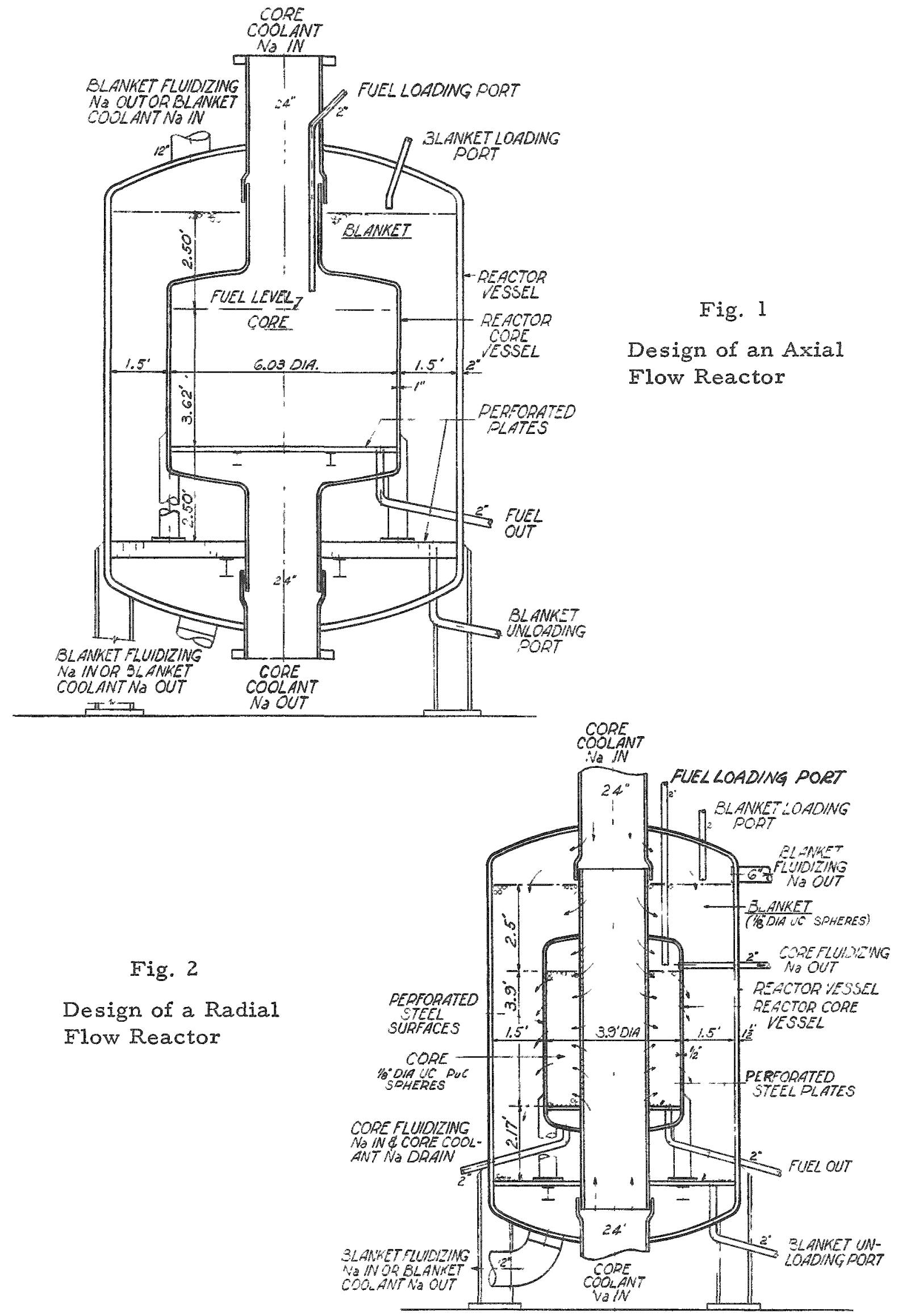


The Brookhaven fast settled bed breeder gives promise of short doubling times and of large cost reductions in fuel fabrication and fuel management. The general advantages of mobile fuels have been recognized by a number of laboratories both in the United States and abroad. However, we realize that a great deal of research and development work is required before we can talk about the SBR as a practical system. Most of the unresolved questions relate to the integrity of the fuel lumps in a reactor environment. The fuel development and testing program at Brookhaven will be described today in a separate paper by $G$. Pancer and $R$. Powers.

The stability of a settled bed reactor with respect to geometry is also an important question. At various stages of reactor operation, the fuel may be in a fluidized state, a loosely packed settled state, or in a densely packed settled state. Fuel packing densities depend on size and shape of both fuel and containex, on fuel size distributions, and on the method of bed compaction. For spherical particles, close packed densities ranging from about $60^{\circ}$ to $65^{\prime \prime}$ have been reported, depending on the size of the container in ball diameters and the method of packing. These numbers may be compared with the close packed limit of $74 \%$ for equal-diameter spheres in a rhombohedral array.

The effect of container walls on local packing densities is shown in Fig. 3. The curves obtained by Benunati and Brosilow, show average voidage within a smooth-walled cylinder into which uniformly sized lead shot

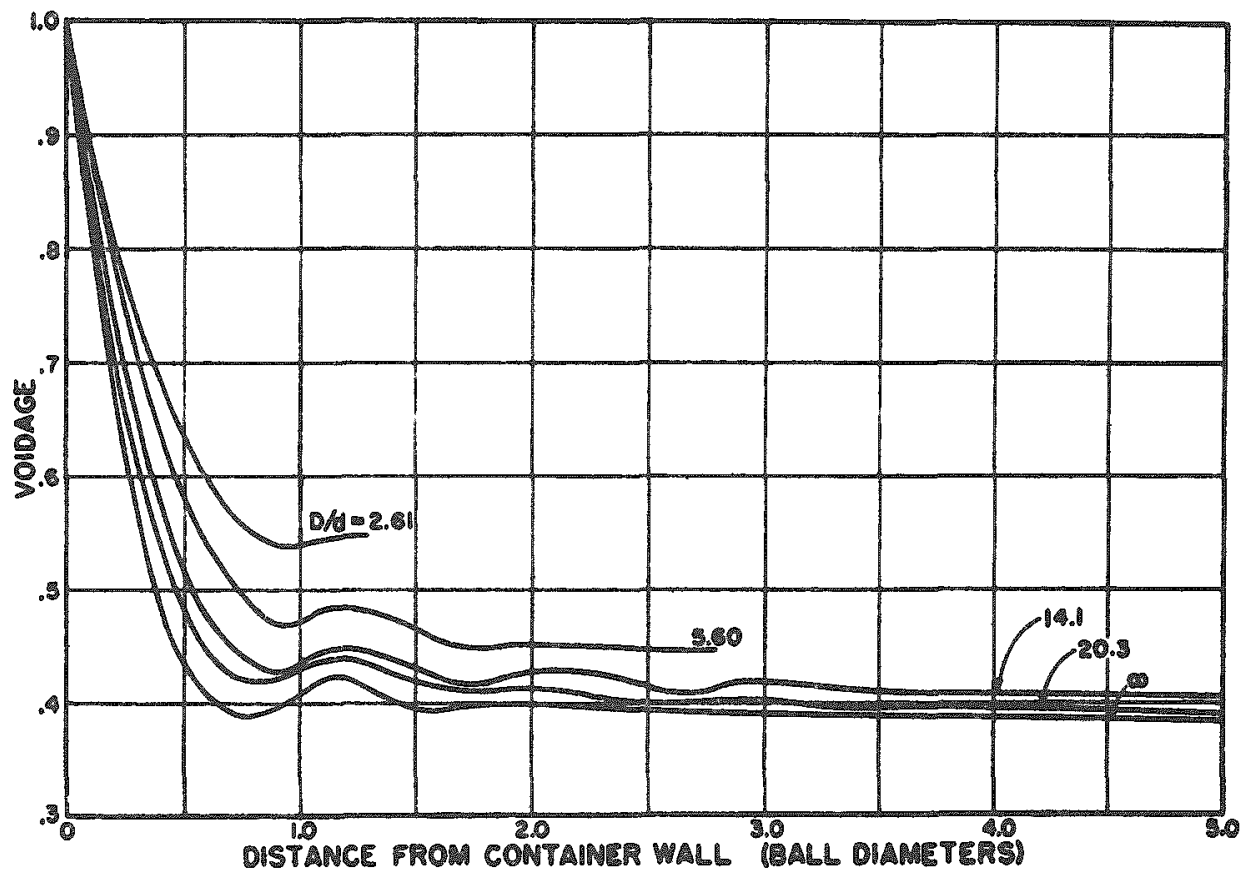

Fig. 3. Integrated Void Fractions in Beds of Uniform Spheres for Various $\mathrm{D} / \mathrm{d}$ Ratios 
had been poured. The asymptotic packing fraction for these "loose" loadings varies from about $61 \%$ for large beds to less than $50 \%$ at certain low ratios $\mathrm{D} / \mathrm{d}$ of container to ball diameter. The oscillations in density which damp out at a distarce of about 4 ball diameters are also apparent. Benanati has shown that these local oscillations in bed density would also occur in a settled bed near internal structures such as control rods. G. D. Scott has found that when packing densities are plotted against reciprocal diameter, a straight line is obtained which can be extrapolated to infinite bed size. Scott obtains asymptotic packing fractions of $60 \%$ for the "loose random packing" which results when steel balls are gentiy rolled into spherical and cylindrical containexs, and $64 \%$ tor the "dense random packing" which results from subsequent shaking and tapping of the container.

Experimental resuits obtained at BNI indicate that the degree of randomness of a densely packed bed depends on the method of compaction as well as on the size and shape of the container. If a flat-bottomed container is vibrated while uniformly sized spheres are slowly poured into it, a hexagonal base is laid down which is as perfect as permitted by the generally nonmatching boundaxies of the container. On this base, a stable rhombohedrally packed cone tends to rise, which can persist to very large bed heights. An exampie of the degree of order that can be obtained is indicated in Fig. 4, which shows the surfaces of a hexagonal container filled with $\frac{1}{8}$-in.-diameter steel shot in this manner. The bottom shows the nearly perfect packing obtained with matching container walls, while the top still shows remarkable ordex at a height approaching the diameter of the container. To investigate such effects in detail, more accurate methods of measuring the densities of settled beds than presently available are needed. A promising method developedat BNL involves the use of small diameter cylinders as gauges which translate a small drop in settled bed height into a large drop in liquid height of the gauge. A magnification of volume change by a factor of 250 has been obtained in this way.

To demonstrate the stability and control of a settled bed reactor, we are planning an early, zero-power, cold critical experiment at Brookhaven. A two-region, water-moderated critical facility will be used, consisting of a centraily located settled bed within an MTR-type assembly. Highly enriched aluminum fuel lumps would be used in the initial experiments to mock up the settled bed fue1, but proposed ceramic fuels would also be tested when they become available. The assembly would be used as much to obtain hydraulics, control, and operational information as well as to check the stability of settled bed configurations. At a later date, a low-power, hot critical experiment is contemplated which would use ceramic fuel lumps and a liquid metal coolant. A variety of control methods are possible in a settled bed reactor from simple addition, subtraction, or reshuffling of fuel and fertile material to partial or complete reprocessing and reconstitution of the fuel. A novel method of control of sodium-cooled reactors, suggested W. Winsche of BNL, would involve dissolving some lithium in the sodium 

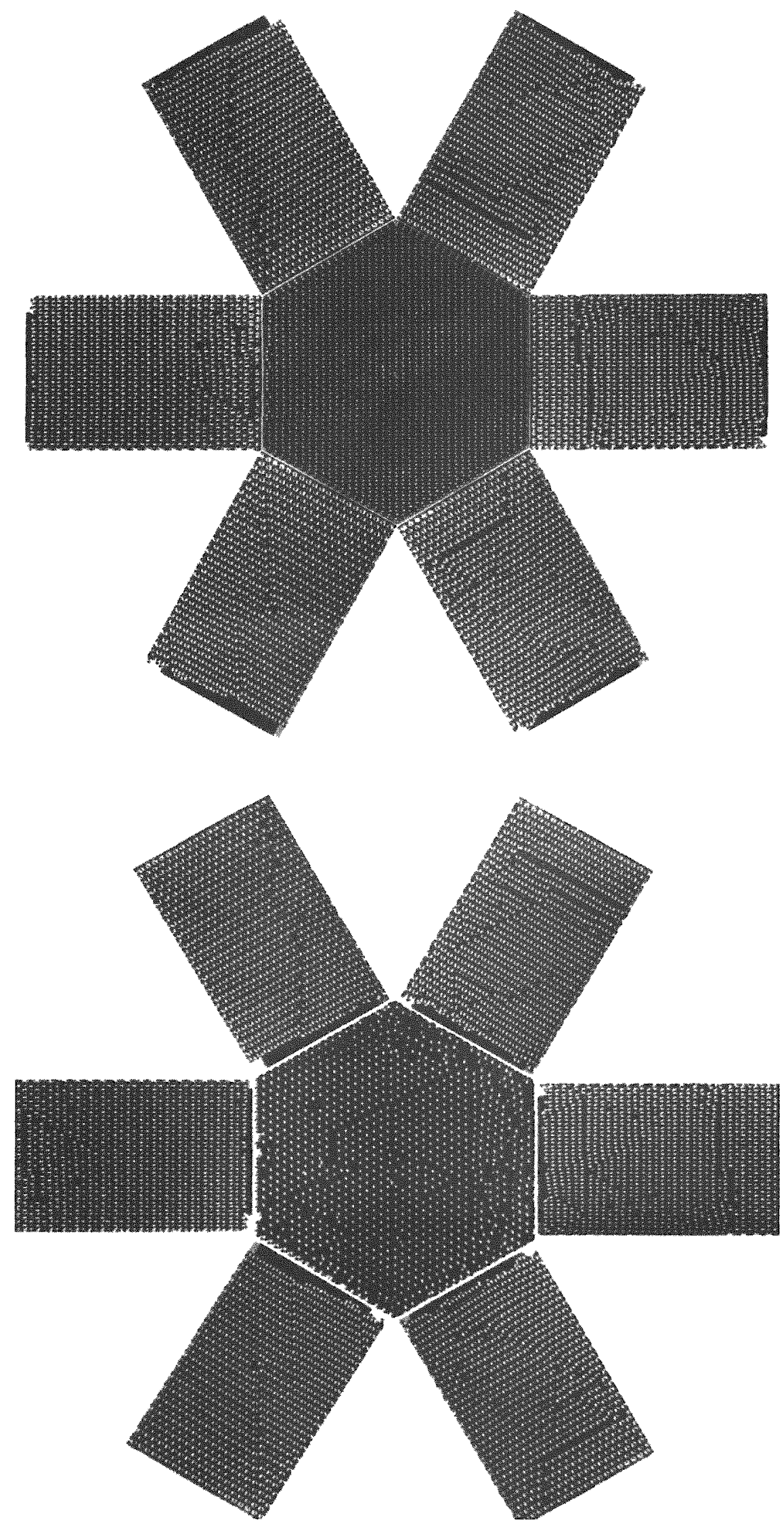

Fig. 4. Example of the Degree of Order Obtainable in a Container Filled with Steel Shot 
coolant. In fast reactors we find that the reactivity change is of the same magnitude as the volume percent of $\mathrm{Li}^{6}$ in the coolant. With ordinary lithium the reactivity swing for the same lithium concentration is, of course, smaller, but the loss in breeding ratio is greatly reduced.

A number of analogies have been drawn between the properties of packed beds and those of simple liquids and solids. Figure 5 shows the radial density distribution of random close-packed spheres as obtained by G. D. Scott. The analogy with the radial distribution of atoms obtained by neutron or $\mathrm{X}$-ray diffraction patterns for simple liquids is quite striking. Similarly, the ratio of solid to liquid densities for the rare gases are close to the ratio $74 / 64$ of regular close packing to randorn close packing of spheres.

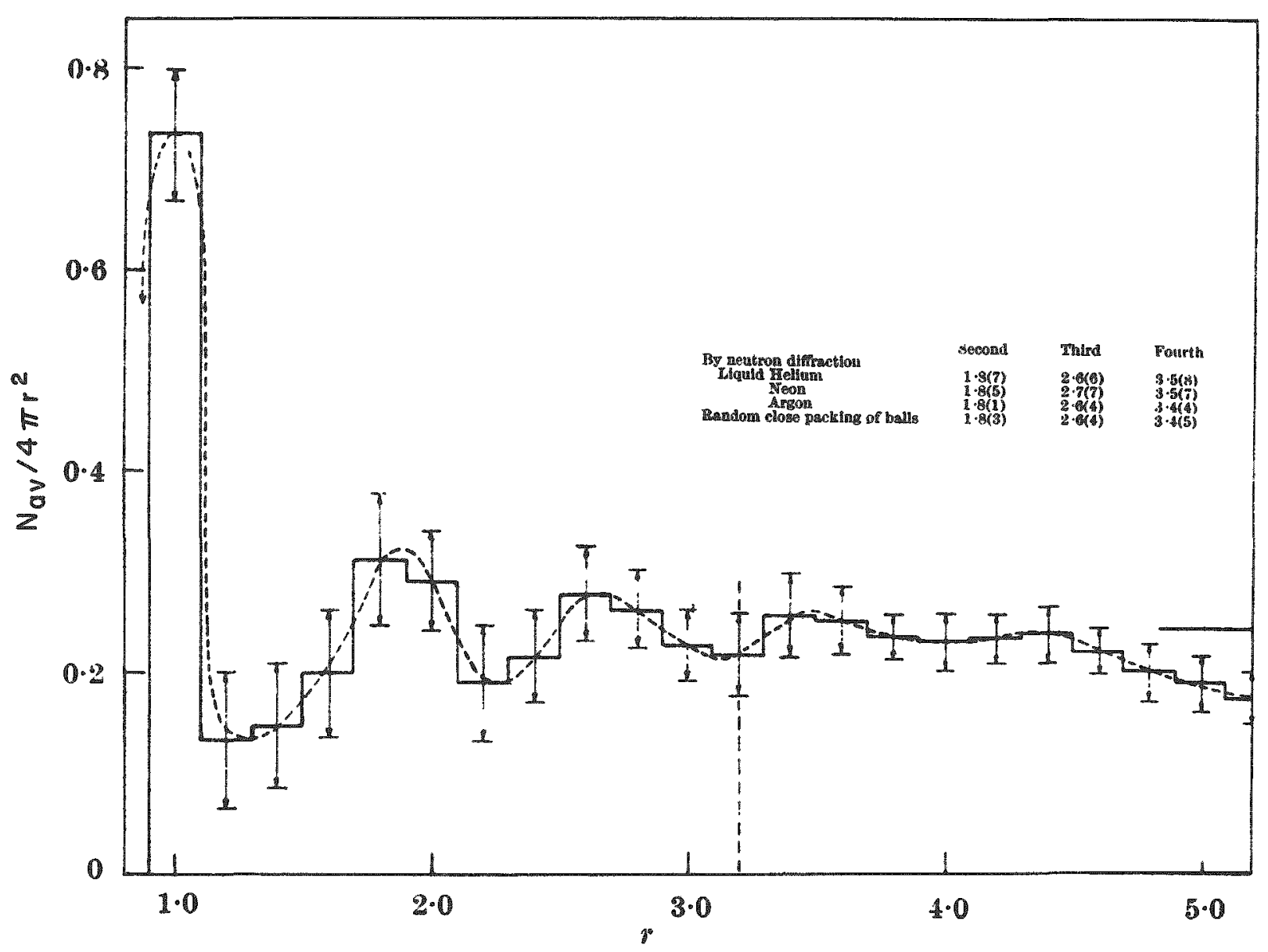

Fig. 5. Radial Density Distribution of Random Close-packed Spheres 


\section{References}

1. L. P. Hatch, W. H. Regan, J. R. Powell, and L. Green. Evaluation Study on Fluidized Fuel Element Reactor, Brookhaven Nuclear Engineering Department Report, Apríl 10, 1959.

2. Evaluation of Large Power Reactor Systems Utilizing Settled Bed Fuels, BNL-5372 (March 28, 1961).

3. W. A. Robba, Editor, The Settled Bed Reactor Status Report, BNL-713 (Jan. 1962).

Note: Discussion of this paper follows Paper 7, page 612. 


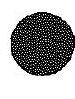




\title{
NUCLEAR PARAMETER STUDIES OF THE \\ FAST SETTLED BED REACTOR
}

\author{
M. M. Levine and A. Aronson
}

Brookhaven National Laboratory

Upton, L. I., New York

(Paper presented by Mr. Levine)

In the preceding discussion by Chernick, the principles of the Settled Bed Reactor concept have been introduced and described. The material to follow is a discussion of specific nuclear performance characteristics of some fast breedex reactors employing these principles.

The two general reactor configurations which have been chosen for demonstration of the settled bed reactor concept are the axial and the radial flow designs shown in Figs. 1 and 2 . The fuel occupies $60 \%$ of the volume of a fueledregion and consists of $\frac{1}{8}$-in.-diameter spheres initially containing $U^{238} \mathrm{C}$ and $P u^{239} \mathrm{C}$ in the core and $U^{238} \mathrm{C}$ in the blanket. The coolant is sodium except where otherwise indicated, and it occupies the remaining $40 \%$ of the volume of the fueledregions. The fuel density has been taken as $12.8 \mathrm{~g} / \mathrm{cc}$. A thermal power of the order of $835 \mathrm{MW}$ is planned. Higher powers will be considered in later work.

Static Characteristics and Burnup

\section{Axial Flow Reactors}

For much of the work the axial flow reactors have been represented as spheres with 1.5-ft-thick blankets. Multigroup diffusion-theory calculations with the AIM code using the cross sections of Yiftah, Okrent, and Moldauer were performed for core diameters over the range from 4.5 to $6.0 \mathrm{ft}$. It is clear that the power obtainable will depend on core size. One design for an axial flow reactor gave a thermal power of $825 \mathrm{MW}$ for a core volume of $182 \mathrm{ft}^{3}$ and a height-to-diameter ratio of 0.6 . All the data to be reported here are based on an initial $k_{\text {eff }}=1.01$.

Figure 3 shows the required enrichment in the core as a function of core diameter for spherical cores. From this the Pu ${ }^{239}$ loading turns out to vary from $750 \mathrm{~kg}$ at a 4.5 -ft diameter to $1570 \mathrm{~kg}$ at a 6.0-ft diameter.

The initial breeding ratios are shown in Figs. 4 and 5 for spherical cores. The parasitic captures are small because the coolant volume fraction is low and there is no structural material except for the vessel 


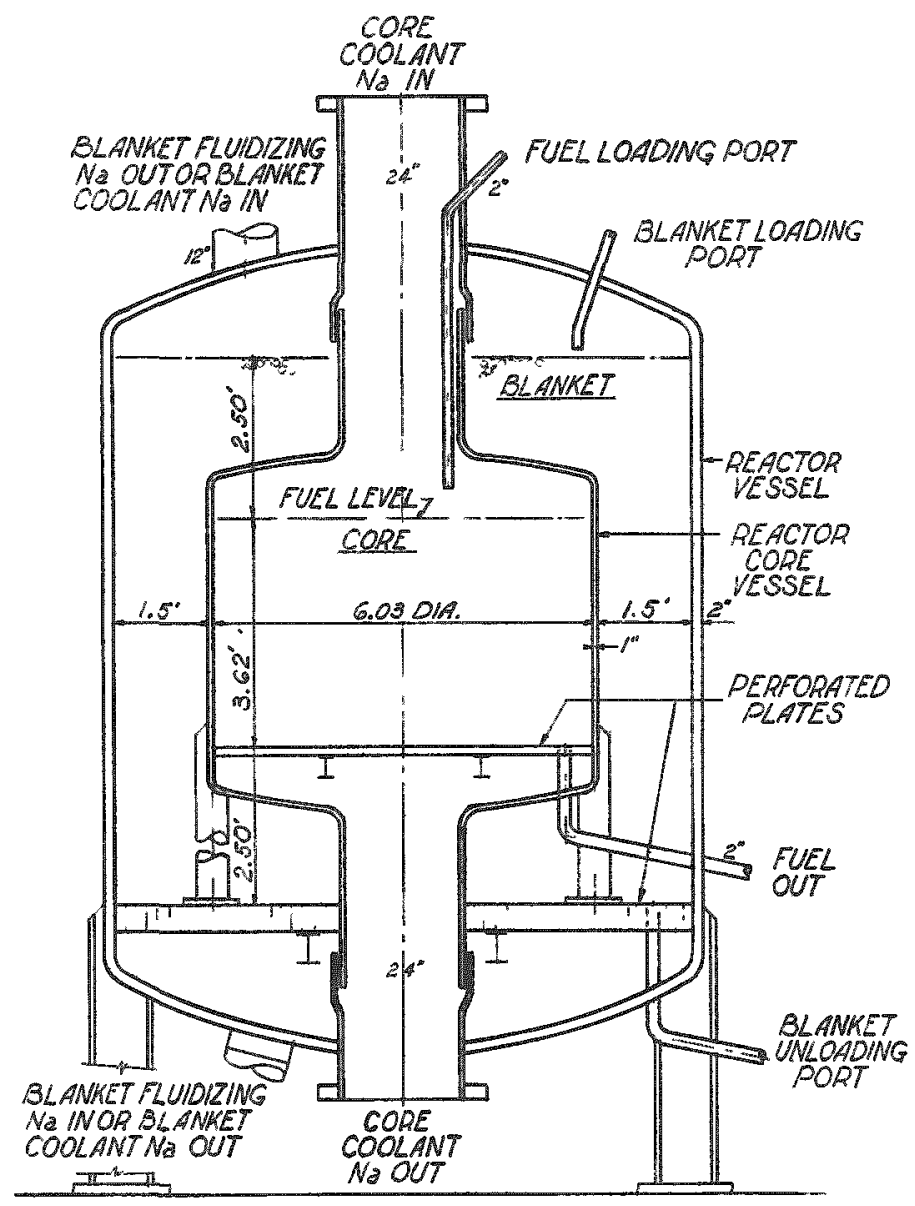

Fig. 1

Design of An Axial Flow Reactor

Fig. 2

Design of A Radial Flow Reactor

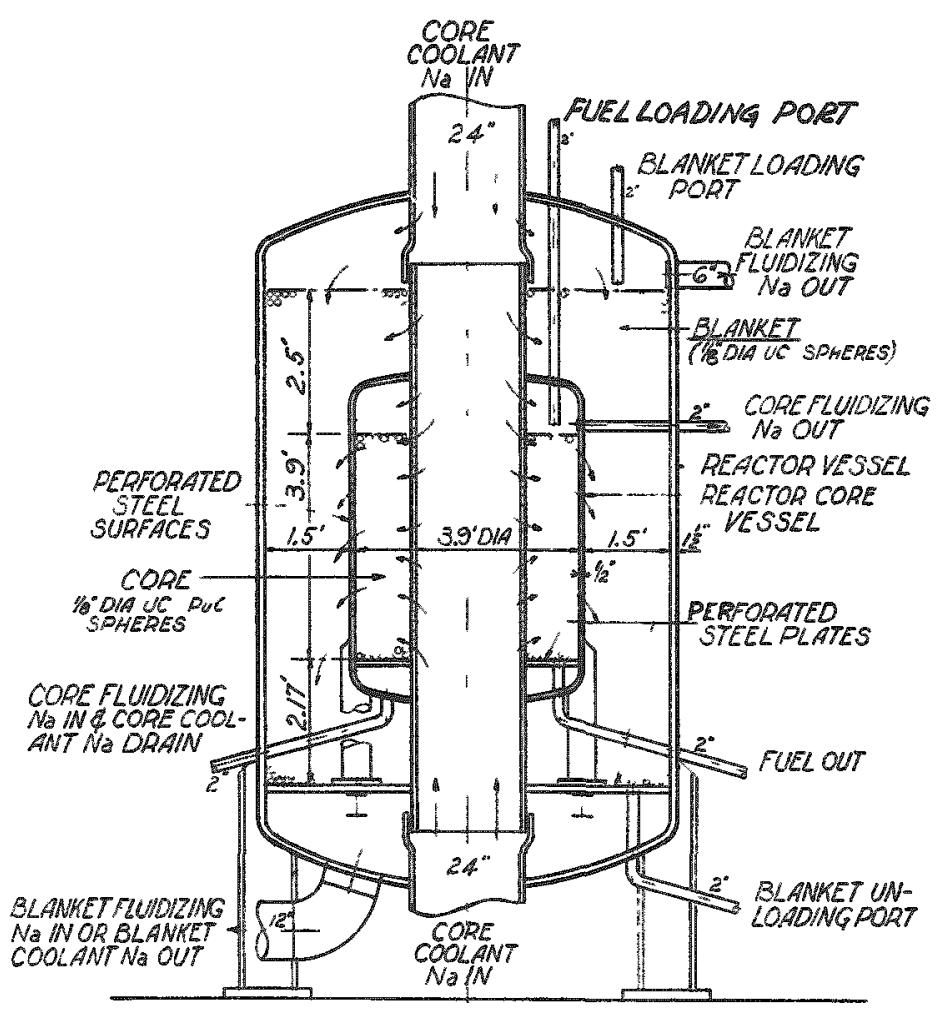




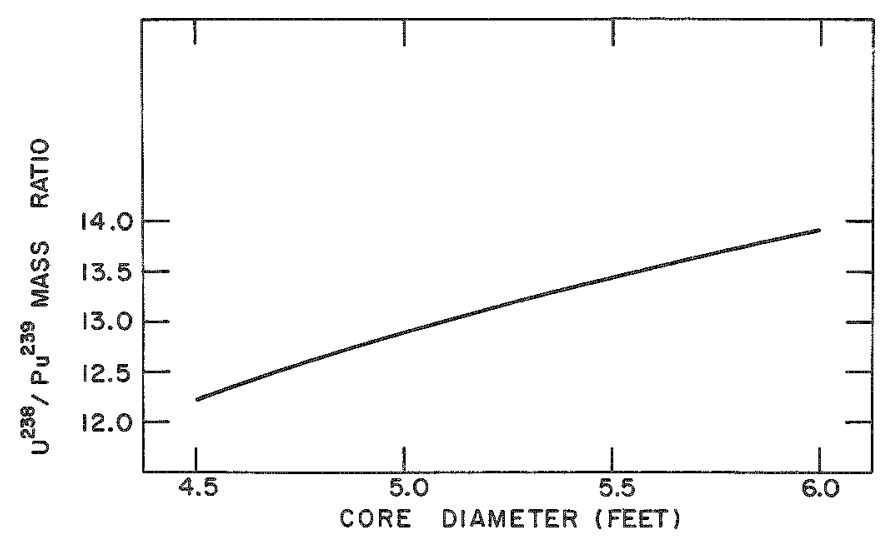

Fig. 3. Settled Bed Reactor with Carbide Fuel, Reflected Spherical Cores: YOM Cross Sections; $k_{e f f}=1.01$.

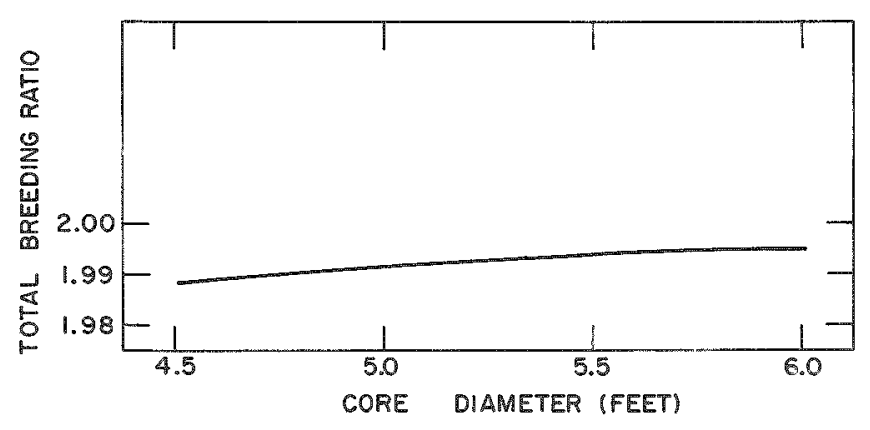

Fig. 4

Settled Bed Reactor with Carbide Fuel, Reflected Spherical Cores; YOM Cross Sections; $k_{e f f}=1.01$.

Fig. 5

Settled Bed Reactor with Carbide Fuel, Reflected Spherical Cores: YOM Cross Sections: $k_{\text {eff }}=1.01$.

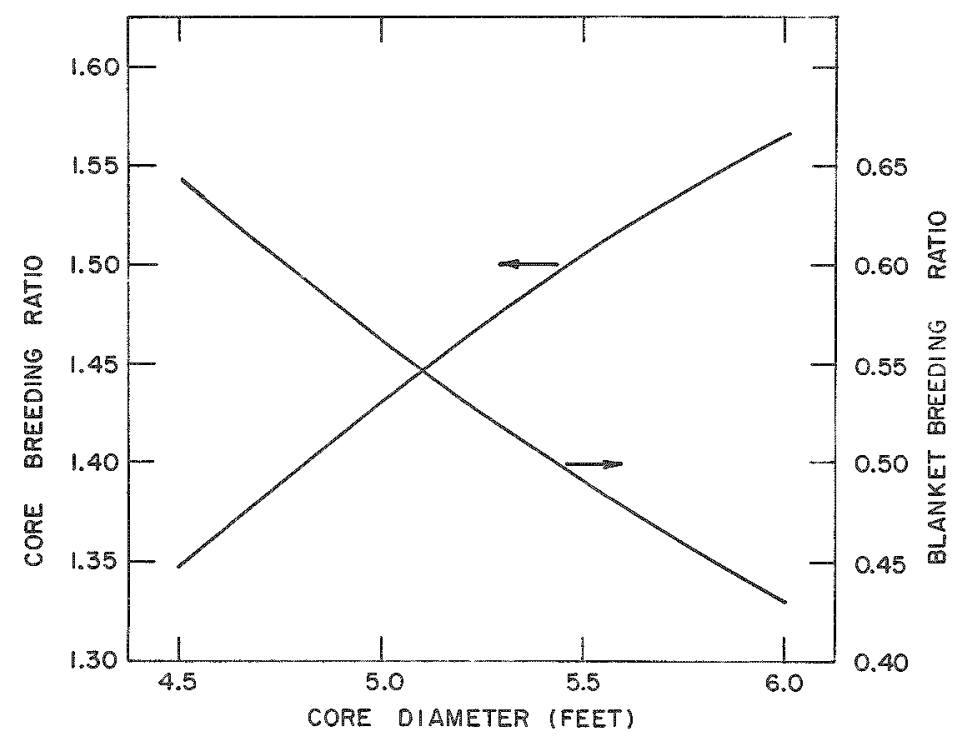


walls. Furthermore, for the same reasons the spectrum is hard enough to yield an average $\alpha$ for $P u^{239}$ of about 0.18 and of about 0.25 of the core fissions in $\mathrm{U}^{238}$.

Power peaking factors go from about 2.3 to 2.5 over this size range. These values are for right circular cylinders having the same reflector savings as found from the spheres.

Because of the high core breeding ratio, the reactivity of these axial flow reactors will rise with irradiation. Indeed, burnup calculations with the SIZZLE code show that for spherical cores of diameter between 4.5 and 7 ft the reactivity rises by about 7 to $7 \frac{1}{2} \%$ for 700 days of operation at $825 \mathrm{MW}$. This corresponds to burnup values of 25,000 to 60,000 MWD/metric ton of metal in the 6.0- and 4.5-ft-diameter cores respectively. At the end of this period the reactivity is still rising at the rate of about $7 \%$ per thousand days. These results were based on the assumption of frequent stirring of the core and blanket to make the fuel burnup more nearly uniform and to prevent preferential plutonium buildup near the inner edge of the blanket. This stirring, to increase the uniformity of burnup, can be accomplished simply by fluidization of the bed for some period of time and then re-settling, and is an attractive feature of the settled-bed operation.

The degree of mixing as a function of fluidization time and other fluidization parameters is important for several reasons. One is that it affects the uniformity of burnup. Also, because compensation for reactivity changes can be accomplished by periodic addition or removal of enriched or unenriched fuel spheres or even by addition or removal of spheres containing poison, the degree of mixing of newly added spheres with the rest of the bed is important for reactivity control and for local power-generation rates.

\section{Radial Flow Reactor}

Only one size was studied for this reactor. The diameter of the central coolant pipe was $2 \mathrm{ft}$, the core thickness $0.885 \mathrm{ft}$, and the blanket thickness $1.5 \mathrm{ft}$. The core height was $3.82 \mathrm{ft}$.

Because of the more complicated leakage problem in this reactor, it was necessary to supplement the one-dimensional calculations with two-dimensional results obtained with the 20 GRAND code. Since this was limited to 6 energy groups, the calculation scheme used 16 group one-dimensional calculations to obtain flux-weighted cross sections for the two-dimensional work. In many cases the flux shapes obtained from the latter were used to obtain transverse bucklings for re-insertion into the 16-group one-dimensional calculations. 
For an initial keff $=1.01$, the enrichment corresponded to $\mathrm{N}_{28} / \mathrm{N}_{49}=8.2$ and a $\mathrm{Pu}^{239}$ loading of $713 \mathrm{~kg}$. Here the initial breeding ratio was 1.79 , with 0.89 coming from the core. The high surface-tovolume ratio of the core is responsible for the shift of breeding from the core to the blanket; also, the spectrum is considerably softened by the presence of the large amount of sodium in the pipe. The overall power peaking factor is 1.52 .

In 700 days of operation at $825 \mathrm{MW}$, the reactivity dropped by $3 \%$ when the core and blanket were well stirred. However, it may be advantageous in this case not to stir the blanket, but to allow preferential plutonium buildup near the inner edge. This reduces the reactivity drop to only $1 \%$ for the same period of operation.

For this exposure - $80,000 \mathrm{MW}$ per ton of metal in the core - the fission product poisoning amounted to about $2 \%$ in reactivity if all the fission products are retained. A considerable fraction of the gaseous fission products could be released by a simple grinding and reconstitution of the fuel spheres. This would not have much effect on reactivity, however, but might help considerably in restoring physical strength to the fuel. Even a high-temperature baking-out process might release a significant fraction of the volatile fission products by diffusion.

Temperature Coefficients

\section{Sodium Coefficient}

In the axial flow reactors the leakage out of the core is sufficiently small so that the main effect of sodium expansion is a hardening of the spectrum. This gives rise to a positive temperature coefficient as is familiar in large cores. Figure 6 shows the sodium temperature coefiricient as a function of core size for spheres representing the axial flow reactors. The spherical cores considered here are equivalent approximately to right cylinders. Flattened cylinders would have more leakage and therefore improved sodium coefficients. This would also shift some of the breeding from the core to the blanket, helping the burnup behavior.

In the radial flow reactors the sodium coefficient is negative since the leakage enhancement is greater than the spectrum hardening when sodium expands. The sodium coefficient has contributions as follows: $-2.35 \times 10^{-6} /{ }^{\circ} \mathrm{C}$ for the core, $-2.42 \times 10^{-6} /{ }^{\circ} \mathrm{C}$ for the blanket, and $-17.6 \mathrm{x}$ $10^{-6} /{ }^{\circ} \mathrm{C}$ for the central pipe. The sodium coefficient increases in magnitude as the sodium density decreases in the radial flow reactor, but is more nearly constant for the axial flow reactor. 


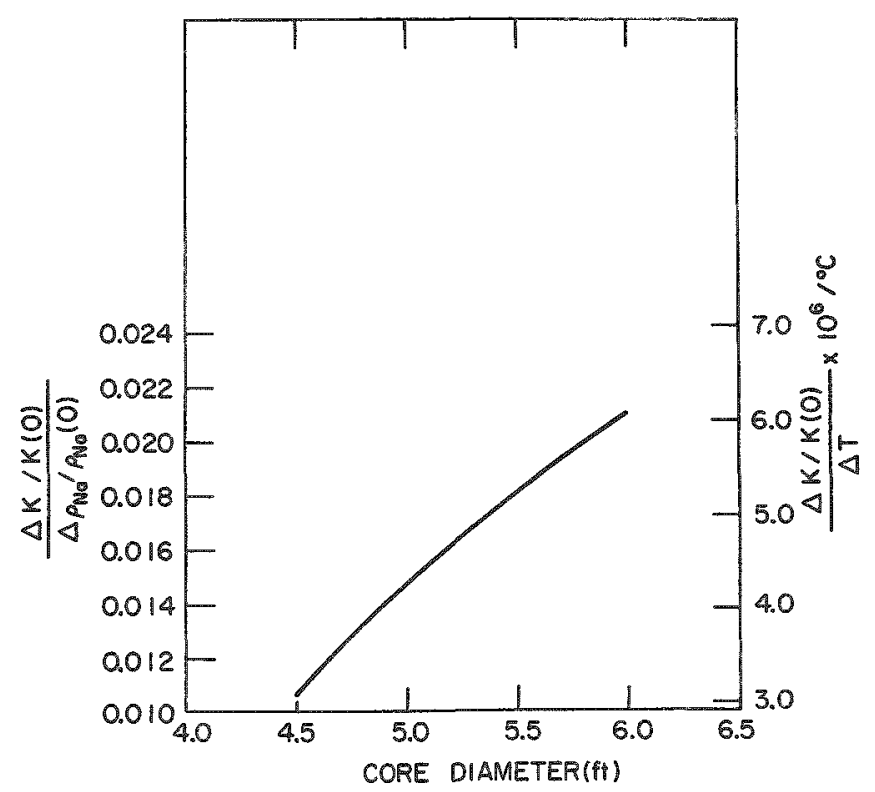

Fig. 6. Sodium Coefficient of Reactivity vs. Core Diameter.
In the case of total sodiium loss, the reactivity rises in the axial flow reactors. For the 4.5-ft-diameter core, $\Delta k=0.013$, and for the 6-ft-diameter core $\Delta k=0.022$. As in the case of the sodium temperature coefficient, there would be improvement from flattening the core.

The reactivity change due to sodium loss in the radial flow reactor is harder to estimate because of the large leakage through the central coolant pipe. However, for a reduction of sodium density to one-fourth of its normal value, the radial flow reactor loses $\Delta \mathrm{k}=0.067 \mathrm{in}$ reactivity.

\section{Doppler Temperature Coefficients}

The average fuel temperature under operating conditions is expected to be around $1500^{\circ} \mathrm{F}$. For the amount of scattering material present in these reactors, the Doppler effect saturates near this temperature so that the Dopplex temperature coefficient, while negative, is not large enough to outweigh the positive sodium coefficient in the axial flow reactors described so far. More will be said below about overcoming this difficulty. In the radial flow reactors the Doppler coefficient is still negative, but weakex than in the axial flow case. Here, however, the sodium coefficient is negative and a strong negative Doppler coefficient is not needed.

The Doppler coefficients were calculated with a version of the GAM code modified to use Greebler's method [(Physics of East Reactors, Vol III, p. 226 (1961)], but allowing for sodium scattering resonances in calculating scattering per atom. Figure 7 shows the reactivity losses due to Doppler effects of $U^{238}$ and $P u^{239}$ combined. The contribution of $U^{238}$ is negative and that of $\mathrm{Pu}^{239}$ positive. The magnitude of the $\mathrm{U}^{238}$ contribution is about 8 times that of Pu ${ }^{239}$ in the axial flow reactors and only about 2 in the radial flow reactor.

Effects of interference between $\mathrm{U}^{238}$ and $\mathrm{Pu}^{239}$ resonances has not yet been examined. 


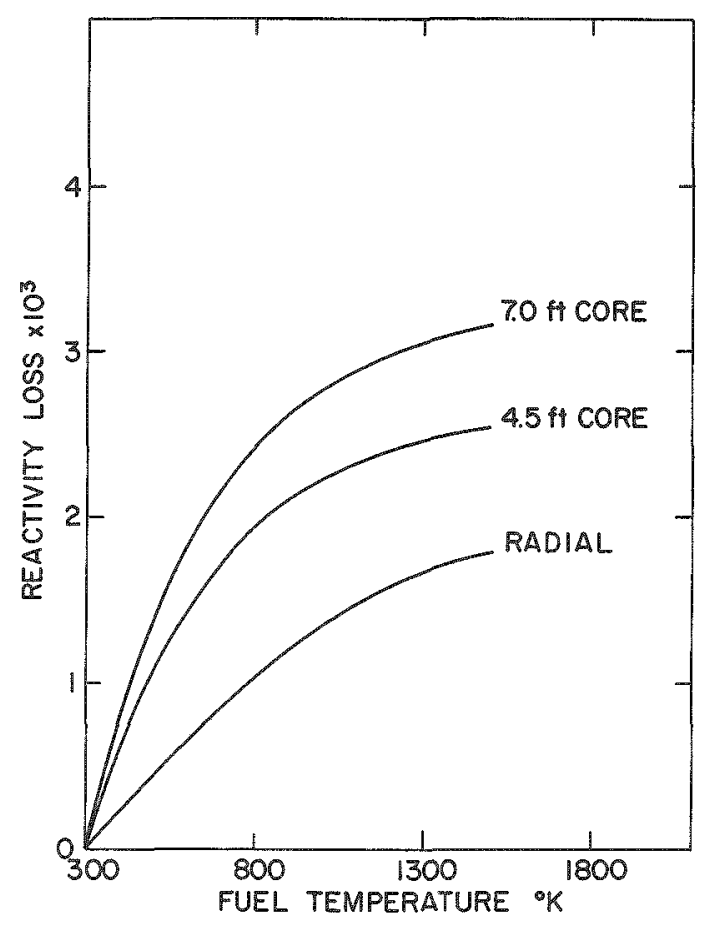

Fig. 7. Doppler Effect
Composition Changes in the Axial Flow Reactors

Substitution of lead or bismuth for the sodium coolant in these reactors was found to have very little effect, mainly because of the low volume fractions of coolant.

On the other hand, increasing the volume fraction of sodium in the core has a strong effect and is in many respects beneficial. It increases the core leakage, thereby shifting some of the breeding from the core to the blanket. This reduction of core breeding ratio will improve the reactivity change vs. burnup properties of the reactors. Furthermore, the sodium temperature coefficient is improved and the Pu 239 loading decreased. These data are exhibited in Tables $1,2,3$, and 4 .

So far, no specific design has been chosen for accomplishing these changes of volume fraction. It may be done by having sodium coolant pipes running through the bed; or the fuel regions may be partitioned to allow sub-zones of sodium or of fertile material. Or still other ways may yet be suggested.

Table 1

$P^{239}$ LOADING (kg) VS. CORE DIAMETER AND SODIUM VOLUME FRACTION

\begin{tabular}{l|l|l}
\hline$D(f t)$ & 4.5 & 6.0 \\
\hline$V_{N a}$ & & \\
\hline 0.26 & 827 & 1780 \\
0.4 & 750 & 1560 \\
0.55 & 670 & 1350 \\
0.7 & 600 & 1150 \\
\hline
\end{tabular}

Table 2

CORE BREEDING RATIO VS. CORE DIAMETER AND SODIUM VOLUME FRACTION

\begin{tabular}{l|l|l}
\hline$D(f t)$ & 4.5 & 6.0 \\
\hline$V_{\mathrm{Na}}$ & & \\
\hline 0.26 & 1.51 & 1.70 \\
0.4 & 1.35 & 1.57 \\
0.55 & 1.10 & 1.36 \\
0.7 & 0.80 & 1.04 \\
\hline
\end{tabular}


TOTAL BREEDING RATIO VS. CORE DIAMETER AND SODIUM VOLUME FRACTION

\begin{tabular}{l|l|l}
\hline $\mathcal{D}(\mathrm{ft})$ & 4.5 & 6.0 \\
\hline $\mathrm{V}_{\mathrm{Na}}$ & & \\
\hline 0.26 & 2.03 & 2.04 \\
0.4 & 1.99 & 1.99 \\
0.55 & 1.93 & 1.93 \\
0.7 & 1.83 & 1.83 \\
\hline
\end{tabular}

$\mathrm{dk} / \mathrm{dTNa}$ IN UNITS OF $10^{-6} /{ }^{\circ} \mathrm{C}$

\begin{tabular}{l|l|l}
\hline $\mathrm{D}(\mathrm{ft})$ & 4.5 & 6.0 \\
\hline $\mathrm{V}$ & & \\
\hline 0.26 & +2.6 & +4.2 \\
0.4 & +3.1 & +6.2 \\
0.5 & +2.8 & +6.8 \\
0.6 & +1.4 & +7.2 \\
0.7 & -2.8 & +5.4 \\
0.75 & -9.4 & +2.8 \\
\hline
\end{tabular}

Effects of Shifts of Bed Density

All the studies described so far assumed that the solid fraction of a randomly packed bed of spheres is $60 \%$. This is only a nominal value, however. As discussed by Chernick, large randomly packed beds may exhibit a loose packing with a solid fraction of about $61 \%$ and a tight packing of as much as $64 \%$. And even such averages as these for large beds may not apply locally where there may be point-to-point fluctuations in density.

Two effects of changes of bed density have been considered: a) the effect on reactivity of a change in average bed density during operation of the reactor, and b) the effect of point-to-point fluctuations in density.

a) For a typical axial flow reactor it was found that a settling which gives a change in solid fraction from 0.600 to 0.602 for the entire core gives a $10 \%$ reactivity increase, based on an effective delayed neutron fraction of 0.0035 . Preliminary measurements have indicated that bed density may fluctuate as much as this from one settling to the next in beds of several thousands or several tens of thousands of balls. However, because the coolant flow is expected to lock the bed in place, little density change is expected during flow. Furthermore, the reactors will have of the order of $10^{8}$ to $10^{9}$ balls, and it appears unlikely that a local settling would be propagated through the entire bed. It seems more likely that fluctuation of bed density for large randomly packed beds will be proportional to the reciprocal square root of the number of balls in the bed. If this is so, then changes in average bed density will be negligible. 
The settling problem for the radial flow reactor is actually more complicated than what has been indicated here. Since the flow, and therefore the drag forces on spheres, is radially outward, any settling during reactor operation will also most likely be outward also. This would give a reactivity decrease, but the magnitude has not yet been estimated.

b) Point-to-point fuctuations in density were studied by means of one-dimensional calculations for layered reactors with the volume fractions in the layers drawn from a Gaussian distribution of solid fractions around a mean value of $60 \%$. To get a $10 \%$ reactivity effect, it was necessary to assume fluctuations having a standard deviation of $0=0.01$ for layers containing $N=6 \times 10^{5}$ balls, that is, $\sigma \sqrt{N} \sim 8$. However, preliminary results for bed packings indicate that the actual value of $\sigma \sqrt{N}$ is less than one-tenth of this. More information is needed on whether, and how closely, the bed statistics follow a Gaussian distribution law. However: as long as density fluctuations average out over distances of the oxder of neutron migration lengths, there should be little effect, and this seems to be the case.

Note Discussion of this paper follows Paper 7 , page 612 . 


\author{
$G$ Pancer and $R$. Powers \\ Brookhaven National Laboratory \\ Upton, L. I., New York \\ (Paper presented by Mr. Powers)
}

1. General Considerations

As presently conceived, the settled bed reactor concept requires $\frac{1}{8}$-in -diameter spherical fuel pellets of fuel of high thermal conductivity that are compatible with the sodium coolant and stainless steel reactor walls at temperatures exceeding $650^{\circ} \mathrm{C}$.

Because broken pellets may lead to unpredictable decreases in void volume and thus potentially to large reactivity increases, it is necessary for pellets to be unusually strong over the lifetime of the fuel The desirability of exceeding burnups of 50,000 MWD/T without excessive fuel swelling puts a further limitation on possible fuel materials Also, in order to be able to fluidize the settled bed periodically for reshuffling fuel, the particles must not sinter together under irradiation

On the basis of the above demands on the settled-bed fuel material, UC-PuC with a $U / P u$ ratio of $7 / 1$ has been provisionally selected as reference fuel, and UC as blanket material Carbon transfer from pellets to a stainless steel reactor vessel is to be minimized by using stolchiometric or slightly hypostoichiometrac UC in the research program UntiI an alphahandling facility is obtained, work on fuel materials at BNL is presently concerned with uranium compounds

\title{
2 Capsule Studies
}

The suitability of UC-PuC fuel for the settled bed reactor concept will depend on whether or not it sinters during irradiation A series of capsule tests is beginning to test the sintering behavior of $\mathrm{UC}$ under irradiation and under load conditions that might be expected in a settled bed reactor

(a) Fuel Procurement and Evaluation. Commercial availability of $\frac{1}{8}$-in.-diameter spheres of UCwas investigated Material prepared by either arc melting or powder metallurgy was obtained from several vendors, and examined metallographically and chemically at BNL Typical metallography of carbide prepared from $\mathrm{UO}_{2}$ and carbon by the low pressure plasma arc process is shown in Figs. I-4 The first two figures show close to stoichometric UC (average carbon content of $475 \%$ ). Figure 1 shows platelets of $U_{2}$ in a UC matrix. This material would be unsuitable for use in a sodrum-filled stainless steel capsule because of carbon transfer through the sodium to the capsule walls. Figure 2 shows single-phase stoichiometric UC 
(1)

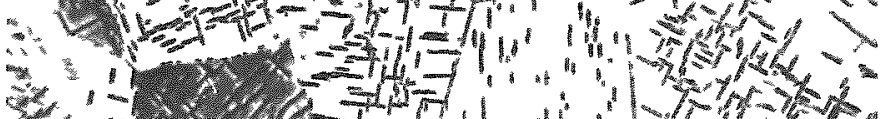

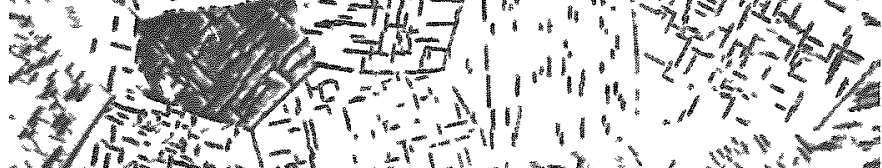

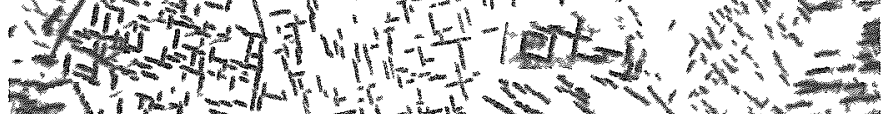
Fin

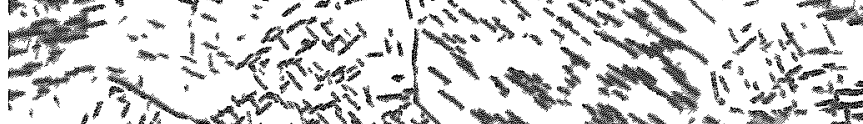

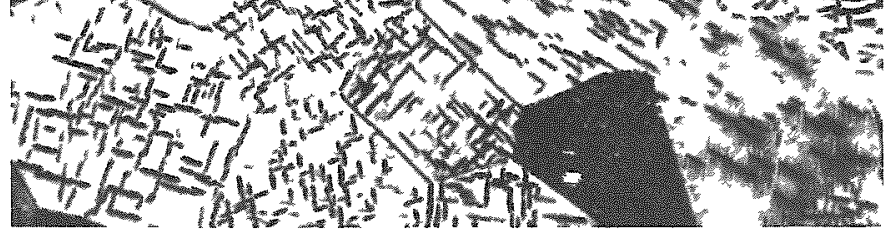

Fig. 2

Single Phase Stoichiometric UC

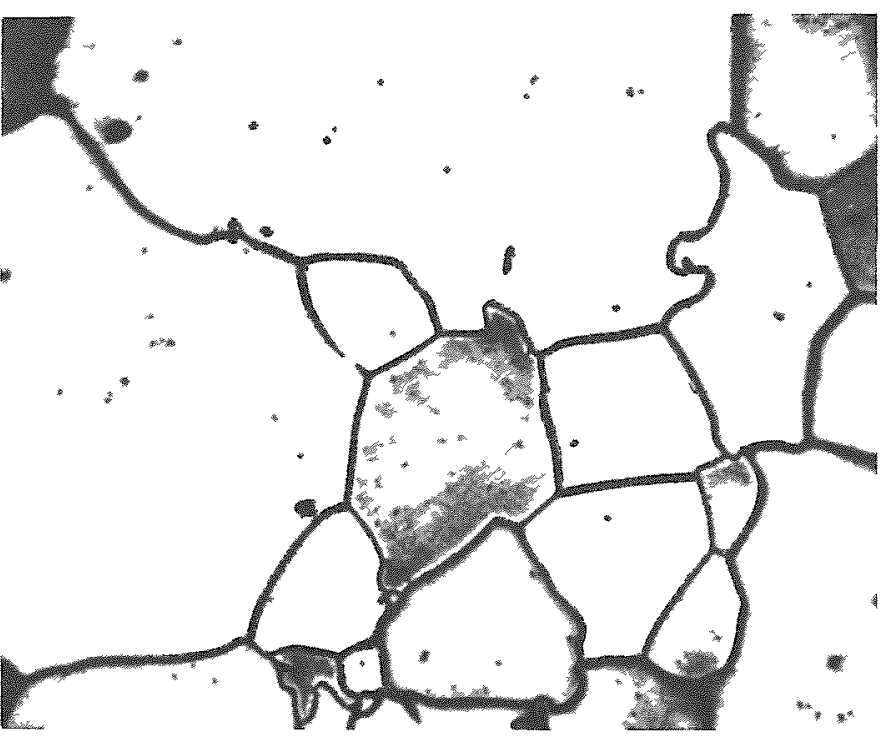

$\mathrm{UC}$ Containing Platelets of $\mathrm{UC}_{2}$

Fig. 1 


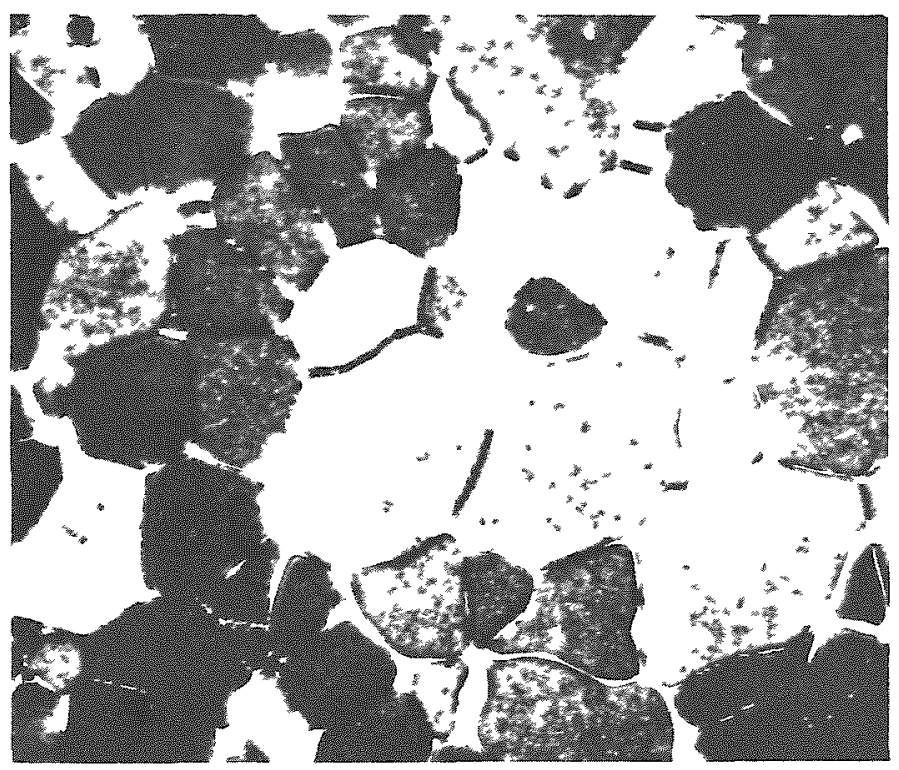

Fig. 4

Hypostoichiometric UC Approximately $44 \% \mathrm{C}$ with most of Uranium at the Grain Boundaries

The material depicted in Fig. 3 contains $4.65 \%$ carbon and shows a trace of uranium metal at the grain boundaries, whereas that in Fig. 4 shows somewhat less carbon and increased amounts of uranium metal.

The appearance of selected spheres of UC is shown in Fig. 5.
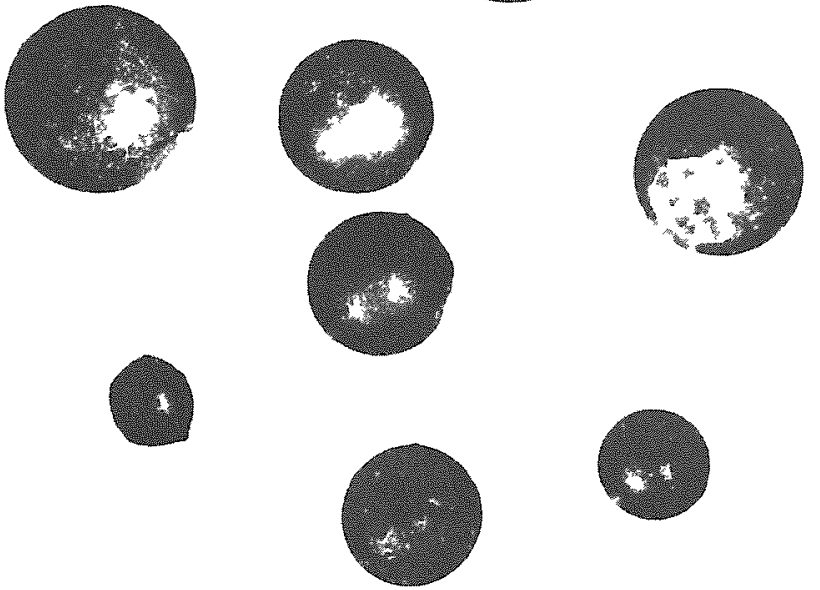

Fig. 5. Selected UC Spheres Fabricated by Arc Melting Process 
(b) Fuel Testing: Crushing (drop weight) tests carried out on the as-received carbide pellets showed the arc-melted carbide to be considerably stronger than that produced by powder metallurgy.

Typical UC pellets were exposed to $\mathrm{NaK}$ at $1400^{\circ} \mathrm{F}$ for one month with a one-pound static load placed on a column of these pellets during test. Other loaded samples were continuously thermal cycled between $300^{\circ}$ and $1400^{\circ} \mathrm{F}$, also for one month.

Chemical and metallurgical examination of samples after these tests showed no adverse effects to fuel produced either by arc melting or powder metallurgy under these test conditions, and no sintering was observed.

(c) Capsule Design: It is estimated that the forces acting on the fuel particles due to the total pressure drop across the fuel bed and to the weight of the bed itself are approximately equal to a $1-1 b$. force on a fuel particle To duplicate the se conditions the column of $\frac{1}{8}$-in,-diameter spheres in the in-pile capsule is loaded with a $1-1 b$. dead weight. The spheres are held in a perforated length of tubing 0.18 -in. ID. This eliminated the need for a complex spring loading system to maintain a constant force on the fuel particles

The capsule unit (Fig. 6) is made up of two independent concentric tubes, each approximately 25 in. long. Both tubes or containers are fabricated of stainless steel. The primary or inner capsule contains the fuel and liquid metal. The secondary or outer capsule is finned for heat removal, the annulus between the two units being filled with He.

The primary capsule contains enough NaK to cover the fuel. A stainless steel-sheathed heater and thermocouples are submerged in NaK inside the primary capsule. These are used for auxiliary heat and temperature indication and control. The penetrations for these leads through the primary container are sealed by a high-temperature furnace braze. A radiograph of the fueled section of the primary in-pile capsule is shown in Fig. 7 .

The resistance wire leads which terminate just outside the primary capsule are joined to stainless steel-sheathed Ni conductor wire.

The activity of the helium flowing between the two capsules is monitored to indicate any failure of the primary capsule, Forced air that removes heat from the secondary container is also monitored to indicate a possible failure of both capsules.

The capsule design required a vertical facility in the reactor (Fig. 8). A hole approximately 4 -in. in diameter and $8 \mathrm{ft}, 4$ in. long was 
$-\quad 325^{\circ}-$
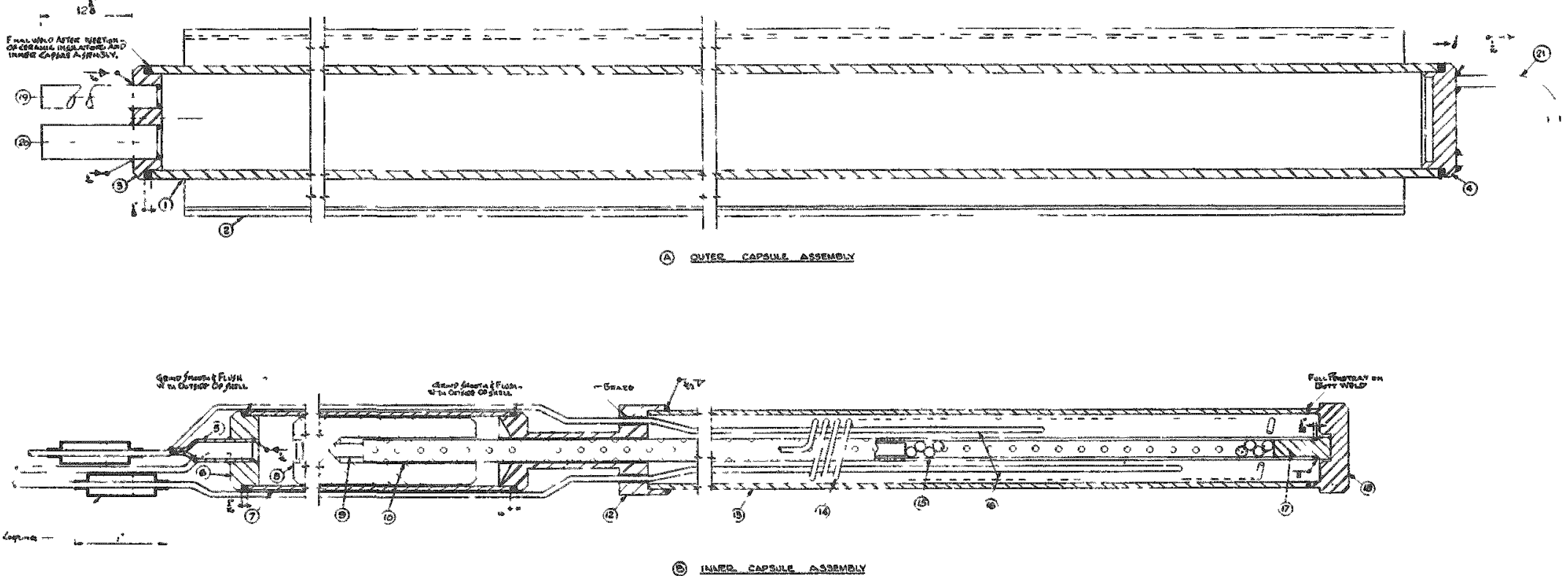

Fig. 6. Primary and Secondary Capsule Design
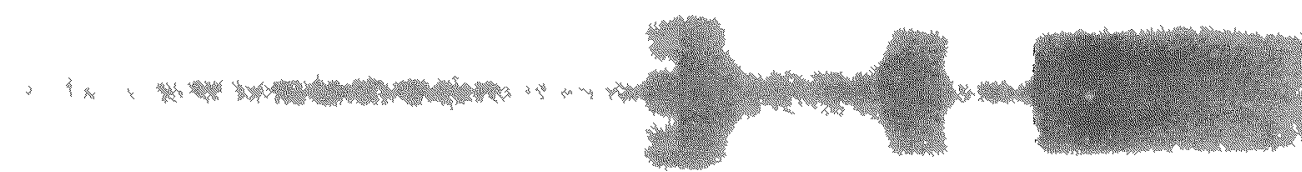

Fig. 7. Radiograph of Fuel Primaxy Capsule

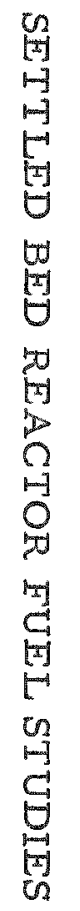


consequently drilled in the Brookhaven Graphite Reactor since none of the existing reacior facilities were adequate, and lined with an air-cooled aluminum thimble. The capsule is inserted into the thimble to a depth of approximately $8 \mathrm{ft}$. The reactor cooling system or an external blower can be used to supply air. A pictorial arrangement of the facility is shown in Fig. 8 .

Normally only the capsule is removed. In event of an emergency the entire capsule and thimble may be taken out of the reactor.

The capsule design and operating parameters are given in the following table:

Fue1

Enrichment

Thermal flux (perturbed)

Fueled length

Fission heat

Auxiliary heat(electric)

Maximum auxiliary heat

NaK operation temp.

(fuel surface temp.)

Design capsule pressure $\frac{1}{8}$-in. diameter UC spheres

$20 \% \mathrm{U}^{235}$

$3.5 \times 10^{12} \mathrm{n} / \mathrm{cm}^{2}-\mathrm{sec}$

4 in.

$382 \mathrm{~W}(1300 \mathrm{BTU} / \mathrm{hr})$

$352 \mathrm{~W}(1200 \mathrm{BTU} / \mathrm{hr})$

$703 \mathrm{~W}(2100 \mathrm{BTU} / \mathrm{hr})$

$760^{\circ} \mathrm{C}\left(1400^{\circ} \mathrm{F}\right)$

$100 \operatorname{psia}\left[815^{\circ} \mathrm{C}\left(1500^{\circ} \mathrm{E}\right)\right]$

(d) Capsule "i: The first capsule loading consists of a stack of 35 spheres of UC produced by the Vitro low-pressure arC process and having a nominal carbon content of $4.7 \%$. Twenty-seven of these spheres were loaded in the as-received condition - with the surface covered by a thin blue film. The remaining eight pellets were given an additional heat treatment of $2 \mathrm{hr}$ at $1710-1715^{\circ} \mathrm{C}$ under a vacuum of $10^{-4}$ torr These samples were held in a tantalum gettered tungsten crucible and resulted in shiny spheres.

(e) Capsule Testing Program:

1. BNI Capsules: It is planned to irradiate Capsule "1 for 4 months. After its removal, a similar capsule containing experimental $\mathrm{U}-\mathrm{Zr}-\mathrm{C}$ compositions will be irradiated. Meanwhile, a second hole is to be drilled in the BNL reactor for capsule work.

2. High Flux Capsules: To test fuel compositions at high burnup, an MTR capsule similar to that used in the BNL studies is to be designed and constructed. 


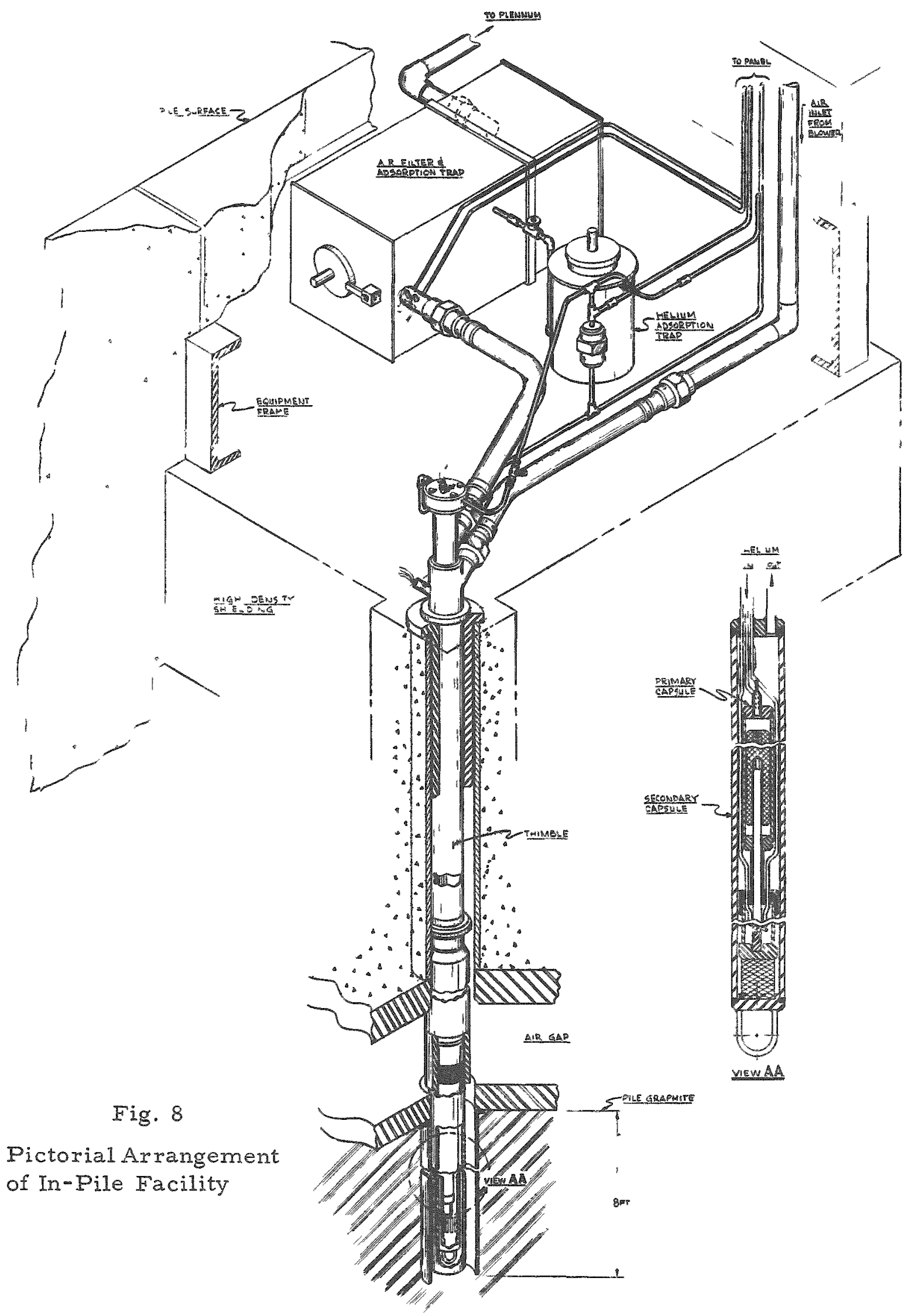


Preliminary design of a capsule for the High Flux Beam Reactor is expected to be completed well before the startup of this reactor.

BNL Fuel Development

In addition to fuel procurement from outside vendors, fuel studies have begun at BNL.

The initial objective of this program will be to determine the extent of the single-phase $U-Z r$ carbide region with particular emphasis on the lower carbon phase boundary.

It is hoped to develop a U-Zr-C fuel for which the present exacting carbon control in fuel preparation may be relaxed and still result in a single-phase substoichiometric $\mathrm{U}-\mathrm{Zr}-\mathrm{C}$ fuel. By raising the melting point and possibly the strength of the fuel by alloying, the chance of fuel particle sintering and breakage under irradiation may be reduced.

Discussion of Papers
Presented by Miss. Chernicl, Levine, and Powers

MR. DIETRICH (General Nuclear Engineering):

The papers on the Fast Settled Bed Reactor are now open for discussion.

MR. SMIDT (Karlsruhe):

We have found the engineering details of these kinds of reactors to be very, very difficult. We assumed that the pressure drop should not exceed a value of about the order of 5 atm., because of mechanical reasons, not so much because of pumping power. If we went to a very flat pancake or relatively thin annulus and tried to find that core design in which we could have an equal flow of fuel balls in all parts of the core, we had an equal loss of fuel everywhere. The machines became very, very complicated and we did not go on.

MR. VISNER (Combustion Engineering):

I was wondering what the loss in breeding gain was in the design with radial flow where you have a 2 -ft chimney coming down the center.

MR. CHERNICK

The loss of breeding ratio was calculated to be 0.2 . There is a breeding blanket above the core, of course, so that not all the neutrons 
streaming out through the sodium are lost, but there is still this 2 -ft reg1on. It has been suggested that maybe you could have an internal breeder, again complicating the geometry if you want to get back this 02 . But this increases some of the engineering problems. Certainly we do not regard these reactors as completely engineered. Nevertheless, it is possible that we may come up with a feasible way of compacting the beds. We are going to perform a 2-region critical experiment in about a year or so, in which we will have simple swimming pool-type fuel elements as the driver reactor, and we will have a small test cylinder, perhaps six inches $i_{3}$ diameter. It will be a cheap experiment in terms of the number of particles we will need and also the pressure drop. We will do this, not so much to get information on safety, which is certainly a prime consideration, but to find how a reactor like this operates, how you may control it, what are the hydraulics like? It seems to me very reasonable to go to a cold experiment in which we will not use sodium, but water, together with aluminum-uranium balls, mainly to get at the characteristics of this reactor in a relatively cheap way.

MR. HAFELE (Kaxisruhe):

We have found it easy to prepare and fabricate small spheres with a diameter of about $8-9 \mathrm{~mm}$. But we found it very expensive to have a routine check whether the cladding of such balls was properly done, Would you comment?

MR.POWERS:

Initially we had not started to clad the spheres, although there is quite a bit of pressure from the physics group to retan fission products.

\section{MR. HAEELE:}

If you accept dirty cermets, that is one thing, but if you don't, then we found that the testing of the welding of the cladding was the heaviest setback.

MR. POWERS.

I believe that for this size of sphere you would have to rely on a vapor plating process to provide an integrated coat over the pellets. The spheres that we have used for the preliminary tests were prepared by a relatively easy process. but the uniformity was not desirable in the sense that there was quite a spread of diameters. Radiography of the samples on a mass basis would probably be quite difficult, but we have done it on the spheres that we have used and found these spheres to have small, uniformly central voids. In any case, I think you have to use a statistical approach. 
MR. EDLUND (Babcock \& Wilcox):

To what level is this project funcied at the present time, and what future plans do you have for experimental work?

MR, CHERNICK:

We have a program in which we are trying to tie together some of the work in metallurgy, physics, and so on. That is it. We think it is much too early to go to the project stage. We are serious about a critical experiment of the type I mentioned. We think this would be a reasonable way to start in. Somewhere beyond that there might be a hot critical experiment in which we would use sodium, but this is quite a distance off. We have many, many steps before we shall be convinced that it is worth going very much further with this fuel. We don't know whether the monocarbide spheres, unclad, are indeed what we want.

MR. CHARAK (Argonne):

I was wondering if you believe that the downflow concept seriously compromises the safety aspects of the system in terms of depending on flow reversal for decay heat removal.

\section{MR. CHERNICK:}

We certainly have not gotten to the point of worrying about decay heat removal yet. Nevertheless, I do not see that this is necessarily a difficult thing to do. Here is a fuel which you can actually move in a fluid to wherever you want it. We think downflow is actually quite dramatic in locking in the particles. A tremendous force is holding in the particles even in velocities of a couple of feet per second, because very high pressure drops, of the order of 100 psi or so through the system, are involved. The large locking forces constitute a safety factor. But we have not looked at the decay problem in detail.

MR. SESONSKE (Purdue University):

I wonder about the effect of very small particles formed by particle degradation. There could be reactivity effects, a change in the hydraulics, and a possibility of hot spots due to changes in the heat removal picture.

MR. CHERNICK:

We have considered that you will have small breakage of particles: we guess that they will normally be swept out. But your point is well taken. 


\author{
SESSION VI \\ October 9,1963 \\ SAFETY - GENERAL SUMMARY \\ Chairman: D. OKRENT \\ Argonne National Laboratory \\ Secretaxy: B. J. Toppel \\ Argonne National Laboratory
}


616 
COMPARISON OF THE DYNAMIC AND SAFETY CHARACTERISTICS OF FAST AND FAST-COUPLED REACTOR SYSTEMS

\author{
Anthony V. Campise \\ Atomics International \\ Canoga Park, California
}

I. Introduction

Nuclear characteristics of fast reactors differ from thermal reactors in several aspects:

1. no light moderating material in core;

2. large fuel inventory in core:

3. short prompt-neutron lifetime;

4. basic nature of reactivity coefficients.

Due to the lack of moderating material, fast reactor cores are highdensity systems. Operation in a fast neutron spectrum, in which microscopic nuclear cross sections are small compared to thermal values, necessitates large critical masses. Maintaining criticality with fastneutrons results in a very small prompt-neutron lifetime, one of the characteristics of these fast systems. In view of the above properties of fast reactors, it is possible to postulate a maximum credible accident leading to a nuclear explosion. Even though this postulated accident is obtained on pessimistic assumption, concern for public welfare demands adequate experiments for the demonstration of safe operation of fast reactors. The superior neutron economy of fast reactor systems is one of the main factors leading to the research and development of this type of power plant. However, in view of the safety consideration, this type of plant may be many years in the future.

Demonstrated safety and reliability of the thermal sodium-cooled graphite-moderated reactor experiment (SRE) and the Hallam Nuclear Power Facility provide the necessary reason to use this technology in future advanced sodium systems. Thus, the problem is resolved to the possibility of using the demonstrated safety of the SGR and the superior neutron economy of fast reactor systems. The answer to this problem was first formulated by Avery at ANI, demonstrated in a ZPR-III experiment, and later applied successfully in the AETR critical assembly. This concept has been termed a coupled reactor system. Basically, this system contains a portion of a fast reactor (which is subcritical) and a portion of a thermal reactor (also subcritical). Criticality is obtained by placing these two reactor regions together.

This type of reactor is proposed as the earliest demonstration of safety and performance for a large, dilute fast reactor. This reactor concept 
could successfully resolve the above-mentioned pertinent problems of fast reactor technology. The concept is of prime importance to AI's present sodium reactor program. This proposed reactor plant would use AI's SRE experience, SGR design effort, years of sodium component work, and attractive nuclear properties of sodium-cooled fast reactor systems whose nuclear properties were determined in AETR fast criticals.

The following notes on the reactor stability and relative safety of coupled systems will indicate how the demonstrated safety of the SGR can be used. The static nuclear charactexistics of fast-coupled reactor have been adequately demonstrated in the above-mentioned criticals and will not be discussed in this paper. The relative economics of these systems are now being studied and will be available at a later date.

\section{General Integral Theory and Approximations}

\section{A. Coupled Reactor Theory}

The following is a general review of pertinent parameters associated with fast-coupled reactor theory. This subject is more adequately covered by Avery. (1)

The basic reactor concept considered will be a fast reactor section (Region - 11) surrounded by a thermal section (Region -22). By definition, the fission rates $S_{1}$ and $S_{2}$ are as follows:

$$
\begin{aligned}
& S_{1}=k_{11} S_{1}+k_{12} S_{2} ; \\
& S_{2}=k_{21} S_{1}+k_{22} S_{2},
\end{aligned}
$$

where the $k_{i j}$ are "coupling" factors which give the neutrons from $j$ to $i$. From (1) and (2) we define the criticality condition of a coupled reactor:

$$
\begin{aligned}
& \left(1-k_{11}\right)\left(1-k_{22}\right)=k_{12} k_{21} \\
& S_{2} / S_{1}=\left(\frac{1-k_{11}}{k_{12}}\right) .
\end{aligned}
$$

Consider one source neutron in the fast section with a coupling factor $k_{11}$.

The multiplication of that neutron is

$$
1+k_{11}+\left(k_{11}\right)^{2}+\left(k_{11}\right)^{3}+\cdots-+=\frac{1}{1-k_{11}}
$$


Consider a source neutron in the thermal section having a coupling factor $k_{2 \mathbb{E}}$, so that the multiplication of this neutron is

$$
1+k_{22}+\left(k_{22}\right)^{2}+\left(k_{22}\right)^{3}+\cdots++=\frac{1}{1-k_{22}}
$$

The total multiplication $M T$ is then

$$
M_{T}=\left(\frac{1}{1-k_{11}}\right)+\left(\frac{1}{1-k_{22}}\right)
$$

Therefore, the partial multiplication which is equal to the partial reactivity or, as Avery(1) stated, reactivity partition in this system is defined as

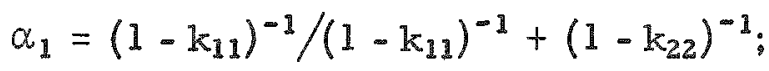

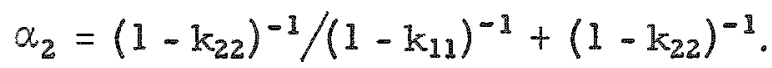

The $k_{i j}$ are obtained from the solution of relevant multigroup models for each factor.

Following formulations by Håfele, (2) we now define* the inhoux equation for coupled-reactor systems (details of formulation contained in Ref. 2):

$$
\left[\left(k_{11} m_{1} \gamma-e^{\omega \ell_{11}}\right)\left(k_{22} m_{2} \gamma-e^{\omega \ell_{22}}\right)-k_{12} k_{21} m_{1} m_{2} \gamma^{2}\right]=0
$$

or, collecting terms,

$$
\left(k_{13} k_{22}-k_{12} k_{23}\right) m_{1} m_{2} \gamma^{2}-k_{22} m_{2} \gamma e^{\omega b_{11}}-k_{11} m_{1} \gamma e^{\omega b_{22}}+e^{\omega\left(l_{11}+l_{22}\right)}=0 .
$$

By assuming

$$
\gamma \simeq 1.0 ; b_{11} \ll b_{22}
$$

we have

$$
e^{\omega l_{22}} \simeq \frac{\left(k_{12} k_{21}-k_{11} k_{22}\right) m_{1} m_{2}+k_{22} m_{2}}{\left(1-k_{11} m_{1}\right)}
$$

For steady-state conditions, $m_{1}=m_{2}=1$, which is shown as follows. Therefore (13) becomes

* Definitions are given in the Nomenclature. 


$$
e^{\omega \ell_{22}} \simeq \frac{\left(k_{12} k_{21}-k_{11} k_{22}\right)+k_{22}}{\left(1-k_{11}\right)}
$$

From (3) we have

$$
e^{\omega l_{22}} \simeq \frac{\left(1-k_{11}\right)\left(1-k_{22}\right)+k_{22}\left(1-k_{11}\right)}{\left(1-k_{11}\right)} \simeq 1.0
$$

so that

$$
\omega l_{22} \simeq 0
$$

Since $\ell_{22}$ is always positive we have that, at steady state,

$$
\omega=0 \text {, }
$$

or that the reactor is on a infinite period:

$$
T=\infty
$$

However, from (10) if $k_{11} m_{1} \gamma \gg 1.0$, so that $\omega l_{22} \gg 1$, we get

$$
k_{11} m_{1}(1-\beta)-1=\omega b_{22}
$$

which is the behavior of a decoupled fast reactor core. Thus, if a large amount of reactivity is inserted into the fast section in a very short period of time, the coupled system will behave as a fast reactor, overriding the thermal neutron delaying effect.

However, if coupling is maintained, then the effective promptneutron lifetime of the coupled system is given by (at steady state)

$$
l_{\text {eff }}=\alpha_{1} l_{11}+\alpha_{2} l_{22}
$$

From condition (12) we have that

$$
l_{\text {eff }} \simeq \alpha_{2} b_{22}
$$

so that the coupled system has an effective lifetime approximately that of the thermal component.

Assuming that flows of the sodium coolant in the fast and thermal sections are equal, we define the effective temperature coefficient of a coupled system as

$$
(\mathrm{T} . \mathrm{C})_{\mathrm{eff}}=\alpha_{1}(\mathrm{~T} \cdot \mathrm{C})_{1}+\alpha_{2}(\mathrm{~T} \cdot \mathrm{C})_{2}
$$


Having defined the necessary parameters for a coupled reactor system, we will examine the stability of this core for small power perturbations.

\section{B. Stability}

The following assumptions are made in writing the kinetic description of reactor fuel and of coolant and moderator temperatures required to investigate the closed-loop temperature and power behavior of coupled reactor systems.

\section{Assumptions}

a. Constant coolant flow is maintained in cores.

b. Lineaxized reactor kinetic equations apply.

c. No heat is generated in coolant or moderator.

d. Coolant, fuel, and moderator temperatures rise linearly.

e. Heat is transferred from lump average fuel temperature to lump average coolant to lump average moderator temperature.

\section{Fast Section}

1. Fuel temperature:

$$
H_{f}^{f} \frac{d \bar{T}_{f}^{f}}{d t}=P^{f}-h^{f} A_{f}^{f}\left(\bar{T}_{i}^{f}-\bar{T}_{c}^{f}\right)
$$

2. Coolant temperature:

$$
H_{C}^{f} \frac{d \bar{T}_{c}^{f}}{d t}=h^{f} A_{f}^{f}\left(\bar{T}_{f}^{f}-\bar{T}_{c}^{f}\right)-F^{f}\left(T_{o}^{f}-T_{i n}^{f}\right) .
$$

\section{Thermal Section}

3. Fuel temperature:

$$
\mathrm{H}_{f}^{\mathrm{T}} \frac{\mathrm{d} \overline{\mathrm{T}}_{f}^{\mathrm{T}}}{\mathrm{dt}}=\mathrm{P}^{\mathrm{T}}-\mathrm{h}^{\mathrm{T}} \mathrm{A}_{f}^{\mathrm{T}}\left(\overline{\mathrm{T}}_{\hat{f}}^{\mathrm{T}}-\overline{\mathrm{T}}_{\mathrm{c}}^{\mathrm{T}}\right) \text {. }
$$


4. Coolant temperature:

$$
H_{c}^{T} \frac{d \bar{T}}{d t}=h^{T} A_{f} T\left(\bar{T}_{f}^{T}-T_{c}^{T}\right)-F^{T}\left(\bar{T}_{0}^{T}-T_{i n}^{T}\right)
$$

5. Moderator temperature:

$$
\mathrm{H}_{\mathrm{M}}^{\mathrm{T}} \frac{\mathrm{d} \overline{\mathrm{T}}_{\mathrm{m}}^{\mathrm{T}}}{\mathrm{dt}}=h_{\mathrm{m}} \mathrm{A}_{\mathrm{m}}^{\mathrm{T}}\left(\overline{\mathrm{T}}_{\mathrm{c}}^{\mathrm{T}}-\overline{\bar{T}}_{\mathrm{M}}^{\mathrm{T}}\right)
$$

6. Reactivity: From (21), we have

$$
(T \cdot C)_{e f f}=\alpha_{1}(T \cdot C)_{2}+\alpha_{2}(T \cdot C)_{2}
$$

so that the effective reactivity of a coupled system, including effects from fuel, coolant, and moderator in different sections, becomes

$$
\delta \rho=\alpha_{1} \alpha_{\frac{f}{I}}^{f} \delta \overline{\mathrm{T}}_{\mathrm{f}}^{f}+\alpha_{2} \alpha_{f}^{\mathrm{T}} \delta \overline{\mathrm{T}}_{f}^{\mathrm{T}}+\alpha_{1} \beta_{c}^{f} \delta \overline{\mathrm{T}}_{\mathrm{c}}^{f}+\alpha_{2} \beta_{\mathrm{c}}^{\mathrm{T}} \delta \overline{\mathrm{T}}_{\mathrm{c}}^{\mathrm{T}}+\alpha_{2} \gamma_{\mathrm{m}} \delta \overline{\mathrm{T}}_{\mathrm{m}}
$$

Taking Laplace transforms of equations $22-28$ we have

\section{East Section}

Euel:

$$
H_{p}^{f} s \Delta \bar{T}_{f}^{f}(s)=\Delta P^{f}(s)-h^{f} A_{f}^{f}\left[\Delta \bar{T}_{f}^{f}(s)-\Delta T_{c}^{f}(s)\right] .
$$

Coolant:

$$
\mathrm{H}_{c}^{f}(s) \Delta \mathrm{T}_{c}^{f}(s)=h^{f} A_{f}^{f}\left[\Delta \overline{\mathrm{T}}_{f}^{f}(s)-\Delta \overline{\mathrm{T}}_{c}^{f}(s)\right] \text {. }
$$

Thermal Section

Fuel:

$$
H_{f}^{T} s \Delta \bar{T}_{f}^{T}(s)=\Delta P^{T}(s)-h^{T} A_{f}^{T}\left[\Delta T_{f}^{T}(s)-\Delta T_{c}^{T}(s)\right] .
$$

Coolant:

$$
H_{c}^{T} s \Delta T_{c}^{T}(s)=h^{T} A_{f}^{T}\left[\Delta \bar{T}^{T}(s)-\Delta \bar{T}_{c}^{T}(s)\right]-F^{T}\left[\Delta T_{0}^{T}-\Delta T_{i n}^{T}\right] .
$$




\section{Moderator}

$$
\begin{aligned}
& \mathrm{H}_{\mathrm{M}} \mathrm{s} \Delta \overline{\mathrm{T}}_{\mathrm{m}}(\mathrm{s})=\mathrm{h}_{\mathrm{m}}^{\mathrm{T}} \mathrm{A}_{\mathrm{m}}^{\mathrm{T}}\left[\Delta \overline{\mathrm{T}}_{\mathrm{c}}^{\mathrm{T}}-\Delta \overline{\mathrm{T}}_{\mathrm{m}}\right] \\
& \Delta \rho(s)=\alpha_{1} \alpha_{f}^{f} \Delta \overline{\mathrm{T}}_{f}^{\mathrm{f}}(\mathrm{s})+\alpha_{2} \alpha_{f}^{\mathrm{T}} \Delta \overline{\mathrm{T}}_{f}^{\mathrm{T}}(\mathrm{s}) \div \alpha_{1} \beta_{\mathrm{c}}^{\mathrm{f}} \overline{\Delta \mathrm{T}}_{\mathrm{c}}^{\mathrm{f}}(\mathrm{s})+\alpha_{2} \beta_{\mathrm{c}}^{\overline{\mathrm{T}}_{\mathrm{T}}} \overline{\mathrm{T}}_{\mathrm{c}}^{\mathrm{f}}(\mathrm{s})+\alpha_{2} \gamma_{\mathrm{m}}^{\mathrm{T}} \Delta \mathrm{T}_{\mathrm{m}}^{\mathrm{T}} . \\
& \text { a. }\left(T_{0}-T_{\text {in }}\right) / 2=\bar{T}_{C} \text {; } \\
& \text { b. } \tau_{f}^{f}=\left(H_{f}^{f}\right)^{-1} ; \tau_{c}^{f}=h^{f} A_{f}^{f} / H_{f}^{f} ; \\
& \text { c. } \lambda_{1}^{f}=h A_{f} / H_{C}: \lambda_{2}^{f}=F^{f} / H_{C}^{f} \text {; } \\
& \text { d. } \tau_{f}^{T}=\left(H_{f}^{T}\right)^{-1} ; \tau_{c}^{T}=h^{T} A^{T} / H_{f}^{T} \text {; } \\
& \text { e. } \lambda_{1}^{T}=h A^{T} / H_{c}^{T} ; \lambda_{2}^{T}=F^{T} / H_{c}^{T} ; \lambda_{m}=F^{T} / h_{m}^{T} A_{m}^{T} \text {; } \\
& \text { f. } P^{T} / P^{f}=\frac{1-k_{11}}{k_{12}}=K \text {; }
\end{aligned}
$$

and collecting terms, we have

\section{Fast Section}

Fuel:

$$
\Delta \overline{\mathrm{T}}_{f}^{f}(\mathrm{~s})=\left[\frac{\tau_{f}^{f}\left(s+\lambda_{1}^{f}+\lambda_{2}^{f}\right)}{\left(s+\tau_{c}^{f}\right)\left(s+\lambda_{1}^{f}+\lambda \frac{f}{2}\right)-\tau_{c}^{f} \lambda_{1}^{f}}\right] \Delta P^{f}(s) .
$$

Coolant:

$$
\Delta \overline{\mathrm{T}}_{c}^{f}(s)=\left[\frac{\lambda_{1} \tau_{f}^{f}}{\left(s+\tau_{c}^{f}\right)\left(s+\lambda_{1}^{f}+\lambda_{2}^{f}\right)-\tau_{c}^{f} \lambda_{1}^{f}}\right] \Delta \mathrm{P}^{f}(s) .
$$


Fuel:

$\Delta T_{f}^{T}(s)=\left[\frac{T_{f}^{T}\left(s+\lambda_{1}^{T}+\lambda_{2}^{T}\right)}{\left(s+\tau_{c}^{T}\right)\left(s+\lambda_{1}^{T}+\lambda_{2}^{T}\right)-\tau_{c}^{T} \lambda_{1}^{T}}\right] K \Delta P^{f}(s)$

Coolant:

$$
\Delta \bar{T}_{c}^{T}=\left[\frac{T_{f}^{T}\left(s+\lambda_{1}^{T}+\lambda_{2}^{T}\right)}{\left(s+\tau_{c}^{T}\right)\left(s+\lambda_{1}^{T}+\lambda_{2}^{T}\right)-\tau_{c}^{T} \lambda_{1}^{T}}\right] K \Delta P^{f}(s)
$$

Moderator:

$$
\Delta \bar{T}_{m}^{T}=\frac{\lambda_{m}}{s+\lambda_{m}}\left[\frac{\lambda_{1}^{T}{ }_{f}^{T}}{\left(s+T_{c}^{T}\right)\left(s+\lambda_{1}^{T}+\lambda_{2}^{T}\right)-T_{c}^{T} \lambda_{1}^{T}}\right] K \Delta P^{f}(s) .
$$

Using the basic feedback diagram

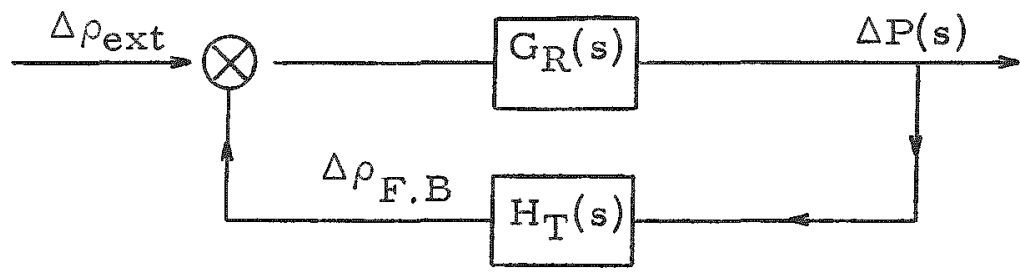

we define $\mathrm{H}_{\mathrm{T}}(\mathrm{s})$ as the feedback power coefficient, which is equal to

$$
H_{T}(s)=H_{f}^{f}(s)+H_{f}^{T}(s)+H_{c}^{f}(s)+H_{c}^{T}(s)+H_{m}(s) .
$$

From (40) we note that $\mathrm{H}_{\mathrm{T}}(\mathrm{s})$ has the form

$$
H_{T}(s)=\Delta \rho_{F} \cdot B(s) / \Delta P(s)
$$

Since reactivity may be written as

$$
\Delta \rho_{F} B_{B}(s)=\Delta \rho_{f}^{f}(s)+\Delta \rho_{f}^{T}(s) .
$$


we have

$$
\begin{aligned}
& H_{T}(s)=\alpha_{1} \alpha_{f}^{f}\left[\frac{T_{f}^{f}\left(s+\lambda_{1}^{f}+\lambda_{2}^{f}\right)}{\left(s+\tau_{c}^{f}\right)\left(s+\lambda_{1}^{f}+\lambda_{2}^{f}\right)-\tau_{c}^{f} \lambda_{1}^{f}}\right] \\
& +\alpha_{2} \beta_{c}^{f}\left[\frac{\lambda_{1}^{f} \tau_{f}^{f}}{\left(s+\tau_{c}^{f}\right)\left(s+\lambda_{1}^{f}+\lambda_{2}^{f}\right)-\tau_{c}^{f} \lambda_{1}^{f}}\right] \\
& +\alpha_{2} \alpha_{f}^{T}\left[\frac{\tau_{f}^{T}\left(s+\lambda_{1}^{T}+\lambda_{2}^{T}\right)}{\left(s+\tau_{c}^{f}\right)\left(s+\lambda_{1}^{T}+\lambda_{2}^{T}\right)-\tau_{c}^{T} \lambda_{1}^{T}}\right] \\
& +a_{2} \beta_{c}^{T} K\left[\frac{\lambda_{1}^{T} T_{f}^{T}}{\left(s+T_{c}^{T}\right)\left(s+\lambda_{1}^{T}+\lambda_{2}^{T}\right)-\tau_{c}^{T} \lambda^{T}}\right] \\
& +\alpha_{2} \gamma_{m} K\left(\frac{\lambda_{m}}{s+\lambda_{m}}\right)\left[\frac{\lambda_{1}^{T} T_{1}^{T}}{\left(s+\tau_{c}^{T}\right)\left(s+\lambda_{1}^{T}+\lambda_{2}^{T}\right)-\tau_{c}^{T} \lambda_{1}^{T}}\right] .
\end{aligned}
$$

Equation (44) is the dynamic power coefficient of a coupled reactor system for the given listed assumptions.

For simplicity we now assume that the response time is the same in the fast and thermal regions, i.e.,

$$
\frac{\tau_{f}^{f}\left(s+\lambda_{1}^{f}+\lambda_{2}^{f}\right)}{\left(s+\tau_{c}^{f}\right)\left(s+\lambda_{1}^{f}+\lambda_{2}^{f}\right)-\tau_{c}^{f} \lambda_{1}^{f}} \simeq \frac{\tau_{f}^{T}\left(s+\lambda_{1}^{T}\right)+\lambda_{2}^{T}}{\left(s+\tau_{c}^{T}\right)\left(s+\lambda_{1}^{T}+\lambda_{2}^{T}\right)-\tau_{c}^{T} \lambda_{1}^{T}}
$$

and

$$
\frac{\lambda_{1}^{f} \tau_{f}^{f}}{\left(s=\tau_{c}^{f}\right)\left(s+\lambda_{1}^{f}+\lambda_{2}^{f}\right)-\tau_{c}^{f} \lambda_{1}^{f}} \simeq \frac{\lambda_{1}^{T} \tau_{f}^{T}}{\left(s+\tau_{c}^{T}\right)\left(s+\lambda_{1}^{T}+\lambda_{2}^{T}\right)-\tau_{c}^{T} \lambda_{1}^{T}}
$$


Collecting terms, we have

$$
\begin{aligned}
\mathrm{H}_{\mathrm{T}}(\mathrm{s}) \simeq & \left(\alpha_{1} \alpha_{f}^{f}+\alpha_{2} \alpha_{f}^{T} \mathrm{~K}\right)\left[\frac{\tau_{f}^{f}\left(s+\lambda_{1}^{f}+\lambda_{2}^{f}\right)}{\left(s+\tau_{c}^{f}\right)\left(s+\lambda_{1}^{f}+\lambda_{2}^{f}\right)-\tau_{c}^{f} \lambda_{1}^{f}}\right] \\
& +\left[\alpha_{1} \beta_{c}^{f}+\alpha_{2} \beta_{c}^{T} K+\alpha_{2} \gamma_{\mathrm{m}} \mathrm{K}\left(\frac{\lambda_{\mathrm{m}}}{s+\lambda_{\mathrm{m}}}\right)\right] \\
& {\left[\frac{\lambda_{1}^{f} \tau_{f}^{f}}{\left(s+\tau_{c}^{f}\right)\left(s+\lambda_{1}^{f}+\lambda_{2}^{f}\right)-\tau_{c}^{f} \lambda_{1}^{f}}\right] }
\end{aligned}
$$

To study the steady-state power coefficient, we set $s=0$ and

$$
\mathrm{H}_{\mathrm{SS}}(0) \simeq\left(\alpha_{1} \alpha_{\underline{f}}^{f}+\alpha_{2} \alpha_{f}^{\mathrm{T}} \mathrm{K}\right) \mathrm{C}_{1}+\left(\alpha_{1} \beta_{\mathrm{c}}^{\mathrm{f}}+\alpha_{2} \beta^{\mathrm{T}} \mathrm{K}+\alpha_{2} \gamma_{\mathrm{m}} \mathrm{K}\right) \mathrm{C}_{2}
$$

where

$$
\begin{aligned}
& \mathrm{C}_{1}=\frac{\tau_{f}^{f}\left(\lambda_{1}^{f}+\lambda_{2}^{f}\right)}{\tau_{c}^{f} \lambda_{2}^{f}} \\
& C_{2}=\frac{\lambda_{1}^{f} \tau_{f}^{f}}{\tau_{c}^{f} \lambda_{2}^{f}} .
\end{aligned}
$$

\section{Case I (Weak Coupling):}

Using typical SGR design values for the fast region, we have

$$
\begin{aligned}
& \mathrm{C}_{1} \simeq 0.967 ; \alpha_{\mathrm{f}}^{f} \simeq-0.33 \times 10^{-5} \mathrm{\delta k} /{ }^{\circ} \mathrm{F} ; \\
& \mathrm{C}_{2} \simeq 0.194 ; \hat{c}_{\mathrm{c}}^{f} \simeq 0.87 \times 10^{-6} \mathrm{\delta k} /{ }^{\circ} \mathrm{F} .
\end{aligned}
$$

For the thermal section we used SRE values (see NAA-SR-3763): 


$$
\begin{aligned}
& \alpha_{f}^{T} \simeq-1.1 \times 10^{-5} \delta \mathrm{k} /{ }^{\circ} \mathrm{F} ; \\
& \beta_{c}^{T} \simeq 0.3 \times 10^{-5} \delta \mathrm{k} /{ }^{\circ} \mathrm{F} ; \\
& \gamma_{\mathrm{m}}^{\mathrm{T}} \simeq 1.3 \times 10^{-\mathrm{E}} \delta \mathrm{k} /{ }^{\circ} \mathrm{F} .
\end{aligned}
$$

For the coupling factors we used Avery's (weak coupling) values, where

$$
\mathrm{k}_{12}=0.94 ; \mathrm{k}_{22}=0.23 ; \mathrm{k}_{12}=0.46 ; \mathrm{k}_{21}=0.10
$$

and

$$
\mathrm{K}=0.13 ; \alpha_{1}=0.928 ; \alpha_{2}=0.072 \text {, }
$$

so that

$$
\mathrm{H}_{\mathrm{SS}} \simeq-0.285 \times 10^{-5} \mathrm{\delta \textrm {k }} / \mathrm{MW} \text {. }
$$

The system is stable at steady state.

In Case II we assume the same temperature conditions and coefficients. However, most of the reactivity is in the thermal section. This case is texmed strong coupling.

$$
\begin{gathered}
\text { Setting } \\
k_{12}=0,6, k_{2 Z}=0.75 ; k_{12}=0.5 ; \mathrm{K}=0.80,
\end{gathered}
$$

we have

$$
\mathrm{H}_{\mathrm{SS}}=-0.487 \times 10^{-5} \delta \mathrm{k} / \mathrm{MW} \text {. }
$$

This system is stable at steady state and has a more negative powex coefficient by $170 \%$. The reason for this stronger power coefficient is traced to the coupling factor. With more reactivity in the thermal section, the larger thermal Doppler reactivity coefficient has a greater effect on the power coefficient. In Case I the Doppler coefficient of the thermal section accounted for only 3.2 per cent of the fuel reactivity feedback, whereas in Case II the thermal Doppler accounted for $81.1 \%$ of the fuel feedback.

With a strongly coupled system (more reactivity in thermal section), a less negative or even a positive Doppler coefficient in the fast region may be tolerated. This would allow for small, highly enriched fast sections.

For Case III we first study a weakly coupled system with a positive Doppler coefficient in the fast region. 


$$
\begin{aligned}
& \text { Case III } \\
& k_{11}=0.94 ; k_{22}=0.23 ; k_{12}=0.462 ; K=0.13 .
\end{aligned}
$$

From equation (51) we have

$$
\mathrm{H}_{\mathrm{sS}} \simeq+0.302 \times 10^{-5} \delta \mathrm{k} / \mathrm{MW} \text {. }
$$

This system is unstable at steady state.

Using strong coupling in Case IV:

$$
k_{11}=0.6 ; k_{22}=0.75 ; k_{12}=0.5 ; K=0.80
$$

and a positive fast. Doppler, we have

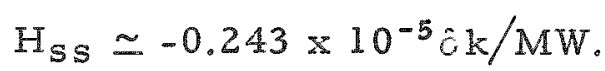

In Case IV the system is stable even with a positive fast Doppler coefficient in the fast region.

Reviewing the above cases, we note that the coupled reactor concept allows certain corrective measures to the Doppler feedback to be taken. This is very important in view of uncertainties in the fast Doppler coefficient, In a critical fast system, the measured Doppler resulting from a future R\&D critical program must be tolerated even if determinations show the Doppler coefficient smaller than computed. However, due to the much larger Doppler coefficient in a SGR, the coupled concept allows a degree of adjustment to the effective Doppler feedback not possible in all fast systems. Thus, stable coupled systems are easy to achieve.

\section{Relative Reactor Safety}

In discussing the aspects of relative reactor safety of fastcoupled reactor systems, we refer back to the inhour equation for this system. Since reactor safety necessarily involves the nonlinear behavior of reactor kinetics, the above feedback treatment is inadequate and in error.

From equation (9) we have that the inhour equation for fastcoupled reactors is as follows:

$$
\left(k_{11} m_{1} \gamma-e^{\omega l_{11}}\right)\left(k_{22} m_{2} \gamma-e^{\omega l} 22\right)-k_{12} k_{21} m_{1} m_{2} \gamma^{2}=0
$$

In this discussion the explicit time dependence of the neutron flux is ignored. This treatment was suggested by Häfele.(2) Only step reactivity input will be 
studied and the resulting period computed. The reason for the above condition is that it allows an analytical result to be computed without the aid of digital computers.

Two assumptions are made in obtaining a formulation easily suited to hand calculations

$$
\text { 1. } \gamma \simeq 1.0 \simeq 1-\left[\beta_{\text {eff }} \omega /(\omega+\bar{\lambda})\right] \text {. }
$$

Since $\bar{\lambda}=0.08$ for one delayed-neutron treatment, if $\omega \gg \bar{\lambda}$ then $\gamma \simeq 1-\beta_{\text {eff. }}$. If we treat a $\mathrm{Pu}^{239}-\mathrm{U}^{238}$ fast section and a $\mathrm{U}^{235}-\mathrm{U}^{238}$ thermal section,

$$
\beta_{\text {eff }}=\alpha_{1} \beta_{1}+\alpha_{2} \beta_{2} \text {. }
$$

For weak coupling,

$$
\alpha_{1}=0.928 ; \alpha_{2}=0.072 ; \beta_{1}=0.004 ; \beta_{2}=0.007,
$$

and we have

$$
\beta_{\text {eff }} \text { (weak coupling) }=0.0042 \text {. }
$$

For strong coupling,

$$
\alpha_{I}=0.385 ; \alpha_{2}=0.615
$$

so that

$$
\beta_{\text {eff }} \text { (strong coupling) }=0.0058 \text {. }
$$

In these cases

$$
\left(1-\beta_{\text {eff }}\right)=0.9958 ; \text { and } 0.9942 \text {, }
$$

respectively, so that the first assumption is valid.

The second assumption involved prompt-neutron lifetime:

$$
\text { 2. } l_{12} \ll b_{22}
$$

This assumption implies that the reactor is prompt critical. A typical fast prompt-neutron lifetime is

$$
\ell_{11} \simeq 10^{-7} \mathrm{sec} .
$$


For the SRE $\ell_{22}=5 \times 10^{-4}$ sec so that the second as sumption is valid. With these assumptions the inhour equation for coupled reactors is reduced to

$$
e^{\omega k_{22}} \simeq \frac{\left(k_{12} k_{21}-k_{11} k_{22}\right) m_{1} m_{2}+k_{22} m_{2}}{\left(1-k_{11} m_{1}\right)}
$$

Before discussing numerical calculations we will examine equation (59). Using Häfele's treatment, we defined $m_{1}$ and $m_{2}$ for step inputs in the fast section as

$$
\begin{aligned}
& \mathrm{m}_{3}=1+\delta \mathrm{k}+(\overline{\mathrm{T} \cdot \mathrm{C}})_{1}(\overline{\delta \mathrm{T}})_{1} \\
& \mathrm{~m}_{2}=1+(\overline{\mathrm{T} \cdot \mathrm{C}})_{2}(\overline{\delta \mathrm{T}})_{2}
\end{aligned}
$$

where $(\overline{\delta T})$ is the average temperature change, $+(\overline{\mathrm{T} \cdot \mathrm{C}})_{j}(\overline{\delta T})_{j}$ is the temperature reactivity feedback, and $\delta \mathrm{k}$ the step reactivity input. The quantities $m_{1}$ and $m_{2}$ are termed reactivity multiplication factors, and $m_{1}=m_{2}=1$ is the steady-state condition.

We would hope to terminate a given transient short of gross fuel melting in fast section if possible. Therefore, in evaluating the relative safety of coupled systems, a reactivity of zero is achieved by elevating fuel temperatures in fast and thermal sections. In view of present fast reactor design efforts, $(\overline{\delta T})=10^{3}{ }^{\circ} \mathrm{F}$ was chosen as the distance in degrees to gross fuel melting. A $\delta k=0.01$ was also chosen for these studies. Therefore, the results obtained refer to the above assumptions and are to be considered as an initial effort in the study of the relative safety of coupled systems.

From equation (59) we note the conditions satisfying the above safety criterion are

$$
\left|k_{22} m_{2}+\left(k_{12} k_{21}-k_{11} k_{22}\right) m_{1} m_{2}\right| \leq\left|1-k_{11} m_{1}\right|
$$

In the following discussions the fuel temperature coefficient of the thermal section will be held constant and equal to

$$
(\overline{\mathrm{T} . \mathrm{C}})_{2}=-1.1 \times 10^{-5} \delta \mathrm{k} /{ }^{\circ} \mathrm{E}
$$

so that

$$
m_{2}=1-\left(1.1 \times 10^{-5}\right) \times 10^{3} \simeq 0.989 .
$$

By observation we set $m_{1}=1,0$, so that from equation (62) for the weakly coupled concept, for which

$$
k_{11}=0.94 ; k_{22}=0.23 ; k_{12}=0.462 ; k_{21}=0.10
$$


so we have

$$
0.0593 \leq 0.060 \text {. }
$$

Therefore, if

$$
m_{1} \text { and } m_{2}<1.0,
$$

the total system is convergent. If $m_{1}=1.0$ and $m_{z}=1.0$, the system is stable. If $m_{1}=1.0$ and $m_{2}>1.0$, we have

$$
\begin{aligned}
& \left|\mathrm{m}_{2}\right|\left|\mathrm{k}_{22}+\left(\mathrm{k}_{12} \mathrm{k}_{21}-\mathrm{k}_{11} \mathrm{k}_{22}\right)\right| \leq \| 1-\mathrm{k}_{11} \mid \\
& \left|0.06 \mathrm{~m}_{2}\right| \leq 0.06 \mid
\end{aligned}
$$

so that if $m_{1}>1.0$ the system is divergent. In summaxy,
Condition
$\underline{\text { Relative Safety }}$
1. $m_{1}=m_{2}=1.0$
Stable
2. $m_{1}>1.0, m_{2}=1.0$
Divergent
3. $m_{1}=1.0 ; m_{2}>1.0$
Divergent
4. $\mathrm{m}_{1}=1.0 ; \mathrm{m}_{2}<1.0$
Convergent
5. $m_{1}$ and $m_{2}>1,0$
Convergent

From conditions 4 and 5 we can obtain insight into magnitude and sign of temperature feedback required in the fast region to prevent gross fuel melting. For $m_{1}=1.0$ we have

$$
m_{1}=1+\delta k+(T \cdot C)_{1}(\overline{\delta T})_{1}
$$

or

$$
\delta \mathrm{k}=-(\mathrm{T}, \mathrm{C})_{\mathrm{I}}(\overline{\delta T})_{\mathrm{l}}
$$

For example, if $\delta k=0.01$ and $(\overline{\delta T})_{1}=10^{3}$, we have

$$
(\mathrm{T}, \mathrm{C})_{\mathrm{I}} \simeq-10^{-5} \delta \mathrm{k} /{ }^{\circ} \mathrm{F} \text {. }
$$

If $(\overline{\delta T})=10^{3}$ for $\mathrm{m}_{2}$, we have

$$
m_{2}=1-(T \cdot C)(\overline{\delta T})_{2} .
$$


Assuming

$$
\frac{\mathrm{P}^{\mathrm{T}}}{\mathrm{P}^{f}} \simeq \frac{(\overline{\delta \mathrm{T}})_{2}}{(\delta \mathrm{T})_{1}} \simeq \frac{1-k_{11}}{k_{12}} ; \overline{\delta T}_{2} \simeq 0.13 \delta \mathrm{T}_{1},
$$

so that $\mathrm{m}_{2}<1.0$, then

$$
m_{2}=1+(T \cdot C)_{2}(0.13) \times 10^{3} .
$$

For the SRE

$$
\begin{aligned}
(\mathrm{T} . \mathrm{C})_{2} & \simeq-1.1 \times 10^{-5} \delta \mathrm{k} /{ }^{\circ} \mathrm{F} ; \\
\mathrm{m}_{2} & =0.998 .
\end{aligned}
$$

Thus $(\mathrm{T}, \mathrm{C})_{1}=-10^{5} \mathrm{\delta k} /{ }^{\circ} \mathrm{F} ;(\mathrm{T}, \mathrm{C})_{2}=-1.1 \times 10^{-5} \delta \mathrm{k} /{ }^{\circ} \mathrm{F}$ for a $8 \mathrm{k}=0.01$ would result in a convergent system. Accordingly, the temperature rise can be limited to below the fuel melting temperature with very reasonable values of the Doppler coefficient. From condition (5) we note that, since $m_{1}=m_{2}=1.0$ results in a stable system, the condition $m_{1}, m_{2}<1.0$ results in a convergent system. Therefore, coefficients equal to or more negative than the above values would result in safe conditions in the fast-coupled reactor system.

However, the above comments apply to accidents involving dropping a fuel element (on-line refueling) or moving control rods. That area of events termed maximum credible accidents will now be reviewed.

III. General Discussions of Safety and Maximum Credible Accidents

All of the following accidents are hypothetical events for sodiumthermal, fast, and fast-coupled reactor cores. In order that we may better understand the comparis on between the various mentioned sodium-cooled reactors, the following accident conditions are assumed for all reactors. Even though the following conditions are based on pessimistic assumptions, the public welfare demands a thorough investigation of these phenomena.

\section{Accident Conditions}

A. The power level in the reactor core is too high, causing sodium boiling in the center of reactor. Molten fuel from the center of reactor core drops down into lower part of core and solidifies. This produces a core with a lower section that is abnormally denser in fuel and leaves a void region at the core center. The upper portion of the core now drops under gravity, rapidly increasing reactivity. 
B. Sodium is temporarily expelled from the core by boiling, then rushes back moments later at higher velocity. During these brief intervals, fuel elements have undergone a degree of failure and the entering sodium carries meltdown fragments of the elements into core, increasing reactivity.

Thermal Reactor:

$$
\begin{aligned}
& \ell_{\mathrm{p}}=5 \times 10^{-4} \mathrm{sec} ; \alpha_{f}=-1.1 \times 10^{-5} \delta \mathrm{k} /{ }^{\circ} \mathrm{F} ; \delta \mathrm{k}=0.07 ; \\
& P_{0}=1200 \mathrm{MWT} ; \beta_{\text {eff }}=0.007 .
\end{aligned}
$$

Conditions \#1 and \#2

For a single group of delayed neutrons, we have

$$
\rho \simeq \frac{\ell_{\mathrm{p}} \omega}{k_{\mathrm{eff}}}+\frac{\beta \omega}{\omega+\lambda}
$$

If $\omega \gg 1.0$, we have

$$
\ell_{p} \omega \simeq(\rho-\beta) k_{e f f}
$$

or

$$
\omega \simeq \frac{\rho-\beta}{b_{p}} k_{e f f}
$$

The effective reactivity in the system leading to conditions 1 and 2 is

$$
\delta \mathrm{k} \simeq 0.07-3 \times 10^{3}\left(1.1 \times 10^{-5}\right) \simeq 0.037 \simeq \$ 5.0,
$$

where

$$
\left(\delta T_{f}\right) \simeq 3 \times 10^{3} \circ \mathrm{F} .
$$

The period of the neutron flux increase for this system is $17 \mathrm{msec}$. A knowledge of the details of the fuel and coolant during a power transient requires the use of digital computers; accordingly, only the integrated power over $\sim 400$ msec will be computed. It will be assumed that the safety rods scram $\sim 400 \mathrm{msec}$ after the start of the transient. 


$$
P_{\text {total }} \simeq P_{0} \int_{0}^{t} e^{\omega t} d t
$$

where $\omega$ is given by equation (77) and $P_{\text {total }} \simeq 73.9 \times 10^{3} \mathrm{MW}-$ sec.

Using conditions 1 and 2 for a fast reactor having identical parameters with only the prompt lifetime equal to $l_{p}=10^{-7} \mathrm{sec}$, we obtain $P_{\text {total }}=3.7 \times 10^{8} \mathrm{MW}-\mathrm{sec}$. As indicated by the total integrated powers for the fast and thermal cases, the mechanical scram system is useless in saving the reactor core. Thus both cores are lost. It is possible to postulate physical disassembly of the fast reactor due to secondary critical. However, in case of the thermal system, subcritical must occur by fuel melting away from the center of the core. This phenomenon has never been experimentally determined, and the time lapse during melting is unknown. Therefore pressurization of the system might lead to core disassembly terminating the transient. In either case, core disassembly terminates the power transient. Therefore, having a prompt-neutron lifetime equal to $10^{-5}$ or $10^{-7} \mathrm{sec}$ has a small effect, and loss of the core is the end result. Until additional experimental data are available showing the behavior of melting fuel in fast and thermal reactors as well as data on the resulting reactivity changes with demonstrations of the termination transient conditions, all statements on final safety are pure conjecture. Bearing in mind the usefulness of making assumptions concerning fuel melting, the following comments will help set design criteria for a fast-coupled reactor.

\section{As sumption \#1}

"Rapid" fuel melting in a fast reactor caused by a power transient forms a high-density central mass of fuel, thus increasing the reactivity and ultimately causing core disassembly with the possibility of breaching the containment vessel.

"Slow" melting in a thermal sodium reactor causes fuel to drop out of the core, thereby decreasing reactivity.

\section{Consequence $\# 1$}

By means of the above assumption, a fast-coupled system would be designed to take advantage of the behavior of melting fuel in a thexmal re actor. This could be accomplished in the following way:

1. Low-melting fuel in thermal section;

2. high power generation in thermal section. 
Under assumption $\# 1$ it is concluded that fast reactor safety is questionable in view of the short prompt lifetime.

\section{As sumption \#2}

"Slow" fuel melting of fast reactor fuel causes fuel to drop out of the core, thus decreasing reactivity.

Consequence \#2

By means of the above assumption \#2, the design of a fast-coupled system would size the "coupling" to obtain a prompt-neutron lifetime that would cause "slow" fuel disassembly due to effective lifetime of a fastcoupled system.

\section{Assumption \#3}

Prompt critical fast reactor cores can result in nuclear explosions and breaching of the primary containment.

Consequence \#3

Fast section of coupled concept would be sized never to be prompt critical on fast neutron. (Reasonable or $-\delta k=5$ or $10 \%$ would be used.)

All possible assumptions have not been studied, however. The above are generally typical of various assumptions made concerning relative reactor safety.

IV. Summary and Conclusions

The following conclusions have been obtained from these studies,

Stability

1. Stable fast-coupled reactor systems are possible and can be designed by use of current technology.

2. The larger Doppler coefficient of thermal sodium reactors can be used to improve the effective Doppler coefficient of fast-coupled systems.

3. A positive Doppler coefficient can be tolerated in the fast section of fast-coupled systems termed strongly coupled (large thermal sections). 


\section{Relative Safety}

1. Small prompt reactivity steps that normally lead to fuel melting in fast systems result in less melting in fast-coupled systems.

2. Control in the fast or thermal portion of a coupled reactor depends on fuel temperature coefficients.

3. More stable conditions result from control near or in thermal section (outer region of fast section).

\section{Safety and Maximum Credible Accident}

1. Depending on the final shutdown mechanism in fast and thermal reactor cores, a fast-coupled system can be designed to use this mechansim.

2. If prompt critical fast reactor cores lead to nuclear explosions, a fast-coupled system, even with weak coupling, can be designed to prevent this event from occurring.

3. If rapid fast reactor fuel melting leads to secondary criticality, a fast-coupled system can lengthen the time for melting by increasing the prompt-neutron lifetime. In summary, it has been demonstrated that the fast-coupled concept can be designed to exhibit inherent stability. By properly coupling fast and thermal reactor portions, an improved Doppler feedback is obtained as compared with all-fast systems. In the area of reactor safety and maximum credible accidents, the fast-coupled concept offers a means of obtaining improved neutron economy over all-thermal systems while not compromising reactor safety features.

Present $\mathbb{R} \& D$ programs are investigating the problem areas involved in reactor safety of fast and thermal systems. Fuel melting, secondary criticality, and potential nuclear explosion are being studied. It is difficult at present to realize the results of these efforts. It has been demonstrated both in the SRE and the Hallam Nuclear Facility that sodium-cooled thermal reactors can be designed with inherent stability required for safe power operation. Coupled concept is a means of further improving the neutron economy of this concept without sacxificing the already demonstrated operational safety characteristics of present sodium-cooled thermal systems.

\section{Nomenclature}

$S_{1} \quad$ Source of neutrons in fast section

$\mathrm{S}_{2} \quad$ Source of neutrons in thermal section

$k_{11} \quad$ Coupling factor fast section

$k_{22}$ Coupling factor thermal section. 
$k_{21} \quad$ Coupling factor thermal to fast

$\mathrm{k}_{12} \quad$ Coupling factor fast to thermai

$\alpha_{1} \quad$ Reactivity partition in fast section

$\alpha_{2} \quad$ Reactivity partition in thermal section

$m_{1} \quad$ Reactivity multiplication factor in tast section

$\mathrm{m}_{2} \quad$ Reactivity multiplication factox in thermal section

$\ell_{13} \quad$ Fast section prompt-neutron lifetime

$l_{22} \quad$ Thermal section prompt-neutron lifetime

0 Reciprocal reactor period

T Reactor period

$F \quad$ Delayed-neutron fraction

$\overline{T \cdot C}$ Average temperature coefficient

$\mathrm{H}_{f} \quad$ Heat capacity of fuel

$\bar{T}_{f} \quad$ Average temperature of tuel

$P \quad$ Reactor power

hA Heat transfer coefficient

$\mathrm{T}_{0} \quad$ Outlet temperature

$\mathrm{I}_{\mathrm{m}}$ Average temperature of moderator

$\overline{\mathrm{T}}_{\mathrm{C}} \quad$ Average coolant temperature

$T_{\text {in }}$ Inlet temperature

$\mathrm{H}_{C} \quad$ Heat capacity of coolant.

F Flow rate times heat capacity of coolant

$\mathrm{H}_{\mathrm{m}} \quad$ Heat capacity of moderator

$P_{T} \quad$ Total power

$P_{0} \quad$ Initial power

$\rho \quad$ Reactivity

$k_{\text {eff }}$ Effective multuplication constant 


\section{References}

1. R. Avery, Theory of Coupled Reactors, Proc. 2nd UN Conf. on Peaceful Uses of Atomic Energy, Geneva, Switzerland, 12, 182 (1958).

2. W. Häfele, Effective Lifetime and Temperature Coefficient of Coupled Fast-Thermal Systems, SM-18/1.

$\frac{\text { Discussion of Paper }}{\text { Presented by Mr. Campise }}$

MR. WOLFE (General Electric):

I have several comments. First of all, with regard to improving the lifetime, as I mentioned on Monday I think that the longer lifetime in the worst hypothetical accident is a disadvantage because for any given ramp rate of reactivity it allows you to put in more reactivity before you disas semble.

MR. CAMPISE:

If this is in a coupled system, you allow the fuel in the thermal section to melt and bring you subcritical before you get into a typical fast reactor accident.

MR. WOLEE:

We have been looking at coupled systems, not from the standpoint of getting the neutron lifetime, but in a superheater, because the fast section of the superheater had certain advantages with regard to chosen materials. When you look at these coupled systems, you find that if you want to have a substantial amount of power in the fast core, then the reactivity in the fast core is large, and reactivity change in the thermal core does not affect the reactivity of the system very much. For instance, the increased Doppler effect in the thermal section is really not there, if you have an accident where you are melting down the fast core and increasing xeactivity rapidly in that core.

\section{MR. CAMPISE:}

This is true in a weakly coupled system. However, if most of the reactivity is in the thermal section, this is not true. 
MR. WOLEE:

If you have most of the reactivity in the thermal section, you end up having most of the power there, too.

MR。CAMPISE:

Right, and the split is what you have to choose as far as increase in the neutron economy over an all-thermal system.

MR。WOLFE:

The point I am trying to make is that to get a split where you make a substantial improvement in the economy, you end up buying all the troubles of the fast reactor systems.

MR. CAMPISE:

I don't think so if you stick around the 50-50 split.

MR. WOLFE:

The power split gives you trouble in these systems in one further way. In general, you want a much higher power density in the fast section. When you look at the power split in these two systems, you find that you end up with quite bad power spikes between the two sections. These require you to put in complicated buffer regions to prevent thermal neutrons from going to the fast region, and fast neutrons back to the thermal.

MR, CAMPISE:

We do maintain a decoupler region, which we also use for control. As far as the power spikes are concerned, we can greatly minimize it by going to the concept of flat power cores.

MR. WOLEE:

On the basis of a large amount of work over several years we have not been able to find any real safety advantage from a thermal-fast system where you were producing a substantial amount of power in the fast section. We would disagree with your conclusion.

MR. CAMPISE:

We would not design a weakly coupled reactor. We would tend to first design for the 50-50 split, which we believe is not a laxge extrapolation from present technology. 
MR, AVERY (Argonne):

I have a question on what you call the weakly coupled system. In the one we studied, we conjectured, but never calculated, that the main Doppler effect might occur from transmission of low-energy resonance neutrons through the natural uranium buffer zone, from the thermal to the fast part. I wonder if your calculations give any information on this point.

MR. CAMPISE:

We have planned calculations by perturbation theory to try and break down the Doppler feedback, but at present I cannot answer your question.

MR. FISCHER (Argonne):

I have done some experimenting and studying coupled fast the rmal systems as regards safety. I think what Mr. Wolfe says is extremely important, that if you have a major accident where you have a meltdown and you are adding reactivity at 50 or 100 dollars a second, you are in much worse trouble with a coupled system, initially at least, than with a fast system. This is because your lifetime slows down the doubling of the power while you are adding reactivity at a very large rate. In a fast system the power-rise is more rapid and your disassembly starts much earlier. This is extremely important when $k$ excess is getting rather large. Here, you are badly hurt by a coupled system, at least in this initial phase.

MR. CAMPISE:

We are getting into an area where the maximum credible accident has to be defined. I contend that in a strongly coupled system where you have an appreciable amount of reactivity in your thermal section, and therefore an appreciable amount of power in the thermal section, we can argue that melting of thermal fuel rather than fast fuel will shut you down.

MR. FISCHER:

I am saying that if you get in any situation where you are adding, say 50 dollars per second, whether you have the thermal section or not, you are in trouble due to the long lifetime.

MR. CAMPISE:

We have to define the accident. I am not ready to assume that an accident has occurred. I would rather take an operational philosophy of what might occur in view of what we already know about our system. 
MR, FISCHER:

Let me go on. If you have a low-power accident, where you have a feedback mechanism such as you were talking about, with, say, the two Doppler coefficients you have, then if you take a linear feedback model, you find that the feedback has a one-over-lifetime dependence in the feedback. Again this hurts you when you have a coupled system with the long lifetime.

I studied the Avery-type system with the fairly sophisticated AX-I code and got some rather interesting results. There is still a third undesirable consequence of the long lifetime for major accidents. When the reactor is shutting down, you lose reactivity in the fast section first, and you have a larger fraction of thermal reactivity. This increases the neutron lifetime, and the power falls off more slowly, which is worse.

MR. CAMPISE:

We have a different concept of a coupled reactor. Our conceptual designs have a roughly 50-50 split in power, and entail a very small fast section, whereas the thermal section is quite large.

MR。FISCHER:

I still would be interested in using the coupled idea to help promote a better fast reactor. We ran accident calculations both for a fast system and an essentially equivalent coupled fast-thermal system. We found that the total yields of the accident were essentially comparable for these two sys tems, in spite of the troubles I brought up. There is one other aspect which is quite important, however. The kinetic energy of the accident, which to me means essentially the damage potential, differs. One finds that the long lifetime during the burst of the coupled system is a distinct help; the pressures are immensely reduced, the reactor has a much longex time during the burst to disassemble itself, and the kinetic energies turn out to be significantly lower, for the coupled fast-thermal system. 
$\bullet$

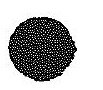




\title{
NEUTRON CONTROLLED EXPANSION FUEL FOR FAST BREEDER REACTORS
}

\author{
F.M. Heck \\ Westinghouse Electric Corporation \\ Atomic Power Division \\ Pittsburgh, Pennsylvania
}

Introduction

On the basis of present evidence, fast breeder reactors can potentially produce economically competitive power. In the long view, fast breeder reactors are an essential complement to burner reactors. Designing fast breeder reactors, like any other engineering job, requires balancing the advantages against the disadvantages to arrive at those engineering compromises which are necessary to produce a workable, economic total plant. The engineering problems involved in designing larger fast breeder power plants have not yet been fully explored; there are, undoubtedly, many problems in the design of large fast breeder plants which have not yet been defined. This monograph outlines an approach to the solution of one set of problems in the engineering design of fast breeder power plants.

The problem of interest here is that of achieving a safe and controllable fuel for a fast breeder reactor. This problem is best viewed in the context of certain requirements and compromises which seem to be required by the large fast breeder. A summary of these requirements and compromises will be used to introduce the particular problems and the solutions offered by the Westinghouse fuel-assembly design.

In order to avoid excessive inventories of fissile material (relative to thermal reactors), fast breeder reactors must have high power release per unit volume in order that the specific power ( $\mathrm{kW} / \mathrm{kg}$ ) of the fissile material be comparable with that achieved in thermal reactors. From heattransfer considerations, a requirement of high specific power leads to finely divided fuel. For solid fuel reactors, this means small-diameter rods or thin plates and a close packing of the rods or plates within the core With small-diameter rods, the surface heat flux produced by the high specific power indicates liquid metal coolants as the best heattransfer media.

Core power densities for fast breeder reactors are in the neighborhood of $400 \mathrm{kWt} /$ liter and above, whereas typical thermal reactors have core power densities below $100 \mathrm{~kW} /$ iter. Thus, fast breeder reactor cores are smaller in volume by a factor of 4 or 5 than are thermal reactors of the same energy output. The use of liquid metal coolants permits low pressure in the primary system while simultaneously achieving high-temperature 
steam conditions. Steam at $1000^{\circ} \mathrm{F}$ can be produced from a fast breeder reactor while retaining several hundred degrees of subcooling in the hottest channel of the core.

These advantages are not achieved without penalty. The fabrication cost per $\mathrm{kg}$ of fuel is appreciably higher for the small-diameter rods required by the fast breeder core than for the laxger rods which can be effectively utilized in the thermal reactor core. The higher fabrication cost requires the fuel to achieve very high burnup levels in order to be economically competitive. Liquid metal technology is not as well developed as water technology; hence there are more unknowns involved, and a higher probability of design exrors exists. The high degree of subcooling in the fast reactor core allows a large temperature rise across the core because the restraining effect of the heat of vaporization is not present. The low heat capacity of the liquid metals requires larger $\Delta T$ or large flow. A large temperature rise across the core introduces problems of thermal shock. The low film drop associated with liquid metal heat transfer aggravates this thermal shock problem.

The prompt-neutron lifetime characteristic of fast reactors is several orders of magnitude smaller than that for a thermal reactor; consequently, the reactor power periods associated with prompt criticality are correspondingly shorter. The effective delayed-neutron fraction is reduced because of the small importance of slow neutrons. The possibility of very short reactor periods makes the control requirements for the fast breeder much more stringent than those for the thermal reactor.

The most promising fissile material for fast breeder reactors is plutonium. The effective delayed-neutron fraction of plutonium in a typical fast breeder reactor is in the order of one-half that of the effective delayed-neutron fraction in a thermal all-uranium core. This aggravates the control problems by making it easier to achieve prompt criticality in the fast reactor than in the thermal reactor.

Typical temperature coefficients of reactivity are lower than those characteristic of a thermal reactor. This further aggravates the control problem.

Nonmetallic Fuel

The use of ceramic fuels may provide answers to many of the problems outlined above. The ability of the oxides to sustain high burnups with minimum distortion has been established in samples. The ability of the carbides to sustain high burnup is not yet as firmly established. The temperature coefficient for the coolant (sodium) is more negative in ceramic cores than in metallic or cermet cores of the same physical size. The lower thermal conductivity of the ceramic fuel causes the effective fuel 
temperature to increase more rapidly during a power transient than in metallic fuels. Thus, the Doppler effect is enhanced. Quite high temperatures can be sustained by the ceramic fuels without damage to the core, since no structural dependence is placed upon the oxide fuel material.

Plutonium-uranium carbide may offer the same advantages as the mixed oxide with the additional virtue of providing higher fuel density and thermal conductivity than the oxide. The burnup behavior of these materials is not as well established as that of the oxide fuel, nor is the fabrication technology developed to the same degree as for the oxide. The fuel system proposed herein was first developed on the basis of the plutoniumuranium oxides. However, it is quite amenable to the use of the carbides. nitrides, and other fuel materials.

The nonmetallic fuels have disadvantages as well as advantages. For the same volume fraction of fuel in a core, the nonmetallic fuels have lower conversion ratios than the high-uranium alloy metal cores. The oxides have lower conversion ratios than either the carbides or the nitrides.

The nonmetallic fuels present a special set of problems related to fuel movement. The thermal expansion behavior of nonmetallic fuels is not simply calculable as is the thermal expansion of metal fuels. The nonmetallic fuels may shrink on heating as a result of a resintering process. Cracks resulting from thermal stress may cause the subsequent thermal expansion to be zero over some temperature range, through which the thermal expansion of the fuel is accomplishing nothing but closing the cracks. During a transient, nonmetallic fuel may crumble and move from its initial location down into the radial gap between the fuel pellets and the clad, or into any central void region which may exist in the lower pellets Plutonium oxide may diffuse preferentially in a uranium oxide matrix as a result of the axial temperature gradient, thus altering the concentration of plutonium in the fuel material. Similar problems may exist with the carbides and nitrides.

Some or all of these phenomena may not be important, but the whole cannot be completely swept aside in the design considerations of fast breeder reactors at the present time. The severity of the consequences of a small but unexpected increase in reactivity is greater than those associated with an equal increase in thermal reactor.

The reactivity effects associated with the postulated anomalous fuel-movement phenomena mentioned above can be reduced by two techniques. The core can be designed as "pancake" core. This is a core in which the height $H$ is appreciably smaller than the diameter $D$ of the core. For an $H / D$ ratio approaching infinity (an infinite cylinder), axial fuel densification results almost entirely in an increase in $k_{20}$. For small 
$H / D$ ratios (approaching a slab), the material $k_{0}$ stillincreases, but the leakage probability also increases as a result of the relatively large change in buckling produced by the change in core height. Thus, for small H/D ratios, the increase in $k_{\infty}$ is partially compensated for by the increased leakage. The result then is that fuel densification (or expansion) has much less effect on the reactivity in a core with a small $\mathrm{H} / \mathrm{D}$ ratio. Increasing the total volume of the core also reduces the effectiveness of uniform fuel movement. If the core geometry does not adequately limit the effects of the postulated anomalous fuel movement, compartmentation of the fuel rod can be utilized to limit mechanically gross fuel movement.

In the design stages of a reactor program, the magnitude of the anomalous fuel-movement problem can be reduced to any required degree by some combination of compartmentation and core geometry.

Having solved this problem, a new problem arises. By designing the core in such a fashion that any undesirable fuel movement has an insignificant effect on reactivity, it follows that any movement which would have been desirable, i.e., direct expansion with temperature, has also been made insignificant.

This represents a sexious problem in designing fast breeder reactors, because the only remaining prompt temperature coefficient is that resulting from Doppler effect. The Doppler coefficient is not large and is extremely difficult to compute with accuracy. It is virtually as difficult to measure the Doppler coefficient accurately as it is to calculate it. Because of a large uncertainty in the magnitude of the value of the Doppler coefficient, and because the Doppler coefficient at this stage seems to be the only mechanism in ceramic fuels for terminating a fast transient, it is essential that either a method be available for increasing the magnitude of the Doppler coefficient so that the minimum magnitude, considering the uncertainty, is sufficiently large; or some other mechanism must be provided to supplement the Doppler coefficient in terminating fast transients. The Doppler coefficient can be increased in a fast breeder reactor, but only at the expense of reducing the breeding ratio.

\section{Controlled Expansion}

A fuel assembly has been developed which retains the advantages of the ceramic fuels and which permits the use of compartmentation and/or geometry to control undesirable fuel movement, while simultaneously having a predictable negative temperature coefficient resulting from thermal expansion. This assembly can be adapted to other nonmetallic Iuels with little modification and can further be adapted with some modification for use with metallic or cermet fuels where simple thermal expansion of the metallic or cermet fuel does not provide sufficient control. This fuel assembly is called the controlled-expansion fuel assembly. An example 

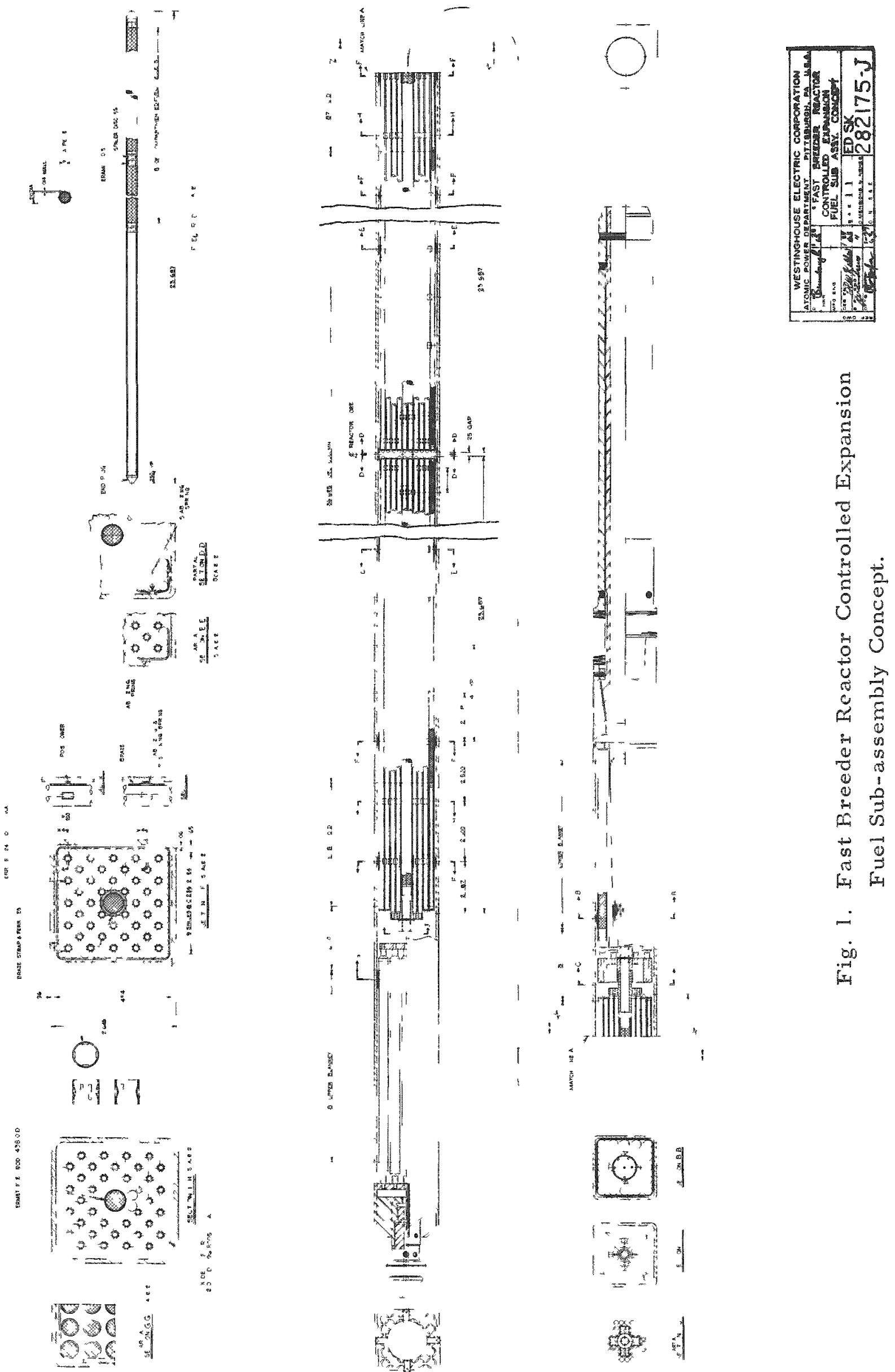
of the application of this fuel assembly for a small reactor core is illustrated in Fig. 1.

The fuel rods are assembled into two bundles in line with each other, each bundle being one-half of the core height. The two bundles of ceramic fuel rods are connected by a central cermet rod which acts as a continuous structural member through the core. Four center fuel rods are deleted from the lattice to allow space for the large center cermet rod, which is attached to the ceramic fuel rod bundle sections only at the top and bottom ends of the fuel bundles, i.e., at the core extremities.

The fuel is in the form of pellets in compartmented stainless steel fuel tubes. The compartments adjacent to the core center contain the fuel pellets, and the larger compartment at the outer ends of the fuel tubes is a fission gas chamber. The compartmentation is accomplished by steel rings fastened to the inside of the fuel clad, and separated from the fuel material by ceramic spacer discs. The ceramic spacer discs prevent the fuel from moving between compartments and insulate the retaining rings from the high-temperature portion of the fuel. The steel rings and the loose spacer discs permit fission gas to flow freely.

The fuel rods are arranged on a rectangular pitch, and their position relative to each other is maintained by steel ferrules brazed to the fuel rod clad and spaced axially every 4 to 6 in.

The central cermet rod is a uniform dispersion of oxide fuel in stainless steel, clad with a thin layer of stainless steel. In this design, the stainless steel clad is bonded directly to the cermet rod. Special spacer ferrules are brazed in the center of the fuel bundles and spaced axially in line with the small spacer ferrules. By means of spring-like tabs on the special center ferrules, the cermet rod is supported radially throughout the core, so that relative axial movement between the cermet rod and the surrounding oxide fuel bundles is permitted.

The support of the two half-bundles of oxide fuel rods is accomplished by the center cermet rod. Special end fittings are provided to which the oxide fuel rod bundles are attached. The lower fitting is attached in turn to the lower axial blanket rods, which in turn are attached to the end fitting welded to lower end of the wrapper tube. Alternative designs provide for the cermet rod to run the full length of the oxide fuel rods plus the two axial blankets. This additional length increases the effectiveness of the cermet expansion. Lateral positioning of the assembly, within the wrapper tube, is accomplished by positione $x$ and stabilizing spring straps brazed to the outside of the peripheral fuel rods. The fuelbundle design illustrated here, exclusive of the cermet rod and the stabilizing springs, is similar to a fuel rod assembly successfully used in the Yankee reactor. 
The positions of the ferrules, in any two adjacent coolant channels through a fuel bundle, are staggered to promote a high degree of mixing within the fuel bundle The presence of the ferrules themselves produces sufficient turbulence to further promote mixing. As a result, a nearly uniform temperature is achieved through the coolant at each level in a given bundle.

During fast transients, the cermet temperature increases more rapidly than that of the oxide fuel clad. This results in pulling apart the two halves of the fuel bundle to a greater extent than would be the case for the same cermet temperature in steady-state conditions. This pulling apart of the two half-bundles is effectively splitting the core in half at the center and results in a reduction in reactivity even though the geometry of the core might make uniform fuel expansion relatively ineffective.

The pulling apart of the two halves of the bundle is equivalent to removing fuel from the center, a region of high importance, and adding the same fuel to the periphery of the core, a region of low importance. Simultaneously, additional sodium is added at the center of the core. This addition of sodium reduces the gain from the fuel movement in most small cores, because sodium has a negative void coefficient. In large cores, the void coefficient for sodium in the center of the core is usually positive, so that the addition of sodium reduces reactivity. Therefore, the effectiveness of the fast CEX response is somewhat greater in large cores than in small cores.

Because of the time lag between the production of heat in the fuel and the transport to the clad. the expansion of the clad around the oxide lags further behind expansion of the cermet as the reactor period gets shortex Thus, the effectiveness of the cermet expansion increases as the period of the reactor decreases This increase in effectiveness with shortening of the reactor period is not exhibited by other inherent mechanisms. This is a distinct advantage of this concept. The reactivity change associated with bringing the reactor up to power is lower than would be obtained for a straight thermal expansion coefficient which has the same fast negative reactivity response.

Controlled Bowing

The fuel assembly illustrating the controlled-expansion concept (see Fig. 1) also contains a separate concept which is called controlled bowing. Either the controlled-bowing or the controlled-expansion concepts can be used separately. For many designs, however, they complement each other.

Fuel-bundle bowing is a serious problem in fast reactors. The normal behavior of a fuel bundle is to bow toward the hotter portion of the 
reactor. If the top and bottom of the fuel bundle are located with respect to adjacent fuel bundles, the effect of bowing of the fuel bundle is a movement of fuel toward a more reactive configuration. This produces a positive power coefficient of reactivity which, under some circumstances, makes a fast reactor core autocatalytic. This was observed to happen in EBR-I.

Bowing can be restrained by interposing pads of solid material between adjacent fuel assemblies. This type of restraint produces high stresses because the material of the fuel bundle is forced to move in opposition to its natural unrestrained motion. The Westinghouse controlledbowing concept takes advantage of the

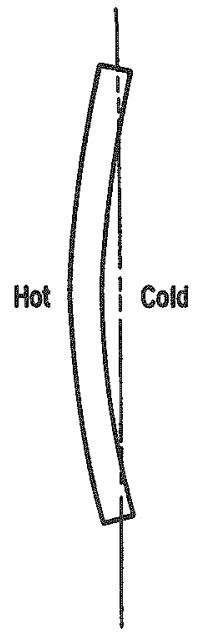

UnPestrained full Bunde

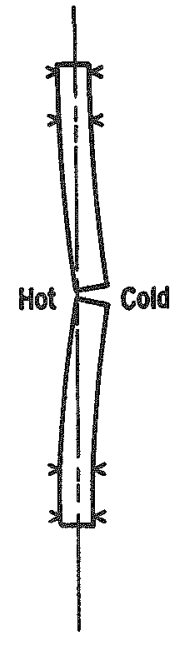

Controlled Split Bundie normal thermal bowing of the fuel bundle by causing the natural thermal bowing to move the fuel away from the hot spot rather than toward it. The principle involved is illustrated in Fig. 2 . An unrestrained fuel bundle is shown with a hot and a cold side; the characteristic shape is illustrated. Opposite is an illustration of a Westinghouse controlled-bowing split bundle. It can clearly be seen that the average fuel movement is away from the hot side. This is accomplished by splitting the fuel bundle into two sections, the upper and lower halves. The outer extremities of each half-bundle are restrained by stiff springs at two axial locations near the outer extremities of the halfbundles. The result is that the outer extremities are restrained to remain in line with the fuel can. Hence, the inner part of the half bundle, i.e., the part near the center of the core, is cantilevered from the ends. The normal curvature, resulting from thermal bowing, bends the inner portions of the fuel bundle halves away from the hot zone of the core. The hotter region of the core is characteristically a region of higher fuel importance; therefore, the controlled-bowing effect results in a reduction in reactivity as the power gradient across the bundle increases. The amount of bowing for a given design can be altered by changing the amount of restraint in a bundle and by changing the clad material around the fuel rods.

Figure 1 illustrates some of the engineering requirements for a controlled-bowing design. Sufficient clearance must be provided between the normal location of the fuel bundle and the wrapper can to permit the bundle bowing to occur. The positioning springs at the upper and lower end of the assembly are stiff, heavy springs to maintain the alignment of the fuel bundle. Additional soft springs are provided along the length of the fuel bundles to reduce any tendency for vibration to occur. 
Design Considerations

A decision to adopt the neutron-controlled expansion fuel-assembly design does not significantly affect the basic core design. The choice of fuel material, as well as the optimum diameter for the rods or pellets to be used, is made on the same basis as that for a more conventional assembly with full-length rods. One exception to this is the fact that a small fraction of the fuel volume is lost due to the presence of the cermet rod. In Fig. I this represents about $3 \%$ of the fuel. It is desirable, although not essential, to use a clad material with a lower thermal expansion coefficient if a choice is available. This is of advantage in the large "pancake" cores where uniform fuel expansion has a small effect of reactivity.

The cermet rod can be sized to fit the space available in a given lattice after a quantity of fuel rods is removed. There are sone limitations on the size of the cermet rod, but the available range is quite broad. Therefore, the fuel-rod pitch can be chosen relatively independent of the fact that the neutron-controlled expansion fuel assembly is being used.

In brief, the nuclear, thermal, and hydraulic design considerations associated with the fuel rods are basically independent of whether the design is to be the neutron-controlled expansion fuel assembly or the conventional full-length bundle assembly.

\section{Design Considerations for the Cermet}

For mechanical reasons, it is desirable to have the average cermet expansion at least equal to the average expansion of the pellet clad in steady-state condition. This insures that in steady state, the two halves of the bundle will not be forced together, i.e., that the central gap in steady state will always be equal to or greater than the gap at the time of fabrication. The equal expansion curve shown in Fig. 3 relates the fuel loading in the cermet to the cermet radius for the condition of equal expansion in the cermet and in the pellet clad for the reference design of Fig. 1.

The parameter alpha that is the ordinate in Fig. 3 is the ratio of the volumetric heat-generation rate in the cermet to that in the oxide pellets. If the enxichment of the oxide in the cermet is equal to that in the oxide of the fuel pellets, then alpha is just the volumetric loading of the oxide in the cermet. If the enrichment of the cermet is different from that in the pellets, then alpha must be multiplied by the ratio of pellet enrichment to cermet enrichment to get the volumetric loading of the cermet. To simplify the data in Fig. 3, alpha was calculated on the basis of an unclad cermet rod. A correction is required for the presence of the clad. If $R$ is the total radius ( $R$ of $F i g .3$ ) and $R_{1}$ is the actual cermet radius $\left(R_{1}\right.$ less than $R$ ), then the actual value of alpha for the clad cermet is obtained by multiplying the alpha from Fig. 3 by 


$$
\left(\frac{R}{R_{1}}\right)^{2}\left(\frac{k_{s}}{k_{c}}\right)\left[\left(\frac{k_{s}}{k_{c}}-2\right)\left(\frac{R_{1}}{R}\right)^{2}+2\right]^{-1} .
$$

In the above multiplier, $\mathrm{k}_{\mathrm{s}}$ is the thermal conductivity in the clad and $k_{\mathrm{C}}$ is the thermal conductivity of the cermet. This multiplier adjusts the alpha in such a fashion that the volume-averaged temperature is the same with the clad as without. Fig. 4 is a plot of this correction.

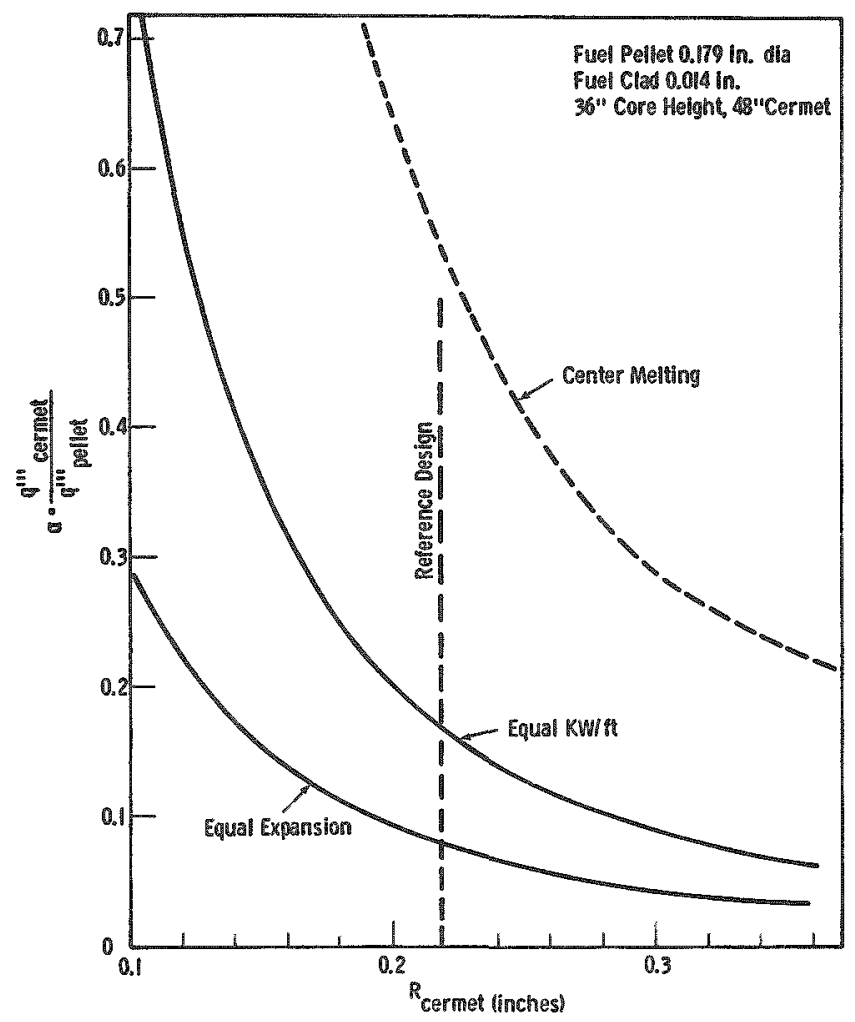

Fig. 3

Cermet Design Limits

Fig. 4

Multiplier for Cermet Volumetric Heat Generation Rate Due to Presence of Clad

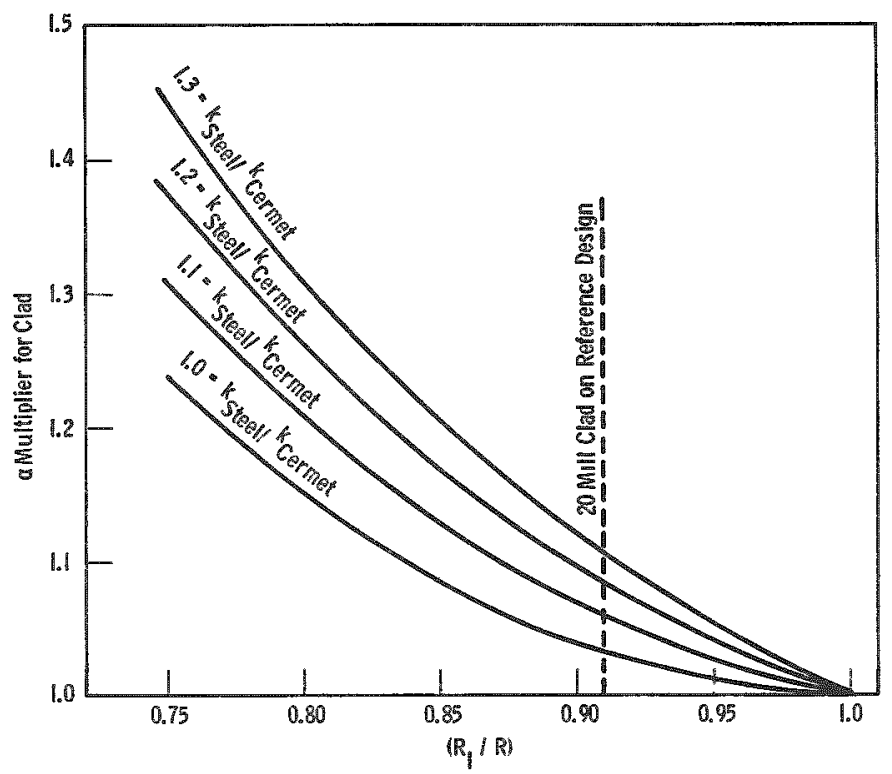


The reactivity coefficients attributable to the cermet temperature will always be higher than that attributable to the pellet clad temperature, Therefore, at the minimum mechanical design criterion, equal average temperatures, the design is well above the stability limit. The introduc. tion of reactivity will always result in a reduction of reactivity, because the expansion of the cermet will be at least as great as the expansion of the clad. and the magnitude of the reactivity coefficient of the cermet is greater than that of the clad.

Thus, the minimum mechanical limit provides a design which is stable. For the design illustrated in Fig. 1, which is a 3-ft-diameter, 3-ft-high core, the temperature coefficient of reactivity for the cermet is in excess of $-15 \times 10^{-6}{ }^{\circ} \mathrm{C}$. The net temperature coefficient of reactivity for the clad is less than $2 \times 10^{-6}{ }^{\circ} \mathrm{C}$.

Because of the high thermal conductivity of the cermet relative to ceramic pellets, the power-generation rate (in $\mathrm{kW} / \mathrm{ft}$ ) in the cermet pin can be somewhat higher than in the ceramic pellets in spite of the lower melting temperature of the cermet. The cermet rod in the assembly is used as a structural member. Therefore, it seems unwise to operate it at as high a power generation rate in $\mathrm{kW} / \mathrm{ft}$ as would be appropriate for a free cermet pin A reasonable approach seems to be to restrict the power-generation rate of the cermet to be no higher than that in the ceramic pellet fuel rods The equal $\mathrm{kW} / \mathrm{ft}$ curve of Fig. 3 illustrates the upper limit established for the design of the cermet expansion control member

The ratro of the temperature rise in the pellet to that in the cermet is inversely proportional to the ratio of the conductivities and directly proportional to the ratio of the radii. Thus. if the pellets are designed to operate at $4400^{\circ} \mathrm{F}$ with a $2300^{\circ} \mathrm{F}$ rise at the hot spot. then the cermet would operate at about $1300^{\circ} \mathrm{F}$ with $950^{\circ} \mathrm{F}$ sodium at the hot spot. if the cermet loading provides for a power-generation rate in $\mathrm{kW} / \mathrm{ft}$ equal to that of the fuel.

This provides for about a $400 \%$ increase in steady-state power before inciprent center melting of the cermet occurs (gross damage to the fuel pellets occurs at this level). For comparison purposes, the curve of steady-state incipient center melting is shown in Fig. 3 and the large margin can be seen. The recommended design region lies between the two solid curves of Fig 3

A burnup of $100,000 \mathrm{MWD} / \mathrm{ton}$ is of the order of $10 \%$ burnup of uranium and plutonium atoms Consequently. the effective fuel loading of the cermet will drop less than $10 \%$ over the life of the fuel assembly. This can easily be accommodated within the available design region. 
The mechanical design restrictions on the size of the cermet rod must be specified in terms of the steady-state loading, the maximum transient loading, and the rate of application of the transient load. Typical half fuel bundles can be expected to weigh of the order of 20 to $50 \mathrm{lb}$, and with sodium flowing upward through the fuel assembly, the actual dead weight on the cermet rod will be quite small. Thus, the steady-state strength requirements are quite small. The transient effects cannot be clearly defined without an intensive study of the characteristics of the particular core in which the fuel assembly is to be loaded.

Engineering reasonableness would suggest that the rods be not less than $0.15 \mathrm{in}$. in radius; the rod should not be greater than $0.375 \mathrm{in}$. in radius because of the large volume occupied by rods of larger size. Consequently, a reasonable design area would appear to be cermet rod diameters ranging from $0.3 \mathrm{in}$. to about $0.6 \mathrm{in}$. Over this range, the volumetric fraction of oxide in the cermet will be from 0.05 to about 0.2 , at equal enrichments for the cermet and the fuel pellets. If the enrichment of the oxide in the cermet is made greater than that of the pellets, the volumetric loading of the cermet is correspondingly reduced. The range of 5 to $20 \mathrm{v} / 0$ oxide in a stainless steel cermet can be comfortably accommodated by present fabricating techniques.

\section{Stability Analysis}

The systems-analysis groups have studied the stability characteristics of this assembly for a small fast breeder reactor core. This is a difficult case, since for this particular core the Doppler coefficient is estimated to be less than $-0.5 \times 10^{-6}{ }^{\circ} \mathrm{C}$. Several different mathematical models for the analysis of this assembly have been used. Lumped models in a linear version for hand calculation and a nonlinear version for use with the analog computer have been devised. These models have demonstrated the stability of the assembly over the design range of interest.

The characteristics of the fuel assembly depend upon the difference between the volume-averaged temperature of the cermet and the volume-averaged temperature of the pellet clad. These two quantities need to be computed more accurately than is required by pheonomena which depend only on a single temperature. In oxder to depict more accurately the actual time behavior of a core incorporating the neutron-controlled expansion fuel assembly, additional work is being carried out to develop better mathematical models for use with the analog computing facilities. By using a PACE computer operating at slower than real time, and coupling this with the GPS computer operating faster than real time, with a memory, the actual temperature distributions as a function of time can be determined. The purpose of the more elaborate model is to define more accurately the reactivity limitations and control requirements. Some preliminary results are shown in Figs. 5 and 6. 


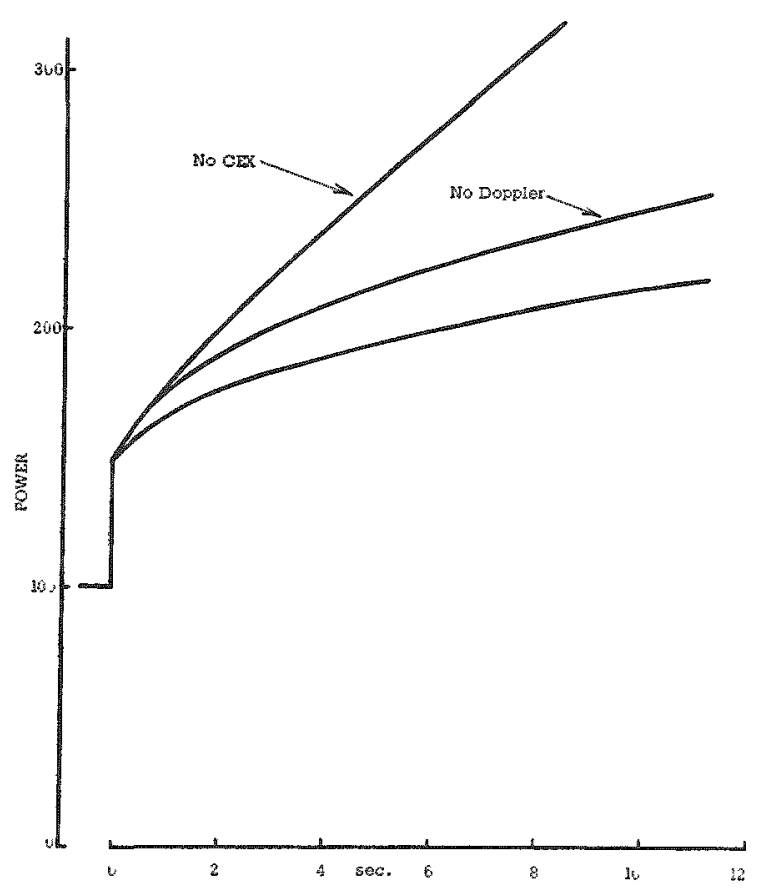

Fig. 5

Preliminary Transient Analysis. Instantaneous insertion of 40 cents, no control. Zero sodium coefficient. Negative Doppler coefficient.

Fig. 6

Preliminary Transient Analysis. Instantaneous insertion of 40 cents, no control. Positive sodium coefficient. Positive Doppler coefficient.

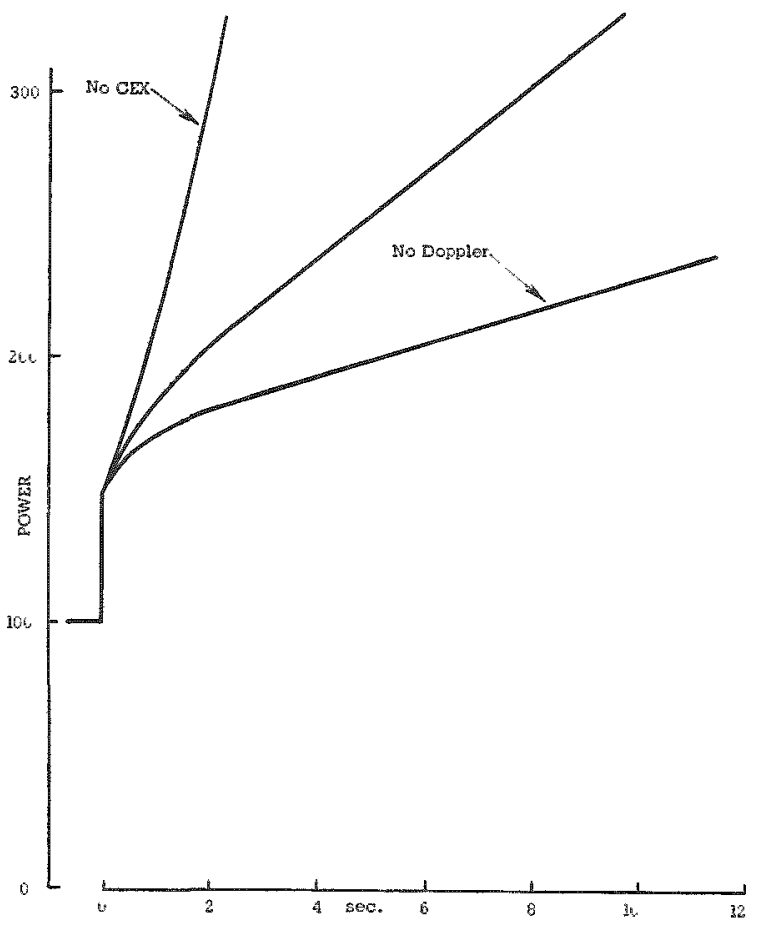




\section{Acknowledgments}

The author wishes to express his appreciation for the design work carried out by Mr. H. Keller, and for the preliminary transient analysis performed by Mr.J. Godts and Mr. W. Gunson.

Discussion of Paper

Presented by Mr. Heck

MR. HALL (Los Alamos):

I missed something when you were talking about the relative phase shift of time delay between your control element and the reactor period.

MR. HECK:

There is essentially no time delay between the generation of heat in the cermet and the rise in temperature of the cermet. There is a time delay between the generation of heat in the fuel pellet and the rise in temperature of the fuel clad. 
MR. HALL:

This is then a delay in the expansion?

MR. HECK:

Right.

MR. HALL:

Why is this good?

MR. HECK:

As the reactor period gets shorter, the cermet will expand relative to the clad more than it will in steady-state conditions. The clad will eventually catch up later, but initially, following a severe burst, the cermet will expand faster than the clad, giving a somewhat improved initial condition compared with the steady-state calculations.

MR. SMITH (Argonne):

If the center rod should melt, is there a danger that the upper por tion of the core may drop, and maybe the lower?

MR. HECK:

Certainly, if the fuel assembly is designed in this fashion and no provision is made for restraining it. Some of the design alternatives contain a connection between the upper fuel bundle and the can which keeps the rod in tension at all times: if the rod fails. it withdraws the upper bundle slightly. Our calculations show that the clad is probably already melted before we reach center melting in the cermet, so we do not really feel that this is something that is likely to happen.

MR. SMITH

You do this with the spring system then? You guard against this possibility?

MR. HECK:

Yes. 
MR. STATHOPLOS (United Nuclear Corp.):

I wonder if you could amplify a little bit on the internal structure of your ceramic rods. I didn't quite get how you would do the spacing. MR. HECK:

Internal to the clad there is a steel ring. On top of this sets a ceramic insulating pellet. This system was used in Yankee Core $I$, which has compartments in it. Also the brazed ferrule bundle design is basically the same as the Yankee Core I fuel assembly. Thus we believe that that part of it has been pretty well checked out, with the exception of the difference between water and sodium.

MR. STATHOPLOS:

You are not worried about this spacing building in void in the core?

MR. HECK:

There is a penalty in inventory clearly by doing this. We only do it in order to prevent fuel movement.

MR. STATHOPLOS:

I was not worried about the increase in the inventory. I was more concerned about holes you have introduced in the reactor being filled with fuel, for example, by cracking.

MR. HECK:

I don't think that is the case with our construction. Fission gases may go past, but there is no opening through the middle of it. If you melted the fuel completely, it might dribble down. But I think some other things would have gone wrong long before that.

MR. THOMPSON (MIT):

Have you thought of turning over this element and running it the other way so that if the center rod which serves to split the two sections were to melt, the core would continue to fall apart?

MR. HECK:

When you say turn it over and run it the other way, you mean downflow? 
MR. THOMPSON:

Not downflow: suspended from the top.

MR. HECK。

Yes. We have looked at suspending it from the top. This will work very well provided that during flow the bundle does not float due to pressure drop. In very many cases it does seem to float. We have also looked at suspending it from the top with downflow. Although we like the downflow system very much, we worry about some of the other aspects.

MR.ZALESKI (EURATOM):

What happens with the fuel if you have 150 or 200 per cent of nominal power?

MR. HECK:

I would hope that we had moved control rods before we got there.

MR, ZALESKI:

In that case, do you need this expansion?

MR. HECK:

These analyses were intended to indicate the relative effect of expansion compared with the Doppler effect. It also looks as though it makes the reactor somewhat more stable during fast transients. We do not intend to imply that we would sit and let that happen.

MR ZALESKI:

I think it is very important to use a realistic criteria to establish whether or not you need it. Of course, the power evolution is nicer with the coefficient than without. But does it really help in a major accident?

MR. HECK.

It seems to have a noticeable influence on stability behavior. For cores in which the Doppler coefficient were positive this expansion effect could be quite significant. 
MR. DIETRICH (General Nuclear Engineering):

Yesterday we were concerned that in the blowing apart of the core under a severe accident, there may be a probability of blowing material into a region of higher importance. We drew some reassurance Irom the proposition that ordinarily the regions of high importance are the regions of high power density. Doesn't it seem that we have now puta region where the power density is essentially zero into a little gap of high importance, and would this have an adverse effect on the very severe accidents?

MR. HECK:

We don't think it would. The fuel worth is flat across the gap. MR. KAZI (United Nuclear):

Do you expect any difficulty with 100,000-MWD burnup of the ceramic materials?

MR. HECK:

We don't anticipate any.

MR. KAZI:

It seems to me that in order to get a reasonable temperature rise, you would have a fair amount of fuel in your cermet.

MR. HECK:

No, because with compartmented fuel rods the fuel is restrained to move with the clad. In effect, the only temperature that we need to exceed is the temperature of the clad. It does not look at all difficult to get well above the clad temperature.

MR. KAZI:

Could you go all of the way and just use cermet fuel elements? MR: HECK:

Yes, except that they do not breed. We were describing a breeding fuel assembly. 
MR. de STORDEUR (EURATOM):

Did you think of putting a spring between the two halves, and using $U^{235}$ metal pieces to hold your end pieces in place? In the case where you would have a power excursion, the $U^{235}$ would melt first, and bang you would go.

MR. HECK:

When we first looked at this particular kind of array, we were afraid, as a matter of fact, that this is exactly what would happen. When we checked, we found that the cermet rod as we would design it for operational purposes would stand up after the fuel had very grossly failed. We are examining the desirability of having a spring-like affair at one end of the bundle, in any case, as an additional saleguard in case of some failure other than a melting through of the rod. 
662 


\section{FACTORS INVOLVED IN THE TIME TO START UP FAST REACTORS, INCLUDING THE MEASUREMENT OF SUBCRITICAL REACTIVITY}

D. C. G. Smith

Dounreay Experimental Reactor Establishment

Thurso, Scotland

$\underline{\text { Introduction }}$

The time taken to start up a fast reactor power plant will be determined by a wide variety of factors. The operating engineer requires rapid startup, not only for the purpose of reducing scheduled shutdown time, but to minimize lost time following an unscheduled shutdown. The designer requires rapid startup to maintain high load factor, but has to compromise with the effects of thermal shock. The safety assessors will have a tendency towards a "slow but sure" attitude.

The present memorandum largely ignores all aspects other than those specifically the concern of the physicists. In particular, the reliability of interlocks to prevent the wrong sequence of insertion of high-speed shutoff rods and control rods is ignored. The less controversial case of thermal shock is considered in a very simplified manner. One important factor in minimizing the startup time is the monitoring of subcritical reactivity; this is discussed in the second half of the paper.

Factors Influencing Permissible Rate of Reactivity Addition

It is convenient to divide the startup of a fast reactor power plant into three regions:

(i) from shutdown to about $1 \% \Delta \mathrm{k} / \mathrm{k}$ below delayed critical;

(ii) from $1 \% \Delta \mathrm{k} / \mathrm{k}$ below delayed critical to the point where temperature effects will modify impressed reactivity changes, say $1 \%$ of full power and $100 \%$ flow rate:

(iii) from $1 \%$ to $100 \%$ of full power at full flow.

Regions (i) and (iii) are those in which the reactor power may be kept in stable equilibrium. Below delayed critical, the neutron source will inject neutrons, each of which initiates a chain reaction of finite length, so that the equilibrium power is uniquely determined by the source strength and subcritical reactivity. In the high-power regions, any perturbation will be followed by a change in power level which will cancel the perturbation (assuming the presence of negative reactivity feedback). The middle region is one of unstable equilibrium, the reactor requiring continuous trimming to maintain constant power when it is just critical. 


\section{Region Boundaries}

The lower limit of power in region (i) will depend on the magnitude of the source strength and the maximum amount by which the reactor is shut down. It is probable that for large fast reactors, containing 1 tonne of plutonium, the $\mathrm{Pu}^{240}$ spontaneous fission source will provide from $2-5 \times 10^{8} \mathrm{n} / \mathrm{sec}$, and it is estimated that at $5 \times 10^{-2} \Delta \mathrm{k} / \mathrm{k}$ (or $\left.\$ 10\right)$ the reactor power level will be very roughly $20-50 \mathrm{~mW}$.

The boundary between regions (i) and (ii) is flexible, but it is suggested that a subcritical level of $\$ 2$ is a reasonable point at which to make a fairly accurate check on the subcritical reactivity, prior to changing the rate of reactivity insertion before achieving criticality (see below). This would be equivalent to a reactor power of about 100-200 $\mathrm{mW}$.

The boundary between (ii) and (iii) will not be critical; the reactivity feedback will make a very small impression at $1 \%$ of full power if the coolant flow rate is at maximum, and a larger but still small effect if power and coolant flow rate are increased together. The setting of the boundary will not materially affect the present discussion, and the level has been arbitrarily set at $1 \%$ of full power, i.e., $10 \mathrm{MW}$ for a $1000-\mathrm{MW}$ reactor.

\section{Region (i)}

Provided the reactor remains subcritical at all times, there is no limit to the safe rate of reactivity addition in region (i). It is, nevertheless, essential that accident conditions be catered for. It is proposed that the maximum rate of reactivity addition in this region should be limited to that level which would cause the period meter to indicate a reactor period of not less than $100 \mathrm{sec}$ (i.e., an apparent doubling time not less than 70 sec). While the reactor is subcritical by more than $10^{-3}(\$ 0.2)$, the power and reactivity are related:

$$
P(d P / d t)^{-1}=R(d R / d t)^{-1}=\text { the apparent reactor period. }
$$

For a given rate of reactivity addition, the apparent period will decrease as the subcritical reactivity decreases. By setting a lower limit to the period, the reactor may be protected against becoming delayed critical with a high rate of reactivity addition.

This safeguard should be combined with a limit on the amount of reactivity available in the rods being moved at this stage and also with an upper limit to the power level which may be about $200 \mathrm{~mW}$ and the trip could be set at $\frac{1}{2}$ W. Then three unlikely and independent failures are necessary before damage to the reactor can occur. 


\section{Region (ii)}

If a ramp reactivity insertion is applied at the commencement of this region, then there is the possibility that a large amount of excess reactivity may have been added before temperatures in the reactor invoke reactivity feedback. For this reason the reactivity insertion rate is much more limited than for the first region (see below). There is no mechanism for terminating the power rise if the reactor goes critical with a short period until thermal effects are effective (i.e., region iii). Very roughly $P=P_{0} \exp (R \lambda t)$ and $R=\alpha t$, where $\lambda$ is the reciprocal of the effective lumped delayed-neutron time constant $(10 \mathrm{sec}), R$ is the total reactivity and $\alpha$ is the rate of reactivity insertion. Thus $P=P_{0} \exp \left(\alpha \lambda t^{2}\right)$, and a very sudden increase will occur as $\alpha \lambda t^{2}$ becomes comparable with unity.

In choosing the maximum allowable value of $\alpha$, it is convenient to determine, first, the maximum step reactivity addition which can be tolerated when the reactor is critical at low power. It is then possible to find the corresponding ramp rate of reactivity which, when applied at the commencement of region (ii), yields the same total excess reactivity at the time the system becomes critical. The relationship between step and ramp reactivity additions is illustrated in Fig. 1, which was obtained from a precise formulation of the neutron kinetic equations, with the use of an analogue computer. In both types of reactivity addition the transient is terminated by temperature effects, but whereas following a step change the reactor will settle at a steady power (assuming that this is below the accident level), a ramp change will continue to force the power up, but at a rate which is so much slower than the initial transient that it is still essentially equivalent to a step.

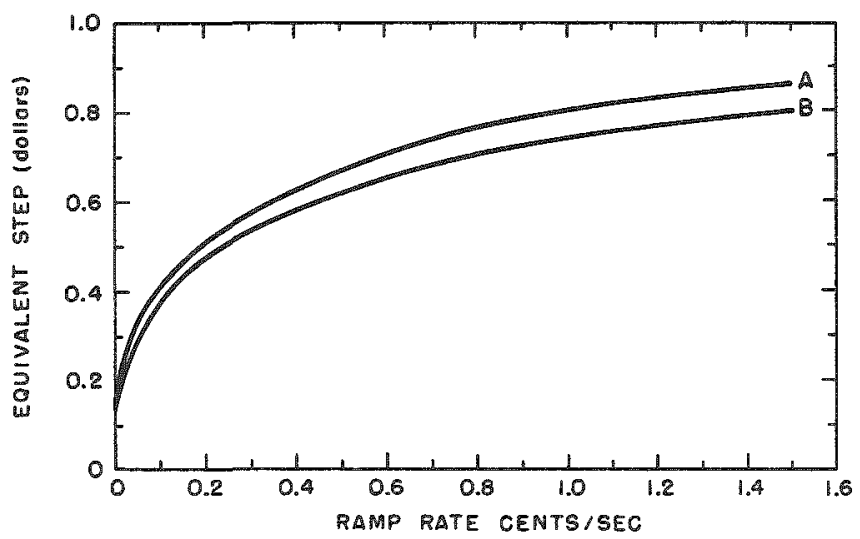

Fig. 1. Reactivity Step for Which the Time Required for the Power to Run by (A) 6 Decades (B) 9 Decades Is the Same as for the Corresponding Ramp Rate.

(A) Assuming a 9 Decade Rise before Feedback Limints Further Rise.

(B) Assuming a 6 Decade Rise before Feedback Limits Further Rise. 
If the reactor can stand reactivity additions up to one dollar from low power, e.g., because of a large prompt negative Doppler effect together with other expansion effects, it is clear from the figure that quite large values of $\alpha$ are tolerable.

Region (iii)

Although it is feasible to permit a limited amount of reactivity to be added at a comparatively fast rate in region (i), the rate of addition for region (ii) is restricted. It would be difficult to devise a satisfactory procedure which would permit reactivity to be added in region (iii) at a rate which exceeded that for region (ii), especially as similar control rods may be used to cover the two regions.

Given any practical rate of increase of power in region (iii), the reactivity feedback will have ample time to be fully operative so that if the feedback is linear with power, as is usually the case, the shape of the powertime curve will be that of the added reactivity-time curve.

If thermal shock to the reactor and auxiliary plant may be ignored, the limiting rate of power increase may be set by the condition that the reactor period should not be less than $100 \mathrm{sec}$, i.e., the maximum rate of increase in power must not exceed $1 \% / \mathrm{sec}$. The lower limit of region (iii) has been set at $1 \%$ of full power, so that at this stage the maximum rate of increase in power will be $10^{-4}$ of full power per sec. If a linear rate of reactivity addition is applied, this would require 3 hr to go from $1 \%$ to full power.

A more realistic condition would be to maintain where possible a constant fractional rate of increase in power and, for the case of linear power coefficient, this requires a rate of reactivity addition which increases with powe1. In the absence of other limitations the range from $1 \%$ to full power would then take $8 \mathrm{~min}$ if the rate of $1 \% / \mathrm{sec}$ were maintained. Since the maximum rate of addition is limited to that acceptable for region (ii), then, when this limit is reached, the rate of increase in power will also be restricted and the figure of $8 \mathrm{~min}$ may not be attained.

So we have assumed for the limits for region (iii) that the reactivity feedback is linear. One condition for linearity is constant flow rate. Typical feedback will comprise two terms; one dependent only on power $P$, and the second on the ratio of power to coolant flow rate, $P / u$ :

$$
\mathrm{H}=\mathrm{aP}+(\mathrm{bP} / \mathrm{u})
$$

For constant $u, H$ varies linearly with $P$, but if $b \gg$ a and $P / u$ is maintained constant, then the reactivity feedback is constant, i.e., independent of power. In this condition the reactor may be brought to full power by 
controlling the coolant flow rate without external reactivity changes. The limits in this case will be set by conditions other than reactor kinetics (thermal shock, etc.).

A practical reactor will not fulfil the condition that $b \gg$, and, in any case, to establish the condition that power may be controlled by flow rate requires that, at the low values of power and flow rate, the temperature distribution in the reactor is fully established, and this will take time if thermal shock is to be avoided.

To use variable flow rate in the approach to full power may be desirable from other points of view, e.g., automatic control, but until more is known about the specific reactor the optimum schedule of flow rate and power changes cannot be specified.

For the present purpose, full flow rate will be assumed as a first step. Qualitative arguments will then be advanced to indicate roughly how reduction in the time to full power may be achieved without running the risk of thermal shock.

Practical Limits for Rates of Reactivity Addition

Region (i)

With the usual nomenclature, the subcritical power level is given by

$$
P=P_{0} / R_{S}, P / P=-R / R_{S}
$$

where $R_{S}$ is the subcritical reactivity and $\dot{P} / P$ is the reciprocal of the apparent reactor period. If we take $100 \mathrm{sec}$ as a reasonable value for the latter, and $\$ 10$ to $\$ 2$ as the range of $R_{S}$ in this region, the maximum value of $\dot{R}$ is $(1 / 100) \times \$ 2=2 \not / s e c$. To change from a shutdown margin of $\$ 10$ to $\$ 2$ at a rate of $2 \mathrm{k} / \mathrm{sec}$ would take $400 \mathrm{sec}$. This is the time to negotiate region (i).

\section{Region (ii)}

If it may be assumed that the just-critical reactor can safely withstand a step addition of $\$ 0.5$, then a ramp rate of $3 \times 10^{-3} \$ / \mathrm{sec}$ is permissible (see Fig. 1). The reactivity range to be covered is from $\$ 2$ subcritical to $\$ 0.1$ above delayed critical, the latter causing the reactor to diverge with a period of 100 sec. When this stage has been reached, no further reactivity addition will be required until temperature effects neutralise the $\$ 0.1$ excess reactivity: region (iii) is then entered. 
The time taken for the reactivity to be added at $3 \times 10^{-3} \$ / \mathrm{sec}$ will be 700 sec; during this time, the reactor power will have risen to about 5-10 W. If region (ii) extends from $10^{-1}$ to $10^{7} \mathrm{~W}$, a further 6 decades will need to be covered after the reactivity addition has been stopped, the reactor diverging with a 100-sec period. The total time required to cover region (ii) will be $11+24 \mathrm{~min}=35 \mathrm{~min}$.

\section{Region (iii)}

It will be evident from what follows that the minimum time required to negotiate region (iii) will probably be determined by consideration of thermal shock. As this is outside the scope of this note it will be ignored for the present, and the limit will be assumed to be set by the rate of reactivity addition. This will give a minimum time for region (iii) which would only be attainable if the turbine, etc., could take a very high rate of loading.

It was observed earliex that a linear rate of reactivity addition would be unnecessarily slow for the whole of region (iii) and that a constant fractional rate of increase would be advantageous.

Assume a linear power coefficient and full coolant flow rate so that the feedback reactivity is always proportional to the power. Let $H$ be the feedback reactivity when the power has reached $P$. Then, if $P$ is made to increase exponentially, $H$ must also do so, and

$$
P=P_{0} \exp (w t)
$$

and

$$
H=H_{0} \exp (w i)
$$

To maintain this condition,

$$
\dot{H}=w H,
$$

and $\dot{H}$ is a maximum when $H$ is maximum, i.e., at neaxly full power.

Taking $w^{-1}$ as 100 sec and the value of $H$ at nearly full power as (typically) $\$ 2$, then $\dot{H}_{\max }=2 \times 10^{-2} \$ / \mathrm{sec}$, which is faster than the acceptable ramp rate of region (ii), so that cancellation of feedback by control rod insertion would not be permissible. If the maximum rate of insertion of control rod is set at $3 \times 10^{-3} \$ / \mathrm{sec}$, then this would be sufficient to cancel the reactivity feedback when $\dot{H}=3 \times 10^{-3} \mathrm{~s} / \mathrm{sec}$, corresponding to $\mathrm{H}=\$ 0.3$ which, in turn, corresponds to $\mathrm{P}=0.15 \mathrm{P}$ max. Therefore the exponential increase in power would fail to be maintained above $0.15 \mathrm{x}$ full powe $\mathrm{x}$ for the conditions assumed above. 
Given a constant period of $100 \mathrm{sec}$, it would take $300 \mathrm{sec}$ to go from 0.01 to $0.15 \times$ full power, and the reactivity change would be $\$ 0.3$. The residual $\$ 1.7$ required to cancel the feedback from 0.15 to full power would have to be inserted at the maximum permissible rate of $3 \times 10^{-3} \mathrm{~s} / \mathrm{sec}$ and would require

$1.7 /\left(3 \times 10^{-3}\right) \mathrm{sec}=600 \mathrm{sec}$.

Total time for region (iii) $=300+600=900 \mathrm{sec}$ or $15 \mathrm{~min}$.

Total time for region (i), (ii), and (iii) $=7+35+15=57 \mathrm{~min}$.

It is to be noted that with this schedule the time to change from $1 \%$ to full power is only 15 min and, where reactivity is being added at maximum rate, the rate of power increase is $10 \%$ of full power per minute.

The total startup time has been considered to be made up from contributions from three regions. Working backwards it was seen that region (iii) took 15 min provided that reactivity could be added at $0.3 \% / \mathrm{sec}$ and that the corresponding rate of power rise was acceptable. Region (ii) took $25 \mathrm{~min}$, this being determined by the permissible rate of reactivity addition. Region (i) took only $7 \mathrm{~min}$, but this was assuming a rate of reactivity addition of $2 \mathrm{k} / \mathrm{sec}$. A slow rate for region (i) could contribute an undesirably long component to the startup time, especially if the shutdown reactivity margin is more than $\$ 10$.

If the fast rate of reactivity addition is to be employed, it is neces sary that the subcritical reactivity margin is monitored.

A second reason for knowing the state of the subcritical reactor is that the amount of shutdown reactivity required includes a safety factor which guards against accidental criticality during shutdown operations such as fuel loading. This safety margin has sometimes been the largest component of the subcritical reactivity. The larger the subcritical reactivity, the longer the startup time. The increase also adds to the engineering problems of providing a large number of control rods.

Measurements of Subcritical Reactivity

A number of methods for measuring subcritical reactivity have been proposed, some simple, others involving complex apparatus. The simplest way is to utilise the relationship between the subcritical reactivity and power. Alternative methods utilise a known perturbation of the artificial neutron source strength or of the subcritical reactivity. The former could be achieved by pulsing or modulating the excitation beam which is aimed at a neutron-emitting target within the reactor. The latter method could be 
similar to the oscillating rod currently used in measurements of the reactor response function. In either case the measured quantity would be some function of reactor power.

The relationship between reactor power and a source strength or reactivity perturbation is shown in the appendix to be

$$
P=S / Q
$$

for varying source strength or

$$
P=P_{0} R_{1} / Q
$$

for varying reactivity $R_{\mathbb{l}}$ (not to be confused with $R$ the shutdown reactivity). In both cases $Q$ is given by

$$
Q=R+\frac{s 1}{\beta}+\sum_{n} \frac{\alpha_{n} s}{s+\lambda_{n}}
$$

The first term is the subcritical reactivity; the second is the contribution from prompt neutrons; the third is the response of a reactor at the point of delayed critical (this is the reciprocal of the "gain" $\left(G_{0}\right)$ used in reactor kinetic studies). If reactivity is measured in dollars, $G_{0}$ varies from 0 at low frequencies to 1 at high frequencies. Here $s$ is the generalized frequency function, which may be replaced by the angular frequency i 0 if the perturbation is sinusoidal:

$$
Q(s)=R+\sum_{n} \frac{\alpha_{n} s}{s+\lambda_{n}}
$$

or

$$
Q(\omega)=R+\sum_{n} \frac{i \alpha_{n} \omega}{i \omega+\lambda_{n}} .
$$

$Q$ is illustrated in Fig. 2.

At steady-state conditions, $Q=R$ and $P=S / R$, so that, by knowing the power and source strength, $R$ may be found. This simple method has been used with success on the Dounreay Fast Reactor (DFR) and is referred to as Method 1 in Table I. 


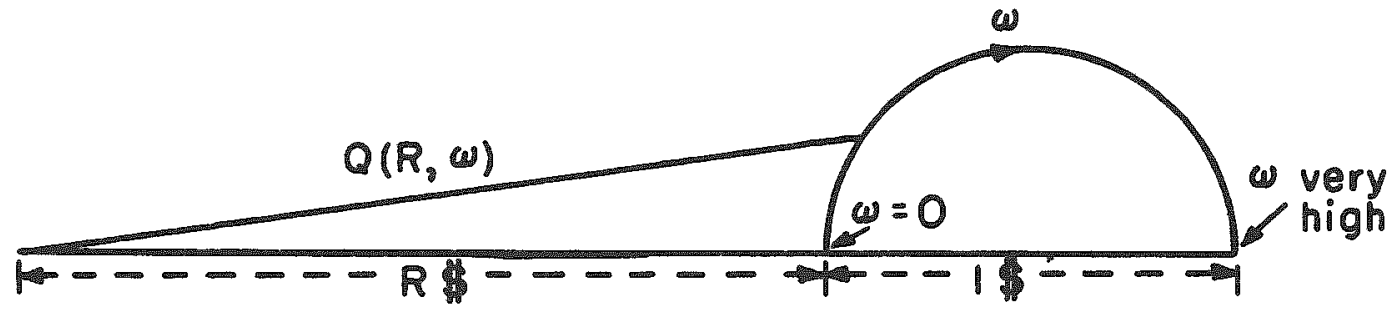

Fig. 2. Relationship between Changes in Reactor Power and Perturbation by Source or Reactivity

Table I

COMPARISONS OF VARIOUS METHODS FOR MEASURING SUBCRITICAL REACTIVITY IN FAST REACTORS

\begin{tabular}{|c|c|c|c|c|c|}
\hline Method & Perturbation & Measured Quantity & $\begin{array}{l}\text { Perturbation } \\
\text { Magnitude to } \\
\text { be known }\end{array}$ & Comments & Sensitivity at $\$ 20$ Shudown la) \\
\hline 1 & - & Reactor Power & - & $\begin{array}{l}\text { Necessary to know the } \\
\text { source strength (1.e., } \\
\text { the constant } S \text { in the } \\
\text { expression } R=S / P \text { ) }\end{array}$ & $5 \%$ \\
\hline 2 & $R \sin 0 t$ & $\begin{array}{l}\text { Reactor Power } \\
\text { Modulation Depth }\end{array}$ & No & $\begin{array}{l}\text { Insensitive except close } \\
\text { to critically. }\end{array}$ & $\begin{array}{l}10^{-4} \text { for sine-wave periurbation } \\
\text { peak to peak } 4 \times 10^{-2} \text {. }\end{array}$ \\
\hline 3 & $R \sin \omega t$ & $\begin{array}{l}\text { Phase Difference } \\
\text { between Power and } \\
\text { Perturbation }\end{array}$ & No & $\begin{array}{l}\text { Insensitive except close } \\
\text { to criticality. }\end{array}$ & $0.07^{\circ}$ \\
\hline 4 & Reactivity Step & $\begin{array}{l}\text { Fractional Change } \\
\text { in Reactor Power }\end{array}$ & Yes & $\begin{array}{l}\text { Still fairly insensitive } \\
\text { excent close to criticality. }\end{array}$ & $\begin{array}{l}10^{-3} \text { for } \Delta R=\$ 0.5 . \text { Error of } 5 \% \\
\text { in } \Delta R \text { would also lead to error } \\
\text { of } \$ 1 \text { at } R=\$ 20\end{array}$ \\
\hline 5 & $\begin{array}{l}\text { Impulse change in } \\
\text { source strength }\end{array}$ & $\begin{array}{l}\text { Rate of Change of } \\
\text { Power }\end{array}$ & No & $\begin{array}{l}\text { Not suitable for fast } \\
\text { reactors. Effect is too } \\
\text { fast to measure. }\end{array}$ & - \\
\hline 6 & $\begin{array}{l}\text { Sine wave perturbation } \\
\text { of source }\end{array}$ & $\begin{array}{l}\text { Reactor Power } \\
\text { Modulation Depth }\end{array}$ & No & $\begin{array}{l}\text { Intense source required } \\
\text { to counter the effect of } \\
\text { steady source. At high } \\
\text { frequencies, controlled } \\
\text { source must be sufti- } \\
\text { ciently intense to give } \\
\text { good statistics. }\end{array}$ & $\begin{array}{l}5 \% \text { change in oscillating com- } \\
\text { ponent of reactor power, but in } \\
\text { the presence of steadv-state } \\
\text { signal, both cornonents having } \\
\text { large statistical fluctuations at } \\
\mathrm{R}=\$ 20 \text {. }\end{array}$ \\
\hline
\end{tabular}

(a) This column compares the ability to measure the shutdown reactivity $R$ to within $\$ 1$ at $R-\$ 20$. The figure quoted is the accuracy required in the primary measurement in order to achieve this sensitivity.

Measurements on DFR

In order to monitow the reactivity effect of fuel-element changes, the neutron flux was monitored during shutdown by a large $\mathrm{BF}_{3}$ proportional counter, temporarily installed for the element interchange in April 1963.

The counter was situated in a vacant ion-chamber hole, where its counting rate, at $10 \%$ shutdown, was nearly $300 \mathrm{cps}$.

Two scalers, with different bias-voltage settings, were used, and changes in amplifier gain or spurious counts due to noise, etc., were revealed by a change in the ratio of counts recorded on them. When the ratio of the scaler counting rates changed by more than a few per cent, the bias 
voltages were effectively reset to their original values by comparing log-log plots of the integral bias curves. (The shape of the latter did not change detectably during the two weeks the counter was installed.)

\section{Results of Monitoring the April, 1963 Shutdown:}

The relationship between reactivity $R$ and counting rate $C$ was taken to be $1 / C=$ constant $\times R$. The constant of proportionality was determined by raising two shut-off rods whose reactivity was known.

The unit of reactivity used for operational purposes is the 'effective inch,' approximately $5 \times 10^{-4}$ or 50 milliniles. It was desirable to maintain a precision of about $1 \%$ in measuring the counting rate during the shutdown, corresponding to an uncertainty of about 2 effective inches (\$0.15) in the subcritical margin.

The interchange of about 90 standard elements (mostly replacing IIB and IIC by IIB elements) plus a few special elements was monitored. The results showed a steady trend in the subcritical reactivity, but with three discontinuities.

(i) An apparent large loss of reactivity (11 effective inches) on removal of an element containing $30 \mathrm{~g}$ of beryllium is attributable to the loss of source neutrons due to the $\operatorname{Be}^{9}(\gamma, \mathrm{n} 2 \alpha)$ reaction.

(ii) There was an apparent loss of reactivity (2 effective inches or 100 milliniles) during the time that engineering work was being carried out, but no element changes made.

(iii) One other reactivity loss was apparently larget than expected: 4 instead of an expected 2 effective inches. If the apparent loss of reactivity due to removal of the beryllium is ignored, the excess reactivity of the recharged core, predicted by the monitor, was 15.8 effective inches, whereas the actual value was 22.5 effective inches. The exror of 7 effective inches $(\$ 0.5)$, although not large from the operational point of view, could probably have been reduced by simple improvements in the equipment.

\section{Other Methods of Measurement}

In view of the importance of the measurement of subcritical reactivity, a number of alternative methods have been considered; these are given in Table I.

The measurements fall into two classes, varying the source strength or varying the reactivity, but there are variations within each class. 
Source Constant, Reactivity Variable

Method 2

The reactivity is varied sinusoidally and the peak-to-peak magnitude of the oscillating component of reactor power is measured, $M=2 P / P_{0}$ of equation 6 (see Appendix). This is done at high frequency $M_{H}$ (too high for delayed neutrons to be effective) and at low frequency $M_{L}$ (low enough for delayed neutrons to be fully effective). Then $M_{H}=A(R+1)^{-1}$ and $M_{L}=A R^{-1}$, where $A$ is a constant depending on the magnitude of the oscillating reactivity. Eliminating $A,\left(M_{L} / M_{H}\right)-1=R^{-1}$, and this enables $R$ to be determined in a manner which is independent of arbitrary constants. A practical difficulty is that it is difficult to obtain much more than $\$ 0.04$ for the peak-to-peak oscillating reactivity, so that for $R=\$ 20, M_{L}$ and $M_{H}$ would only be $\approx 10^{-3}$. To know $\mathrm{R}$ to $\mathrm{x}_{\%}^{0}$ would therefore require $\mathrm{M}$ to be measured to $\left(2 \times 10^{-3}\right) \times \%$, which makes the method of doubtful value.

\section{Method 3}

Alternatively, the phase difference between the oscillating reactor power and the stimulus may be measured. This has a maximum value given by $\sin \theta=\frac{1}{2}\left(R+\frac{1}{2}\right)^{-1}$ (see Fig. 1). For $R=\$ 20$, this would give $f=1.4^{\circ}$, which is also impracticably small.

\section{Method 4}

Measurement of the effect of a known incremental change in $R$ may be made at constant frequency. If the increment is $\Delta R$ and the measurement is made at $w=0$ (steady state), then the fractional change in reactor power would be $1 / R(R+1)$ times the increment. [A slight improvement would be obtained if the measurement were made at high frequencies, the multiplying factor being $1 / R$ instead of $1 /(R+1)$, but this advantage is of little consequence when $R>10$, which is the region of interest.]

The advantage of this method is that $\Delta \mathrm{R}$ can be quite large, as it can be obtained by the movement of a control rod between fixed limits, and $\$ 0.5$ is quite feasible so that, if $R=\$ 20$, then the fractional change in power will be $2.5 \%$. This is about 10 times more than the equivalent peakto-peak value of Method 2. The method has the disadvantage of being dependent on a knowledge of incremental reactivity addition although, for an operational reactor, the worth of a control rod is usually adequately known. 


\section{Source Variable, Reactivity Constant}

\section{Method 5}

Method 5 employs impulse perturbation of the source. Equation 4 of the Appendix show that for very rapid changes, of the order of the prompt-neutron lifetime, the power and source are related by

$$
\frac{P}{S}=\left(\frac{S 1}{\beta}-R+1\right)^{-1}
$$

For an impulse change in $S$, the power will rise instantaneously and then fall exponentially towards its original value, the exponent being a measure of the subcriticality $R$. This method is satisfactorily used in thermal reactors, but would be extremely difficult in fast reactors where $1 / \beta$ is much less than a millisecond. It has been used on fast critical fuelled with $U^{235}$. For a plutonium-fuelled reactor having a large spontaneous fission source, a very much larger pulsed source will need development.

\section{Method 6}

Cyclic perturbation of source. This method is similar to Method 2 with the possible advantage that modulated sources may be developed having intensities which would provide larger modulations than is practicable with reactivity oscillators. The large spontaneous fission source from $\mathrm{Pu}^{240}$ will increase the required source strength. If the modulation depth can be made close to unity, i.e., the magnitude of the controllable source is very large compared with the steady source term (e.g., from spontaneous fission), then the change in the amplitude of the power oscillation when measured at low and high frequencies will be $5 \%$ for a measurement accuracy of $\$ 1$ in $\$ 20$. This percentage change will be reduced linearly with the value of the modulation depth obtainable in practice. The reduction may be very large. A controllable source having an average strength of $10^{9} \mathrm{n} / \mathrm{sec}$ would only be of the same order as the spontaneous source. The intensity of the variable source would need to be sufficiently high for the detector to follow sinusoidal variations at frequencies of several cycles per second with a sufficient number of counts per cycle to give reasonable statistical accuracy. In principle, one can count an ill-defined sine wave for many cycles and so achieve accuracy, but this is less satisfactory.

The corresponding phase measurement (cf Method 3) would be insensitive for the same reasons as Method 3, namely, that the reactivity $R$, which is frequency-independent, has a dilution effect. For $R=\$ 20$, the phase difference between power and source variation would be $1.4^{\circ}$. 


\section{Discussion of Subcritical Reactivity Measurement Methods}

Of the methods described in this section, there 15 not one which offers much hope of being able to measure the subcritical reactivity to within $\$ 1$ at $R=\$ 20$, except the steady-state method used for DFR and, possibly, Method 4. The monitoring of subcritical power is a sufficiently accurate method to ensure that gross errors in loading are detectable during operational changes made at shutdown.

Durang the critical approach, a further check on the reactivity condition may be obtained by use of Method 4. The subcritical power is measured before and after the movement of a control rod through an increment of $\$ 0.5$. If this 1 s done at $\$ 2$ from delayed critical, a rapid assessment of the subcritical margin may be obtained. This has the advantage of being independent of the neutron source value, but assumes that the control rod calibration is known. Throughout the life of the reactor the worth of the control rods must be checked (e.g., by period measurements); it is probable that this will be done after each shutdown and could serve to calibrate the incremental reactivity change. The considerable advantage of this method lies in the fact that no special equipment has to be installed in the reactor, and it provides an independent check on the estimate from direct measurement of subcritical power (Method 1). The latter is independent of any reactivity standard but depends on a calibration constant which is affected by changes in effective source strength. For an operational reactor plant, unexpected changes in source strength are likely to be negligibly small.

It is concluded that adequate methods are available for monitoring subcritrcal reactivity. The rate at which reactivity may be added while the reactor is subcritical is therefore not limited to that applicable at the point of delayed critical In addition, the subcritical margin which is necessary for safe operation at shutdown may be reduced to less than the figure necessary should the subcritical state have to be guaranteed without continuous measurement.

Conclusion

The time taken to start up a fast reactor power plant (see Fig. 3) will be determined by the following

(1) the acceptable rate of increase in the loading of the generating equipment;

(ii) the maximum permassible rate of reactivity addition at the point of delayed critical,

(i1i) the ability to monitor the subcritical reactivity in order to exceed the previous limit while the reactor is subcritical. 


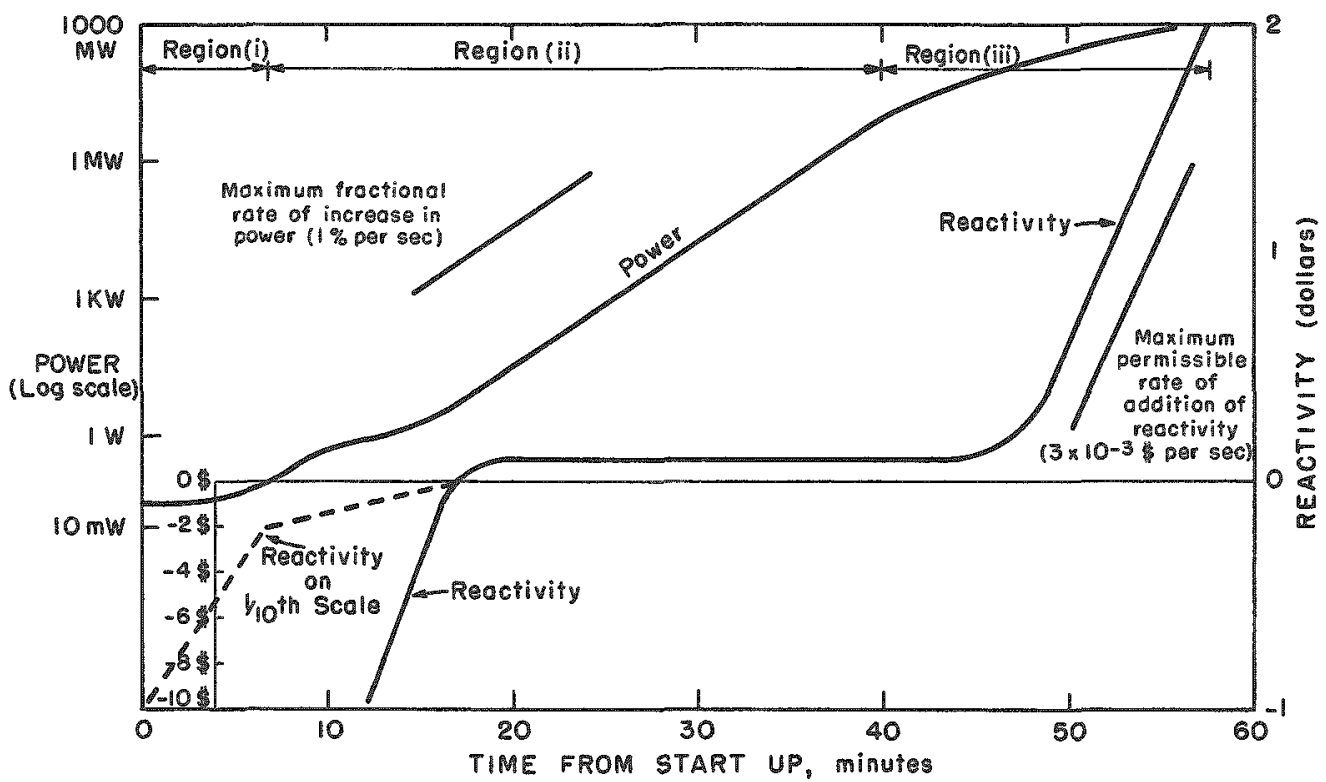

Fig. 3. Start-up Schedule for Fast Reactor

Of the methods available for monitoring the subcriticality, the steadystate subcritical power is adequate while the reactor is a long way from critical.

During the subsequent critical approach, the reactor should be held at about $\$ 2$ or $\$ 3$ and the change in power measured as a control rod is moved by known amount (about $\$ 0.5$ ). This provides an independent check on the previous method.

As a specific example, assuming that a fast reactivity addition rate of $0.02 \$ / \mathrm{sec}$ is permissible during subcriticality, and that the generating plant may be loaded at a rate corresponding to full load in $15 \mathrm{~min}$, the reactor may be at full power within one hour from being at $\$ 20$ subcritical.

\section{Acknowledgement}

Acknowledgement is due to Mr.J. C. Bates who was responsible for the subcritical reactivity monitoring on DFR. 


\section{Appendix}

With the usual nomenclature, the reactor kinetic equations may be written as

$$
\begin{aligned}
& 1 \mathrm{dP} / \mathrm{dt}=(\rho-\beta) P+\sum \lambda_{i} C_{i}+S_{j} \\
& \partial C_{i} / \partial t=\beta_{i} P-\lambda_{i} C_{i} .
\end{aligned}
$$

Let $P=P_{0} \exp (s t)$ and $C_{i}=C_{10} \exp (s t)$, Then

$$
C_{i}=\frac{\beta_{i}}{s+\lambda_{i}} P
$$

Case 1. Source varying, reactivity constant.

From Equation (1),

$$
\begin{aligned}
& 1 s P-(\rho-\beta) P=P \sum \frac{\lambda_{i} \beta_{i}}{s+\lambda_{i}}+s_{i} \\
& \begin{aligned}
\frac{P}{S} & =\left[-\rho+s I+\beta-\sum \frac{\lambda_{i} \beta_{i}}{s+\lambda_{i}}\right]^{-1} \\
& =\left[-\beta+s I+\beta \sum \frac{\alpha_{i} s}{s+\lambda_{i}}\right]^{-1}=Q^{-1} .
\end{aligned}
\end{aligned}
$$

Case 2. Source constant, reactivity varying.

\section{Let}

$$
\begin{aligned}
& \rho=\rho_{0}+\rho_{i} \exp (s t) \text { where } \rho_{1} \ll \rho_{0}: \\
& P=P_{0}+P_{I} \exp (s t) \text { where } P_{1} \ll P_{0}
\end{aligned}
$$

Equation (1) may be rewritten as

$$
1 s P_{l} \exp (s t)=\left[\rho_{0}+\rho_{1} \exp (s t)\right]\left[P_{0}+P_{I} \exp (s t)\right]-\beta P+\sum \lambda_{i} C_{i}+s^{\prime}
$$

Clearing the brackets, ignoring terms not varying with time, and using Equation (3) but considering only its time-varying part, we find 


$$
\begin{aligned}
& 1 \mathrm{~s} P_{1}=\rho_{1} P_{0}+\left(\rho_{0}-\beta\right) P_{1}+P_{1} \sum \frac{\lambda_{i} \beta_{i}}{s+\lambda_{i}} \\
& P_{1}\left[s 1-\rho_{0}+\beta-\sum \frac{\lambda_{i} \beta_{i}}{s+\lambda_{i}}\right]=\rho_{1} P_{0} \\
& \frac{P_{1}}{P_{0} \rho_{1}}=\left[-\rho_{0}+s 1+\beta \sum \frac{\alpha_{i} s}{s+\lambda_{i}}\right]^{-1}=Q^{-1} .
\end{aligned}
$$

Thus $Q$ is the same for the two cases.

The first of the three terms represents the subcritical reactivity (the negative sign will then disappear). The second term is the promptneutron term and may be neglected for fast reactors. The third term is the delayed-neutron term, which, taken with $s=i d$ is the familiar "in hour" equation.

For dynamic purposes it is usually more convenient to use reactivity in units of the delayed-neutron fraction. If $R$ is the subcritical reactivity (in dollars) and $s 1$ is neglected, then

$$
Q=R+\sum_{i} \frac{\alpha_{i} s}{s+\lambda_{i}}
$$

and $R_{1}$ is the impressed cyclic reactivity, corresponding to $p_{1}$ in Equation (7). For sinusoidal changes at high frequency, of for fairly prompt effects $Q \rightarrow R+1$ since the sum $\rightarrow 1$. For low frequencies or slow changes, $Q \rightarrow R$ 。 
Discussion of Paper

Presented by Mr. Smith

MR. TAVERNIER (BelgoNucleaire)

The method you are using here is essentially an alpha technique. What is the typical measuring tume you would need in order to make a given measurement of subcriticality to a given accuracy?

MR。SMITH: counter.

It took us about 10 min to get some several $\times 10^{4}$ counts with $a \mathrm{BF}_{3}$ MR, TAVERNIER:

Do you make the measurement in one reactivity step or do you go step by step? I ask this because we have been thinking for some time, of taking direct ratio measurements at various times after pulses and converting this into reactivity measurements. From theoretical calculation it would appear that at one megacycle frequency, with adequate counters you can get good statistics within a second or two.

MR. SMITH。

I think we are talking about two different things. You are talking about a puise method. That is not the one we have used at Dounreay. I do not think the pulse method will work very well for fast reactors. Particularly when you have a high plutonium -240 content, the size of the pulse you are going to need to overcome the steady $\mathrm{Pu}^{240}$ contribution is going to be very difficult to get, and you will pay an awful lot of money to get your source target into the reactor. Something very simple like my proposal works quite well.

MR.THOMPSON (MIT)。

There have been either several accidents or near accidents in the United States and other places because of the basic problem that you are going from a less conservative regime to a more conservative one as you go from step one to step two to step three. An error on the part of the operator, leaving it too long an motion type two, after you have moved into reactivity zone three, can result in meltdown of the coxe. It would be worth considerable effort to see how you can limit this regime and take it out of the hands of the operator. The pressure to rush on to power can lead to major trouble. 
MR. SMITH:

Yes. I think there are two points here. One is the one you have made. One can also go the other way around. When the Dounreay reactor was designed may years ago, they thought of the most miserable things that could happen and adopted an extremely slow approach to criticality. It takes us hours. The man who should be in charge of that reactor is so bored he may really not be paying proper attention. 


\section{GENERAL REVIEW AND SUMMARY}

MR。OKRENT (Argonne):

Rather than have a detailed review, we will have a few five minute comments, and then throw the floor open for discussion. I think I will ask Dr. Dietrich if he will be the first to comment.

MR, DIETRICH (General Nuclear Engineering):

I think the title of the conference, that is, a conference on breeding, economics and safety in large fast power reactors was well-chosen. If one thing has come out, I think it is that there is a very strong relationship among these questions. The safety problem has not been solved yet in the sense that one can design a reactor which is safe and which does not, in order to be safe, compromise things in the direction of lower breeding ratio, or higher cost. So long as this is so, you cannot really say when we will have a fast breeder that will solve the problem of fuel resources. I think I would take a different point of view from some that have been expressed here and say it seems to me that the development of a fast reactor is an urgent matter on the basis of development of fuel resources. This is because we do not now know how long it may be before we have a safe fast reactor with a short doubling time and good economics.

Looking at the components of the safety question, it appears to me, from what I have heard here, that there are two very important problems: the question of the positive sodium coefficient, and the uncertainties in Doppler coefficient, including the reactions of the positive sodium coefficient on the design in such a way as to affect the Doppler coefficient. It is probable that these uncertaintres, great as they are in the physics, will be resolved, more or less, by methods that we know about now. It will probably take a lot of work

It also seems to me that the real culprit is this sodium void coefficient, because if it were not for that consideration, presumably one could get a good negative Doppler coefficient without too much trouble, even. though there might be a good deal of uncertainty as to just how negative it was. It seems that you get out of this sodium problem either by proving it is not a problem, through accident analyses, or through designing for high leakage. The latter procedure seems to be a difficult way out and, furthermore, gives the possibilities of producing local positive effects, even if you do achieve in some designs a gross negative void coefficient.

The really sticky part of the whole business comes when you look at just what the accidents are When we are faced with this kind of a 
problem, we usually turn our thoughts toward experiments. But because we must conserve our efforts and money, we have to exercise our ingenuity before we start the experiments, rather than hoping that somehow we will get smarter in the process of carrying them out and be able to exercise some ingenuity in the analysis of them after they are done.

One responsibility we all ought to accept as designers of reactors is to forget about the ACRS in this sense, and act as our own ACRS. We must put ourselves in the position of having to make the final decision that a reactor is safe and build it so that it will not present a hazard to people who should not have such hazards imposed upon them. It seems to me that this is the only way that we can exercise our ingenuity, through recognition of the real problems, rather than by trying to out-guess either the ACRS, the Division of Licensing and Regulations, or whatever other overseeing body there may be.

\section{MR. OKRENT:}

I would like to ask Dr. Hall for his comments.

MR. HALL (Los Alamos):

I am heartily in accord with Mr. Dietrich's remarks about the regulatory staff of the AEC or the ACRS.

One of the things that impressed me was the amount of effort that is going into the meltdown and the subsequent accident, the secondary criticality problem. Indeed this is a very serious one. A great deal of reliance is being placed on the AX-I code. This is certainly good for idealized conditions, but I think the people using this code are very aware of its limitations and of the approximations that are made in order to get any kind of a system to describe. The total energy that evolves is usually about 100 or 300 or even 1,000 pounds of high-explosive equivalent. What is pertinent, however, is not that number, but the kinetic energy, which is also derivable from the AX-I code. In general, for most accidents which are imagined, the kinetic energy is the order of a few per cent of the total energy release. I think this is something that has been neglected and really should be a part of the analysis.

There are proposals to understand what the initial or starting conditions for the calculations should be. Of course, the TREAT reactor is a direct result of these discussions, an attempt to find out what is the meltdown configuration of fuel elements having undergone a transient. Another proposal is that of Dr. Tavernier, which is intended to improve the techniques for this. But primarily it is not enough to pulse a fuel element and look to see what happens afterwards. I think the dynamics of this, the kinetic observations, are extremely important, and they also are 
extremely difficult and expensive. But techniques such as the gamma-ray camera, which was tried by Argonne and ran into background troubles, would be extremely useful in trying to understand what are the conditions of a meltdown. It may well be, for example, that a fuel element with appreciable fis sion product inventory would foam and froth, rather than run down as a pool of mercury. Thus the secondary criticality problem may not exist the way it is imagined in the AX calculations. The simulation studies that were reported by Huebotter were idealized because they certainly do not take into account any changed properties of the fuel. They simulate the uranium by using lead instead. I am sure APDA is well aware of the limitations of this, and that it is a first very small step on this road.

We must be concerned with the safety factors and the cost of introducing safety factors. General Electric has proposed a fast reactor with oxide fuel, in which they would degrade the spectrum with beryllium in order to be assured of a Doppler coefficient favorable to an excursion. But this results in a degradation of the neutron economy, and, consequently, of the economic position of this reactor. This is a price which Dr. Cohen was very clear in stating that he was willing to pay in order to be sure he got the program off to a good start. It may well be that this is the thing that has to be done, but there should be other ways to avoid any severe economic penalty for including safety.

I do not think the safety features of containment, for example, are at all burdensome to total reactor designs, and I have not noticed any reluctance on the part of power plant engineers to include adequate containment.

There seems to be some kind of a reluctance to include adequate operating staff. This is one thing that gives some concern. This session has not been devoted to operating problems. Rather than just being sure that the reactor as built and the plant as built contain all of the safety features, we have to think about the position of that plant 10 or maybe 20 years from now when the design crew has long since gone. Maybe the third string is in there, people who have long since forgotten all of the discussions and considerations that went into the design. That is one of the reasons why it is highly desirable to have inherent features which are not dependent on administrative control.

MR. OKRENT:

I should like to mention a few things that stick out in my mind, after listening to the various papers. First, we still suffer from a major need for good capture cross sections. It might be well if the people here exercise what influence they can on those who set priorities for cross-section measurements. Cross sections constitute our major need in determining the important reactivity coefficients. 
Until recently, the emphasis has been on cross sections which are critical for thermal reactor performance. It is time to change this emphasis.

In the absence of such measurements, we should begin very good, sophisticated experiments in existing critical facilities to give us a handle, however incomplete, on such important things like the effect of $\mathrm{Pu}^{240}$, vanadium, or niobium on the sodium coefficient.

There has been a lack of papers at this meeting dealing with reactors having high sodium volume fractions. People did not present what the performance of such a reactor is, nor how it is penalized as compared with a reactor having $45 \%$ sodium. There do seem to be gains from the point of view of sodium coefficients, at least for uniform core removal. It is clear there can be penalties on performance, but this was not discussed.

An area where considerable research work is warranted in the future is the transient behaviour of sodium during an accident. We heard a description of the way water has a pulsing flow. My understanding of the present state of knowledge is that theoretical methods in fiuid flow and heal transfer are not adequate to handle such phenomena in sodium. There is need of basic work on just how sodium will behave under transient conditions. This topic has been of long-term interest, but it has certainly become much more vital with the advent of the possibility of large, positive sodium void effects.

Another area that warrants some effort is the evaluation of accidents leading to explosive disassembly in a detailed fashion, using as good a representation at each stage as is feasible. In other words, one calculates the onset of the accident for the specific reactor in detail: either sodium is ejected, or melting occurs, or what have you. The proper Doppler coefficient is used, and as good a geometric representation is made as practical; etc. If, by using pessimistic (but not ridiculously pessimistic) assumptions, one can decide that the range of explosive yields is containable, a long step forward will have been made. There will still remain the question of protection of the economic investment, of course. This may be insurable - but the rates could become prohibitive, if an accident involving total loss of a large fast power reactor plant occurred.

We still have really not defined what is meant by a "large reactor." Not too long ago $500 \mathrm{MWe}$ was large. We would all have had much less to talk about this week had the size stayed there. People are now working hard to design a 1000-MWe reactor, and the utilities are thinking in terms of 1500-MWe plants. Inevitably one is going to have to compare one large reactor with two smaller ones. This area of study has been lacking at this meeting. It may be that the apparent gains in capital investment by going to the 1000-MWe reactor may not be worth the losses that we incur by 
compromising the breeding ratio, by raising the containment costs, or by introducing problems in reactor-siting. Two 500-MWe reactors tied to the same heat removal system may be better. I do not know.

Interesting and encouraging results are given in the work reported by Dr. Cohen and Mr, Zaleski on the possibility of living with some kind of a positive sodium effect. It seems to me that one possibly important thing in making comparisons of the safety of two reactor designs, as influenced by the sodium void effect, may be as follows: Does the presence of a considerably positive sodium void effect, on the one hand, as contrasted with a design having an essentially zero sodium coefficient, make the possibility of large scale meltdown really probable? Where it was something hypothetical before, does it become reasonably credible? If so, the meltdown accident becomes a much more significant thing than it was previously. One is forced to study the meltdown question in sufficient detail to be satisfied that containment is not violated.

MR. SMITH (Winfrith):

I think there has been loose talk about the sodium coefficient, that is, a confusion between sodium temperature coefficient and the sodium void coefficient. Of course we are really interested in a power coefficient, which is neither of the se things. It's harder to measure or to calculate. In the particular case of the sodium void coefficient, I think that really we have to go along the lines of a rather detailed accident analysis, that $\mathrm{Mr}$. Cohen among others did. The question as posed by the Chairman just now, in the form in which he presented it, is a little meaningless. You were suggesting there was a difference between a reactor with a zero sodium void coefficient and one with a positive sodium void coefficient. It seems to me that both these reactors will have a positive coefficient for the displacement of part of the sodium. If you make a void in the center of the core or near the center of the core, you are likely to get a positive reactivity increase, This to me seems to be the thing we are worrying about, not so much the case where you get 100 per cent sodium loss, since in practice that core has melted down anyway and you have a much bigger reactivity gain on your hands. In such a case nobody really cares what the sodium loss coefficient would be.

I feel we have to be a little bit more careful in defining what we say in separating out the temperature coefficients. In this connection, I don't think that a small positive temperature coefficient is even an operational embarrassment. The accidents associated with possible coefficients resulting from voiding or part voiding of the reactor, can cause dangerous situations if they are highly positive. In this respect, I think the larger sodium coolant volumes which we spoke of earlier could be a positive handicap. They might make the contribution from the moving part of the sodium even more positive, and you would get a safety loss rather than a gain. 
MR.ZALESKI (EURATOM):

I agree with Dr. Smith. It is very important to know the distribution of the sodium coefficient reactivity worth. Even with a zero void coefficient with sodium, you have positive parts. If I have understood what Dr. Smith said, that we are only concerned with power coefficients in the accidents, it seems to me that for some kinds of accidents the isothermal coefficient may also be important. Maybe for the flow perturbation. Generally speaking, it is dangerous to take too simplified a picture of these coefficients. It is better to take a reactor and to see what's happening with this reactor following some realistic scheme, and then only look to the maximum accident, but for all kinds of maximum accidents. If some destruction or some melting occurs, then it's difficult to speak about reactivity coefficients for the undestroyed reactor. The coefficients of reactivity for the undestroyed reactor are not the same.

Finally, the positive sodium coefficient may have some influence on the maximum accident, but the preliminary study that we have done seems to show that this positive coefficient cannot introduce a very strong maximum accident. We can't see how a strong positive sodium coefficient can introduce a large maximum accident, larger than we can forsee from some meltdown of the core.

MR. COHEN (General Electric):

I would like to comment a little bit on what we should expect to accomplish in a meeting of this kind. I think that perhaps there is a great deal of absolutely unjustified gloom about what seems to me to be the prospects of a very attractive reactor type. When we looked for ourselves, we examined the prospects for a fast reactor and considered whether this was a reactor which had promise. The condition that we set for it was that fuel cost would be less than one mill per kilowatt-hour. We thought the capital costs would be basically the same or close to the same as those of other advanced reactor types when it was developed. We were fairly happy to see that the reactor would have a very modest positive breeding ratio. I believe it was less than 1.1 .

Now, I have always noticed that in economic studies people are interested in breeding, always count breeding twice. Once they take a count of the plutonium credit and then they look at the breeding ratio afterwards as a separate number. The fact of the matter is that this is not true. If you look at it from the economic standpoint, you see that breeding has a certain advantage, and in the long range, the real long range, it has an advantage from the standpoint of the conservation of natural resources. There is not a particle of evidence that we are in trouble on natural resources for a long time. There is considerable evidence that the economics 
of nuclear reactors is the determining bottleneck in the expansion of nuclear power. So let us take our problems one at a time,

The first problem is to get a fast nuclear reactor which is economic. Therefore, let us look at the cost. I am perfectly persuaded that the breeding ratio will, in the course of time, creep up. We are sitting here full of ignorance on all the consequences on all the coefficients, with very little data, and I do not think we should try to draw too firm conclusions as to what directions we should go. For example, the statement that beryllium in a reactor is an absolutely horrible compromise in the entire potential of the system - I cannot go along with that at a11. I think the system has good potential, and we expect to make money on it.

\section{MR. OKRENT:}

While you are collecting your thoughts, I might amplify on my last remark. What I was wondering was, is it possible, for example, that partial expulsion from the core in a few subassemblies might produce a reactivity excursion leading to full core melting in the presence of the positive sodium coefficient whereas it doesn't in what I'll call a more desirable reactor. This is not something that I have evaluated. It is an aspect I was raising for consideration.

MR.SPINRAD (Argonne):

If I may, I would like to comment on two of the questions that the Chairman raised. One is a point that has been discussed in a number of ways the question of high or low sodium volume content. A few weeks ago Dr. Persiani and I did a little plotting of whatever consistent data we could find on this point. What we discovered was that for a given volume of fuel in a given type of system, regardless of whether there was a lot or little sodium, the coefficient was zero. The less sodium there was in the reactor above that point, the less positive sodium coefficient there was, and also the less negative below that point. Therefore, if the power of the reactor is limited primarily by the power capability of the fuel elements, as is generally believed, the sodium volume fraction is not a partrcularly significant parameter in determining the power capability which is achievable whth a negative coefficient

The other point that the Chaurman also raised, I think was at least partially and qualitatively commented on in the paper I presented, so I would like to repeat the point again A number of the designs which have appeared in the Argonne evaluation of the extrapolated fast reactor sys tems are indeed designs of systems which are basically modular reactors encapsulated in a common blanket. There is apparently no reason that wo can see for the perturbation on the economics if this is the way it has to be because of the difficulty of getting fuel in one lump. The perturbation on the economics can arise from two causes. 
One, the required increased enrichment of having leakier systems by this means, which is something we achieved in any case in the smaller reactor sizes, and second, having extra control systems that are somewhat more expensive than a single unit control might be. I would be very surprised if, taking the Canadian convention of a development shorn of the cost which we are trying to achieve, these amount to more than a few million dollars per thousand megawatt reactor, which is not that much. It is a significant penalty, but it is not the sort of thing that I think we would find overriding if it is necessary. For this reason, it may well be that the question of the size effect on sodium coefficient is not of much greater significance than is, for example, the significance of alternative containment types.

MR. NIMS (Atomic Power Development Associates):

I would like to comment on one of the questions that you just raised about the problem of local bolling. There are probably about three things that you would be concerned about in a system where you have a positive sodium coefficient. One of them would be the local boiling problem. For the values we calculate now of what this sodium worth might be, I don't think that local boiling is a problem. One or two subassemblies contribute only something on the order of 10 cents worth of reactivity, and the normal feedback of the system will handle this.

A second problem is that of stability. If you have a system which has a positive component, you might be concerned about stability; but in this case I believe we have a number of larger effects with similar time constants which are more than capable of overriding any sodium density effects.

The real problem is the one in which you raise the general temperatures up to the point where you are about ready to boil in one channel. Once this happens, it spreads very rapidly (in milliseconds probably), it voids essentially the whole core, and you add reactivity at rates of several hundred dollars a second. If this can happen, then you have a meltdown accident which is no longer quite as unrealistic as the hypothetical accidents that we have worried about. There has always been a certain amount of conservative optimism in a lot of the assumptions made about instantaneous melting, gravity collapse of the top surface, and so forth. But if this boiling condition takes place, then I think you have a very real situation on your hands where you can insert several hundred dollars a second and where you will get a very substantial energy release.

There is one other point that has been made very clear here from the discussions in the last two or three days: there is a good deal of uncertainty in the calculations of sodium worth, Doppler, and spectrum. Of course, we were aware of this for some time. It is obvious that we need some experiments and preferably critical experiments. I think during the 
past few months we have gotten some good information from the ZPR-III facility At the present time, I am not aware of what additional information is going to be available or when it will be available or what's planned. Argonne does have a ZPR-VI facility but this is a strictly $U^{235}$ affair. Can you tell us a little about what we might expect in the way of large plutonium cores, and on what sort of time scale?

MR AVERY (Argonne):

Although ZPR-VI is as of now limited to $U^{235}$, our plans are to include study of the problems that have been of primary concern at this meeting, primarily, the Doppler coefficient and the sodrum void coefficient. In terms of the sodium void coefficient, we are well aware that we will be studying it in a $U^{235}$ system, but we feel that if we can get agreement between theory and experiment in such systems, we wlll have gone a long way towards verifying the systematics. Then I think the only remaining cross sections that will be relevant will be those of capture and fission of the plutonium isotopes themselves.

Argonne has plans and proposals for a large scale plutonium facility, the ZPPR, in which we would certainly hope to make experiments on large plutonium systems, and hopefully even systems with sizable zones of plutonium of higher isotopic content

In terms of the Doppler effect measurements, Dr. Fischer will present a paper tomorrow on some measurements made recently with $Z P R-I I I$. The results certainly appear to be quite encouraging, and we will be again making a number of such measurements with both ZPR-III and ZPR-VI In the case of $Z P R-V I$, it will be primarily to determine the effect of the size of rod samples on Doppler coefficient measurements, since it is the extrapolation of the homogeneous to heterogeneous systems in terms of Doppler effect that really serves as the basis for many of the planned measurements on FARET and SEFOR on Doppler effect

I would like to also make a couple of comments if I may With ZPR-III we do have sizable discrepancies for two quantities; lifetime, and the boron danger coefficient, both of which are very sensitive to the lowenergy end of the spectrum. Much of the information that we have heard at this meeting is based on cross sections yieldng such discrepancies

The larger systems we are talking about will have far more neutrons in the low-energy end. What the implication is I do not think anyone knows. Whether this is an indication of a very bad error or not certanly is not clear. It seems that in any phenomenon involving the very low energy end of the spectrum, that is, neutrons having survived many possibilities of removal along the way, rather small exrors along the way 
could have a rather profound effect. I think we should be very conscious of this fact and I think we may be in for a number of surprises

The second area that I would like to make a comment on is one of the so-called large or maximum accidents. It seems to me that the crucial item in such accidents where we assume we have a large reactivity insextion rate, usually assumed to be some consequence of a meltown that has started by some other means, is the first differential movement which will affect the energy release. If we go through a very quick negative effect, such as given by the negative Doppler effect, the calculations indicate that one tends to shut down with a rather relatively small energy release, whereas if one assumes, for example, very small positive Doppler effects, so that the first movements are positive, this can give very, very large energy releases. I assume the same is true in the sodium void coefficient. Certainly one can hypothesize movements of sodium which could be very fast: if they were positive, one could envisage very bad results. In this connection one should mention the evidence from the TREAT experiments that the fuel does tond to foam; if this phenomenon is a very real one, one might expect that in any substantial failure all of the fuel might really foam out and essentially fill up the voids. If in doing this sodium can be forced out very rapidy without moving the fuel itself, it is not clear exactly what happens. This could lead to rather drastic results if it were accompanied by a positive soduum void coefficient.

I think there has been sort of a tacit assumption that all of these very small fuel movements, such as might be involved in filling up coolant gaps, are not really relevant. We talk about Doppler coefficients and sodium void coefficients, but I do not think we can neglect the possibility that the very small local movements of fuel might have small reactivity changes (and I think we are only talking about matters of a few cents) which, if they were positive, could again lead to very bad results. We really do have to study the detailed mechanisms of local movements and the effects on the Doppler and sodium void coefficients in the immediate time we have a very rapid meltdown.

MR. SMITH (Dounreay):

I would like to come back to a point that we should take care of particular accidents, not be too general in our approach. I would like to consider two accidents, both of which are improbable, by that I mean sooner or later they will happen. One will be the loss or partial loss of coolant flow rate, while the other accident is a redistribution of a fuel, perhaps in a pebble bed or perhaps in a subassembly, in other words, a slight change which leads to a step addition of reactivity. Examination shows it is desirable insofar as the first kind of accident is concerned, to have a positive Doppler coeficient plus a small delayed neutron fraction 
For the second accident, you want a negative Doppler coefficient and a big delayed neutron fraction. This indicates to me that we must be very careful in looking at the whole range of accidents in relation to a specific reactor, and not be too general.

MR. WOLFE (General Electric):

Can you explain that first accident?

MR. SMITH:

If flow rate falls, the coolant temperature will rise and in our ex: ample the overall negative coolant coefficient will reduce reactivity. The consequent power reduction leads to an overall reduction in the average fuel temperature. With a negative fuel coefficient, reactivity would be inserted to prolong the transient, whereas a positive fuel coefficient will cause (initially) the power to fall more rapidly. This is not taken as an example to advocate a positive fuel coefficient, but to demonstrate that specific accidents must be related to specific reactors and that too much emphasis on one aspect of reactor safety can distort the overall position.

MR. ZALESKI:

When the flow is decreasing and for the load that we used, we found that it is better to have a larger negative Doppler effect. I cannot say what happens with the positive Doppler effect. I have not made the calculation.

MR. MENEGHETTI (Argonne):

As was mentioned earlier, the calculated spectra and what would seem to be the experimental spectrum for ZPR-III assemblies do not agree because of the lifetime discrepancy. It is generally felt that, in the first part, this is due to a discrepancy in the low energy tail, which is what would be important for Doppler effects. However, Jim White and I recentlv noticed that what is important as far as the lifetime is concerned is not the low energy tail, but a slight shift in the maximum.

MR. DAVEY (Argonne):

I agree with Dr. Meneghetti. The shift is not necessarily in the low energy tail. But, it requires a large move of spectrum in the region of say a few hundred keV. I think that the discrepancies might indicate, and I would emphasize might indicate, a discrepancy below $10 \mathrm{keV}$.

Another point goes back to the point you made, Mr. Chairman, that the question of trying to influence people who measure cross sections to measure the ones we need. The discrepancies in lifetime and boron-10 
reactivity found using the YOM cross sections on the ZPR-III assemblies exist for. I think it is fair to say, all the systems investigated; those with fairly hard spectra, those with fairly soft spectra. But I do not think it should be assumed that the troubles we may run into in understanding Doppler effect will remain in understanding low energy cross sections. I think it is quite possible that we are in dire need of understanding high energy cross sections, possibly inelastic cross sections in the range of, perhaps about $1 \mathrm{MeV}$.

MR. HAFELE (Karlsruhe):

There is one point left, a point of deep concern, namely the possibility of large-scale fuel element testing with fast neutrons. I understand that several groups are preparing fuel element tests with fast neutrons trying to irradiate one, two, or three pins. I think that if that works, and it is still difficult, it is not enough. We have to have large scale fuel element testing because fission gas release and the fission gas pickup in a plenum on the one hand, and the cladding material problem on the other hand, is very basic. I would like to renew a proposal which Dr. Spinrad made two years ago in Vienna, when he said that perhaps in some cooperative program there could be a fast-neutron material test reactor for large scale fuel elements. 


\author{
SESSION VII \\ October 10,1963 \\ THEORY OF DOPPLER EFFECT \\ Chairman: P. GREEBLER \\ General Electric Company, APED \\ Secretary: W. Y. Kato \\ Argonne National Laboratory
}




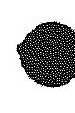




\author{
D. B. Adler and F. T. Adler \\ Department of Physics \\ University of Illinois \\ Urbana, Illinois
}

(Paper presented by Mrs. D. B. Aaler)

Introduction

The high-resolution measurements of the slow neutron fission and total cross sections $(1,2)$ during the late $1950^{\prime}$ s has led to considerable interest in the Wigner-Eisenbud multilevel-multichannel formalism. $(3,4)$ Along this line Moore and Reich $(5-7)$ have applied a specialization of the Wigner-Eisenbud formalism to the analysis of the resonance structure of $\mathrm{U}^{233}$. Although this treatment assumes that only one neutron channel and one or two fission channels are open, it includes a large number of channels for the capture process. Although the approach could in principle be generalized, it is of practical use for the fitting of cross sections only if the number of assumed fission channels is small. Reich and Moore have also noted that the radiative capture cross section in the multilevel case is not the sum of single-level Breit-Wigner terms.

Shore and Sailor(1) have analyzed the low-lying resonances in $\mathrm{U}^{235}$ (at 0 to $2 \mathrm{eV}$ ) and have found that although the capture component of the resonances is symmetrical, the fission component of the same reaction is asymmetrical. This indicates clearly the need for a multilevel description of the fission cross section.

The general Wigner-Eisenbud resonance theory was applied to the study of neutron resonances in fissile elements for the first time by Vogt. $(8,9)$ In his approach, Vogt neglected all but a small group of resonances. His expressions are, however, somewhat complicated for the purpose of analyzing neutron cross-section data. Moreover, the practical problem of calculating Doppler-broadened cross sections based on this formalism remained open and would have presented great computational complexities.

A simple and direct approach to the Wigner-Eisenbud theory has been obtained, leading to results which are useful for the analysis of nuclear cross-section data, as well as for the practical purpose of reactor calculations, in particular for the calculation of Doppler-broadened cross sections in the low-energy region, and for the calculation of temperature coefficients. 
One interesting consequence is that the temperature coefficient of $\mathrm{U}^{235}$ for the eV region shows a marked change if the single-level fit is replaced by the preliminary results obtained from the multilevel-multichannel fitting procedure.

The aim of this report is to outline the analytical method used to obtain the formulae for the cross sections of fissile elements. In particular, it will be shown how the results for the multilevel-multichannel case can be specialized to the Eeshbach-Porter-Weisskopf (10) expressions, and finally, that further specializations reduce the cross sections to the case of superposed Breit-Wigner single-level terms. The results presented in this paper are final expressions for low-energy neutron cross sections of heavy elements, obtained from the Wigner-Eisenbud theory without special assumptions in the form of superposition of interfering resonance terms (of the Breit-Wigner type). The coefficients of the resonance terms are either constants or slowly varying functions of the energy.

1. Collision Matrix and Level Matrix

We recall here the definition of the collision matrix:

$$
U_{c c^{\prime}}=e^{i\left(\phi_{c}+\phi_{c^{\prime}}\right)}\left\{\delta_{c c^{\prime}}+i \sum_{\lambda \lambda^{\prime}} A_{\lambda \lambda^{\prime}} \sqrt{\Gamma_{\lambda c} \Gamma_{\lambda^{\prime} c^{\prime}}}\right\}
$$

as given by Vogt, (8) for example, where $\phi_{c}$ and $\phi_{c^{\prime}}$ are the phase-shifts for channels $c$ and $c^{\prime}, \Gamma_{\lambda_{c}}$ is the partial width for decay of the $\lambda$-th 1 evel into the c-channel, and $A_{\lambda \lambda}$, is the Wigner level matrix. $(3,4)$

For s-wave neutrons and for hard-sphere elastic scattering, the diagonal elements of the collision matrix are given by

$$
U_{n n}=e^{-2 i k a}\left\{1 \div i \sum_{\lambda \lambda^{\prime}} A_{\lambda \lambda^{\prime}} \sqrt{\Gamma_{\lambda n^{n}} \Gamma_{\lambda ! n}}\right\}
$$

where $k$ is the neutron momentum in $\mathrm{cm}^{-1}$, a is the nuclear radius, and $\Gamma_{\lambda_{n}}$ is the partial neutron width for decaying of the $\lambda_{\text {-th }}$ level into the $n$ channel. The generic off-diagonal element of $U$ is given by

$$
U_{n c}=e^{-i k a+i \phi_{c}}\left\{i \sum_{\lambda \lambda^{\prime}} A_{\lambda \lambda_{1}} \sqrt{\Gamma_{\lambda n^{\prime}} \lambda_{\lambda^{\prime}}}\right\} \text {. }
$$

We will consider two separate cases for the Wigner level matrix:

(a) A is diagonal (many-level model);

(b) A is not diagonal (multilevel-multichannel model). 
Case a

In this case, the generic element of $A^{-1}$ is

$$
A-\frac{1}{\lambda} \lambda^{\prime}=\delta_{\lambda \lambda},\left(E \lambda^{-E}-i \Gamma_{\lambda}\right)
$$

where $E_{\lambda}$ is the $\lambda$-th level and $\Gamma_{\lambda}$ is the corresponding half-width. In particular,

$$
2 \Gamma_{\lambda}=\Gamma_{\lambda n}+\Gamma_{\lambda \gamma}+\Gamma_{\lambda f}
$$

where for resonance absorbers $\Gamma_{\lambda f} \equiv 0$. One has immediately:

$$
A_{\lambda \lambda^{\prime}}=\delta_{\lambda \lambda^{\prime}}\left(E_{\lambda}-E-i \Gamma_{\lambda}\right)^{-1}
$$

For brevity in our calculations, we will use the notation

$$
W_{\lambda}=E_{\lambda}-E-i \Gamma_{\lambda} ; W_{\lambda}^{*}=E_{\lambda}-E+i \Gamma_{\lambda}
$$

Case b

In this case

$$
A_{\lambda \lambda^{\prime}}^{-1}=\left(E_{\lambda}-E\right) \delta_{\lambda \lambda^{\prime}}-i \Gamma_{\lambda \lambda^{\prime}}
$$

with

$$
2 \Gamma_{\lambda \lambda^{\prime}}=\sum_{c} \sqrt{\Gamma_{\lambda c} \Gamma_{\lambda^{\prime} c}}
$$

and

$$
2 \Gamma_{\lambda \lambda}=\sum_{c} \Gamma_{\lambda c} \equiv \Gamma_{\lambda}
$$

With the same meaning as for case (a).

In this case, we have shown(11) that one can diagonalize and invert $\mathrm{A}^{-1}$, obtaining for the matrix $\mathrm{A}$ a linear superposition of "single level" Breit-Wigner-type denominators.

The transformation $S$ which diagonalizes $\mathrm{A}^{-1}$ is an orthogonal complex transformation, satisfying the equation

$$
A^{-1} S=S D^{-1} \text {, }
$$


ADIER and ADLER

where

$$
D_{k n}^{-1}=\left(c_{k}-E\right) \delta_{k n}
$$

is the diagonal form of $A^{-1}$ and where the $c_{k}$ are the complex eigenvalues of $\mathrm{A}^{-1}$. From $(8)$ one has

$$
\mathrm{A}=\mathrm{SDS}^{-1}
$$

or, explicitly,

$$
A_{\lambda \lambda^{\prime}}=\sum_{k} \frac{S_{\lambda k^{\prime}} S_{\lambda^{\prime} k}}{c_{k}-E} .
$$

This procedure is general and does not require any simplifying assumption regarding the detailed structure of the matrix $\mathrm{A}^{-1}$. In particular, the transformation $S$ involves only measured resonance parameters, such as resonance levels and off-diagonal elements of $\mathrm{A}^{-1}$. Therefore $\mathrm{S}$ is a slowly varying function of the energy, in the sense that it is a function of resonance parameters which are either constant or slowly varying functions of the energy.

The result expressed by (11) can be taken as a generalized form of equation (4'). In contrast with (4'), equation (11) contains the superposition of all the Breit-Wigner denominators determined by (9). It appears natural to attribute to the eigenvalue $c_{k}$ the meaning of $k$-th resonance level, in particular to define

$$
\begin{aligned}
& \mu_{k}=\operatorname{Re} c_{k} \text { as a shifted energy level; } \\
& -\nu_{k}=\operatorname{Im} c_{k} \text { as a modified half-width. }
\end{aligned}
$$

Inserting $A$ into the generic element of the collision matrix, one obtains for either case:

$$
\begin{aligned}
& U_{c c^{\prime}}=e^{i\left(\phi_{c}+\phi_{c^{\prime}}\right)}\left\{\delta_{c c^{\prime}}+i \sum_{\lambda} \frac{\sqrt{\Gamma_{\lambda c} \Gamma_{\lambda c^{\prime}}}}{E_{\lambda}-E-i \Gamma_{\lambda}}\right\} \\
& U_{c c^{\prime}}=e^{i\left(\phi_{c}+\phi_{c^{\prime}}\right)}\left\{\delta_{c c^{\prime}}+i \sum_{J} \frac{\left(\tilde{\Gamma}_{J c} \tilde{\Gamma}_{J c^{\prime}}\right)^{1 / 2}}{c_{J}-E}\right\},
\end{aligned}
$$

where the symbolic square root $\left(\tilde{\Gamma}_{\mathrm{JC}}\right)^{1 / 2}$ is defined as

$$
\left(\tilde{\Gamma}_{J c}\right)^{1 / 2}=\sum_{\lambda} S_{\lambda J} \sqrt{\Gamma_{\lambda c}} .
$$


This procedure leads to the desired expression for the neutron cross sections, in the form of superpositions of interacting single-level cross sections, with coefficients which are either constant or slowly varying functions of the energy. The parameters defined in eq. (14) are complex parameters which can be taken as constant over each resonance line. In particular,

$$
\tilde{\Gamma}_{\mathrm{Jn}}=\sum_{\lambda \lambda^{\prime}} s_{\lambda J} s_{\lambda{ }^{\prime} J} \sqrt{\Gamma_{\lambda n} \Gamma_{\lambda ! n}}
$$

will be used in the form

$$
\tilde{\Gamma}_{\mathrm{J} n}=\alpha_{\mathrm{J}}+i \beta_{\mathrm{J}}
$$

With the intent of unifying the expressions for the cross sections, both for cases (a) and (b), the general derivation will be carried on for the case (b), with the understanding that for case (a) $s_{\lambda J}=\delta_{\lambda J}$; hence, $\beta_{J}=0$ and $c_{J}=E_{J}-i \Gamma_{J J}$.

\section{Total Cross Section}

In terms of $U_{n n}$, we define

$$
\sigma_{n T}(E)=\frac{\pi}{k^{2}} \sum_{J} g^{J} \operatorname{Re}\left(1-U_{n n}^{J}\right)
$$

where $\mathrm{g}^{\mathrm{J}}$ is the statistical factor for the total angular momentum $J$. For simplicity we will use only one value of $g^{J}$, and hence an average statistical factor $g$ (averaged over two values of $J$, as it is the case for $s-w a v e s$ ). Hence, we analyze

$$
\sigma_{n T}(E)=\frac{\pi}{k_{2}} g \operatorname{Re}\left(1-U_{n n}\right) .
$$

By denoting

$$
i \sum_{J} \frac{\tilde{\Gamma}_{J_{n}}}{W_{J}}=\psi+i \phi,
$$

where $W_{J}$ is defined by eq. (5) and the index $J$ is here and in the following the level index, one obtains from eq. (13)

$$
\operatorname{Re}\left(1-U_{n n}\right)=(1+\psi) \cos 2 k a+\phi \sin 2 k a,
$$

where $\phi_{n}=-k a$ is the conventional s-wave hard-sphere scattering phase shift. From (18), one obtains explicitly for $\psi$ and $\phi$ the "resonance" expressions 
ADLER and ADLER

$$
\begin{aligned}
& \psi=\sum_{J} \frac{\alpha_{J} \nu_{J}+\beta_{J}\left(\mu_{J}-E\right)}{\left|W_{J}\right|^{2}} \\
& \phi=\sum_{J} \frac{\alpha_{J}\left(\mu_{J}-E\right)-\beta_{J} \nu_{J}}{\left|W_{J}\right|^{2}},
\end{aligned}
$$

which, combined with (19) and (17), lead to the total cross section in the general "Breit-Wigner"-type form:

$$
\sigma_{n T}(E)=c\left\{2 g \frac{(1-\cos 2 k a)}{E}+\frac{1}{\sqrt{E}} \sum_{J} \frac{\nu_{J} G_{J}(T)}{\mid} \frac{\left(\mu_{J}-E\right) H_{J}{ }^{(T)}}{\left|W_{J}\right|^{2}}\right\},
$$

where $c=6.52 \times 10^{5} \mathrm{~b} \times \mathrm{eV}$. Here, the first term represents the conventional potential scattering while the summation (over all resonance levels $\mathrm{J}$ ) represents a superposition of interfering single levels. The parameters $G(T)$ and $H(T)$ are given by

$$
\begin{aligned}
& G_{J}^{(T)}=\alpha_{J}^{\circ} \cos 2 \mathrm{ka}+\beta_{J}^{\circ} \sin 2 \mathrm{ka} ; \\
& \mathrm{H}_{J}^{(T)}=\beta_{J}^{\circ} \cos 2 \mathrm{ka}-\alpha_{\mathrm{J}}^{\circ} \sin 2 \mathrm{ka},
\end{aligned}
$$

where

$$
\alpha_{J}^{\circ}=\frac{2 g}{\sqrt{E}} \alpha_{J} ; \beta_{J}^{\circ}=\frac{2 g}{\sqrt{E}} \beta_{J}
$$

It is interesting to realize that the general structure of (21) does not differ from the customary structure of a superposition of single-level cross sections. The difference between the multilevel-multichannel case (b) and the many-level case (a) arises from the $\beta_{J}^{\circ}$ terms in $G(T)$ and $H_{J}(T)$. For the case (a), in fact, these reduce to

$$
G_{J}^{(T)}=\Gamma_{J_{n}}^{0} \cos 2 \mathrm{ka} ; H_{J}^{(T)}=-\Gamma_{J_{n}}^{\circ} \sin 2 \mathrm{ka} \text {. }
$$

\section{Reaction Cross Sections}

The inelastic cross section for the reaction $n \rightarrow c$ is given by

$$
\sigma_{n c}(E)=\frac{\pi}{k^{2}} \cdot g\left|U_{n . c}\right|^{2}
$$

Using eq. (13), one can write:

$$
\left|U_{n c}\right|^{2}=\sum_{J J^{\prime}}\left(\tilde{\Gamma}_{J_{n}} \tilde{\Gamma}_{J^{\prime} n^{\prime}} \tilde{\Gamma}_{J c} \tilde{\Gamma}_{J^{\prime} c}\right)^{1 / 2} /\left(W_{J^{\prime}} W_{J^{\prime}}^{*}\right)
$$


and after some intermediate calculations obtain

$$
\frac{g}{\sqrt{E}}\left|U_{n c}\right|^{2}=\sum_{J} \frac{1}{\left|W_{J}\right|^{2}}\left(\nu_{J} G_{J}^{(c)}+\left(\mu_{J}-E\right) H_{J}^{(c)}\right),
$$

where

$$
\begin{aligned}
& G_{J}^{(c)}=\sum_{J^{\prime}} \frac{\left(\nu_{J^{\prime}}+\nu_{J^{\prime}}\right) \phi_{J^{\prime}}^{(c)}-\left(\mu_{J^{\prime}}-\mu_{J^{\prime}}\right) \sigma_{J^{\prime}}(c)}{\left(\mu_{J^{\prime}}-\mu_{J}\right)^{2}+\left(\nu_{J}+\nu_{J^{\prime}}\right)^{2}} ; \\
& H_{J}^{(c)}=\sum_{J^{\prime}} \frac{\left(\mu_{J^{\prime}}-\mu_{J^{\prime}}\right) \phi_{J^{\prime}}+\left(\nu_{J^{\prime}}+\nu_{J^{\prime}}\right) \sigma_{J^{\prime}}}{\left(\mu_{J^{\prime}}-\mu_{J^{\prime}}\right)^{2}+\left(\nu_{J^{\prime}}+\nu_{J^{\prime}}\right)^{2}}
\end{aligned}
$$

and

$$
\begin{aligned}
& \dot{\phi}_{J^{\prime}}^{(c)}=\frac{2 g}{\sqrt{E}} \operatorname{Re}\left(\tilde{\Gamma}_{J n} \widetilde{\Gamma}_{J^{\prime}{ }^{\prime}} \tilde{\Gamma}_{J c} \tilde{\Gamma}_{J^{\prime}}\right) \\
& \sigma_{J^{\prime} J^{\prime}}^{(c)}=\frac{2 g}{\sqrt{E}} \operatorname{Im}\left(\tilde{\Gamma}_{J_{n}} \tilde{\Gamma}_{J^{\prime} n} \tilde{\Gamma}_{J c} \tilde{\Gamma}_{J^{\prime} c}\right) .
\end{aligned}
$$

From (26) one has

$$
\sigma_{n c}(E)=\frac{c}{\sqrt{E}} \sum_{J} \frac{G_{J}^{(c)} \nu_{J}+H_{J}^{(c)}\left(\mu_{J}-E\right)}{\left|W_{J}\right|^{2}}
$$

where $c=6.52 \times 10^{5} \mathrm{~b} \times \mathrm{eV}$. The reaction cross section given by (29) is a superposition of Breit-Wigner type with interferences. This is an entirely new result as compared with the well-known superposition of single BreitWigner levels which one obtains by adding single-level cross sections.

The presence of the interference term, $\mathrm{H}_{J}\left(\mu_{J}-E\right)$ introduces an asymmetry in the $J$-th resonance line, which corresponds to the noticeable asymmetries shown in the measurements. Thatinteractions of levels and channels, as described by the Wigner and Eisenbud method, would lead to the explanation of the experimental asymmetries had been foreseen by several authors $(5)$ and numerically demonstrated by Vogt. (8)

The present approach represents a further step, insofar as it gives the explicit analytical structure of the "resonance line" simply in terms of a sum of symmetrical and asymmetrical Breit-Wigner-type terms, as represented in (29). 
It is worthwhile to remark that the asymmetrical part which in the single-level picture is present only in the total and scattering cross sections as the interference term between potential and resonance scattering, here represents a generalized interference between levels and channels. For the case (a), such a term still appears as an interference between levels only. In fact, in this case one has

$$
\sigma_{J^{\prime}}^{(c)}=0 ; \phi(c)=\sqrt{\Gamma_{J^{\prime}}^{0} J^{\prime} \Gamma_{J^{\prime} n}^{0} \Gamma^{\prime} c^{\Gamma} J^{\prime} c^{\prime}}
$$

leading to

$$
G_{J}^{(c)}=\sum_{J^{\prime}} \frac{\left(\nu_{J}+\nu_{J^{\prime}}\right) \phi J_{J^{\prime}}^{(c)}}{\left(\mu_{J^{\prime}}-\mu_{J}\right)^{2}+\left(\nu_{J^{\prime}}-\nu_{J^{\prime}}\right)^{2}} H_{J}^{(c)}=\sum_{J^{\prime}} \frac{\left(\mu_{J^{\prime}}-\mu_{J}\right) \phi J_{J^{\prime}}}{\left.(c)-\mu_{J}\right)^{2}+\left(\nu_{J}+\nu_{J^{\prime}}\right)^{2}}
$$

We define this case as "overlapping many levels" in contrast with the "multilevel-multichannel" case. This case corresponds to the FeshbachPorter-Weisskopf formulation (i0) which in the past has been shown not to be quite adequate to represent the fission cross sections. It is only when one has reasons to neglect $\mathrm{H}_{\mathrm{J}} \mathrm{c}$ that (29) reduces to the familiar form of the reaction cross section obtained by superposition of single levels of symmetrical form. Since $\mathrm{H}_{\mathrm{J}}(\mathrm{c})=0$ means

$$
\sum_{J^{\prime} \neq J} \frac{\sqrt{\Gamma_{J^{\prime} \mathrm{n}} \Gamma_{J^{\prime} \mathrm{C}}}}{\mu_{J^{\prime}}-\mu_{J}} \approx 0
$$

this leads to

$$
G_{J}^{(c)}=\frac{\Gamma_{\mathrm{Jn}} \Gamma_{\mathrm{Jc}}}{2 \nu_{J}}
$$

i.e., the reaction cross section only contains the direct contribution from each single level. Particular assumptions lead to this case, which we define as the "superposed single levels" case; one is Bethe's assumption about the random signs of the $\sqrt{\Gamma_{\mathrm{Jn}}} ;(3)$ another, the case of well-spaced levels with narrow widths:

$$
\Gamma_{\lambda} \ll\left|E_{\lambda}-E_{\lambda}\right|\left(\lambda \neq \lambda^{\prime}\right)
$$

4. Derivation of the Scattering Cross Section

In terms of the collision matrix, the scattering cross section is defined by

$$
\sigma_{n n}(E)=\frac{\pi}{k^{2}} g\left|1-U_{n n}\right|^{2}
$$


where, using the notation of eqs. (18) and (19),

$$
\mathrm{U}_{n n}=(\cos 2 \mathrm{ka}-\mathrm{i} \sin 2 \mathrm{ka})(1+i+i) \text {. }
$$

From this one has immediately

$$
\left|1-U_{n n}\right|^{2}=2 \operatorname{Re}\left(1-U_{n n}\right)+2 \xi^{\prime}+\xi^{2}+t^{2} .
$$

Because the functions: and contain only the characteristic parameters describing the total cross section, eq. (36) leads to a definition of the scattering cross section purely in terms of total cross-section parameters. Although the derivation given below is in itself trivial, the significance of this result is worthy of comment.

From the point of view of lundamentals, this result is to be expected as a consequence of the dispersion relations (12) between real and imaginary part of the scattering amplitude (for $s$ waves). This means one can always obtain the Hilbert transform of $R e\left(1-U_{n n}\right)$ at each point $E$ and evaluate at each $E$,

$$
\left|1-U_{n n}\right|^{2}=\left[R e\left(1-U_{n n}\right)\right]^{2}-\left(\operatorname{Im} U_{n n}\right)^{2} .
$$

The present formulation, however, in defining explicitly the energy dependence of $U_{n n}[$ as in eq. (18)], establishes the explicit energy dependence of $\left|1-U_{n n}\right|^{2}$, in terms of the same characteristic parameters which define the function $R e\left(1-U_{n n}\right)$. Practically, one has the advantage of having available at method which gives the explicit energy dependence of the scattering cross section purely by information obtained from measurements of the total cross section. The consequences of this result can be of experimental and theoretical interest. Among the latter is the possibility of gauging numerically specific assumptions as to the role of the neutron width in the multichannelmultilevel formalism, by comparison of these results with scattering cross section obtained by difference between total and absorption.

We here report the results obtained by expanding (36) in terms of resonance levels [using eq. (20)]:

$$
\begin{aligned}
& \sigma_{n}(E)=c\left\{\frac{2 g(1-\cos 2 k a)}{E}\right. \\
& +\frac{1}{\sqrt{E}} \sum_{J} \frac{1}{\left|W_{J}\right|^{2}}\left[\nu_{J}\left(G_{J}^{(T)}-\omega_{J}^{o}\right)+\left(\mu_{J}-E\right)\left(H_{J}^{(T)}-i_{J}^{o}\right)\right] \\
& \left.-\sum_{J} \frac{1}{\left|W_{J}\right|^{2}}\left[\nu_{J} G_{J}^{(n)}-\left(\mu_{J}-E\right) H_{J}^{(n)}\right]\right\} .
\end{aligned}
$$


Although the first two terms on the right-hand side of (37) contain the parameters given by (22) and (23) for the total cross section, and thereforevia $G(T)$ and $H(T)$, the interferences with the potential scattexing, the third term of (37) purely represents the effects of interfering levels and channels. One has

$$
\begin{aligned}
& G_{J}^{(n)}=\frac{1}{2 g} \sum_{J^{\prime}} \frac{\left(\nu_{J}+\nu_{J^{\prime}}\right) \phi_{J^{\prime}}^{0}+\left(\mu_{J^{\prime}}-\mu_{J}\right) \sigma_{J^{\prime}}^{0}}{\left(\mu_{J^{\prime}}-\mu_{J^{\prime}}\right)^{2}+\left(\nu_{J}+\nu_{J^{\prime}}\right)^{2}} ; \\
& \mathrm{HJ}^{(n)}=\frac{1}{2 g} \sum_{J^{\prime}} \frac{\left(\mu_{J^{\prime}}-\mu_{J}\right) \phi_{J^{\prime}}^{0}-\left(\nu_{J}+\nu_{J^{\prime}}\right) \sigma_{J^{\prime}}^{0}}{\left(\mu_{J^{\prime}}-\mu_{J^{\prime}}\right)^{2}+\left(\nu_{J}+\nu_{J^{\prime}}\right)^{2}},
\end{aligned}
$$

with

$$
\begin{aligned}
& \phi_{J^{\prime} J^{\prime}}^{\circ}=\alpha_{J^{\circ}}^{\circ} \alpha_{J^{\prime}}+\beta_{J^{\prime}}^{\circ} \beta_{J^{\prime}}^{\circ} \\
& \sigma_{J^{\prime}}^{\circ}=\alpha_{J^{\prime}}^{\circ} \beta_{J^{\prime}}^{\circ}-\alpha_{J^{\prime}}^{\circ} \beta_{J^{\prime}}^{\circ}
\end{aligned}
$$

where the structural analogy of (38) with (27) is obvious. As discussed for total and reaction cross sections, for the case (a), (38) reduces to

$$
\begin{aligned}
& G_{J}^{(n)}=\frac{1}{2 g} \sum_{J^{\prime}} \frac{\left(\nu_{J}+\nu_{J^{\prime}}\right) \Gamma J_{n^{\prime}}^{0} \Gamma_{J^{\prime} n}}{\left(\mu_{J^{\prime}}-\mu_{J}\right)^{2}+\left(\nu_{J}+\nu_{J^{\prime}}\right)^{2}} ; \\
& \mathrm{H}_{J}^{(n)}=\frac{1}{2 g} \sum_{J^{\prime}} \frac{\left(\mu_{J^{\prime}}-\mu_{J}\right) \Gamma_{J^{\prime}} \Gamma^{0} J^{\prime} n}{\left(\mu_{J^{\prime}}-\mu_{J}\right)^{2}+\left(\nu_{J^{\prime}}+\nu_{J^{\prime}}\right)^{2}},
\end{aligned}
$$

and finally for the case of noninteracting levels (superposed single levels),

$$
G_{J}^{(n)}=\frac{1}{4 g} \frac{\left(\Gamma_{J_{n}}^{0}\right)^{2}}{\Gamma_{J}} ; H_{J}^{(n)}=0
$$

Final Remarks

The basic symbols and characteristic parameters used in this paper are listed in Tables $I$ and II, where a distinction is made between the cases (a) (OML), (b) (ML-MC), and the special case of superposed single levels (SSL). The unified cross-section formulae are listed in Table III. Due to their simplicity, these formulae lend themselves to the particular task of fitting experimental data. For this purpose, leastsquares-fitting routines have been developed; the method used is basically the Gauss method of linearization. For each type of cross section, 
TableI

LIST OF SYMBOLS

\begin{tabular}{|c|c|c|c|c|}
\hline Definition & $M L-M C$ & OML & SSL & Dimension \\
\hline $\begin{array}{l}\text { Resonance } \\
\text { Parameters }\end{array}$ & $\begin{array}{l}\mu_{J}=\operatorname{Re}\left(\mathrm{c}_{\mathrm{J}}\right) ; \\
\nu_{\mathrm{J}}=-\operatorname{Im}(\mathrm{cJ}) ; \\
\left(\mathrm{c}_{\mathrm{J}}-\Sigma_{\lambda}\right) s_{\lambda \mathrm{i}}=-\mathrm{i} \sum_{\lambda^{\mathrm{i}}} I_{\lambda \lambda^{\prime}} \mathrm{s}_{\lambda^{\prime} \mathrm{i}}\end{array}$ & \multicolumn{2}{|l|}{$\begin{array}{l}\mu_{J}-E_{J} \\
\nu_{J}-\Gamma_{J} \\
\Gamma_{\lambda \lambda^{\prime}}=\delta_{\lambda \lambda}, \Gamma_{\lambda}\end{array}$} & $\mathrm{eV}$ \\
\hline $\begin{array}{l}\text { Resonance (BW) } \\
\text { Derominators }\end{array}$ & $W_{J}=\mu_{J}-i \nu_{J}-E$ & \multicolumn{2}{|c|}{$W_{J}=E_{J}-i i_{J}-E$} & eV \\
\hline Neuiron Width & $\widetilde{\Gamma}_{J n}=\sum_{\lambda \lambda^{\prime}} S_{\lambda J} S_{\lambda^{\prime} J} \sqrt{\Gamma_{\lambda n} I_{\lambda^{\prime} n}}$ & \multicolumn{2}{|l|}{$\tilde{\Gamma}_{J_{n}}=\Gamma_{I_{n}}$} & eV \\
\hline $\begin{array}{l}\text { Reduced } \\
\text { Neutron Width }\end{array}$ & $\tilde{I}_{J n}^{B}=\sum_{\lambda \lambda^{\prime}} S_{\lambda J} S_{\lambda \cdot J} \sqrt{\Gamma_{\lambda n^{Q} \Gamma_{\lambda^{\prime} n}}}$ & \multicolumn{2}{|c|}{$\tilde{\Gamma}^{0} J_{n}=\Gamma_{J_{n}}^{0}=\frac{2 g}{\sqrt{E}} I_{J n}$} & $(\mathrm{oV})^{12}$ \\
\hline $\begin{array}{l}\text { Neutron } \\
\text { Parameters } \\
\text { (total + scattering) }\end{array}$ & $\begin{array}{l}\alpha_{J}^{o}=\operatorname{Re}\left(\widetilde{\Gamma}_{J_{n}}^{\circ}\right) \\
\beta_{J}=\operatorname{Im}\left(\tilde{\Gamma}_{J_{n}}^{\circ}\right)\end{array}$ & \multicolumn{2}{|l|}{$\begin{array}{l}\alpha_{\mathrm{J}}^{\circ}=\Gamma \rho_{\mathrm{J}} \\
\rho_{\mathrm{J}}-0\end{array}$} & $(e V)^{1 / 2}$ \\
\hline $\begin{array}{l}\text { Noutron } \\
\text { Paramoters } \\
\text { (scattering) }\end{array}$ & 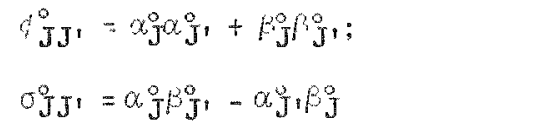 & 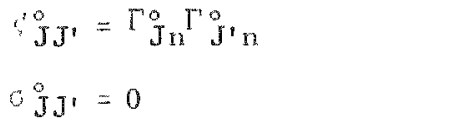 & $\begin{array}{l}\hat{J}_{J 1}^{0}=\delta_{J J}\left(\Gamma_{J n}^{0}\right)^{2} \\
\sigma_{J}^{0}=0\end{array}$ & $\mathrm{eV}$ \\
\hline $\begin{array}{l}\text { Reaction Width } \\
(J \rightarrow C)\end{array}$ & $\left(\tilde{\Gamma}_{J c}\right)^{12}=\sum_{\lambda} S_{\lambda J}\left(\Gamma_{\lambda c}\right)^{1 / 2}$ & \multicolumn{2}{|c|}{$\left(\tilde{I}_{\mathrm{JC}}\right)^{1^{2}}=\sqrt{\Gamma_{\mathrm{J}_{C}}}$} & $(\mathrm{eV})^{12}$ \\
\hline $\begin{array}{l}\text { Ruration }(c) \\
\text { Parameters }\end{array}$ & 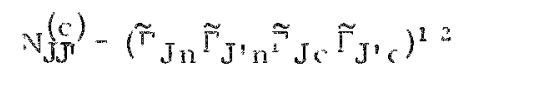 & $\sqrt{ }(c)-\sqrt{J^{1} J^{2} J n^{2} J C^{\Gamma} J^{\prime} c}$ & $\mathrm{NJJ}_{\mathrm{J}}^{(\mathrm{c})}=\mathrm{JJ} \Gamma_{\mathrm{Jn}} \mathrm{I}_{\mathrm{JC}}$ & $(\mathrm{eV})^{2}$ \\
\hline $\begin{array}{l}\text { Reduced } \\
\text { Redetion (c) } \\
\text { Petemeters }\end{array}$ & 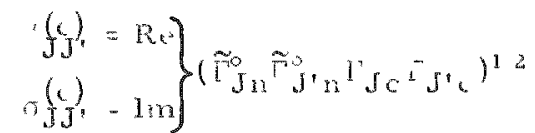 & 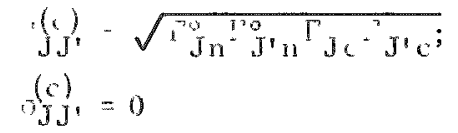 & $\begin{array}{l}(c)=J^{1} J^{1} J \\
J(c)=0\end{array}$ & $\left(0^{2} V\right)^{32}$ \\
\hline
\end{tabular}


Table LI

CHARACTERISTIC PARAMETERS $\left[\left.\left.\right|_{\mathrm{J} J}\right|^{2}=\left(\mu_{\mathrm{J}}-\mu_{\mathrm{J}}\right)^{2}+\left(\nu_{\mathrm{J}}+\nu_{\mathrm{J}}\right)^{2}\right]$

\begin{tabular}{|c|c|c|c|c|}
\hline Defintion & $M L-M C$ & OMI. & SSI & Dizurension \\
\hline$G_{J}^{(T)}$ & $\alpha_{J}^{\circ} \cos 2 k a+\beta_{J}^{\circ} \sin 2 k a$ & $\Gamma_{\mathrm{n}}^{\circ} \cos 2 \mathrm{ka}$ & $\Gamma \mathrm{J}_{n} \cos 2 \mathrm{ka}$ & $(a V)^{1 / 2}$ \\
\hline $\mathrm{HJ}^{(\mathrm{T})}$ & $\beta_{\mathfrak{J}}^{o} \cos 2 k a-\alpha_{\mathfrak{J}}^{O} \sin 2 k a t$ & $-\Gamma_{\mathrm{Jn}}^{\circ} \sin 2 \mathrm{ka}$ & $-\Gamma_{\mathrm{n}}^{\infty} \sin 2 \mathrm{ka}$ & $(\mathrm{eV})^{1 / 2}$ \\
\hline$G(n)$ & $\frac{1}{2 g} \sum_{J^{\prime}} \frac{\left(\nu_{I}+\nu_{J^{\prime}}\right) \phi_{J^{\prime}}^{\alpha}-\left(\mu_{J 1}-\mu_{J}\right) \sigma_{J J}}{\left|\rho_{J} I^{\prime}\right|^{2}}$ & 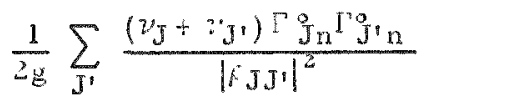 & $\frac{\left(\Gamma_{\mathrm{Jn}}\right)^{2}}{4 \mathrm{~g} \Gamma_{J}}$ & {$[0]$} \\
\hline$H_{J}^{(n)}$ & $\frac{1}{2 g} \sum_{J^{\prime}} \frac{\left(\mu_{J}-\mu_{J}\right) \phi_{J J}^{\circ}-\left(\nu_{J}+\nu_{J^{\prime}}\right) \sigma_{J J}^{\circ}}{\left|\rho_{J J}\right|^{2}}$ & $\frac{1}{2 g} \sum_{J^{\prime}} \frac{\left(\mu_{J}-\mu_{J}\right) \Gamma J^{\prime} \Gamma^{\prime} J^{\prime} n}{\left|J_{J}\right|^{2}}$ & 0 & [0] \\
\hline $\mathrm{G}_{J}^{(c)}$ & $\sum_{J \prime} \frac{\left(v_{J}+v_{J}\right) \rho_{J^{\prime}}(c)-\left(\mu_{J}-\mu_{J}\right) v_{J} J^{\prime}}{\mid \rho_{J} J^{\prime}}$ & 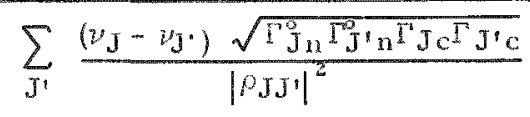 & $\frac{\Gamma_{\mathrm{J}}^{\circ} \Gamma_{\mathrm{Jc}}}{2 \Gamma_{\mathrm{J}}}$ & $(e V)^{12}$ \\
\hline$H_{f}(c)$ & 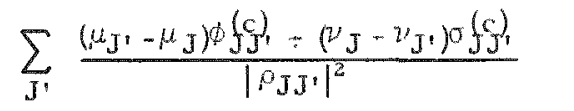 & 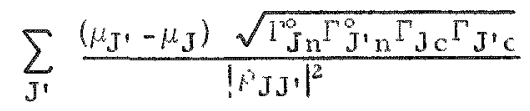 & 0 & $(\mathrm{eV})^{12}$ \\
\hline
\end{tabular}

Table III

GENERAL CROSS-SECTION WORMULAE

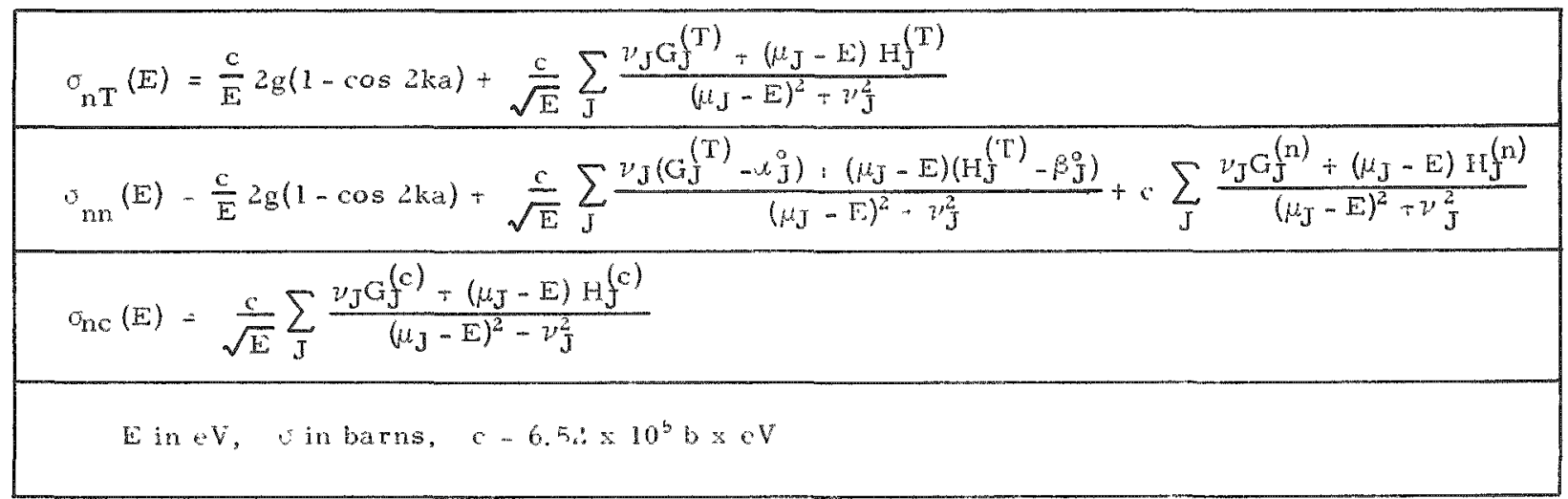


and for each level, one obtains a set of four parameters: $\mu_{J}, \nu_{J}, G_{J}$, and $\mathrm{H}_{\mathrm{J}}$, or $\mu_{\mathrm{J}}, \nu_{\mathrm{J}}, \alpha_{\mathrm{J}}$, and $\beta_{\mathrm{J}}$. (13) The definitions of these characteristic parameters and their errors are sufficient information for the practical purpose of evaluation of cross sections. Since in the least-squares-fitting program the temperature effects are taken into account, the parameters obtained can be used for evaluation of Doppler-broadened cross sections at any given temperature. One of the advantageous features of the formalism consists in the fact that one can apply directly the conventional Doppler-broadening techniques, thus obtaining the cross section as a superposition of lineshape functions. For example, for a reaction cross section one has

$$
\sigma_{n c}(E ; T)=\frac{c}{\sqrt{E}} \sum_{J}\left\{\frac{G_{J}^{(c)}}{\nu_{J}} \dot{\psi}\left(x_{J} ; T\right)+\frac{H_{J}(c)}{\nu_{J}} \chi(x J ; T)\right\} .
$$

where $\mathrm{xJ}=\left(\mathrm{E}-\mu_{\mathrm{J}}\right) / \nu_{J}$, and $\gamma$ and $\chi$ are the conventionally defined line-shape functions. (14)

For what concerns the application of the formalism to the direct evaluation of cross sections from known resonance parameters, a perturbation (15) method has been found, which is rapidly convergent, whenever

$$
\left|A_{\lambda \lambda^{-1}}^{1}\left(1-\delta_{\lambda \lambda^{\prime}}\right) / A_{\lambda \lambda}^{-1}\right| \cdots 1 \text {. }
$$

The same perturbation method can be applied to obtain from the fitting of experimental data an analysis of the level matrix.

\section{$\underline{\text { References }}$}

1. F. J. Shore and V. L. Sailor, Phys. Rev, 112, 191 (1958).

2. D. J. Hughes, Neutron Cross Sections, BNL-325.

3. A. M. Lane and R. G. Thomas, Revs. Mod. Phys. 30,257 (1958).

4. E. P. Wigner and L. Eisenbud, Phys. Rev. 72, 29 (1947).

5. C. W. Reich and M. S. Moore, Phys. Rev, 111, 929 (1c58).

6. M. S. Moore and C. W. Reich, Phys. Rev. 118, 718 (1960).

7. M. S. Moore, Nuclear Sci, and Engin. 13, 18 (1962).

8. E. Vogt, Phys. Rev. 112, 203 (1958).

9. E. Vogt, Phys. Rev. 118, 724 (1960). 
10. H. Feshbach, C. E. Porter, and V. F. Weisskopf, Phys. Rev. 96,448 (1954).

11. D. B. Adler and F. T. Adler, Am. Nucl. Soc. Transactions 5 (1), 53 (1962).

12. D. B. Adler and F. T. Adlex, ibid., 6 (1), 38 (1963).

13. F. T. Adler and D. B. Adler, ibid., 6 (1), 37 (1963).

14. F. T. Adler, J. Nuclear Eng. Parts A/B 14, 209 (1961).

15. D. B. Adlex and F. T. Adler, Am. Nucl. Soc. Transactions 5 (2), 407 $(1962)$

$$
\frac{\text { Discussion of Paper }}{\text { Presented by Mrs. Adler }}
$$

MR. GRAVES (United Nuclear Sorp.):

Could you give your thoughts on how much change you might expect in calculated Doppler coeflicionts for the reactors we are talking about if the multilevel instead of the single-level formula is used? I would have suspected it would be a small effect.

MR, ADLER:

Unfortunately we did not have time to show our curves for $\mathrm{U}^{233}, \mathrm{U}^{235}$, and $\mathrm{Pu}$ in the 0 to $15 \mathrm{eV}$ range. In this range we have even observed changes in sign. This, of course, depends on a variety of things.

MR. CHERNICK (Brookhaven):

Dr. Adler, the parameters you used are weakly energy dependent. Would you explain a little more what dificulty this causes, if any, in the lower-energy resonances?

MRS. ADLER:

Since the matrix elements are defined in terms of the basic resonance parameters, they have the same energy dependence as the basic resonance parameters. These basic resonance parameters contain the momentum of the incoming neutron because they are related to the boundary conditions satisfied by the wave functions which basically define the resonance levels. This dependence can be evaluated if one wants to start from a given model. Now, as long as one takes a short interval of energy, one does not commit a great $\sin$ if one evaluates these elements at each single 
level. It is only when from a given range of energy one continues the effect of a previous group of resonances to a higher energy that one has to think of an analytical calculation, a sort of data expansion of these parameters into a higher energy, because obviously something like a square root of the momentum changes considerably. However, these variations are certainly so small around each level that we can neglect them. And a feature which is common to these low-energy cases with high-energy problems is that the separation of the sharp resonance functions of the problem from the slowly varying functions of the energy of the problem, is always desirable in the sense that it permits to gauge the role of the resonance terms versus very smooth varying functions of the energy.

MR. LEVINE (Brookhaven):

When you calculate the interference effects of neighboring resonances on this one, I presume you pick out how many resonances you have to consider?

MRS. ADLER:

Yes.

MR. LEVINE:

Do you have to consider more or fewer of them when you go to higher energies or does that make much difference?

MRS. ADLER:

I honestly cannot answer. I think this is very much a choice based on common sense, and how to handle the data. Even in the fitting of lowenergy data, one has to use a certain degree of common sense in picking the data. It is very dangerous, for instance, to put the extreme of the energy interval ending near a small bump rather than the valley of a sharply defined resonance. This is because what I call small bumps (namely, peaks) are wide and sometimes spread along in the main growth of the main peak. They might occasionally not really represent a resonance, but there might be just a constructive effect of interfering functions. In fact, if we take two levels containing the interfering part and evaluate the position of all possible maxima, there are situations in which the distance of the level and the combination of the parameters are such that you can find in the middle a little maximum, a little bump. 


\title{
SOME CALCULATIONS CONCERNING THE INFLUENCE OF RESONANCE OVER LAPPING ON THE DOPPLER
}

\section{EFFECT IN A DILUTE FAST REACTOR}

J. Codd and P. J. Collins

Atomic Energy Establishment

Winfrith, England

(Paper presented by Mr. Codd)

\begin{abstract}
Approximate calculations are made of the Doppler change in neutron absorption at $1 \mathrm{keV}$ when the overlapping of plutonium-239 resonances, and of plutonium-239 and uranium-238 resonances, are separately taken into account. The validity of the assumption of constant collision density near a $U^{238}$ resonance is also examined. The calculations refer to an infinite medium with composition representative of a large plutonium-uranium oxide-fueled reactor.
\end{abstract}

\section{Introduction}

This paper describes some calculations concerning three particular topics in the theory of the Doppler coefficient of dilute fast reactors. The calculations are made for an infinite homogeneous medium with composition typical of a mixed plutonium-uranium oxide-fueled reactor. In such a system most of the Doppler coefficient is likely to come from the lowenergy part of the neutron spectrum, and for the purpose of the calculations $1 \mathrm{keV}$ has been selected as a representative energy. The problems investigated are as follows:

\section{(1) Overlapping of $\mathrm{Pu}^{239}$ Resonances}

In the energy region concerned, the plutonium resonances are expected to be weakly overlapping. A convenient method of calculating the plutonium Doppler coefficient is thus to treat the resonances as discrete and then apply a correction for overlapping. A preliminary investigation of overlapping is described in Section 2. The Doppler effect in a Pu ${ }^{239}$ resonance is calculated as a function of energy separation from a neighbouring resonance.

(2) Overlapping of $\mathrm{Pu}^{239}$ and $\mathrm{U}^{238}$ Resonances

Preliminary calculations for this problem(1) have been repeated with the assumption of a higher value of the $\mathrm{Pu}^{239}$ strength function 
(see Section 4 ). The effect of fluctuations in the neutron and fission widths, assuming Porter-Thomas distributions, is shown to change the results considerably (see Section 5). Some remarks on the possible effect of the overlapping on the Doppler coefficient are included in Section 6.

(3) The Behaviour of the Collision Density near a U ${ }^{238}$ Resonance

In the customary method of calculating effective cross sections, the collision density (flux times total cross section) is assumed constant through a group of resonances. The actual energy and temperature dependence of the collision density near a $U^{238}$ resonance, which may affect the absorption in neighbouring $\mathrm{Pu}^{239}$ resonances, is investigated in Section 7.

\section{Reactor Composition and Resonance Data}

An infinite homogeneous mixture of material representative of the core of a dilute oxide-fueled fast reactor was chosen for the calculations. The atomic proportions relative to $\mathrm{Pu}^{239}$ are as follows:

$\begin{array}{lr}\text { Pu } & 1 \\ U^{239} & 7 \\ \text { Iron } & 32 \\ \text { Sodium } & 8 \\ \text { Oxygen } & 16\end{array}$

The background nonresonant scattering cross section was taken to be $75 \mathrm{~b}$ per $\mathrm{U}^{238}$ atom, and $525 \mathrm{~b}$ per $\mathrm{Pu}^{239}$ atom.

The following mean $S$-wave resonance parameters have been assumed in the calculations:

$$
\begin{aligned}
& \frac{\mathrm{U}^{238}}{\left\langle\Gamma_{\gamma}\right\rangle}=0.025 \mathrm{eV} ;\left\langle\Gamma_{n}{ }^{0}\right\rangle=0.002 \mathrm{eV} ;\langle\mathrm{D}\rangle=18.3 \mathrm{eV} . \\
& \frac{\mathrm{Pu}^{23 \%}}{\left\langle\Gamma_{\gamma}\right\rangle}=0.038 \mathrm{eV} ; \\
& \left\langle\Gamma_{\mathrm{f}}\right\rangle=0.076 \mathrm{eV} ; \\
& \left\langle\Gamma_{\mathrm{n}}{ }^{\circ}\right\rangle=0.00072 \mathrm{eV} \text { for } \mathrm{J}=0 \\
& \left\langle\Gamma_{\mathrm{n}}^{0}\right\rangle=0.00024 \mathrm{eV} \text { for } \mathrm{J}=1 \\
& \langle\mathrm{D}\rangle=7.2 \mathrm{eV} \text { for } \mathrm{J}=0 ; \\
& \langle\mathrm{D}\rangle=2.4 \mathrm{eV} \text { for } \mathrm{J}=1 .
\end{aligned}
$$


The $\left\langle\Gamma_{n}{ }^{0}\right\rangle$ values correspond to a strength function of $1.0 \times 10^{-4}$, as compared with the value (1) $0.87 \times 10^{-4}$.

In the calculations of Section 5 the neutron and fission width are assumed to have a chi-squared distribution with 1 and 3 degrees of freedom, respectively.

There is considerable uncertainty in the mean parameters for plutonium, and other choices would have been possible.

\section{Overlapping of $\mathrm{Pu}^{239}$ Resonances at $1 \mathrm{keV}$}

Overlapping of resonances can be treated by the 'method A' approximations (2) which are usually made at high energies. For $\mathrm{Pu}^{239}$ in the energy region of $1 \mathrm{keV}$ the method A treatment is of doubtful validity, and this section gives the results of some numerical calculations.

For the present purposes the method A approximations for the Doppler-broadened resonance shape $\Psi(\tilde{s}, \mathrm{x})$ are

$$
\begin{aligned}
& \text { (i) } \Psi(\dot{\xi}, x) / \hat{\beta} \cdots 1 ; \\
& \text { (ii) } \Psi(\dot{\xi}, x) \simeq \frac{\sqrt{\pi}}{2} \xi e^{-\xi^{2} x^{2} / 4},
\end{aligned}
$$

where $\xi=\Gamma / \Delta$, with $\Gamma$ the natural width, $\Delta$ the Doppler width, and $\beta=\sigma_{\mathrm{b}} / \sigma_{0}$.

These assumptions give an approximation to the usual $J$ function, which takes into account the temperature dependence of the resonance absorption:

$$
J(\xi, \beta)=\int_{0}^{\infty} \frac{\Psi(\xi, x)}{\beta+\Psi(\xi, x)} d x \div \frac{\pi}{2 \beta}\left[1-\frac{\xi}{2 \beta} \sqrt{(\pi / 2)}\right] .
$$

Table 1 gives computed values of $J$ for a $\mathrm{Pu}^{239}$ resonance of the $\mathrm{J}=1$ sequence with mean parameters. The approximation (3.2) is accurate to $0.5 \%$ in $\mathrm{J}$, but gives an error of more than $30 \%$ in $\Delta \mathrm{J}$.

The absorption in two $\mathrm{Pu}^{239}$ resonances has been calculated numerically as a function of their relative separation by the method described in Ref. 1. The Doppler change in absorption, $\Delta A_{9}$, normalized to unity for large separations, is plotted in Fig. 1 for a temperature change of 300 to $600^{\circ} \mathrm{K}$. Mean parameters for spin $J=1$ have been taken for both resonances. 
Table 1

\begin{tabular}{l|c|c}
\multicolumn{2}{c}{$\mathrm{J}(\xi, \beta)$ FOR Pu AT 1 keV } \\
\hline & $J(\xi, \beta)$ & $\begin{array}{c}\text { Method A } \\
\text { Approximation }\end{array}$ \\
\hline $300^{\circ} \mathrm{K}$ & 0.35604 & 0.35453 \\
$600^{\circ} \mathrm{K}$ & 0.35817 & 0.35739 \\
$\Delta \mathrm{J}$ & 0.00213 & 0.00286 \\
\hline
\end{tabular}

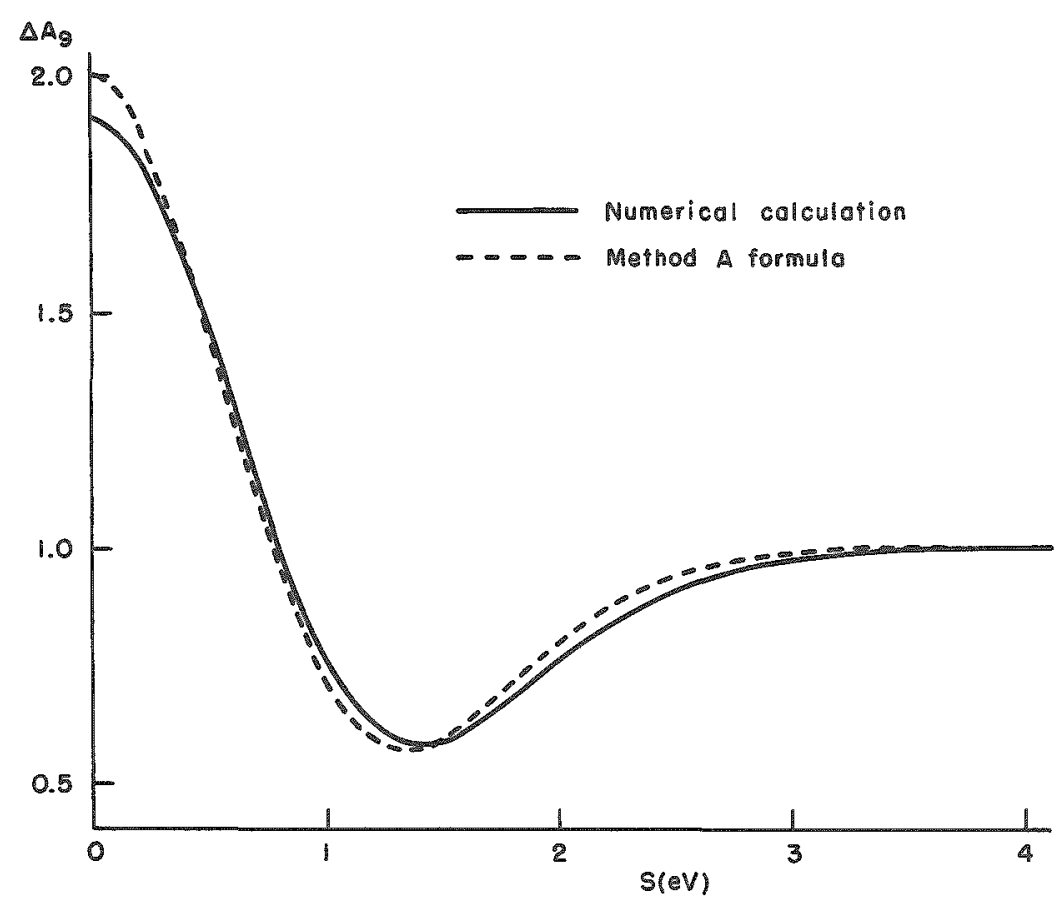

Fig. 1. Dependence of the Doppler Change in Absorption $\left(\triangle \mathrm{A}_{9}\right)$ in a $\mathrm{Pu}^{239}$ Resonance on the Separation(s) from a Neighbouring $\mathrm{Pu}^{239}$ Resonance for a Temperature Change of $300^{\circ} \mathrm{K}$ to $600^{\circ} \mathrm{K}$. Energy $1 \mathrm{keV}$.

For the given case the overlapping is seen to have an appreciable effect and produces a decrease of about $10 \%$ in the Doppler change in absorption at the assumed mean spacing (for $J=1$ ) of $2.4 \mathrm{eV}$.

The approximations (3.1) lead to the following formula for the overlapping effect:

$$
\frac{\Delta \mathrm{A}_{9}(S)}{\Delta \mathrm{A}_{9}(\infty)}=1+\frac{\Delta_{2} e^{-\mathrm{y}_{1}}-\Delta_{1} e^{-\mathrm{y}_{2}}}{\Delta_{2}-\Delta_{1}}
$$


with $y=S^{2} /\left(2 \Delta^{2}\right)$ and $S$ the separation. The subscripts 1 and 2 refer to temperatures $T_{1}$ and $T_{2}$. This is also plotted in Fig. 1 for comparison.

The results suggest that the method $A$ approximations give a fairly accurate estimate of the variation of the Doppler effect with separation, although the magnitude may be appreciably in error.

4. Overlapping of $\mathrm{Pu}^{239}$ and $\mathrm{U}^{238}$ Resonances under the Assumption of Mean Resonance Widths

The absorption $\mathrm{Ag}_{9}$ in a single $\mathrm{Pu}^{239} \mathrm{~s}$-wave resonance with spin $J=1$ was calculated as a function of separation from a $U^{238}$ resonance at $1 \mathrm{keV}$, for two temperatures, 300 and $600^{\circ} \mathrm{K}$. The resonances were as sumed to have a Doppler-broadened single-level Breit-Wigner shape. The plutonium and uranium were assumed to be intimately mixed, so that both resonances broaden when the temperature rises.

As an approximation, the collision density was taken to be constant through the resonances so that the flux varies as the reciprocal of the total cross section of the medium. Apart from a normalization constant which depends on the absolute level of the flux, the absorption $A_{0}$ in the $\mathrm{Pu}^{239}$ resonance is given by the integral

$$
A_{g}=\int_{R E S} \Sigma_{a g}(E) / \Sigma_{t}(E) d E_{g}
$$

where $\Sigma_{a 9}$ is the macroscopic $P u^{239}$ absorption cross section, and $\Sigma^{2}$ is the macroscopic total cross section at energy $E$. $\Sigma_{t}$ includes contributions from $\mathrm{Pu}^{239}, \mathrm{U}^{238}$, and the scattering materials present. The integral was evaluated numerically (see Ref. 1 for further details). The Doppler. change in $A_{9}$ is given by

$$
\Delta A_{g}=A_{9}\left(600^{\circ} \mathrm{K}\right)-A_{9}\left(300^{\circ} \mathrm{K}\right)
$$

The results for $\mathrm{A}_{9}$ and $\Delta \mathrm{A}_{9}$ are plotted against the energy separation of the resonances in Figs. 2 and 3 , respectively. For convenience of presentation, $A_{9}$ and $\Delta A_{9}$ have both been normalized to unity at large separation, i.e., for an isolated $\mathrm{Pu}^{239}$ resonance.

Figure 2 shows that $A_{9}$ is appreciably reduced when the resonances are close together. This is a consequence of the local depression in the neutron flux spectrum caused by the $U^{238}$ resonance.

On the other hand, Fig. 3 shows that the Doppler change $\Delta \mathrm{Ag}_{g}$ is large and positive for very closely spaced resonances, but becomes large and negative for separations between about 1 and $3 \mathrm{eV}$. This behaviour 
may be explained by the perturbation in the local flux spectrum when the $\mathrm{U}^{238}$ resonance broadens. This causes a change in the absorption in the $\mathrm{Pu}^{239}$ resonance, in addition to that resulting from the broadening of the $\mathrm{Pu}{ }^{239}$ resonance itself.

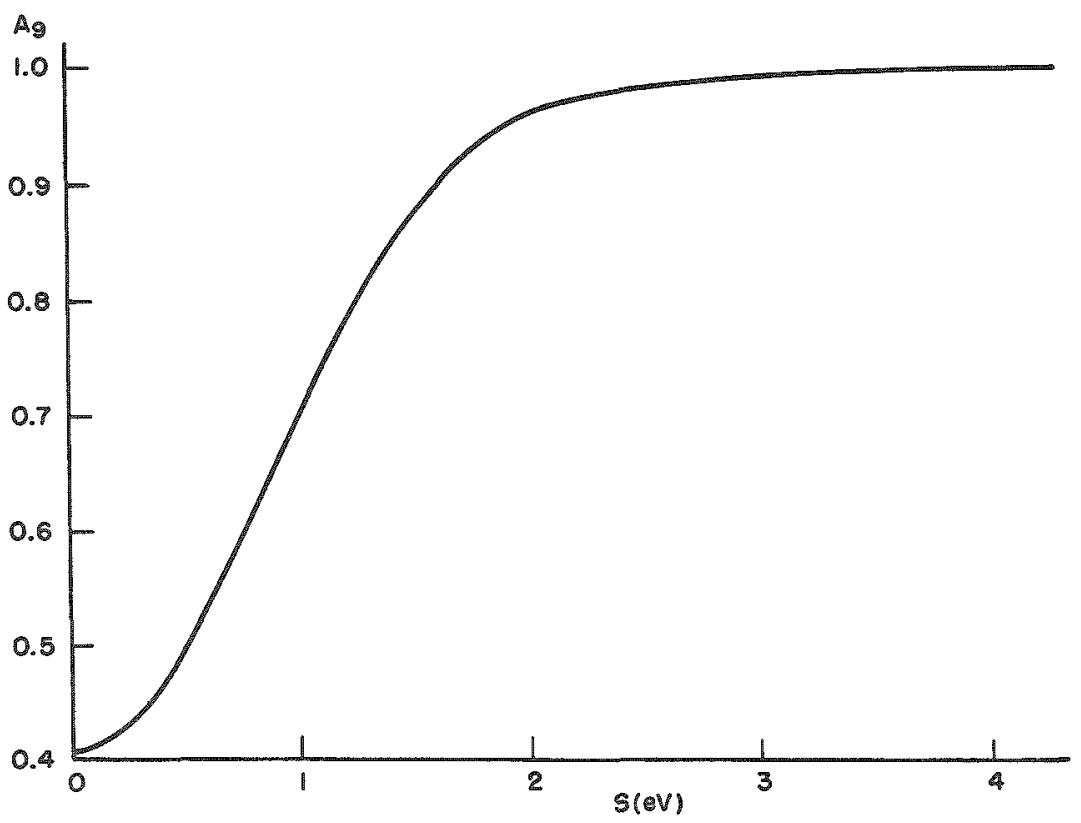

Fig. 2. Dependence of Absorption (Ag) in a $\mathrm{Pu}^{239}$ Resunance at $1 \mathrm{keV}$ on the Separation(s) from a Neighbourng $U^{238}$ Resonance for a Temperature of $300^{\circ} \mathrm{K}$

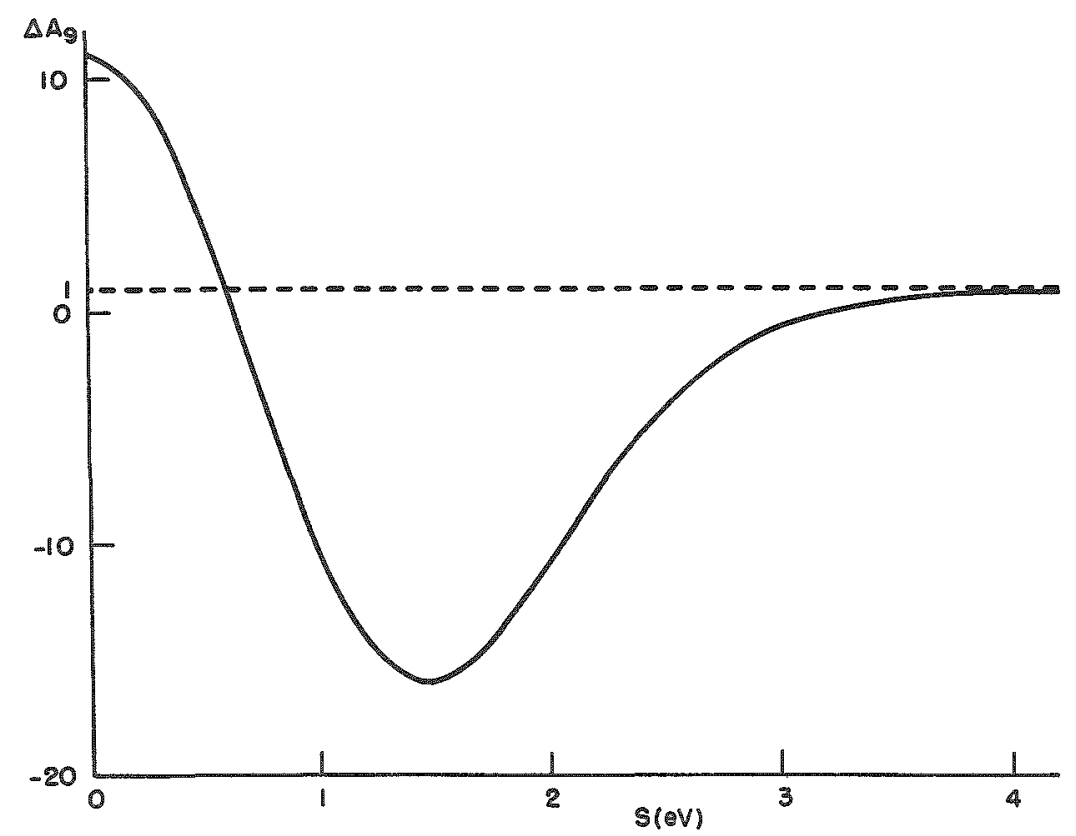

Fig. 3. Dependence of the Doppler Change in Absorption ( $\triangle \mathrm{Ag}$ ) in a $\mathrm{Pu}^{239}$ Resonance at $1 \mathrm{keV}$ on the Separation(s) from a Neighbouring $\mathrm{U}^{238}$ Resonance for a Temperature Change of $300^{\circ} \mathrm{K}$ to $600^{\circ} \mathrm{K}$ 
A mean value for $\Delta A_{9}$, denoted by $\overline{\Delta A}_{g}$, was estimated by assuming a constant level density of $\mathrm{Pu}^{239}$ resonances, and averaging the results of Fig. 3 over the range $S=-9.15$ to $S=+9.15 \mathrm{eV}$, i.e., over a range equal to the mean $\mathrm{U}^{238}$ level spacing. It is noted that in consequence of using the assumption of constant collision density the curve will be symmetrical about the $S=0$ axis. The result was normalized to unit flux just above the resonances, giving

$$
\overline{\Delta A}_{g}=-0.062 \text {. }
$$

The corresponding value for an isolated $\mathrm{Pu}^{239}$ resonance is

$$
\overline{\Delta A}_{9}=+0053 \text {. }
$$

These results suggest that the Doppler change in the average $\mathrm{Pu}^{239}$ absorption near $1 \mathrm{keV}$ is changed from positive to negative in consequence of the broadening of the $U^{238}$ resonances. This conclusion is amended, however, when allowance is made for the fluctuation of resonance widths (see Section 5)

The result $(43)$ was found to agree within about $1 \%$ with that obtained by an approximate analytical formula due to J. L. Rowlands (A.E.E. Winfrith, private communication). He obtained the following expressions for the mean $\mathrm{Pu}^{239}$ and $\mathrm{U}^{238}$ absorptions per atom per unit energy interval, allowing for overlapping of $\mathrm{Pu}^{239}$ and $\mathrm{U}^{238}$ resonances (but not of $\mathrm{Pu}^{239}$ resonances themselves):

$$
\begin{aligned}
& \bar{A}_{9}=\sigma_{b_{9}}{ }^{\top}{ }_{a 9} J_{9}\left(1-\Gamma_{8} J_{8} / D_{8}\right) / D_{9:} \\
& \bar{A}_{8}=\sigma_{b 8} \Gamma_{98} J_{8}\left(1-\Gamma_{9} J_{9} / D_{9}\right) / D_{8} .
\end{aligned}
$$

The suffices 8 and 9 refer to $U^{238}$ and $P u^{239}$. The quantity $\sigma_{b}$ denotes the nonresonant scattering cross section of the medium per absorber nucleus. Also, $J$ is the usual $J(\xi, \beta)$ function, and $\Gamma_{2}, \Gamma$, and $D$ denote the mean $a b-$ sorption and total widths, and the level spacing, respectively. The above expressions were obtained by assuming the following approximation to the macroscopic total cross section:

$$
\begin{aligned}
z_{t} & =\Sigma_{b}+z_{t 8}+z_{t 9} \\
& \div\left(z_{b}+\Sigma_{t 8}\right)\left(z_{b}+\Sigma_{t 9}\right) / \Sigma_{b}
\end{aligned}
$$

provided $\Sigma_{t 8} \Sigma_{t 9} / \Sigma_{\mathrm{b}} \Sigma_{\mathrm{t}} 、<1$. Here $\Sigma_{\mathrm{b}}, \Sigma_{\mathrm{t} 8}$, and $\Sigma_{\mathrm{tg}}$ denote, respectively, the macroscopic total nonresonant cross section of the medium, and the macroscopic total resonant cross sections of $\mathrm{U}^{238}$ and $\mathrm{Pu}^{239}$. 
5. The Overlapping of $\mathrm{Pu}^{239}$ and $\mathrm{U}^{238}$ Resonances with Allowance for the Fluctuation of Widths

The calculations described in the previous section suggested that the Doppler change in the $\mathrm{Pu}^{239}$ absorption at $1 \mathrm{keV}$, for the particular system investigated, is changed from positive to negative by the broadening of $U^{238}$ resonances. Mean resonance parameters were assumed. Since the result will depend on the relative sizes of the $\mathrm{Pu}^{239}$ and $\mathrm{U}^{238}$ resonances, an investigation was made of the effect of fluctuations in the neutron and fission widths, assuming appropriate Porter-Thomas distributions.

The calculations were based on generalized forms of (4.4) and (4.5):

$$
\begin{aligned}
& \overline{\mathrm{A}}_{9}=\sigma_{\mathrm{b}_{9}}\left\langle\Gamma_{\mathrm{a} 9} \mathrm{~J}_{9}\right\rangle\left(1-\left\langle\Gamma_{8} J_{8}\right\rangle / \mathrm{D}_{8}\right) / \mathrm{D}_{9} ; \\
& \overline{\mathrm{A}}_{8}=\sigma_{\mathrm{b}_{8}}\left\langle\Gamma_{\left.\mathrm{a}_{8} \mathrm{~J}_{8}\right\rangle\left(1-\left\langle\Gamma_{9} J_{9}\right\rangle / \mathrm{D}_{9}\right) / \mathrm{D}_{8},}\right.
\end{aligned}
$$

where $\langle f\rangle$ denotes the average of any quantity $f$ over the Porter-Thomas distributions. The averaging was carried out by the method of $R e f .4$ by use of two Winfrith IBM 7090 computer programmes, ERIC II and JANY. Contributions from both the $J=0$ and $J=1$ sequences of $P u^{239} \mathrm{~s}$-wave resonances were included.

The results for $\bar{A}_{9}$ and $\bar{A}_{8}$ are given in Tables 2 and 3 below. For comparison, results are also included based on mean neutron and fission widths. $\overline{\mathrm{A}}_{9}$ denotes the average $\mathrm{Pu}^{239}$ absorption per unit energy interval per $\mathrm{Pu}^{239}$ atom, normalized to unit flux just above the resonances, with a similar meaning for $\overline{\mathrm{A}}_{8} \cdot \overline{\Delta A}_{9}$ and $\overline{\Delta A}_{8}$ denote the Doppler changes in the absorption between 300 and $600^{\circ} \mathrm{K}$. The results refer to the system described in Section 2 and a neutron energy of $1 \mathrm{keV}$.

Table 2

\begin{tabular}{|c|c|c|c|c|}
\hline \multirow{2}{*}{$\begin{array}{l}\text { Widths } \\
\text { Used }\end{array}$} & \multicolumn{2}{|c|}{$\begin{array}{c}\text { With Resonance } \\
\text { Overlapping }\end{array}$} & \multicolumn{2}{|c|}{$\begin{array}{c}\text { Isolated } \mathrm{Pu}^{239} \\
\text { Resonances }\end{array}$} \\
\hline & Mean & Fluctuating & Mean & Fluctuating \\
\hline$\overline{\mathrm{A}}_{9}\left(300^{\circ} \mathrm{K}\right)$ & 10.656 & 9.103 & 11.507 & 9.668 \\
\hline$\overline{\mathrm{A}_{9}}\left(600^{\circ} \mathrm{K}\right)$ & 10.576 & 9.142 & 11.575 & 9.793 \\
\hline$\overline{\Delta A}_{9}$ & -0.080 & +0.039 & +0.068 & +0.125 \\
\hline
\end{tabular}

PLUTONIUM ABSORPTION AT $1 \mathrm{keV}$ PER Pu ${ }^{239}$ ATOM 
Table 3

URANIUM ABSORPTION AT 1 keV PER U238 ATOM

\begin{tabular}{|c|c|c|c|c|}
\hline \multirow{2}{*}{$\begin{array}{l}\text { Widths } \\
\text { Used }\end{array}$} & \multicolumn{2}{|c|}{$\begin{array}{c}\text { With Resonance } \\
\text { Overlapping }\end{array}$} & \multicolumn{2}{|c|}{$\begin{array}{l}\text { Isolated } \mathrm{U}^{238} \\
\text { Resonances }\end{array}$} \\
\hline & Mean & Fluctuating & Mean & Fluctuating \\
\hline $\bar{A}_{8}\left(300^{\circ} \mathrm{K}\right)$ & 1.532 & 1.132 & 1.569 & 1.159 \\
\hline $\bar{A}_{8}\left(600^{\circ} \mathrm{K}\right)$ & 1.790 & 1.274 & 1.834 & 1.304 \\
\hline$\Delta A_{8}$ & +0.258 & +0.142 & +0.265 & +0.145 \\
\hline
\end{tabular}

The results show that allowance for the fluctuation of resonance widths has an appreciable effect on both the absorption and its Doppler change. For example, with mean widths, $\overline{\Delta A}_{9}$ is changed from +0.068 to -0.080 when the overlapping of $\mathrm{Pu}^{2.39}$ resonances by $\mathrm{U}^{238}$ resonances is allowed for there being a change in sign as discussed in Section 4. (These figures differ from the values quoted in Section 4, owing to the inclusion of both $J=0$ and $J=1$ states.) When the fluctuation of wjdths is taken into account, the corresponding change in $\overline{\Delta A}_{0}$ is from to. 125 to to.039, thus a reduction by $70 \%$ but not a change in sign.

In contrast with the results for plutonium, the effect of overlapping for the $U^{238}$ absorption and its Doppler change is small, as might be expected. As with plutonum, however, the effect of the fluctuation in resonance widths is quite maxked This effect is unfavourable from the point of view of the Doppler coefficient $\overline{\Delta A}_{g}$ is increased, while $\overrightarrow{\triangle A}_{8}$ is decreased

6. $\mathrm{Pu}^{239}-\mathrm{U}^{238}$ Resonance Overlapping in Relation to the Doppler

Coefficient of a Reactor

We consider for simplicity the Doppler change in reactivity of a large bare reactor when the fuel temperature is raised by a prescribed amount, $\Delta T$. According to first-order multigroup perturbation theory, the contribution to the reactivity change from a particular energy group is given by

$$
\Delta \mathrm{R}=\phi\left(\nu \Delta \tilde{\Sigma}_{f}-\phi^{*} \Delta \tilde{\Sigma}_{\mathrm{a}}\right)
$$

Here $\phi$ is the integrated group flux, normalized to unit total fission source from all groups, and t $^{*}$ is the group adjoint flux normalized to unity for fission spectrum neutrons. $\Delta \tilde{\Sigma}_{f}$ and $\Delta \Sigma_{\text {a }}$ denote the changes in the macroscopic effective group cross sections for fission and absorption, resulting from temperature rise in the fuel. 
We assume that the adjoint flux for the low-energy groups of a large fast reactor (small leakage) may be approximated by

$$
\begin{aligned}
\phi^{*} & =n \\
& =\nu \widetilde{\Sigma}_{f} / \widetilde{\Sigma}_{a} .
\end{aligned}
$$

This approximation was suggested by consideration of the fundamental mode multigroup adjoint equations and was checked against some results for $\phi^{*}$ included in Ref. 3. It is also assumed that the temperature dependence of the capture to fission ratio can be reglected.

With these approximations it is possible to express $(6.1)$ in the form

$$
\Delta R=\left(\phi \nu \widetilde{\Sigma}_{f}\right) f_{8}\left(\frac{\overline{\Delta A}_{9}}{\bar{A}_{9}}-\frac{\overline{\Delta A}_{8}}{\bar{A}_{8}}\right) \text {. }
$$

Here $f_{8}$ denotes the fraction of neutrons absorbed in $\mathrm{U}^{238}$ for the energy group under consideration. $\bar{A}_{8}$ and $\bar{A}_{9}$ are the absorptions in $U^{238}$ and $\mathrm{Pu}^{239}$ for this energy group, and $\overline{\Delta A}_{8}$ and $\overline{\Delta A}_{9}$ are the corresponding Doppler changes. The bar over $A_{8}$ and $A_{9}$ indicates that an average is taken over the energy separations of $U^{238}$ and $P u^{239}$ resonances, as in the calculations of Section 5. In view of the way $\phi$ is normalized, the factor

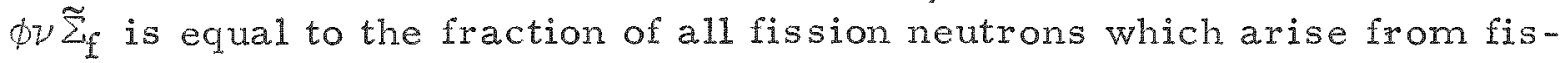
sions in the group. The contribution from the group to the total Doppler coefficient is $\Delta R / \Delta T$.

It should be observed that when resonance over lapping is taken into account, and the resonances of both isotopes are assumed to broaden with temperature rise, the component of the Doppler coefficient associated with the term $\overline{\triangle A}_{9} / \bar{A}_{9}$ in $(6.3)$ is not the "Pu ${ }^{239}$ Doppler coefficient" as conventionally defined. A similar reservation applies to the term $\overline{\triangle A}_{8} / \bar{A}_{8}$. The reason is that $\overline{\Delta A}_{9} / \bar{A}_{q}$, for example, is now a function of both the $\mathrm{Pu}^{239}$ and $\mathrm{U}^{238}$ temperatures, whereas the conventional "Pu ${ }^{239}$ Doppler coefficient" refers to a rise in the $\mathrm{Pu}^{239}$ temperature alone. The component of the Doppler coefficient associated with the term $\overline{\Delta A}_{9} / \bar{A}_{9}$ in $(6.3)$ might be called the "Pu" 239 absorption Doppler coefficient" to distinguish it from the conventional "Pu ${ }^{239}$ Doppler coefficient," and likewise for $\mathrm{U}^{238}$. The two coefficients are identicaI when $\mathrm{Pu}^{239}-\mathrm{U}^{238}$ resonance overlapping is not taken into account.

The results in Tables 2 and 3 of Section 5 may be used to give some indication of the influence of $\mathrm{Pu}^{239}-\mathrm{U}^{238}$ xesonance overlapping on the Doppler coefficient from an energy group near $1 \mathrm{keV}$. In Table 4 we give values of the last factor in (6.3), which determines the sign of the Doppler coefficient. 
Table 4

FRACTIONAL CHANGES IN Pu ${ }^{239}$ AND U ${ }^{238}$ ABSORPTIONS AT $1 \mathrm{kEV}$, EOR TEMPERATURE RISE $300-600^{\circ} \mathrm{K}$

\begin{tabular}{c|c|c}
\hline $\begin{array}{c}\text { Doppler } \\
\text { Change }\end{array}$ & $\begin{array}{c}\text { With } \\
\text { Overlapping }\end{array}$ & $\begin{array}{c}\text { Without } \\
\text { Overlapping }\end{array}$ \\
\hline$\overline{\Delta A}_{9} / \bar{A}_{9}$ & +0.0043 & +0.0129 \\
$\overline{\Delta A}_{8} / \bar{A}_{8}$ & +0.1253 & +0.1256 \\
$\frac{\overline{\Delta A}_{9}}{\bar{A}_{9}}-\frac{\overline{\Delta A}_{8}}{\bar{A}_{8}}$ & -0.1210 & -0.1127 \\
\hline
\end{tabular}

The negative values of the last factor in (6.3) show that the calculated Doppler coefficient near $1 \mathrm{keV}$ is negative. The main effect of the $\mathrm{Pu}^{239}-\mathrm{U}^{238}$ resonance overlapping is to reduce the term $\overline{\triangle A}_{9} / \bar{A}_{9}$ by about two thirds, as shown in the first Ine of Table 4 . The corresponding reduction in $\overline{\Delta A}_{8} / \bar{A}_{8}$ is comparatively insignificant. The net effect of these changes is to increase the magnitude of the Doppler coefficient near $1 \mathrm{keV}$ by about $7 \%$, as shown by the last line of Table 4

The factor $f_{g}$ in $(63)$ has also been estumated from the results in Tables 2 and 3 , noting that $\bar{A}_{8}$ and $\bar{A}_{g}$ as given are the absorptions per atom of each isotope, and the atomic ratio $U / P u$ is 7.1 . For the case of temperature $300^{\circ} \mathrm{K}$, and fluctuations in widths allowed for, $f_{8}$ is found to increase from 0456 to 0465 due to resonance overlapping. From this source the Doppler coefficient near $1 \mathrm{keV}$ would thus be increased by about $2 \%$.

It is more difficult to predict the elfect of resonance overlapping on the remauning factor in $(63)$, namely, $d v \Sigma_{f}$ It is possible that the $r e-$ duction in the absorptions shown in Tables 2 and 3 will cause the multigroup spectrum to be softened, thus increasing $\rho$ On the other hand, $\widetilde{\Sigma}_{f}$ will be expected to decrease. It is not unlukely that the product ov $\widetilde{\Sigma}_{1}$ will increase slightly but detailed multigroup calculations would be necessary to settle the point.

Apart from this uncertainty the above considerations suggest that $P u^{239}-U^{238}$ resonance overlapping mat increase the magnitude of the fuel Doppler coefficient near $1 \mathrm{keV}$ by nearly $10 \%$ The most marked effect, however, is the reduction in the contribution of the plutonium absorption term by two-thirds In view of earlier remarks in this section, the latter result does not apply to the conventional "Pu" Doppler coefficient," which is defined in relation to a rise in the $\mathrm{Pu}^{239}$ temperature alone. The present work has been confined to the case in which the temperature of both $\mathrm{Pu}^{239}$ and $U^{238}$ are changed simultaneously 
7. Variation in Collision Density Due to Resonances

For the calculations in previous sections the collision density was assumed to be constant over an energy range containing several resonances. The accuracy of this approximation has been studied through use of the computer programme RESIOW. The programme solves the slowing down equation (7.1) for an infinite homogeneous medium consisting of several nuclides each of which may contain resonances. The collision density $F(u)$ above the highest energy resonance is taken to be constant in lethargy:

$F(u)=\Sigma_{t}(u) \phi(u)=\sum_{i=1}^{N} \int_{u-U_{m i}}^{u} d u^{\prime} \Sigma_{s c i}\left(u^{\prime}\right) \phi\left(u^{\prime}\right) \frac{\exp \left(u^{\prime}-u\right)}{1-\alpha_{i}}$,

with $\alpha=\left(\frac{A-I}{A+1}\right)^{2}, U_{m}=\ln (1 / \alpha)$, and $A$ is the mass number. The summation is over the $\mathrm{N}$ nuclides $i$ of the system.

The perturbation in collision density due to a single $U^{238}$ resonance at $1 \mathrm{keV}$ with mean parameters is shown in Fig. 4. The resonance is narrow compared with $U_{m}\left(U^{238}\right)$, the maximum lethargy decrement on elastic scattering for $U^{238}$. Figure 4 shows clearly the effects of $U^{238}$ resonance scattering and absorption. Within the first collision interval from the resonance, the collision density increases as a consequence of $\Gamma_{n}$ being appreciably greater than $\Gamma_{\gamma}$, coupled with the effect of light-element moderating materials. The collision density falls sharply at the end of the first collision interval and shows a change of slope at the end of the second.

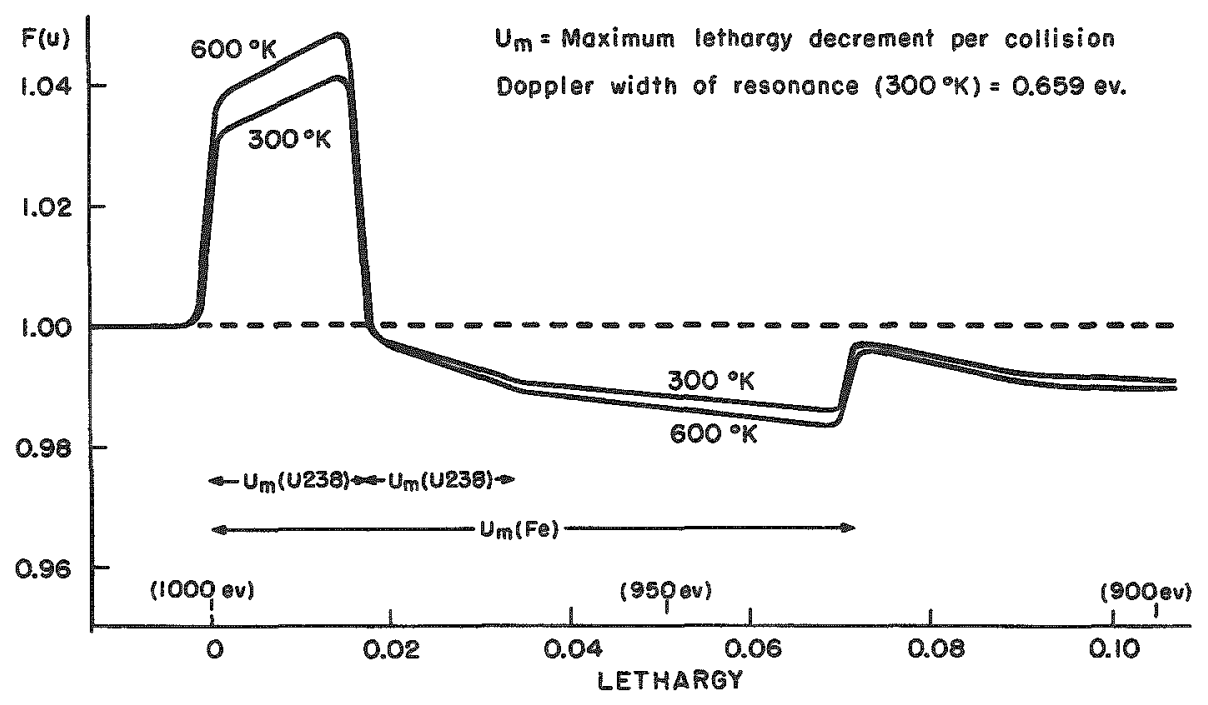

Fig. 4. Perturbation in Collision Density Due to a Single $\mathrm{U}^{238}$ Resonance at $1 \mathrm{keV}$ 
A marked increase occurs after one collision interval for iron which is present in high proportion in the system. At some distance from the resonance, the collision density at $600^{\circ} \mathrm{K}$ assumes a lower value than at $300^{\circ} \mathrm{K}$, due to the increased absorption at the higher temperature.

The absorption per unit energy in the $U^{238}$ resonance obtained from the calculated flux is compared in Table 5 with the 'narrow resonance' (NR) value.

Table 5

ABSORPTION IN A U ${ }^{238}$ RESONANCE AT $1 \mathrm{keV}$

\begin{tabular}{l|c|c}
\hline & Solution of (7.1) & $\begin{array}{c}\text { NR } \\
\text { Approximation }\end{array}$ \\
\hline $\mathrm{A}_{8}\left(300^{\circ} \mathrm{K}\right)$ & 1.594 & 1.569 \\
$\mathrm{~A}_{8}\left(600^{\circ} \mathrm{K}\right)$ & 1.868 & 1.834 \\
$\Delta \mathrm{A}_{8}$ & 0.274 & 0.265 \\
\hline
\end{tabular}

The perturbation in collision density due to a single $\mathrm{Pu}^{239}$ resonance is much less than for $\mathrm{U}^{238}$, due to the lower peak height and lower concentration.

The variation in collision density near the $U^{238}$ resonance is seen from Table 5 to increase the Doppler change in absorption in $\mathrm{U}^{238}$ itself by only a few per cent. The effect on neighbouring Pu ${ }^{239}$ resonances, however, could be more significant. These effects are being studied further.

\section{Conclusions}

(1) The variation of the Doppler increase in absorption with spacing between two average $\mathrm{Pu}^{239}$ resonances near $1 \mathrm{keV}$ is given to within a few per cent by the approximate method of calculation appropriate to high energies. The absolute value, however, is about $30 \%$ too high.

(2) The effect of overlapping of $\mathrm{Pu}^{239}$ and $\mathrm{U}^{238}$ resonances is estimated, for a particular system, to reduce the absorption in $\mathrm{Pu}^{239}$ and $\mathrm{U}^{238}$ near $1 \mathrm{keV}$ by about $16 \%$ and $2 \%$, respectively. The Doppler increment in absorption, for temperature rise from 300 to $600^{\circ} \mathrm{K}$, is reduced by about $70 \%$ for $\mathrm{Pu}^{239}$ and $2 \%$ for $\mathrm{U}^{238}$.

Application of the results suggests that the fuel Doppler coefficient from an energy group near $1 \mathrm{keV}$ might be increased in magnitude by roughly $10 \%$. Since the treatment assumes that the temperature rises in 
both $P u^{239}$ and $U^{238}$ simultaneously, the results do not provide information on the individual $\mathrm{Pu}{ }^{239}$ and $\mathrm{U}^{238}$ Doppler coefficients, defined with respect to a temperature rise in each isotope separately.

Statistical fluctuations in the neutron and fission widths are shown to be important. They tend to enhance the Pu ${ }^{239}$ Doppler effect, but to reduce the $\mathrm{U}^{238}$ effect.

(3) The usual approximation of constant collision density is adequate for estimating the absorption in an average $U^{238}$ resonance at $1 \mathrm{keV}$, and its Doppler change. Further investigation will be necessary, however, to assess whether the actual variation of the collision density with energy and temperature will affect appreciably the absorption in neighbouring $\mathrm{Pu}^{239}$ resonances.

\section{References}

1. Codd, J., and Collins, P. J., Plutonium 239 and Uranium 238 Resonance Interaction Effects in a Dilute Fast Reactor, E.A.E.S. Symposium on Advances in Reactor Theory, Karlsruhe, April 1963.

2. Nicholson, R. B., The Doppler Effect in Fast Reactors, APDA-139 (1960).

3. Bhide, M. G., and Hummel, H. H., Calculations of the Doppler Coefficient of Large Ceramic-fueled Fast Reactors, ANL-6601 (1962).

4. Ferziger, J. H., Greebler, P., Kelley, M. D., and Walton, J. W., Resonance Integral Calculations for Evaluation of Doppler Coefficients. The RAPTURE Code, GEAP-3923 (1962).

Discussion of Paper

Presented by Mr. Codd

MR. OTT (Karlsruhe):

Did you investigate the energy dependence of the interaction only near one keV?

MR. CODD:

In the paper presented only near one keV, but we have done recent calculations up to about $100 \mathrm{keV}$. As Mr. Hwang may discuss in more 
detail this afternoon, the overlapping effect gets smaller below one keV, gets bigger as you go to higher energies, and seems to have a maximum at about $20 \mathrm{keV}$.

MR. OTT:

We have found this too. In what shape have you used your $U^{238}$ resonance in the study of the collision density?

MR. CODD:

A single Breit-Wigner Doppler-broadened shape for the $U^{238}$ resonance.

MR NICHOLSON (University of Wisconsin).

As I understand it, the figures that you show are for the situation where both the $U^{238}$ and the plutonium are changing temperature together: is that correct?

MR. CODD。

Yes, I referred to the fuel coefficient.

\section{MR NICHOLSON;}

I wonder if you would care to comment on the case where the two are separate, as in a critical experiment. I think it would be interesting to the critical experiment people, whether or not this effect is important, were the $\mathrm{U}^{235}$ to heat separately from the $\mathrm{U}^{238}$.

MR. CODD.

I think what happens is that if the two materials are heated separately, the Doppler coefticient of the fissile material is virtually unchanged. If, instead, you hear the $U^{238}$ separately. I think its Doppler coefficient temperature is increased. In other words, the effect can be assigned to the Doppler effect in the $\mathrm{U}^{238}$.

MR. DAVEY (Argonne)

I understand you have a very detailed description of the slowing down below $20 \mathrm{keV}$. May I ask how you intend to estimate the fraction of the flux reaching $20 \mathrm{keV}$ ? 


\section{MR.CODD:}

Here we do not attempt to improve the method of calculation. Above that energy we shall rely on the best available multigroup methods at the time we come to use these programs. Our source will be provided by other methods. This new treatment will depend very much on the accuracy of the nuclear data in the high-energy region, and the method of calculation, which I think you pointed out yesterday. 
AN IMPROVED METHOD OF DOPPLER-EFEECT CALCULATION FOR FISSILE MATERIALS IN THE INTERMEDIATE ENERGY REGION

\author{
R. N. Hwang \\ Argonne National Laboratory \\ Argonne, Illinois
}

I. Introduction

Two methods generally known as "Method A" and "Method B"(2) have been used almost exclusively up to the present time for calculations of the Doppler effect in the unresolved region. For fissile materials, however, both methods become questionable in the intermediate energy region because of the interference interactions of resonances.

Two types of interference interactions in the intermediate energy region are of great concern. The first type, which will be referred to as the overlapping effect, is attributed to the interaction of neighboring resonances of a particular fissile isotope. This effect was not treated accurately in the previous calculations. The second type, which has been neglected completely in previous treatments, $(1,2)$ is attributed to the interaction between resonances of two different isotopes in the mixture. The effect of these interactions gives rise to the change of the fine structure of the neutron flus and, consequently, may bring about a substantial reduction in the Doppler broadening obtained by previous methods.

Extensive studies have been made of the interference effect. The problem becomes extremely difficult when two types of interference interactions occur simultaneously. As the first step, an improved method in which two types of interactions are considered separately has been developed.

II. General Characteristics of Interference Interaction

A. Effects of an Interfering Resonance

Assume that the narrow-resonance approximation and singlelevel equation are valid. The rate of reaction of isotope 1 at $k^{\text {th }}$ resonance is proportional to $\mathrm{J*}$, defined as

$$
J *=\frac{1}{2} \int_{-\infty}^{\infty} \frac{\psi_{1 k} d x_{1}}{E_{1}+\psi_{1 k}+A_{2 k^{\prime}} \psi_{2 k^{\prime}}}
$$

where the interfering resonance $\mathrm{k}^{\prime}$ is either the neighboring resonance or a resonance of isotope 2 in the mixture, $A_{2 k}$ the ratio of the peak 
macroscopic cross sections of $\mathrm{k}^{\text {th }}$ and $\mathrm{k}^{\text {th }}$ resonances, and $\psi_{\mathrm{k}}$ the usual $\psi$-function defined as

$$
\psi_{1 k}\left(x_{1}, \varepsilon_{1}\right)=\frac{1}{2 \sqrt{\pi}} \xi_{1}^{\infty} \frac{e^{-\frac{\xi_{1}^{2}}{4}\left(x_{1}-y\right)^{2}} d y}{1+y^{2}}
$$

$\mathrm{J} *$ would become equal to the usual $\mathrm{J}$-function if there were no interference.

The effects of the interfering term $\mathbf{A}_{2 k^{\prime}} \psi_{2 k^{\prime}}$ on $J^{*}$ and $\delta J^{*}$, the change in $J^{*}$ due to the rise in temperature, can be summarized as follows:

(1) The magnitude of $\mathrm{J}^{*}$ is always smaller than or equal to $\mathrm{J}$, and the minimum value of $J^{*}$ occurs when $k$ and $k^{\prime}$ resonances coincide. $J$ * approaches $J$ rapidly when the separation between resonances becomes large.

(2) At an elevated temperature, $\psi_{2 k}$ does not vary as sharply as its value at the initial temperature as the separation increases. As a result, $\delta J^{*}$ varies from positive to negative before it finally approaches $\delta J$ at large separation.

\section{B. Quantities $\delta H$ and $\delta I$}

In studying the effects of interference interactions, it is most convenient to introduce two important quantities:

\section{(1) $\delta \mathrm{H}$}

Define

$$
H=J^{*}-J_{0}
$$

so that

$$
\delta H=\delta J *-\delta J
$$

Physically, SH represents the increment or decrement of $\delta J$ caused by the interference interaction. 
(2) $\delta I$

Define

$$
I=\int_{-\infty}^{\infty} \psi_{1 k^{2}} \psi_{2 k^{\prime}} d E
$$

and

$$
\delta I=I_{\text {at }} T-I_{\text {at }} T_{0}{ }^{\prime}
$$

where $T_{0}$ is taken as room temperature and $T$ is any elevated temperature.

The integral I has the following characteristics:

(1)- By using the Faltung theorem (5) of Fourier transforms, it can be shown readily that $I$ is related to the $\psi$-function defined by Eq. 2 , i.e.,

$$
\begin{aligned}
I & =\frac{\Gamma_{1 k} \Gamma_{2 k^{\prime}}}{4 \Delta} \int_{-\infty}^{\infty} \exp \left(-2 P^{2}-\frac{\Gamma_{1 k}+\Gamma_{2 k^{\prime}}}{\Delta}|P|\right) \exp \left(\frac{2 \mu}{\Delta} p_{i}\right) d P \\
& =\frac{\Gamma_{1 k} \Gamma_{2 k^{\prime}} \pi}{2\left(\Gamma_{1 k}+\Gamma_{2 k^{\prime}}\right)} \psi\left(\frac{2 \mu}{\Gamma_{1 k}+\Gamma_{2 k^{\prime}}}, \frac{\Gamma_{1 k}+\Gamma_{2 k^{\prime}}}{\sqrt{2} \Delta}\right),
\end{aligned}
$$

where

$$
\begin{aligned}
& \mu=\text { separation }=E_{2 k^{\prime}}-E_{1 k} \\
& \Delta=\text { Doppler width }
\end{aligned}
$$

$\Gamma_{1 k}, \Gamma_{2 k^{\prime}}=$ total widths of $k$ and $k^{\prime}$, respectively.

(2) $\int_{-\infty}^{\infty} I \mathrm{~d} \mu=\frac{\pi^{2} \Gamma_{1 \mathrm{k}} \Gamma_{2 \mathrm{k}^{\prime}}}{4}$

which is temperature-independent.

A code for evaluating the $\mathrm{J}^{*}$-function has been available for some time at ANL so that $\delta H$ can be evaluated quite readily. Extensive studies show that, for any set of parameters, the quantity $\delta \mathrm{H}$ generally exhibits a $v$-shaped behavior along the separation $\mu$. Figure 1 illustrates how $\delta \mathrm{H}$ and another interesting quantity $\left(-\frac{\mathrm{A}_{2}}{\beta_{1}^{2}} \frac{\delta \mathrm{I}}{\Gamma_{1 \mathrm{k}}}\right)$ vary along the 
separation $\mu$. The striking difference between $\delta \mathrm{H}$ and $\left(-\frac{A_{2}}{\beta_{1}^{2}} \frac{\delta I}{\Gamma_{1 k}}\right)$ is that the net area enclosed by the latter is zero according to Eq. 6, whereas the net area enclosed by the former is not. In a limiting case, $\delta \mathrm{H}$ is related to $\delta \mathrm{I}$ by

$$
\delta H \cong\left(-\frac{A_{2}}{\beta_{1}^{2}} \frac{\delta I}{\Gamma_{1 k}}\right)
$$

if

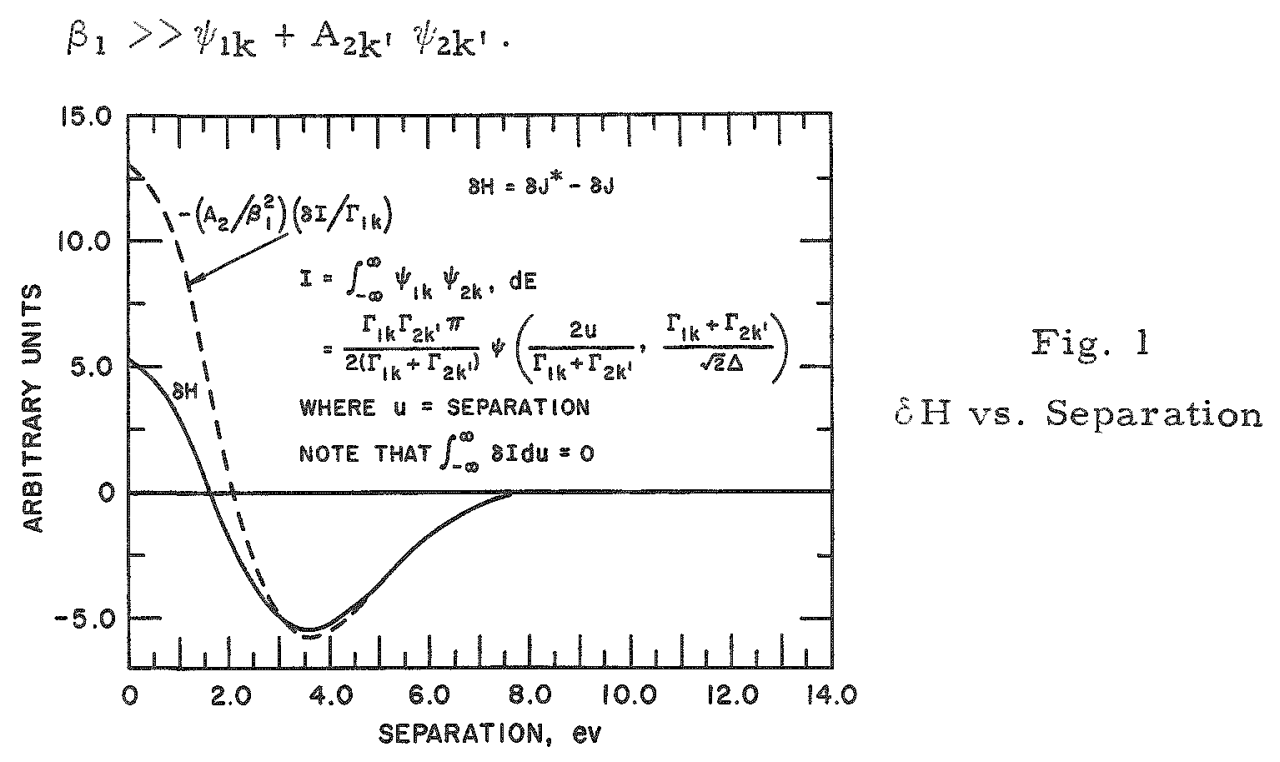

The validity of Eq. 7 can be shown quite readily by series expansion. This last condition is well-satisfied for fissile isotopes either in the high energy region or at large separation. These $\mathrm{v}$-shaped characteristics of $\delta H$ and $\delta I$ are believed to be responsible for interference interactions between resonances.

\section{The Doppler Change of the Effective Cross Section in the Presence of Interference Interaction}

From Eq. 3, the change in effective cross section for a particular process $\mathrm{x}$ of isotope 1 due to the rise in temperature can be written as

$$
\left.\delta \tilde{\sigma}_{\mathrm{X}}=\left\langle\frac{\Sigma_{\mathrm{tN}}}{N_{1}}\right\rangle \sum_{\substack{\text { spin } \\
\text { states } \\
J}} \frac{1}{\left\langle S_{\mathrm{I}}\right\rangle_{\mathrm{J}}}\left\{\left\langle\Gamma_{\mathrm{X}} \delta \mathrm{J}\right\rangle \begin{array}{l}
\text { over widths } \\
\text { of } 1
\end{array}+\left[\bar{I}_{\mathrm{x}} \delta \mathrm{H}\right\rangle \gamma_{\begin{array}{l}
\text { over widths } \\
\text { of } 1 \text { and } 2
\end{array}}\right]_{\begin{array}{l}
\text { over sep - } \\
\text { aration }
\end{array}}\right\} \text {, }
$$


where

$$
\begin{aligned}
\left\langle\frac{2 t N}{N}\right\rangle & =\text { background scattering cross section per atom of isotope } 1 \\
\left\langle S_{1}\right\rangle_{J} & =\text { average level spacing of isotope } 1 \\
\Gamma_{\mathrm{X}} & =\text { level width of the process } \mathrm{x} \\
\langle\rangle_{g}[] & =\text { statistical average over widths and separation, respectively }
\end{aligned}
$$

The averaging process in Eq. 8 requires specification of the statistical distribution functions for each unknown parameter The chisquared distribution of degree of freedom of $\nu_{\text {. }}$

$$
\mathrm{P}\left(\mathrm{y}_{s} \nu\right)=\frac{(\nu / 2)}{\Gamma(\nu / 2)}(\nu / 2 \mathrm{y})^{\nu / 2-1} \mathrm{e}^{-\nu / 2 \mathrm{y}}
$$

where $\mathrm{y}$ is the ratio of the local-to-average value of a parameter, is generally used for all neutron and fission widths involved The first term on the right-hand side of Eq. 8 is essentially the well-known "Method B" expression $(2)$ which can be readily evaluated by means of the method suggested by Greebler (7) The problem is then to evaluate the quantity

$$
\left[\left\langle\Gamma_{x} \mathrm{oH}\right\rangle\right]
$$

The method of evaluation depends on the type of interference interaction under consideration.

III. The Overlapping Effect

If two neighboring resonances are designated by $k$ and $k$. respec-

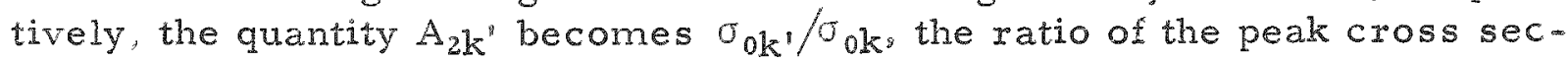
tion of $k^{\prime}$ and $k$ resonances. The subscript $l$ and 2 will he dropped in the following discussions.

\section{A Determination of the Distribution Function of Levels}

The function $\Omega(\mu)$ is defined as the probability of finding any level within an interval d $\mu$ at a distance $\mu$ from a given level, where $\mu$ is the separation between $k$ and $k$ ' resonances, $\Omega(\mu)$ is assumed to be the solution of an integral equation of the form $(3)$

$$
\Omega(y)=P_{\nu}(y)+\int_{0}^{y} P_{\nu}(t) \Omega(y-t) d t
$$


where

$$
\mathrm{y}=|u| /\langle\mathrm{s}\rangle_{\mathrm{J}}
$$

and $P_{\nu}$ is defined by Eq. 9.

Equation 10 is known as the Faltung type of integral equation $(4)$ and can be solved most readily by the use of Laplace transform. In the present work, it is assumed that $\nu=8$ for all fissile materials under consideration. The solution of Eq. 10 is then

$$
\Omega(y)=1-e^{-8 y}-2 \sin 4 y e^{-4 y}
$$

Figure 2 shows that $\Omega(y)$ exhibits characteristics almost similar to a unit step function. The effect is thus to prevent the spacing between two neighboring resonances from becoming too close together. Consequently, a part of the positive area under $\delta \mathrm{H}$ is eliminated by $\Omega(\mathrm{y})$ when averaged, so that $[\delta H]$ is expected to be negative.

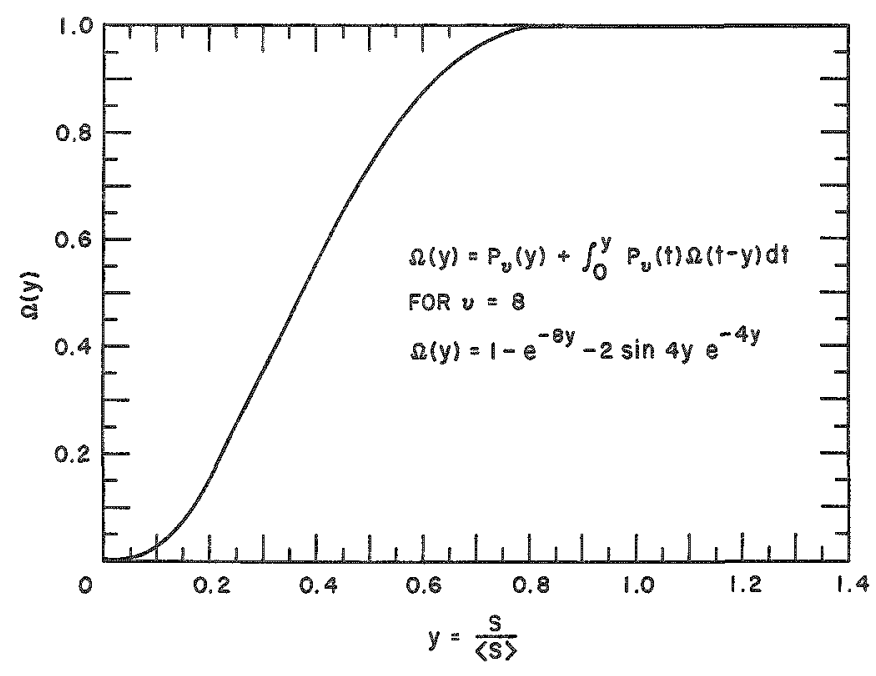

Fig. 2

Level Distribution Function

B. Evaluation of $\left[\left\langle\Gamma_{x} \delta H\right\rangle\right]$

In a large fast reactor, the potential scattering cross section per unit atom of the fissile isotope is expected to be much higher than the peak cross sections $\sigma_{0 k}$ and $\sigma_{0 k}$, even in the intermediate region. It is, therefore, reasonable to expand the integrand of $J$ and $J *$ into a power series so that

$$
\left[\left\langle\Gamma_{\mathrm{X}} \delta \mathrm{H}\right\rangle\right]=-\left[\left\langle\frac{\Gamma_{\mathrm{X}}}{\Gamma_{\mathrm{lk}}} \frac{\mathrm{A}_{2}}{\beta^{2}} \delta I\right\rangle\right]+\mathrm{R}_{\mathrm{g}}
$$

where $R$ represents the higher-order correction terms. 
Each term in Eq. 12 consists of an integral of the form

$$
\int_{-\infty}^{\infty} \psi_{k}^{m} \psi_{k}^{n} d E
$$

In particular, the first-order term has already been given by Eq. 5 . By changing the order of integration, one has

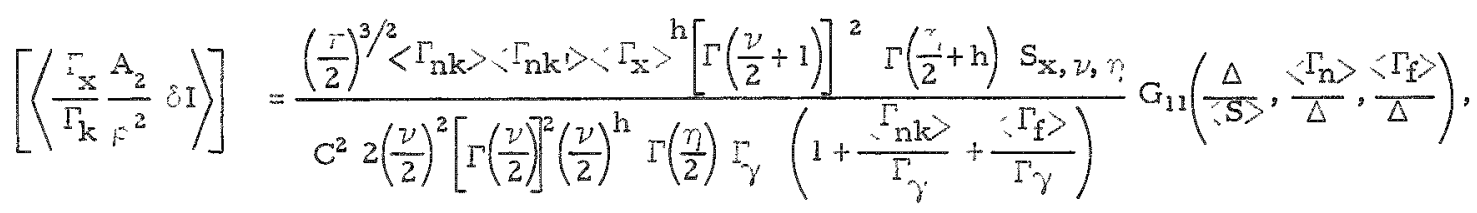

where

$$
\begin{aligned}
& c=\frac{\left\langle\frac{\Sigma_{t N}}{N_{1}}\right\rangle}{4 \pi \pi^{2} g_{k}} \\
& \mathrm{~S}_{\mathrm{x}, \nu, \eta}=\left(1+\frac{\left\langle\Gamma_{\mathrm{n}}\right\rangle}{\Gamma_{\gamma}}+\frac{\left\langle\Gamma_{\mathrm{f}}\right\rangle}{\Gamma_{\gamma}}\right) \int_{0}^{\infty} \frac{\mathrm{e}^{-\mathrm{S}} \mathrm{dS}}{\left(1+\frac{\left\langle\Gamma_{\mathrm{n}}\right\rangle}{\Gamma_{\gamma}} \mathrm{s}\right)^{(\nu / 2)+1}\left(1+\frac{\left.\Gamma_{\mathrm{f}}\right\rangle_{\mathrm{S}}}{\Gamma_{\gamma}}\right)^{(\eta / 2)+\mathrm{h}}} \\
& h=0 \text { if } x \text { is radiative capture } \\
& =1 \text { if } x \text { is fission }
\end{aligned}
$$

subscripts $n, f, \gamma=$ neutron, fission, and capture, respectively

$\eta_{0} \nu=$ degrees of freedom for fission and neutron widths, respectively

$\Gamma(Z)=$ gamma function

$$
G_{11}\left(\frac{\Delta}{\langle S\rangle}, \frac{\left.\Gamma_{\mathrm{n}}\right\rangle}{\Delta}, \frac{\left\langle\Gamma_{\mathrm{f}}\right\rangle}{\Delta}\right) \cong\left(\frac{2^{5 / 2}}{\sqrt{\pi}}\right) \int_{0}^{\infty} \Omega(\mathrm{y}) \mathrm{dy} \int_{0}^{\infty} \mathrm{e}^{-2 \mathrm{P}^{2}-\frac{2 \gamma}{\Delta} \mathrm{P}} \cos \frac{2 \mu}{\Delta} \mathrm{p} \mathrm{dP}
$$

so long as the radiative capture width $5<$ Doppler width $\triangle$. 
A code based on Romberg integration scheme has been developed for evaluating $G_{11}$. The terms of higher order, in general, will not be of much practical significance. In order to see the relative magnitude of each term, a special case will be discussed.

At the upper end of the intermediate energy region, the $\psi$-function approaches its Gaussian form. A generalized expression can be obtained for all terms in Eq. 12, i.e.,

$$
\begin{aligned}
& {\left[\left\langle\frac{\Gamma_{\mathrm{x}}}{\Gamma_{\mathrm{k}}} \frac{\mathrm{A}_{2}^{\mathrm{n}}}{\beta^{\mathrm{m}+\mathrm{n}}} \int_{-\infty}^{\infty} \psi_{\mathrm{k}}^{\mathrm{m}} \psi_{\mathrm{k}^{\prime}}^{\mathrm{n}} \mathrm{dE}\right\rangle\right]=}
\end{aligned}
$$

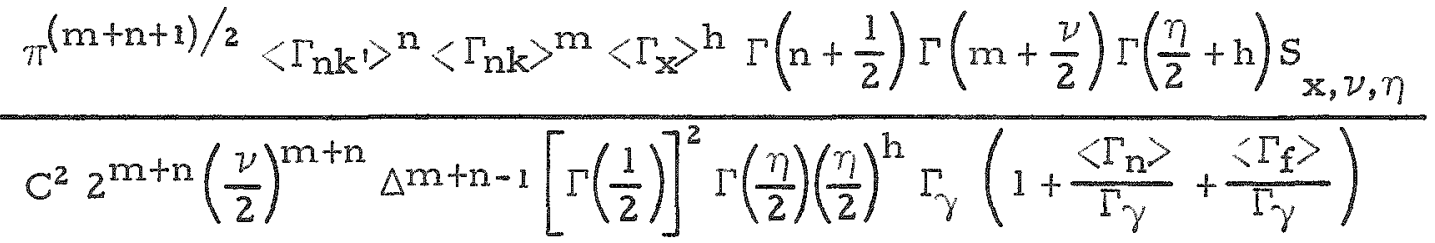

$$
\begin{aligned}
& F_{m n}\left(\frac{\Delta}{\langle S\rangle}\right)
\end{aligned}
$$

The function $F_{m n}\left(\frac{\Delta}{\langle S\rangle}\right)$ is defined as

$$
\begin{aligned}
F_{m n}\left(\frac{\Delta}{\langle S\rangle}\right)= & 2 \int_{0}^{\infty} \Omega(y) e^{-\frac{\delta^{2}}{\Delta^{2}} \frac{m n}{m+n}} d y \\
= & \sqrt{\pi} \frac{\Delta}{\langle S\rangle} \sqrt{\frac{m+n}{m n}}\left\{1-\exp \left(\frac{16 \Delta^{2}}{\langle S\rangle^{2}} \frac{m+n}{m n}\right) \operatorname{Erfc}\left(\frac{4 \Delta}{\langle S\rangle} \sqrt{\frac{m+n}{m n}}\right)\right. \\
& -2\left[\sin 8 \frac{\Delta^{2}}{\langle S\rangle^{2}} \frac{m+n}{m n}\left(C\left(\frac{8 \Delta^{2}}{\langle S\rangle^{2}} \frac{m+n}{m n}\right)+S\left(\frac{8 \Delta^{2}}{\langle S\rangle^{2}} \frac{m+n}{m n}\right)-1\right)\right. \\
& \left.\left.+\cos \frac{8 \Delta^{2}}{\langle S\rangle^{2}} \frac{m+n}{m n}\left(C\left(\frac{8 \Delta^{2}}{\langle S\rangle^{2}} \frac{m+n}{m n}\right)-S\left(\frac{8 \Delta^{2}}{\langle S\rangle^{2}} \frac{m+n}{m n}\right)\right)\right]\right\}
\end{aligned}
$$


where

$$
C(x)=\frac{1}{\sqrt{2 \pi}} \int_{0}^{x} \frac{\cos x t d t}{t^{1 / 2}}
$$

and

$$
S(x)=\frac{1}{\sqrt{27}} \int_{0}^{x} \frac{\sin x t d t}{t^{1 / 2}}
$$

are Fresnel integrals.

$F_{m n}\left(\frac{\Delta}{S}\right)$ is a sharply decreasing function of $\Delta /, S>$ when $\left.\Delta / S\right\rangle$ is small and approaches its asymptotic form:

$$
F_{m n} \cong \sqrt{\pi} \frac{\Delta}{S>} \sqrt{\frac{m+n}{m n}}-\frac{3}{4}
$$

when

$$
\Delta / S>\sim 1
$$

Note that the overlap correction lactor $e^{(S)}$ defined by Lane et al ${ }^{(1)}$ is jusl

$$
e^{(S)}=-\left(1-\Delta \frac{\partial}{u_{i}}\right) F_{11}
$$

which assumes an asymptotic value of $3 / 4$ according to Eq. 17.

The first-order term given by Eq. 15 differs from Eq. 13 only in the function $F_{11}$. Figure 3 shows quantities $e^{(S)}$ and $c(S)$ plotted along (S) $/ \triangle$ for $U^{235}$ with spin $J=3$, where

$$
c^{(S)}=-\left(1-\Delta \frac{0}{\partial \Delta}\right) G_{11}
$$

is the corresponding term by more rigorous calculation. The deviation of $e^{(S)}$ from $\epsilon(S)$ is not too significant, as is shown in Fig. 3. Hence, Eq. 15 is believed to hold reasonably well even at the lower end of the intermediate energy region.

Calculations have been made for both $\mathrm{U}^{235}$ and $\mathrm{Pu}^{239}$ and the results are listed in Table I and Table II, respectively. The first-order 
overlapping term was obtained by use of Eq. 3. Higher-ordor terms were not significant in all cases considered. Equation 15 could be used for higher-order corrections should they become significant. Equation 8 is essentially a combination of "Method $A$ " and "Method $B$ " in which both overlapping and nonoverlapping parts are evaluated more accurately.

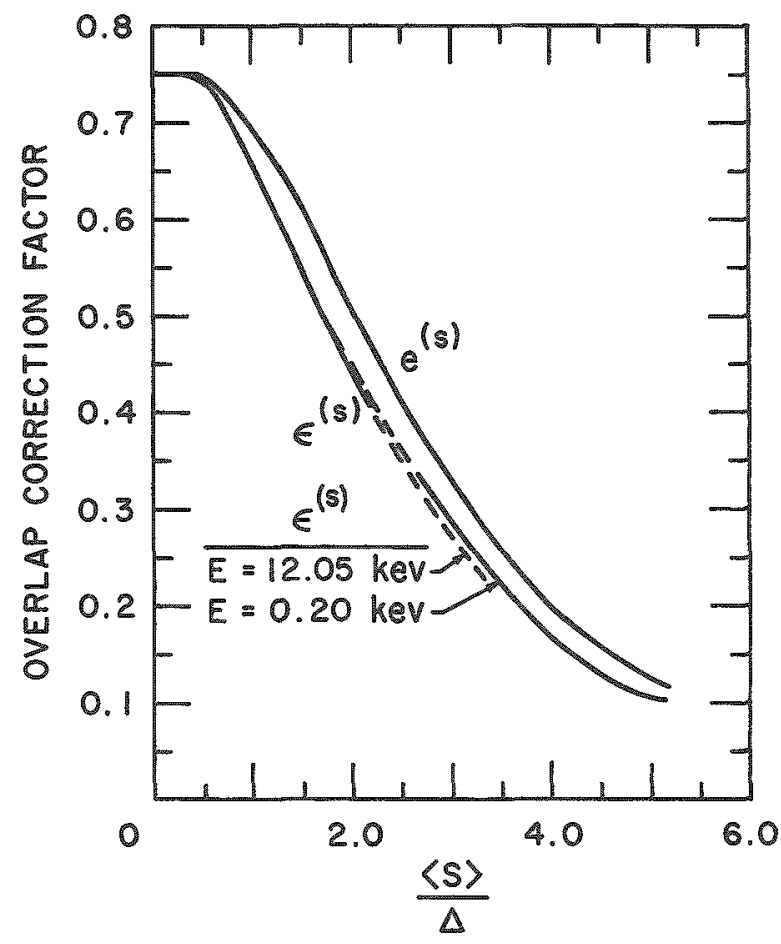

Fig. 3

Overlap Correction Factor

Table I

$u^{235}$

\begin{tabular}{|c|c|c|c|c|c|c|}
\hline \multirow{3}{*}{ Energy ikev! } & \multicolumn{3}{|c|}{$\delta \tilde{\sigma}_{f}$} & \multicolumn{3}{|c|}{$\delta \tilde{\sigma}_{\gamma}$} \\
\hline & $\begin{array}{l}\text { Vo overlap } \\
\text { Method Bi }\end{array}$ & With Overlap & $\%$ Reduction & $\begin{array}{l}\text { Wo Overlap } \\
\text { Method Bi }\end{array}$ & With Overlap & $\%$ Reduction \\
\hline & \multicolumn{6}{|c|}{ a. $T=300750^{\circ} \mathrm{K}$} \\
\hline $\begin{array}{r}12.05 \\
6.50 \\
2.25 \\
0.61 \\
0.20 \\
\end{array}$ & $\begin{array}{l}0.0035 \\
0.0091 \\
0.0428 \\
0.2291 \\
0.6870 \\
\end{array}$ & $\begin{array}{l}0.0023 \\
0.0061 \\
0.0290 \\
0.1648 \\
0.5121 \\
\end{array}$ & $\begin{array}{l}33.8 \\
33.5 \\
33.2 \\
28.1 \\
250 \\
\end{array}$ & $\begin{array}{l}0.0016 \\
0.0043 \\
0.0220 \\
0.1326 \\
0.4402 \\
\end{array}$ & $\begin{array}{l}0.0010 \\
0.0029 \\
0.0152 \\
0.0982 \\
0.3426 \\
\end{array}$ & $\begin{array}{l}36.0 \\
33.8 \\
31.1 \\
26.0 \\
22.2 \\
\end{array}$ \\
\hline & \multicolumn{6}{|c|}{ b. $T=300-15000 \mathrm{~K}$} \\
\hline $\begin{array}{r}12.05 \\
6.50 \\
2.25 \\
0.61 \\
0.20\end{array}$ & $\begin{array}{l}0.0053 \\
0.0140 \\
0.0669 \\
0.3718 \\
1.1820\end{array}$ & $\begin{array}{l}00035 \\
0.0099 \\
0.0452 \\
0.2610 \\
0.8550\end{array}$ & $\begin{array}{l}33.5 \\
32.7 \\
32.5 \\
29.8 \\
27.7\end{array}$ & $\begin{array}{l}0.0024 \\
0.0065 \\
0.034 \\
0.2110 \\
0.7451\end{array}$ & $\begin{array}{l}0.0016 \\
0.0043 \\
0.0233 \\
0.1519 \\
0.5570\end{array}$ & $\begin{array}{l}34.3 \\
33.2 \\
32.0 \\
28.0 \\
25.1\end{array}$ \\
\hline
\end{tabular}

Parameters: $\left\langle\frac{\sum+N}{N_{1}}\right\rangle-200$ blatom; $\nu=1, \eta=2$

$\left\langle\Gamma_{f}\right\rangle \quad 120 \mathrm{mV} ; \Gamma_{\gamma}=33 \mathrm{mV} ; \frac{\left\langle\Gamma_{\mathrm{p}}^{0}\right\rangle}{\langle\mathrm{S}\rangle}-10^{-4}$

$\langle s\rangle_{3} \quad 1.72 \mathrm{eV}:\langle\mathrm{s}\rangle_{4} \quad 1.34 \mathrm{eV}$

$S$ wave only. 
Table II

$\mathrm{Pu}^{239}$

\begin{tabular}{|c|c|c|c|c|c|c|}
\hline \multirow{3}{*}{ Energy tkev } & \multicolumn{3}{|c|}{$\delta \tilde{\sigma}_{f}$} & \multicolumn{3}{|c|}{$\delta \tilde{\sigma} \gamma$} \\
\hline & $\begin{array}{l}\text { No Overlap } \\
\text { Method B! }\end{array}$ & With Overlap & \% Reduction & $\begin{array}{l}\text { No Overlap } \\
\text { Method Bi) }\end{array}$ & With Overlap & Reduction \\
\hline & \multicolumn{6}{|c|}{ a. $T=300-750^{\circ} \mathrm{K}$} \\
\hline $\begin{array}{l}9.1 \\
6.5 \\
2.25 \\
0.61 \\
\end{array}$ & $\begin{array}{l}0.0111 \\
0.0190 \\
0.0000 \\
0.3800\end{array}$ & $\begin{array}{l}0.0081 \\
0.0144 \\
0.0753 \\
0.3462 \\
\end{array}$ & $\begin{array}{r}27.1 \\
24.5 \\
16.4 \\
8.9 \\
\end{array}$ & $\begin{array}{l}0.0061 \\
0.0110 \\
0.0503 \\
0.2600 \\
\end{array}$ & $\begin{array}{l}0.0043 \\
0.0083 \\
0.0421 \\
0.2382 \\
\end{array}$ & $\begin{array}{r}29.0 \\
24.7 \\
15.9 \\
8.4 \\
\end{array}$ \\
\hline & \multicolumn{6}{|c|}{ b. $T=300-1500 \mathrm{~K}$} \\
\hline $\begin{array}{l}9.1 \\
6.5 \\
2.25 \\
0.61\end{array}$ & $\begin{array}{l}0.0172 \\
0.0290 \\
0.1000 \\
0.6500\end{array}$ & $\begin{array}{l}0.0123 \\
0.0215 \\
0.1135 \\
0.5767\end{array}$ & $\begin{array}{l}28.3 \\
26.0 \\
18.9 \\
11.3\end{array}$ & $\begin{array}{l}0.0094 \\
0.0170 \\
00800 \\
0.4400\end{array}$ & $\begin{array}{l}0.00656 \\
0.0126 \\
0.0648 \\
0.3928\end{array}$ & $\begin{array}{l}29.8 \\
26.0 \\
20.2 \\
10.7\end{array}$ \\
\hline
\end{tabular}

Parameters: $\left\langle\frac{\sum+N}{N_{1}}\right\rangle \quad 200$ blatom; $v-1 ; \eta 2$

$\left\langle\Gamma_{f}^{p}\right\rangle=99 \mathrm{mV} \cdot \Gamma_{y} \quad 39 \mathrm{mV} ; \frac{\Gamma_{n}^{0}}{\langle S\rangle}-10^{-4}$.

$\langle\mathrm{S}\rangle_{0}=11.58 \mathrm{eV} ;\langle\mathrm{S}\rangle_{1} \quad 3.86 \mathrm{eV}$

S wave only.

IV. Interference Interactions between Fertile and Fissile Isotopes

All earlier calculations $(1,2,7)$ for the Doppler effect of a fissile isotope in the unresolved region were based on the assumption that the interference effect due to the fertile isotope in the mixture would be washed out over a statistical average of many resonances. This assumption is certainly valid at the high energy region if there is no correlation between levels of two different isotopes. Since the quantily $H$ approaches its asymptotic value

$$
\left(-\frac{A_{2}}{\beta_{1}^{2}} \frac{I}{\Gamma_{1 k}}\right)
$$

at high energy, the statistical average over a random separation is, in fact, temperature independent according to Eq. 6. However, the quantity $H$ can no longer be represented by its asymptotic form in the intermediate energy region, where $A_{2}$ becomes comparable to or even larger than $F_{1}$. A net gain in the negative area enclosed by the curve $\delta H$ along the separation $\mu$ is observed as pointed out earlier by Codd and Collins.(6) Consequently, a net reduction in $\delta \widetilde{\sigma}_{x}$ is to be expected when averaged over a random separation.

A straightforward evaluation of $\left[\left\langle\Gamma_{\mathrm{x}} \delta \mathrm{H}\right\rangle\right]$ is extremely difficult in this case, if not impractical. In order to simplify the problem of numerical integration and utilize the existing code, the following approximation has been developed. 
Since the quantity $A_{2}$ is expected to be very large in the intermediate energy region, it is possible to replace $J *$ by

$$
\mathrm{J}^{*} \cong \frac{1}{2} \int_{-\infty}^{\infty} \frac{\psi_{1 k} \mathrm{dx}}{\beta_{1}+\mathrm{A}_{2 k^{\prime}} \psi_{2 k^{\prime}}}
$$

so long as the separation is small. On the other hand, since $A_{2 k^{\prime}} \psi_{2 k^{\prime}}$ is a sharply decreasing function of the separation, J* rapidly approaches its asymptotic form so that $\delta H$ can be represented by Eq. 7 at large separation. By using Eq. 20, Eq. 7, and Eq. 9, and changing the order of integration, $\left[\left\langle\Gamma_{\mathrm{X}} \delta \mathrm{H}\right\rangle\right]$ can be expressed separately by two much simplified expressions as follows (see Appendix):

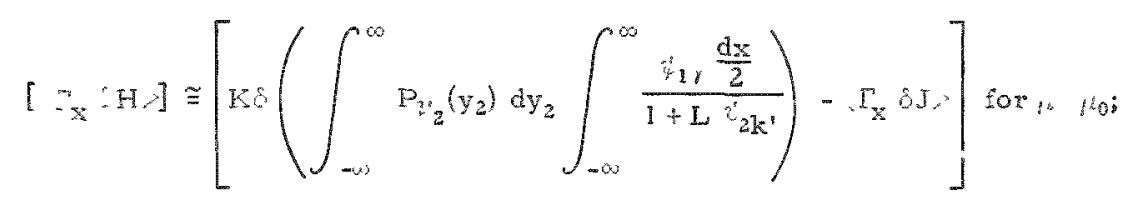

for $\mu<\mu_{0}$;

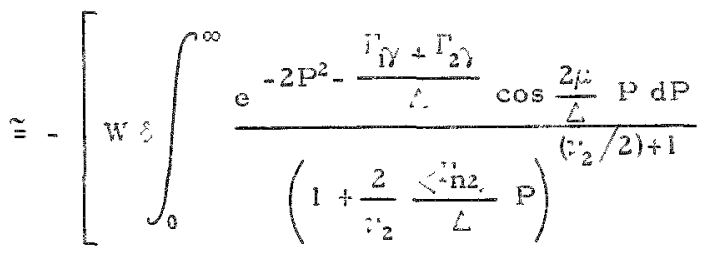

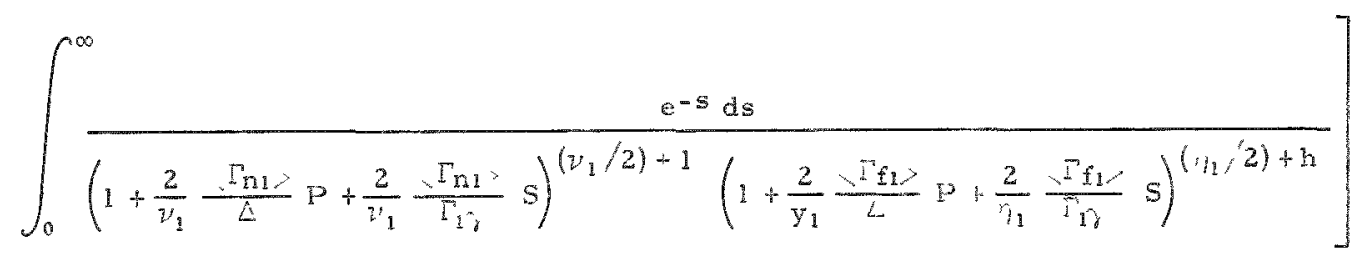

for $\mu>\mu_{0}$

where $\mu_{0}$ is taken as the largest separation at which Eq. 21 and Eq. 22 agree, and

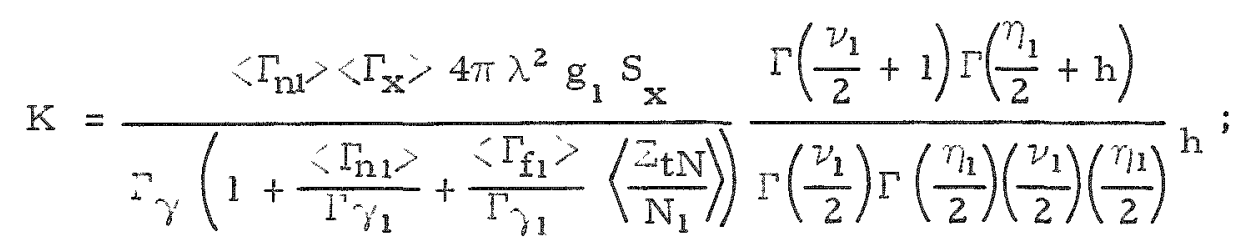




$$
W=\frac{N_{2}}{N_{1}}\left(\frac{4 \pi \pi^{2}}{\left\langle\frac{\Sigma_{t N}}{N_{1}}\right\rangle}\right)^{2} g_{1} g_{2} \frac{\pi\left\langle\Gamma_{n 2}\right\rangle\left\langle\Gamma_{n 1}\right\rangle\left\langle\Gamma_{x}\right\rangle}{2 \Delta \Gamma_{\gamma_{1}}} \frac{\left.\Gamma \frac{1 \nu_{2}}{2}+1\right) \Gamma\left(\frac{\nu_{1}}{2}+1\right) \Gamma\left(\frac{\eta_{1}}{2}+h\right)}{\Gamma\left(\frac{\nu_{2}}{2}\right) \Gamma\left(\frac{\nu_{1}}{2}\right) \Gamma\left(\frac{\eta_{1}}{2}\right)\left(\frac{\nu_{1}}{2}\right)\left(\frac{\nu_{2}}{2}\right)\left(\frac{\eta_{1}}{2}\right)^{h}}
$$

$\Gamma_{\gamma_{1}} \Gamma_{\gamma_{2}}=$ radiative capture widths for isotopes 1 and 2 , respectively:

$$
\begin{aligned}
& \psi \gamma=\psi\left(x, \frac{\Gamma \gamma}{\Delta}\right) \\
& \nu_{1}, \eta_{1}, \nu_{2}=\text { degrees of freedom for the neutron and fission widths of } \\
& =0 \text { for capture: } \\
& L=\left(\frac{N_{2}}{N_{1}}\right)\left(\frac{4 \pi \hbar^{2} g_{2}}{\left\langle\frac{\Sigma_{t N}}{N_{1}}\right\rangle}\right) \frac{\Gamma_{n 2}}{\Gamma_{2 k}}
\end{aligned}
$$
isotope 1 and the neutron widths of isotope 2 , respectively:

Equation 21 is valid for all $\Delta>>>\Gamma_{\gamma}$. In the actual calculation, the first integral of Eq. 21 inside the bracket [] can be replaced by a set of discrete neutron widths, as suggested by Greebler. (7) In the present work, a set of 10 neutron widths is used. The double integral in Eq. 22 can be evaluated numerically by the same integration scheme used in the overlap calculations. Hence, it is possible to obtain a graph with $\left\langle\Gamma_{x} \delta H\right\rangle$ plotted vs. the separation whenever average parameters are given. Finally, for a random separation, the quantity $\left[\left\langle\Gamma_{\mathrm{r}} \delta \mathrm{H}\right\rangle\right]$ is just twice the net area enclosed by $\left\langle\Gamma_{\mathrm{X}} \delta \mathrm{H}\right\rangle$ along $\mathrm{u}$ divided by $\left\langle\mathrm{S}_{\mathbf{2}}\right\rangle_{\mathrm{J}}$, the average spacing of isotope 2.

Calculations have been made for a system of a homogeneous mixture of $\mathrm{Pu}^{239} \mathrm{U}^{238}$ with enrichment ratio of $1: 5$ and background scattering cross section of 300 ber plutonium atom. The results are tabulated in Table III. Figure 7 shows the relative reduction of the effective cross section at $300^{\circ} \mathrm{K}$ along the separation for three different energies. Figures 4,5, and 6 show the corresponding Doppler changes along the separation $\mu$. Since the interference effect characterized by the $\mathrm{v}$-shaped curve has a larger width along the separation, the relative reduction in the Doppler change seems to increase with energy at first. The effect will gradually vanish at still higher energy as $\left\langle\Gamma_{x} H\right\rangle$ approaches its asymptotic value. On the other hand, the $\mathrm{v}$-shaped curve becomes narrower as energy decreases. Consequently, the effect on the Doppler change will decrease with energy 
over a random separation. It seems to indicate that the maximum interference effect occurs somewhere in the intermediate energy region.

Table III

$x$ FOR PU239 WITH INTERFERENCE CORRECTION

Parameters Used:

Spin State J $=1$ only. $\left\langle\frac{\Sigma_{t N}}{N_{1}}\right\rangle=300$ b per atom of $\mathrm{Pu}^{239}$.

Temperature: $300-600 \% ; \quad \nu=1 ; \quad \eta=2$.

$\left\langle\Gamma_{\eta}\right\rangle=0.099 \mathrm{mV} ; \quad \Gamma_{\gamma}=0.039 \mathrm{mV} . \quad\left\langle\Gamma_{n}\right\rangle=\left\langle\Gamma_{n}^{0}\right\rangle \sqrt{E}$

$\frac{\left\langle\Gamma_{n}^{O}\right\rangle}{\langle S\rangle}=10^{-4} ; \quad\langle S\rangle_{J=1}=3.86 \mathrm{eV}$.

\begin{tabular}{c|c|c|c|c|c|c}
\hline \multirow{2}{*}{ E keV } & \multicolumn{3}{|c|}{ Fission } & \multicolumn{3}{c}{ Capiure } \\
\cline { 2 - 7 } & $\delta \tilde{\sigma}_{\text {f0 }}$ (Mathod B) & $\delta \tilde{\sigma}_{f}$ & Reduction (\%) & $\delta \tilde{\sigma}_{\gamma_{0}}$ Method B) & $\delta \tilde{\sigma}$ & Reduction (\%) \\
\hline 6.5 & 0.0087 & -0.002695 & 130.98 & 0.00459 & -0.001544 & 131.19 \\
2.25 & 0.0413 & 0.0009739 & 07.64 & 0.0259 & 0.0005825 & 97.75 \\
0.61 & 0.193 & 0.10814 & 43.97 & 0.13715 & 0.06747 & 50.81 \\
\hline
\end{tabular}

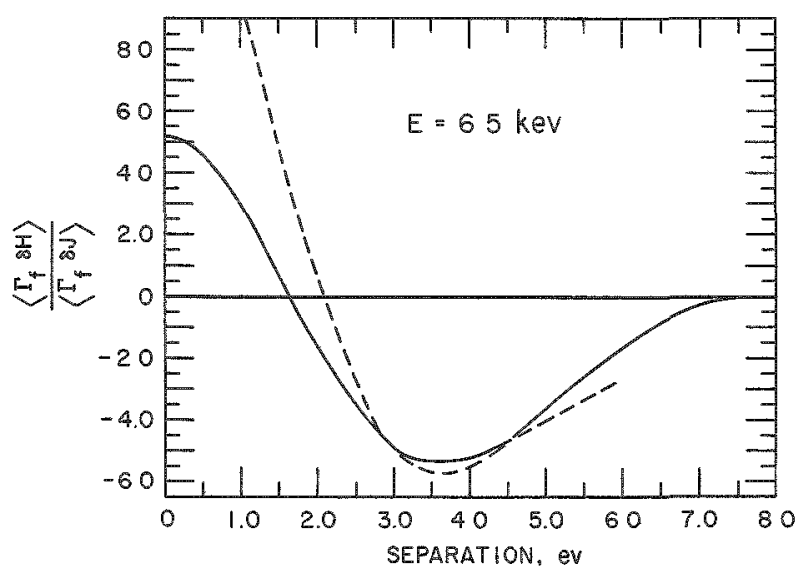

Eig. 4. Doppler Change along the Separation for $E=6.5 \mathrm{kev}$

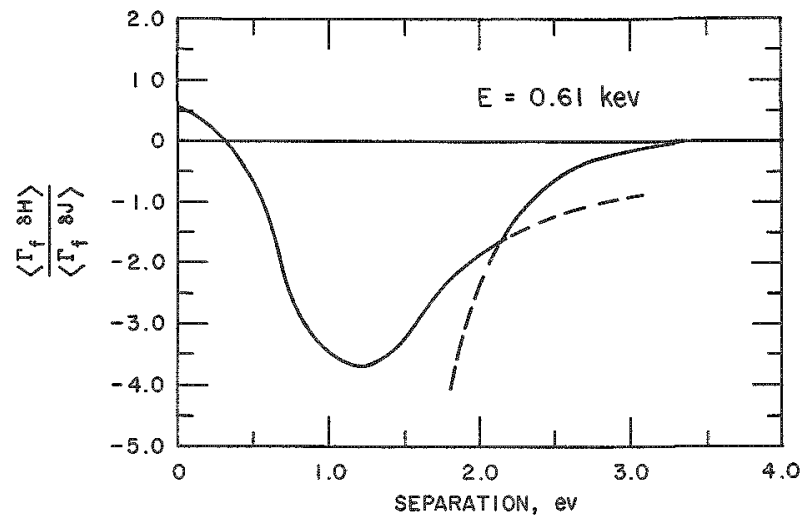

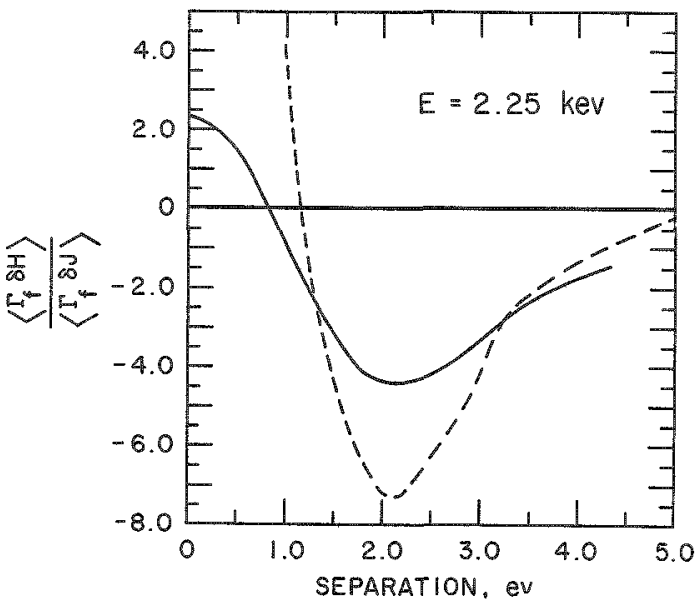

Fig. 5. Doppler Change along the Separation for $E=2.25 \mathrm{kev}$
Fig. 6

Doppler Change along the Separation for $E=0.61 \mathrm{kev}$ 


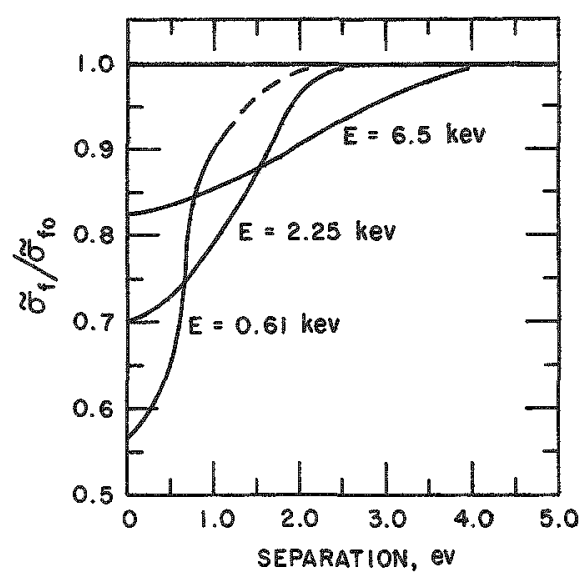

Fig. 7

$\tilde{\sigma}_{f} / \widetilde{\sigma}_{f_{0}}$ at $300^{\circ} \mathrm{K}$

where

$\tilde{\sigma}_{f}$ - effective fission cross section with interference

$\tilde{\sigma}_{f_{0}}$ - effective fission cross section without interference

V. Conclusion

The net effect of both types of interference interactions is to reduce or even make negative the Doppler coefficient of fissile isotopes in the intermediate energy region. The second type of interaction, which vanishes at high neutron energy, seems to be more important than the overlapping effect in the intermediate energy region. The drawback of the present work is that the combined effects of two types of interferences have not been taken into account. The second type of interference will undoubtedly reduce the overlapping effect also if they occur simultaneously. For $\mathrm{Pu}^{239}$ the overlapping effect is relatively unimportant in the interme diate energy region and can be neglected. For $U^{235}$, however, both effects may become significant. 


\section{Appendix}

By noting the convolution properties of $\psi$-function, $\psi_{1} k$ can be written in the following form:

$$
\begin{aligned}
\psi_{1 \mathrm{k}} & =\frac{\xi_{1}}{2 \sqrt{\pi}} \int_{-\infty}^{\infty} \frac{e^{-\frac{\xi_{1}^{2}}{4}\left(\mathrm{x}_{1}-\mathrm{y}\right)^{2}}}{1+\mathrm{y}^{2}} \mathrm{dy} \\
& =\frac{\xi_{1}}{2} \int_{-\infty}^{x} e^{-\eta^{2}-\frac{\Gamma_{1 k}}{\Delta}|\eta|} e^{-i \frac{2\left(E-E_{1 k}\right)}{\Delta} \eta} \mathrm{d} \eta
\end{aligned}
$$

By changing the oxder of integration and averaging over $P_{\nu_{1}}\left(\frac{\Gamma n l}{\langle\Gamma n l\rangle}\right)$ and $P_{\eta 1}\left(\frac{\Gamma \in I}{\langle\Gamma\{1\rangle}\right)$, the expression $\left\langle\Gamma_{\mathrm{X}} \delta \mathrm{H}\right\rangle$ is reduced to Eq. 21.

\section{References}

1. A. M. Lane, J. E. Lynn, and J. S. Story, An Estimation of Doppler Effect in Fast Neutron Reactors, AERE T M 137 (1956).

2. R. B. Nicholson, Doppler Effect in Fast Reactors, APDA-139 (1960).

3. C. C. Graves, A Review of Analytical and Experimental Work Pertinent to the Doppler Effect, UNC-5034 (1962).

4. F. G. Tricomi, Integral Equations, Interscience Publishers, Inc., (1957).

5. I. N. Sneddon, Fourier Transforms, McGraw-Hill Book Co,, Inc., New York (195ॉ).

6. J. Codd and P. J. Collins, Plutonium-239 and Uranium-238 Resonance Interaction Effects in a Dilute Fast Reactor, E.A.E.S. Symposium on Advances in Reactor Theory (1963).

7. P. Greebler and E. Goldman, Doppler Calculations for Large Fast Reactors, GEAP-4092 (1962). 


$\frac{\text { Discussion of Paper }}{\text { Presented by Mr. Hwang }}$

MR. FISCHER (Atomic Power Development Assoc.):

As far as I see, the correction fraction $H$ takes only into account the overlap between two neighboring resonances, and you would neglect the overlap between secondary and the higher-order neighbors. Is this correct?

MR, HWANG:

No, $\delta H$ is defined as the general term, if we assume there is only one interference interaction at a time.

MR. FISCHER:

My calculations show that in the Doppler effect the first-order correction is negative for higher spacings, and becomes positive for very small spacings. Now, if the resonances are very close together, there must be a considerable effect of higher-order interference between the resonances, and so it is consistent with the general idea that by the total overlap the Dopplex coefficient must be smaller.

MR. HWANG:

If I understand your question. $\delta H$ is the general term. If we were only talking about the interference between the neighboring resonances for fissile material, we can expand $\delta H$ into a series, in which $I$ also take into account the higher-order corrections. As for this interference between $\mathrm{U}^{238}$ and $\mathrm{Pu}^{239}$, we cannot expand this into a series because $\mathrm{A}_{2}$ is quite large, sometimes even larger than the beta factor.

MR。LOEWENSTEIN (Argonne):

I wonder if you are in agreement with Dr. Codd's reply to Mr. Nicholson's question [see discussion of Paper VII-2].

MR. HWANG:

Yes. The question was, if we fix the temperature of fertile material, and raise the temperature in fissile material only, as is the case in the critical assembly where the fissile and fertile materials are physically separated, what is the effect? In that case, I would say this, if we hold $\psi_{2}$ constant in Eq. I we would not have the additional interference from the fertile material due to the temperature rise. Of course, we do have some 
localized increase in scattering. We would expect some decrease, but not as much as the change in $\mathrm{J}^{*}$ or $\Delta \mathrm{J} *$.

The question also depends on the ratio of fertile to fissile material. But one thing, though, the striking difference is that the change in cross section would reduce, but would never become negative. The reason is we do not have the spread in $\psi_{2}$. If we hold $\psi_{2}$ constant, then, the total effect amounts to a localized increase in the potential cross section. As a result, the change in Doppler broadening is going to reduce somewhat but will not change sign.

MR. OTT (Karlsruhe):

I also want to make a comment on the question of Dr. Nicholson. I think that separation of materials in a critical facility, if the separation is faixly small, of the order of one millimeter, should not affect the Doppler effect very much, because the neutrons which produce the Doppler effect have mean free paths of the order of the scattering length.

MR. NICHOLSON (University of Wisconsin):

I am not quite sure whether the last answer to my previous question covered the whole problem. As I understand it, Dr. Hwang's term "J" refers to the calculation relating to the capture and fission, say, in the $\mathrm{U}^{235}$. But how about the effect of broadening of the $\mathrm{U}^{235}$ resonances on the capture in $\mathrm{U}^{238}$ ?

MR. HWANG:

I think Dr. Codd considered that point. The effect is small because the magnitude of the curve depends on the magnitude of $\mathrm{A}_{2}$ which is very small in the intermediate energy region if you have a dilute reactor. I don't think that term would come in too significantly.

MR. STORRER (EURATOM):

I would like to say, in answer to Mr. Nicholson's question this point is one of the main objects of the paper I am going to give this afternoon.

MR. CODD (Winfrith):

When Mr. Nicholson asked me his question, I said that the Doppler coefficient of the fuel in a critical assembly might be slightly increased. What I had in mind here was the fact that there would be an interaction of the plutonium broadening on, say, the $U^{238}$, which will tend to make the plutonium coefficient a little more negative. I think this would refer to 
the sort of system I was talking about. The effect might be different in a different critical assembly with more plutonium relative to the $\mathrm{U}^{238}$ 


\title{
EFFECT OF PARAMETER VARIATION IN
}

DOPPLER EFEECT CALCULATIONS

\author{
H. H. Hummel and A. L. Rago \\ Argonne National Laboratory
}

Argonne, Illinois

(Paper presented by Mr. Hummel)

In order to obtain a better understanding of the uncertainty in Doppler effect calculations because of uncertainty in nuclear data, a series of calculations has been carried out in which parameters were arbitrarily varied. Resonance integral calculations were done in the narrow-resonance approximation. No account was taken of multilevel effects in fissile material or of interference effects between $\mathrm{U}^{238}$ and $\mathrm{Pu}^{239}$ resonances. Distribution functions for neutrons and fission widths were taken into account by using ten discrete values for neutron width and five for fission width. $\%$ functions were calculated as described by O'Shea and Thacher.(1) Variation was carried for $s$-wave parameters only, as the $p$ wave was found to have only a small influence on the Doppler effect.

The calculations were performed for a fundamental mode flux by means of the ELMOE program. (2) The coarse-group cross-section set used was a 22-group one constructed by using the first 13 groups of the YOM set(3) and adding 9 groups for lower energies. For the 13 groups of higher energy ( 11 in the case of $U^{238}$ and Pu ${ }^{240}$ ), the YOM cross sections were used except that $\nu$ and $\alpha$ of $\mathrm{Pu}^{239}$ and $\sigma \gamma$ of $\mathrm{U}^{238}$ were altered to fit more recent data. (4-7) For the low-energy region, the following parameters were used:

$\mathrm{Pu}^{239}$

s wave

( $\left.\Gamma_{\mathrm{n}}^{0}\right) / \mathrm{D}$ was varied between 0.7 and $1.5 \times 10^{-4}$, which covers the range of uncertainty suggested by Bollinger et al. (8)

The channels per fission, $\eta$, were varied between 1 and 3 . Because this was found to have a strong influence on $\alpha,\left\langle\Gamma_{f}\right\rangle$ was also varied from the value of $0.099 \mathrm{eV}$ which was used as a standard value. For $\eta=1$ values for $\left\langle\Gamma_{f}\right\rangle$ of 0.099 and $0.130 \mathrm{eV}$ and for $\eta=3$ values of 0.099 and $0.080 \mathrm{eV}$ were used. This was considered to cover a reasonable range of $\alpha$ as shown in Table I. (F) was not varied as a function of energy since the energy range in which the Pu ${ }^{239}$ Doppler effect is significant is small compared with excitation energies of the compound nucleus. (9)

$$
\Gamma_{\gamma} \text { was chosen to be } 0.039 \mathrm{eV} .(8)
$$


Table I

$$
\begin{gathered}
a\left(=\sigma \gamma / \sigma_{\hat{\rho}}\right) \text { AT } 2500^{\circ} \mathrm{K} \text { AS A FUNCTION OF CHANNELS PER FISSION } \\
\text { AND AVERAGE FISSION WIDTH } \$ \text {-WAVE ONLY }
\end{gathered}
$$

$\sigma_{\mathrm{p}}=300 \mathrm{~b} ;\left\langle{ }_{\mathrm{n}}^{-0}\right\rangle / \mathrm{D}=1 \times 10^{-4}$

\begin{tabular}{l|c|c|c|c|c}
\hline \multirow{2}{*}{$E, k e V$} & \multicolumn{2}{|c|}{$\eta=1$} & $\eta_{0}$ & \multicolumn{2}{|c}{$\eta^{\prime}=3$} \\
\cline { 2 - 6 } & $\left\langle\Gamma_{\mathfrak{f}}\right\rangle=0.099 \mathrm{eV}$ & $\left\langle\Gamma_{\mathfrak{f}}\right\rangle=0.130 \mathrm{eV}$ & $\left\langle\Gamma_{f}\right\rangle=0.099 \mathrm{eV}$ & $\left\langle\Gamma_{\mathfrak{f}}\right\rangle=0.080 \mathrm{eV}$ & $\left\langle\Gamma_{\mathfrak{f}}\right\rangle=0.099 \mathrm{eV}$ \\
\hline 6.50 & 0.712 & 0.589 & 0.560 & 0.610 & 0.507 \\
2.25 & 0.765 & 0.636 & 0.590 & 0.632 & 0.525 \\
0.61 & 0.827 & 0.690 & 0.619 & 0.650 & 0.543 \\
0.18 & 0.865 & 0.723 & 0.635 & 0.665 & 0.553 \\
0.061 & 0.870 & 0.720 & 0.636 & 0.065 & 0.553 \\
\hline
\end{tabular}

Level spacing was usually taken to be $11.6 \mathrm{eV}$ for $\mathrm{J}=0$ and $3.86 \mathrm{eV}$ for $J=1$, based on the work of Bollinger et al. (8) The effect on cross sections of using the level spacing found by Egelstaff, (10) assumed equivalent to $7.2 \mathrm{eV}$ for $\mathrm{J}=0$ and $2.4 \mathrm{eV}$ for $\mathrm{J}=2$, was also investigated.

Calculations were made for isolated resonances at the following energies, in keV: $6.5,2.25,0.61,0.18$, and 0.061 . Results of these calculations are given in Table II. Group average values were found by interpolation. The fission cross section down to $9.1 \mathrm{keV}$ was taken from YOM, (3) and temperature variation of fission and capture cross sections of $\mathrm{Pu}^{239}$ were assumed to be negligible above this energy.

Based on earlier work of $R$. Hwang, an overlap correction was applied for $\mathrm{Pu}^{239}$ which reduced $\delta \sigma_{\mathrm{f}}$ and $\delta \sigma_{\gamma}$ at $6.5 \mathrm{keV}$ by about $10 \%$ and was negligible at lower energies. This correction would have negligible influence on the calculated Doppler effect. Subsequent refinements of this work(11) have indicated that the se corrections are too small and that $\delta \sigma_{\mathrm{f}}$ and $\delta \sigma \gamma$ should have been multiplied by factors of about 0.74 at $6.5 \mathrm{keV}$, 0.83 at $2.25 \mathrm{keV}$, and 0.89 at $0.61 \mathrm{keV}$. This would probably have the effect of reducing the plutonium Doppler effect by about $10 \%$ over the values reported here.

Heterogeneity effects were not taken into account for $\mathrm{Pu}^{239}$. The homogeneous $\sigma \gamma$ was based on scattering cross sections of $10 \mathrm{~b}$ for steel, uranium, and plutonium, of $3 \mathrm{~b}$ for sodium, of $3.8 \mathrm{~b}$ for oxygen, and of $4.7 \mathrm{~b}$ for carbon.

\section{p wave}

The $\mathrm{p}$-wave contribution to the $\mathrm{Pu}^{239}$ Doppler effect was found to be negligible and was not taken into account. A temperature-independent correction was added to the calculated $s$-wave cross section to bring it into agreement with measured values. This addition was $0.7 \mathrm{~b}$ for $\jmath_{\mathrm{f}}$ and $0.4 \mathrm{~b}$ 
Table II

S-WUAV CALCULATIONS OF PU239 CROSS SECTIONS. NO OVERLAP CORRECTION.

$\left\langle\Gamma_{f}\right\rangle=0.009 \mathrm{eV} ; \quad\left\langle\Gamma_{n}^{0}\right\rangle / D \times 10^{4}-1.0 \quad$ Level spacing $J_{0}=11.6 \mathrm{eV} ; \quad J_{1}=3.86 \mathrm{eV}$, unless otherwise noted.

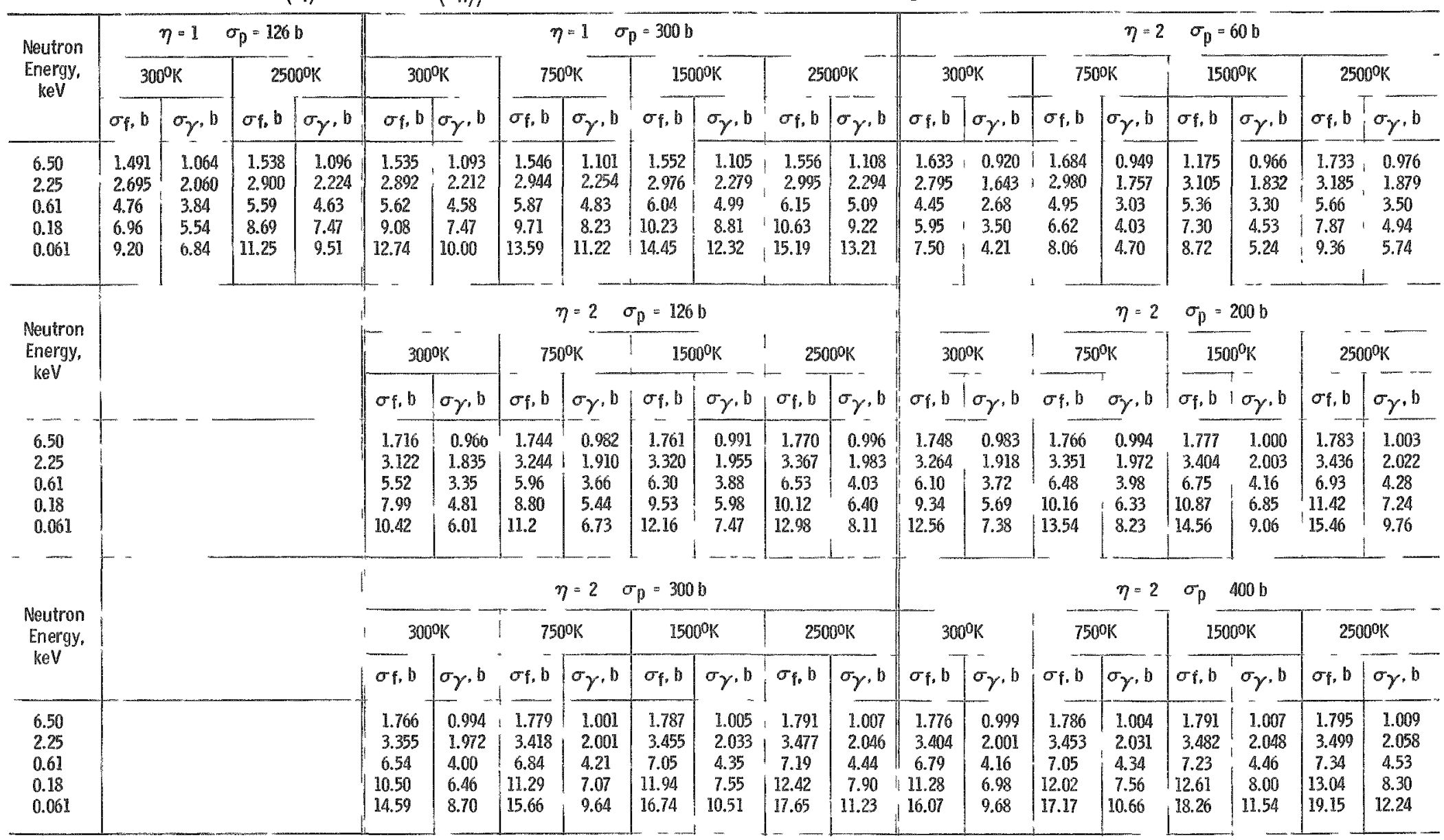


$\left\langle\Gamma_{f}\right\rangle-0.099 \mathrm{eV} ; \quad\left\langle\Gamma_{n}^{0}\right\rangle / D \times 10^{4}-1.0$

$\left\langle\Gamma_{\mathrm{f}}\right\rangle \quad 0130 \mathrm{eV} ; \quad\left\langle\Gamma_{n}^{0}\right\rangle / D \times 10^{4} \quad 1.0$

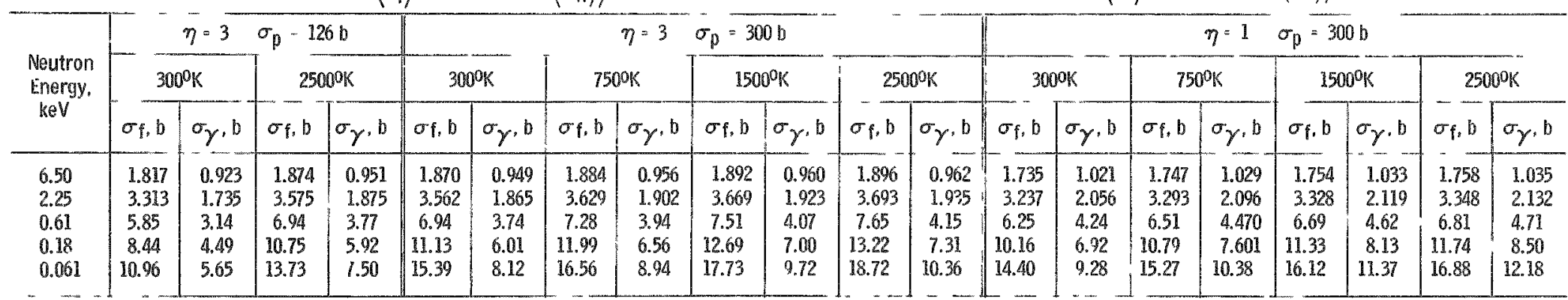

$\left\langle\Gamma_{\mathrm{f}}\right\rangle=0.099 \mathrm{eV}$

$\left\langle\Gamma_{n}^{0}\right\rangle / D \times 10^{4}=0.7$

$\left\langle\Gamma_{\mathrm{f}}\right\rangle=0.099 \mathrm{eV}: \quad\left\langle\Gamma_{n}^{0}\right\rangle / D \times 10^{4} 0.83$

$\left\langle\Gamma_{f}\right\rangle=0.099 \mathrm{eV} ; \quad\left\langle\Gamma_{n}^{0}\right\rangle / 0 \times 10^{4}=1.2$

\begin{tabular}{|c|c|c|c|c|c|c|c|c|c|c|c|c|c|c|c|c|c|c|c|c|}
\hline \multirow{3}{*}{$\begin{array}{l}\text { Neutron } \\
\text { Energy, } \\
\text { keV }\end{array}$} & \multicolumn{4}{|c|}{$\eta=2 \quad \sigma_{p}=300 \mathrm{~b}$} & \multicolumn{8}{|c|}{$\eta-2 \quad \sigma_{p}-300 b$} & \multicolumn{8}{|c|}{$\eta=2 \quad \sigma_{p}-300 b$} \\
\hline & \multicolumn{2}{|c|}{$3000 \mathrm{~K}$} & \multicolumn{2}{|c|}{$2500^{\circ} \mathrm{K}$} & \multicolumn{2}{|c|}{$300^{\circ} \mathrm{K}$} & \multicolumn{2}{|c|}{$750^{\circ} \mathrm{K}$} & \multicolumn{2}{|c|}{$15000 \mathrm{~K}$} & \multicolumn{2}{|c|}{$2500^{\circ} \mathrm{K}$} & \multicolumn{2}{|c|}{$300^{\circ} \mathrm{K}$} & \multicolumn{2}{|c|}{$750^{\circ} \mathrm{K}$} & \multicolumn{2}{|c|}{$1500^{\circ} \mathrm{K}$} & \multicolumn{2}{|c|}{$2500^{\circ} \mathrm{K}$} \\
\hline & $\sigma_{f, b}$ & $\sigma_{\gamma}, b$ & $\sigma_{f, b}$ & $\sigma_{y}, b$ & $\sigma f, b$ & $\sigma_{\gamma}, b$ & $\sigma f, b$ & $\sigma_{y} \cdot b$ & $\sigma f, b$ & $\sigma_{y}, b$ & $\sigma r, b$ & $\sigma_{\gamma}, b$ & $\sigma f, b$ & $\sigma_{y}, b$ & $\sigma f, b$ & $\sigma_{y}, b$ & $\sigma f, b$ & $\sigma_{\gamma}, b$ & $\sigma f, b$ & $\sigma_{\gamma}, b$ \\
\hline 6.50 & 1.381 & 0.801 & 1.395 & 0.809 & 1.557 & 0.890 & 1.567 & 0.895 & 1.572 & 0.898 & 1.575 & 0.900 & 1.988 & 1.101 & 2.005 & 1.110 & 2.014 & 1.115 & 2.020 & 1.118 \\
\hline 2.25 & 2.583 & 1.561 & 2.654 & 1.605 & 2.934 & 1.750 & 2.982 & 1.780 & 3.010 & 1.797 & 3.026 & 1.807 & 3.804 & 2.203 & 3.885 & 2.252 & 3.934 & 2.281 & 3.963 & 2.297 \\
\hline 0.61 & 5.04 & 3.16 & 5.43 & 3.43 & 5.72 & 3.54 & 5.96 & 3.71 & 6.12 & 3.82 & 6.23 & 3.89 & 7.41 & 4.47 & 7.79 & 4.73 & 8.06 & 4.91 & 8.23 & 5.02 \\
\hline 0.18 & 8.25 & 5.186 & 9.52 & 6.16 & 9.28 & 5.77 & 0.93 & 6.29 & 10.46 & 6.68 & 10.84 & 6.96 & 11.79 & 7.16 & 12.73 & 7.89 & 13.52 & 8.46 & 14.11 & 8.88 \\
\hline 0.061 & 11.66 & 7.105 & 13.91 & 9.00 & 13.00 & 7.84 & 13.93 & 8.67 & 14.85 & 9.42 & 15.62 & 10.03 & 16.26 & 9.58 & 17.47 & 10.63 & 18.72 & 11.64 & 19.78 & 12.47 \\
\hline
\end{tabular}

$\left\langle\Gamma_{\mathrm{f}}\right\rangle \quad 0.099 \mathrm{eV}$;

$\left\langle\Gamma_{n}^{0}\right\rangle / 0 \times 10^{4}=1.5$

$\left\langle\Gamma_{\mathrm{f}}\right\rangle=0.080 \mathrm{eV} ;$

$\left\langle\Gamma_{n}^{0}\right\rangle / D \times 10^{4}-1.0$

\begin{tabular}{|c|c|c|c|c|c|c|c|c|}
\hline \multirow{3}{*}{$\begin{array}{l}\text { Neutron } \\
\text { Energy. } \\
\text { keV }\end{array}$} & \multicolumn{4}{|c|}{$\eta=2 \quad \sigma_{p}=300 \mathrm{~b}$} & \multicolumn{4}{|c|}{$\eta=3 \quad \sigma_{p}=300 \mathrm{~b}$} \\
\hline & \multicolumn{2}{|c|}{$3000 \mathrm{~K}$} & \multicolumn{2}{|c|}{$2500^{\circ K}$} & \multicolumn{2}{|c|}{$3000 \mathrm{~K}$} & \multicolumn{2}{|c|}{$2500^{\circ} \mathrm{K}$} \\
\hline & $\sigma_{f, b}$ & $\sigma_{\gamma}, \mathrm{b}$ & $\sigma_{f}, \mathrm{~b}$ & $\sigma_{\gamma}, b$ & $\sigma_{\mathrm{f}, \mathrm{b}}$ & $\sigma_{y, b}$ & $\sigma_{f, b}$ & $\sigma_{\gamma} \cdot b$ \\
\hline 6.50 & 2.281 & 1.239 & 2.324 & 1.262 & 1.670 & 1.022 & 1.693 & 1.036 \\
\hline 2.25 & 4.404 & 2.504 & 4.620 & 2.629 & 3.212 & 2.025 & 3.332 & 2.101 \\
\hline 0.61 & 8.56 & 5.08 & 9.65 & 5.79 & 6.31 & 4.09 & 6.97 & 4.54 \\
\hline 0.18 & 13.50 & 8.07 & 16.37 & 10.17 & 10.10 & 6.58 & 12.10 & 8.05 \\
\hline 0.061 & 18.48 & 10.73 & 22.60 & 14.07 & 13.84 & 8.85 & 17.12 & 11.41 \\
\hline
\end{tabular}

Egelstaff spacing $J_{0}-7.2 \quad J_{1} 2.4$

$\left\langle\Gamma_{f}\right\rangle=0.099 \mathrm{eV} ; \quad\left\langle\Gamma_{n}^{0}\right\rangle / \mathrm{D} \times 10^{4}-1.0$

\begin{tabular}{|c|c|c|c|c|c|c|c|}
\hline & & & $\eta=2$ & $\sigma_{p}-30$ & & & \\
\hline \multicolumn{2}{|c|}{$3000 \mathrm{~K}$} & \multicolumn{2}{|c|}{$7500 \mathrm{~K}$} & \multicolumn{2}{|c|}{$1500^{\circ} \mathrm{K}$} & \multicolumn{2}{|c|}{$25000 \mathrm{~K}$} \\
\hline$\sigma_{f, b}$ & $\sigma_{\gamma}, \mathrm{b}$ & $\sigma_{f, b}$ & $\sigma_{\gamma}, b$ & $\sigma_{f}, b$ & $\sigma_{\gamma}, b$ & $\sigma_{\mathfrak{f}, b}$ & $\sigma_{\gamma}, b$ \\
\hline 2.039 & 1.193 & 2.048 & 1.199 & 2.054 & 1.202 & 2.058 & 1.204 \\
\hline 3.794 & 2.312 & 3.843 & 2344 & 3.872 & 2.362 & 3.889 & 2.372 \\
\hline 7.40 & 4.67 & 7.66 & 4.86 & 7.83 & 4.97 & 7.93 & 504 \\
\hline 12.20 & 772 & 12.96 & 8.33 & 13.54 & 8.78 & 13.96 & 9.09 \\
\hline 1736 & 10.65 & 18.54 & 11.71 & 19.67 & 12.65 & 20.58 & 13.38 \\
\hline
\end{tabular}


for $\sigma_{\gamma}$ at $6.5 \mathrm{keV}$, and zero at lower energies. This correction is rather arbitrary since the data for $\sigma_{f}$ are rather scattered in this energy range and there is little information on $\sigma \gamma$.

$\mathrm{U}^{238}$

\section{s-wave}

Below 1 keV, resolved resonance parameters were taken from BNL-325.(12)

Calculations have also been done with the use of more recent data on resolved resonances extending to $1.8 \mathrm{keV}$. (13) A Porter-Thomas distribution of neutron widths was used. $\left\langle\Gamma_{\mathrm{n}}^{0}\right\rangle / \mathrm{D}$ was varied from 0.78 to 1.13 , which covers what is believed to be the range of uncertainty. D was fixed at $18.5 \mathrm{eV}$, and $\Gamma_{\gamma}$ was taken to be $0.0246 \mathrm{eV}$. Results are given in Table III for the BNL-325 parameters and in Table IV for the data of Firk et al. (13) The flux weighting for the resolved resonances in these calculations with the available code is $1 / E$, which leaves something to be desired. It is planned in the new version of ELMOE on the CDC-3600 to insert resolved resonances directly into the appropriate fine group. For the values of $\sigma_{p}=40 \mathrm{~b}$ in Table III, a weighting with a representative flux was performed by hand. The values for $\sigma_{\mathrm{p}}=40 \mathrm{~b}$ seem to be reasonably well in line with those at other op's, so that the error in flux weighting may not be too important.

Table III

s-WAVE COMTRIBUTION To $\sigma_{\gamma}$ of $\mathrm{U}^{238}$, BARNS

BAN -325 Data!12

\begin{tabular}{|c|c|c|c|c|c|c|c|c|c|c|c|c|c|c|}
\hline \multirow{3}{*}{ Group } & \multirow{3}{*}{\multicolumn{2}{|c|}{ Lit, hev }} & \multicolumn{12}{|c|}{ Unresolved Region: $\left(\Gamma_{n}^{0}\right) / D=0.94 \times 30^{-4} ; 0=185 \mathrm{ev} ; \Gamma_{Y}=0.0246 \mathrm{ev}$} \\
\hline & & & \multicolumn{3}{|c|}{$\sigma_{p}=40 \mathrm{~b}$} & \multicolumn{3}{|c|}{$\sigma_{p}=40 b$} & \multicolumn{3}{|c|}{$\sigma_{p}=600$} & \multicolumn{3}{|c|}{$\sigma_{p}=100 \mathrm{~b}$} \\
\hline & & & 3000 & $7500 \mathrm{~K}$ & $750-3000$ & $300 \% \mathrm{~K}$ & $7500 \mathrm{~K}$ & $750-300^{9}$ & $300^{\circ} \mathrm{K}$ & $750^{\circ} \mathrm{K}$ & $750-300^{\circ}$ & $300^{\circ} \mathrm{K}$ & $750 \% \mathrm{~K}$ & $750-3000$ \\
\hline 12 & $15.0-25.0$ & 20.0 & 0.1553 & 0.1635 & 0.0082 & 0.1613 & 0.1683 & 0.0070 & 0.1684 & 0.1737 & 0.0053 & 0.1750 & 0,1787 & 0.0037 \\
\hline 13 & $9.1-$ & $12.0 \mathrm{i}$ & 0.2246 & 0.2413 & 0.0167 & 0.2368 & 0.2516 & 0.0148 & 0.2517 & $0.263 \%$ & 0.0120 & 0.2666 & 0.2753 & 0.0087 \\
\hline 14 & 4.0 . & $6.5 !$ & 0.3321 & 0.3674 & 0.033 & 0.3576 & 0.3905 & 0.0329 & 0.3907 & 0.4194 & 0.0287 & 0.0264 & 0.4490 & 0.0226 \\
\hline 15 & $2.8-$ & (3.4) & 0.469 & 0.536 & 0.067 & 0.517 & 0.584 & 0.067 & 0.585 & 0.647 & 0.062 & 0.664 & 0.718 & 0054 \\
\hline \multirow[t]{2}{*}{16} & 2.0 & (2.5) & 0.537 & 0.625 & 0.038 & 0.600 & 0.689 & 0.089 & 0.690 & 0.776 & 0006 & 0.800 & 0.873 & 0.078 \\
\hline & & (2.1) & 0.576 & 0.676 & 0.100 & 0.048 & 0.750 & 0.102 & 0.752 & 0.854 & 0.102 & 0.833 & 0.978 & 0.095 \\
\hline 17 & $1.4-$ & (1.7) & 0.623 & 0.739 & 0.116 & 0.707 & 0.827 & 0.120 & 0.839 & 0.952 & 1. 122 & 0.987 & 1.106 & 0.119 \\
\hline 18 & 1.0 & $1.2^{2}$ & 0.698 & 0.839 & 0.141 & 0.891 & 0.952 & $0.15 !$ & 0.957 & 1.119 & 0.161 & 1.167 & 1.331 & 0.164 \\
\hline 19 & 0.55 & & 0.831 & 1,014 & 0.183 & 0.927 & 1.117 & 0.190 & 1.183 & 1,417 & 0.234 & 1.495 & 1.756 & 0.261 \\
\hline 20 & 0.30 & & 0.727 & 0.893 & 0.171 & 0.912 & 1.120 & 0.208 & 1.041 & 1.258 & 1).217 & 1.318 & 1.557 & 0239 \\
\hline 21 & 0.10 & & 1,086 & 1.258 & 0.172 & 1.122 & 1.350 & 0.228 & 1,623 & 1.907 & 0,284 & 2.172 & 2.567 & 0.395 \\
\hline 22 & 0.03 & & 1.562 & 1,664 & 0.102 & 1.827 & 1.951 & 0.124 & 2.268 & 2.446 & 0.178 & 2.984 & 3.262 & 0.279 \\
\hline \multirow{2}{*}{ Grous } & \multirow{2}{*}{\multicolumn{2}{|c|}{$\underline{E}, \mathrm{keV}$}} & \multicolumn{3}{|c|}{$\sigma_{p}-40 b$} & \multicolumn{3}{|c|}{$\sigma_{p}=40 \mathrm{~b}$} & \multicolumn{3}{|c|}{$\sigma_{0}=600$} & \multicolumn{3}{|c|}{$o_{p}=1000$} \\
\hline & & & $15000 \mathrm{~K}$ & $25000 \%$ & $2500-1500$ & $15000 \mathrm{~K}$ & $25000 \%$ & $2500-15000$ & $15000 \mathrm{~K}$ & $2500^{\circ} \mathrm{K}$ & $2500-1500^{\circ}$ & $1500^{\circ} \mathrm{K}$ & $25000 \%$ & $2500-1500^{\circ}$ \\
\hline$\overline{12}$ & $15.0-25.0$ & 20.0 & 0.1687 & 0.1719 & 0.0032 & 0.1727 & 0.1753 & 0.00 & 0.17 & 0.1789 & 0,00 & 0.1808 & 0.1821 & 0.0013 \\
\hline 13 & 9.1 & 120 & 0.2525 & 0.2599 & 0.0074 & 0.2612 & 0.2675 & 0.00 & 0.2713 & 0.2760 & 0.00 & 0.2806 & 02838 & 0.0032 \\
\hline 14 & $4.0-$ & 36.51 & 0.3927 & 0.4100 & 0.0773 & 0.4134 & 0.4288 & 0.0154 & 0.4386 & 0.4511 & 0.0125 & 0.463 & 0.4725 & 00001 \\
\hline 15 & 2.8 & 3.4. & 0.588 & 0.626 & 0.038 & 0.637 & 0.699 & 0.032 & 0.692 & 0.723 & 0.031 & 0.755 & 0.780 & 0.025 \\
\hline I6 & $2.0-$ & 2.5 & 0.695 & 0.747 & 0,052 & 0.757 & 0.807 & 0.050 & 0.841 & 0.86 & 0.045 & 0.934 & 0.971 & 0.037 \\
\hline & & (2.1) & 0.758 & 0.819 & 0.061 & 0.831 & 0.891 & 0.060 & 0.932 & 0.987 & 0.055 & 1.046 & 1.0993 & 0.047 \\
\hline 17 & 1.4- & $18.7 \mathrm{i}$ & 0.835 & 0.909 & 0.074 & 0.924 & 0.997 & 0.073 & 1.048 & 1.117 & 0069 & 1.193 & 1.254 & 0.061 \\
\hline 18 & $1.0=$ & 01,2 & 0.962 & 1.658 & 0.096 & 1.080 & 1.178 & 0.098 & 1.249 & 1.347 & 0.098 & $1.45 \%$ & 1.548 & 0.091 \\
\hline 19 & 0.55 & & 1.184 & 1.324 & 0.140 & 1.262 & 1.402 & 0.14 & 1.621 & 1.783 & 0.162 & 1.972 & 2.136 & 0.164 \\
\hline 20 & 0.30 & & 1.034 & 1.282 & 0.128 & 1.312 & 1.439 & 0.128 & 1.044 & 1.589 & 0.145 & 1.752 & 1.898 & 0.146 \\
\hline 21 & 0.10 & & 1.449 & 1.603 & 0.182 & 1.602 & 1.83 & 0.22 & 2.210 & 2,490 & 0.280 & 2.976 & 3.342 & 0.367 \\
\hline 22 & 0.03 & & 1.782 & 1.008 & 0.126 & 2.104 & 2.266 & 0.162 & 2.666 & 2.895 & 0.229 & 3.602 & 3.954 & 0.352 \\
\hline
\end{tabular}

(Energies in parentheses are assumed averages for the group in the unresolved region. For Group 16 , an average of the values at 2.5 and 2.1 keV was used." 
Table IV

$\sigma_{\gamma}$ OF U $\mathrm{U}^{238}$ FROM DATA OF FIRK et al.,(13) BARNS $\sigma_{p}=40 \mathrm{~b} ; \quad \Gamma_{\gamma}=0.0246 \mathrm{eV}$

\begin{tabular}{c|c|c|c|c|c|c|c}
\hline Group & $E_{\mathrm{L}, \mathrm{keV}}$ & $300^{\circ} \mathrm{K}$ & $750^{\circ} \mathrm{K}$ & $\delta(300-750)$ & $1500^{\circ} \mathrm{K}$ & $2500^{\circ} \mathrm{K}$ & $\delta(1500-2500)$ \\
\hline 17 & $1.4-2.0$ & 0.722 & 0.860 & 0.138 & 0.973 & 1.059 & 0.086 \\
18 & 1.0 & 0.900 & 1.039 & 0.139 & 1.146 & 1.224 & 0.077 \\
19 & 0.55 & 0.823 & 0.985 & 0.162 & 1.134 & 1.257 & 0.123 \\
20 & 0.30 & 0.677 & 0.796 & 0.119 & 0.902 & 0.989 & 0.081 \\
21 & 0.10 & 1.243 & 1.405 & 0.162 & 1.589 & 1.769 & 0.180 \\
22 & 0.03 & 1.839 & 1.967 & 0.125 & 2.120 & 2.285 & 0.165 \\
\hline
\end{tabular}

p-wave

$\left\langle\Gamma_{\mathrm{n}}^{0}\right\rangle / \mathrm{D}$ was fixed at $1.5 \times 10^{-4}$, which seemed to give a reasonable p-wave contribution to the capture cross section. Level spacing was taken to be $18.5 \mathrm{eV}$ for $\mathrm{J}=1 / 2$ and $9.25 \mathrm{eV}$ for $\mathrm{J}=3 / 2$. $\Gamma_{\gamma}$ was assumed to be $0.0246 \mathrm{eV}$. Calculations of the $\mathrm{p}$-wave contribution to $\sigma \gamma$ for $a$ of $40 \mathrm{~b}$ are given in Table $\mathrm{V}$.

Table I

p-WAVE CONTRIBUTION TO U239 or. BARNS

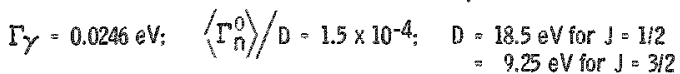

\begin{tabular}{|c|c|c|c|c|c|c|c|c|c|c|c|c|c|}
\hline \multirow{3}{*}{ Group } & & & & & \multicolumn{4}{|c|}{$\sigma_{p}=40 \mathrm{~b}$} & & & & & \\
\hline & \multirow{2}{*}{$\begin{array}{l}\text { Eave. } \\
\text { keV }\end{array}$} & \multicolumn{3}{|c|}{$3000 \mathrm{~K}$} & \multicolumn{3}{|c|}{$7500 \mathrm{~K}$} & \multicolumn{3}{|c|}{$15000 \%$} & \multicolumn{3}{|c|}{$25000 \mathrm{~K}$} \\
\hline & & $J=1 / 2$ & $J=3 / 2$ & Total & $J=1 / 2$ & $J=3 / 2$ & Total & $\mathrm{J}=1 / 2$ & $J=3 / 2$ & Total & $J=1 / 2$ & $J=3 / 2$ & Tota! \\
\hline 12 & 20.0 & 0.0842 & 0.2317 & 0.3159 & 0.0849 & 0.2341 & 0.3190 & 0.0853 & 0.2352 & 0.3205 & 0.0853 & 0.2348 & 0.3201 \\
\hline 13 & 12.0 & 0.0932 & 0.2353 & 0.3285 & 0.0942 & 0.2380 & 0.3322 & 0.0947 & 0.2393 & 0.3340 & 0.0949 & 0.2398 & 0.3347 \\
\hline 14 & 6.5 & 0.0931 & 0.2138 & 0.3069 & 0.0942 & 0.2164 & 0.3106 & 0.0948 & 0.2178 & 0.3126 & 0.0951 & 0.2186 & 0.3137 \\
\hline 15 & 3.4 & & & $(0.250)$ & & & & & & & & & \\
\hline 16 & 2.5 & & & 0.2201 & & & & & & & & & \\
\hline - & 2.25 & 0.0703 & 0.1462 & 0.2165 & 0.0712 & 0.1482 & 0.2194 & 0.0717 & 0.1492 & 0.2209 & 0.0720 & 0.1497 & 0.2217 \\
\hline 17 & 1.7 & & & $(0.180)$ & & & & & & & & & \\
\hline 18 & 1.2 & & & 60.150 & & & & & & & & & \\
\hline
\end{tabular}

(Values in parentheses are estimated. Temperature dependence neglected below Group 14.)

$P u^{240}$

\section{s-wave}

Assumed parameters are $\left\langle\Gamma_{n}^{0}\right\rangle / D=2 \times 10^{-4}, D=11.0 \mathrm{eV}$, $\Gamma \gamma=0.034 \mathrm{eV}$, and $\sigma_{p}=600 \mathrm{~b}$ for the composition used here. Results are given in Table VI for groups 12-22. For groups $1-11$ values of YOM were used, which assumed that the $P u^{240} \sigma \gamma$ was $1 \frac{3}{4}$ times that of $U^{238}$. 


\section{Table VI}

$$
\sigma_{\gamma} \text { OF PU } 240, \text { BARNS }
$$

$\left\langle\Gamma_{\mathrm{n}}^{0}\right\rangle / \mathrm{D}=2 \times 10^{-4} ; \quad \sigma_{\mathrm{p}}=600 \mathrm{~b}$.

\begin{tabular}{|c|c|c|c|c|c|c|}
\hline \multirow{2}{*}{ Group } & \multirow{2}{*}{$\begin{array}{c}\text { Eave, } \\
\text { keV }\end{array}$} & \multicolumn{4}{|c|}{ Calculated s-wave Contribution } & \multirow{2}{*}{$\begin{array}{c}\text { Assumed } \\
\text { p-wave } \\
\text { Contribution }\end{array}$} \\
\hline & & $300^{\circ} \mathrm{K}$ & $750^{\circ} \mathrm{K}$ & $1500^{\circ} \mathrm{K}$ & $2500^{\circ} \mathrm{K}$ & \\
\hline 12 & 20.0 & 0.4219 & 0.4240 & 0.4252 & 0.4259 & 0.62 \\
\hline 13 & 12.0 & 0.6618 & 0.6672 & 0.6703 & 0.6721 & 0.62 \\
\hline 14 & 6.5 & 1.117 & 1.133 & 1.143 & 1.148 & 0.60 \\
\hline 15 & 3.4 & 1.887 & 1.936 & 1.966 & 1.985 & 0.50 \\
\hline 16 & 2.3 & 2.539 & 2.631 & 2.690 & 2.726 & 0.44 \\
\hline 17 & 1.7 & 3.156 & 3.301 & 3.395 & 3.455 & 0.36 \\
\hline 18 & 1.2 & 3.992 & 4.228 & 4.387 & 4.490 & 0.30 \\
\hline 19 & 0.77 & 5.244 & 5.656 & 5.947 & 6.143 & \\
\hline 20 & 0.42 & 7.215 & 7.996 & 8.588 & 9.008 & \\
\hline 21 & 0.18 & 10.15 & 11.63 & 12.88 & 13.84 & \\
\hline 22 & 0.061 & 13.63 & 15.81 & 17.94 & 19.77 & \\
\hline
\end{tabular}

p-wave

The contributions used, assumed independent of temperature, are given in Table VI. They can be described only as guesses which gave about the same relative $s$ - and $p$-wave contributions as were found for $U^{238}$ and which gave a total capture cross section which blended in smoothly with that assumed at higher temperature.

Stainless Steel

$\sigma y$ in the lower energy region was based on data given in YOM(3) and are given in Table VII. No self-shielding of capture resonances is as sumed.

Table VII

○. OF TYPE 304 STAINLESS STEEL (SS)

AND OF SODIUM, BARNS

\begin{tabular}{|c|c|c|c|c|c|c|c|}
\hline Group & $\mathrm{E}_{\mathrm{L}}, \mathrm{keV}$ & $\begin{array}{c}\text { or of } \\
\text { Stainless } \\
\text { Steel }\end{array}$ & $\begin{array}{c}\sigma_{\gamma} \text { of } \\
\text { Sodium }\end{array}$ & Group & $E_{\mathbb{L},}, k e V$ & $\begin{array}{c}\text { oy of } \\
\text { Stainless } \\
\text { Steel }\end{array}$ & $\begin{array}{l}\text { Or. of } \\
\text { Sodium }\end{array}$ \\
\hline 14 & $4.0-9.1$ & 0.022 & - & 19 & 0.55 & 0.016 & 0.003 \\
\hline 15 & 2.8 & 0.016 & 0.444 & 20 & 0.30 & 0.022 & 0.004 \\
\hline 16 & 2.0 & 0.014 & 0.019 & 21 & 0.10 & 0.031 & 0.006 \\
\hline 17 & 1.4 & 0.020 & 0.003 & 22 & 0.03 & 0.059 & 0.010 \\
\hline 18 & 1.0 & 0.044 & 0.003 & & & & \\
\hline
\end{tabular}


Sodium

Capture in the $2.85-\mathrm{keV}$ resonance was based on $\Gamma_{0}=1 \mathrm{eV} .(3) \mathrm{Al}$ though capture cross sections in the vicinity of the resonance were inserted in individual fine groups in the ELMOE calculations, effective coarse-group cross sections did not change much from one case to another. Representative values are given in Table VII.

Heterogeneity Correction

Heterogeneity effects were taken into account with the usual equivalence theory. with the corrected scattering cross section per fuel atom as

$$
\sigma_{p}=\sigma_{p_{0}}+\frac{1-C}{2 R N_{0}}
$$

where $\sigma_{p_{0}}$ pertains to the fuel, $R$ is rod radius $(\mathrm{cm}), N_{0}$ is fuel atoms $/ \mathrm{cc}$ of fuel $x_{1} 10^{-24}$, and $C$ is the Dancoff correction. The latter was evaluated both by the method of Bell(14) and by that of Dancoff and Ginsburg, (15) with the use in the latter case of the prescription of Fukai(16) for a hexagonal lattice. There was not a great deal of difference. The results from Bell's method were used. It was believed at first that because of the strong sodium resonance at $2.85 \mathrm{keV}$, geometric effects might play a significant role in the Doppler effect. However, it appears that this is not the case because of the strong flux depression associated with the sodium resonance and also because of the lack of sensitivity of $\delta \sigma \gamma / \delta \mathrm{T}$ of $\mathrm{U}^{238}$ to $\sigma_{\mathrm{p}}$ (see Table III).

The values of of for a $\frac{1}{4}-$ in. oxide rod $(\rho=10 \mathrm{~g} / \mathrm{cc})$ in a reactor with $33 \mathrm{v} / 0$ fuel, $17 \mathrm{v} / \mathrm{o}^{\mathrm{p}}$ steel, and $50 \mathrm{v} / \mathrm{o}$ sodium obtained as described above are given in Table VIII. Doppler effects calculated with these cross sections did not differ significantly from those in which a constant $\sigma_{p}$ of 40 b was used.

Table VIII

$\sigma_{p}(b)$ FOR $\frac{1}{4}-I N$. OXIDE RODS $(\rho=10.0 \mathrm{~g} / \mathrm{cc})$

$33 \mathrm{v} / \mathrm{O}$ fuel, $17 \mathrm{v} / \mathrm{O}$ steel, $50 \mathrm{v} / \mathrm{O} \mathrm{Na}$

\begin{tabular}{|c|c|c|c|c|c|}
\hline $\mathrm{E}, \mathrm{keV}$ & Sodium & No Sodium & $E, k e V$ & Sodium & No Sodium \\
\hline 20.0 & 44 & 40 & 2.5 & 54 & 40 \\
\hline 12.0 & 44 & & 1.7 & 44 & \\
\hline 6.5 & 47 & & $0.55-1.0$ & 43 & \\
\hline 3.8 & 58 & & $0.30-0.55$ & 43 & \\
\hline 3.4 & 78 & & $0.10-0.30$ & 43 & \\
\hline 3.0 & 89 & & $0.03-0.10$ & 43 & \\
\hline 2.7 & 77 & 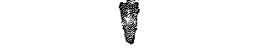 & & & 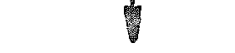 \\
\hline
\end{tabular}


Results of Calculations

\section{Parameter Variations}

A series of calculations was carried out (with the results given in Table IX) for an oxide-fueled reactor with an atom ratio of fertile to fissile material of 7 . The fertile material was $U^{238}$, except for the case indicated by the last entry in Table IX, in which sufficient $U^{238}$ was replaced by Pu ${ }^{240}$ to give a $\mathrm{Pu}{ }^{240} / \mathrm{Pu}^{239}$ atom ratio of 0.5 . The core composition was assumed to be $32 \mathrm{v} / 0$ fuel $(\rho=10.9 \mathrm{~g} / \mathrm{cc}), 16 \mathrm{v} / \mathrm{o}$ steel, and $52 \mathrm{v} / \mathrm{o}$ sodium.

Table 这

CALCULATIONS OF THE DOPPLER EFFECT FOR S-WAVE PARAMETER VARIATIONS FOR 7.1 OXIDE REACTOR

$\sigma_{\beta}$ of $\mathrm{Pu}^{239}=300 \mathrm{~b}, \quad \sigma_{p}$ of $\mathrm{U}^{238}=40 \mathrm{~b} ; \quad \sigma_{p}$ of $\mathrm{Pu}^{240}=600 \mathrm{~b}$

BNL-325 parameters for U235

\begin{tabular}{|c|c|c|c|c|c|c|c|c|c|c|c|c|}
\hline $\begin{array}{r}\text { Pu } \\
\left.\langle\Gamma\rangle^{0}\right\rangle^{10} \\
\times 10^{4}\end{array}$ & $\underset{\mathrm{eV}}{\left\langle T_{i}\right\rangle}$ & $\begin{array}{l}\text { Channels } \\
\text { per Fission }\end{array}$ & $\begin{array}{l}U^{233} \\
\left\langle\Gamma^{0}\right\rangle / 10 \\
x 10^{4}\end{array}$ & $\begin{array}{l}\text { Pu } 240 \\
\left\langle\Gamma_{0}^{0}\right\rangle / 0 \\
\times 10^{4}\end{array}$ & Sodium & $\begin{array}{c}-70 \mathrm{k} \\
300-750^{\circ} \mathrm{K}\end{array}$ & $\begin{array}{l}\text { Plutonium } \\
\text { Effect, o }\end{array}$ & $\begin{array}{c}-\% \delta \mathrm{k} \\
750-1500^{\circ} \mathrm{K}\end{array}$ & $\begin{array}{l}\text { Plutonium } \\
\text { Effect, } \%\end{array}$ & $\begin{array}{c}-\% \delta k \\
1500-2500^{\circ} \mathrm{K}\end{array}$ & $\begin{array}{l}\text { Plutonum } \\
\text { Effect, } \%\end{array}$ & $\begin{array}{c}-v_{0} \delta k \\
300-2500 \% k\end{array}$ \\
\hline \multirow[t]{2}{*}{1.0} & 0.099 & 1 & 0.94 & $=$ & Yes & 0.735 & 15 & 0.515 & 15 & 0.364 & 14 & 1.614 \\
\hline & 0.130 & 1 & 0.94 & - & $\begin{array}{l}\text { No } \\
\text { Yes }\end{array}$ & $\begin{array}{l}0.316 \\
0.793\end{array}$ & $\begin{array}{l}13 \\
13\end{array}$ & $\begin{array}{l}0.212 \\
0.564\end{array}$ & $\begin{array}{l}12 \\
12\end{array}$ & $\begin{array}{l}0.139 \\
0.392\end{array}$ & $\begin{array}{l}11 \\
12\end{array}$ & $\begin{array}{l}0.667 \\
1.749\end{array}$ \\
\hline 1.0 & & & & & No & 0.336 & 11 & 0.229 & 10 & 0.148 & 10 & 0.713 \\
\hline \multirow[t]{2}{*}{1.2} & 0.099 & 2 & 0.94 & - & Yes & 0.731 & 19 & 0.527 & 18 & 0.371 & 17 & 1.629 \\
\hline & & & & & $\mathrm{No}$ & 0.324 & 16 & 0.224 & 13 & 0.146 & 13 & 0.6994 \\
\hline \multirow[t]{2}{*}{1.0} & 0.099 & 2 & 0.94 & - & Yes & 0.740 & 18 & 0.535 & 17 & 0.384 & 14 & 1.660 \\
\hline & & & & & No & 0.327 & 14 & 0.226 & 12 & 0.150 & 10 & 0.703 \\
\hline 1.0 & 0.099 & 3 & 0.94 & - & $\begin{array}{l}\text { Yes } \\
\text { Mo }\end{array}$ & - & & - & & - & & $\begin{array}{l}1.754 \\
0.738\end{array}$ \\
\hline 1.0 & 0.080 & 3 & 0.94 & - & Yes & - & & - & & - & & $\begin{array}{l}1.645 \\
0.691\end{array}$ \\
\hline 0.7 & 0.099 & 2 & 0.94 & - & $\begin{array}{l}\text { Yes } \\
\text { No }\end{array}$ & - & & - & & - & & $\begin{array}{l}1.842 \\
0.743\end{array}$ \\
\hline \multirow[t]{2}{*}{0.83} & 0.099 & 2 & 0.94 & - & Yes & 0.836 & 16 & 0.576 & 15 & 0.377 & 12 & 1.789 \\
\hline & & & & & No & 0.351 & 13 & 0.238 & 11 & 0.148 & 9 & 0.733 \\
\hline 1.5 & 0.099 & 2 & 0.94 & - & $\begin{array}{l}\text { Yes } \\
\text { No }\end{array}$ & $=$ & & $=$ & & - & & $\begin{array}{l}1.543 \\
0.670\end{array}$ \\
\hline \multirow[t]{2}{*}{1.0} & 0.099 & 2 & 0.78 & - & Yes & 0.719 & & 0.514 & & 0.369 & & 1.602 \\
\hline & & & & & No & 0.315 & & 0.213 & & 0.143 & & 0.671 \\
\hline \multirow[t]{2}{*}{1.0} & 0.099 & 2 & 1.13 & - & Yes & 0.753 & & 0.549 & & 0.397 & & 1.698 \\
\hline & & & & & $\mathrm{No}$ & 0.335 & & 0.233 & & 0.158 & & 0.726 \\
\hline \multirow[t]{2}{*}{1.0} & 0.099 & 2 & 0.94 & 2.0 & Yes & 0.518 & 22 & 0.369 & 20 & 0.263 & 17 & 1.150 \\
\hline & & & & & No & 0.212 & 17 & 0.145 & 15 & 0.095 & 12 & 0.453 \\
\hline
\end{tabular}

Level Spacing Pu239 $\mathrm{J}=0 \quad 11.6 \mathrm{eV}$

$\mathrm{U}^{238} \mathrm{~J}=\mathrm{I} / 2 \mathrm{2} \quad \mathrm{I} .5 \mathrm{eV}$.

The reactivity changes tabulated are the net negative changes after the positive $P u^{239}$ contribution has been subtracted from the negative $\mathrm{U}^{238}$ and $\mathrm{Pu}{ }^{240}$ contribution. The columns labeled "plutonium effect" are the positive $\mathrm{Pu}{ }^{239}$ effect expressed as percent of the total negative $\left(\mathrm{U}^{238}+\mathrm{Pu}^{240}\right)$ effect.

As is seen, none of the variations, which are believed to be representative of the ranges of uncertainty of the parameters involved, resulted in a very large change in the Doppler effect. The positive Pu ${ }^{239}$ effect was never found to be very large and will be further reduced by $U^{238}$ interference. (17) Work by Hwang(11) indicates that the reduction will be 50-80\% of the original value. 
All the results in Table IX were calculated through use of the BNL-325 parameters (12) for $\mathrm{U}^{238}$. A calculation was carried out by perturbation theory with use of the data of Firk et al., (13) denoted as FLM. It was found that with these data a reduction in the total Doppler effect of $12 \%$ of the original value occurred, the reduction in the $\mathrm{U}^{238}$ alone being $10 \%$. This result is somewhat surprising since the average resonance parameters reported by FLM agree well with those resulting from the BNL parameters. Further perturbation calculations have been carried. out with data from the USSR. (18) Study of these data has not been completed. It appears that the data lead to larger Doppler effects than those obtained in this paper.

One somewhat unexpected result obtained in Table IX was that variation in $\left\langle\Gamma_{\mathrm{n}}^{0}\right\rangle / \mathrm{D}$ for $\mathrm{Pu}{ }^{239}$ did not change the Doppler effect very much, although rather large changes in $\delta \sigma_{f}$ and $\delta \sigma_{\gamma}$ occurred. However, $\sigma_{f}$ and $\sigma_{\gamma}$ also changed in the same way, introducing changes in the lowenergy adjoint function which tended to cancel out the other change; that is, the reactivity change $\delta k_{j}$ for group $j$ with suitable normalization of the flux $\phi_{j}$ and adjoint flux $\phi_{j}^{+}\left(\sum_{j} \phi_{j} \nu \Sigma_{f j}=1\right.$ and $\sum_{j} \beta_{j} \phi_{j}^{+}=1$, where $\beta_{j}$ is fraction of the fission spectrum in group $j$ ) is

$$
\delta k_{j}=\left(\nu \delta \Sigma_{f}\right)_{j} \phi_{j}-\left[\left(\delta \Sigma_{f}\right)_{j}+\left(\delta \Sigma_{\gamma}\right)_{j}\right] \phi_{j} \phi_{j}^{+}
$$

An increase in $\sigma_{f j}$ and $\sigma_{\gamma j}$ for fissile material will tend to raise $\phi_{j}^{+}$, as

$$
\phi_{j}^{+}=\frac{\nu \Sigma_{f j}+\phi_{j+1}^{+} \Sigma(j \rightarrow j+1)}{\Sigma_{f j}+\Sigma \gamma_{j}+D_{j} B^{2}+\Sigma(j \rightarrow j+1)},
$$

assuming a $D B^{2}$ leakage and $j \rightarrow j+1$ transfer only, which is true in the region of lower energy. An increase in $\phi_{j}^{+}$exerts a negative effect which counters the positive effect of increasing $\delta \sigma_{f j}$. An increase in $\delta \sigma_{\gamma j}$ also exerts a negative effect; however, $\delta \sigma_{\gamma} / \delta \sigma_{f}$ was not significantly changed with the variation of $\left\langle\Gamma_{\mathrm{n}}^{0}\right\rangle / \mathrm{D}$.

Although level spacing has not been varied in the Doppler effect calculations, $\delta \sigma_{f}$ and $\delta \sigma \gamma$ for the level spacing of Bollinger have been compared in Table $\mathrm{X}$ with those found by Egelstaff. It appears that the latter will probably give a slightly smaller Doppler effect. The overlap correction, not yet applied to the data in Table II, will be larger for the Egelstaff spacing.

It is seen that the reduction in Doppler effect because of the presence of $\mathrm{Pu}^{240}$, amounting to about $30 \%$, is much greater than any change introduced by the parameter variations. This was apparently due to the fact that $P u^{240}$ was calculated to have a large low-energy absorption cross section, while the ratio of $\delta \sigma_{\gamma}$ to $\sigma_{\gamma}$ was smaller than for $U^{238}$. It was 
found that the addition of fission products corresponding to $5 \%$ burnup plus $1.6 \mathrm{v} / \mathrm{o}$ molybdenum (representing a refractory liner for the stainless steel clad) gave a further reduction of about $30 \%$, so that the resulting Doppler effects were about half those in Table IX for the cases without $\mathrm{Pu}^{240}$.

Table X

$\delta \sigma_{f}$ AND $\delta \sigma \gamma$ FOR BOLLINGER (B) AND

EGELSTAFE (E) LEVEL SPACINGS

s-wave only. No overlap correction.

$\eta=2 ; \quad\left\langle\Gamma_{f}\right\rangle=0.099 \mathrm{eV} ; \quad\left\langle\begin{array}{l}0 \\ n\end{array}\right\rangle / D=1 \times 10^{4} ; \quad \sigma_{p}=300 \mathrm{~b}$.

\begin{tabular}{|c|c|c|c|c|c|c|c|c|}
\hline \multirow{3}{*}{$E, k e V$} & \multicolumn{4}{|c|}{$300-750^{\circ} \mathrm{K}$} & \multicolumn{4}{|c|}{$1500-2500^{\circ} \mathrm{K}$} \\
\hline & \multicolumn{2}{|c|}{$\delta \sigma_{i}, b$} & \multicolumn{2}{|c|}{$\delta \sigma_{\gamma}, b$} & \multicolumn{2}{|c|}{$\delta \sigma_{f}, b$} & \multicolumn{2}{|c|}{$\delta \sigma_{\gamma}, \mathrm{b}$} \\
\hline & $\mathrm{B}$ & $E$ & $\mathrm{~B}$ & $E$ & $\mathrm{~B}$ & E & $\mathrm{B}$ & $\Xi$ \\
\hline 6.5 & 0.013 & 0.007 & 0.007 & 0.006 & 0.004 & 0.004 & 0.002 & 0.002 \\
\hline 2.25 & 0.063 & 0.049 & 0.039 & 0.032 & 0.022 & 0.017 & 0.013 & 0.010 \\
\hline 0.61 & 0.30 & 0.26 & 0.21 & 0.19 & 0.14 & 0.10 & 0.09 & 0.07 \\
\hline 0.18 & 0.79 & 0.76 & 0.61 & 0.61 & 0.48 & 0.42 & 0.35 & 0.31 \\
\hline 0.061 & 1.07 & 1.18 & 0.94 & 1.06 & 0.91 & 0.91 & 0.72 & 0.73 \\
\hline
\end{tabular}

It appears from this work that the principal problem in the Doppler effect is not uncertainty in the calculations, but the question of how to produce an effect of useful magnitude in the presence of the necessary parasitic capture, particularly with sodium absent. Another problem in the case of oxide fuel is that it may be desirable to operate with the fuel largely molten in order to increase the power density. At such a high operating temperature for the fuel, the available Doppler effect would be drastically reduced.

It is true that the Doppler effect can be increased by enhancing the low-energy flux through the addition of moderator. In this case the crucial question is how much the breeding ratio is lowered by softening the spectrum. It may be that more information on the capture cross sections of the plutonium isotopes will be needed before this question can be answered adequately.

Doppler Effect as a Function of Composition

In Table XI are given Doppler effects for oxide- and carbide-fueled reactors of various enrichment. The fuel in this case contains no $P u^{240}$. Some of these compositions duplicate those for which calculations were made in ANL-6601,(19) which used cross sections of Greebler et a1. (20) 
The results obtained here are not greatly different from the previous ones. The positive effect of $\mathrm{Pu}^{239}$ is less than it was found to be before. Calculations have also been made for oxide fuel for higher enrichments up to a $\mathrm{U}^{238}: \mathrm{Pu}^{239}$ ratio of 2 . It is seen that the positive Pu ${ }^{239}$ effect is still less than half the negative $U^{238}$ one at this ratio, whereas both have become quite small because of the small amount of low-energy flux present. It seems unlikely that a positive Doppler effect will ever become a problem in a fast power reactor in which capture occurs mainly in fertile and fissile material.

Table XI

DOPPLER EFFECT OF OXIDE- AND CARBIDE-FUELED REACTORS AS A FUNCTION OF U23\%PU 239 RATIO $(52$ v/o Na)

\begin{tabular}{|c|c|c|c|c|c|c|c|c|}
\hline & \multirow[t]{2}{*}{ U239Pu239 } & & \multicolumn{3}{|c|}{ Total $(-6 \mathrm{k})$} & \multicolumn{3}{|c|}{$\begin{array}{c}\text { Positive Pu } 239 \text { Effect as } \\
\text { Percentage of Negative } \\
\text { U238 Effect }\end{array}$} \\
\hline & & & $300-750$ & $750-1500$ & $1500-2500$ & $300-750$ & $750-1500$ & $1500-2500$ \\
\hline \multirow[t]{5}{*}{$\begin{array}{l}\text { Oxide (32 v/o fuel, } \\
16 \text { v/o steel) }\end{array}$} & 9 & $\begin{array}{c}\mathrm{Na} \\
\mathrm{No} \mathrm{Na}\end{array}$ & $\begin{array}{l}1.17 \\
0.66\end{array}$ & $\begin{array}{l}0.87 \\
0.38\end{array}$ & $\begin{array}{l}0.59 \\
0.25\end{array}$ & $\begin{array}{l}14 \\
11\end{array}$ & $\begin{array}{l}13 \\
10\end{array}$ & $\begin{array}{l}13 \\
10\end{array}$ \\
\hline & 7 & $\begin{array}{c}\mathrm{Na} \\
\mathrm{No} \mathrm{Na}\end{array}$ & $\begin{array}{l}0.72 \\
0.33\end{array}$ & $\begin{array}{l}0.53 \\
0.23\end{array}$ & $\begin{array}{l}0.38 \\
0.15\end{array}$ & $\begin{array}{l}19 \\
14\end{array}$ & $\begin{array}{l}17 \\
13\end{array}$ & $\begin{array}{l}15 \\
11\end{array}$ \\
\hline & 5 & $\mathrm{Na}$ & 0.38 & 0.28 & 0.20 & 24 & 23 & 19 \\
\hline & 3 & $\mathrm{Na}$ & 0.092 & 0.068 & 0.045 & 42 & 38 & 38 \\
\hline & 2 & $\mathrm{Na}$ & 0.032 & 0.025 & 0.016 & 44 & 41 & 41 \\
\hline \multirow[t]{2}{*}{$\begin{array}{l}\text { Carbide } 130 \text { v/o } \\
\text { fuel, } 18 \text { vo steel) }\end{array}$} & 9 & $\begin{array}{c}\mathrm{Na} \\
\text { No Na }\end{array}$ & $\begin{array}{l}0.82 \\
0.38\end{array}$ & $\begin{array}{l}0.59 \\
0.25\end{array}$ & $\begin{array}{l}0.40 \\
0.17\end{array}$ & $\begin{array}{l}14 \\
11\end{array}$ & $\begin{array}{r}12 \\
9\end{array}$ & $\begin{array}{r}12 \\
9\end{array}$ \\
\hline & 7 & $\begin{array}{c}\mathrm{Na} \\
\text { No Na }\end{array}$ & $\begin{array}{l}0.66 \\
0.22\end{array}$ & $\begin{array}{l}0.36 \\
0.15\end{array}$ & $\begin{array}{l}0.25 \\
0.10\end{array}$ & $\begin{array}{l}18 \\
13\end{array}$ & $\begin{array}{l}16 \\
12\end{array}$ & $\begin{array}{l}15 \\
10\end{array}$ \\
\hline
\end{tabular}

Designs are possible in which significant positive effects can be produced. To see how this can come about, assume $\phi_{j}^{+}$for the low-energy region to be approximated by $\left(k_{\infty}\right)_{j}=\nu_{j} /\left(1+\alpha_{j}\right)$, where $\alpha_{j}=\Sigma_{\gamma j} / \Sigma_{f j}$. Equation (1) with these assumptions can be rewritten

$$
\frac{\delta k_{j}}{\phi j}=\nu_{j} \delta \sigma_{f j}\left[1-\frac{1+\alpha_{j}^{\prime}}{1+\alpha_{j}}\right] \text {, }
$$

where

$$
\alpha^{\prime}=\frac{\delta \Sigma_{\gamma j}}{\delta \Sigma_{f j}}
$$

If capture occurs only in fissile material, and if the capture-tofission ratio increases with temperature, which in general it will, a negative Doppler effect will be obtained if leakage effects can be neglected, as was assumed here. On the other hand, if a large amount of capture is 
present in materials other than the fissile material, $\alpha_{j}>\alpha_{j}^{\prime}$ and a positive effect is produced. If no Doppler effect is associated with this other capture, a positive reactor Doppler effect will be produced.(21) This is a problem in critical assemblies in which fissile material and the resonance capturer are physically separated, or it may be that the capturing material would not give a Doppler effect in any event.

An example of how the Doppler effect is influenced by a change in parasitic capture is given in Table XII, in which calculations have been made for a critical assembly with a $\mathrm{C} / \mathrm{Pu}{ }^{239}$ ratio of about 50 , which yields a hard intermediate spectrum. Heating of $\mathrm{Pu}^{239}$ only is assumed, and the $\mathrm{Pu}^{239}$ Doppler effect is seen to be strongly influenced by the presence or absence of $U^{238}$. A positive effect is still obtained with no $U^{238}$ because of the effect of leakage on the $10 \mathrm{w}$-energy adjoint function. Interference effects of $\mathrm{U}^{238}$ on $\mathrm{Pu}{ }^{239}$ resonances are not taken into account in these calculations and are expected to diminish the positive $\mathrm{Pu}{ }^{239}$ effect. This could not necessarily be relied on in a critical experiment, however, because of the physical separation of the fuel. The effects produced are sensitive to the channels per fission assumed. Experiments of this type appear to be the most useful in obtaining information about the parameters of $\mathrm{Pu}^{239}$.

Table XII

CRITICAL EXPERIMENT DOPPLER EFFECT CALCULATIONS FOR HIGH CARBON ASSEMBLIES

$$
\begin{gathered}
\text { Atoms } / \mathrm{cc} \times 10^{-24} \\
\mathrm{C} 0.0534 \\
\text { Stainless Steel } 0.00624 \\
U^{238} 0.0025 \\
P_{u}^{239} 0.00106 \\
\sigma_{\mathrm{p}}\left(\mathrm{U}^{238}\right)=100 \mathrm{~b}: \quad\left\langle\Gamma_{\mathrm{n}}^{0}\right\rangle / \mathrm{D}=0.94 \times 10^{-4} \\
\sigma_{\mathrm{p}}\left(\mathrm{Pu}^{239}\right)=300 \mathrm{~b} \quad\left\langle\Gamma_{\mathrm{n}}^{0}\right\rangle / \mathrm{D}=2 \times 10^{-4} \\
\left\langle\Gamma_{\mathrm{f}}\right\rangle=0.099 \mathrm{eV}
\end{gathered}
$$

$\leftarrow \delta \mathrm{k}$ in $\%, 300-750^{\circ} \mathrm{K}$ heating of $\mathrm{Pu}^{239}$ only.

\begin{tabular}{c|c|c|c}
\hline \multicolumn{2}{c|}{$\eta=1$} & \multicolumn{2}{|c}{$\eta=2$} \\
\hline$U^{238}$ & No $U^{238}$ & $U^{238}$ & No $U^{238}$ \\
\hline 0.235 & -0.214 & 0.467 & 0.125 \\
\hline
\end{tabular}


The Dopplex effect is increased at a given fuel-enrichment ratio by increasing the ratio of sodium to fuel. It was found that for a $7: 1$ $\mathrm{U}^{238} / \mathrm{Pu}^{239}$ fuel with $70 \mathrm{~V} / \mathrm{o}$ sodium, $18 \mathrm{v} / 0$ fuel, and $12 \mathrm{~V} / \mathrm{o}$ steel, the Doppler - \% ok from 1500 to $2500^{\circ} \mathrm{K}$ was 0.80 for oxide and 0.52 for car bide, representing increases of about a factor of 2 over results for $52 \mathrm{v} /$ o sodium. The Doppler effect would then decrease by a factor of about 5 on loss of sodium.

\section{$\underline{\text { References }}$}

1. O'Shea, D. M., and Thacher, H. C., Trans. Am. Nuc. Soc. $6,(1)$, 36 (June 1963).

2. Humme1, H. H., and Rago, A. L., Physics of Fast and Intermediate Reactors, Vol. I, p. 231, International Atomic Energy Agency, Vienna (1962).

3. Yiftah, S., Okrent, D., and Moldauer, P. A., Fast Reactor Cross Sections, Pergamon Press, New York (1960).

4. Smith, A. B., Physics of Fast and Intermediate Reactors, Vol. I, p. 29. International Atomic Energy Agency, Vienna (1962).

5. Neiler, J. H., ibid., Vol. I, p. 95.

6. Gibbons, J. H., et al., Phys. Rev. 122, 182 (1961).

7. Hopkins, J. C., and Diven, B. C., Physics of Fast and Intermediate Reactors, Vol. I, p. 112, International Atomic Energy Agency, Vienna (1962).

8. Bollinger, L. M., et al., Proc. 2nd UN Intnl. Conf. on Peaceful Uses of Atomic Energy, Geneva, Switzerland, 15, 121 (1958).

9. Moldauer, P.A., Private communication.

10. Egelstaff, P. A., et al., J. Nuc. Energy, 6, 303 (1958).

11. Hwang, R. N., Proceedings of this conference.

12. Hughes, D. J., et al., BNL-325 suppl., 3rd Ed. (1960).

13. Firk, F.W. K., Lynn, J. E., and Moxon, M. C., Nuc. Phys. 41 , $614(1963)$. 
14. Bell, G. I., Nuclear Sci. \& Eng. 5, 138 (1959),

15. Dancoff, S. M., and Ginsburg, M., P-215 (1944).

16. Fukai, V, Nuclear Sci. \& Eng. 9, 310 (1961).

17. Codd, J., and Collins, P., Proceedings of this conference.

18. Abagyan, L. P., et al., Group Constants of Fast and Intermediate Neutrons for the Designing of Nuclear Reactors, Physical Power Engineering Inst., State Committee for Use of Atomic Energy, USSR (1962).

19. Bhide, M. G。, and Hummel, H.H., Calculations of the Doppler Coefficient of Large Ceramic-fueled Fast Reactors, ANL-6601 (Aug 1962).

20. Greebler, P., et al., GEAP-3646 (1961).

21. According to R. B. Nicholson, this key role of $\phi_{j}^{+}$was first pointed out by H. A. Bethe in connection with the KAPL assemblies.

\section{Acknowledgment}

Extensive assistance with the computational work was provided by Mrs. Stella Dean and Miss Lois Meyer.

Discussion of Paper

Presented by Mr. Hummel

MR. CAMPISE (Atomics International):

Did you carry your enrichment to a point where you did get a cross= over in these Dopplex coefficients?

MR. HUMMEL:

No. It was getting so small already I kind of lost interest in it. We never really succeeded in getting a positive effect. You see, the $U^{238}$ effect comes in at a little higher energy than the plutonium effect, so that as you go to higher enrichment where the spectrum gets harder and harder, this sort of counter-balances the effect. I am not sure you will ever get a positive effect. 
MR. SPINRAD (Argonne):

Presumably as an inference from the Pu ${ }^{240}$ effect you mentioned that you would expect fission products also to decrease the Doppler effect markedly. Have you made any estimates of this for highly burned fuel?

MR. HUMMEL:

I don't remember exactly what the results are, but I would say maybe about a 10 to 20 per cent further effect would be reasonable.

MR. WOLFE (General Electric):

What is that reduction in Doppler effect due to the Pu ${ }^{240}$ content? Can that be restated as saying it is a reduction due to a changed ratio of $\mathrm{U}^{238}$ to $\mathrm{Pu}^{239}$ ?

MR. HUMMEL:

No, it is mostly that the calculated $\mathrm{Pu}^{240}$ cross section is a lot bigger than the $U^{238}$ cross section, and you do not get any Doppler effect to speak of out of the Pu. ${ }^{240}$. But it gobbles up low energy neutrons.

MR. GREEBLER:

Would it be fair to say we are just making low energy neutrons less important if we put in fission products or $\mathrm{Pu}^{240}$ ?

MR. HUMMEL:

That would be another way to state it, yes.

MR. SPINRAD:

I wonder whether it would be fair to attempt a summary impression of the results you have presented, which is that the general Doppler effect of these assemblies is rather less sensitive to what you might consider reasonable variations in nuclear parameters than all of the discussion of the errors in parameters themselves has indicated.

MR. HUMMEL:

That is my feeling. Of course, I do not know anything about multilevel effects. Something I have overlooked may come to light later, like this plutonium-U $U^{238}$ effect. 
MR. FOSSOUL (BelgoNucleaire):

May I ask you what sort of cross section you used for these calculations, and the way you used the fundamental nuclear data to make a set at another temperature?

MR. HUMMEL.

What we did was to calculate resonance integrals at those energies by the method Hwang described. We actually used more detailed groups and plotted $\delta \Sigma_{\hat{f}}$ and $\delta \Sigma_{\gamma}$ as functions of energy and interpolated. We used a 22-group cross-section set which used the Yiftah cross-sections groups 1 to 11 with a couple of modifications, like changing the alpha of plutonium-239. In the lower-energy region we used cross sections based on these resonance integral calculations which included essentially method B calculations, with a slight overlap correction, which did not affect the results any. There should have been more overlap correction for plutonium, which would reduce the plutonium results by about 10 per cent overall. The calculation was carried out simply by making a fundamental mode calculation at different temperatures, the same ones that Paul Greebler originally used, $300,750,1500$, and $2500^{\circ} \mathrm{K}$. We calculated the critical buckling at one temperature and then put the cross sections in for a different temperature, and observed what the $\delta \mathrm{k}$ was. 
•

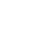




\title{
DEPENDENCE OF FAST REACTOR DOPPLER COEFFICIENTS ON NUCLEAR DATA UNCERTAINTIES
}

\author{
R, Froelich, K, Ott, and J. J. Schmidt \\ Institut für Neutronenphysik and Reaktortechnik \\ Kernforschungszentrum, Karlsruhe, Germany \\ (Paper presented by $\mathrm{Mr}$. Schmidt)
}

I. Introduction

This report shows for one typical example how much the reliable theoretical prediction of fast reactor Doppler coefficients is influenced by the inaccuracies of the nuclear data involved. We will not discuss inaccuracies of the theory itself.

We restrict ourselves to a discussion of isothermal Doppler coefficients as defined in the Doppler coefficient theory paper of the authors (1) of a certain reference reactor for a certain reference temperature, Furthermore, to avoid unnecessary complication, the resonance part of the total cross section of sodium and stainless steel is omitted in the expression for the local Doppler coefficient. This means neglecting interaction effects between scatterer and fuel resonances.

In section II we discuss the present knowledge and uncertainties of relevant nuclear resonance data for $\mathrm{U}^{238}$ and $\mathrm{Pu}^{239}$ in theory and experiment; in section III some Doppler coefficient results for the reference reactor mentioned before are considered as a function of changes in the nuclear data.

II. Present Knowledge of Relevant Nuclear Resonance Data for $\mathrm{U}^{238}$ and

The basic nuclear data involved in calculations of fast reactor Doppler coefficient are neutron strength functions, average resonance halfwidths, and average level spacings. Because of the high centrifugal barrier for higher neutron orbital angular momenta, only $s$ - and $p$-wave neutrons contribute significantly to the Doppler coefficient.

Table 1 summarizes the present knowledge and uncertainties of statistical $\mathrm{s}$ - and $\mathrm{p}$-wave resonance data for $\mathrm{U}^{238}$ and $\mathrm{Pu}^{23 \%}$. This table is the result of a critical review of available experimental data and predictions of nuclear theory. Most symbols used are well-known: $S_{0}$ and $S_{1}$ denote $s$ - and $p$-wave neutron strength functions: $\nu_{f}$ the number of degrees of freedom in the fission width $\chi^{2}$-distribution. The uncertainties indicated should throughout be considered as arising from physical estimates rather than from mathematical deduction. 


$$
\begin{aligned}
& \mathrm{U}^{238}, 1=0 \\
& S_{0}=1.0 \times 10^{-4} \pm 20 \% \\
& \bar{F} \gamma_{0}=24.6 \mathrm{meV} \pm 10 \% \text {. } \\
& \overline{\mathrm{D}}_{\mathrm{J}=1 / 2}=18.5 \mathrm{eV} \pm 10 \%=\overline{\mathrm{D}}_{\text {observed }} \\
& U^{238}, 1=1 \\
& S_{1}=1.0 \times 10^{-4}+\sim 200 \% \text { for } \mathrm{J}=\frac{1}{2}, \frac{3}{2} \text {. } \\
& \bar{\Gamma} \gamma_{1}=\bar{\Gamma} \gamma_{0}=24.6 \mathrm{meV}_{-}^{+} 150 \% \text { for } J=\frac{1}{2}, \frac{3}{2} . \\
& \overline{\mathrm{D}}_{\mathrm{J}=1 / 2}=18.5 \mathrm{eV} \pm 10 \% \\
& \overline{\mathrm{D}}_{\mathrm{J}=3 / 2}=10.1 \mathrm{eV} \pm 10 \% \text {. }
\end{aligned}
$$

$$
\begin{aligned}
& S_{0}=1.0 \times 10^{-4}+40 \% \\
&-30 \% \\
& \text { for } J=0,1 . \\
& \bar{\Gamma} \gamma_{0}=38.7 \mathrm{meV} \pm 15 \% \text { for } J=0,1 .
\end{aligned}
$$

$\overline{\mathrm{D}}_{\text {observed }}=2.64 \pm 20 \%$.

$$
\begin{array}{ll}
\overline{\mathrm{D}}_{\mathrm{J}=0} & =10.12 \mathrm{eV} \pm 20 \% \\
\overline{\mathrm{D}}_{\mathrm{J}=1} & =3.57 \mathrm{eV} \pm 20 \% .
\end{array}
$$

$P^{239}, I=1$

$$
\begin{aligned}
\mathrm{S}_{1} & =1.0 \times 10^{-4}+200 \% \\
& -30 \% \text { for } \mathrm{J}=0,1,2 . \\
\bar{\Gamma} \gamma_{1} & =\bar{\Gamma} \gamma_{0}=38.7 \mathrm{meV} \pm 15 \% \text { for } \mathrm{J}=0,1,2 . \\
\overline{\mathrm{D}}_{\mathrm{J}=0} & =10.12 \mathrm{eV} \pm 20 \% . \\
\overline{\mathrm{D}}_{\mathrm{J}=1} & =3.57 \mathrm{eV} \pm 20 \% . \\
\mathrm{D}_{\mathrm{J}=2} & =2.40 \mathrm{eV} \pm 20 \% .
\end{aligned}
$$

$\mathrm{Pu}^{239} ;$ fission data

$$
\begin{aligned}
\bar{\Gamma}_{f_{0}} & =\bar{\Gamma}_{f_{1}} \text { for } J=0,1,2 . \\
\nu_{f} & =1,3 . \\
\alpha(E) & =\bar{\alpha}(E) \pm 20 \% .
\end{aligned}
$$


Four main information sources were used:

a. analysis of isolated resonances ( $\mathrm{s}$-wave data):

b. statistical theory analysis of average cross-section measurements in the keV region (mainly p-wave daia):

c. predictions of the optical model (neutron strength functions);

d. predictions of the Fermi gas model (spin and energy dependence of the average level spacing).

We explain briefly the main results.

1. $\mathrm{U}^{238}, 1=0$

Careful resonance measurements and analysis mainly from Columbia $(2,3)$ and Harwell $(4-6)$ data up to about $2 \mathrm{keV}$ establish a rather reliable set of statistical $s$-wave data for $U^{238}$ However, a $\pm 20 \%$ error in $S_{0}$ is indicated mainly due to insufficient statistics

2. $\mathrm{U}^{238} \cdot 1=1$

$\mathrm{p}$-wave strength functions obtained from earlier theoretical fits to average cross sections, $(7,8)$ and from different optical model calculations $(9-12)$ disagree by a factor about three. The statistical theory fits yielded $S_{1} \cong 1 \times 10^{-4}$, whereas Krueger and Margolis, $(11,12)$ using a diffuse complex potential with surface absorption and spin-orbit coupling, find $S_{1}=3 \times 10^{-4}$ This value is presently believed to be the most reliable optical model prediction for $\mathrm{S}_{1}\left(\mathrm{U}^{238}\right)$; for $\mathrm{Krueger}$ and Margolis with their potential could obtain a very good $f_{1} t$ to the Duke University measurements $(13)$ of $S_{1}$ for nuclei around $A=100$.

There is some, but vague, indication $(8,14)$ that the p-wave capture width is higher than that of the $\mathrm{s}$-wave by a factor about 2.5. These arguments explain the upper Immits assigned in the table to $S_{1}$ and $\Sigma \gamma_{1}$.

$$
3 \quad \mathrm{Pu}^{239} \mathrm{I}=0
$$

The two most important series of resonance measurements have been performed at Argonne(15) and at Harwello(16-18) They show good agreement in the s-wave strength function and average capture width. They are, however, seriously discrepant in the mean level spacing, the average fission width, and the fission width distribution observed. The Argonne fission-width data are compatzble with a $\chi^{2}$-distribution wath $\nu_{f}=1$. whereas the Harwell data are only consistent with $v_{f}$ about 3. 
FROELICH et al.

The large errors assigned, particularly to $S_{0}^{49}$, are partly due to the se discrepancies, partly to the insufficient statistics because of the small number of resonances investigated up to now $(\sim 20$ up to $\sim 50 \mathrm{eV})$. It is seen that the experimental s-wave data of $\mathrm{Pu}^{239}$ are much more unreliable than those of $\mathrm{U}^{238}$.

$$
\text { 4. } \mathrm{Pu}^{239}, 1=1
$$

$\mathrm{P}$-wave information for $\mathrm{Pu}^{239}$ is so far completely lacking. Thus, preliminarily p-wave strength-functions, and capture and fission widths were assumed to be independent of the respective compound nucleus spin and were set equal to the respective s-wave quantities. Optical model systematics suggests that $\mathrm{U}^{238}$ and $\mathrm{Pu}^{239}$, as close neighbours, should have similar $\mathrm{p}$-wave strength function values and errors.

$$
\text { 5. } \overline{\Gamma \gamma}(\mathrm{E})
$$

$\bar{\Gamma} \gamma$ changes its resonance value only by a few percent when going to $100 \mathrm{keV}$, thus remaining within the experimental uncertainty of the resonance value. Therefore, $\bar{\Gamma} \gamma$ has been taken constant at all energies.

$$
\text { 6. } \underline{\bar{\Gamma}_{f}(E)}
$$

The quantity $\alpha=\sigma_{\gamma} / \sigma_{f}$ and its energy dependence are best suited for estimates of $\bar{\Gamma}_{f}$ and its energy dependence; for $\alpha$ depends only weakly on the correct values of the strength function. The energy dependence of $\alpha\left(\mathrm{Pu}^{239}\right)$ in the Doppler energy region has been extensively discussed by one of the authors $(19,20)$ elsewhere; it is reproduced in Fig. 1.

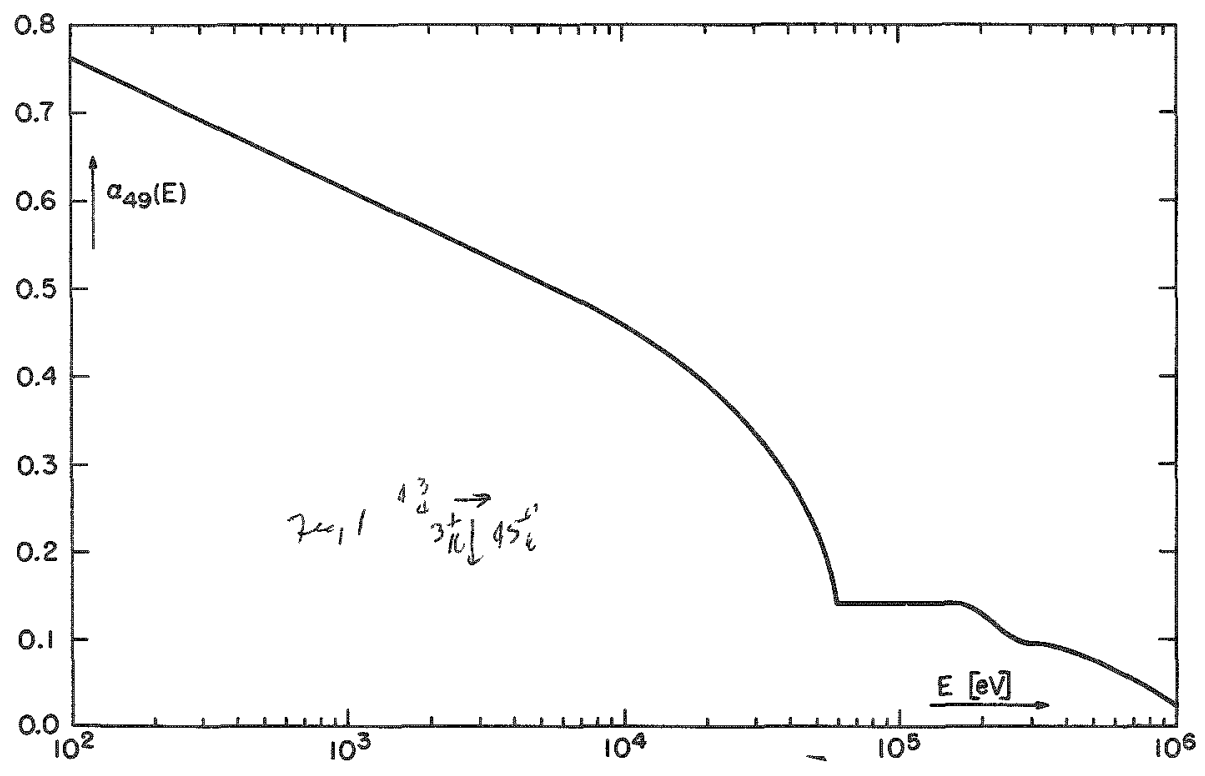

Fig. 1. $\alpha_{49}(E)$ between $100 \mathrm{eV}$ and $1 \mathrm{MeV}$ 
We recall our former conclusion, that in the Doppler energy region the average uncertainty of the $\alpha$-values found is about $\pm 20 \%$. We have looked into the variation of $\bar{\Gamma}_{f}$ when changing $\alpha$ in the range of its accuracy and supposing $x^{2}$-distributions for $\Gamma_{f}$ with $\nu_{f}=1$ or 3 according to the discrepant resonance experiments. Figure 2 shows $\bar{\Gamma}_{f}(E)$ for the six relevant $\left(\nu_{f}, \alpha\right)$-combinations; the combination $\nu_{f}=1, \alpha=\bar{\alpha}$ is qualified "best." $(21)$ The qualitative shape of the different $\bar{\Gamma}_{f}(E)$ curves is about the same, whereas, quantitatively, great differences appear. However, the influence of the $\bar{\Gamma}_{f}$ uncertainties on the Doppler coefficient will be shown to be not so serious as one would believe from Fig. 2 .

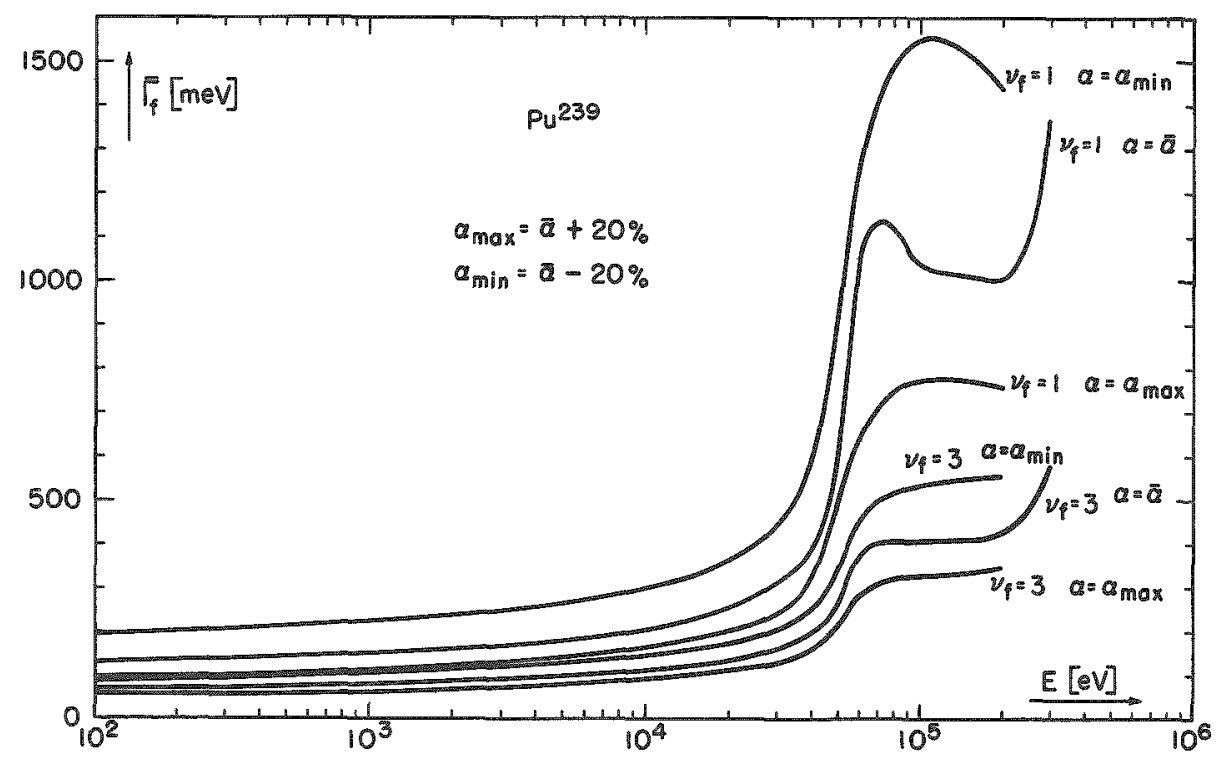

Eig. 2. $\bar{\Gamma}_{f}(\mathbb{E})$ for $\operatorname{Six}\left(\nu_{f}, \alpha\right)$-Combinations

7. $\overline{\mathrm{D}}(\mathrm{E}, J)$

Energy and spin dependences of the average level spacing are evaluated from the Fermi gas model of the nucleus corresponding to the work of Bethe, (22) Newton, (23) and Cameron.(24) The neutron transmission through the nuclear surface, hence the neutron strength functions, are assumed to depend only on the neutron orbital angular momentum, and not on channel and compound nucleus spins. This corresponds to vanishing spin-orbit force. Then average neutron widths and level spacings have the same compound nucleus spin dependences.

A more extensive discussion of the present theoretical and experimental knowledge of the statistical resonance properties of $U^{238}$ and $\mathrm{Pu}^{239}$ is given in Chapter 4 of Reference 21. 
III. Results and Discussion

We consider isothermal Doppler coefficients of a reference reactor consisting of the following material mixture:

$\begin{array}{lr}\mathrm{Pu}^{239} \mathrm{O}_{2}: & 5 \mathrm{v} / 0 ; \\ \mathrm{U}^{238} \mathrm{O}_{2}: & 25 \mathrm{v} / 0 ; \\ \text { Sodium: } & 40 \mathrm{v} / 0 ; \\ \text { Stainless Steel: } & 30 \mathrm{v} / 0 ;\end{array}$

with

$$
\mathrm{y}=\frac{\mathrm{U}^{238} \mathrm{O}_{2} \text {-volume }}{\mathrm{Pu}^{239} \mathrm{O}_{2} \text {-volume }}=5 .
$$

The stainless steel (SS) chosen consists of

$65 \mathrm{w} / \mathrm{OFe} ; 16 \mathrm{w} / \mathrm{O} \mathrm{Cr} ; 16 \mathrm{w} / 0 \mathrm{Ni} ; 3 \mathrm{w} / \mathrm{OMO}$.

As reference temperature we take $800^{\circ} \mathrm{K}$. The reference set of nuclear data consists of the average values given in Table 1 with $\nu_{f}=1$ and $\alpha=\bar{\alpha}$. Figure 3 and Tables 2 and 3 are obtained for the se reference conditions.

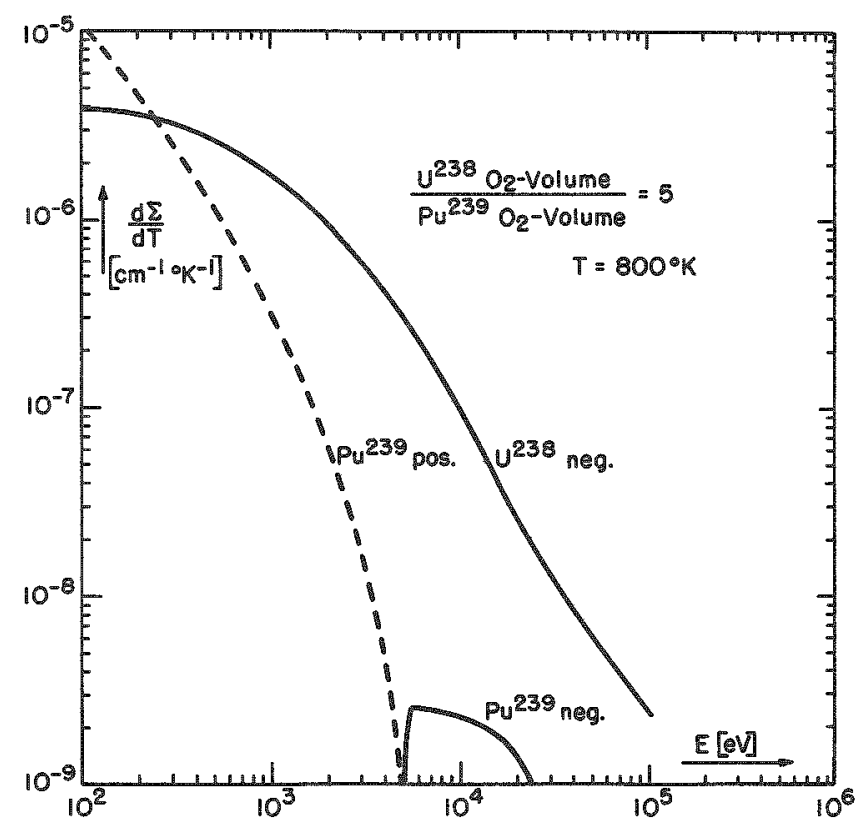

Fig. 3. Local Doppler Coefficients for $\mathrm{U}^{238}$ and $\mathrm{Pu}^{239}$ 
Table 2

ISOTHERMAL GROUP DOPPLER COEFFICIENTS

Average nuclear data; $\frac{\mathrm{U}^{238 \mathrm{O}_{2} \text {-volume }}}{\mathrm{Pu}^{239 \mathrm{O}_{2} \text {-volume }}}=5 ; \mathrm{T}=800^{\circ} \mathrm{K}$

\begin{tabular}{|c|c|c|c|c|c|c|c|}
\hline \multirow[b]{2}{*}{ Group } & \multirow[b]{2}{*}{$\begin{array}{c}E_{1}-E_{H} \\
{[\mathrm{keV}]}\end{array}$} & \multicolumn{2}{|c|}{$\mathrm{Pu}^{239}$} & \multicolumn{2}{|c|}{$\mathrm{v}^{238}$} & \multicolumn{2}{|c|}{ Total } \\
\hline & & {$\left[10^{-7} / \frac{d k}{d T}\right]$} & $\begin{array}{c}9 \% \text { of } \\
\left(\frac{d k}{d T}\right)_{\text {tolal }}^{49}\end{array}$ & $\frac{\frac{d k}{d T}}{\left[10^{-7 / 6 K}\right]}$ & $\begin{array}{c}\% \text { of } \\
\left(\frac{d k}{d T}\right)_{\text {total }}^{28}\end{array}$ & $\frac{\frac{d k}{d T}}{\left[10^{-1 / 0 \%]}\right]}$ & $\begin{array}{c}\% \text { of } \\
\left(\frac{d y}{d i}\right)_{\text {total }}\end{array}$ \\
\hline $9-10$ & $40.7-110$ & 0.111 & +2.1 & -0.931 & 3.3 & -0.820 & 3.5 \\
\hline $11-12$ & $15-40.7$ & -0.052 & -1.0 & -2.296 & 8.2 & -2.348 & 30.1 \\
\hline $13-14$ & $5.5-15$ & -0.094 & -1.8 & -5.274 & 18.7 & -5.368 & 23.1 \\
\hline 15 & $2.1-5.5$ & 0.182 & +3 & -6.779 & 24.1 & -6.597 & 28.4 \\
\hline 16 & $0.1-2.1$ & 4.719 & +97.0 & -12.846 & 45.7 & -8.127 & 34.9 \\
\hline
\end{tabular}

Table 3

ISOTHERMAL DOPPLER COEFFICIENT UNCERTAINTIES

\begin{tabular}{|c|c|c|c|c|c|c|}
\hline \multirow[b]{2}{*}{ Case } & \multicolumn{2}{|c|}{$P_{4} 239$} & \multicolumn{2}{|c|}{$\mathrm{y}^{238}$} & \multicolumn{2}{|c|}{ Total } \\
\hline & $\frac{\frac{d \mathrm{~d}}{d \mathrm{dT}}}{\left[10^{-6} 6 \mathrm{ok}\right]}$ & $\begin{array}{l}\text { Relative } \\
\text { Error } \\
\%\end{array}$ & $\frac{\frac{d k}{d T}}{\left[10^{-6 / 0 K}\right]}$ & $\begin{array}{l}\text { Relative } \\
\text { Error } \\
\%\end{array}$ & $\frac{\frac{d k}{d T}}{\left[10^{-6 / \%} k\right]}$ & $\begin{array}{l}\text { Relative } \\
\text { Error } \\
\%\end{array}$ \\
\hline Average nuclear data & 0.487 & 0 & -2.813 & 0 & -2.326 & 0 \\
\hline $\bar{S} 8^{28}+20 \%$ & 0.455 & -6.6 & -3.234 & +15.0 & -2.779 & +19.5 \\
\hline$s_{0}^{28}=20 \%$ & 0.477 & -2.1 & -2.342 & -16.7 & -1.865 & -19.8 \\
\hline $\bar{S} 49+40 \%$ & 1.316 & +170.2 & -2.813 & 0 & -1.497 & -35.6 \\
\hline $\bar{S}_{0}^{49}-30 \%$ & 0.041 & -91.6 & -2.813 & 0 & -2.772 & -19.2 \\
\hline$\nu_{f}=1 ; \bar{a}+20 \%$ & 0.273 & -43.9 & -2.813 & 0 & -2.540 & +9.2 \\
\hline$v_{f}=1: \bar{d}-20 \%$ & 0.757 & +55.4 & -2.813 & 0 & -2.056 & -11.7 \\
\hline$v_{f}-3: \bar{g}+20 \%$ & 0.421 & -13.6 & -2.813 & 0 & -2.392 & +2.8 \\
\hline$v_{f}=3 ; \bar{\alpha}-20 \%$ & 0.286 & -41.3 & -2.813 & 0 & -2.527 & $\$ 8.6$ \\
\hline $\bar{F}_{Y I=1}^{28}=60 \mathrm{meV}$ & 0.487 & 0 & -2.974 & +5.7 & -2.487 & +7.0 \\
\hline$s_{1}^{28}=3 \times 10^{-4}$ & 0.487 & 0 & -3.333 & +18.5 & -2.846 & -22.5 \\
\hline
\end{tabular}

Figure 3 shows local Doppler coefficients for $U^{238}$ and $P u^{239}$ as a function of the neutron energy. Apparently $\mathrm{Pu}^{239}$ reveals the highest Doppler effect in the small energy region below $1 \mathrm{keV}$, whereas the $\mathrm{U}^{238}$ Doppler effect is important over a much larger energy range. In the region of higher keV it becomes some orders of magnitude larger than the Pu ${ }^{239}$ Doppler effect.

These features appear also in the isothermal group Doppler coefficients given in Table 2. The group of lowest energy covers $97 \%$ of the total $\mathrm{Pu}^{239}$ Doppler coefficient. This fraction would be somewhat smaller 
if there were no interaction between $\mathrm{U}^{238} \mathrm{~s}$-wave and $\mathrm{Pu}^{239}$ resonances. Important contributions to the $U^{238}$ Doppler coefficient come also from groups of higher energy. The Doppler coefficiont contributions of $\mathrm{Pu}^{239}$ there cancel each other partly.

Table 3 shows uncertainties in the isotopic and total isothermal Doppler coefficients of the reference reactor due to nuclear data inaccuracies. The most important errors in the total Doppler coefficient arise from the errors in the s-wave strength functions of both nuclei. The particularly great errors in $S_{0}^{49}$, hence in the $P u^{239}$ and total Doppler coefficients, are mainly due to the insufficiency of available $\mathrm{Pu}^{239}$ resonance experiments. They become smaller, if one considers that $\mathrm{U}^{238}$ and $\mathrm{Pu}^{239}$, as close neighbours, should have about the same s-wave strength functions and that $S_{0}^{28}$ is better known from experiment than $S_{0}^{49}$.

Nuclear data changes of one isotope have generally no effect on the Dopplex coefficient of the other isotope; a small coupling exists for $\mathrm{S}_{0}^{28}$ changes.

Inaccuracies in the fission data cause errors in the total Doppler coefficient of the order of $\pm 10 \%$. Most probably there is only one fission channel ir $P^{239}$ resonance fission. $(21)$ Then a better knowledge of $\alpha$ would still reduce these errors further.

The influence of $U^{238} \mathrm{p}$-wave data on the total Doppler coefficient is obviously rather weak: a $200 \%$ increase in $S_{1}^{28}$ causes only a $20 \%$ increase in the total Doppler coefficient. Optical model $(11,12)$ and statistical theory calculations $(25)$ presently favour the value $3 \times 10^{-4}$ for $S_{1}^{28}$. P-wave data for $\mathrm{Pu}^{239}$ are even more unimportant; thus their influence has not been investigated.

Summarizing the above arguments we conclude: the Doppler coefficient for a fast reactor with $y=5$ can rather safely be calculated to an accuracy of about $\pm 20 \%$.

\section{References}

1. R. Froelich, K. Ott, and J. J. Schmidt, "Calculations of Doppler Coefficients of Dilute Fast Reactors," see this Proceedings.

2. J. L. Rosen, CU-185 (1959).

3. J.L. Rosen et al., Phys, Rev. 118, 687 (1960).

4. F. W. K. Firk et al., TNCC(UK) -44 (1959).

5. F.W.K. Firk et al., Proc. Int. Conf. on Nuclear Structure, Kingston, Canada (1960), p.93. 
6. F, W, K, Firk et al1, Nuc1, Phys, 41, 614 (1963).

7. C. A. Uttley and R, M. Jones, Proc, EANDC-Symposium on Neutron Time- of-Flight Methods, Saclay (1961), p. 109.

8. E. G. Bilpuch et a1. Annals of Physics 10, 455 (1960).

9. H. Feshbach et al., Phys, Rev, 96, 448 (1954).

10. E. J. Campbell et al., TID-5820 (1960),

11. T. K, Krueger and B. Margolis, TID-13904 (1961).

12. T, K, Krueger and B, Margolis, Nucl. Phys.28, 578 (1961).

13. L.W.Weston et al., Annals of Physics 10, 477 (1960).

14. L. M. Bollinger et 21., Phys, Rev。105, 661 (1957).

15. L. M. Bollinger et 21., Proc of the 2nd UN Intnl. Conf. on Peaceful Uses of Atomic Energy, Geneva, Switzerland, 15, 127 (1958).

16. F, J. M. Farley, J. Nucl。 Energy 3, 33 (1956).

17. P. A. Egelstaff et al, J, Nucl. Energy 6, 303 (1958)。

18. P. A. Egelstaff and D. J. Hughes, Progr. Nuc1. Energy 11, 55 (1956).

19. J. J. Schmidt, Vienna Semmar on the Physics of Fast and Intermediate Reactors, August (1961), Paper SM 18/4.

20. J.J. Schmidt, KFR 120 (EANDC-E-35 U)(1962).

21. J. I Schmıdt, EANDC $-E-46$ L (1963).

22. H. A. Bethe Revs. Mod, Phys 9, 69 (1937).

23. T. D. Newton, Can. J. Phys. 34.804 (1956); Errata: Can. J. Phys. 35. 1400 (1957).

24. A. G. W, Cameron, Can, J.Phys, 35,666 (1957).

25. J.E. Lynn, NRDC-138 (EANDC-UK-21"U")(1963), p. 26, to be published in Nuc1. Phys

\section{Discussion of Paper Presented by Mr. Schmidt}

MR. CHERNICK (Brookhaven):

Some time ago we tried fitting of the $p$-wave data. It was very difficult to choose what ratio strength functions to use, and we certainly did not fit over the entire range of the known capture cross sections, about 10 to $30 \mathrm{keV}$ in the range where primarily the p-wave is important. Could you tell us a little more about Lynn's fit; have the group data changed; why do they get such a good fit now? 
MR. SCHMIDT:

There is only a short note in the United Kingdom Index report which does not indicate what Lynn has really done. It gives only numbers. We are investigating this at Karlsruhe.

MR. CHERNICK:

It would be of great interest to know whether Lynn gets a continuous fit or if this is just mean-free-square data.

MR. SCHMIDT:

I cannot judge on it for the moment. I think because all $\mathrm{p}$-wave strength function predictions, from all sorts of optical models, are rather consistent with this value of three. I am more confident of this value than the value one concludes from experiments.

MR. CARPENTER (Atomics Internationa1):

I would like to question that value of $\alpha$, which was taken equal to $\bar{\alpha}$, with a \pm 20 per cent error on it. We feel that certainly the strong structure in $\alpha$ should be taken into account. When you look at the error of the value of $\alpha$ as a function of energy, we guess it would be more like \pm 100 per cent or worse at individual energy points. Although this wouldn' $t$ be as important for the calculation for plutonium, we feel that it turned out to be quite important for the calculation for $U^{235}$.

MR. SCHMIDT:

I would say that the values of $\bar{\alpha}$ to which I refer are mainly the results of measurements in certain reactor spectra, so they cannot show fluctuations from one resonance to another resonance. They can only be average values of a larger range of energy which covers some 100 resonances.

MR.CARPENTER:

I will certainly agree with the average of $\bar{\alpha}_{0}$ My question was concerned with the fact that you took $\alpha$ as a function of energy equal to that same value of $\bar{\alpha}$, rather than considering the fluctuations in relative capture to fission rates in various resonances. When the calculation of the Doppler effect is a function of energy, because of the strong fluctuations in the capture to fission ratios at various resonances, for certain resonances you will get a much stronger positive effect than for other resonances; in fact, I believe in about a third of the resonances, where we have the resonance parameters for plutonium-239, we calculate a negative effect 
because of the very high $\alpha$ in these resonances When these are taken into account, as they fluctuate from resonance to resonance, you get a very different answer than when you just take an average $\alpha$ for the whole region

MR SCHMIDT

The theory which is essentially a statistical one is not meant for a single resonance The statistical averaging over the fission width distrubution takes into account the fluctuations you have just mentioned,

MR ZALESKI (EURATOM):

Dr Schmidt made some conclusion about the uncertanty of Doppler effect calculation due to the uncertainty of the measured nuclear data Do you have some feeling about uncertanties due to the imperfect calculation methods?

MR OTT

I would like to say something about the errors which might be introduced by the theory It is necessary to have a better one Such errors should be fairly small for high energies, but are relatively large in the neighborhood of $100 \mathrm{eV}$

MR. SCHMIDT.

I would guess that if you used only average cross sections instead of effective cross sections, which take into account the microscopic flux structure, then you could easily get a difference of 15 or so in the theoret ical prediction, because these differences of the cross sections directly go into the calculation of the flux, and its adjoint 



\section{CALCULATION OF DOPPLER COEFFICIENTS OF DILUTE FAST REACTORS}

R. Froelich, K. Ott, and J. J. Schmidt

Institut für Neutronenphysik und Reaktortechnik

Kernsforschungszentrum, Karlsruhe, Germany

(Paper presented by Mr. Ott)

I. Introduction

The theory of Doppler coefficients in fast reactors was first developed for the strongly overlapping resonances in the high-energy region essentially by Goertzel et al. (1) Bethe, (2) Lane et a1.,(3) the calculations being for fairly enriched fast neutron reactors. The neutron spectrum in these reactors is very hard, and the Doppler effect is mainly important in an energy region in which the resonances of the fertile and fissile nuclei are strongly overlapping.

In this case, the following three assumptions are well fulfilled:

1. The mean free path of the Doppler coefficient-producing neutrons is large compared with the heterogeneities, so that the systems can be treated as homogeneous.

2. The mean energy loss per collision is large compared with the mean distance between the resonances, so that the collision density can be assumed to be constant over some resonances. As a consequence of this the neutron flux is of the shape

$$
E \phi(E)=\frac{1}{\Sigma_{\text {total }}}=\frac{1}{\Sigma_{\text {pot }}+\Sigma_{\text {res }}}
$$

3. The total potential cross section $\Sigma$ pot is large compared with the total resonance cross section $\Sigma_{\text {res }}$, so that the denominator in (1) can be expanded, and the flux shape inside the resonances has the following form

$$
E \phi(E) \sim c_{0}-c_{1} \Sigma_{\text {res }}
$$

As the consequence of the fact that the flux depression inside each resonance (the microscopic flux depression) is proportional to the resonance shape, given by the $\psi$-function, the interaction effects of independent resonance series cancel their contributions to the Doppler coefficient, as can easily be proved. The Doppler coefficient DC can then be written as a sum over the contributions of the different isotopes 


$$
D C=\sum_{n} D C_{n}
$$

In ceramic-fueled fast reactors, which, moreover, use fuel of low enrichment, the Doppler coefficient mainly comes from the low-energy end of the spectrum, that is, from the neighbourhood of $1 \mathrm{keV}$. In this energy region, the expansion (2) is no longer valid and the spectrum (1) must be used. However, considering that the sum over all resonances in the denominator of (1) must be taken, it is nearly impossible to carry out any practical calculations.

Fortunately, the low-energy resonances permit, to some extent, another simplification: the overlapping of the resonances of the same series can be neglected. If one neglects beyond this also the Doppler effect influence - coming from the still remaining overlapping between resonances of different series - the Doppler coefficient can again be written in form (3), and each $D C_{n}$ is essentially a sum over the temperature derivatives of the well known I-functions for single resonances. All the calculations of Doppler coefficients for dilute fast reactors have in principle made use of this concept: Nicholson, (4) Greebler, (5) Hummel, (6) Fossoul, $(7)$ and the authors of this paper. (8)

Recently, Codd and Collins $(9)$ showed for a few specific resonances that the interaction effect in the low-energy region is of great importance for the Doppler coefficients of dilute fast reactors. Their method, however, which is quite sufficient for examples, seems too tedious for routine calculations of Doppler coefficients.

Looking for possibilities of taking into account the distribution functions of level width and spacings, we have developed the following method for the calculation of Doppler coefficients of dilute fast reactors. This paper gives only a rough survey. The mathematical details, which are somewhat lengthy, will be given shortly in a report in which the quantitative importance of the different contributions will be discussed.

\section{The Local Doppler Coefficient}

We make use of the first two assumptions referred to above: the homogeneous treatment, and the constancy of the collision density in small energy intervals.

The description may be simplified if one distinguishes the "local Doppler coefficient, "which is a function of energy only, and the Doppler coefficient of a reactor, which is to be calculated from the local DC by normal averaging with the energy-and space-dependent neutron flux. 
The main difficulty in the theory of Doppler coefficients is the calculation of the basic quantity (and its temperature derivative):

$$
\frac{d}{d T}\left\langle\vec{J}_{x}\right\rangle_{D \Gamma}=\frac{d}{d T}\left\langle\int_{\Delta E} \frac{\Sigma_{\text {res } x}}{\sum_{\text {pot }}+\Sigma_{x e s}} d E\right\rangle_{D \Gamma},
$$

where d/dT denotes the derivative with respect to the absolute temperature and the brackets assign the averaging over the distribution functions of the spacings $D$ and widths $\Gamma$ of all resonances. $\Sigma_{p o t}$ is the total potential cross section: $\Sigma_{\text {res }}$ and $\Sigma_{\text {res }} \mathrm{x}$ are the total and $\mathrm{x}-\mathrm{type}-\mathrm{res}$ onance cross sections ( $x=$ fission or capture).

In dilute fast ceramic-fueled reactors all series of resonances in the fertile and fissile isotopes can be classified in two groups:

I. $\mathrm{U}^{238}: \mathrm{I}=0$ - series

II. U $\mathrm{U}^{238}$ : higher 1 -values

$\mathrm{Pu}^{239}$ : all quantum numbers

$U^{235}:$ a.ll quantum numbers.

For all the series in group II the resonance peak cross sections for real temperatures are small compared with $\Sigma_{\text {pot }}\left(\equiv \Sigma_{\mathrm{p}}\right)$ and only for the $\mathrm{U}^{238}$ $(1=0)\left[\equiv U_{0}\right]$-series this is not the case.

Therefore we expand the series of group II in the former way and for group I we can use the fact, that the resonances of the $U_{0}$-series are well separated in the low-energy region.

Furthermore, to minimize the error coming from neglecting terms of higher order. we do not expand around $\Sigma$ pot, but around a modified cross section

$$
\Sigma_{\text {pot }}+\Sigma_{S}^{*}
$$

and determine $\Sigma_{S}^{*}$ so that the second term of the expansion around $\Sigma_{p}+\Sigma_{S}^{*}$ vanishes. Using this, we can calculate the Doppler coefficient essentially using the same terms as in the Doppler coefficient theory without resonance interaction.

At first we split the series of resonance cross sections into group I (uranium $I=0: U_{0}$ ) and group II (others) (in the following each $\Sigma$ denotes the cross section of an individual resonance series): 


$$
\Sigma_{\text {res }} \equiv \Sigma_{r}=\Sigma_{r}\left(U_{0}\right)+\sum_{S} \Sigma_{r}(S)
$$

where $S$ is an index, which indicates the "other" series. Then

$$
\begin{aligned}
\bar{J}_{\mathrm{X}} & =\int_{\Delta E} \frac{\Sigma_{\mathrm{xX}}\left(\mathrm{U}_{0}\right)}{\bar{\Sigma}_{\mathrm{p}}+\Sigma_{\mathrm{r}}} \mathrm{dE}+\sum_{\mathrm{S}} \int_{\Delta E} \frac{\Sigma_{\mathrm{rX}}(\mathrm{S})}{\Sigma_{\mathrm{p}}+\Sigma_{\mathrm{r}}} \mathrm{dE} \\
& =\bar{J}_{\mathrm{X}}\left(\mathrm{U}_{0}\right)+\sum_{\mathrm{S}} \overline{\mathrm{J}}_{\mathrm{X}}(\mathrm{S}) .
\end{aligned}
$$

Now, because the resonances of group II are small compared with $\Sigma_{p}$, the denominator of each term can be expanded (after adding and subtracting a certain quantity $\left.\Sigma_{U_{0}^{*}}^{*}\right):$

$$
\bar{J}_{X}\left(U_{0}\right)=\int_{\Delta E} \frac{\Sigma_{Y X}\left(U_{0}\right)}{\Sigma_{p}+\Sigma_{U_{0}}^{*}+\Sigma_{Y}\left(U_{0}\right)}\left\{1-\frac{\sum_{S} \Sigma_{Y}(S)-\Sigma_{U_{0}}^{*}}{\Sigma_{P}+\Sigma_{U_{0}}^{*}+\Sigma_{r}\left(U_{0}\right)}\right\} d E .
$$

The series of group II appear in (7) only in the numerator, so that averaging over the distribution functions of level widths and spacings of the group II resonances can be carried out. The second expansion term vanishes, if one defines

$$
\Sigma_{U_{0}}^{*}=\left\langle\sum_{S} \Sigma_{r}(S)\right\rangle_{D \Gamma(\text { all } S)}
$$

and there remains

$$
\left\langle\bar{J}_{X}\left(U_{0}\right)\right\rangle=\left\langle\int_{\Delta E} \frac{\Sigma_{\mathrm{rX}}\left(U_{0}\right)}{\Sigma_{\mathrm{p}}+\Sigma_{U_{0}}^{*}+\Sigma_{\mathrm{r}}\left(U_{0}\right)} d E\right\rangle_{D \Gamma\left(U_{0}\right)} .
$$

Similarly, the other terms can be expanded:

$$
\bar{J}_{X}(S)=\int_{\Delta E} \frac{\Sigma_{r X}(S)}{\nabla_{p}+\Sigma_{S}^{*}+\Sigma_{r}\left(U_{0}\right)}\left\{1-\frac{\sum_{S} \Sigma_{x}(S)-\Sigma_{S}^{*}}{\Sigma_{p}+\Sigma_{S}^{*}+\Sigma_{r}\left(U_{0}\right)}\right\} d E_{,}
$$


where the averaging leads to a vanishing second term if one defines

$$
\Sigma_{S}^{*}=\frac{\left\langle\Sigma_{r x}(S) \sum_{S^{\prime}} \Sigma_{r}\left(S^{\prime}\right)\right\rangle_{D \Gamma\left(a 11 S^{\prime}\right)}}{\left\langle\Sigma_{r x}(S)\right\rangle_{D \Gamma(S)}}
$$

which depends on temperature, contrary to (8). Thus one gets for each $S$ the result (because of the independence of different series)

$$
\left\langle\bar{J}_{\mathrm{X}}(S)\right\rangle=\left\langle\Sigma_{\mathrm{rX}}(\mathrm{S})\right\rangle_{\mathrm{D} \Gamma(S)}\left\langle\int_{\Delta E} \frac{\mathrm{dE}}{\Sigma_{\mathrm{p}}+\Sigma_{S}^{*}+\Sigma_{\mathrm{r}}\left(U_{0}\right)}\right\rangle_{D \Gamma\left(U_{0}\right)}
$$

Now, (9) plus all terms (12) are to be differentiated with respect to temperature:

$$
\begin{aligned}
& \frac{\mathrm{d}}{\mathrm{dT}}\left\langle\overline{J_{\mathrm{X}}}\right\rangle=\frac{\mathrm{d}}{\mathrm{dT}}\left\langle\int_{\Delta \mathrm{E}} \frac{\Sigma_{\mathrm{TX}}\left(\mathrm{U}_{0}\right)}{\Sigma_{\mathrm{P}}+\Sigma_{U_{0}}^{*}+\Sigma_{\mathrm{r}}\left(U_{0}\right)} \mathrm{dE}\right\rangle_{D \Gamma\left(\mathrm{U}_{0}\right)} \\
& +\sum_{S}\left\langle\Sigma_{\mathrm{rx}}(S)\right\rangle_{D \Gamma(S)} \frac{\mathrm{d}}{\mathrm{d} T}\left[\left\langle\int_{\Delta \mathrm{E}} \frac{\mathrm{dE}}{\Sigma_{\mathrm{P}}+\Sigma_{S}^{*}+\Sigma_{\mathrm{r}}\left(U_{0}\right)}\right\rangle\right]_{D \Gamma\left(U_{0}\right)_{\Sigma_{S}^{*}=\text { const }}} \\
& +\sum_{S} \frac{d}{d T}\left\langle\Sigma_{r X}\left(S^{\prime}\right) \sum_{S^{\prime}} \Sigma_{r}\left(S^{\prime}\right)\right\rangle_{D \Gamma\left(a 11 S^{\prime}\right)}\left\langle\int_{\Delta E} \frac{d E}{\left(\Sigma_{p}+\Sigma_{S^{*}}^{*}+\Sigma_{r}\left(U_{0}\right)\right)^{2}}\right\rangle_{D \Gamma\left(U_{0}\right)}
\end{aligned}
$$

The physical meanings of these three terms, especially in comparison with the respective terms without resonance interaction are the following:

The first term gives the Doppler coefficient of $U^{238}$, where the other resonance series add the cross section $\Sigma_{U_{0}}^{*}$ to $\Sigma_{p}$ to account for the fact that the $\mathrm{U}^{238}$ resonances have to compete not only with the potential, but also with all other cross sections.

The second term describes the influence of the $\mathrm{U}^{238}: 1=0$ resonances on all others. One can shift the first bracket into the numerator of the integral and interpret this expression as the $\Sigma_{\mathrm{rX}}(\mathrm{S})$ reaction rate reduced by the temperature-dependent $U^{238}: 1=0$ flux depressions. This term vanishes at very high energy because the flux depression then has the form (2) and the average becomes independent of temperature. It vanishes also at very low energies, because then the boundaries of the 
flux dips are caused by the far wings of the resonances, and therefore are also independent of temperature. This term does not appear in the usual theory.

The third term describes the usual Doppler effect of the small resonances, but it differs from the usual one in the last bracket:

$$
\left\langle\int_{\Delta E} \frac{d E}{\left(\Sigma_{p}+\Sigma_{S}^{*}+\Sigma_{r}\left(U_{0}\right)\right)^{2}}\right\rangle_{D \Gamma\left(U_{0}\right)} \text { instead of } \frac{\Delta E}{\left\langle\Sigma_{\text {total }}\right\rangle^{2}}
$$

and therefore contains also a remarkable interaction effect.

III. The Doppler Coefficient of a Reactor

The local Doppler coefficient is defined as

$\frac{\mathrm{d} \Sigma}{\mathrm{dT}}=-\frac{1}{\int_{\Delta E} \frac{\mathrm{dE}}{\Sigma_{\mathrm{t}}}}\left[\sum_{\text {capt }} \frac{\mathrm{d}}{\mathrm{dT}} \int_{\Delta E} \frac{\Sigma_{\mathrm{rx}}}{\Sigma_{\mathrm{t}}} \mathrm{dE}-\sum_{\text {fission }}(\nu-1) \frac{\mathrm{d}}{\mathrm{dT}} \int_{\Delta E} \frac{\Sigma_{\mathrm{rx}}}{\Sigma_{\mathrm{t}}} \mathrm{dE}\right]$,

where the first sum contains all capture and the second all fission resonance series. The assignment of the averaging over the distribution function is omitted in (15). Further, the adjoint function of the fission neutrons is set equal to the adjoint function of the Doppler coefficientproducing neutrons.

For the local temperature $T$ we use, in the following calculations, the temperature $\overline{\mathrm{T}}_{\mathrm{L}}$, the average over a rod cross section. To account for the temperature distribution over the fuel rod, one has to calculate a modified mean temperature depending also on the parameters of the fuel rod temperature distribution as proposed by Greebler. (10) In a cylindrical core the distribution of this local temperature $\overline{\mathrm{T}}_{\mathrm{L}}$ can be separated into an axial and a radial distribution. In the axial direction we use a suitable average value, and in the radial direction we include the distribution in the perturbation formula.

As the temperature to which the reactivity change refers we choose (arbitrarily) the overall mean fuel temperature in the core, $\bar{T}$. Then the Doppler coefficient of the reactor is given by

$$
\frac{d k}{d \bar{T}}=\frac{1}{N} \int \frac{d \Sigma}{d \bar{T}_{L}} \frac{d \bar{T}_{L}}{d \bar{T}} \phi \phi^{+} r d r d E,
$$


where

$$
N=\int \chi\left(E^{\prime}\right) \nu \Sigma_{f} \phi^{+}\left(E^{\prime}, r\right) \phi(E, r) d E^{\prime} d E r d r
$$

and approximately

$$
\bar{T}_{L}=\bar{T}+1 / 2 \Delta T-\Delta T\left(\frac{x}{R}\right)^{2},
$$

where $R$ is the core radius and $\Delta T$ the temperature difference between core axis and boundary.

The quantity $d \bar{T}_{I} / d \bar{T}$, which transforms the "local" into the "reference" temperature, depends on the shape of temperature increase. Especially for adiabatic excursions, one gets

$$
\frac{d \bar{T}_{L}}{d t} \sim S(r) \int_{0}^{t}\left[\phi\left(t^{\prime}\right)-\phi_{0}\right] d t^{\prime}
$$

and

$$
\frac{d \bar{T}}{d t} \sim \bar{s} \int_{0}^{t}\left[\phi\left(t^{\prime}\right)-\phi_{0}\right] d t^{\prime}
$$

which gives

$$
\frac{d \bar{T}_{L}}{d \bar{T}}=\frac{S(r)}{\bar{S}}
$$

proportional to the fission rate distribution $S(r)$.

IV. Results and Discussion

We have calculated the Doppler coefficient of two reactors for different temperatures, temperature distributions, and sodium densities. The detailed composition of the two systems, which have a $\mathrm{U}^{238}$ to $\mathrm{Pu}^{239}$ ratio of 5 and 7 , is given in the paper of Ott and Schröter in Session 1.

Figure 1 shows the contribution of $U^{238}$ in the $y=7$ reactor to the local DC $\left(\frac{d \Sigma}{d T}\right)$ for different temperatures. At low and high energies, $\frac{d \Sigma}{d T}$ tends to vanish, as is well known. Also, the broad resonances of sodium and the structural material decrease the Doppler effect. The temperature 
dependence at high energies is of the well-known $T^{-3 / 2}$ form; at low energies there does not exist a simple temperature law.

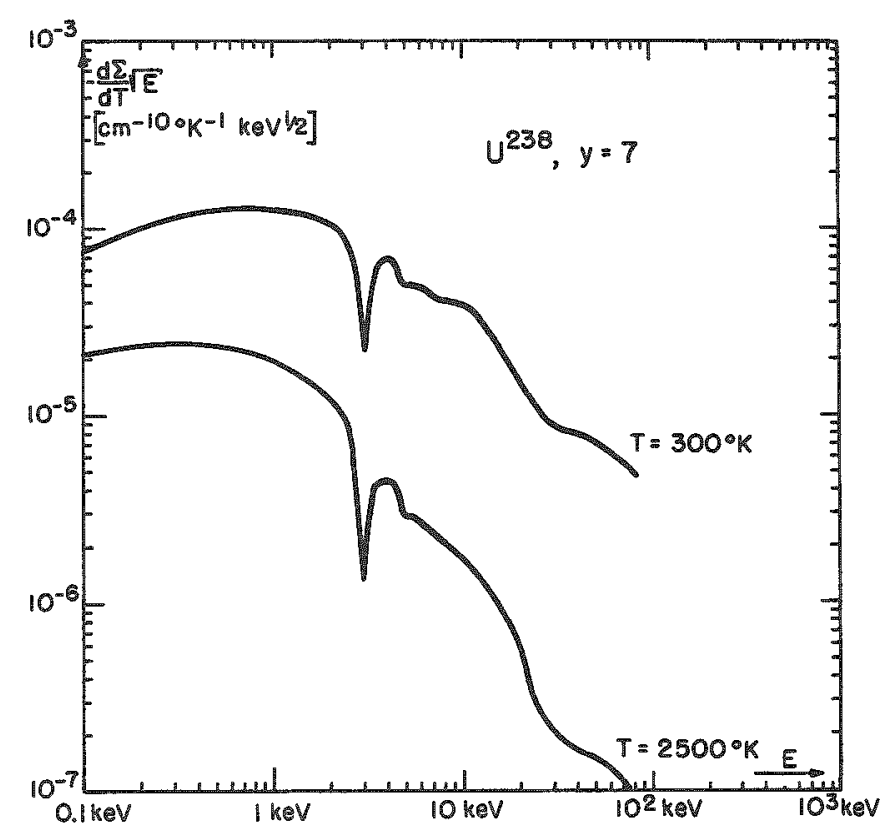

Fig. 1

Contribution of $\mathrm{U}^{238}$ to the Local Doppler Coefficient when $\mathrm{y}=7$.

Figure 2 gives the effect of the resonance interaction on the $\mathrm{Pu}^{239}$ contribution at different temperatures on a linear scale in order to show the changes on sign along the energy axis. In the dashed curves the resonance interaction is neglected, whose relative influence increase with increasing temperature up to a change in sign, as can be seen from Fig. 2.

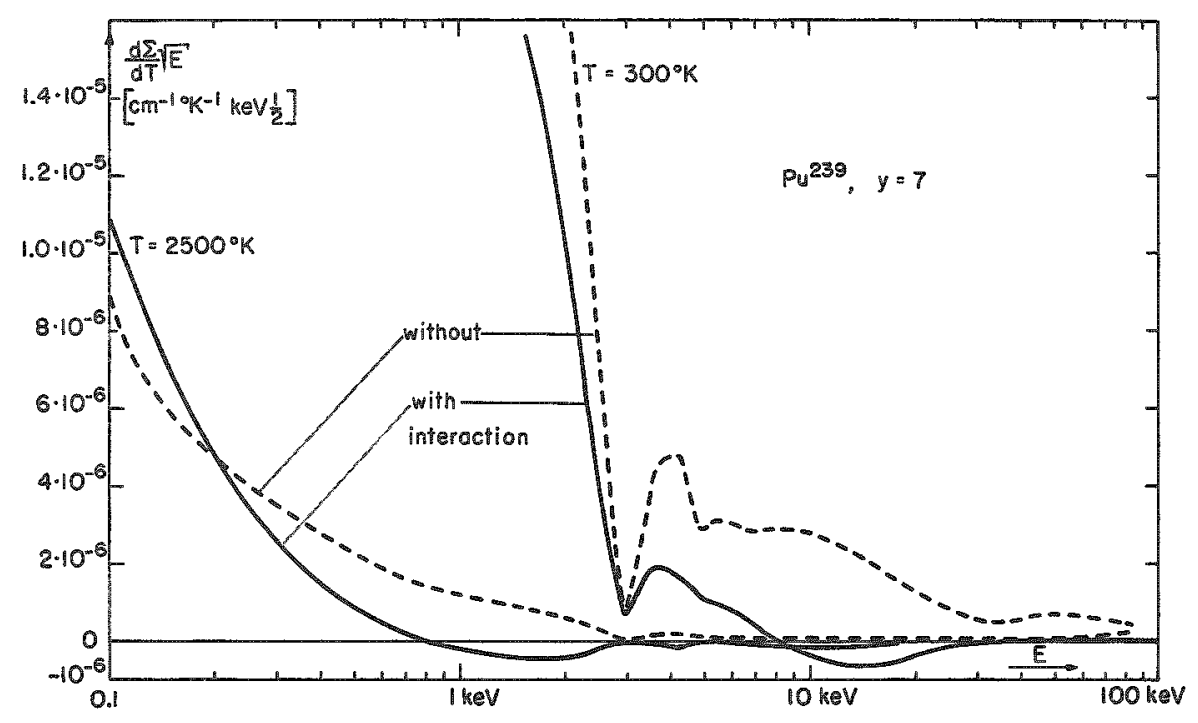

Fig. 2. Effect of Resonance Interaction on the $\mathrm{Pu}^{239}$ Contribution to the Doppler Coefficient 
Figures 3 and 4 give the energy dependence of the local DC for $y=7$ and 5. For decreasing energy, the local DC decreases and changes sign, which is indicated by dashing. With increasing $y$ and increasing temperature the energy point, at which the sign changes, tends to become lower because of the increase of the $U^{238}$ effect.

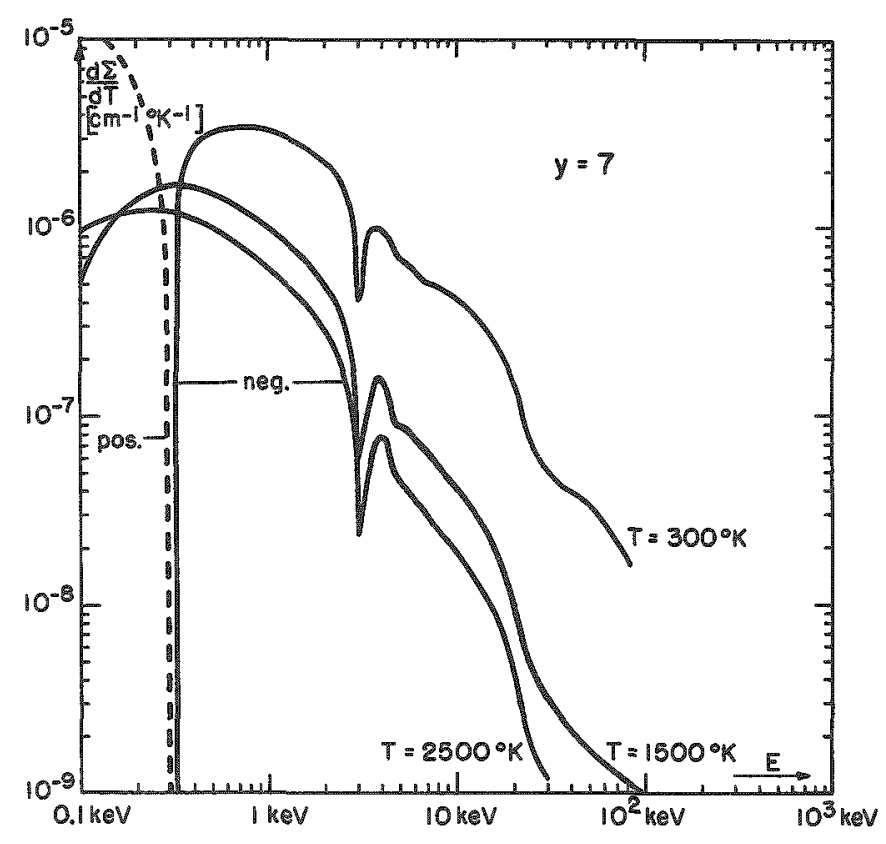

Fig. 3

Energy Dependence of the Local Doppler Coefficient when $y=7$

Fig. 4

Energy Dependence of the Local Doppler Coefficient when $y=5$

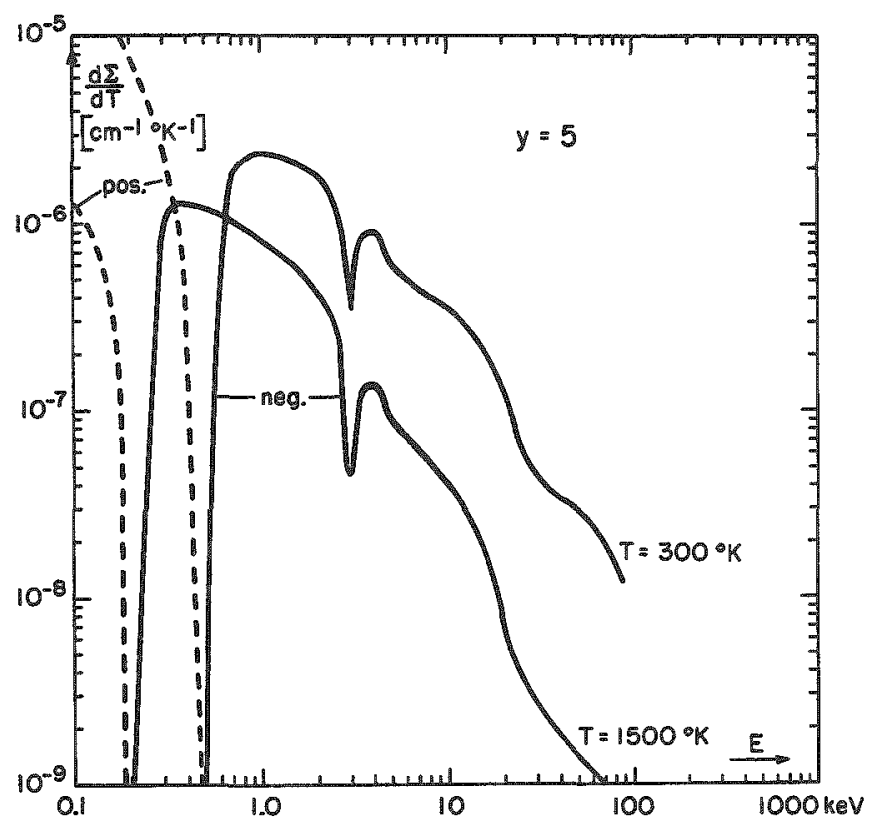

All these curves show that the sodium resonance decreases the Doppler effect by about a factor of three. When the Doppler coefficient of a reactor is calculated, a further decrease is found from the flux dip caused by the large sodium resonance, so that in a first approximation the 
contribution from the energy range of the sodium resonance is cancelled. After a sodium loss, this energy region contributes a full Doppler effect (see Table 1).

Table 1

\begin{tabular}{c|c|c|c}
\hline $\begin{array}{c}\text { Lower } \\
\text { E-limit, keV }\end{array}$ & Group & $\begin{array}{c}y=7 \\
40 \% \text { Sodium }\end{array}$ & $\begin{array}{c}y=7 \\
\text { No Sodium }\end{array}$ \\
\hline 110 & 8 & 0 & 0 \\
67 & 9 & $-1.96 \times 10^{-8}$ & $-2.01 \times 10^{-8}$ \\
41 & 10 & $-3.29 \times 10^{-8}$ & $-4.31 \times 10^{-8}$ \\
25 & 11 & $-3.18 \times 10^{-8}$ & $-2.98 \times 10^{-8}$ \\
15 & 12 & $-1.03 \times 10^{-7}$ & $-9.69 \times 10^{-8}$ \\
9.1 & 13 & $-1.67 \times 10^{-7}$ & $-1.59 \times 10^{-7}$ \\
5.5 & 14 & $-1.68 \times 10^{-7}$ & $-1.55 \times 10^{-7}$ \\
2.1 & 15 & $-3.95 \times 10^{-7}$ & $-6.74 \times 10^{-7}$ \\
100 & 16 & $-2.30 \times 10^{-6}$ & $-1.04 \times 10^{-6}$ \\
\hline & \\
$\left(\frac{\mathrm{dk}}{\mathrm{dT}}\right)_{\text {isothermal }}\left(\overline{\mathrm{T}}=1500^{\circ} \mathrm{K}\right)$ & for each group.
\end{tabular}

In the calculation of the DC by means of the perturbation formula (16), we have used a first guess only for the low-energy end of the spectrum, which will now be improved. We have used the multigroup spectrum calculated with a YOM cross section set up to $500 \mathrm{eV}$, and between 500 and $100 \mathrm{eV}$ a suitable Fermi age spectrum for the fundamental mode calculated with the effective cross sections for this composition and $300^{\circ} \mathrm{K}$. The contribution below $100 \mathrm{eV}$ was neglected.

Table 2 gives the isothermal DC for the $y=7$ reactor with and without sodium. The temperature dependence can very well be described

Table 2

\begin{tabular}{r|c|c}
\hline $\bar{T}\left({ }^{\circ} \mathrm{K}\right)$ & $y=7$ & $y=7$ \\
& $40 \%$ Sodium & No Sodium \\
\hline 300 & $-1.34 \times 10^{-5}$ & $-1.26 \times 10^{-5}$ \\
600 & $-7.42 \times 10^{-6}$ & $-6.04 \times 10^{-6}$ \\
900 & $-5.16 \times 10^{-6}$ & $-3.90 \times 10^{-6}$ \\
1500 & $-3.22 \times 10^{-6}$ & $-2.22 \times 10^{-6}$ \\
2100 & $-2.34 \times 10^{-6}$ & $-1.52 \times 10^{-6}$ \\
\hline
\end{tabular}

$\left(\frac{\mathrm{dk}}{\mathrm{dT}}\right)_{\text {is othermal }}$ 
by a $T^{-\gamma}$ law, where $\gamma$ depends on the reactor composition. We have found for the $y=7$ reactor and various sodium densities the results given in Table 3, for which we have used law

$$
\operatorname{DC}(\overline{\mathrm{T}})=\mathrm{C}\left(\frac{\overline{\mathrm{T}}_{0}}{\overline{\mathrm{T}}}\right)^{\gamma}, \quad \overline{\mathrm{T}}_{0}=300^{\circ} \mathrm{K} \text {. }
$$

Table 3

\begin{tabular}{|c|c|c|c|}
\hline \multirow{2}{*}{} & \multicolumn{3}{|c|}{ Relative Sodium Density } \\
\cline { 2 - 4 } & $100 \%$ & $50 \%$ & $0 \%$ \\
\hline $\mathrm{C}$ & $-1.34 \times 10^{-5}$ & $-1.28 \times 10^{-5}$ & $1.26 \times 10^{-5}$ \\
$\gamma$ & 0.89 & 0.96 & 1.06 \\
\hline
\end{tabular}

The influence of temperature distribution is of the order of $10-20 \%$, as found by Greebler, (10) One can account for this effect and reduce to a pseudo-isothermal DC if one uses an effective mean temperature

$$
\overline{\mathrm{T}} \approx \frac{\overline{\mathrm{T}}_{\mathrm{L}}(0)+\overline{\mathrm{T}}}{2}
$$

as it is found for parabolic temperature distributions with various spreads $\Delta \mathrm{T}$.

\section{References}

1. G. Goertze1, H. Eeshbach, H. Yamauchi. H. Hurwitz, and G. Roe. United Nations International Conference on the Peaceful Uses of Atomic Energy, Geneva, Switzerland, 5, 472 (1955).

2. H. A. Bethe, APDA-119 (1957).

3. A. M. Lane, J. E Lynn, and J S. Story, AERE T/M 137 (1956).

4. R.B. Nicholson, APDA-139 (1960).

5. P. Greebler, Vienna Seminar on the Physics of Fast and Intermediate Reactors, paper SM 18/59 (1961).

6. M. G. Bhide and H. H. Hummel, ANL-6601 (1962).

7. E. A. Fossoul, Vienna Seminar on the Physics of Fast and Intermediate Reactors, paper SM 18/10 (1961). 
8. R. Froelich, K. Ott, and J. J. Schmidt, EAES-Symposium on Advances in Reactor Theory, Karlsruhe, April 1963, P/III, 1.

9. J. Codd and P. J. Collins, ibid., P/III, 3.

10. P. Greebler and E. Goldman, GEAP-4092 (1962).

Discussion of Paper

Presented by Mr. Ott

MR. CAMPISE (Atomics International):

Have you looked at $\mathrm{U}^{235}$ and $\mathrm{U}^{238}$ systems?

MR. OTT:

No, but we are doing this.

MR. TAVERNIER (BelgoNucleaire):

Was the Doppler coefficient you showed weighted by the importance?

MR. OTT:

Yes.

MR. STORRER (EURATOM):

One point which has so far not been discussed is the fine energy structure of the adjoint flux. It is quite obvious, I think, that not only the flux, but also the adjoint flux has a fine energy structure which so far has not been taken into account in Doppler-effect calculations. I feel it is not important, but intuition can sometimes fool us. We are right now looking into this problem.

I think that the gross structure should also be looked at a little more closely. The reactivity effect due to Doppler is proportional to $\eta$ times the worth of the fast neutron minus the worth of the neutron at the energy at which it is absorbed. This number can in some cases be a small difference between large quantities.

MR. ZALESKI (EURATOM):

Does Dr. Ott agree with Dr. Codd about the interference correction? It seems to me he shows that the interference correction is very 
sma11. Dr. Codd shows that it can be $70 \%$ taking into account the fluctuation. We have made some statistical evaluation on actual measured values of resonances and found even larger numbers for statistics between $500 \mathrm{eV}$ and $3.5 \mathrm{keV}$ than just for the $1 \mathrm{keV}$ region. It seems to me that Dr. Ott's values are much smaller. I would be happy to have some comment on this point.

MR. OTT.

The expansion used does not make use of the assumption of small interaction effects. Therefore, the results are correct as long as the expansion conditions are fulfilled.

MR. NICHOLSON (University of Wisconsin):

Dr. Ott has attacked the rather interesting problem of the variation in temperature across the reactor. The general idea here, of course is that the resonance cross sections depending on temperature will be different at one point or another in the reactor. However, there is also the point that the theory assumes that the neutron flux is inversely proportional to the total cross section. Now, in that the mean free paths are rather large, the total cross section which is really involved in this assumption is the total cross section averaged over some region of space in the neighborhood of a local point. This, on the whole, was probably not very important, and one can actually use here again the actual local temperature as was done. When one gets too near the edge of the core however I begin to wonder about this. In the blanket, right at the edge of the core, there is a step change, you might say, in temperature. Also. the material in the blanket is not changing temperature very much. Do you think that this could be important?

MR OTT.

First, I would like to comment on your last point. The temperature rise in the blanket is very small, and therefore we have not integrated over the blanket and have neglected the small Dopplex coefficient part coming from the blanket. Now, as to the first point, you are right in saying that there is contradiction between assuming a constant collision density and using a temperature distribution over the reactor But this contradiction should be small because the temperature variation per mean free path is fairly small. Mean free paths are of the order of $5 \mathrm{~cm}$, and the temperature variation over $5 \mathrm{~cm}$ is not very large, especially if you consider that the main part of the Doppler coefficient comes from the inner part, where the variation of the temperature is still smaller, and gocs up to zero in the middle of the reactor. Therefore, I think that the correction which could be done would be very small. 


\title{
METHODS OF EVALUATION OF EFFECTIVE CROSS SECTION FOR DOPPLER COEFFICIENT CALCULATION IN LARGE
}

FAST BREEDER REACTORS

\author{
C. Graves \\ United Nuclear Corporation \\ White Plains, New York
}

The study described in this paper is part of a program on fast reactor safety conducted at United Nuclear Corporation and sponsored by the USAEC. The present paper is concerned with the Doppler effect in fast reactors. Other work on the program has been directed towards surveys and topical studies of reactor heat transfer, instability, and feedback mechanisms

In a previous study (Ref. 1) under the program, a survey was made of analytical and experimental work on the Doppler effect, with particular emphasis on material pertinent to the large dilute fast reactors. This paper will report on the status and some results of work on several of the areas recommended for further study in Reference 1. The work can be separated into two general parts: 1) a study of some of the uncertainties in the methods of predicting effective cross sections, and 2) calculation of correction factors to permit rapid calculation of effective cross sections for various fertile and fissıle materials

The principal concern of the work on uncertainties is the effect of resonance overlap in the unresolved resonance region on the predicted Doppler coefficient. The effect of resonance overlap on effective cross sections was first treated by Feshbach et al (Ref 2). Their formula accounted for the effects of overlap of Doppler-broadened resonances within a given sequence (e.g., given material, neutron orbital angular momentum, and spin of compound nucleus), as well as accidental overlap of resonances from different sequences. However, the Feshbach formula is limited to conditions for which the fluctuations in the total cross section are small. At lower neutron energies, for which the fluctuations are large, there appeared to be no suitable method available to account for overlap effects, particularly accidental overlap. Accordingly, a digital computer code, MARC, was prepared to calculate effective cross sections for a mixture of resonance absorbers. The code performs integrations over groups of resonances assuming constant total collision density over the group. The location and widths of the various resonances are obtained by sampling methods assuming the various widths and spacings are statistically independent. These data serve as input to the code, which then computes effective cross sections. Preparation of input data for a. $\mathrm{Pu}^{239}-\mathrm{U}^{238}$ mixture is currently in process. Additional work is also in process to indicate the effects of variation in the fission-width distribution on temperature coefficients of effective cross sections 
For purposes of discussing correction factors to permit more rapid calculation of effective cross sections, one can consider three regions of neutron energy. First, there is the lower neutron-energy region, in which the Doppler-broadened resonances have been assumed isolated and in which the fluctuations in the total cross section are relatively large. For example, the local increase in total cross section for an average resonance may be twice the background cross section for some reactor compositions. The first study of this region for fast reactors was made by Nicholson (Ref. 3). At the higher neutron energies, the effects of overlap should be included, but the fluctuations in total cross section are small. This was the region investigated by Feshbach et al. Finally, there is the poorly defined intermediate-energy region in which overlap effects should be included, but in which the fluctuations of total cross section are too large to permit use of the Feshbach formula.

Figure 1 gives some of the equations for calculating the effective cross sections $\bar{\mu}_{\mathrm{x}}$ for the isolated resonance portion of the unresolved resonance region. The calculation is seen to involve averaging of the ratio of point values of the cross section for reaction $x$ and the total cross section, and of the reciprocal of the total cross section. The total cross section appears because the assumption of constant total collision density was used in the analysis. Using the Doppler-broadened, Breit-Wigner single-level formulas, Equation 2 of Fig. 1 is obtained, in which $J(\xi, \beta)$ is the familiar function used in calculating effective resonance integrals, $\langle D\rangle$ is the average level spacing, and $\vec{I}_{x}$ is the width for reaction $x$. The average of $\Gamma_{\mathrm{X}} \mathrm{J}(\xi, \beta)$ is over the distribution of neutron and fission widths as indicated in Equation 3, where $y_{n}$ and $y_{f}$ are the ratios of the local to average neutron and fission widths, respectively, and $P(y) d y$ is the probability that $\mathrm{y}$ is between $\mathrm{y}$ and $\mathrm{y}+\mathrm{dy}$.

$$
\begin{aligned}
& \tilde{\mu}_{\mathrm{x}}=\frac{\left\langle\frac{\mu_{\mathrm{x}}}{\mu_{\mathrm{t}}}\right\rangle}{\left\langle\frac{1}{\mu_{\mathrm{t}}}\right\rangle}=\tilde{\mu}_{\mathrm{t}}\left\langle\frac{\mu_{\mathrm{x}}}{\mu_{\mathrm{t}}}\right\rangle \\
& \tilde{\mu}_{\mathrm{XS}}=\frac{\tilde{\mu}_{\mathrm{t}}}{\langle\mathrm{D}\rangle}\left\langle\Gamma_{\mathrm{X}} J(\xi, \beta)\right\rangle \\
& \left\langle\Gamma_{\mathrm{X}} \mathrm{J}(\xi, \beta)\right\rangle=\int_{0}^{\infty} \int_{0}^{\infty} \mathbb{P}\left(\mathrm{y}_{\mathbf{n}}, \nu_{\mathrm{n}}\right) \mathrm{P}\left(\mathrm{y}_{\mathfrak{f}}, \nu_{\mathrm{f}}\right)\left[\Gamma_{\mathrm{X}} \mathrm{J}(\xi, \beta)\right] d y_{\mathbf{n}} \mathrm{dy} \\
& \mathbb{P}(\mathrm{y}, \nu)=\frac{\left(\frac{\nu}{2}\right)}{\Gamma\left(\frac{\nu}{2}\right)}\left(\frac{\nu}{2} \mathrm{y}\right)^{(\nu / 2)-1} \mathrm{e}^{-(\nu / 2) \mathrm{y}}
\end{aligned}
$$

Fig. 1. Effective Cross Sections for Isolated Resonances in Unresolved Resonance Region. 
A digital computer code, BIGSUM, was prepared in FORTRAN to perform this averaging for the case in which the chi-squared distribution (see Equation 4 of Fig. 1) is used. The code has been used to calculate effective cross sections over a fairly wide range of conditions. In the calculations to date, a $\nu_{n}$ of 1 , giving the Porter-Thomas distribution, was used for the neutron widths and a $\nu_{f}$ of 2 was used for the fission widths. The objective was to have the results in general form to permit application to any fertile or fissile material. Accordingly, the results are expressed as shown in Fig. 2. In Equation 5, $f$ is the self-shielding factor, the ratio of the effective cross section $\tilde{\mu}_{\mathrm{X}}$ to the unshielded cross section $\left\langle\mu_{x}\right\rangle$. f is expressed as a product of three factors. The first factor is the ratio of the effective total cross section $\tilde{\mu}_{t}$ to the constant background total cross section $\mu_{t N}$, used in calculating $\beta$ in the $J$ function. One of the typical assumptions used is equivalent to setting this factor equal to unity and setting $\mu_{\mathrm{t} N}$ equal to the potential scattering cross section of the mixture. In the second factor, the asterisks indicate calculation with average values of the neutron and fission widths. This factor gives the self-shielding factor if all levels had the same widths. The third factor is a correction factor to account for the distribution of the widths about their average values. This factor plays a role similar to the correction factors used in calculating unshielded cross sections. It involves ratios of the average value of a function to the value of the function at the average value of its argument.

$$
\begin{aligned}
& f=\frac{\tilde{\mu}_{t}}{\mu_{t N}}\left[\frac{J\left(\xi^{*}, \beta^{*}\right)}{\frac{\pi}{2 \beta^{*}}}\right] \mathbb{Y}_{\mathrm{X}} \\
& Y_{X}=\left[\frac{\left\langle\Gamma_{X} J(\xi, \beta)>\right.}{\left\langle\Gamma_{\mathrm{X}}>J\left(\xi^{*}, \beta^{*}\right)\right.}\right]\left[\frac{\frac{\left\langle\Gamma_{\mathrm{n}}\right\rangle\left\langle\Gamma_{\mathrm{X}}\right\rangle}{\langle\Gamma\rangle}}{\left\langle\frac{\Gamma_{\mathrm{X}} \Gamma_{\mathrm{X}}}{\Gamma}\right\rangle}\right] \\
& \mathbf{Y}_{\mathrm{X}}=\mathbf{f}_{\mathrm{X}}\left(\frac{\left\langle\Gamma_{\mathrm{n}}\right\rangle}{\Gamma_{\gamma}}, \frac{\left\langle\Gamma_{\mathrm{P}}\right\rangle}{\Gamma_{\gamma}}, \xi^{*}, \xi^{*} / \beta^{*}\right) \\
& \xi *=\frac{\left\langle\Gamma_{\mathbf{n}}\right\rangle+\left\langle\Gamma_{\mathbf{f}}\right\rangle+\Gamma_{\gamma}}{\Delta} \\
& \frac{\xi^{*}}{\beta^{*}}=\frac{4 \pi \lambda^{2} g<\Gamma_{\mathrm{n}^{\prime}}}{\left(\frac{\mu_{\mathrm{tN}}}{\rho}\right)^{\Delta}}
\end{aligned}
$$

Fig. 2. Self-shielding Factor for Isolated Resonances in Unresolved Resonance Region 
For the case of a fissile material, $\mathrm{Y}_{\mathrm{X}}$ is a function of four parameters. A particular set is given in Equation 7, involves the ratio of the average neutron width to the radiative capture width, $\frac{\left\langle\Gamma_{n}\right\rangle}{\Gamma \gamma}$, the ratio of the average fission width to the radiative capture width, $\frac{\left.\zeta \Gamma_{f}\right\rangle}{\Gamma \gamma}$, and the parameters $\xi^{*}$ and $\frac{\xi^{*}}{\beta^{*}}$, given by Equations 8 and 9 . Here, $\xi^{*}$ is the ratio of the total level width (using average values) to the Doppler width. $\frac{\rho^{*}}{\beta^{*}}$ is a measure of the average fluctuation in the total cross section resulting from the resonances in the particular sequence considered.

In Fig. 3, Equation 10 is the Eeshbach formula for the temperature coefficient of the effective cross section, and Equation 11 is the corresponding equation for the self-shielding factor. This figure is introduced primarily to point out the terms $e$ and $\phi$ in these equations which account for the effect of overlap within a sequence. These have been calculated through use of the Wigner distribution for level spacing. Both e and $\phi$ approach zero at large width. Hence, a correction factor for overlap within a sequence can be obtained as shown in Equation 13.

$$
\begin{aligned}
& \frac{\partial \tilde{\mu}_{\mathrm{X}}}{\partial \mathrm{T}}=\frac{\cos 2 \delta \ell<\mathrm{D}\rangle\left\langle\mu_{\mathrm{X}}\right\rangle\left\langle\mu_{\mathrm{C}}\right\rangle}{\left.\sqrt{8 \pi} \mathrm{T} \Delta<\mu_{\mathrm{t}}\right\rangle} \quad\left[\mathrm{Ex}_{\mathrm{X}}-\mathrm{e}\right] \\
& f=\frac{\tilde{\mu}_{\mathrm{X}}}{\left\langle\mu_{\mathrm{X}}{ }^{\prime}\right.}=1-\frac{\left.2.5 \times 10^{3} \cos 2 \delta_{\mathrm{l}} 9<\Gamma_{\mathrm{n}}\right\rangle}{\left(\frac{238}{\mathrm{M}_{\mathrm{S}}}\right)^{1 / 2}\left(\frac{\left\langle\mu_{\mathrm{t}}\right\rangle}{\rho}\right) \mathrm{E}^{3 / 2}\left(\frac{\mathrm{T}}{300}\right)^{3 / 2}}\left[\mathrm{E}_{\mathrm{X}}-\phi\right] \\
& E_{\mathrm{X}}=\frac{\left\langle\frac{\Gamma_{\mathrm{n}}^{2} \Gamma_{\mathrm{X}}}{\Gamma}\right\rangle}{\left\langle\Gamma_{\mathrm{n}}\right\rangle\left\langle\frac{\Gamma_{\mathrm{n}} \Gamma_{\mathrm{X}}}{\Gamma}\right\rangle} \\
& \frac{\tilde{\mu}_{X}^{\prime}-<\mu_{X}>}{\tilde{\mu}_{X}-<\mu_{X^{>}}}=1-\frac{\phi\left(\frac{<D}{\Delta}\right)}{E_{X}}
\end{aligned}
$$

Fig. 3. Effective Cross Sections for Weak, Overlapping Resonances in Unresolved Resonance Region

Figure 4 shows a simplified approach to the problem of calculating effective cross sections for isolated resonances. If it is assumed that the asymptotic formula for $\psi$ holds, $\frac{2 \beta}{\pi} J(\xi, \beta)$ can be expressed by the series 
shown in Equation 15 for $\frac{\nu \pi}{2} \frac{\xi}{\beta}$ less than 1 . Substituting Equation 15 in Equation 14, rearranging, and performing the required averaging, we obtain Equation 16. $Z_{1 x}$ is a function of $A$ and $B$ as shown in Equation 17. Equation 17 reduces to the Feshbach formula with $\phi=0$ if the series in Equation 15 is truncated to the term involving $\frac{\xi}{\beta}$ and $\mu_{\mathrm{tN}}$ is set equal to $\tilde{\mu}_{t}$. The $\cos 2 \delta l$ factor appears in this equation as a correction for interference between potential and resonance scattering. Inclusion of the $Z_{1 x}$ and higher-order terms gives a correction for the effect of increase in fluctuations of the total cross section on the effective cross section.

$$
\begin{aligned}
& \tilde{\mu}_{\mathrm{X}}=\frac{\tilde{\mu}_{\mathrm{t}}}{\langle\mathrm{D}\rangle}<\Gamma_{\mathrm{X}} \mathrm{J}(\xi, \beta)> \\
& \frac{2}{\pi} \beta J(\xi, \beta)=1-\sqrt{\frac{\pi}{8}}\left(\frac{\xi}{\beta}\right)+\frac{\pi}{\sqrt{48}}\left(\frac{\xi}{\beta}\right)^{2}-\frac{\pi^{3 / 2}}{16}\left(\frac{\xi}{\beta}\right)^{2}+\ldots \\
& \frac{\tilde{\mu}_{\mathrm{X}}}{<\mu_{\mathrm{X}}}=\frac{\tilde{\mu}_{\mathrm{t}}}{\mu_{\mathrm{tN}}}\left\{1-\left[\frac{\cos 2 \delta_{\ell}<\mu_{\mathrm{c}}>E_{\mathrm{X}}<\mathrm{D}>}{\mu_{\mathrm{tN}} \Delta}\right]\right. \\
& \begin{array}{l}
+[ \\
+\ldots\}
\end{array} \\
& \mathrm{z}_{1 \mathrm{X}}=\frac{\left\langle\frac{\Gamma_{\mathrm{n}}^{3} \Gamma_{x}}{\Gamma}\right\rangle\left\langle\frac{\Gamma_{\mathrm{n}} \Gamma_{\mathrm{x}}}{\Gamma}\right\rangle}{\left\langle\frac{\Gamma_{\mathrm{n}}^{2} \Gamma_{x}}{\Gamma}\right\rangle^{2}}
\end{aligned}
$$

Fig. 4. Approximate Expression for Self-shielding Factor for Isolated Resonances in Unresolved Resonance Region

The general characteristics of the factors used in calculating effective cross sections for isolated resonances in the unresolved resonance region are illustrated in Figs. 5 through 8 . The subscripts $\gamma$ and $f$ refer to radiative capture and fission, respectively.

In Fig. $5, \frac{2 \beta^{*}}{\pi} \mathrm{J}\left(\xi^{*}, \beta^{*}\right)$ is plotted versus $\frac{\xi^{*}}{\beta^{*}}$. For fertile materials, $\xi^{*}$ of interest ranges from about 0.04 to 0.1 , and the $\frac{\xi^{*}}{\beta^{*}}$ of interest ranges up to about 2 . In this region there is a small spread in the curves with $\xi *$. 


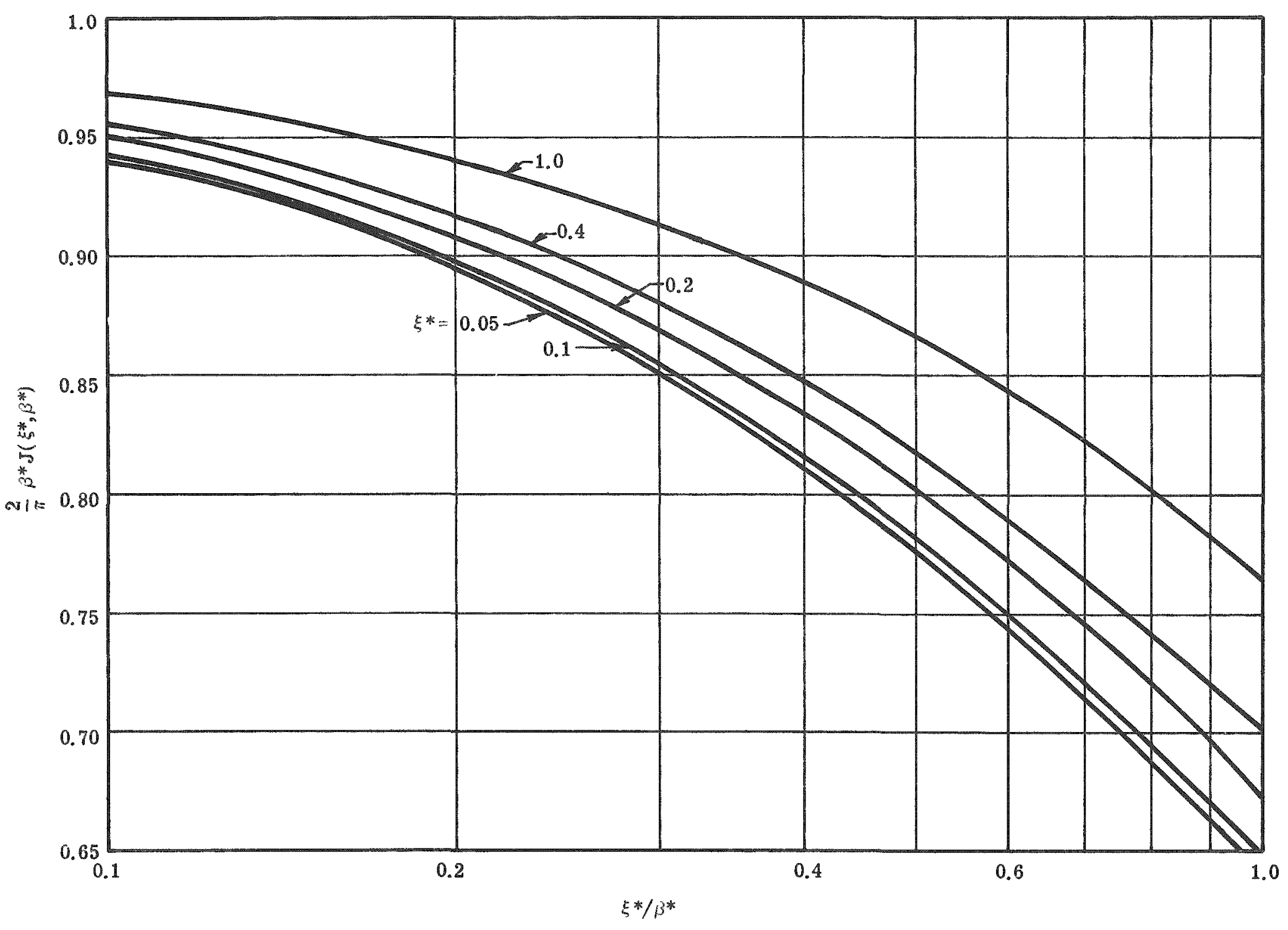

Fig. 5. $\frac{2}{\pi} \beta^{* J}(\xi *, \beta *)$ vs $\xi * / \beta^{*}$ 


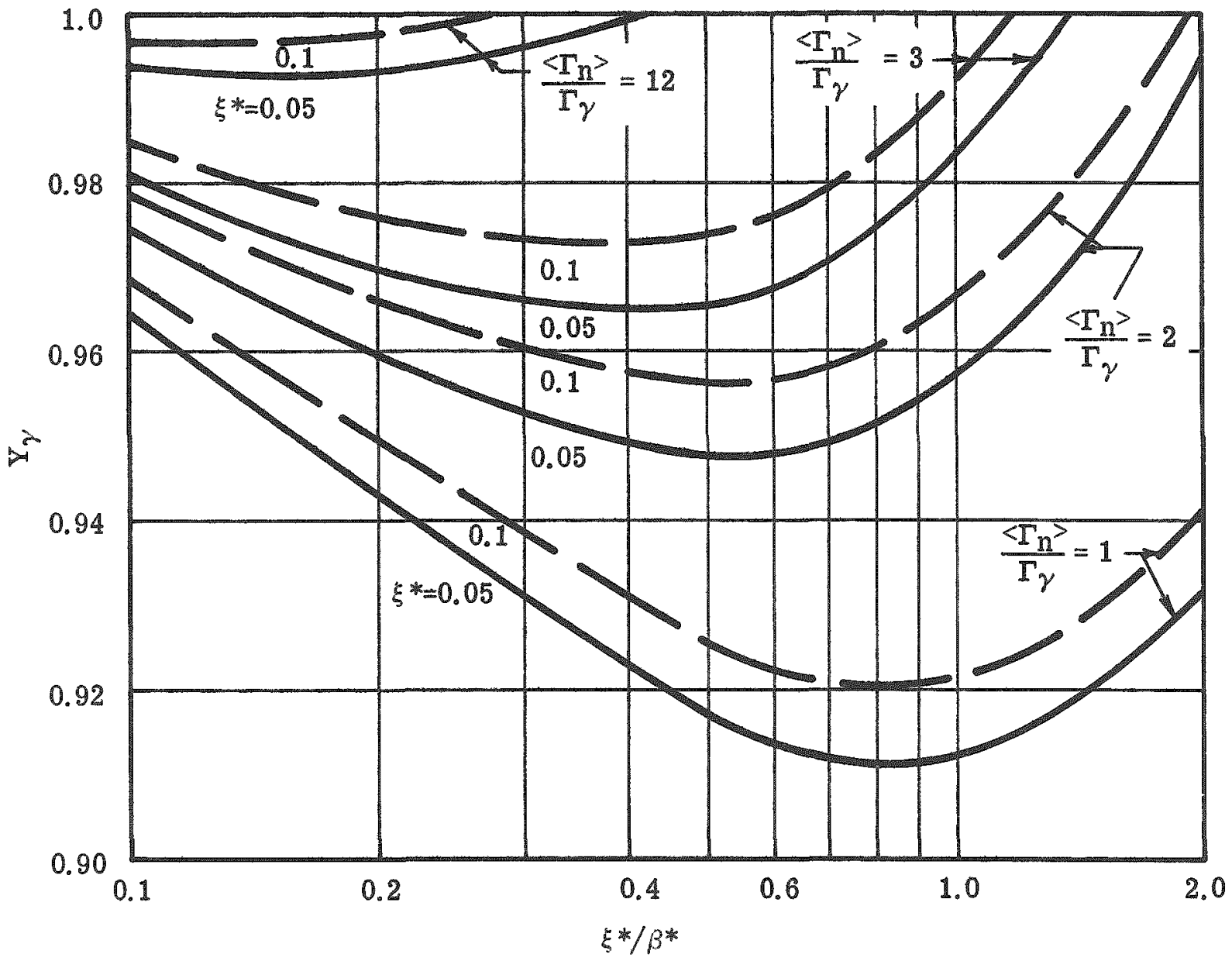

Fig. 6. Effects of $\left\langle\Gamma_{n}\right\rangle / \Gamma \gamma, \xi * / \beta^{*}$ and $\xi^{*}$ on Correction Factor $\mathrm{Y}_{\gamma}$ for Fertile Material

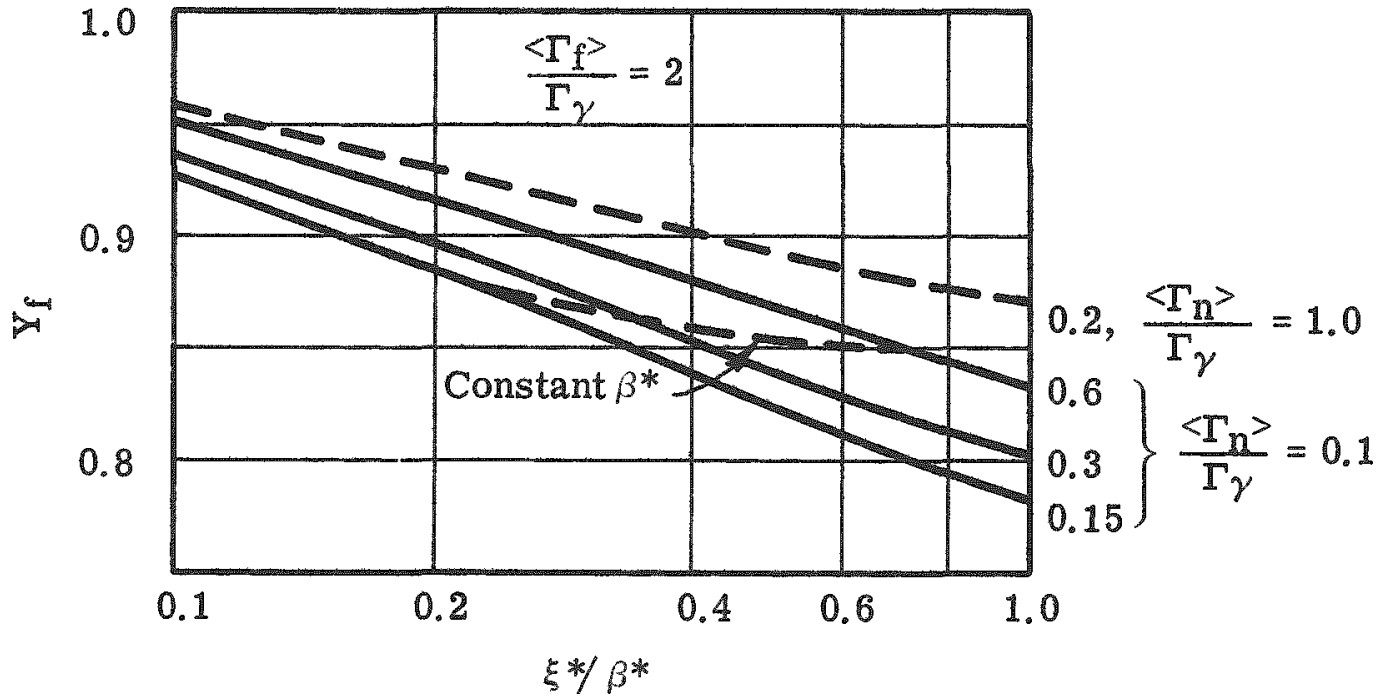

Fig. 7. Effect of $\left\langle\Gamma_{\mathrm{n}}\right\rangle / \Gamma_{\gamma}, \xi * / \beta *$ and $\xi *$ on $\mathrm{Y}_{\mathrm{f}}$ for Fissile Material with $\left\langle\Gamma_{f}\right\rangle / \Gamma_{\gamma}=2$ 


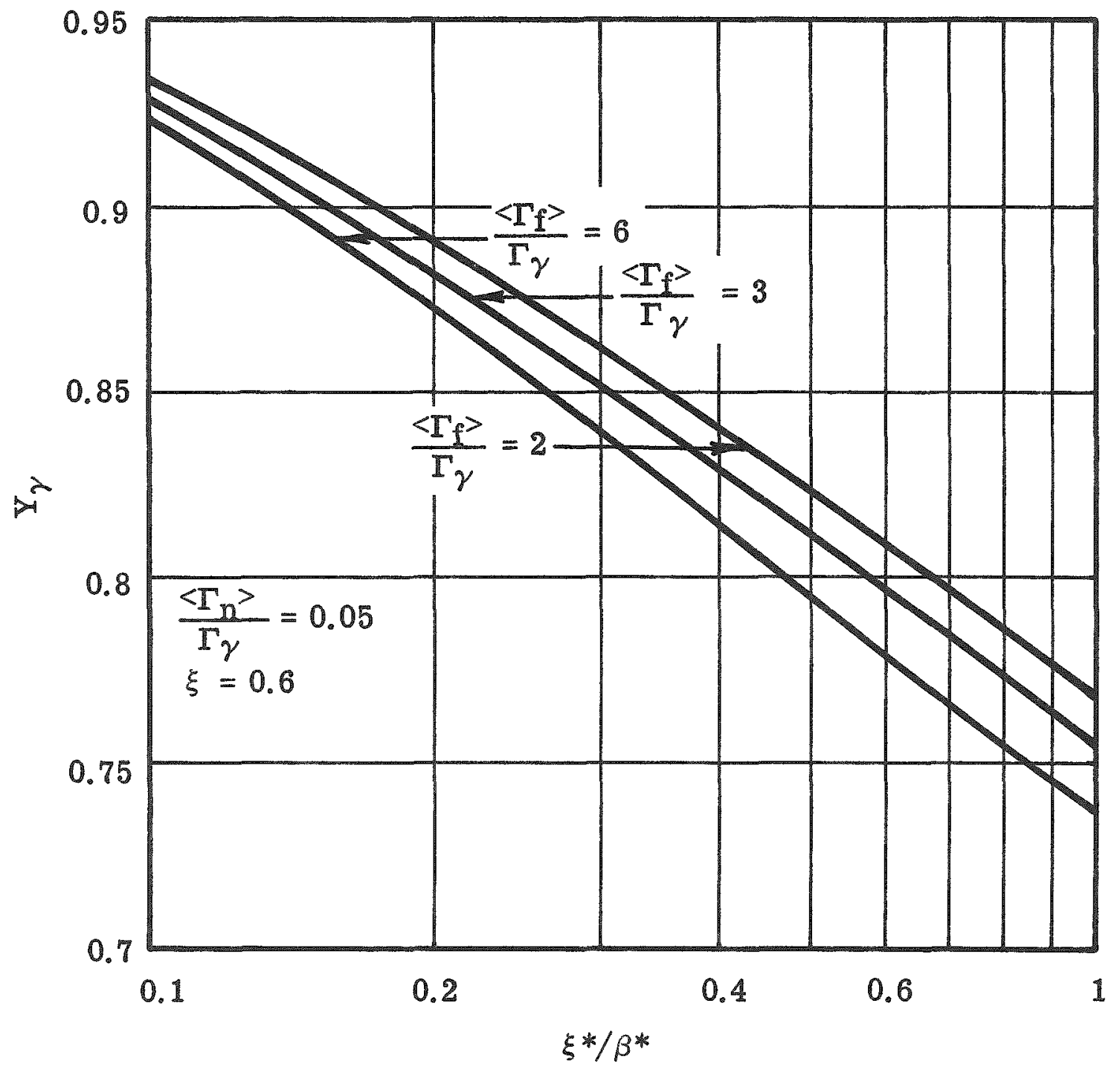

Fig. 8. Effect of $、 \Gamma_{f}>/ \Gamma_{\gamma}$ on $Y_{\gamma}$ for Fertile Material

For $\mathrm{U}^{235}$ and $\mathrm{Pu}^{239}$, the range of interest is from about 0.1 to 0.5 for $\xi^{*}$ and up to about 1 for $\xi^{*} / \beta^{*}$. It should be noted that the use of the asymptotic formula for $\psi$, the shape of the Doppler-broadened cross section, corresponds to $\frac{2 \beta^{*}}{\pi} \mathrm{J}\left(\xi^{*}, \beta^{*}\right)$ versus $\frac{\xi^{*}}{\beta^{*}}$, reducing to a single curve.

Figure 6 illustrates the trend in the correction factor $\mathrm{Y}_{\gamma}$ for a fertile material. Here $Y_{\gamma}$ is plotted versus $\xi^{*} / \beta^{*}$ for various values of $A=、 \Gamma_{n}>/ \Gamma_{\gamma}$ and $\xi^{*}$. For $U^{238}$ and $s$-waves, for example, A varies from about 2 at $1 \mathrm{keV}$ to about 12 at $30 \mathrm{keV}$. Note the small variation in $\mathrm{Y}_{\gamma}$ over the full range of interest (less than about $5 \%$ for given $A$ ). Over the same range, $\frac{2 \beta^{*}}{\pi} \mathrm{J}\left(\xi^{*}, \beta^{*}\right)$ varies by about $50 \%$. Sample calculations indicate that the contribution of $Y_{\gamma}$ to the change in the self-shielding factor with temperature generally is quite small for $\mathrm{U}^{238}$. 
Figure 7 illustrates the variation in $\mathrm{Y}_{\mathrm{f}}$ for a fissile material having a $\Gamma_{f} / \Gamma_{\gamma}$ of 2 . For $P_{u}{ }^{239}$, the,$\Gamma_{n}>/ \Gamma_{\gamma}$ of interest varies from roughly 0.1 at $0.2 \mathrm{keV}$ to about 1 at $2 \mathrm{keV}$. The variation in $Y_{f}$ is much more pronounced than the variation shown on the previous figure. In some sample calculations the variation in $Y_{f}$ contributed roughly $50 \%$ to the change in $f$ with temperature. The variation in $Y_{f}$ with change in fuel temperature only is illustrated by the curve labeled "constant $\beta *$."

Figure 8 illustrates the effect of $B=\left\langle\Gamma_{f}\right\rangle / \Gamma_{\gamma}$ on $Y_{\gamma} \cdot$ Values of $\mathrm{B}$ between 2 and 4 bracket estimated values for $\mathrm{Pu}{ }^{239}$ and $\mathrm{U}^{235}$.

The variations in $Y_{\gamma}$ and $Y_{f}$ with the various parameters are relatively small. Two point-interpolation procedures with a limited number of tabulated values of the parameters $\Gamma_{\mathrm{n}}>/ \Gamma_{\gamma}, \Gamma_{\mathrm{f}} / \Gamma_{\gamma}, *$, and $\varepsilon^{*} / \beta^{*}$ have been found sufficient to evaluate $Y_{f}$ and $Y_{\gamma}$ to the accuracy required in typical calculations.

Calculations of the change in the effective cross sections with temperatures have been made by a variety of assumptions and methods. Figures 9 and 10 illustrate some of the results. In the figures, the increase in the effective cross section $\tilde{\mu}_{X}$ in going from $500^{\circ} \mathrm{K}$ to $1500^{\circ} \mathrm{K}$ divided by $\tilde{\mu}_{X}$ at $500^{\circ} \mathrm{K}$ is plotted versus neutron energy. Values of $\xi * / \beta *$ and $\xi *$ at $500^{\circ} \mathrm{K}$ arc also given at several values of neutron energy. The calculations are restricted to s-wave contributions. A mixture of $\mathrm{Pu}^{239}, \mathrm{U}^{238}$, and scattering material was chosen such that the potential scattering per atom was $100 \mathrm{~b}$ for $\mathrm{U}^{238}$ and $400 \mathrm{~b}$ for $\mathrm{Pu}^{239}$.

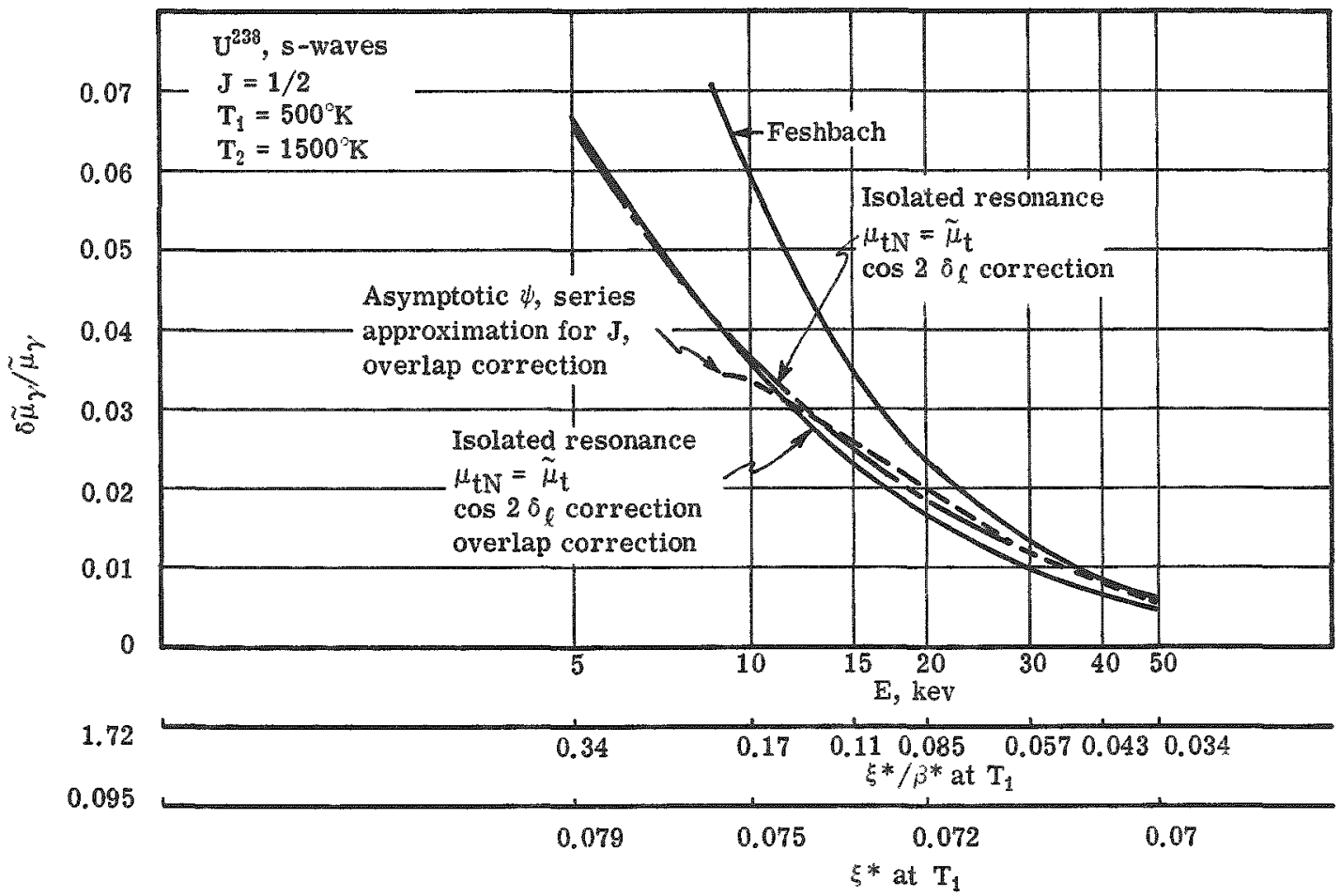

Fig. 9. Variation in Effective Radiative Capture Cross Section for U238 with Neutron Energy and Temperature 


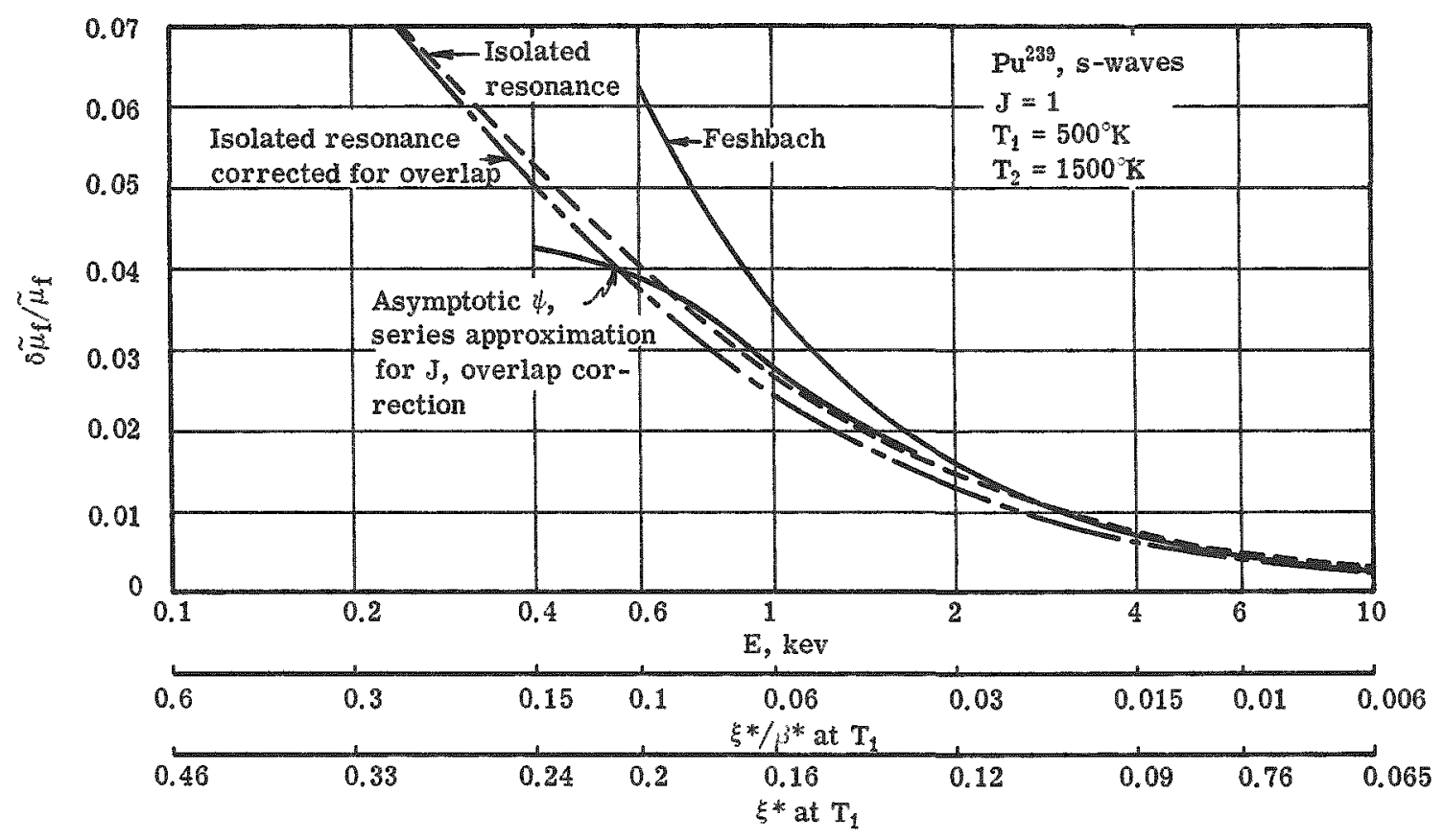

Fig. 10. Variation in Effective Fission Cross Section for $\mathrm{Pu}^{239}$ with Neutron Energy and Temperature

In Fig. $9, \frac{\delta \tilde{\mu}_{\gamma}}{\tilde{\mu}_{\gamma}}$, the fractional change in the effective radiative capture cross section, is plotted for $\mathrm{U}^{238}$. The results obtained from Equation 11 by the BIGSUM code include an approximate correction for resonance overlap through the use of $\tilde{\mu}_{t}$ in place of $\mu_{t N}$. An approximate correction for interference between potential and resonance scattering is also included through the use of $\cos 2 \delta \ell$ in evaluating $\beta *$. Neglect of this interference effect by setting $\cos 2 \delta_{\ell}$ equal to unity would increase $\frac{\delta \tilde{\mu}_{\gamma}}{\tilde{\mu}_{\gamma}}$ by a few percent at $5 \mathrm{keV}$ and about 30 percent at $50 \mathrm{keV}$. The results of applying the overlap correction, Equation 13 to Equation 11 , are also illustrated. A relatively small effect was found over most of the energy range. At the lower neutron energies, the conditions are well outside those assumed in the derivation of the Feshbach formula. The major reason for the large deviation in this region is the errox introduced by the first-order expansion in the total cross-section fluctuation used in the analysis. At the higher-energy end, the BIGSUM results suggest that use of the asymptotic formula for $\psi$ in the Feshbach analysis gives an overestimate of the Doppler effect. However, this region has a small effect on the overall Doppler coefficient for typical large, dilute fast reactors. The results obtained with the approximate formula given by Equation 16 are presented for the case where the expansion of $\frac{2 \beta}{\pi} J(\xi, \beta)$ has been truncated to include only the $Z_{1 x}$ term. Correction for resonance overlap with Equation 13 has also been used. The agreement with the BIGSUM results is reasonable up to a $\xi * / \beta *$ of about 0.1 . 
Figure 10 shows results for $\mathrm{Pu}^{239}, \mathrm{~s}$-waves, and levels with $\mathrm{J}=1$. These calculations have not been completed and do not include the effect of interaction. Again the Feshbach formula deviates markedly from the BIGSUM results at lower energies, as expected. The approximate formula based on the truncated series expansion for $J(\xi, \beta)$ gives reasonable agreement with the BIGSUM results up to a $\xi * / \beta *$ of about 0.1 .

In summary, the $Y_{\mathrm{X}}$ correction factors have been obtained in terms of general parameters over ranges sufficient to permit calculations of effective cross sections for any fertile or fissile material. The results are, of course, subject to questions concerning adequacy of the single-level formulas, the particular choice of fission-width distribution, and overlap effects in the low neutron-energy region. The results will be published shortly in UNC-5064. The data can probably best be used with a digital computer code employing an interpolation procedure. However, general graphs will also be presented to permit reasonably rapid hand calculations. Approximate formulas are given, including effects of resonance overlap, interference between potential and resonance scattering, and increased fluctuations, of the total cross section.

\section{$\underline{\text { References }}$}

1. Charles C. Graves, A Review of Analytical and Experimental Work Pertinent to the Doppler Effect in Fast Reactors, UNC-5034 (1962).

2. H, Feshbach, G, Goertzel, and H. Yamauchi, Estimation of Doppler Effect in Fast Reactors, Nuc. Sci. and Eng., 1, 4-20 (1956).

3. R. B. Nicholson, Doppler Effect in Fast Reactors, APDA-139 (1960).

$$
\frac{\text { Discussion of Paper }}{\text { Presented by Mr.Graves }}
$$

MR.SCHMIDT (Karlsruhe):

Do I understand correctly that you have to calculate effective cross sections for each reactor from the beginning, and even for each reactor zone, which is of a different composition from another one?

MR. GRAVES:

These are not group-average cross sections. These are effective cross sections about the neighborhood of a given energy for homogeneous mixtures. They do not include the change for heterogeneity, for example, 
the change in $\sigma_{\mathrm{p}}$. These results were made so that you would not have to form averages every time you run a calculation. Essentially what I was hoping for was that these might be equivalent to a $\mathrm{J}$ function. They would be more limited because they are dependent on particular distribution. Nevertheless the object was that here would be a set of functions as a function of 4 parameters, which do not vary over very wide ranges, which could put in the machine with interpolation procedures. A two-point interpolation procedure is all I need in a table of functions. It also turns out that the changes in these are so simple functionally that the report includes graphs so you can do calculations by hand. The trouble with it is somebody may come along and say a multilevel formula changes everything. If that happens, then these are useless. If those corrections are small, this is fine. I have corrections, but most turned out to be small. The object was to get something to permit rapid calculations using more conventional formulas. 


\title{
DOPPLER EFFECT IN FAST REACTORS FOR NONUNIFORM HEATING AND TEMPERATURE DISTRIBUTION
}

\author{
Emile A. Fossoul \\ BelgoNucleaire \\ Brussels, Belgium
}

\section{Introduction}

Because of the small importance attached to spatial heterogeneity effects in fast reactors, it is possible to consider a system which is a homogeneous mixture of atoms subjected to different temperatures and to different heating laws.

The case considered here is one in which atoms of constituent isotopes are not homogeneously mixed. The Doppler effect of a particular isotope in this system can be determined for both region $A$ of overlapping resonances and region $B$ of isolated resonances.

Let the total atoms of the isotope considered be composed of an homogeneous mixture of $n$ sets $1,2, \ldots, m, \ldots, n$ having tempera tures $T_{1}, T_{2}, \ldots, T_{m}, \ldots, T_{n}$, respectively.

Let the effective macroscopic cross section of that isotope for the particular process considered be $\tilde{\mu}\left(T_{1}, T_{2}, \ldots, T_{m}, \ldots, T_{n}\right)$ and let $T\left(T_{1}, T_{2}, \ldots, T_{m}, \ldots, T_{n}\right)$ be the reference temperature.

When the reactor heats up, the $n$ sets have temperature variations $\delta \mathrm{T}_{1}, \delta \mathrm{T}_{2}, \ldots, \delta \mathrm{T}_{\mathrm{m}} \ldots ., \delta \mathrm{T}_{\mathrm{n}}$ corresponding to a variation

$$
\delta T=\frac{\partial T}{\partial T_{1}} \delta T_{1}+\frac{\partial T}{\partial T_{2}} \delta T_{2}+\ldots+\frac{\partial T}{\partial T_{m}} \delta T_{m}+\ldots+\frac{\partial T}{\partial T_{n}} \delta T_{n}
$$

The variation $\delta \tilde{\mu}$ of the effective cross section is given by

$$
\delta \tilde{\mu}=\frac{\partial \tilde{\mu}}{\partial T_{1}} \delta T_{1}+\frac{\partial \tilde{\mu}}{\partial T_{2}} \delta T_{2}+\ldots+\frac{\partial \tilde{\mu}}{\partial T_{m}} \delta T_{m}+\ldots+\frac{\partial \tilde{\mu}}{\partial T_{n}} \delta T_{n},
$$

so that

$$
\frac{\delta \tilde{\mu}}{\delta \mathrm{T}}=\frac{\partial \tilde{\mu}}{\partial \mathrm{T}_{1}} \frac{\delta \mathrm{T}_{1}}{\delta \mathrm{T}}+\frac{\partial \tilde{\mu}}{\partial \mathrm{T}_{2}} \frac{\delta \mathrm{T}_{2}}{\delta \mathrm{T}}+\ldots+\frac{\partial \tilde{\mu}}{\partial \mathrm{T}_{\mathrm{m}}} \frac{\delta \mathrm{T}_{\mathrm{m}}}{\delta \mathrm{T}}+\ldots+\frac{\partial \tilde{\mu}}{\partial \mathrm{T}_{\mathrm{n}}} \frac{\delta \mathrm{T}_{\mathrm{n}}}{\delta \mathrm{T}}
$$

and 


$$
\begin{aligned}
\mathrm{T} \frac{\delta \tilde{\mu}}{\delta \mathrm{T}}= & \frac{\mathrm{T}}{\mathrm{T}_{1}} \frac{\delta \mathrm{T}_{1}}{\delta \mathrm{T}}\left(\mathrm{T}_{1} \frac{\partial \tilde{\mu}}{\partial \mathrm{T}_{1}}\right)+\ldots+\frac{\mathrm{T}}{\mathrm{T}_{2}} \frac{\delta \mathrm{T}_{2}}{\delta \mathrm{T}}\left(\mathrm{T}_{2} \frac{\partial \tilde{\mu}}{\partial \mathrm{T}_{2}}\right)+\ldots \\
& +\frac{\mathrm{T}}{\mathrm{T}_{\mathrm{m}}} \frac{\delta \mathrm{T}_{\mathrm{m}}}{\delta \mathrm{T}}\left(\mathrm{T}_{\mathrm{m}} \frac{\partial \tilde{\mu}}{\partial \mathrm{T}_{\mathrm{m}}}\right)+\ldots+\frac{\mathrm{T}}{\mathrm{T}_{\mathrm{n}}} \frac{\delta \mathrm{T}_{\mathrm{n}}}{\delta \mathrm{T}}\left(\mathrm{T}_{\mathrm{n}} \frac{\partial \tilde{\mu}}{\partial \mathrm{T}_{\mathrm{n}}}\right) .
\end{aligned}
$$

Finally, let $\rho$ be the atomic density of the isotope considered and $\rho_{1}, \rho_{2}, \ldots, \rho_{m}, \ldots, \rho_{n}$ the partial density corresponding to the sets $1,2, \ldots, m, \ldots, n$. The problem now reduces to a determination of the coefficient $T_{m} \frac{\partial \tilde{\mu}}{\partial T_{m}}$ corresponding to the heating of the set $n$ alone. For simplicity, the Doppler width $\Delta$ is taken as the temperature variable instead of $T$. Since $\Delta$ is proportional to the square root of $T$, one has the relation

$$
T \frac{\partial}{\partial T}=\frac{\Delta}{2} \frac{\partial}{\partial \Delta} .
$$

2. Region A, Overlapping Resonances

We may write

$$
\mathrm{T} \frac{\partial \tilde{\mu}}{\partial \mathrm{T}}=-\frac{\mathrm{T}}{\left\langle\mu_{\mathrm{t}}\right\rangle} \frac{\partial\left\langle\mu \mu_{\mathrm{t}}\right\rangle}{\partial \mathrm{T}}=-\frac{\Delta}{2\left\langle\mu_{\mathrm{t}}\right\rangle} \frac{\partial\left\langle\mu_{\left.\mu_{\mathrm{t}}\right\rangle}\right.}{\partial \Delta} \text {. }
$$

where $\mu_{t}$ is the total cross section of the medium, and $\mu$ and $\mu_{t}$ are functions of $\Delta_{1}, \Delta_{2}, \ldots, \Delta_{m}, \ldots, \Delta_{n}$.

Following step by step the calculation of the homogeneous case in temperature but taking into account the respective masses and different temperatures, one obtains

$$
\frac{\partial}{\partial \Delta}\left\langle\mu \mu_{\mathrm{t}}\right\rangle=\frac{\partial}{\partial \Delta}\left\langle\left(\mu_{\text {res }}+\mu_{\text {int }}\right) \mu\right\rangle .
$$

because $\left\langle\mu_{\text {pot }} \mu\right\rangle=\mu$ pot $\langle\mu\rangle$, independent of $\Delta$.

Indices as follows will be used:

$\mathrm{x}$ the reaction considered;

$i$ the set considered for $\mu_{X}$;

$j$ the set considered for $\mu_{t}$;

$s$ the sequence of resonances;

Then,

$k$ the resonance. 


$$
\begin{aligned}
& \left\langle\mu_{x}\left(\mu_{\text {res }}+\mu_{\text {int }}\right)\right\rangle=\left\langle\sum_{i} \sum_{j}\left(\sum_{s} \rho_{i}^{(s)} \sum_{k} \sigma_{o x k} \psi_{1 k i}\right)\right. \\
& \left.\left[\sum_{s} \rho_{j}^{(s)} \sum_{k} \sigma_{o c k}\left(\cos 2 \delta_{1} \psi_{k j}+\sin 2 \delta_{1} \Phi_{k j}\right)\right]\right) \\
& \left.\psi_{k i}=\psi^{\prime} q_{k}, t_{i}\right) ; q_{k}=\frac{2}{\Gamma_{k}}\left(E-E_{k}\right) ; \\
& \Phi_{k i}=\Phi\left(q_{k}, t_{i}\right) ; t_{i}=\left(\frac{\Delta i}{\Gamma_{k}}\right)^{2} . \\
& \left\langle\mu_{\mathrm{X}}\left(\mu_{\text {res }}+\mu_{\text {int }}\right)\right\rangle=\sum_{s} \sum_{s^{\prime}} \sum_{i} \sum_{j} \rho_{i}^{(s)_{\rho}}\left(s^{\prime}\right)
\end{aligned}
$$

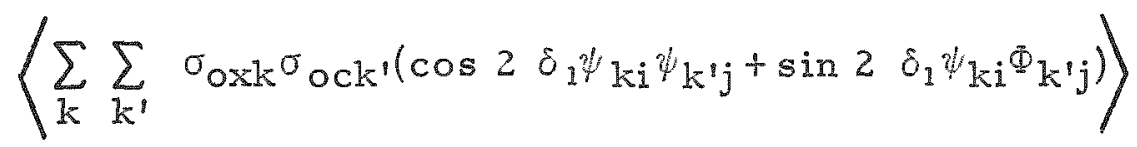

$$
\begin{aligned}
& =\sum_{s} \sum_{i} \sum_{j} \rho_{i}^{(s)} \rho_{j}(s)\left\langle\sum_{k} \sigma_{o x k} \sigma_{o c k}\left(\cos 2 \delta_{1} \psi_{k i} \psi_{k j}+\sin 2 \delta_{1} \psi_{k i} \Phi_{k j}\right)\right\rangle
\end{aligned}
$$

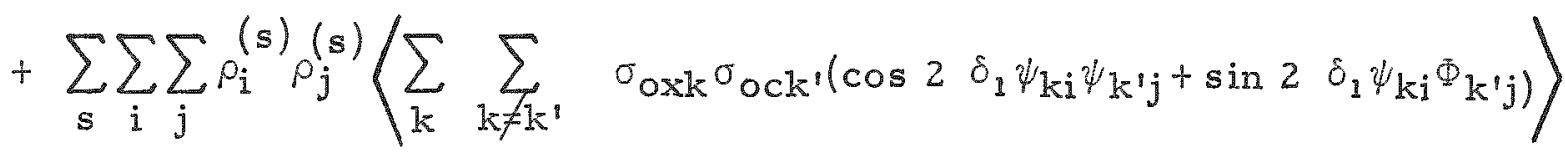

if there is no correlation between $s$ and $s^{\prime}$. For the averaging process with respect to energy, one has to evaluate four types of integral

$$
\int \psi_{k i} \psi_{k j} d E ; \quad \int \psi^{\prime} k^{\Phi^{\Phi}}{ }_{k j} d E ; \int \psi_{k i} \psi_{k j} d E ; \int \psi_{k i} \Phi_{k}{ }_{k j} d E .
$$

If $\psi$ is assumed to have the asymptotic form

$$
\psi_{\mathrm{ki}}=\frac{1}{2} \sqrt{\frac{\pi}{t_{i}}} \exp \left[-\frac{q_{k}^{2}}{4 t_{i}}\right]=\frac{\sqrt{7}}{2}\left(\frac{\Gamma_{k}}{\Delta_{i}}\right) \exp \left[-\left(\frac{E-E_{k}}{\Delta_{i}}\right)^{2}\right]
$$

and the integration is extended at infinity due to the local character of $\psi$ and $\Phi$, one has 
FOSSOUL

$$
\begin{aligned}
& \int_{-\infty}^{+\infty} \psi_{k i} \psi_{k j} d E=\frac{\pi}{4} \frac{\Gamma_{k}^{2}}{\Delta_{i} \Delta_{j}} \int_{-\infty}^{+\infty} e^{-\left(\frac{x}{\Delta_{i}}\right)^{2}-\left(\frac{x}{\Delta_{j}}\right)^{2}} d x=\frac{\pi}{4} \frac{\Gamma_{k}^{2}}{\Delta_{i} \Delta_{j}} \\
& \int_{-\infty}^{+\infty} \int^{-x^{2}\left(\frac{1}{\Delta_{i}^{2}}+\frac{1}{\Delta_{j}^{2}}\right)} d x=\frac{\pi}{4} \frac{\Gamma_{k}^{2}}{\Delta_{i} \Delta_{j}} \sqrt{\frac{1}{\Delta_{i}^{2}}+\frac{1}{\Delta_{j}^{2}}} \\
& =\left(\frac{\pi}{2}\right)^{3 / 2} \frac{\Gamma_{k}^{2}}{2 \sqrt{\frac{\Delta_{i}^{2}+\Delta_{j}^{2}}{2}}}=\left(\frac{\pi}{2}\right)^{3 / 2} \frac{\Gamma_{k}^{2}}{2 \Delta_{i j}},
\end{aligned}
$$

where

$$
\Delta_{i j}=\sqrt{\frac{\Delta_{i}^{2}+\Delta_{j}^{2}}{2}}
$$

We have

$$
\int_{-\infty}^{+\infty} \psi_{\mathrm{ki}} \Phi_{\mathrm{kj}} \mathrm{dE}=0 .
$$

Further,

$$
\begin{aligned}
& \int_{-\infty}^{+\infty} \psi_{k i} \psi_{k^{\prime} j} d E=\frac{\pi}{4} \frac{\Gamma_{k} \Gamma_{k}}{\Delta_{i} \Delta_{j}} \int_{-\infty}^{+\infty} \exp \left[-\left(\frac{E-E_{k}}{\Delta_{i}}\right)^{2}-\left(\frac{E-E_{k}}{\Delta_{j}}\right)^{2}\right] d E \\
& =\frac{\pi}{4} \frac{\Gamma_{k} \Gamma_{k^{\prime}}}{\Delta_{i} \Delta_{j}} \int_{-\infty}^{+\infty} \exp -\left[\left(\frac{E-E_{k}}{\Delta_{i}}\right)^{2}+\left(\frac{E-E_{k}}{\Delta_{j}}\right)^{2}+\frac{2\left(E-E_{k}\right)\left(E-E_{k^{\prime}}\right)}{\Delta_{j}^{2}}+\left(\frac{E_{k^{\prime}}-E_{k^{\prime}}}{\Delta_{j}}\right)^{2}\right] d E \\
& =\frac{\pi}{4} \frac{\Gamma_{k} \Gamma_{k^{\prime}}}{\Delta_{i} \Delta_{j}} \int_{-\infty}^{+\infty} \exp \left\{\frac{\left(E_{k}-E_{k^{\prime}}\right)^{2}}{-\Delta_{j}^{2}}\left[1-\frac{\frac{1}{\Delta_{j}^{2}}}{\frac{1}{\Delta_{i}^{2}}+\frac{1}{\Delta_{j}^{2}}}\right]\right\}
\end{aligned}
$$




$$
\begin{aligned}
& \exp \left\{\left(\frac{1}{\Delta_{i}^{2}}+\frac{1}{\Delta_{j}^{2}}\right)\left[\left(E-E_{k}\right)+\frac{E_{k}-E_{k}}{-\Delta_{j}^{2}} \frac{1}{\frac{1}{\Delta_{i}^{2}}+\frac{1}{\Delta_{j}^{2}}}\right]^{2}\right\} d E \\
& =\frac{\pi}{4} \frac{\Gamma_{\mathrm{k}} \Gamma_{\mathrm{k}}}{\Delta_{\mathrm{i}} \Delta_{\mathrm{j}}} \exp \left\{\frac{\left(E_{\mathrm{k}}-E_{\mathrm{k}^{\prime}}\right)^{2}}{\Delta_{\mathrm{j}}^{2}}\left[\frac{\Delta_{i}^{2}+\Delta_{\mathrm{j}}^{2}-\Delta_{i}^{2}}{\Delta_{i}^{2}+\Delta_{j}^{2}}\right]\right\} \sqrt{\frac{\pi}{\frac{1}{\Delta_{i}^{2}}+\frac{1}{\Delta_{j}^{2}}}} \\
& =\frac{\pi}{4} \frac{\Gamma_{k^{\prime}} \Gamma_{k^{\prime}}}{\Delta_{i} \Delta_{j}} \exp \left[-\frac{\left(E_{k}-E_{k^{\prime}}\right)^{2}}{2 \frac{\Delta_{i}^{2}+\Delta_{j}^{2}}{2}}\right] \sqrt{\frac{\pi}{\frac{1}{\Delta_{i}^{2}}+\frac{1}{\Delta_{j}^{2}}}} \\
& =\left(\frac{\pi}{2}\right)^{3 / 2} \frac{\Gamma_{k} \Gamma_{k^{\prime}}}{\sqrt[2]{\frac{\Delta_{i}^{2}+\Delta_{j}^{2}}{2}}} \exp \left[-\frac{\left(E_{k}-E_{k^{\prime}}\right)^{2}}{2\left(\frac{\Delta_{i}^{2}+\Delta_{j}^{2}}{2}\right)}\right]=\left(\frac{\pi}{2}\right)^{3 / 2} \frac{\Gamma_{k} \Gamma_{k^{\prime}}}{2 \Delta_{i j}} \exp \left[-\frac{\left(E_{k}-E_{k^{\prime}}\right)^{2}}{2 \Delta_{i j}^{2}}\right] .
\end{aligned}
$$

In addition,

$$
\int_{-\infty}^{+\infty} \psi_{k i} \Phi_{k \prime j} d E=0
$$

due to the odd character of $\Phi_{k \prime}$, which cancels the mutual contributions from both sides of the resonance $k$.

Hence,

$$
\begin{aligned}
& \int\left\langle\mu_{x}\left(\mu_{\text {res }}+\mu_{\text {int }}\right)\right\rangle \mathrm{d} E=\sum_{s} \sum_{i} \sum_{j} \rho_{i}^{(s)} \rho_{j}^{(s)} \\
& \left\langle\sum_{k} \sigma_{o x k} \sigma_{o c k} \cos 2 \delta_{1} \frac{\Gamma_{k}^{2}}{2 \Delta_{i j}}\left(\frac{\pi}{2}\right)^{3 / 2}\right\rangle+\sum_{s} \sum_{i} \sum_{j} \rho_{i}^{(s)} \rho_{j}^{(s)} \\
& \left\langle\sum_{k} \sum_{k \neq k^{\prime}} \sigma_{o x k^{2} \sigma o c k} \cos 2 \delta_{1} \frac{\Gamma_{k} \Gamma_{k^{\prime}}}{2 \Delta_{i j}}\left(\frac{\pi}{2}\right)^{3 / 2} \exp \left[-\frac{\left(E_{k}-E_{k^{\prime}}\right)^{2}}{2 \Delta_{i j}^{2}}\right]\right\rangle
\end{aligned}
$$


and the average value is finally given by dividing the former integral by the average spacing of the sequence:

$\sum_{s} \sum_{i} \sum_{j} \rho_{i}^{(s)} \rho_{j}^{(s)} \frac{1}{2 \Delta_{i j}}\left(\frac{\pi}{2}\right)^{3 / 2} \frac{\cos 2 \delta_{1}}{\langle s\rangle_{s}}\left\{\left\langle\sigma_{o x} \sigma_{o c} \Gamma^{2}\right\rangle+\left\langle\sigma_{o x} \Gamma\right\rangle\left\langle\sigma_{o c} \Gamma\right\rangle\left\langle e^{-\frac{\delta^{2}}{2 \Delta_{i j}^{2}}}\right\rangle\right\}$,

so that

$$
\begin{aligned}
& \frac{\partial \int\left\langle\mu_{x}\left(\mu_{\text {res }}+\mu_{\text {int }}\right)>d E\right.}{\partial \Delta_{m}} \\
& =\frac{1}{2}\left(\frac{\pi}{2}\right)^{3 / 2} \frac{\partial}{\partial \Delta_{m}}\left\{\sum_{s} \sum_{i} \sum_{j} \frac{\rho_{i}^{(s)} \rho_{j}^{(s)}}{\langle s\rangle_{s}} \frac{\cos 2 \delta_{1}}{\Delta_{i j}}\right. \\
& \left.\left[\left\langle\sigma_{o x} \sigma_{o c} \Gamma^{2}\right\rangle+\left\langle\sigma_{o x} \Gamma\right\rangle\left\langle\sigma_{o c} \Gamma\right\rangle\left\langle e^{-\frac{\delta^{2}}{2 \Delta_{i j}^{2}}}\right\rangle\right]\right\} \\
& =\frac{1}{2}\left(\frac{\pi}{2}\right)^{3 / 2} \sum_{s} \sum_{i} \sum_{j}\left\{-\frac{1}{\Delta_{i j}^{2}} \frac{\rho_{i}^{(s)} \rho_{j}^{(s)}}{\langle s\rangle_{s}} \cos 2 \delta_{1}\right. \\
& {\left[\left\langle\sigma_{o x} \sigma_{o c} \Gamma^{2}\right\rangle+\left\langle\sigma_{o x} \Gamma\right\rangle\left\langle\sigma_{o c} \Gamma\right\rangle\left\langle e^{-\frac{\delta^{2}}{2 \Delta_{i j}^{2}}}\right\rangle\right]} \\
& \left.+\frac{1}{\Delta_{i j}} \frac{\rho_{i}^{(s)} \rho_{j}^{(s)}}{\langle S\rangle_{s}} \cos 2 \delta_{1}\left\langle\sigma_{o x} \Gamma\right\rangle\left\langle\sigma_{o c} \Gamma\right\rangle \frac{\partial}{\partial \Delta_{i j}}\left\langle e^{-\frac{\delta^{2}}{2 \Delta_{i j}^{2}}}\right)\right\} \frac{\partial \Delta_{i j}}{\partial \Delta_{m}} \\
& =-\frac{1}{2}\left(\frac{\pi}{2}\right)^{3 / 2} \sum_{s} \sum_{i} \sum_{j} \frac{1}{\Delta_{i j}^{2}} \frac{\partial \Delta_{i j}}{\partial \Delta_{m}} \frac{\rho_{i}^{(s)} \rho_{j}^{(s)}}{\langle s\rangle_{s}} \cos 2 \delta_{1}
\end{aligned}
$$


Then,

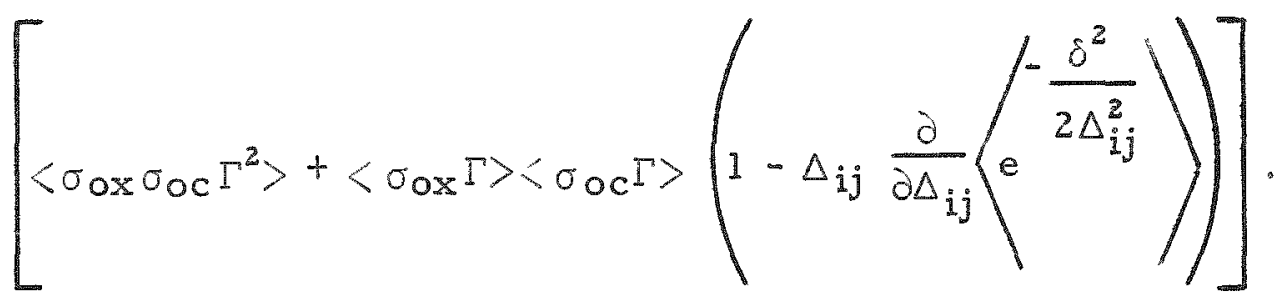

$$
\begin{gathered}
T_{m} \frac{\partial \tilde{\mu}_{x}}{\partial T_{m}}=\frac{-\Delta_{m}}{2\left\langle\mu_{t}\right\rangle} \frac{\partial\left\langle\mu_{x} \mu_{t}\right\rangle}{\partial \Delta_{m}}=\frac{\Delta m}{4\left\langle\mu_{t}\right\rangle}\left(\frac{\pi}{2}\right)^{3 / 2} \sum_{s} \sum_{i} \sum_{j} \frac{1}{\Delta_{i j}^{2}} \frac{\partial \Delta_{i j}}{\partial \Delta_{m}} \frac{\rho_{i}^{(s)} \rho_{j}^{(s)}}{\langle s\rangle_{s}} \\
\quad \cos 2 \delta_{1}\left\langle\sigma_{o x} \Gamma\right\rangle\left\langle\sigma_{o c} \Gamma\right\rangle\left[E_{x}^{(s)}-e^{(s)}\right],
\end{gathered}
$$

where

Since

$$
E_{x}^{(s)}=\frac{\left\langle\sigma_{o x} \sigma_{o c} \Gamma^{2}\right\rangle}{\left\langle\sigma_{o x} \Gamma\right\rangle\left\langle\sigma_{o c}\right\rangle^{s}} \quad e^{(s)}=-\left(1-\Delta \frac{\partial}{\partial \Delta}\left\langle e^{-\frac{\delta^{2}}{2 \Delta^{2}}}\right)\right)
$$

$$
\left\langle\sigma_{o x} \Gamma\right\rangle=\frac{2}{\pi}\langle S\rangle\left\langle\sigma_{x}\right\rangle: \quad\left\langle\sigma_{o c} \Gamma\right\rangle=\frac{2}{\pi}\langle S\rangle\left\langle\sigma_{c}\right\rangle,
$$

we have

$$
\begin{aligned}
& T_{m} \frac{\partial \tilde{\mu}_{x}}{\partial T_{m}}=\frac{1}{\left.\sqrt{8-\Delta_{m}\langle\mu t}\right\rangle} \sum_{s} \sum_{i} \sum_{j}\left(\frac{\Delta m}{\Delta i j}\right)^{2} \frac{\partial \Delta_{i j}}{\partial \Delta_{m}} \rho_{i}^{(s)} \rho_{j}^{(s)} \\
& \cos 2 \delta_{1}\langle s\rangle_{s}\left\langle\sigma_{x}\right\rangle_{s}\left\langle\sigma_{C}\right\rangle_{s}\left[E_{x}^{(s)}-e^{(s)}\right] .
\end{aligned}
$$

Defining

$$
R_{x}^{(s)}=\cos 2 \delta_{1}\left\langle\sigma_{c}\right\rangle_{s}\left\langle\sigma_{x}\right\rangle_{s}\langle s\rangle_{s}\left[E_{x}^{(s)}-e^{(s)}\right]
$$

and supposing $e^{(s)}$ independent of $\Delta$ in a certain domain of variation, one has

$$
T_{m} \frac{\partial \tilde{\mu}_{x}}{\partial T_{m}}=\frac{1}{\sqrt{8 \pi} \Delta_{m}\langle\mu t\rangle} \sum_{s} \sum_{i} \sum_{j}\left(\frac{\Delta m}{\Delta i j}\right)^{2} \frac{\partial \Delta_{i j}}{\partial \Delta_{m}} \rho_{i}^{(s)} \rho_{j}^{(s)} R_{x}^{(s)}
$$

Summation over $\mathrm{i}$ and $\mathrm{j}$

The variable wath two indices. $\Delta_{i j}$, has the following components: 


$$
\begin{aligned}
& \Delta 1 \\
& \sqrt{\frac{\Delta_{1}^{2}+\Delta_{2}^{2}}{2}} \cdot \sqrt{\frac{\Delta_{1}^{2}+\Delta_{1}^{2}}{2}} \cdot \sqrt{\frac{\Delta_{1}^{2}+\Delta_{n}^{2}}{2}} \\
& \sqrt{\frac{\Delta_{2}^{2}+\Delta_{1}^{2}}{2}} \\
& \Delta_{2} \cdot \sqrt{\frac{\Delta_{2}^{2}+\Delta_{m}^{2}}{2}} \cdot \sqrt{\frac{\Delta_{2}^{2}+\Delta_{n}^{2}}{2}} \\
& \sqrt{\frac{\Delta_{n}^{2}+\Delta_{1}^{2}}{2}} \sqrt{\frac{\Delta_{m}^{2}+\Delta_{2}^{2}}{2}} \cdots \Delta_{m} \cdots \sqrt{\frac{\Delta_{m}^{2}+\Delta_{n}^{2}}{2}} \\
& \sqrt{\frac{\Delta_{n}^{2}+\Delta_{1}^{2}}{2}} \quad \sqrt{\frac{\Delta_{n}^{2}+\Delta_{2}^{2}}{2}} \cdot \sqrt{\frac{\Delta_{n}^{2}+\Delta_{n}^{2}}{2}} \cdots \Delta_{n} .
\end{aligned}
$$

The representation of $\Delta_{i j}$ is symmetric and the principal diagonal elements are $\Delta_{i}$

$$
\frac{\partial \Delta_{i j}}{\partial \Delta m} \text { has zero components except for } i=m \text { and } j=m \text {. Thus, }
$$

when

$$
i=j=m, \frac{\partial \Delta i j}{\partial \Delta_{m}}=\frac{\partial \Delta_{m}}{\partial \Delta m}=1 ;
$$

when

$$
i=m, \frac{\partial \Delta_{i j}}{\partial \Delta_{m}}=\frac{\partial \Delta_{m j}}{\partial \Delta_{m}}=\frac{\partial}{\partial \Delta_{m}} \sqrt{\frac{\Delta_{m}^{2}+\Delta_{j}^{2}}{2}}=\frac{1}{2 \Delta_{m j}} \frac{2 \Delta_{m}}{2}=\frac{\Delta_{m}}{2 \Delta_{m j}} ;
$$

when

$$
j=m, \frac{\partial \Delta_{i j}}{\partial \Delta_{m}}=\frac{\partial \Delta_{i m}}{\partial \Delta_{m}}=\frac{\partial}{\partial \Delta m} \sqrt{\frac{\Delta_{i}^{2}+\Delta_{m}^{2}}{2}}=\frac{1}{2 \Delta_{i m}} \frac{2 \Delta_{m}}{2}=\frac{\Delta_{m}}{2 \Delta_{i m}} .
$$

The representation thus becomes 


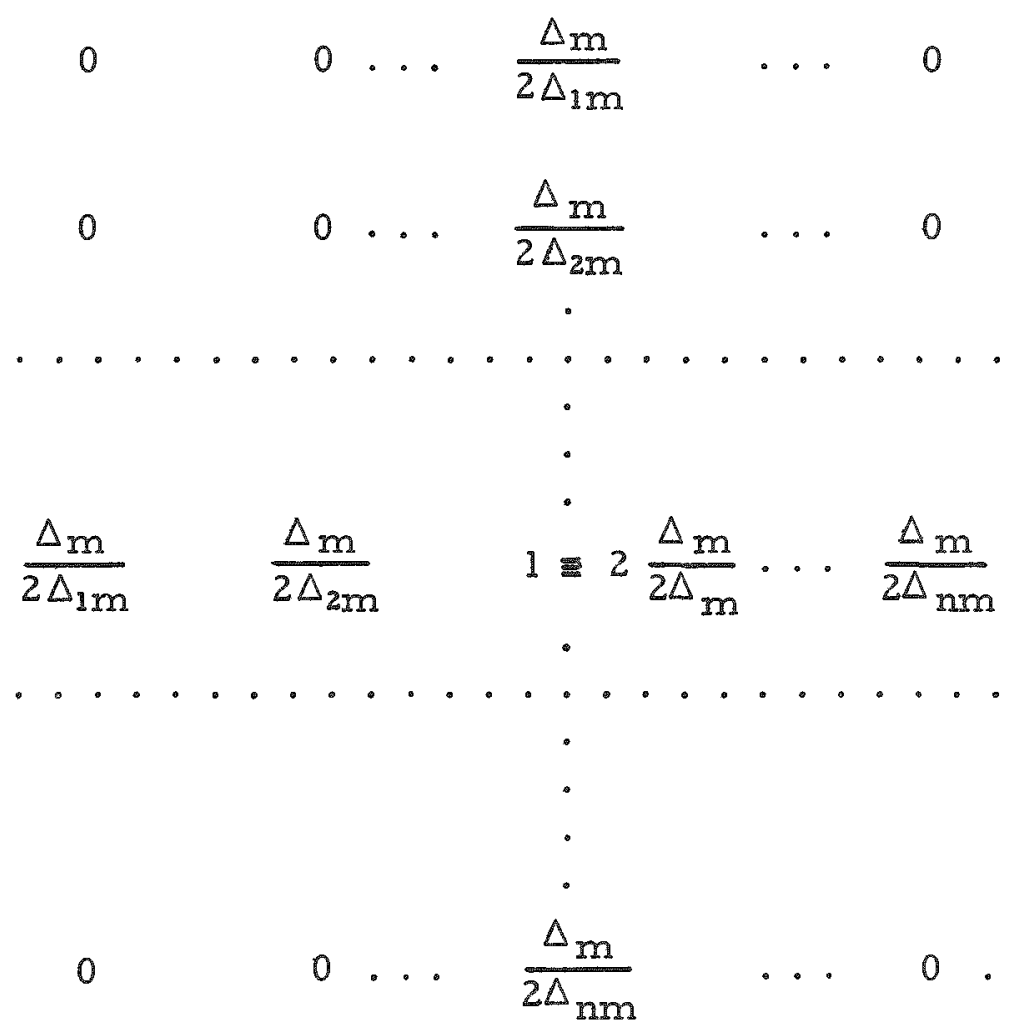

Accordingly,

$$
T_{m} \frac{\partial \tilde{\mu}}{\partial T_{m}}=\frac{1}{\sqrt{8 \pi} \Delta_{m}\left\langle\mu_{t}\right\rangle} \sum_{s} R_{x}^{(s)} \sum_{i} \sum_{j} \rho_{i}^{(s)} \rho_{j}^{(s)}\left(\frac{\Delta_{m}}{\Delta_{i j}}\right)^{2} \frac{\partial \Delta_{i j}}{\partial \Delta_{m}}
$$

and, inasmuch as

$$
\sum_{i} \sum_{j} \rho_{i}^{(s)} \rho_{j}^{(s)}\left(\frac{\Delta_{m}}{\Delta_{i j}}\right)^{2} \frac{\partial \Delta_{i j}}{\partial \Delta_{m}}=2 \sum_{j} \rho_{m}^{(s)} \rho_{j}^{(s)}\left(\frac{\Delta_{m}}{\Delta_{j m}}\right)^{2}\left(\frac{\Delta_{m}}{2 \Delta_{j m}}\right)=\rho_{m} \sum_{j} \rho_{j}\left(\frac{\Delta_{m}}{\Delta_{j m}}\right)^{3} .
$$

we have

$$
T_{m} \frac{\partial \tilde{\mu}}{\partial T_{m}}=\frac{1}{\sqrt{8 \pi} \Delta_{m}\left\langle\mu_{t}\right\rangle} \sum_{s} R_{x}^{(s)} \rho_{m}^{(s)} \sum_{j} \rho_{j}^{(s)}\left(\frac{\Delta_{m}}{\Delta_{j m}}\right)^{3} .
$$

This leads from the reactivity standpoint to

$$
T_{m} \frac{\partial k_{E}}{\partial T_{m}}=\frac{W_{H^{\Phi}} \Phi_{E}}{\sqrt{8 \pi} \Delta_{m}<\mu_{t}}>\sum_{s} \rho_{m}^{(s)} \sum_{j} \rho_{j}^{(s)}\left(\frac{\Delta_{m}}{\Delta_{j m}}\right)^{3} Q_{E}^{(s)}
$$


and, finally, to

$$
T \frac{o k_{E}}{o T}=\frac{W_{H} \Phi_{E}}{\sqrt{8 \pi}<\mu_{t}>} \sum_{s} Q_{E}^{(s)} \sum_{i} \rho_{i}^{(s)} \sum_{j} \rho_{j}^{(s)}\left(\frac{\Delta_{i}^{2}}{\Delta_{i j}^{3}}\right)\left(\frac{T}{T_{i}}\right)\left(\frac{\delta T_{i}}{\delta T}\right) .
$$

When all $\Delta_{i}$ are equal to $\Delta$, one gets

$$
\Delta_{i j}=\Delta
$$

and

$$
T \frac{\partial k_{E}}{\partial T}=\frac{W_{H} \Phi_{E}}{\sqrt{8 \pi} \Delta\left\langle\mu_{t}>\right.} \sum_{s} Q_{E}^{(s)} \sum_{i} \rho_{i}^{(s)} \sum_{j} \rho_{j}^{(s)}\left(\frac{T}{T_{i}}\right)\left(\frac{\delta T_{i}}{\delta T}\right) .
$$

Since

$$
\rho^{(s)}=\sum_{j} \rho_{j}^{(s)}
$$

we have

$$
\begin{aligned}
T \frac{\partial k_{E}}{\partial T} & =\frac{W_{H^{\Phi}} E}{\sqrt{8 \pi \Delta<\mu_{t}}>} \sum_{s} \sum_{i} \rho_{i}^{(s)}\left(\frac{T}{T_{i}}\right)\left(\frac{\delta T_{i}}{\delta T}\right) \rho^{(s)} Q_{E}^{(s)} \\
& =\left(T \frac{\delta k_{E}}{\delta T}\right)_{\text {homog }} \sum_{i} \frac{\rho_{i}^{(s)}}{\rho^{(s)}}\left(\frac{T}{T_{i}}\right)\left(\frac{\delta T_{i}}{\delta T}\right) .
\end{aligned}
$$

If the reference temperature $T$ also corresponds to $\Delta$,

$$
T \frac{\delta k_{E}}{\delta T}=\left(T \frac{\partial k_{E}}{\partial T}\right)_{\text {homog }} \sum_{i} \frac{\rho(s)}{\rho}\left(\frac{\delta T_{i}}{\delta T}\right) \text {. }
$$

3. Region $B$, Isolated Unresolved Resonances

$$
T_{m} \frac{\partial k_{E}}{\partial T_{m}}=\Phi_{E}\left\{\left[\nu W_{H}-W_{E}\right] T_{m} \frac{\partial \tilde{\mu}_{f}}{\partial T_{m}}-W_{E} T_{m} \frac{\partial \tilde{\mu}_{\gamma}}{\partial T_{m}}\right\} .
$$


Now

$$
\tilde{\mu}_{f}=\sum_{i}\left\langle\mu_{f}>f_{i} \frac{\rho_{i}}{\rho} ; \quad \tilde{\mu}_{\gamma}=\sum_{i}\left\langle\mu_{\gamma}>f_{i} \frac{\rho_{i}}{\rho} ; \quad \tilde{\mu}_{a}=\sum_{i} \frac{\rho_{i}}{\rho}\left\langle\mu_{a}>f_{i} .\right.\right.\right.
$$

Then

$$
\begin{aligned}
\mathrm{T}_{\mathrm{m}} \frac{\partial \mathrm{k}_{\mathrm{E}}}{\partial \mathrm{T}_{\mathrm{m}}} & =\Phi_{E}\left\{\left[\frac{\nu W_{\mathrm{H}}}{1+\alpha}-\mathrm{W}_{\mathrm{E}}\right] \mathrm{T}_{\mathrm{m}} \frac{\partial \tilde{\mu}_{\mathrm{a}}}{\partial \mathrm{T}}\right\} \\
& =\Phi_{\mathrm{E}}\left\{\left[\frac{\nu \mathrm{W}_{\mathrm{H}}}{1+\alpha}-\mathrm{W}_{\mathrm{E}}\right] \mathrm{T}_{\mathrm{m}} \sum_{\mathrm{i}} \frac{\rho_{\mathrm{i}}}{\rho}\left\langle\mu_{\mathrm{a}}\right\rangle \frac{\partial f_{\mathrm{i}}}{\partial \mathrm{T}_{\mathrm{m}}}\right\} \\
& =\sum \frac{\pi}{2\langle\mathrm{~S}\rangle_{\mathrm{s}}}\left\langle\sigma_{0 \mathrm{a}}\left[\frac{\nu \mathrm{W}_{\mathrm{H}}}{1+\alpha(\mathrm{s})}-\mathrm{W}_{\mathrm{E}}\right] \Gamma \mathrm{T}_{\mathrm{m}} \sum_{\mathrm{s}} \rho_{\mathrm{i}} \frac{\partial \mathrm{f}_{\mathrm{i}}}{\partial \mathrm{T}_{\mathrm{m}}}\right\rangle_{\mathrm{s}}
\end{aligned}
$$

Now,

$f_{i}=\frac{2}{\pi \Gamma} \int_{-\infty}^{+\infty} \frac{\psi_{i}}{1+\frac{\sum_{j} \rho_{j} \sigma_{\text {ot }} \psi_{i}}{\mu_{t N}}} d E=\frac{2 \beta}{\pi \Gamma} \int_{-\infty}^{+\infty} \frac{\psi_{i}}{\beta+\sum_{j}\left(\frac{\rho_{j}}{\rho}\right) \psi_{j}} d E_{;}$

$\frac{\partial f_{i}}{\partial T_{m}}=\frac{2 \beta}{\pi \Gamma} \int_{-\infty}^{+\infty} \frac{\partial}{\partial T_{m}}\left(\frac{\psi_{i}}{\beta+\sum_{j}\left(\frac{\rho_{j}}{\rho}\right) \psi_{j}}\right) d E_{;} \quad \beta=\frac{\mu_{t N}}{\rho \sigma_{o t}} ;$

$\frac{\partial}{\partial T_{m}}\left(\frac{\psi_{i}}{\beta+\sum_{j}\left(\frac{\rho_{j}}{\rho}\right) \psi_{j}}\right)=\frac{\partial \psi_{i}}{\partial T_{m}} \frac{1}{\beta+\sum_{j}\left(\frac{\rho_{j}}{\rho}\right) \psi_{j}}+\psi_{i}\left[\frac{-1}{\left[\beta+\sum_{j}\left(\frac{\rho j}{\rho}\right) \psi_{j}\right]^{2}}\right]\left(\frac{\rho_{m}}{\rho}\right) \frac{d \psi_{m}}{d T_{m}}$

For $i=m$, this quantity becomes 
FOSSOUL

$$
\frac{\partial \psi_{m}}{\partial T_{m}} \frac{\left[\beta+\sum_{j}\left(\frac{\rho_{j}}{\rho}\right) \psi_{j}-\psi_{m}\left(\frac{\rho m}{\rho}\right)\right]}{\left[\beta+\sum_{j}\left(\frac{\rho j}{\rho}\right) \psi_{j}\right]^{2}}
$$

and for i $\neq m$ it becomes

$$
\left(\frac{\rho_{\mathrm{m}}}{\rho}\right) \frac{\partial \psi_{\mathrm{m}}}{\partial \mathrm{T}} \frac{-\psi_{\mathrm{i}}}{\left[\beta+\sum_{\mathrm{j}}\left(\frac{\rho \mathrm{j}}{\rho}\right) \psi_{\mathrm{j}}\right]^{2}}
$$

We obtain

$$
\begin{aligned}
& \sum_{i} \rho_{i} \frac{\partial f_{i}}{\partial T_{m}}=\frac{2 \beta}{\pi \Gamma} \int_{-\infty}^{+\infty} \frac{\partial \mu_{m}}{\partial T_{m}} \frac{\rho_{m} \beta+\rho_{m} \sum\left(\frac{\rho_{j}}{\rho}\right) \psi_{j}-\sum_{i} \rho_{i} \psi_{i}\left(\frac{\rho_{m}}{\rho}\right)}{\left[\beta+\sum\left(\frac{\rho_{j}}{\rho}\right) \psi_{j}\right]^{2}} d E \\
& =\frac{2 \beta}{\pi \Gamma} \int_{-\infty}^{+\infty}\left(\frac{\rho_{\mathrm{m}}}{\rho}\right) \frac{\mathrm{d} \psi_{\mathrm{m}}}{\mathrm{dT} \mathrm{m}} \frac{\rho \beta}{\left[\beta+\sum_{j}\left(\frac{\rho_{\mathrm{j}}}{\rho}\right) \psi_{\mathrm{j}}\right]^{2}} \mathrm{dE} \\
& =\frac{2 \beta}{\pi \Gamma} \int_{-\infty}^{+\infty} \rho_{m} \frac{\partial \psi_{m}}{\partial T}\left[\frac{\beta}{\beta+\Sigma_{j}\left(\frac{\rho j}{\rho}\right) \psi_{j}}\right]^{2} d E=\frac{2 \beta}{\pi \Gamma} \int_{-\infty}^{+\infty} \rho_{m} \frac{\partial \psi_{m}}{\partial T_{m}} \frac{\beta}{\left(\beta+\psi_{m}\right)^{2}}\left[\frac{\beta+\psi_{m}}{\beta+\sum_{j}\left(\frac{\rho j}{\rho}\right) \psi_{j}}\right]^{2} d E .
\end{aligned}
$$

As

$$
\frac{\partial}{\partial \mathrm{T}_{\mathrm{m}}}\left[\frac{\psi_{\mathrm{m}}}{\beta+\psi_{\mathrm{m}}}\right]=\frac{\partial \dot{\gamma}_{\mathrm{m}}}{\partial \mathrm{T}_{\mathrm{m}}}\left(\frac{1}{\beta+\psi_{\mathrm{m}}}\right)+\psi_{\mathrm{m}}\left[\frac{-1}{\left(\beta+\psi_{\mathrm{m}}\right)^{2}}\right] \frac{\partial \psi_{\mathrm{m}}}{\partial \mathrm{T}_{\mathrm{m}}}=\frac{\partial \psi_{\mathrm{m}}}{\partial \mathrm{T}_{\mathrm{m}}} \frac{\beta}{\left(\beta+\psi_{\mathrm{m}}\right)^{2}}
$$

one gets

$$
\sum_{i} \rho_{i} \frac{\partial f_{i}}{\partial T_{m}}=\frac{2 \beta}{\pi \Gamma} \int_{-\infty}^{+\infty} \rho_{m} \frac{\partial}{\partial T_{m}}\left(\frac{\psi m}{\beta+\psi_{m}}\right)\left[\frac{\beta+\psi_{m}}{\beta+\sum\left(\frac{\rho_{j}}{\rho}\right) \psi_{j}}\right]^{2} d E,
$$

814 


$$
\begin{aligned}
\mathrm{T}_{\mathrm{m}} \frac{\partial \mathrm{k}_{\mathrm{E}}}{\partial \mathrm{T}_{\mathrm{m}}}= & \sum_{\mathrm{s}} \frac{\pi}{2} \frac{\Phi_{\mathrm{E}}}{\langle\mathrm{S}\rangle_{\mathrm{s}}}\left\langle\sigma_{0 \mathrm{a}}\left[\frac{\nu \mathrm{W}_{\mathrm{H}}}{1+\alpha(\mathrm{s})}-\mathrm{W}_{\mathrm{E}}\right] \Gamma \rho_{\mathrm{m}} \frac{2 \beta}{\pi \Gamma}\right. \\
& \left.\int_{-\infty}^{+\infty} \mathrm{T}_{\mathrm{m}} \frac{\partial}{\partial \mathrm{T}_{\mathrm{m}}}\left(\frac{\xi_{\mathrm{m}}}{\beta+\psi_{\mathrm{m}}}\right)\left[\frac{\beta+\psi_{\mathrm{m}}}{\beta+\sum_{\mathrm{j}}\left(\frac{\rho_{\mathrm{j}}}{\rho}\right){ }^{\psi} \mathrm{j}}\right]^{2} \mathrm{dE}\right\rangle_{\mathrm{s}}
\end{aligned}
$$

and finally

$$
\begin{aligned}
T \frac{\partial k_{E}}{\partial T}= & \sum \frac{\pi}{2} \frac{\Phi_{E}}{s S\rangle_{s}}\left\langle\sigma_{0 a}\left[\frac{\nu W_{H}}{1+\alpha(s)}-W_{E}\right] \frac{2 \beta}{\pi}\right. \\
& \left.\int_{-\infty}^{+\infty} d E \sum \rho_{i}\left[\frac{\beta+\psi_{i}}{\beta+\sum_{j}\left(\frac{\rho_{j}}{\rho}\right) \psi_{j}}\right]^{2} T_{i} \frac{\partial}{\partial T_{i}}\left(\frac{\psi_{i}}{\beta+\psi_{i}}\right)\left(\frac{T}{T_{i}}\right)\left(\frac{\delta T_{i}}{\delta T}\right)\right\rangle
\end{aligned}
$$

If all $\mathrm{T}_{\mathrm{i}}$ are equal to $\mathrm{T}, \psi_{\mathrm{i}}=\psi_{\mathrm{j}}=\psi_{\text {, and }}$

$$
\begin{aligned}
T \frac{\partial k_{E}}{\partial T} & =\sum_{s} \frac{\pi}{2} \frac{\bar{\Phi}_{E}}{\langle S\rangle_{s}}\left\langle\sigma_{0 a}\left[\frac{\nu W_{H}}{1+\alpha(s)}-W_{E}\right] \frac{2 \beta}{\pi}\right. \\
& \left.\int_{-\infty}^{+\infty} d E \sum_{i} \rho_{i} T \frac{\partial}{\partial T}\left(\frac{\psi}{\beta+\psi}\right)\left(\frac{\partial T_{i}}{\partial T}\right)\right\rangle_{s} \\
& =\left(T \frac{\partial k_{E}}{\partial T}\right)_{\text {homog }} \sum_{i}\left(\frac{\rho_{i}}{\rho}\right)\left(\frac{\delta T_{i}}{\delta T}\right) .
\end{aligned}
$$

4. General Formulae

In the general case the Doppler coefficient is given by the expressions

A.

$$
T \frac{\partial k_{E}}{\partial T}=\frac{W_{H} \Phi_{E}}{\sqrt{8 \pi}<\mu_{t}>\Delta} \sum_{s} \sum_{i} \rho_{i}^{(s)}\left(\frac{T}{T_{i}}\right)\left(\frac{\delta T_{i}}{\delta T}\right) \sum_{j} \rho_{j}^{(s)}\left(\frac{\Delta}{\Delta_{i}}\right)\left(\frac{\Delta_{i}}{\Delta_{i j}}\right)^{3} Q_{E}^{(s)}
$$

or 


$$
T \frac{\partial k_{E}}{\partial T}=\frac{W_{H^{\Phi}}{ }^{\Phi}}{\sqrt{8 \pi} \Delta<\mu_{t}>} \sum_{s} \sum_{i} \rho_{i}^{(s)}\left(\frac{T}{T_{i}}\right)^{3 / 2}\left(\frac{\delta T_{i}}{\delta T}\right) \sum_{j} \rho_{j}^{(s)}\left(\frac{2 T_{i}}{T_{i}+T_{j}}\right)^{3 / 2} Q_{E}^{(s)}
$$

because

$\Delta_{i j}=\sqrt{\frac{\Delta_{i}^{2}+\Delta_{j}^{2}}{2}} ; \quad \Delta_{i j}^{2}=\frac{\Delta_{i}^{2}+\Delta_{j}^{2}}{2} ; \quad T_{i j}=\frac{T_{i}+T_{j}}{2}$.

B.

$$
\begin{aligned}
& T \frac{\partial k_{E}}{\partial T}=\sum_{s} \frac{\Phi_{E}}{\langle S\rangle_{s}}\left\langle\sigma_{0 a}\left[\frac{\nu W_{H}}{1+\alpha(s)}-W_{E}\right] \beta\right. \\
& \left.\int_{-\infty}^{+\infty} \sum_{i} \rho_{i}^{(s)}\left(\frac{T}{T_{i}}\right)\left(\frac{\delta T_{i}}{\delta T}\right)\left[\frac{\beta+\psi_{i}}{\beta+\sum_{j}\left(\frac{\rho_{j}}{\rho}\right) \psi_{j}}\right]^{2} T_{i} \frac{\partial}{\partial T_{i}}\left(\frac{\psi_{i}}{\beta+\psi_{i}}\right) d E\right)
\end{aligned}
$$

or

$$
\begin{aligned}
\mathrm{T} \frac{\partial \mathrm{k}_{\mathrm{E}}}{\partial \mathrm{T}}= & \sum_{\mathrm{s}} \frac{\Phi_{\mathrm{E}}}{\langle\mathrm{S}\rangle_{\mathrm{s}}}\left\langle\sigma_{0 \mathrm{a}}\left[\frac{\nu \mathrm{W}_{\mathrm{H}}}{1+\alpha(\mathrm{s})}-\mathrm{W}_{\mathrm{E}}\right] \beta \sum_{\mathrm{i}} \rho_{\mathrm{i}}^{(\mathrm{s})}\left(\frac{\mathrm{T}}{\mathrm{T}_{\mathrm{i}}}\right)\left(\frac{\delta \mathrm{T}_{\mathrm{i}}}{\delta \mathrm{T}}\right)\right. \\
& \left.\int_{-\infty}^{+\infty}\left[\frac{\beta+\psi_{\mathrm{i}}}{\beta+\sum_{\mathrm{j}}\left(\frac{\rho}{\rho}\right) \psi_{j}}\right]^{2} \mathrm{~T}_{\mathrm{i}} \frac{\partial}{\partial \mathrm{T}_{\mathrm{i}}}\left(\frac{\psi_{\mathrm{i}}}{\beta+\psi_{\mathrm{i}}}\right) \mathrm{dE}\right\rangle
\end{aligned}
$$

With the approximation

$$
\frac{\beta+\psi_{i}}{\beta+\sum_{j}\left(\frac{\rho_{j}}{\rho}\right) \psi_{j}} \cong 1
$$

Which is true rigorously if $\psi_{i}=\psi_{j}=\psi$, and putting

$$
\frac{\partial f_{i}}{\partial T_{i}}=\frac{2 \beta}{\Gamma T_{j}} \int_{-\infty}^{+\infty} \frac{\partial}{\partial T_{i}}\left(\frac{\psi_{i}}{\beta+\psi_{i}}\right) d E
$$


we have

$$
T \frac{\partial k_{E}}{\partial T}=\sum_{s} \frac{\pi}{2} \frac{\Phi_{E}}{\langle S\rangle_{s}}\left\langle\sigma_{0 a}\left[\frac{\nu W_{H}}{1+\alpha(s)}-W_{E}\right] \Gamma T \sum_{i} \rho_{i}^{(s)}\left(\frac{\delta T_{i}}{\delta T}\right) \frac{\partial f_{i}}{\partial T_{i}}\right\rangle_{s} .
$$

If the approximation is not used, one must put

$$
\frac{\partial f_{i}}{\partial T_{i}}=\frac{2 \beta}{\pi \Gamma} \int_{-\infty}^{+\infty}\left[\frac{\beta+\psi_{i}}{\beta+\frac{\sum}{j}\left(\frac{\rho_{j}}{\rho}\right) \psi_{j}}\right]^{2} \frac{\partial}{\partial T_{i}}\left(\frac{\psi_{i}}{\beta+\psi_{i}}\right) d E
$$

to keep the same general form.

\section{Conclusions}

The extension of Nicholson's formulae to calculate the Doppler effect in fast reactors presents no difficulty if the medium can be assumed to be neutronically homogeneous but with uneven temperature distributions. This is true both in region $A$ of overlapping resonances as well as in region $B$ of isolated resonances. The general expressions of the Doppler temperature coefficient have strong formal analogies with the corresponding isothermal expressions. Such formulae are useful from the standpoint of temperature and heating rates during a hypothetical power excursion, principally for a zero-power fast critical assembly in which the constituent elements are physically separated.

In the region $A$ as in region $B$ there is proportionality between $T \frac{\partial k}{\partial T}$ and the sum of the products of the partial atomic densities $\rho_{i}$ by their own heating $\delta \mathrm{T}_{1}$. This must be somehow corrected, however.

In case $A_{0}$ that of overlapping resonances, there appears under the double summation $\operatorname{sign} \frac{1}{2}\left(\frac{T}{T_{i}+T_{j}}\right)^{3 / 2}$, which depends on the definition of the reference temperature $T$ and on the different temperatures of the atom sets considered. The expression of the isothermal case $\rho^{2} Q$ becomes

$$
\sum_{i} \rho_{i}\left(\frac{\delta T_{i}}{\delta T}\right) \frac{1}{2} \sum_{j} \rho_{j}\left(\frac{T}{T_{i}+T_{j}}\right)^{3 / 2} Q
$$

In case $B$, that of isolated unresolved resonances, the isothermal expression $\rho \frac{\partial f}{\partial T}$ becomes 


$$
\Sigma \rho\left(\frac{\delta T_{i}}{\delta T}\right) \frac{\partial f_{i}}{\partial T_{i}}
$$

where $\frac{\partial f_{i}}{\partial T_{i}}$ differs from the usual formula:

$$
\frac{\partial f_{i}}{\partial T_{i}}=\frac{2 \beta}{\pi \Gamma} \int_{-\infty}^{+\infty} \frac{\partial}{\partial T_{i}}\left(\frac{\psi_{i}}{\beta+\psi_{i}}\right) d E,
$$

by a factor

$$
\left[\frac{\beta+\psi_{i}}{\beta+\sum_{j}\left(\frac{\rho_{j}}{\rho}\right) \psi_{j}}\right]^{2}
$$

But the main conclusion is certainly that for a power excursion leading to nonuniform heating of the fuel initially at a uniform temperature, the initial Doppler power coefficient (expressed as a temperature coefficient by adopting as reference temperature $T$, the temperature to which the fuel would rise if uniformly heated,) is given for a particular isotope by the relation

$$
T \frac{\partial k}{\partial T}=\left(T \frac{\partial k}{\partial T}\right)_{i \text { sothermal }} \sum_{i}\left(\frac{\rho_{i}}{\rho}\right)\left(\frac{\delta T_{i}}{\delta T}\right),
$$

that is, proportional to:

1) the quantities heated;

2) the temperature rise of these quantities.

Incidentally, one can observe that the reference temperature does not play any absolute role in these developments. It is always possible to adopt as reference temperature the most convenient temperature for the problem considered.

The work described herein, which was sponsored by the EURATOMCEA Association, was initiated last year, when the problem was raised to define a maximum acceptable enrichment for the plutonium fuel of the fast critical facility MASURCA if one were to avoid any positive prompt energetic coefficient in a very large range of reactor core compositions and sizes.

Another important conclusion, which can be deduced from the simple relation relating the Doppler energetic coefficient of a particular reactor mockup to the Doppler energetic coefficient of the reactor simulated, is the following. 
The measurement of the Doppler energetic coefficient of several mockups of the same particular reactor can lead not only to the isothermal Dopplex coefficient, but also to the determination of the separate contributions of the different isotopes to this coefficient (fissile-fertile).

This kind of measurement seems the most attractive in the case in which one could heat up the entire fuel, and the fuel oniy, of the mockup in a very short time, and then get a direct measurement of the reactivity effect associated with the energy release.

Such an experiment appears possible by using neutronic heating of the reactor. As an example, injection of $10^{14}$ neutrons in the fundamental mode into a delayed critical reactor containing $500 \mathrm{~kg}$ of $\mathrm{U}^{235}$ as fuel, would lead to a temperature rise of the order of $10^{\circ} \mathrm{C}$. For a typical reactivity coefficient of the fuel of $+1 \times 10^{-50} \mathrm{C}$, such a temperature use leads to a prompt injection of about $1 \not$ reactivity over delayed critical.

As concerns the way of introducing so many neutrons in so short a time into a reactor, it can be done by feeding from the outside of the assembly the fast neutron emitted by a pulsed Godiva type reactor. Such a burst has a width of $50 \mu \mathrm{s}$, giving a pulse in the reactor due to prompt multiplication of about $100 \mu \mathrm{s}$. Immediately aftex this pulse, the power of the reactor rises to a new power level which varies slowly with time. The power trace can then be interpreted as a measure of the reactivity effect.

\section{Bibliography}

1. Nicholson, R. B. The Doppler Effect in Fast Reactors, APDA 139 (June 1960).

2. Fossoul, E. A., Effect Doppler dans les réacteur rapides à spectre dur, BN 6109-15 (Sept.1961). (Internal report).

Discussion of Paper

Prescnted by Mr. Fossoul

MR. HETRICK (University of Arizona):

This morning Dr. Ott described a calculation which involved the temperature distribution. I believe this simply referred to the position in the core, and could not account for temperatures varying from one part of a fuel rod to another. Is that right? 
MR. OTT (Karlsruhe):

Two average procedures are used. First, an average procedure in the small space region, which means an average over a cross section of the fuel rod. This can be done in the way described by Dr. Greebler. Then one has to average over the core distribution, which I discussed in my paper.

MR. HETRICK:

What might be done in case one wished to explore temperatures changing as a function of time?

MR. FOSSOUL:

This is a dificult problem. The real analytical problem would mean to take the derivative, not with respect to the temperature, but with respect to the distribution. Because of the higher mathematics involved in such an approach, we took the discrete model.

MR. EDW ARDS (Dounreay):

I'm still not clear whether this treatment and also that of Dr. Ott. apply only to temperature distributions which are gradual compared with the mean free path, or not. It seems to me that both fuel plates in a zeroenergy assembly, and also pin sizes in a power reactor, are in fact small compared with the mean free path.

MR. FOSSOUL:

Yes. You can make an average mixture of blue atoms, red atoms, and yellow atoms which actually have different temperatures.

MR. OTT:

The mean free patin of the neutrons is very large compared with the diameter of the small fuel rods in fast reactors. Therefore, the Dopplerproducing neutrons do the averaging, and we have only to do the average which is not done by the neutrons. This means the average over the cross temperature profile.

MR. DAVEY (Argonne):

May I ask what enrichment you decided was permissible to get a zero Doppler coefficient over the wide range of spectra?

MR. FOSSOUL:

I think $25 \%$ 
SESSION VIII

October 10,1963

\section{DOPPLER EXPERIMENTS}

Chairman: H.H. HUMMEL Argonne National Laboratory

Secretary: W. Y. Kato

Argonne National Laboratory 
MEASUREMENTS OF THE DOPPLER COEFFICIENT IN

LARGE FAST POWER REACTORS USING A

FAST CRITICAL ASSEMBLY AND AN

EXPERIMENTAL EAST REACTOR

\title{
PART I. THEORETICAL ASPECTS OF THE MEASUREMENT OF THE DOPPLER COEFFICIENT IN A CRITICAL FACILITY
}

\author{
F. Storrer, A. Khairallah, and J. Ozeroff* \\ EURATOM CEA (Cadarache) \\ Bouches du Rhone, France \\ (Paper presented by Mr. F. Storrer)
}

\section{Introduction}

In this paper, two methods of measuring the Doppler coefficient are discussed.

In the reactivity method a sample is heated and the resulting change of the reactivity is measured. This can be done either by a static or by an oscillation experiment. The composition of the sample can either be the same as that of the surrounding core, or different from it. In some cases, it should be surrounded by a buffer zone.

In the activation method a sample of either fissile or fertile material is heated and after some irradiation its activity is compared with that of a cold sample which was irradiated under the same conditions. Again, this sample may be surrounded by a buffer zone.

Some theoretical aspects along with some practical aspects of both methods are discussed. It is shown that by using several types of experiments, different components of the Doppler coefficient can be measured. The experimental difficulties to be expected with both methods are also discussed.

One important result presented in this paper is the following. It has been commonly accepted so far (1) that, if in the reactivity method only a very small sample is heated, only part of the Doppler coefficient, i.e. the cross-section component, is measured. In this paper, it is proved. that even for a very small sample, the full Doppler coefficient is meas ured, of course weighted by the sample volume, by the flux, and by the importance.

* On leave from General Electric Co., Vallecitos Atomic Power Laboratory, Pleasanton, California. 
II. Reactivity Method

Since heating the whole core in a critical assembly would be impractical, we investigate the reactivity change upon heating a sample.

A. Theory of Sample Measurements with Sample of the Same Composition as the Core

We consider the problem of calculating the change in reactivity due to the Doppler broadening of nuclear resonances in a reactor when only a small volume (or sample) of the reactor is heated. The reactor is assumed to be otherwise uniform and homogeneous, and the sample has the same composition.

1. Discussion of the Problem and Intuitive Treatment

Reactivity variations are proportional to variations of reaction rates, properly weighted by the importance, which is a function. of space and energy. In this discussion, we shall investigate variations of the reaction rate for a reaction with a cross section $\sigma_{\mathrm{X}}(\mathrm{E}, \mathrm{T})$ that is a function of energy $E$ and temperature $T$. We shall denote the total cross section by $\sigma$ and let $\phi$ denote the flux.

We consider first an infinite homogeneous medium at uniform temperature. The reaction rate over a volume $V$ per unit energy at energy $\mathrm{E}$ is given by

$$
J=\int_{V} \phi \sigma_{\mathrm{X}} d V=V \phi \sigma_{\mathrm{X}}
$$

The macroscopic flux was assumed flat in space since the spatial dependence of the macroscopic flux or of the importance has no bearing on the problem under investigation. Since we are interested in the Doppler coefficient, which is a derivative with respect to temperature, we take the temperature derivative of $\mathrm{J}$ :

$$
J^{\prime}=\mathrm{V}\left(\phi \sigma_{\mathrm{x}}^{\prime}+\phi^{\prime} \sigma_{\mathrm{x}}\right)
$$

or, using the differential notation,

$$
\mathrm{dJ}=\mathrm{V}\left(\dot{\phi} \mathrm{d} \sigma_{\mathrm{x}}+\sigma_{\mathrm{x}} \mathrm{d} \phi\right)
$$

This is an expression for the variation of the reaction rate, up to first ordex in the tempexature variation. In the next section, the case of large temperature changes will be considered. The llux perturbation d $\phi$ (or spectrum perturbation if its energy dependence is considered) can be 
either positive or negative. The neutrons involved in the perturbation are termed "hot," to contrast with the "cold" neutrons of the unperturbed flux.

Consider now the case in which only a very small sample of volume $V_{s}$ is heated by an amount $d T$ while the surrounding medium remains at the initial temperature. "Very small" means that practically all neutrons in this sample suffered their last collision in the surrounding region. The change in reaction rate in this sample is obviously given by

$$
\mathrm{dJ}_{\mathrm{S}}=\mathrm{V}_{\mathrm{s}} \phi \mathrm{d} \sigma_{\mathrm{X}}
$$

since practically all neutrons in the sample are "cold."

It was commonly accepted that in a reactivity measurement with a small sample one measures only the cross-section component $\phi \mathrm{d} \sigma_{\mathrm{X}}$ of the Doppler coefficient and not the spectral component $\sigma_{\mathrm{x}} \mathrm{d} \phi$. This was particularly disturbing since the ratio of these two components, which is unity at high energies where the resonances overlap, varies widely with energy and temperature in a softer spectrum.

This commonly accepted view is, however, erroneous, since the reactivity is proportional to the total reaction rate, i.e., including that in the surrounding region. It turns out that, still for a very small sample, the change in that reaction rate in the surrounding region is given exactly by

$$
\mathrm{d} J_{\mathrm{R}}=\mathrm{V}_{\mathrm{s}} \sigma_{\mathrm{x}} \mathrm{d} \phi
$$

so that for the total reaction rate

$$
\mathrm{dJ}=\mathrm{dJ} J_{\mathrm{s}}+\mathrm{d} J_{\mathrm{R}}=\mathrm{V}_{\mathrm{s}}\left(\phi \mathrm{d} \sigma_{\mathrm{X}}+\sigma_{\mathrm{x}} \mathrm{d} \phi\right)
$$

or exactly the same as (3) when normalized to the volume of the heated region. This comes from the fact that the sample sends out "hot" neutrons which react with the surrounding medium. Thus a small sample experiment gives the complete Doppler coefficient.

This general conclusion is true for a sample of any size. When the sample is not very small, the cross-section component $V_{S} \phi d \sigma_{X}$ still comes from the sample only, but the spectral component $V_{\mathrm{S}} \sigma_{\mathrm{X}} \mathrm{d} \phi$ comes partly from the sample and partly from the surrounding medium.

It now remains to prove the statement contained in (5), namely, that the spectral component, integrated over space, of dJ due to the heating of a sample of volume $V_{S}$ is always given by $V_{s} \sigma_{X} d \phi$, where $\mathrm{d} \phi$ has the same meaning as in (3). A rigorous proof is given in the next section. Here a more or less intuitive proof is presented. Such an 
intuitive treatment, which led us to perform the more rigorous work, will also be used in later sections of this paper.

This proof is based on the principle of superposition and can be divided in two statements:

a) Each volume of heated sample produces a number of hot neutrons proportional to its volume, independently of whether the surrounding medium is hot or cold.

b) It does not make any difference whether a hot neutron interacts with a hot or a cold medium.

\section{Proof of Statement a}

The source of hot neutrons is obviously the interaction of the unperturbed flux $\phi$ with the perturbed cross section $d \sigma$. For a heated sample of volume $V_{S}$, the source $S$ is given in strength by

$$
S=-V_{s} \phi d \sigma
$$

which is indeed proportional to $\mathrm{V}_{\mathrm{s}}$. Any interaction of the perturbed flux with d $\sigma$ would give rise to a second-order source.

\section{Proof of Statement b}

As is, for example, shown by (3), d $\phi$ is to be multiplied by the unperturbed cross section $\sigma_{\mathrm{x}}$, since any interaction of hot neutrons d $\phi$ with do would lead to a second-order effect.

In order to illustrate these statements, again let us consider (7). For an infinitesimally small sample of volume dV the source of hot neutrons is given by

$$
d S=-\phi d \sigma d V \text {. }
$$

The flux perturbation at a distance $r$ from the sample is given by

$$
d \phi(r)=\frac{e^{-\sigma r}}{4 \pi r^{2}} d S,
$$

and the flux perturbation integrated over the whole volume, $\delta \dot{\phi}$, is given by

$$
\delta \phi=\int_{0}^{\infty} 4 \pi r^{2} d \phi(r) d r=\frac{d S}{\sigma}=-\frac{\phi d \sigma}{\sigma} d V
$$


When a large volume $V$ is heated, one can use the superposition principle:

$$
\delta \phi=-\frac{\phi d \sigma}{\sigma} \mathrm{V},
$$

and when $V$ tends to infinity, the flux perturbation is constant in space and becomes

$$
\alpha \phi=\frac{\delta \phi}{V}=-\frac{\phi d \sigma}{\sigma}
$$

As given by (12), d $\phi$ is of course the same as in (3). Equation (12) can be written as $d(\phi \sigma)=0$, which expresses the usual assumption that the collision density, which for a homogeneous medium is the same as the slowingdown source, is independent of temperature.

\section{Exact Treatment for Arbitrary Sample Volumes and Arbitrary Temperature Changes}

We again consider a reactor of volume $V$ and within it a sample of volume $V^{\prime}$. In the volume $V$, the temperature is $T$ and the macroscopic total and absorption cross sections are $\sigma$ and $\sigma_{a}$; in $V^{\prime}$, the temperature is $T^{\prime}$ (with $T^{\prime}>T$ ) and the cross sections are $\sigma^{\prime}$ and $\sigma_{2}^{\prime}$, the changes being due to the increased Doppler broadening. We suppose that the macroscopic flux and importance function do not vary over the region around the sample volume; a straightforward correction can be made if this is not the case. The slowing-down source, $q_{0}$, is then spatially uniform. The number of neutrons entering a narrow energy interval dE at $E$ is $q_{0} d E$. Since we are considering only the neutrons in the narrow range dE, any event, scattering or absorption, removes neutrons, and we may use the transport equation for a purely absorption medium with the total cross section playing the role of the absorption cross section.

\section{a. One-dimensional Case}

In the one-dimensional case, an exact solution of the transport equation is easily obtained. The equation is $(2)$

$$
\mu \frac{d \psi}{d z}+\sigma \psi=\frac{q_{0}}{v}
$$

where $\psi$ is the directional flux per unit energy, $\mu$ is the cosine of the angle between the neutron velocity vector and the $z$ axis, and $v$ the velocity. If we solve this equation for a one-dimensional sample of thickness $2 \mathrm{a}$ in the geometry indicated in Fig. 1 and integrate $\psi(\mathrm{z}, \mu)$ over all angles, we obtain the following expressions for the flux: 

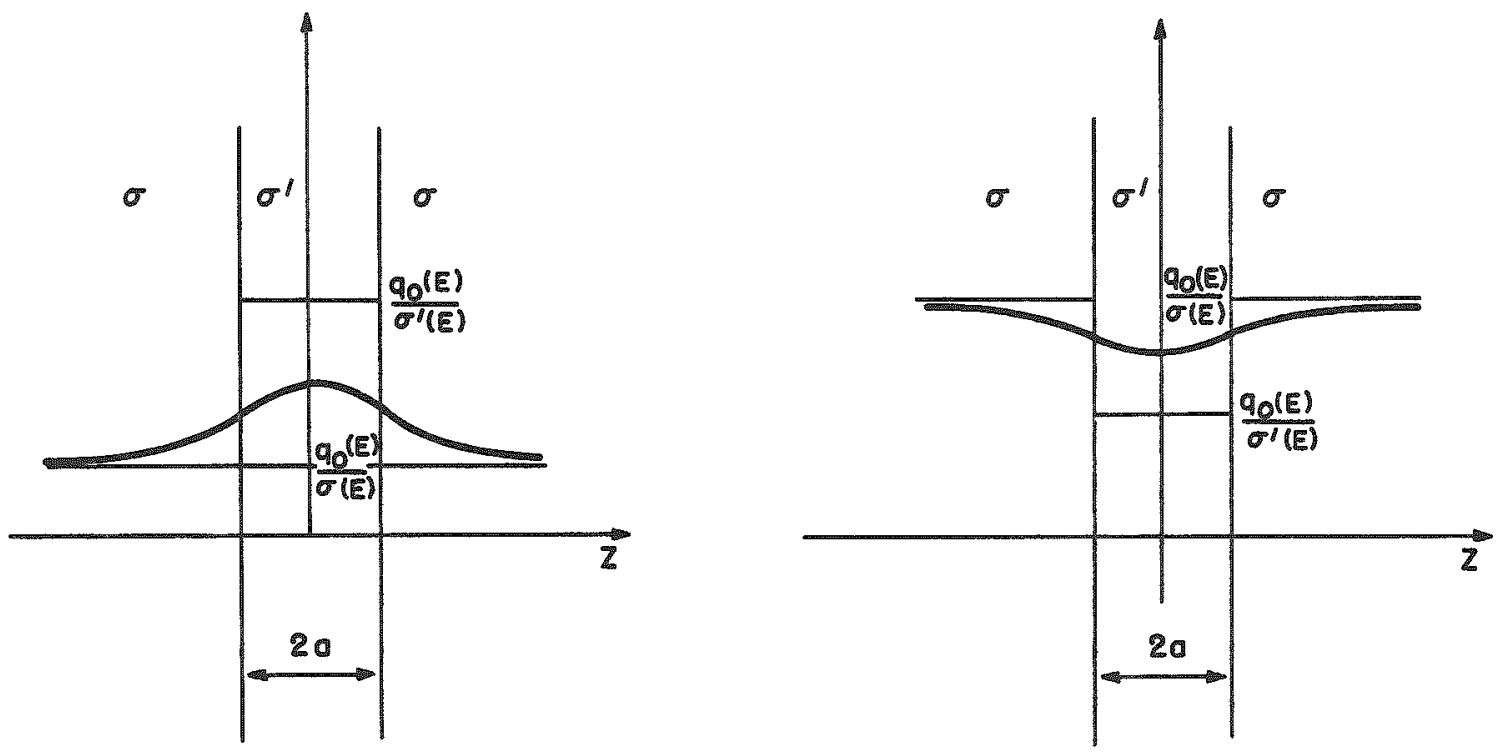

Fig. 1. (Left) $\phi(E)$ for $\sigma^{\prime}(E)<\sigma(E)$; (Right) $\phi(E)$ for $\sigma^{\prime}(E)>\sigma(E)$. for $|z|<a$,

$$
\phi^{\prime}(z)=\frac{q_{0}}{\sigma^{\prime}}\left\{1-\frac{\sigma-\sigma^{\prime}}{\sigma} \frac{E_{2}\left[\sigma^{\prime}(z+a)\right]+E_{2}\left[\sigma^{\prime}(z-a)\right]}{2}\right\} ;
$$

for $|z|>a_{0}$

$$
\phi^{\prime}(z)=\frac{q_{0}}{\sigma}\left\{1-\frac{\sigma^{\prime}-\sigma}{\sigma^{\prime}} \frac{E_{2}[\sigma(z-a)]-E_{2}\left[\sigma(z+a)+2 a \sigma^{\prime}\right]}{2}\right\} .
$$

Figure 1 shows that, when there is temperature heterogeneity, there is a fine structure in the collision density, as well in space as in energy, even though the slowing-down source has no such fine structure. Before the temperature change was made, the flux was, of course,

$$
\phi(z)=q_{0} / \sigma .
$$

Therefore, the changes in reaction rates are given by

$$
\Delta \mathrm{J}_{\text {sample }}=\int_{\mathrm{V}^{\prime}}\left[\phi^{\prime}(z) \sigma_{\mathrm{a}}^{\prime}-\phi(\mathrm{z}) \sigma_{\mathrm{a}}\right] \mathrm{dV}^{\prime} \text { in the sample }
$$

and

$$
\Delta J_{\text {reactor }}=\int_{V}\left[\phi^{\prime}(z)-\phi(z)\right] \sigma_{a} d V \text { in the reactor. }
$$


By means of the expressions (2) and (3) for $\phi$ and $\phi^{\prime}$, we obtain*

$$
\begin{aligned}
& \Delta J_{\text {sample }}=q_{0}\left(\frac{\sigma_{a}^{\prime}}{\sigma^{\prime}}-\frac{\sigma_{a}}{\sigma}\right) 2 a+q_{0} \frac{\sigma_{a}^{\prime}}{\sigma^{\prime}} \frac{\sigma^{\prime}-\sigma}{\sigma^{\prime} \sigma}\left[\frac{1}{2}-E_{3}\left(2 a \sigma^{\prime}\right)\right] ; \\
& \Delta J_{\text {reactor }}=-q_{0} \frac{\sigma_{a}}{\sigma} \frac{\sigma^{\prime}-\sigma}{\sigma^{\prime} \sigma}\left[\frac{1}{2}-E_{3}\left(2 a \sigma^{\prime}\right)\right] .
\end{aligned}
$$

It is of interest to examine these expressions. The first term in $\Delta \mathrm{J}$ sample is the change in reaction rate in the sample if the flux were in equilibrium with the sample temperature at all points in the sample volume. The second term is the correction due to the spatial transient in the sample while the term for $\Delta J_{\text {reactor }}$ is due to the spatial transient in the part of the reactor surrounding the sample. If the temperature change is not too large, these last two terms are nearly equal and of opposite sign. They therefore cancel one another and what remains for the total change in reaction rate is

$$
\Delta J=\Delta J_{\text {sample }}+\Delta J_{\text {reactor }}=q_{0} 2 a\left(\frac{\sigma_{a}^{\prime}}{\sigma^{\prime}}-\frac{\sigma_{a}}{\sigma}\right),
$$

that is, a reaction rate change computed as if the flux were in equilibrium with the sample temperature. If we consider very small samples $\left(a \sigma^{1}<<1\right)$, for which the magnitude of the noncancellation of the transient terms would be the greatest, we obtain, since $E_{3}(x) \simeq \frac{1}{2}-x+0\left(x^{2}\right)$,

$$
\Delta J=2 a q_{0}\left(\frac{\sigma_{a}^{\prime}}{\sigma^{\prime}}-\frac{\sigma_{a}}{\sigma}\right)\left(1+\frac{\sigma^{\prime}-\sigma}{\sigma}\right) \text {. }
$$

Even in this case, the magnitude of the noncancellation of the transients is only of magnitude $\left(\sigma^{\prime}-\sigma\right) / \sigma$ as compared with unity. As stated earlier, this can be neglected if the temperature change is not too large. This conclusion holds, a fortiori, for larger samples.

The above discussion has been concerned only with the neutrons in a narrow energy range. If we are to compute the change in reaction rate produced by the Doppler broadening of nuclear resonances, it is necessary to integrate the expressions for $\Delta J$ over the resonances. The same general conclusions will be shown to hold for the integrated reaction rates; this will be done in the following sections in which we consider more general geometries.

*The functions $E_{2}(x)$ and $E_{3}(x)$ are defined in Ref. 2 . 
b. Three-dimensional Case with Arbitrary Geometry

We have seen in the one-dimensional case that, if we are considering the neutrons in a narrow energy range $\mathrm{d} \boldsymbol{E}$ in a resonance, it is possible to calculate the spatial dependence of the flux in detail, and this allows us to conclude that the lack of equilibrium in the neutron-energy spectrum of the sample is automatically compensated by the changed reaction rate outside the sample. No such detailed solution is available in the general three-dimensional case; however, similar conclusions may be reached by using the concepts of escape probabilities as these are defined in Ref. 2 .

Consider again a reactor of volume $V$ and a heated sample of volume $V^{\prime}$. The number of neutrons (per unit time, per unit energy at energy $E$ ) which escape from $V^{\prime}$ into $V$ and suffer a collision. there is given by

$$
q_{0} V^{\prime} P_{0}\left(\sigma^{\prime}\right)
$$

where $P_{0}$ is the escape probability of the sample. (We assume that $V$ is sufficiently large that all neutrons which escape from $V^{\prime}$ will suffer a collision in V.) Hence, the number of neutrons per unit time which escape $V^{\prime}$ and are absorbed in $V$ is given by

$$
q_{0} V^{\prime} P_{0}\left(\sigma^{\prime}\right)\left(\sigma_{a} / \sigma\right)
$$

Similarly, the number of neutrons per unit time which escape from $V$ into $\mathrm{V}^{\prime}$ and suffer a collision there is given by

$$
q_{0} V^{\prime} P_{0}\left(\sigma^{\prime}\right)\left(\sigma^{\prime} / \sigma\right)
$$

and the number of these absorbed in $V^{\prime}$ is given by

$$
q_{0} V^{\prime} P_{0}\left(\sigma^{\prime}\right)\left(\sigma^{\prime} / \sigma\right)\left(\sigma_{a}^{\prime} / \sigma^{\prime}\right)
$$

Using the above results, we may now write the following expressions for the reaction rates $J$ and $J^{\prime}$ in the two media:

In the Sample V:

before heating:

$$
J^{\prime}=q_{0} V^{\prime} \sigma_{a} / \sigma
$$

after heating:

$$
J^{\prime}=\left[q_{0} V^{\prime}-q_{0} V^{\prime} P_{0}\left(\sigma^{\prime}\right)+q_{0} V^{\prime} P_{0}\left(\sigma^{\prime}\right) \frac{\sigma^{\prime}}{\sigma}\right] \frac{\sigma^{\prime}}{\sigma^{\prime}} .
$$


Hence, the change $\Delta J_{V}^{\prime}$ upon heating is

$$
\Delta J_{V^{\prime}}^{\prime}=q_{0} V^{\prime}\left\{\left[1-P_{0}\left(\sigma^{\prime}\right)+P_{0}\left(\sigma^{\prime}\right) \frac{\sigma^{\prime}}{\sigma}\right] \frac{\sigma_{a}^{\prime}}{\sigma^{\prime}}-\frac{\sigma_{a}}{\sigma}\right\} .
$$

In the Rest of the Reactor V,

before heating:

$$
J=q_{0} V \quad \sigma_{a} / \sigma
$$

after heating:

$$
J=\left[q_{0} V-q_{0} V^{\prime} P_{0}\left(\sigma^{\prime}\right) \frac{\sigma^{\prime}}{\sigma}+q_{0} V^{\prime} P_{0}\left(\sigma^{\prime}\right)\right] \frac{\sigma_{a}}{\sigma} .
$$

Thus the change $\Delta J_{V}$ in the rest of the reactor is

$$
\Delta J_{V}=q_{0} V^{\prime} P_{0}\left(\sigma^{\prime}\right) \frac{\sigma-\sigma^{\prime}}{\sigma} \frac{\sigma_{a}}{\sigma}
$$

The total variation in the reaction rate due to heating the sample from temperature $T$ to $T^{\prime}$ then becomes

$$
\Delta J=\Delta J_{V^{\prime}}^{\prime}+\Delta J_{V}=q_{0} V^{\prime}\left(\frac{\sigma_{a}^{\prime}}{\sigma^{\prime}}-\frac{\sigma_{a}}{\sigma}\right)\left[1+\frac{\sigma^{\prime}-\sigma}{\sigma} P_{0}\left(\sigma^{\prime}\right)\right] .
$$

This expression is valid for any sample size and shape and for any temperature change. It must now be integrated over the resonances at all energies in order to obtain the total change in reaction rate in the reactor due to heating of the sample. As before, we notice that if, inside the sample, the spectrum was the equilibrium spectrum corresponding to temperature $T^{\prime}$ - that would be the case if the whole reactor was heated to temperature $T^{\prime}$ - the contribution of the sample $V^{\prime}$ to the change in reaction rate would be given by the first term of expression (13), namely

$$
\Delta J_{\infty}=q_{0} V^{\prime}\left(\frac{\sigma_{a}}{\sigma^{\prime}}-\frac{\sigma_{a}}{\sigma}\right)
$$

Therefore, expression (13) includes the "homogeneous" case (the whole reactor heated) by equating the escape probability $P_{0}\left(\sigma^{\prime}\right)$ to zero. The interaction between the hot sample and the cold reactor gives rise to a correction term, which depends on the mean escape probability $P_{0}\left(\sigma^{\prime}\right)$ and therefore on the size of the sample, and also, via $\sigma^{\prime}-\sigma$, on the temperature difference $\Delta T=T^{\prime}-T$. Note, however, that it appears from expression (13) that, up to first order, i.e., for a small temperature change, the change in reaction rate, and therefore the Doppler coefficient, 
is the same for a sample of any size as for the whole reactor (with, of course, a proper weighting by volume). That is because the correction term, namely,

$$
\left(\frac{\sigma_{a}^{\prime}}{\sigma^{\prime}}-\frac{\sigma_{a}}{\sigma}\right) \frac{\sigma^{\prime}-\sigma}{\sigma} P_{0}\left(\sigma^{\prime}\right)
$$

which is the product of two first-order terms, is a second-order term.

This appears more clearly upon expanding expres sion (13) in the two extreme cases:

(a) The whole reactor, characterized by $P_{0}\left(\sigma^{\prime}\right)=0$, in which case expression (13) may be written as

$$
\Delta J_{\infty} \sim \sigma_{p} \frac{\Delta \sigma_{a}}{\left(\Gamma / \Gamma \gamma \sigma_{a}+\sigma_{p}\right)^{2}}\left[1-\frac{\Gamma / \Gamma \gamma \Delta \sigma_{a}}{\left(\Gamma / \Gamma \gamma \Delta \sigma_{a}+\sigma_{p}\right)}+O\left(\Delta \sigma_{a}^{2}\right)\right]
$$

(b) The very small sample (small compared with the total mean free path at any energy), characterized by $P_{0}\left(\sigma^{\prime}\right)=1$, in which case expression (13) reduces simply to

$$
\Delta J_{s} \sim \sigma_{p} \frac{\Delta \sigma_{a}}{\left(\Gamma / \Gamma \gamma \sigma_{a}+\sigma_{p}\right)^{2}}
$$

In these expressions

$$
\left\{\begin{aligned}
\Delta \sigma_{a} & =\sigma_{a}^{\prime}-\sigma_{a}=\Gamma \gamma / \Gamma\left(\sigma^{\prime}-\sigma\right) \\
\sigma_{p} & =\sigma^{\prime}-\Gamma / \Gamma \gamma \sigma_{a}^{\prime}=\sigma-\Gamma / \Gamma \gamma \sigma_{a} .
\end{aligned}\right.
$$

and (16):

Two more conclusions appear from expressions (15)

1) For the small sample $\Delta J_{S}$ is a linear function of $\Delta \sigma_{a}$ while $\Delta J_{\infty}$ for the whole reactor is not.

2) For a large temperature change, $\Delta \mathrm{J}_{\mathrm{S}}$ is smaller or larger than $\Delta J_{\infty}$, depending on whether, at energy $E, \Delta \sigma_{a}$ is $<0$ or $>0$. Since the major part of the Doppler effect of a resonance comes from the wings of the resonance, i.e., from a region in which $\Delta \sigma_{a}$ is $>0$, it is to be expected that the Doppler effect per unit volume measured with a small sample should be larger than in a measurement where the whole reactor is heated. 
The same conclusion holds for a large sample (large compared with the total mean free path at any energy), characterized by

$$
P_{0}\left(\sigma^{\prime}\right)=\frac{1}{\bar{d} \sigma^{\prime}}
$$

(where $\bar{l}$ is the mean chord), in which case expression (13) reduces to

$$
\Delta J_{b} \sim \sigma_{p} \frac{\Delta \sigma_{a}}{\left(\Gamma / \Gamma_{\gamma} \sigma_{a}+\sigma_{p}\right)^{2}}\left[1-\left(1-\frac{L_{p} / \bar{l}}{\left(\Gamma / \Gamma_{\gamma} \sigma_{a}+\sigma_{p}\right)}\right) \frac{\Gamma / \Gamma \gamma \Delta \sigma_{a}}{\left(\Gamma / \Gamma_{\gamma} \sigma_{a}+\sigma_{p}\right)}+O\left(\Delta \sigma_{a}^{2}\right)\right] \text {, }
$$

where $L_{p}=\frac{1}{\sigma_{p}}$, total potential scattering mean free path, including all materials.

Since the mean escape probability $P_{0}\left(\sigma^{\prime}\right)$ is a decreasing function of the dimensions of the heated sample, it is to be expected that the relative importance of the correction term should decrease as the sample size increases.

In the following paragraphs we evaluate the magnitude of the correction term in the two cases of a very small sample and of a large sample, compared with the main term which corresponds to an infinite heated sample (the whole reactor is heated) for isolated resonances and overlapping resonances.

\section{c. Integration Over Energy - Isolated Resonances}

An isolated resonance in a given medium and at a given temperature is characterized by two parameters:

$$
\beta=\sigma_{\mathrm{p}} / \sigma_{0} ; \quad t=4 \mathrm{E}_{0} \mathrm{kT} / \Gamma^{2} \mathrm{~A} \text {. }
$$

When written in terms of the Doppler broadened resonance shape $\psi(x, t)$ and integrated ove $x$ resonance, the change in reaction rate in the three cases of the whole reactor, the small sample, and the large sample are, respectively, proportional to the integrals

$$
\begin{aligned}
& \Delta \mathrm{J}_{\infty} \sim \mathrm{I}=\int_{0}^{\infty}\left(\frac{\psi_{2}}{\psi_{2}+\beta}-\frac{\psi_{1}}{\psi_{1}+\beta}\right) \mathrm{dx} ; \\
& \Delta \mathrm{J}_{\mathrm{S}} \sim \mathrm{I}_{\mathrm{S}}=\beta \int_{0}^{\infty} \frac{\dot{\psi}_{2}-\psi_{1}}{\left(\psi_{1}+\beta\right)^{2}} \mathrm{dx}=\mathrm{I}+\Delta \mathrm{I}_{\mathrm{S}} ;
\end{aligned}
$$




$$
\begin{aligned}
\Delta \mathrm{J}_{\mathrm{b}} \sim \mathrm{I}_{\mathrm{b}} & =\int_{0}^{\infty}\left(\frac{\psi_{2}}{\psi_{2}+\beta}-\frac{\psi_{1}}{\psi_{1}+\beta}\right) \mathrm{dx}+\frac{L_{\mathrm{p}}}{\bar{l}} \int_{0}^{\infty}\left(\frac{\psi_{2}}{\psi_{2}+\beta}-\frac{\psi_{1}}{\psi_{\mathrm{l}}+\beta}\right)^{2} \\
& =\mathrm{I}+\frac{\mathrm{L}_{\mathrm{p}}}{\bar{l}} \Delta \mathrm{I}_{\mathrm{b}} .
\end{aligned}
$$

In these expressions,

$$
x=\frac{E-E_{0}}{\Gamma / 2}
$$

$\psi_{1}$ corresponds to temperature $\mathrm{T}$, and $\psi_{2}$ corresponds to temperature $T+\Delta T$.

1) Numerical calculations have been made of the integrals $I, \Delta I_{S}$, and $\Delta I_{b}$ as defined by equations (18), (19) and (20). The calculations have been made for various t's and $\beta$ 's and for constant ratios $\Delta \mathrm{T} / \mathrm{T}=\Delta \mathrm{t} / \mathrm{t}(0<\Delta \mathrm{T} / \mathrm{T}<0.5)$. The corresponding curves are shown in Fig. $2 \mathrm{a}, 2 \mathrm{~b}$, and $2 \mathrm{c}$.

2) In Fig. $3 a$ and $3 b$, are shown the curves of $\Delta I_{S} / I$ and $\Delta \mathrm{I}_{\mathrm{b}} / \mathrm{I}$, that is, the relative magnitude of the correction term in the case of small samples and of large samples. The curves $\Delta \mathrm{I}_{\mathrm{b}} / \mathrm{I}$ must be multiplied by $L_{\mathrm{p}} / \bar{l} \leq 1 / 5$.

3) By interpolation between the curves of Fig. 2a, 2b, and $2 c$, we have calculated the mean change in reaction rate for the small sample, the large sample, and the infinite sample, as a function of $\Delta T / T$, averaged over the first 56 resolved resonances of $U^{238}$ (up to $1 \mathrm{keV}$ ), as well as for the two cases

(a) pure $U^{238}$ at room temperature: $\sigma_{\mathrm{p}}=10 \mathrm{~b}$;
$\mathrm{T}=300^{\circ} \mathrm{K}$;

(b) $\mathrm{U}^{238}$ in a typical dilute fast reactor at $300^{\circ} \mathrm{C}$ :

$$
\sigma_{\mathrm{p}}=60 \mathrm{~b} ; \mathrm{T}=600^{\circ} \mathrm{K} \text {. }
$$

For the infinite sample we have $\bar{I} \sim \Sigma\left(\Gamma_{\gamma} I\right)$

For the small sample, $\overline{\mathrm{I}}_{\mathrm{S}} \sim \Sigma\left(\Gamma_{\gamma} \mathrm{I}\right)+\Sigma\left(\Gamma \gamma \Delta \mathrm{I}_{\mathrm{S}}\right)$

For the larger sample, $\overrightarrow{\mathrm{I}}_{\mathrm{b}} \sim \Sigma\left(\Gamma_{\gamma} \mathrm{I}\right)+\Sigma\left(\Gamma_{\gamma} \Delta \mathrm{I}_{\mathrm{b}}\right)$.

The integrals $I, \Delta I_{S}$, and $\Delta I_{b}$ are defined in equations (18), (19), and (20). The results are shown in $F$ ig. $4 a$ and $4 b$. On the same graph is drawn the tangent at $\Delta \mathrm{T} / \mathrm{T}=0$ to the curve corresponding to the infinite sample, which gives the Doppler coefficient of the reactor. One notices that the 


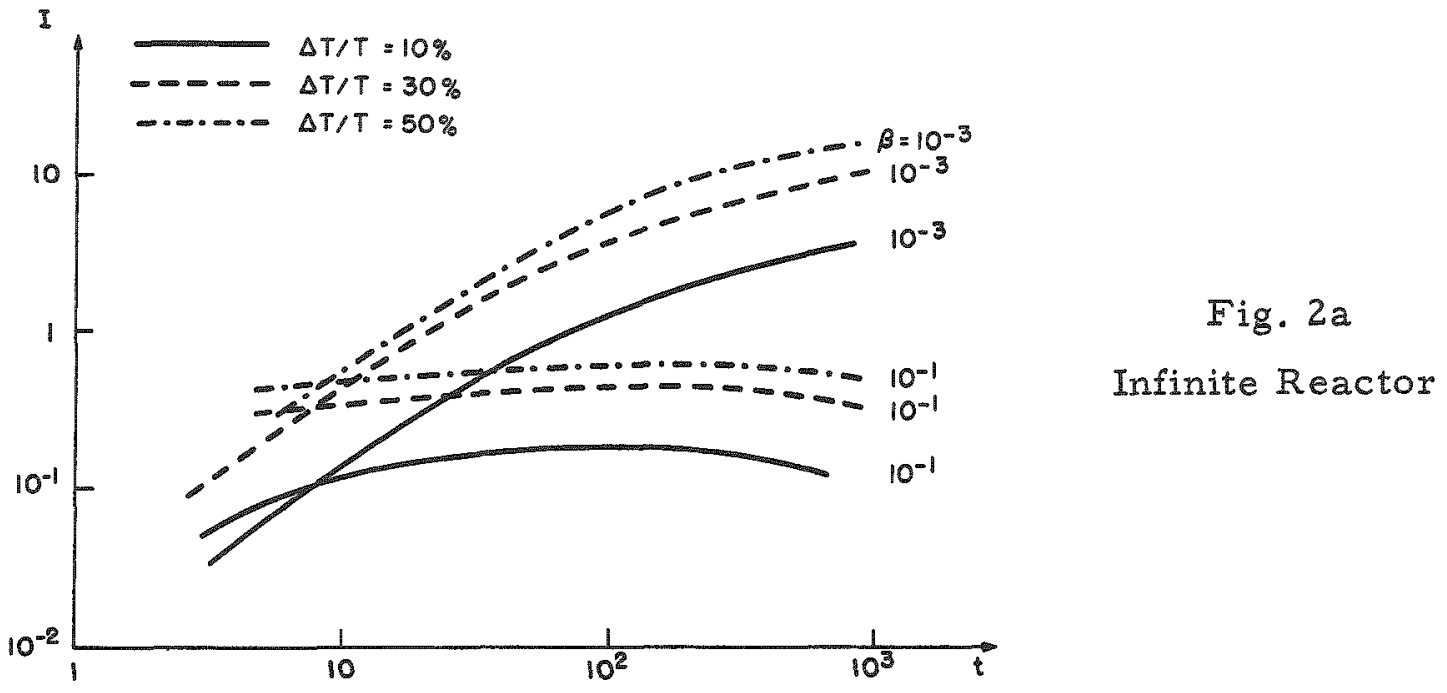

Fig. 2b

Correction for

Small Samples
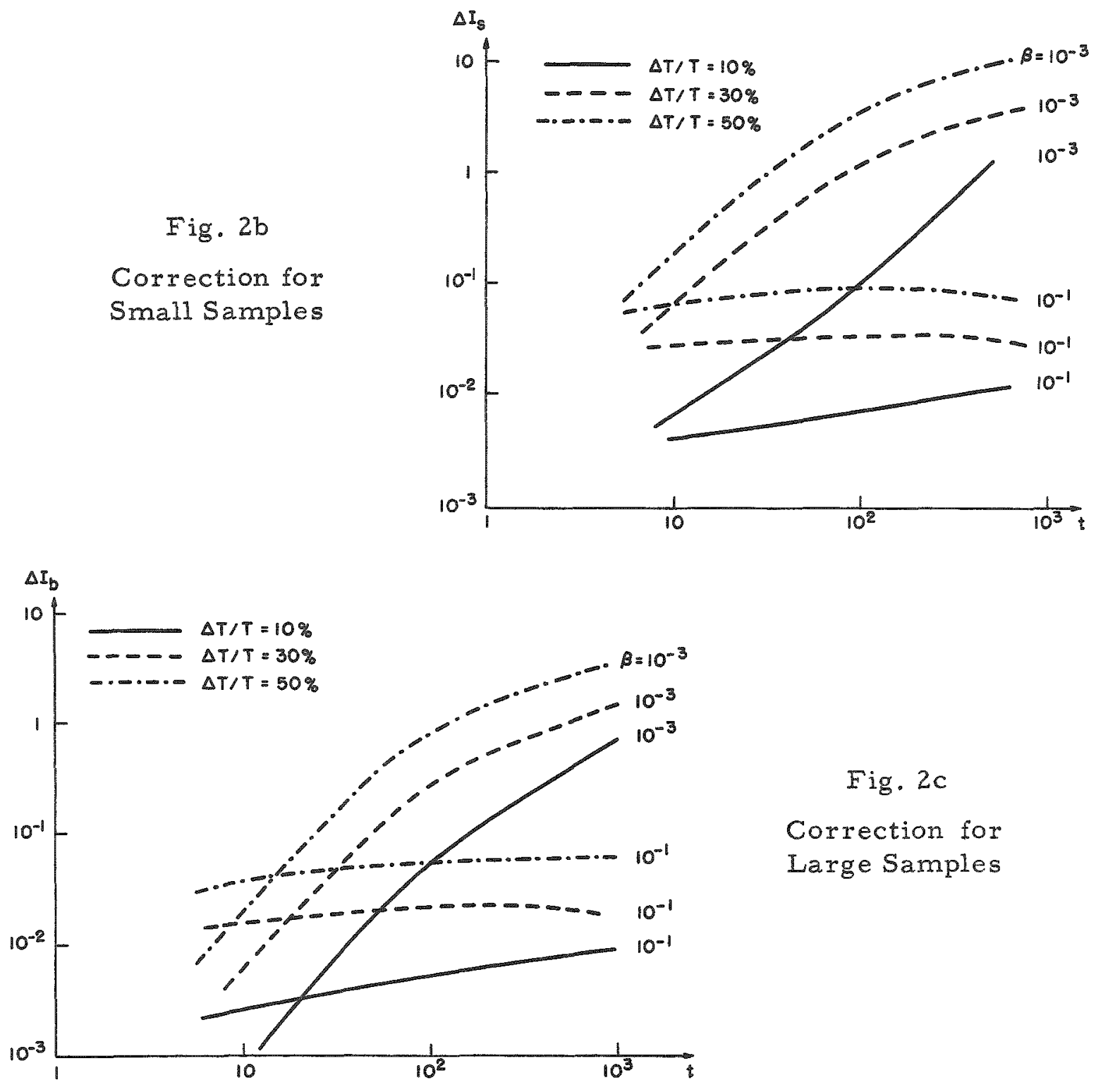
STORRER et al.

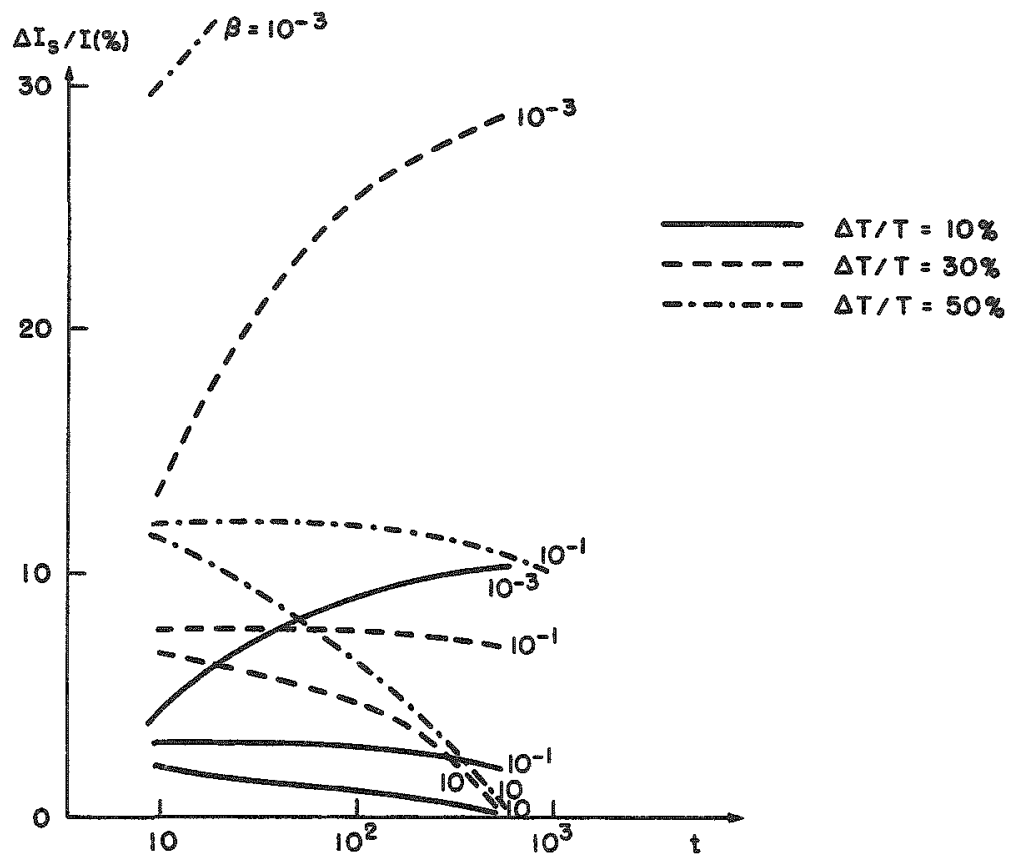

Fig. $3 a$

Relative Correction for a Small Sample

Fig. 3b

Relative Correction for a Large Sample
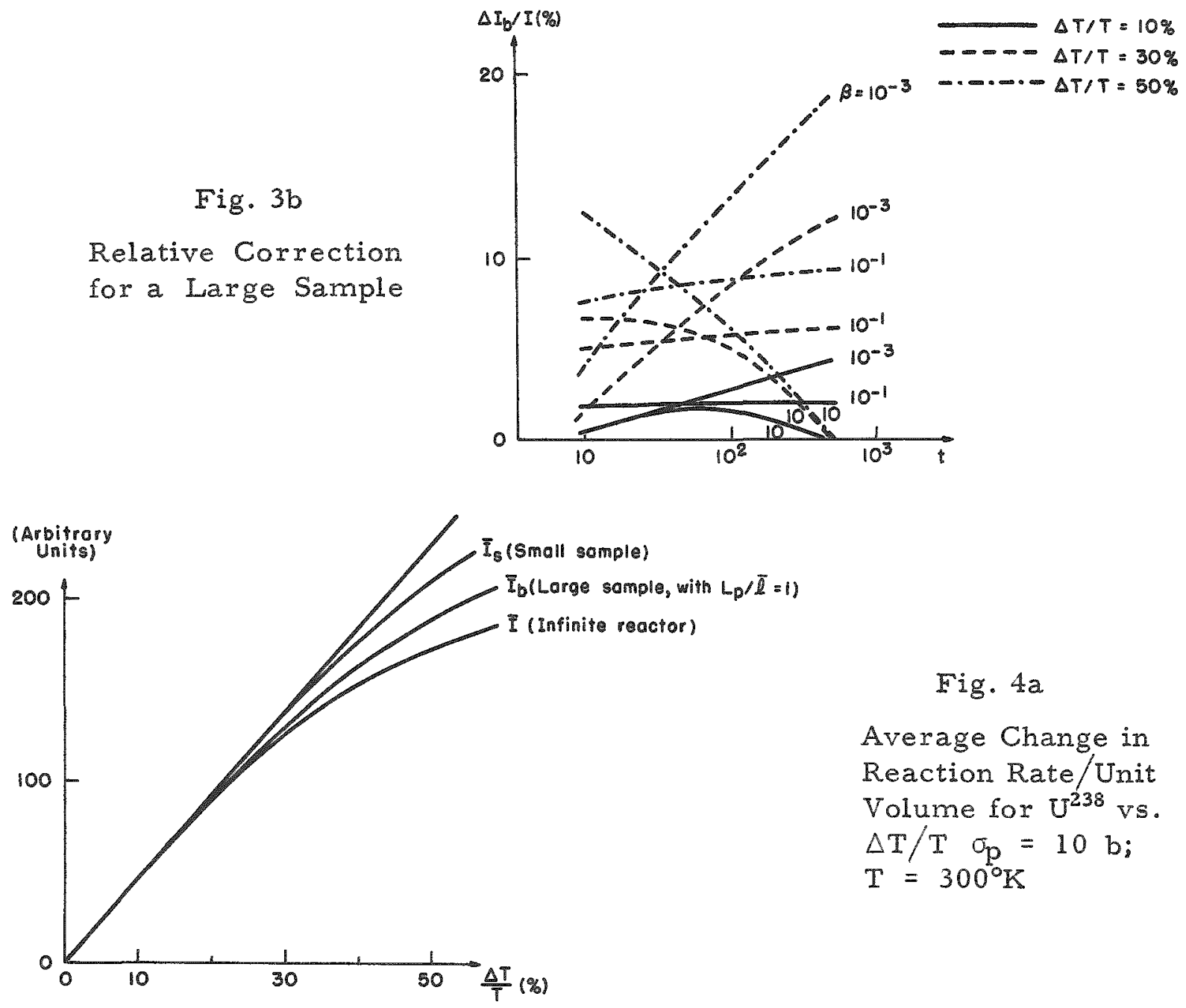

Fig. $4 a$

Average Change in Reaction Rate/Unit Volume for $\mathrm{U}^{238} \mathrm{vs}$. $\Delta \mathrm{T} / \mathrm{T} \quad \sigma_{\mathrm{p}}=10 \mathrm{~b}$ $\mathrm{T}=300^{\circ} \mathrm{K}$ 


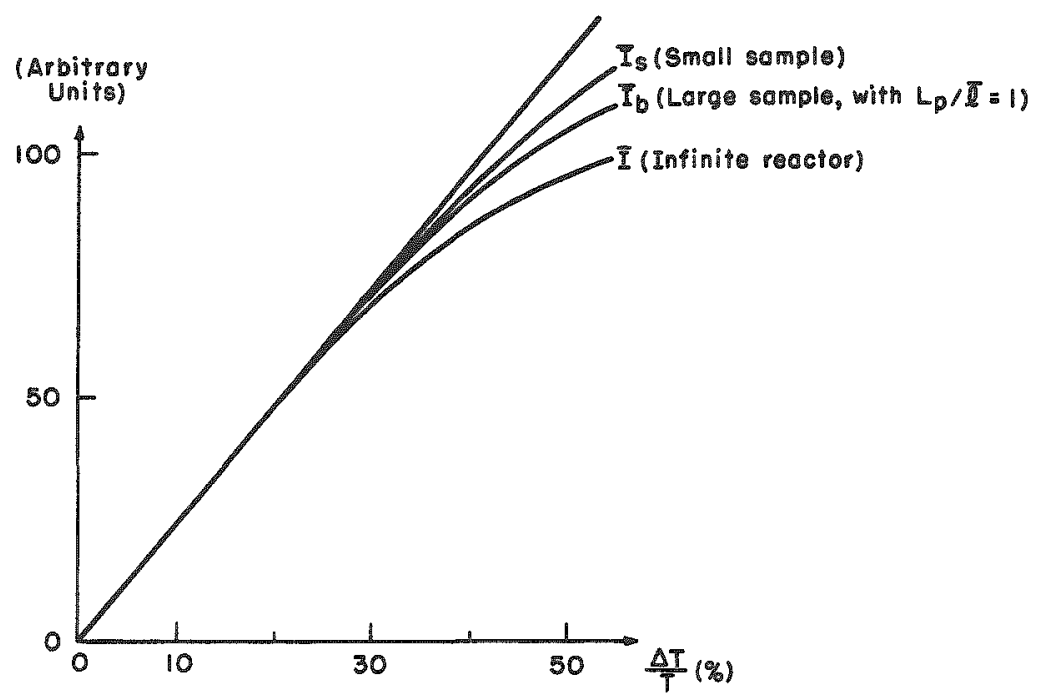

Fig. 4 b

Average Change in Reaction Rate/Unit Volume for $\mathrm{U}^{238} \mathrm{vs}$. $\Delta \mathrm{T} / \mathrm{T} \quad \sigma_{\mathrm{p}}=60 \mathrm{~b}$; $\mathrm{T}=600^{\circ} \mathrm{K}$

curves $\overline{\mathrm{I}}_{\mathrm{S}}$ and $\overline{\mathrm{I}}_{\mathrm{b}}$ are above the curve $\overline{\mathrm{I}}$ (as already expected) and that they are closer to the tangent over a much wider range of $\Delta T / T$. This means that the measurement of the Doppler coefficient with a heated sample in a cold reactor would give a result which is closer to the actual value the smaller the sample. This is, unfortunately, counterbalanced by the fact that the smaller the sample, the larger the temperature difference $\Delta T / T$ must be in order to achieve a measurable effect.

4) Using the curves of Fig. $4 \mathrm{a}$ and $4 \mathrm{~b}$, we have calculated mean relative correction terms averaged over the first 56 resolved resonances of $\mathrm{U}^{238}$ (up to $1 \mathrm{keV}$ ):

$$
\Delta \bar{I}_{\mathrm{S}} / \bar{I} \text { and } \Delta \bar{I}_{\mathrm{b}} / \bar{I}_{\text {. }}
$$

The results, shown in Fig. 5, lead to the following conclusions: in the academic case of a very small sample, the correction term increases rapidly with $\Delta \mathrm{T} / \mathrm{T}$, almost inearly. For $\Delta \mathrm{T} / \mathrm{T}=50 \%$, the relative magnitude of the correction term is about $20 \%$.

Fig. 5

Average Correction Term for $\mathrm{U}^{238}(0-1 \mathrm{keV})$ vs. $\Delta \mathrm{T} / \mathrm{T}$

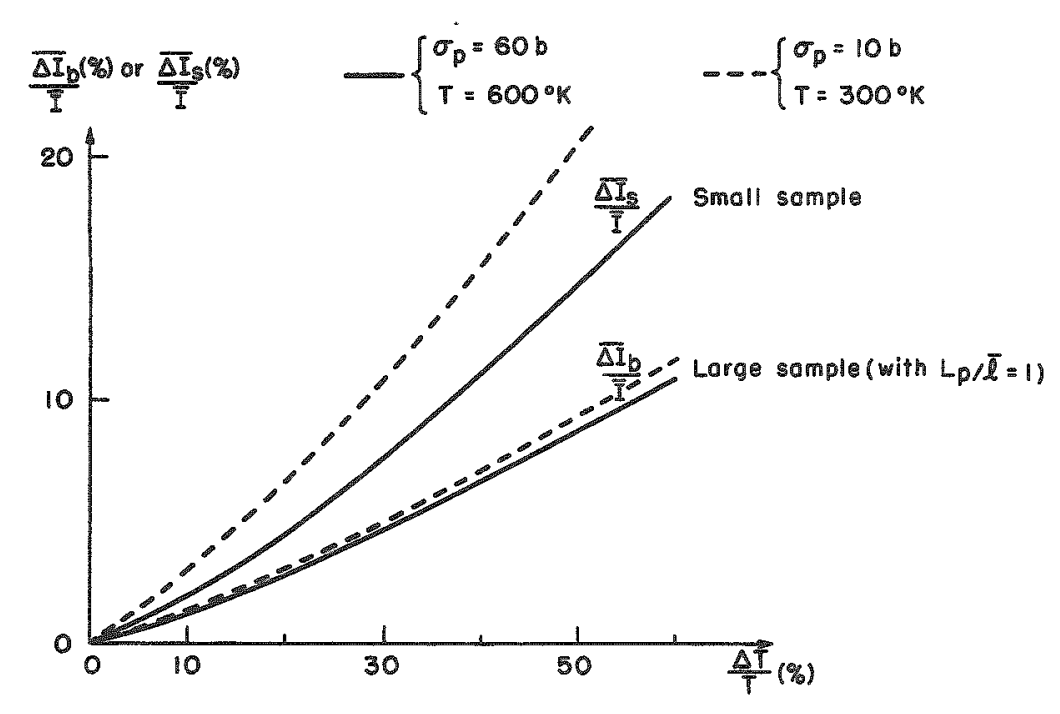


In the practical case of a large sample, the correction term increases with $\Delta T / T$ less rapidly than in the preceding case. The curves of Fig. 5 correspond to $L_{\mathrm{p}} / \bar{l}=1$. To obtain the real correction term of a large sample $\left(L_{\mathrm{p}} / \bar{l} \leq 1 / 5\right)$, these curves must be multiplied by $L_{p} / \bar{l} \leq 1 / 5$. One then obtains, for instance for $\Delta T / T=50 \%$, a correction term equal to $\left(L_{\mathrm{p}} / \bar{l}\right)(10 \%) \leq 2 \%$, which, compared with the other experimental sources of error, represents a negligible error.

5) For higher energies, the error is even less than that calculated in the above. Figure 6 represents the relative correction term $\left(\overline{\Delta I_{b} / I}\right)$ as a function of energy. This quantity is calculated for the mean resonance parameters of $U^{238}$ at each energy. Figure 6 shows that the relative correction terms decrease more or less rapidly as energy increases. Notice that the curves $\left(\overline{\Delta I_{b} / I}\right)$ corresponding to the large sample must again be multiplied by $L_{\mathrm{p}} / \bar{l} \leq 1 / 5$.

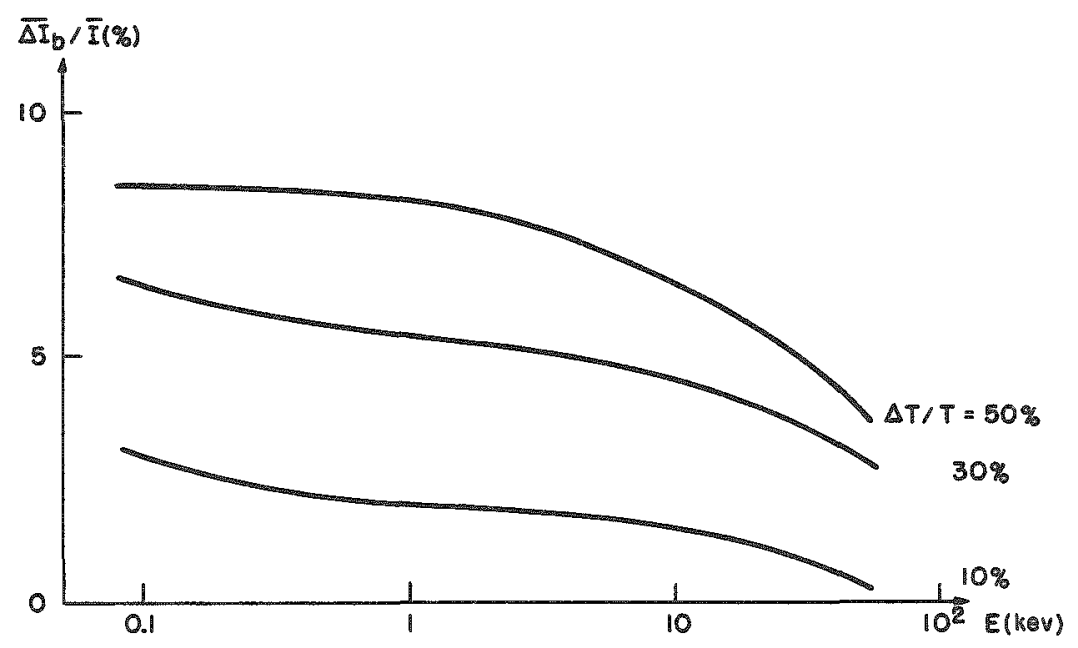

Fig. 6

Correction Term for Average $\mathrm{U}^{238}$ Resonance vs. Energy for a Large Sample (with $L_{\mathrm{p}} / \bar{l}=1$ ) $T=600^{\circ} \mathrm{K} ; \sigma_{\mathrm{p}}=60 \mathrm{~b}$

6) For the same mean resonance parameters of $U^{238}$ (see Fig. 7) gives the magnitude of the correction terms as a function of the reference temperature $T$, at different energies, and for $\sigma_{\mathrm{p}}=60 \mathrm{~b}$

Fig. 7

Correction Term for Average $\mathrm{U}^{238}$ Resonance, at $0.1-1-10 \mathrm{keV}$ for a Large Sample (with $\left.L_{\mathrm{p}} / \bar{l}=1\right)$, and for $\Delta \mathrm{T} / \mathrm{T}=50 \% ; \sigma_{\mathrm{p}}=60 \mathrm{~b}$

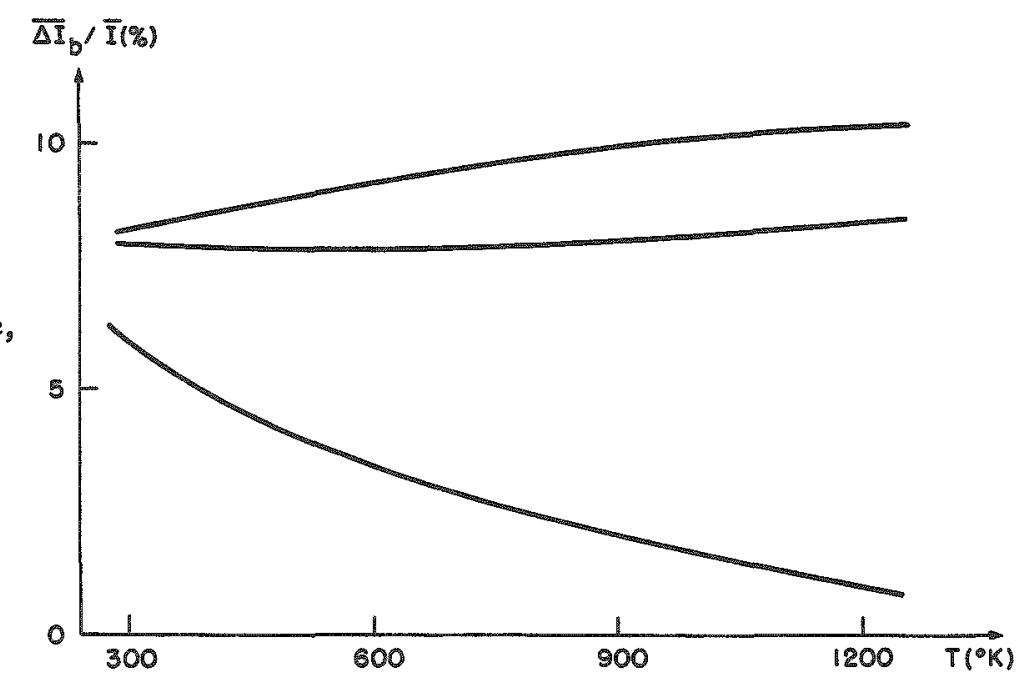


(typical dilute fast reactor) and $\Delta \mathrm{T} / \mathrm{T}=50 \%$. Figure 7 shows that at higher temperatures the correction terms must be of the same order of magnitude, or perhaps a little smaller than the values calculated in the above.

\section{d. Integration over Energy - Overlapping Resonances}

In the energy region of overlapping resonances, one has to compare average values of the reaction rate, i.e.,

$$
\left\langle\frac{\sigma_{a}^{\prime}}{\sigma^{\prime}}-\frac{\sigma_{a}}{\sigma}\right\rangle \text { for the infinite reactor, }
$$

and

$$
\left\langle\frac{\sigma_{\mathrm{a}}}{\sigma^{\prime}}-\frac{\sigma_{\mathrm{a}}}{\sigma}\right\rangle+\frac{\mathrm{L}_{\mathrm{p}}}{\bar{l}}\left\langle\left(\frac{\sigma_{\mathrm{a}}^{\prime}}{\sigma^{\prime}}-\frac{\sigma_{\mathrm{a}}}{\sigma}\right)^{2}\right\rangle \text { for a large sample, }
$$

so that the relative magnitude of the correction term is given by

$$
\frac{L_{\mathrm{p}}}{\bar{l}_{\mathrm{b}}} \frac{\left\langle\left(\frac{\sigma_{\mathrm{a}}^{1}}{\sigma^{1}}-\frac{\sigma_{\mathrm{a}}}{\sigma}\right)^{2}\right\rangle}{\left\langle\frac{\sigma_{\mathrm{a}}^{\prime}}{\sigma^{\prime}}-\frac{\sigma_{\mathrm{a}}}{\sigma}\right\rangle}
$$

where the brackets $\langle>$ imply averaging over energy and over the statistical distribution of resonance widths and spacings. Provided that the resonances are strongly overlapping, so that

$$
\frac{\sigma-\langle\sigma\rangle}{\langle\sigma\rangle}<\langle 1
$$

it is easily found that

$$
\begin{aligned}
& \left\langle\frac{\sigma_{a}^{1}}{\sigma^{\prime}}-\frac{\sigma_{a}}{\sigma}\right\rangle \simeq \frac{\left\langle\sigma_{a}^{2}\right\rangle-\left\langle\sigma_{a}^{2}\right\rangle}{\langle\sigma\rangle^{2}} ; \\
& \left\langle\left(\frac{\sigma_{a}^{1}}{\sigma^{\prime}}-\frac{\sigma_{a}}{\sigma}\right)^{2}\right\rangle \simeq \frac{\left\langle\sigma_{a}^{2}\right\rangle+\left\langle\sigma_{a}^{2}\right\rangle-2\left\langle\sigma_{a} \sigma_{a}^{\prime}\right\rangle}{\langle\sigma\rangle^{2}}
\end{aligned}
$$

Following usual methods, one finds that

$$
\left\langle\sigma_{\mathrm{a}}^{2}\right\rangle=\left(\frac{\pi}{2}\right)^{3 / 2} \frac{\omega}{2 \Delta}\left\{\left\langle\sigma_{a_{0}}^{2} \Gamma^{2}\right\rangle_{\mathrm{w}}+\left\langle\sigma_{a_{0}} \Gamma\right\rangle_{\mathrm{w}}^{2}\left\langle e^{-\frac{\delta^{2}}{2 \Delta^{2}}}\right\rangle_{\mathrm{s}}\right\}
$$




$$
\begin{aligned}
& \left\langle\sigma_{\mathrm{a}}^{\mathrm{i} 2}\right\rangle=\left(\frac{\pi}{2}\right)^{3 / 2} \frac{\omega}{2 \Delta^{\prime}}\left\{\left\langle\sigma_{\mathrm{a}_{0}}^{2} \Gamma^{2}\right\rangle_{\mathrm{w}}+\left\langle\sigma_{\mathrm{a}_{0}} \Gamma\right\rangle_{\mathrm{w}}^{2}\left\langle\mathrm{e}^{\left.-\frac{\delta^{2}}{2 \Delta^{\prime 2}}\right\rangle_{\mathrm{s}}}\right\}_{;}\right. \\
& \left\langle\sigma_{\mathrm{a}} \sigma_{\mathrm{a}}^{\prime}\right\rangle=\left(\frac{\pi}{2}\right)^{3 / 2} \frac{\omega}{\sqrt{2\left(\Delta^{2}+\Delta^{\prime 2}\right)}}\left\{\left\langle\sigma_{\mathrm{a}_{0}}^{2} \Gamma^{2}\right\rangle_{\mathrm{w}}+\left\langle\sigma_{\mathrm{a}_{0}} \Gamma\right\rangle_{\mathrm{w}}^{2}\left\langle e^{\left.-\frac{\delta^{2}}{\Delta^{2}+\Delta^{12}}\right\rangle_{\mathrm{s}}}\right\} .\right.
\end{aligned}
$$

In the preceding expressions, $\omega$ is the mean density of levels, $\Delta$ and $\Delta^{\prime}$ are the Doppler widths at temperatures $T$ and $T^{\prime}$, and $\delta$ is the spacing between a resonance and any other resonance. The brackets $<\rangle_{\mathrm{w}}$ indicate an average over the distribution of resonances widths, and \langle\rangle$_{\mathrm{s}}$ an average over the distribution $\Omega(\delta)$ of the spacings $\delta$.

The relative correction term may then be written as

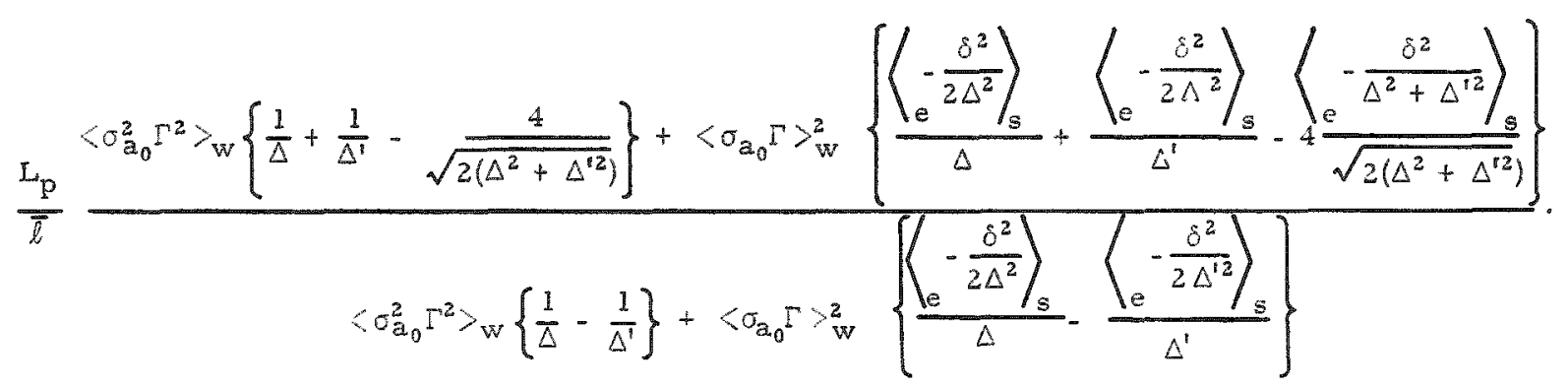

The average over the distribution of spacings can be evaluated analytically only in two extreme cases.

1) If the spacings $S$ between neighbouring resonances are assumed to have a random or Poisson distribution $\left(\chi^{2}\right.$ with $\left.\nu=2\right)$ so that

$$
\Omega(\delta)=\langle S\rangle^{-1}=\text { constant, }
$$

it is found that

$$
\begin{aligned}
& \left\langle e^{-\frac{\delta^{2}}{2 \Delta^{2}}}\right\rangle_{s}=\left(\frac{\pi}{2}\right)^{1 / 2} \frac{2 \Delta}{\langle S\rangle} \\
& \left\langle e^{-\frac{\delta^{2}}{2 \Delta^{12}}}\right\rangle_{s}=\left(\frac{\pi}{2}\right)^{1 / 2} \frac{2 \Delta^{\prime}}{\left\langle S^{\prime}\right\rangle} \\
& \left\langle e^{-\frac{\delta^{2}}{\Delta^{2}+\Delta^{12}}}\right\rangle_{s}=\left(\frac{\pi}{2}\right)^{1 / 2} \frac{\sqrt{2\left(\Delta^{2}+\Delta^{12}\right)}}{\langle S\rangle} .
\end{aligned}
$$


2) If the spacings $S$ are assumed to have a uniform distribution $\left(\chi^{2}\right.$ with $\left.\nu=\infty\right)$ so that

$$
\Omega(s)=\sum_{1}^{\infty} \partial(\delta-n\langle s\rangle)
$$

where $\partial$ is the Dirac $\delta$-function, it is found, provided that $\langle s\rangle / \Delta \ll 1$ (which is the case in the region of strongly overlapping resonances),

$$
\begin{aligned}
& \left\langle e^{-\frac{\delta^{2}}{2 \Delta^{2}}}\right\rangle_{s} \simeq\left(\frac{\pi}{2}\right)^{1 / 2} \frac{2 \Delta}{\langle s\rangle}-1 \\
& \left\langle e^{-\frac{\delta^{2}}{2 \Delta^{12}}}\right\rangle_{s} \simeq\left(\frac{\pi}{2}\right)^{1 / 2} \frac{2 \Delta^{\prime}}{\langle S\rangle}-1
\end{aligned}
$$

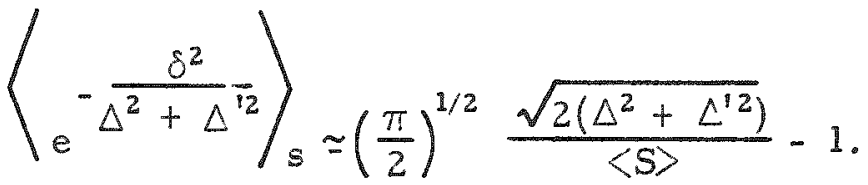

In both cases, it is straightforward to see that the relative correction term reduces to

$$
\frac{L_{p}}{\bar{l}} \frac{\frac{1}{\Delta}+\frac{1}{\Delta}-\frac{4}{\sqrt{2\left(\Delta^{2}+\Delta^{12}\right)}}}{\frac{1}{\Delta}-\frac{1}{\Delta^{1}}}
$$

or

$$
\frac{L_{p}}{\bar{l}} \frac{\sqrt{1+\alpha}+1-2 \sqrt{\frac{1+\alpha}{1+\alpha / 2}}}{\sqrt{1+\alpha}-1}
$$

where

$$
\alpha=\frac{T^{\prime}-T}{T}=\frac{\Delta T}{T}
$$

The above result is surprisingly independent of energy and of the distribution of resonance widths. This is, of course, due to the particular extreme distributions chosen for the spacings. A more realistic distribution ( $\chi^{2}$ with $\nu=10$, for instance) would give rise to dependence on 
energy by means of $\left\langle\sigma_{a_{0}}^{2} \Gamma^{2}\right\rangle \mathrm{w}$ and $\left\langle\sigma_{a_{0}} \Gamma\right\rangle{ }_{\mathrm{w}}^{2}$, but it is believed that the above approximations give the main part of the correction term.

Some numerical values axe given here for $L_{\mathrm{p}} / \bar{l}=1$.

\begin{tabular}{l|r}
$\Delta \mathrm{T} / \mathrm{T}$ & Correction \\
\hline $10 \%$ & $4 \%$ \\
$30 \%$ & $10 \%$ \\
$50 \%$ & $15 \%$
\end{tabular}

These values must be multiplied by $L_{\mathrm{p}} / \bar{l} \leq 1 / 5$. Hence, the relative magnitude of the correction term in the high-energy region is $\leq 3 \%$ for $\Delta \mathrm{T} / \mathrm{T} \simeq 50 \%$. This value is slightly larger than that found for isolated resonances in $\mathrm{U}^{238}$, but it remains of the same order of magnitude.

Assuming that the magnitude of the correction term is a monotonous function of the "overlapping, "we may now conclude that it is negligible for any energy range, and thus in any kind of spectrum. B. Sample of Composition Different from That of the Surrounding

1. General Formulation of the Doppler Coefficient, Including Interference Effects

If one takes into account possible interferences between individual isotopes in the overall Doppler coefficient, the reactivity change due to Doppler broadening in a homogeneous medium is given by a properly weighted integral over volume and over energy of a quantity which we can write as

$-W(E)\left(\frac{\sigma_{8}+\Gamma \gamma / \Gamma \Delta \sigma_{8}}{\sigma+\Delta \sigma_{8}+\Delta \sigma_{9}}-\frac{\sigma_{8}}{\sigma}\right)+\left(\eta(E) W_{f}-W(E)\right)\left(\frac{\sigma_{g}+\frac{\Gamma \gamma+\Gamma f}{\Gamma} \Delta \sigma_{g}}{\sigma+\Delta \sigma_{8}+\Delta \sigma_{9}}-\frac{\sigma_{g}}{\sigma}\right):$

where the symbols have the following meaning:

$W(E)$ worth of a neutron of energy $E$;

$W_{f}$ average worth of a fission neutron;

$\eta(E) \quad \eta$ for the fissile material at energy $E$; 
$\sigma_{;} \sigma_{8} ; \sigma_{9}$ total cross section, including all materials; cross section of the fertile nuclide $\left(\mathrm{U}^{238}\right)$; cross section of the fissile nuclide $\left(\mathrm{Pu}^{239}\right)$, at the initial temperature and at energy $E$ for the reactions of interest.

$\Delta \sigma_{8}, \Delta \sigma_{9}$ variations of $\sigma_{8}$ and $\sigma_{9}$ upon heating.

Expression (1) is based on the usual assumption of constant collision density, which leads to a flux which is inversely proportional to the total cross section. This expression consists of two independent terms: the contributions of the reaction rates in $\mathrm{U}^{238}$ and $\mathrm{Pu}^{239}$, respectively, but the contributions of $\Delta \sigma_{8}$ and $\Delta \sigma_{9}$ cannot be considered separately. These separations can, however, be performed for small temperature changes, in which case expression (1) can be linearized:

$$
\begin{aligned}
& -W(E)\left[\frac{\Gamma \gamma / \Gamma}{\sigma} \frac{\partial \sigma_{8}}{\partial T}-\frac{\sigma_{8}}{\sigma^{2}} \frac{\partial \sigma_{8}}{\partial T}-\frac{\sigma_{8}}{\sigma^{2}} \frac{\partial \sigma_{9}}{\partial T}\right] \Delta \mathrm{T} \\
& +\left[\eta(E) W_{f}-W(E)\right]\left[\frac{\frac{\Gamma \gamma+\Gamma f}{\Gamma}}{\sigma} \frac{\partial \sigma_{9}}{\partial T}-\frac{\sigma_{9}}{\sigma^{2}} \frac{\partial \sigma_{9}}{\partial T}-\frac{\sigma_{9}}{\sigma^{2}} \frac{\partial \sigma_{8}}{\partial T}\right] \Delta T .
\end{aligned}
$$

After the volume and energy integration, and after dividing by $\Delta T$, one obtains for the Doppler coefficient six terms corresponding to the six terms of (2), which we write as

$$
\text { Doppler coefficient }=A_{8}+A_{88}+A_{98}+A_{9}+A_{99}+A_{89}
$$

where $A_{8}$ and $A_{9}$ are the "cross-section" components of the Dopplex coefficient, respectively, for $\mathrm{U}^{238}$ and $P \mathrm{u}^{239}$. The $\mathrm{A}_{i j}{ }^{\prime}$ s represent spectral components; the first index refers to the heated nuclide, and the second index refers to the nuclide for which the reaction rate is considered. Hence, $A_{88}$ and $A_{99}$ represent the usual spectral components, neglecting interference. The quantity $A_{89}$ is the interference component evaluated first by Codd and Collins. Ags is another interference component which is thought to be negligible, but which was included for the sake of completeness.

Thus, the Doppler coefficient, which relates only to small temperature changes, is the sum of six components.

What can be considered as the contributions to the Doppler coefficient of $\mathrm{U}^{238}$ and $\mathrm{Pu}{ }^{239}$, respectively? If one chooses the reaction rate as the criterion, as could be suggested by (1) or (2), one should use

$$
\left.\begin{array}{ll}
\text { for } U^{238}: & A_{8}+A_{88}+A_{98} \\
\text { for } P^{239}: & A_{9}+A_{99}+A_{89}
\end{array}\right\} \text {. }
$$


should use

If one chooses the heated nuclide as the criterion, one

$$
\left.\begin{array}{ll}
\text { for } U^{238}: & A_{8}+A_{88}+A_{89} \\
\text { for } P u^{239}: & A_{9}+A_{99}+A_{98}
\end{array}\right\} \text {. }
$$

According to definition (4), the interference term $A_{89}$ studied by Codd and Collins is a plutonium coefficient, and according to definition (5) it is a uranium coefficient. We tend to prefer definition (5), for reasons which will become apparent in the next paragraph.

\section{Application}

As was seen in $A$, when the sample has the same composition as the surrounding medium, one measures the total Doppler coefficient,

$$
\text { (Doppler coefficient) } t=A_{8}+A_{88}+A_{98}+A_{9}+A_{99}+A_{89}
$$

whatever the size of the heated sample.

If we now consider an imaginary situation in which, in a homogeneous medium, only the atoms of $U^{238}$ are heated, one would measure the $\mathrm{U}^{238}$ contribution to the total Doppler coefficient (DC):

$$
(D C)_{8}=A_{8}+A_{88}+A_{89}
$$

and similarly for $\mathrm{Pu}^{239}$

$$
(\mathrm{DC})_{9}=\mathrm{A}_{9}+\mathrm{A}_{99}+\mathrm{A}_{98}
$$

This corresponds to the definition (5) of the preceding paragraph.

If in a homogeneous medium containing both $U^{238}$ and $P u^{239}$, plus other materials, a very small sample, or many very small samples, of $U^{238}$ were heated, one can easily conclude from a reasoning similar to that used in A. I., that the measured reactivity change, when normalized to the heated amount of $\mathrm{U}^{238}$, would give (DC) 8 , as given by (2). Similarly, one would measure (DC), by heating samples of $\mathrm{Pu}^{239}$.

These conclusions are rigorously true only for small temperature variations and for very small samples.

Let us now examine the implications of these two conditions. 


\section{a) Small Temperature Variation}

As was discussed in $B .1$, the components $(D C)_{8}$ and (DC), can be linearly combined only in the case of small temperature variations. However, by using a reasoning similar to that in A.2, one may expect that even for a $\triangle T$ of several hundred degrees, the errors in the measured values of $(D C)_{8}$ and $(D C)_{9}$ would not exceed a few percent.

\section{b) Very Small Samples}

It is clear that the sample(s) would be small enough if a neutron which interacted with a sample would have a probability close to unity of suffering its next collision in the surrounding homogeneous medium.

It is not immediately obvious what size of sample can be tolerated, since the mean free path varies from a fraction of a millimeter to many centimeters for neutrons in the peak or the wings of the resonances. Since it does not seem practical to heat many samples, the sample should be large enough to yield a measurable reactivity change. We are presently investigating this problem of allowable sample size by theoretical means. This problem might also be tackled experimentally by using samples of different sizes.

Qualitatively, one can say that if a not-very-small sample of $\mathrm{U}^{238}$ was heated, the contributions to $\mathrm{A}_{88}$ coming from the peaks of the $U^{238}$ resonances would be slightly enhanced, while the corresponding contributions to $A_{89}$ would be slightly decreased. These errors for $A_{88}$ and $A_{89}$ are probably negligible except at those energies at which the peaks of the resonances of $\mathrm{Pu}^{239}$ and $\mathrm{U}^{238}$ coincide. As was shown by Codd and Collins, these energies give positive contributions to $A_{89}$.

Errors due to perturbations of the macroscopic flux, resulting mainly from the lack of fissions in the sample without plutonium, do not become important unless the sample is very large, since the neutrons diffuse appreciably in their slowing down to the resonance region. Anyway, these errors could be corrected by an adequate normalization of the experimental results.

samples.

Similar conclusions can be reached for plutonium

We repeat the main conclusion, which is only tentative at this time: Experiments with samples of pure $\mathrm{U}^{238}$ or $\mathrm{Pu}^{239}$ probably give good measurements of, respectively, $(D C)_{8}$ and $(D C)_{9}$, except for the contributions of the energies at which the mean free paths for the reactions of interest in both the fertile and fissile nuclides are not large compared 
with the linear dimensions of the sample. It is indeed only for these energies that the heterogeneity introduced by the sample changes the competition of both nuclides for the neutrons which have collided with the hot sample. It remains to be investigated how large these errors are relative to the total effect.

The measurements reported at this Conference in papers VIII- 3 and VIII-4 are of the type discussed in this section. Hence, they probably yield good values of the separate contributions of the fertile and the fissile nuclides to the Doppler coefficient, except for the possible sources of errors which were mentioned.

It is important to note that with a sample of fertile material the interference effect $\mathrm{A}_{89}$, as discussed by Codd and Collins, would be included, while with a sample of fissile material it is an eventual interference effect Agg $_{9}$ which would be included.

One last comment: while the considerations about very small samples may have only academic interest when the samples have the same composition as the surrounding medium, they are, of course, essential when the samples have a different composition, because in that case the samples must be very small.

\section{Experimental Techniques}

The accuracy of the reactivity method can be improved by the oscillation technique, in which one can either oscillate the temperature of the sample or periodically interchange a hot and a cold sample. The attainable accuracy has been discussed by many authors, and various means have been suggested to minimize the expansion or its effect on the reactivity. This work will not be reviewed here, but only a few aspects of the experimental techniques will be discussed.

\section{Temperature Dependence of the Doppler Coefficient}

As was shown in the preceding sections, the Doppler coefficient at temperature $T_{0}$ can be determined by heating a sample by an amount $\triangle T$ above the temperature $T_{0}$ of the surrounding medium, or by oscillating the sample temperature around $T_{0}$. It was also shown that the reactivity variation so obtained, when normalized to the volume of the heated sample, departed less from linearity than if the whole core was heated. Hence, the temperature dependence of the Doppler coefficient can only be obtained if the reference temperature $T_{0}$ is also varied. There is, of course, no need to heat the whole core. It is sufficient to heat a buffer zone thick enough so that the sample interacts only with a material in which the microscopic spectrum is unaffected by the temperature of the rest of the core. It should be noted that thermal expansion of the buffer zone is not a source of error. 
It seems technologically possible to measure in such a way the Doppler coefficient up to temperatures of the order of $1000^{\circ} \mathrm{C}$. This is probably sufficient, since more than half of the total Doppler investment is used up at that temperature.

\section{Minimization of the Expansion Coefficient}

Many methods have been suggested to minimize the expansion or its effect on the reactivity. We suggest a more radical method that consists in experimenting on a medium with $k_{\infty}=1$. This can be achieved by choosing an adequately low enrichment or by adding boron to a medium representative of the core of the power reactor which is being studied. The assembly should, of course, be made critical with a suitable driver zone.

The fact that such an experiment does not yield directly the is othermal Doppler coefficient of some power reactor is, in our opinion, not a major disadvantage. Indeed, the main objective of a Doppler experiment, as of most integral experiments, is to yield a measurement which can be compared with a calculated result and not to yield a quantity which can be directly used as such.

\section{Assembly Containing No $\mathrm{U}^{238}$}

It would be highly desirable to perform an experiment with a dilute, soft-spectrum, plutonium as sembly containing no $\mathrm{U}^{238}$, for instance plutonium diluted with graphite.

The following quantities, pertaining to that assembly, could then be measured:

if a plutonium sample is heated, $A_{9}+A_{99}$

if a very small $\mathrm{U}^{238}$ sample is heated, $\mathrm{A}_{89}$.

\section{Determination of the Spectrum}

Whenever the Doppler coefficient is measured for a medium not representative of a power reactor, as in sections 2 or 3 above, the objective is to obtain an experimental quantity which can be compared with its calculated value, so that the adequacy of the theory or of the basic data can be ascertained. The correlation of theory and experiment would, of course, be greatly facilitated if the spectrum could be measured. It is, therefore, of the utmost importance to be able to measure the spectrum in the energy range which is most important for the Doppler effect, i.e., from $10 \mathrm{keV}$ down to about $100 \mathrm{eV}$. As of now, there exists no method to measure spectra in that range. 


\section{Heterogeneity}

It was always assumed so far that the medium is perfectly homogeneous. For reasons of flexibility the requixed core composition in a critical assembly is obtained by the juxtaposition of individual simulation pieces having different compositions. Thus, there will be a greater heterogeneity in a critical assembly than in a power reactor. We are presently investigating this problem. It may be necessary to use especially made homogeneous material, or smaller simulation pieces than in the rest of the assembly, for the sample and eventually also for the buffer zone.

III. Activation Method

A. General Formulation of the Activation, Including Interference Effects

If a homogeneous medium is heated uniformly the changes in reaction rates at a given energy are given by:

for captures in the fertile material,

$$
\frac{\sigma_{8}+\Gamma \gamma / \Gamma \Delta \sigma_{8}}{\sigma+\Delta \sigma_{8}+\Delta \sigma_{9}}-\frac{\sigma_{8}}{\sigma}
$$

for fissions in the fissile material,

$$
\frac{\sigma_{f_{g}}+\Delta \sigma_{f_{9}}}{\sigma+\Delta \sigma_{8}+\Delta \sigma_{9}}-\frac{\sigma_{f_{9}}}{\sigma}
$$

for captures in the fissile material,

$$
\frac{\sigma_{c q}+\Delta \sigma_{c q}}{\sigma+\Delta \sigma_{8}+\Delta \sigma_{9}}-\frac{\sigma_{c q}}{\sigma}
$$

where

$$
\Delta \sigma_{9}=\Delta \sigma_{\text {fq }}+\Delta \sigma_{\text {cq }}+\Delta \sigma_{\text {sq }}
$$

and $f$ and $c$ refer to fission and capture, respectively.

For small temperature changes these expressions can again be linearized, and one obtains for the change in reaction rates per unit temperature increase

$$
\mathrm{R}_{8}=\mathrm{B}_{8}+\mathrm{B}_{88}+\mathrm{B}_{98}
$$




$$
\begin{aligned}
& R_{f 9}=B_{f 9}+B_{f 99}+B_{f 89} \\
& R_{C 9}=B_{C 9}+B_{C 99}+B_{C 89 .} \\
& B . \quad \text { Application }
\end{aligned}
$$

Assume that one can measure the individual reaction rates and their relative changes with reasonable accuracy, for instance by measuring the activity of the reaction products after some time of constant power operation. Assume a homogeneous medium in which a very small sample of $U^{238}$ is inserted.

1. If the sample is heated while the surrounding medium remains cold, the change in sample activity would be given by $\underline{B}_{8}$, which is the "cross-section" component of $R_{8}$. It was indeed demonstrated in II. A.1. that, for a very small sample, the spectral components ( $B_{88}$ and $B_{98}$ ) would be missing in the reaction rate of the sample.

2. If the surrounding medium - or an adequately thick buffer zone - is heated while the sample remains cold, the reaction rate of the sample would also change, but in this case only the spectral components $B_{88}+B_{98}$ would be measured.

3. If both the surrounding medium and the sample are heated, the change in reaction rate in the sample would be given by

$$
R_{8}=B_{8}+B_{88}+B_{98} \text {. }
$$

Note that the interference term is $B_{98}$, while in the reactivity method with a $U^{238}$ sample the interference term was $A_{89}$.

In principle, similar measurements can be made with a plutonium sample. By measuring the activity of the fission products one could measure

$$
B_{f 9 ;} \quad B_{f 99}+B_{f 89} ; \quad B_{f 9}+B_{f 99}+B_{f 89} .
$$

If the capture rate in plutonium could be determined, one could measure

$$
\mathrm{B}_{\mathrm{C} 9 ;} \quad \mathrm{B}_{\mathrm{C} 99}+\mathrm{B}_{\mathrm{C} 89} ; \quad \mathrm{B}_{\mathrm{C} 9}+\mathrm{B}_{\mathrm{C} 99}+\mathrm{B}_{\mathrm{C} 89} \text {. }
$$

However, this is probably an impossible task. 


\section{Experimental Techniques}

Practical application of the activation method may be extremely difficult, particularly in view of the relatively small variations of the reaction rates which should be measured. Such experiments will probably be shortly undertaken in the mixed spectrum critical experiment at General Electric Vallecitos.*

We feel that their great potential warrants a considerable experimental effort, even though success is not assured.

We present here some considerations on the experimental techniques.

\section{Sample Size}

In the activation method the samples should always be very small. This is not a disadvantage, since the specific activation is the quantity to be measured. In the reactivity method the use of a very $s$ mall sample is a disadvantage, since the reactivity change is directly related to the volume of the sample.

\section{Size of the Buffer Zone}

The thickness of the buffer zone should be such that practically all neutrons interacting with the sample at the resonance energies suffered their last collision in a part of the buffer zone whose microscopic spectrum is not affected by the temperature of the surrounding medium. It is, of course, dependent on the composition of the core.

\section{Experimental Procedure}

In view of the smallness of the relative changes in the reaction rates to be measured, it is essential to use a reference sample so that fluctuations in the power level of the assembly during the course of an experiment are automatically corrected for. For example, in the measurement of $\mathrm{B}_{8}$ one should use two identical $\mathrm{U}^{238}$ samples, heater included, at symmetrical locations. A first experiment is performed by heating one of the samples, and the ratio of the activations of the two samples is then measured. In a second experiment the other sample is heated, while the first one remains cold, so that systematic errors corresponding to the two locations can be corrected for.

*G. T. Petersen, personal communication. 


\section{Measurement of the Reaction Rates}

Herein lies, of course, the great difficulty of this method, since the relative changes in the reaction rates are in practice of the order of a few percent. Radiochemical techniques probably cannot be made accurate enough. The reaction rate can probably best be measured by the activation of the capture products of $\mathrm{U}^{238}$, and of the fission products of $\mathrm{Pu}^{239}$. It is very doubtful that the capture rate in $\mathrm{Pu}^{239}$ could be measured with adequate accuracy.

\section{Means of Improving the Sensitivity of the Method}

Although the total reaction rate varies by a few percent, the relative change of the fraction of the reaction rate which corresponds to the energies for which the Doppler effect is largest (say below $10 \mathrm{keV}$ ) is greater by a factor of about five. Thus the accuracy of the method could be improved if the spectrum was artificially softened so as to minimize the fraction of neutrons above $10 \mathrm{keV}$. We are presently investigating this possibility.

\section{Assembly Containing No $\mathrm{U}^{238}$}

This could be, for instance, an assembly of plutonium diluted with graphite. The following quantities could then be measured:

with a Pu sample,

$B_{f 9,}$ if the sample is heated;

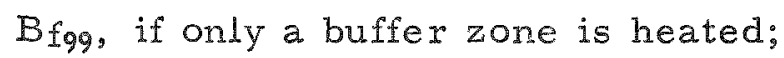

$B_{f_{9}}+B_{f 99,}$ if sample and buffer are heated;

with a $U^{238}$ sample,

$B_{98}$ if a buffer zone is heated.

7. Other Remarks

a. Since reaction rates, and not reactivities, are directly measured, thermal expansion of the sample has no effect on the measurement.

b. Note also that for the same reason the neutron importance plays no role in the interpretation of such an experiment. 
c. Such an experiment is not necessarily performed in a critical assembly. An exponential assembly may be adequate, or even any experiment in which there is a constant or slowly varying slowing-down source.

d. As in the reactivity-type experiment, it is possible to measure the temperature dependence of the different $B^{\prime} s$ by heating the buffer zone.

IV. Conclusion

A critical facility certainly is a most appropriate tool for experimental determinations of the Doppler coefficient. Because of its great flexibility of composition, a critical facility can yield the value of the Doppler coefficient over a wide range of compositions and, as was discussed, can also yield individual components of the total Doppler coefficient. If the activation method can be made to work, there would be obtained separately the "cross section" and spectral components of the total Doppler coefficient. The knowledge of these individual components will certainly facilitate the interpretation of the experiments, particularly if some discrepancy is observed between calculated and experimental results.

The temperature dependence of the Doppler coefficient can also be measured, by heating a buffer zone to the adequate temperature. It might, however, be technologically difficult to exceed temperatures of about $1000^{\circ} \mathrm{C}$. This is not a major disadvantage, since more than half of the total Doppler investment is used up at that temperature, and since the temperature dependence experimentally determined up to that temperature can be used as a basis for extrapolation above that temperature.

In a critical assembly one can also measure the influence of the presence of sodium on the Doppler coefficient. This last point is certainly of the utmost importance, particularly since sodium void coefficients are likely to become positive in some cases, and since loss of sodium may strongly affect the Doppler coefficient.

\section{References}

1. G. Goertzel, and C. Klahr, NDA $14-127$ (June 29, 1956).

2. See, for example, K. M. Case, F. de Hoffmann, and G. Placzek, Introduction to the Theory of Neutron Diffusion, U. S. Govt. Printing Office, Washington 25,D.C.(1953). 


\title{
MEASUREMENTS OF THE DOPPLER COEFEICIENT IN \\ LARGE FAST POWER REACTORS USING A \\ FAST CRITICAL ASSEMBLY AND AN \\ EXPERIMENTAL FAST REACTOR
}

PART II. USE OF RAPSODIE FOR THE MEASUREMENT OF THE DOPPILE COEFFICIENT OF FAST POWER REACTORS

\author{
J. Ladet, I. Puig, and L. Stevens \\ EURATOM CEA (Cadarache) \\ Bouches du Rhone, France \\ (Paper presented by Mr. F. Storrer)
}

\section{Generalities}

The necessity of an experimental program for the measurement of Doppler coefficients comparable with those prevailing in large fast power reactors has become the general concern of the teams responsible for the design of this type of reactor in various countries.

SEFOR and FARET are the first materializations of that need and may rightly be considered as important stages of the American program.

As far as the French program is concerned, RAPSODIE, which is to diverge in 1966, is a perfect instrument for such experiments and offers the possibility of yielding results in due course, i.e., before detailed projects of prototype power reactors are undertaken.

Measurements would be done on a 400-1iter core to be placed in RAPSODIE. This dimension results from a compromise between technological possibilities and the necessity for reactor physics conditions similar to those of power reactors.

We may briefly point out that, from the mechanical point of view, the modifications necessary to the insertion of a 400-1iter core into RAPSODIE have been provided for in the building plans (high-pressure coolant zone of large dimension, extension of the control rod stroke, etc.).

The core would consist of approximately 200 assemblies, i.e., about 4 times as many as in the initial one, and each assembly would contain fissile material over the entire active height (900 mm), which would remain unchanged. There would be no upper and lower axial blankets.

The main criteria which have led the design of such a core are: 
1) A Reactor Physics Criterion:

Obtaining a high fuel percentage, as high as $68 \%$, so as to get a fertile to fissile ratio comparable to that of large power reactors (roughly 7 to 8 ).

\section{2) A Thermal Criterion:}

Driving towards thermal conditions comparable to those in large reactors, e.g., for the hottest rod:

central temperature of $2500^{\circ} \mathrm{C}$;

cladding temperature of $600^{\circ} \mathrm{C}$;

thermal conductivity of the oxide of $55 \mathrm{~W} /{ }^{\circ} \mathrm{C}$.

3) A Dynamics Criterion:

Minimizing the effects due to axial fuel expansion and to the radial core expansion in order to isolate the Doppler effect to a certain extent.

In order to reduce power level, it is not possible to keep a power reactor geometry. It is necessary to increase the rod diameter and to admit a certain restriction of central temperatures.

The only solutions liable to satisfy the above mentioned conditions are

7 rods per assembly (15.4-mm diameter);

1 rod per assembly (44.4-mm diameter).

Vertical partitioning of fuel is contemplated, so as to avoid additive expansion of the rod, the cladding being kept at a constant temperature during measurements, as explained later.

The fuel pellets would be placed on refractory metal "dishes" fitted to the cladding. Figures 1 and 2 show diagrammatically two possible solutions. The first offers the advantage of using less structural materials, but the more elaborate implementation is a drawback. Actually there would be 5 pellets per "dish," and 9 "dishes" per fuel rod. The shape of the pellets (see Fig. 3) would favour a free expansion of the fuel centerline. 


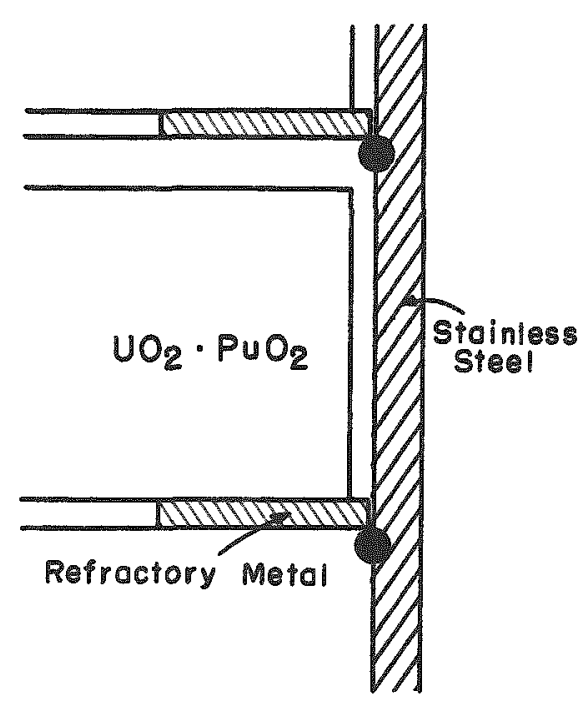

Fig. 1. Fuel Pellet Configuration in Cladding

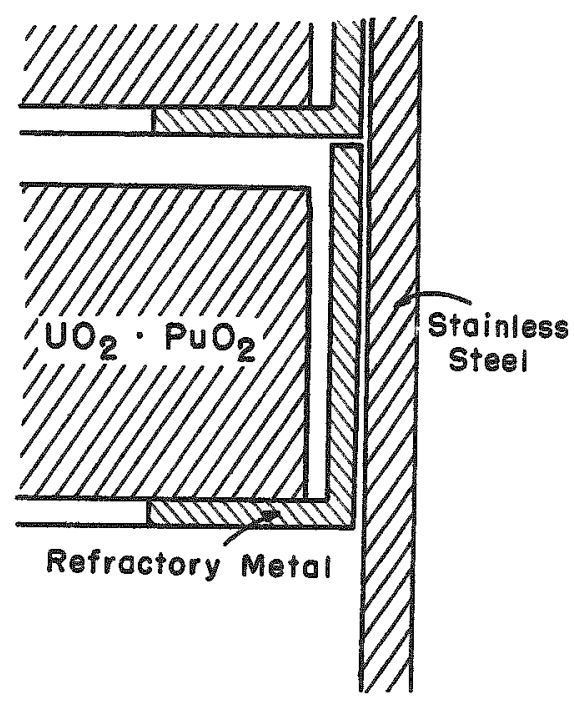

Fig. 2. Alternative Fuel Configuration in Cladding

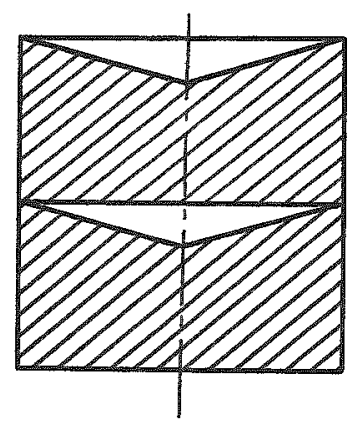

Fig. 3. Pellet Shape

The table below summarizes the thermal specifications for the 7 and 1 -rod solutions.

Core of "7 Rods"

Core of "1 Rod"

Total reactor power (MW)

Average specific power $\left(\mathrm{W} / \mathrm{cm}^{3}\right)$

30

6.5

75

17.5

Conductivity integral at the

hot spot $(W / \mathrm{cm})$

38

62

Coolant flow $\left(\mathrm{m}^{3} / \mathrm{h}\right)$

720

720

Temperature rise of coolant in

core $\left({ }^{\circ} \mathrm{C}\right)$

140

28

Specific power $(\mathrm{W} / \mathrm{cm})$

240

380

Thermal Resistivity

$\mathrm{R}_{\mathrm{C}}$ of sodium film $\left({ }^{\circ} \mathrm{C}-\mathrm{cm} / \mathrm{W}\right)$

0.021

0.008

$\mathrm{R}_{\mathrm{g}}$ of sodium cladding $\left({ }^{\circ} \mathrm{C}-\mathrm{cm} / \mathrm{W}\right)$

0.058

0.034

$R_{j}$ of sodium clearance-cladding-fuel

$\left({ }^{\circ} \mathrm{C}-\mathrm{cm} / \mathrm{W}\right)$

0.62

0.215

$\mathrm{R}_{\mathrm{T}}$ of sodium fuel $\left({ }^{\circ} \mathrm{C}-\mathrm{cm} / \mathrm{W}\right)$

The table calls for the following comments:

The "1-rod" solution has the advantage of greater versatility: obtaining very high central temperatures $\left(2100^{\circ} \mathrm{C}\right)$ with low total powers $(6.5 \mathrm{MW})$. 
Even if the one-rod solution was chosen, it might be interesting to do another series of measurements using $15.4-\mathrm{mm}$ rods. In this way it would be possible to frame the results obtained by SEFOR, (23.3-mm diameter) which is useful in view of the small number of experimental measurements on Doppler effect.

Summarizing the conditions required that the Doppler effect be prevailing, we have:

Either a high flow rate (indeed the ratio of the coolant to Doppler effect is inversely proportional to flow) or a flow to power ratio adjusted so as to keep the coolant temperature constant.

Large-diameter rods, in order to make the relative rise of fuel temperature larger than the coolant temperature rise.

A high heat resistivity. This factor is difficult to control, but gets less important as rod diameter increases.

The danger coefficients for the "1-rod" core were calculated by the method of $k$ eff difference between 2 states of the reactor $(2$-dimensional ALCI code). The model divides the core into 6 zones of equal height and simulates the upper and the lower transition zones and radial blanket (see Fig. 4).

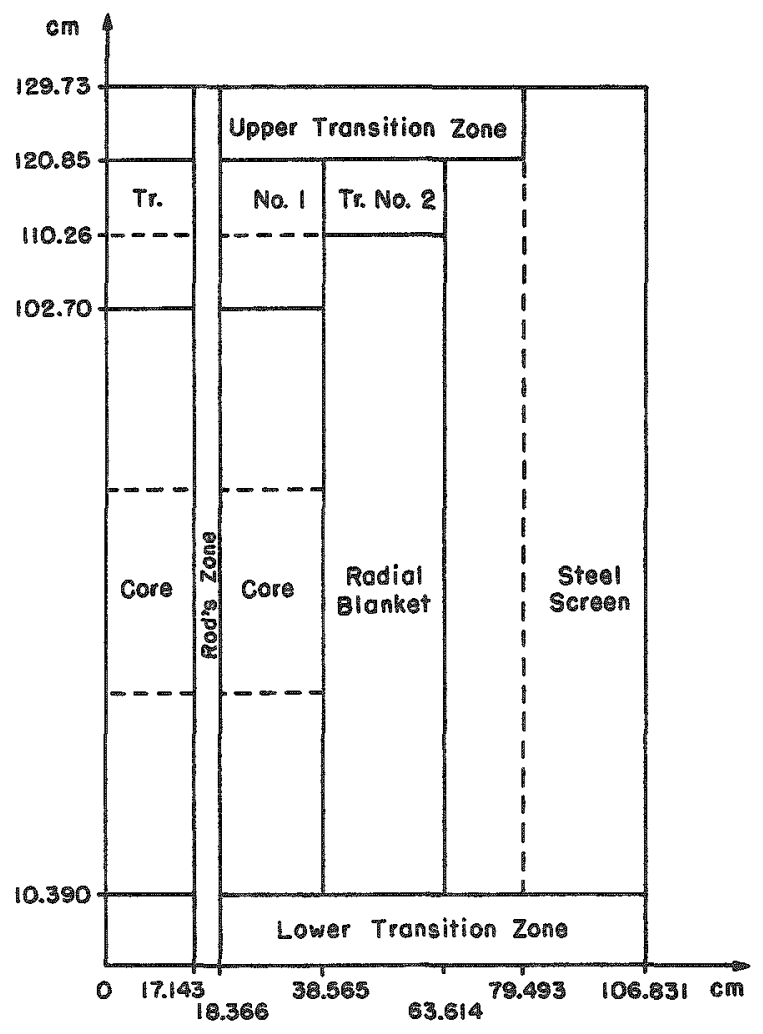

Fig. 4

RAPSODIE; 400-liter, 1-Rod Core. Model Used for Computation at $525^{\circ} \mathrm{C}$ (Fuel at $1000^{\circ} \mathrm{C}$ ) 
Coefficients for the "7-rod" core were not calculated, but were taken as equal to the "1-rod" coefficients.

The table below summarizes the values of the isothermal reactivity coefficients. The table shows that the axial cladding-expansion coefficient is much larger in value than either the sodium coefficient or the Doppler coefficient. In the measurement methods to be used, it will be necessary to try and minimize this effect by keeping the sodium and cladding temperatures as constant as possible.

\begin{tabular}{lc}
\hline Temperature Coefficients, pcm $/{ }^{\circ} \mathrm{C} *$ & "1- and 7 -Rod" Cores \\
\hline Doppler coefficient $\left(\mathrm{K}_{\mathrm{T}}\right)$ & -0.29 \\
Coefficient of Sodium $\left(\mathrm{K}_{\mathrm{V}}\right)$ & -0.16 \\
Axial cladding expansion coefficient $\left(\mathrm{K}_{\mathrm{G}}\right)$ & -0.53 \\
\hline
\end{tabular}

$* 1 \mathrm{pcm} \equiv 10^{-5} \mathrm{k}$.

It should also be pointed out that the radial blanket contribution to the sodium coefficient $\left(-0.08 \mathrm{pcm} /{ }^{\circ} \mathrm{C}\right)$ is important and stands for half the value of that coefficient.

Finally, the table does not show the value of the overall coefficient due to radial expansion of the core. This effect will also be minimized by keeping sodium temperature constant during the measurements.

It must be noted that the measurements which are to be detailed presently are gross measurements, which may cover the entire range of fuel temperature.

Two Doppler coefficient measurement methods will be described: the "static method" and the "oscillation method."

II. Static Method

This paragraph describes the principle of the static method and considers the various exrors which may influence the measurement.

\section{A. Principle}

The "static method" consists in going from a stable power level $\mathrm{q}_{1}$ to another stable power level $\mathrm{q}_{2}$ by inserting a reactivity $\delta \mathrm{k}$ while keeping the coolant inlet temperature constant. The reactor power coefficient $\mathrm{K}_{\mathrm{p}}$, in $\mathrm{pcm} / \mathrm{MW}$, is then

$$
K_{p}=\frac{\delta k}{q_{1}-q_{2}}
$$


LADET et al.

The coefficient $K_{p}$ is composed of the power coefficients due

to fuel:Dopplex effect and fuel axial displacement;

to cladding expansion;

to coolant;

to structures.

As said earlier, parasitic effects will have to be minimized in order to isolate the Doppler effect. Thus:

The coolant temperature gradient will be kept constant by regulation of the sodium flow.

The cladding thermal resistance should be kept much smaller than the fuel thermal resistance.

The arrangement of the fuel inside cladding should eliminate axial displacements of the fuel.

Large structure coefficients should also be avoided by suitable mechanical design.

We assume that a series of measurements will be made between $1 \mathrm{MW}$ and the nominal reactor power $\mathrm{q}_{2}$. The successive power increases, $\Delta q=q_{2}-q_{1}$, correspond to an integral variation of $4.35 \mathrm{~W} / \mathrm{cm}$; this corresponds to a $125^{\circ} \mathrm{C}$ rise in the temperature drop within the fuel. These are average values for the core. The table below shows the characteristics for one measurement:

Core of 7 Pins Core of 1 Pin

Nominal power $(\mathrm{MW}) *$

49

7

Conductivity integral average variation

for one measurement $(W / \mathrm{cm})$

4.4

4.4

Temperature-drop average variation

within fuel for one measurement $\left({ }^{\circ} \mathrm{C}\right)$

125

125

Power variation for one measurement

(MW)

Reactivity to be inserted for one

measurement (pcm)

$\sim 50$

$\sim 25$

* Power corresponding to a $30-\mathrm{W} / \mathrm{cm}$ average conductivity integral. 
From a series of measurements we obtain a Doppler power coefficient curve as a function of the conductivity integral. It is to be noted that the fuel surface temperature will vary from one measurement to the other, although coolant temperatures are kept constant. This is due to the resistance of the clearance between fuel and cladding.

The following calculations were done with use of the abovementioned values and the core characteristics given in the section titled "Generalities." Parasitic effects due to the structures were considered to be negligible.

B. Calculation of the Power Coefficient and the Inherent Errors

Let

n

L

$q_{1}$ and $q_{2}$

$\mathrm{R}_{\mathrm{T}}$

$R_{J_{Z}}, R_{G_{Z}}, R_{C_{Z}}$

$\mathrm{RTG}_{2}, \mathrm{RGU}_{Z}$

$\mathrm{K}_{\mathrm{T}}$

$\mathrm{K}_{\mathrm{G}}$

$\mathrm{K}_{\mathrm{U}}$

$\delta k$

$\overline{\mathrm{U}}_{\mathbf{Z}}$

We then get the number of rods in the core

the core height, cm

the initial and final power levels for one measurement, $W$ the thermal resistance of fuel per unit length at a power level $q_{z},{ }^{\circ} \mathrm{C}-\mathrm{cm} / \mathrm{W}$

the thermal resistances of the clearance between fuel and cladding, of cladding, and of sodium film per unit length at a power level $\mathrm{q}_{z},{ }^{\circ} \mathrm{C}-\mathrm{cm} / \mathrm{W}$

the overall fuel-to-cladding and cladding-to-coolant thermal resistances per unit length at a power level $q_{z}$, ${ }^{\circ} \mathrm{C}-\mathrm{cm} / \mathrm{W}$

the overall isothermal temperature coefficient of the fuel, $\mathrm{cm} /{ }^{\circ} \mathrm{C}$

the overall isothermal temperature coefficient of the cladding, $\mathrm{cm} /{ }^{\circ} \mathrm{C} . \mathrm{K}_{\mathrm{G}}$ includes the term due to cladding radial expansion and that due to axial expansion, which produces displacement of fuel.

the overall isothermal temperature coefficient of the coolant, $\mathrm{cm} /{ }^{\circ} \mathrm{C} . \mathrm{K}_{\mathrm{U}}$ includes not only the coolant effect of the core, but also that of the radial blanket and of the transition zones.

the reactivity necessary to go from $q_{1}$ to $q_{2}, \mathrm{~cm}$

the average coolant temperature at a power level $\mathrm{q}_{\mathrm{z}},{ }^{\circ} \mathrm{C}$. 


$$
\begin{aligned}
\delta k= & -K_{T}\left\{\bar{U}_{2}-\bar{U}_{1}+\left[\left(\frac{1}{2} R_{T_{2}}+R_{J_{2}}+R_{G_{2}}+R_{C_{2}}\right) \frac{q_{2}}{L n}-\left(\frac{1}{2} R_{T_{1}}+R_{J_{1}}+R_{G_{1}}+R_{C_{1}}\right) \frac{q_{1}}{L n}\right]\right\} \\
& -K_{G}\left\{\bar{U}_{2}-\bar{U}_{1}+\left[\left(\frac{1}{2} R_{G_{2}}+R_{C_{2}}\right) \frac{q_{2}}{L n}-\left(\frac{1}{2} R_{G_{1}}+R_{C_{1}}\right) \frac{q_{1}}{L n}\right]\right\} \\
& -K_{U}\left(\bar{U}_{2}-\bar{U}_{1}\right)
\end{aligned}
$$

or, approximately,

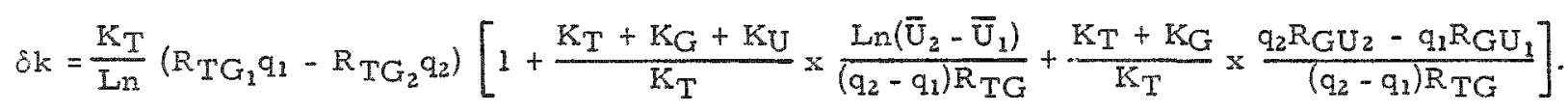

\section{Errors Inherent to the Method of Measurement}

If it is desired to isolate the power coefficient $K_{p}$ due to the fuel-to-cladding temperature drop and consequently to the Doppler effect, one obtains

$\frac{\delta \mathrm{k}}{q_{1}-q_{2}}=K_{p}\left[1+\frac{K_{T}+K_{G}+K_{U}}{K_{T}} \times \frac{L_{n}\left(\bar{U}_{2}-\bar{U}_{1}\right)}{\left(q_{2}-q_{1}\right) R_{T G}}+\frac{K_{T}+K_{G}}{K_{T}} \times \frac{q_{2} R_{G U_{2}}-q_{1} R_{G} U_{1}}{\left(q_{2}-q_{1}\right) R_{T G}}\right]$.

Then the following errors must be considered:

$$
-\epsilon_{U}=\left[1+\frac{K_{T}+K_{U}}{K_{T}}\right] \frac{L_{n}\left(\bar{U}_{2}-\vec{U}_{1}\right)}{\left(q_{2}-q_{1}\right) R_{T G}}
$$

is the error due to average temperature change of the coolant. This error would be immaterial if

the inlet temperature of the coolant were kept strictly constant;

the flow rates were accurately adjusted to the power level to keep the coolant temperature rise within the core constant;

flow distortions were negligible.

It may be noticed that between two consecutive measurements the coolant temperature rise hardly varies, roughly $4^{\circ} \mathrm{C}$ for the "1-rod" solution and $20^{\circ} \mathrm{C}$ for the " 7 -rod" solution. The error mainly due to changes of the core inlet temperature is assumed to be $\overline{\mathrm{U}}_{2}-\overline{\mathrm{U}}_{1}=1^{\circ} \mathrm{C}$.

The canning effect error is given by

$$
-\epsilon_{G}=\left[1+\frac{K_{G}}{K_{T}}\right] \frac{q_{2} R_{G U}-q_{1} R_{G U}}{R_{T G}\left(q_{2}-q_{1}\right)}
$$


The temperature of the canning changes even though the coolant temperatures are kept constant. This error is minimized by making the canningcoolant thermal resistance small as compared with the fuel-canning thermal resistance.

These errors are given in the following table. The importance of the errors $\epsilon_{G}$ and $\epsilon_{U}$ is due to the axial expansion and ensuing dis placement of the fuel.

\begin{tabular}{lccc}
\hline & Error in $\%$ & 7 -Pin Core & 1-Pin Core \\
\hline$\epsilon_{U}$ & $\bar{U}_{2}-\bar{U}_{1}=1^{\circ} \mathrm{C}$ & 3.3 & 4.1 \\
$\epsilon_{G}$ & 8 & 5.2 \\
\hline
\end{tabular}

D. Experimenta1 Errors

Since $\delta \mathrm{k}$ and $\mathrm{q}_{2}-\mathrm{q}_{1}$ contain experimental errors, one has

$$
\frac{\Delta \mathrm{K}_{\mathrm{p}}}{\mathrm{K}_{\mathrm{p}}}=\frac{\Delta \delta \mathrm{k}}{\delta \mathrm{k}}+\frac{\Delta\left(\mathrm{q}_{2}-\mathrm{q}_{1}\right)}{\left(\mathrm{q}_{2}-\mathrm{q}_{1}\right)} \text {. }
$$

The power changes for the 1- and 7-rod cases will be assumed to be 1 and $7 \mathrm{MW}$, respectively. The reactivity to be inserted is approximately $25 \mathrm{pcm}$ in one case and 50 in the other.

$\Delta \delta \mathrm{k} / \delta \mathrm{k}$ is assumed to be between $2 \%$ (optimistic value) and $5 \%$ (pessimistic value). This assumption entails that the experimental rod has been previously calibrated at power by an oscillation method for instance.

$\Delta\left(q_{2}-q_{1}\right) /\left(q_{2}-q_{1}\right)$ can be split into:

a calibrating error which increases with the operating power, since the power indicator has been calibrated at zero power. This is a systematical error;

an error due to instrumental noise, nonlinearities etc., by a compensation method for the measurement of $q_{2}-q_{1}$. One has $\Delta\left(q_{2}-q_{1}\right) /\left(q_{2}-q_{1}\right)=1 \%$ if $q_{2}-q_{1}=1 \mathrm{MW}$.

The following table shows different errors. 
LADET et al.

\begin{tabular}{|c|c|c|c|}
\hline & Error in \% & 7-Pin Core & 1-Pin Core \\
\hline$\triangle \delta \mathrm{k}$ & optimistic value & $2 \%$ & $2 \%$ \\
\hline$\overline{\delta k}$ & pessimistic value & $5 \%$ & $5 \%$ \\
\hline$\Delta\left(q_{2}-q_{1}\right)$ & calibrating error & $\left(2+3 \frac{q}{q_{n}}\right) \%$ & $\left(2+3 \frac{q}{q_{n}}\right) \%$ \\
\hline$\overline{\left(q_{2}-q_{1}\right)}$ & statistical error & $1 \%$ & $1 \%$ \\
\hline
\end{tabular}

E. Conclusions

1. Total Error

The total error was taken to be

$\frac{\Delta \mathrm{K}_{\mathrm{p}}}{\mathrm{K}_{\mathrm{p}}}=\epsilon_{\mathrm{G}}\left[\frac{\Delta\left(\mathrm{q}_{2}-\mathrm{q}_{1}\right)}{\mathrm{q}_{2}-\mathrm{q}_{1}}\right]_{\text {Calibration }}+\sqrt{\epsilon_{\mathrm{U}}^{2}+\left(\frac{\Delta \delta \mathrm{k}}{\delta \mathrm{k}}\right)^{2}+\left[\frac{\Delta\left(\mathrm{q}_{2}-\mathrm{q}_{1}\right)}{\mathrm{q}_{2}-\mathrm{q}_{1}}\right]_{\text {statistical }}}$,

in which the canning effect and the power calibration errors are taken as systematic. The total exror could be decreased by an experimental correction of the term $\epsilon_{\mathrm{G}}$. The following table shows the total error.

\begin{tabular}{lcc}
\hline Total Error in $\%$ & 7 -Pin Core & 1 -Pin Core \\
\hline optimistic value & $14+3 \frac{\mathrm{q}}{\mathrm{q}_{\mathrm{n}}}$ & $12+3 \frac{\mathrm{q}}{\mathrm{q}_{\mathrm{n}}}$ \\
pessimistic value & $16+3 \frac{\mathrm{q}}{\mathrm{q}_{\mathrm{n}}}$ & $14+3 \frac{\mathrm{q}}{\mathrm{q}_{\mathrm{n}}}$ \\
\hline
\end{tabular}

2. Utilization of the Results

The measurements may be used for the calculation of the isothermal Doppler coefficients $\mathrm{K}_{\mathrm{T}}$ by means of the relation

$$
K_{T}=K_{p} \times \frac{L_{n}}{R_{T}} .
$$

Let

$$
\frac{\Delta K_{T}}{K_{T}}=\sqrt{\left(\frac{\Delta K_{p}}{K_{p}}\right)^{2}+\left(\frac{\Delta R_{T}}{R_{T}}\right)^{2}}
$$

where $R_{T}$ is the fuel-canning thermal resistance per unit length. The error 
$\frac{\Delta R_{T}}{R_{T}}$ is estimated to be of the order of $20 \%$, so that

$$
\frac{\Delta K_{T}}{K_{T}}=\sqrt{\left(\frac{\Delta K_{p}}{K_{p}}\right)^{2}+(20 \%)^{2}}
$$

The total exrox on the isothermal coefficient lies between 21 and $25 \%$ if $\mathrm{K}_{\mathrm{U}} / \mathbf{K}_{\mathrm{T}}=0$

The measurements may also be used as a basis for the calculation of the power coefficients of large reactors. For constant conductivity intervals, spectra, and fissile to fertile ratio, the Doppler coefficient can easily be extrapolated.

It must, however, be noticed, that the measured power coefficient is entirely dependent upon the fuel-canning temperature drop. Let the average temperature increases of the fuel, the canning, and the coolant for a constant-flow power increase of $1 \mathrm{MW}$ be designated by $\Delta \overline{\mathrm{T}}, \Delta \overline{\mathrm{T}}_{\mathrm{G}}, \Delta \overline{\mathrm{U}}$, respectively. The Doppler power coefficient is $\mathrm{K}_{\mathrm{T}} \Delta \overline{\mathrm{T}}$, but $(\Delta \bar{T}-\Delta \bar{T} G)$ is actually measured. In order to extend the results of the measurements to large reactors in which the coolant temperature rise across the core is of the order of $150-200^{\circ} \mathrm{C}$, a correction $\Delta \bar{T} G / \Delta \bar{T}$ of the order of $15 \%$ will have to be made. In order to make this correction the knowledge of the average fuel temperature rise is needed.

\section{Oscillation Method}

\section{A. Generalities}

The oscillation method consists in measuring the total power feedback function of the reactor. This function,

$$
H=P K(i \omega)=\frac{1}{G}-\frac{1}{G_{0}}
$$

is deduced from the direct measurement of the zero-power transfer function $\mathrm{G}_{0}$ and the power transfer function $\mathrm{G}$.

Not only does $H$ comprise the Doppler effect, but also the coolant and structure effects. One uses flow and temperature conditions so as to optimize the Doppler effect. However, it is impossible to eliminate completely the other effects as, for instance, in the static method. In good conditions the sodium effect amounts to $10 \%$ of the total effect. In this rough study the effect of deformation of the core structure was assumed to be negligible. 
After subtraction of the coolant effect and taking into account the fuel temperature response to a power change, one obtains the Doppler temperature coefficient as a function of frequency.

The fitting of a one-time-constant model to this function yields the static value. It is to be noted that a total coefficient is obtained.

The fuel and temperature responses in the different reactor zones are calculated as a function of flow, power distribution, sodium inlet temperature, etc., by a digital code which is part of a stability program.

The functions $G$ and $G_{0}$ are measured by the classical pile oscillator method.

\section{B. Calculated Cases}

To confirm the exactness of the method and to check the influence of the different parameters, some preliminary calculations of the power coefficients and the coolant to Doppler ratio were made. The reactor was assumed to be divided into zones, each of which was characterized by its power distribution, its Doppler, coolant, and structure temperature coefficients, and its coolant velocity. The inputs were reactivity, sodium inlet temperature, and flow. The code computed the temperature distribution in the fuel, the canning, and the coolant for the different zones. The corresponding power coefficients were then calculated and, finally, the open-loop transfer function. Stability was then checked by the Nyquist criterion.

\section{A limited parametric study was made with the following} parameters:

the number of pins per assembly, i.e., the pin diameter;

the total contact coefficient $H$;

the coolant flow:

the ratio of Doppler to coolant temperature coefficients, the latter being kept constant.

The distribution of the sodium and Doppler coefficients throughout the different reactor zone was given in the introduction of the second part of this paper (see Fig. 4).

The following table shows the values of the parameters used in the different cases. The last column, which gives values of

$$
\frac{\Delta K_{p}}{K_{p}}=\frac{K_{N a}+\text { Doppler }-K_{\text {Doppler }}}{K_{\text {Doppler }}} \text {, }
$$


is a measure of the efficiency in isolating the Doppler coefficient. Case number 6 was not calculated at the same time as the others. The value of $\Delta \mathrm{K}_{\mathrm{p}} / \mathrm{K}_{\mathrm{p}}$ was found by comparison with another study made previously. Figures 5 to 9 show the power feedbacks for the cases 1 to 5 .

The results of the table demonstrate clearly that it is possible to reduce the sodium effect to less than $10 \%$ of the total.

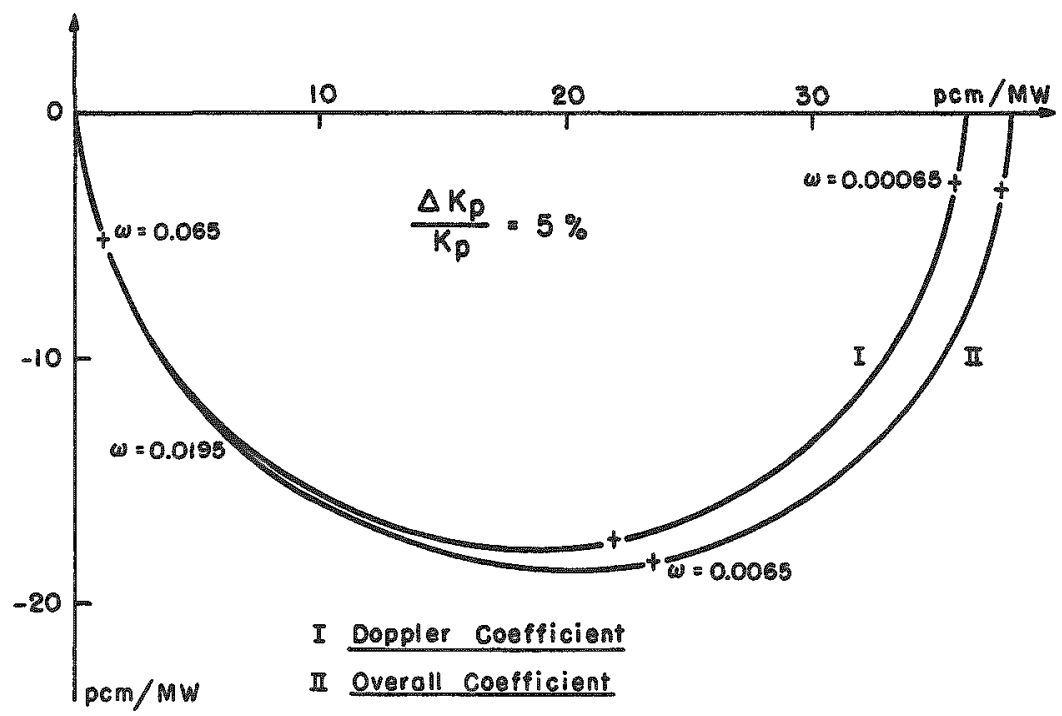

Fig. 5. Power Coefficient at $6.5 \mathrm{MW} . \mathrm{H}=0.1 \mathrm{~W} /{ }^{\circ} \mathrm{C} \mathrm{x} \mathrm{cm}$; $\Delta \mathrm{T}=28^{\circ} \mathrm{C} ;$ Doppler $=-0.29 \mathrm{pcm} /{ }^{\circ} \mathrm{C}$

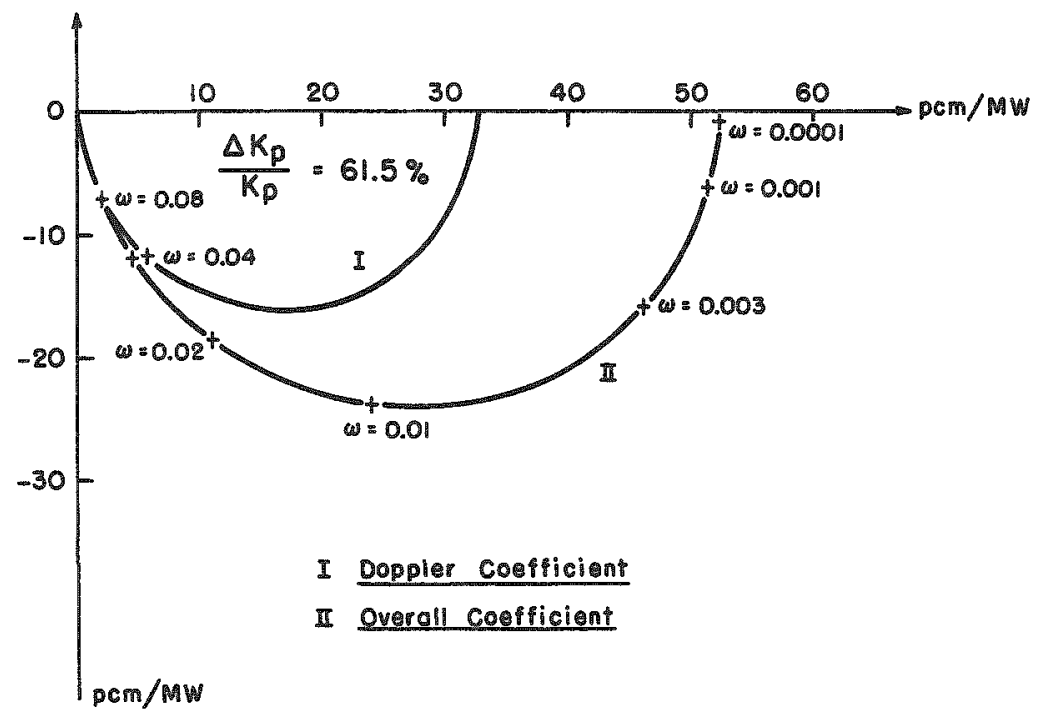

Fig. 6. Power Coefficient at $1 \mathrm{MW}, \mathrm{H}=1 \mathrm{~W} /{ }^{\circ} \mathrm{C} \mathrm{x} \mathrm{cm}{ }^{2}$; $\Delta \mathrm{T}=56^{\circ} \mathrm{C} ;$ Doppler $=-0.29 \mathrm{pcm} /{ }^{\circ} \mathrm{C}$ 


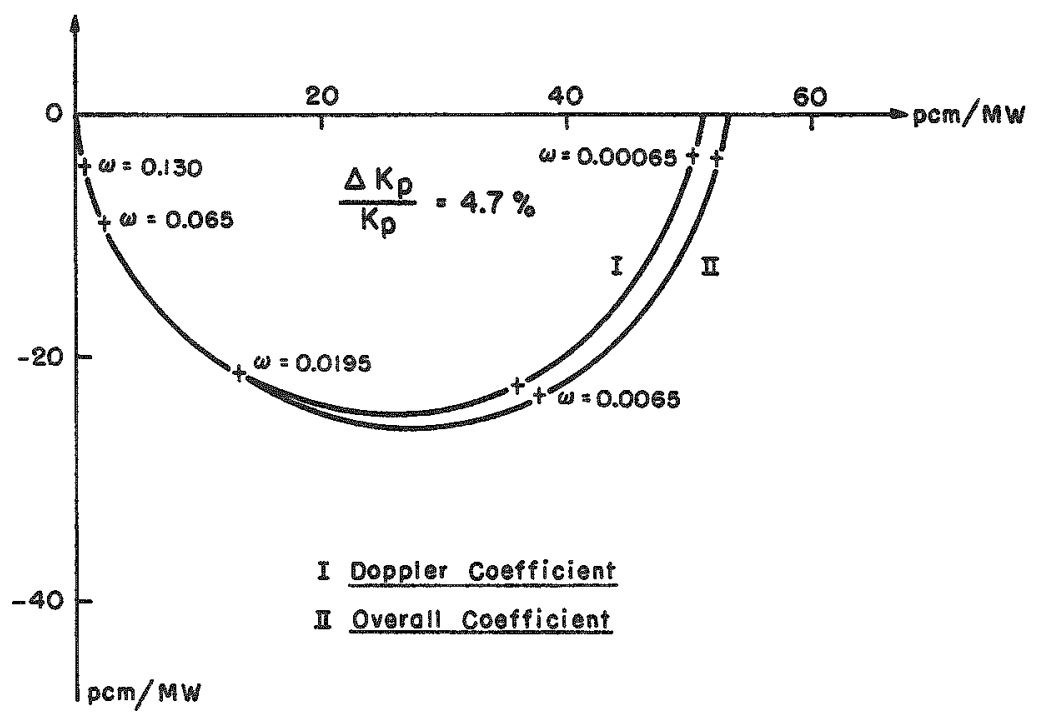

Fig. 7. Power Coefficient at $6.5 \mathrm{MW} . \mathrm{H}=1 \mathrm{~W} /{ }^{\circ} \mathrm{C} \mathrm{x} \mathrm{cm}{ }^{2}$; $\Delta \mathrm{T}=28^{\circ} \mathrm{C} ;$ Doppler $=-0.58 \mathrm{pcm} /{ }^{\circ} \mathrm{C}$

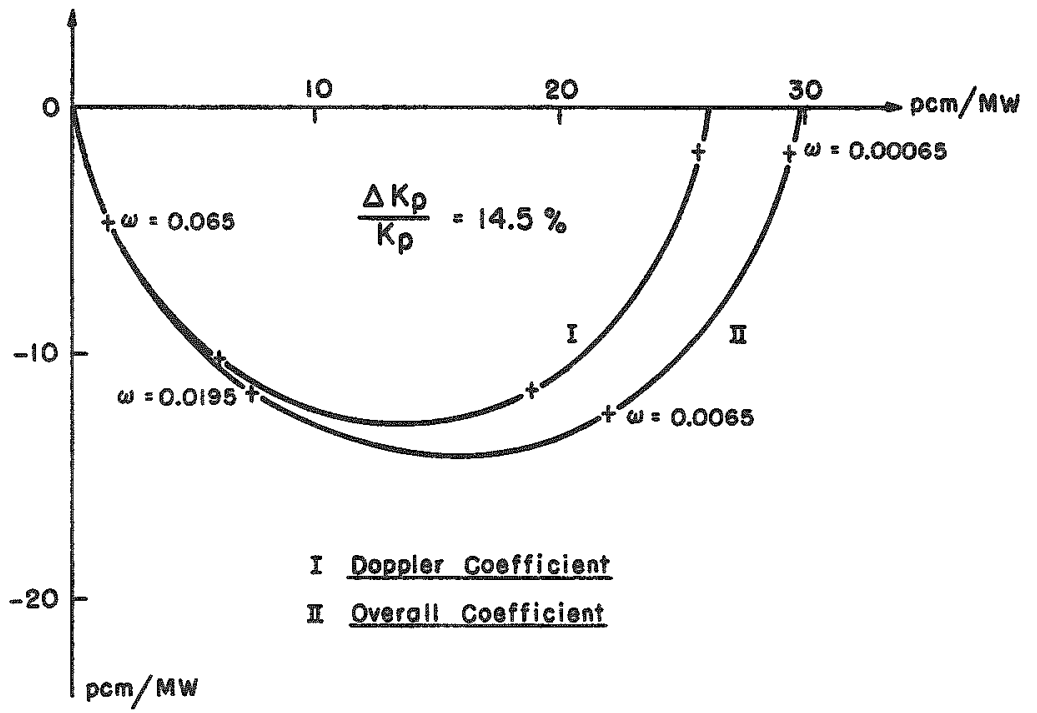

Fig. 8. Power Coefficient at $6.5 \mathrm{MW} . \mathrm{H}=1 \mathrm{~W} /{ }^{\circ} \mathrm{C} \mathrm{x} \mathrm{cm}{ }^{2}$; $\Delta \mathrm{T}=56^{\circ} \mathrm{C} ;$ Doppler $=-0.29 \mathrm{pcm} /{ }^{\circ} \mathrm{C}$ 


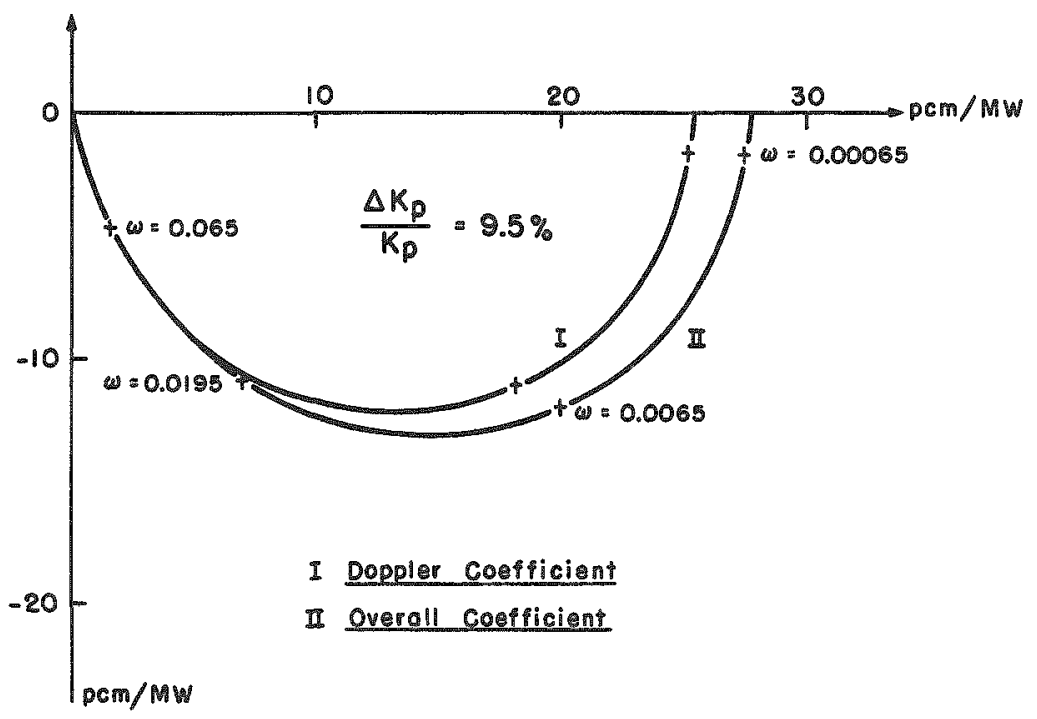

Fig. 9. Power Coefficient at $6.5 \mathrm{MW} . \mathrm{H}=1 \mathrm{~W} /{ }^{\circ} \mathrm{C} \mathrm{x} \mathrm{cm}$; $\Delta \mathrm{T}=28^{\circ} \mathrm{C} ;$ Doppler $=-0.29 \mathrm{pcm} /{ }^{\circ} \mathrm{C}$

A realistic case for a 400-liter core in RAPSODIE would probably be situated between the reference case and the cases 4 and 5 .

Not considering the fact that a 7 -pin assembly is more realistic, this case is less interesting for the measurement of the Doppler effect its elf.

RAPSODIE; 400-liter Core, 1 -Pin Model, $\mathrm{K}_{\mathrm{Na}}=-0.16 \mathrm{pcm} /{ }^{\circ} \mathrm{C}$

\begin{tabular}{|c|c|c|c|c|c|}
\hline Case & $\begin{array}{c}\mathrm{P} \\
(\mathrm{MW})\end{array}$ & $\begin{array}{c}\mathrm{H} \\
\left(\mathrm{W} /{ }^{\circ} \mathrm{C}-\mathrm{cm}^{2}\right)\end{array}$ & $\begin{array}{c}\Delta \mathrm{T} \approx \frac{\mathrm{P}}{\text { flow }} \\
\left({ }^{\circ} \mathrm{C}\right)\end{array}$ & $\begin{array}{c}\text { Isothermal } \\
\text { Doppler } \\
\text { Coefficient } \\
\left(\text { pcm } /{ }^{\circ} \mathrm{C}\right)\end{array}$ & $\frac{\Delta \mathrm{K}_{\mathrm{p}}}{\mathrm{K}_{\mathrm{p}}}$ \\
\hline 1 (reference) & 6.5 & 1 & 28 & -0.29 & 9.5 \\
\hline 2 & 6.5 & 1 & 56 & -0.29 & 14.5 \\
\hline 3 & 1 & 1 & 56 & -0.29 & 61.5 \\
\hline 4 & 6.5 & 1 & 28 & -0.58 & 4.7 \\
\hline 5 & 6.5 & 0.1 & 28 & -0.29 & 5.0 \\
\hline $6(7-p i n)$ & 30 & 1 & $(130)$ & -0.29 & $(13.5)$ \\
\hline
\end{tabular}

C. Accuracy

The following factors influence the error calculation:

the errors of $G$ and $G_{0}$ for which $P k(i \omega)$ is deducted;

the error in the value of the average power $P$; 
the error in the coolant effect;

the error made when extrapolating $\mathrm{K}_{\mathrm{Dopp}}(\omega=0)$;

the error of the calculated fuel temperature response to a power change (the latter error depends among other things, upon the knowledge of the fuel heat capacity, the heat transfer constants, and the power distribution in the reactor).

The accuracies for the cases 1 and 6 of the previous table were calculated on the basis of the following assumptions:

error of $G_{0}: 2 \%$ and $2^{\circ}$;

error of $\mathrm{G}$ : $5 \%$ and $5^{\circ}$;

error of P: $2 \%$;

error of $\mathrm{K}(\mathrm{i} \omega)$ for $\mathrm{Na}$ : $50 \%$;

the error of the extrapolated value $\omega=0$ is assumed to be half the error on the individual points (graphical or least squares analysis);

exror of the temperature response: $20 \%$.

The results are given in the following table.

\begin{tabular}{|c|c|c|}
\hline & Case 1 & Case 6 \\
\hline error of $H-P K(i \omega)$ & $11.5 \%$ & $13.5 \%$ \\
\hline error of the amplitude of $K(i \omega)_{\text {tot }}$ & $12.5 \%$ & $15 \%$ \\
\hline error of $K(\omega=0)_{\text {tot }}$ & $6 \%$ & $7.5 \%$ \\
\hline error of $K(\omega=0)_{\text {Dopp }}$ & $8 \%$ & $10 \%$ \\
\hline $\begin{array}{l}\text { error of Doppler temperature } \\
\text { coefficient }\end{array}$ & $16 \%$ & $17 \%$ \\
\hline
\end{tabular}

The relative error of $H=G^{-1}-G_{0}^{-1}$ increases with frequency. It increases also at very low frequencies because of experimental difficulties, such as reactor drift. The absolute error is almost independent of frequency.

\section{Remarks}

At low frequencies, i.e., $\omega<0.3 \mathrm{rad} / \mathrm{sec}$ in case number 6 , the function $K(i \omega)$ is perfectly described by one time constant:

$$
K(i \omega)=\frac{K(0)}{i+\tau \omega_{i}}
$$


where $T$ is the ratio of the heat capacity $C$ to the total fuel transfer coeficient $\mathrm{H}$,

$$
\begin{aligned}
& \frac{1}{H t}=\frac{1}{8 \pi k}+\frac{1}{2 \pi R H_{C}} ; \quad H_{C}=0.1 \rightarrow 1 \mathrm{~W} / \mathrm{cm}^{2}-{ }^{\circ} \mathrm{C} \text {. } \\
& \text { fuel fuel canning } \\
& \text { contact }
\end{aligned}
$$

In case 6 (see previous table) the error of the measured points of $K$ (iw) is approximately $14 \%$ and $8^{\circ}$. The resulting errors of $\mathrm{K}(0)$ and $T$ would be 7 and $10 \%$, respectively.

The relation $\tau=\mathrm{C} / \mathrm{H}$ then yields $\mathrm{H}$, the heat capacity $\mathrm{C}$ being relatively well known.

At small flow rates the blanket effect becomes relatively large, and $K(i \omega)$ is described by two time constants and the analysis becomes more complicated.

A direct and more accurate measurement of the Doppler effect would be possible by installation of thermocouples in the fuel (especially in the $45 \mathrm{-mm}$ rods). In this case the result becomes independent upon the heat transmission. However, the execution is technologically difficult, and the increase in accuracy may not be worthwhile.

In order to obtain the net Doppler effect, the sodium effect must be deducted from the measured feedback function. An experimental value of this correction could be obtained by measurements at constant power and varying flow rates. The Dopplex effect is then obtained by extrapolation. However, the flow change, the fuel temperature influence the Doppler effect itself in two ways:

by the heat exchange coefficient;

by the canning temperature.

An approximate value for the sodium effect can further be obtained either by calculation or by direct measurements. This may be done by local perturbation or by complete emptying of the vessel, the latter being a nonlinear effect.

IV. Conclusions

We now compare the two methods which have been described and another method which can be applied to the same core: the reactivity step method (ref. 2 ). 


\section{A. Ease of Execution}

The static method necessitates only the functional reactor instrumentation. It is to be noted that the regulating rod must be calibrated as accurately as possible by means of an oscillation method.

The oscillation method is actually a transfer function meas urement. At very low frequencies, however, it might prove to be difficult to keep the inlet temperature constant.

Special instruments are required for the step method.

\section{B. Information Obtained}

The static method yields directly the power coefficient; the isothermal coefficient is obtained by calculation unless thermocouples are used in the rods.

The same is true for the oscillation method, but due to the experimental knowledge of the heat transfer coefficient, the uncertainty of the temperature coefficient is somewhat smaller.

Finally, the step method yields an "energy" coefficient which can be reduced to an isothermal coefficient after evaluation of the heat capacity.

\section{Accuracy}

The following table summarizes the accuracy obtainable with the different methods.

\begin{tabular}{lcc}
\hline Method & Power Coefficient & Isothermal Coefficient \\
\hline Static & 16 to $18 \%$ & 21 to $25 \%$ \\
Oscillation & 8 to $10 \%$ & 16 to $17 \%$ \\
Step & $10 \%$ & \\
& (energy coefficient) & \\
\hline
\end{tabular}

\section{Conclusions}

It is estimated that the Doppler effect can be measured, e.g. in a small reactor like RAPSODIE, by different methods (static, oscillation, sub-or over-prompt critical step) with a sufficient accuracy to allow the construction of large fast reactors without taking any major risks as to their stability. 
The above calculated accuracies could yield by extrapolation an approximate value of the Doppler coefficient in large reactors. Since no instrumentation difficulties can be foreseen, the only problem is an economical one, i.e., the investment of approximately $300 \mathrm{~kg}$ of $\mathrm{Pu}^{239}$ in a 400 -1iter core. However, the plutonium could be reused due to its small activity.

Due to the integral character and to the fact that they are executed in real operating conditions, the measurements discussed above may be considered complementary to those carried out with zero-power mockups.

\section{References}

1. C. P. Zaleski and L. Vautrey, Le réacteur rapide surgénérateur Rapsodie, (Vienna 1961).

2. K. Ott, Theory of Delayed Supercritical Excursions to Measure Doppler Coefficients, I.N.R. Kernforschungszentrum Karls ruhe

3. W. Håefele, Prompte Ueberkritische Leistrungsexkursionen in Schnellen Reaktoren, Nukleonik, 5, 201 (1963).

$\frac{\text { Discussion of Paper }}{\text { Presented by Mr. Storrer }}$

MR. KATO (Argonne):

Would you give us an estimate of what you think the change in activation rate would be for a specific change in temperature?

MR.STORRER:

We found out about the activation quite recently and have had no time to make detailed calculations. This technique will probably be tried at $G E$ by Peterson for their mixed-spectrum experiment. It is a difficult proposition to measure. But I think it is worthwhile trying.

The trouble is that you measure also the activation of neutrons at very high energies which are Doppler independent. Otherwise, it would be a very good method since the relative change in effective cross section in the low energy region is quite large. But you have those other neutrons which bother you. 
MR. HETRICK (University of Arizona):

I guess I believe your arguments about the small sample, and the fact that you get both terms that way, although I think you physical argument is not so convincing in itself, because if hot neutrons come back from a small sample, you would think their effect would be pretty much washed out in the surrounding reactor.

MR. STORRER:

I think your feeling is wrong. 


\title{
REACTIVITY COEFEICIENT MEASUREMENTS IN FARET
}

\author{
Paul J. Persiani \\ Argonne National Laboratory \\ Argonne, Illinois
}

The Fast Reactor Test Facility (FARET) is being designed to generate physics and engineering data required in the development of large fast power breeders. Although certain physics measurements may be obtained from critical experiments, these do not necessarily simulate the actual conditions of operating temperatures, fuel design, reactor coolant, and coolant flow rates. The design will provide an appropriate reactor environment in which high-power density and high-temperature systems can be investigated, as well as to resolve the problem of major unknown power reactivity parameters.

The facility will be capable of accepting reactor experiments with a heat generation of $50 \mathrm{MWt}$ and a maximum mean outlet temperature for the reactor coolant up to $650^{\circ} \mathrm{C}$. It is designed to accommodate a variety of reactor configurations, coolant flow rates, and sizes operating over the full range of power levels $(0$ to $50 \mathrm{MW}$ ) and at a variety of temperatures. The reactor vessel will allow inserting a core having a 6 -ft $(180-\mathrm{cm})$ diameter.

This paper will be concerned only with physics phase of the expeximental program, and in particular with the measurement of the fueltemperature coefficient which is comprised of the expansion and Doppler effect.

The immediate objective of the experimental program planned for FARET is the measurement of the Doppler coefficient which can be assigned to a reference power reactor. To allow these measurements to be made for several reference reactor systems, zoned loadings will be utilized. The zoned loading will consist of a relatively small test zone in the center of the reactor, which in turn is surrounded by a buffer region, followed by a driver and reflector section.

The test zone would simulate the composition, structure, and neutron spectrum of the reference reactor. The buffer region serves to equilibrate the neutron spectrum in the test zone to that of a corresponding zone in the reference system. The dxiver section will be uranium metal-fueled and will furnish whatever additional reactivity is required to achieve criticality.

The utility of the zoned-loading technique with a metallic driver is based on many factors. The cost per experiment for a large reactor is considerably reduced because of the smaller size of the test zone. The fuelcoolant-structure composition may be varied without changing the complete 
core assembly. The buffer and driver regions may be readily varied in size to obtain the appropriate conditions for different test-zone compositions. For a ceramic test zone with reactivity effects to be measured, a metallic driver section would assure the safety of the experiment by virtue of its reliable prompt-negative expansion reactivity feedback.

The fuel temperature of the test zone is varied to obtain a static meas urement of the corresponding change in reactivity. The measured reactivity coefficient of the test zone can then be extrapolated to the full-size reference reactor by the theoretically determined ratio of the Doppler coefficient of the reference test zone to that of the total core.

Several methods are being explored to confine a significant temper ature change in the fuel elements of the FARET test zone. The magnitude of the temperature change must be large enough to give an interpretable Doppler reactivity neasurement.

One of these methods, requiring a minimum of developmental effort, is the varying power-level experiment without a fuel-clad gas gap. In this method fuel pellets are slip-fitted inside the clad. The fuel temperature change is dependent on the power density in the rod, the conductivity, and radius of the fuel pin. For thermal conduction the average fuel temperature increase is proportional to $q_{h} r^{2}\left[1-\left(q l / q_{h}\right)\right] / k$, where $q h$ and $q l$ are the heats generated in a rod, of radius $r$ and conductivity $k$, at the high and low power level, respectively. The temperature changes of the coolant and clad in the test zone is reduced by varying the coolant flow rate in direct proportion to the change in the power level. The initial temperatures in the buffer and driver region are maintained essentially constant by the increased coolant flow.

The initial experiment in the FARET zoned system is the investigation of the Doppler effect for an oxide-fueled, uranium-blanketed reference reactor. The poor conductivity of the uranium oxide allows a significant temperature increase in the fuel pin.

To measure the Dopplex reactivity effect for a large, plutoniumenriched uranium oxide system having a $\mathrm{U}^{238} / \mathrm{Pu}^{239}$ atom ratio of $7 / 1$, a series of multiregion core configurations were studied. The above enrichment for a reactor system having a $\mathrm{Pu}-\mathrm{UO}_{2} / \mathrm{Na} / \mathrm{SS}$ composition of $32 / 52 / 16$ by volume per cent is taken as being typical of a large system of approximately 2000 liters with a length-to-diameter $(\mathrm{L} / \mathrm{D})$ ratio of $1 / 2$, and a $3 \mathrm{ft}(91.8-\mathrm{cm})$ height. The Doppler coefficient, or rather the Doppler component, of the fuel-temperature coefficient for this system was computed to be $-7.3 \times 10^{-6} \Delta \mathrm{k}_{\mathrm{eff}} / \Delta \mathrm{T}^{\circ} \mathrm{C}$ at $750^{\circ} \mathrm{K}$. This is considered to be of significant magnitude to generate a measurable reactivity effect in the FARET zoned system. 
In investigating the various experimental configurations, a criterion used was that a reactivity change arising from a change in the test-zone fuel tempexature be within some acceptable error of the reactivity change in the corresponding region of the reference reactor, when subjected to the same perturbation. This assures one that the importance spectrum as well as the neutron spectrum is reasonably reproduced.

The results of neutron spectrum matching for some configurations are presented in Table 1. The volumes of the test zone for the 7.4 - and 5.3-in. radii are 102 and 52.4 liters, respectively. The zoned core system is further identified in the table by the outer radius of the buffer region and by the outer radius of the metallic driver region. The remaining volume of the FARET system contains sodium-cooled stainless steel subassemblies. The stainless steel hexagonal subassemblies are $2.32 \mathrm{in}$. $(5.90 \mathrm{~cm})$ across the flats, the same as used for EBR-II.

Table ]

COMPUTED GROUP FLUX INT THE TEST ZONE OF A 2OM-IITE OXIDE REFERENCE CORE AND FARET ZONED SYSTEMS

\begin{tabular}{|c|c|c|c|c|c|c|c|c|c|}
\hline \multirow{2}{*}{ Group } & \multirow{2}{*}{$\begin{array}{l}\text { Energy } \\
\text { EL (keV) }\end{array}$} & \multirow{2}{*}{$\begin{array}{l}\text { Reference } \\
\text { Core } \\
\text { Test Zone }\end{array}$} & & \multicolumn{4}{|c|}{$\begin{array}{l}\text { 7.4-in.-radius } \\
\text { Test Zone }\end{array}$} & \multicolumn{2}{|c|}{$\begin{array}{l}\text { 5.3-n-radius } \\
\text { Test Zone }\end{array}$} \\
\hline & & & \multicolumn{2}{|r|}{$13.7 / 27.5^{a}$} & $8(\%)$ & $13.7 / 15.8^{\mathrm{a}}$ & $8 \%$ & $11.6 / 13.7^{2}$ & $\delta(\%)$ \\
\hline $\begin{array}{l}12 \\
13 \\
14 \\
15 \\
16 \\
17 \\
18\end{array}$ & $\begin{array}{c}15.0 \\
0.1 \\
4.0 \\
1.0 \\
0.3 \\
0.1 \\
0.03\end{array}$ & $\begin{array}{l}0.0623 \\
0.0391 \\
0.0305 \\
0.0379 \\
0.0105 \\
0.0020 \\
0.0004\end{array}$ & 1 & $\begin{array}{l}0.0613 \\
0.0375 \\
0.0285 \\
0.0332 \\
0.0087 \\
0.0016 \\
0.0003\end{array}$ & $\begin{array}{l}-1.6 \\
-4 \\
-6 \\
-13 \\
-17 \\
-20 \\
-25\end{array}$ & $\begin{array}{l}0.0663 \\
0.0413 \\
0.0320 \\
0.0396 \\
0.0144 \\
0.0024 \\
0.0003\end{array}$ & $\begin{array}{l}-6.4 \\
+5.6 \\
-5 \\
+4.5 \\
+8.5 \\
+20 \\
+25\end{array}$ & $\begin{array}{l}0.0659 \\
0.0404 \\
0.0311 \\
0.0377 \\
0.0109 \\
0.0024 \\
0.0005\end{array}$ & $\begin{array}{r}+5.7 \\
+3.5 \\
+2.0 \\
-0.5 \\
+4.0 \\
+20 \\
+25\end{array}$ \\
\hline & $\begin{array}{l}\text { ums } \\
\text { nsity } \\
\text { one } \\
\text { ? }\end{array}$ & 0.041 & : & & & & & & \\
\hline
\end{tabular}

In the case of the Doppler effect the reproduction of the neutron spectrum is confined to the energy groups in the region from 9 to $0.1 \mathrm{keV}$. It is estimated that about 60 per cent of the Doppler effect is contributed by the neutrons in the energy region from 9 to $1 \mathrm{keV}$ and about 30 per cent in the energy range from 1 to $0.1 \mathrm{keV}$. The comparison is made with the region-integrated group flux.

The plutonium content in the buffer for the $13.7-\mathrm{in} .(34.8-\mathrm{cm}) \mathrm{UO}_{2}$ buffer and $27.5-i n .(69.3-\mathrm{cm})$ metallic driver system, is in the form of metal rods to enhance the cooling in this zone. This system produces a somewhat harder spectrum than that in the reference core test zone. The 
computed averaged Doppler coefficients, however, were within 20 per cent of the coefficients in the reference region for a variety of temperature changes.

Another condition imposed on the FARET experiment is that the diameter of the fuel rod used in the test zone be as close to the rod size that would be used in the actual power reactor but still allow the desired fueltemperature change. There is no limit to the fuel-temperature change that can be obtained in the varying power-level experiment, since one can increase the size of the rod. However, in order to greatly reduce possible heterogeneity effects on the Doppler coefficient, the size of rod should be maintained as small as possible.

To optimize the reactivity signal in the experiment, a fueltemperature change in the test zone of greater than $500^{\circ} \mathrm{C}$ is desired. A heat transfer analysis indicates that for a $\frac{1}{2}$-in. $(1.27-\mathrm{cm})$-diameter pin and a power density of $0.044 \mathrm{MW} /$ liter in the test zone, a temperature rise of $500^{\circ} \mathrm{C}$ is marginal.

Therefore, in order to increase the power density in the test zone, the zoned system was compacted to the outer radii of 15.8 and $13.7 \mathrm{in}$. In addition, only depleted $\mathrm{UO}_{2}$ rods have been placed in the buffer region. This increase in power density allows temperature increases to exceed $500^{\circ} \mathrm{C}$ for the $\frac{1}{2}-i n .(1.27-\mathrm{cm})$-diameter pin.

The group flux in the test zone for the depleted $\mathrm{UO}_{2}$ buffer systems is found to be more representative of the reference system. In the range of interest the variation is less than $10 \%$. The spectrum is softer, but the averaged Dopplex coefficient is still within 20 per cent of the coefficient ir. the reference region. The calculated coefficients at several temperatures for the 7.4-in. test-zone systems are presented in Table 2.

The reactivity coefficients as a function of temperature for the systems described are also presented in Fig. 1. As far as the Doppler coefficient is concerned, the two FARET zoned systems appear to be almost identical. The significance of this comparison is that the temperature dependence is expected to be maintained very close to that in the test zone of the reference core.

The depleted $\mathrm{UO}_{2}$ buffer region proves advantageous in two respects. First, the metallic plutonium fuel elements in the buffer zone are eliminated. Second, the depleted oxide buffer tends to flatten the group flux in the test zone. This situation is more representative of the flux distribution in the reference core test zone. This effect can be seen by a comparison of the flur distribution, presented in Figs. 2 and 3, for the two zoned systems. 
Table 2

AVERAGE DOPPLER COEFEICIENT AS A FUNCTION OF TEMPERATURE

\begin{tabular}{c|c|c|c}
\hline \multirow{2}{*}{$\begin{array}{c}\text { Temperature } \\
\left({ }^{\circ} \mathrm{K}\right)\end{array}$} & \multicolumn{3}{|c}{$\Delta \mathrm{k}_{\text {eff }} / \mathrm{T} \times 10^{6}$} \\
\cline { 2 - 4 } & $(\mathrm{T}-300)$ & $(\mathrm{T}-750)$ & $(\mathrm{T}-1500)$ \\
\hline \multicolumn{4}{c}{ Reference Core Test Zone Region } \\
\hline 300 & - & - & - \\
750 & 1.84 & - & - \\
2500 & 1.31 & 1.00 & 0.55 \\
\hline
\end{tabular}

FARET Test Zone, 7.4/13.7/27.5 (T.Z./B.R./D) a System

\begin{tabular}{c|c|c|c}
\hline 300 & - & - & - \\
750 & 1.69 & - & - \\
1500 & 1.14 & 0.82 & - \\
2500 & 0.88 & 0.67 & 0.56 \\
\hline
\end{tabular}

FARET Test Zone, 7.4/13.7/15.8 (T.Z./B.R./D) ${ }^{a}$ System

\begin{tabular}{c|c|c|c}
\hline 300 & - & - & - \\
750 & 1.53 & - & - \\
1500 & 1.08 & 0.81 & - \\
2500 & 0.84 & 0.67 & 0.56 \\
\hline
\end{tabular}

a $T \cdot Z_{\text {. }}$ test zone; $B \cdot R$. = buffer region; $D=$ driver

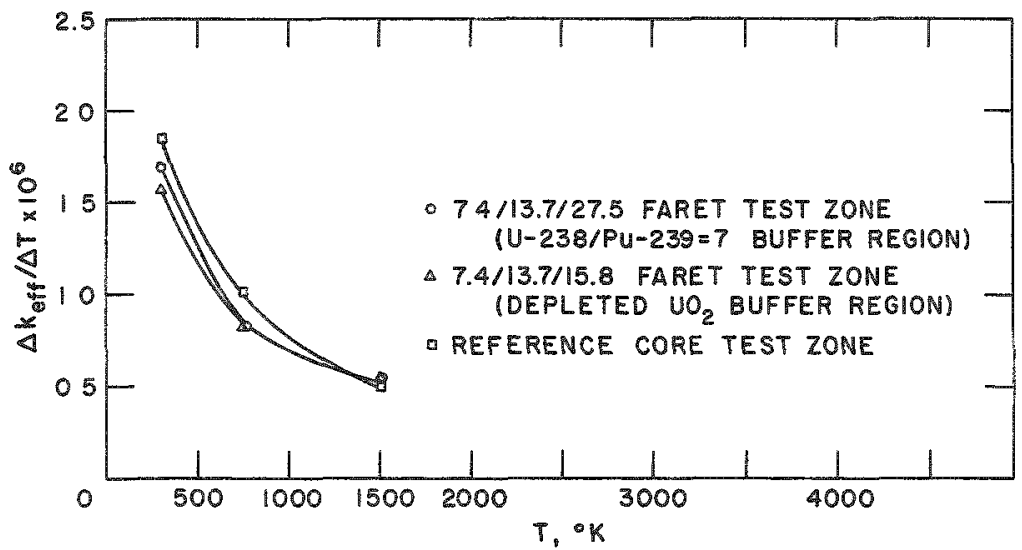

Fig. 1. Doppler Coefficient as a Function of Temperature 


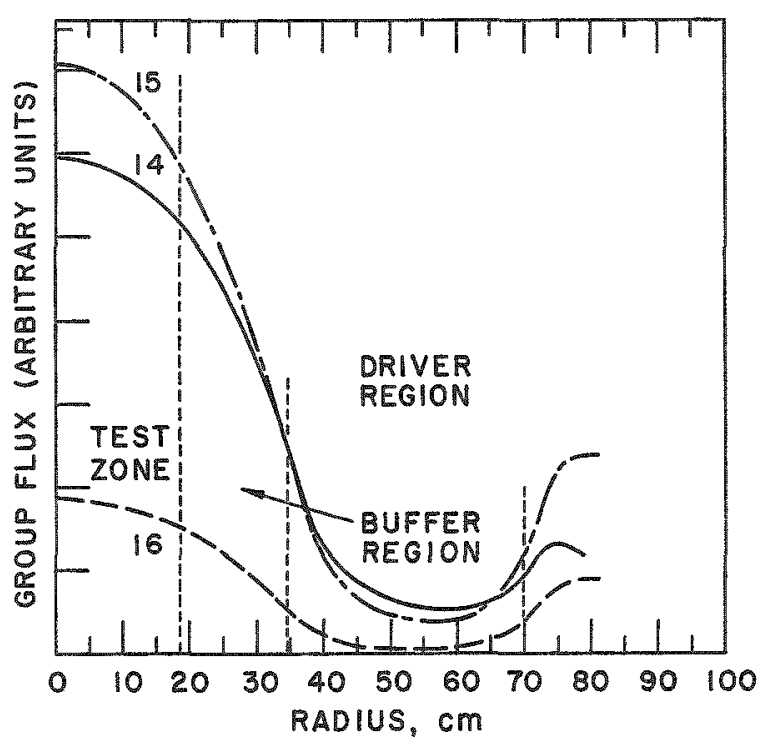

Fig. 2

Group Flux as a Function of Radius in the $7.4 / 13.7 / 27.5$ FARET Zoned System $\left(\mathrm{U}^{238} / \mathrm{Pu}^{239}=7\right.$ Buffex Region)

Fig. 3

Group Flux as a Function of Radius in the 7.4/13.7/15.8 FARET Zoned System (Depleted Oxide Buffer)

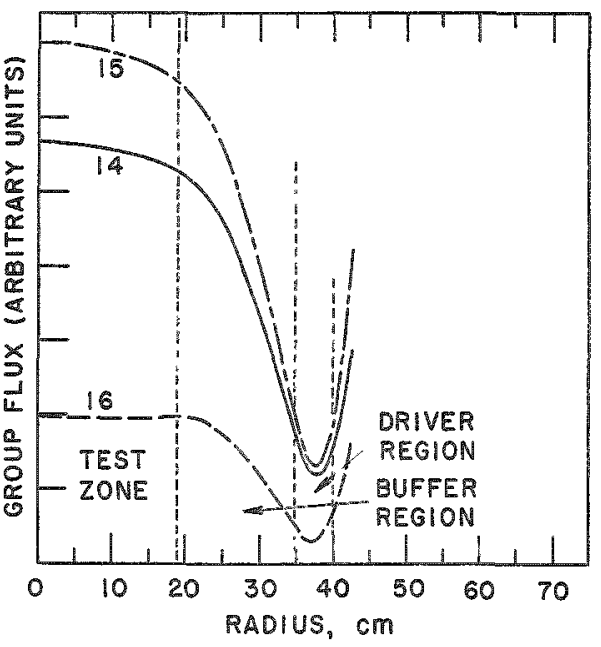

The replacement of the depleted oxide buffer region with other filters is still under study, the objective being simultaneously to increase the power density in the test zone and to improve further the group-flux matching and temperature dependence of the Doppler coefficient.

The problem of isolating an interpretable Doppler effect in a reactivity measurement depends on the contributions to the signal by effects due to fuel expansion in the test zone, and the temperature changes of coolant and structure in the whole system. In Figs. 4 and 5 , the averaged sodium and fuel expansion coefficients are plotted for the entire zoned system. Referring to Fig. 4, although the sodium coefficients are comparable with the Doppler coefficient in the test zone, the average temperature rise of the coolant is kept small and, as will be shown later, is expected to give a negligible contribution to the total measured signal. It should be mentioned that the overall sodium coefficient for the system is negative. 


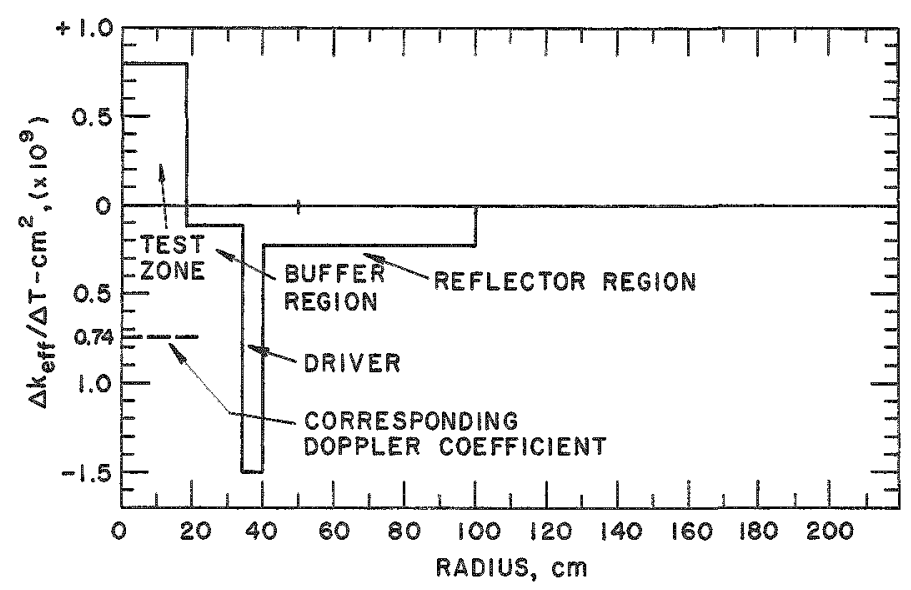

Fig. 4

Averaged Sodium Expansion Coefficient per Unit of Radial Area versus Radius for the 7.4/13.7/15.8 FARET Zoned System (Depleted $\mathrm{UO}_{2}$ Buffer Region)

Fig. 5

Averaged Fuel Expansion Coefficient per Unit of Radial Area versus Radius for the 7.4/13.7/15.8 FARET Zoned System (Depleted $\mathrm{UO}_{2}$ Buffex Region)

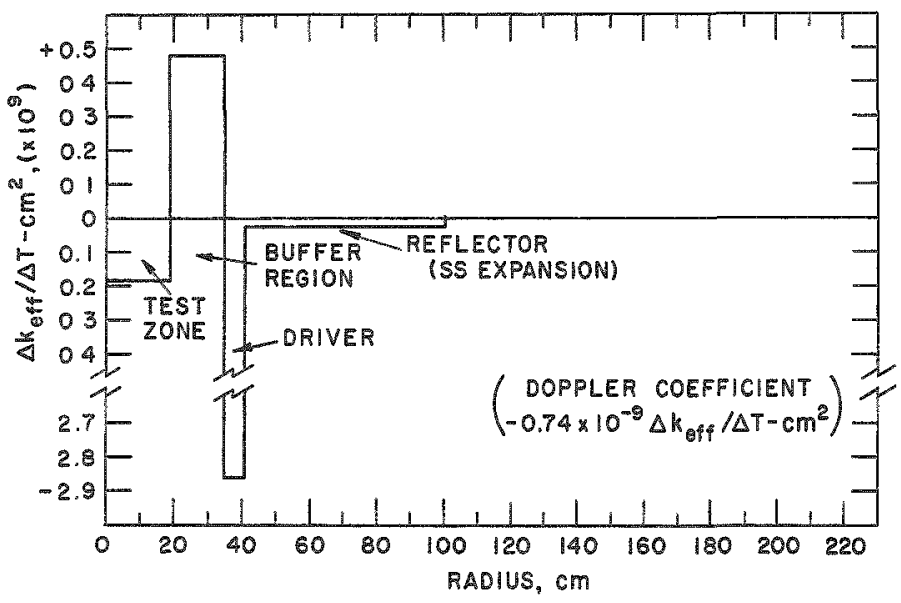

In Fig. 5, the areas of interest are the test zone, buffer, and driver region. The fuel expansion in the test zone is $25 \%$ of the Doppler coefficient. This is based on the assumption that the oxide fuel will expand in a normal manner. The oxide expansion coefficient in the buffer region is positive, and its magnitude is comparable with the Doppler coefficient. The positive sign is due to the increase in the neutron radial leakage from a region of low importance to regions of much higher importance. The negative fuelexpansion coefficient in the driver zone is expected to be some four times as great as the Doppler coefficient in the test zone.

Although these coefficients appear to be non-negligible, an error analysis indicates that the Dopplex reactivity effect, based on a $500^{\circ} \mathrm{C}$ fuel temperature rise in the test zone, can be observed with error of $20 \%$.

Fuel-temperature changes greater than $500^{\circ} \mathrm{C}$ are expected; however, to be conservative, the error analysis presented in Table 3 is based on a $\triangle \mathrm{T}$ of $500^{\circ} \mathrm{C}$. In the first column is a list of the computed reactivity coefficients which need to be considered in isolating the test-zone Doppler effect from the observed measurement. The errors associated with the coefficients are estimates based on variations observed in the calculation of many zoned 
Table 3

ERROR ANALYSIS FOR THE VARYING POWER LEVEL EXPERIMENT $(5 \mathrm{MW} \rightarrow 50 \mathrm{MW})$

\begin{tabular}{|c|c|c|c|}
\hline \multicolumn{2}{|c|}{$\begin{array}{c}\text { Reactivity Coefficients } 750^{\circ} \mathrm{K} \\
13.7 / 15.8 \text { Depleted } \mathrm{UO}_{2} \text { Buffer, } \\
\Delta \mathrm{k}_{\mathrm{eff}} / \triangle \mathrm{T}^{\circ} \mathrm{C} \times 10^{6}\end{array}$} & $\begin{array}{l}\text { Average } \\
\text { Temperature } \\
\text { Rise, } \Delta \mathrm{T}^{\circ} \mathrm{C}\end{array}$ & $\begin{array}{c}\text { Expected Contribution } \\
\text { To Reactivity Measurement, } \\
\times 10^{6}\end{array}$ \\
\hline \multicolumn{4}{|l|}{ Doppler } \\
\hline $\begin{array}{l}\text { Buffer Zone } \\
\text { Driver }\end{array}$ & $\begin{array}{l}-1.50 \pm 0.5 \\
-0.20 \pm 0.06\end{array}$ & $\begin{array}{l}22 \pm 2 \\
28 \pm 3\end{array}$ & $\begin{array}{l}-33 \pm 11 \\
-5.6 \pm 2\end{array}$ \\
\hline \multicolumn{4}{|l|}{ Fuel Expansion } \\
\hline $\begin{array}{l}\text { Test Zone } \\
\text { Buffer Zone } \\
\text { Driver } \\
\text { SS Reflector } \\
\text { Expansion }\end{array}$ & $\begin{aligned}-0.20 & \pm 0.07 \\
+1.28 & \pm 0.40 \\
-3.6 & \pm 1.0 \\
-0.7 & \pm 0.2\end{aligned}$ & $\begin{aligned} 500 & \pm 50 \\
22 & \pm 2 \\
28 & \pm 3 \\
2 & \pm 0.2\end{aligned}$ & $\begin{aligned}-100 & \pm 36 \\
+28 & \pm 9 \\
-100 & \pm 30 \\
-1.8 & \pm 1.0\end{aligned}$ \\
\hline \multicolumn{4}{|c|}{ Sodium Expansion } \\
\hline $\begin{array}{l}\text { Test Zone } \\
\text { Filter Zone } \\
\text { Driver } \\
\text { Reflector }\end{array}$ & $\begin{aligned}+0.9 & \pm 0.3 \\
-0.3 & \pm 0.1 \\
-1.9 & \pm 0.4 \\
-8 & \pm 1.6\end{aligned}$ & $\begin{array}{l}2 \pm 1 \\
2 \pm 1 \\
2 \pm 1 \\
2 \pm 1\end{array}$ & $\begin{aligned}+1.8 & \pm 1.0 \\
-0.6 & \pm 0.1 \\
-3.8 & \pm 2.0 \\
-16 & \pm 8.6\end{aligned}$ \\
\hline
\end{tabular}

Total $-232 \pm 50 \times 10^{-6}$

Test zone Doppler reactivity change at $750^{\circ} \mathrm{K}-410 \times 10^{-6}$

systems having a variety of spectra, including systems studied with slightly different cross-section sets. On the average, the errors were judged to be about $30 \%$. The second column lists the average temperature change which can be expected in varying the power level from $5 \mathrm{MWt}$ to $50 \mathrm{MWt}$. The errors associated with these temperature changes are estimates which at present axe judged to be reasonable.

The expected Doppler reactivity change for a $500^{\circ} \mathrm{C}$ fuel temperature increase in the test zone is $-410 \times 10^{-6}$. The standard deviation then amounts to about $12 \%$. Assuming that a series of determinations on the experimental value of $-642 \times 10^{-6}$ yields an error of $10 \%$, the Doppler coefficient can be determined within $20 \%$.

To establish more firmly the extraneous contributions and associated errors, a consistency check can be made by comparing measured reactivity values with the expected values at various temperatures.

The contribution due to any bowing effect has not as yet been studied. However, since the core is being designed to be as rigid as is possible, the 
effect should be negligible when compared with the fuel-expansion effect in the test zone and driver region.

The estimated errors are by no means final. Efforts toward improvements, such as performing critical experiments to determine sodium coefficients, the fuel expansion effects, and associated errors, will be made in assigning deviations with a good degree of confidence. As mentioned, studies will be continued toward the design of a zoned system where a temperature change greater than $500^{\circ} \mathrm{C}$ can be obtained with fuel pin diameters less than $\frac{1}{2}$ in. $(1.27 \mathrm{~cm})$.

\section{Discussion of Paper Presented by Mr. Persiani}

MR. CHERNICK (Brookhaven):

I am not sure whether this question belongs at this point or after the next paper. It is again fairly general. It reters to both papers. There are two types of experiments that might be done, that I really have not heard much about. It seems to me a fast reactor experiment, to get Doppler coefficients, and temperature coefficients in general, is going to be expensive. One should not try to do marginal experiments in a case like this, but should spend some money, if necessary. There are two things that I have in mind. First the heated rod experiment is expensive, but you can go over a large range of temperatures. Although you have to insulate the rods, etc., you can follow them on the way up and on the way down, and try to get a really good measurement rather than this point-type measurement in which you may heateverything up. The usual difficulties will come in trying to interpret the measurement later. Perhaps we can forestall some of these things for once by planning a rather large series of experiments, and perhaps not on the type of reactor you are going to build. You may want some moderators which are quite different, just to test the theory on.

The second question has to do with safety. In the old days no one was afraid to do excursion tests. Apparently the EBR-I meltdown has dampened everybody's enthusiasm for such tests. Nevertheless, it seems to me this is important from the safety viewpoint. Haven't we learned enough about fast reactors so that we can do excursions again? I think it is quite pertinent that we understand what happens in an excursion which is not the same as what happens under a steady rise in temperature, that is, under whatever excursions you can feel you can take in these reactors.

MR. WOLFE (General Electric):

I would like to comment on Mr. Chernick's comment and on the last paper. In reference to the SEFOR program, we intend to build a reactor of 
$20 \mathrm{MWt}$ which will have large fuel rods. We can duplicate the temperature distribution in these large fuel rods in accordance with what we would expect in the fuel rod of a power reactor running at a much higher power density. We intend to build a core which is some $30 \mathrm{in.}$ in diameter, and homogeneous in the sense that it will consist of nothing but these fuel rods. The physics of the reactor is so arranged that to within a reasonable approximation we will get about the same spectrum we expect in the large power reactor, and about the same temperature distribution across the core. It should be about the same Doppler effect. Unfortunately, in making this mock up, we will not be able to duplicate the sodium coefficient.

With this reactor we then intend to measure the Doppler coefficient both isothermally and up to temperatures of central melting in the center. We will get the temperature coefficient throughout the core, and with temper ature distributions characteristic of a large reactor. After we thoroughly understand these measurements, we then intend to put the reactor on transients. In response to Mr. Chernick's question, we do intend in the final series of experiments to put the reactor on prompt critical excursions to demonstrate that we understand the kinetics of the reactor and to prove that these reactors axe indeed safe.

I do not think that the SEFOR or FARET programs are in competition with the critical experiment programs. They give different types of information.

MR. STORRER (Euratom):

They are complementary, not mutually exclusive.

MR. WOLEE:

The FARET reactor is going to get some information that we do not get in regard to being able to duplicate the sodium coefficients and get a more exact duplication of the fuel conditions in the zone core. On the other hand, I think our measurements will be clearer to interpret in terms of the Doppler coefficient alone, and we do not expect to get the extraneous temperature factors that seem to be of concern in this paper.

MR. ZALESKI (Euratom):

I have a short comment to make about the statement of Mr. Chernick. I think that effectively the question is which is the best kind of experiment for measuring Doppler effects. I agree with Dr. Storrer that probably critical experiments will be more rich and more flexible than power experiments. I think that nevertheless some power experiments may be uselul also. In respect to what Dr. Wolfe was saying, temperature distribution enters and 
also some difficulty may arise from precision or accuracy of some types of measurements on critical experiments. Nevertheless I think this power experiment is only a complementary experiment to the critical one. It would be nice to verify one or two points by some power experiments. The question may be raised as to what price we would be willing to pay for complementary experiments. Is it necessary to build a special reactor, or even two reactors, for this or is it not possible to make some experiments with existing reactors like EBR-II, Enrico Fermi, or Rapsodie? In this latter case I think you can get 80 or 90 per cent of the information that you could gain on this subject with specially built reactors. In this case, you would pay only for one or two special cores, not a special reactor. Of course, for excursion measurements, you need a special reactor. But I am not convinced that it is quite necessary to do these measurements, for such measurements are not very representative of what will happen with large fast reactors. If you have a special small reactor, the fuel would not be exactly the same, some special features, mechanical features, will be quite different. It is very difficult to extrapolate just straightforwaxdly from experiments with 400-liter or 1000-liter cores to one of 8000 liters. Some theory is required to go from one to the other. You can base this theory on Dopplex coefficient and reactivity coefficient measurements better than just on an excursion to give you a general view of the problem.

MR. PERSIANI (Argonne):

I agree that you don't need to build a very expensive facility to meas ure the Doppler coefficient and its pattern. We hope EARET will be an irradiation facility as well, that is, as far as the lifetime of the facility itself is concerned. I would say that maybe 80 per cent of it would be devoted to studies of fuels. I think we are badly in need of that facility. But initially we expect to make these Doppler coefficient attempts.

MR. CHERNICK:

I would just like to answer Dr. Zaleski. What I called the hot fuel experiment could very well be a zero-power experiment, one at very low power. Secondly, in addition to more theories, we need some verification of our present theories. I think this is more easily done in a controlled. critical experiment or facility of this nature than it can be done in the large reactor.

MR. WOLFE:

I would like to take up the second comment that Dr. Zaleski made. We would be willing to spend money strictly for a reactor to put on an excursion and get the 20 per cent verification that Dr. Zaleski talked about. 
I think he is right that we can get 80 per cent of the information from static measurements. And as for the $20 \%$, if everything is right, when you are all done, you say, "You see, I told you so, it really was not worth doing." On the other hand, that 20 per cent, that is the reason for doing the other 80 per cent of the reactor. The reactor is designed to do other things afterward, but if we do a three-year program to verify the Doppler, and put it on these excursions, and show the Doppler will indeed terminate what would be a large energy release excursion, we will think that the money is well spent. We do not encourage people to build 5 or 6 or 10 of them. I do point out that in the water reactor field there have been a number of these built, and as I under. stand it they are still being built. They still haven't milked all of the infor mation that they think they ought to have from them. 


\title{
EXPERIMENTAL RESULTS FOR U ${ }^{238}$ DOPPLER MEASUREMENTS
} IN FAST REACTOR SPECTRA

\author{
G. J. Fischer, H. H. Hummel, \\ J. R. Folkrod, and D. A. Meneley \\ Argonne National Laboratory \\ Argonne, Illinois \\ (Paper presented by Mr. Fischer)
}

\begin{abstract}
Doppler reactivity measurements have been made for uranium-238 heating in two-zoned core loadings of the ZPR-III fast critical as sembly. The central zone of the first loading, in which the measurements were made, had the composition of a 5,000-liter uranium monocarbide fast power breeder. The second central-zoneloading differed from the first by replacement of $40 \%$ of the sodium with graphite. This change gave an increased Doppler reactivity effect and a second point for theoretical comparison.
\end{abstract}

The design of the Doppler element and of the core loading, both of which were planned to minimize non-Doppler reactivity effects, is described. Supplementary experiments which show that non-Doppler reactivity effects must represent at most a small part of the observed reactivity change are discussed. An experiment which tested the importance of resonance depression of the neutron flux incident upon the element, due to adjacent $U^{238}$ in the central-zone loading, is described and results are given.

Calculational comparisons with these experimental results using recently developed ANL cross sections are reported. They show close agreement with the first experiment and poorer agreement with the softer spectrum case.

Introduction

Zoned-loading techniques were employed with the ZPR-III reactor to produce two soft-spectrum central-zone loadings in which measurements of the $U^{238}$ Doppler effect were made. The central zone of the first assembly, ZPR-III Assembly 43, had the composition of a 5,000-1iter, uranium monocarbide fast power breeder, with a sodium volume fraction of $48 \%$ and with stainless steel clad and structure. 
The calculated real and adjoint fluxes at the center of the central zone matched those of the actual large reactor to within a few percent for all energy intervals, and the quality of this spectral match remained good. within a few percent for approximately $18 \mathrm{~cm}$ of the $24-\mathrm{cm}$ radius of the zone. The virtually flat radial distribution helped to minimize the importance of any uncertainties in radial position, while the $86-\mathrm{cm}$ height of this cylindrical system produced axial flux distributions which were flat to within $5 \%$ over the length of the Doppler elements which were used in the work.

The central zone of the second assembly, $43 \mathrm{~A}$, was created by replacing $40 \%$ of the sodium with plates of graphite. This second assembly, which had an increased Doppler reactivity response because of the larger fraction of neutrons in the few-kilovolt neutron-energy region, provided a second experimental Doppler measurement point for theoretical analysis. The compositions of the se two assemblies are given in Table $I$.

Table I

COMPOSITION OF ASSEMBLIES $43 \& 43 \mathrm{~A}$, ATOMS $/ \mathrm{cm}^{3}\left(\mathrm{x} 10^{-24}\right)$

\begin{tabular}{|c|c|c|c|}
\hline & & Assy 43 & Assy $43 \mathrm{~A}$ \\
\hline \multirow[t]{2}{*}{ Central Zone } & $U^{235}$ & 0.00114 & 0.00114 \\
\hline & $\mathrm{U}^{238}$ & 0.00732 & 0.00732 \\
\hline $\mathrm{R}=24.4 \mathrm{~cm}$ & Carbon & 0.00833 & 0.02499 \\
\hline \multirow[t]{3}{*}{$H=86.4 \mathrm{~cm}$} & Sodium & 0.01061 & 0.00636 \\
\hline & Stainless & & \\
\hline & Steel & 0.01657 & 0.01413 \\
\hline Filter & $U^{238}$ & 0.02172 & 0.01448 \\
\hline $\mathrm{R}=31.4 \mathrm{~cm}$ & Carbon & 0.02916 & 0.04166 \\
\hline Driver & $U^{235}$ & 0.00467 & 0.00467 \\
\hline $\mathrm{R}=45.5 \mathrm{~cm}$ & $U^{238}$ & 0.00032 & 0.00032 \\
\hline$=44.7 \mathrm{~cm}(43 \mathrm{~A})$ & Carbon & 0.03333 & 0.03333 \\
\hline \multirow[t]{2}{*}{$\mathrm{H}=86.4 \mathrm{~cm}$} & Stainless & & \\
\hline & Steel & 0.03325 & 0.03325 \\
\hline
\end{tabular}

The geometrical arrangement of these experiments is shown schematically in Figs. 1 and 2. The physical locations of the two Doppler elements during an experiment can be seen in Fig. 1. The ele ments were located in matrix channels above and below the reactor centerline. The location of the element when in the "out" position was chosen to be so far out that the reactivity importance in that position was negligible, particularly with respect to whether it was hot or cold. Location of the element was indicated at the reactor console both by 
selsyn dial indicators, which could read to a fraction of a millimeter, and by signal lights operating from microswitches. Tests of reproducibility of location of the "in" position demonstrated that this position could be repeated to within $0.025 \mathrm{~mm}$ with no dificulty. A similar precision was as sumed for the "out" position location.

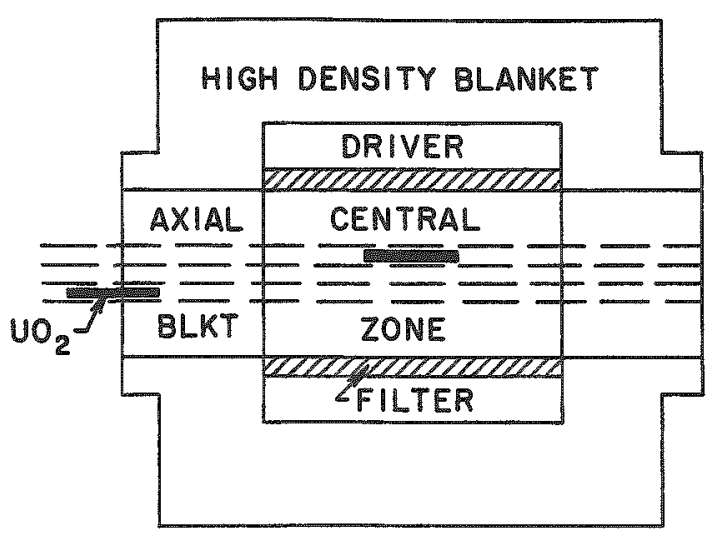

Fig. 1. Assembly 43 of ZPR-III

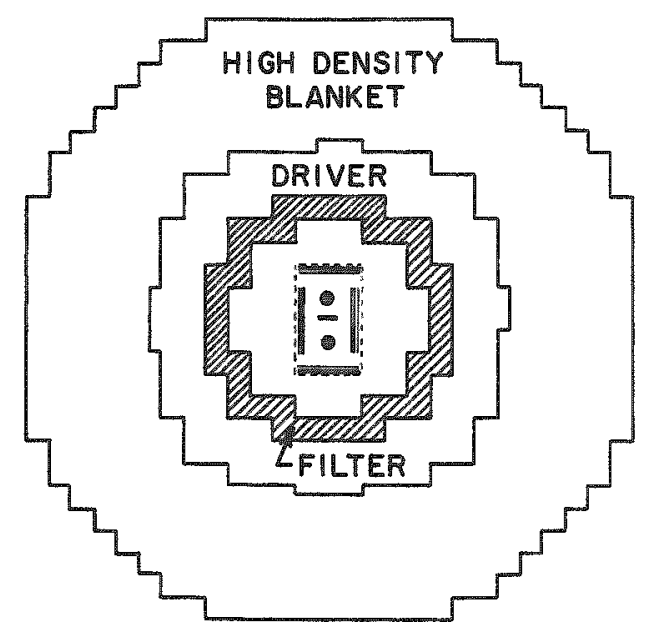

Fig. 2. Assembly 43 of ZPR-III

The procedure of the experiment was to exchange repeatedly the position of the two elements while maintaining the power level of the reactor essentially constant at about $10 \mathrm{~W}$ and to measure the reactivity worth of each exchange at the core center until a fit of the data obtained gave a confidence of about $5 \times 10^{-8} \mathrm{\Delta k} / \mathrm{k}$ for the measurement. The roles of hot element in the hot-vs-cold test were exchanged, and the cold-vs-cold measurements were repeated in order to improve confidence is the measurements. Figure 3 indicates the nature of the data for one of the runs. The ordinates for the two runs have been moved relative to each other a number

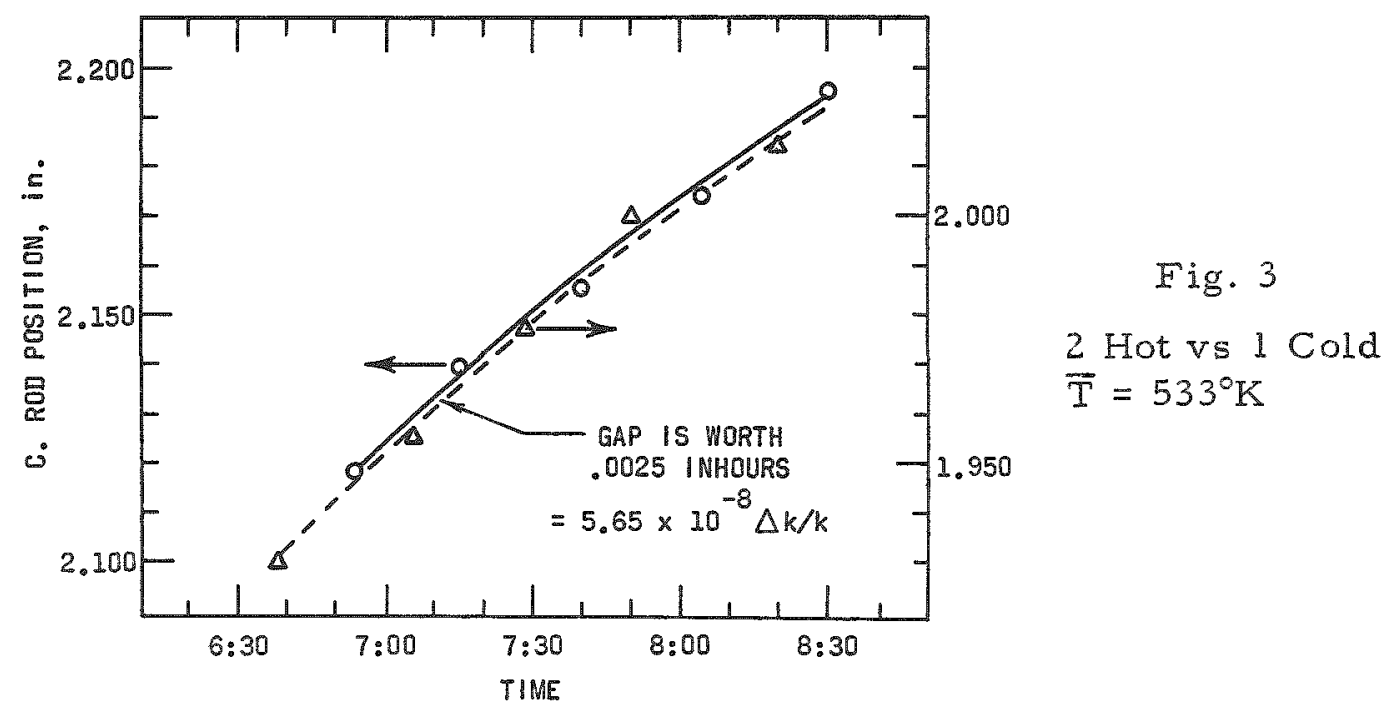


FISCHER et al.

of scale divisions in order to demonstrate the fit of the data points to the two curves which have the same curvature, representing the slow drift of the reactor during the course of the measurement.

Because it was very important that the reactivity changes not be masked by other undesirable effects, such as those due to expansion and heating of the reactor, considerable pains were taken in the design of the Doppler element, of the reactor loading, and in the planning of supplementary experiments to demonstrate that the reactivity changes observed were truly due to the Doppler effect. These features of the experiment will be described throughout the paper.

\section{Doppler Element}

The design of the Doppler element which was used for these experiments is shown in Fig. 4. The uranium contained within the inner heater tube was in the form of $\mathrm{UO}_{2}$ pellets of $70 \%$ theoretical density, sintered with a light bond of sodium metasilicate. Each of the eleven pellets of an element was $3.7 \mathrm{~cm}$ in diameter and $2.5 \mathrm{~cm}$ high. Thermocouples located $2 \mathrm{~cm}$ from each end and at the center continuously measured the temperature along the center line of the cylinder defined by the $\mathrm{UO}_{2}$ pellets during the experiments. The use of an external heater had the desirable consequence that the equilibrium temperature was constant across the diameter of the element. A vacuum between the heated element and the outer shell, plus a highly polished inner surface of the outer shell, made it possible to maintain a temperature of $800^{\circ} \mathrm{K}$ throughout the $\mathrm{UO}_{2}$ with less than a $100-W$ heat loss. A stream of air across the outer shell minimized heat exchange to the reactor matrix.

An important feature of the element design was that the heater cylinder which surrounded the $\mathrm{UO}_{2}$ pellets was free to expand axially over the pellets because of the bellows at one end. Since the outer stainless steel shell of the Doppler element remained almost at room temperature, this shell and the internal porcelain supports acted to preserve the cold dimension of the $\mathrm{UO}_{2}$ pellets. The intentional sintering of the pellets to $70 \%$ density provided a means of elastic intermotion of the $\mathrm{U}(238) \mathrm{O}_{2}$ granules, with only a relatively small thrust on the porcelain supports. Heat was transferred from the heater tube to the $\mathrm{UO}_{2}$ by argon gas which filled the inner chamber at low pressure when at room temperature. Repeated bench-test heat cycling, accompanied by $X$-ray pictures of the elements when both hot and cold, demonstrated the stability of the axial dimension. X radiographs taken before and after the ZPR-III experiments gave no evidence of physical change during the course of the experiments. 


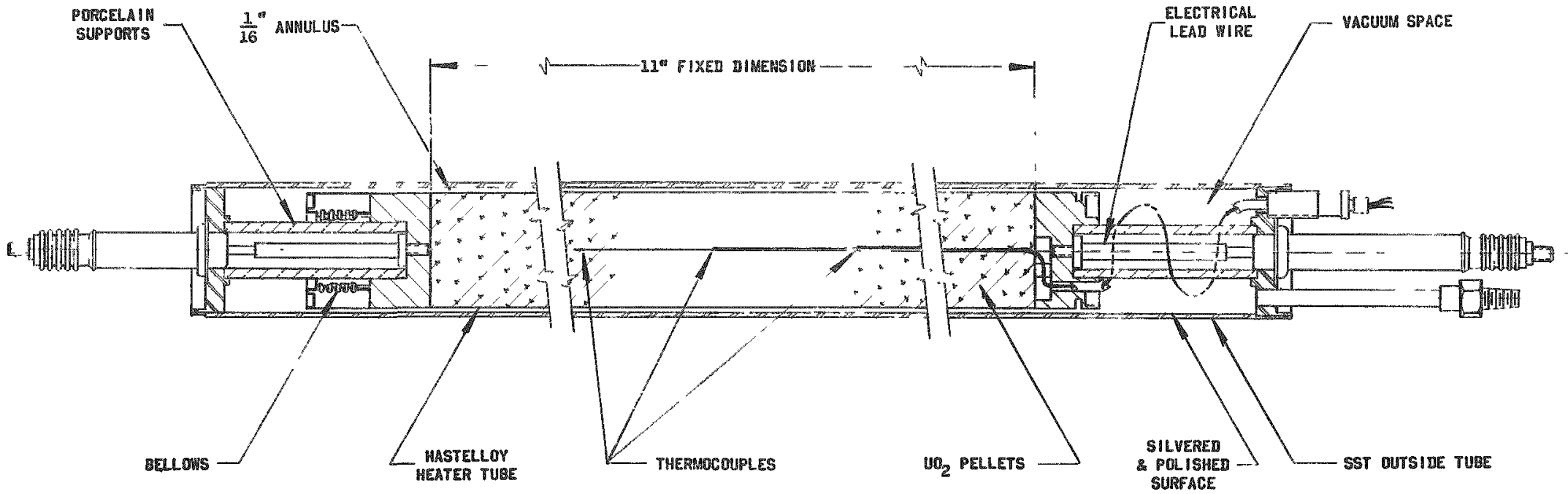

Fig. 4. Doppler Temperature Coefficient Capsule 
FISCHER et al.

Experimental Results of Doppler Reactivity Measurements

The experiments which were performed to check the amount of nonDoppler reactivity seen by these measurements will be described in the next section; the basic data will be given here. The first step in the main experiment consisted of determining the difference of reactivity which occurred when Doppler element No. 1, with its $U(238) \mathrm{O}_{2}$ at $800^{\circ} \mathrm{K}$, replaced Doppler element No. 2, with its $\mathrm{U}(238) \mathrm{O}_{2}$ cold $\left(300^{\circ} \mathrm{K}\right)$, at the axial center of assembly 43, as represented in Fig. 1. The second step consisted of repeating the above measurement with both Doppler elements cold $\left(300^{\circ} \mathrm{K}\right)$. The net reactivity difference of these two measurements represents the reactivity change at the center of the assembly due to heating the $\mathrm{U}(238) \mathrm{O}_{2}$ of element No. 1 from $300^{\circ} \mathrm{K}$ to $800^{\circ} \mathrm{K}$.

The first two lines of Table II give these results and show that a reactivity loss of 0.402 inhour occurred as a result of this temperature change. When the role of hot element was reversed and element No. 2 was heated to $800^{\circ} \mathrm{K}$, a net reactivity loss of 0.398 inhour occurred, corrected for a $1 \%$ mass difference. The agreement of the two results to less than $1 \%$ may be fortuitous and probably should not be taken too seriously, although Table III demonstrates the same kind of agreement for the $U^{238}$ Doppler experiment with the second zoned loading. Until further experiments justify such optimism, a precision of approximately $5 \%$ would seem a more reasonable assignment for the present results.

Table II

$U^{238}$ DOPPLER REACTIVITY COEFFICIENT IN ZPR-III ASSEMBLY 43; 5,000- $\ell$ UC SPECTRUM

1 Hot vs $2 \operatorname{Cold}\left(\overline{\mathrm{T}}=800^{\circ} \mathrm{K}\right)$

1 Cold vs 2 Cold

2 Hot vs $1 \operatorname{Cold}\left(\overline{\mathrm{T}}=800^{\circ} \mathrm{K}\right)$

1 Cold vs 2 Cold

$$
\begin{array}{lcr}
1 \text { Hot vs 2 Cold }\left(\overline{\mathrm{T}}=800^{\circ} \mathrm{K}\right) & \frac{\Delta \rho, \text { Ih }}{-0.251} \\
1 \text { Cold vs 2 Cold } & & \frac{+0.151}{-0.402} \\
& \text { Total } & -0.549 \\
\text { 2 Hot vs 1 Cold }\left(\overline{\mathrm{T}}=800^{\circ} \mathrm{K}\right) & & +0.151 \\
1 \text { Cold vs 2 Cold } & \text { Total } & -0.398 \\
1 \text { Cold vs 2 Cold } & & (+0.138) \\
\text { Expt. }=-0.90 \times 10^{-5} \Delta \mathrm{k} / \mathrm{k}=-0.462 \times 10^{-5} \Delta \mathrm{k} / \mathrm{k} / \mathrm{kg} \mathrm{U} \mathrm{U}^{238}
\end{array}
$$$$
1 \text { Cold vs } 2 \text { Cold }
$$$$
\text { Calc. }=-1.00 \times 10^{-5} \Delta \mathrm{k} / \mathrm{k}(1-\mathrm{D} \text { calculation })
$$ 
Table III

$\mathrm{U}^{238}$ DOPPLER REACTIVITY COEFFICIENT

IN ZPR-III ASSEMBLY 43A;

VERY SOFT SPECTRUM

\begin{tabular}{|c|c|c|}
\hline & & $\Delta \rho$, In \\
\hline \multirow[t]{2}{*}{$\begin{array}{l}1 \text { Cold vs } 2 \text { Cold } \\
1 \text { Hot vs } 2 \text { Cold }\left(\bar{T}=800^{\circ} \mathrm{K}\right)\end{array}$} & & $\begin{array}{l}+0.090 \\
-0.593 \\
\end{array}$ \\
\hline & Total & -0.683 \\
\hline \multirow[t]{2}{*}{$\begin{array}{l}1 \text { Cold vs } 2 \text { Cold } \\
2 \text { Hot vs } 1 \text { Cold }\left(\overline{\mathrm{T}}=800^{\circ} \mathrm{K}\right)\end{array}$} & & $\begin{array}{l}+0.088 \\
-0.765 \\
\end{array}$ \\
\hline & & $\begin{array}{l}-0.677 \\
-0.981\end{array}$ \\
\hline \multirow[t]{2}{*}{$\begin{array}{l}2 \text { Hot vs } 1 \text { Cold }\left(\bar{T}=553^{\circ} \mathrm{K}\right) \\
1 \text { Cold vs } 2 \text { Cold }\end{array}$} & & $\begin{array}{l}-0.437 \\
+0.089\end{array}$ \\
\hline & & -0.348 \\
\hline
\end{tabular}

The second cold-vs-cold result of -0.138 inhour represents a considerably less reliable measurement and has been arbitrarily left out of the present analysis. The average of these reactivity losses was equal to $-0.90 \times 10^{-5} \Delta \mathrm{k} / \mathrm{k}$ for the $1.96 \mathrm{~kg}$ of $\mathrm{U}^{238}$ of the Doppler element. The calculated reactivity change of $-1.00 \times 10^{-5} \Delta \mathrm{k} / \mathrm{k}$ for this experiment was obtained by using a 1 -dimensional diffusion-theory model of the ZPR assembly and employing a recent 22 -group ANL cross-section set. (3) $\mathrm{U}^{238}$ capture cross sections were evaluated as a function of temperature for $\sigma_{\mathrm{p}}=40 \mathrm{~b}$. This value of $\sigma_{\mathrm{p}}$ was appropriate for these experiments.

Table III gives a summary of the increased Doppler reactivity change seen when the experiment was performed in the softer spectrum of assembly $43 \mathrm{~A}$. Again the two separate measurements agree well with each other. In this case, however, the calculated result agrees less satisfactorily with the experiment. It is interesting that the theoretical calculation in one sense predicts more low-energy neutrons than are found experimentally, just the opposite of the problem which has bothered theoreticians in trying to match experimental fission ratios and neutron lifetimes. A more careful study of resonance self-shielding of the Doppler element may change this computed, relatively preliminary result.

A second experiment with assembly $43 \mathrm{~A}$ consisted of reducing the temperature of the hot sample from $800^{\circ} \mathrm{K}$ to $553^{\circ} \mathrm{K}$, giving a net reactivity loss of 0.348 inhour, without a repeat check. 
Besides the problem of separation of non-Doppler reactivity effects from the results just given, which will be discussed below, there are several aspects of this experiment which perhaps should be pointed out. The first is that this is a hot-sample-in-a-cold-reactor experiment.

A paper by Storrer(1) at this conference and a paper by Ozeroff, Khairallah, and Neviere(2) discuss some of the implications of this form of experiment. Secondly, the Doppler element employed in these measurements was large enough to produce a change of several percent in the gross flux in the neutron-energy region of importance to Doppler reactivity changes, so that first order perturbation analysis would be unreliable.

The importance of flux depression at the $U^{238}$ resonances by the $U^{238}$ present in the central-zone makeup materials was estimated by another experiment with assembly 43. Figure 2 rather crudely shows that for the experiments of Tables II and III, the materials of the $2 \times 2$-in. drawers immediately around the Doppler elements were re-bunched so that all of the sodium cans $(3.2 \mathrm{~cm}$ thick for assembly 43 and $1.9 \mathrm{~cm}$ thick for assembly 43A) were adjacent to the Doppler elements, while the $U^{238}$ and other materials of the drawer were kept in their previous relation but moved to the outer edge of the drawers. This geometry provided approximately one mean free path for collision for neutrons of the energy range of interest to Doppler reactivity changes. A measurement to test the importance of resonance depression consisted of repeating the assembly 43 experiment, of Table II, with the bunching arrangement of those drawers exactly reversed, to give a "worst case" condition. The results of this experiment are briefly reported in Table IV, indicating approximately a $5 \%$ reactivity difference for the change.

\section{Table IV}

\section{TESTS OF ASSEMBLY 43 RESULTS}

Boron Test

1 Hot vs 2 Cold (Net)

Calc. Residual Doppler

Probable Non-Doppler

Resonance Flux Dep. Test

2 Hot vs 1 Cold (Net)

Prev. Result

$U^{238}$ Expansion (43A)

Dummy at Center (43A)

Element at Center (43A)

Element at Out Position

$$
\begin{aligned}
& -0.077 \mathrm{Ih} \\
& -0.066 \mathrm{Ih} \\
& \sim-0.011 \mathrm{Ih}(\sim 3 \%)
\end{aligned}
$$

-0.382 Ih

-0.400 Ih $(5 \%)$

$+0.0086 \mathrm{Ih} / \mathrm{mm}$

$+0.002 \mathrm{Ih} / \mathrm{mm}$

$+0.003 \mathrm{Ih} / \mathrm{mm}$

$-0.005 \mathrm{Ih} / \mathrm{mm}$ 
A number of experiments were performed in order to evaluate the size and importance of possible spurious non-Doppler reactivity effects occurring within the results reported above. One type of experiment evaluated the significance of uncertainties or errors in location of the position of the Dopplex element during the reactivity measurements. The last three listings of Table IV indicate that errors of $1 \mathrm{~mm}$ for these three types of positional error lead to reactivity changes which are of the order of $1 \%$ of the total reactivity changes obtained with the assembly 43 Doppler studies. Since measurements have shown that positional locations within a fraction of a millimeter were readily reproducible, this source of experimental uncertainty must be quite small.

Although the discussion of the design and construction of the Doppler element used for these experiments indicates that considerable pains were taken to remove as far as possible any axial expansion of the $\mathrm{U}(238) \mathrm{O}_{2}$ of the elements, it remained important to evaluate the significance of this expansion if by some means it could occur. In order to evaluate this importance, one of the Doppler elements was removed and a "clean geometry" experiment was set up in its place. Standard ZPR-III plates of depleted uranium were mounted in a fixed position in one of the halves of the split-halves reactor, to the same depth as that of the Doppler element when located at the center. An equal number of plates was mounted in a mirror position in the other half, but within a thin aluminum tube which passed through the entire axis of the central zone. The thin aluminum tube was then connected to the drive mechanism built for the Doppler elements.

With this kind of very simple geometry it was then possible to say that a carefully measured worth of the displacement of the aluminum tube from its proper position measured only the reactivity effect of this kind of "expansion" of the $\mathrm{U}^{238}$. This experiment with assembly $43 \mathrm{~A}$ for which a total Dopplex reaclivity change was -0.680 inhour, showed that a $1-\mathrm{mm}$ displacement represented a reactivity change of only 0.0086 inhour. Thus, if the design of the Doppler element failed so that the $\mathrm{UO}_{2}$ was perfectly free to expand in the flux distribution of assembly $43 \mathrm{~A}$, and if a crude cquivalence was assumed between the two reactivity worths per $\mathrm{mm}$ of each type of expansion, somewhat more than a $1 \%$ reactivity difference would have occurred per $\mathrm{mm}$ of expansion. The temperature rise of the $\mathrm{UO}_{2}$ elements would have caused a motion of the center of mass of the $\mathrm{UO}_{2}$ somewhat more than $1 \mathrm{~mm}$ if an average temperature coefficient of expansion of $10 \times 10^{-6}$ is assumed.

A rather important test, described as the Boron Test in Table IV, added further to the confidence that the results of Tables II and III were largely of true Doppler origin. The idea of this test was to use boron to 
surround the Doppler element which was to be heated during the course of an experiment; the boron should greatly perturb the flux and importance of neutrons in the energy region of a few kilovolts, which is the major source of the Doppler effect, while leaving largely unperturbed the remainder of the spectrum. Thus, the non-Doppler reactivity effects due to heating the Doppler element would be largely unperturbed while the Doppler reactivity change should be greatly reduced. In particular, a moderate amount of boron in the form of natural boron carbide was used; theoretical calculations which had shown good agreement with experiment on assembly 43 were used with satisfactory confidence to predict the small amount of residual true Doppler reactivity change to be expected.

As Table IV shows, this experiment predicts that non-Doppler reactivity effects amount to approximately $3 \%$ of the reactivity change seen on assembly 43 .

A final comment should be made about the importance of the binding of the $U^{238}$ atoms in a crystalline lattice to the magnitude of the reactivity changes observed. If one accepts the predictions based on the Debye model as given in GEAP -4092 , then use of the favored Debye $\theta=600^{\circ} \mathrm{K}$ predicts a $2.7 \%$ correction is to be made, while use of the suggested upper limit choice of $\theta=900^{\circ} \mathrm{K}$ predicts a $4.7 \%$ correction to the results of the experiments. It is important for those planning future experiments to note that these crystal effects are important only over the lower part of the temperature interval used here, so that they could have been largely removed by the simple expedient of raising the temperature of the "cold" element by approximately $200^{\circ}$.

\section{Acknowledgement}

John Long and Joe Gasidlo of the ZPR-III staff are to be sincerely thanked, both for watching over the ZPR-III reactor during the series of measurements of this paper, and for contributing a number of valuable suggestions of experimental procedure. Fred Thalgott was exceptionally helpful and encouraging. Joe Handwerk and Dave White developed and produced the low density $\mathrm{U}(238) \mathrm{O}_{2}$ which was used in the Doppler elements.

Among the technicians who assisted in this program, W. Windmiller, L. Hansen, and Thayne Huntsman were exceptionally helpful as well as highly skilled in the operation of ZPR-III. John Harmon deserves great thanks for his contributions to the assembling and testing of the Doppler elements. 
1. F. Storrer et al., Measurements of the Doppler Coefficient in Large Fast Power Reactors Using a Fast Critical Assembly and an Experimental Fast Reactor, Part I. Theoretical Aspects of the Measurement of the Doppler Coefficient in a Critical Facility. This conference.

2. W. Ozeroff, M. Khairallah, and M. Neviere, Symposium on Advances in Reactor Theory, Karlsruhe, April, 1963, Paper III-2.

3. H. H. Hummel and A. L. Rago, Effect of Parameter Variation in Doppler Effect Calculations. This conference.

Discussion of Paper Presented by Mr. Fischer

There were no questions or comments. 
896 


\title{
FAST SPECTRUM DOPPLER MEASUREMENTS
}

OF $\mathrm{Th}^{232}, \mathrm{U}^{238}$, AND U $\mathrm{U}^{235}$ METALS

\author{
T. H. Springer and S. G. Carpenter \\ Atomics International \\ Canoga Park, California.
}

(Paper presented by Mr. Springer)

Several attempts $(1,2)$ have been made in the past few years to meas ure the Doppler coefficient of various materials in fast neutron systems. These investigations have resulted in the establishment of upper-limit values only. By using an improved oscillator technique (3) wherein reactivity sensitivities of the order of $2 \times 10^{-8} \Delta \mathrm{k} / \mathrm{k}$ are attainable with reasonable ease, it has been possible to extend measurements down to the point at which effects, presumably due to Doppler broadening of the resonances, can be adequately evaluated.

Measurements have been made with the Advanced Epithermal Thorium Reactor (AETR) critical assembly, $(4,5)$ a split-table type of machine in which multiregion cores are constructed. The spectrum in the spherical inner region, where core parameters are studied, can be made typical of that which would exist in a fast power reactor by the addition of appropriate amounts of moderators, structural materials, and aluminum (to simulate sodium), in addition to fuel and fertile material. This test region is, in turn, surrounded by concentric spherical zones which consist (proceeding in an outward direction) of (1) a buffer region which effectively extends the size of the test region when the latter is $\mathrm{U}^{233}$ fueled, (2) a thorium decoupler which prevents thermal neutrons from leaking into the inner sphere, (3) a polyethylene-moderated $\mathrm{U}^{235}$-fueled thermal driver, and (4) a polyethylene reflector. The thermal driver zone, where $\sim 84 \%$ of the fissions occur, affords the assembly the safety and operating advantages of a thermal reactor and a small critical mass. The effective diameter of the test region, including the buffer zone (when it is employed), is generally on the order of $24 \mathrm{in.}$

Initial Doppler effect measurements $(6)$ were made with a cylindrical slug of thorium metal ( $0.464 \mathrm{in}$. OD by $4 \mathrm{in}$. long) in core 9 , which consisted of a $\mathrm{U}^{233}$-fueled test region. The maximum slug temperature attained was $500^{\circ} \mathrm{C}$. Recently, these measurements have been extended to higher temperatures $\left(935^{\circ} \mathrm{C}\right.$ for thorium) and to other materials, namely, $\mathrm{U}^{238}, \mathrm{U}^{235}$, and iron. The latter results have been obtained with core 10 , which closely approximates core 9 except that $U^{235}$ fuel is used in place of $U^{233}$.

The neutron spectrum, spatially flattened in the region in which the measurements were made, has the calculated shape shown in Figs. 1 and 2 
for cores 9 and 10 , respectively. The median fission energy $E_{m}$, defined so that $\int_{0}^{E_{m}} \Sigma_{f}(E) \phi(E) d E=\int_{E_{m}}^{\infty} \Sigma_{f}(E) \phi(E) d E$, has taken on the values 190 and $195 \mathrm{keV}$, respectively, at the core center.

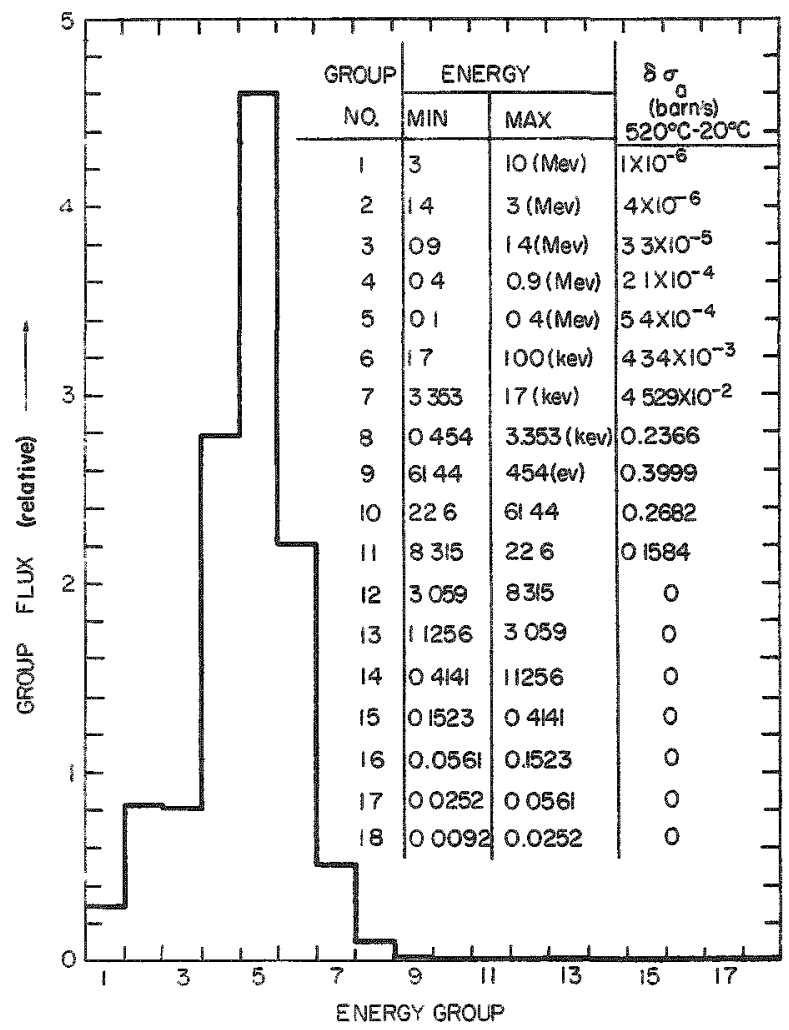

Fig. 1. Group Flux Distribution, Core 9

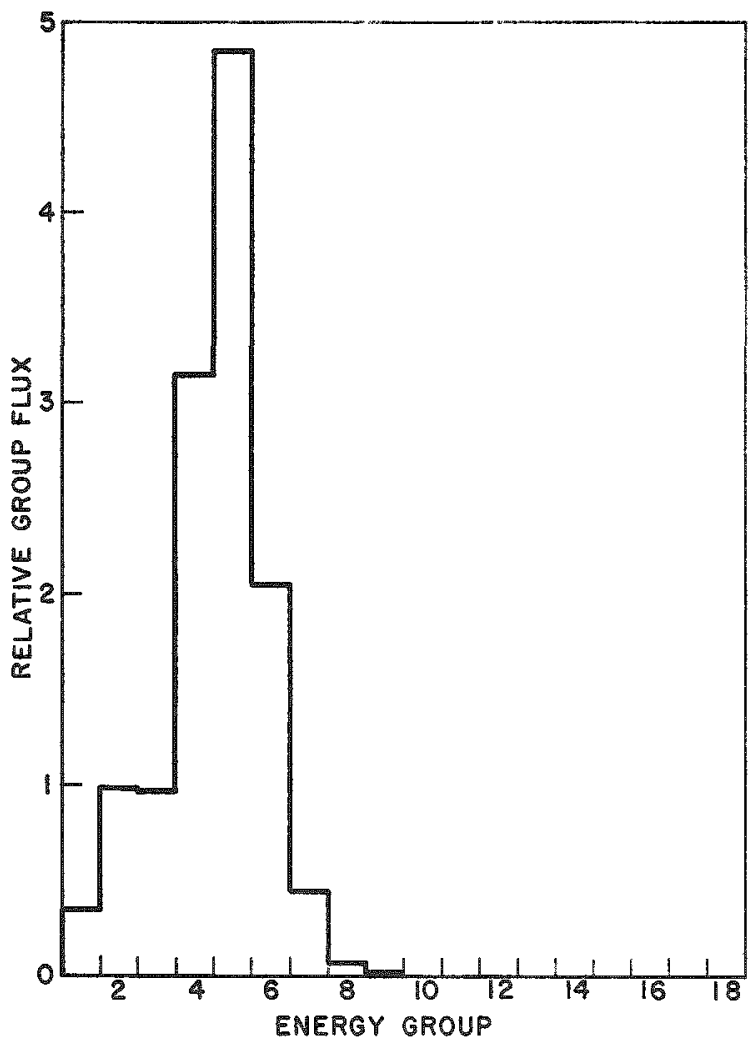

Fig. 2 Calculated Neutron Flux in Core 10

The ARES computer code, written by Fillmore(7) has been used to carry out, concurrently with the experimental work, an analytical study of the Doppler effect for the particular physical conditions under consideration. The calculation is based upon the works of Dresner, Chernick, Vernon, Goldstein, Adler, Nordheim, and Cohen. The code calculates resonance integrals in the resolved region by a nonlinear combination of the narrow-resonance-infinite-mass (NRIM) and the narrow-resonance (NR) approximations without interference from potential scattering. In the unresolved region, the resonance integrals are obtained from the NR approximation only, with the use of average resonance parameters. The $1 / \mathrm{v}$ contributions are also evaluated and added to the above values. The resonance integrals are printed out by preselected energy groups, of which as many as 50 may be specified. The group cross sections are obtained by dividing the integral by the appropriate lethargy interval. For a given value of $\mathrm{S} / \mathrm{M}$, the calculation is first done for $0^{\circ} \mathrm{K}$; then Doppler broadening of both resolved and unresolved resonances is applied (corresponding to some other specified temperature) by means of tabulated correction 
factors. The changes $\delta \sigma_{a}$ in the group cross sections between two specified temperatures (see, for example, the table shown in Fig. 1) are combined with fluxes and adjoints obtained from a standard-type diffusion code ("AIM-6")(8) in a perturbation calculation which yields the expected reactivity change.

For core 9 , for example, it turns out that, although the $\delta \sigma_{a}$ values are largest in the energy groups containing resolved resonances, the magnitude of the group flux there is small; consequently it is particularly noteworthy that more than $50 \%$ of the Doppler effect in this spectrum comes from the unresolved region $(\sim 37 \%$ from the energy group extending from $3.353 \mathrm{keV}$ to $17 \mathrm{keV}, \sim 13 \%$ from the group extending from $17 \mathrm{keV}$ to $100 \mathrm{keV}$, and $\sim 4 \%$ from that extending from $100 \mathrm{keV}$ to $400 \mathrm{keV}$ ). A similax distribution for core 10 is to be expected.

Doppler-broadened group cross sections for $20^{\circ} \mathrm{C}$ (the reference temperature at which experimental values are measured) have been used to calculate, on an absolute basis, the reactivity worth of the slugs relative to a void. The resulting values (shown in Table I) compare favorably with the measured values, thus providing additional confidence in the experimental determinations.

Table I

\begin{tabular}{c|c|c|c|c}
\hline Material & $\begin{array}{c}\text { Core } \\
\text { No. }\end{array}$ & $\begin{array}{c}\rho \text { (calculated), } \\
\phi\end{array}$ & $\begin{array}{c}\rho(\text { measured }), \\
\phi\end{array}$ & $\frac{1}{\rho} \frac{\mathrm{d} \rho}{\mathrm{dt}},{ }^{\circ} \mathrm{C}^{-1}$ \\
\hline $\mathrm{Th}$ & 9 & -0.148 & -0.132 & $-7.18 \times 10^{-5}$ \\
$\mathrm{Th}$ & 10 & -0.133 & -0.114 & $-5.55 \times 10^{-5}$ \\
$\mathrm{U}^{235}$ & 10 & - & +1.569 & $-5.58 \times 10^{-6}$ \\
$\mathrm{U}^{238}$ & 10 & -0.0749 & -0.0873 & $-1.10 \times 10^{-4}$ \\
\hline
\end{tabular}

The experimental method consists of oscillating in and out of the core center in a square-wave fashion two identical samples, one of which can be uniformly heated, and the other maintained at or near a constant temperature of $20^{\circ} \mathrm{C}$. The oscillator techniques and data-analysis procedures were previously developed for material reactivity determination in the AETR. (3) The methods used to heat the sample and mitigate heat losses to the reactor core are similar to those used by A. R. Baker and T.A. J. Jaques. (2) In the present experiment a slug of the material of interest is inserted into a heater consisting of a tube wound with nichrome wire, the tube being a machinable ceramic or, in some cases, anodized aluminum. The heater is, in turn, encapsulated in a 3/4-in.-OD by 5-in.long stainless steel tubular container, which is evacuated and sealed in order to provide thermal insulation. Temperature determinations are made by means of stainless steel-sheathed, $\mathrm{MgO}$-insulated, chromel-alumel 
thermocouples which exit from the capsule, along with the heater wires, through hermetic seals. A steady-state temperature of some specified value (presently limited to approximately $1000^{\circ} \mathrm{C}$ for the ceramic-type heater) is attained by continuously supplying electrical power to the heater to balance heat losses resulting primarily from thermal radiation. The experimental procedure is to place two capsules, both containing the same slug material, into separate pockets in a special oscillator bar which can be pneumatically fired on a preset timing cycle in such a way that first one capsule and then, alternately, the other, is at the core center position; thus, temperature-dependent reactivity effects are determined between structurally identical samples. When in position, the capsules can be provided with forced-air cooling over their outer surface in order to minimize core heating.

The values for the measured reactivity changes (i.e., the algebraic difference between the room-temperature reactivity value and those values obtained at some elevated temperature) are given, along with their associated statistical uncertainties, in Figs. 3 and 4 . No corrections to the measurements have been applied. The results shown in Fig. 4 include, among the standard data, reactivity values obtained under conditions in which the slug is surrounded by a thorium blanket averaging about $3 / 8$ in. in thickness. No definite effect from this change was detected, at least within the

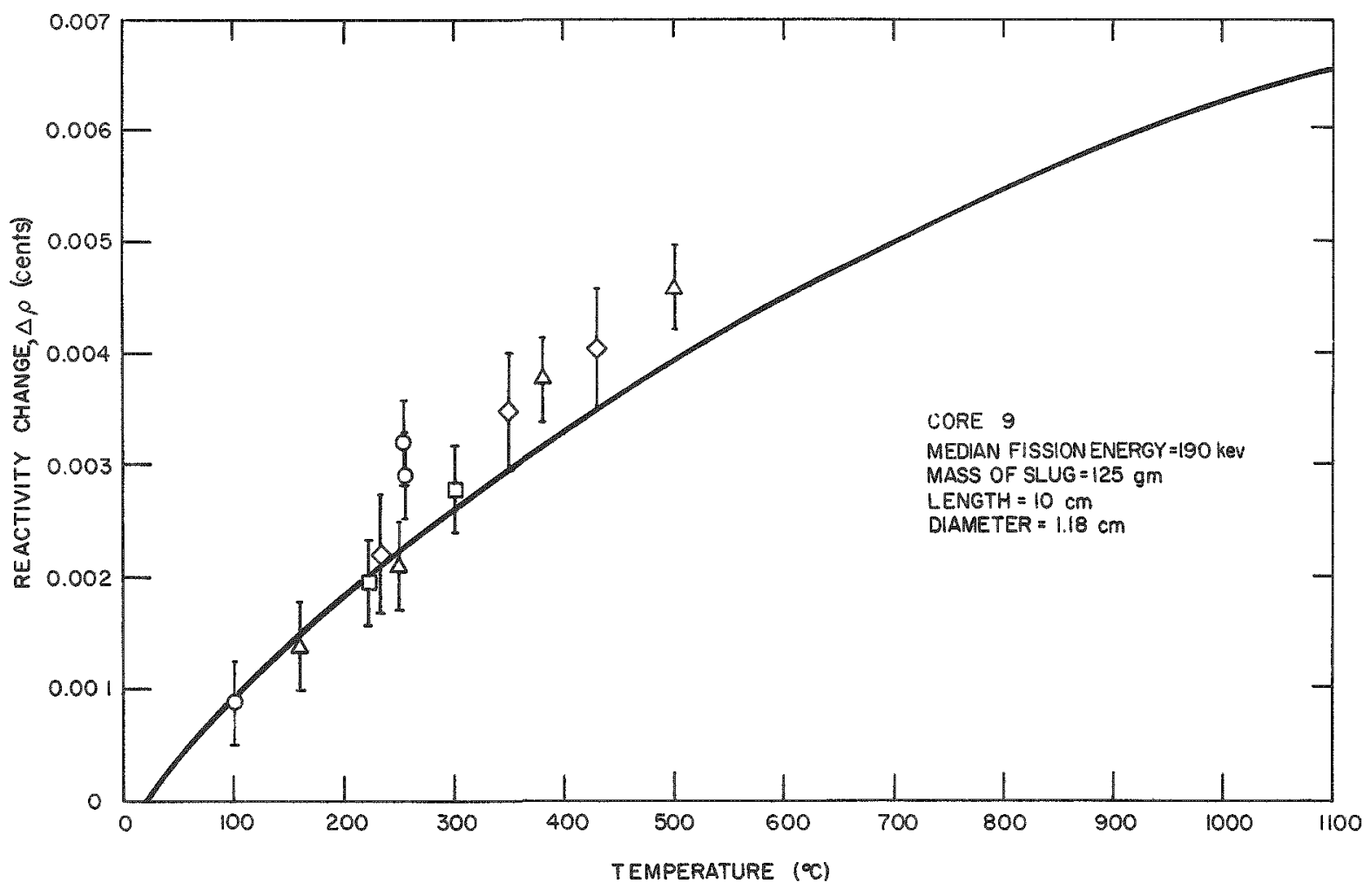

Fig. 3. Doppler Effect in Thorium Metal 


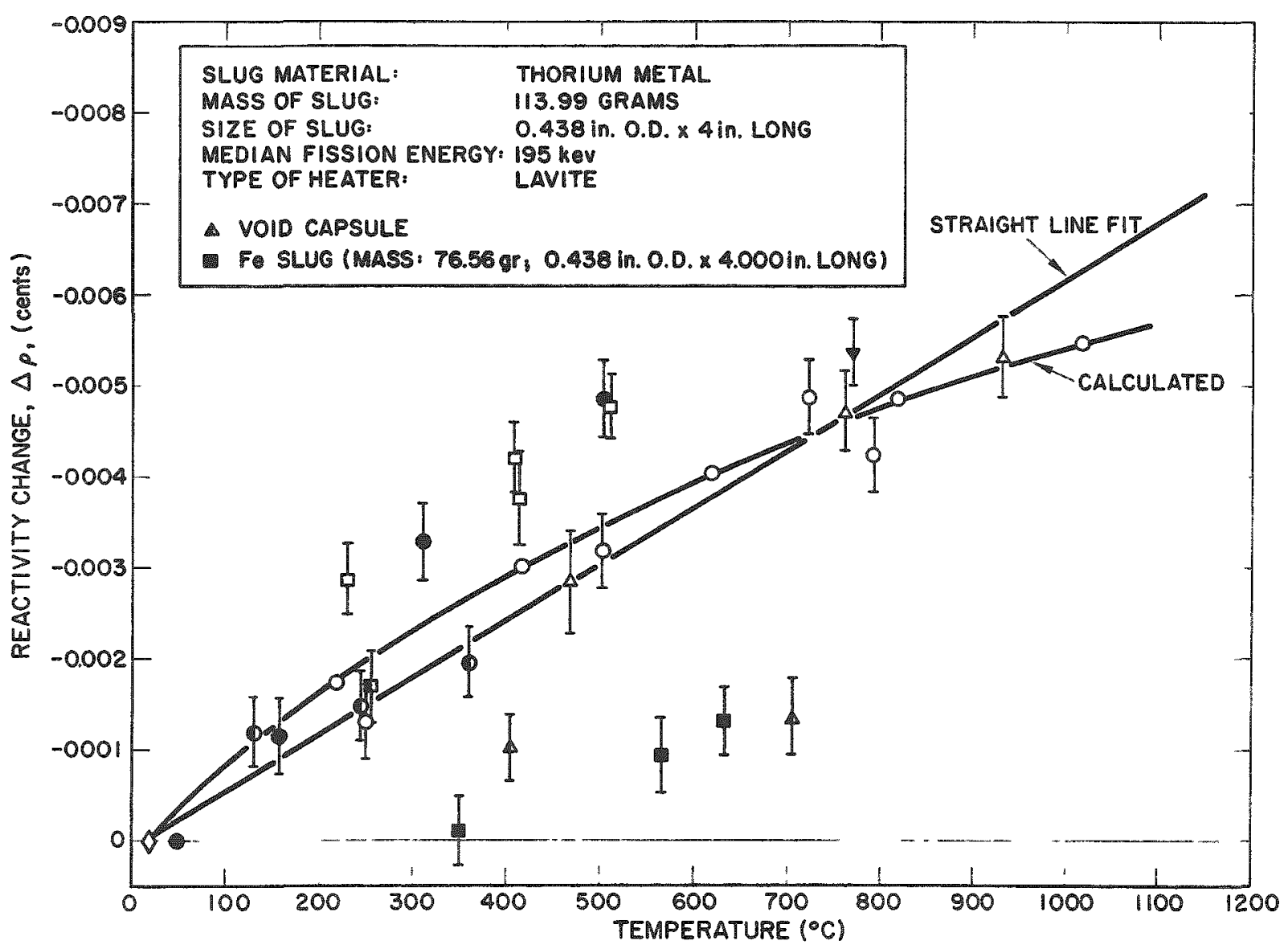

Fig. 4. The Doppler Effect in Thorium, Core 10

experimental uncertainties. The results of the calculation corroborate this conclusion. The fact that two sets of measurements appear to show a different curve may ensue from the method in which the data are presented. Since all the plotted values are obtained by difference determinations, statistical errors in the reference temperature measurements will, perforce, cause all subsequent data to deviate from the normal curve. In the figure a straight line has been fitted by the least-squares method to the lower values only. A weighted average of all the data gives for the quantity $1 / \rho \mathrm{d} \rho / \mathrm{d} t$, where " $\rho$ " is the reactivity and " $t$ " is the temperature, the value $-5.55 \times 10^{-5} /{ }^{\circ} \mathrm{C}$. The corresponding result for core 9 yielded $-7.18 \times 10^{-5} /{ }^{\circ} \mathrm{C}$ for thorium. Also included in Fig. 4 are the reactivity changes obtained when an empty ceramic heater is oscillated first at room temperature and then at various higher temperatures. These results, when viewed in conjunction with the iron slug oscillation, imply that some correction due to capsule heating may be necessary. At the same time, it is concluded that the changes in the scattering properties of the slugs, if they exist, are unimportant. A similar conclusion was obtained previously by oscillating a lead sample in core 9.

Because the results for $U^{238}$ and $U^{235}$ have only very recently been obtained, no graphical representation of the data is available ai this time. 
It is worthwhile pointing out, however, that, although the ARES code predicts a positive Doppler effect for $U^{235}$ for this particular spectrum, negative values of considerable magnitude were actually observed. Based on a straight-line fit for the data, a value for $1 / \rho$ d $\rho / d t$ has been tentatively estimated at $-5.58 \times 10^{-6} /{ }^{\circ} \mathrm{C}$ for this material. This result leads to the prediction of a decrease in reactivity with increasing temperature for a fullscale fast reactor possessing a similar flux and importance spectrum. The advantages of this condition with regard to the safety of certain fast reactors is readily apparent. There is considerable confidence at this time that the values are reasonably accurate, particularly with regard to the sign of the effect. Some theoretical support for this conclusion is available. In the present ARES code the resonance parameters are entered up to about $61 \mathrm{eV}$ for $\mathrm{U}^{235}$. Of four AIM-6 groups which include these resonances, usually three and sometime four give a negative effect. The higher-energy group is strongly negative. For groups above $61 \mathrm{eV}$, a constant value for $\alpha$ is entered $(\sim 0.5)$, and this forces a positive Doppler effect. It is unreasonable to expect that the actual Doppler effect changes abruptly at the point where resonance parameters cease to be available, and therefore some other analytical techniques must be used. Recent data from Rensselaer Polytechnic Institute show continuous structure for $\alpha$ in the region from $8 \mathrm{eV}$ to $2.4 \mathrm{keV}$. This structure, as well as the fact that the ratio $\phi_{\mathrm{f}}^{*} / \phi^{*}$ is less than unity, may produce a negative effect. The Doppler effect in $\mathrm{U}^{238}$ appears to agree well with the calculated values. The quantity $1 / \rho$ $\mathrm{d} \rho / \mathrm{dt}$ is estimated at $-1.10 \times 10^{-4} /{ }^{\circ} \mathrm{C}$ as based on a straight-line fit.

The problem of primary importance has been to determine conclusively whether or not the observed reactivity effects can indeed be attributed primarily to the Doppler broadening of the resonances rather than to other experimental or physical phenomena. That the effect is largely associated with the temperature rise in the sample slug alone has been demonstrated since the reactivity difference between capsules containing all of the normal components except the slug is, within the statistical uncertainty, not a strong function of temperature up to a temperature of $700^{\circ} \mathrm{C}$.

One of the areas of major uncertainty lies in the density unshielding phenomenon. It has been shown by means of oscillating an alloyed slug that this effect in thorium is unimportant, but no experimental data for the uranium slugs are available at this time.

Corrections relating to heat losses to the core and linear motion of the slug within a region where the neutron flux and importance may not be as spatially flat as predicted have thus far proved to be negligible. Further investigations into these and other effects are, however, continuing. 


\section{$\underline{\text { References }}$}

1. W. Y. Kato and D. K. Butler, Nuclear Sci. and Eng., 5, 320-330 (1959).

2. A. R. Baker and T. A. J. Jaques, A Measurement of the Contribution of the Doppler Effect to the Temperature Coefficient of Reactivity in a Fast Reactor, AERE R/M 168 (June 1958).

3. S. G. Carpenter and V. E. Kistler, Trans. Am. Nuclear Soc. 5,68 (1962).

4. H. A. Morewitz et al., Trans. Am. Nuclear Soc., 4, 55 (1961).

5. I. A. Mountford and V. E. Kistler, Trans. Am. Nuclear Soc., 5, 68 (1962).

6. The results are expected to be published in the October 1963 issue of Nucl. Sci. and Eng.

7. F. L. Fillmore, Trans. Am. Nuclear Soc., 5, 57 (1962).

8. H. P. Flatt and D. C. Baller, The AIM-6 Code; NAA Program Description (1961), available upon request.

$\frac{\text { Discussion of Paper }}{\text { Presented by Mr. Springer }}$

MR. HUMMEL:

Are there any questions?

MR. LONG (Argonne):

A comment. Your $U^{235}$ negative result is not only of great interest to reactor people, but is perhaps even of more interest to people involved in critical assembly work, where the fuel is heated possibly separately. I hope that your measurements and analyses can be extended to include plutonium also.

MR. SCHMIDT (Karls ruhe):

Have you any explanation for this negative effect including its large size? 
MR. SPRINGER:

This information was obtained very, very recently. If you get a sigma effect for particular groups in the resolved region, we can find a negative value. We suppose that this might extend up on into a little higher region.

MR。SCHMIDT:

It is difficult to understand. Is it perhaps a consequence of a certain interaction effect with $U^{238}$ resonances, and a consequence of the fact that you have approximately 25 or so levels between the $U^{235}$ and $U^{238}$ resonances?

MR. SPRINGER:

Perhaps Dr. Carpenter may be able to answer this better.

MR. CARPENTER (Atomics International):

The resonance structure in alpha at the low energies is very considerable. Recent data from Rensselaer show the value going up to as high as 10 and a large fraction of the time it is above 2 and 3 for this particular core. If the alpha in the resonance is as high as 1.2 , you will calculate a negative Doppler coefficient for approximately two-thirds of the resolved resonances; this is true for this core. In plutonium, and we took but a quick look, it was only true for about one-third of the resonances; for $U^{233}$, almost no resonances. We believe that if this resonance structure continues, and the data show that it does up to approximately $3 \mathrm{keV}$, these groups will continue to give negative values. I might mention there are only two other measurements of the Doppler coefficient of $U^{235}$ that I know of. They were done in the PPA assemblies. One was done at $134 \mathrm{keV}$ (median fission energy) and the other at slightly lower energy. The Doppler coefficients for $U^{235}$ in these cores were also negative. Our core has thorium as the fertile material. There is very little $\mathrm{U}^{238}$ in it.

MR. SCHMIDT:

Perhaps there might be another explanation. If you neglect for the moment that the capture widths and the fission widths have different distributions, where the capture width was essentially a delta type distribution, and the fission width distribution might vary between $\nu=1$ and $\nu=4$ in the degrees of freedom, then, if you neglect these effects, if the average fission width is smaller than the average capture width, then I would at once believe this is right. At least as a framework of one other theory. In other words, if the average capture width is higher than the average fission width in $U^{235}$, in that region where the Doppler coefficient of $\mathrm{U}^{235}$ is most important, then I would believe that one would find a 
negative Doppler coefficient. You know of the present discrepancy in resonance measurements. It gives a difference in the whole region of $20 \%$ in the fission cross section. This naturally makes a difference in the fission widths. It is just this difference of $20 \%$ which can make the average fission widths higher than the average capture widths or can make them smaller. Perhaps if this experiment is right, there would be a decision for that fission cross section measurement which gives you smaller values and, therefore, the smaller fission widths.

MR. HUMMEL:

Do you feel that the fission width can vary drastically over a small energy region?

MR. SCHMIDT:

There is a Livermore report which gives drastically reduced fission widths compared with other reports. 
906 
MR. HUMMEL:

I would like to call on Paul Greebler to summarize the morning session.

MR. GREEBLER: (General Electric):

In this morning's session, considerable emphasis was placed on uncertainties in the Doppler calculations due to uncertainties in data. Mostly this was concerned with uncertainties in the resonance parameters. We inferred that these uncertainties might contribute as much as a \pm 20 per cent uncertainty on the overall Doppler coefficient calculation. Most of this appears to be due to the uncertainty in the strength function for $\mathrm{U}^{238}$.

Now, there are other uncertainties which were not covered this morning, but were discussed at considerable length yesterday afternoon. These are the uncertainties in cross section which relate to the calculation of the neutron spectrum and of the neutron adjoint spectrum, such as the alpha values of the plutonium and the elastic and inelastic transfer cross sections. These are uncertainties which would contribute considerably more than \pm 20 per cent to the uncertainty in the Doppler calculation.

It appears that the problem of resonance overlap is now under very good control. Some fairly sophisticated methods were presented for calculations on both the self-overlap, the overlap of the plutonium resonances on themselves in the region of intermediate overlapping, and also the interisotope overlapping, the $\mathrm{U}^{238}$ and $\mathrm{Pu}{ }^{239}$ overlap. There are several computer programs now under development which will calculate Doppler-broadened cross sections by means of a direct flux averaging using a transport or many-group, multigroup method, as Dr. Codd described. Such computer programs will automatically take the overlap effect into account and will permit the additional complexity of a 3-isotope resonance absorber.

Methods have also been presented which take into account the temperature distributions and their effect on the calculated Doppler coefficient. Under development also are programs which will permit us to evaluate the effect of the neutron importance as a weighting function in calculating the average Doppler-broadened cross section and change of cross section with temperature. We believe now that this effect will be a small one, but we will be able to check this directly very soon.

Most of the investigators who have calculated the overlap effect find that it is a small effect, something of the oxder of 10 per cent of the overall 
Doppler coefficient. However, there is not unanimity on that point, and we have the counteracting results which indicate that the effect may be more nearly a 50 or 60 per cent increment of the Doppler coefficient.

In a similar vein, there are some calculational results at Argonne and $G E$ which indicate that the loss of sodium produces a very sizable reduction in the Doppler coefficient, whereas we have the results from Karlsruhe, reported by Dr. Ott, which indicate a much smaller loss in the Doppler coefficient when sodium is lost. In both of these areas, the overlap and the influence of sodium loss on the Doppler effect, the physical phenomena involved are fairly straightforward, and we should in the near future resolve these questions.

The possible effect of the multi-level formalism on the calculated Doppler coefficient remains an uncertainty. This probably will not affect the Doppler coefficient of a power reactor where most of the Doppler effect is due to the fertile material, because we expect the multi-level effect, if any, will be felt only by the fissile material.

A method was presented which will make it much simpler to evaluate the effect of the multi-level formalism on the Doppler coefficient contributed by the fissile isotope. One problem which remains in using this method is that of obtaining resonance parameter distributions at the higher energies of interest to the fast reactors which are in conformity with the multi-level scheme, rather than resonance parameters which have been fitted to a Breit-Wigner single-level formula.

Finally, we cannot disregard processes which may be important to the Doppler effect, but which we have not yet recognized in our calculations. In this regard, we must turn to the experimentalist to give us guidance as to where such effects may occur. I think the results that we saw in the preceding paper, with the fairly sizable negative temperature coefficient for $\mathrm{U}^{235}$, may be just the sort of thing that starts us thinking as to what processes may be occurring that would have caused this effect. It is most heartening that we do now have Doppler measurements and many more underway, and that at least for the fertile materials we are getting results from these early measurements which are in quite good agreement with the calculations.

MR. HUMMEL:

There is one further note I would like to add to the results of this morning. I noticed that the results from Karlsruhe on the effect of parameter uncertainty seem to be considerably bigger than the ones I calculated for the same parameter uncertainty, so that is one more computational difficulty that we will have to try to clear up, added to several that Paul Greebler has already mentioned. 
Concerning our program at Argonne, I think there are several different facets to what we would like to do experimentally. One thing that would be of immediate interest would be to try to study the rod-size effects, to see whether or not this theory that we are relying on for resonance integral calculations for heterogeneous systems is really good enough for Doppler effect calculations. We are trying to plan some experimental test of this to see, for instance, whether we can go up to a 1 -in. rod, or even larger than that, without running into errors that might invalidate the experiment to some extent. We are also interested in pushing ahead with plutonium as fast as we can. One thing that we can do right away in ZPR-VI is to study $U^{235}$, mixtures of $U^{235}$ and $U^{238}$, and separately heat samples of $U^{235}$ and $U^{238}$, in various degrees of heterogeneity to look for interference effects on the resonances of $U^{235}$. We are anxious to take a look at this $U^{235}$ effect that Springer and Carpenter have brought up. Certainly it is a puzzling thing. I do not see the explanation for it right now. I would like to see more discus sion on this point, particularly the point that Dr. Schmidt raised a little while ago about the fission width maybe being very small over certain energy regions. Our local nuclear expert, Peter Moldauer, maintains there is no reason to suppose the fission width is going to vary very much over a few hundred electron volts, because of the fact that this energy is small compared with compound nucleus energies. Perhaps the Adlers can give us some feeling for this, whether it makes any sense to assume that the fission width can vary sharply over several hundred electron volts, as an explanation for this. Paul Greebler suggested a little while ago that perhaps it might be a phenomenon where the statistics just were not very good, and that you have a few resonances with large values of alpha which were causing a negative effect which is not adequately explained by the statistical model.

I think we would like to press ahead on ZPR-III with the zone-loading measurements with plutonium, as soon as the facility is available for this. We do not have a plutonium element available yet. We could just heat up the 238 in a plutonium-loaded system, and get a $U^{238}$ Doppler effect.

We are also going ahead with fixing up an element, perhaps elements, of several different enrichments of uranium oxide, mixed uranium oxide and plutonium oxide, which we could then heat in ZPR-III. If ZPR-III is not available for this, we can put these elements in ZPR-VI and get some interesting information, even though the low-energy adjoint function and flux would not agree in the $U^{235}$ fuel assembly with what we would have in the plutonium fuel as sembly.

Another area of experimentation that is of interest is varying the spectrum of the adjoint function by adding parasitic capturing materials or moderating materials. These are various fundamental studies which might throw some light on the resonance parameters. 


\section{question?}

Dr. Schmidt, did you have some comment on the fission width

MR. SCHMIDT (Karls ruhe):

I will try to outline qualitatively a third possibility for the negative Doppler coefficient. Of all fissionable nuclei, the fission cross section of $\mathrm{U}^{235}$ shows the strongest asymmetries, that is, the strongest interaction effects between different fission resonances. The most important effect is that interaction effect is not destructive interference, but more constructive interference. The capture resonances, as is well known, show the interaction effect, but as a consequence of the many exit channels in capture, these interaction effects tend to cancel such that the capture cross section between the resonances is very small. The resonances in themselves are symmetric, but there are great differences between these dips in the cross section. Factors may axise as great as 10 or 20 in value. I think this is consistent with what Dr. Carpenter just said, that they observed alpha values at Rensselaer in the resonance region, that are a consequence of this effect. Now, as these resonances are varied by changes of temperature, then it might be possible that the relative increase in the capture is greater than relative increase in the fission. This might explain the effect.

MR. ADLER (University of Illinois):

I would like to concur with Dr. Schmidt. We have found in our calculations this very effect. There is another thing to say about the Doppler broadening qualitatively. Even if the lines are asymmetric, the same thing Dr. Schmidt said holds. The reason for that is the primary contribution to the capture line will be broadened by the psi function by the symmetric part, even if there is a small asymmetric part; whereas in the fission line, the asymmetric part is strong. You would have a Doppler broadening described by the famous line shape function, the asymmetric one, chi, which would give a much less large contribution. For this we have now preliminary numerical results.

MR. STORRER (Euratom):

Thexe may be other possible explanations for the effect observed by Mr. Carpenter for $U^{235}$, also. Dr. Schmidt mentioned that a possible thing that was measured was the interference between the $U^{235}$ and the thorium, which is around it. We should remember though, it is the interference of the fissile material on the fertile material since only the fissile material was heated, and it is just the opposite of the interference effect which was presented by Dr. Codd. So, there is a possible explanation, that there is a sizable interference from the fissile on the fertile material. Maybe another possible effect would be internal self-shielding in the samples, which was mentioned by Dr. Springer himself. When you say that the sample is small 
compared to the mean-free path, it is small in one direction and not in the other direction. That is one point. Number two, if it is small compared to the mean-free path, it certainly is very large compared to some mean-free paths, particularly in the peaks of the resonances.

Then there is something else I would like to say. When you talk about measuring Doppler coefficients. I think one very necessary thing, at least a very useful thing, would be to be able to measure the spectrum in this energy region; and so far below $50 \mathrm{keV}$, I think we have been very unsuccessful. If you want to correlate different experiments, I think it is essential that we are able to measure the spectrum below $50 \mathrm{keV}$, possibly by time-of-flight methods.

One more thing. That is that in Doppler coefficient measurements, one should try to measure also the interference between the sodium void coefficient and the Doppler coefficient, That is also an essential thing, that one should measure with and without sodium.

MR. HUMMEL:

I am afraid you have made a good point about the spectrum measurements. Unfortunately, at present the Doppler effect is the most sensitive low energy spectral indication that we have.

MR. CAMPISE (Atomics International):

I wonder, since Argonne is one of the few organizations that will have both a critical facility and a power reactor, whether an integrated program of measuring first the Doppler in a homogeneous setup, then a heterogeneous setup, and then finally putting power into FARET and measuring it with power transients, is planned?

MR. HUMMEL:

Well, it is certainly intended that the critical experiment measurements and power reactor measurements will be complementary. We feel that an extensive program of critical measurements is necessary in any case.

MR. PATTENDEN (Harwell):

Brooks of Harwell is making measurements of eta as a function of neutron energy, for $U^{235}$. In fact, all the measurements are made, and he is just doing the final analysis of the results now; they should be available in a month's time, I would say. His measurements will go up to a few hundred electron volts, but in the higher energy region, the individual resonances will not be resolved. We are also thinking of a similar type of 
experiment, that is a sort of eta measurement, with varying sample temperature. This in connection with $\mathrm{Pu}^{239}$. 
ADLER, D. B.

University of Illinois

ADLER, F. T.

University of Illinois

AKCASU, Z.

University of Michigan

ALEXANDER, L. G.

Oak Ridge National Laboratory

ALEXANDERSON, E. L.

Power Reactor Development Co.

ALLIO, R. J.

Westinghouse (APD)

AMOROSI, A.

Atomic Power Development

Associates, Inc.

ARNOLD, M. J.

UKAEA (Winfrith)

AXFORD, R. A.

Northwestern University

BAILEY, R. E.

Purdue University

BARRINGER, H. S.

Babcock \& Wilcox Co.

BENEDICT, $M$.

Massachusetts Institute of

Technology

BRADISH, J. P. Marquette University
BRANYAN, C. E. Atomic Power Development

Associates, Inc.

CAMPISE, A. V.

Atomics International

CARMICHAEL, B. M.

Los Alamos Scientific Laboratory

CARPENTER, S. G.

Atomics International

CAUMETTE, P.

EURATOM-CEA (ANL-Idaho)

CHERNICK, J.

Brookhaven National Laboratory

CLEMENT, J.D.

Nuclear Materials and

Equipment Corp.

CODD, J。

UKAEA (Winfrith)

COHEN, K. P.

General Electric Co. (APED)

COLLINS, G. D.

General Electric Co. (APED)

CREAGAN, R。 J.

Westinghouse (APD)

CRITOPH, E.

Atomic Energy of Canada, Ltd.

CROSS, B. McGraw-Hill

* Incomplete 
DAVIDSON, J。K.

Allis-Chalmers Mfg. Co.

DELICATE, W. S.

Babcock \& Wilcox Co.

deSTORDEUR, A. N.

EURATOM (Brussels)

DIETRICH, J.R.

General Nuclear Engineering Co.

DOYLE, T. A.

EURATOM

EDLUND, M. C.

Babcock \& Wilcox Co.

EDWARDS, A. G.

UKAEA (ANL-Idaho)

EGGEN, D. T.

Atomics International

ENGELMANN, P.

Karls ruhe

FERGUSON, A. T. G.

UKAEA (Harwell)

FISCHER, E. A.

Atomic Power Development

Associates, Inc.

ELORA, J.W.

USAEC, Denver

FOSSOUL, E. A.

BelgoNucleaire (Brussels)

FRIEDLAND, A.J.

Atomic Power Development

Associates, Inc.

FURET, J.

CEA, Paris
GARG, S. B.

India Atomic Energy

Commission (APDA)

GOLDMAN, A.J.

United Nuclear Corp.

GRAVES, C. C.

United Nuclear Corp.

GREEBLER, P.

General Electric Co. (APED)

GUERON, J.

EURATOM (Brussels)

HÁFELE, W。

Karlsruhe

HALI, D. B.

Los Alamos Scientific Laboratory

HANNUM, W, H.

Los Alamos Scientific Laboratory

$\mathrm{HECK}, \mathrm{F}, \mathrm{M}$.

Westinghouse (APD)

HETRICK, D. L.

University of Arizona

HOFMANN, P. L,

General Electric Co. (HAPO)

HORST, K. M.

General Electric Co. (APED)

HUEBOTTER, P. R.

Atomic Power Development

Associates, Inc.

HUNGERFORD, H. E.

Purdue University

ISBIN, H. S.

University of Minnesota. 
JACKSON, G. O.

UKAEA (Risley)

JAMRACK, W. P.

UKAEA (Windscale)

JAYE, S.

General Atomic

JENS, W. H. Atomic Power Development Associates, Inc.

KATI, S. L.

India Atomic Energy

Commission (ORNL)

KAZI, A. H.

United Nuclear Corp.

KEHOE, R. B.

UKAEA (Risley)

KLEMA, E. D.

Northwestern University

KLOTZ, C. E.

Allis-Chalmers Mfg. Co.

KUMMERER, K.

Karlsruhe

LANE, J.A.

Oak Ridge National Laboratory

LEARY, J.

Los Alamos Scientific Laboratory

LEITZ, F. J.

General Electric Co. (APED)

LEVINE, M. M.

Brookhaven National Laboratory

LEWELLEN, J. W. Allis-Chalmers Mfg. Co.
LOEWE, W.E.

IIT Research Institute

MacPHERSON, H. G.

Oak Ridge National Laboratory

MacMILLAN, J. H.

Babcuck \& Wilcox Co.

MAKENS, R. F.

USAEC, Idaho Operations Office

MANN, J. E.

UKAEA (Risley)

MCCARTHY, W. J., Jr.

Power Reactor Development Co.

MCNELLY, M. J.

General Electric Co. (APED)

MENKE, J. R.

United Nuclear Corp.

MILEY, G. H.

University of Illinois

MINTON, G. H.

Westinghouse (APD)

MOREWITZ, H. A.

Atomics International

NICHOLSON, R. B.

University of Wisconsin

NIMS, J。B., Jr.

Atomic Power Development

Associates, Inc.

NODERER, L. C. Combustion Engineering, Inc.

NOMURA, T. JAERI, Tokyo 


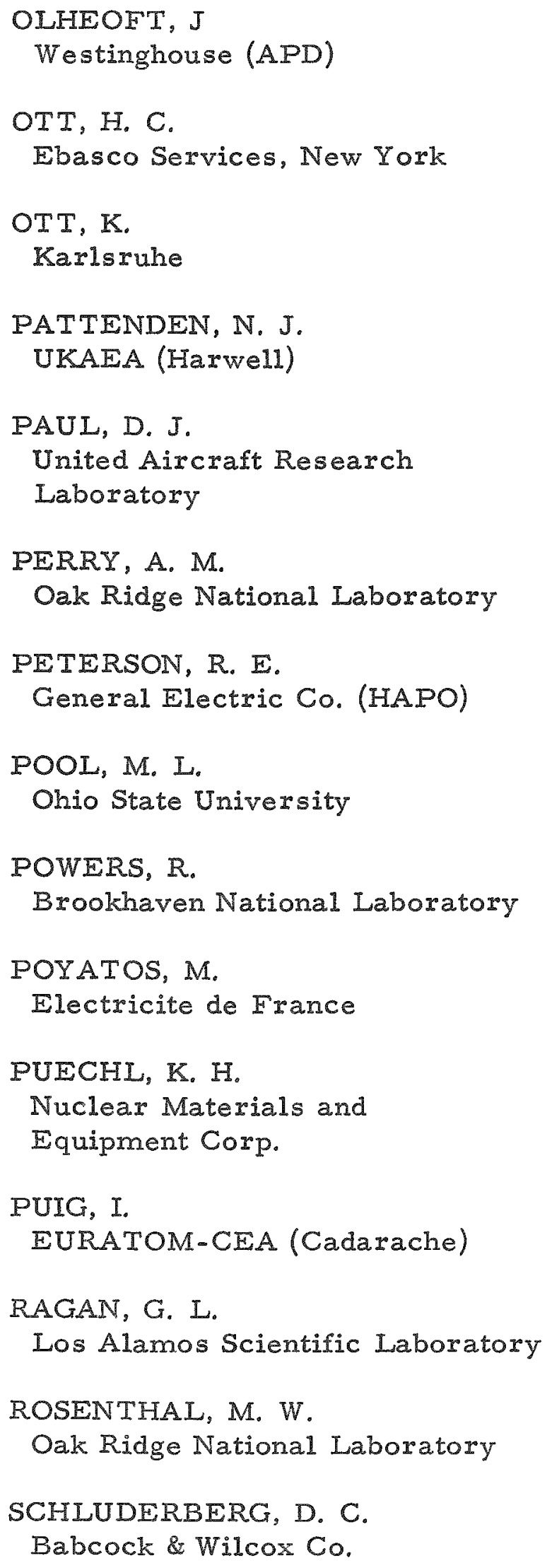

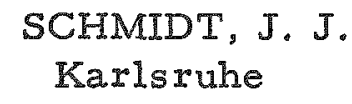

SMITH, R. D. UKAEA (Winfrith)

SOFER, G. A. United Nuclear Corp.

SPRINGER, T. H. Atomics International

STATHOPLOS, A. United Nuclear Corp.

STERN, F.M. Combustion Engineering, Inc.

STOKER, D. J. Atomics International

STORRER, F. P. EURATOM-CEA (Cadarache)

STRASSER, A.A. United Nuclear Corp. 
SUSSKIND, $\mathrm{H}$.

Brookhaven National Laboratory

SZAWLEWICZ, S. A.

USAEC, Washington

TAVERNIER, G. C.

BelgoNucleaire (Brussels)

THIEBERGER, R.

Israel Atomic Energy Commission

THOMPSON, T. J.

Massachusetts Institute of

Technology

TOWER, S. N.

Westinghouse (APD)

ULLMANN, J.W.

Oak Ridge National Laboratory

VISNER, S.

Combustion Engineering, Inc.

VOGELSANG, W. F.

Westinghouse (APD)

WARD, A. G.

Atomic Energy of Canada, Ltd.

WEINBERG, A. M.

Oak Ridge National Laboratory
WELSHANS, L. M.

USAEC, Washington

WENSCH, G.W.

USAEC, Washington

WIESEI, R.

AB Atomenergi, Sweden

WRIGHT, J. $\mathrm{H}$.

Westinghouse (APD)

WOLFE, $B$.

General Electric (APED)

YAMADA, T.

Electrotechnical Laboratory, Japan

ZAGOTTA, W. E.

IIT Research Institute

ZALESKI, C. P. EURATOM-CEA (Cadarache)

ZEBROSKI, E。L.

General Electric (APED)

ZINN, W. H.

Combustion Engineering, Inc. 